\title{
Summary Report of Commercial Reactor Criticality Data for Three Mile Island Unit 1
}

by

Larry B. Wimmer

Prepared for:

U.S. Department of Energy

Yucca Mountain Site Characterization Office

P.O. Box 30307

North Las Vegas, Nevada 89036-0307

Prepared by:

Bechtel SAIC Company, LLC

1180 Town Center Drive

Las Vegas, Nevada 89144

Under Contract Number

DE-AC08-01RW12101 


\section{DISCLAIMER}

This report was prepared as an account of work sponsored by an agency of the United States Government. Neither the United States Government nor any agency thereof, nor any of their employees, nor any of their contractors, subcontractors or their employees, makes any warranty, express or implied, or assumes any legal liability or responsibility for the accuracy, completeness, or any third party's use or the results of such use of any information, apparatus, product, or process disclosed, or represents that its use would not infringe privately owned rights. Reference herein to any specific commercial product, process, or service by trade name, trademark, manufacturer, or otherwise, does not necessarily constitute or imply its endorsement, recommendation, or favoring by the United States Government or any agency thereof or its contractors or subcontractors. The views and opinions of authors expressed herein do not necessarily state or reflect those of the United States Government or any agency thereof. 


\section{Bechtel SAIC Company, LLC.}

\section{Summary Report of Commercial Reactor Criticality Data}

for Three Mile Island Unit 1

TDR-UDC-NU-000004 REV 01

August 2001

Prepared by:

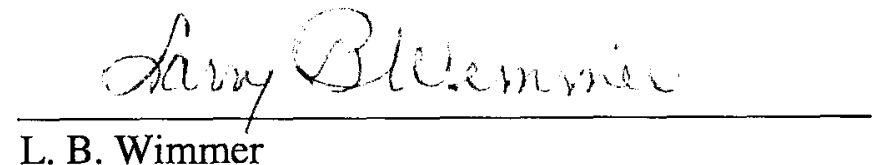

Waste Package Criticality

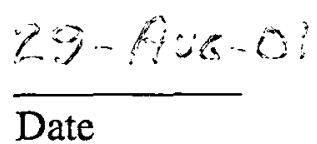

Date

Reviewed by:

Mahendra k. punatar

M. K. Punatar

Technical Checker, Waste Package Criticality

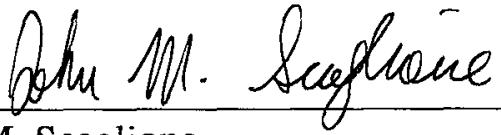

J. M. Scaglione

Compliance Checker, Waste Package Criticality
August 29,200

Date

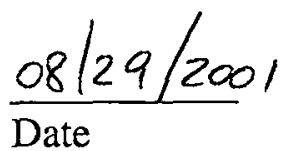

Approved by:

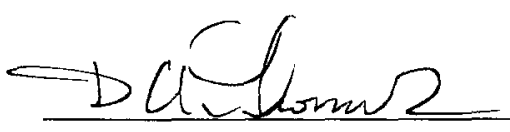

D. A. Thomas

Manager, Waste Package Criticality
$09 / 13 / 2001$

Date 
INTENTIONALLY LEFT BLANK 


\section{CHANGE HISTORY}

Revision Interim

Number Change No.

Date

April 1998

August 2001

\section{Description of Change}

Initial issuance

This document replaces an earlier version of this document (statepoints 59 through 61) of the same title issued under DI B00000000-017175705-00069 Rev. 00. Additional statepoints (62 through 74) are included in this revision. Updated burnup and thermal hydraulic information tables for series " $D$ ", and " $E$ " fuel have been included. 
INTENTIONALLY LEFT BLANK 


\section{ACKNOWLEDGMENT}

The author (preparer) would like to express his thanks to Exelon Nuclear for their assistance with gathering and verifying the information used to model the critical statepoint conditions for the Three Mile Island Unit 1 reactor. The author would also like to thank the AmerGen Energy Company for granting permission to publish this information. 
INTENTIONALLY LEFT BLANK 
ACKNOWLEDGEMENT …............................................................................................... vii

ACRONYMS \& ABBREVIATIONS................................................................................

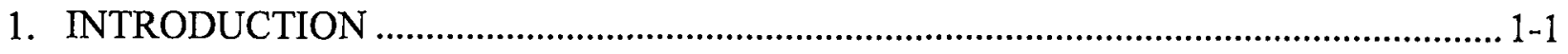

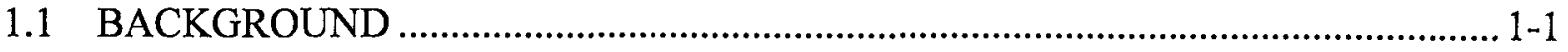

1.2 OBJECTIVE

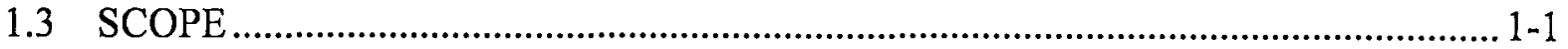

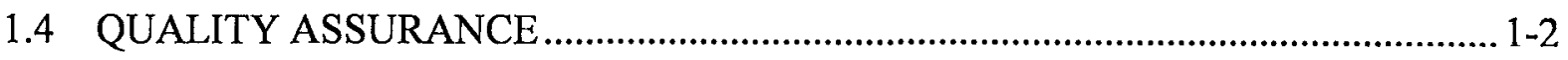

2. REACTOR AND FUEL DESIGN INFORMATION........................................................2-1

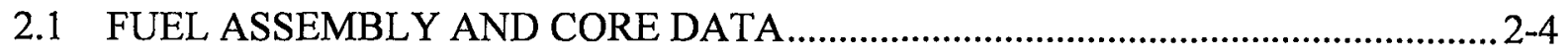

2.2 MARK-B4 AND -B4Z FUEL ASSEMBLY ..................................................... 2-6

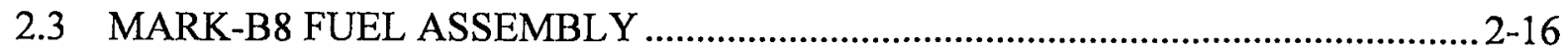

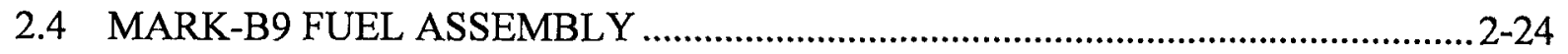

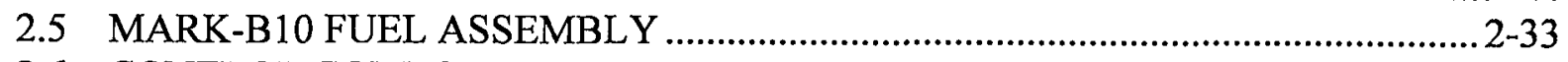

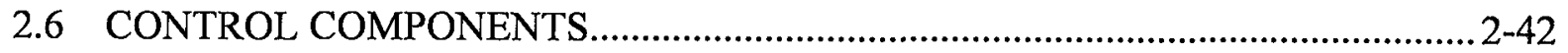

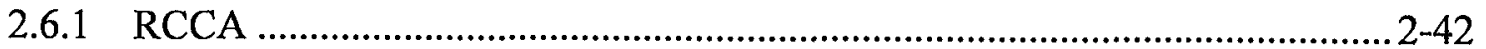

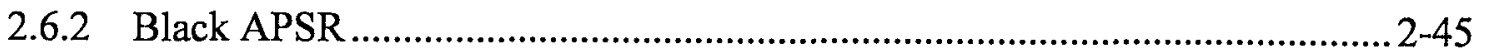

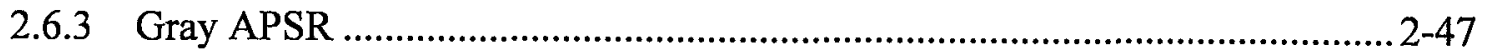

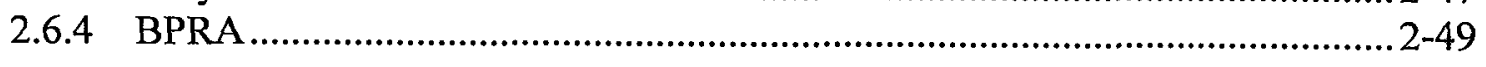

3. FUEL CYCLE DESIGN INFORMATION .................................................................

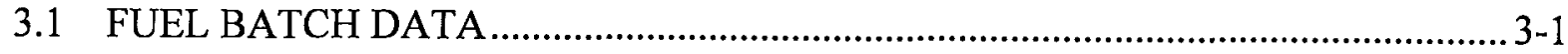

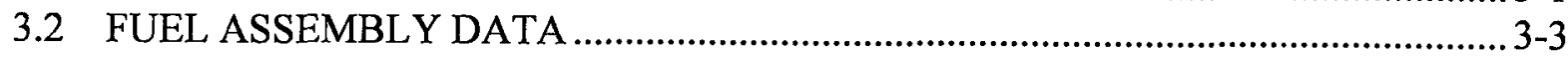

4. CORE OPERATIONS AND STATEPOINT INFORMATION ..........................................

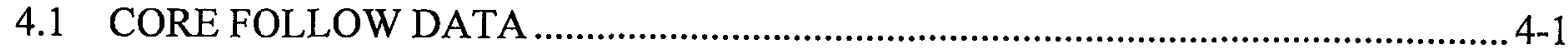

4.2 CORE FOLLOW-CONTROL ROD HISTORY AND BORON DATA .................. 4-127

4.3 STATEPOINT CRITICAL CONDITIONS AND MEASUREMENTS .................... 4-144

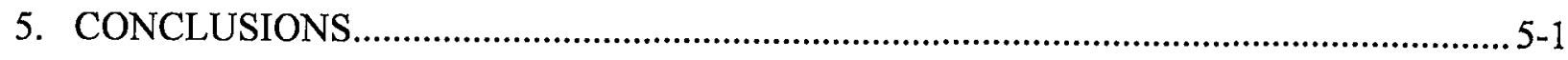

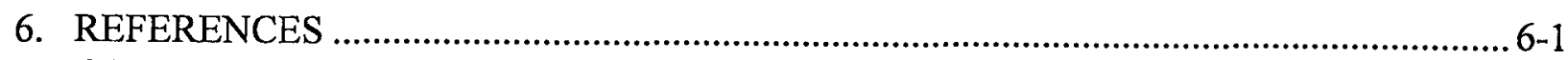

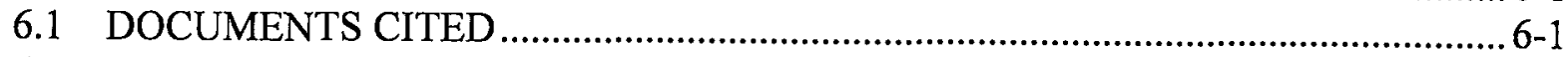

6.2 CODES, STANDARDS, REGULATIONS, AND PROCEDURES ……….................. 6-1

6.3 SOURCE DATA, LISTED BY DATA TRACKING NUMBER ................................. 6-1

APPENDIX A - FUEL ROD DATA FOR ASSEMBLIES NJ05YU \& NJ070G ....................... A-1 
INTENTIONALLY LEFT BLANK 


\section{FIGURES}

Page

2-1. Radial View of the Reactor Vessel and Internals along Core Midplane ............................2-2

2-2. Fuel Assembly Radial View Layout and Dimensions.........................................................2-3

2-3. Mark-B4 Assembly Axial Dimensions by Region .......................................................... 2-11

2-4. Mark-B4Z Assembly Axial Dimensions by Region........................................................2-12

2-5. Mark-B4 \& -B4Z Assembly Axial Dimensions for Guide Tubes.................................... 2-13

2-6. Mark-B4 \& -B4Z Assembly Axial Dimensions for Instrument Tube............................... 2-14

2-7. Mark-B4 \& -B4Z Assembly Axial Dimensions for Standard Fuel Rod ............................ 2-15

2-8. Mark-B8 Assembly Axial Dimensions by Region ........................................................ 2-20

2-9. Mark-B8 Assembly Axial Dimensions for Guide Tubes .............................................. 2-21

2-10. Mark-B8 Assembly Axial Dimensions for Instrument Tube …………….....................2-22

2-11. Mark-B8 Assembly Axial Dimensions for Standard Fuel Rod ...................................... 2-23

2-12. Mark-B9 Assembly Axial Dimensions by Region .......................................................... 2-28

2-13. Mark-B9 Assembly Axial Dimensions for Guide Tubes ............................................... 2-29

2-14. Mark-B9 Assembly Axial Dimensions for Instrument Tube ........................................2-30

2-15. Mark-B9 Assembly Axial Dimensions - Standard Fuel Rod ........................................ 2-31

2-16. Mark-B9 Assembly Axial Dimensions - Gadolinia Fuel Rod........................................ 2-32

2-17. Mark-B10 Assembly Axial Dimensions by Region .........................................................2-2-37

2-18. Mark-B10 Assembly Axial Dimensions for Guide Tubes ..............................................2-38

2-19. Mark-B10 Assembly Axial Dimensions for Instrument Tube ......................................2-39

2-20. Mark-B10 Assembly Axial Dimensions for Standard Fuel Rod .................................... 2-40

2-21. Mark-B10 Assembly Axial Dimensions for Gadolinia Fuel Rod .................................2-41

2-22. Mark-B Assembly Axial Dimensions for RCCA 0 \% Withdrawn.................................2-43

2-23. Mark-B Assembly with RCCA, Black, and Gray APSR $100 \%$ Withdrawn ..................2-44

2-24. Mark-B Assembly Axial Dimensions with Black APSR $0 \%$ Withdrawn.......................2-46

2-25. Mark-B Assembly Axial Dimensions with Gray APSR 0 \% Withdrawn .......................2-48

2-26. Mark-B Assembly Axial Dimensions for BPRA Inserted............................................... 2-50

3-1. One-Eighth Core Loading for TMI-1 (0.0 - 256.0 EFPD), Cycle 1A …………................3-4

3-2. One-Eighth Core Loading for TMI-1 (256.0 - 466.4 EFPD), Cycle 1B............................ 3-5

3-3. One-Eighth Core Loading for TMI-1, Cycle 2.............................................................. 3-6

3-4. One-Eighth Core Loading for TMI-1, Cycle 3 ............................................................... 3-7

3-5. One-Eighth Core Loading for TMI-1, Cycle 4.......................................................... 3-8

3-6. One-Eighth Core Loading for TMI-1, Cycle 5 ..............................................................

3-7. One-Eighth Core Loading for TMI-1, Cycle 6 ............................................................. 3-10

3-8. One-Eighth Core Loading for TMI-1, Cycle 7 ................................................................. 3-11

3-9. One-Eighth Core Loading for TMI-1, Cycle 8 ……................................................. 3-12

3-10. One-Eighth Core Loading for TMI-1, Cycle 9............................................................ 3-13

3-11. One-Eighth Core Loading for TMI-1, Cycle 10 …..................................................14

3-12. One-Eighth Core Loading for TMI-1, Cycle 11 .................................................... 3-15

3-13. TMI-1, Cycle 1 Fuel Assembly Identification \& Location ............................................... 3-16

3-14. TMI-1, Cycle 2 Fuel Assembly Identification \& Location ............................................ 3-17

3-15. TMI-1, Cycle 3 Fuel Assembly Identification \& Location .............................................. 3-18

3-16. TMI-1, Cycle 4 Fuel Assembly Identification \& Location ............................................ 3-19 


\section{FIGURES}

Page

3-17. TMI-1, Cycle 5 Fuel Assembly Identification \& Location...............................................3-20

3-18. TMI-1, Cycle 6 Fuel Assembly Identification \& Location...............................................3-21

3-19. TMI-1, Cycle 7 Fuel Assembly Identification \& Location................................................

3-20. TMI-1, Cycle 8 Fuel Assembly Identification \& Location...............................................3-23

3-21. TMI-1, Cycle 9 Fuel Assembly Identification \& Location..............................................3-24

3-22. TMI-1, Cycle 10 Fuel Assembly Identification \& Location...........................................3-25

3-23. TMI-1, Cycle 11 Fuel Assembly Identification \& Location................................................3-26

4-1. TMI-1 Active Fuel Length for Statepoint Analysis ...........................................................4-2

A-1. TMI-1 Cycle 9 Assembly NJ05YU Location …………..............................................A-2

A-2. TMI-1 Cycle 10 Assembly NJ05YU and NJ070G Location ...........................................

A-3. TMI-1 Fuel Rod Locations in Assembly NJ05YU ....................................................

A-4. TMI-1 Fuel Rod Locations in Assembly NJ070G ....................................................... 


\section{TABLES}

Page

2-1. Dimensions from Core Center to Outside Surface of Pressure Vessel ...............................2-1

2-2. Three Mile Island Unit 1 Fuel Assembly/Core Data ............................................................2-5

2-3. Mark-B4 \& -B4Z Assembly VFs by Region .................................................................... 2-6

2-4. Mark-B4 \& -B4Z Assembly VFs with RCCAs 0\% Withdrawn .........................................2-7

2-5. Mark-B4 \& -B4Z Assembly VFs with RCCAs \& APSRs 100\% Withdrawn ......................2-7

2-6. Mark-B4 \& -B4Z Assembly VFs with Black APSRs 0 \% Withdrawn ................................2-8

2-7. Mark-B4 \& -B4Z Assembly VFs with Gray APSRs $0 \%$ Withdrawn...................................2-8

2-8. Mark-B4 \& -B4Z Assembly VFs with BPRAs Inserted..................................................2-9

2-9. Mark-B4 \& -B4Z Fuel Rod VFs by Region ......................................................................2-9

2-10. Mark-B8 Assembly VFs by Region.............................................................................2-16

2-11. Mark-B8 Assembly VFs with RCCAs 0\% Withdrawn ...................................................2-16

2-12. Mark-B8 Assembly VFs with RCCAs \& APSRs 100\% Withdrawn................................2-17

2-13. Mark-B8 Assembly VFs with Gray APSRs $0 \%$ Withdrawn ..........................................2-17

2-14. Mark-B8 Assembly VFs with BPRAs Inserted ..............................................................2-18

2-15. Mark-B8 Fuel Rod VFs by Region ............................................................................2-18

2-16. Mark-B9 Assembly VFs by Region........................................................................2-24

2-17. Mark-B9 Assembly VFs with RCCAs 0\% Withdrawn ...................................................2-24

2-18. Mark-B9 Assembly VFs with RCCAs \& Gray APSRs 100\% Withdrawn......................2-25

2-19. Mark-B9 Assembly VFs with Gray APSRs $0 \%$ Withdrawn ..........................................2-25

2-20. Mark-B9 Assembly VFs with BPRAs Inserted ................................................................2-26

2-21. Mark-B9 Standard Fuel Rod VFs by Region.............................................................2-26

2-22. Mark-B9 Gadolinia Fuel Rod VFs by Region .............................................................2-27

2-23. Mark-B10 Assembly VFs by Region............................................................................2-33

2-24. Mark-B10 Assembly VFs with RCCAs 0\% Withdrawn ...............................................2-33

2-25. Mark-B10 Assembly VFs with RCCAs and Gray APSRs 100\% Withdrawn ................2-34

2-26. Mark-B10 Assembly VFs with Gray APSRs 0 \% Withdrawn ........................................2-34

2-27. Mark-B10 Assembly VFs with BPRAs Inserted ............................................................2-35

2-28. Mark-B10 Standard Fuel Rod VFs by Region.............................................................2-36

2-29. Mark-B10 Gadolinia Fuel Rod VFs by Region .............................................................2-36

3-1. Fuel Assembly/Fuel Rod/Cycle Description for Cycles 1 through 11 ...............................3-1

3-2. Statepoint Information for Cycles 1 through 11 ........................................................... $3-2$

3-3. Fuel Assembly Locations by Cycle ……………...........................................................3-28

3-4. TMI-1 FA and Control Component Locations …….....................................................3-32

4-1. Assembly Axial Nodal Spacing .....................................................................................

4-2. Burnup and TH Feedback Parameters by Axial Node for Assembly B 15 ........................4-3

4-3. Burnup and TH Feedback Parameters by Axial Node for Assembly B21 .........................4-4

4-4. Burnup and TH Feedback Parameters by Axial Node for Assembly B21a........................4-5

4-5. Burnup and TH Feedback Parameters by Axial Node for Assembly B28 ……..................4-6

4-6. Burnup and TH Feedback Parameters by Axial Node for Assembly B29 …......................4-7

4-7. Burnup and TH Feedback Parameters by Axial Node for Assembly C8 ...........................4-8

4-8. Burnup and TH Feedback Parameters by Axial Node for Assembly C15 ........................4-9 


\section{TABLES}

Page

4-9. Burnup and TH Feedback Parameters by Axial Node for Assembly C15a 4-10

4-10. Burnup and TH Feedback Parameters by Axial Node for Assembly C20 4-11

4-11. Burnup and TH Feedback Parameters by Axial Node for Assembly C21 4-12

4-12. Burnup and TH Feedback Parameters by Axial Node for Assembly C25 4-13

4-13. Burnup and TH Feedback Parameters by Axial Node for Assembly C28 4-14

4-14. Burnup and TH Feedback Parameters by Axial Node for Assembly C29 4-15

4-15. Burnup and TH Feedback Parameters by Axial Node for Assembly D8 4-16

4-16. Burnup and TH Feedback Parameters by Axial Node for Assembly D15

4-17. Burnup and TH Feedback Parameters by Axial Node for Assembly D15a 4-18

4-18. Burnup and TH Feedback Parameters by Axial Node for Assembly D20 4-19

4-19. Burnup and TH Feedback Parameters by Axial Node for Assembly D20a 4-20

4-20. Burnup and TH Feedback Parameters by Axial Node for Assembly D21

4-21. Burnup and TH Feedback Parameters by Axial Node for Assembly D25

4-22. Burnup and TH Feedback Parameters by Axial Node for Assembly D25a 4-23

4-23. Burnup and TH Feedback Parameters by Axial Node for Assembly D27 4-24

4-24. Burnup and TH Feedback Parameters by Axial Node for Assembly D27a 4-25

4-25. Burnup and TH Feedback Parameters by Axial Node for Assembly D28 .4-26

4-26. Burnup and TH Feedback Parameters by Axial Node for Assembly E8 4-27

4-27. Burnup and TH Feedback Parameters by Axial Node for Assembly E15. 4-28

4-28. Burnup and TH Feedback Parameters by Axial Node for Assembly E20 4-30

4-29. Burnup and TH Feedback Parameters by Axial Node for Assembly E20a 4-32

4-30. Burnup and TH Feedback Parameters by Axial Node for Assembly E20b 4-33

4-31. Burnup and TH Feedback Parameters by Axial Node for Assembly E20c 4-35

4-32. Burnup and TH Feedback Parameters by Axial Node for Assembly E21 4-36

4-33. Burnup and TH Feedback Parameters by Axial Node for Assembly E25 .4-38

4-34. Burnup and TH Feedback Parameters by Axial Node for Assembly E27 4-39

4-35. Burnup and TH Feedback Parameters by Axial Node for Assembly E28

4-36. Burnup and TH Feedback Parameters by Axial Node for Assembly F2

4-37. Burnup and TH Feedback Parameters by Axial Node for Assembly F4 .4-42

4-38. Burnup and TH Feedback Parameters by Axial Node for Assembly F6 $.4-43$

4-39. Burnup and TH Feedback Parameters by Axial Node for Assembly F10 .4-44

4-40. Burnup and TH Feedback Parameters by Axial Node for Assembly F12 $.4-45$

4-41. Burnup and TH Feedback Parameters by Axial Node for Assembly F14 $.4-46$

4-42. Burnup and TH Feedback Parameters by Axial Node for Assembly F14a 4-48

4-43. Burnup and TH Feedback Parameters by Axial Node for Assembly F17 $.4-49$

4-44. Burnup and TH Feedback Parameters by Axial Node for Assembly F17a 4-50

4-45. Burnup and TH Feedback Parameters by Axial Node for Assembly F19 4-51

4-46. Burnup and TH Feedback Parameters by Axial Node for Assembly F23

4-47. Burnup and TH Feedback Parameters by Axial Node for Assembly F25 $.4-53$

4-48. Burnup and TH Feedback Parameters by Axial Node for Assembly F27 4-54

4-49. Burnup and TH Feedback Parameters by Axial Node for Assembly G2 4-55

4-50. Burnup and TH Feedback Parameters by Axial Node for Assembly G4 4-56

4-51. Burnup and TH Feedback Parameters by Axial Node for Assembly G6 


\section{TABLES}

Page

4-52. Burnup and TH Feedback Parameters by Axial Node for Assembly G10 .....................4-59

4-53. Burnup and TH Feedback Parameters by Axial Node for Assembly G12 .....................4-60

4-54. Burmup and TH Feedback Parameters by Axial Node for Assembly G12a ...................4-61

4-55. Burnup and TH Feedback Parameters by Axial Node for Assembly G14 .....................4-62

4-56. Burnup and TH Feedback Parameters by Axial Node for Assembly G17 .....................4-63

4-57. Burnup and TH Feedback Parameters by Axial Node for Assembly G19 .....................4-64

4-58. Burnup and TH Feedback Parameters by Axial Node for Assembly G23 .....................4-65

4-59. Burnup and TH Feedback Parameters by Axial Node for Assembly G25 .....................4-66

4-60. Burnup and TH Feedback Parameters by Axial Node for Assembly G25a ...................4-67

4-61. Burnup and TH Feedback Parameters by Axial Node for Assembly G27 .....................4-68

4-62. Burnup and TH Feedback Parameters by Axial Node for Assembly G27a ...................4-69

4-63. Burnup and TH Feedback Parameters by Axial Node for Assembly H6 ........................4-70

4-64. Burnup and TH Feedback Parameters by Axial Node for Assembly H10 .....................4-72

4-65. Burnup and TH Feedback Parameters by Axial Node for Assembly H10a ...................4-72

4-66. Burnup and TH Feedback Parameters by Axial Node for Assembly H10b ...................4-74

4-67. Burnup and TH Feedback Parameters by Axial Node for Assembly H12 .....................4-75

4-68. Burnup and TH Feedback Parameters by Axial Node for Assembly H12a ....................4-77

4-69. Burnup and TH Feedback Parameters by Axial Node for Assembly H14 ......................4-77

4-70. Burnup and TH Feedback Parameters by Axial Node for Assembly H17 .....................4-79

4-71. Burnup and TH Feedback Parameters by Axial Node for Assembly H19 .....................4-79

4-72. Burnup and TH Feedback Parameters by Axial Node for Assembly H2O .....................4-81

4-73. Burnup and TH Feedback Parameters by Axial Node for Assembly H23 .....................4-81

4-74. Burnup and TH Feedback Parameters by Axial Node for Assembly H25 .....................4-83

4-75. Burnup and TH Feedback Parameters by Axial Node for Assembly H25a ...................4-83

4-76. Burnup and TH Feedback Parameters by Axial Node for Assembly H27 ......................4-84

4-77. Burnup and TH Feedback Parameters by Axial Node for Assembly H27a ...................4-84

4-78. Burnup and TH Feedback Parameters by Axial Node for Assembly J2 .........................4-85

4-79. Burnup and TH Feedback Parameters by Axial Node for Assembly J10.......................4-86

4-80. Burnup and TH Feedback Parameters by Axial Node for Assembly J12 .......................4-88

4-81. Burnup and TH Feedback Parameters by Axial Node for Assembly J12a.....................4-89

4-82. Burnup and TH Feedback Parameters by Axial Node for Assembly J14 ........................4-90

4-83. Burnup and TH Feedback Parameters by Axial Node for Assembly J17 .......................4-91

4-84. Burnup and TH Feedback Parameters by Axial Node for Assembly J17a.....................4-93

4-85. Burnup and TH Feedback Parameters by Axial Node for Assembly J19.......................4-94

4-86. Burnup and TH Feedback Parameters by Axial Node for Assembly J20.......................4-95

4-87. Burnup and TH Feedback Parameters by Axial Node for Assembly J23 .......................4-96

4-88. Burnup and TH Feedback Parameters by Axial Node for Assembly J25 .......................4-97

4-89. Burnup and TH Feedback Parameters by Axial Node for Assembly J25a......................4-98

4-90. Burnup and TH Feedback Parameters by Axial Node for Assembly J27 .......................4-99

4-91. Burnup and TH Feedback Parameters by Axial Node for Assembly J27a....................4-100

4-92. Burnup and TH Feedback Parameters by Axial Node for Assembly K2 .....................4-101

4-93. Burnup and TH Feedback Parameters by Axial Node for Assembly K6 .....................4-102

4-94. Burnup and TH Feedback Parameters by Axial Node for Assembly K10 ..................... $4-104$ 


\section{TABLES}

Page

4-95. Burnup and TH Feedback Parameters by Axial Node for Assembly K10a ..................4-105

4-96. Burnup and TH Feedback Parameters by Axial Node for Assembly K12 ...................4-107

4-97. Burnup and TH Feedback Parameters by Axial Node for Assembly K14 ...................4-108

4-98. Burnup and TH Feedback Parameters by Axial Node for Assembly K17 ...................4-110

4-99. Burnup and TH Feedback Parameters by Axial Node for Assembly K19 ...................4-111

4-100. Burnup and TH Feedback Parameters by Axial Node for Assembly K19a ................4-113

4-101. Burnup and TH Feedback Parameters by Axial Node for Assembly K20 .................4-114

4-102. Burnup and TH Feedback Parameters by Axial Node for Assembly K23 ..................4-115

4-103. Burnup and TH Feedback Parameters by Axial Node for Assembly K25 .................4-116

4-104. Burnup and TH Feedback Parameters by Axial Node for Assembly K27 .................4-118

4-105. Burnup and TH Feedback Parameters by Axial Node for Assembly K27a ...............4-119

4-106. Burnup and TH Feedback Parameters by Axial Node for Assembly L2 ....................4-121

4-107. Burnup and TH Feedback Parameters by Axial Node for Assembly L6.....................4-121

4.108. Burnup and TH Feedback Parameters by Axial Node for Assembly L10 ..................4-122

4-109. Burnup and TH Feedback Parameters by Axial Node for Assembly L12 ..................4-122

4-110. Burnup and TH Feedback Parameters by Axial Node for Assembly L14..................4-123

4-111. Burnup and TH Feedback Parameters by Axial Node for Assembly L17 ..................4-123

4-112. Burnup and TH Feedback Parameters by Axial Node for Assembly L19.................4-124

4-113. Burnup and TH Feedback Parameters by Axial Node for Assembly L20...................4-124

4-114. Burnup and TH Feedback Parameters by Axial Node for Assembly L23..................4-125

4-115. Burnup and TH Feedback Parameters by Axial Node for Assembly L27 ..................4-125

4-116. Burnup and TH Feedback Parameters by Axial Node for Assembly L28...................4-126

4-117. Rod Insertion Time by Axial Node for Assembly B15 ……..................................4-127

4-118. Rod Insertion Time by Axial Node for Assembly B21 …....................................4-128

4-119. Rod Insertion Time by Axial Node for Assembly B21 a ..........................................4-128

4-120. Rod Insertion Time by Axial Node for Assembly B29 ........................................... $4-129$

4-121. Rod Insertion Time by Axial Node for Assembly C21 ..............................................4-129

4-122. Rod Insertion Time by Axial Node for Assembly C28 .........................................4-130

4-123. Rod Insertion Time by Axial Node for Assembly C29 …….....................................4-130

4-124. Rod Insertion Time by Axial Node for Assembly D15 …........................................1-131

4-125. Rod Insertion Time by Axial Node for Assembly D15a .........................................

4-126. Rod Insertion Time by Axial Node for Assembly D20a ...........................................4-132

4-127. Rod Insertion Time by Axial Node for Assembly D21 ............................................4-132

4-128. Rod Insertion Time by Axial Node for Assembly D25a ...........................................4-133

4-129. Rod Insertion Time by Axial Node for Assembly D28 ............................................

4-130. Rod Insertion Time by Axial Node for Assembly F2 ........................................... 4-134

4-131. Rod Insertion Time by Axial Node for Assembly F4 ............................................ $4-134$

4-132. Rod Insertion Time by Axial Node for Assembly F23 ............................................. 4-135

4-133. Rod Insertion Time by Axial Node for Assembly G2 ……................................... 4-135

4-134. Rod Insertion Time by Axial Node for Assembly G10 …......................................4-136

4-135. Rod Insertion Time by Axial Node for Assembly G27a ..........................................4-136

4-136. Rod Insertion Time by Axial Node for Assembly H10a ......................................... 4-137

4-137. Rod Insertion Time by Axial Node for Assembly H10b .......................................... 4-137 


\section{TABLES}

Page

4-138. Rod Insertion Time by Axial Node for Assembly H12

$4-138$

4-139. Rod Insertion Time by Axial Node for Assembly H23 4-138

4-140. Rod Insertion Time by Axial Node for Assembly H27a

4-141. Rod Insertion Time by Axial Node for Assembly J20

4-142. Rod Insertion Time by Axial Node for Assembly J 27 4-140

4-143. Rod Insertion Time by Axial Node for Assembly J27a .4-140

4-144. Rod Insertion Time by Axial Node for Assembly K6 4-141

4-145. Rod Insertion Time by Axial Node for Assembly K12 4-141

4-146. Rod Insertion Time by Axial Node for Assembly K27 4-142

4-147. All-Rods-Out-Critical-Boron Data for TMI-1 4-143

4-148. Statepoint Data for TMI-1 - Measured Critical Conditions. 4-145

4-149. Statepoint Data for TMI 1 - Shutdown and Startup Dates 4-145

A-1. Assembly \& Fuel Rod Uranium Loading (Assemblies NJ05YU \& NJ070G) ..................A-6 A-2. Fuel Rod Geometric \& Material Data (Assemblies NJ05YU \& NJ070G) ...........................6 A-3. Fuel Pellet Uranium Isotopic (wt \%) A-7

A-4. Fuel Rod Data for Assembly NJ05YU ……………….......................................... A-7

A-5. Fuel Rod Data for Assembly NJ070G A-18 
INTENTIONALLY LEFT BLANK 


\section{ACRONYMS AND ABBREVIATIONS}

\section{Acronyms}

\begin{tabular}{|c|c|}
\hline $\begin{array}{l}\text { APSR } \\
\text { AROCB }\end{array}$ & $\begin{array}{l}\text { Axial Power Shaping Rod } \\
\text { all rods out critical boron }\end{array}$ \\
\hline $\mathrm{BOC}$ & beginning of cycle \\
\hline BPRA & Burnable Poison Rod Assemblies \\
\hline $\begin{array}{l}\text { CRC } \\
\text { CRGT }\end{array}$ & $\begin{array}{l}\text { Commercial Reactor Criticality } \\
\text { control rod guide tube }\end{array}$ \\
\hline DOE & U. S. Department of Energy \\
\hline DP & datapoint \\
\hline DTN & Data Tracking Number \\
\hline EFPD & effective full power days \\
\hline EOC & end of cycle \\
\hline FA & fuel assembly \\
\hline FCF & Framatome Cogema Fuels \\
\hline $\mathrm{DD}$ & inside diameter \\
\hline $\mathrm{NRC}$ & U.S. Nuclear Regulatory Commission \\
\hline OCRWM & Office of Civilian Radioactive Waste Management \\
\hline OD & outside diameter \\
\hline ppmB & parts per million Boron \\
\hline QARD & Quality Assurance Requirements and Description \\
\hline RCCA & Rod Cluster Control Assembly \\
\hline SP & statepoint \\
\hline $\mathrm{TD}$ & Theoretical Density \\
\hline $\mathrm{TH}$ & thermal-hydraulic \\
\hline TMI-1 & Three Mile Island Unit 1 \\
\hline VF & Volume Fraction \\
\hline WD & withdrawn \\
\hline
\end{tabular}


INTENTIONALLY LEFT BLANK 


\section{INTRODUCTION}

The Summary Report of Commercial Reactor Criticality Data for Three Mile Island Unit 1 contains the detailed information necessary to perform commercial reactor criticality (CRC) analyses for the fuel used in the Three Mile Island Unit 1 (TMI-1) reactor.

\subsection{BACKGROUND}

The United States Department of Energy (DOE) Office of Civilian Radioactive Waste Management (OCRWM) is developing a methodology for criticality analysis to support disposal of commercial spent nuclear fuel in a geologic repository. A topical report on the disposal criticality analysis methodology was submitted to the United States Nuclear Regulatory Commission (NRC) for formal review in March 2001 (YMP 2000). This summary report provides data that was and will be used in analyses that will support the validation of parts of the disposal criticality analysis methodology. This technical report is prepared per Technical Work Plan TWP-EBS-MD-000004, Technical Work Plan for: Waste Package Design Description for LA (BSC 2001).

\subsection{OBJECTIVE}

The objective of the Summary Report of Commercial Reactor Criticality Data for Three Mile Island Unit $l$ is to present the CRC data for the TMI-1 reactor. Results from the CRC evaluations will support the development and validation of the neutronics models used for criticality analyses involving commercial spent nuclear fuel. These models and their validation are discussed in the Disposal Criticality Analysis Methodology Topical Report (YMP 2000).

\subsection{SCOPE}

The scope of this Summary Report is the presentation of data required to perform 16 statepoint (SP) calculations from Cycles 1 through 11 of TMI-1. A statepoint is a point during a reactor startup when criticality is achieved and the measured critical conditions are obtained. Potential statepoints include initial reactor startup for each cycle and during the cycle following reactor shutdown. The only interface for the development of the information in this document is with Framatome ANP, formally Framatome Cogema. Fuels (FCF). FCF independently requested and received permission from AmerGen Energy, the owner of TMI-1, to publish the information related to SP measurements that is recorded in this document. All the information contained in this report is documented in Framatome ANP/FCF calculation files (DTN: MO0106SPADEP01.007). The data provided in these references were obtained from various other reports, calculations, and drawings developed under a NRC accepted quality assurance program (Framatome Cogema Fuels 2000), and the data has supported prior licensing submittals. The technical information taken from reference (DTN: MO0106SPADEP01.007) are considered accepted data by the DOE issuance of a concurrence letter (Mellington 1999). The data therefore are considered acceptable for quality affecting activities and for use in analyses affecting procurement, construction, or fabrication. 


\subsection{QUALITY ASSURANCE}

The development of the this report has been subject to the United States Department of Energy OCRWM Quality Assurance Requirements and Description (QARD) (DOE 2000) controls. The information, provided in this report, will be used to develop the methodology for evaluation of the potential Monitored Geologic Repository engineered barrier system. A number of classification analyses (e.g., CRWMS M\&O 1999) have identified components of the engineered barrier system as items important to radiological safety and waste isolation.

No scientific and engineering software or computational software was used in the development of this report. Electronic management of data w/as accomplished in accordance with the controls specified in the Technical Work Plan (BSC 2001).

The work that is to be performed to support the License Application using this information will be performed in accordance with the then current versions of the QARD and NRC regulations. All information used for the License Application will be from acceptable sources and will be developed in accordance with the QARD requirements and NRC regulations. 


\section{REACTOR AND FUEL DESIGN INFORMATION}

This section provides general material and geometry data for modeling the Three Mile Island Unit 1 (TMI-1) reactor and core for cycles 1 through 11 . Figures 2-1 through 2-26 provide pictorial representations of various components that must be modeled. A radial view of the core and reactor internals is presented in Figure 2-1. There are 177 fuel assemblies in the reactor core region. A radial representation of a single fuel assembly is provided in Figure 2-2. The core liner, core barrel, thermal shield, and pressure vessel cladding are represented as stainless steel 304 (304SS). The pressure vessel is CS533, Type B carbon steel (ASTM A533/A533M-93, Standard Specification for Pressure Vessel Plates, Alloy Steel, Quenched and Tempered, Manganese-Molybdenum and Manganese-Molybdenum-Nickel). Table 2-1 provides dimensions from the center of the core (from Assembly 1, Assembly 2, Assembly 3, ..., Assembly 8, core liner, etc. - Figure 2-1) to the outside surface of the pressure vessel.

Table 2-1. Dimensions from Core Center to Outside Surface of Pressure Vessel

\begin{tabular}{|c|c|c|}
\hline Description & Thickness (cm) & Outer Dimensions $(\mathrm{cm})$ \\
\hline Core Center & - & 0.0 \\
\hline $1 / 2$ Fuel Assembly 1 & 10.84072 & 10.84072 \\
\hline Water & 0.12954 & 10.97026 \\
\hline Fuel Assembly 2 & 21.68144 & 32.65170 \\
\hline Water & 0.12954 & 32.78124 \\
\hline Fuel Assembly 3 & 21.68144 & 54.46268 \\
\hline Water & 0.12954 & 54.59222 \\
\hline Fuel Assembly 4 & 21.68144 & 76.27366 \\
\hline Water & 0.12954 & 76.40320 \\
\hline Fuel Assembly 5 & 21.68144 & 98.08464 \\
\hline Water & 0.12954 & 98.21418 \\
\hline Fuel Assembly 6 & 21.68144 & 119.89562 \\
\hline Water & 0.12954 & 120.02516 \\
\hline Fuel Assembly 7 & 21.68144 & 141.70660 \\
\hline Water & 0.12954 & 141.83614 \\
\hline Fuel Assembly 8 & 21.68144 & 163.51758 \\
\hline Water & 0.27442 & 163.79200 \\
\hline Core Liner & 1.905 & 165.697 \\
\hline Water & 13.373 & 179.070 \\
\hline Core Barrel & 5.080 & 184.150 \\
\hline Water & 2.540 & 186.690 \\
\hline Thermal Shield & 5.080 & 191.770 \\
\hline Water & 24.925 & 216.695 \\
\hline Pressure Vessel Clad & 0.478 & 217.173 \\
\hline Pressure Vessel & 21.433 & 238.606 \\
\hline
\end{tabular}




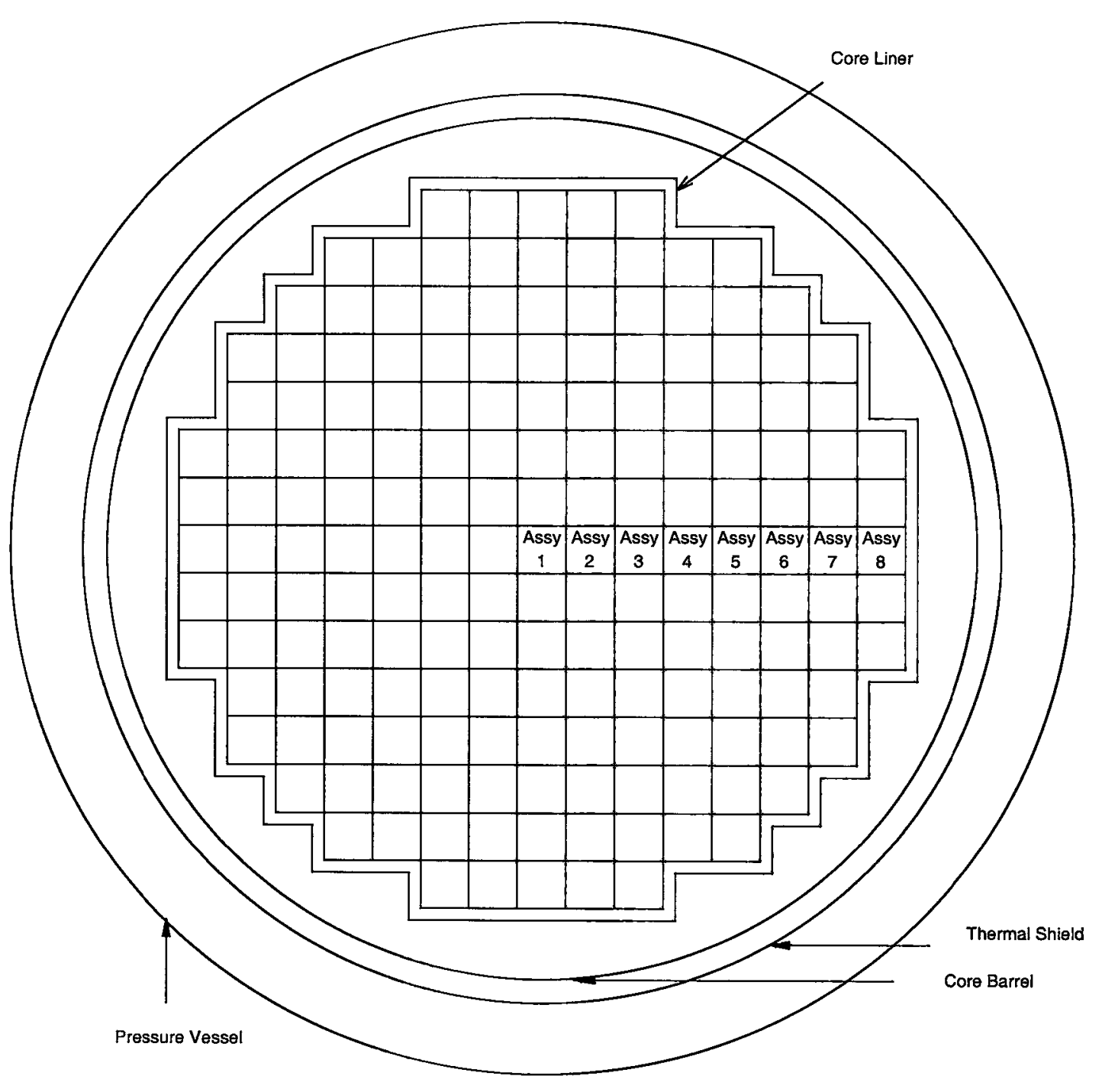

Figure 2-1. Radial View of the Reactor Vessel and Internals along Core Midplane 

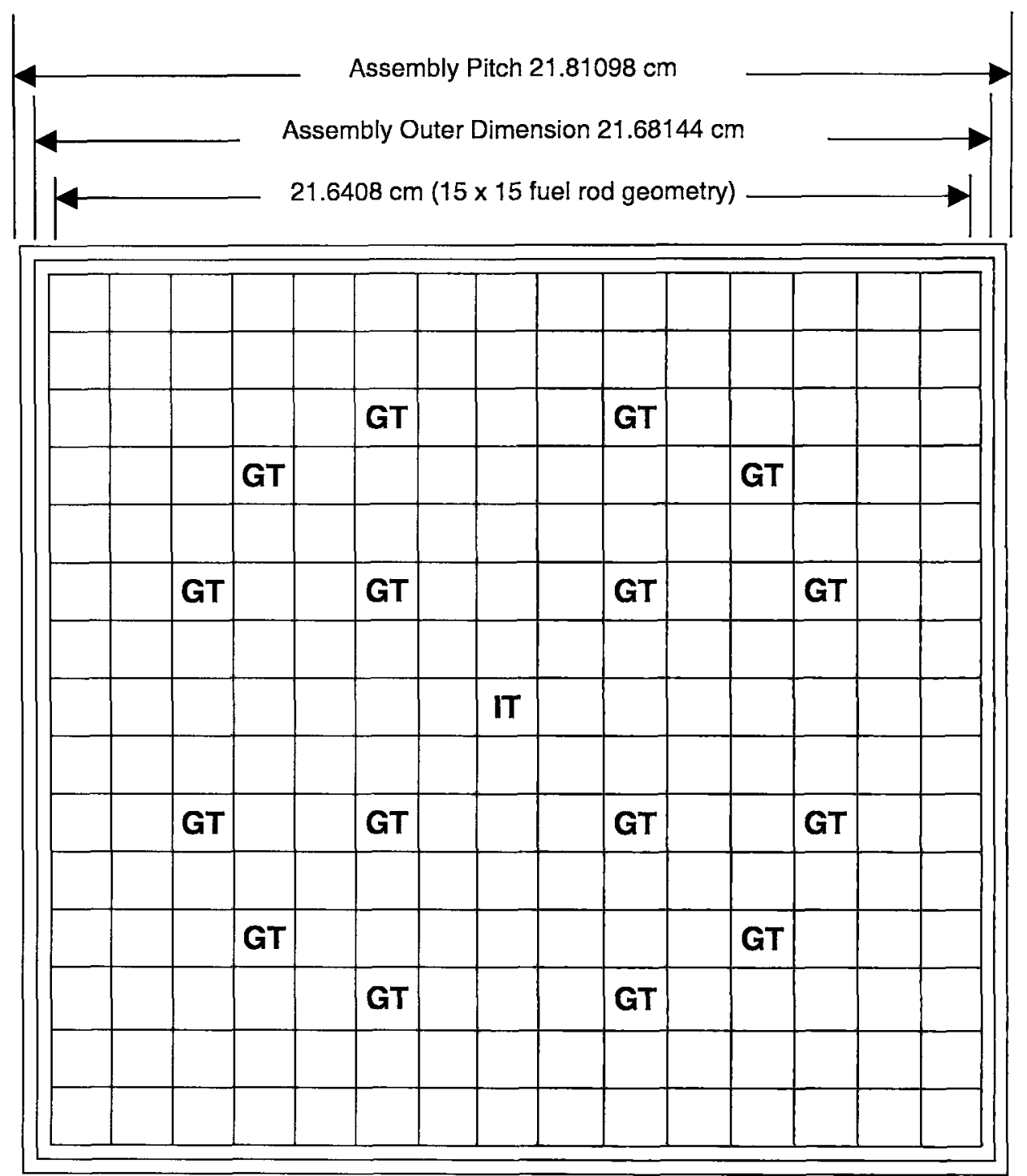

GT Guide Tube

IT Instrument Tube

Fuel Rod Cell

Figure 2-2. Fuel Assembly Radial View Layout and Dimensions 


\subsection{FUEL ASSEMBLY AND CORE DATA}

This section provides general material and geometry data for modeling the Babcock \& Wilcox Mark-B fuel assemblies. The TMI-1 reactor core contains 177 fuel assemblies. The material and geometric data for the Mark-B4 fuel assembly is representative of the material and geometric data applicable to the Mark-B2 and Mark-B3 fuel assemblies. The data will be used in modeling each fuel assembly type for burnup calculations and the reactor criticality calculations.

Fuel Assembly Design - Babcock \& Wilcox / FCF Mark-B Fuel Assemblies - Each fuel assembly consists of 208 fuel rods, 16 guide thimble tubes and 1 instrumentation thimble tube arranged within a supporting structure. The instrumentation thimble is located in the center position. The guide thimbles provide channels for insertion of either a rod cluster control assembly (RCCA), a neutron source assembly, a burnable poison rod assembly (BPRA) or an axial power shaping rod (APSR) assembly. If no component is inserted, the guide tube thimbles are filled with water. The guide tubes are connected to the grids, and the top and bottom nozzles. Figure 2-2 indicates a cross-section of the fuel assembly.

Two hundred and eight fuel rods are mechanically joined in a square array to form a fuel assembly. The fuel rods are supported at intervals along their length by grid assemblies, which maintain the lateral spacing between the rods throughout the design life of the assembly. The grids at the top and the bottom are made of Inconel-718 and the intermediate grids are made of Zircaloy-4. The Mark-B4 fuel assembly is an exception. In the Mark-B4 fuel design, the six intermediate spacer grids are made of Inconel-718. The fuel rods consist of natural or slightly enriched uranium dioxide ceramic cylindrical pellets contained in Zircaloy-4 tubing which is plugged and seal welded at the ends to encapsulate the fuel. All fuel rods are pressurized with helium.

The bottom end fitting (nozzle) is a box-like structure and made of 304SS. The top end fitting (nozzle) assembly functions as an upper structural element of the fuel assembly in addition to providing a protective housing for the RCCA, BPRA or other components. The top end fitting (nozzle) forging is stainless steel.

Table 2-2 summarizes fuel assembly and reactor core data for modeling the TMI-1 reactor for cycles 1 through 11 . Additional fuel cycle design, core operations, and reactor criticality statepoint information are provided in Sections 3 and 4. 
Table 2-2. Three Mile Island Unit 1 Fuel Assembly/Core Data

\begin{tabular}{|c|c|c|c|c|}
\hline Parameter & $\begin{array}{c}\text { Mark-B2/-B3 } \\
\text { Mark-B4/-B4Z }\end{array}$ & Mark-B8 & Mark-B9 & Mark-B10 \\
\hline Fuel Assembly Array size & \multicolumn{4}{|l|}{$15 \times 15$} \\
\hline Number of Fuel Rod $\left(N_{f}\right) /$ Assy & \multicolumn{4}{|l|}{208} \\
\hline Number of Guide Tubes ( $\mathrm{N}_{\mathrm{GT}}$ ) / Assy & \multicolumn{4}{|l|}{16} \\
\hline Number of Instrument Tubes $\left(\mathrm{N}_{\mathrm{IT}}\right)$ / Assy & \multicolumn{4}{|l|}{1} \\
\hline Number of Assemblies in Core & \multicolumn{4}{|l|}{177} \\
\hline System Pressure - psia & \multicolumn{4}{|c|}{$2200 \mathrm{psi} / 1.52 \times 10^{7} \mathrm{~Pa}$} \\
\hline Active Fuel Height $(H)$ & $360.172 \mathrm{~cm}$ & $360.172 \mathrm{~cm}$ & $357.111 \mathrm{~cm}$ & $357.111 \mathrm{~cm}$ \\
\hline Rod Pitch & \multicolumn{4}{|l|}{$1.44272 \mathrm{~cm}$} \\
\hline Assembly Pitch $(P)$ & \multicolumn{4}{|l|}{$21.81098 \mathrm{~cm}$} \\
\hline Fuel Rod Clad Material & \multicolumn{4}{|l|}{ Zircaloy-4 } \\
\hline Fuel Rod Clad $O D^{a}\left(O D_{c}\right)$ & \multicolumn{4}{|l|}{$1.0922 \mathrm{~cm}$} \\
\hline Fuel Rod Clad ID' $\left(I_{C}\right)$ & \multicolumn{4}{|l|}{$0.95758 \mathrm{~cm}$} \\
\hline Fuel Pellet OD & $0.936244 \mathrm{~cm}^{\mathrm{c}}$ & $0.936244 \mathrm{~cm}$ & $0.9398 \mathrm{~cm}$ & $0.9398 \mathrm{~cm}$ \\
\hline Fuel Rod End Cap Material & \multicolumn{4}{|l|}{ Zircaloy-4 } \\
\hline Guide Tube Material & \multicolumn{4}{|l|}{ Zircaloy-4 } \\
\hline Guide Tube OD (ODGT) & \multicolumn{4}{|l|}{$1.3462 \mathrm{~cm}$} \\
\hline Guide Tube ID (IDGT) & \multicolumn{4}{|l|}{$1.26492 \mathrm{~cm}$} \\
\hline Instrument Tube Material & \multicolumn{4}{|l|}{ Zircaloy -4} \\
\hline Instrument Tube $\mathrm{OD}(\mathrm{OD} / \mathrm{T})$ & $1.38193 \mathrm{~cm}$ & $1.25222 \mathrm{~cm}$ & $1.25222 \mathrm{~cm}$ & $1.25222 \mathrm{~cm}$ \\
\hline Instrument Tube ID (ID & \multicolumn{4}{|l|}{$1.12014 \mathrm{~cm}$} \\
\hline
\end{tabular}

NOTES: ${ }^{\mathrm{a}} \mathrm{OD}=$ outer diameter

${ }^{\mathrm{b}} \mathrm{ID}=$ inner diameter

${ }^{c}$ Refer to Table 3-1 for fuel pellet OD for an individual fuel batch.

Figure 2-2 provides a radial view of a single fuel assembly indicating the locations of the guide tubes, instrument tube, and fuel rods. A fuel assembly contains six intermediate spacer grids and two Inconel end spacer grids. The upper-end spacer grid is above the active fuel region and the lower-end spacer grid is below the active fuel region. The six intermediate spacer grids are inside the active fuel region.

Figures 2-3 through 2-26 provide axial dimensions, by region, for the Mark-B fuel assemblies. Regions 1 through 4 and 10 through 12 are represented as homogenized regions of stainless steel and water. The volume fractions (VF) for regions 1 through 4 are applicable to the $30 \mathrm{~cm}$ directly above the bottom of the upper grid plate and regions 10 through 12 are applicable to the $30 \mathrm{~cm}$ directly below the top of the lower grid plate. 
Regions 5 through 9 contain various combinations of guide tubes, instrument tube, and fuel rods, as well as other materials (stainless steel, Inconel, Zircaloy, and water). The fraction of guide tubes, instrument tube, and fuel rod assemblies will be represented explicitly in these regions. The other materials will be homogenized within the remaining portions of the regions. The water inside the guide tubes and instrument tube will be represented explicitly within the respective tubes. The volume fractions of other materials, by region, for the Mark-B fuel assemblies are indicated. The fuel assembly volume fractions are provided with and without control components. The Mark-B4 assembly, reported in Section 2.2, represents the dimensions and volume fractions of the Mark-B2, Mark-B3, and Mark-B4 fuel assemblies.

Fuel Rod - Regions 6, 7, and 9 contain various amounts of stainless steel and Zircaloy that are representative of the end caps, spacer, and springs. In addition, these regions contain helium and fission gases, as well as the Zircaloy cladding. Note that these regions of the fuel rod assembly do not contain fuel pellets. Region 8 is the active fuel region and contains fuel pellets, Zircaloy fuel cladding, and helium gas in the gaps. The fuel rod assembly volume fractions for materials in Regions 6 through 9 are provided.

\subsection{MARK-B4 AND -B4Z FUEL ASSEMBLY}

Figures 2-3 and 2-4 provide region boundaries and axial locations for intermediate spacer grids. The intermediate spacer grid height is $3.81 \mathrm{~cm}$ for each of the six grids for the Mark-B4. For the Mark-B4Z fuel assemblies, each of the six intermediate spacer grids is $5.08 \mathrm{~cm}$. Tables $2-3$ through 2-8 provides volume fractions for Mark-B4 and -B4Z fuel assemblies with and without control components.

Table 2-3. Mark-B4 \& -B4Z Assembly VFs by Region

\begin{tabular}{|c|c|c|c|c|}
\hline Region & 304SS & Inconel 718 & Zircaloy-4 & Water \\
\hline 1 & 0.0578 & 0.0 & 0.0 & 0.9422 \\
\hline 2 & 0.1381 & 0.0 & 0.0 & 0.8619 \\
\hline 3 & 0.2491 & 0.0 & 0.0 & 0.7509 \\
\hline 4 & 0.3418 & 0.0 & 0.0 & 0.6582 \\
\hline 5 & 0.2756 & 0.0441 & 0.0081 & 0.6722 \\
\hline 6 & 0.0 & 0.0457 & 0.0069 & 0.9474 \\
\hline 7 & 0.0 & 0.0 & 0.0 & 1.0 \\
\hline $8 / \mathrm{B} 4$ & 0.0 & 0.005757609 & 0.0 & 0.994242391 \\
$8 / \mathrm{B} 4 \mathrm{Z}$ & 0.0 & 0.0 & 0.008165257 & 0.991834743 \\
\hline 9 & 0.1656 & 0.0306 & 0.0125 & 0.7913 \\
\hline 10 & 0.2848 & 0.0 & 0.0 & 0.7152 \\
\hline 11 & 0.2400 & 0.0 & 0.0 & 0.7600 \\
\hline 12 & 0.0300 & 0.0 & 0.0 & 0.9700 \\
\hline
\end{tabular}

NOTE: All components except fuel rods, instrument tube and guide tubes 
Table 2-4. Mark-B4 \& -B4Z Assembly VFs with RCCAs 0\% Withdrawn

\begin{tabular}{|c|c|c|c|c|}
\hline Region & 304SS & Inconel 718 & Zircaloy-4 & Water \\
\hline 1 & 0.0934 & 0.0 & 0.0 & 0.9066 \\
\hline 2 & 0.1945 & 0.0 & 0.0 & 0.8055 \\
\hline 3 & 0.3481 & 0.0 & 0.0 & 0.6519 \\
\hline 4 & 0.3748 & 0.0 & 0.0 & 0.6252 \\
\hline 5 & 0.2981 & 0.0441 & 0.0081 & 0.6497 \\
\hline 6 & 0.0 & 0.0457 & 0.0069 & 0.9474 \\
\hline 7 & 0.0 & 0.0 & 0.0 & 1.0 \\
\hline 8/B4 & 0.0 & 0.005757609 & 0.0 & 0.994242391 \\
\hline /B4Z & 0.0 & 0.0 & 0.008165257 & 0.991834743 \\
\hline 9 & 0.1656 & 0.0306 & 0.0125 & 0.7913 \\
\hline 10 & 0.2848 & 0.0 & 0.0 & 0.7152 \\
\hline 11 & 0.2400 & 0.0 & 0.0 & 0.7600 \\
\hline 12 & 0.0300 & 0.0 & 0.0 & 0.9700 \\
\hline
\end{tabular}

NOTE: All components except fuel rods, instrument tube, guide tubes, and control tubes.

Table 2-5. Mark-B4 \& -B4Z Assembly VFs with RCCAs \& APSRs $100 \%$ Withdrawn

\begin{tabular}{|c|c|c|c|c|c|}
\hline Region & 304SS & Inconel 718 & Zircaloy-4 & Ag-ln-Cd $^{\mathbf{a}}$ & Water \\
\hline 1 & 0.0638 & 0.0 & 0.0 & 0.0262 & 0.9100 \\
\hline 2 & 0.1442 & 0.0 & 0.0 & 0.0262 & 0.8296 \\
\hline 3 & 0.2553 & 0.0 & 0.0 & 0.0262 & 0.7185 \\
\hline 4 & 0.3481 & 0.0 & 0.0 & 0.0262 & 0.6257 \\
\hline 5 & 0.2820 & 0.0451 & 0.0083 & Explicit & 0.6646 \\
\hline 6 & 0.0 & 0.0457 & 0.0069 & 0.0 & 0.9474 \\
\hline 7 & 0.0 & 0.0 & 0.0 & 0.0 & 1.0 \\
\hline $8 /$ B4 & 0.0 & 0.005757609 & 0.0 & 0.0 & 0.994242391 \\
\hline /B4Z & 0.0 & 0.0 & 0.008165257 & 0.0 & 0.991834743 \\
\hline 9 & 0.1656 & 0.0306 & 0.0125 & 0.0 & 0.7913 \\
\hline 10 & 0.2848 & 0.0 & 0.0 & 0.0 & 0.7152 \\
\hline 11 & 0.2400 & 0.0 & 0.0 & 0.0 & 0.7600 \\
\hline 12 & 0.0300 & 0.0 & 0.0 & 0.0 & 0.9700 \\
\hline
\end{tabular}

NOTES: All components except fuel rods, instrument tube, guide tubes, and control tubes.

a For Gray APSR, the material is Inconel 600 
Table 2-6. Mark-B4 \& -B4Z Assembly VFs with Black APSRs $0 \%$ Withdrawn

\begin{tabular}{|c|c|c|c|c|}
\hline Region & 304SS & Inconel-718 & Zircaloy-4 & Water \\
\hline 1 & 0.1096 & 0.0 & 0.0 & 0.8904 \\
\hline 2 & 0.2212 & 0.0 & 0.0 & 0.7788 \\
\hline 3 & 0.2828 & 0.0 & 0.0 & 0.7172 \\
\hline 4 & 0.3748 & 0.0 & 0.0 & 0.6252 \\
\hline 5 & 0.2960 & 0.0441 & 0.0081 & 0.6518 \\
\hline 6 & 0.0 & 0.0457 & 0.0069 & 0.9474 \\
\hline 7 & 0.0 & 0.0 & 0.0 & 1.0 \\
\hline $8 /$ B4 & 0.0 & 0.005757609 & 0.0 & 0.994242391 \\
\hline $8 / B 4 Z$ & 0.0 & 0.0 & 0.008165257 & 0.991834743 \\
\hline 9 & 0.1656 & 0.0306 & 0.0125 & 0.7913 \\
\hline 10 & 0.2848 & 0.0 & 0.0 & 0.7152 \\
\hline 11 & 0.2400 & 0.0 & 0.0 & 0.7600 \\
\hline 12 & 0.0300 & 0.0 & 0.0 & 0.9700 \\
\hline
\end{tabular}

NOTE: All components except fuel rods, instrument tube, guide tubes, and control tubes.

Table 2-7. Mark-B4 \& -B4Z Assembly VFs with Gray APSRs $0 \%$ Withdrawn

\begin{tabular}{|c|c|c|c|c|}
\hline Region & 304SS & Inconel-718 & Zircaloy-4 & Water \\
\hline 1 & 0.1081 & 0.0 & 0.0 & 0.8919 \\
\hline 2 & 0.2212 & 0.0 & 0.0 & 0.7788 \\
\hline 3 & 0.2828 & 0.0 & 0.0 & 0.7172 \\
\hline 4 & 0.3748 & 0.0 & 0.0 & 0.6252 \\
\hline 5 & 0.2959 & 0.0441 & 0.0081 & 0.6519 \\
\hline 6 & 0.0 & 0.0457 & 0.0069 & 0.9474 \\
\hline 7 & 0.0 & 0.0 & 0.0 & 1.0 \\
\hline $8 /$ B4 & 0.0 & 0.005757609 & 0.0 & 0.994242391 \\
\hline / B4Z & 0.0 & 0.0 & 0.008165257 & 0.991834743 \\
\hline 9 & 0.1656 & 0.0306 & 0.0125 & 0.7913 \\
\hline 10 & 0.2848 & 0.0 & 0.0 & 0.7152 \\
\hline 11 & 0.2400 & 0.0 & 0.0 & 0.7600 \\
\hline 12 & 0.0300 & 0.0 & 0.0 & 0.9700 \\
\hline
\end{tabular}

NOTE: All components except fuel rods, instrument tube, guide tubes, and control tubes. 
Table 2-8. Mark-B4 \& -B4Z Assembly VFs with BPRAs Inserted

\begin{tabular}{|c|c|c|c|c|}
\hline Region & 304SS & Inconel 718 & Zircaloy-4 & Water \\
\hline 1 & 0.0699 & 0.0 & 0.0 & 0.9301 \\
\hline 2 & 0.1827 & 0.0 & 0.0 & 0.8173 \\
\hline 3 & 0.2937 & 0.0 & 0.0069 & 0.6994 \\
\hline 4 & 0.3890 & 0.0 & 0.0120 & 0.5990 \\
\hline 5 & 0.2874 & 0.0450 & 0.0083 & 0.6593 \\
\hline 6 & 0.0 & 0.0457 & 0.0069 & 0.9474 \\
\hline 7 & 0.0 & 0.0 & 0.0 & 1.0 \\
\hline $8 /$ B4 & 0.0 & 0.005757609 & 0.0 & 0.994242391 \\
\hline / B4Z & 0.0 & 0.0 & 0.008165257 & 0.991834743 \\
\hline 9 & 0.1656 & 0.0306 & 0.0125 & 0.7913 \\
\hline 10 & 0.2848 & 0.0 & 0.0 & 0.7152 \\
\hline 11 & 0.2400 & 0.0 & 0.0 & 0.7600 \\
\hline 12 & 0.0300 & 0.0 & 0.0 & 0.9700 \\
\hline
\end{tabular}

NOTE: All components except fuel rods, instrument tube, guide tubes, and control tubes.

Mark-B4 and -B4Z Fuel Rods- The 208 fuel rods are contained in regions 6 through 9. Region 8 is modeled explicitly. Regions 6,7 , and 9 contain various amounts of stainless steel and Zircaloy in the fuel rod that are representative of the plenum springs and end caps. In addition, these regions also contain helium and fission gases, as well as the Zircaloy cladding.

Figure 2-7 indicates the axial view of the fuel rod. The B4 and B4Z fuel rods are represented as a cylinder with $1.0922 \mathrm{~cm}$ diameter with $Y_{\text {bottom }}=5.69 \mathrm{~cm}$ and $Y_{\text {top }}=396.056 \mathrm{~cm}$, where $Y=0$ is at the bottom of region 9. Table 2-9 provides the Mark-B4 and $-\mathrm{B} 4 \mathrm{Z}$ fuel rod volume fractions.

Table 2-9. Mark-B4 \& -B4Z Fuel Rod VFs by Region

\begin{tabular}{|c|c|c|c|c|}
\hline Region & Stainless Steel & Zircaloy-4 & Cladding $^{\text {a }}$ & Helium \\
\hline 6 & 0.0 & 0.3344 & 0.1940 & 0.4716 \\
\hline 7 & 0.0810 & 0.0439 & 0.2313 & 0.6438 \\
\hline 8 & $\begin{array}{l}\text { Explicit Idealization } \\
\text { Fuel Clad: Zircaloy } \mathrm{OD}=1.0922 \mathrm{~cm}, \mathrm{ID}=0.95758 \mathrm{~cm} \\
\text { Fuel Pellet: } \mathrm{UO}_{2} \mathrm{OD}^{\mathrm{b}}=0.936244 \mathrm{~cm}, 95 \% \text { Theoretical Density } \\
\text { Helium in gaps }\end{array}$ & \multicolumn{1}{|l}{} \\
\hline 9 & 0.1230 & 0.1926 & 0.2163 & 0.4681 \\
\hline
\end{tabular}

NOTES: ${ }^{a}$ The Zircaloy cladding extends the entire length of the fuel rod but does not include the end caps.

befer to Table 3-1 for fuel pellet OD for an individual fuel batch. 
Mark-B4 and -B4Z Guide Tubes- Figure 2-5 indicates an axial view of the guide tubes.

- Material $=$ Zircaloy -4

- 16 guide tubes, $\mathrm{OD}=1.3462 \mathrm{~cm}, \mathrm{ID}=1.26492 \mathrm{~cm}$

- Length $=397.035 \mathrm{~cm}$

- $Y_{\text {bottom }} \approx 6.032 \mathrm{~cm}$

- $\mathrm{Y}_{\text {top }} \approx 403.067 \mathrm{~cm}$

Mark-B4 and -B4Z Instrument Tube- Figure 2-6 indicates an axial view of the instrument tube.

- Material $=$ Zircaloy-4

- One tube, $\mathrm{OD}=1.38193 \mathrm{~cm}, \mathrm{ID}=1.12014 \mathrm{~cm}$

- Length $=389.890 \mathrm{~cm}$

- $Y_{\text {bottom }} \approx 3.175 \mathrm{~cm}$

- $\mathrm{Y}_{\text {top }} \approx 393.065 \mathrm{~cm}$ 


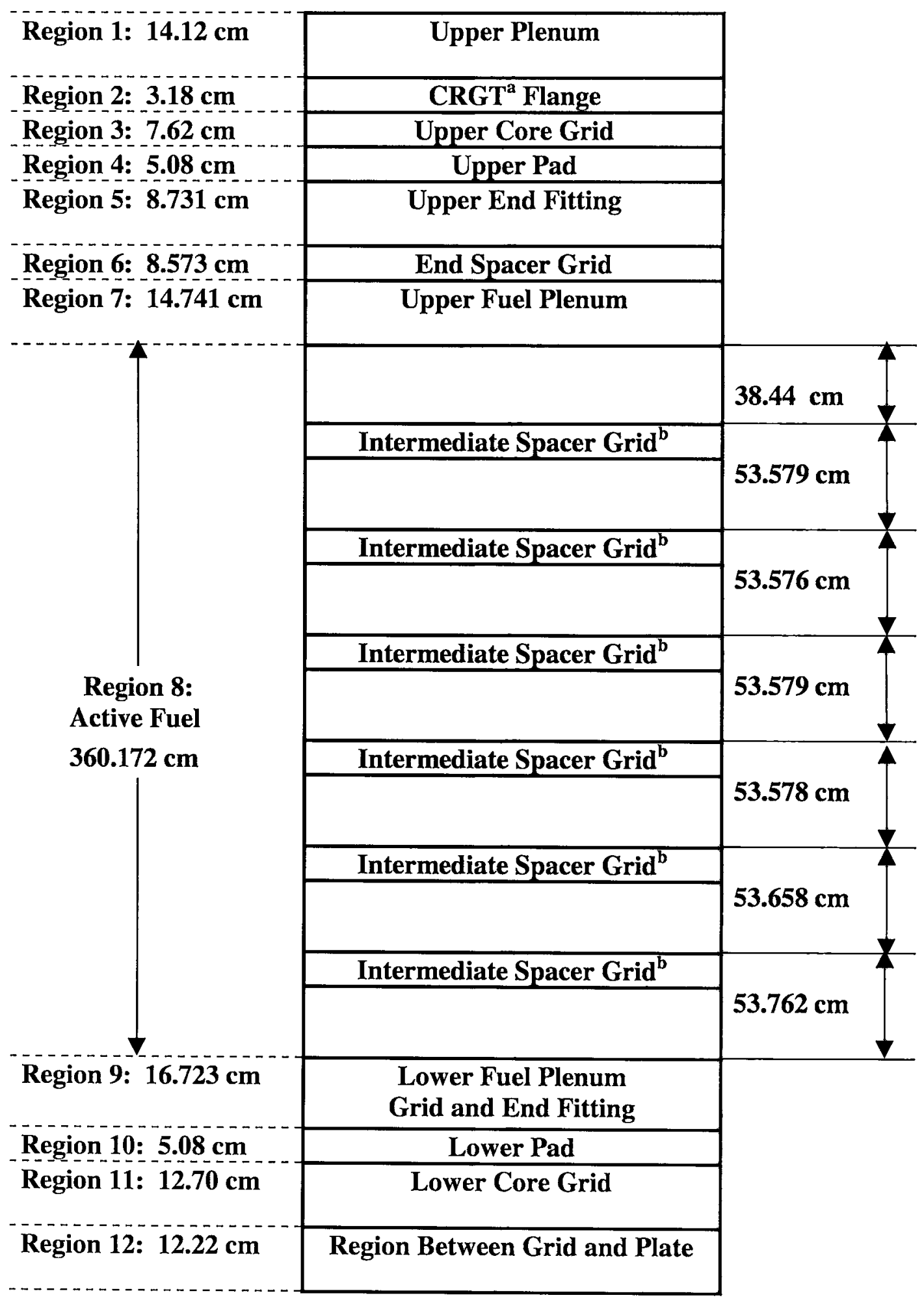

NOTES: ${ }^{a}$ Control Rod Guide Tube (CRGT)

${ }^{\mathrm{b}}$ Intermediate spacer grid height is $3.81 \mathrm{~cm}$

Figure 2-3. Mark-B4 Assembly Axial Dimensions by Region 


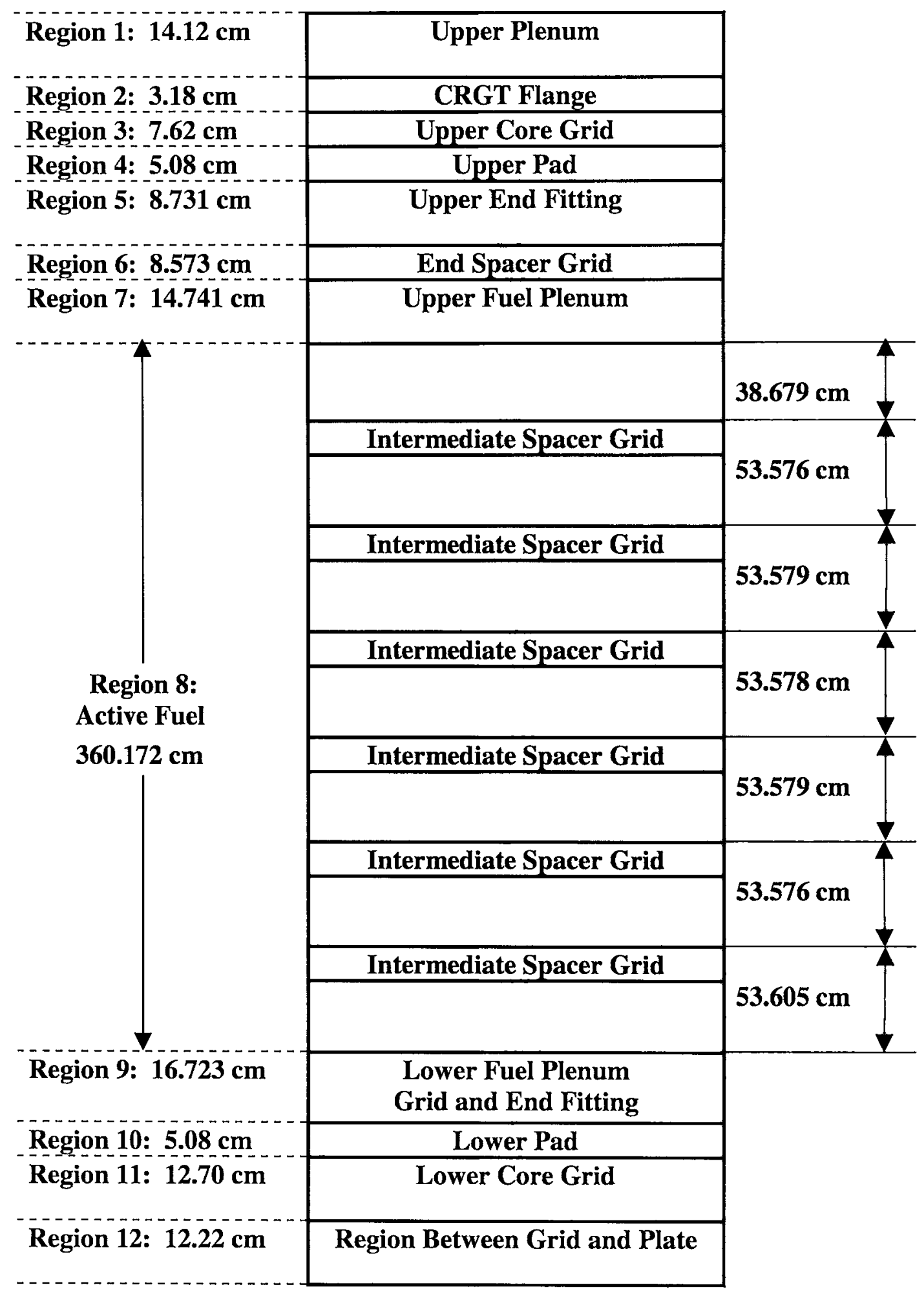

NOTE: Intermediate spacer grid height is $5.08 \mathrm{~cm}$

Figure 2-4. Mark-B4Z Assembly Axial Dimensions by Region 


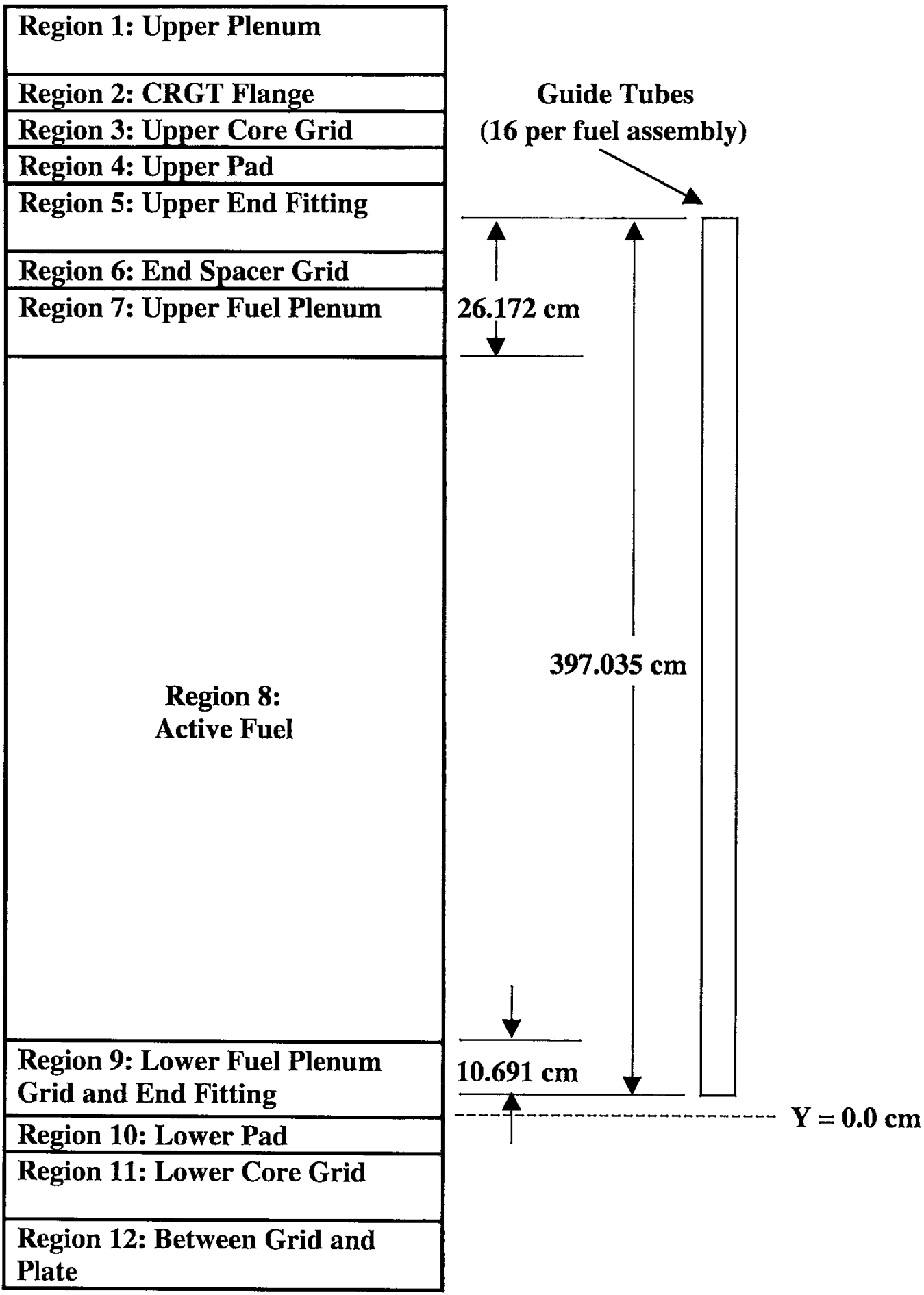

Figure 2-5. Mark-B4 \& -B4Z Assembly Axial Dimensions for Guide Tubes 


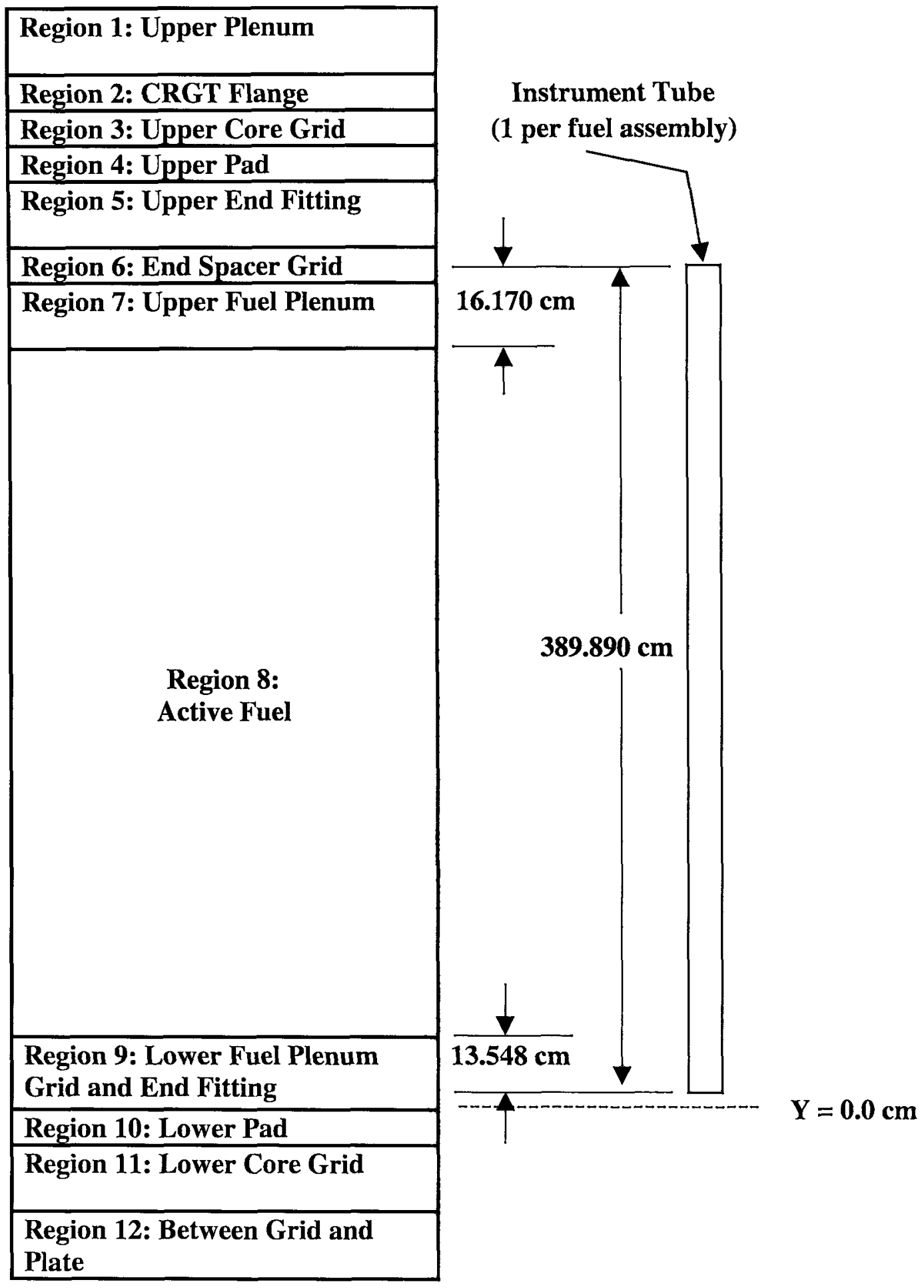

Figure 2-6. Mark-B4 \& -B4Z Assembly Axial Dimensions for Instrument Tube 


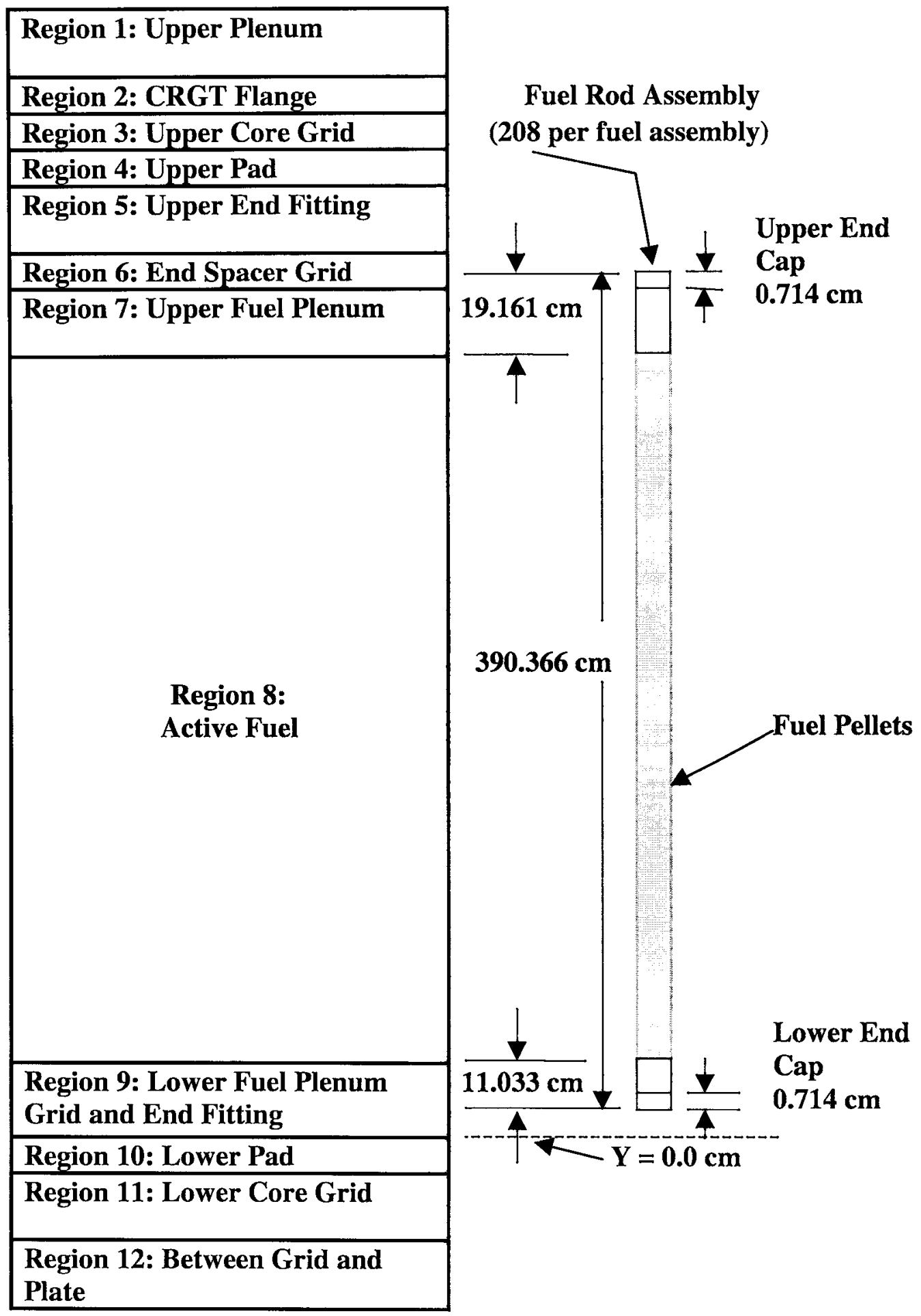

NOTE: $Y=0.0 \mathrm{~cm}$ at the bottom of Region 9

Figure 2-7. Mark-B4 \& -B4Z Assembly Axial Dimensions for Standard Fuel Rod 


\subsection{MARK-B8 FUEL ASSEMBLY}

Figure 2-8 provides region boundaries and axial locations for intermediate spacer grids. The intermediate spacer grid height is $4.826 \mathrm{~cm}$ for each of the six grids. Tables 2-10 through 2-14 provide volume fractions for the Mark-B8 fuel assemblies with and without control components.

Table 2-10. Mark-B8 Assembly VFs by Region

\begin{tabular}{|c|c|c|c|c|c|c|}
\hline Region & 304SS & 308SS & 316SS & Inconel 718 & Zircaloy-4 & Water \\
\hline 1 & 0.0578 & 0.0 & 0.0 & 0.0 & 0.0 & 0.9422 \\
\hline 2 & 0.1381 & 0.0 & 0.0 & 0.0 & 0.0 & 0.8619 \\
\hline 3 & 0.2491 & 0.0 & 0.0 & 0.0 & 0.0 & 0.7509 \\
\hline 4 & 0.3418 & 0.0 & 0.0 & 0.0 & 0.0 & 0.6582 \\
\hline 5 & 0.2831 & 0.0 & 0.0011 & 0.0460 & 0.0067 & 0.6632 \\
\hline 6 & 0.0095 & 0.0 & 0.0 & 0.0 & 0.0 & 0.9905 \\
\hline 7 & 0.0 & 0.0 & 0.0 & 0.0342 & 0.0007 & 0.9650 \\
\hline 8 & 0.0 & 0.0 & 0.0 & 0.0 & 0.0091420 & 0.9908580 \\
\hline 9 & 0.1675 & 0.0011 & 0.0 & 0.0255 & 0.0113 & 0.7947 \\
\hline 10 & 0.2848 & 0.0 & 0.0 & 0.0 & 0.0 & 0.7152 \\
\hline 11 & 0.2400 & 0.0 & 0.0 & 0.0 & 0.0 & 0.7600 \\
\hline 12 & 0.0300 & 0.0 & 0.0 & 0.0 & 0.0 & 0.9700 \\
\hline
\end{tabular}

NOTE: All components except fuel rods, instrument tube, and guide tubes.

Table 2-11. Mark-B8 Assembly VFs with RCCAs 0\% Withdrawn

\begin{tabular}{|c|c|c|c|c|c|c|}
\hline Region & 304SS & 308SS & 316SS & Inconel 718 & Zircaloy-4 & Water \\
\hline 1 & 0.0934 & 0.0 & 0.0 & 0.0 & 0.0 & 0.9066 \\
\hline 2 & 0.1945 & 0.0 & 0.0 & 0.0 & 0.0 & 0.8055 \\
\hline 3 & 0.3481 & 0.0 & 0.0 & 0.0 & 0.0 & 0.6519 \\
\hline 4 & 0.3748 & 0.0 & 0.0 & 0.0 & 0.0 & 0.6252 \\
\hline 5 & 0.3056 & 0.0 & 0.0011 & 0.0460 & 0.0067 & 0.6407 \\
\hline 6 & 0.0095 & 0.0 & 0.0 & 0.0 & 0.0 & 0.9905 \\
\hline 7 & 0.0 & 0.0 & 0.0 & 0.0342 & 0.0007 & 0.9650 \\
\hline 8 & 0.0 & 0.0 & 0.0 & 0.0 & 0.0091420 & 0.9908580 \\
\hline 9 & 0.1675 & 0.0011 & 0.0 & 0.0255 & 0.0113 & 0.7947 \\
\hline 10 & 0.2848 & 0.0 & 0.0 & 0.0 & 0.0 & 0.7152 \\
\hline 11 & 0.2400 & 0.0 & 0.0 & 0.0 & 0.0 & 0.7600 \\
\hline 12 & 0.0300 & 0.0 & 0.0 & 0.0 & 0.0 & 0.9700 \\
\hline
\end{tabular}

NOTE: All components except fuel rods, instrument tube, and guide tubes. 
Table 2-12. Mark-B8 Assembly VFs with RCCAs \& APSRs $100 \%$ Withdrawn

\begin{tabular}{|c|c|c|c|c|c|c|c|}
\hline Region & 304SS & 308SS & 316SS & Inconel 718 & Zircaloy-4 & Inconel 600 & Water \\
\hline 1 & 0.0638 & 0.0 & 0.0 & 0.0 & 0.0 & 0.0262 & 0.9100 \\
\hline 2 & 0.1442 & 0.0 & 0.0 & 0.0 & 0.0 & 0.0262 & 0.8296 \\
\hline 3 & 0.2553 & 0.0 & 0.0 & 0.0 & 0.0 & 0.0262 & 0.7185 \\
\hline 4 & 0.3481 & 0.0 & 0.0 & 0.0 & 0.0 & 0.0262 & 0.6257 \\
\hline 5 & 0.2896 & 0.0 & 0.0011 & 0.0470 & 0.0068 & Explicit & 0.6554 \\
\hline 6 & 0.0095 & 0.0 & 0.0 & 0.0 & 0.0 & 0.0 & 0.9905 \\
\hline 7 & 0.0 & 0.0 & 0.0 & 0.0342 & 0.0007 & 0.0 & 0.9650 \\
\hline 8 & 0.0 & 0.0 & 0.0 & 0.0 & 0.0091420 & 0.0 & 0.9908580 \\
\hline 9 & 0.1675 & 0.0011 & 0.0 & 0.0255 & 0.0113 & 0.0 & 0.7947 \\
\hline 10 & 0.2848 & 0.0 & 0.0 & 0.0 & 0.0 & 0.0 & 0.7152 \\
\hline 11 & 0.2400 & 0.0 & 0.0 & 0.0 & 0.0 & 0.0 & 0.7600 \\
\hline 12 & 0.0300 & 0.0 & 0.0 & 0.0 & 0.0 & 0.0 & 0.9700 \\
\hline
\end{tabular}

NOTE: All components except fuel rods, instrument tube, and guide tubes.

Table 2-13. Mark-B8 Assembly VFs with Gray APSRs $0 \%$ Withdrawn

\begin{tabular}{|c|c|c|c|c|c|c|}
\hline Region & 304SS & 308SS & 316SS & Inconel 718 & Zircaloy-4 & Water \\
\hline 1 & 0.1081 & 0.0 & 0.0 & 0.0 & 0.0 & 0.8919 \\
\hline 2 & 0.2212 & 0.0 & 0.0 & 0.0 & 0.0 & 0.7788 \\
\hline 3 & 0.2828 & 0.0 & 0.0 & 0.0 & 0.0 & 0.7172 \\
\hline 4 & 0.3748 & 0.0 & 0.0 & 0.0 & 0.0 & 0.6252 \\
\hline 5 & 0.3033 & 0.0 & 0.0011 & 0.0460 & 0.0067 & 0.6430 \\
\hline 6 & 0.0095 & 0.0 & 0.0 & 0.0 & 0.0 & 0.9905 \\
\hline 7 & 0.0 & 0.0 & 0.0 & 0.0342 & 0.0007 & 0.9650 \\
\hline 8 & 0.0 & 0.0 & 0.0 & 0.0 & 0.0091420 & 0.9908580 \\
\hline 9 & 0.1675 & 0.0011 & 0.0 & 0.0255 & 0.0113 & 0.7947 \\
\hline 10 & 0.2848 & 0.0 & 0.0 & 0.0 & 0.0 & 0.7152 \\
\hline 11 & 0.2400 & 0.0 & 0.0 & 0.0 & 0.0 & 0.7600 \\
\hline 12 & 0.0300 & 0.0 & 0.0 & 0.0 & 0.0 & 0.9700 \\
\hline
\end{tabular}

NOTE: All components except fuel rods, instrument tube, guide tubes, and control tubes. 
Table 2-14. Mark-B8 Assembly VFs with BPRAs Inserted

\begin{tabular}{|c|c|c|c|c|c|c|}
\hline Region & 304SS & 308SS & 316SS & Inconel 718 & Zircaloy-4 & Water \\
\hline 1 & 0.0699 & 0.0 & 0.0 & 0.0 & 0.0 & 0.9301 \\
\hline 2 & 0.1827 & 0.0 & 0.0 & 0.0 & 0.0 & 0.8173 \\
\hline 3 & 0.2937 & 0.0 & 0.0 & 0.0 & 0.0069 & 0.6994 \\
\hline 4 & 0.3890 & 0.0 & 0.0 & 0.0 & 0.0120 & 0.5990 \\
\hline 5 & 0.2950 & 0.0 & 0.0011 & 0.0470 & 0.0068 & 0.6500 \\
\hline 6 & 0.0095 & 0.0 & 0.0 & 0.0 & 0.0 & 0.9905 \\
\hline 7 & 0.0 & 0.0 & 0.0 & 0.0342 & 0.0007 & 0.9650 \\
\hline 8 & 0.0 & 0.0 & 0.0 & 0.0 & 0.0091420 & 0.9908580 \\
\hline 9 & 0.1675 & 0.0011 & 0.0 & 0.0255 & 0.0113 & 0.7947 \\
\hline 10 & 0.2848 & 0.0 & 0.0 & 0.0 & 0.0 & 0.7152 \\
\hline 11 & 0.2400 & 0.0 & 0.0 & 0.0 & 0.0 & 0.7600 \\
\hline 12 & 0.0300 & 0.0 & 0.0 & 0.0 & 0.0 & 0.9700 \\
\hline
\end{tabular}

NOTE: All components except fuel rods, instrument tube, guide tubes, and control tubes.

Mark-B8 Fuel Rods: The 208 fuel rods are contained in regions 6 through 9 . Region 8 is modeled explicitly. Regions 6, 7 and 9 contain various amounts of stainless steel and Zircaloy in the fuel rod that are representative of the plenum springs and end caps. In addition, these regions also contain helium and fission gases, as well as the Zircaloy cladding.

Standard Mark-B8 Fuel Rods: The Standard B8 fuel rod is represented as a cylinder with $1.0922 \mathrm{~cm}$ diameter with $Y_{\text {bottom }}=4.277 \mathrm{~cm}$ and $Y_{\text {top }}=395.081 \mathrm{~cm}$, where $Y=0$ is at the bottom of region 9. Figure 2-11 indicates the axial view of the fuel rod. The fuel rod assembly volume fractions for materials in these regions are provided in Table 2-15.

Table 2-15. Mark-B8 Fuel Rod VFs by Region

\begin{tabular}{|c|c|c|c|c|}
\hline Region & 302SS & A286 Alloy SS & Zircaloy-4 & Helium \\
\hline 6 & 0.0749 & 0.0 & 0.3714 & 0.5537 \\
\hline 7 & 0.1408 & 0.0 & 0.2359 & 0.6232 \\
\hline 8 & $\begin{array}{l}\text { Explicit Idealization } \\
\text { Fuel Clad: Zircaloy } \mathrm{OD}=1.0922 \mathrm{~cm}, I \mathrm{D}=0.95758 \mathrm{~cm} \\
\text { Fuel Pellet: } \mathrm{UO}_{2} \mathrm{OD}=0.936244 \mathrm{~cm}, 95 \% \text { Theoretical Density } \\
\text { Helium in gaps }\end{array}$ & 0.6590 & 0.2733 \\
\hline 9 & 0.0 & 0.0677 & 0.650
\end{tabular}


Mark-B8 Guide Tubes - (Figure 2-9 indicates an axial view of a guide tube)

Material $=$ Zircaloy -4

16 guide tubes, $\mathrm{OD}=1.3462 \mathrm{~cm}, \mathrm{ID}=1.26492 \mathrm{~cm}$

Length $=398.739 \mathrm{~cm}$

$\mathrm{Y}_{\text {bottom }} \approx 3.851 \mathrm{~cm}$

$\mathrm{Y}_{\text {top }} \approx 402.590 \mathrm{~cm}$

Mark-B8 Instrument Tube - (Figure 2-10 indicates an axial view of an instrument tube)

Material $=$ Zircaloy -4

One tube, $\mathrm{OD}=1.25222 \mathrm{~cm}, \mathrm{DD}=1.12014 \mathrm{~cm}$

Length $=394.295 \mathrm{~cm}$

$\mathrm{Y}_{\text {bottom }} \approx 1.608 \mathrm{~cm}$

$\mathrm{Y}_{\text {top }} \approx 395.903 \mathrm{~cm}$ 


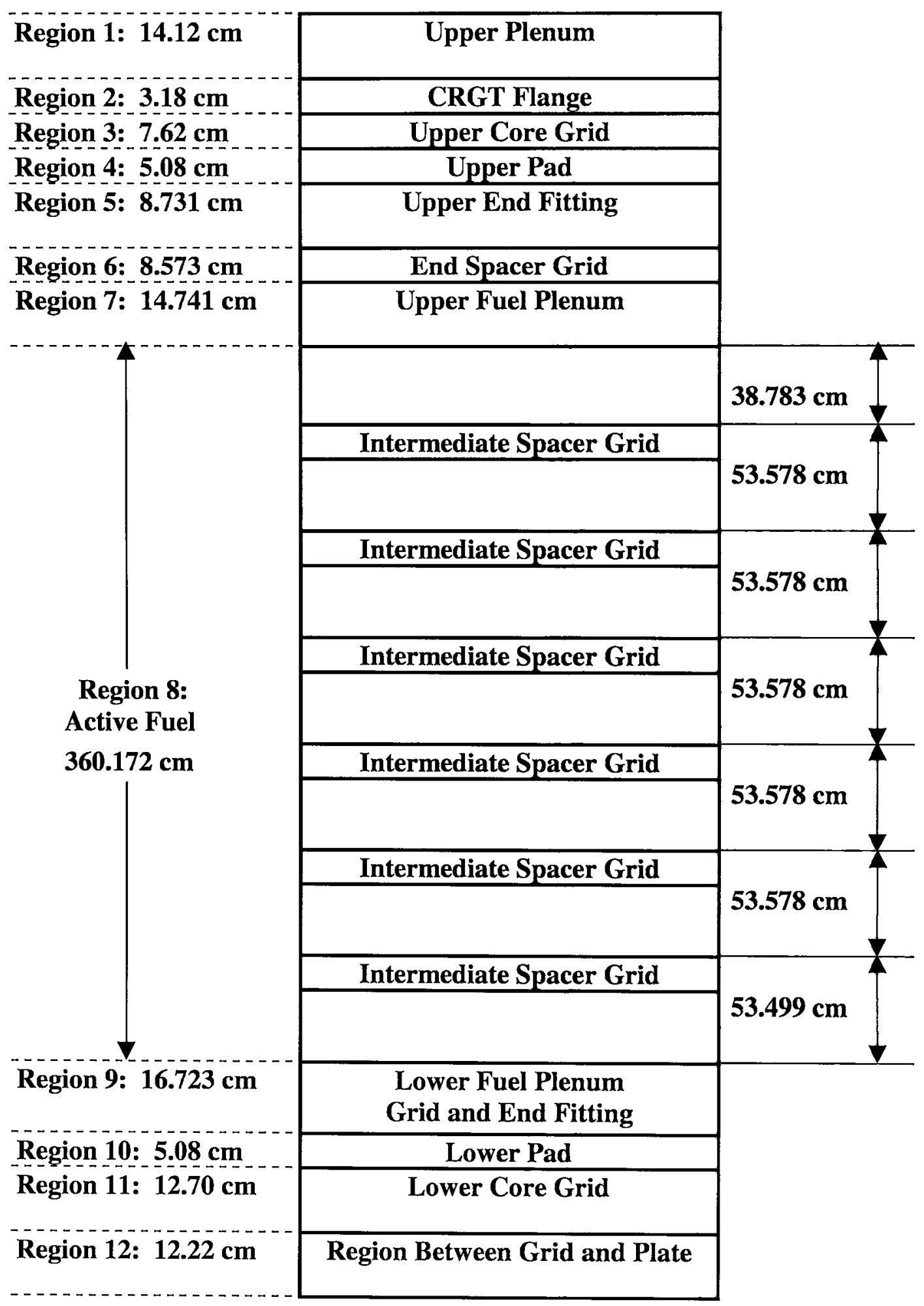

NOTE: Intermediate spacer grid height is $4.826 \mathrm{~cm}$

Figure 2-8. Mark-B8 Assembly Axial Dimensions by Region 


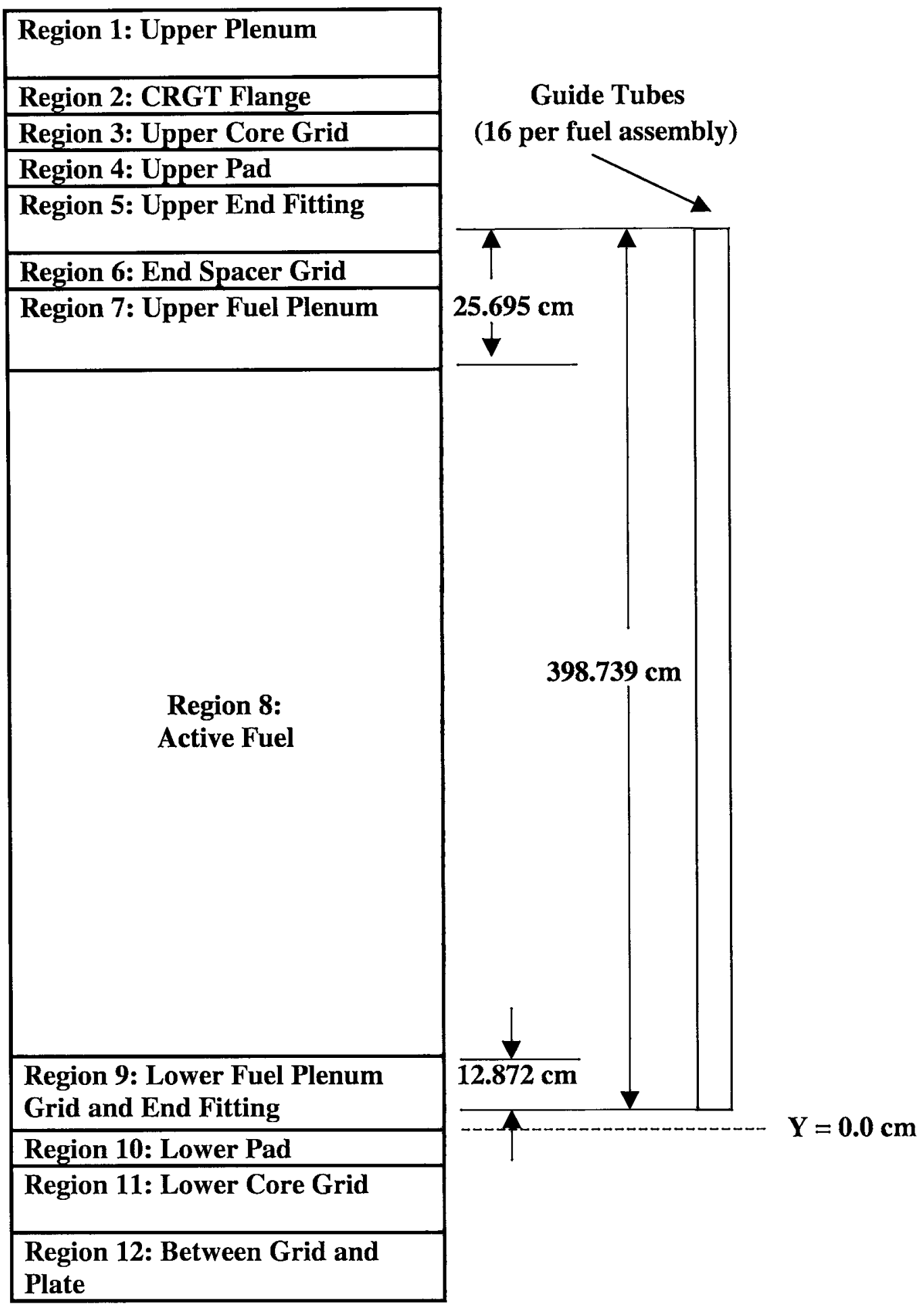

Figure 2-9. Mark-B8 Assembly Axial Dimensions for Guide Tubes 


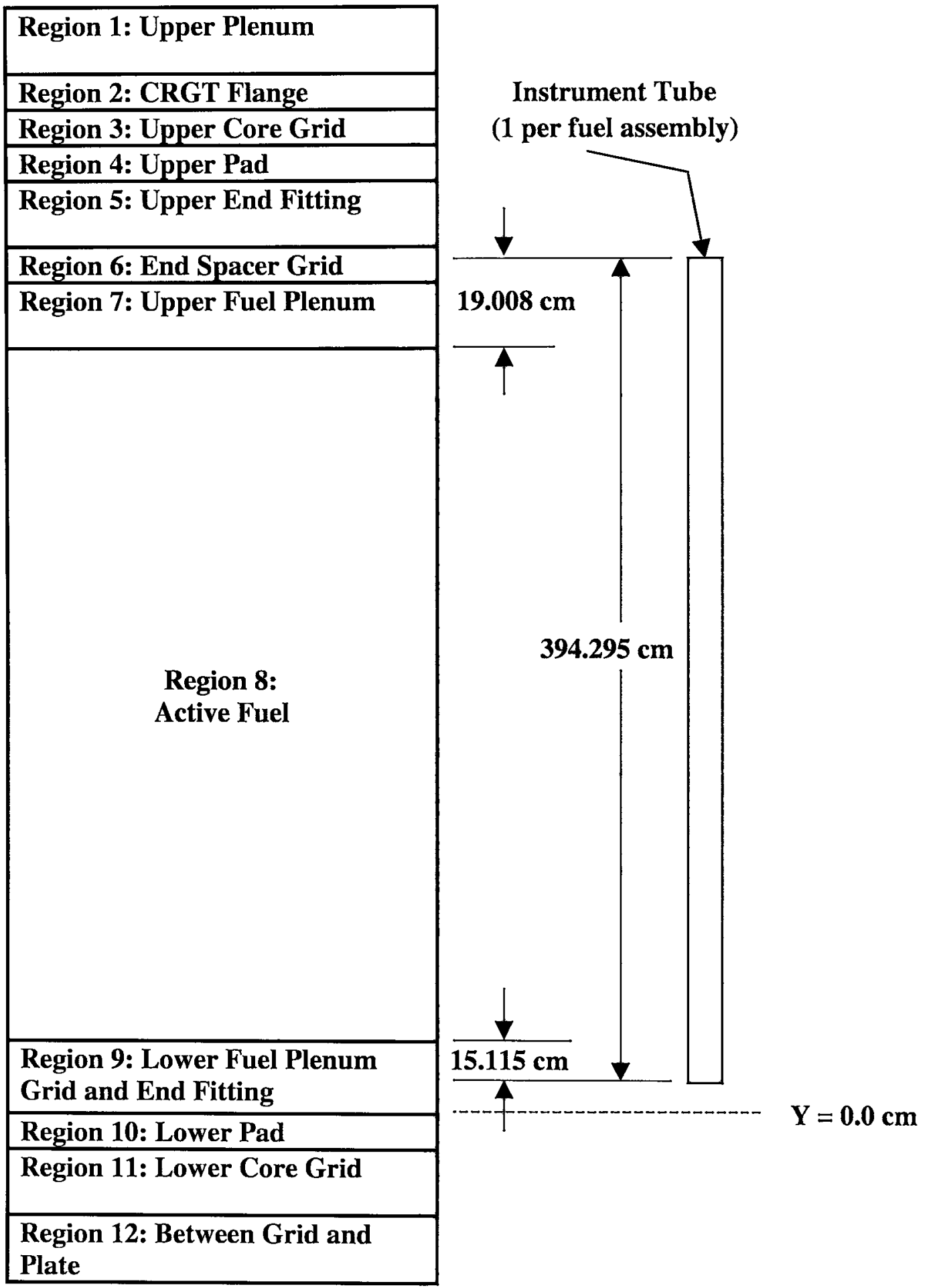

Figure 2-10. Mark-B8 Assembly Axial Dimensions for Instrument Tube 


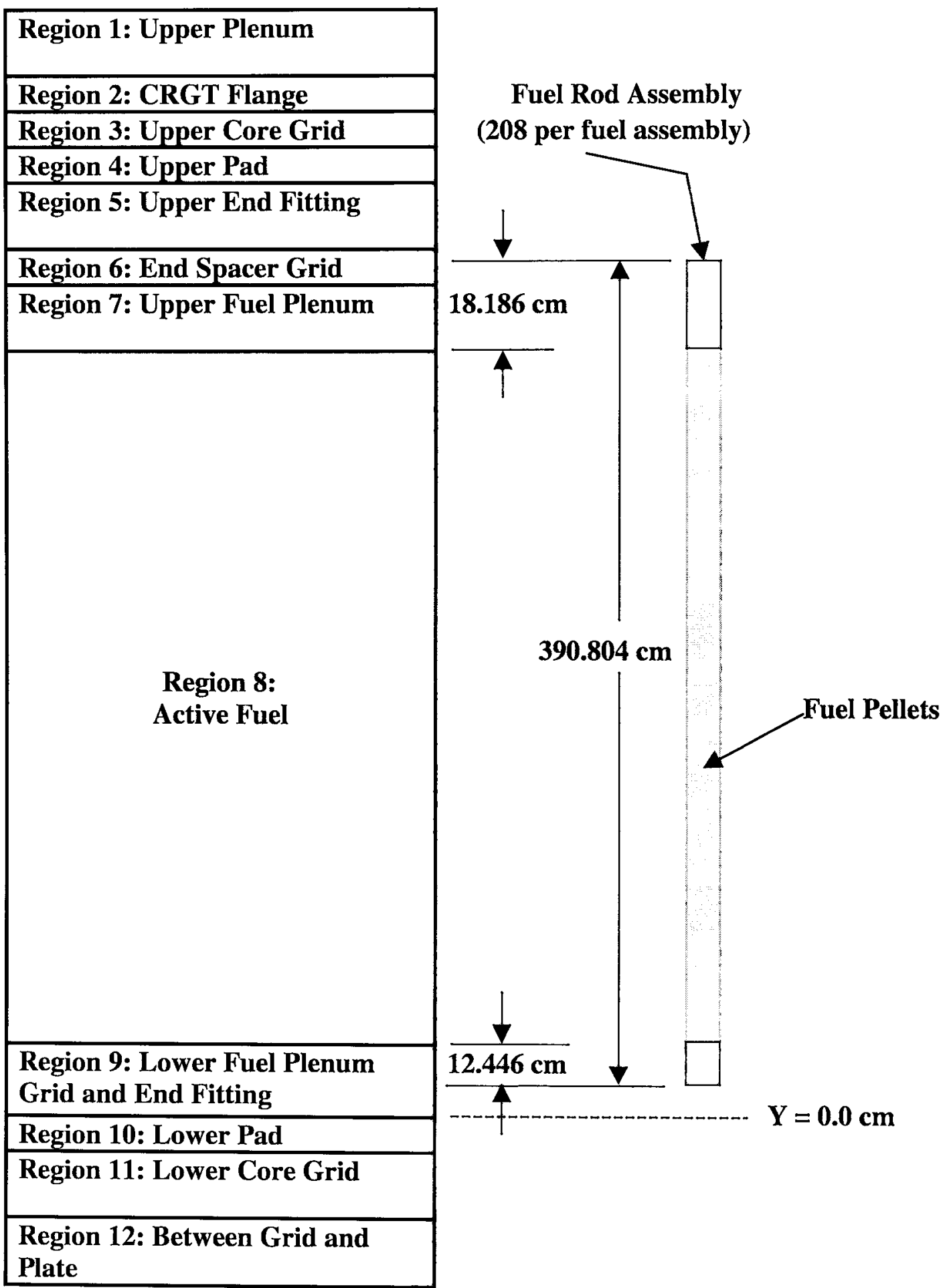

Figure 2-11. Mark-B8 Assembly Axial Dimensions for Standard Fuel Rod 


\subsection{MARK-B9 FUEL ASSEMBLY}

Figure 2-12 provides region boundaries and axial locations for intermediate spacer grids. The intermediate spacer grid height is $4.826 \mathrm{~cm}$ for each of the six grids. Tables 2-16 through 2-20 provides volume fractions for Mark-B9 fuel assemblies with and without control components.

Table 2-16. Mark-B9 Assembly VFs by Region

\begin{tabular}{|c|c|c|c|c|c|}
\hline Region & 304SS & 316SS & Inconel 718 & Zircaloy-4 & Water \\
\hline 1 & 0.0578 & 0.0 & 0.0 & 0.0 & 0.9422 \\
\hline 2 & 0.1381 & 0.0 & 0.0 & 0.0 & 0.8619 \\
\hline 3 & 0.2491 & 0.0 & 0.0 & 0.0 & 0.7509 \\
\hline 4 & 0.3418 & 0.0 & 0.0 & 0.0 & 0.6582 \\
\hline 5 & 0.2795 & 0.0011 & 0.0456 & 0.0068 & 0.6670 \\
\hline 6 & 0.0070 & 0.0 & 0.0 & 0.0 & 0.9930 \\
\hline 7 & 0.0 & 0.0 & 0.0342 & 0.0007 & 0.9650 \\
\hline 8 & 0.0 & 0.0 & 0.0 & 0.0091560 & 0.9908440 \\
\hline 9 & 0.1655 & 0.0 & 0.0242 & 0.0416 & 0.7686 \\
\hline 10 & 0.2848 & 0.0 & 0.0 & 0.0 & 0.7152 \\
\hline 11 & 0.2400 & 0.0 & 0.0 & 0.0 & 0.7600 \\
\hline 12 & 0.0300 & 0.0 & 0.0 & 0.0 & 0.9700 \\
\hline
\end{tabular}

NOTE: All components except fuel rods, instrument tube and guide tubes.

Table 2-17. Mark-B9 Assembly VFs with RCCAs 0\% Withdrawn

\begin{tabular}{|c|c|c|c|c|c|}
\hline Region & 304SS & 316SS & Inconel 718 & Zircaloy-4 & Water \\
\hline 1 & 0.0934 & 0.0 & 0.0 & 0.0 & 0.9066 \\
\hline 2 & 0.1945 & 0.0 & 0.0 & 0.0 & 0.8055 \\
\hline 3 & 0.3481 & 0.0 & 0.0 & 0.0 & 0.6519 \\
\hline 4 & 0.3748 & 0.0 & 0.0 & 0.0 & 0.6252 \\
\hline 5 & 0.3018 & 0.0011 & 0.0456 & 0.0068 & 0.6446 \\
\hline 6 & 0.0070 & 0.0 & 0.0 & 0.0 & 0.9930 \\
\hline 7 & 0.0 & 0.0 & 0.0342 & 0.0007 & 0.9650 \\
\hline 8 & 0.0 & 0.0 & 0.0 & 0.0091560 & 0.9908440 \\
\hline 9 & 0.1655 & 0.0 & 0.0242 & 0.0416 & 0.7686 \\
\hline 10 & 0.2848 & 0.0 & 0.0 & 0.0 & 0.7152 \\
\hline 11 & 0.2400 & 0.0 & 0.0 & 0.0 & 0.7600 \\
\hline 12 & 0.0300 & 0.0 & 0.0 & 0.0 & 0.9700 \\
\hline
\end{tabular}

NOTE: All components except fuel rods, instrument tube, guide tubes and control tubes. 
Table 2-18. Mark-B9 Assembly VFs with RCCAs \& Gray APSRs $100 \%$ Withdrawn

\begin{tabular}{|c|c|c|c|c|c|c|}
\hline Region & 304SS & 316SS & Inconel 718 & Zircaloy-4 & Inconel 600 & Water \\
\hline 1 & 0.0638 & 0.0 & 0.0 & 0.0 & 0.0262 & 0.9100 \\
\hline 2 & 0.1442 & 0.0 & 0.0 & 0.0 & 0.0262 & 0.8296 \\
\hline 3 & 0.2553 & 0.0 & 0.0 & 0.0 & 0.0262 & 0.7185 \\
\hline 4 & 0.3481 & 0.0 & 0.0 & 0.0 & 0.0262 & 0.6257 \\
\hline 5 & 0.2859 & 0.0011 & 0.0467 & 0.0069 & Explicit & 0.6594 \\
\hline 6 & 0.0070 & 0.0 & 0.0 & 0.0 & 0.0 & 0.9930 \\
\hline 7 & 0.0 & 0.0 & 0.0342 & 0.0007 & 0.0 & 0.9650 \\
\hline 8 & 0.0 & 0.0 & 0.0 & 0.0091560 & 0.0 & 0.9908440 \\
\hline 9 & 0.1655 & 0.0 & 0.0242 & 0.0416 & 0.0 & 0.7686 \\
\hline 10 & 0.2848 & 0.0 & 0.0 & 0.0 & 0.0 & 0.7152 \\
\hline 11 & 0.2400 & 0.0 & 0.0 & 0.0 & 0.0 & 0.7600 \\
\hline 12 & 0.0300 & 0.0 & 0.0 & 0.0 & 0.0 & 0.9700 \\
\hline
\end{tabular}

NOTE: All components except fuel rods, instrument tube, guide tubes and control tubes.

Table 2-19. Mark-B9 Assembly VFs with Gray APSRs $0 \%$ Withdrawn

\begin{tabular}{|c|c|c|c|c|c|}
\hline Region & 304SS & 316SS & Inconel 718 & Zircaloy-4 & Water \\
\hline 1 & 0.1081 & 0.0 & 0.0 & 0.0 & 0.8919 \\
\hline 2 & 0.2212 & 0.0 & 0.0 & 0.0 & 0.7788 \\
\hline 3 & 0.2828 & 0.0 & 0.0 & 0.0 & 0.7172 \\
\hline 4 & 0.3748 & 0.0 & 0.0 & 0.0 & 0.6252 \\
\hline 5 & 0.2996 & 0.0011 & 0.0456 & 0.0068 & 0.6469 \\
\hline 6 & 0.0070 & 0.0 & 0.0 & 0.0 & 0.9930 \\
\hline 7 & 0.0 & 0.0 & 0.0342 & 0.0007 & 0.9650 \\
\hline 8 & 0.0 & 0.0 & 0.0 & 0.0091560 & 0.9908440 \\
\hline 9 & 0.1655 & 0.0 & 0.0242 & 0.0416 & 0.7686 \\
\hline 10 & 0.2848 & 0.0 & 0.0 & 0.0 & 0.7152 \\
\hline 11 & 0.2400 & 0.0 & 0.0 & 0.0 & 0.7600 \\
\hline 12 & 0.0300 & 0.0 & 0.0 & 0.0 & 0.9700 \\
\hline
\end{tabular}

NOTE: All components except fuel rods, instrument tube, guide tubes and control tubes. 
Table 2-20. Mark-B9 Assembly VFs with BPRAs Inserted

\begin{tabular}{|c|c|c|c|c|c|}
\hline Region & 304SS & 316SS & Inconel 718 & Zircaloy-4 & Water \\
\hline 1 & 0.0699 & 0.0 & 0.0 & 0.0 & 0.9301 \\
\hline 2 & 0.1827 & 0.0 & 0.0 & 0.0 & 0.8173 \\
\hline 3 & 0.2937 & 0.0 & 0.0 & 0.0069 & 0.6994 \\
\hline 4 & 0.3890 & 0.0 & 0.0 & 0.0120 & 0.5990 \\
\hline 5 & 0.2913 & 0.0011 & 0.0466 & 0.0069 & 0.6540 \\
\hline 6 & 0.0070 & 0.0 & 0.0 & 0.0 & 0.9930 \\
\hline 7 & 0.0 & 0.0 & 0.0342 & 0.0007 & 0.9650 \\
\hline 8 & 0.0 & 0.0 & 0.0 & 0.0091560 & 0.9908440 \\
\hline 9 & 0.1655 & 0.0 & 0.0242 & 0.0416 & 0.7686 \\
\hline 10 & 0.2848 & 0.0 & 0.0 & 0.0 & 0.7152 \\
\hline 11 & 0.2400 & 0.0 & 0.0 & 0.0 & 0.7600 \\
\hline 12 & 0.0300 & 0.0 & 0.0 & 0.0 & 0.9700 \\
\hline
\end{tabular}

NOTE: All components except fuel rods, instrument tube, guide tubes and control tubes.

Mark-B9 Fuel Rods: There are 208 fuel rods in each fuel assembly, some of which contain Gadolinia. The assemblies that contain Gadolinia fuel rods consist of 204 standard fuel rods and 4 Gadolinia fuel rods. All fuel rods are contained in regions 6 through 9 . Part of region 8 is modeled explicitly. Regions 6, 7,8 and 9 contain various amounts of stainless steel and Zircaloy in the fuel rod that are representative of the plenum springs and end caps. In addition, these regions also contain helium and fission gases, as well as the Zircaloy cladding.

Standard Mark-B9 Fuel Rods: The Standard B9 fuel rod is represented as a cylinder with $1.0922 \mathrm{~cm}$ diameter with $Y_{\text {bottom }}=4.277 \mathrm{~cm}$ and $Y_{\text {top }}=395.081 \mathrm{~cm}$, where $Y=0$ is at the bottom of Region 9. Figure 2-15 indicates the axial view of the fuel rod. The fuel rod assembly volume fractions for materials in these regions are provided in Table 2-21.

Table 2-21. Mark-B9 Standard Fuel Rod VFs by Region

\begin{tabular}{|c|c|c|c|c|}
\hline Region & 302SS & A286 Alloy & Zircaloy-4 & Helium \\
\hline 6 & 0.0751 & 0.0 & 0.3714 & 0.5535 \\
\hline 7 & 0.1388 & 0.0 & 0.2351 & 0.6261 \\
\hline 8 & \multicolumn{4}{|c|}{$\begin{array}{l}\text { Explicit Idealization from } Y=16.723 \mathrm{~cm} \text { to } Y=373.834 \mathrm{~cm} \\
\text { Fuel Clad: Zircaloy } O D=1.0922 \mathrm{~cm}, I D=0.95758 \mathrm{~cm} \\
\text { Fuel Pellet: } \mathrm{UO}_{2} \mathrm{OD}=0.9398 \mathrm{~cm}, 95 \% \text { Theoretical Density } \\
\text { Helium in gaps }\end{array}$} \\
\hline & \multicolumn{4}{|c|}{ From $Y=373.834 \mathrm{~cm}$ to $Y=376.895 \mathrm{~cm}(\Delta Y=3.061 \mathrm{~cm})$} \\
\hline & 0.1366 & 0.0 & 0.2314 & 0.6320 \\
\hline 9 & 0.0 & 0.0678 & 0.6587 & 0.2736 \\
\hline
\end{tabular}


Gadolinia Mark-B9 Fuel Rods: Figure 2-16 indicates the axial view of the fuel rod. The Gadolinia B9 fuel rod is represented as a cylinder with $1.0922 \mathrm{~cm}$ diameter with $Y_{\text {bottom }}=4.460$ $\mathrm{cm}$ and $Y_{\text {top }}=395.643 \mathrm{~cm}$, where $\mathrm{Y}=0$ is at the bottom of region 9 . TMI-1 batches $12 \mathrm{C}$ and $12 \mathrm{E}$ have 4 Gadolinia rods in each fuel assembly. The Gadolinia rods have $2 \mathrm{wt} \% \mathrm{Gd}_{2} \mathrm{O}_{3}$ and $4.19 \mathrm{wt} \%{ }^{235} \mathrm{U}$ in the central $321.185 \mathrm{~cm}$ region. The upper and lower $17.963 \mathrm{~cm}$ contain a blanket of natural Uranium. The fuel rod assembly volume fractions for materials in these regions of the Gadolinia fuel rod are provided in Table 2-22.

Table 2-22. Mark-B9 Gadolinia Fuel Rod VFs by Region

\begin{tabular}{|c|c|c|c|c|}
\hline Region & 30255 & A286 Alloy & Zircaloy-4 & Helium \\
\hline 6 & 0.0842 & 0.0 & 0.5018 & 0.4140 \\
\hline 7 & 0.1330 & 0.0 & 0.2314 & 0.6356 \\
\hline 8 & \multicolumn{4}{|c|}{$\begin{array}{l}\text { Explicit Idealization from } Y=16.723 \mathrm{~cm} \text { to } Y=373.834 \mathrm{~cm} \\
\text { Fuel Clad: Zircaloy } O D=1.0922 \mathrm{~cm}, I D=0.95758 \mathrm{~cm} \\
\text { From } Y=16.723 \mathrm{~cm} \text { to } Y=34.686 \mathrm{~cm} \text { Natural } U_{2} \text { fuel Pellet, } O D=0.9398 \mathrm{~cm} \\
\text { From } Y=34.686 \mathrm{~cm} \text { to } Y=355.871 \mathrm{~cm} \text { UO }{ }_{2}-\text { Gadolinia fuel pellet, } O D=0.9398 \mathrm{~cm} \\
\text { From } Y=355.871 \mathrm{~cm} \text { to } Y=373.834 \mathrm{~cm} \text { Natural } U_{2} \text { fuel Pellet, } O D=0.9398 \mathrm{~cm} \\
\text { Fuel Pellets: } 95 \% \text { Theoretical Density } \\
\text { Helium in Gaps }\end{array}$} \\
\hline & \multicolumn{4}{|c|}{ From $Y=373.834 \mathrm{~cm}$ to $Y=376.895 \mathrm{~cm}(\Delta Y=3.061)$} \\
\hline & 0.1303 & 0.0 & 0.2314 & 0.6383 \\
\hline 9 & 0.0 & 0.0687 & 0.6871 & 0.2442 \\
\hline
\end{tabular}

Mark-B9 Guide Tubes-Figure 2-13 indicates an axial view of a guide tube.

- Material $=$ Ziraloy-4

- 16 guide tubes, $\mathrm{OD}=1.3462 \mathrm{~cm}, \mathrm{ID}=1.26492 \mathrm{~cm}$

- Length $=391.554 \mathrm{~cm}$

- $\mathrm{Y}_{\text {bottom }} \approx 9.766 \mathrm{~cm}$

- $\mathrm{Y}_{\text {top }} \approx 401.320 \mathrm{~cm}$

Mark-B9 Instrument Tube-Figure 2-14 indicates an axial view of an instrument tube.

- Material = Ziraloy-4

- One tube, $\mathrm{OD}=1.25222 \mathrm{~cm}, \mathrm{ID}=1.12014 \mathrm{~cm}$

- Length $=392.925 \mathrm{~cm}$

- $\mathrm{Y}_{\text {bottom }} \approx 1.608 \mathrm{~cm}$

- $\mathrm{Y}_{\text {top }} \approx 394.533 \mathrm{~cm}$ 


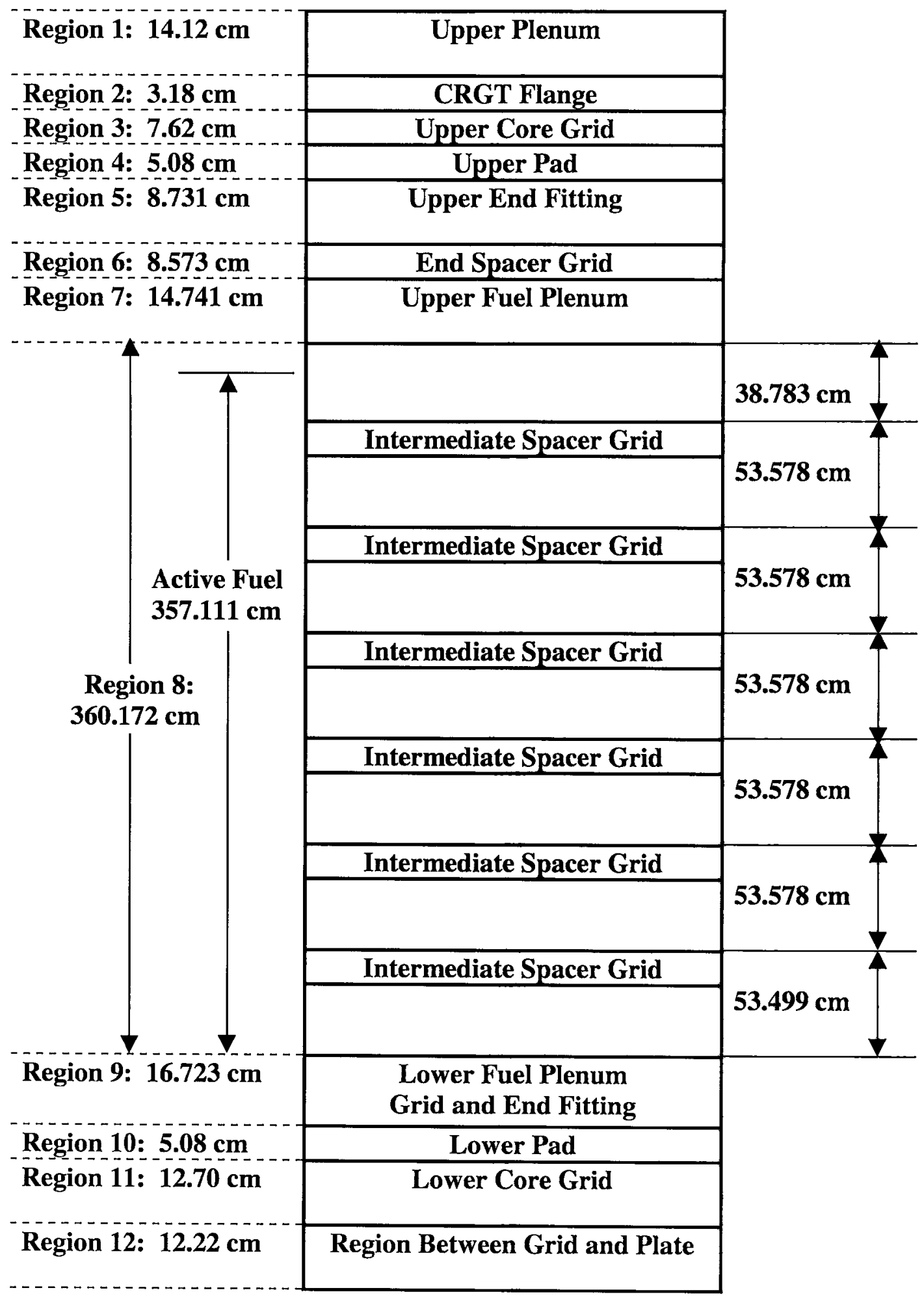

NOTE: Intermediate spacer grid height is $4.826 \mathrm{~cm}$

Figure 2-12. Mark-B9 Assembly Axial Dimensions by Region 


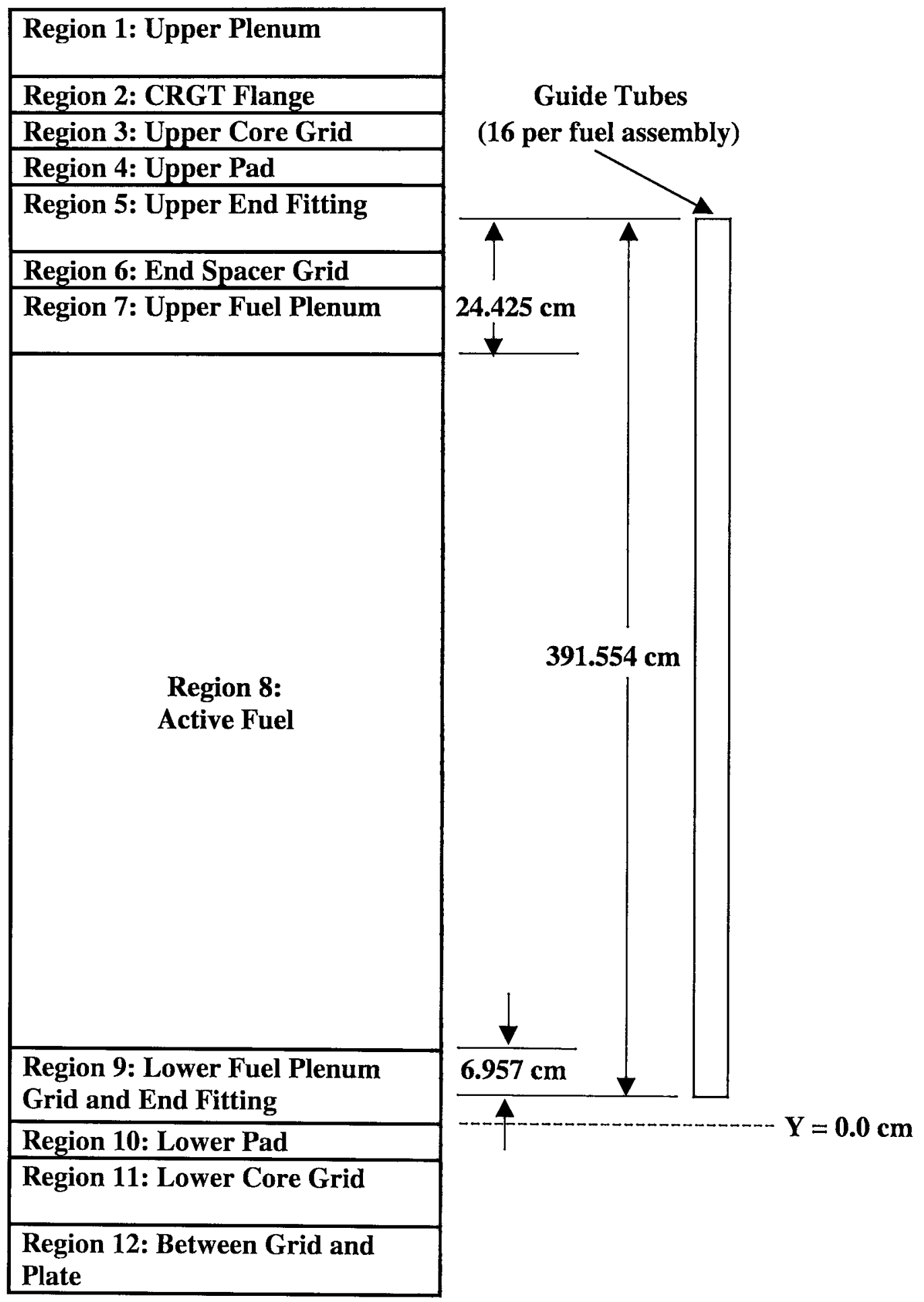

Figure 2-13. Mark-B9 Assembly Axial Dimensions for Guide Tubes 


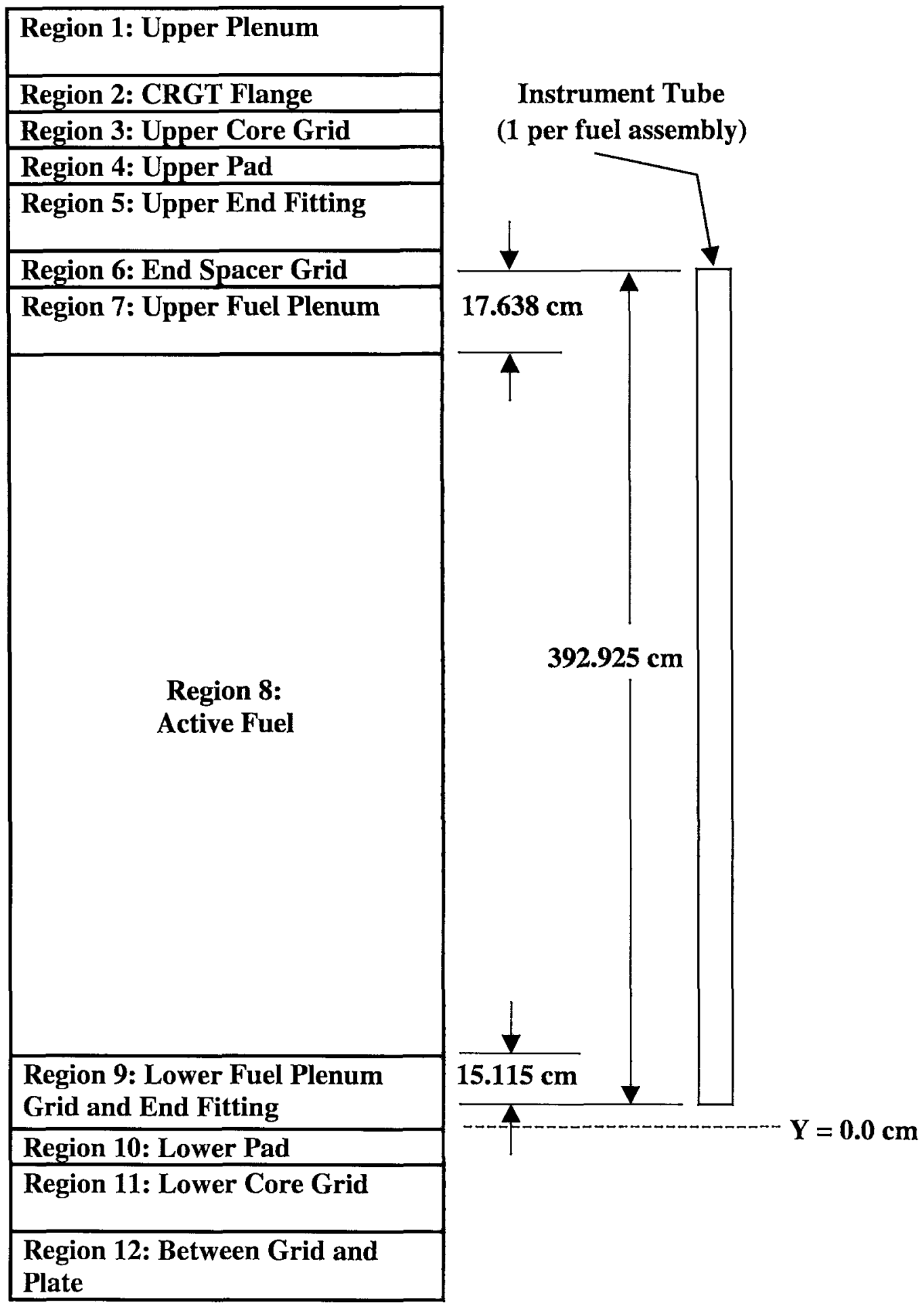

Figure 2-14. Mark-B9 Assembly Axial Dimensions for Instrument Tube 


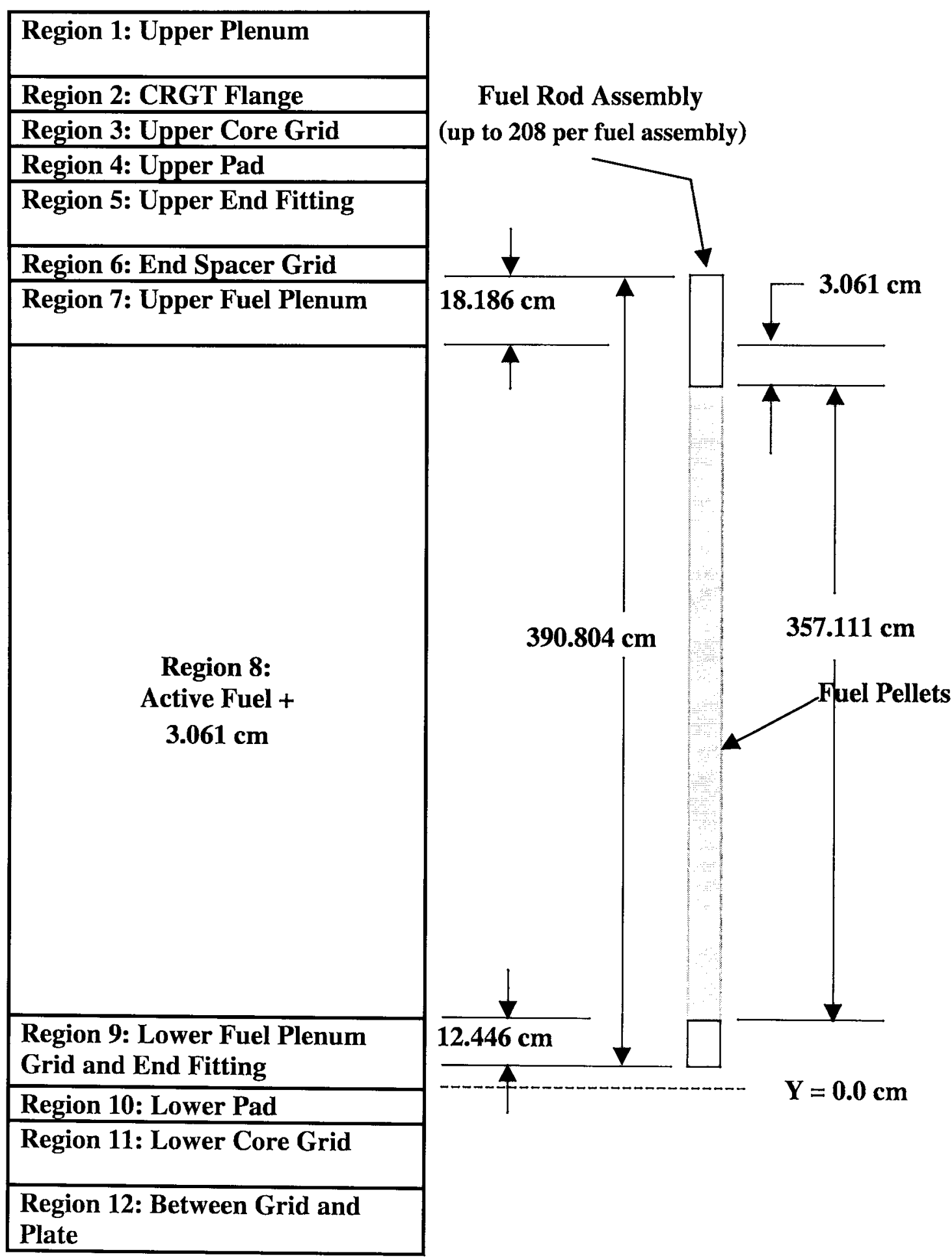

Figure 2-15. Mark-B9 Assembly Axial Dimensions - Standard Fuel Rod 


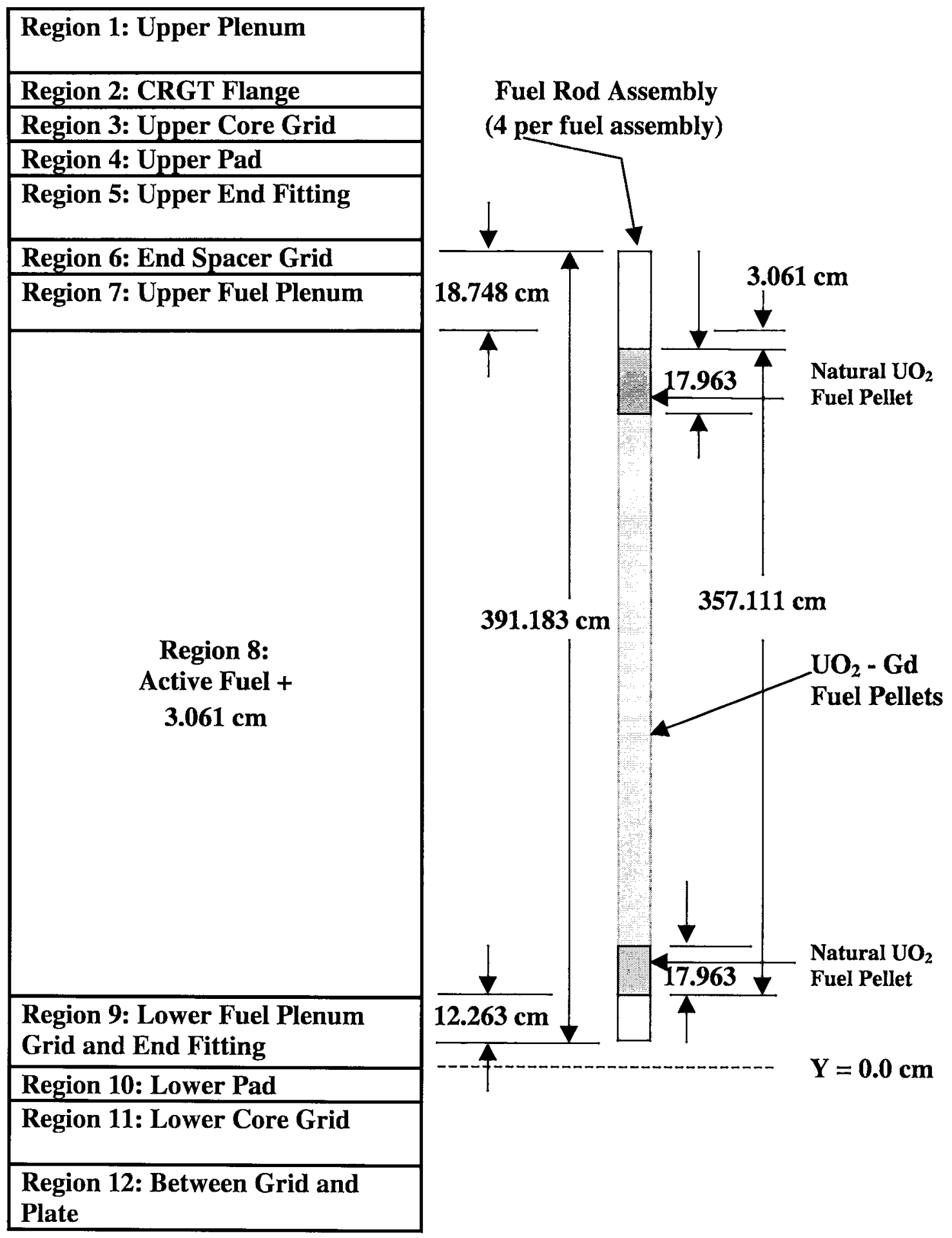

Figure 2-16. Mark-B9 Assembly Axial Dimensions - Gadolinia Fuel Rod 


\subsection{MARK-B10 FUEL ASSEMBLY}

Figure 2-17 provides region boundaries and axial locations for intermediate spacer grids. The intermediate spacer grid height is $4.826 \mathrm{~cm}$ for each of the six grids. Tables 2-23 through 2-27 provide volume fractions for Mark-B10 fuel assemblies with and without control components.

Table 2-23. Mark-B10 Assembly VFs by Region

\begin{tabular}{|c|c|c|c|c|c|c|}
\hline Region & 304SS & 316SS & Inconel 718 & Inconel X-750 & Zircaloy-4 & Water \\
\hline 1 & 0.0578 & 0.0 & 0.0 & 0.0 & 0.0 & 0.9422 \\
\hline 2 & 0.1381 & 0.0 & 0.0 & 0.0 & 0.0 & 0.8619 \\
\hline 3 & 0.2491 & 0.0 & 0.0 & 0.0 & 0.0 & 0.7509 \\
\hline 4 & 0.3418 & 0.0 & 0.0 & 0.0 & 0.0 & 0.6582 \\
\hline 5 & 0.2781 & 0.0011 & 0.0331 & 0.0067 & 0.0076 & 0.6733 \\
\hline 6 & 0.0070 & 0.0 & 0.0 & 0.0 & 0.0 & 0.9930 \\
\hline 7 & 0.0 & 0.0 & 0.0342 & 0.0 & 0.0007 & 0.9650 \\
\hline 8 & 0.0 & 0.0 & 0.0 & 0.0 & 0.0091560 & 0.9908440 \\
\hline 9 & 0.1655 & 0.0 & 0.0242 & 0.0 & 0.0420 & 0.7683 \\
\hline 10 & 0.2848 & 0.0 & 0.0 & 0.0 & 0.0 & 0.7152 \\
\hline 11 & 0.2400 & 0.0 & 0.0 & 0.0 & 0.0 & 0.7600 \\
\hline 12 & 0.0300 & 0.0 & 0.0 & 0.0 & 0.0 & 0.9700 \\
\hline
\end{tabular}

NOTE: All components except fuel rods, instrument tube and guide tubes.

Table 2-24. Mark-B10 Assembly VFs with RCCAs $0 \%$ Withdrawn

\begin{tabular}{|c|c|c|c|c|c|c|}
\hline Region & 304SS & 316SS & Inconel 718 & Inconel X-750 & Zircaloy-4 & Water \\
\hline 1 & 0.0934 & 0.0 & 0.0 & 0.0 & 0.0 & 0.9066 \\
\hline 2 & 0.1945 & 0.0 & 0.0 & 0.0 & 0.0 & 0.8055 \\
\hline 3 & 0.3481 & 0.0 & 0.0 & 0.0 & 0.0 & 0.6519 \\
\hline 4 & 0.3748 & 0.0 & 0.0 & 0.0 & 0.0 & 0.6252 \\
\hline 5 & 0.3005 & 0.0011 & 0.0331 & 0.0067 & 0.0076 & 0.6509 \\
\hline 6 & 0.0070 & 0.0 & 0.0 & 0.0 & 0.0 & 0.9930 \\
\hline 7 & 0.0 & 0.0 & 0.0342 & 0.0 & 0.0007 & 0.9650 \\
\hline 8 & 0.0 & 0.0 & 0.0 & 0.0 & 0.0091560 & 0.9908440 \\
\hline 9 & 0.1655 & 0.0 & 0.0242 & 0.0 & 0.0420 & 0.7683 \\
\hline 10 & 0.2848 & 0.0 & 0.0 & 0.0 & 0.0 & 0.7152 \\
\hline 11 & 0.2400 & 0.0 & 0.0 & 0.0 & 0.0 & 0.7600 \\
\hline 12 & 0.0300 & 0.0 & 0.0 & 0.0 & 0.0 & 0.9700 \\
\hline
\end{tabular}

NOTE: All components except fuel rods, instrument tube, guide tubes and control tubes. 
Table 2-25. Mark-B10 Assembly VFs with RCCAs and Gray APSRs $100 \%$ Withdrawn

\begin{tabular}{|c|c|c|c|c|c|c|c|}
\hline Region & $\mathbf{3 0 4 S S}$ & $\mathbf{3 1 6 5 S}$ & $\begin{array}{c}\text { Inconel } \\
\mathbf{7 1 8}\end{array}$ & $\begin{array}{c}\text { Inconel } \\
\mathbf{X}-750\end{array}$ & Zircaloy-4 & Inconel 600 & Water \\
\hline 1 & 0.0638 & 0.0 & 0.0 & 0.0 & 0.0 & 0.0262 & 0.9100 \\
\hline 2 & 0.1442 & 0.0 & 0.0 & 0.0 & 0.0 & 0.0262 & 0.8296 \\
\hline 3 & 0.2553 & 0.0 & 0.0 & 0.0 & 0.0 & 0.0262 & 0.7185 \\
\hline 4 & 0.3481 & 0.0 & 0.0 & 0.0 & 0.0 & 0.0262 & 0.6257 \\
\hline 5 & 0.2845 & 0.0011 & 0.0339 & 0.0069 & 0.0078 & Explicit & 0.6658 \\
\hline 6 & 0.0070 & 0.0 & 0.0 & 0.0 & 0.0 & 0.0 & 0.9930 \\
\hline 7 & 0.0 & 0.0 & 0.0342 & 0.0 & 0.0007 & 0.0 & 0.9650 \\
\hline 8 & 0.0 & 0.0 & 0.0 & 0.0 & 0.0091560 & 0.0 & 0.9908440 \\
\hline 9 & 0.1655 & 0.0 & 0.0242 & 0.0 & 0.0420 & 0.0 & 0.7683 \\
\hline 10 & 0.2848 & 0.0 & 0.0 & 0.0 & 0.0 & 0.0 & 0.7152 \\
\hline 11 & 0.2400 & 0.0 & 0.0 & 0.0 & 0.0 & 0.0 & 0.7600 \\
\hline 12 & 0.0300 & 0.0 & 0.0 & 0.0 & 0.0 & 0.0 & 0.9700 \\
\hline
\end{tabular}

NOTE: All components except fuel rods, instrument tube, guide tubes and control tubes.

Table 2-26. Mark-B10 Assembly VFs with Gray APSRs $0 \%$ Withdrawn

\begin{tabular}{|c|c|c|c|c|c|c|}
\hline Region & $\mathbf{3 0 4 S S}$ & $\mathbf{3 1 6 S S}$ & Inconel 718 & $\begin{array}{c}\text { Inconel } \\
\text { X-750 }\end{array}$ & Zircaloy-4 & Water \\
\hline 1 & 0.1081 & 0.0 & 0.0 & 0.0 & 0.0 & 0.8919 \\
\hline 2 & 0.2212 & 0.0 & 0.0 & 0.0 & 0.0 & 0.7788 \\
\hline 3 & 0.2828 & 0.0 & 0.0 & 0.0 & 0.0 & 0.7172 \\
\hline 4 & 0.3748 & 0.0 & 0.0 & 0.0 & 0.0 & 0.6252 \\
\hline 5 & 0.2983 & 0.0011 & 0.0331 & 0.0067 & 0.0076 & 0.6532 \\
\hline 6 & 0.0070 & 0.0 & 0.0 & 0.0 & 0.0 & 0.9930 \\
\hline 7 & 0.0 & 0.0 & 0.0342 & 0.0 & 0.0007 & 0.9650 \\
\hline 8 & 0.0 & 0.0 & 0.0 & 0.0 & 0.0091560 & 0.9908440 \\
\hline 9 & 0.1655 & 0.0 & 0.0242 & 0.0 & 0.0420 & 0.7683 \\
\hline 10 & 0.2848 & 0.0 & 0.0 & 0.0 & 0.0 & 0.7152 \\
\hline 11 & 0.2400 & 0.0 & 0.0 & 0.0 & 0.0 & 0.7600 \\
\hline 12 & 0.0300 & 0.0 & 0.0 & 0.0 & 0.0 & 0.9700 \\
\hline
\end{tabular}

NOTE: All components except fuel rods, instrument tube, guide tubes and control tubes. 
Table 2-27. Mark-B10 Assembly VFs with BPRAs Inserted

\begin{tabular}{|c|c|c|c|c|c|c|}
\hline Region & 304SS & 316SS & Inconel 718 & $\begin{array}{c}\text { Inconel } \\
\mathbf{X - 7 5 0}\end{array}$ & Zircaloy-4 & Water \\
\hline 1 & 0.0699 & 0.0 & 0.0 & 0.0 & 0.0 & 0.9301 \\
\hline 2 & 0.1827 & 0.0 & 0.0 & 0.0 & 0.0 & 0.8173 \\
\hline 3 & 0.2937 & 0.0 & 0.0 & 0.0 & 0.0069 & 0.6994 \\
\hline 4 & 0.3890 & 0.0 & 0.0 & 0.0 & 0.0120 & 0.5990 \\
\hline 5 & 0.2899 & 0.0011 & 0.0338 & 0.0069 & 0.0078 & 0.6604 \\
\hline 6 & 0.0070 & 0.0 & 0.0 & 0.0 & 0.0 & 0.9930 \\
\hline 7 & 0.0 & 0.0 & 0.0342 & 0.0 & 0.0007 & 0.9650 \\
\hline 8 & 0.0 & 0.0 & 0.0 & 0.0 & 0.0091560 & 0.9908440 \\
\hline 9 & 0.1655 & 0.0 & 0.0242 & 0.0 & 0.0420 & 0.7683 \\
\hline 10 & 0.2848 & 0.0 & 0.0 & 0.0 & 0.0 & 0.7152 \\
\hline 11 & 0.2400 & 0.0 & 0.0 & 0.0 & 0.0 & 0.7600 \\
\hline 12 & 0.0300 & 0.0 & 0.0 & 0.0 & 0.0 & 0.9700 \\
\hline
\end{tabular}

NOTE: All components except fuel rods, instrument tube, guide tubes and control tubes.

Standard Mark-B10 Fuel Rods-There are 208 fuel rods in the fuel assembly. In assemblies that contain Gadolinia rods, there are either 200 or 204 standard fuel rods. The fuel rods are contained in regions 6 through 9. Part of region 8 is modeled explicitly. Regions 6, 7, 8 and 9 contain various amounts of stainless steel and Zircaloy in the fuel rod that are representative of the plenum springs and end caps. In addition, these regions contain helium and fission gases, as well as the Zircaloy cladding.

The Standard B10 fuel rod is represented as a cylinder with $1.0922 \mathrm{~cm}$ diameter with $Y_{\text {bottom }}=$ $4.277 \mathrm{~cm}$ and $Y_{\text {top }}=395.081 \mathrm{~cm}$, where $Y=0$ is at the bottom on region 9. Figure $2-22$ indicates the axial view of the fuel rod. The fuel rod assembly volume fractions for materials in these regions for the Standard Mark B10 are presented in Table 2-28.

Gadolinia Mark-B10 Fuel Rods: The Gadolinia B10 fuel rod is represented as a cylinder with $1.0922 \mathrm{~cm}$ diameter with $Y_{\text {bottom }}=4.460 \mathrm{~cm}$ and $Y_{\text {top }}=395.643 \mathrm{~cm}$. TMI-1 batches $13 \mathrm{C}$ and $13 \mathrm{E}$ have 4 Gadolinia fuel rods each, and fuel batch $13 \mathrm{~F}$ has $8 \mathrm{Gadolinia}$ rods per assembly. The Gadolinia rods have $2 \mathrm{wt} \% \mathrm{Gd}_{2} \mathrm{O}_{3}$ and $4.19 \mathrm{wt} \%{ }^{235} \mathrm{U}$ in the central $321.185 \mathrm{~cm}$ and natural uranium in the upper and lower $17.963 \mathrm{~cm}$ blanket regions. Figure 2-23 indicates the axial view of the fuel rod. The fuel rod assembly volume fractions for materials in these regions of the Gadolinia Mark B10 assembly are presented in Table 2-29. 
Table 2-28. Mark-B10 Standard Fuel Rod VFs by Region

\begin{tabular}{|c|c|c|c|c|}
\hline Region & 302SS & A286 Alloy & Zircaloy-4 & Helium \\
\hline 6 & 0.0751 & 0.0 & 0.3714 & 0.5535 \\
\hline 7 & 0.1388 & 0.0 & 0.2351 & 0.6261 \\
\hline 8 & \multicolumn{2}{|c|}{$\begin{array}{l}\text { Explicit Idealization from } \mathrm{Y}=16.723 \mathrm{~cm} \text { to } \mathrm{Y}=373.834 \mathrm{~cm} \\
\text { Fuel Clad: Zircaloy } \mathrm{OD}=1.0922 \mathrm{~cm}, \mathrm{ID}=0.95758 \mathrm{~cm}\end{array}$} \\
& $\begin{array}{l}\text { Fuel Pellet: } \mathrm{UO}_{2} \mathrm{OD}=0.9398 \mathrm{~cm}, 95 \% \text { Theoretical Density } \\
\text { Helium in gaps }\end{array}$ \\
\cline { 2 - 5 } & \multicolumn{3}{|c|}{ From $\mathrm{Y}=373.834 \mathrm{~cm}$ to $\mathrm{Y}=376.895 \mathrm{~cm}(\Delta \mathrm{Y}=3.061 \mathrm{~cm})$} \\
\cline { 2 - 5 } & 0.1366 & 0.0 & 0.2314 & 0.6320 \\
\hline 9 & 0.0 & 0.0678 & 0.6587 & 0.2736 \\
\hline
\end{tabular}

Table 2-29. Mark-B10 Gadolinia Fuel Rod VFs by Region

\begin{tabular}{|c|c|c|c|c|}
\hline Region & 302SS & A286 Alloy & Zircaloy-4 & Helium \\
\hline 6 & 0.0842 & 0.0 & 0.5018 & 0.4140 \\
\hline 7 & 0.1330 & 0.0 & 0.2314 & 0.6356 \\
\hline 8 & \multicolumn{4}{|c|}{$\begin{array}{l}\text { Explicit Idealization from } Y=16.723 \mathrm{~cm} \text { to } Y=373.834 \mathrm{~cm} \\
\text { Fuel Clad: Zircaloy } O D=1.0922 \mathrm{~cm}, I D=0.95758 \mathrm{~cm} \\
\text { From } Y=16.723 \mathrm{~cm} \text { to } Y=34.686 \mathrm{~cm} \text {, fuel pellet with Natural } U_{2}, O D=0.9398 \mathrm{~cm} \\
\text { From } Y=34.686 \mathrm{~cm} \text { to } Y=355.871 \mathrm{~cm} \text { UO }_{2} \text { - Gadolinia fuel pellet, } O D=0.9398 \mathrm{~cm} \\
\text { From } Y=355.871 \mathrm{~cm} \text { to } Y=373.834 \mathrm{~cm} \text {, fuel pellet with Natural } \mathrm{UO}_{2}, O D=0.9398 \mathrm{~cm} \\
\text { Fuel Pellets: } 95 \% \text { Theoretical Density } \\
\text { Helium in Gaps }\end{array}$} \\
\hline & \multicolumn{4}{|c|}{ From $Y=373.834 \mathrm{~cm}$ to $Y=376.895 \mathrm{~cm}(\Delta Y=3.061 \mathrm{~cm})$} \\
\hline & 0.1303 & 0.0 & 0.2314 & 0.6383 \\
\hline 9 & 0.0 & 0.0687 & 0.6871 & 0.2442 \\
\hline
\end{tabular}

Mark-B10 Guide Tubes- Figure 2-20 indicates an axial view of the guide tubes.

- Material = Ziraloy-4

- 16 guide tubes, $\mathrm{OD}=1.3462 \mathrm{~cm}, \mathrm{ID}=1.26492 \mathrm{~cm}$

- Length $=392.049 \mathrm{~cm}$

- $Y_{\text {bottom }} \approx 9.766 \mathrm{~cm}$

- $\mathrm{Y}_{\text {top }} \approx 401.815 \mathrm{~cm}$

Mark-B10 Instrument Tube- Figure 2-21 provides an axial view of the instrument tube.

- Material $=$ Ziraloy-4

- One tube, $\mathrm{OD}=1.25222 \mathrm{~cm}, \mathrm{ID}=1.12014 \mathrm{~cm}$

- Length $=392.925 \mathrm{~cm}$

- $Y_{\text {bottom }} \approx 1.608 \mathrm{~cm}$

- $Y_{\text {top }} \approx 394.533 \mathrm{~cm}$ 


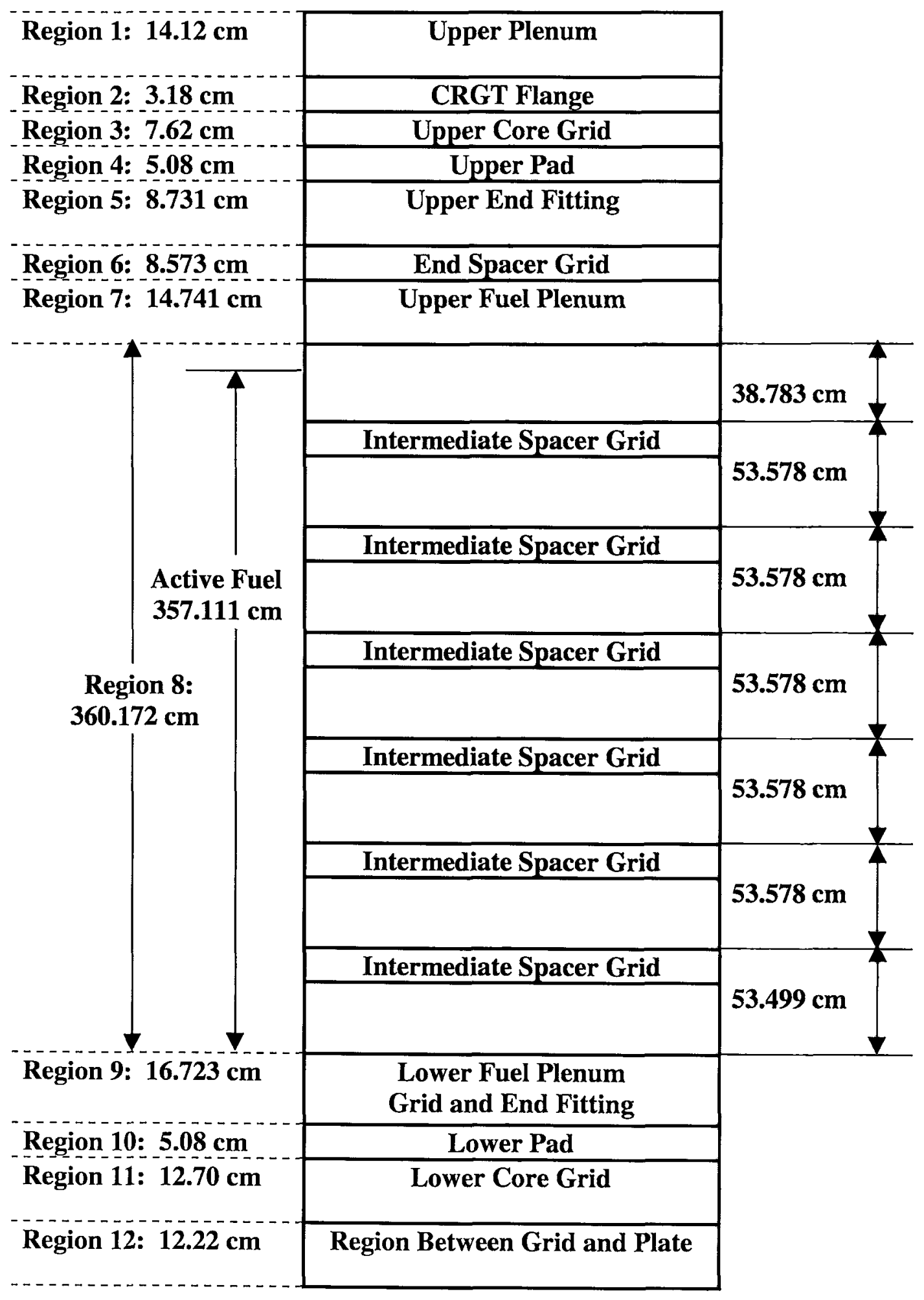

NOTE: Intermediate spacer grid height is $4.826 \mathrm{~cm}$

Figure 2-17. Mark-B10 Assembly Axial Dimensions by Region 


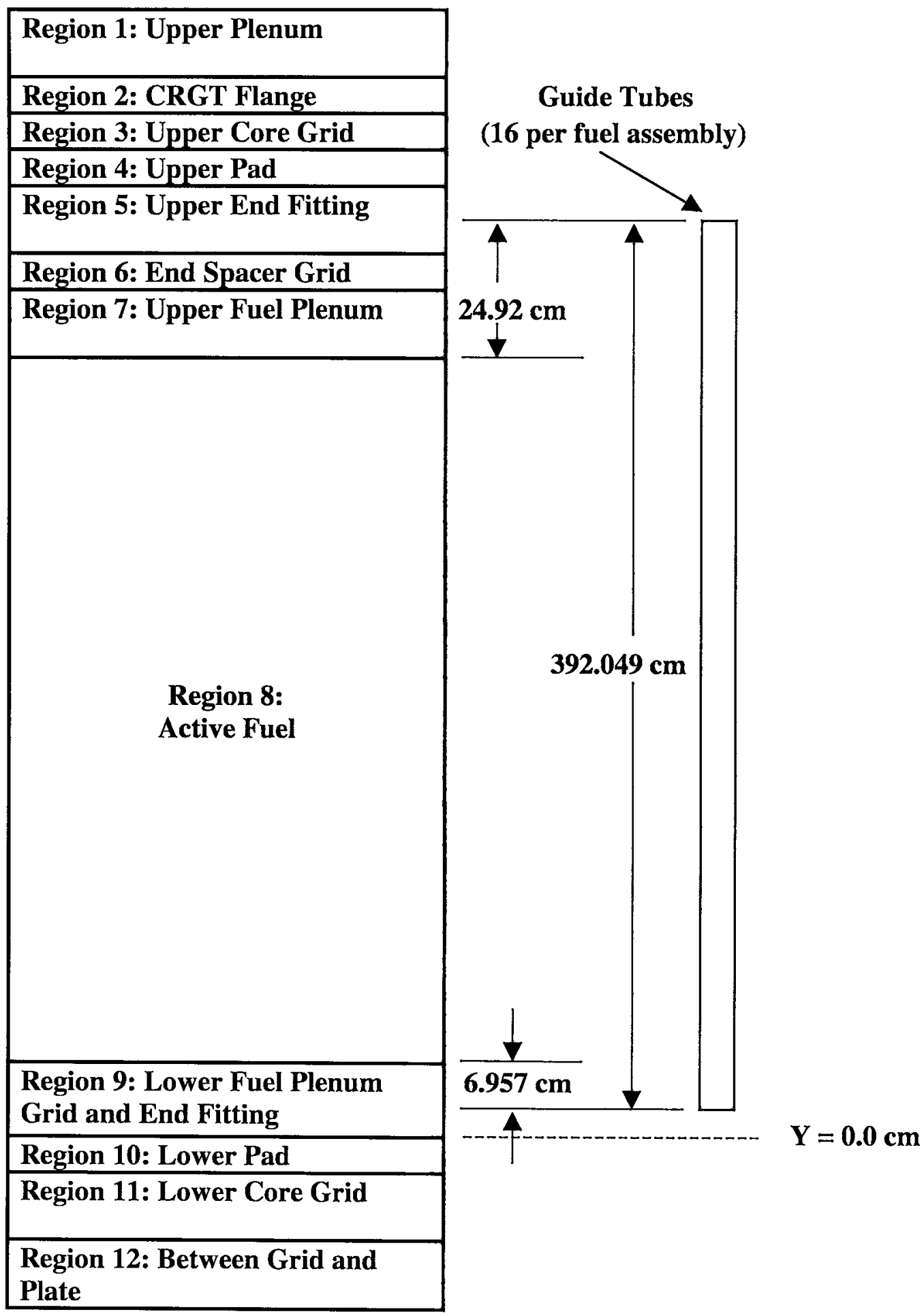

Figure 2-18. Mark-B10 Assembly Axial Dimensions for Guide Tubes 


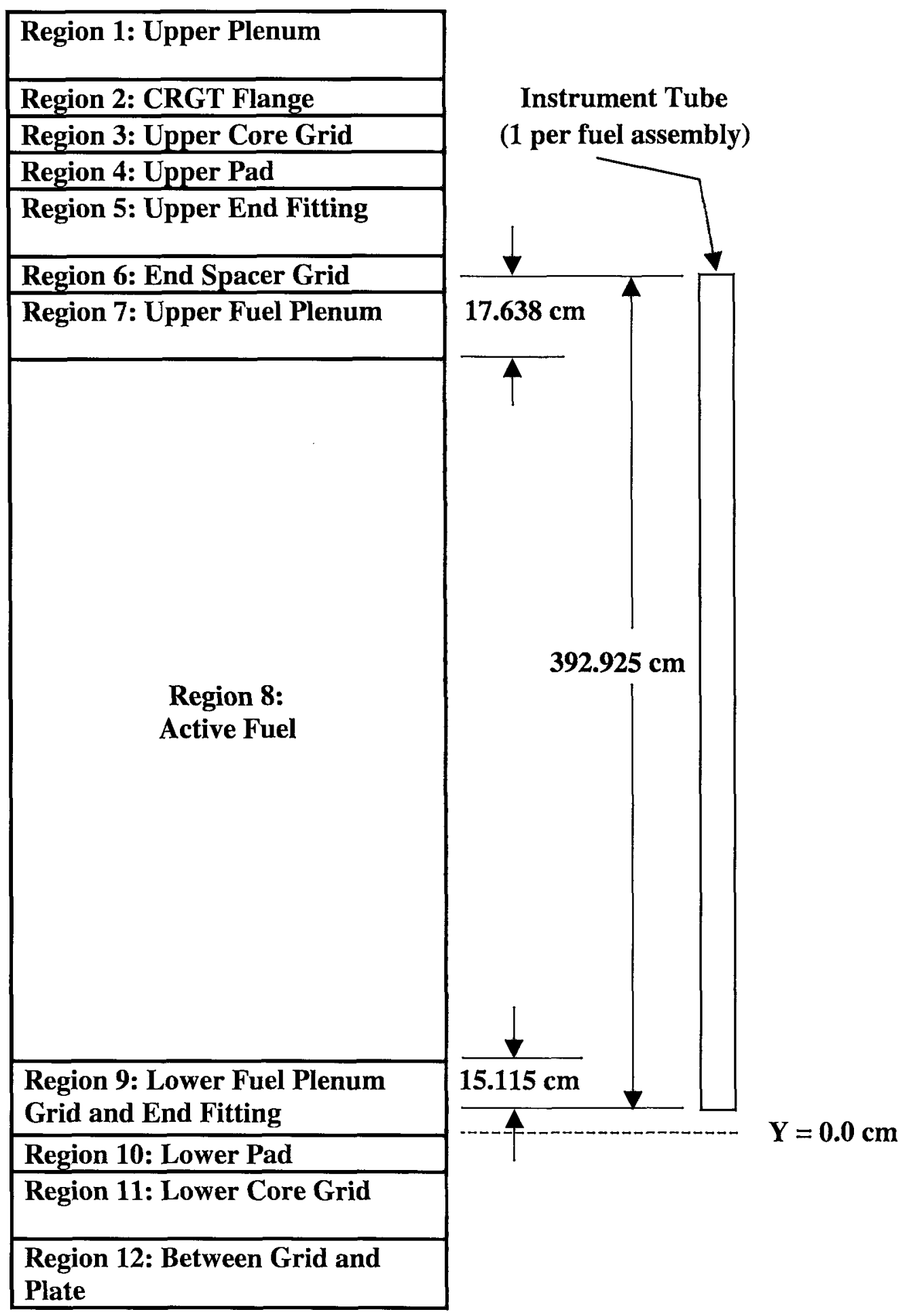

Figure 2-19. Mark-B10 Assembly Axial Dimensions for Instrument Tube 


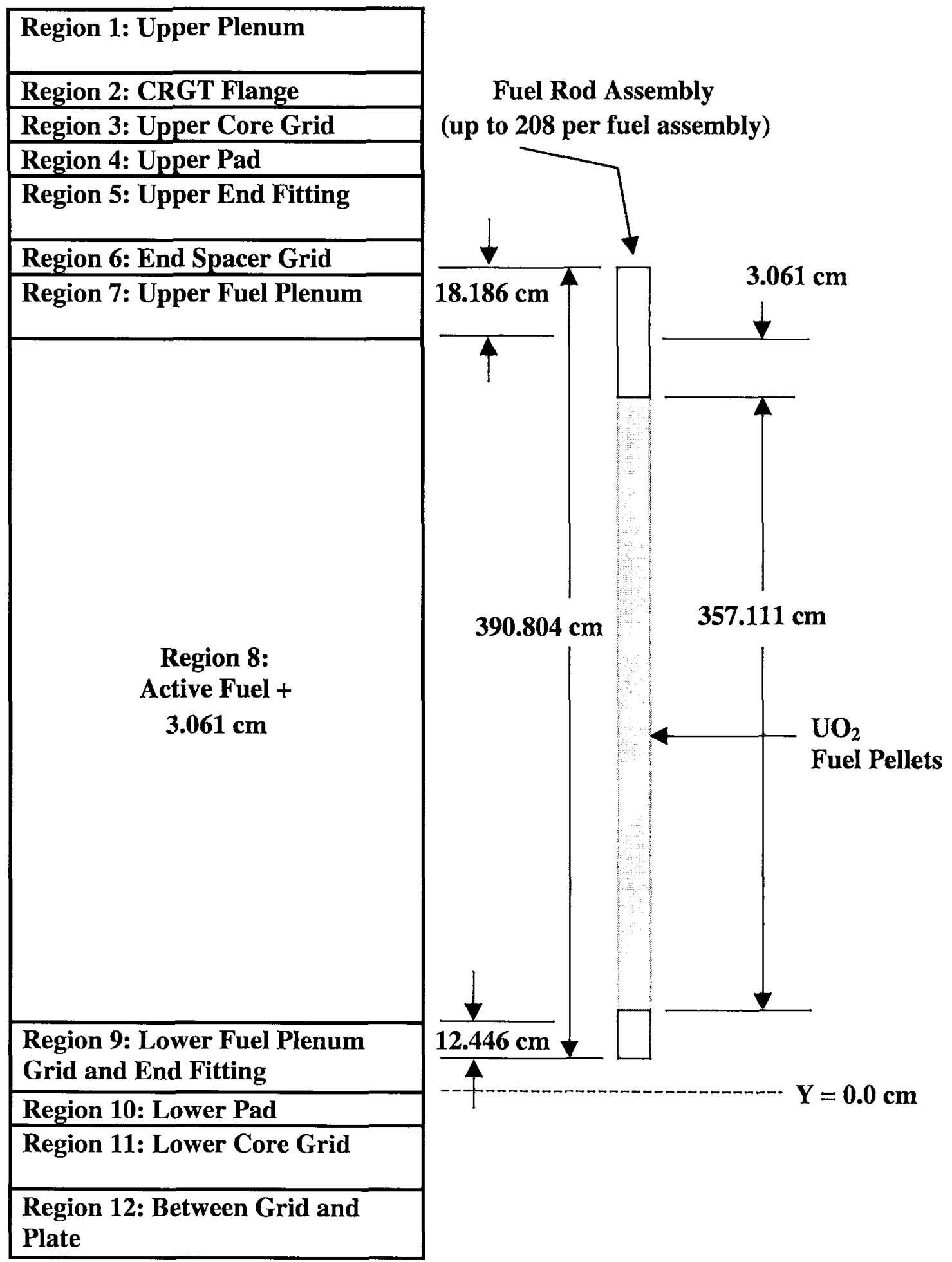

Figure 2-20. Mark-B10 Assembly Axial Dimensions for Standard Fuel Rod 


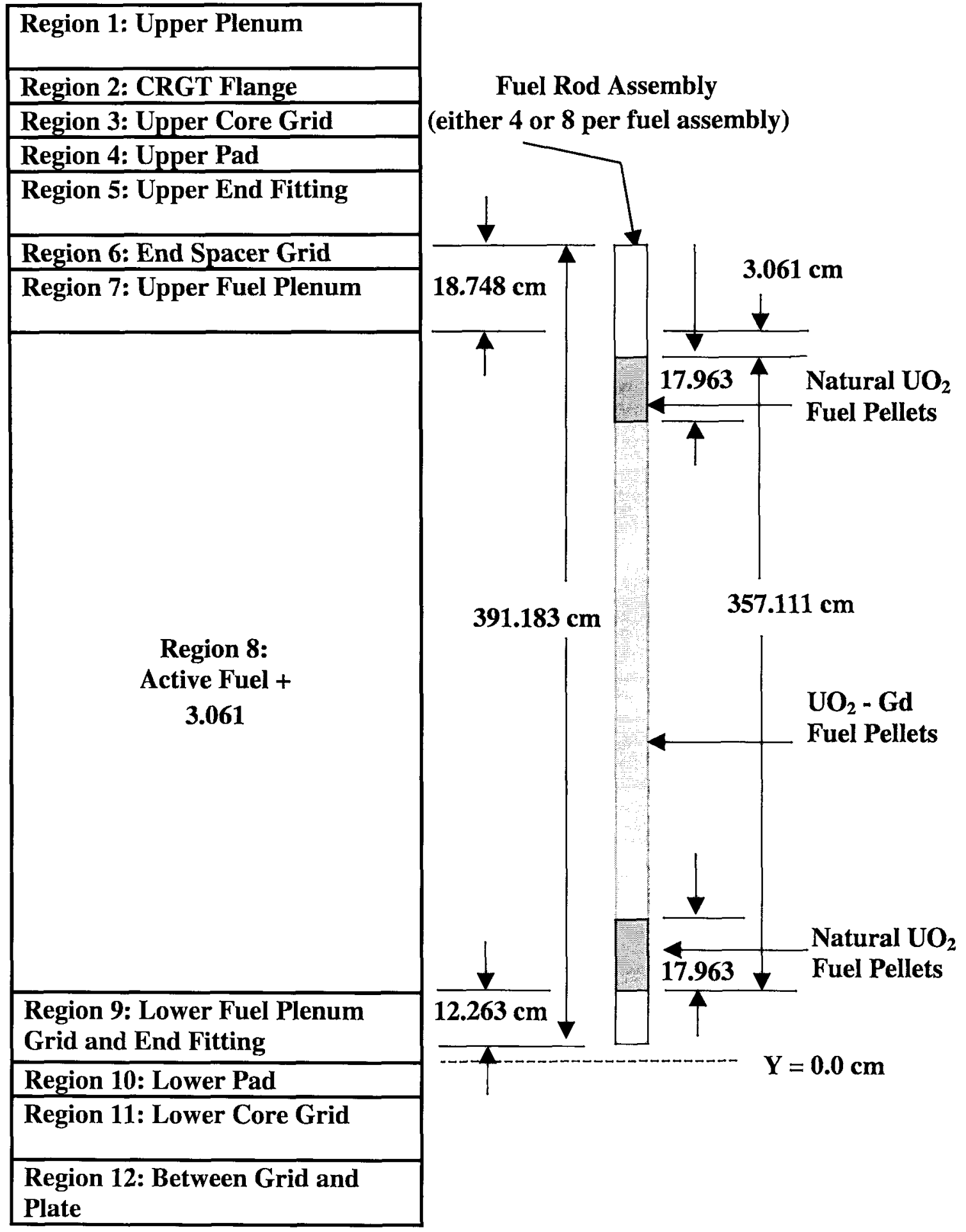

Figure 2-21. Mark-B10 Assembly Axial Dimensions for Gadolinia Fuel Rod 


\subsection{CONTROL COMPONENTS}

Figures 2-22 through 2-26 provide axial dimensions for RCCAs with rods at $0 \%$ and $100 \%$ withdrawn, for black APSRs, for gray APSRs, and for BPRAs. Geometric, material and volumetric data for each item is included.

\subsubsection{RCCA}

RCCA Volume Fractions- The rods are represented explicitly in the active fuel, upper fuel plenum, and end spacer grid regions, and to the ends of the control rod cladding in the upper endfitting region. The remainders of the materials are homogenized in regions 1 through 5 . The volume fractions of these materials (including non-RCCA materials) for RCCAs with rods at $0 \%$ withdrawn are provided in Tables 2-4, 2-11, 2-17, and 2-24.

For rods $100 \%$ withdrawn, the cladding and absorber material inside the cladding are represented explicitly through region 5 (upper end fitting). This extends beyond the guide tubes. Materials for the remaining regions are homogenized. The volume fractions of these materials (including non-RCCA materials) with rods $100 \%$ withdrawn are given in Tables 2-5, 2-12, 2-18, and 2-25.

Figures 2-22 (0 \% withdrawn) and 2-23 (100\% withdrawn) provide axial dimensions for control rods.

\section{RCCA Materials and Volume Fractions}

- Lower end plug - 304SS

- Cladding - 304SS

- Absorber - Ag-In-Cd

- Spring spacer - 304SS, volume fraction of spacer spring/plug inside of clad $=0.3090$ 304SS and $0.6910 \mathrm{Gas}$

- Upper plug and spider assembly - 304SS

\section{RCCA}

- Number of rods per assembly

- Pellet Material

- Percent of Pellet Materials

- Pellet Density

- Pellet OD

- Clad Material

- Clad OD

- Clad ID
16

Ag-In-Cd

$\mathrm{Ag}$ (79.8\%), In (15.0\%), Cd (5.0\%)

$10.17 \mathrm{~g} / \mathrm{cc}$

$0.99568 \mathrm{~cm}$

304SS

$1.11760 \mathrm{~cm}$

$1.01092 \mathrm{~cm}$ 


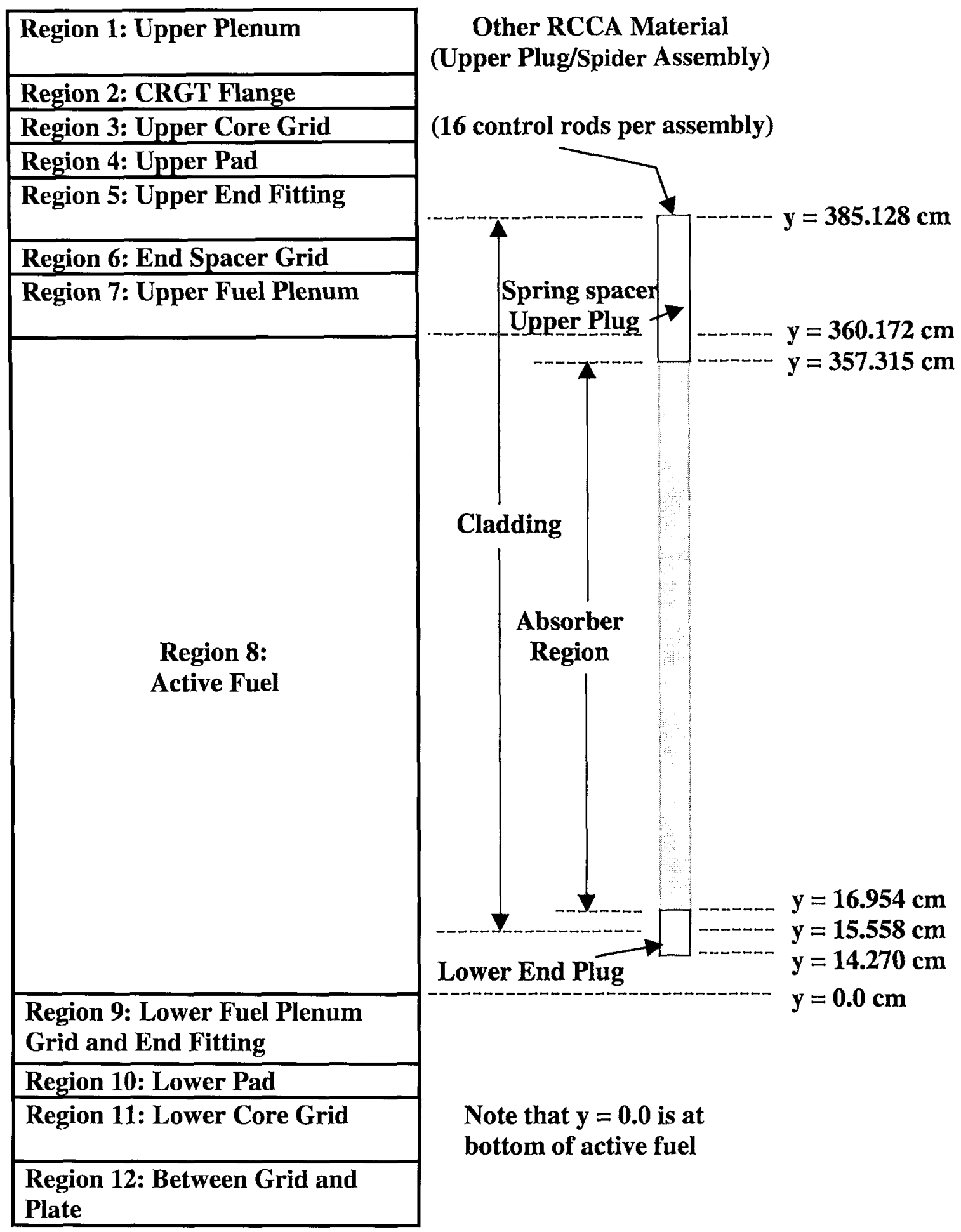

Figure 2-22. Mark-B Assembly Axial Dimensions for RCCA $0 \%$ Withdrawn 


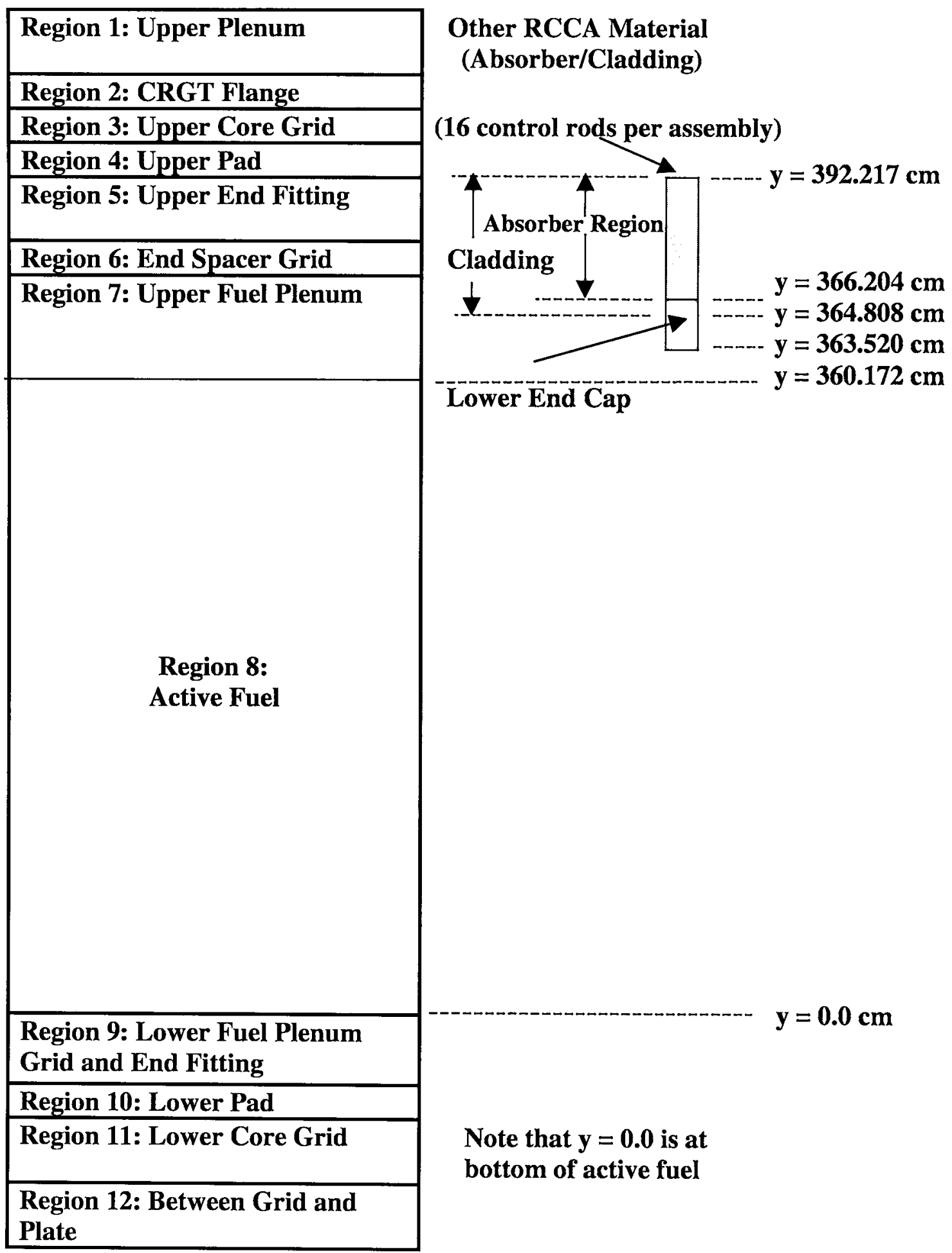

Figure 2-23. Mark-B Assembly with RCCA, Black, and Gray APSR $100 \%$ Withdrawn 


\subsubsection{Black APSR}

Black APSR Volume Fraction-The rods and other materials inside the cladding are represented explicitly in the active fuel, upper fuel plenum, end spacer grid regions, and to the ends of the APSR cladding in the upper end-fitting region. The remainders of the materials are homogenized in regions 1 through 5. Table 2-6 provides the volume fractions of these materials (including non-APSR materials) for black APSRs with rods at 0\% withdrawn. Figure 2-24 provides the axial dimensions for black APSRs. Black APSRs at $100 \%$ withdrawn should be treated the same as RCCAs that are $100 \%$ withdrawn (Figure 2-23).

\section{Black APSR Materials}

- Lower end plug-304SS

- Cladding - 304SS

- Absorber - Ag-In-Cd

- Spacer - Zircaloy (annular design), volume $=0.3818 \mathrm{~cm}^{3}$ for each of 16 rods (for a total of $6.11 \mathrm{~cm}^{3}$ )

- Intermediate plug $-304 \mathrm{SS}$, volume $=1.0094 \mathrm{~cm}^{3}$ for each of 16 rods (for a total of $16.15 \mathrm{~cm}^{3}$ )

- Upper end plug \& spider assembly - 304SS

\section{Black APSRs and RCCAs}

- Pellet Material

- Fraction of Pellet Materials

- Pellet Density

- Pellet OD

- Clad Material

- Clad OD

- Clad ID
Ag-In-Cd

$\mathrm{Ag}(79.8 \%), \operatorname{In}(15.0 \%), \mathrm{Cd}(5.0 \%)$

$10.17 \mathrm{~g} / \mathrm{cc}$

$0.99568 \mathrm{~cm}$

304SS

$1.11760 \mathrm{~cm}$

$1.01092 \mathrm{~cm}$ 


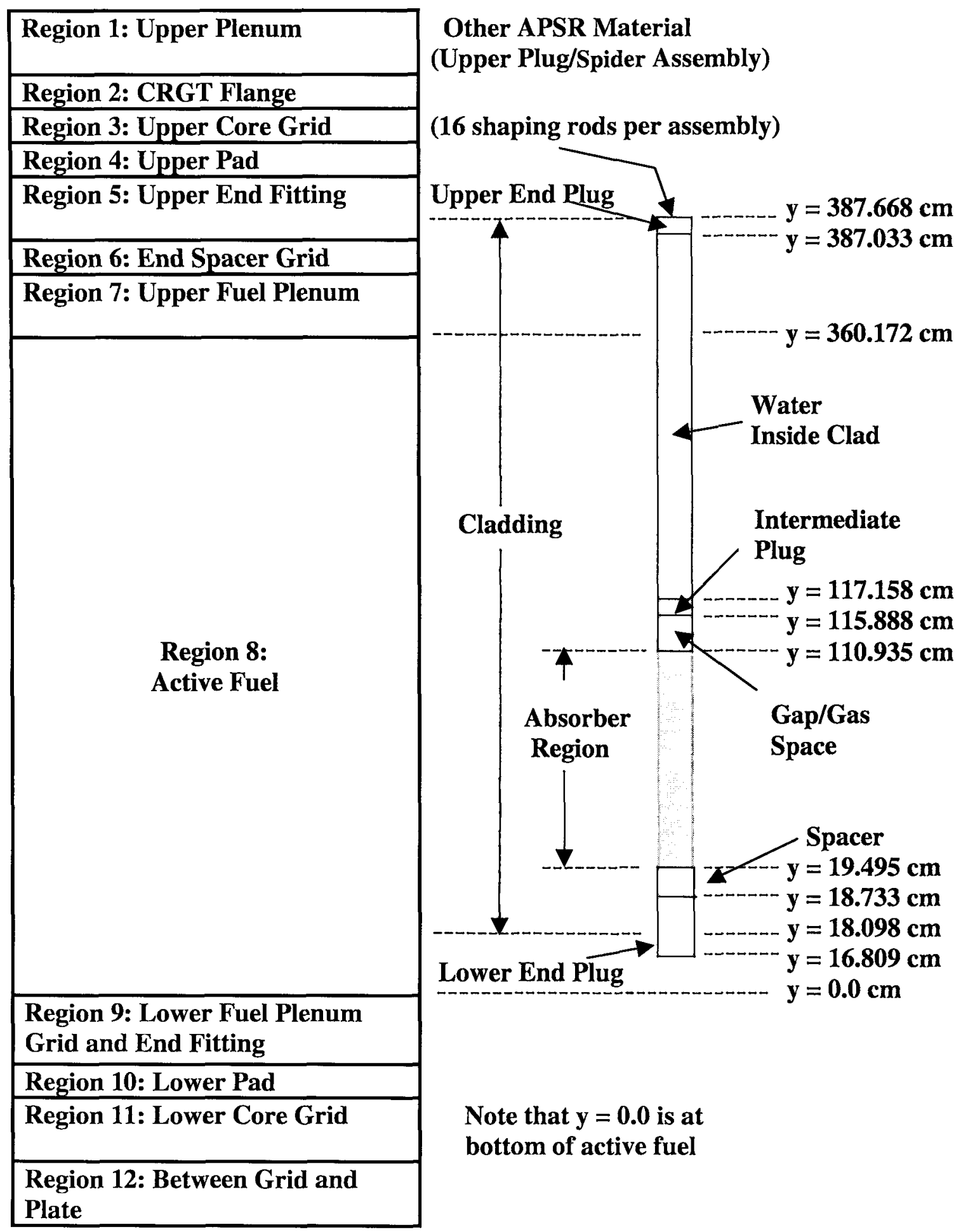

Figure 2-24. Mark-B Assembly Axial Dimensions with Black APSR $0 \%$ Withdrawn 


\subsubsection{Gray APSR}

Gray APSR Volume Fractions-The rods and other materials inside the cladding are represented explicitly in the active fuel, upper fuel plenum, end spacer grid regions, and to the ends of the APSR cladding in the upper end-fitting region. The remainders of the materials are homogenized in regions 1 through 5. The volume fractions for these materials (including nonAPSR materials) for gray APSRs with rods at $0 \%$ withdrawn are provided in Tables 2-7, 2-13, 219 , and 2-26. Figure 2-25 provides the axial dimensions for gray APSRs. Gray APSRs at $100 \%$ withdrawn should be treated the same as RCCAs that are 100\% withdrawn (Figure 2-23).

\section{Gray APSR Materials}

- Lower end plug - 304SS

- Cladding - 304SS

- Gray Neutron Absorber - Inconel 600

- Intermediate plug $-304 \mathrm{SS}$ volume $=1.4191 \mathrm{~cm}^{3}$ for each rod

- Upper end plug \& spider assembly - 304SS

\section{Gray APSRs}

- Number of rods per assembly

- Rod Material

- Rod OD

- Clad material

- Clad OD

- Clad ID
16 Inconel 600 , density $8.3 \mathrm{~g} / \mathrm{cm}^{3}$ $0.95250 \mathrm{~cm}$ $304 \mathrm{SS}$

$1.11760 \mathrm{~cm}$

$0.98044 \mathrm{~cm}$ 


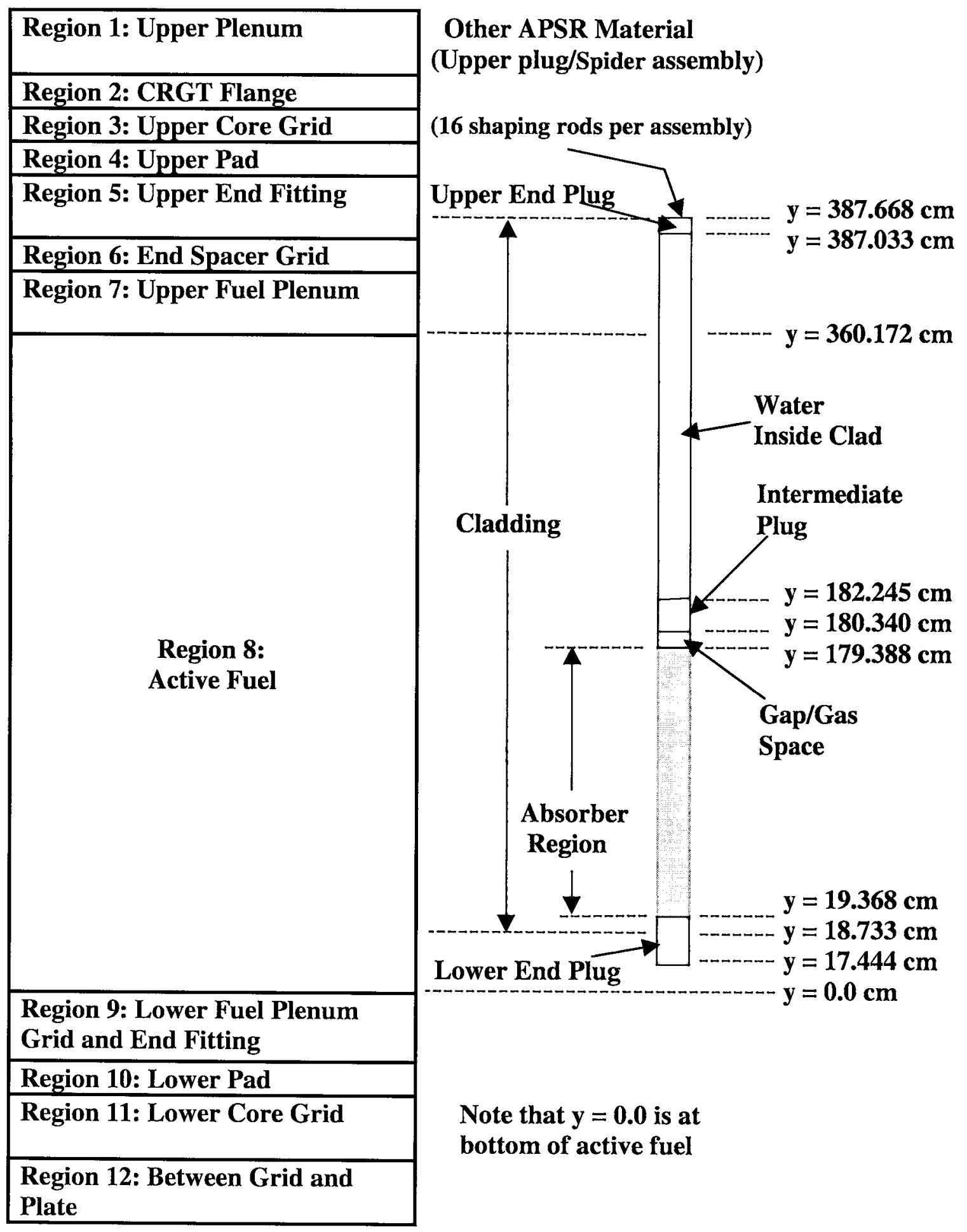

Figure 2-25. Mark-B Assembly Axial Dimensions with Gray APSR $0 \%$ Withdrawn 


\subsubsection{BPRA}

BPRA Volume Fractions-The burnable absorber and other materials inside the cladding are represented explicitly through region 5 (upper end fitting). This extends beyond the guide tubes. Materials for the remaining regions are homogenized. The volume fractions of these materials (including non-BPRA materials) are provided in Tables 2-8, 2-14, 2-20, and 2-27. Figure 2-26 provides the axial dimensions for BPRAs.

\section{BPRA Materials and Volume}

- Upper End Plug

- Lower End Plug

- Clad (Tubing)

- Clad OD

- Clad ID

- Spring

- Burnable Poison Pellets

- Pellet Density

- Pellet OD

- Coupling Spider

\section{Zircaloy-4 \\ Zircaloy-4 \\ Zircaloy-4 \\ $1.0922 \mathrm{~cm}$ \\ $0.9144 \mathrm{~cm}$ \\ 304SS}

$\mathrm{B}_{4} \mathrm{C}$ in an alumina $\left(\mathrm{Al}_{2} \mathrm{O}_{3}\right)$ matrix

$3.7 \mathrm{~g} / \mathrm{cc}$

$0.8636 \mathrm{~cm}$

304SS

Volume Fraction of Spring Inside the Clad
- 304SS Spring
0.2090
- Helium Gas
0.7910

BPRAs-The number of burnable poison rods per lump burnable poison cluster is 16 . 


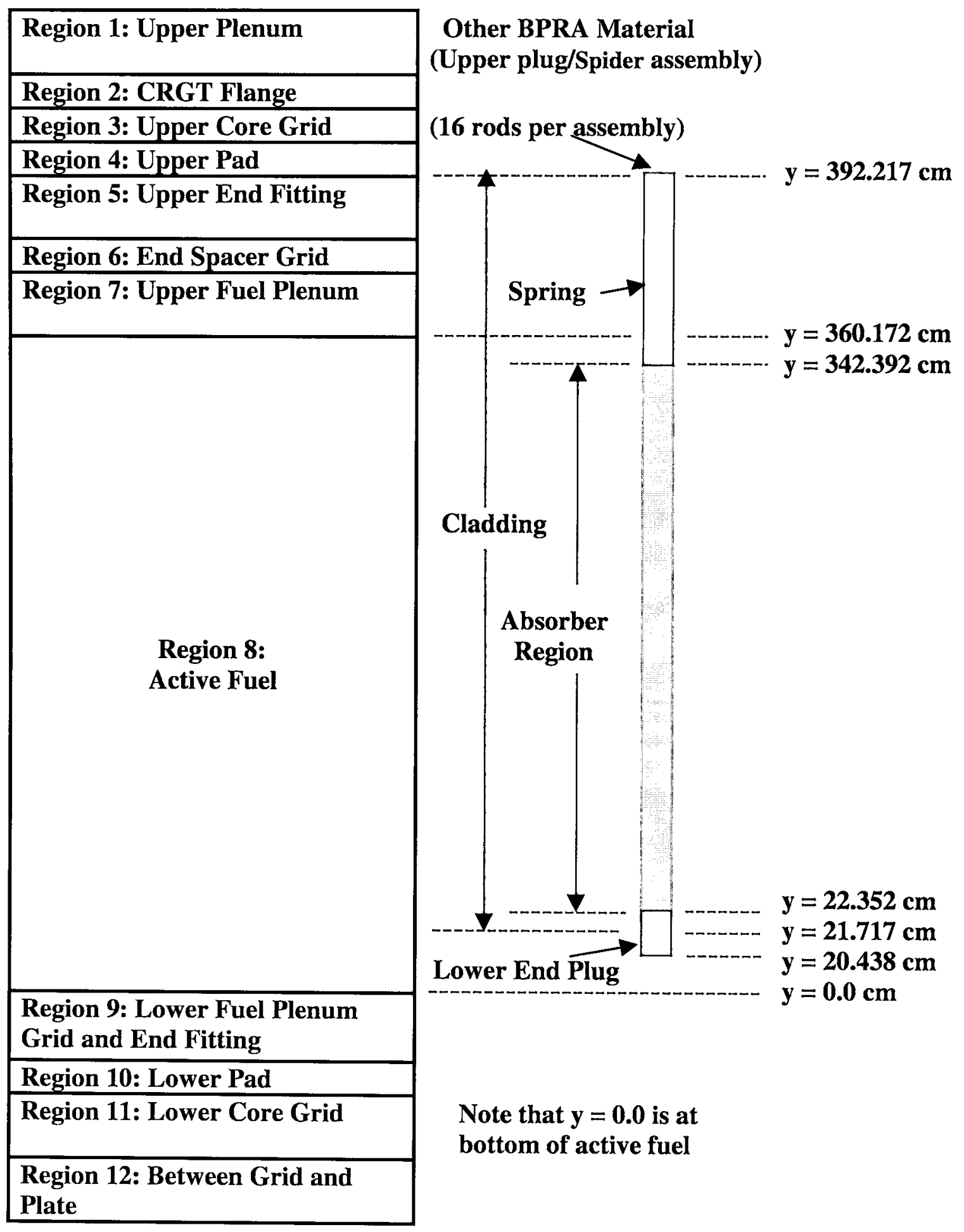

Figure 2-26. Mark-B Assembly Axial Dimensions for BPRA Inserted 


\section{FUEL CYCLE DESIGN INFORMATION}

This section provides fuel assembly design data for Cycles 1 through 11 of the Three Mile Island Unit 1 (TMI-1) reactor. Material and geometry data for the fuel assembly components along with cycle length data is presented in Section 3.1. The fuel assembly locations for each cycle, fuel enrichments, burnable absorber enrichments, and control rod bank locations are presented in Section 3.2.

\subsection{FUEL BATCH DATA}

Material and geometry data for each fuel batch in cycles 1 through 11 are given in Table 3-1. This includes the cycle in which the fuel was first loaded, the fuel assembly type, the enrichment, the kilograms of uranium in each fuel assembly batch, the diameter of the fuel pellets, and the type of fuel assembly grid material. The axial dimensions of the Mk-B2, MkB3, and Mk-B4 fuel assemblies are represented by the Mark-B4 fuel assembly design shown in Figure 2-3. The type of axial power shaping rods (APSRs) used in each cycle is provided along with information concerning the use of burnable poison rods and the mode of operation (operated with regulating control rod banks inserted). The radial dimensions of the fuel clad, instrument tube, and guide tube are presented in Table 2-2. Material and radial dimensions of the RCCAs, the black and gray APSRs, and the BPRAs are provided in Section 2.6. This data should be used in modeling each fuel assembly type for burnup calculations and the reactor criticality calculations for the statepoints (SPs) defined in Table 3-2.

Table 3-1. Fuel Assembly/Fuel Rod/Cycle Description for Cycles 1 through 11

\begin{tabular}{|c|c|c|c|c|c|c|c|c|c|c|}
\hline Cycle & $\begin{array}{c}\text { Fresh } \\
\text { Fuel } \\
\text { Batch }\end{array}$ & $\begin{array}{c}\text { Assembly } \\
\text { Type }\end{array}$ & $\begin{array}{l}\text { Wt \% } \\
{ }^{235} \mathrm{U}\end{array}$ & $\begin{array}{c}\% \\
\text { TD }\end{array}$ & $\begin{array}{c}\mathrm{KgU} / \\
\text { Assembly }\end{array}$ & $\begin{array}{c}\text { Fuel Pellet } \\
\text { OD } \\
\text { (cm) }\end{array}$ & $\begin{array}{c}\text { Fuel } \\
\text { Assembly } \\
\text { Grid } \\
\text { Material } \\
\end{array}$ & $\begin{array}{c}\text { APSRs } \\
\text { Blackd } \\
\text { Gray }\end{array}$ & $\begin{array}{c}\text { Burnable } \\
\text { Poison } \\
\text { Cycle } \\
\end{array}$ & $\begin{array}{c}\text { Rodded } \\
\text { Cycle }\end{array}$ \\
\hline \multirow[t]{3}{*}{1} & 1 & Mk-B2 & 2.06 & 92.5 & 463.63 & 0.93980 & Inconel 718 & Black & Yes & Yes \\
\hline & 2 & Mk-B3 & 2.75 & 92.5 & 463.63 & 0.93980 & Inconel 718 & Black & Yes & Yes \\
\hline & 3 & Mk-B3 & 3.05 & 92.5 & 463.63 & 0.93980 & Inconel 718 & Black & Yes & Yes \\
\hline 2 & 4 & Mk-B4 & 2.64 & 93.5 & 468.63 & 0.939038 & Inconel 718 & Black & No & Yes \\
\hline 3 & 5 & Mk-B4 & 2.85 & 94.0 & 463.63 & 0.93853 & Inconel 718 & Black & No & Yes \\
\hline 4 & 6 & Mk-B4 & 2.85 & 94.0 & 463.63 & 0.93853 & Inconel 718 & Black & No & No \\
\hline 5 & 7 & Mk-B4 & 2.85 & 94.0 & 463.63 & 0.93853 & Inconel 718 & Black & No & No \\
\hline \multirow[t]{2}{*}{6} & $8 \mathrm{~A}$ & Mk-B4 & 2.95 & 95.0 & 463.63 & 0.936244 & Inconel 718 & Gray & Yes & No \\
\hline & $8 B, C, D$ & Mk-B4 & 2.85 & 94.0 & 463.6 & 0.93853 & Inconel 718 & Gray & Yes & No \\
\hline \multirow[t]{3}{*}{7} & $9 A$ & Mk-B4 & 2.85 & 95.0 & 463.63 & 0.936244 & Inconel 718 & Gray & Yes & No \\
\hline & $9 \mathrm{~B}$ & Mk-B4 & 2.95 & 95.0 & 463.6 & 0.936244 & Inconel 718 & Gray & Yes & No \\
\hline & $9 \mathrm{C}$ & Mk-B4Z & 3.63 & 95.0 & 463.6 & 0.936244 & Zircaloy-4 & Gray & Yes & No \\
\hline \multirow[t]{6}{*}{8} & $10 \mathrm{~A}$ & Mk-B4 & 2.85 & 95.0 & 463.63 & 0.936244 & Inconel 718 & Gray & Yes & No \\
\hline & $10 \mathrm{~B}$ & Mk-B4Z & 3.63 & 95.0 & 463.63 & 0.936244 & Zircaloy-4 & Gray & Yes & No \\
\hline & $10 \mathrm{~B}$ & Mk-B8 & 3.63 & 95.0 & 463.63 & 0.936244 & Zircaloy-4 & Gray & Yes & No \\
\hline & $10 C$ & Mk-B4 & 2.85 & 95.0 & 463.63 & 0.936244 & Inconel 718 & Gray & Yes & No \\
\hline & $10 \mathrm{D}$ & Mk-B8 & 3.63 & 95.0 & 463.63 & 0.936244 & Zircaloy-4 & Gray & Yes & No \\
\hline & $10 \mathrm{E}$ & Mk-B8 & 3.63 & 95.0 & 463.63 & 0.936244 & Zircaloy-4 & Gray & Yes & No \\
\hline
\end{tabular}


Table 3-1. Fuel Assembly/Fuel Rod/Cycle Description for Cycles 1 through 11 (Cont.)

\begin{tabular}{|c|c|c|c|c|c|c|c|c|c|c|}
\hline Cycle & $\begin{array}{c}\text { Fresh } \\
\text { Fuel } \\
\text { Batch }\end{array}$ & $\begin{array}{c}\text { Assembly } \\
\text { Type }\end{array}$ & $\begin{array}{c}\text { Wt\% } \\
235\end{array}$ & $\begin{array}{c}\% \\
\text { TD }\end{array}$ & $\begin{array}{c}\text { KgU/ } \\
\text { Assy. }\end{array}$ & $\begin{array}{c}\text { Fuel Pellet } \\
\text { OD (cm) }\end{array}$ & $\begin{array}{c}\text { Assembly } \\
\text { Grid } \\
\text { Material }\end{array}$ & $\begin{array}{c}\text { APSRs } \\
\text { Black/ } \\
\text { Gray }\end{array}$ & $\begin{array}{c}\text { Burnable } \\
\text { Poison } \\
\text { Cycle }\end{array}$ & $\begin{array}{c}\text { Rodded } \\
\text { Cycle }\end{array}$ \\
\hline 9 & $11 \mathrm{~A}$ & Mk-B8 & 3.63 & 95.0 & 463.605 & 0.936244 & Zircaloy-4 & Gray & Yes & No \\
\hline & $11 \mathrm{~B}$ & W-LTA & 3.90 & 95.0 & 463.605 & 0.929386 & Zircaloy-4 & Gray & Yes & No \\
\hline & $11 \mathrm{C}$ & Mk-B8V & 4.00 & 95.0 & 463.605 & 0.936244 & Zircaloy-4 & Gray & Yes & No \\
\hline & $11 \mathrm{D}$ & Mk-B8 & 3.63 & 95.0 & 463.63 & 0.936244 & Zircaloy-4 & Gray & Yes & No \\
\hline & $11 \mathrm{E}$ & Mk-B8 & 4.00 & 95.0 & 463.605 & 0.936244 & Zircaloy-4 & Gray & Yes & No \\
\hline 10 & $12 \mathrm{~A}$ & Mk-B8V & 4.00 & 95.0 & 463.60 & 0.936244 & Zircaloy-4 & Gray & Yes & No \\
\hline & $12 \mathrm{~B}$ & Mk-B9 & 4.65 & 95.0 & 463.66 & 0.939800 & Zircaloy-4 & Gray & Yes & No \\
\hline & $12 \mathrm{C}$ & Mk-B9Gd & $4.65^{\mathrm{a}}$ & 95.0 & 463.45 & 0.939800 & Zircaloy-4 & Gray & Yes & No \\
\hline & $12 \mathrm{D}$ & Mk-B9 & 4.75 & 95.0 & 463.66 & 0.939800 & Zircaloy-4 & Gray & Yes & No \\
\hline & $12 \mathrm{E}$ & Mk-B9Gd & $4.75^{\mathrm{a}}$ & 95.0 & 463.45 & 0.939800 & Zircaloy-4 & Gray & Yes & No \\
\hline 11 & $13 \mathrm{~A}$ & Mk-B8V & 4.00 & 95.0 & 463.60 & 0.936244 & Zircaloy-4 & Gray & Yes & No \\
\hline & $13 \mathrm{~B}$ & Mk-B10 & 4.23 & 95.0 & 463.66 & 0.939800 & Zircaloy-4 & Gray & Yes & No \\
\hline & $13 \mathrm{C}$ & Mk-B10Gd & $4.23^{\mathrm{b}}$ & 95.0 & 463.45 & 0.939800 & Zircaloy-4 & Gray & Yes & No \\
\hline & $13 \mathrm{D}$ & W-LTA & 4.25 & 95.0 & 457.02 & 0.929386 & Zircaloy-4 & Gray & Yes & No \\
\hline & $13 \mathrm{E}$ & Mk-B10Gd & $4.55^{\mathrm{b}}$ & 95.0 & 463.45 & 0.939800 & Zircaloy-4 & Gray & Yes & No \\
\hline & $13 \mathrm{~F}$ & Mk-B10Gd & $4.55^{\mathrm{c}}$ & 95.0 & 463.23 & 0.939800 & Zircaloy-4 & Gray & Yes & No \\
\hline
\end{tabular}

NOTES: ${ }^{a}$ The fuel assemblies have 4 gadolinia rods with $2.0 \mathrm{wt} \% \mathrm{Gd}_{2} \mathrm{O}_{3}$ and $4.19 \mathrm{wt} \%{ }^{235} \mathrm{U}$

${ }^{b}$ The fuel assemblies have 4 Gadolinia rods with $2.0 \mathrm{wt} \% \mathrm{Gd}_{2} \mathrm{O}_{3}$ and $3.59 \mathrm{wt} \%{ }^{235} \mathrm{U}$

${ }^{\circ}$ The fuel assemblies have 8 Gadolinia rods with $2.0 \mathrm{wt} \% \mathrm{Gd}_{2} \mathrm{O}_{3}$ and $3.59 \mathrm{wt} \%{ }^{235} \mathrm{U}$

The length of each fuel cycle, expressed as effective-full-power-days (EFPD), is provided in Table 3-2. The time during each cycle where SP criticality data was measured is indicated.

Table 3-2. Statepoint Information for Cycles 1 through 11

\begin{tabular}{|c|c|c|c|c|}
\hline Cycle & $\begin{array}{c}\text { Full Power } \\
\text { (MWt) }\end{array}$ & $\begin{array}{c}\text { End-of-Cycle } \\
\text { EFPD }\end{array}$ & $\begin{array}{c}\text { Statepoint } \\
\text { Number }\end{array}$ & $\begin{array}{c}\text { Measurement } \\
\text { EFPD }\end{array}$ \\
\hline 1 & 2535 & 466.4 & SP59 & 0.0 \\
\hline 5 & 2535 & 302.4 & SP60 & 0.0 \\
\hline 6 & & & SP61 & 114.4 \\
\hline & 2535 & 421.2 & SP62 & 0.0 \\
\hline 7 & & & SP63 & 69.1 \\
\hline & 2568 & 474.7 & SP64 & 0.0 \\
\hline & & & SP65 & 30.0 \\
\hline 8 & 2568 & & SP66 & 107.8 \\
\hline 9 & 2568 & 508.8 & SP67 & 0.0 \\
\hline 10 & 2568 & 639.4 & SP68 & 0.0 \\
\hline & & 660.3 & SP69 & 0.0 \\
\hline & & & SP70 & 27.0 \\
\hline & & & SP71 & 143.0 \\
\hline 11 & 2568 & 683.0 & SP72 & 209.0 \\
\hline & & & SP73 & 0.0 \\
\hline & & & SP74 & 615.0 \\
\hline
\end{tabular}




\subsection{FUEL ASSEMBLY DATA}

The fuel assembly loading for each cycle is presented in Figures 3-1 through 3-12. The figures are representative of a one-eighth core, where the fuel assembly at the center of the core is in location $\mathrm{H} 8$. The data on the figures indicate the location of the fuel assemblies in the current cycle, the location in a previous cycle (if applicable), the cycle that the fuel was first inserted, and the fuel batch number for each fuel assembly. The enrichment of ${ }^{235} \mathrm{U}$ (by batch), the locations of BPRAs, the weight percent of $\mathrm{B}_{4} \mathrm{C}$ in each BPRA, and the location of the various control rod banks are also indicated.

Each fuel assembly is given a unique alphanumeric designation that is used in tracking the assembly through its entire period of operation. This includes both the times that each fuel assembly was in the reactor during reactor operation (i.e., producing power) and the time spent in a non-power producing mode (e.g., in the reactor during shutdown or in the spent fuel pool).

Starting with the letter " $A$ " for Cycle 1, each subsequent cycle is assigned a unique letter designation (" $B$ " for Cycle 2, "C" for Cycle 3, etc. to " $L$ " for Cycle 11). Each of the oneeighth core locations is assigned a unique number. Since the TMI-1 reactor contains 177 fuel assemblies, the one-eighth core symmetry reduces the number of assemblies to 29 . The locations in the figure are numbered 1 through 29 . Starting at the center of the core, H8 is location number 1 . Location numbers 2 through 8 are assigned to H9 through H15. Proceeding from left to right (then down), $\mathrm{K} 9$ becomes location 9, K15 becomes location 15, L10 becomes location 16, M11 becomes location 22, etc., to 013 becomes location 29.

Using this nomenclature, the assemblies in Cycle 1 are labeled A1 (for H8) through A29 (for O13). For subsequent cycles, a complete set of labels is not required since a combination of burned and fresh fuel is used.

Figures 3-13 through 3-23 were constructed by applying this nomenclature to the fuel assembly location data given in Figures 3-1 through 3-12. These figures also provide additional data that is needed for modeling fuel cycles. It is observed in examining Figures 3-13 through 3-23 that a further designation is assigned to the fuel assembly identification. For example, in Cycle 7 (Figure 3-19), assemblies G12 and G12a are in position K12. In the next cycle (Figure 3-20), assembly G12 is in position H10 and assembly G12a is in position M11. The assemblies take on different fuel cycle paths. 


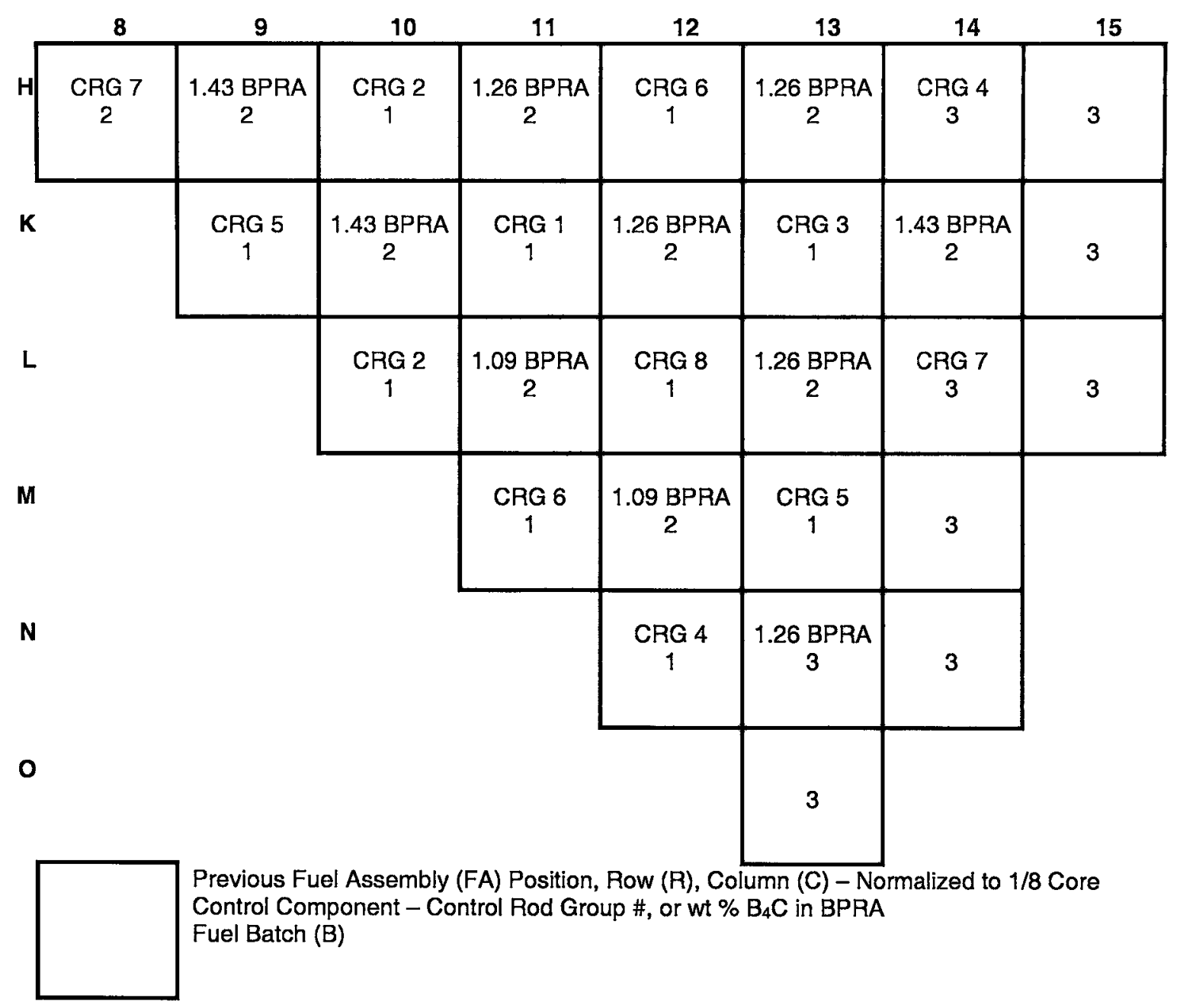

The difference between Cycle $1 A$ and Cycle $1 B$ is the location of the RCCAs.

\begin{tabular}{|c|c|c|c|}
\hline Cycle & Batch/FA & wt $\%{ }^{235} \mathrm{U}$ & \# FA \\
\hline \multirow{2}{*}{1} & $1 / \mathrm{MK}-\mathrm{B} 2$ & 2.06 & 56 \\
\cline { 2 - 4 } & $2 / \mathrm{MK}-\mathrm{B} 3$ & 2.75 & 61 \\
\cline { 2 - 4 } & $3 / \mathrm{MK}-\mathrm{B} 3$ & 3.05 & 60 \\
\hline \multicolumn{3}{|c}{} & 177 \\
\hline
\end{tabular}

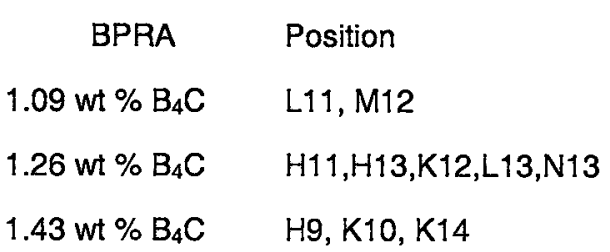

Banks 1-4 Safety $\mathrm{H} 10, \mathrm{H} 14, \mathrm{~K} 11, \mathrm{~K} 13, \mathrm{~L} 10, \mathrm{~N} 12$

Bank 5 Regulating $\mathrm{K} 9, \mathrm{M} 13$

Bank 6 Regulating $\mathrm{H} 12, \mathrm{M} 11$

Bank 7 Regulating $\mathrm{H} 8, \mathrm{~L} 14$

Bank 8 (Black APSR)

L12

Figure 3-1. One-Eighth Core Loading for TMI-1 (0.0-256.0 EFPD), Cycle 1A 


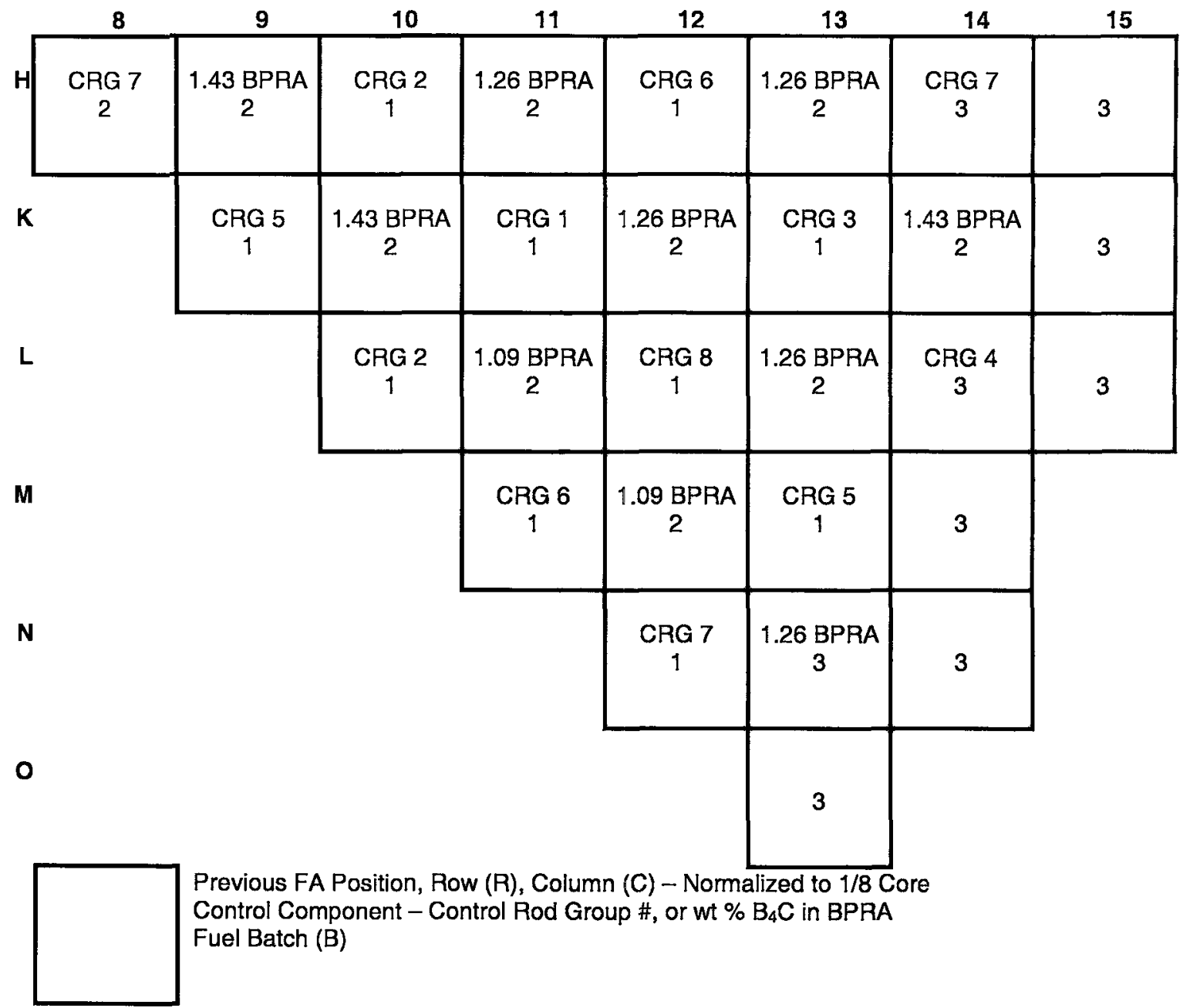

The difference between Cycle $1 \mathrm{~A}$ and Cycle $1 \mathrm{~B}$ is the location of the RCCAs.

\begin{tabular}{|c|c|c|c|}
\hline Cycle & Batch/FA & wt $\%^{235} \mathrm{U}$ & \# FA \\
\hline \multirow{3}{*}{1} & $1 / \mathrm{MK}-\mathrm{B} 2$ & 2.06 & 56 \\
\cline { 2 - 4 } & $2 / \mathrm{MK}-\mathrm{B} 3$ & 2.75 & 61 \\
\cline { 2 - 4 } & $3 / \mathrm{MK}-\mathrm{B} 3$ & 3.05 & 60 \\
\hline \multicolumn{3}{|c}{} & 177 \\
\hline
\end{tabular}

$\begin{array}{cl}\text { BPRA } & \text { Position } \\ 1.09 \text { wt } \% \mathrm{~B}_{4} \mathrm{C} & \text { L11, M12 } \\ 1.26 \text { wt } \% \mathrm{~B}_{4} \mathrm{C} & \mathrm{H} 11, \mathrm{H} 13, \mathrm{~K} 12, \mathrm{~L} 13, \mathrm{~N} 13 \\ 1.43 \text { wt } \% \mathrm{~B}_{4} \mathrm{C} & \mathrm{H} 9, \mathrm{~K} 10, \mathrm{~K} 14\end{array}$

Banks $1-4$ Safety

H10, K11, K13, L10, L14

Bank 5 Regulating

$\mathrm{K} 9, \mathrm{M} 13$

Bank 6 Regulating

H12, M11

Bank 7 Regulating

$\mathrm{H} 8, \mathrm{H} 14, \mathrm{~N} 12$

Bank 8 (Black APSA)

L12

Figure 3-2. One-Eighth Core Loading for TMI-1 (256.0 - 466.4 EFPD), Cycle 1B 


\begin{tabular}{|c|c|c|c|c|c|c|c|c|}
\hline & 8 & 9 & 10 & 11 & 12 & 13 & 14 & 15 \\
\hline$H$ & $\begin{array}{c}\mathrm{H} 8 \\
\text { CRG } 4 \\
2\end{array}$ & $\begin{array}{c}\mathrm{H9} \\
2\end{array}$ & $\begin{array}{c}\mathrm{H} 13 \\
\text { CRG } 6 \\
2\end{array}$ & $\begin{array}{c}\mathrm{K} 12 \\
2\end{array}$ & $\begin{array}{c}\mathrm{N} 13 \\
\text { CRG } 3 \\
3\end{array}$ & $\begin{array}{c}\mathrm{H} 14 \\
3\end{array}$ & $\begin{array}{c}\mathrm{H} 15 \\
\text { CRG } 5 \\
3\end{array}$ & 4 \\
\hline K & & $\begin{array}{c}\mathrm{N} 13 \\
\text { CRG } 3 \\
3\end{array}$ & $\begin{array}{c}\text { L14 } \\
3\end{array}$ & $\begin{array}{c}\mathrm{K} 14 \\
\text { CRG } 1 \\
2\end{array}$ & 4 & $\begin{array}{c}\text { M12 } \\
\text { CRG } 7 \\
2\end{array}$ & $\begin{array}{c}\mathrm{K} 10 \\
2\end{array}$ & 4 \\
\hline$L$ & & & $\begin{array}{c}\text { K12 } \\
\text { CRG } 5 \\
2\end{array}$ & $\begin{array}{c}\overline{L 11} \\
2\end{array}$ & $\begin{array}{c}\text { M14 } \\
\text { CRG } 8 \\
3\end{array}$ & $\begin{array}{c}\text { L13 } \\
2\end{array}$ & $\begin{array}{c}\text { CAG } 4 \\
4\end{array}$ & 4 \\
\hline $\mathbf{M}$ & & & & $\begin{array}{c}\mathrm{O} 13 \\
\text { CRG } 3 \\
3\end{array}$ & $\begin{array}{c}\mathrm{K} 15 \\
3\end{array}$ & $\begin{array}{c}\text { L15 } \\
\text { CRG } 2 \\
3\end{array}$ & 4 & \\
\hline $\mathbf{N}$ & & & & & $\begin{array}{c}H 11 \\
\text { CRG } 6 \\
2\end{array}$ & $\begin{array}{c}\text { N14 } \\
3\end{array}$ & 4 & \\
\hline 0 & & & & & & 4 & & \\
\hline & & \multicolumn{7}{|c|}{$\begin{array}{l}\text { Previous FA Position, Row (A), Column (C) - Normalized to } 1 / 8 \text { Core } \\
\text { Control Component - Control Rod Group \#, or wt \% } \mathrm{B}_{4} \mathrm{C} \text { in BPRA } \\
\text { Fuel Batch (B) }\end{array}$} \\
\hline
\end{tabular}

\begin{tabular}{|c|c|c|c|}
\hline Cycle & Batch/FA & wt $\%{ }^{235} \mathrm{U}$ & \# FA \\
\hline 2 & $2 / \mathrm{MK}-\mathrm{B} 3$ & 2.75 & 61 \\
\cline { 2 - 4 } & $3 / \mathrm{MK}-\mathrm{B} 3$ & 3.05 & 60 \\
\cline { 2 - 4 } & $4 / \mathrm{MK}-\mathrm{B} 4$ & 2.64 & 56 \\
\hline \multicolumn{2}{|l}{} & 177 \\
\hline
\end{tabular}

No BPRAs

$\begin{array}{ll}\text { Banks 1-4 Safety } & \mathrm{H} 8, \mathrm{H} 12, \mathrm{~K} 9, \mathrm{~K} 11, \mathrm{~L} 14, \mathrm{M} 11, \mathrm{M} 13 \\ \text { Bank 5 Regulating } & \mathrm{H} 14, \mathrm{~L} 10 \\ \text { Bank 6 Regulating } & \mathrm{H} 10, \mathrm{~N} 12 \\ \text { Bank 7 Transient } & \mathrm{K} 13 \\ \text { Bank 8 (Black APSR) } & \mathrm{L} 12\end{array}$

Figure 3-3. One-Eighth Core Loading for TMI-1, Cycle 2 


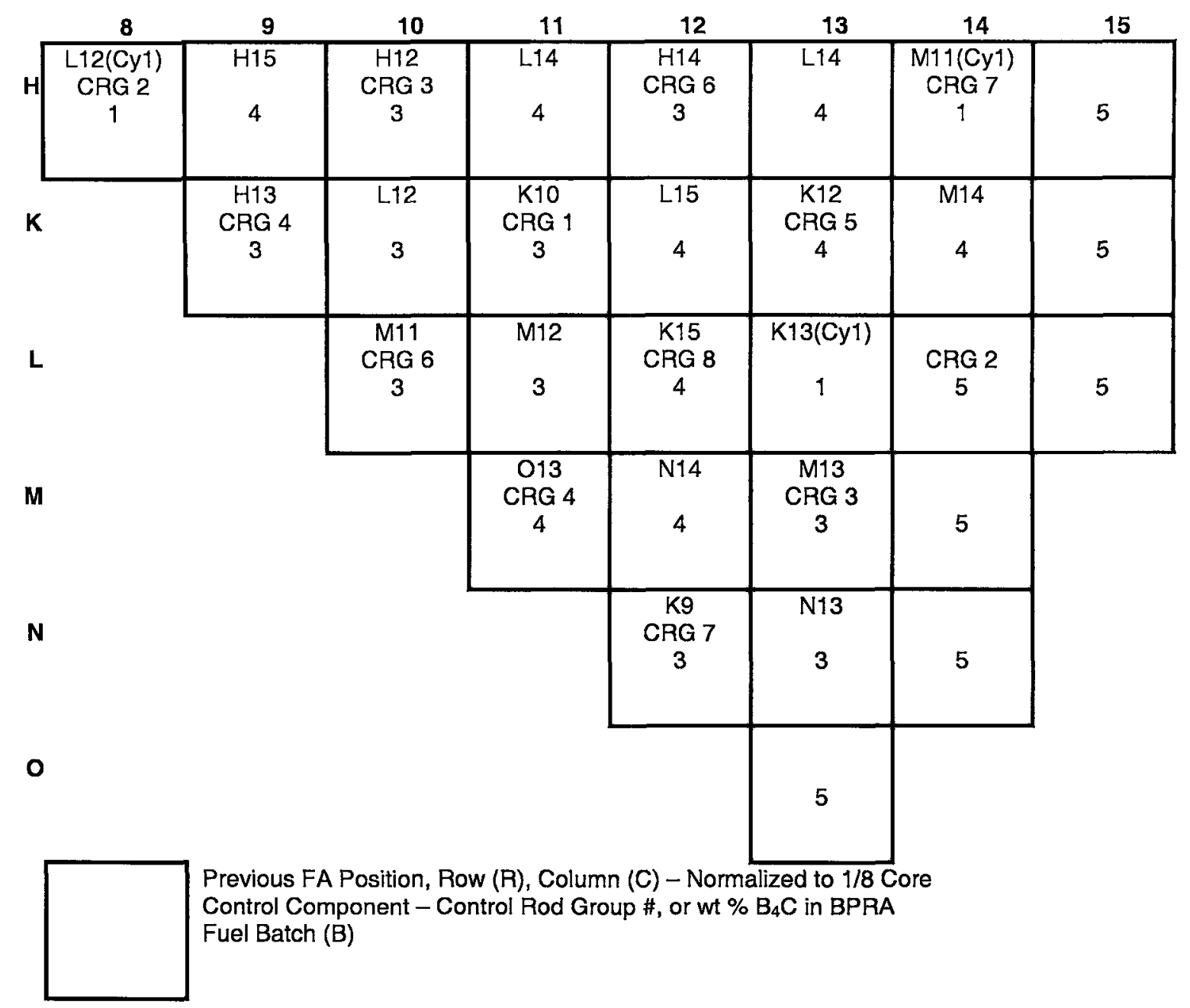

\begin{tabular}{|c|c|c|c|}
\hline Cycle & Batch/FA & wt $\%{ }^{235} \mathrm{U}$ & \# FA \\
\hline \multirow{3}{*}{3} & $1 / \mathrm{MK}-\mathrm{B} 2$ & 2.06 & 13 \\
\cline { 2 - 4 } & $3 / \mathrm{MK}-\mathrm{B} 3$ & 3.05 & 60 \\
\cline { 2 - 4 } & $4 / \mathrm{MK}-\mathrm{B} 4$ & 2.64 & 56 \\
\cline { 2 - 4 } & $5 / \mathrm{MK}-\mathrm{B} 4$ & 2.85 & 48 \\
\hline \multicolumn{2}{|c}{} & 177 \\
\hline
\end{tabular}

No BPRAs

Banks 1-4 Safety

Bank 5 Regulating

Bank 6 Regulating

Bank 7 Transient

Bank 8 (Black APSR)
$\mathrm{H} 8, \mathrm{H} 10, \mathrm{~K} 9, \mathrm{~K} 11, \mathrm{~L} 14, \mathrm{M} 11, \mathrm{M} 13$

$\mathrm{K} 13$

$\mathrm{H} 12, \mathrm{~L} 10$

$\mathrm{H} 14, \mathrm{~N} 12$

L12

Figure 3-4. One-Eighth Core Loading for TMI-1, Cycle 3 


\begin{tabular}{|c|c|c|c|c|c|c|c|}
\hline 8 & 9 & 10 & 11 & 12 & 13 & 14 & 15 \\
\hline H $\begin{array}{c}\text { L12(Cy } 1) \\
\text { CRG } 3 \\
1\end{array}$ & $\begin{array}{c}\mathrm{H} 9 \\
4\end{array}$ & $\begin{array}{c}\mathrm{K} 15 \\
\text { CRG } 5 \\
5\end{array}$ & $\begin{array}{c}\text { M13(Cy1) } \\
1\end{array}$ & $\begin{array}{c}\mathrm{H} 15 \\
\text { CRG } 5 \\
5\end{array}$ & $\begin{array}{c}\text { L12(Cy1) } \\
1\end{array}$ & $\begin{array}{c}\text { M11 } \\
\text { CRG } 7 \\
4\end{array}$ & 6 \\
\hline K & $\begin{array}{c}\mathrm{K} 15 \\
\text { CRG } 4 \\
5\end{array}$ & $\begin{array}{c}\mathrm{H} 11 \\
4\end{array}$ & $\begin{array}{c}\text { L14 } \\
\text { CRG } 2 \\
5\end{array}$ & $\begin{array}{c}\text { L12 } \\
4\end{array}$ & $\begin{array}{c}\mathrm{N} 14 \\
\text { CRG } 6 \\
5\end{array}$ & $\begin{array}{c}\mathrm{K} 13 \\
4\end{array}$ & 6 \\
\hline L & & $\begin{array}{c}\text { O13 } \\
\text { CRG } 7 \\
5\end{array}$ & $\begin{array}{c}\text { M12 } \\
4\end{array}$ & $\begin{array}{c}\text { L15 } \\
\text { CRG } 8 \\
5\end{array}$ & $\begin{array}{c}\text { L13(Cy2) } \\
2\end{array}$ & $\begin{array}{c}\text { CRG } 3 \\
6\end{array}$ & 6 \\
\hline $\mathbf{M}$ & & & $\begin{array}{c}\text { N12(Cy1) } \\
\text { CRG } 5 \\
1\end{array}$ & $\begin{array}{c}\text { M14 } \\
5\end{array}$ & $\begin{array}{c}\text { K14 } \\
\text { CRG } 1 \\
4\end{array}$ & 6 & \\
\hline $\mathbf{N}$ & & & & $\begin{array}{c}\mathrm{K} 12 \\
\text { CRG } 7 \\
4\end{array}$ & 6 & 6 & \\
\hline 0 & & & & & $\begin{array}{c}\mathrm{K} 12 \\
4\end{array}$ & & \\
\hline & \multicolumn{7}{|c|}{$\begin{array}{l}\text { Previous FA Position, Row (R), Column (C) - Normalized to } 1 / 8 \text { Core } \\
\text { Control Component - Control Rod Group \#, or wt } \% \mathrm{~B}_{4} \mathrm{C} \text { in BPRA } \\
\text { Fuel Batch (B) }\end{array}$} \\
\hline
\end{tabular}

\begin{tabular}{|c|c|c|c|}
\hline Cycle & Batch/FA & wt $\%{ }^{235} \mathrm{U}$ & \# FA \\
\hline \multirow{4}{*}{4} & $1 / \mathrm{MK}-\mathrm{B} 2$ & 2.06 & 13 \\
\cline { 2 - 4 } & $2 / \mathrm{MK}-\mathrm{B} 3$ & 2.75 & 8 \\
\cline { 2 - 4 } & $4 / \mathrm{MK}-\mathrm{B} 4$ & 2.64 & 56 \\
\cline { 2 - 4 } & $5 / \mathrm{MK}-\mathrm{B} 4$ & 2.85 & 48 \\
\cline { 2 - 4 } & $6 / \mathrm{MK}-\mathrm{B} 4$ & 2.85 & 52 \\
\hline \multicolumn{3}{|c}{} & 177 \\
\hline
\end{tabular}

No BPRAs

Banks 1-4 Safety $\quad$ H8, K9, K11, L14, M13

Bank 5 Regulating $\quad \mathrm{H} 10, \mathrm{H} 12, \mathrm{M} 11$

Bank 6 Regulating $\mathrm{K} 13$

Bank 7 Transient $\quad H 14, L 10, N 12$

Bank 8 (Black APSR) L12

Figure 3-5. One-Eighth Core Loading for TMI-1, Cycle 4 


\begin{tabular}{|c|c|c|c|c|c|c|c|c|}
\hline & 8 & 9 & 10 & 11 & 12 & 13 & 14 & 15 \\
\hline $\mathrm{H}$ & $\begin{array}{c}\mathrm{N} 12 \\
\text { CRG } 3 \\
4\end{array}$ & $\begin{array}{c}\mathrm{H} 10 \\
5\end{array}$ & $\begin{array}{c}\mathrm{H} 15 \\
\text { CRG } 5 \\
6\end{array}$ & $\begin{array}{c}\mathrm{H} 14 \\
4\end{array}$ & $\begin{array}{c}\text { L14 } \\
\text { CRG } 5 \\
6\end{array}$ & $\begin{array}{c}\text { H12 } \\
5\end{array}$ & $\begin{array}{c}\text { L14 } \\
\text { CRG } 7 \\
6\end{array}$ & 7 \\
\hline$K$ & & $\begin{array}{c}\mathrm{M} 14 \\
\mathrm{CRG} 4 \\
6\end{array}$ & $\begin{array}{c}\mathrm{K} 11 \\
5\end{array}$ & $\begin{array}{c}\mathrm{N} 14 \\
\mathrm{CRG} 2 \\
6\end{array}$ & $\begin{array}{c}M 12 \\
5\end{array}$ & $\begin{array}{c}\text { K15 } \\
\text { CRG } 6 \\
6\end{array}$ & $\begin{array}{c}\mathrm{K} 12 \\
4\end{array}$ & 7 \\
\hline L & & & $\begin{array}{c}\text { M14 } \\
\text { CRG } 7 \\
6\end{array}$ & $\begin{array}{c}L 12 \\
5\end{array}$ & $\begin{array}{c}\text { L15 } \\
\text { CRG } 8 \\
6\end{array}$ & $\begin{array}{c}\mathrm{L11} \\
4\end{array}$ & $\begin{array}{c}\text { CRG } 3 \\
7\end{array}$ & 7 \\
\hline $\mathbf{M}$ & & & & $\begin{array}{c}\mathrm{K9} \\
\mathrm{CRG} 5 \\
5\end{array}$ & $\begin{array}{c}\mathrm{K} 13 \\
5\end{array}$ & $\begin{array}{c}\text { N13 } \\
\text { CRG } 1 \\
6\end{array}$ & 7 & \\
\hline $\mathbf{N}$ & & & & & $\begin{array}{c}\mathrm{O} 13 \\
\mathrm{CRG} 7 \\
4\end{array}$ & 7 & 7 & \\
\hline 0 & & & & & & $\begin{array}{c}\mathrm{L10} \\
5\end{array}$ & & \\
\hline & & \multicolumn{7}{|c|}{$\begin{array}{l}\text { Previous FA Position, Row (A), Column (C) - Normalized to } 1 / 8 \text { Core } \\
\text { Control Component - Control Rod Group \#, or wt \% } B_{4} C \text { in BPRA } \\
\text { Fuel Batch (B) }\end{array}$} \\
\hline
\end{tabular}

\begin{tabular}{|c|c|c|c|}
\hline Cycle & Batch/FA & wt $\%{ }^{235} \mathrm{U}$ & \# FA \\
\hline \multirow{3}{*}{5} & $4 / \mathrm{MK}-\mathrm{B} 4$ & 2.64 & 25 \\
\cline { 2 - 4 } & $5 / \mathrm{MK}-\mathrm{B} 4$ & 2.85 & 48 \\
\cline { 2 - 4 } & $6 / \mathrm{MK}-\mathrm{B} 4$ & 2.85 & 52 \\
\cline { 2 - 4 } & $7 / \mathrm{MK}-\mathrm{B} 4$ & 2.85 & 52 \\
\hline \multicolumn{3}{|c}{} & 177 \\
\hline
\end{tabular}

No BPRAs

Banks 1 - 4 Safety

Bank 5 Regulating

Bank 6 Regulating

Bank 7 Transient

Bank 8 (Black APSA)
H8, K9, K11, L14, M13

H10, H12, M11

K13

H14, L10, N12

L12

Figure 3-6. One-Eighth Core Loading for TML-1, Cycle 5 


\begin{tabular}{|c|c|c|c|c|c|c|c|c|}
\hline & 8 & 9 & 10 & 11 & 12 & 13 & 14 & 15 \\
\hline$H$ & $\begin{array}{c}\text { M13 } \\
\text { CRG } 4 \\
6 B\end{array}$ & $\begin{array}{c}1.03 \text { BPRA } \\
8 \mathrm{~A}\end{array}$ & $\begin{array}{c}\text { K09 } \\
\text { CRG } 2 \\
6 B\end{array}$ & $\begin{array}{c}1.03 \mathrm{BPRA} \\
8 \mathrm{~B}\end{array}$ & $\begin{array}{c}\text { K13 } \\
\text { CRG } 7 \\
6 B\end{array}$ & $\begin{array}{c}0.8 \text { BPRA } \\
8 \mathrm{~B}\end{array}$ & $\begin{array}{c}\text { M13 } \\
\text { CRG } 6 \\
6 B\end{array}$ & $\begin{array}{c}\mathrm{H} 12 \\
6 \mathrm{~B}\end{array}$ \\
\hline K & & $\begin{array}{c}\text { L10 } \\
\text { CRG } 2 \\
6 B\end{array}$ & $\begin{array}{c}1.03 \mathrm{BPRA} \\
8 \mathrm{~B}\end{array}$ & $\begin{array}{c}\mathrm{L} 12 \\
\mathrm{CRG} 4 \\
6 \mathrm{~B}\end{array}$ & $\begin{array}{c}1.03 \mathrm{BPRA} \\
8 \mathrm{~B}\end{array}$ & $\begin{array}{c}\text { M14 } \\
\text { CRG } 5 \\
7\end{array}$ & $\begin{array}{c}0.2 \text { BPRA } \\
8 B\end{array}$ & $\begin{array}{c}\text { L14 } \\
7\end{array}$ \\
\hline $\mathbf{L}$ & & & $\begin{array}{c}\mathrm{H} 14 \\
\text { CRG } 6 \\
6 \mathrm{~B}\end{array}$ & $\begin{array}{c}1.03 \text { BPRA } \\
8 A\end{array}$ & $\begin{array}{c}\mathrm{K} 11 \\
\mathrm{CRG} 8 \\
6 \mathrm{~B}\end{array}$ & $\begin{array}{c}0.8 \mathrm{BPRA} \\
8 \mathrm{~B}\end{array}$ & $\begin{array}{c}\text { K15 } \\
\text { CRG } 1 \\
7\end{array}$ & $\begin{array}{c}\text { N13 } \\
7\end{array}$ \\
\hline $\mathbf{M}$ & & & & $\begin{array}{c}\mathrm{H} 10 \\
\text { CRG } 5 \\
6 B\end{array}$ & $\begin{array}{c}0.8 \text { BPRA } \\
8 B\end{array}$ & $\begin{array}{c}\text { N14 } \\
\text { CRG } 3 \\
7\end{array}$ & $8 B$ & \\
\hline $\mathbf{N}$ & & & & & $\begin{array}{c}\mathrm{K} 13 \\
\mathrm{CRG} 7 \\
6 \mathrm{~B}\end{array}$ & $\begin{array}{c}0.2 \mathrm{BPRA} \\
8 \mathrm{~B}\end{array}$ & $\begin{array}{c}\text { L15 } \\
7\end{array}$ & \\
\hline 0 & & & & & & $\begin{array}{c}\mathrm{H} 15 \\
7\end{array}$ & & \\
\hline & & \multicolumn{7}{|c|}{$\begin{array}{l}\text { Previous FA Position, Row (B), Column (C) - Normalized to } 1 / 8 \text { Core } \\
\text { Control Component - Control Rod Group \#, or wt \% } \mathrm{B}_{4} \mathrm{C} \text { in BPRA } \\
\text { Fuel Batch (B) }\end{array}$} \\
\hline
\end{tabular}

\begin{tabular}{|c|c|c|c|}
\hline Cycle & Batch/FA & wt $\%^{235} \mathrm{U}$ & \# FA \\
\hline \multirow{3}{*}{6} & $6 / \mathrm{MK}-\mathrm{B} 4$ & 2.85 & 49 \\
\cline { 2 - 4 } & $7 / \mathrm{MK}-\mathrm{B} 4$ & 2.85 & 52 \\
\cline { 2 - 4 } & $8 \mathrm{~A} / \mathrm{MK}-\mathrm{B} 4$ & 2.95 & 12 \\
\cline { 2 - 4 } & $8 \mathrm{~B} / \mathrm{MK}-\mathrm{B} 4$ & 2.85 & 64 \\
\hline \multicolumn{3}{|c}{} & 177 \\
\hline
\end{tabular}

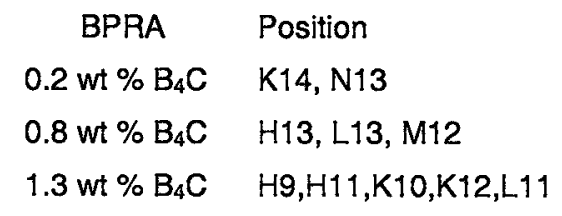

Banks 1-4 Safety H8, H10, K9, K11, L14, M13

Bank 5 Regulating K13, M11

Bank 6 Regulating H14, L10

Bank 7 Regulating $\mathrm{H} 12, \mathrm{~N} 12$

Bank 8 (Gray-APSR) L12

Figure 3-7. One-Eighth Core Loading for TMI-1, Cycle 6 


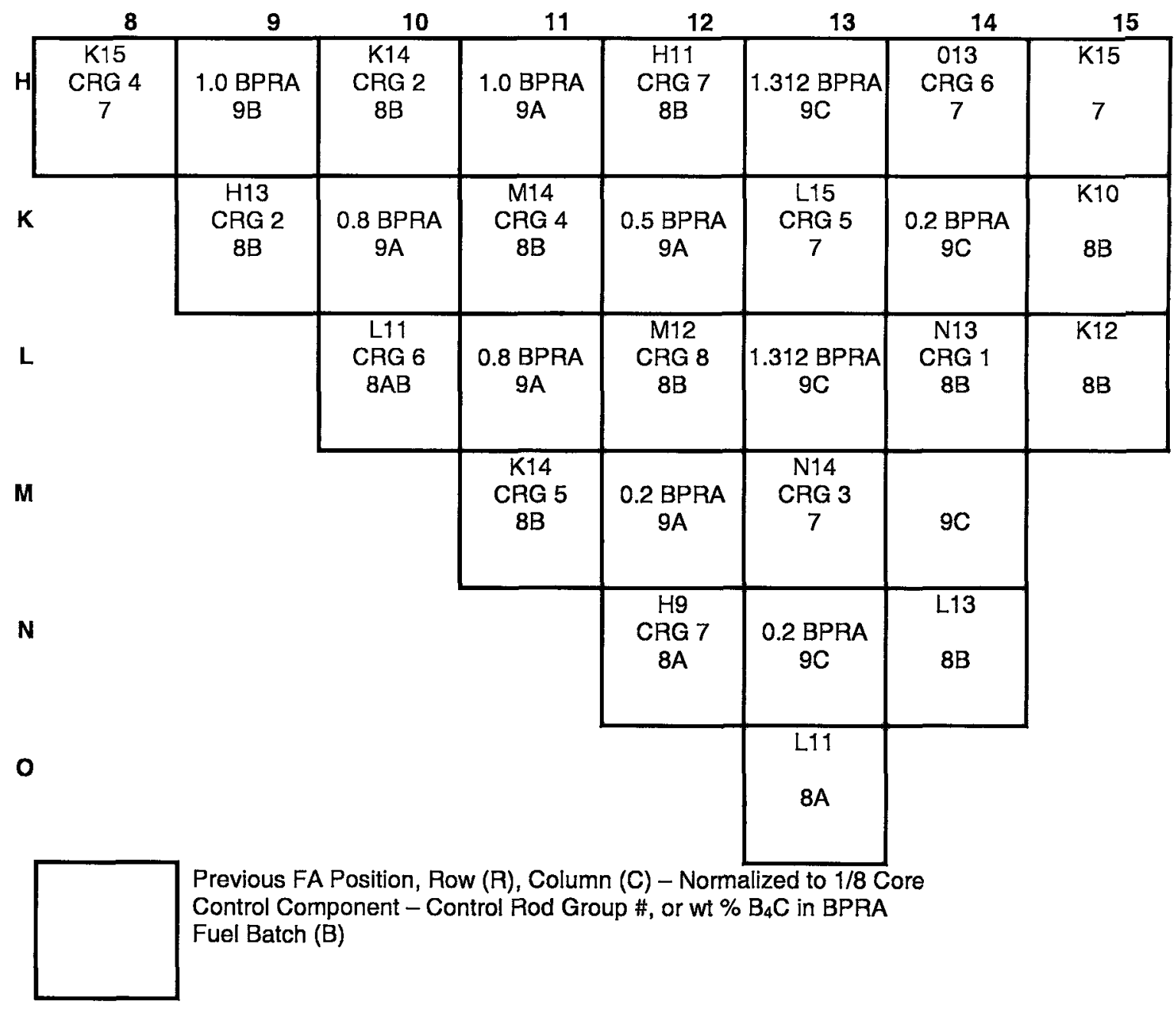

\begin{tabular}{|c|c|c|c|}
\hline Cycle & Batch/FA & wt $\%{ }^{235} \mathrm{U}$ & \# FA \\
\hline 7 & $7 / \mathrm{MK}-\mathrm{B} 4$ & 2.85 & 25 \\
\cline { 2 - 4 } & 8A / MK-B4 & 2.95 & 12 \\
\cline { 2 - 4 } & $8 \mathrm{~B} / \mathrm{MK}-\mathrm{B} 4$ & 2.85 & 64 \\
\cline { 2 - 4 } & $9 \mathrm{~A} / \mathrm{MK}-\mathrm{B} 4$ & 2.85 & 36 \\
\cline { 2 - 4 } & $9 \mathrm{~B} / \mathrm{MK}-\mathrm{B} 4$ & 2.95 & 4 \\
\cline { 2 - 4 } & $9 \mathrm{C} / \mathrm{MK}-\mathrm{B} 4 Z$ & 3.63 & 36 \\
\hline
\end{tabular}

$\begin{array}{cl}\text { BPRA } & \text { Position } \\ 0.2 w t \% \mathrm{~B}_{4} \mathrm{C} & \mathrm{K} 14, \mathrm{M} 12, \mathrm{~N} 13 \\ 0.5 w t \% \mathrm{~B}_{4} \mathrm{C} & \mathrm{K} 12, \\ 0.8 w t \% \mathrm{~B}_{4} \mathrm{C} & \mathrm{K} 10, \mathrm{~L} 11 \\ 1.0 w t \% \mathrm{~B}_{4} \mathrm{C} & \mathrm{H} 9, \mathrm{H} 11 \\ 1.312 w t \% \mathrm{~B}_{4} \mathrm{C} & \mathrm{H} 13, \mathrm{~L} 13\end{array}$

Banks 1-4 Safety

H8, H10, K9, K11, L14, M13

Bank 5 Regulating

$\mathrm{K} 13, \mathrm{M} 11$

Bank 6 Regulating

H14, L10

Bank 7 Regulating

$\mathrm{H} 12, \mathrm{~N} 12$

Bank 8 (Gray -APSR)

L12

Figure 3-8. One-Eighth Core Loading for TMI-1, Cycle 7 


\begin{tabular}{|c|c|c|c|c|c|c|c|}
\hline 8 & 9 & 10 & 11 & 12 & 13 & 14 & 15 \\
\hline H $\begin{array}{c}\text { K15 (Cy6) } \\
\text { CRG } 4 \\
7\end{array}$ & $\begin{array}{l}\mathrm{M} 14 \\
9 \mathrm{C}\end{array}$ & $\begin{array}{c}\text { K12 } \\
\text { CRG } 2 \\
9 A\end{array}$ & $\begin{array}{c}0.5 \text { BPRA } \\
10 A\end{array}$ & $\begin{array}{c}\mathrm{H} 9 \\
\mathrm{CRG} 7 \\
9 \mathrm{~B}\end{array}$ & $\begin{array}{c}1.7 \text { BPRA } \\
10 B\end{array}$ & $\begin{array}{c}\text { N13 } \\
\text { CRG } 6 \\
9 C\end{array}$ & $\begin{array}{l}\mathrm{N} 14 \\
8 \mathrm{C}\end{array}$ \\
\hline $\mathbf{K}$ & $\begin{array}{c}\mathrm{H} 13 \\
\text { CRG } 2 \\
9 \mathrm{C}\end{array}$ & $\begin{array}{c}1.303 \text { BPRA } \\
10 \mathrm{~B}\end{array}$ & $\begin{array}{c}\mathrm{M} 12 \\
\mathrm{CRG} 4 \\
9 \mathrm{~A}\end{array}$ & $\begin{array}{c}1.7 \text { BPRA } \\
10 B\end{array}$ & $\begin{array}{c}\text { L13 } \\
\text { CRG } 5 \\
9 C\end{array}$ & $\begin{array}{c}0.5 \text { BPRA } \\
10 \mathrm{~B}\end{array}$ & $\begin{array}{l}\mathrm{L} 15 \\
8 \mathrm{C}\end{array}$ \\
\hline L & & $\begin{array}{c}\text { H11 } \\
\text { CRG } 6 \\
9 A\end{array}$ & $\begin{array}{c}1.7 \text { BPRA } \\
10 B\end{array}$ & $\begin{array}{c}\text { K10 } \\
\text { CRG } 8 \\
9 A\end{array}$ & $\begin{array}{c}1.7 \text { BPRA } \\
10 B\end{array}$ & $\begin{array}{c}\text { CRG } 1 \\
10 A\end{array}$ & $\begin{array}{l}\mathrm{L} 11 \\
9 \mathrm{~A}\end{array}$ \\
\hline $\mathbf{M}$ & & & $\begin{array}{c}\text { K12 } \\
\text { CRG } 5 \\
9 A\end{array}$ & $\begin{array}{c}1.7 \text { BPRA } \\
10 B\end{array}$ & $\begin{array}{c}\text { K14 } \\
\text { CRG } 3 \\
9 \mathrm{C}\end{array}$ & $10 B$ & \\
\hline $\mathbf{N}$ & & & & $\begin{array}{c}\text { N13 } \\
\text { CPG } 7 \\
9 \mathrm{C}\end{array}$ & $\begin{array}{c}0.5 \text { BPRA } \\
10 B\end{array}$ & $\begin{array}{c}\text { L14(Cy6) } \\
7 C\end{array}$ & \\
\hline 0 & & & & & $\begin{array}{l}\mathrm{M14} \\
9 \mathrm{C}\end{array}$ & & \\
\hline & \multicolumn{7}{|c|}{$\begin{array}{l}\text { Previous FA Position, Row }(\mathrm{R}) \text {, Column }(\mathrm{C}) \text { - Normalized to } 1 / 8 \text { Core } \\
\text { Control Component - Control Rod Group } \# \text {, or wt \% } \mathrm{B}_{4} \mathrm{C} \text { in BPRA } \\
\text { Fuel Batch }(B)\end{array}$} \\
\hline
\end{tabular}

\begin{tabular}{|c|c|c|c|}
\hline Cycle & Batch/FA & wt $\%{ }^{235} \mathrm{U}$ & \# FA \\
\hline 8 & $6 / \mathrm{MK}-\mathrm{B} 4$ & 2.85 & 1 \\
\cline { 2 - 4 } & $7 / \mathrm{MK}-\mathrm{B} 4$ & 2.85 & 9 \\
\cline { 2 - 4 } & $8 \mathrm{~B} / \mathrm{MK}-\mathrm{B} 4$ & 2.85 & 12 \\
\cline { 2 - 4 } & $9 \mathrm{~A} / \mathrm{MK}-\mathrm{B} 4$ & 2.85 & 35 \\
\cline { 2 - 4 } & $9 \mathrm{~B} / \mathrm{MK}-\mathrm{B} 4$ & 2.95 & 4 \\
\cline { 2 - 4 } & $9 \mathrm{C} / \mathrm{MK}-\mathrm{B} 4 \mathrm{Z}$ & 3.63 & 36 \\
\cline { 2 - 4 } & $10 \mathrm{~A} / \mathrm{MK}-\mathrm{B} 4$ & 2.85 & 12 \\
\cline { 2 - 4 } & $10 \mathrm{~B} / \mathrm{MK}-\mathrm{B} 4 \mathrm{~B}, \mathrm{~B} 8$ & 3.63 & 68 \\
\hline \multicolumn{3}{|c}{} & 177 \\
\hline
\end{tabular}

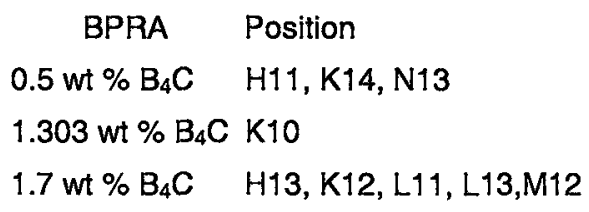

Banks 1-4 Safety

$\mathrm{H} 8, \mathrm{H} 10, \mathrm{~K} 9, \mathrm{~K} 11, \mathrm{~L} 14, \mathrm{M} 13$

Bank 5 Regulating

$\mathrm{K} 13, \mathrm{M} 11$

$\mathrm{H} 14, \mathrm{~L} 10$

$\mathrm{H} 12, \mathrm{~N} 12$

Bank 7 Regulating

$\mathrm{L} 12$

Figure 3-9. One-Eighth Core Loading for TMI-1, Cycle 8 


\begin{tabular}{|c|c|c|c|c|c|c|c|}
\hline 8 & 9 & 10 & 11 & 12 & 13 & 14 & 15 \\
\hline H $\begin{array}{c}\text { K15(Cy6) } \\
\text { CRG } 4 \\
7\end{array}$ & $\begin{array}{c}1.7 \text { BPRA } \\
11 \mathrm{~A}\end{array}$ & $\begin{array}{c}\text { N13 } \\
\text { CRG } 2 \\
10 B\end{array}$ & $\begin{array}{l}\text { M14 } \\
10 B\end{array}$ & $\begin{array}{c}\mathrm{K} 12 \\
\mathrm{CRG} 7 \\
10 \mathrm{~B}\end{array}$ & $\begin{array}{c}1.1 \mathrm{BPRA} \\
11 \mathrm{~B}\end{array}$ & $\begin{array}{c}\mathrm{H} 13 \\
\mathrm{CRG} 6 \\
10 B\end{array}$ & $\begin{array}{c}\text { N14 } \\
7\end{array}$ \\
\hline $\mathbf{K}$ & $\begin{array}{c}\text { M14 } \\
\text { CAG } 2 \\
10 B\end{array}$ & $\begin{array}{c}1.7 \mathrm{BPRA} \\
11 \mathrm{~A}\end{array}$ & $\begin{array}{c}\text { L11 } \\
\text { CRG } 4 \\
10 B\end{array}$ & $\begin{array}{c}1.7 \text { BPRA } \\
11 \mathrm{C}\end{array}$ & $\begin{array}{c}\mathrm{L} 13 \\
\mathrm{CAG} 5 \\
10 B\end{array}$ & $\begin{array}{c}0.8 \text { BPRA } \\
11 \mathrm{C}\end{array}$ & $\begin{array}{l}\mathrm{K} 15 \\
8 \mathrm{~B}\end{array}$ \\
\hline $\mathbf{L}$ & & $\begin{array}{c}\mathrm{K} 12 \\
\mathrm{CRG} 6 \\
10 \mathrm{~B}\end{array}$ & $\begin{array}{c}2.1 \text { BPRA } \\
11 \mathrm{C}\end{array}$ & $\begin{array}{c}\text { M12 } \\
\text { CRG } 8 \\
10 B\end{array}$ & $\begin{array}{c}2.1 \text { BPRA } \\
11 \mathrm{C}\end{array}$ & $\begin{array}{c}\text { CRG } 1 \\
11 \mathrm{~A}\end{array}$ & $\begin{array}{c}\text { M13(Cy6) } \\
7\end{array}$ \\
\hline $\mathbf{M}$ & & & $\begin{array}{c}\mathrm{K} 10 \\
\text { CRG } 5 \\
10 \mathrm{~B}\end{array}$ & $\begin{array}{c}2.1 \text { BPRA } \\
11 \mathrm{C}\end{array}$ & $\begin{array}{c}\mathrm{K} 14 \\
\mathrm{CRG} 3 \\
10 \mathrm{~B}\end{array}$ & $11 \mathrm{C}$ & \\
\hline $\mathbf{N}$ & & & & $\begin{array}{c}\text { N13 } \\
\text { CRG } 7 \\
10 B\end{array}$ & $\begin{array}{c}0.0 \text { BPRA } \\
11 \mathrm{C}\end{array}$ & $\begin{array}{l}\mathrm{L} 14 \\
10 \mathrm{~A}\end{array}$ & \\
\hline 0 & & & & & $\begin{array}{l}\mathrm{K} 10 \\
10 \mathrm{~B}\end{array}$ & & \\
\hline & \multicolumn{7}{|c|}{$\begin{array}{l}\text { Previous FA Position, Row (R), Column }(C)-\text { Normalized to } 1 / 8 \text { Core } \\
\text { Control Component - Control Rod Group \#, or wt \% } B_{4} C \text { in BPRA } \\
\text { Fuel Batch }(B)\end{array}$} \\
\hline
\end{tabular}

\begin{tabular}{|c|c|c|c|}
\hline Cycle & Batch/FA & wt $\%{ }^{235} \mathrm{U}$ & \# FA \\
\hline \multirow{3}{*}{} & $7 / \mathrm{MK}-\mathrm{B} 4$ & 2.85 & 13 \\
\cline { 2 - 4 } & $8 \mathrm{~B} / \mathrm{MK}-\mathrm{B} 4$ & 2.85 & 8 \\
\cline { 2 - 4 } & $10 \mathrm{~A} / \mathrm{MK}-\mathrm{B} 4$ & 2.85 & 8 \\
\cline { 2 - 4 } & $10 \mathrm{~B} / \mathrm{MK}-\mathrm{B} 4 \mathrm{Z}$ & 3.63 & 68 \\
\cline { 2 - 4 } & $11 \mathrm{~A} / \mathrm{MK}-\mathrm{B} 8$ & 3.63 & 20 \\
\cline { 2 - 4 } & $11 \mathrm{~B} / \mathrm{W}-\mathrm{LTA}$ & 3.90 & 4 \\
\cline { 2 - 4 } & $11 \mathrm{C} / \mathrm{MK}-\mathrm{B} 8 \mathrm{~V}$ & 4.00 & 56 \\
\hline
\end{tabular}

$\begin{array}{rl}\text { BPRA } & \text { Position } \\ 0.0 \text { wt \% } \mathrm{B}_{4} \mathrm{C} & \mathrm{N} 13 \\ 0.8 \mathrm{wt} \% \mathrm{~B}_{4} \mathrm{C} & \mathrm{K} 14 \\ 1.1 \mathrm{wt} \% \mathrm{~B}_{4} \mathrm{C} & \mathrm{H} 13 \\ 1.7 \text { wt \% } \mathrm{B}_{4} \mathrm{C} & \mathrm{H} 9, \mathrm{~K} 10, \mathrm{~K} 12 \\ 2.1 \mathrm{wt} \% \mathrm{~B}_{4} \mathrm{C} & \mathrm{L} 11, \mathrm{~L} 13, \mathrm{M} 12\end{array}$

Banks 1 - 4 Safety

$\mathrm{H} 8, \mathrm{H} 10, \mathrm{~K} 9, \mathrm{~K} 11, \mathrm{~L} 14, \mathrm{M} 13$

Bank 5 Regulating

K13, M11

Bank 6 Regulating

H14, L10

Bank 7 Regulating

$\mathrm{H} 12, \mathrm{~N} 12$

Bank 8 (Gray -APSR)

L12

Figure 3-10. One-Eighth Core Loading for TMI-1, Cycle 9 


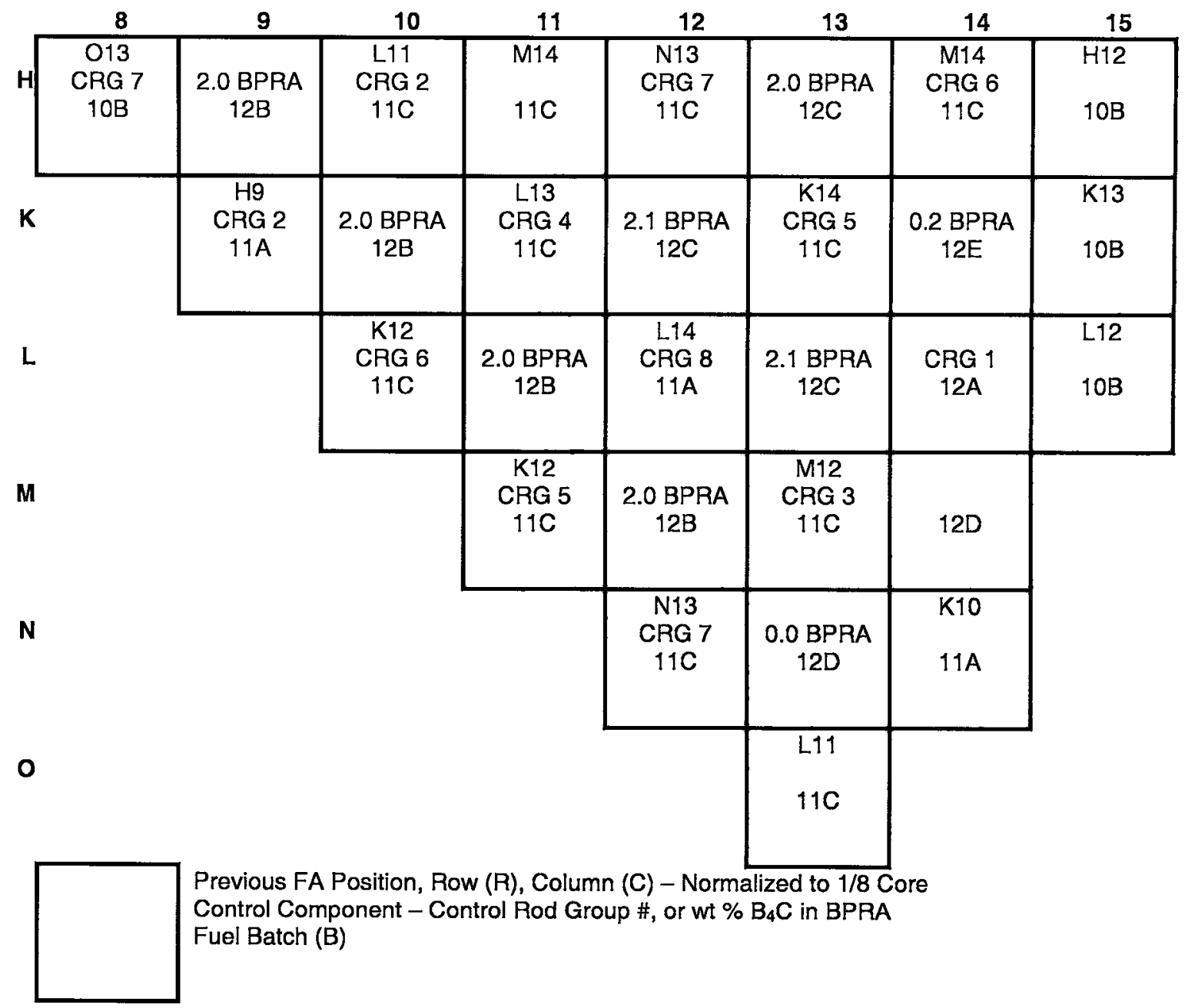

\begin{tabular}{|c|c|c|c|}
\hline Cycle & Batch/FA & wt $\%{ }^{235} U$ & \#FA \\
\hline \multirow[t]{8}{*}{10} & 10B / MK-B8 & 3.63 & 21 \\
\hline & $11 \mathrm{~A} / \mathrm{MK}-\mathrm{B8}$ & 3.63 & 20 \\
\hline & $11 \mathrm{C} / \mathrm{MK}-\mathrm{B} 8 \mathrm{~V}$ & 4.00 & 56 \\
\hline & $12 \mathrm{~A} / \mathrm{MK}-\mathrm{B} 8 \mathrm{~V}$ & 4.00 & 8 \\
\hline & 12B / MK-B9 & 4.65 & 28 \\
\hline & $12 \mathrm{C} / \mathrm{MK}-\mathrm{B} 9 \mathrm{Gd}$ & 4.65 & 20 \\
\hline & 12D / MK-B9 & 4.75 & 16 \\
\hline & $12 \mathrm{E} / \mathrm{MK}-\mathrm{B} 9 \mathrm{Gd}$ & 4.75 & 8 \\
\hline & & & 177 \\
\hline
\end{tabular}

$\begin{array}{cl}\text { BPRA } & \text { Position } \\ 0.0 w t \% B_{4} C & N 13 \\ 0.2 w t \% B_{4} C & K 14 \\ 2.0 w t \% B_{4} C & H 9, H 13, K 10, L 11, M 12 \\ 2.1 w t \% B_{4} C & K 12, L 13\end{array}$

Banks $1-4$ Safety

$\mathrm{H} 10, \mathrm{~K} 9, \mathrm{~K} 11, \mathrm{~L} 14, \mathrm{M} 13$

Bank 5 Regulating

$\mathrm{K} 13, \mathrm{M} 11$

Bank 6 Regulating

$\mathrm{H} 14, \mathrm{~L} 10$

Bank 7 Regulating

$\mathrm{H} 8, \mathrm{H} 12, \mathrm{~N} 12$

Bank 8 (Gray -APSR)

L12

Figure 3-11. One-Eighth Core Loading for TMI-1, Cycle 10 


\begin{tabular}{|c|c|c|c|c|c|c|c|c|}
\hline & 8 & 9 & 10 & 11 & 12 & 13 & 14 & 15 \\
\hline $\mathrm{H}$ & $\begin{array}{c}\text { O13(Cy9) } \\
\text { CRG } 7 \\
10 B\end{array}$ & $\begin{array}{c}2.0 \mathrm{BPRA} \\
13 \mathrm{~A}\end{array}$ & $\begin{array}{c}\text { K10 } \\
\text { CRG 2 } \\
12 B\end{array}$ & $\begin{array}{l}\text { N13 } \\
12 \mathrm{D}\end{array}$ & $\begin{array}{c}\mathrm{H} 13 \\
\mathrm{CHG} 7 \\
12 \mathrm{C}\end{array}$ & $\begin{array}{c}2.3 \mathrm{BPRA} \\
13 \mathrm{D}\end{array}$ & $\begin{array}{c}\mathrm{H9} \\
\text { CRG } 6 \\
12 \mathrm{~B}\end{array}$ & $\begin{array}{l}013 \\
11 \mathrm{C}\end{array}$ \\
\hline K & & $\begin{array}{c}\text { L13 } \\
\text { CRG } 2 \\
12 C\end{array}$ & $\begin{array}{c}\text { 3.5 BPRA } \\
13 \mathrm{~B}\end{array}$ & $\begin{array}{c}\text { K14 } \\
\text { CRG 4 } \\
12 E\end{array}$ & $\begin{array}{c}3.5 \text { BPRA } \\
13 \mathrm{C}\end{array}$ & $\begin{array}{c}\text { M12 } \\
\text { CRG } 5 \\
12 B\end{array}$ & $13 \mathrm{~F}$ & $\begin{array}{l}\text { N14 } \\
11 \mathrm{~A}\end{array}$ \\
\hline$L$ & & & $\begin{array}{c}\text { L13 } \\
\text { CPG } 6 \\
12 C\end{array}$ & $\begin{array}{c}3.5 \text { BPRA } \\
13 \mathrm{~B}\end{array}$ & $\begin{array}{c}\text { K12 } \\
\text { CRG } 8 \\
12 \mathrm{C}\end{array}$ & $\begin{array}{c}3.5 \text { BPRA } \\
13 \mathrm{E}\end{array}$ & $\begin{array}{c}\text { CRG } 1 \\
13 A\end{array}$ & $\begin{array}{c}\text { M13(Cy9) } \\
10 \mathrm{~B}\end{array}$ \\
\hline $\mathbf{M}$ & & & & $\begin{array}{c}\text { K10 } \\
\text { CRG } 5 \\
12 B\end{array}$ & $\begin{array}{c}\text { 3.5 BPRA } \\
13 \mathrm{~B}\end{array}$ & $\begin{array}{c}\text { M14 } \\
\text { CRG 3 } \\
12 \mathrm{D}\end{array}$ & $\begin{array}{l}\text { L11 } \\
12 B\end{array}$ & \\
\hline $\mathbf{N}$ & & & & & $\begin{array}{c}\text { N13 } \\
\text { CRG } 7 \\
12 D\end{array}$ & $13 G$ & $13 \mathrm{~F}$ & \\
\hline 0 & & & & & & $\begin{array}{c}\text { H14(Cy9) } \\
10 E\end{array}$ & & \\
\hline & & \multicolumn{7}{|c|}{$\begin{array}{l}\text { Previous FA Position, Row (R), Column (C) - Normalized to } 1 / 8 \text { Core } \\
\text { Control Component - Control Rod Group \#, or wt \% } \mathrm{B}_{4} \mathrm{C} \text { in BPRA } \\
\text { Fuel Batch (B) }\end{array}$} \\
\hline
\end{tabular}

\begin{tabular}{|c|c|c|c|}
\hline Cycle & Batch/FA & wt $\%^{235} \mathrm{U}$ & \# FA \\
\hline \multirow{4}{*}{11} & $10 \mathrm{~B} / \mathrm{MK}-\mathrm{B} 8$ & 3.63 & 13 \\
\cline { 2 - 4 } & $11 \mathrm{~A} / \mathrm{MK}-\mathrm{B} 8$ & 3.63 & 8 \\
\cline { 2 - 4 } & $11 \mathrm{C} / \mathrm{MK}-\mathrm{B} 8 \mathrm{~V}$ & 4.00 & 4 \\
\cline { 2 - 4 } & $12 \mathrm{~B} / \mathrm{MK}-\mathrm{B} 9$ & 4.65 & 28 \\
\cline { 2 - 4 } & $12 \mathrm{C} / \mathrm{MK}-\mathrm{B} 9 \mathrm{Gd}$ & 4.65 & 20 \\
\cline { 2 - 4 } & $12 \mathrm{D} / \mathrm{MK}-\mathrm{Bg}$ & 4.75 & 16 \\
\cline { 2 - 4 } & $12 \mathrm{E} / \mathrm{MK}-\mathrm{B} 9 \mathrm{Gd}$ & 4.75 & 8 \\
\hline
\end{tabular}

\begin{tabular}{|c|c|c|c|}
\hline Cycle & Batch/FA & wt $\%{ }^{235} \mathrm{U}$ & \# FA \\
\hline \multirow{4}{*}{11} & $13 \mathrm{~A} / \mathrm{MK}-\mathrm{B} 8 \mathrm{~V}$ & 4.00 & 12 \\
\cline { 2 - 4 } & $13 \mathrm{~B} / \mathrm{MK}-\mathrm{B} 10$ & 4.23 & 24 \\
\cline { 2 - 4 } & $13 \mathrm{C} / \mathrm{MK}-\mathrm{B} 10 \mathrm{Gd}$ & 4.23 & 8 \\
\hline & $13 \mathrm{D} / \mathrm{W}-\mathrm{LTA}$ & 4.25 & 4 \\
\hline $13 \mathrm{E} / \mathrm{MK}-\mathrm{B} 10 \mathrm{Gd}$ & 4.55 & 8 \\
\hline & $13 \mathrm{~F} / \mathrm{MK}-\mathrm{B} 10 \mathrm{Gd}$ & 4.55 & 16 \\
\hline & $13 \mathrm{G} / \mathrm{MK}-\mathrm{B} 10$ & 4.00 & 8 \\
\hline \multicolumn{3}{|c}{} & 177 \\
\hline
\end{tabular}

Banks $1-4$ Safety

Bank 5 Regulating

Bank 6 Regulating

Bank 7 Regulating

Bank 8 (Gray -APSR)
$\mathrm{H} 10, \mathrm{~K} 9, \mathrm{~K} 11, \mathrm{~L} 14, \mathrm{M} 13$

$\mathrm{K} 13, \mathrm{M} 11$

H14, L.10

$\mathrm{H} 8, \mathrm{H} 12, \mathrm{~N} 12$

$\mathrm{L} 12$

$\begin{array}{rl}\text { BPRA } & \text { Position } \\ 2.0 w t \% \mathrm{~B}_{4} \mathrm{C} & \mathrm{H} 9 \\ 2.3 w t \% \mathrm{~B}_{4} \mathrm{C} & \mathrm{H} 13 \\ 3.5 \mathrm{wt} \% \mathrm{~B}_{4} \mathrm{C} & \mathrm{K} 10, \mathrm{~K} 12, \mathrm{~L} 11, \mathrm{~L} 13, \mathrm{M} 12\end{array}$

3.5 wt \% $\mathrm{B}_{4} \mathrm{C} \quad \mathrm{K} 10, \mathrm{~K} 12, \mathrm{~L} 11, \mathrm{~L} 13, \mathrm{M} 12$

Figure 3-12. One-Eighth Core Loading for TMI-1, Cycle 11 


\begin{tabular}{|c|c|c|c|c|c|c|c|c|}
\hline & 8 & 9 & 10 & 11 & 12 & 13 & 14 & 15 \\
\hline $\mathrm{H}$ & A1 & A2 & A3 & A4 & A5 & A6 & A7 & A8 \\
\hline K & & A9 & A10 & A11 & A12 & A13 & A14 & A15 \\
\hline $\mathbf{L}$ & & & A16 & A17 & A18 & A19 & A20 & A21 \\
\hline M & & & & A22 & A23 & A24 & A25 & \\
\hline $\mathbf{N}$ & & & & & A26 & A27 & A28 & \\
\hline 0 & & & & & & A29 & & \\
\hline
\end{tabular}
A Cycle 1
B Cycle 2
C Cycle 3
D Cycle 4
E Cycle 5
F Cycle 6
G Cycle 7
H Cycle 8
$J$ Cycle 9
K Cycle 10
L Cycle 11

Figure 3-13. TMl-1, Cycle 1 Fuel Assembly Identification \& Location 


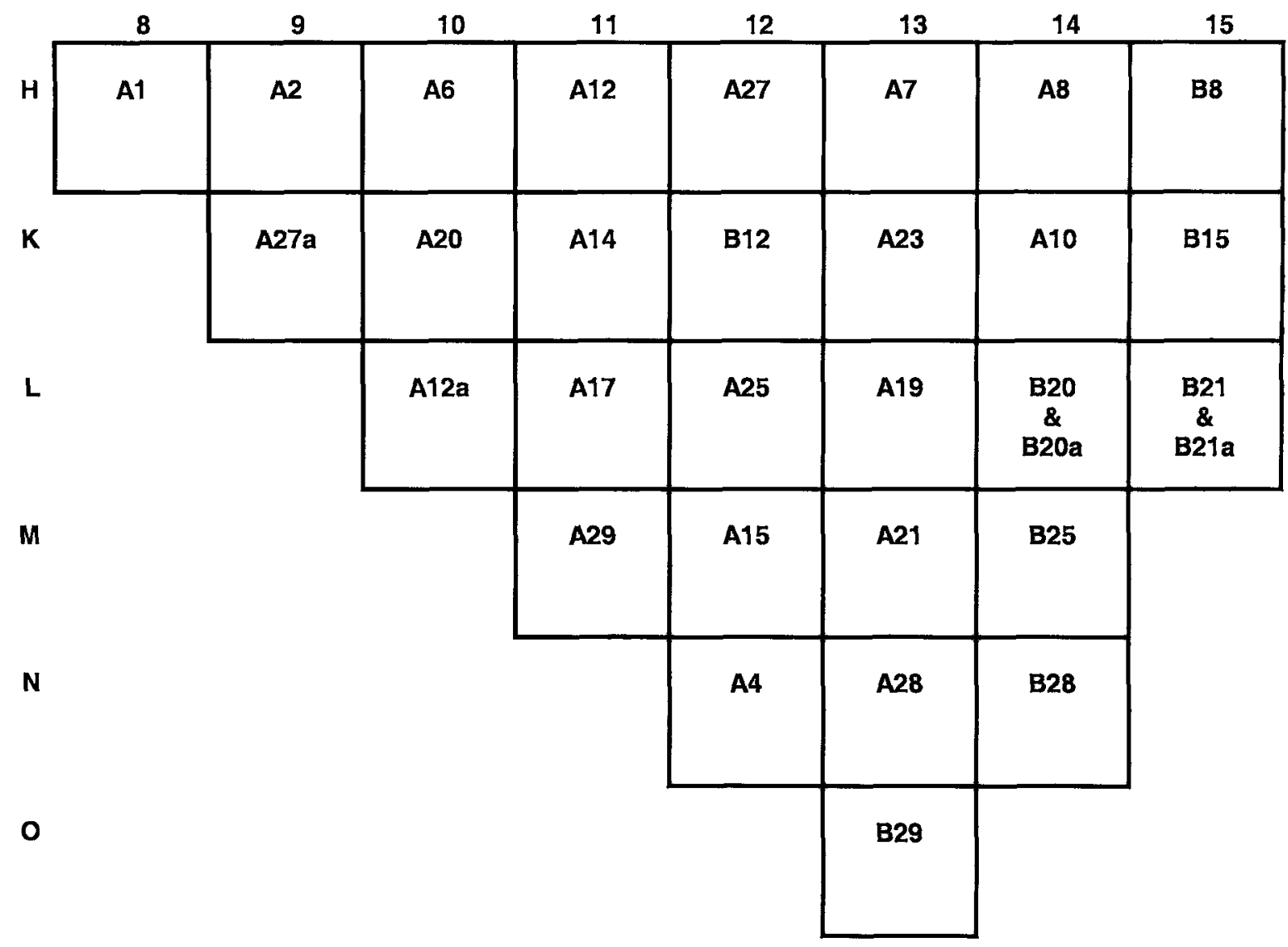

A Cycle 1

B Cycle 2

C Cycle 3

D Cycle 4

E Cycle 5

F Cycle 6

G Cycle 7

H Cycle 8

J Cycle 9

K Cycle 10

L Cycle 11

Figure 3-14. TMI-1, Cycle 2 Fuel Assembly Identification \& Location 


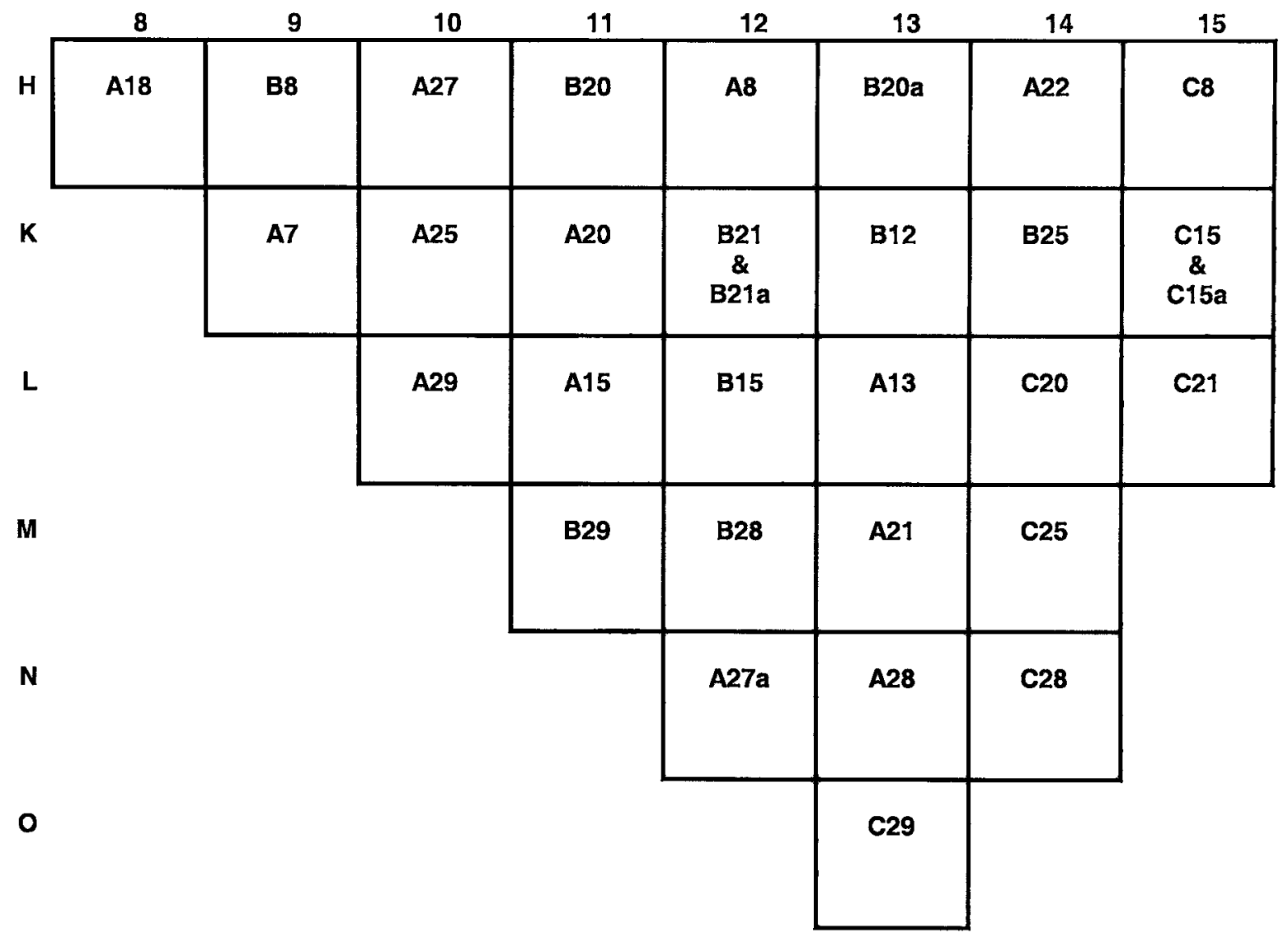
A Cycle 1
B Cycle 2
C Cycle 3
D Cycle 4
E Cycle 5
F Cycle 6
G Cycle 7
H Cycle 8
$\checkmark$ Cycle 9
K Cycle 10
L Cycle 11

Figure 3-15. TMI-1, Cycle 3 Fuel Assembly Identification \& Location 


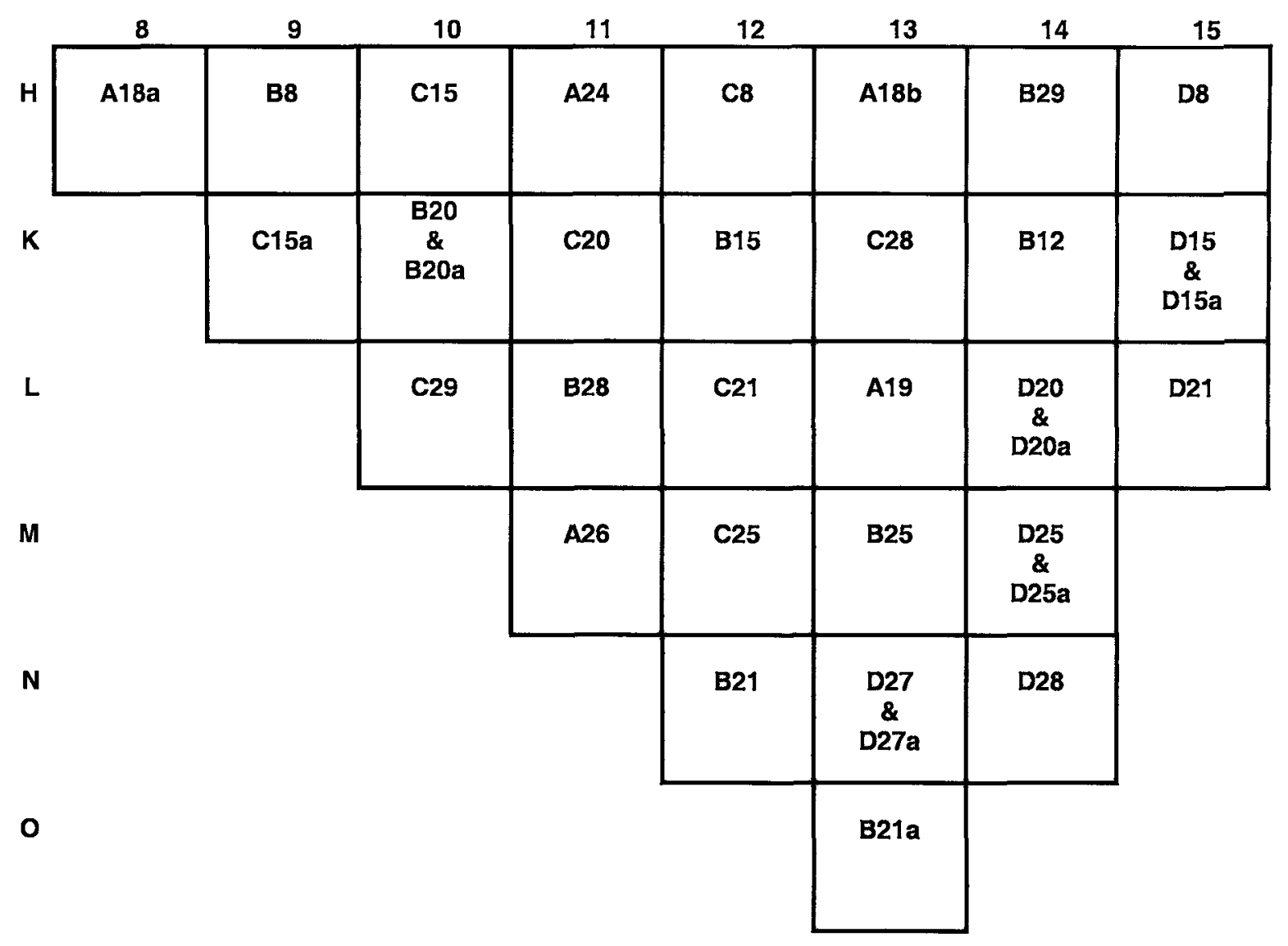
A Cycle 1
B Cycle 2
C Cycle 3
D Cycle 4
E Cycle 5
$\mathrm{F} \quad$ Cycle 6
G Cycle 7
H Cycle 8
$J$ Cycle 9
K Cycle 10
L Cycle 11

Figure 3-16. TMI-1, Cycle 4 Fuel Assembly Identification \& Location 


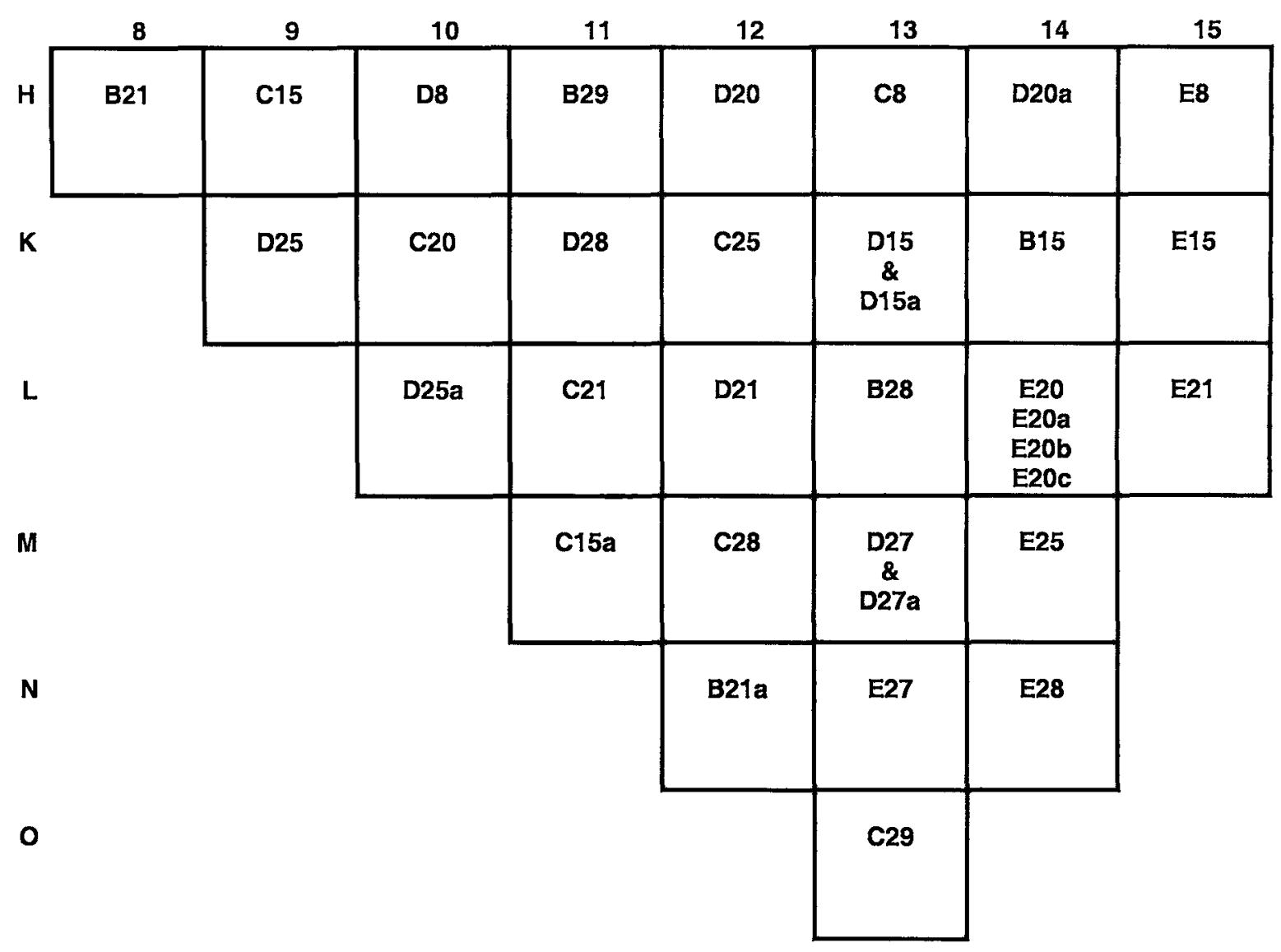
A Cycle 1
B Cycle 2
C Cycle 3
D Cycle 4
E Cycle 5
$\mathrm{F}$ Cycle 6
G Cycle 7
H Cycle 8
$J$ Cycle 9
K Cycle 10
L. Cycle 11

Figure 3-17. TMI-1, Cycle 5 Fuel Assembly Identification \& Location 


\begin{tabular}{|c|c|c|c|c|c|c|c|c|}
\hline & 8 & 9 & 10 & 11 & 12 & 13 & 14 & 15 \\
\hline H & D27a & F2 & D25 & F4 & D15 & F6 & D27 & D20 \\
\hline K & & D25a & F10 & D21 & $\mathrm{F} 12$ & E25 & $\begin{array}{c}\mathrm{F} 14 \\
\& \\
\text { F14a }\end{array}$ & $\begin{array}{c}E 20 \\
E 20 a \\
E 20 b \\
E 20 c\end{array}$ \\
\hline L & & & D20a & $\begin{array}{c}\text { F17 } \\
\& \\
\text { F17a }\end{array}$ & D28 & F19 & E15 & E27 \\
\hline M & & & & D8 & F23 & E28 & F25 & \\
\hline $\mathbf{N}$ & & & & & D15a & F27 & E21 & \\
\hline 0 & & & & & & E8 & & \\
\hline
\end{tabular}

Figure 3-18. TMI-1, Cycle 6 Fuel Assembly Identification \& Location 


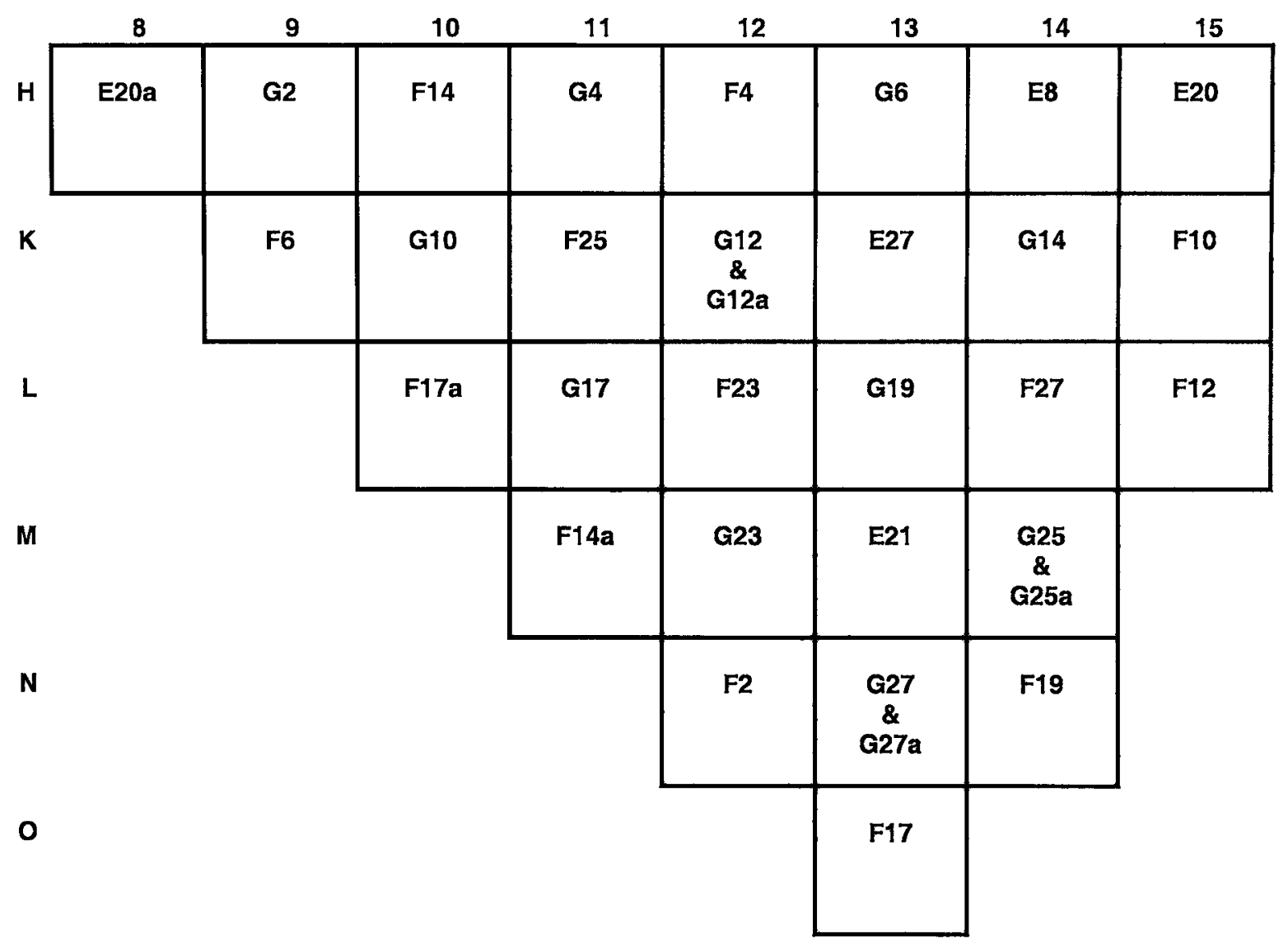

Notes: One G17 assembly was removed at end of Cycle 7

Four F19 assemblies were removed at end of Cycle 7

Figure 3-19. TMI-1, Cycle 7 Fuel Assembly Identification \& Location 


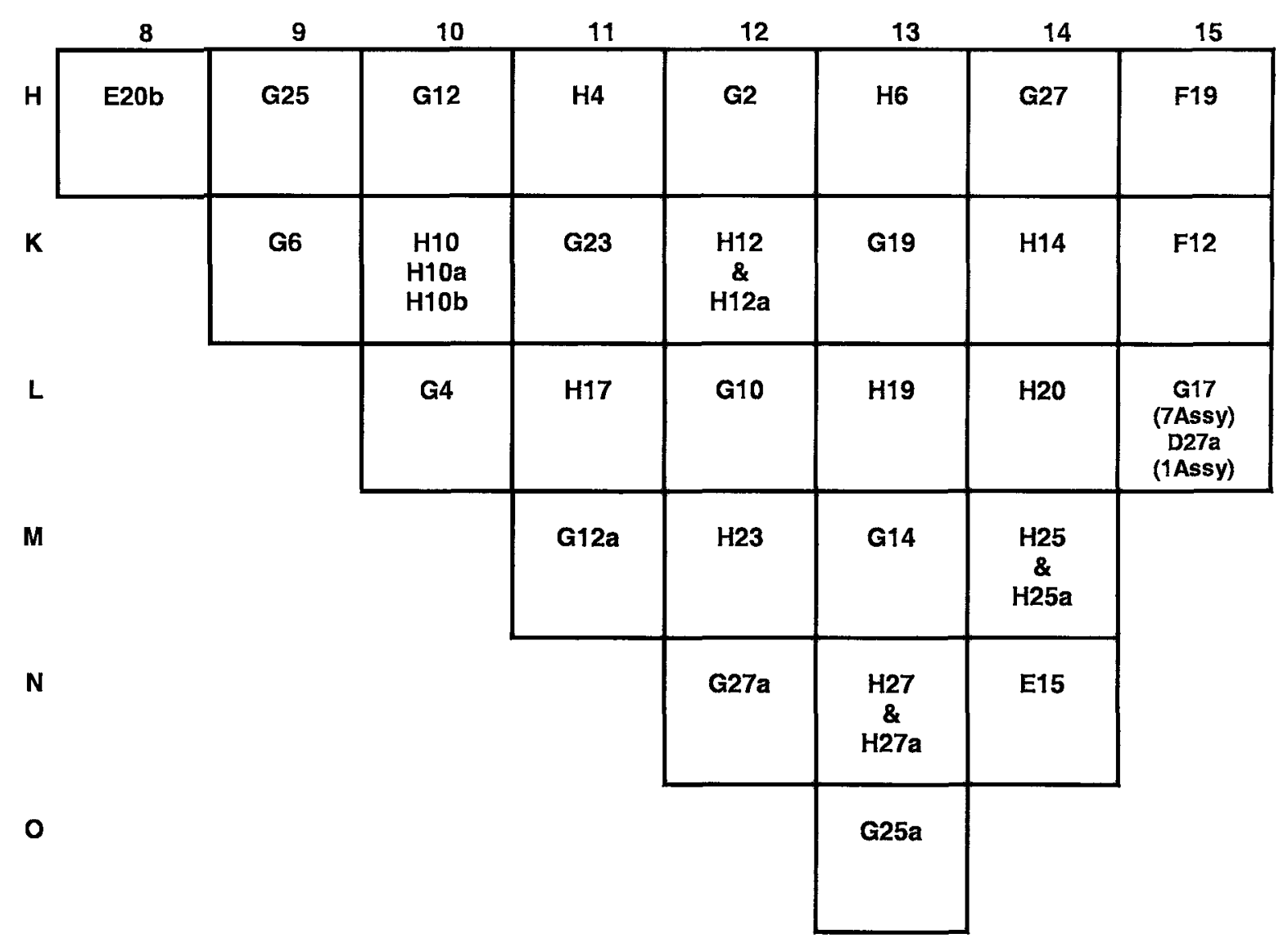

Figure 3-20. TMI-1, Cycle 8 Fuel Assembly Identification \& Location 


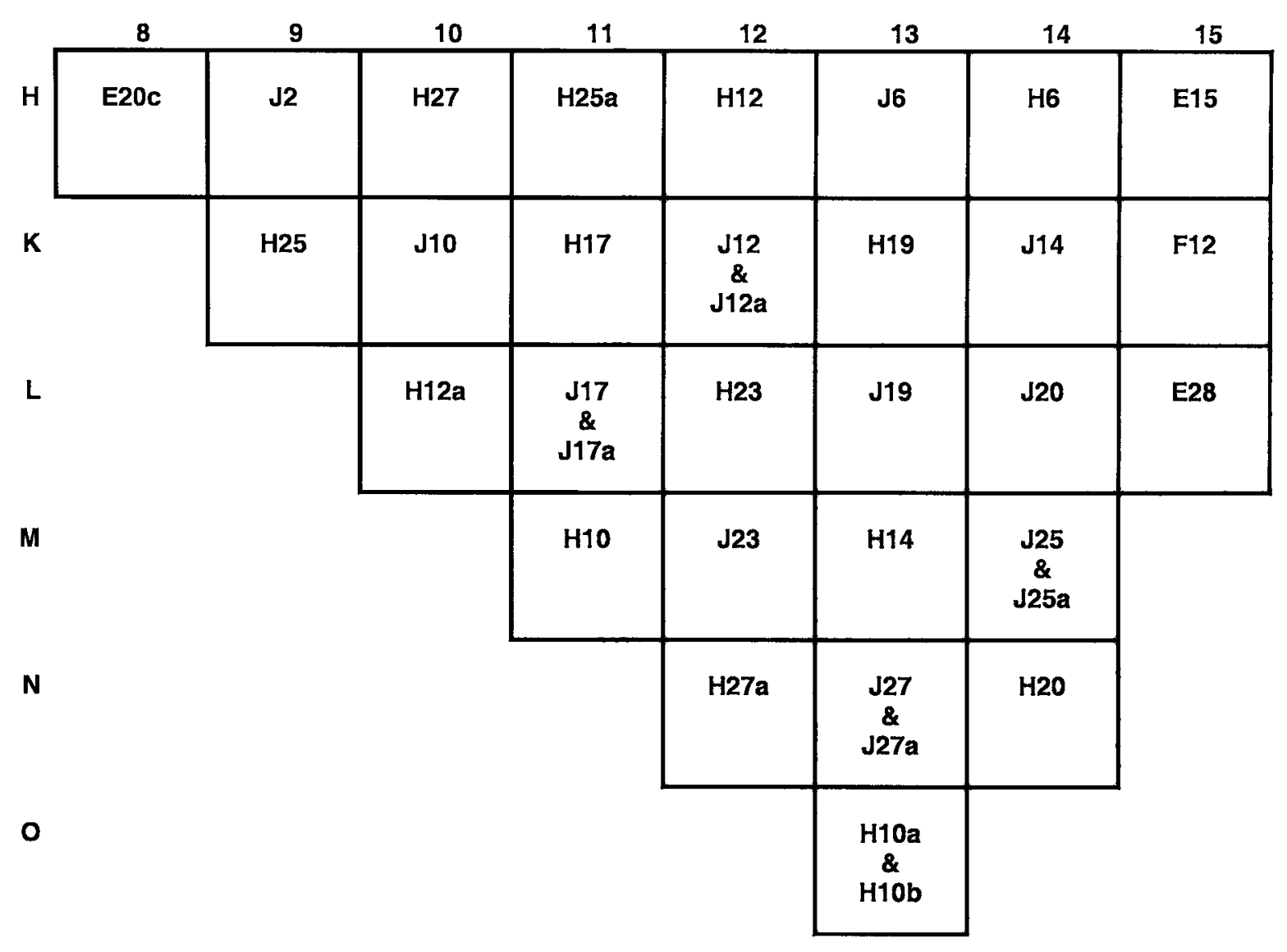

Figure 3-21. TMI-1, Cycle 9 Fuel Assembly Identification \& Location 


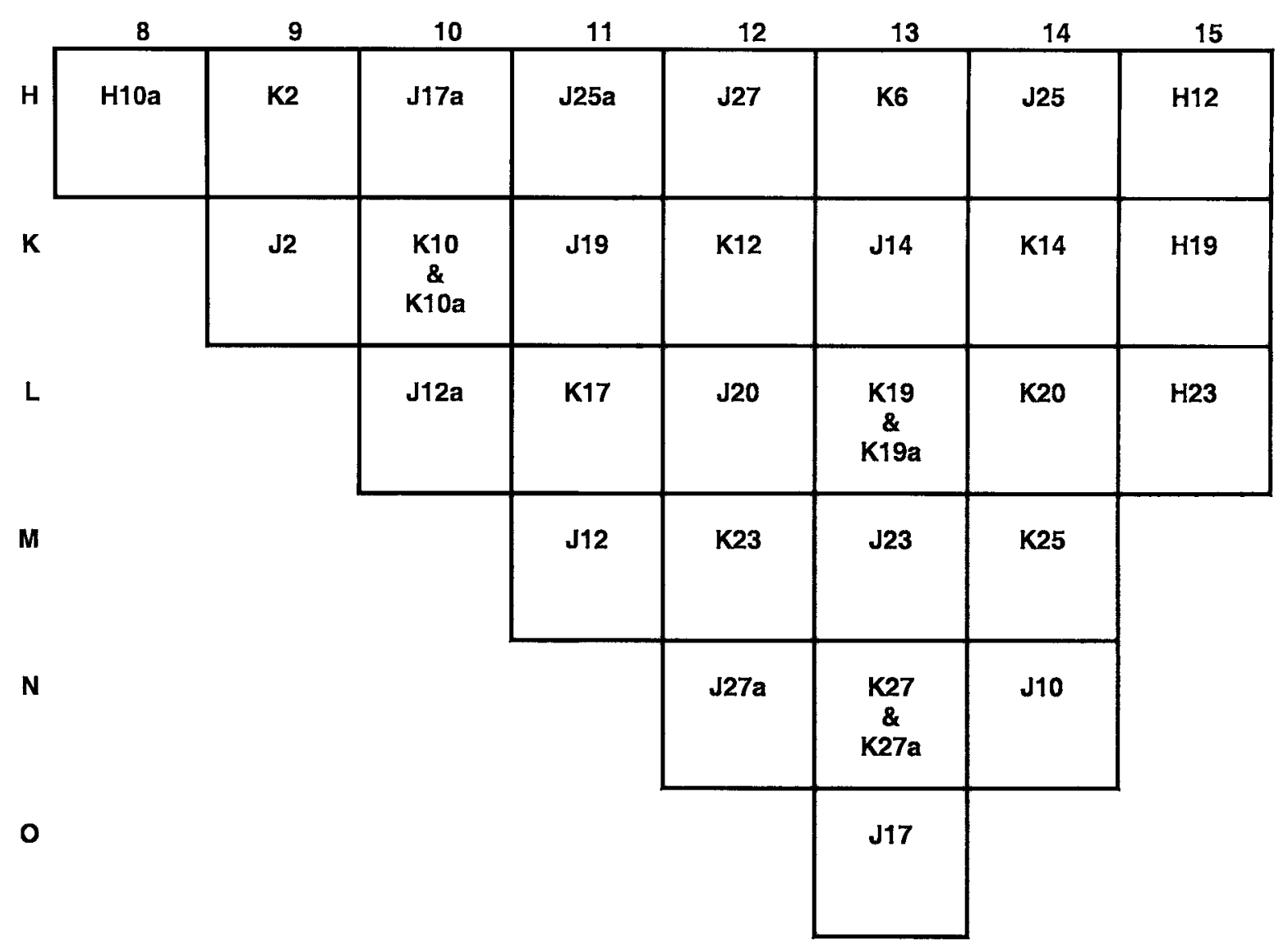
A Cycle 1
B Cycle 2
C Cycle 3
D Cycle 4
E Cycle 5
$F \quad$ Cycle 6
G Cycle 7
H Cycle 8
$J$ Cycle 9
K Cycle 10
L Cycle 11

Figure 3-22. TMI-1, Cycle 10 Fuel Assembly Identification \& Location 


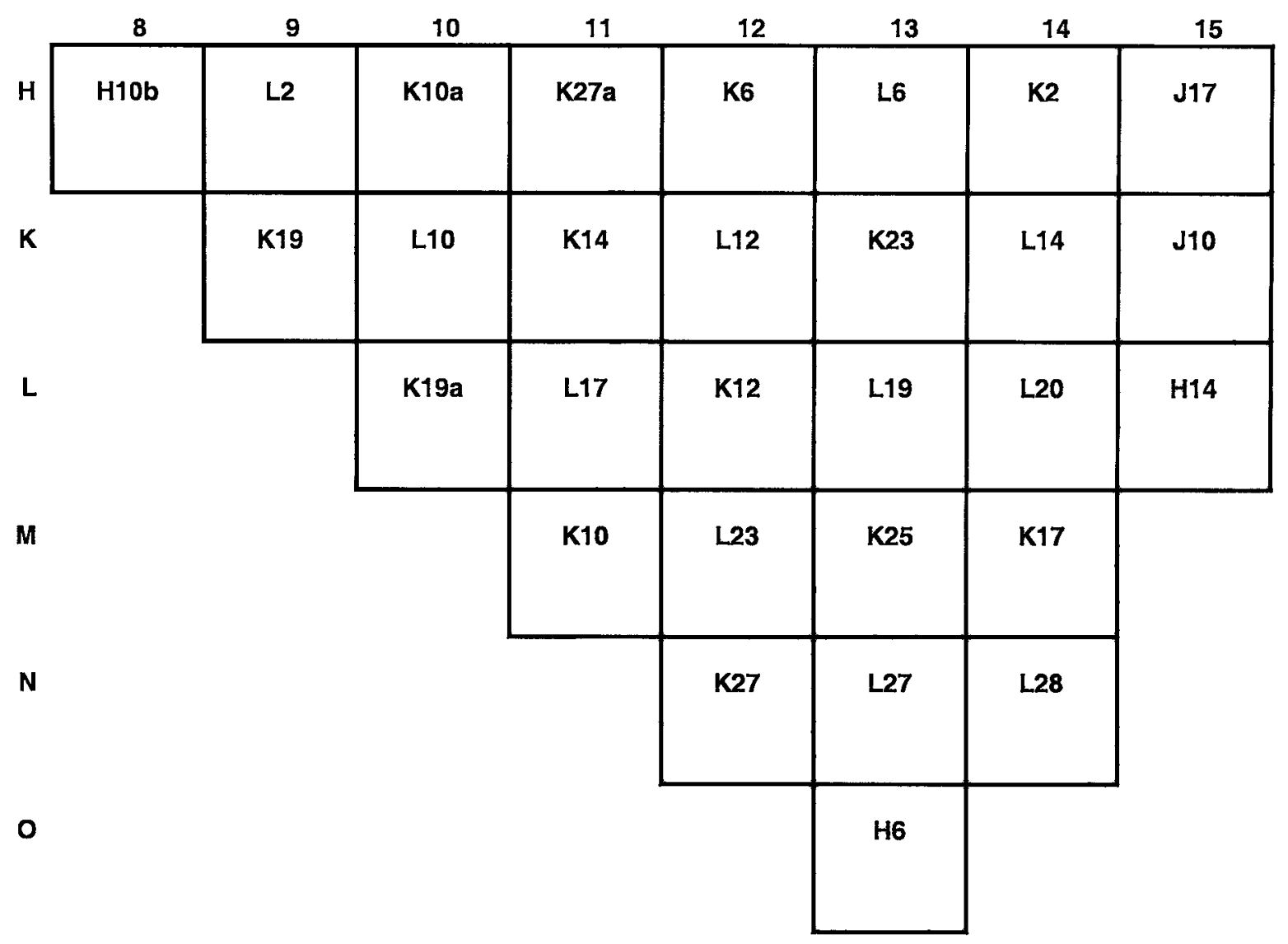

Figure 3-23. TMI-1, Cycle 11 Fuel Assembly Identification \& Location 
To aid in the burnup calculations, and thus the generation of isotopic data for the SP calculations, the information provided in Figures 3-1 through 3-23 was reduced to two tables. Table 3-3 traces each fuel assembly (and subsequent split by shuffling symmetric components to more than one location, if applicable) by assembly identification and cycle from the time the assembly was first inserted in the reactor to the time it was discharged (or remained past cycle 11). The coordinates given in the figures (e.g., H8, K12) indicate the location of each assembly in each cycle.

Table 3-4 is a repeat of portions of Table 3-3 where control rod bank indicators (e.g., CR7 for bank 7, CR8 for bank 8 or APSRs) are given for those assemblies that contained control rods during cycle operation. Control rod banks 1 through 4 are for safety and are withdrawn during operation. Control rod bank 5 is also withdrawn during operation.

Control rod insertion must be modeled in the burnup calculations for those assemblies and axial locations where rods are inserted. Additional data for rod insertion time by axial node is given is Section 4 . The wt $\%$ of $\mathrm{B}_{4} \mathrm{C}$ in the burnable poison rod assembly is provided by assembly and by cycle. 
Table 3-3. Fuel Assembly Locations by Cycle

\begin{tabular}{|c|c|c|c|c|c|c|c|}
\hline $\begin{array}{c}\text { Assembly } \\
\text { Number }\end{array}$ & $\begin{array}{c}\text { Batch } \\
\text { Number }\end{array}$ & Cycle 1A & Cycle 1B & Cycle 2 & Cycle3 & Cycle 4 & Cycle 5 \\
\hline \multicolumn{8}{|l|}{ Cycle 1} \\
\hline $\mathrm{A} 1$ & 2 & $\mathrm{H}-08$ & $\mathrm{H}-08$ & $\mathrm{H}-08$ & & & \\
\hline $\mathrm{A} 2$ & 2 & $\mathrm{H}-09$ & $\mathrm{H}-09$ & $\mathrm{H}-09$ & & & \\
\hline A3 & 1 & $\mathrm{H}-10$ & $\mathrm{H}-10$ & & & & \\
\hline A4 & 2 & $\mathrm{H}-11$ & $\mathrm{H}-11$ & $\mathrm{~N}-12$ & & & \\
\hline A5 & 1 & $\mathrm{H}-12$ & $\mathrm{H}-12$ & & & & \\
\hline A6 & 2 & $\mathrm{H}-13$ & $\mathrm{H}-13$ & $\mathrm{H}-10$ & & & \\
\hline $\mathrm{A} 7$ & 3 & $\mathrm{H}-14$ & $\mathrm{H}-14$ & $\mathrm{H}-13$ & K-09 & & \\
\hline $\mathrm{AB}$ & 3 & $\mathrm{H}-15$ & $\mathrm{H}-15$ & $\mathrm{H}-14$ & $\mathrm{H}-12$ & & \\
\hline A9 & 1 & K-09 & K-09 & & & & \\
\hline A10 & 2 & $\mathrm{~K}-10$ & $\mathrm{~K}-10$ & $\mathrm{~K}-14$ & & & \\
\hline A11 & 1 & $\mathrm{~K}-11$ & $\mathrm{~K}-11$ & & & & \\
\hline A12 & 2 & $\mathrm{~K}-12$ & $\mathrm{~K}-12$ & $\mathrm{H}-11$ & & & \\
\hline A12a & 2 & $\mathrm{~K}-12$ & $\mathrm{~K}-12$ & $L-10$ & & & \\
\hline A13 & 1 & $\mathrm{~K}-13$ & $\mathrm{~K}-13$ & & $\mathrm{~L}-13$ & & \\
\hline A14 & 2 & $\mathrm{~K}-14$ & $\mathrm{~K}-14$ & $\mathrm{~K}-11$ & & & \\
\hline $\mathrm{A} 15$ & 3 & $K-15$ & $K-15$ & $\mathrm{M}-12$ & $L-11$ & & \\
\hline A16 & 1 & $L-10$ & $L-10$ & & & & \\
\hline $\mathrm{A} 17$ & 2 & $\mathrm{~L}-11$ & $L-11$ & $L-11$ & & & \\
\hline A18 & 1 & $L-12$ & $L-12$ & & $\mathrm{H}-08$ & & \\
\hline A18a & 1 & $\mathrm{~L}-12$ & $\mathrm{~L}-12$ & & & $\mathrm{H}-08$ & \\
\hline $\mathrm{A} 18 \mathrm{~b}$ & 1 & $\mathrm{~L}-12$ & $L-12$ & & & $\mathrm{H}-13$ & \\
\hline A19 & 2 & $\mathrm{~L}-13$ & $\mathrm{~L}-13$ & $L-13$ & & $L-13$ & \\
\hline A20 & 3 & $\mathrm{~L}-14$ & $\mathrm{~L}-14$ & $\mathrm{~K}-10$ & $\mathrm{~K}-11$ & & \\
\hline A21 & 3 & $\mathrm{~L}-15$ & $L-15$ & $M-13$ & $\mathrm{M}-13$ & & \\
\hline A22 & 1 & $\mathrm{M}-11$ & $M-11$ & & $\mathrm{H}-14$ & & \\
\hline A23 & 2 & $\mathrm{M}-12$ & $\mathrm{M}-12$ & $K-13$ & & & \\
\hline A24 & 1 & $\mathrm{M}-13$ & $\mathrm{M}-13$ & & & $\mathrm{H}-11$ & \\
\hline A25 & 3 & $\mathrm{M}-14$ & $\mathrm{M}-14$ & $\mathrm{~L}-12$ & $\mathrm{~K}-10$ & & \\
\hline A26 & 1 & $\mathrm{~N}-12$ & $\mathrm{~N}-12$ & & & $\mathrm{M}-11$ & \\
\hline A27 & 3 & $\mathrm{~N}-13$ & $\mathrm{~N}-13$ & $\mathrm{H}-12$ & $\mathrm{H}-10$ & & \\
\hline A27a & 3 & $\mathrm{~N}-13$ & $\mathrm{~N}-13$ & K-09 & $\mathrm{N}-12$ & & \\
\hline A28 & 3 & $\mathrm{~N}-14$ & $\mathrm{~N}-14$ & $\mathrm{~N}-13$ & $\mathrm{~N}-13$ & & \\
\hline A29 & 3 & $0-13$ & $0-13$ & $\mathrm{M}-11$ & $L-10$ & & \\
\hline \multicolumn{8}{|l|}{ Cycle 2} \\
\hline B8 & 4 & & & $\mathrm{H}-15$ & $\mathrm{H}-09$ & $\mathrm{H}-09$ & \\
\hline $\mathrm{B} 12$ & 4 & & & $\mathrm{~K}-12$ & $K-13$ & $\mathrm{~K}-14$ & \\
\hline B15 & 4 & & & $\mathrm{~K}-15$ & $L-12$ & $\mathrm{~K}-12$ & $\mathrm{~K}-14$ \\
\hline $\mathrm{B} 20$ & 4 & & & $L-14$ & $\mathrm{H}-11$ & $\mathrm{~K}-10$ & \\
\hline $\mathrm{B} 20 \mathrm{a}$ & 4 & & & $L-14$ & $\mathrm{H}-13$ & $\mathrm{~K}-10$ & \\
\hline B21 & 4 & & & $L-15$ & $\mathrm{~K}-12$ & $\mathrm{~N}-12$ & $\mathrm{H}-08$ \\
\hline B21a & 4 & & & $L-15$ & $\mathrm{~K}-12$ & $0-13$ & $\mathrm{~N}-12$ \\
\hline $\mathrm{B} 25$ & 4 & & & $M-14$ & $K-14$ & $M-13$ & \\
\hline $\mathrm{B} 28$ & 4 & & & $\mathrm{~N}-14$ & $\mathrm{M}-12$ & $L-11$ & $L-13$ \\
\hline B29 & 4 & & & $0-13$ & $\mathrm{M}-11$ & $\mathrm{H}-14$ & $\mathrm{H}-11$ \\
\hline
\end{tabular}


Table 3-3. Fuel Assembly Locations by Cycle (Cont.)

\begin{tabular}{|c|c|c|c|c|c|c|c|c|}
\hline $\begin{array}{l}\text { Assembly } \\
\text { Number }\end{array}$ & $\begin{array}{c}\text { Batch } \\
\text { Number }\end{array}$ & Cycle3 & Cycle 4 & Cycle 5 & Cycle 6 & Cycle 7 & Cycle 8 & Cycle 9 \\
\hline \multicolumn{9}{|l|}{ Cycle 3} \\
\hline C8 & 5 & $\mathrm{H}-15$ & $\mathrm{H}-12$ & $\mathrm{H}-13$ & & & & \\
\hline C15 & 5 & $\mathrm{~K}-15$ & $\mathrm{H}-10$ & $\mathrm{H}-09$ & & & & \\
\hline C15a & 5 & $\mathrm{~K}-15$ & K-09 & $\mathrm{M}-11$ & & & & \\
\hline C20 & 5 & $L-14$ & $\mathrm{~K}-11$ & $\mathrm{~K}-10$ & & & & \\
\hline C21 & 5 & $L-15$ & $L-12$ & $L-11$ & & & & \\
\hline C25 & 5 & $M-14$ & $\mathrm{M}-12$ & $\mathrm{~K}-12$ & & & & \\
\hline $\mathrm{C} 28$ & 5 & $\mathrm{~N}-14$ & $\mathrm{~K}-13$ & $\mathrm{M}-12$ & & & & \\
\hline C29 & 5 & $0-13$ & $L-10$ & O-13 & & & & \\
\hline \multicolumn{9}{|l|}{ Cycle 4} \\
\hline D8 & 6 & & $\mathrm{H}-15$ & $\mathrm{H}-10$ & $\mathrm{M}-11$ & & & \\
\hline D15 & 6 & & $\mathrm{~K}-15$ & $\mathrm{~K}-13$ & $\mathrm{H}-12$ & & & \\
\hline D15a & 6 & & $\mathrm{~K}-15$ & $\mathrm{~K}-13$ & $\mathrm{~N}-12$ & & & \\
\hline D20 & 6 & & $\mathrm{~L}-14$ & $\mathrm{H}-12$ & $\mathrm{H}-15$ & & & \\
\hline D20a & 6 & & $L-14$ & $\mathrm{H}-14$ & $L-10$ & & & \\
\hline D21 & 6 & & $L-15$ & $L-12$ & $\mathrm{~K}-11$ & & & \\
\hline D25 & 6 & & $\mathrm{M}-14$ & K-09 & $\mathrm{H}-10$ & & & \\
\hline $\mathrm{D} 25 \mathrm{a}$ & 6 & & $\mathrm{M}-14$ & $L-10$ & K-09 & & & \\
\hline D27 & 6 & & $\mathrm{~N}-13$ & $M-13$ & $\mathrm{H}-14$ & & & \\
\hline D27a & 6 & & $\mathrm{~N}-13$ & $\mathrm{M}-13$ & $\mathrm{H}-08$ & & $L-15$ (1 assy) & \\
\hline D28 & 6 & & $\mathrm{~N}-14$ & $\mathrm{~K}-11$ & $L-12$ & & & \\
\hline \multicolumn{9}{|l|}{ Cycle 5} \\
\hline $\mathrm{E8}$ & 7 & & & $\mathrm{H}-15$ & $0-13$ & $\mathrm{H}-14$ & & \\
\hline E15 & 7 & & & $\mathrm{~K}-15$ & $L-14$ & & $\mathrm{~N}-14$ & $\mathrm{H}-15$ \\
\hline E20 & 7 & & & $L-14$ & $\mathrm{~K}-15$ & $\mathrm{H}-15$ & & \\
\hline E20a & 7 & & & $L-14$ & $\mathrm{~K}-15$ & $\mathrm{H}-08$ & & \\
\hline $\mathrm{E} 20 \mathrm{~b}$ & 7 & & & $L-14$ & $\mathrm{~K}-15$ & & $\mathrm{H}-08$ & \\
\hline E20c & 7 & & & $L-14$ & $\mathrm{~K}-15$ & & & $\mathrm{H}-08$ \\
\hline E21 & 7 & & & $L-15$ & $\mathrm{~N}-14$ & $M-13$ & & \\
\hline E25 & 7 & & & $M-14$ & $\mathrm{~K}-13$ & & & \\
\hline $\mathrm{E} 27$ & 7 & & & $\mathrm{~N}-13$ & $L-15$ & $\mathrm{~K}-13$ & & \\
\hline E28 & 7 & & & $\mathrm{~N}-14$ & $\mathrm{M}-13$ & & & $L-15$ \\
\hline \multicolumn{9}{|l|}{ Cycle 6} \\
\hline $\mathrm{F} 2$ & $8 \bar{A}$ & & & & $\mathrm{H}-\mathrm{Og}$ & $\mathrm{N}-12$ & & \\
\hline $\mathrm{F} 4$ & $8 B$ & & & & $\mathrm{H}-11$ & $\mathrm{H}-12$ & & \\
\hline F6 & $8 B$ & & & & $\mathrm{H}-13$ & K-09 & & \\
\hline F10 & $8 \mathrm{~B}$ & & & & $\mathrm{~K}-10$ & $\mathrm{~K}-15$ & & \\
\hline F12 & $8 B$ & & & & $\mathrm{~K}-12$ & $L-15$ & $\mathrm{~K}-15$ & $\mathrm{~K}-15$ \\
\hline F14 & $8 \mathrm{~B}$ & & & & $\mathrm{~K}-14$ & $\mathrm{H}-10$ & & \\
\hline $\mathrm{F} 14 \mathrm{a}$ & $8 \mathrm{~B}$ & & & & $\mathrm{~K}-14$ & $\mathrm{M}-11$ & & \\
\hline F17 & $8 A$ & & & & $L-11$ & $0-13$ & & \\
\hline F17a & $8 \mathrm{~A}$ & & & & $\mathrm{~L}-11$ & $L-10$ & & \\
\hline F19 & $8 \mathrm{~B}$ & & & & $L-13$ & $\mathrm{~N}-14$ & $\mathrm{H}-15$ & \\
\hline F23 & $8 \mathrm{~B}$ & & & & $\mathrm{M}-12$ & $L-12$ & & \\
\hline $\mathrm{F} 25$ & $8 \mathrm{~B}$ & & & & $\mathrm{M}-14$ & $\mathrm{~K}-11$ & & \\
\hline F27 & $8 \mathrm{~B}$ & & & & $\mathrm{~N}-13$ & $L-14$ & & \\
\hline
\end{tabular}


Table 3-3. Fuel Assembly Locations by Cycle (Cont.)

\begin{tabular}{|c|c|c|c|c|c|c|}
\hline $\begin{array}{l}\text { Assembly } \\
\text { Number }\end{array}$ & $\begin{array}{c}\text { Batch } \\
\text { Number }\end{array}$ & Cycle 7 & Cycle 8 & Cycle 9 & Cycle 10 & Cycle 11 \\
\hline \multicolumn{7}{|l|}{ Cycle 7} \\
\hline G2 & $9 \mathrm{~B}$ & $\mathrm{H}-09$ & $\mathrm{H}-12$ & & & \\
\hline G4 & $9 \mathrm{~A}$ & $\mathrm{H}-11$ & $L-10$ & & & \\
\hline G6 & $9 \mathrm{C}$ & $\mathrm{H}-13$ & K-09 & & & \\
\hline G10 & $9 \mathrm{~A}$ & $\mathrm{~K}-10$ & $L-12$ & & & \\
\hline G12 & $9 A$ & $\mathrm{~K}-12$ & $\mathrm{H}-10$ & & & \\
\hline G12a & $9 \mathrm{~A}$ & $\mathrm{~K}-12$ & $\mathrm{M}-11$ & & & \\
\hline G14 & $9 \mathrm{C}$ & $\mathrm{K}-14$ & $\mathrm{M}-13$ & & & \\
\hline $\mathrm{G} 17$ & $9 A$ & $\mathrm{~L}-11$ & $L-15$ (7 assy) & & & \\
\hline $\bar{G} 19$ & $9 \mathrm{C}$ & $L-13$ & $\mathrm{~K}-13$ & & & \\
\hline G23 & $9 \mathrm{~A}$ & $\mathrm{M}-12$ & $\mathrm{~K}-11$ & & & \\
\hline G25 & $9 \mathrm{C}$ & $\mathrm{M}-14$ & $\mathrm{H}-09$ & & & \\
\hline G25a & $9 \mathrm{C}$ & $M-14$ & O-13 & & & \\
\hline G27 & $9 \mathrm{C}$ & $\mathrm{N}-13$ & $\mathrm{H}-14$ & & & \\
\hline G27a & $9 \mathrm{C}$ & $\mathrm{N}-13$ & $\mathrm{~N}-12$ & & & \\
\hline \multicolumn{7}{|l|}{ Cycle 8} \\
\hline $\mathrm{H} 4$ & $10 \mathrm{~A}$ & & $\mathrm{H}-11$ & & & \\
\hline $\mathrm{H} 6$ & $10 \mathrm{~B}$ & & $\mathrm{H}-13$ & $\mathrm{H}-14$ & & $0-13$ \\
\hline $\mathrm{H} 10$ & $10 \mathrm{~B}$ & & $K-10$ & $\mathrm{M}-11$ & & \\
\hline $\mathrm{H} 10 \mathrm{a}$ & $10 \mathrm{~B}$ & & $\mathrm{~K}-10$ & $0-13$ & $\mathrm{H}-08$ & \\
\hline $\mathrm{H} 10 \mathrm{~b}$ & $10 \mathrm{~B}$ & & $\mathrm{~K}-10$ & 0.13 & & $\mathrm{H}-08$ \\
\hline $\mathrm{H} 12$ & $10 \mathrm{~B}$ & & $\mathrm{~K}-12$ & $\mathrm{H}-12$ & $\mathrm{H}-15$ & \\
\hline $\mathrm{H} 12 \mathrm{a}$ & $10 \mathrm{~B}$ & & $\mathrm{~K}-12$ & $L-10$ & & \\
\hline $\mathrm{H} 14$ & $10 \mathrm{~B}$ & & $\mathrm{~K}-14$ & $M-13$ & & $L-15$ \\
\hline $\mathrm{H} 17$ & $10 \mathrm{~B}$ & & $L-11$ & $\mathrm{~K}-11$ & & \\
\hline H19 & $10 \mathrm{~B}$ & & $L-13$ & $\mathrm{~K}-13$ & K-15 & \\
\hline $\mathrm{H} 2 \mathrm{O}$ & $10 \mathrm{~A}$ & & $L-14$ & $\mathrm{~N}-14$ & & \\
\hline $\mathrm{H} 23$ & $10 \mathrm{~B}$ & & $\mathrm{M}-12$ & $L-12$ & $L-15$ & \\
\hline $\mathrm{H} 25$ & $10 \mathrm{~B}$ & & $M-14$ & K-09 & & \\
\hline $\mathrm{H} 25 \mathrm{a}$ & $10 \mathrm{~B}$ & & $M-14$ & $\mathrm{H}-11$ & & \\
\hline $\mathrm{H} 27$ & $10 \mathrm{~B}$ & & $\mathrm{~N}-13$ & $\mathrm{H}-10$ & & \\
\hline $\mathrm{H} 27 \mathrm{a}$ & $10 \mathrm{~B}$ & & $\mathrm{~N}-13$ & $\mathrm{~N}-12$ & & \\
\hline \multicolumn{7}{|l|}{ Cycle 9} \\
\hline J2 & $11 \mathrm{~A}$ & & & $\mathrm{H}-09$ & K-09 & \\
\hline J6 & $11 \mathrm{~B}$ & & & $\mathrm{H}-13$ & & \\
\hline J10 & $11 \mathrm{~A}$ & & & $\mathrm{~K}-10$ & $\mathrm{~N}-14$ & $K-15$ \\
\hline J12 & $11 \mathrm{C}$ & & & $\mathrm{K}-12$ & $\mathrm{M}-11$ & \\
\hline $\mathrm{J12a}$ & $11 \mathrm{C}$ & & & $\mathrm{K}-12$ & $L-10$ & \\
\hline J14 & $11 \mathrm{C}$ & & & $\mathrm{K}-14$ & $\mathrm{~K}-13$ & \\
\hline $\mathrm{J} 17$ & $11 \mathrm{C}$ & & & $L-11$ & $0-13$ & $\mathrm{H}-15$ \\
\hline$J 17 a$ & $11 \mathrm{C}$ & & & $L-11$ & $\mathrm{H}-10$ & \\
\hline $\mathrm{J} 19$ & $11 \mathrm{C}$ & & & $L-13$ & $\mathrm{~K}-11$ & \\
\hline $\mathrm{J} 2 \mathrm{O}$ & $11 \mathrm{~A}$ & & & $L-14$ & $L-12$ & \\
\hline J23 & $11 \mathrm{C}$ & & & $M-12$ & $M-13$ & \\
\hline J25 & $11 \mathrm{C}$ & & & $\mathrm{M}-14$ & $\mathrm{H}-14$ & \\
\hline J25a & $11 \mathrm{C}$ & & & $\mathrm{M}-14$ & $\mathrm{H}-11$ & \\
\hline J27 & $11 \mathrm{C}$ & & & $\mathrm{N}-13$ & $\mathrm{H}-12$ & \\
\hline J27a & $11 \mathrm{C}$ & & & $\mathrm{N}-13$ & $\mathrm{~N}-12$ & \\
\hline
\end{tabular}


Table 3-3. Fuel Assembly Locations by Cycle (Cont.)

\begin{tabular}{|c|c|c|c|c|c|c|}
\hline $\begin{array}{c}\text { Assembly } \\
\text { Number }\end{array}$ & $\begin{array}{c}\text { Batch } \\
\text { Number }\end{array}$ & Cycle 7 & Cycle 8 & Cycle 9 & Cycle 10 & Cycle 11 \\
\hline \multicolumn{7}{|l|}{ Cycle 10} \\
\hline $\mathrm{K} 2$ & $12 \mathrm{~B}$ & & & & $\mathrm{H}-0 \mathrm{O}$ & $\mathrm{H}-14$ \\
\hline K6 & $12 \mathrm{C}$ & & & & $\mathrm{H}-13$ & $\mathrm{H}-12$ \\
\hline K10 & $12 \mathrm{~B}$ & & & & $\mathrm{~K}-10$ & $\mathrm{M}-11$ \\
\hline $\mathrm{K} 10 \mathrm{a}$ & $12 \mathrm{~B}$ & & & & $\mathrm{~K}-10$ & $\mathrm{H}-10$ \\
\hline $\mathrm{K} 12$ & $12 \mathrm{C}$ & & & & $\mathrm{K}-12$ & $L-12$ \\
\hline $\mathrm{K} 14$ & $12 \mathrm{E}$ & & & & $K-14$ & $\mathrm{~K}-11$ \\
\hline $\mathrm{K} 17$ & $12 B$ & & & & $L-11$ & $\mathrm{M}-14$ \\
\hline $\mathrm{K} 19$ & $12 \mathrm{C}$ & & & & $L-13$ & K-09 \\
\hline K19a & $12 \mathrm{C}$ & & & & $L-13$ & $L-10$ \\
\hline K20 & $12 \mathrm{~A}$ & & & & $L-14$ & \\
\hline K23 & $12 \mathrm{~B}$ & & & & $M-12$ & $\mathrm{~K}-13$ \\
\hline K25 & 12D & & & & $M-14$ & $M-13$ \\
\hline K27 & $12 \mathrm{D}$ & & & & $\mathrm{N}-13$ & $\mathrm{~N}-12$ \\
\hline $\mathrm{K} 27 \mathrm{a}$ & $12 \mathrm{D}$ & & & & $\mathrm{N}-13$ & $\mathrm{H}-11$ \\
\hline \multicolumn{7}{|l|}{ Cycle 11} \\
\hline L2 & $13 A$ & & & & & $\mathrm{H}-09$ \\
\hline L6 & $13 D$ & & & & & $\mathrm{H}-13$ \\
\hline L10 & $13 \mathrm{~B}$ & & & & & $\mathrm{~K}-10$ \\
\hline $\mathrm{L} 12$ & $13 C$ & & & & & $\mathrm{~K}-12$ \\
\hline L14 & $13 \mathrm{~F}$ & & & & & $\mathrm{~K}-14$ \\
\hline L17 & $13 \mathrm{~B}$ & & & & & $L-11$ \\
\hline L19 & $13 \mathrm{E}$ & & & & & $L-13$ \\
\hline L20 & $13 A$ & & & & & $L-14$ \\
\hline$\llcorner 23$ & $13 B$ & & & & & $\mathrm{M}-12$ \\
\hline $\mathrm{L} 27$ & $13 G$ & & & & & $\mathrm{~N}-13$ \\
\hline L28 & $13 \mathrm{~F}$ & & & & & $\mathrm{~N}-14$ \\
\hline
\end{tabular}


Table 3-4. TMI-1 FA and Control Component Locations

\begin{tabular}{|c|c|c|c|c|c|c|c|}
\hline $\begin{array}{l}\text { Assembly } \\
\text { Number }\end{array}$ & \begin{tabular}{|c|} 
Batch \\
Number \\
\end{tabular} & Cycle 1A & Cycle 1B & Cycle 2 & Cycle3 & Cycle 4 & Cycle 5 \\
\hline \multicolumn{8}{|l|}{ Cycle 1} \\
\hline A1 & 2 & $\mathrm{H}-08 / \mathrm{CR}-7$ & $\mathrm{H}-08 / \mathrm{CR}-7$ & $\mathrm{H}-08$ & & & \\
\hline A2 & 2 & $\mathrm{H}-09 / 1.43$ BPRA & $\mathrm{H}-09 / 1.43$ BPRA & $\mathrm{H}-09$ & & & \\
\hline A3 & 1 & $\mathrm{H}-10$ & $\mathrm{H}-10$ & & & & \\
\hline A4 & 2 & $\mathrm{H}-11 / 1.26$ BPRA & $\mathrm{H}-11 / 1.26$ BPAA & $\mathrm{N}-12 / \mathrm{CR}-6$ & & & \\
\hline A5 & 1 & $\mathrm{H}-12 / \mathrm{CR}-6$ & $\mathrm{H}-12 / \mathrm{CR}-6$ & & & & \\
\hline A6 & 2 & $\mathrm{H}-13 / 1.26$ BPRA & $\mathrm{H}-13 / 1.26$ BPRA & $\mathrm{H}-10 / \mathrm{CR}-6$ & & & \\
\hline A7 & 3 & $\mathrm{H}-14$ & $\mathrm{H}-14 / \mathrm{CR}-7$ & $\mathrm{H}-13$ & $\mathrm{~K}-09$ & & \\
\hline A8 & 3 & $\mathrm{H}-15$ & $\mathrm{H}-15$ & $\mathrm{H}-14 / \mathrm{CR}-5$ & $\mathrm{H}-12 / \mathrm{CR}-6$ & & \\
\hline A9 & 1 & K-09/CR-5 & K-09/CR-5 & & & & \\
\hline A10 & 2 & K-10/1.43 BPRA & K-10/1.43 BPRA & $\mathrm{K}-14$ & & & \\
\hline A11 & 1 & $\mathrm{~K}-11$ & $\mathrm{~K}-11$ & & & & \\
\hline $\mathrm{A} 12$ & 2 & K-12/1.26 BPRA & $\mathrm{K}-12 / 1.26 \mathrm{BPRA}$ & $\mathrm{H}-11$ & & & \\
\hline A12a & 2 & $\mathrm{~K}-12 / 1.26$ BPRA & K-12/1.26 BPRA & $\mathrm{L}-10 / \mathrm{CR}-5$ & & & \\
\hline A13 & 1 & $\mathrm{~K}-13$ & $\mathrm{~K}-13$ & & $L-13$ & & \\
\hline A14 & 2 & K-14/1.43 BPRA & $\mathrm{K}-14 / 1.43$ BPRA & $\mathrm{K}-11$ & & & \\
\hline A15 & 3 & $\mathrm{~K}-15$ & $\mathrm{~K}-15$ & $\mathrm{M}-12$ & $L-11$ & & \\
\hline A16 & 1 & $L-10$ & $L-10$ & & & & \\
\hline A17 & 2 & L-11/1.09 BPRA & $L-11 / 1.09$ BPAA & $\mathrm{L}-11$ & & & \\
\hline A18 & 1 & L-12/CR-8 & $\mathrm{L}-12 / \mathrm{CR}-8$ & & $\mathrm{H}-08$ & & \\
\hline A18a & 1 & L-12/CR-8 & L-12/CR-8 & & & $\mathrm{H}-08$ & \\
\hline$A 18 b$ & 1 & $\mathrm{~L}-12 / \mathrm{CR}-8$ & L-12/CR-8 & & & $\mathrm{H}-13$ & \\
\hline A19 & 2 & L-13/1.26 BPRA & L-13/1.26 BPRA & $L-13$ & & $L-13$ & \\
\hline A20 & 3 & L-14/CR-7 & $L-14$ & $\mathrm{~K}-10$ & $\mathrm{~K}-11$ & & \\
\hline A21 & 3 & $\mathrm{~L}-15$ & $L-15$ & $\mathrm{M}-13$ & $\mathrm{M}-13$ & & \\
\hline A22 & 1 & $M-11 / C R-6$ & M-11/CR-6 & & $\mathrm{H}-14 / \mathrm{CR}-7$ & & \\
\hline A23 & 2 & $\mathrm{M}-12 / 1.09 \mathrm{BPRA}$ & $\mathrm{M}-12 / 1.09$ BPRA & K-13/CR-7 & & & \\
\hline A24 & 1 & $M-13 / C R-5$ & M-13/CR-5 & & & $\mathrm{H}-11$ & \\
\hline A25 & 3 & $\mathrm{M}-14$ & $\mathrm{M}-14$ & L-12/CR-8 & $\mathrm{K}-10$ & & \\
\hline A26 & 1 & $\mathrm{~N}-12$ & $\mathrm{~N}-12 / \mathrm{CR}-7$ & & & M-11/CA-5 & \\
\hline A27 & 3 & $\mathrm{~N}-13 / 1.26$ BPRA & $\mathrm{N}-13 / 1.26$ BPRA & $\mathrm{H}-12$ & $\mathrm{H}-10$ & & \\
\hline A27a & 3 & $\mathrm{~N}-13 / 1.26$ BPRA & $\mathrm{N}-13 / 1.26$ BPRA & K-09 & $\mathrm{N}-12 / \mathrm{CR}-7$ & & \\
\hline A28 & 3 & $\mathrm{~N}-14$ & $\mathrm{~N}-14$ & $\mathrm{~N}-13$ & $\mathrm{~N}-13$ & & \\
\hline A29 & 3 & $0-13$ & $0-13$ & $\mathrm{M}-11$ & L-10/CA-6 & & \\
\hline \multicolumn{8}{|l|}{ Cycle 2} \\
\hline B8 & 4 & & & $\mathrm{H}-15$ & $\mathrm{H}-09$ & $\mathrm{H}-09$ & \\
\hline B12 & 4 & & & $\mathrm{~K}-12$ & K-13/CR-5 & $\mathrm{K}-14$ & \\
\hline B15 & 4 & & & $\mathrm{~K}-15$ & $\mathrm{~L}-12 / \mathrm{CR}-8^{\mathrm{a}}$ & $\mathrm{K}-12$ & $\mathrm{~K}-14$ \\
\hline B20 & 4 & & & $L-14$ & $\mathrm{H}-11$ & $\mathrm{~K}-10$ & \\
\hline B20a & 4 & & & $L-14$ & $\mathrm{H}-13$ & $\mathrm{~K}-10$ & \\
\hline B21 & 4 & & & $L-15$ & $\mathrm{~K}-12$ & $\mathrm{~N}-12 / \mathrm{CR}^{-7^{\mathrm{a}}}$ & $\mathrm{H}-08$ \\
\hline B21a & 4 & & & & & $0-13$ & $\mathrm{~N}-12 / \mathrm{CR}-7^{\mathrm{a}}$ \\
\hline B25 & 4 & & & $\mathrm{M}-14$ & $\mathrm{~K}-14$ & $\mathrm{M}-13$ & \\
\hline B28 & 4 & & & $\mathrm{~N}-14$ & $M-12$ & $L-11$ & $L-13$ \\
\hline B29 & 4 & & & $0-13$ & $M-11$ & $\mathrm{H}-14 / \mathrm{CR}^{-7^{\mathrm{a}}}$ & $\mathrm{H}-11$ \\
\hline
\end{tabular}


Table 3-4. TMI-1 FA and Control Component Locations (Cont.)

\begin{tabular}{|c|c|c|c|c|c|c|c|c|}
\hline $\begin{array}{c}\text { Assembly } \\
\text { Number }\end{array}$ & $\begin{array}{c}\text { Batch } \\
\text { Number }\end{array}$ & Cycle3 & Cycle 4 & Cycle 5 & Cycle 6 & Cycle 7 & Cycle 8 & Cycle 9 \\
\hline \multicolumn{9}{|l|}{ Cycle 3} \\
\hline $\mathrm{CB}$ & 5 & $\mathrm{H}-15$ & $\mathrm{H}-12 / \overline{\mathrm{CR}}-5$ & $\mathrm{H}-13$ & & & & \\
\hline C15 & 5 & $\mathrm{~K}-15$ & $\mathrm{H}-10 / \mathrm{CR}-5$ & $\mathrm{H}-09$ & & & & \\
\hline C15a & 5 & $K-15$ & K-09 & $M-11 / C R-5$ & & & & \\
\hline $\mathrm{C} 20$ & 5 & $\mathrm{~L}-14$ & K-11 & $\mathrm{K}-10$ & & & & \\
\hline C21 & 5 & $\mathrm{~L}-15$ & L-12/CR-8 ${ }^{\mathrm{a}}$ & $L-11$ & & & & \\
\hline C25 & 5 & $\mathrm{M}-14$ & $M-12$ & $\mathrm{~K}-12$ & & & & \\
\hline $\mathrm{C} 28$ & 5 & $\mathrm{~N}-14$ & $\mathrm{~K}-13 / \mathrm{CR}-6^{\mathrm{a}}$ & $M-12$ & & & & \\
\hline C29 & 5 & $0-13$ & $\mathrm{~L}-10 / \mathrm{CR}-7^{\mathrm{a}}$ & $0-13$ & & & & \\
\hline \multicolumn{9}{|l|}{ Cycle 4} \\
\hline D8 & 6 & & $\mathrm{H}-15$ & $\mathrm{H}-10 / \mathrm{CR}-5$ & $\mathrm{M}-11 / \mathrm{CR}-5$ & & & \\
\hline D15 & 6 & & $\mathrm{~K}-15$ & K-13/CR-6 & $\mathrm{H}-12 / \mathrm{CR}-7^{\mathrm{a}}$ & & & \\
\hline D15a & 6 & & $\mathrm{~K}-15$ & $\mathrm{~K}-13$ & $\mathrm{~N}-12 / \mathrm{CR}-7^{\mathrm{a}}$ & & & \\
\hline D20 & 6 & & L-14 & $\mathrm{H}-12 / \mathrm{CR}-5$ & $\mathrm{H}-15$ & & & \\
\hline D20a & 6 & & $L-14$ & $\mathrm{H}-14 / \mathrm{CR}-7^{\mathrm{a}}$ & $\mathrm{L}-10 / \mathrm{CR}-6$ & & & \\
\hline $\bar{D} 21$ & 6 & & $L-15$ & $L-12 / C R-8^{a}$ & $\mathrm{~K}-11$ & & & \\
\hline D25 & 6 & & $M-14$ & K-09 & $\mathrm{H}-10$ & & & \\
\hline D25a & 6 & & $\mathrm{M}-14$ & $L-10 / C R-7^{a}$ & K-09 & & & \\
\hline D27 & 6 & & $\mathrm{~N}-13$ & $M-13$ & $\mathrm{H}-14 / \mathrm{CR}-6$ & & & \\
\hline D27a & 6 & & $\mathrm{~N}-13$ & $\mathrm{M}-13$ & $\mathrm{H}-08$ & & & \\
\hline D28 & 6 & & $\mathrm{~N}-14$ & $\mathrm{~K}-11$ & $L-12 / C R-8^{a}$ & & & \\
\hline \multicolumn{9}{|l|}{ Cycle 5} \\
\hline E8 & 7 & & & $\mathrm{H}-15$ & $0-13$ & $\begin{array}{ll}\mathrm{H}-14 / \mathrm{CR}-6 \\
\end{array}$ & & \\
\hline E15 & 7 & & & K-15 & $L-14$ & & $\mathrm{~N}-14$ & $\mathrm{H}-15$ \\
\hline E20 & 7 & & & $L-14$ & $\mathrm{~K}-15$ & $\mathrm{H}-15$ & & \\
\hline E20a & 7 & & & $L-14$ & $\mathrm{~K}-15$ & $\mathrm{H}-08$ & & \\
\hline E20b & 7 & & & $L-14$ & $\mathrm{~K}-15$ & & $\mathrm{H}-08$ & \\
\hline E20c & 7 & & & $\mathrm{~L}-14$ & $\mathrm{~K}-15$ & & & $\mathrm{H}-08$ \\
\hline E21 & 7 & & & $\mathrm{~L}-15$ & $\mathrm{~N}-14$ & $M-13$ & & \\
\hline E25 & 7 & & & $\mathrm{M}-14$ & K-13/CR-5 & & & \\
\hline E27 & 7 & & & $\mathrm{~N}-13$ & $L-15$ & K-13/CA-5 & & \\
\hline E28 & 7 & & & $\mathrm{~N}-14$ & $M-13$ & & & $L-15$ \\
\hline \multicolumn{9}{|l|}{ Cycle 6} \\
\hline F2 & $8 A$ & & & & $\mathrm{H}-09 / 1.03$ BPRA & $\mathrm{N}-12 / \mathrm{CR}-7^{\mathrm{a}}$ & & \\
\hline $\mathrm{F4}$ & $8 \mathrm{~B}$ & & & & $\mathrm{H}-11 / 1.03$ BPRA & $\mathrm{H}-12 / \mathrm{CR}-7^{\mathrm{a}}$ & & \\
\hline F6 & $8 \mathrm{~B}$ & & & & $\mathrm{H}-13 / 0.8$ BPRA & $\mathrm{K}-09$ & & \\
\hline F10 & $8 \mathrm{~B}$ & & & & $\mathrm{~K}-10 / 1.03$ BPRA & $\mathrm{K}-15$ & & \\
\hline $\mathrm{F} 12$ & $8 \mathrm{~B}$ & & & & $\mathrm{~K}-12 / 1.03$ BPRA & $L-15$ & K-15 & $\mathrm{K}-15$ \\
\hline $\mathrm{F} 14$ & $8 \mathrm{~B}$ & & & & $\mathrm{~K}-14 / 0.2$ BPRA & $\mathrm{H}-10$ & & \\
\hline F14a & $8 B$ & & & & $\mathrm{~K}-14 / 0.2$ BPRA & $M-11 / C R-5$ & & \\
\hline F17 & $8 \bar{A}$ & & & & $L-11 / 1.03$ BPRA & $0-13$ & & \\
\hline $\mathrm{F} 17 \mathrm{a}$ & $8 \mathrm{~A}$ & & & & L-11/1.03 BPRA & L-10/CR-6 & & \\
\hline F19 & $8 \mathrm{~B}$ & & & & $L-13 / 0.8$ BPRA & $\mathrm{N}-14$ & $\mathrm{H}-15$ & \\
\hline F23 & $8 \mathrm{~B}$ & & & & $\mathrm{M}-12 / 0.8 \mathrm{BPRA}$ & $\mathrm{L}-12 / \mathrm{CR}-8^{\mathrm{a}}$ & & \\
\hline F25 & $8 \mathrm{~B}$ & & & & $M-14$ & $\mathrm{~K}-11$ & & \\
\hline F27 & 8B & & & & $\mathrm{N}-13 / 0.2$ BPRA & $L-14$ & & \\
\hline
\end{tabular}


Table 3-4. TMI-1 FA and Control Component Locations (Cont.)

\begin{tabular}{|c|c|c|c|c|c|c|}
\hline $\begin{array}{l}\text { Assembly } \\
\text { Number }\end{array}$ & $\begin{array}{c}\text { Batch } \\
\text { Number }\end{array}$ & Cycle 7 & Cycle 8 & Cycle 9 & Cycle 10 & Cycle 11 \\
\hline \multicolumn{7}{|l|}{ Cycle 7} \\
\hline G2 & $9 \mathrm{~B}$ & $\mathrm{H}-09 / 1.0$ BPRA & $\mathrm{H}-12 / \mathrm{CR}-7^{\mathrm{a}}$ & & & \\
\hline G4 & $9 A$ & $\mathrm{H}-11 / 1.0 \mathrm{BPRA}$ & L-10/CR-6 & & & \\
\hline G6 & $9 \mathrm{C}$ & $\mathrm{H}-13 / 1.312 \mathrm{BPRA}$ & $\mathrm{K}-09$ & & & \\
\hline G10 & $9 A$ & $\mathrm{~K}-10 / 0.8 \mathrm{BPRA}$ & $L-12 / C R-8^{a}$ & & & \\
\hline G12 & $9 A$ & $\mathrm{~K}-12 / 0.5$ BPRA & $\mathrm{H}-10$ & & & \\
\hline G12a & $9 A$ & $\mathrm{~K}-12 / 0.5$ BPRA & $M-11 / C$ A-5 & & & \\
\hline G14 & $9 \mathrm{C}$ & K-14/0.2 BPRA & $\mathrm{M}-13$ & & & \\
\hline G17 & $9 \mathrm{~A}$ & $\mathrm{~L}-11 / 0.8 \mathrm{BPRA}$ & $L-15$ & & & \\
\hline G19 & $9 \mathrm{C}$ & L-13/1.312 BPRA & $\mathrm{K}-13 / \mathrm{CR}-5$ & & & \\
\hline G23 & $9 A$ & M-12/0.2 BPRA & $\mathrm{K}-11$ & & & \\
\hline G25 & $9 \mathrm{C}$ & $\overline{M-14}$ & $\mathrm{H}-09$ & & & \\
\hline G25a & $9 \mathrm{C}$ & $\mathrm{M}-14$ & $0-13$ & & & \\
\hline G27 & $9 \mathrm{C}$ & $\mathrm{N}-13 / 0.2$ BPRA & $\mathrm{H}-14 / \mathrm{CR}-6$ & & & \\
\hline G27a & $9 \mathrm{C}$ & $\mathrm{N}-13 / 0.2$ BPRA & $\mathrm{N}-12 / \mathrm{CR}-7^{\mathrm{a}}$ & & & \\
\hline \multicolumn{7}{|l|}{ Cycle 8} \\
\hline $\mathrm{H} 4$ & $10 \mathrm{~A}$ & & $\mathrm{H}-11 / 0.5$ BPRA & & & \\
\hline $\mathrm{H} 6$ & $10 \mathrm{~B}$ & & $\mathrm{H}-13 / 1.7$ BPRA & $\mathrm{H}-14 / \mathrm{CR}-6$ & & $0-13$ \\
\hline $\mathrm{H} 10$ & 10B & & K-10/1.303 BPRA & $\mathrm{M}-11 / \mathrm{CR}-5$ & & \\
\hline $\mathrm{H} 10 \mathrm{a}$ & $10 \mathrm{~B}$ & & $\mathrm{~K}-10 / 1.303$ BPRA & $0-13$ & $\mathrm{H}-08 / \mathrm{CR}^{-7^{\mathrm{a}}}$ & \\
\hline $\mathrm{H} 10 \mathrm{~b}$ & $10 \mathrm{~B}$ & & K-10/1.303 BPRA & $0-13$ & & $\mathrm{H}-08 / \mathrm{CA}-7^{\mathrm{a}}$ \\
\hline $\mathrm{H} 12$ & $10 \mathrm{~B}$ & & $\mathrm{~K}-12 / 1.7$ BPRA & $\mathrm{H}-12 / \mathrm{CR}^{-} 7^{\mathrm{a}}$ & $\mathrm{H}-15$ & \\
\hline $\mathrm{H} 12 \mathrm{a}$ & $10 \mathrm{~B}$ & & K-12/1.7 BPRA & L-10/CR-6 & & \\
\hline $\mathrm{H} 14$ & 10B & & K-14/0.5 BPRA & $M-13$ & & $L-15$ \\
\hline $\mathrm{H} 17$ & 10B & & L-11/1.7 BPRA & $\mathrm{K}-11$ & & \\
\hline $\mathrm{H} 19$ & $10 \mathrm{~B}$ & & L-13/1.7 BPRA & $\mathrm{K}-13 / \mathrm{CR}-5$ & $\mathrm{~K}-15$ & \\
\hline $\mathrm{H} 2 \mathrm{O}$ & $10 \mathrm{~A}$ & & $L-14$ & $\mathrm{~N}-14$ & & \\
\hline $\mathrm{H} 23$ & $10 \mathrm{~B}$ & & M-12/1.7 BPRA & $L-12 / C R-8^{a}$ & $L-15$ & \\
\hline $\mathrm{H} 25$ & 10B & & $M-14$ & $\mathrm{~K}-09$ & & \\
\hline $\mathrm{H} 25 \mathrm{a}$ & $10 \mathrm{~B}$ & & $\mathrm{M}-14$ & $\mathrm{H}-11$ & & \\
\hline $\mathrm{H} 27$ & $10 \mathrm{~B}$ & & $\mathrm{~N}-13 / 0.5$ BPRA & $\mathrm{H}-10$ & & \\
\hline $\mathrm{H} 27 \mathrm{a}$ & $10 \mathrm{~B}$ & & $\mathrm{~N}-13 / 0.5$ BPRA & $\mathrm{N}-12 / \mathrm{CR}-7^{\mathrm{a}}$ & & \\
\hline \multicolumn{7}{|l|}{ Cycle 9} \\
\hline $\mathrm{J} 2$ & $11 \mathrm{~A}$ & & & H-09/1.7 BPRA & K-09 & \\
\hline$\sqrt{6}$ & $11 \mathrm{~B}$ & & & $\mathrm{H}-13 / 1.1$ BPRA & & \\
\hline$J 10$ & $11 \mathrm{~A}$ & & & $\mathrm{~K}-10 / 1.7$ BPRA & $\mathrm{N}-14$ & $K-15$ \\
\hline $\mathrm{J} 12$ & $11 \mathrm{C}$ & & & K-12/1.7 BPRA & $\mathrm{M}-11 / \mathrm{CR}-5$ & \\
\hline $\mathrm{J12a}$ & $11 \mathrm{C}$ & & & $\mathrm{K}-12 / 1.7$ BPRA & $L-10$ & \\
\hline J14 & $11 \mathrm{C}$ & & & K-14/0.8 BPRA & K-13/CR-5 & \\
\hline $\mathrm{J} 17$ & $11 \mathrm{C}$ & & & L-11/2.1 BPRA & $0-13$ & $\mathrm{H}-15$ \\
\hline J17a & $11 \mathrm{C}$ & & & L-11/2.1 BPRA & $\mathrm{H}-10$ & \\
\hline$J 19$ & $11 \mathrm{C}$ & & & L-13/2.1 BPRA & $\mathrm{K}-11$ & \\
\hline $\mathrm{J} 20$ & $11 \mathrm{~A}$ & & & $L-14$ & L-12/CR- $8^{a}$ & \\
\hline$\sqrt{23}$ & $11 \mathrm{C}$ & & & $\mathrm{M}-12 / 2.1 \mathrm{BPRA}$ & $M-13$ & \\
\hline $\mathrm{J} 25$ & $11 \mathrm{C}$ & & & $\mathrm{M}-14$ & $\mathrm{H}-14 / \mathrm{CR}-6$ & \\
\hline J25a & $11 \mathrm{C}$ & & & $\mathrm{M}-14$ & $\mathrm{H}-11$ & \\
\hline J27 & $11 \mathrm{C}$ & & & $\mathrm{N}-13 / 0.0$ BPRA & $\mathrm{H}-12 / \mathrm{CR}^{-7^{2}}$ & \\
\hline $\mathrm{J} 27 \mathrm{a}$ & $11 \mathrm{C}$ & & & $\mathrm{N}-13 / 0.0 \mathrm{BPRA}$ & $\mathrm{N}-12 / \mathrm{CR}^{-7^{\mathrm{a}}}$ & \\
\hline
\end{tabular}


Table 3-4. TMI-1 FA and Control Component Locations (Cont.)

\begin{tabular}{|c|c|c|c|c|c|c|}
\hline $\begin{array}{l}\text { Assembly } \\
\text { Number }\end{array}$ & $\begin{array}{c}\text { Batch } \\
\text { Number }\end{array}$ & Cycle 7 & Cycle 8 & Cycle 9 & Cycle 10 & Cycle 11 \\
\hline \multicolumn{7}{|l|}{ Cycle 10} \\
\hline $\mathrm{K} 2$ & $12 \mathrm{~B}$ & & & & H-09/2.0 BPRA & $\mathrm{H}-14 / \mathrm{CR}-6$ \\
\hline $\mathrm{K} 6$ & $12 \mathrm{C}$ & & & & $\mathrm{H}-13 / 2.0$ BPRA & $\mathrm{H}-12 / \mathrm{CR}^{-7^{\mathrm{a}}}$ \\
\hline $\mathrm{K} 10$ & $12 \mathrm{~B}$ & & & & K-10/2.0 BPRA & $M-11 / C R-5$ \\
\hline K10a & $12 \mathrm{~B}$ & & & & K-10/2.0 BPRA & $\mathrm{H}-10$ \\
\hline $\mathrm{K} 12$ & $12 \mathrm{C}$ & & & & K-12/2.1 BPRA & $L-12 / C R-8^{a}$ \\
\hline $\mathrm{K} 14$ & $12 E$ & & & & K-14/0.2 BPRA & $\mathrm{K}-11$ \\
\hline K17 & $12 \mathrm{~B}$ & & & & L-11/2.0 BPRA & $\mathrm{M}-14$ \\
\hline K19 & $12 \mathrm{C}$ & & & & L-13/2.1 BPRA & K-09 \\
\hline $\mathrm{K} 19 \mathrm{a}$ & $12 \mathrm{C}$ & & & & L-13/2.1 BPRA & $L-10 / C R-6$ \\
\hline $\mathrm{K} 2 \mathrm{O}$ & $12 A$ & & & & $L-14$ & \\
\hline $\mathrm{K} 23$ & 12B & & & & $\mathrm{M}-12 / 2.0 \mathrm{BPRA}$ & K-13/CR-5 \\
\hline K25 & $12 \mathrm{D}$ & & & & $\mathrm{M}-14$ & $\mathrm{M}-13$ \\
\hline K27 & $12 \mathrm{D}$ & & & & $\mathrm{N}-13 / 0.0$ BPRA & $\mathrm{N}-12 / \mathrm{CR}-7^{\mathrm{a}}$ \\
\hline $\mathrm{K} 27 \mathrm{a}$ & $12 \mathrm{D}$ & & & & $\mathrm{N}-13 / 0.0$ BPRA & $\mathrm{H}-11$ \\
\hline \multicolumn{7}{|l|}{ Cycle 11} \\
\hline $\mathrm{L} 2$ & $13 \mathrm{~A}$ & & & & & H-09/2.0 BPRA \\
\hline L6 & 13D & & & & & $\mathrm{H}-13 / 2.3$ BPRA \\
\hline L10 & 13B & & & & & K-10/3.5 BPRA \\
\hline L12 & $13 \mathrm{C}$ & & & & & $\mathrm{K}-12 / 3.5 \mathrm{BPRA}$ \\
\hline L14 & $13 \mathrm{~F}$ & & & & & $\mathrm{~K}-14$ \\
\hline L17 & 13B & & & & & $L-11 / 3.5$ BPRA \\
\hline L19 & $13 \mathrm{E}$ & & & & & L-13/3.5 BPRA \\
\hline L20 & $13 \mathrm{~A}$ & & & & & $L-14$ \\
\hline L23 & $13 B$ & & & & & $\mathrm{M}-12 / 3.5 \mathrm{BPRA}$ \\
\hline$\llcorner 27$ & $13 G$ & & & & & $\mathrm{~N}-13$ \\
\hline L28 & $13 \mathrm{~F}$ & & & & & $\mathrm{~N}-14$ \\
\hline
\end{tabular}

NOTE: ${ }^{\mathrm{a}}$ The rod history is provided in Section 4. 
INTENTIONALLY LEFT 


\section{CORE OPERATIONS AND STATEPOINT INFORMATION}

This section provides core operations data for the burnup calculations that are required to generate isotopic concentrations for the statepoint evaluations. The measured critical conditions for the statepoints (SPs) evaluated are also contained in this section.

\subsection{CORE FOLLOW DATA}

The use of commercial reactor criticality data for model validation requires detailed knowledge of how the reactor was operated for the lifetime of every fuel assembly contributing to the criticality database. This is necessary to adequately model the conditions for burnup calculations at each axial location of each fuel assembly represented in the reactor core for each SP evaluation. Thus, core follow calculations based on core operation data are used to provide local conditions as a function of time to be used for all burnup calculations performed in support of the SP evaluations. In addition, measured global data such as rod insertions and boron letdown data are also provided.

The core follow calculations provide three-dimensional thermal-hydraulic (TH) feedback and burnup data. Tables 4-2 through 4-116 provide axial burnup profiles for each assembly at each SP along with axial fuel temperature and moderator specific volume distributions to be used in the burnup calculations between SPs. Fuel assembly nodal burnup values are provided at a given SP or datapoint (DP). The fuel temperature and the moderator specific volume are obtained from approximately half way to the previous SP (or DP) at full power. The data in Tables 4-2 through 4-116 is given by axial node location. Starting from the top of the reactor core (node 1) the nodal spacing is as indicated in Table 4-1. Active fuel and fuel assemblies modeled in the depletion and statepoint analysis are provided in Figure 4-1. Note that Cycles 1 through 4 are modeled with an active fuel height of $360.4515 \mathrm{~cm}$, Cycles 5 through 9 are modeled with an active fuel height of $360.172 \mathrm{~cm}$, and Cycles 10 and 11 are modeled with an active fuel height of $357.111 \mathrm{~cm}$.

Table 4-1. Assembly Axial Nodal Spacing

\begin{tabular}{|c|c|c|c|}
\hline \multirow{2}{*}{$\begin{array}{c}\text { Axial } \\
\text { Node }\end{array}$} & \multicolumn{3}{|c|}{ Node Spacing (cm) } \\
\cline { 2 - 4 } & Cycles 1-4 & Cycles 5 - 9 & Cycles 10 - 11 \\
\hline 1 (top) & 20.2057 & 20.0660 & 17.4780 \\
\hline 2 & 20.0025 & 20.0025 & 20.0025 \\
\hline 3 & 20.0025 & 20.0025 & 20.0025 \\
\hline 4 & 20.0025 & 20.0025 & 20.0025 \\
\hline 5 & 20.0025 & 20.0025 & 20.0025 \\
\hline 6 & 20.0025 & 20.0025 & 20.0025 \\
\hline 7 & 20.0025 & 20.0025 & 20.0025 \\
\hline 8 & 20.0025 & 20.0025 & 20.0025 \\
\hline 9 & 20.0025 & 20.0025 & 20.0025 \\
\hline 10 & 20.0025 & 20.0025 & 20.0025 \\
\hline 11 & 20.0025 & 20.0025 & 20.0025 \\
\hline
\end{tabular}


Table 4-1. Assembly Axial Nodal Spacing (Cont.)

\begin{tabular}{|c|c|c|c|}
\hline \multirow{2}{*}{$\begin{array}{c}\text { Axial } \\
\text { Node }\end{array}$} & \multicolumn{3}{|c|}{ Node Spacing (cm) } \\
\cline { 2 - 4 } & Cycles 1 - 4 & Cycles 5 - 9 & Cycles 10 - 11 \\
\hline 12 & 20.0025 & 20.0025 & 20.0025 \\
\hline 13 & 20.0025 & 20.0025 & 20.0025 \\
\hline 14 & 20.0025 & 20.0025 & 20.0025 \\
\hline 15 & 20.0025 & 20.0025 & 20.0025 \\
\hline 16 & 20.0025 & 20.0025 & 20.0025 \\
\hline 17 & 20.0025 & 20.0025 & 20.0025 \\
\hline 18 (bottom) & 22.2057 & 20.0660 & 19.5940 \\
\hline Active Fuel Height & 360.4515 & 360.172 & 357.111 \\
\hline
\end{tabular}

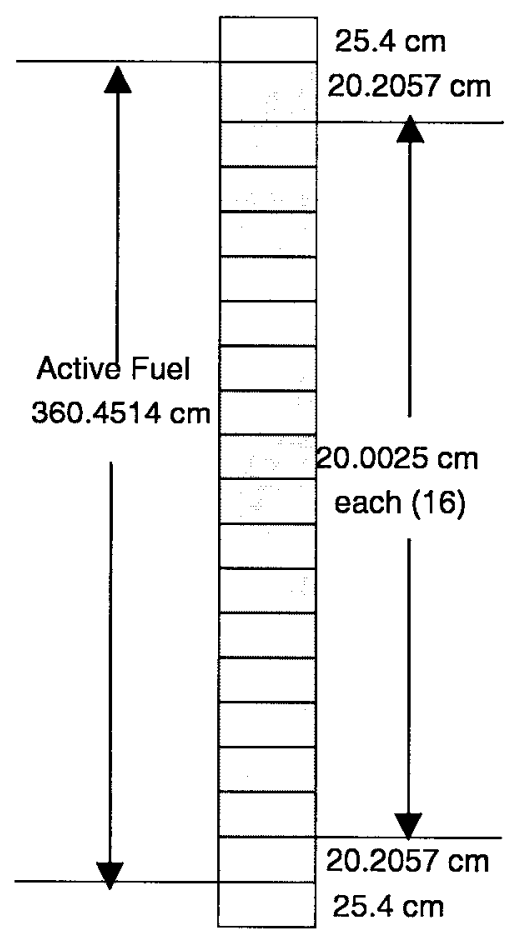

Cycles 1 through 4

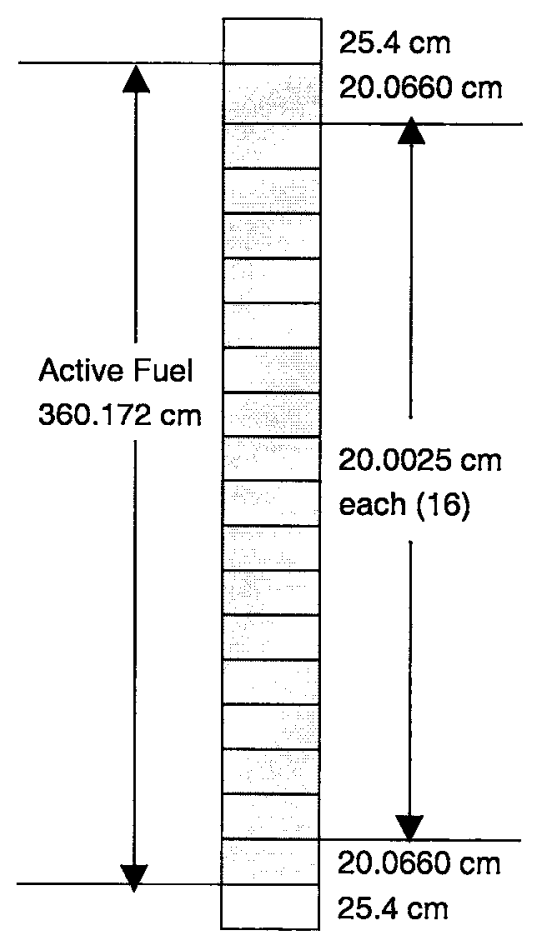

Cycles 5 through 9

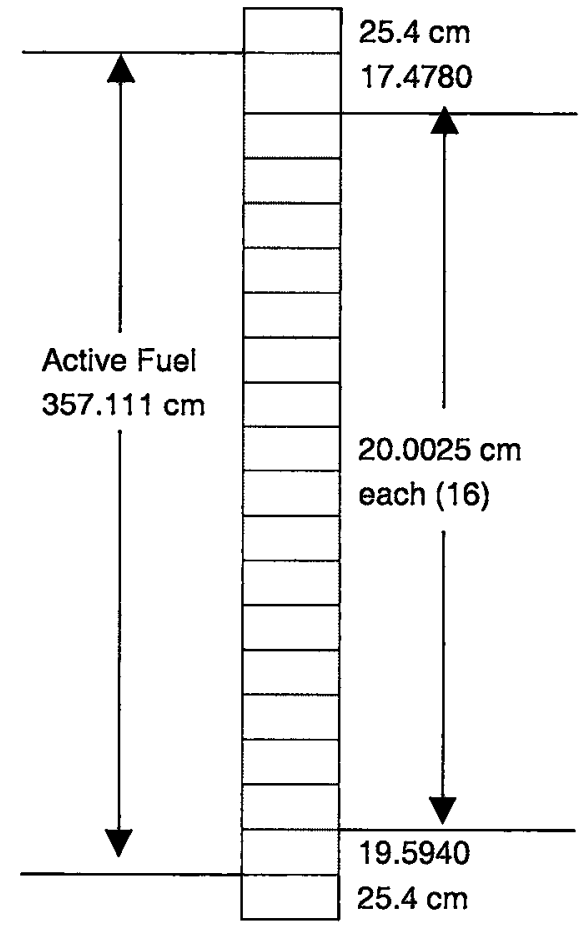

Cycles 10 through 11

Figure 4-1. TMl-1 Active Fuel Length for Statepoint Analysis

The following information applies to Tables 4-2 through 4-116:

- Burnup is measured in GWd/MTU.

- T-Fuel is Fuel Temperature measured in ${ }^{\circ} \mathrm{F}$.

- Spec. Vol. is Moderator Specific Volume measured in $\mathrm{ft}^{3} / \mathrm{lbm}$. 
Table 4-2. Burnup and TH Feedback Parameters by Axial Node for Assembly B15

\begin{tabular}{|c|c|c|c|c|c|c|c|c|c|}
\hline \multirow[b]{2}{*}{$\begin{array}{l}\text { Axial } \\
\text { Node }\end{array}$} & \multicolumn{3}{|c|}{ Datapoint (257.0 Cy2) } & \multicolumn{3}{|c|}{ Datapoint (142.8 Cy3) } & \multicolumn{3}{|c|}{ Datapoint (287.5 Cy3) } \\
\hline & $\begin{array}{c}\text { Burnup } \\
\text { (GWd/MTU) }\end{array}$ & $\begin{array}{l}\text { T-Fuel } \\
\left({ }^{\circ} \mathrm{F}\right)\end{array}$ & $\begin{array}{c}\text { Spec. Vol. } \\
\left(\mathrm{tt}^{3} / \mathrm{lbm}\right)\end{array}$ & $\begin{array}{c}\text { Burnup } \\
\text { (GWd/MTU) }\end{array}$ & $\begin{array}{l}\text { T-Fuel } \\
\left({ }^{\circ} \mathrm{F}\right)\end{array}$ & $\begin{array}{l}\text { Spec. Vol. } \\
\left(\mathrm{ft}^{3} /[\mathrm{bm})\right.\end{array}$ & $\begin{array}{c}\text { Burnup } \\
\text { (GWd/MTU) }\end{array}$ & $\begin{array}{c}\text { T-Fuel } \\
\left({ }^{\circ} \mathrm{F}\right)\end{array}$ & $\begin{array}{l}\text { Spec. Vol. } \\
\left(\mathrm{tt}^{3} / \mathrm{lbm}\right)\end{array}$ \\
\hline & $\mathrm{EOC}^{\mathrm{B}} \mathrm{Cy} 2$ & $131.6 \mathrm{Cy} 2$ & $131.6 \mathrm{Cy} 2$ & $142.8 \mathrm{Cy3}$ & $69.0 \mathrm{Cy} 3$ & $69.0 \mathrm{Cy} 3$ & EOC Cy3 & 209.4 Су3 & 209.4 Cy3 \\
\hline 1 & 2.757 & 846.7 & 0.0238 & 5.870 & 1091.7 & 0.0249 & 9.365 & 1079.5 & 0.0248 \\
\hline 2 & 4.585 & 994.7 & 0.0238 & 9.542 & 1289.3 & 0.0248 & 14.670 & 1221.1 & 0.0247 \\
\hline 3 & 5.563 & 1065.0 & 0.0237 & 11.485 & 1375.3 & 0.0246 & 17.246 & 1267.1 & 0.0245 \\
\hline 4 & 6.003 & 1094.2 & 0.0236 & 12.368 & 1399.8 & 0.0244 & 18.303 & 1272.3 & 0.0243 \\
\hline 5 & 6.184 & 1104.6 & 0.0235 & 12.731 & 1401.0 & 0.0242 & 18.697 & 1266.7 & 0.0241 \\
\hline 6 & 6.253 & 1107.6 & 0.0234 & 12.849 & 1393.3 & 0.0240 & 18.819 & 1260.8 & 0.0239 \\
\hline 7 & 6.276 & 1107.9 & 0.0233 & 12.814 & 1379.6 & 0.0238 & 18.775 & 1256.1 & 0.0238 \\
\hline 8 & 6.276 & 1107.3 & 0.0232 & 12.609 & 1345.5 & 0.0236 & 18.449 & 1244.9 & 0.0236 \\
\hline 9 & 6.264 & 1106.1 & 0.0231 & 11.814 & 1225.0 & 0.0234 & 16.972 & 1189.1 & 0.0234 \\
\hline 10 & 6.249 & 1104.8 & 0.0230 & 10.002 & 1060.4 & 0.0233 & 13.893 & 1040.0 & 0.0233 \\
\hline 11 & 6.247 & 1104.3 & 0.0229 & 9.694 & 1033.9 & 0.0232 & 13.262 & 1014.8 & 0.0232 \\
\hline 12 & 6.266 & 1105.0 & 0.0228 & 9.740 & 1037.4 & 0.0231 & 13.302 & 1014.4 & 0.0231 \\
\hline 13 & 6.300 & 1106.4 & 0.0227 & 10.007 & 1069.2 & 0.0230 & 13.771 & 1032.8 & 0.0230 \\
\hline 14 & 6.323 & 1106.8 & 0.0226 & 11.147 & 1233.9 & 0.0229 & 15.957 & 1136.6 & 0.0229 \\
\hline 15 & 6.279 & 1102.1 & 0.0225 & 12.458 & 1363.5 & 0.0228 & 18.348 & 1246.9 & 0.0228 \\
\hline 16 & 6.014 & 1080.9 & 0.0225 & 12.293 & 1370.6 & 0.0226 & 18.288 & 1264.0 & 0.0226 \\
\hline 17 & 5.150 & 1014.8 & 0.0224 & 10.694 & 1300.2 & 0.0225 & 16.170 & 1228.5 & 0.0225 \\
\hline 18 & 3.125 & 849.5 & 0.0223 & 6.669 & 1085.3 & 0.0224 & 10.419 & 1072.4 & 0.0224 \\
\hline
\end{tabular}

\begin{tabular}{|c|c|c|c|c|c|c|c|c|c|}
\hline \multirow[b]{2}{*}{$\begin{array}{l}\text { Axial } \\
\text { Node }\end{array}$} & \multicolumn{3}{|c|}{ Datapoint (126.6 Cy4) } & \multicolumn{3}{|c|}{ Statepoint 60 (0.0 Cy5) } & \multicolumn{3}{|c|}{ Statepoint 61 (114.4 Cy5) } \\
\hline & $\begin{array}{c}\text { Burnup } \\
\text { (GWd/MTU) }\end{array}$ & $\begin{array}{l}\text { T-Fuel } \\
\left({ }^{\circ} \mathrm{F}\right)\end{array}$ & $\begin{array}{c}\text { Spec. Vol. } \\
\left(\mathrm{ft}^{3} / \mathrm{lbm}\right)\end{array}$ & \begin{tabular}{|c|} 
Burnup \\
(GWd/MTU)
\end{tabular} & $\begin{array}{c}\text { T-Fuel } \\
\left({ }^{\circ} \mathrm{F}\right)\end{array}$ & $\begin{array}{l}\text { Spec. Vol. } \\
\left(\mathrm{ft}^{3} / \mathrm{lbm}\right)\end{array}$ & $\begin{array}{c}\text { Burnup } \\
\text { (GWd/MTU) }\end{array}$ & $\begin{array}{l}\text { T-Fuel } \\
\left({ }^{\circ} \mathrm{F}\right)\end{array}$ & $\begin{array}{c}\text { Spec. Vol. } \\
\left(\mathrm{ft}^{3} / \mathrm{lbm}\right)\end{array}$ \\
\hline & $126.6 \mathrm{Cy} 4$ & 71.2 Cy 4 & 71.2 Cy 4 & $\mathrm{BOC}^{\mathrm{b}} \mathrm{Cy} 5$ & 203.4 Cy 4 & 203.4 Cy 4 & 114.4 Cy 5 & 58.2 Cy5 & 58.2 Cy5 \\
\hline 1 & 11.740 & 970.0 & 0.0247 & 14.84 & 983.3 & 0.0246 & 16.242 & 810.3 & 0.0229 \\
\hline 2 & 18.319 & 1090.7 & 0.0246 & 22.77 & 1068.7 & 0.0245 & 24.968 & 879.8 & 0.0228 \\
\hline 3 & 21.518 & 1131.1 & 0.0244 & 26.493 & 1089.2 & 0.0244 & 29.145 & 900.6 & 0.0228 \\
\hline 4 & 22.846 & 1143.8 & 0.0243 & 27.957 & 1086.9 & 0.0242 & 30.836 & 905.3 & 0.0227 \\
\hline 5 & 23.343 & 1145.5 & 0.0241 & 28.464 & 1079.5 & 0.0241 & 31.439 & 904.5 & 0.0226 \\
\hline 6 & 23.474 & 1141.9 & 0.0239 & 28.584 & 1073.0 & 0.0239 & 31.589 & 899.8 & 0.0225 \\
\hline 7 & 23.368 & 1135.2 & 0.0238 & 28.476 & 1069.2 & 0.0238 & 31.484 & 894.7 & 0.0224 \\
\hline 8 & 22.910 & 1123.3 & 0.0236 & 28.015 & 1067.3 & 0.0236 & 31.039 & 895.3 & 0.0224 \\
\hline 9 & 21.377 & 1122.0 & 0.0235 & 26.485 & 1073.4 & 0.0235 & 29.614 & 916.2 & 0.0223 \\
\hline 10 & 18.528 & 1162.7 & 0.0233 & 23.714 & 1098.9 & 0.0233 & 27.073 & 962.5 & 0.0222 \\
\hline 11 & 18.032 & 1170.5 & 0.0232 & 23.188 & 1100.9 & 0.0232 & 26.627 & 980.7 & 0.0221 \\
\hline 12 & 18.221 & 1172.5 & 0.0230 & 23.381 & 1100.8 & 0.0231 & 26.828 & 986.4 & 0.0220 \\
\hline 13 & 18.821 & 1172.9 & 0.0229 & 24.012 & 1100.7 & 0.0229 & 27.399 & 979.2 & 0.0219 \\
\hline 14 & 20.861 & 1142.2 & 0.0228 & 26.018 & 1085.4 & 0.0228 & 29.187 & 942.2 & 0.0218 \\
\hline 15 & 22.834 & 1085.8 & 0.0226 & 27.874 & 1061.1 & 0.0227 & 30.765 & 898.0 & 0.0218 \\
\hline 16 & 22.357 & 1046.3 & 0.0225 & 27.254 & 1050.6 & 0.0225 & 29.908 & 871.8 & 0.0217 \\
\hline 17 & 19.610 & 995.9 & 0.0224 & 24.028 & 1024.2 & 0.0224 & 26.304 & 843.0 & 0.0216 \\
\hline 18 & 12.643 & 883.1 & 0.0223 & 15.712 & 934.8 & 0.0223 & 17.211 & 776.2 & 0.0216 \\
\hline
\end{tabular}

NOTES: $\quad{ }^{a} E O C=$ End of cycle

${ }^{b} \mathrm{BOC}=$ Beginning of cycle 
Table 4-3. Burnup and TH Feedback Parameters by Axial Node for Assembly B21

\begin{tabular}{|c|c|c|c|c|c|c|c|c|c|}
\hline \multirow{2}{*}{$\begin{array}{c}\text { Axial } \\
\text { Node }\end{array}$} & \multicolumn{2}{|c|}{ Datapoint (257.0 Cy2) } & \multicolumn{2}{c|}{ Datapoint (142.8 Cy3) } & \multicolumn{3}{c|}{ Datapoint (287.5 Cy3) } \\
\cline { 2 - 11 } & $\begin{array}{c}\text { Burnup } \\
\text { (GW/MTU) }\end{array}$ & $\begin{array}{c}\text { T-Fuel } \\
\text { ( }{ }^{\circ} \text { F) }\end{array}$ & $\begin{array}{c}\text { Spec. Vol. } \\
\left(\mathrm{ft}^{3} / \mathrm{lbm}\right)\end{array}$ & $\begin{array}{c}\text { Burnup } \\
(\mathrm{GWd} / \mathrm{MTU})\end{array}$ & $\begin{array}{c}\text { T-Fuel } \\
\left({ }^{\circ} \mathrm{F}\right)\end{array}$ & $\begin{array}{c}\text { Spec. Vol. } \\
\left(\mathrm{ft}^{3} / \mathrm{lbm}\right)\end{array}$ & $\begin{array}{c}\text { Burnup } \\
(\mathrm{GW} / \mathrm{MTU})\end{array}$ & $\begin{array}{c}\text { T-Fuel } \\
\left({ }^{\circ} \mathrm{F}\right)\end{array}$ & $\begin{array}{c}\text { Spec. VoI. } \\
\left(\mathrm{ft}^{3} / \mathrm{lbm}\right)\end{array}$ \\
\hline 1 & 2.237 & 797.4 & 0.0236 & 5.158 & 1084.4 & 0.0254 & 8.567 & 1073.8 & 0.0252 \\
\hline 2 & 3.781 & 923.6 & 0.0235 & 8.540 & 1297.0 & 0.0253 & 13.644 & 1224.0 & 0.0251 \\
\hline 3 & 4.643 & 988.8 & 0.0235 & 10.511 & 1399.7 & 0.0251 & 16.320 & 1283.3 & 0.0249 \\
\hline 4 & 5.054 & 1017.0 & 0.0234 & 11.472 & 1429.5 & 0.0248 & 17.472 & 1293.3 & 0.0247 \\
\hline 5 & 5.235 & 1027.7 & 0.0233 & 11.883 & 1431.8 & 0.0246 & 17.912 & 1288.7 & 0.0245 \\
\hline 6 & 5.310 & 1031.3 & 0.0232 & 12.037 & 1425.6 & 0.0244 & 18.068 & 1283.1 & 0.0243 \\
\hline 7 & 5.336 & 1031.9 & 0.0231 & 12.053 & 1414.6 & 0.0242 & 18.081 & 1278.8 & 0.0241 \\
\hline 8 & 5.334 & 1031.3 & 0.0230 & 11.943 & 1396.0 & 0.0240 & 17.928 & 1273.0 & 0.0239 \\
\hline 9 & 5.315 & 1029.7 & 0.0230 & 11.653 & 1361.1 & 0.0238 & 17.494 & 1258.2 & 0.0237 \\
\hline 10 & 5.289 & 1027.6 & 0.0229 & 11.268 & 1325.5 & 0.0236 & 16.914 & 1235.9 & 0.0235 \\
\hline 11 & 5.272 & 1026.1 & 0.0228 & 11.106 & 1314.8 & 0.0234 & 16.671 & 1226.3 & 0.0234 \\
\hline 12 & 5.279 & 1026.0 & 0.0227 & 11.151 & 1323.4 & 0.0233 & 16.739 & 1227.0 & 0.0232 \\
\hline 13 & 5.305 & 1027.0 & 0.0227 & 11.347 & 1348.5 & 0.0231 & 17.048 & 1236.4 & 0.0231 \\
\hline 14 & 5.327 & 1027.7 & 0.0226 & 11.676 & 1387.6 & 0.0229 & 17.602 & 1257.6 & 0.0229 \\
\hline 15 & 5.285 & 1023.5 & 0.0225 & 11.874 & 1409.4 & 0.0228 & 17.985 & 1278.5 & 0.0228 \\
\hline 16 & 5.033 & 1003.8 & 0.0224 & 11.469 & 1397.2 & 0.0226 & 17.517 & 1280.0 & 0.0226 \\
\hline 17 & 4.253 & 942.6 & 0.0224 & 9.880 & 1320.9 & 0.0225 & 15.379 & 1240.2 & 0.0225 \\
\hline 18 & 2.533 & 798.9 & 0.0223 & 6.117 & 1095.6 & 0.0224 & 9.876 & 1080.6 & 0.0224 \\
\hline
\end{tabular}

\begin{tabular}{|c|c|c|c|c|c|c|c|c|c|}
\hline \multirow[b]{2}{*}{$\begin{array}{l}\text { Axial } \\
\text { Node }\end{array}$} & \multicolumn{3}{|c|}{ Datapoint (126.6 Cy4) } & \multicolumn{3}{|c|}{ Statepoint 60 (0.0 Cy5) } & \multicolumn{3}{|c|}{ Statepoint 61 (114.4 Cy5) } \\
\hline & \begin{tabular}{|c|} 
Burnup \\
(GWd/MTU)
\end{tabular} & $\begin{array}{l}\text { T-Fuel } \\
\left({ }^{\circ} \mathrm{F}\right)\end{array}$ & $\begin{array}{c}\text { Spec. Vol. } \\
\left(\mathrm{ft}^{3} / \mathrm{lbm}\right)\end{array}$ & $\begin{array}{c}\text { Burnup } \\
\text { (GWd/MTU) }\end{array}$ & $\begin{array}{c}\text { T-Fuel } \\
\left({ }^{\circ} \mathrm{F}\right)\end{array}$ & $\begin{array}{c}\text { Spec. Vol. } \\
\left(\mathrm{ft}^{3} / \mathrm{lbm}\right)\end{array}$ & \begin{tabular}{|c|} 
Burnup \\
(GWd/MTU)
\end{tabular} & $\begin{array}{c}\text { T-Fuel } \\
\left({ }^{\circ} \mathrm{F}\right)\end{array}$ & $\begin{array}{c}\text { Spec. Vol. } \\
\left(\mathrm{tt}^{3} / \mathrm{lbm}\right)\end{array}$ \\
\hline & $126.6 \mathrm{Cy} 4$ & 71.2 Cy 4 & $71.2 \mathrm{Cy} 4$ & BOC Cy 5 & $203.4 \mathrm{Cy} 4$ & 203.4 Cy4 & 114.4 Cy5 & 58.2 Cy 5 & 58.2 Cy 5 \\
\hline 1 & 9.976 & 841.0 & 0.0246 & 12.029 & 889.9 & 0.0246 & 14.202 & 922.7 & 0.0230 \\
\hline 2 & 16.638 & 1057.0 & 0.0245 & 20.726 & 1051.2 & 0.0245 & 23.749 & 976.5 & 0.0229 \\
\hline 3 & 20.312 & 1124.1 & 0.0244 & 25.144 & 1083.8 & 0.0243 & 28.438 & 973.4 & 0.0228 \\
\hline 4. & 21.909 & 1144.0 & 0.0242 & 26.931 & 1083.8 & 0.0242 & 30.288 & 959.2 & 0.0227 \\
\hline 5 & 22.525 & 1150.5 & 0.0240 & 27.594 & 1078.5 & 0.0240 & 30.942 & 944.5 & 0.0226 \\
\hline 6 & 22.729 & 1150.2 & 0.0239 & 27.809 & 1073.4 & 0.0239 & 31.123 & 931.9 & 0.0225 \\
\hline 7 & 22.710 & 1144.7 & 0.0237 & 27.796 & 1069.9 & 0.0237 & 31.076 & 923.0 & 0.0224 \\
\hline 8 & 22.469 & 1134.9 & 0.0236 & 27.552 & 1067.5 & 0.0236 & 30.813 & 919.8 & 0.0224 \\
\hline 9 & 21.945 & 1124.1 & 0.0234 & 26.999 & 1066.0 & 0.0234 & 30.265 & 923.8 & 0.0223 \\
\hline 10 & 21.327 & 1116.8 & 0.0233 & 26.329 & 1064.8 & 0.0233 & 29.612 & 932.0 & 0.0222 \\
\hline 11 & 21.095 & 1110.7 & 0.0231 & 26.046 & 1062.5 & 0.0232 & 29.324 & 936.9 & 0.0221 \\
\hline 12 & 21.208 & 1106.2 & 0.0230 & 26.136 & 1060.5 & 0.0230 & 29.378 & 936.4 & 0.0220 \\
\hline 13 & 21.561 & 1103.0 & 0.0229 & 26.502 & 1060.1 & 0.0229 & 29.682 & 930.2 & 0.0219 \\
\hline 14 & 22.084 & 1095.2 & 0.0227 & 27.061 & 1060.1 & 0.0228 & 30.156 & 919.0 & 0.0219 \\
\hline 15 & 22.300 & 1077.1 & 0.0226 & 27.285 & 1059.0 & 0.0227 & 30.288 & 907.7 & 0.0218 \\
\hline 16 & 21.494 & 1047.2 & 0.0225 & 26.361 & 1053.0 & 0.0225 & 29.247 & 899.2 & 0.0217 \\
\hline 17 & 18.730 & 995.3 & 0.0224 & 23.104 & 1025.7 & 0.0224 & 25.714 & 884.1 & 0.0216 \\
\hline 18 & 12.000 & 874.7 & 0.0223 & 14.990 & 929.7 & 0.0223 & 16.813 & 821.7 & 0.0216 \\
\hline
\end{tabular}


Table 4-4. Burnup and TH Feedback Parameters by Axial Node for Assembly B21a

\begin{tabular}{|c|c|c|c|c|c|c|c|c|c|}
\hline \multirow[b]{2}{*}{$\begin{array}{l}\text { Axial } \\
\text { Node }\end{array}$} & \multicolumn{3}{|c|}{ Datapoint (257.0 Cy2) } & \multicolumn{3}{|c|}{ Datapoint (142.8 Cy3) } & \multicolumn{3}{|c|}{ Datapoint (287.5 Cy3) } \\
\hline & $\begin{array}{c}\text { Burnup } \\
\text { (GWd/MTU) }\end{array}$ & $\begin{array}{c}\text { T-Fuel } \\
\left({ }^{\circ} \mathrm{F}\right)\end{array}$ & $\begin{array}{l}\text { Spec. Vol. } \\
\left(\mathrm{ft}^{3} / / \mathrm{bm}\right)\end{array}$ & $\begin{array}{c}\text { Burnup } \\
\text { (GWd/MTU) }\end{array}$ & $\begin{array}{l}\text { T-Fuel } \\
\left({ }^{\circ} \mathrm{F}\right)\end{array}$ & $\begin{array}{c}\text { Spec. Vol. } \\
\left(\mathrm{ft}^{3} / \mathrm{lbm}\right)\end{array}$ & $\begin{array}{c}\text { Burnup } \\
\text { (GWd/MTU) }\end{array}$ & $\begin{array}{l}\text { T-Fuel } \\
\left({ }^{\circ} \mathrm{F}\right)\end{array}$ & $\begin{array}{c}\text { Spec. Vol. } \\
\left(\mathrm{ft}^{3} / \mathrm{lbm}\right)\end{array}$ \\
\hline & EOC Cy2 & $131.6 \mathrm{Cy} 2$ & 131.6 Cy2 & 142.8 Су 3 & $69.0 \mathrm{Cy} 3$ & $69.0 \mathrm{Cy} 3$ & EOC Суз & 209.4 Cy3 & $209.4 \mathrm{Cy} 3$ \\
\hline 1 & 2.237 & 797.4 & 0.0236 & 5.158 & 1084.4 & 0.0254 & 8.567 & 1073.8 & 0.0252 \\
\hline 2 & 3.781 & 923.6 & 0.0235 & 8.540 & 1297.0 & 0.0253 & 13.644 & 1224.0 & 0.0251 \\
\hline 3 & 4.643 & 988.8 & 0.0235 & 10.511 & 1399.7 & 0.0251 & 16.320 & 1283.3 & 0.0249 \\
\hline 4 & 5.054 & 1017.0 & 0.0234 & 11.472 & 1429.5 & 0.0248 & 17.472 & 1293.3 & 0.0247 \\
\hline 5 & 5.235 & 1027.7 & 0.0233 & 11.883 & 1431.8 & 0.0246 & 17.912 & 1288.7 & 0.0245 \\
\hline 6 & 5.310 & 1031.3 & 0.0232 & 12.037 & 1425.6 & 0.0244 & 18.068 & 1283.1 & 0.0243 \\
\hline 7 & 5.336 & 1031.9 & 0.0231 & 12.053 & 1414.6 & 0.0242 & 18.081 & 1278.8 & 0.0241 \\
\hline 8 & 5.334 & 1031.3 & 0.0230 & 11.943 & 1396.0 & 0.0240 & 17.928 & 1273.0 & 0.0239 \\
\hline 9 & 5.315 & 1029.7 & 0.0230 & 11.653 & 1361.1 & 0.0238 & 17.494 & 1258.2 & 0.0237 \\
\hline 10 & 5.289 & 1027.6 & 0.0229 & 11.268 & 1325.5 & 0.0236 & 16.914 & 1235.9 & 0.0235 \\
\hline 11 & 5.272 & 1026.1 & 0.0228 & 11.106 & 1314.8 & 0.0234 & 16.671 & 1226.3 & 0.0234 \\
\hline 12 & 5.279 & 1026.0 & 0.0227 & 11.151 & 1323.4 & 0.0233 & 16.739 & 1227.0 & 0.0232 \\
\hline 13 & 5.305 & 1027.0 & 0.0227 & 11.347 & 1348.5 & 0.0231 & 17.048 & 1236.4 & 0.0231 \\
\hline 14 & 5.327 & 1027.7 & 0.0226 & 11.676 & 1387.6 & 0.0229 & 17.602 & 1257.6 & 0.0229 \\
\hline 15 & 5.285 & 1023.5 & 0.0225 & 11.874 & 1409.4 & 0.0228 & 17.985 & 1278.5 & 0.0228 \\
\hline 16 & 5.033 & 1003.8 & 0.0224 & 11.469 & 1397.2 & 0.0226 & 17.517 & 1280.0 & 0.0226 \\
\hline 17 & 4.253 & 942.6 & 0.0224 & 9.880 & 1320.9 & 0.0225 & 15.379 & 1240.2 & 0.0225 \\
\hline 18 & 2.533 & 798.9 & 0.0223 & 6.117 & 1095.6 & 0.0224 & 9.876 & 1080.6 & 0.0224 \\
\hline
\end{tabular}

\begin{tabular}{|c|c|c|c|c|c|c|c|c|c|}
\hline \multirow[b]{2}{*}{$\begin{array}{l}\text { Axial } \\
\text { Node }\end{array}$} & \multicolumn{3}{|c|}{ Datapoint (126.6 Cy4) } & \multicolumn{3}{|c|}{ Statepoint $60(0.0$ Cy5) } & \multicolumn{3}{|c|}{ Statepoint 61 (114.4 Cy5) } \\
\hline & $\begin{array}{c}\text { Burnup } \\
\text { (GWd/MTU) }\end{array}$ & $\begin{array}{l}\text { T-Fuel } \\
\left({ }^{\circ} \mathrm{F}\right)\end{array}$ & $\begin{array}{c}\text { Spec. Vol. } \\
\left(\mathrm{ft}^{3} / \mathrm{lbm}\right)\end{array}$ & $\begin{array}{c}\text { Burnup } \\
\text { (GWd/MTU) }\end{array}$ & $\begin{array}{l}\text { T-Fuel } \\
\left({ }^{\circ} \mathrm{F}\right)\end{array}$ & $\begin{array}{l}\text { Spec. Vol. } \\
\left(\mathrm{ft}^{3} / \mathrm{bm}\right)\end{array}$ & $\begin{array}{c}\text { Burnup } \\
\text { (GWd/MTU) }\end{array}$ & $\begin{array}{l}\text { T-Fuel } \\
\left({ }^{\circ} \mathrm{F}\right)\end{array}$ & $\begin{array}{c}\text { Spec. Vol. } \\
\left(\mathrm{ft}^{3} / \mathrm{lbm}\right)\end{array}$ \\
\hline & 126.6 Cy4 & 71.2 Cy 4 & $71.2 \mathrm{Cy} 4$ & BOC Cy 5 & $203.4 \mathrm{Cy} 4$ & $203.4 \mathrm{Cy} 4$ & 114.4 Cy5 & 58.2 Cy5 & 58.2 Cy5 \\
\hline 1 & 9.550 & 756.3 & 0.0234 & 10.976 & 782.0 & 0.0235 & 12.189 & 832.6 & 0.0231 \\
\hline 2 & 15.297 & 832.5 & 0.0234 & 17.527 & 850.5 & 0.0234 & 19.792 & 979.3 & 0.0230 \\
\hline 3 & 18.381 & 869.0 & 0.0233 & 20.998 & 869.9 & 0.0234 & 24.170 & 1009.8 & 0.0229 \\
\hline 4 & 19.753 & 884.1 & 0.0232 & 22.507 & 872.4 & 0.0233 & 26.019 & 1012.2 & 0.0228 \\
\hline 5 & 20.298 & 889.7 & 0.0232 & 23.091 & 870.4 & 0.0232 & 26.710 & 1006.4 & 0.0227 \\
\hline 6 & 20.491 & 890.4 & 0.0231 & 23.295 & 867.7 & 0.0231 & 26.925 & 995.4 & 0.0226 \\
\hline 7 & 20.504 & 888.5 & 0.0230 & 23.313 & 865.9 & 0.0231 & 26.892 & 980.7 & 0.0225 \\
\hline 8 & 20.333 & 885.5 & 0.0229 & 23.150 & 865.4 & 0.0230 & 26.650 & 968.6 & 0.0225 \\
\hline 9 & 19.887 & 883.6 & 0.0229 & 22.713 & 867.0 & 0.0229 & 26.159 & 968.2 & 0.0224 \\
\hline 10 & 19.310 & 883.1 & 0.0228 & 22.144 & 869.8 & 0.0228 & 25.592 & 980.1 & 0.0223 \\
\hline 11 & 19.075 & 881.0 & 0.0227 & 21.905 & 870.5 & 0.0228 & 25.390 & 995.7 & 0.0222 \\
\hline 12 & 19.147 & 877.5 & 0.0227 & 21.969 & 869.5 & 0.0227 & 25.511 & 1011.0 & 0.0221 \\
\hline 13 & 19.444 & 872.1 & 0.0226 & 22.258 & 867.4 & 0.0226 & 25.844 & 1019.7 & 0.0220 \\
\hline 14 & 19.942 & 862.5 & 0.0225 & 22.741 & 863.7 & 0.0226 & 26.312 & 1014.7 & 0.0219 \\
\hline 15 & 20.210 & 848.2 & 0.0225 & 22.970 & 858.9 & 0.0225 & 26.446 & 999.1 & 0.0218 \\
\hline 16 & 19.542 & 828.1 & 0.0224 & 22.190 & 851.5 & 0.0224 & 25.466 & 977.3 & 0.0217 \\
\hline 17 & 17.047 & 793.8 & 0.0224 & 19.361 & 831.0 & 0.0224 & 22.209 & 939.4 & 0.0216 \\
\hline 18 & 10.897 & 724.1 & 0.0223 & 12.413 & 764.2 & 0.0223 & 14.290 & 844.2 & 0.0216 \\
\hline
\end{tabular}


Table 4-5. Burnup and TH Feedback Parameters by Axial Node for Assembly B28

\begin{tabular}{|c|c|c|c|c|c|c|c|c|c|}
\hline \multirow[b]{2}{*}{$\begin{array}{l}\text { Axial } \\
\text { Node } \\
\end{array}$} & \multicolumn{3}{|c|}{ Datapoint (257.0 Cy2) } & \multicolumn{3}{|c|}{ Datapoint (142.8 Cy3) } & \multicolumn{3}{|c|}{ Datapoint (287.5 Cy3) } \\
\hline & $\begin{array}{c}\text { Burnup } \\
\text { (GWd/MTU) }\end{array}$ & $\begin{array}{c}\text { T-Fuel } \\
\left({ }^{\circ} \mathrm{F}\right)\end{array}$ & $\begin{array}{c}\text { Spec. Vol. } \\
\left(\mathrm{ft}^{3} / \mathrm{lbm}\right)\end{array}$ & $\begin{array}{c}\text { Burnup } \\
\text { (GWd/MTU) }\end{array}$ & $\begin{array}{c}\text { T-Fuel } \\
\left({ }^{\circ} \mathrm{F}\right)\end{array}$ & $\begin{array}{c}\text { Spec. Vol. } \\
\left(\mathrm{ft}^{3} / \mathrm{lbm}\right)\end{array}$ & \begin{tabular}{|c|} 
Burnup \\
(GWd/MTU)
\end{tabular} & $\begin{array}{l}\text { T-Fuel } \\
\left({ }^{\circ} \mathrm{F}\right)\end{array}$ & $\begin{array}{c}\text { Spec. Vol. } \\
\left(\mathrm{ft}^{3} / \mathrm{lbm}\right)\end{array}$ \\
\hline & EOC Cy2 & $131.6 \mathrm{Cy} 2$ & $131.6 \mathrm{Cy} 2$ & $142.8 \mathrm{Cy} 3$ & $69.0 \mathrm{Cy} 3$ & 69.0 Су3 & EOC Cy3 & $209.4 \mathrm{Cy} 3$ & $209.4 \mathrm{Cy} 3$ \\
\hline 1 & 2.423 & 813.7 & 0.0237 & 5.230 & 1048.0 & 0.0249 & 8.376 & 1050.4 & 0.0249 \\
\hline 2 & 4.091 & 946.4 & 0.0237 & 8.553 & 1231.6 & 0.0248 & 13.187 & 1181.3 & 0.0247 \\
\hline 3 & 5.082 & 1017.6 & 0.0236 & 10.377 & 1306.8 & 0.0246 & 15.596 & 1224.8 & 0.0246 \\
\hline 4 & 5.638 & 1054.6 & 0.0235 & 11.285 & 1326.9 & 0.0244 & 16.686 & 1228.1 & 0.0244 \\
\hline 5 & 5.935 & 1072.4 & 0.0234 & 11.707 & 1324.9 & 0.0242 & 17.179 & 1221.1 & 0.0242 \\
\hline 6 & 6.073 & 1079.8 & 0.0233 & 11.866 & 1315.1 & 0.0240 & 17.370 & 1214.4 & 0.0240 \\
\hline 7 & 6.126 & 1082.0 & 0.0232 & 11.864 & 1300.0 & 0.0239 & 17.373 & 1209.3 & 0.0239 \\
\hline 8 & 6.129 & 1081.6 & 0.0231 & 11.705 & 1272.9 & 0.0237 & 17.159 & 1201.5 & 0.0237 \\
\hline 9 & 6.099 & 1079.4 & 0.0231 & 11.326 & 1228.6 & 0.0235 & 16.606 & 1182.7 & 0.0236 \\
\hline 10 & 6.051 & 1076.1 & 0.0230 & 10.828 & 1183.7 & 0.0234 & 15.870 & 1155.0 & 0.0234 \\
\hline 11 & 6.013 & 1073.2 & 0.0229 & 10.575 & 1166.0 & 0.0233 & 15.505 & 1142.3 & 0.0233 \\
\hline 12 & 6.007 & 1072.2 & 0.0228 & 10.583 & 1172.7 & 0.0232 & 15.533 & 1143.6 & 0.0232 \\
\hline 13 & 6.033 & 1073.1 & 0.0227 & 10.817 & 1203.1 & 0.0230 & 15.913 & 1158.0 & 0.0230 \\
\hline 14 & 6.061 & 1074.4 & 0.0226 & 11.278 & 1260.9 & 0.0229 & 16.690 & 1192.0 & 0.0229 \\
\hline 15 & 6.013 & 1070.8 & 0.0225 & 11.727 & 1318.6 & 0.0227 & 17.499 & 1235.3 & 0.0228 \\
\hline 16 & 5.718 & 1049.8 & 0.0225 & 11.634 & 1343.5 & 0.0226 & 17.528 & 1257.5 & 0.0226 \\
\hline 17 & 4.836 & 984.4 & 0.0224 & 10.182 & 1286.9 & 0.0225 & 15.610 & 1227.7 & 0.0225 \\
\hline 18 & 2.901 & 828.8 & 0.0223 & 6.337 & 1076.4 & 0.0223 & 10.049 & 1071.5 & 0.0224 \\
\hline
\end{tabular}

\begin{tabular}{|c|c|c|c|c|c|c|c|c|c|}
\hline \multirow[b]{2}{*}{$\begin{array}{l}\text { Axial } \\
\text { Node }\end{array}$} & \multicolumn{3}{|c|}{ Datapoint (126.6 Cy4) } & \multicolumn{3}{|c|}{ Statepoint $60(0.0$ Cy5) } & \multicolumn{3}{|c|}{ Statepoint 61 (114.4 Cy5) } \\
\hline & $\begin{array}{c}\text { Burnup } \\
\text { (GWd/MTU) }\end{array}$ & $\begin{array}{c}\text { T-Fuel } \\
\left({ }^{\circ} \mathrm{F}\right) \\
\end{array}$ & $\begin{array}{c}\text { Spec. Vol. } \\
\left(\mathrm{ft}^{3} / \mathrm{lbm}\right)\end{array}$ & $\begin{array}{c}\text { Burnup } \\
\text { (GWd/MTU) }\end{array}$ & $\begin{array}{c}\text { T-Fuel } \\
\left({ }^{\circ} \mathrm{F}\right) \\
\end{array}$ & $\begin{array}{l}\text { Spec. Vol. } \\
\left(\mathrm{ft}^{3} / \mathrm{lbm}\right)\end{array}$ & $\begin{array}{c}\text { Burnup } \\
\text { (GWd/MTU) }\end{array}$ & $\begin{array}{c}\text { T-Fuel } \\
\left({ }^{\circ} \mathrm{F}\right)\end{array}$ & $\begin{array}{l}\text { Spec. Vol. } \\
\left(\mathrm{ft}^{3} / \mathrm{bm}\right)\end{array}$ \\
\hline & 126.6 Cy4 & $71.2 \mathrm{Cy} 4$ & 71.2 Cy 4 & BOC Cy 5 & 203.4 Cy 4 & 203.4 Cy4 & 114.4 Cy5 & 58.2 Cy5 & 58.2 Cy 5 \\
\hline 1 & 10.742 & 976.7 & 0.0247 & 13.826 & 989.8 & 0.0246 & 15.701 & 876.0 & 0.0230 \\
\hline 2 & 16.951 & 1122.0 & 0.0246 & 21.527 & 1090.8 & 0.0245 & 24.348 & 953.3 & 0.0230 \\
\hline 3 & 20.111 & 1175.6 & 0.0244 & 25.290 & 1115.9 & 0.0244 & 28.550 & 973.5 & 0.0229 \\
\hline 4 & 21.531 & 1188.2 & 0.0243 & 26.852 & 1112.8 & 0.0242 & 30.304 & 974.6 & 0.0228 \\
\hline 5 & 22.139 & 1187.7 & 0.0241 & 27.461 & 1103.5 & 0.0240 & 30.984 & 967.8 & 0.0227 \\
\hline 6 & 22.335 & 1182.2 & 0.0239 & 27.638 & 1095.7 & 0.0239 & 31.152 & 956.2 & 0.0226 \\
\hline 7 & 22.255 & 1173.3 & 0.0237 & 27.548 & 1090.8 & 0.0237 & 30.980 & 936.4 & 0.0225 \\
\hline 8 & 21.859 & 1156.2 & 0.0236 & 27.122 & 1086.0 & 0.0236 & 30.411 & 913.7 & 0.0224 \\
\hline 9 & 21.089 & 1131.2 & 0.0234 & 26.225 & 1076.1 & 0.0234 & 29.405 & 914.1 & 0.0223 \\
\hline 10 & 20.262 & 1116.5 & 0.0233 & 25.193 & 1064.0 & 0.0233 & 28.379 & 931.9 & 0.0222 \\
\hline 11 & 19.916 & 1110.2 & 0.0232 & 24.732 & 1057.5 & 0.0232 & 27.984 & 952.5 & 0.0222 \\
\hline 12 & 20.031 & 1106.8 & 0.0230 & 24.809 & 1054.6 & 0.0230 & 28.165 & 976.1 & 0.0221 \\
\hline 13 & 20.541 & 1108.8 & 0.0229 & 25.351 & 1055.6 & 0.0229 & 28.812 & 993.3 & 0.0220 \\
\hline 14 & 21.377 & 1112.9 & 0.0228 & 26.315 & 1063.2 & 0.0228 & 29.792 & 984.8 & 0.0219 \\
\hline 15 & 22.014 & 1094.0 & 0.0226 & 27.043 & 1065.9 & 0.0227 & 30.409 & 961.4 & 0.0218 \\
\hline 16 & 21.671 & 1058.4 & 0.0225 & 26.603 & 1057.9 & 0.0225 & 29.751 & 935.2 & 0.0217 \\
\hline 17 & 19.136 & 1008.4 & 0.0224 & 23.605 & 1031.7 & 0.0224 & 26.338 & 900.8 & 0.0216 \\
\hline 18 & 12.338 & 892.7 & 0.0223 & 15.454 & 941.3 & 0.0223 & 17.276 & 822.6 & 0.0216 \\
\hline
\end{tabular}


Table 4-6. Burnup and TH Feedback Parameters by Axial Node for Assembly B29

\begin{tabular}{|c|c|c|c|c|c|c|c|c|c|}
\hline \multirow[b]{2}{*}{$\begin{array}{l}\text { Axial } \\
\text { Node }\end{array}$} & \multicolumn{3}{|c|}{ Datapoint (257.0 Cy2) } & \multicolumn{3}{|c|}{ Datapoint (142.8 Cy3) } & \multicolumn{3}{|c|}{ Datapoint (287.5 Cy3) } \\
\hline & $\begin{array}{c}\text { Burnup } \\
\text { (GWd/MTU) }\end{array}$ & $\begin{array}{c}\text { T-Fuel } \\
\left({ }^{\circ} \mathrm{F}\right)\end{array}$ & $\begin{array}{c}\text { Spec. Vol. } \\
\left(\mathrm{ft}^{3} / \mathrm{lbm}\right)\end{array}$ & $\begin{array}{c}\text { Burnup } \\
\text { (GWd/MTU) }\end{array}$ & $\begin{array}{c}\text { T-Fuel } \\
\left({ }^{\circ} \mathrm{F}\right)\end{array}$ & $\begin{array}{l}\text { Spec. Vol. } \\
\left(\mathrm{ft}^{3} / \mathrm{lbm}\right)\end{array}$ & $\begin{array}{c}\text { Burnup } \\
\text { (GWd/MTU) }\end{array}$ & $\begin{array}{c}\text { T-Fuel } \\
\left({ }^{\circ} \mathrm{F}\right)\end{array}$ & $\begin{array}{c}\text { Spec. Vol. } \\
\left(\mathrm{ft}^{3} / \mathrm{lbm}\right)\end{array}$ \\
\hline & EOC Cy2 & $131.6 \mathrm{Cy} 2$ & $131.6 \mathrm{Cy} 2$ & 142.8 Cy3 & 69.0 Cy 3 & 69.0 Cy3 & EOC Cy3 & 209.4 Cy3 & $209.4 \mathrm{Cy} 3$ \\
\hline 1 & 2.490 & 820.8 & 0.0239 & 5.521 & 1088.1 & 0.0252 & 8.927 & 1075.1 & 0.0251 \\
\hline 2 & 4.195 & 955.5 & 0.0238 & 9.033 & 1285.6 & 0.0250 & 14.048 & 1215.4 & 0.0249 \\
\hline 3 & 5.278 & 1033.1 & 0.0237 & 11.056 & 1367.8 & 0.0248 & 16.687 & 1260.6 & 0.0247 \\
\hline 4 & 5.985 & 1079.8 & 0.0236 & 12.161 & 1386.3 & 0.0246 & 17.941 & 1261.0 & 0.0245 \\
\hline 5 & 6.387 & 1104.5 & 0.0235 & 12.696 & 1379.9 & 0.0244 & 18.493 & 1251.1 & 0.0243 \\
\hline 6 & 6.564 & 1114.1 & 0.0234 & 12.890 & 1367.7 & 0.0242 & 18.685 & 1242.9 & 0.0242 \\
\hline 7 & 6.624 & 1116.6 & 0.0233 & 12.892 & 1350.2 & 0.0240 & 18.674 & 1237.2 & 0.0240 \\
\hline 8 & 6.628 & 1116.1 & 0.0232 & 12.731 & 1322.5 & 0.0238 & 18.453 & 1229.7 & 0.0238 \\
\hline 9 & 6.596 & 1113.8 & 0.0231 & 12.375 & 1282.2 & 0.0237 & 17.946 & 1214.2 & 0.0236 \\
\hline 10 & 6.548 & 1110.6 & 0.0230 & 11.944 & 1244.3 & 0.0235 & 17.330 & 1193.5 & 0.0235 \\
\hline 11 & 6.510 & 1107.7 & 0.0229 & 11.714 & 1229.3 & 0.0234 & 17.008 & 1183.0 & 0.0233 \\
\hline 12 & 6.505 & 1106.7 & 0.0228 & 11.732 & 1237.4 & 0.0232 & 17.050 & 1184.1 & 0.0232 \\
\hline 13 & 6.532 & 1107.6 & 0.0227 & 11.964 & 1267.2 & 0.0231 & 17.419 & 1196.3 & 0.0231 \\
\hline 14 & 6.559 & 1108.7 & 0.0226 & 12.362 & 1315.6 & 0.0229 & 18.071 & 1221.2 & 0.02 .29 \\
\hline 15 & 6.509 & 1105.1 & 0.0226 & 12.674 & 1356.8 & 0.0228 & 18.629 & 1249.1 & 0.0228 \\
\hline 16 & 6.207 & 1084.5 & 0.0225 & 12.417 & 1363.4 & 0.0226 & 18.405 & 1260.9 & 0.0226 \\
\hline 17 & 5.286 & 1019.1 & 0.0224 & 10.837 & 1299.7 & 0.0225 & 16.340 & 1229.5 & 0.0225 \\
\hline 18 & 3.209 & 854.2 & 0.0223 & 6.783 & 1087.7 & 0.0224 & 10.565 & 1074.9 & 0.0224 \\
\hline
\end{tabular}

\begin{tabular}{|c|c|c|c|c|c|c|c|c|c|}
\hline \multirow[b]{2}{*}{$\begin{array}{l}\text { Axial } \\
\text { Node }\end{array}$} & \multicolumn{3}{|c|}{ Datapoint (126.6 Cy4) } & \multicolumn{3}{|c|}{ Statepoint $60(0.0$ Cy5 $)$} & \multicolumn{3}{|c|}{ Statepoint 61 (114.4 Cy5) } \\
\hline & $\begin{array}{c}\text { Burnup } \\
\text { (GWd/MTU) }\end{array}$ & $\begin{array}{c}\text { T-Fuel } \\
\left({ }^{\circ} \mathrm{F}\right)\end{array}$ & $\begin{array}{c}\text { Spec. Vol. } \\
\left(\mathrm{ft}^{3} / \mathrm{lbm}\right)\end{array}$ & $\begin{array}{c}\text { Burnup } \\
\text { (GWd/MTU) }\end{array}$ & $\begin{array}{c}\text { T-Fuel } \\
\left({ }^{\circ} \mathrm{F}\right)\end{array}$ & $\begin{array}{c}\text { Spec. Vol. } \\
\left(\mathrm{ft}^{3} / \mathrm{lbm}\right)\end{array}$ & $\begin{array}{c}\text { Burnup } \\
\text { (GWd/MTU) }\end{array}$ & $\begin{array}{c}\text { T-Fuel } \\
\left({ }^{\circ} \mathrm{F}\right) \\
\end{array}$ & $\begin{array}{c}\text { Spec. Vol. } \\
\left(\mathrm{ft}^{3} / \mathrm{bm}\right)\end{array}$ \\
\hline & $126.6 \mathrm{Cy} 4$ & $71.2 \mathrm{Cy} 4$ & $71.2 \mathrm{Cy} 4$ & BOC Cy 5 & 203.4 Cy 4 & $203.4 \mathrm{Cy} 4$ & 114.4 Cy5 & 58.2 Cy5 & 58.2 Cy 5 \\
\hline 1 & 10.033 & 788.9 & 0.0241 & 11.754 & 844.1 & 0.0242 & 14.040 & 947.2 & 0.0232 \\
\hline 2 & 16.385 & 961.4 & 0.0241 & 19.833 & 987.9 & 0.0242 & 23.105 & 1018.0 & 0.0231 \\
\hline 3 & 19.79 & 1014.5 & 0.0240 & 23.880 & 1017.2 & 0.0240 & 27.519 & 1025.8 & 0.0230 \\
\hline 4 & 21.372 & 1027.9 & 0.0238 & 25.623 & 1016.7 & 0.0239 & 29.375 & 1014.7 & 0.0229 \\
\hline 5 & 22.05 & 1031.3 & 0.0237 & 26.338 & 1011.4 & 0.0238 & 30.097 & 999.2 & 0.0228 \\
\hline 6 & 22.288 & 1031.0 & 0.0236 & 26.584 & 1006.8 & 0.0237 & 30.304 & 983.3 & 0.0227 \\
\hline 7 & 22.293 & 1029.7 & 0.0235 & 26.604 & 1004.7 & 0.0235 & 30.260 & 968.3 & 0.0226 \\
\hline 8 & 22.089 & 1029.9 & 0.0234 & 26.432 & 1006.0 & 0.0234 & 30.026 & 958.6 & 0.0225 \\
\hline 9 & 21.639 & 1035.1 & 0.0232 & 26.040 & 1011.7 & 0.0233 & 29.603 & 959.6 & 0.0224 \\
\hline 10 & 21.13 & 1045.0 & 0.0231 & 25.610 & 1020.6 & 0.0232 & 29.180 & 969.3 & 0.0223 \\
\hline 11 & 20.943 & 1054.9 & 0.0230 & 25.505 & 1028.4 & 0.0231 & 29.091 & 979.8 & 0.0222 \\
\hline 12 & 21.094 & 1059.9 & 0.0229 & 25.720 & 1032.9 & 0.0230 & 29.314 & 987.2 & 0.0221 \\
\hline 13 & 21.462 & 1053.2 & 0.0228 & 26.097 & 1031.2 & 0.0228 & 29.678 & 988.2 & 0.0220 \\
\hline 14 & 21.966 & 1032.0 & 0.0227 & 26.541 & 1022.6 & 0.0227 & 30.075 & 980.7 & 0.0219 \\
\hline 15 & 22.24 & 999.2 & 0.0226 & 26.684 & 1009.0 & 0.0226 & 30.135 & 968.7 & 0.0218 \\
\hline 16 & 21.607 & 958.4 & 0.0225 & 25.801 & 990.3 & 0.0225 & 29.114 & 956.2 & 0.0217 \\
\hline 17 & 18.958 & 906.2 & 0.0224 & 22.625 & 957.9 & 0.0224 & 25.595 & 932.4 & 0.0217 \\
\hline 18 & 12.214 & 808.7 & 0.0223 & 14.691 & 871.7 & 0.0223 & 16.729 & 854.6 & 0.0216 \\
\hline
\end{tabular}


Table 4-7. Burnup and TH Feedback Parameters by Axial Node for Assembly C8

\begin{tabular}{|c|c|c|c|c|c|c|c|c|c|}
\hline \multirow[b]{2}{*}{$\begin{array}{l}\text { Axial } \\
\text { Node }\end{array}$} & \multicolumn{3}{|c|}{ Datapoint (142.8 Cy3) } & \multicolumn{3}{|c|}{ Datapoint (287.5 Cy3) } & \multicolumn{3}{|c|}{ Datapoint (126.6 Cy4) } \\
\hline & \begin{tabular}{|c|} 
Burnup \\
(GWd/MTU)
\end{tabular} & $\begin{array}{l}\text { T-Fuel } \\
\left({ }^{\circ} \mathrm{F}\right)\end{array}$ & $\begin{array}{l}\text { Spec. Vol. } \\
\left(\mathrm{ft}^{3} / \mathrm{lbm}\right)\end{array}$ & \begin{tabular}{|c|} 
Burnup \\
(GWd/MTU)
\end{tabular} & $\begin{array}{c}\text { T-Fuel } \\
\left({ }^{\circ} \mathrm{F}\right)\end{array}$ & $\begin{array}{c}\text { Spec. Vol. } \\
\left(\mathrm{ft}^{3} / \mathrm{lbm}\right)\end{array}$ & $\begin{array}{c}\text { Burnup } \\
\text { (GWd/MTU) }\end{array}$ & $\begin{array}{c}\text { T-Fuel } \\
\left({ }^{\circ} \mathrm{F}\right)\end{array}$ & $\begin{array}{c}\text { Spec. Vol. } \\
\left(\mathrm{ft}^{3} / \mathrm{lbm}\right)\end{array}$ \\
\hline & 142.8 Су 3 & 69.0 Cy 3 & $69.0 \mathrm{Cy3}$ & EOC Cy3 & $209.4 \mathrm{Cy} 3$ & 209.4 Су3 & 126.6 Cy4 & 71.2 Cy 4 & 71.2 Cy 4 \\
\hline 1 & 1.448 & 841.6 & 0.0240 & 3.243 & 888.1 & 0.0241 & 5.837 & 1069.4 & 0.0251 \\
\hline 2 & 2.482 & 998.5 & 0.0239 & 5.364 & 1034.0 & 0.0240 & 9.455 & 1242.4 & 0.0250 \\
\hline 3 & 3.110 & 1083.9 & 0.0239 & 6.549 & 1094.6 & 0.0239 & 11.389 & 1313.1 & 0.0248 \\
\hline 4 & 3.452 & 1125.2 & 0.0238 & 7.174 & 1115.2 & 0.0238 & 12.335 & 1335.4 & 0.0246 \\
\hline 5 & 3.633 & 1143.3 & 0.0236 & 7.537 & 1120.4 & 0.0237 & 12.814 & 1336.8 & 0.0244 \\
\hline 6 & 3.722 & 1149.3 & 0.0235 & 7.741 & 1121.2 & 0.0236 & 13.035 & 1331.2 & 0.0242 \\
\hline 7 & 3.761 & 1149.2 & 0.0234 & 7.853 & 1121.4 & 0.0235 & 13.108 & 1322.4 & 0.0240 \\
\hline 8 & 3.772 & 1146.8 & 0.0233 & 7.92 & 1122.1 & 0.0234 & 13.118 & 1311.3 & 0.0238 \\
\hline 9 & 3.771 & 1144.7 & 0.0232 & 7.972 & 1123.4 & 0.0233 & 13.168 & 1302.8 & 0.0236 \\
\hline 10 & 3.776 & 1145.1 & 0.0231 & 8.028 & 1125.4 & 0.0232 & 13.327 & 1302.2 & 0.0235 \\
\hline 11 & 3.803 & 1149.7 & 0.0230 & 8.106 & 1128.3 & 0.0231 & 13.565 & 1306.7 & 0.0233 \\
\hline 12 & 3.855 & 1158.8 & 0.0229 & 8.208 & 1132.5 & 0.0230 & 13.829 & 1312.5 & 0.0231 \\
\hline 13 & 3.930 & 1171.6 & 0.0228 & 8.332 & 1139.4 & 0.0229 & 14.013 & 1311.7 & 0.0230 \\
\hline 14 & 4.033 & 1189.3 & 0.0227 & 8.504 & 1153.5 & 0.0227 & 14.047 & 1295.4 & 0.0228 \\
\hline 15 & 4.173 & 1213.2 & 0.0226 & 8.746 & 1177.0 & 0.0226 & 13.942 & 1257.8 & 0.0227 \\
\hline 16 & 4.200 & 1219.3 & 0.0225 & 8.741 & 1185.2 & 0.0225 & 13.418 & 1201.3 & 0.0225 \\
\hline 17 & 3.631 & 1138.4 & 0.0224 & 7.626 & 1134.0 & 0.0224 & 11.520 & 1120.8 & 0.0224 \\
\hline 18 & 2.161 & 920.5 & 0.0223 & 4.683 & 958.9 & 0.0223 & 7.126 & 961.2 & 0.0223 \\
\hline
\end{tabular}

\begin{tabular}{|c|c|c|c|c|c|c|}
\hline \multirow[b]{2}{*}{$\begin{array}{l}\text { Axlal } \\
\text { Node }\end{array}$} & \multicolumn{3}{|c|}{ Statepoint $60(0.0$ Cy5 $)$} & \multicolumn{3}{|c|}{ Statepoint 61 (114.4 Cy5) } \\
\hline & $\begin{array}{c}\text { Burnup } \\
\text { (GWd/MTU) }\end{array}$ & $\begin{array}{l}\text { T-Fuel } \\
\left({ }^{\circ} \mathrm{F}\right)\end{array}$ & $\begin{array}{c}\text { Spec. Vol. } \\
\left(\mathrm{tt}^{3} / \mathrm{lbm}\right)\end{array}$ & $\begin{array}{c}\text { Burnup } \\
\text { (GWd/MTU) }\end{array}$ & $\begin{array}{c}\text { T-Fuel } \\
\left({ }^{\circ} \mathrm{F}\right)\end{array}$ & $\begin{array}{l}\text { Spec. Vol. } \\
\left(\mathrm{ft}^{3} / \mathrm{lbm}\right)\end{array}$ \\
\hline & BOC Cy5 & 203.4 Cy 4 & 203.4 Cy 4 & 114.4 Cy5 & $58.2 \mathrm{Cy} 5$ & 58.2 Cy5 \\
\hline 1 & 9.231 & 1068.6 & 0.0250 & 11.220 & 930.2 & 0.0233 \\
\hline 2 & 14.442 & 1201.0 & 0.0249 & 17.528 & 1050.0 & 0.0232 \\
\hline 3 & 17.009 & 1239.6 & 0.0247 & 20.648 & 1082.1 & 0.0231 \\
\hline 4 & 18.120 & 1239.3 & 0.0245 & 21.990 & 1083.8 & 0.0230 \\
\hline 5 & 18.610 & 1229.6 & 0.0243 & 22.552 & 1074.8 & 0.0228 \\
\hline 6 & 18.817 & 1220.7 & 0.0241 & 22.754 & 1061.7 & 0.0227 \\
\hline 7 & 18.887 & 1215.0 & 0.0240 & 22.779 & 1047.8 & 0.0226 \\
\hline 8 & 18.904 & 1211.3 & 0.0238 & 22.744 & 1037.7 & 0.0225 \\
\hline 9 & 18.966 & 1209.1 & 0.0236 & 22.781 & 1036.9 & 0.0224 \\
\hline 10 & 19.141 & 1208.1 & 0.0235 & 22.967 & 1044.1 & 0.0223 \\
\hline 11 & 19.408 & 1208.7 & 0.0233 & 23.253 & 1053.6 & 0.0222 \\
\hline 12 & 19.716 & 1210.8 & 0.0232 & 23.573 & 1061.2 & 0.0221 \\
\hline 13 & 19.928 & 1212.5 & 0.0230 & 23.772 & 1063.6 & 0.0220 \\
\hline 14 & 19.948 & 1211.6 & 0.0229 & 23.736 & 1057.5 & 0.0219 \\
\hline 15 & 19.760 & 1205.0 & 0.0227 & 23.435 & 1043.2 & 0.0218 \\
\hline 16 & 19.014 & 1188.9 & 0.0226 & 22.492 & 1022.6 & 0.0217 \\
\hline 17 & 16.515 & 1147.6 & 0.0224 & 19.576 & 986.3 & 0.0217 \\
\hline 18 & 10.509 & 1008.9 & 0.0223 & 12.542 & 877.0 & 0.0216 \\
\hline
\end{tabular}


Table 4-8. Burnup and TH Feedback Parameters by Axial Node for Assembly C15

\begin{tabular}{|c|c|c|c|c|c|c|c|c|c|}
\hline \multirow[b]{2}{*}{$\begin{array}{l}\text { Axial } \\
\text { Node }\end{array}$} & \multicolumn{3}{|c|}{ Datapoint (142.8 Cy3) } & \multicolumn{3}{|c|}{ Datapoint (287.5 Cy3) } & \multicolumn{3}{|c|}{ Datapoint (126.6 Cy4) } \\
\hline & $\begin{array}{c}\text { Burnup } \\
\text { (GWd/MTU) } \\
\end{array}$ & $\begin{array}{c}\text { T-Fuel } \\
\left({ }^{\circ} \mathrm{F}\right)\end{array}$ & $\begin{array}{l}\text { Spec. Vol. } \\
\left(\mathrm{ft}^{3} / \mathrm{lbm}\right)\end{array}$ & $\begin{array}{c}\text { Burnup } \\
\text { (GWd/MTU) }\end{array}$ & $\begin{array}{c}\text { T-Fuel } \\
\left({ }^{\circ} \mathrm{F}\right)\end{array}$ & $\begin{array}{c}\text { Spec. Vol. } \\
\left(\mathrm{ft}^{3} / \mathrm{lbm}\right)\end{array}$ & $\begin{array}{c}\text { Burnup } \\
\text { (GWd/MTU) }\end{array}$ & $\begin{array}{c}\text { T-Fuel } \\
\left({ }^{\circ} \mathrm{F}\right)\end{array}$ & $\begin{array}{c}\text { Spec. Vol. } \\
\left(\mathrm{ft}^{3} / \mathrm{bm}\right)\end{array}$ \\
\hline & $142.8 \mathrm{Cy} 3$ & 69.0 Cy3 & $69.0 \mathrm{Cy} 3$ & EOC Cy 3 & 209.4 Cy3 & 209.4 Cy 3 & $126.6 \mathrm{Cy} 4$ & 71.2 Cy4 & $71.2 \mathrm{Cy} 4$ \\
\hline 1 & 1.575 & 863.7 & 0.0242 & 3.506 & 909.0 & 0.0242 & 6.223 & 1080.1 & 0.0251 \\
\hline 2 & 2.714 & 1033.8 & 0.0241 & 5.815 & 1060.6 & 0.0242 & 10.072 & 1253.6 & 0.0250 \\
\hline 3 & 3.412 & 1126.1 & 0.0240 & 7.108 & 1124.5 & 0.0240 & 12.134 & 1325.5 & 0.0248 \\
\hline 4 & 3.795 & 1172.0 & 0.0239 & 7.772 & 1145.4 & 0.0239 & 13.132 & 1346.5 & 0.0246 \\
\hline 5 & 3.997 & 1191.2 & 0.0238 & 8.130 & 1150.8 & 0.0238 & 13.616 & 1347.1 & 0.0244 \\
\hline 6 & 4.098 & 1197.5 & 0.0236 & 8.330 & 1151.8 & 0.0237 & 13.841 & 1340.6 & 0.0242 \\
\hline 7 & 4.143 & 1197.6 & 0.0235 & 8.443 & 1152.3 & 0.0236 & 13.933 & 1331.7 & 0.0240 \\
\hline 8 & 4.155 & 1195.0 & 0.0234 & 8.510 & 1153.2 & 0.0234 & 13.962 & 1321.2 & 0.0238 \\
\hline 9 & 4.151 & 1192.4 & 0.0233 & 8.557 & 1154.7 & 0.0233 & 13.981 & 1310.6 & 0.0236 \\
\hline 10 & 4.153 & 1192.5 & 0.0232 & 8.608 & 1156.7 & 0.0232 & 14.028 & 1301.2 & 0.0235 \\
\hline 11 & 4.178 & 1197.1 & 0.0231 & 8.682 & 1159.4 & 0.0231 & 14.117 & 1293.2 & 0.0233 \\
\hline 12 & 4.232 & 1206.7 & 0.0230 & 8.785 & 1163.4 & 0.0230 & 14.229 & 1285.5 & 0.0231 \\
\hline 13 & 4.313 & 1220.3 & 0.0228 & 8.912 & 1169.6 & 0.0229 & 14.318 & 1275.5 & 0.0230 \\
\hline 14 & 4.406 & 1236.3 & 0.0227 & 9.058 & 1180.0 & 0.0228 & 14.342 & 1259.8 & 0.0228 \\
\hline 15 & 4.471 & 1248.7 & 0.0226 & 9.158 & 1192.0 & 0.0226 & 14.213 & 1234.8 & 0.0227 \\
\hline 16 & 4.342 & 1233.3 & 0.0225 & 8.909 & 1188.2 & 0.0225 & 13.587 & 1196.4 & 0.0225 \\
\hline 17 & 3.673 & 1141.5 & 0.0224 & 7.662 & 1132.9 & 0.0224 & 11.649 & 1128.8 & 0.0224 \\
\hline 18 & 2.164 & 920.3 & 0.0223 & 4.672 & 956.7 & 0.0223 & 7.211 & 972.8 & 0.0223 \\
\hline
\end{tabular}

\begin{tabular}{|c|c|c|c|c|c|c|}
\hline \multirow{2}{*}{$\begin{array}{c}\text { Axial } \\
\text { Node }\end{array}$} & $\begin{array}{c}\text { Burnup } \\
(\text { GWd/MTU) }\end{array}$ & $\begin{array}{c}\text { T-Fuel } \\
\left({ }^{\circ} \mathrm{F}\right)\end{array}$ & $\begin{array}{c}\text { Spec. Vol. } \\
\left(\mathrm{ft}^{3} / \mathrm{lbm}\right)\end{array}$ & $\begin{array}{c}\text { Burnup } \\
(\mathrm{GWd} / \mathrm{MTU})\end{array}$ & $\begin{array}{c}\text { S.Fuel } \\
\left({ }^{\circ} \mathrm{F}\right)\end{array}$ & $\begin{array}{c}\text { Spec. Vol. } \\
\left(\mathrm{ft}^{3} / \mathrm{lbm}\right)\end{array}$ \\
\hline & BOC Cy5 & $203.4 \mathrm{Cy} 4$ & $203.4 \mathrm{Cy} 4$ & $114.4 \mathrm{Cy} 5$ & 58.2 Cy5 & $58.2 \mathrm{Cy5}$ \\
\hline 1 & 9.667 & 1068.9 & 0.0250 & 12.060 & 977.9 & 0.0234 \\
\hline 3 & 15.112 & 1199.7 & 0.0249 & 18.668 & 1091.3 & 0.0233 \\
\hline 4 & 17.801 & 1235.2 & 0.0247 & 21.810 & 1111.7 & 0.0231 \\
\hline 5 & 18.956 & 1233.3 & 0.0245 & 23.103 & 1104.3 & 0.0230 \\
\hline 6 & 19.445 & 1223.0 & 0.0243 & 23.606 & 1090.0 & 0.0229 \\
\hline 7 & 19.648 & 1213.5 & 0.0241 & 23.779 & 1075.4 & 0.0228 \\
\hline 8 & 19.723 & 1206.8 & 0.0239 & 23.815 & 1063.8 & 0.0227 \\
\hline 9 & 19.743 & 1202.3 & 0.0238 & 23.804 & 1057.0 & 0.0226 \\
\hline 10 & 19.751 & 1198.7 & 0.0236 & 23.795 & 1055.9 & 0.0225 \\
\hline 11 & 19.780 & 1195.4 & 0.0235 & 23.816 & 1058.5 & 0.0223 \\
\hline 12 & 19.852 & 1192.7 & 0.0233 & 23.872 & 1061.9 & 0.0222 \\
\hline 13 & 19.953 & 1190.9 & 0.0232 & 23.942 & 1063.3 & 0.0221 \\
\hline 14 & 20.040 & 1190.2 & 0.0230 & 23.981 & 1061.6 & 0.0220 \\
\hline 15 & 19.906 & 1190.1 & 0.0229 & 23.941 & 1056.5 & 0.0219 \\
\hline 16 & 19.140 & 1188.8 & 0.0227 & 23.701 & 1048.6 & 0.0218 \\
\hline 17 & 16.673 & 1148.9 & 0.0226 & 22.790 & 1036.6 & 0.0217 \\
\hline 18 & 10.647 & 1013.2 & 0.0225 & 19.940 & 1006.9 & 0.0217 \\
\hline
\end{tabular}


Table 4-9. Burnup and TH Feedback Parameters by Axial Node for Assembly C15a

\begin{tabular}{|c|c|c|c|c|c|c|c|c|c|}
\hline \multirow[b]{2}{*}{$\begin{array}{l}\text { Axial } \\
\text { Node } \\
\end{array}$} & \multicolumn{3}{|c|}{ Datapoint (142.8 Cy3) } & \multicolumn{3}{|c|}{ Datapoint (287.5 Cy3) } & \multicolumn{3}{|c|}{ Datapoint (126.6 Cy4) } \\
\hline & $\begin{array}{c}\text { Burnup } \\
\text { (GWd/MTU) }\end{array}$ & $\begin{array}{c}\text { T-Fuel } \\
\left({ }^{\circ} \mathrm{F}\right)\end{array}$ & $\begin{array}{c}\text { Spec. Vol. } \\
\left(\mathrm{ft}^{3} / \mathrm{lbm}\right)\end{array}$ & \begin{tabular}{|c|} 
Burnup \\
(GWd/MTU) \\
\end{tabular} & $\begin{array}{c}\text { T-Fuel } \\
\left({ }^{\circ} \mathrm{F}\right)\end{array}$ & $\begin{array}{l}\text { Spec. Vol. } \\
\left(\mathrm{ft}^{3} / \mathrm{lbm}\right)\end{array}$ & $\begin{array}{c}\text { Burnup } \\
\text { (GWd/MTU) }\end{array}$ & $\begin{array}{c}\text { T-Fuel } \\
\left({ }^{\circ} \mathrm{F}\right)\end{array}$ & $\begin{array}{c}\text { Spec. Vol. } \\
\left(\mathrm{ft}^{3} / \mathrm{lbm}\right)\end{array}$ \\
\hline & $142.8 \mathrm{Cy} 3$ & $69.0 \mathrm{Cy} 3$ & $69.0 \mathrm{Cy3}$ & EOC Cy3 & 209.4 Cy3 & 209.4 Cy 3 & $126.6 \mathrm{Cy} 4$ & 71.2 Cy4 & 71.2 Cy 4 \\
\hline 1 & 1.575 & 863.7 & 0.0242 & 3.506 & 909.0 & 0.0242 & 6.192 & 1076.3 & 0.0252 \\
\hline 2 & 2.714 & 1033.8 & 0.0241 & 5.815 & 1060.6 & 0.0242 & 10.051 & 1252.2 & 0.0250 \\
\hline 3 & 3.412 & 1126.1 & 0.0240 & 7.108 & 1124.5 & 0.0240 & 12.138 & 1326.5 & 0.0248 \\
\hline 4 & 3.795 & 1172.0 & 0.0239 & 7.772 & 1145.4 & 0.0239 & 13.153 & 1348.5 & 0.0246 \\
\hline 5 & 3.997 & 1191.2 & 0.0238 & 8.130 & 1150.8 & 0.0238 & 13.646 & 1349.1 & 0.0244 \\
\hline 6 & 4.098 & 1197.5 & 0.0236 & 8.330 & 1151.8 & 0.0237 & 13.880 & 1343.2 & 0.0242 \\
\hline 7 & 4.143 & 1197.6 & 0.0235 & 8.443 & 1152.3 & 0.0236 & 13.980 & 1335.0 & 0.0240 \\
\hline 8 & 4.155 & 1195.0 & 0.0234 & 8.510 & 1153.2 & 0.0234 & 14.016 & 1325.5 & 0.0238 \\
\hline 9 & 4.151 & 1192.4 & 0.0233 & 8.557 & 1154.7 & 0.0233 & 14.037 & 1315.2 & 0.0236 \\
\hline 10 & 4.153 & 1192.5 & 0.0232 & 8.608 & 1156.7 & 0.0232 & 14.079 & 1305.8 & 0.0235 \\
\hline 11 & 4.178 & 1197.1 & 0.0231 & 8.682 & 1159.4 & 0.0231 & 14.165 & 1298.1 & 0.0233 \\
\hline 12 & 4.232 & 1206.7 & 0.0230 & 8.785 & 1163.4 & 0.0230 & 14.276 & 1291.0 & 0.0231 \\
\hline 13 & 4.313 & 1220.3 & 0.0228 & 8.912 & 1169.6 & 0.0229 & 14.363 & 1280.9 & 0.0230 \\
\hline 14 & 4.406 & 1236.3 & 0.0227 & 9.058 & 1180.0 & 0.0228 & 14.387 & 1264.9 & 0.0228 \\
\hline 15 & 4.471 & 1248.7 & 0.0226 & 9.158 & 1192.0 & 0.0226 & 14.258 & 1239.7 & 0.0227 \\
\hline 16 & 4.342 & 1233.3 & 0.0225 & 8.909 & 1188.2 & 0.0225 & 13.627 & 1200.7 & 0.0225 \\
\hline 17 & 3.673 & 1141.5 & 0.0224 & 7.662 & 1132.9 & 0.0224 & 11.680 & 1132.1 & 0.0224 \\
\hline 18 & 2.164 & 920.3 & 0.0223 & 4.672 & 956.7 & 0.0223 & 7.229 & 975.1 & 0.0223 \\
\hline
\end{tabular}

\begin{tabular}{|c|c|c|c|c|c|c|}
\hline \multirow[b]{2}{*}{$\begin{array}{l}\text { Axial } \\
\text { Node }\end{array}$} & \multicolumn{3}{|c|}{ Statepoint $60(0.0$ Cy5) } & \multicolumn{3}{|c|}{ Statepoint 61 (114.4 Cy5) } \\
\hline & $\begin{array}{c}\text { Burnup } \\
\text { (GWd/MTU) }\end{array}$ & $\begin{array}{c}\text { T-Fuel } \\
\left({ }^{\circ} \mathrm{F}\right)\end{array}$ & $\begin{array}{c}\text { Spec. Vol. } \\
\left(\mathrm{ft}^{3} / \mathrm{lbm}\right)\end{array}$ & $\begin{array}{c}\text { Burnup } \\
\text { (GWd/MTU) }\end{array}$ & $\begin{array}{c}\text { T-Fuel } \\
\left({ }^{\circ} \mathrm{F}\right)\end{array}$ & $\begin{array}{c}\text { Spec. Vol. } \\
\left(\mathrm{tt}^{3} / \mathrm{lbm}\right)\end{array}$ \\
\hline & BOC Cy5 & 203.4 Cy 4 & 203.4 Cy 4 & 114.4 Cy 5 & 58.2 Cy5 & 58.2 Cy5 \\
\hline 1 & 9.598 & 1065.8 & 0.0250 & 11.717 & 952.1 & 0.0233 \\
\hline 2 & 15.067 & 1197.8 & 0.0249 & 18.318 & 1069.7 & 0.0232 \\
\hline 3 & 17.797 & 1234.5 & 0.0247 & 21.603 & 1097.8 & 0.0231 \\
\hline 4 & 18.976 & 1233.0 & 0.0245 & 23.013 & 1095.6 & 0.0230 \\
\hline 5 & 19.478 & 1222.9 & 0.0243 & 23.572 & 1082.8 & 0.0228 \\
\hline 6 & 19.690 & 1213.6 & 0.0241 & 23.739 & 1062.8 & 0.0227 \\
\hline 7 & 19.774 & 1206.9 & 0.0240 & 23.689 & 1032.8 & 0.0226 \\
\hline 8 & 19.801 & 1202.4 & 0.0238 & 23.522 & 1001.1 & 0.0225 \\
\hline 9 & 19.810 & 1198.9 & 0.0236 & 23.379 & 991.0 & 0.0224 \\
\hline 10 & 19.839 & 1196.0 & 0.0235 & 23.374 & 1001.1 & 0.0223 \\
\hline 11 & 19.917 & 1194.0 & 0.0233 & 23.502 & 1021.1 & 0.0222 \\
\hline 12 & 20.031 & 1193.1 & 0.0232 & 23.732 & 1049.1 & 0.0222 \\
\hline 13 & 20.123 & 1192.9 & 0.0230 & 23.955 & 1073.3 & 0.0220 \\
\hline 14 & 20.147 & 1193.0 & 0.0229 & 24.032 & 1075.7 & 0.0219 \\
\hline 15 & 19.988 & 1191.5 & 0.0227 & 23.819 & 1063.0 & 0.0218 \\
\hline 16 & 19.211 & 1184.1 & 0.0226 & 22.882 & 1044.7 & 0.0217 \\
\hline 17 & 16.726 & 1150.2 & 0.0225 & 19.992 & 1010.0 & 0.0217 \\
\hline 18 & 10.679 & 1014.4 & 0.0223 & 12.875 & 900.0 & 0.0216 \\
\hline
\end{tabular}


Table 4-10. Burnup and TH Feedback Parameters by Axial Node for Assembly C20

\begin{tabular}{|c|c|c|c|c|c|c|c|c|c|}
\hline \multirow{2}{*}{$\begin{array}{c}\text { Axial } \\
\text { Node }\end{array}$} & \multicolumn{2}{|c|}{ Datapoint (142.8 Cy3) } & \multicolumn{2}{c|}{ Datapoint (287.5 Cy3) } & \multicolumn{3}{c|}{ Datapoint (126.6 Cy4) } \\
\hline & $\begin{array}{c}\text { Burnup } \\
\text { (GW/MTU) }\end{array}$ & $\begin{array}{c}\text { T-Fuel } \\
\left({ }^{\circ} \mathrm{F}\right)\end{array}$ & $\begin{array}{c}\text { Spec. Vol. } \\
\left(\mathrm{ft}^{3} / \mathrm{lbm}\right)\end{array}$ & $\begin{array}{c}\text { Burnup } \\
(\mathrm{GWd} / \mathrm{MTU})\end{array}$ & $\begin{array}{c}\text { T-Fuel } \\
\left({ }^{\circ} \mathrm{F}\right)\end{array}$ & $\begin{array}{c}\text { Spec. Vol. } \\
\left(\mathrm{ft}^{3} / \mathrm{lbm}\right)\end{array}$ & $\begin{array}{c}\text { Burnup } \\
(\mathrm{GWd} / \mathrm{MTU})\end{array}$ & $\begin{array}{c}\text { T-Fuel } \\
\left({ }^{\circ} \mathrm{F}\right)\end{array}$ & $\begin{array}{c}\text { Spec. Vol. } \\
\left(\mathrm{ft}^{3} / \mathrm{lbm}\right)\end{array}$ \\
\hline 1 & $2.8 \mathrm{Cy3}$ & $69.0 \mathrm{Cy3}$ & $69.0 \mathrm{Cy3}$ & EOC Cy3 & $209.4 \mathrm{Cy3}$ & $209.4 \mathrm{Cy3}$ & 126.6 Cy4 & $71.2 \mathrm{Cy} 4$ & $71.2 \mathrm{Cy} 4$ \\
\hline 2 & 4.171 & 1248.0 & 0.0251 & 8.673 & 1228.4 & 0.0250 & 12.752 & 1198.4 & 0.0248 \\
\hline 3 & 5.166 & 1367.3 & 0.0249 & 10.420 & 1297.0 & 0.0248 & 15.249 & 1265.0 & 0.0247 \\
\hline 4 & 5.688 & 1422.1 & 0.0247 & 11.250 & 1317.4 & 0.0247 & 16.405 & 1283.3 & 0.0245 \\
\hline 5 & 5.952 & 1443.3 & 0.0245 & 11.654 & 1321.0 & 0.0245 & 16.927 & 1283.4 & 0.0243 \\
\hline 6 & 6.078 & 1448.2 & 0.0243 & 11.865 & 1320.7 & 0.0243 & 17.148 & 1277.0 & 0.0241 \\
\hline 7 & 6.123 & 1445.0 & 0.0241 & 11.972 & 1320.7 & 0.0241 & 17.199 & 1267.1 & 0.0239 \\
\hline 8 & 6.104 & 1436.6 & 0.0239 & 12.001 & 1320.9 & 0.0239 & 17.123 & 1252.4 & 0.0237 \\
\hline 9 & 6.038 & 1426.0 & 0.0238 & 11.966 & 1320.5 & 0.0237 & 16.996 & 1235.1 & 0.0236 \\
\hline 10 & 5.970 & 1418.7 & 0.0236 & 11.924 & 1319.8 & 0.0236 & 16.945 & 1223.3 & 0.0234 \\
\hline 11 & 5.963 & 1421.4 & 0.0234 & 11.959 & 1321.3 & 0.0234 & 17.026 & 1216.4 & 0.0232 \\
\hline 12 & 6.038 & 1434.7 & 0.0233 & 12.097 & 1325.7 & 0.0232 & 17.227 & 1211.1 & 0.0231 \\
\hline 13 & 6.180 & 1455.9 & 0.0231 & 12.319 & 1333.1 & 0.0231 & 17.487 & 1206.8 & 0.0229 \\
\hline 14 & 6.344 & 1477.2 & 0.0229 & 12.567 & 1342.8 & 0.0229 & 17.668 & 1199.4 & 0.0228 \\
\hline 15 & 6.412 & 1486.7 & 0.0228 & 12.667 & 1349.9 & 0.0228 & 17.542 & 1178.4 & 0.0227 \\
\hline 16 & 6.156 & 1459.0 & 0.0226 & 12.240 & 1342.1 & 0.0226 & 16.732 & 1143.6 & 0.0225 \\
\hline 17 & 5.204 & 1341.2 & 0.0224 & 10.577 & 1280.7 & 0.0225 & 14.402 & 1082.2 & 0.0224 \\
\hline 18 & 3.110 & 1062.3 & 0.0223 & 6.581 & 1080.1 & 0.0224 & 9.037 & 942.7 & 0.0223 \\
\hline
\end{tabular}

\begin{tabular}{|c|c|c|c|c|c|c|}
\hline \multirow[b]{2}{*}{$\begin{array}{l}\text { Axial } \\
\text { Node }\end{array}$} & \multicolumn{3}{|c|}{ Statepoint 60 (0.0 Cy5) } & \multicolumn{3}{|c|}{ Statepoint 61 (114.4 Cy5) } \\
\hline & $\begin{array}{c}\text { Burnup } \\
\text { (GWd/MTU) }\end{array}$ & $\begin{array}{c}\text { T-Fuel } \\
\left({ }^{\circ} \mathrm{F}\right)\end{array}$ & $\begin{array}{c}\text { Spec. Vol. } \\
\left(\mathrm{tt}^{3} / \mathrm{lbm}\right)\end{array}$ & $\begin{array}{c}\text { Burnup } \\
\text { (GWd/MTU) }\end{array}$ & $\begin{array}{l}\text { T-Fuel } \\
\left({ }^{\circ} \mathrm{F}\right)\end{array}$ & $\begin{array}{c}\text { Spec. Vol. } \\
\left(\mathrm{ft}^{3} / \mathrm{lbm}\right)\end{array}$ \\
\hline & BOC Cy 5 & 203.4 Cy 4 & 203.4 Cy 4 & 114.4 Cy5 & 58.2 Cy5 & 58.2 Cy5 \\
\hline 1 & 11.333 & 1038.5 & 0.0249 & 13.590 & 963.6 & 0.0234 \\
\hline 2 & 17.654 & 1160.8 & 0.0247 & 21.094 & 1073.0 & 0.0233 \\
\hline 3 & 20.766 & 1188.1 & 0.0246 & 24.770 & 1099.1 & 0.0232 \\
\hline 4 & 22.073 & 1183.6 & 0.0244 & 26.283 & 1093.8 & 0.0231 \\
\hline 5 & 22.598 & 1173.6 & 0.0242 & 26.845 & 1079.5 & 0.0229 \\
\hline 6 & 22.798 & 1164.9 & 0.0240 & 27.014 & 1063.5 & 0.0228 \\
\hline 7 & 22.836 & 1158.9 & 0.0239 & 26.998 & 1048.9 & 0.0227 \\
\hline 8 & 22.745 & 1153.9 & 0.0237 & 26.859 & 1040.2 & 0.0226 \\
\hline 9 & 22.568 & 1147.5 & 0.0236 & 26.666 & 1041.2 & 0.0225 \\
\hline 10 & 22.437 & 1140.2 & 0.0234 & 26.545 & 1049.2 & 0.0224 \\
\hline 11 & 22.460 & 1135.0 & 0.0233 & 26.578 & 1058.3 & 0.0223 \\
\hline 12 & 22.634 & 1131.9 & 0.0231 & 26.747 & 1064.6 & 0.0222 \\
\hline 13 & 22.898 & 1131.3 & 0.0230 & 26.979 & 1064.6 & 0.0221 \\
\hline 14 & 23.113 & 1133.7 & 0.0228 & 27.131 & 1057.4 & 0.0220 \\
\hline 15 & 22.999 & 1135.3 & 0.0227 & 26.927 & 1047.2 & 0.0218 \\
\hline 16 & 22.077 & 1130.8 & 0.0226 & 25.853 & 1034.1 & 0.0218 \\
\hline 17 & 19.250 & 1105.8 & 0.0224 & 22.631 & 1002.4 & 0.0217 \\
\hline 18 & 12.383 & 986.2 & 0.0223 & 14.671 & 902.3 & 0.0216 \\
\hline
\end{tabular}


Table 4-11. Burnup and TH Feedback Parameters by Axial Node for Assembly C21

\begin{tabular}{|c|c|c|c|c|c|c|c|c|c|}
\hline \multirow{2}{*}{$\begin{array}{c}\text { Axial } \\
\text { Node }\end{array}$} & \multicolumn{3}{|c|}{ Datapoint (142.8 Cy3) } & \multicolumn{2}{c|}{ Datapoint (287.5 Cy3) } & \multicolumn{3}{c|}{ Datapoint (126.6 Cy4) } \\
\cline { 2 - 10 } & $\begin{array}{c}\text { Burnup } \\
(\mathrm{GWd} / \mathrm{MTU})\end{array}$ & $\begin{array}{c}\text { T-Fuel } \\
\left({ }^{\circ} \mathrm{F}\right)\end{array}$ & $\begin{array}{c}\text { Spec. Vol. } \\
\left(\mathrm{ft}^{3} / \mathrm{lbm}\right)\end{array}$ & $\begin{array}{c}\text { Burnup } \\
(\mathrm{GWd} / \mathrm{MTU})\end{array}$ & $\begin{array}{c}\text { T-Fuel } \\
\left({ }^{\circ} \mathrm{F}\right)\end{array}$ & $\begin{array}{c}\text { Spec. Vol. } \\
\left(\mathrm{ft}^{3} / \mathrm{lbm}\right)\end{array}$ & $\begin{array}{c}\text { Burnup } \\
(\mathrm{GWd} / \mathrm{MTU})\end{array}$ & $\begin{array}{c}\text { T-Fuel } \\
\left({ }^{\circ} \mathrm{F}\right)\end{array}$ & $\begin{array}{c}\text { Spec. Vol. } \\
\left(\mathrm{ft}^{3} / \mathrm{lbm}\right)\end{array}$ \\
\hline 1 & $142.8 \mathrm{Cy3}$ & $69.0 \mathrm{Cy3}$ & $69.0 \mathrm{Cy3}$ & EOC Cy3 & $209.4 \mathrm{Cy3}$ & $209.4 \mathrm{Cy3}$ & $126.6 \mathrm{Cy} 4$ & $71.2 \mathrm{Cy4}$ & $71.2 \mathrm{Cy4}$ \\
\hline 2 & 1.352 & 823.2 & 0.0239 & 3.012 & 864.3 & 0.0239 & 5.665 & 1076.3 & 0.0248 \\
\hline 3 & 2.352 & 973.5 & 0.0238 & 5.044 & 1002.5 & 0.0239 & 9.271 & 1261.5 & 0.0246 \\
\hline 4 & 3.309 & 1098.6 & 0.0236 & 6.757 & 1081.3 & 0.0237 & 12.180 & 1369.6 & 0.0243 \\
\hline 5 & 3.484 & 1116.1 & 0.0235 & 7.043 & 1086.8 & 0.0236 & 12.613 & 1373.8 & 0.0241 \\
\hline 6 & 3.568 & 1121.5 & 0.0234 & 7.191 & 1087.8 & 0.0235 & 12.777 & 1369.7 & 0.0239 \\
\hline 7 & 3.601 & 1120.9 & 0.0233 & 7.271 & 1088.0 & 0.0234 & 12.713 & 1360.1 & 0.0237 \\
\hline 8 & 3.603 & 1117.4 & 0.0232 & 7.310 & 1088.4 & 0.0233 & 12.156 & 1322.5 & 0.0235 \\
\hline 9 & 3.587 & 1113.5 & 0.0231 & 7.327 & 1089.1 & 0.0232 & 10.975 & 1142.0 & 0.0234 \\
\hline 10 & 3.574 & 1111.8 & 0.0230 & 7.344 & 1090.1 & 0.0231 & 10.496 & 1048.7 & 0.0232 \\
\hline 11 & 3.583 & 1114.6 & 0.0229 & 7.386 & 1091.8 & 0.0230 & 10.526 & 1033.7 & 0.0231 \\
\hline 12 & 3.623 & 1122.4 & 0.0228 & 7.460 & 1094.7 & 0.0229 & 10.798 & 1032.8 & 0.0231 \\
\hline 13 & 3.688 & 1133.8 & 0.0227 & 7.560 & 1099.3 & 0.0228 & 11.576 & 1063.1 & 0.0230 \\
\hline 14 & 3.752 & 1145.1 & 0.0227 & 7.652 & 1105.1 & 0.0227 & 12.749 & 1255.4 & 0.0228 \\
\hline 15 & 3.752 & 1147.1 & 0.0226 & 7.641 & 1108.4 & 0.0226 & 12.832 & 1274.7 & 0.0227 \\
\hline 16 & 3.554 & 1120.9 & 0.0225 & 7.292 & 1096.7 & 0.0225 & 12.103 & 1237.4 & 0.0226 \\
\hline 17 & 2.940 & 1033.8 & 0.0224 & 6.165 & 1041.5 & 0.0224 & 10.222 & 1159.9 & 0.0224 \\
\hline 18 & 1.700 & 847.2 & 0.0223 & 3.696 & 883.7 & 0.0223 & 6.236 & 986.5 & 0.0223 \\
\hline
\end{tabular}

\begin{tabular}{|c|c|c|c|c|c|c|}
\hline \multirow[b]{2}{*}{$\begin{array}{l}\text { Axial } \\
\text { Node }\end{array}$} & \multicolumn{3}{|c|}{ Statepoint 60 (0.0 Cy5) } & \multicolumn{3}{|c|}{ Statepoint 61 (114.4 Cy5) } \\
\hline & $\begin{array}{c}\text { Burnup } \\
\text { (GWd/MTU) }\end{array}$ & $\begin{array}{l}\text { T-Fuel } \\
\left({ }^{\circ} \mathrm{F}\right)\end{array}$ & $\begin{array}{c}\text { Spec. Vol. } \\
\left(\mathrm{ft}^{3} / \mathrm{lbm}\right)\end{array}$ & $\begin{array}{c}\text { Burnup } \\
(\mathrm{GWd} / \mathrm{MTU})\end{array}$ & $\begin{array}{c}\text { T-Fuel } \\
\left({ }^{\circ} \mathrm{F}\right)\end{array}$ & $\begin{array}{l}\text { Spec. Vol } \\
\left(\mathrm{ft}^{3} / \mathrm{lbm}\right)\end{array}$ \\
\hline & BOC Cy5 & $203.4 \mathrm{Cy} 4$ & $203.4 \mathrm{Cy} 4$ & 114.4 Cy 5 & 58.2 Cy5 & 58.2 Cy 5 \\
\hline 1 & 9.126 & 1074.4 & 0.0247 & 11.368 & 980.5 & 0.0235 \\
\hline 2 & 14.390 & 1212.5 & 0.0246 & 17.874 & 1112.1 & 0.0234 \\
\hline 3 & 17.026 & 1253.5 & 0.0244 & 21.137 & 1144.5 & 0.0233 \\
\hline 4 & 18.160 & 1254.5 & 0.0242 & 22.519 & 1141.7 & 0.0231 \\
\hline 5 & 18.621 & 1246.0 & 0.0240 & 23.036 & 1127.5 & 0.0230 \\
\hline 6 & 18.782 & 1238.2 & 0.0239 & 23.150 & 1107.1 & 0.0229 \\
\hline 7 & 18.720 & 1233.3 & 0.0237 & 22.958 & 1077.4 & 0.0228 \\
\hline 8 & 18.106 & 1225.9 & 0.0235 & 22.177 & 1049.9 & 0.0227 \\
\hline 9 & 16.278 & 1153.4 & 0.0234 & 20.319 & 1069.1 & 0.0226 \\
\hline 10 & 14.321 & 1025.7 & 0.0233 & 18.499 & 1117.9 & 0.0224 \\
\hline 11 & 14.107 & 1004.4 & 0.0232 & 18.370 & 1147.4 & 0.0223 \\
\hline 12 & 14.351 & 1001.1 & 0.0231 & 18.744 & 1181.1 & 0.0222 \\
\hline 13 & 15.241 & 1010.7 & 0.0230 & 19.738 & 1198.4 & 0.0221 \\
\hline 14 & 17.449 & 1123.4 & 0.0229 & 21.822 & 1156.0 & 0.0220 \\
\hline 15 & 18.503 & 1206.2 & 0.0227 & 22.703 & 1118.2 & 0.0219 \\
\hline 16 & 17.815 & 1215.3 & 0.0226 & 21.836 & 1098.0 & 0.0218 \\
\hline 17 & 15.389 & 1177.9 & 0.0225 & 18.962 & 1059.8 & 0.0217 \\
\hline 18 & 9.736 & 1032.2 & 0.0223 & 12.114 & 932.8 & 0.0216 \\
\hline
\end{tabular}


Table 4-12. Burnup and TH Feedback Parameters by Axial Node for Assembly C25

\begin{tabular}{|c|c|c|c|c|c|c|c|c|c|}
\hline \multirow[b]{2}{*}{$\begin{array}{l}\text { Axial } \\
\text { Node } \\
\end{array}$} & \multicolumn{3}{|c|}{ Datapoint (142.8 Cy3) } & \multicolumn{3}{|c|}{ Datapoint (287.5 Cy3) } & \multicolumn{3}{|c|}{ Datapoint (126.6 Cy4) } \\
\hline & \begin{tabular}{|c|} 
Burnup \\
(GWd/MTU)
\end{tabular} & $\begin{array}{l}\text { T-Fuel } \\
\left({ }^{\circ} \mathrm{F}\right)\end{array}$ & $\begin{array}{l}\text { Spec. Vol. } \\
\left(\mathrm{ft}^{3} / \mathrm{lbm}\right)\end{array}$ & $\begin{array}{c}\text { Burnup } \\
\text { (GWd/MTU) }\end{array}$ & $\begin{array}{l}\text { T-Fuel } \\
\left({ }^{\circ} \mathrm{F}\right)\end{array}$ & $\begin{array}{l}\text { Spec. Vol. } \\
\left(\mathrm{ft}^{3} / \mathrm{lbm}\right)\end{array}$ & $\begin{array}{c}\text { Burnup } \\
\text { (GWd/MTU) }\end{array}$ & $\begin{array}{c}\text { T-Fuel } \\
\left({ }^{\circ} \mathrm{F}\right)\end{array}$ & $\begin{array}{c}\text { Spec. Vol. } \\
\left(\mathrm{ft}^{3} / \mathrm{lbm}\right)\end{array}$ \\
\hline & $142.8 \mathrm{Cy} 3$ & $69.0 \mathrm{Cy} 3$ & $69.0 \mathrm{Cy} 3$ & $\mathrm{EOC} \mathrm{Cy3}$ & 209.4 Cy3 & 209.4 Cy3 & $126.6 \mathrm{Cy} 4$ & $71.2 \mathrm{Cy4}$ & 71.2 Cy4 \\
\hline 1 & 1.917 & 919.0 & 0.0243 & 4.210 & 963.1 & 0.0244 & 6.635 & 1033.5 & 0.0250 \\
\hline 2 & 3.231 & 1109.4 & 0.0243 & 6.832 & 1122.7 & 0.0243 & 10.798 & 1213.4 & 0.0249 \\
\hline 3 & 3.980 & 1203.9 & 0.0241 & 8.187 & 1182.6 & 0.0242 & 13.026 & 1297.9 & 0.0247 \\
\hline 4 & 4.350 & 1244.2 & 0.0240 & 8.794 & 1199.6 & 0.0241 & 14.052 & 1328.5 & 0.0245 \\
\hline 5 & 4.520 & 1257.9 & 0.0239 & 9.063 & 1202.0 & 0.0239 & 14.493 & 1336.7 & 0.0243 \\
\hline 6 & 4.588 & 1259.0 & 0.0237 & 9.185 & 1200.9 & 0.0238 & 14.650 & 1335.1 & 0.0241 \\
\hline 7 & 4.596 & 1253.5 & 0.0236 & 9.232 & 1199.8 & 0.0237 & 14.612 & 1325.8 & 0.0239 \\
\hline 8 & 4.557 & 1243.8 & 0.0235 & 9.219 & 1199.0 & 0.0235 & 14.371 & 1301.2 & 0.0237 \\
\hline 9 & 4.482 & 1232.1 & 0.0233 & 9.158 & 1197.6 & 0.0234 & 14.006 & 1257.6 & 0.0235 \\
\hline 10 & 4.408 & 1223.4 & 0.0232 & 9.094 & 1196.3 & 0.0233 & 13.762 & 1222.9 & 0.0234 \\
\hline 11 & 4.380 & 1222.7 & 0.0231 & 9.090 & 1196.8 & 0.0231 & 13.744 & 1207.3 & 0.0232 \\
\hline 12 & 4.419 & 1231.3 & 0.0230 & 9.171 & 1200.1 & 0.0230 & 13.931 & 1204.0 & 0.0231 \\
\hline 13 & 4.517 & 1247.7 & 0.0229 & 9.326 & 1206.6 & 0.0229 & 14.288 & 1215.7 & 0.0230 \\
\hline 14 & 4.635 & 1266.3 & 0.0227 & 9.506 & 1215.5 & 0.0228 & 14.638 & 1239.6 & 0.0228 \\
\hline 15 & 4.683 & 1274.4 & 0.0226 & 9.572 & 1221.9 & 0.0227 & 14.617 & 1235.8 & 0.0227 \\
\hline 16 & 4.493 & 1249.6 & 0.0225 & 9.236 & 1212.3 & 0.0225 & 13.909 & 1199.6 & 0.0225 \\
\hline 17 & 3.794 & 1155.4 & 0.0224 & 7.957 & 1155.3 & 0.0224 & 11.897 & 1124.9 & 0.0224 \\
\hline 18 & 2.260 & 934.3 & 0.0223 & 4.910 & 977.3 & 0.0223 & 7.383 & 965.0 & 0.0223 \\
\hline
\end{tabular}

\begin{tabular}{|c|c|c|c|c|c|c|}
\hline \multirow[b]{2}{*}{$\begin{array}{l}\text { Axial } \\
\text { Node }\end{array}$} & \multicolumn{3}{|c|}{ Statepoint 60 ( 0.0 Cy5) } & \multicolumn{3}{|c|}{ Statepoint 61 (114.4 Cy5) } \\
\hline & $\begin{array}{c}\text { Burnup } \\
\text { (GWd/MTU) }\end{array}$ & $\begin{array}{c}\text { T-Fuel } \\
\left({ }^{\circ} \mathrm{F}\right)\end{array}$ & $\begin{array}{c}\text { Spec. Vol. } \\
\left(\mathrm{ft}^{3} / \mathrm{lbm}\right)\end{array}$ & $\begin{array}{c}\text { Burnup } \\
\text { (GWd/MTU) }\end{array}$ & $\begin{array}{c}\text { T-Fuel } \\
\left({ }^{\circ} \mathrm{F}\right)\end{array}$ & $\begin{array}{c}\text { Spec. Vol } \\
\left(\mathrm{ft}^{3} / \mathrm{lbm}\right)\end{array}$ \\
\hline & BOC Cy5 & $203.4 \mathrm{Cy} 4$ & 203.4 Cy4 & 114.4 Cy 5 & 58.2 Cy 5 & 58.2 Cy 5 \\
\hline 1 & 9.861 & 1042.7 & 0.0249 & 12.191 & 969.0 & 0.0234 \\
\hline 2 & 15.696 & 1179.9 & 0.0248 & 19.194 & 1080.6 & 0.0233 \\
\hline 3 & 18.645 & 1221.3 & 0.0246 & 22.632 & 1104.6 & 0.0231 \\
\hline 4 & 19.879 & 1223.0 & 0.0244 & 24.045 & 1102.2 & 0.0230 \\
\hline 5 & 20.361 & 1215.7 & 0.0242 & 24.568 & 1090.2 & 0.0229 \\
\hline 6 & 20.522 & 1209.0 & 0.0241 & 24.688 & 1072.4 & 0.0228 \\
\hline 7 & 20.487 & 1204.5 & 0.0239 & 24.535 & 1045.8 & 0.0227 \\
\hline 8 & 20.210 & 1198.3 & 0.0237 & 24.075 & 1015.7 & 0.0226 \\
\hline 9 & 19.664 & 1181.4 & 0.0236 & 23.379 & 1010.9 & 0.0225 \\
\hline 10 & 19.130 & 1158.2 & 0.0234 & 22.820 & 1025.0 & 0.0224 \\
\hline 11 & 18.954 & 1145.8 & 0.0233 & 22.695 & 1045.1 & 0.0223 \\
\hline 12 & 19.105 & 1142.4 & 0.0231 & 22.953 & 1071.8 & 0.0222 \\
\hline 13 & 19.534 & 1148.0 & 0.0230 & 23.508 & 1093.8 & 0.0221 \\
\hline 14 & 20.104 & 1167.2 & 0.0229 & 24.130 & 1091.4 & 0.0220 \\
\hline 15 & 20.277 & 1184.2 & 0.0227 & 24.242 & 1076.2 & 0.0219 \\
\hline 16 & 19.511 & 1184.5 & 0.0226 & 23.306 & 1057.5 & 0.0218 \\
\hline 17 & 16.953 & 1150.3 & 0.0225 & 20.320 & 1021.0 & 0.0217 \\
\hline 18 & 10.811 & 1011.2 & 0.0223 & 13.058 & 907.0 & 0.0216 \\
\hline
\end{tabular}


Table 4-13. Burnup and TH Feedback Parameters by Axial Node for Assembly C28

\begin{tabular}{|c|c|c|c|c|c|c|c|c|c|}
\hline \multirow[b]{2}{*}{$\begin{array}{l}\text { Axial } \\
\text { Node }\end{array}$} & \multicolumn{3}{|c|}{ Datapoint (142.8 Cy3) } & \multicolumn{3}{|c|}{ Datapoint (287.5 Cy3) } & \multicolumn{3}{|c|}{ Datapoint (126.6 Cy4) } \\
\hline & $\begin{array}{c}\text { Burnup } \\
\text { (GWd/MTU) }\end{array}$ & $\begin{array}{c}\text { T-Fuel } \\
\left({ }^{\circ} \mathrm{F}\right)\end{array}$ & $\begin{array}{c}\text { Spec. Vol. } \\
\left(\mathrm{ft}^{3} / \mathrm{lbm}\right)\end{array}$ & $\begin{array}{c}\text { Burnup } \\
\text { (GWd/MTU) }\end{array}$ & $\begin{array}{c}\text { T-Fuel } \\
\left({ }^{\circ} \mathrm{F}\right)\end{array}$ & $\begin{array}{c}\text { Spec. Vol. } \\
\left(\mathrm{ft}^{3} / \mathrm{lbm}\right)\end{array}$ & $\begin{array}{c}\text { Burnup } \\
\text { (GWd/MTU) }\end{array}$ & $\begin{array}{c}\text { T-Fuel } \\
\left({ }^{\circ} \mathrm{F}\right)\end{array}$ & $\begin{array}{c}\text { Spec. Vol. } \\
\left(\mathrm{ft}^{3} / \mathrm{lbm}\right)\end{array}$ \\
\hline & $142.8 \mathrm{Cy} 3$ & $69.0 \mathrm{Cy} 3$ & $69.0 \mathrm{Cy} 3$ & EOC Cy3 & $209.4 \mathrm{Cy} 3$ & 209.4 Суз & $126.6 \mathrm{Cy} 4$ & $71.2 \mathrm{Cy} 4$ & 71.2 Cy4 \\
\hline 1 & 1,178 & 788.1 & 0.0234 & 2.653 & 832.7 & 0.0236 & 4.906 & 1036.1 & 0.0250 \\
\hline 2 & 1.985 & 910.6 & 0.0234 & 4.336 & 954.0 & 0.0236 & 8.081 & 1216.3 & 0.0249 \\
\hline 3 & 2.427 & 972.9 & 0.0233 & 5.184 & 1002.4 & 0.0235 & 9.716 & 1297.5 & 0.0247 \\
\hline 4 & 2.629 & 997.7 & 0.0233 & 5.543 & 1016.5 & 0.0234 & 10.441 & 1329.2 & 0.0245 \\
\hline 5 & 2.710 & 1004.5 & 0.0232 & 5.686 & 1017.9 & 0.0233 & 10.744 & 1339.4 & 0.0243 \\
\hline 6 & 2.732 & 1003.1 & 0.0231 & 5.741 & 1016.2 & 0.0232 & 10.848 & 1340.2 & 0.0241 \\
\hline 7 & 2.721 & 997.5 & 0.0230 & 5.751 & 1014.4 & 0.0232 & 10.834 & 1335.7 & 0.0240 \\
\hline 8 & 2.685 & 989.5 & 0.0230 & 5.728 & 1012.8 & 0.0231 & 10.739 & 1325.1 & 0.0238 \\
\hline 9 & 2.634 & 980.9 & 0.0229 & 5.685 & 1011.2 & 0.0230 & 10.644 & 1311.6 & 0.0236 \\
\hline 10 & 2.586 & 974.4 & 0.0228 & 5.644 & 1010.1 & 0.0229 & 10.659 & 1306.7 & 0.0235 \\
\hline 11 & 2.565 & 972.9 & 0.0228 & 5.635 & 1010.4 & 0.0228 & 10.805 & 1312.2 & 0.0233 \\
\hline 12 & 2.580 & 977.5 & 0.0227 & 5.674 & 1013.2 & 0.0228 & 11.033 & 1321.4 & 0.0231 \\
\hline 13 & 2.631 & 987.4 & 0.0226 & 5.759 & 1018.8 & 0.0227 & 11.239 & 1327.1 & 0.0230 \\
\hline 14 & 2.700 & 999.6 & 0.0226 & 5.866 & 1026.8 & 0.0226 & 11.298 & 1322.4 & 0.0228 \\
\hline 15 & 2.742 & 1007.1 & 0.0225 & 5.924 & 1033.4 & 0.0225 & 11.075 & 1293.8 & 0.0227 \\
\hline 16 & 2.655 & 994.4 & 0.0224 & 5.746 & 1026.7 & 0.0225 & 10.386 & 1238.1 & 0.0225 \\
\hline 17 & 2.261 & 934.8 & 0.0224 & 4.960 & 979.6 & 0.0224 & 8.771 & 1145.5 & 0.0224 \\
\hline 18 & 1.352 & 792.5 & 0.0223 & 3.044 & 840.8 & 0.0223 & 5.379 & 962.6 & 0.0223 \\
\hline
\end{tabular}

\begin{tabular}{|c|c|c|c|c|c|c|}
\hline \multirow[b]{2}{*}{$\begin{array}{l}\text { Axial } \\
\text { Node } \\
\end{array}$} & \multicolumn{3}{|c|}{ Statepoint 60 ( 0.0 Cy5) } & \multicolumn{3}{|c|}{ Statepoint 61 (114.4 Cy5) } \\
\hline & $\begin{array}{c}\text { Burnup } \\
\text { (GWd/MTU) }\end{array}$ & $\begin{array}{c}\text { T-Fuel } \\
\left({ }^{\circ} \mathrm{F}\right)\end{array}$ & $\begin{array}{c}\text { Spec. Vol. } \\
\left(\mathrm{ft}^{3} / \mathrm{lbm}\right)\end{array}$ & $\begin{array}{c}\text { Burnup } \\
\text { (GWd/MTU) }\end{array}$ & $\begin{array}{c}\text { T-Fuel } \\
\left({ }^{\circ} \mathrm{F}\right)\end{array}$ & $\begin{array}{c}\text { Spec. Vol. } \\
\left(\mathrm{ft}^{3} / \mathrm{lbm}\right)\end{array}$ \\
\hline & BOC Cy5 & 203.4 Cy4 & $203.4 \mathrm{Cy} 4$ & 114.4 Cy5 & 58.2 Cy 5 & 58.2 Cy5 \\
\hline 1 & 8.052 & 1053.0 & 0.0250 & 10.121 & 957.9 & 0.0234 \\
\hline 2 & 12.832 & 1190.4 & 0.0248 & 16.077 & 1088.7 & 0.0233 \\
\hline 3 & 15.148 & 1238.0 & 0.0247 & 19.016 & 1129.6 & 0.0231 \\
\hline 4 & 16.084 & 1245.4 & 0.0245 & 20.234 & 1134.4 & 0.0230 \\
\hline 5 & 16.436 & 1241.3 & 0.0243 & 20.681 & 1126.2 & 0.0229 \\
\hline 6 & 16.554 & 1236.3 & 0.0241 & 20.780 & 1109.2 & 0.0228 \\
\hline 7 & 16.557 & 1233.3 & 0.0240 & 20.657 & 1079.3 & 0.0227 \\
\hline 8 & 16.475 & 1231.0 & 0.0238 & 20.353 & 1041.8 & 0.0226 \\
\hline 9 & 16.360 & 1226.9 & 0.0236 & 20.030 & 1029.0 & 0.0225 \\
\hline 10 & 16.340 & 1222.9 & 0.0235 & 19.938 & 1036.7 & 0.0224 \\
\hline 11 & 16.504 & 1224.1 & 0.0233 & 20.139 & 1054.9 & 0.0223 \\
\hline 12 & 16.796 & 1228.9 & 0.0232 & 20.560 & 1084.7 & 0.0222 \\
\hline 13 & 17.065 & 1234.4 & 0.0230 & 21.005 & 1114.6 & 0.0221 \\
\hline 14 & 17.180 & 1240.5 & 0.0229 & 21.231 & 1121.5 & 0.0220 \\
\hline 15 & 16.945 & 1241.7 & 0.0227 & 20.968 & 1112.2 & 0.0219 \\
\hline 16 & 16.047 & 1227.5 & 0.0226 & 19.892 & 1093.4 & 0.0218 \\
\hline 17 & 13.777 & 1175.1 & 0.0224 & 17.150 & 1049.1 & 0.0217 \\
\hline 18 & 8.703 & 1022.4 & 0.0223 & 10.912 & 916.0 & 0.0216 \\
\hline
\end{tabular}


Table 4-14. Burnup and TH Feedback Parameters by Axial Node for Assembly C29

\begin{tabular}{|c|c|c|c|c|c|c|c|c|c|}
\hline \multirow[b]{2}{*}{$\begin{array}{l}\text { Axial } \\
\text { Node }\end{array}$} & \multicolumn{3}{|c|}{ Datapoint (142.8 Cy3) } & \multicolumn{3}{|c|}{ Datapoint (287.5 Cy3) } & \multicolumn{3}{|c|}{ Datapoint (126.6 Cy4) } \\
\hline & $\begin{array}{c}\text { Burnup } \\
\text { (GWd/MTU) }\end{array}$ & $\begin{array}{l}\text { T-Fuel } \\
\left({ }^{\circ} \mathrm{F}\right)\end{array}$ & $\begin{array}{c}\text { Spec. Vol. } \\
\left(\mathrm{ft}^{3} / \mathrm{lbm}\right)\end{array}$ & $\begin{array}{c}\text { Burnup } \\
\text { (GWd/MTU) }\end{array}$ & $\begin{array}{c}\text { T-Fuel } \\
\left({ }^{\circ} \mathrm{F}\right)\end{array}$ & $\begin{array}{l}\text { Spec. Vol. } \\
\left(\mathrm{ft}^{3} / \mathrm{lbm}\right)\end{array}$ & $\begin{array}{c}\text { Burnup } \\
\text { (GWd/MTU) }\end{array}$ & $\begin{array}{l}\text { T-Fuel } \\
\left({ }^{\circ} \mathrm{F}\right)\end{array}$ & $\begin{array}{c}\text { Spec. Vol. } \\
\left(\mathrm{ft}^{3} / \mathrm{lbm}\right)\end{array}$ \\
\hline & 142.8 Су 3 & $69.0 \mathrm{Cy} 3$ & $69.0 \mathrm{Cy3}$ & $\mathrm{EOC} \mathrm{Cy3}$ & 209.4 Cy 3 & $209.4 \mathrm{Cy} 3$ & $126.6 \mathrm{Cy} 4$ & 71.2 Cy4 & $71.2 \mathrm{Cy} 4$ \\
\hline 1 & 1.133 & 779.1 & 0.0233 & 2.577 & 827.6 & 0.0235 & 4.385 & 952.6 & 0.0254 \\
\hline 2 & 1.872 & 891.5 & 0.0233 & 4.148 & 944.4 & 0.0235 & 8.006 & 1281.8 & 0.0252 \\
\hline 3 & 2.250 & 946.1 & 0.0232 & 4.899 & 989.6 & 0.0234 & 10.044 & 1397.1 & 0.0250 \\
\hline 4 & 2.405 & 965.3 & 0.0232 & 5.199 & 1000.7 & 0.0233 & 10.886 & 1425.1 & 0.0248 \\
\hline 5 & 2.453 & 968.0 & 0.0231 & 5.315 & 1000.1 & 0.0233 & 11.195 & 1430.4 & 0.0246 \\
\hline 6 & 2.455 & 964.2 & 0.0230 & 5.355 & 997.0 & 0.0232 & 11.280 & 1428.0 & 0.0243 \\
\hline 7 & 2.432 & 957.3 & 0.0230 & 5.351 & 994.2 & 0.0231 & 11.243 & 1421.3 & 0.0241 \\
\hline 8 & 2.389 & 948.2 & 0.0229 & 5.319 & 991.8 & 0.0230 & 11.127 & 1410.2 & 0.0239 \\
\hline 9 & 2.334 & 938.7 & 0.0228 & 5.268 & 989.5 & 0.0230 & 10.992 & 1395.9 & 0.0237 \\
\hline 10 & 2.283 & 931.5 & 0.0228 & 5.222 & 987.9 & 0.0229 & 10.914 & 1383.3 & 0.0235 \\
\hline 11 & 2.258 & 929.3 & 0.0227 & 5.207 & 988.0 & 0.0228 & 10.923 & 1373.5 & 0.0234 \\
\hline 12 & 2.269 & 933.3 & 0.0227 & 5.241 & 990.9 & 0.0228 & 11.004 & 1367.1 & 0.0232 \\
\hline 13 & 2.320 & 943.5 & 0.0226 & 5.329 & 997.8 & 0.0227 & 11.116 & 1362.5 & 0.0230 \\
\hline 14 & 2.407 & 959.1 & 0.0226 & 5.477 & 1010.7 & 0.0226 & 11.191 & 1353.4 & 0.0229 \\
\hline 15 & 2.514 & 977.1 & 0.0225 & 5.656 & 1027.2 & 0.0225 & 11.131 & 1328.1 & 0.0227 \\
\hline 16 & 2.542 & 981.4 & 0.0224 & 5.662 & 1031.2 & 0.0225 & 10.696 & 1279.9 & 0.0226 \\
\hline 17 & 2.250 & 935.5 & 0.0224 & 5.024 & 991.0 & 0.0224 & 9.264 & 1193.1 & 0.0224 \\
\hline 18 & 1.387 & 798.5 & 0.0223 & 3.157 & 852.4 & 0.0223 & 5.808 & 1006.0 & 0.0223 \\
\hline
\end{tabular}

\begin{tabular}{|c|c|c|c|c|c|c|}
\hline \multirow{2}{*}{$\begin{array}{c}\text { Axial } \\
\text { Node }\end{array}$} & \begin{tabular}{c}
$|c|$ \\
\cline { 2 - 7 } \\
$($ BWd/MTU)
\end{tabular} & $\begin{array}{c}\text { T-Fuel } \\
\left({ }^{\circ} \mathrm{F}\right)\end{array}$ & $\begin{array}{c}\text { Spec. Vol. } \\
\left(\mathbf{t t}^{3} / \mathrm{lbm}\right)\end{array}$ & $\begin{array}{c}\text { Burnup } \\
(\text { GWd/MTU) }\end{array}$ & $\begin{array}{c}\text { Statepoint 61 (114.4 Cy5) } \\
\text { T-Fuel } \\
\left({ }^{\circ} \mathrm{F}\right)\end{array}$ & $\begin{array}{c}\text { Spec. Vol. } \\
\left(\mathrm{ft}^{3} / \mathrm{lbm}\right)\end{array}$ \\
\hline & BOC Cy5 & 203.4 Cy4 & 203.4 Cy4 & 114.4 Cy5 & 58.2 Cy5 & 58.2 Cy5 \\
\hline 1 & 6.905 & 1001.6 & 0.0252 & 7.875 & 759.1 & 0.0225 \\
\hline 2 & 13.037 & 1227.0 & 0.0250 & 14.601 & 823.7 & 0.0224 \\
\hline 3 & 15.979 & 1286.7 & 0.0248 & 17.897 & 850.1 & 0.0224 \\
\hline 4 & 17.029 & 1290.0 & 0.0246 & 19.144 & 859.6 & 0.0223 \\
\hline 5 & 17.371 & 1283.4 & 0.0244 & 19.579 & 860.8 & 0.0223 \\
\hline 6 & 17.454 & 1276.7 & 0.0242 & 19.693 & 857.7 & 0.0222 \\
\hline 7 & 17.419 & 1272.4 & 0.0241 & 19.655 & 852.9 & 0.0222 \\
\hline 8 & 17.300 & 1269.1 & 0.0239 & 19.520 & 849.6 & 0.0221 \\
\hline 9 & 17.129 & 1264.3 & 0.0237 & 19.336 & 850.1 & 0.0220 \\
\hline 10 & 16.985 & 1258.3 & 0.0235 & 19.193 & 854.7 & 0.0220 \\
\hline 11 & 16.938 & 1253.7 & 0.0234 & 19.159 & 861.3 & 0.0219 \\
\hline 12 & 16.993 & 1251.7 & 0.0232 & 19.230 & 867.4 & 0.0219 \\
\hline 13 & 17.115 & 1252.9 & 0.0230 & 19.357 & 870.2 & 0.0218 \\
\hline 14 & 17.227 & 1256.6 & 0.0229 & 19.444 & 867.3 & 0.0218 \\
\hline 15 & 17.169 & 1257.4 & 0.0227 & 19.308 & 856.9 & 0.0217 \\
\hline 16 & 16.584 & 1246.6 & 0.0226 & 18.557 & 836.9 & 0.0217 \\
\hline 17 & 14.560 & 1199.9 & 0.0225 & 16.205 & 799.8 & 0.0216 \\
\hline 18 & 9.384 & 1045.2 & 0.0224 & 10.399 & 721.6 & 0.0216 \\
\hline
\end{tabular}


Table 4-15. Burnup and TH Feedback Parameters by Axial Node for Assembly D8

\begin{tabular}{|c|c|c|c|c|c|c|c|c|c|}
\hline \multirow[b]{2}{*}{$\begin{array}{l}\text { Axial } \\
\text { Node } \\
\end{array}$} & \multicolumn{3}{|c|}{ Datapoint (126.6 Cy4) } & \multicolumn{3}{|c|}{ Statepoint 60 (BOC Cy5) } & \multicolumn{3}{|c|}{ Statepoint 61 (114.4 Cy5) } \\
\hline & $\begin{array}{c}\text { Burnup } \\
\text { (GWd/MTU) }\end{array}$ & $\begin{array}{c}\text { T-Fuel } \\
\left({ }^{\circ} \mathrm{F}\right)\end{array}$ & $\begin{array}{c}\text { Spec. Vol. } \\
\left(\mathrm{ft}^{3} / \mathrm{lbm}\right)\end{array}$ & $\begin{array}{c}\text { Burnup } \\
\text { (GWd/MTU) }\end{array}$ & $\begin{array}{c}\text { T-Fuel } \\
\left({ }^{\circ} \mathrm{F}\right)\end{array}$ & $\begin{array}{l}\text { Spec. Vol. } \\
\left(\mathrm{ft}^{3} / \mathrm{lbm}\right)\end{array}$ & $\begin{array}{c}\text { Burnup } \\
\text { (GWd/MTU) }\end{array}$ & $\begin{array}{c}\text { T-Fuel } \\
\left({ }^{\circ} \mathrm{F}\right)\end{array}$ & $\begin{array}{r}\text { Spec. Vol } \\
\left(\mathrm{ft}^{3} / \mathrm{lbm}\right)\end{array}$ \\
\hline & $126.6 \mathrm{Cy} 4$ & $71.2 \mathrm{Cy} 4$ & $71.2 \mathrm{Cy} 4$ & BOC Cy5 & $203.4 \mathrm{Cy} 4$ & 203.4 Cy4 & 114.4 Cy 5 & 58.2 Cy5 & $58.2 \mathrm{Cy} 5$ \\
\hline 1 & 1.174 & 831.5 & 0.0240 & 2.954 & 897.4 & 0.0241 & 5.595 & 1098.7 & 0.0238 \\
\hline 2 & 2.128 & 1002.9 & 0.0239 & 5.144 & 1055.8 & 0.0240 & 9.237 & 1279.5 & 0.0237 \\
\hline 3 & 2.756 & 1099.1 & 0.0238 & 6.436 & 1121.2 & 0.0239 & 11.153 & 1330.1 & 0.0235 \\
\hline 4 & 3.093 & 1144.5 & 0.0237 & 7.040 & 1140.2 & 0.0238 & 11.969 & 1330.2 & 0.0234 \\
\hline 5 & 3.256 & 1164.6 & 0.0236 & 7.304 & 1142.9 & 0.0237 & 12.272 & 1314.2 & 0.0232 \\
\hline 6 & 3.332 & 1172.2 & 0.0235 & 7.424 & 1141.7 & 0.0236 & 12.362 & 1294.9 & 0.0231 \\
\hline 7 & 3.368 & 1174.9 & 0.0234 & 7.493 & 1140.7 & 0.0235 & 12.378 & 1277.6 & 0.0229 \\
\hline 8 & 3.397 & 1176.0 & 0.0233 & 7.557 & 1141.0 & 0.0233 & 12.394 & 1266.4 & 0.0228 \\
\hline 9 & 3.441 & 1178.1 & 0.0232 & 7.644 & 1142.8 & 0.0232 & 12.454 & 1264.3 & 0.0227 \\
\hline 10 & 3.509 & 1182.1 & 0.0231 & 7.759 & 1145.8 & 0.0231 & 12.563 & 1270.0 & 0.0225 \\
\hline 11 & 3.586 & 1186.8 & 0.0229 & 7.881 & 1149.4 & 0.0230 & 12.686 & 1279.0 & 0.0224 \\
\hline 12 & 3.639 & 1188.8 & 0.0228 & 7.969 & 1152.7 & 0.0229 & 12.767 & 1287.0 & 0.0223 \\
\hline 13 & 3.629 & 1184.1 & 0.0227 & 7.973 & 1154.9 & 0.0228 & 12.749 & 1291.0 & 0.0221 \\
\hline 14 & 3.524 & 1168.7 & 0.0226 & 7.849 & 1154.9 & 0.0227 & 12.580 & 1290.0 & 0.0220 \\
\hline 15 & 3.300 & 1137.8 & 0.0225 & 7.541 & 1148.9 & 0.0226 & 12.195 & 1284.2 & 0.0219 \\
\hline 16 & 2.924 & 1082.4 & 0.0224 & 6.927 & 1127.1 & 0.0225 & 11.399 & 1266.2 & 0.0218 \\
\hline 17 & 2.310 & 987.1 & 0.0224 & 5.709 & 1060.6 & 0.0224 & 9.646 & 1205.0 & 0.0217 \\
\hline 18 & 1.315 & 814.3 & 0.0223 & 3.400 & 890.4 & 0.0223 & 5.928 & 1018.8 & 0.0216 \\
\hline
\end{tabular}

\begin{tabular}{|c|c|c|c|c|c|c|c|c|c|}
\hline \multirow[b]{2}{*}{$\begin{array}{l}\text { Axial } \\
\text { Node }\end{array}$} & \multicolumn{3}{|c|}{ Datapoint (206.7 Cy5) } & \multicolumn{3}{|c|}{ Statepoint 62 (BOC Cy6) } & \multicolumn{3}{|c|}{ Statepoint 63 (69.1 Cy6) } \\
\hline & $\begin{array}{c}\text { Burnup } \\
\text { (GWd/MTU) }\end{array}$ & $\begin{array}{c}\text { T-Fuel } \\
\left({ }^{\circ} \mathrm{F}\right)\end{array}$ & $\begin{array}{c}\text { Spec. Vol. } \\
\left(\mathrm{ft}^{3} / \mathrm{lbm}\right)\end{array}$ & $\begin{array}{c}\text { Burnup } \\
\text { (GWd/MTU) }\end{array}$ & $\begin{array}{l}\text { T-Fuel } \\
\left({ }^{\circ} \mathrm{F}\right)\end{array}$ & $\begin{array}{l}\text { Spec. Vol. } \\
\left(\mathrm{ft}^{3} / \mathrm{lbm}\right)\end{array}$ & $\begin{array}{c}\text { Burnup } \\
\text { (GWd/MTU) }\end{array}$ & $\begin{array}{l}\text { T-Fuel } \\
\left({ }^{\circ} \mathrm{F}\right)\end{array}$ & $\begin{array}{l}\text { Spec. Vol. } \\
\left(\mathrm{ft}^{3} / \mathrm{lbm}\right)\end{array}$ \\
\hline & 206.7 Cy 5 & 153.5 Cy5 & 153.5 Cy 5 & BOC Cy 6 & 252.2 Cy 5 & 252.2 Cy 5 & $69.1 \mathrm{Cy} 6$ & 41.5 Cy 6 & 41.5 Cy6 \\
\hline 1 & 7.799 & 1064.1 & 0.0237 & 10.020 & 965.2 & 0.0236 & 11.170 & 881.0 & 0.0235 \\
\hline 2 & 12.478 & 1205.4 & 0.0236 & 15.617 & 1063.5 & 0.0235 & 17.396 & 990.3 & 0.0234 \\
\hline 3 & 14.754 & 1247.1 & 0.0234 & 18.170 & 1085.4 & 0.0234 & 20.305 & 1046.5 & 0.0233 \\
\hline 4 & 15.642 & 1249.2 & 0.0233 & 19.101 & 1089.4 & 0.0232 & 21.453 & 1085.7 & 0.0232 \\
\hline 5 & 15.931 & 1242.3 & 0.0232 & 19.395 & 1097.3 & 0.0231 & 21.881 & 1110.6 & 0.0231 \\
\hline 6 & 15.992 & 1235.1 & 0.0230 & 19.486 & 1114.9 & 0.0230 & 22.064 & 1127.1 & 0.0229 \\
\hline 7 & 15.983 & 1229.0 & 0.0229 & 19.540 & 1142.5 & 0.0229 & 22.191 & 1139.5 & 0.0228 \\
\hline 8 & 15.981 & 1223.1 & 0.0227 & 19.625 & 1176.5 & 0.0228 & 22.331 & 1148.4 & 0.0227 \\
\hline 9 & 16.031 & 1217.3 & 0.0226 & 19.764 & 1209.1 & 0.0226 & 22.504 & 1152.4 & 0.0225 \\
\hline 10 & 16.140 & 1211.7 & 0.0225 & 19.936 & 1230.3 & 0.0225 & 22.683 & 1150.8 & 0.0224 \\
\hline 11 & 16.268 & 1206.9 & 0.0224 & 20.081 & 1233.4 & 0.0224 & 22.815 & 1144.7 & 0.0223 \\
\hline 12 & 16.365 & 1203.9 & 0.0222 & 20.150 & 1218.3 & 0.0222 & 22.858 & 1136.7 & 0.0222 \\
\hline 13 & 16.374 & 1204.3 & 0.0221 & 20.107 & 1191.8 & 0.0221 & 22.778 & 1128.0 & 0.0221 \\
\hline 14 & 16.242 & 1208.5 & 0.0220 & 19.928 & 1163.9 & 0.0220 & 22.530 & 1115.4 & 0.0219 \\
\hline 15 & 15.891 & 1215.0 & 0.0219 & 19.549 & 1142.5 & 0.0219 & 22.030 & 1093.6 & 0.0218 \\
\hline 16 & 15.065 & 1215.2 & 0.0218 & 18.678 & 1127.7 & 0.0218 & 20.959 & 1059.2 & 0.0217 \\
\hline 17 & 13.006 & 1177.2 & 0.0217 & 16.358 & 1097.7 & 0.0217 & 18.305 & 1005.5 & 0.0217 \\
\hline 18 & 8.206 & 1023.7 & 0.0216 & 10.557 & 973.4 & 0.0216 & 11.836 & 885.5 & 0.0216 \\
\hline
\end{tabular}


Table 4-16. Burnup and TH Feedback Parameters by Axial Node for Assembly D15

\begin{tabular}{|c|c|c|c|c|c|c|c|c|c|}
\hline \multirow[b]{2}{*}{$\begin{array}{l}\text { Axial } \\
\text { Node } \\
\end{array}$} & \multicolumn{3}{|c|}{ Datapoint (126.6 Cy4) } & \multicolumn{3}{|c|}{ Statepoint 60 (BOC Cy5) } & \multicolumn{3}{|c|}{ Statepoint 61 (114.4 Cy5) } \\
\hline & $\begin{array}{c}\text { Burnup } \\
\text { (GWd/MTU) }\end{array}$ & $\begin{array}{c}\text { T-Fuel } \\
\left({ }^{\circ} \mathrm{F}\right)\end{array}$ & $\begin{array}{c}\text { Spec. Vol. } \\
\left(\mathrm{ft}^{3} / \mathrm{lbm}\right)\end{array}$ & $\begin{array}{c}\text { Burnup } \\
\text { (GWd/MTU) }\end{array}$ & $\begin{array}{c}\text { T-Fuel } \\
\left({ }^{\circ} \mathrm{F}\right) \\
\end{array}$ & $\begin{array}{c}\text { Spec. Vol. } \\
\left(\mathrm{ft}^{3} / \mathrm{lbm}\right)\end{array}$ & $\begin{array}{c}\text { Burnup } \\
\text { (GWd/MTU) }\end{array}$ & $\begin{array}{c}\text { T-Fuel } \\
\left({ }^{\circ} \mathrm{F}\right) \\
\end{array}$ & $\begin{array}{c}\text { Spec. Vol. } \\
\left(\mathrm{ft}^{3} / \mathrm{lbm}\right)\end{array}$ \\
\hline & $126.6 \mathrm{Cy} 4$ & 71.2 Cy 4 & 71.2 Cy 4 & BOC Cy 5 & $203.4 \mathrm{Cy} 4$ & 203.4 Cy 4 & 114.4 Cy 5 & 58.2 Cy 5 & 58.2 Cy 5 \\
\hline 1 & 1.231 & 840.2 & 0.0240 & 3.065 & 898.3 & 0.0241 & 5.274 & 1026.8 & 0.0235 \\
\hline 2 & 2.170 & 1006.1 & 0.0239 & 5.198 & 1052.1 & 0.0240 & 8.700 & 1195.4 & 0.0234 \\
\hline 3 & 2.773 & 1098.6 & 0.0238 & 6.431 & 1116.0 & 0.0239 & 10.561 & 1251.9 & 0.0233 \\
\hline 4 & 3.104 & 1144.4 & 0.0237 & 7.026 & 1136.0 & 0.0238 & 11.419 & 1261.9 & 0.0232 \\
\hline 5 & 3.269 & 1165.3 & 0.0236 & 7.294 & 1139.2 & 0.0237 & 11.775 & 1254.4 & 0.0230 \\
\hline 6 & 3.345 & 1173.6 & 0.0235 & 7.416 & 1138.2 & 0.0236 & 11.890 & 1238.2 & 0.0229 \\
\hline 7 & 3.379 & 1176.1 & 0.0234 & 7.483 & 1137.2 & 0.0235 & 11.885 & 1214.9 & 0.0228 \\
\hline 8 & 3.401 & 1176.4 & 0.0233 & 7.538 & 1137.3 & 0.0233 & 11.834 & 1192.7 & 0.0227 \\
\hline 9 & 3.435 & 1177.0 & 0.0232 & 7.607 & 1138.4 & 0.0232 & 11.835 & 1189.3 & 0.0226 \\
\hline 10 & 3.490 & 1179.2 & 0.0230 & 7.698 & 1140.6 & 0.0231 & 11.941 & 1203.5 & 0.0225 \\
\hline 11 & 3.556 & 1182.3 & 0.0229 & 7.798 & 1143.3 & 0.0230 & 12.104 & 1225.2 & 0.0223 \\
\hline 12 & 3.604 & 1183.6 & 0.0228 & 7.876 & 1146.2 & 0.0229 & 12.261 & 1249.8 & 0.0222 \\
\hline 13 & 3.599 & 1179.6 & 0.0227 & 7.888 & 1148.7 & 0.0228 & 12.336 & 1266.8 & 0.0221 \\
\hline 14 & 3.506 & 1165.9 & 0.0226 & 7.786 & 1149.5 & 0.0227 & 12.219 & 1264.9 & 0.0220 \\
\hline 15 & 3.296 & 1136.7 & 0.0225 & 7.505 & 1144.8 & 0.0226 & 11.822 & 1247.8 & 0.0219 \\
\hline 16 & 2.928 & 1082.5 & 0.0224 & 6.909 & 1124.0 & 0.0225 & 10.982 & 1216.9 & 0.0218 \\
\hline 17 & 2.312 & 986.9 & 0.0224 & 5.691 & 1057.2 & 0.0224 & 9.208 & 1149.3 & 0.0217 \\
\hline 18 & 1.311 & 813.4 & 0.0223 & 3.377 & 887.6 & 0.0223 & 5.600 & 971.4 & 0.0216 \\
\hline
\end{tabular}

\begin{tabular}{|c|c|c|c|c|c|c|c|c|c|}
\hline \multirow[b]{2}{*}{$\begin{array}{l}\text { Axial } \\
\text { Node } \\
\end{array}$} & \multicolumn{3}{|c|}{ Datapoint (206.7 Cy5) } & \multicolumn{3}{|c|}{ Statepoint 62 (BOC Cy6) } & \multicolumn{3}{|c|}{ Statepoint $63(69.1$ Cy6) } \\
\hline & $\begin{array}{c}\text { Burnup } \\
\text { (GWd/MTU) }\end{array}$ & $\begin{array}{l}\text { T-Fuel } \\
\left({ }^{\circ} \mathrm{F}\right)\end{array}$ & $\begin{array}{l}\text { Spec. Vol. } \\
\left(\mathrm{ft}^{3} / \mathrm{lbm}\right)\end{array}$ & $\begin{array}{c}\text { Burnup } \\
\text { (GWd/MTU) }\end{array}$ & $\begin{array}{l}\text { T-Fuel } \\
\left({ }^{\circ} \mathrm{F}\right)\end{array}$ & $\begin{array}{l}\text { Spec. Vol. } \\
\left(\mathrm{ft}^{3} / \mathrm{lbm}\right)\end{array}$ & $\begin{array}{c}\text { Burnup } \\
\text { (GWd/MTU) }\end{array}$ & $\begin{array}{c}\text { T-Fuel } \\
\left({ }^{\circ} \mathrm{F}\right)\end{array}$ & $\begin{array}{l}\text { Spec. Vol. } \\
\left(\mathrm{ft}^{3} / \mathrm{lbm}\right)\end{array}$ \\
\hline & 206.7 Cy5 & 153.5 Cy5 & 153.5 Cy5 & BOC Cy6 & 252.2 Cy5 & 252.2 Cy 5 & $69.1 \mathrm{Cy} 6$ & 41.5 Cy6 & 41.5 Cy 6 \\
\hline 1 & 7.248 & 1021.3 & 0.0236 & 9.295 & 943.0 & 0.0236 & 10.069 & 786.6 & 0.0235 \\
\hline 2 & 11.672 & 1162.3 & 0.0235 & 14.627 & 1044.1 & 0.0235 & 16.151 & 897.7 & 0.0234 \\
\hline 3 & 13.928 & 1211.2 & 0.0233 & 17.193 & 1072.8 & 0.0234 & 19.320 & 1045.1 & 0.0233 \\
\hline 4 & 14.898 & 1220.6 & 0.0232 & 18.236 & 1080.8 & 0.0233 & 20.630 & 1100.6 & 0.0232 \\
\hline 5 & 15.269 & 1218.6 & 0.0231 & 18.633 & 1091.2 & 0.0232 & 21.175 & 1128.6 & 0.0231 \\
\hline 6 & 15.373 & 1214.3 & 0.0229 & 18.781 & 1112.0 & 0.0230 & 21.425 & 1147.4 & 0.0230 \\
\hline 7 & 15.336 & 1208.2 & 0.0228 & 18.822 & 1146.2 & 0.0229 & 21.552 & 1162.9 & 0.0228 \\
\hline 8 & 15.229 & 1197.2 & 0.0227 & 18.820 & 1193.4 & 0.0228 & 21.629 & 1177.0 & 0.0227 \\
\hline 9 & 15.176 & 1183.9 & 0.0226 & 18.884 & 1243.1 & 0.0227 & 21.750 & 1186.3 & 0.0226 \\
\hline 10 & 15.280 & 1177.2 & 0.0224 & 19.087 & 1275.5 & 0.0225 & 21.968 & 1185.7 & 0.0224 \\
\hline 11 & 15.470 & 1175.2 & 0.0223 & 19.314 & 1281.1 & 0.0224 & 22.170 & 1176.7 & 0.0223 \\
\hline 12 & 15.679 & 1177.4 & 0.0222 & 19.496 & 1260.5 & 0.0222 & 22.294 & 1161.7 & 0.0222 \\
\hline 13 & 15.836 & 1186.0 & 0.0221 & 19.589 & 1221.6 & 0.0221 & 22.301 & 1142.6 & 0.0221 \\
\hline 14 & 15.790 & 1195.7 & 0.0220 & 19.468 & 1178.5 & 0.0220 & 22.074 & 1121.7 & 0.0219 \\
\hline 15 & 15.410 & 1200.2 & 0.0219 & 19.024 & 1146.3 & 0.0219 & 21.496 & 1097.9 & 0.0218 \\
\hline 16 & 14.500 & 1194.1 & 0.0218 & 18.031 & 1124.9 & 0.0218 & 20.309 & 1064.8 & 0.0217 \\
\hline 17 & 12.387 & 1150.4 & 0.0217 & 15.629 & 1089.4 & 0.0217 & 17.579 & 1012.9 & 0.0217 \\
\hline 18 & 7.725 & 999.2 & 0.0216 & 9.973 & 964.1 & 0.0216 & 11.256 & 890.3 & 0.0216 \\
\hline
\end{tabular}


Table 4-17. Burnup and TH Feedback Parameters by Axial Node for Assembly D15a

\begin{tabular}{|c|c|c|c|c|c|c|c|c|c|}
\hline \multirow[b]{2}{*}{$\begin{array}{l}\text { Axial } \\
\text { Node }\end{array}$} & \multicolumn{3}{|c|}{ Datapoint (126.6 Cy4) } & \multicolumn{3}{|c|}{ Statepoint 60 (BOC Cy5) } & \multicolumn{3}{|c|}{ Statepoint 61 (114.4 Cy5) } \\
\hline & $\begin{array}{c}\text { Burnup } \\
\text { (GWd/MTU) }\end{array}$ & $\begin{array}{l}\text { T-Fuel } \\
\left({ }^{\circ} \mathrm{F}\right)\end{array}$ & \begin{tabular}{|c|} 
Spec. Vol. \\
$\left(\mathrm{ft}^{3} / \mathrm{lbm}\right)$
\end{tabular} & $\begin{array}{c}\text { Burnup } \\
\text { (GWd/MTU) }\end{array}$ & $\begin{array}{c}\text { T-Fuel } \\
\left({ }^{\circ} \mathrm{F}\right)\end{array}$ & $\begin{array}{c}\text { Spec. Vol. } \\
\left(\mathrm{ft}^{3} / \mathrm{lbm}\right)\end{array}$ & $\begin{array}{c}\text { Burnup } \\
\text { (GWd/MTU) }\end{array}$ & $\begin{array}{c}\text { T-Fuel } \\
\left({ }^{\circ} \mathrm{F}\right)\end{array}$ & $\begin{array}{c}\text { Spec. Vol } \\
\left(\mathrm{ft}^{3} / \mathrm{lbm}\right)\end{array}$ \\
\hline & $126.6 \mathrm{Cy} 4$ & 71.2 Cy 4 & $71.2 \mathrm{Cy} 4$ & $\mathrm{BOC} C \mathrm{Cy}$ & 203.4 Cy 4 & $203.4 \mathrm{Cy} 4$ & 114.4 Cy5 & 58.2 Cy 5 & 58.2 Cy 5 \\
\hline 1 & 1.231 & 840.2 & 0.0240 & 3.065 & 898.3 & 0.0241 & 5.274 & 1026.8 & 0.0235 \\
\hline 2 & 2.170 & 1006.1 & 0.0239 & 5.198 & 1052.1 & 0.0240 & 8.700 & 1195.4 & 0.0234 \\
\hline 3 & 2.773 & 1098.6 & 0.0238 & 6.431 & 1116.0 & 0.0239 & 10.561 & 1251.9 & 0.0233 \\
\hline 4 & 3.104 & 1144.4 & 0.0237 & 7.026 & 1136.0 & 0.0238 & 11.419 & 1261.9 & 0.0232 \\
\hline 5 & 3.269 & 1165.3 & 0.0236 & 7.294 & 1139.2 & 0.0237 & 11.775 & 1254.4 & 0.0230 \\
\hline 6 & 3.345 & 1173.6 & 0.0235 & 7.416 & 1138.2 & 0.0236 & 11.890 & 1238.2 & 0.0229 \\
\hline 7 & 3.379 & 1176.1 & 0.0234 & 7.483 & 1137.2 & 0.0235 & 11.885 & 1214.9 & 0.0228 \\
\hline 8 & 3.401 & 1176.4 & 0.0233 & 7.538 & 1137.3 & 0.0233 & 11.834 & 1192.7 & 0.0227 \\
\hline 9 & 3.435 & 1177.0 & 0.0232 & 7.607 & 1138.4 & 0.0232 & 11.835 & 1189.3 & 0.0226 \\
\hline 10 & 3.490 & 1179.2 & 0.0230 & 7.698 & 1140.6 & 0.0231 & 11.941 & 1203.5 & 0.0225 \\
\hline 11 & 3.556 & 1182.3 & 0.0229 & 7.798 & 1143.3 & 0.0230 & 12.104 & 1225.2 & 0.0223 \\
\hline 12 & 3.604 & 1183.6 & 0.0228 & 7.876 & 1146.2 & 0.0229 & 12.261 & 1249.8 & 0.0222 \\
\hline 13 & 3.599 & 1179.6 & 0.0227 & 7.888 & 1148.7 & 0.0228 & 12.336 & 1266.8 & 0.0221 \\
\hline 14 & 3.506 & 1165.9 & 0.0226 & 7.786 & 1149.5 & 0.0227 & 12.219 & 1264.9 & 0.0220 \\
\hline 15 & 3.296 & 1136.7 & 0.0225 & 7.505 & 1144.8 & 0.0226 & 11.822 & 1247.8 & 0.0219 \\
\hline 16 & 2.928 & 1082.5 & 0.0224 & 6.909 & 1124.0 & 0.0225 & 10.982 & 1216.9 & 0.0218 \\
\hline 17 & 2.312 & 986.9 & 0.0224 & 5.691 & 1057.2 & 0.0224 & 9.208 & 1149.3 & 0.0217 \\
\hline 18 & 1.311 & 813.4 & 0.0223 & 3.377 & 887.6 & 0.0223 & 5.600 & 971.4 & 0.0216 \\
\hline
\end{tabular}

\begin{tabular}{|c|c|c|c|c|c|c|c|c|c|}
\hline \multirow[b]{2}{*}{$\begin{array}{l}\text { Axial } \\
\text { Node }\end{array}$} & \multicolumn{3}{|c|}{ Datapoint (206.7 Cy5) } & \multicolumn{3}{|c|}{ Statepoint 62 (BOC Cy6) } & \multicolumn{3}{|c|}{ Statepoint 63 (69.1 Cy6) } \\
\hline & \begin{tabular}{|c|} 
Burnup \\
(GWd/MTU)
\end{tabular} & $\begin{array}{l}\text { T-Fuel } \\
\left({ }^{\circ} \mathrm{F}\right)\end{array}$ & $\begin{array}{c}\text { Spec. Vol. } \\
\left(\mathrm{ft}^{3} / \mathrm{lbm}\right)\end{array}$ & $\begin{array}{c}\text { Burnup } \\
\text { (GWd/MTU) } \\
\end{array}$ & $\begin{array}{l}\text { T-Fuel } \\
\left({ }^{\circ} \mathrm{F}\right)\end{array}$ & $\begin{array}{l}\text { Spec. Vol. } \\
\left(\mathrm{ft}^{3} / \mathrm{lbm}\right)\end{array}$ & $\begin{array}{c}\text { Burnup } \\
\text { (GWd/MTU) }\end{array}$ & $\begin{array}{l}\text { T-Fuel } \\
\left({ }^{\circ} \mathrm{F}\right)\end{array}$ & $\begin{array}{c}\text { Spec. Vol. } \\
\left(\mathrm{ft}^{3} / / \mathrm{bm}\right)\end{array}$ \\
\hline & 206.7 Cy5 & 153.5 Cy 5 & 153.5 Cy5 & BOC Cy6 & 252.2 Cy5 & 252.2 Cy5 & 69.1 Cy6 & 41.5 Cy 6 & 41.5 Cy 6 \\
\hline 1 & 7.248 & 1021.3 & 0.0236 & 9.298 & 943.0 & 0.0236 & 9.979 & 760.4 & 0.0234 \\
\hline 2 & 11.672 & 1162.3 & 0.0235 & 14.631 & 1044.1 & 0.0235 & 16.032 & 868.8 & 0.0233 \\
\hline 3 & 13.928 & 1211.2 & 0.0233 & 17.198 & 1072.8 & 0.0234 & 19.201 & 1013.7 & 0.0232 \\
\hline 4 & 14.898 & 1220.6 & 0.0232 & 18.241 & 1080.8 & 0.0233 & 20.528 & 1071.6 & 0.0231 \\
\hline 5 & 15.269 & 1218.6 & 0.0231 & 18.638 & 1091.2 & 0.0232 & 21.084 & 1101.3 & 0.0230 \\
\hline 6 & 15.373 & 1214.3 & 0.0229 & 18.786 & 1112.0 & 0.0230 & 21.334 & 1119.6 & 0.0229 \\
\hline 7 & 15.336 & 1208.2 & 0.0228 & 18.828 & 1146.2 & 0.0229 & 21.445 & 1131.8 & 0.0228 \\
\hline 8 & 15.229 & 1197.2 & 0.0227 & 18.826 & 1193.4 & 0.0228 & 21.491 & 1139.9 & 0.0226 \\
\hline 9 & 15.176 & 1183.9 & 0.0226 & 18.889 & 1243.1 & 0.0227 & 21.579 & 1142.9 & 0.0225 \\
\hline 10 & 15.280 & 1177.2 & 0.0224 & 19.092 & 1275.5 & 0.0225 & 21.783 & 1139.8 & 0.0224 \\
\hline 11 & 15.470 & 1175.2 & 0.0223 & 19.320 & 1281.1 & 0.0224 & 21.995 & 1133.0 & 0.0223 \\
\hline 12 & 15.679 & 1177.4 & 0.0222 & 19.502 & 1260.5 & 0.0222 & 22.151 & 1124.6 & 0.0222 \\
\hline 13 & 15.836 & 1186.0 & 0.0221 & 19.596 & 1221.6 & 0.0221 & 22.205 & 1114.7 & 0.0220 \\
\hline 14 & 15.790 & 1195.7 & 0.0220 & 19.475 & 1178.5 & 0.0220 & 22.017 & 1102.1 & 0.0219 \\
\hline 15 & 15.410 & 1200.2 & 0.0219 & 19.031 & 1146.3 & 0.0219 & 21.457 & 1082.2 & 0.0218 \\
\hline 16 & 14.500 & 1194.1 & 0.0218 & 18.038 & 1124.9 & 0.0218 & 20.260 & 1047.8 & 0.0217 \\
\hline 17 & 12.387 & 1150.4 & 0.0217 & 15.635 & 1089.4 & 0.0217 & 17.497 & 989.4 & 0.0216 \\
\hline 18 & 7.725 & 999.2 & 0.0216 & 9.978 & 964.1 & 0.0216 & 11.159 & 862.2 & 0.0216 \\
\hline
\end{tabular}


Table 4-18. Burnup and TH Feedback Parameters by Axial Node for Assembly D20

\begin{tabular}{|c|c|c|c|c|c|c|c|c|c|}
\hline \multirow[b]{2}{*}{$\begin{array}{l}\text { Axial } \\
\text { Node }\end{array}$} & \multicolumn{3}{|c|}{ Datapoint (126.6 Cy4) } & \multicolumn{3}{|c|}{ Statepoint 60 (BOC Cy5) } & \multicolumn{3}{|c|}{ Statepoint 61 (114.4 Cy5) } \\
\hline & $\begin{array}{c}\text { Burnup } \\
\text { (GWd/MTU) }\end{array}$ & $\begin{array}{c}\text { T-Fuel } \\
\left({ }^{\circ} \mathrm{F}\right)\end{array}$ & $\begin{array}{l}\text { Spec. Vol. } \\
\left(\mathrm{ft}^{3} / \mathrm{lbm}\right)\end{array}$ & $\begin{array}{c}\text { Burnup } \\
\text { (GWd/MTU) }\end{array}$ & $\begin{array}{l}\text { T-Fuel } \\
\left({ }^{\circ} \mathrm{F}\right)\end{array}$ & $\begin{array}{l}\text { Spec. Vol. } \\
\left(\mathrm{ft}^{3} / \mathrm{bm}\right)\end{array}$ & $\begin{array}{c}\text { Burnup } \\
\text { (GWd/MTU) }\end{array}$ & $\begin{array}{l}\text { T-Fuel } \\
\left({ }^{\circ} \mathrm{F}\right)\end{array}$ & $\begin{array}{c}\text { Spec. Vol. } \\
\left(\mathrm{ft}^{3} / \mathrm{lbm}\right)\end{array}$ \\
\hline & $126.6 \mathrm{Cy} 4$ & $71.2 \mathrm{Cy} 4$ & $71.2 \mathrm{Cy} 4$ & BOC Cy5 & 203.4 Cy 4 & 203.4 Cy 4 & 114.4 Cy5 & 58.2 Cy 5 & 58.2 Cy5 \\
\hline 1 & 1.994 & 988.4 & 0.0249 & 4.762 & 1036.2 & 0.0249 & 7.217 & 1043.0 & 0.0236 \\
\hline 2 & 3.381 & 1207.7 & 0.0248 & 7.746 & 1210.6 & 0.0248 & 11.512 & 1191.4 & 0.0235 \\
\hline 3 & 4.211 & 1322.1 & 0.0246 & 9.350 & 1277.0 & 0.0246 & 13.674 & 1238.0 & 0.0233 \\
\hline 4 & 4.649 & 1377.1 & 0.0245 & 10.095 & 1295.1 & 0.0244 & 14.613 & 1239.2 & 0.0232 \\
\hline 5 & 4.866 & 1401.6 & 0.0243 & 10.426 & 1296.3 & 0.0243 & 14.982 & 1225.2 & 0.0230 \\
\hline 6 & 4.957 & 1411.0 & 0.0241 & 10.572 & 1293.8 & 0.0241 & 15.091 & 1205.5 & 0.0229 \\
\hline 7 & 4.975 & 1411.9 & 0.0239 & 10.632 & 1292.0 & 0.0239 & 15.067 & 1183.1 & 0.0228 \\
\hline 8 & 4.953 & 1406.4 & 0.0237 & 10.641 & 1290.6 & 0.0238 & 14.980 & 1165.1 & 0.0227 \\
\hline 9 & 4.935 & 1398.0 & 0.0236 & 10.632 & 1288.7 & 0.0236 & 14.908 & 1161.5 & 0.0226 \\
\hline 10 & 4.964 & 1392.8 & 0.0234 & 10.656 & 1287.1 & 0.0234 & 14.926 & 1171.6 & 0.0224 \\
\hline 11 & 5.044 & 1392.8 & 0.0233 & 10.742 & 1287.6 & 0.0233 & 15.045 & 1188.5 & 0.0223 \\
\hline 12 & 5.138 & 1395.6 & 0.0231 & 10.865 & 1290.7 & 0.0231 & 15.216 & 1205.8 & 0.0222 \\
\hline 13 & 5.193 & 1397.0 & 0.0229 & 10.965 & 1296.3 & 0.0230 & 15.348 & 1216.1 & 0.0221 \\
\hline 14 & 5.137 & 1389.9 & 0.0228 & 10.956 & 1303.3 & 0.0228 & 15.322 & 1214.2 & 0.0220 \\
\hline 15 & 4.901 & 1362.0 & 0.0227 & 10.704 & 1305.3 & 0.0227 & 14.989 & 1203.2 & 0.0219 \\
\hline 16 & 4.417 & 1299.3 & 0.0225 & 9.994 & 1288.0 & 0.0226 & 14.094 & 1182.3 & 0.0218 \\
\hline 17 & 3.546 & 1177.7 & 0.0224 & 8.384 & 1217.9 & 0.0224 & 11.992 & 1126.0 & 0.0217 \\
\hline 18 & 2.051 & 942.6 & 0.0223 & 5.101 & 1021.7 & 0.0223 & 7.443 & 972.6 & 0.0216 \\
\hline
\end{tabular}

\begin{tabular}{|c|c|c|c|c|c|c|c|c|c|}
\hline \multirow[b]{2}{*}{$\begin{array}{l}\text { Axial } \\
\text { Node }\end{array}$} & \multicolumn{3}{|c|}{ Datapoint (206.7 Cy5) } & \multicolumn{3}{|c|}{ Statepoint 62 (BOC Cy6) } & \multicolumn{3}{|c|}{ Statepoint 63 (69.1 Cy6) } \\
\hline & $\begin{array}{c}\text { Burnup } \\
\text { (GWd/MTU) }\end{array}$ & $\begin{array}{l}\text { T-Fuel } \\
\left({ }^{\circ} \mathrm{F}\right)\end{array}$ & $\begin{array}{l}\text { Spec. Vol. } \\
\left(\mathrm{ft}^{3} / \mathrm{lbm}\right)\end{array}$ & $\begin{array}{c}\text { Burnup } \\
\text { (GWd/MTU) }\end{array}$ & $\begin{array}{l}\text { T-Fuel } \\
\left({ }^{\circ} \mathrm{F}\right)\end{array}$ & $\begin{array}{l}\text { Spec. Vol. } \\
\left(\mathrm{ft}^{3} / \mathrm{lbm}\right)\end{array}$ & $\begin{array}{c}\text { Burnup } \\
\text { (GWd/MTU) }\end{array}$ & $\begin{array}{l}\text { T-Fuel } \\
\left({ }^{\circ} \mathrm{F}\right)\end{array}$ & $\begin{array}{c}\text { Spec. Vol. } \\
\left(\mathrm{tt}^{3} / \mathrm{lbm}\right)\end{array}$ \\
\hline & 206.7 Cy 5 & 153.5 Cy5 & 153.5 Cy5 & BOC Cy 6 & 252.2 Cy 5 & 252.2 Cy 5 & $69.1 \mathrm{Cy} 6$ & $41.5 \mathrm{Cy} 6$ & 41.5 Cy 6 \\
\hline 1 & 9.325 & 1025.6 & 0.0236 & 11.466 & 939.2 & 0.0235 & 11.922 & 686.3 & 0.0222 \\
\hline 2 & 14.590 & 1154.0 & 0.0234 & 17.598 & 1029.6 & 0.0235 & 18.309 & 730.4 & 0.0222 \\
\hline 3 & 17.090 & 1188.5 & 0.0233 & 20.361 & 1046.9 & 0.0233 & 21.205 & 750.3 & 0.0222 \\
\hline 4 & 18.099 & 1189.1 & 0.0232 & 21.413 & 1050.1 & 0.0232 & 22.335 & 762.5 & 0.0221 \\
\hline 5 & 18.459 & 1182.5 & 0.0230 & 21.783 & 1058.2 & 0.0231 & 22.753 & 770.7 & 0.0221 \\
\hline 6 & 18.539 & 1175.6 & 0.0229 & 21.898 & 1077.0 & 0.0230 & 22.899 & 776.2 & 0.0220 \\
\hline 7 & 18.478 & 1168.5 & 0.0228 & 21.910 & 1108.8 & 0.0229 & 22.932 & 779.7 & 0.0220 \\
\hline 8 & 18.342 & 1159.5 & 0.0227 & 21.879 & 1151.7 & 0.0228 & 22.914 & 781.5 & 0.0219 \\
\hline 9 & 18.228 & 1149.7 & 0.0225 & 21.877 & 1196.3 & 0.0226 & 22.915 & 781.4 & 0.0219 \\
\hline 10 & 18.232 & 1142.6 & 0.0224 & 21.967 & 1226.6 & 0.0225 & 22.997 & 779.0 & 0.0219 \\
\hline 11 & 18.363 & 1138.4 & 0.0223 & 22.123 & 1231.2 & 0.0224 & 23.139 & 774.7 & 0.0218 \\
\hline 12 & 18.568 & 1137.5 & 0.0222 & 22.290 & 1210.9 & 0.0222 & 23.285 & 769.2 & 0.0218 \\
\hline 13 & 18.757 & 1140.9 & 0.0221 & 22.404 & 1172.6 & 0.0221 & 23.372 & 762.9 & 0.0217 \\
\hline 14 & 18.788 & 1147.0 & 0.0220 & 22.359 & 1132.4 & 0.0220 & 23.294 & 755.9 & 0.0217 \\
\hline 15 & 18.490 & 1153.0 & 0.0219 & 22.007 & 1102.6 & 0.0219 & 22.895 & 747.5 & 0.0217 \\
\hline 16 & 17.560 & 1153.6 & 0.0218 & 21.018 & 1084.8 & 0.0218 & 21.836 & 735.5 & 0.0216 \\
\hline 17 & 15.172 & 1123.6 & 0.0217 & 18.383 & 1060.0 & 0.0217 & 19.076 & 715.6 & 0.0216 \\
\hline 18 & 9.617 & 987.0 & 0.0216 & 11.888 & 949.7 & 0.0216 & 12.333 & 670.6 & 0.0216 \\
\hline
\end{tabular}


Table 4-19. Burnup and TH Feedback Parameters by Axial Node for Assembly D20a

\begin{tabular}{|c|c|c|c|c|c|c|c|c|c|}
\hline \multirow{2}{*}{$\begin{array}{c}\text { Axial } \\
\text { Node }\end{array}$} & \multicolumn{2}{|c|}{ Datapoint (126.6 Cy4) } & \multicolumn{2}{c|}{ Statepoint 60 (BOC Cy5) } & \multicolumn{3}{c|}{ Statepoint 61 (114.4 Cy5) } \\
\hline & $\begin{array}{c}\text { BWrnup } \\
\text { (GWTU) }\end{array}$ & $\begin{array}{c}\text { T-Fuel } \\
\left({ }^{\circ} \mathrm{F}\right)\end{array}$ & $\begin{array}{c}\text { Spec. Vol. } \\
\left(\mathrm{ft}^{3} / \mathrm{lbm}\right)\end{array}$ & $\begin{array}{c}\text { Burnup } \\
(\mathrm{GWd} / \mathrm{MTU})\end{array}$ & $\begin{array}{c}\text { T-Fuel } \\
\left({ }^{\circ} \mathrm{F}\right)\end{array}$ & $\begin{array}{c}\text { Spec. Vol. } \\
\left(\mathrm{ft}^{3} / \mathrm{lbm}\right)\end{array}$ & $\begin{array}{c}\text { Burnup } \\
(\mathrm{GWd} / \mathrm{MTU})\end{array}$ & $\begin{array}{c}\text { T-Fuel } \\
\left({ }^{\circ} \mathrm{F}\right)\end{array}$ & $\begin{array}{c}\text { Spec. Vol. } \\
\left(\mathrm{ft}^{3} / \mathrm{lbm}\right)\end{array}$ \\
\hline 1 & 1.994 & 988.4 & 0.0249 & 4.762 & 1036.2 & 0.0249 & 5.949 & 892.6 & 0.0233 \\
\hline 2 & 3.381 & 1207.7 & 0.0248 & 7.746 & 1210.6 & 0.0248 & 10.092 & 1064.8 & 0.0232 \\
\hline 3 & 4.211 & 1322.1 & 0.0246 & 9.350 & 1277.0 & 0.0246 & 12.635 & 1117.8 & 0.0231 \\
\hline 4 & 4.649 & 1377.1 & 0.0245 & 10.095 & 1295.1 & 0.0244 & 13.748 & 1131.4 & 0.0230 \\
\hline 5 & 4.866 & 1401.6 & 0.0243 & 10.426 & 1296.3 & 0.0243 & 14.208 & 1129.7 & 0.0229 \\
\hline 6 & 4.957 & 1411.0 & 0.0241 & 10.572 & 1293.8 & 0.0241 & 14.393 & 1122.7 & 0.0228 \\
\hline 7 & 4.975 & 1411.9 & 0.0239 & 10.632 & 1292.0 & 0.0239 & 14.458 & 1115.4 & 0.0226 \\
\hline 8 & 4.953 & 1406.4 & 0.0237 & 10.641 & 1290.6 & 0.0238 & 14.482 & 1113.9 & 0.0225 \\
\hline 9 & 4.935 & 1398.0 & 0.0236 & 10.632 & 1288.7 & 0.0236 & 14.530 & 1123.2 & 0.0224 \\
\hline 10 & 4.964 & 1392.8 & 0.0234 & 10.656 & 1287.1 & 0.0234 & 14.641 & 1140.3 & 0.0223 \\
\hline 11 & 5.044 & 1392.8 & 0.0233 & 10.742 & 1287.6 & 0.0233 & 14.786 & 1155.0 & 0.0222 \\
\hline 12 & 5.138 & 1395.6 & 0.0231 & 10.865 & 1290.7 & 0.0231 & 14.916 & 1162.1 & 0.0221 \\
\hline 13 & 5.193 & 1397.0 & 0.0229 & 10.965 & 1296.3 & 0.0230 & 14.961 & 1158.8 & 0.0220 \\
\hline 14 & 5.137 & 1389.9 & 0.0228 & 10.956 & 1303.3 & 0.0228 & 14.824 & 1143.4 & 0.0219 \\
\hline 15 & 4.901 & 1362.0 & 0.0227 & 10.704 & 1305.3 & 0.0227 & 14.383 & 1119.6 & 0.0218 \\
\hline 16 & 4.417 & 1299.3 & 0.0225 & 9.994 & 1288.0 & 0.0226 & 13.406 & 1087.1 & 0.0217 \\
\hline 17 & 3.546 & 1177.7 & 0.0224 & 8.384 & 1217.9 & 0.0224 & 11.288 & 1025.3 & 0.0216 \\
\hline 18 & 2.051 & 942.6 & 0.0223 & 5.101 & 1021.7 & 0.0223 & 6.923 & 885.7 & 0.0216 \\
\hline
\end{tabular}

\begin{tabular}{|c|c|c|c|c|c|c|c|c|c|}
\hline \multirow[b]{2}{*}{$\begin{array}{l}\text { Axial } \\
\text { Node }\end{array}$} & \multicolumn{3}{|c|}{ Datapoint (206.7 Cy5) } & \multicolumn{3}{|c|}{ Statepoint 62 (BOC Cy6) } & \multicolumn{3}{|c|}{ Statepoint $63(69.1 \mathrm{Cy} 6)$} \\
\hline & $\begin{array}{c}\text { Burnup } \\
\text { (GWd/MTU) }\end{array}$ & $\begin{array}{c}\text { T-Fuel } \\
\left({ }^{\circ} \mathrm{F}\right)\end{array}$ & $\begin{array}{c}\text { Spec. Vol. } \\
\left(\mathrm{ft}^{3} / \mathrm{lbm}\right)\end{array}$ & $\begin{array}{c}\text { Burnup } \\
\text { (GWd/MTU) }\end{array}$ & $\begin{array}{c}\text { T-Fuel } \\
\left({ }^{\circ} \mathrm{F}\right)\end{array}$ & $\begin{array}{l}\text { Spec. Vol. } \\
\left(\mathrm{tt}^{3} / \mathrm{lbm}\right)\end{array}$ & $\begin{array}{c}\text { Burnup } \\
\text { (GWd/MTU) }\end{array}$ & $\begin{array}{c}\text { T-Fuel } \\
\left({ }^{\circ} \mathrm{F}\right)\end{array}$ & $\begin{array}{c}\text { Spec. Vol. } \\
\left(\mathrm{ft}^{3} / \mathrm{lbm}\right)\end{array}$ \\
\hline & 206.7 Cy5 & 153.5 Cy 5 & 153.5 Cy 5 & $\mathrm{BOC} \mathrm{Cy}_{6}$ & 252.2 Cy5 & 252.2 Cy 5 & $69.1 \mathrm{Cy} 6$ & $41.5 \mathrm{Cy} 6$ & 41.5 Cy6 \\
\hline 1 & 7.062 & 831.9 & 0.0233 & 8.381 & 802.7 & 0.0233 & 9.624 & 917.3 & 0.0234 \\
\hline 2 & 12.501 & 1035.8 & 0.0233 & 15.038 & 969.2 & 0.0232 & 16.852 & 1006.1 & 0.0233 \\
\hline 3 & 15.552 & 1110.5 & 0.0232 & 18.482 & 1011.7 & 0.0231 & 20.562 & 1036.3 & 0.0232 \\
\hline 4 & 16.803 & 1124.0 & 0.0230 & 19.820 & 1020.0 & 0.0230 & 22.065 & 1061.3 & 0.0231 \\
\hline 5 & 17.298 & 1124.6 & 0.0229 & 20.349 & 1028.6 & 0.0229 & 22.705 & 1080.4 & 0.0230 \\
\hline 6 & 17.489 & 1122.4 & 0.0228 & 20.578 & 1043.8 & 0.0228 & 23.019 & 1095.1 & 0.0229 \\
\hline 7 & 17.555 & 1120.3 & 0.0227 & 20.709 & 1067.2 & 0.0227 & 23.223 & 1107.7 & 0.0227 \\
\hline 8 & 17.590 & 1119.7 & 0.0226 & 20.834 & 1096.9 & 0.0226 & 23.418 & 1119.0 & 0.0226 \\
\hline 9 & 17.676 & 1122.2 & 0.0225 & 21.030 & 1127.8 & 0.0225 & 23.664 & 1125.9 & 0.0225 \\
\hline 10 & 17.842 & 1126.3 & 0.0224 & 21.294 & 1150.4 & 0.0224 & 23.939 & 1124.4 & 0.0224 \\
\hline 11 & 18.028 & 1127.3 & 0.0223 & 21.518 & 1155.5 & 0.0223 & 24.136 & 1115.5 & 0.0222 \\
\hline 12 & 18.178 & 1125.3 & 0.0221 & 21.650 & 1143.0 & 0.0222 & 24.211 & 1101.3 & 0.0221 \\
\hline 13 & 18.227 & 1121.8 & 0.0220 & 21.644 & 1119.0 & 0.0220 & 24.121 & 1083.5 & 0.0220 \\
\hline 14 & 18.069 & 1116.5 & 0.0219 & 21.412 & 1091.4 & 0.0219 & 23.788 & 1064.2 & 0.0219 \\
\hline 15 & 17.583 & 1110.3 & 0.0218 & 20.855 & 1069.0 & 0.0218 & 23.1111 & 1042.7 & 0.0218 \\
\hline 16 & 16.501 & 1099.3 & 0.0217 & 19.683 & 1052.5 & 0.0217 & 21.772 & 1014.6 & 0.0217 \\
\hline 17 & 14.050 & 1058.4 & 0.0217 & 16.949 & 1022.4 & 0.0217 & 18.759 & 973.9 & 0.0216 \\
\hline 18 & 8.752 & 927.2 & 0.0216 & 10.745 & 909.3 & 0.0216 & 11.957 & 869.5 & 0.0216 \\
\hline
\end{tabular}


Table 4-20. Burnup and TH Feedback Parameters by Axial Node for Assembly D21

\begin{tabular}{|c|c|c|c|c|c|c|c|c|c|}
\hline \multirow[b]{2}{*}{$\begin{array}{l}\text { Axial } \\
\text { Node }\end{array}$} & \multicolumn{3}{|c|}{ Datapoint (126.6 Cy4) } & \multicolumn{3}{|c|}{ Statepoint 60 (BOC Cy5) } & \multicolumn{3}{|c|}{ Statepoint 61 (114.4 Cy5) } \\
\hline & $\begin{array}{c}\text { Burnup } \\
\text { (GWd/MTU) }\end{array}$ & $\begin{array}{c}\text { T-Fuel } \\
\left({ }^{\circ} \mathrm{F}\right)\end{array}$ & $\begin{array}{c}\text { Spec. Vol. } \\
\left(\mathrm{ft}^{3} / \mathrm{lbm}\right)\end{array}$ & $\begin{array}{c}\text { Burnup } \\
\text { (GWd/MTU) }\end{array}$ & $\begin{array}{c}\text { T-Fuel } \\
\left({ }^{\circ} \mathrm{F}\right)\end{array}$ & $\begin{array}{c}\text { Spec. Vol. } \\
\left(\mathrm{ft}^{3} / l \mathrm{bm}\right)\end{array}$ & $\begin{array}{c}\text { Burnup } \\
\text { (GWd/MTU) }\end{array}$ & $\begin{array}{l}\text { T-Fuel } \\
\left({ }^{\circ} \mathrm{F}\right)\end{array}$ & $\begin{array}{c}\text { Spec. Vol. } \\
\left(\mathrm{ft}^{3} / \mathrm{lbm}\right)\end{array}$ \\
\hline & 126.6 Cy 4 & 71.2 Cy 4 & $71.2 \mathrm{Cy} 4$ & BOC Cy5 & $203.4 \mathrm{Cy} 4$ & 203.4 Cy4 & 114.4 Cy5 & 58.2 Cy5 & 58.2 Cy5 \\
\hline 1 & 1.059 & 802.2 & 0.0237 & 2.619 & 847.7 & 0.0238 & 5.124 & 1078.7 & 0.0235 \\
\hline 2 & 1.861 & 942.9 & 0.0236 & 4.432 & 982.7 & 0.0237 & 8.396 & 1270.4 & 0.0234 \\
\hline 3 & 2.369 & 1024.2 & 0.0236 & 5.468 & 1039.9 & 0.0236 & 10.123 & 1335.9 & 0.0232 \\
\hline 4 & 2.649 & 1064.2 & 0.0235 & 5.975 & 1058.8 & 0.0235 & 10.907 & 1345.5 & 0.0231 \\
\hline 5 & 2.791 & 1082.8 & 0.0234 & 6.206 & 1062.8 & 0.0234 & 11.216 & 1333.4 & 0.0230 \\
\hline 6 & 2.855 & 1090.2 & 0.0233 & 6.309 & 1062.3 & 0.0233 & 11.256 & 1308.2 & 0.0228 \\
\hline 7 & 2.878 & 1091.8 & 0.0232 & 6.357 & 1061.3 & 0.0233 & 10.971 & 1242.7 & 0.0227 \\
\hline 8 & 2.883 & 1090.4 & 0.0231 & 6.383 & 1060.7 & 0.0232 & 10.200 & 1023.0 & 0.0226 \\
\hline 9 & 2.892 & 1088.2 & 0.0230 & 6.408 & 1060.6 & 0.0231 & 9.303 & 994.0 & 0.0225 \\
\hline 10 & 2.917 & 1087.1 & 0.0229 & 6.445 & 1061.0 & 0.0230 & 9.167 & 1002.9 & 0.0224 \\
\hline 11 & 2.956 & 1087.3 & 0.0228 & 6.497 & 1062.1 & 0.0229 & 9.292 & 1032.3 & 0.0224 \\
\hline 12 & 2.990 & 1087.5 & 0.0227 & 6.547 & 1064.2 & 0.0228 & 9.839 & 1156.3 & 0.0223 \\
\hline 13 & 2.992 & 1084.9 & 0.0227 & 6.566 & 1066.8 & 0.0227 & 10.689 & 1326.8 & 0.0222 \\
\hline 14 & 2.932 & 1075.4 & 0.0226 & 6.510 & 1068.7 & 0.0226 & 11.257 & 1347.3 & 0.0220 \\
\hline 15 & 2.774 & 1052.7 & 0.0225 & 6.308 & 1065.5 & 0.0225 & 11.101 & 1335.0 & 0.0219 \\
\hline 16 & 2.472 & 1007.9 & 0.0224 & 5.821 & 1046.2 & 0.0225 & 10.397 & 1305.4 & 0.0218 \\
\hline 17 & 1.944 & 923.1 & 0.0224 & 4.774 & 986.5 & 0.0224 & 8.748 & 1229.2 & 0.0217 \\
\hline 18 & 1.087 & 773.6 & 0.0223 & 2.794 & 835.4 & 0.0223 & 5.304 & 1025.3 & 0.0216 \\
\hline
\end{tabular}

\begin{tabular}{|c|c|c|c|c|c|c|c|c|c|}
\hline \multirow[b]{2}{*}{$\begin{array}{l}\text { Axial } \\
\text { Node }\end{array}$} & \multicolumn{3}{|c|}{ Datapoint (206.7 Cy5) } & \multicolumn{3}{|c|}{ Statepoint 62 (BOC Cy6) } & \multicolumn{3}{|c|}{ Statepoint 63 (69.1 Cy6) } \\
\hline & $\begin{array}{c}\text { Burnup } \\
\text { (GWd/MTU) }\end{array}$ & $\begin{array}{c}\text { T-Fuel } \\
\left({ }^{\circ} \mathrm{F}\right)\end{array}$ & $\begin{array}{c}\text { Spec. Vol. } \\
\left(\mathrm{ft}^{3} / \mathrm{lbm}\right)\end{array}$ & $\begin{array}{c}\text { Burnup } \\
\text { (GWd/MTU) }\end{array}$ & $\begin{array}{c}\text { T-Fuel } \\
\left({ }^{\circ} \mathrm{F}\right)\end{array}$ & $\begin{array}{c}\text { Spec. Vol. } \\
\text { (ft'/lbm) }\end{array}$ & $\begin{array}{c}\text { Burnup } \\
\text { (GWd/MTU) }\end{array}$ & $\begin{array}{c}\text { T-Fuel } \\
\left({ }^{\circ} \mathrm{F}\right)\end{array}$ & $\begin{array}{c}\text { Spec. Vol. } \\
\left(\mathrm{ft}^{3} / \mathrm{lbm}\right)\end{array}$ \\
\hline & 206.7 Cy5 & 153.5 Cy5 & 153.5 Cy5 & BOC Cy6 & 252.2 Cy 5 & 252.2 Cy 5 & 69.1 Cy6 & 41.5 Cy 6 & $41.5 \mathrm{Cy} 6$ \\
\hline 1 & 7.296 & 1059.0 & 0.0235 & 9.484 & 966.2 & 0.0239 & 10.641 & 888.3 & 0.0235 \\
\hline 2 & 11.643 & 1212.6 & 0.0234 & 14.776 & 1068.9 & 0.0238 & 16.548 & 997.5 & 0.0234 \\
\hline 3 & 13.780 & 1263.3 & 0.0232 & 17.226 & 1097.6 & 0.0236 & 19.333 & 1051.8 & 0.0233 \\
\hline 4 & 14.674 & 1272.3 & 0.0231 & 18.188 & 1106.1 & 0.0235 & 20.498 & 1088.0 & 0.0232 \\
\hline 5 & 14.990 & 1269.2 & 0.0229 & 18.531 & 1118.5 & 0.0234 & 20.968 & 1112.1 & 0.0231 \\
\hline 6 & 15.005 & 1263.9 & 0.0228 & 18.598 & 1144.8 & 0.0233 & 21.133 & 1130.5 & 0.0230 \\
\hline 7 & 14.635 & 1255.4 & 0.0227 & 18.324 & 1195.5 & 0.0231 & 20.961 & 1152.0 & 0.0229 \\
\hline 8 & 13.424 & 1201.1 & 0.0225 & 17.159 & 1290.8 & 0.0230 & 19.950 & 1192.0 & 0.0227 \\
\hline 9 & 11.588 & 1022.3 & 0.0224 & 15.184 & 1413.0 & 0.0228 & 18.173 & 1251.3 & 0.0226 \\
\hline 10 & 11.318 & 893.1 & 0.0224 & 15.020 & 1470.0 & 0.0227 & 18.053 & 1259.8 & 0.0224 \\
\hline 11 & 11.450 & 989.3 & 0.0223 & 15.196 & 1480.3 & 0.0225 & 18.205 & 1251.0 & 0.0223 \\
\hline 12 & 12.104 & 997.7 & 0.0222 & 15.796 & 1437.7 & 0.0223 & 18.717 & 1224.3 & 0.0222 \\
\hline 13 & 13.687 & 1103.0 & 0.0221 & 17.468 & 1335.5 & 0.0221 & 20.197 & 1166.3 & 0.0220 \\
\hline 14 & 14.942 & 1222.5 & 0.0220 & 18.809 & 1239.1 & 0.0220 & 21.338 & 1113.7 & 0.0219 \\
\hline 15 & 14.934 & 1249.4 & 0.0219 & 18.753 & 1188.6 & 0.0219 & 21.130 & 1082.5 & 0.0218 \\
\hline 16 & 14.191 & 1247.9 & 0.0218 & 17.928 & 1161.2 & 0.0218 & 20.115 & 1048.2 & 0.0217 \\
\hline 17 & 12.197 & 1204.0 & 0.0217 & 15.638 & 1122.5 & 0.0217 & 17.517 & 998.3 & 0.0216 \\
\hline 18 & 7.615 & 1037.2 & 0.0216 & 10.008 & 989.2 & 0.0216 & 11.253 & 881.4 & 0.0216 \\
\hline
\end{tabular}


Table 4-21. Burnup and TH Feedback Parameters by Axial Node for Assembly D25

\begin{tabular}{|c|c|c|c|c|c|c|c|c|c|}
\hline \multirow[b]{2}{*}{$\begin{array}{l}\text { Axial } \\
\text { Node }\end{array}$} & \multicolumn{3}{|c|}{ Datapoint (126.6 Cy4) } & \multicolumn{3}{|c|}{ Statepoint 60 (BOC Cy5) } & \multicolumn{3}{|c|}{ Statepoint 61 (114.4 Cy5) } \\
\hline & $\begin{array}{c}\text { Burnup } \\
\text { (GWd/MTU) }\end{array}$ & $\begin{array}{l}\text { T-Fuel } \\
\left({ }^{\circ} \mathrm{F}\right)\end{array}$ & $\begin{array}{l}\text { Spec. Vol. } \\
\left(\mathrm{ft}^{3} / \mathrm{lbm}\right)\end{array}$ & $\begin{array}{c}\text { Burnup } \\
\text { (GWd/MTU) }\end{array}$ & $\begin{array}{l}\text { T-Fuel } \\
\left({ }^{\circ} \mathrm{F}\right)\end{array}$ & $\begin{array}{l}\text { Spec. Vol. } \\
\left(\mathrm{ft}^{3} / \mathrm{lbm}\right)\end{array}$ & \begin{tabular}{|c|} 
Burnup \\
(GWd/MTU)
\end{tabular} & $\begin{array}{l}\text { T-Fuel } \\
\left({ }^{\circ} \mathrm{F}\right)\end{array}$ & $\begin{array}{l}\text { Spec. Vol. } \\
\left(\mathrm{ft}^{3} / \mathrm{lbm}\right)\end{array}$ \\
\hline & 126.6 Cy 4 & 71.2 Cy 4 & $71.2 \mathrm{Cy} 4$ & BOC Cy5 & $203.4 \mathrm{Cy} 4$ & $203.4 \mathrm{Cy} 4$ & 114.4 Cy 5 & 58.2 Cy5 & 58.2 Cy 5 \\
\hline 1 & 1.643 & 918.3 & 0.0244 & 3.945 & 963.6 & 0.0244 & 6.492 & 1075.9 & 0.0237 \\
\hline 2 & 2.826 & 1114.4 & 0.0243 & 6.511 & 1128.7 & 0.0243 & 10.459 & 1242.7 & 0.0236 \\
\hline 3 & 3.550 & 1218.8 & 0.0242 & 7.920 & 1191.2 & 0.0242 & 12.483 & 1290.9 & 0.0234 \\
\hline 4 & 3.938 & 1268.7 & 0.0241 & 8.583 & 1208.7 & 0.0241 & 13.355 & 1289.2 & 0.0233 \\
\hline 5 & 4.129 & 1290.7 & 0.0239 & 8.876 & 1210.2 & 0.0239 & 13.684 & 1273.2 & 0.0231 \\
\hline 6 & 4.205 & 1298.6 & 0.0238 & 8.998 & 1208.0 & 0.0238 & 13.778 & 1255.4 & 0.0230 \\
\hline 7 & 4.212 & 1298.2 & 0.0236 & 9.035 & 1206.0 & 0.0236 & 13.773 & 1240.9 & 0.0229 \\
\hline 8 & 4.180 & 1291.6 & 0.0235 & 9.020 & 1204.1 & 0.0235 & 13.726 & 1233.3 & 0.0227 \\
\hline 9 & 4.142 & 1281.7 & 0.0233 & 8.978 & 1201.5 & 0.0234 & 13.675 & 1234.4 & 0.0226 \\
\hline 10 & 4.135 & 1273.3 & 0.0232 & 8.949 & 1198.9 & 0.0232 & 13.652 & 1241.7 & 0.0225 \\
\hline 11 & 4.167 & 1269.1 & 0.0231 & 8.968 & 1198.0 & 0.0231 & 13.672 & 1250.3 & 0.0224 \\
\hline 12 & 4.220 & 1268.6 & 0.0230 & 9.029 & 1199.6 & 0.0230 & 13.716 & 1255.9 & 0.0222 \\
\hline 13 & 4.256 & 1268.7 & 0.0228 & 9.098 & 1204.1 & 0.0229 & 13.743 & 1256.0 & 0.0221 \\
\hline 14 & 4.218 & 1263.3 & 0.0227 & 9.101 & 1210.0 & 0.0228 & 13.683 & 1250.9 & 0.0220 \\
\hline 15 & 4.041 & 1241.6 & 0.0226 & 8.916 & 1211.6 & 0.0226 & 13.408 & 1242.3 & 0.0219 \\
\hline 16 & 3.653 & 1189.9 & 0.0225 & 8.338 & 1195.6 & 0.0225 & 12.651 & 1224.4 & 0.0218 \\
\hline 17 & 2.926 & 1083.8 & 0.0224 & 6.968 & 1131.8 & 0.0224 & 10.777 & 1168.4 & 0.0217 \\
\hline 18 & 1.676 & 877.0 & 0.0223 & 4.187 & 948.5 & 0.0223 & 6.654 & 1000.1 & 0.0216 \\
\hline
\end{tabular}

\begin{tabular}{|c|c|c|c|c|c|c|c|c|c|}
\hline \multirow[b]{2}{*}{$\begin{array}{l}\text { Axial } \\
\text { Node }\end{array}$} & \multicolumn{3}{|c|}{ Datapoint (206.7 Cy5) } & \multicolumn{3}{|c|}{ Statepolnt 62 (BOC Cy6) } & \multicolumn{3}{|c|}{ Statepoint 63 (69.1 Cy6) } \\
\hline & $\begin{array}{c}\text { Burnup } \\
\text { (GWd/MTU) }\end{array}$ & $\begin{array}{l}\text { T-Fuel } \\
\left({ }^{\circ} \mathrm{F}\right)\end{array}$ & $\begin{array}{l}\text { Spec. Vol. } \\
\left(\mathrm{ft}^{3} / \mathrm{lbm}\right)\end{array}$ & \begin{tabular}{|c|} 
Burnup \\
(GWd/MTU)
\end{tabular} & $\begin{array}{l}\text { T-Fuel } \\
\left({ }^{\circ} \mathrm{F}\right)\end{array}$ & $\begin{array}{l}\text { Spec. Vol. } \\
\left(\mathrm{ft}^{3} / \mathrm{lbm}\right)\end{array}$ & $\begin{array}{c}\text { Burnup } \\
\text { (GWd/MTU) }\end{array}$ & $\begin{array}{l}\text { T-Fuel } \\
\left({ }^{\circ} \mathrm{F}\right)\end{array}$ & $\begin{array}{l}\text { Spec. Vol } \\
\left(\mathrm{ft}^{3} / / \mathrm{bm}\right)\end{array}$ \\
\hline & 206.7 Cy 5 & 153.5 Cy5 & $153.5 \mathrm{Cy} 5$ & BOC Cy6 & 252.2 Cy5 & 252.2 Cy5 & 69.1 Cy 6 & $41.5 \mathrm{Cy} 6$ & 41.5 Cy 6 \\
\hline 1 & 8.624 & 1038.9 & 0.0236 & 10.779 & 946.1 & 0.0235 & $\$ 1.935$ & 881.7 & 0.0233 \\
\hline 2 & 13.603 & 1175.2 & 0.0235 & 16.656 & 1042.8 & 0.0234 & 18.379 & 976.6 & 0.0232 \\
\hline 3 & 15.984 & 1215.0 & 0.0234 & 19.309 & 1063.2 & 0.0233 & 21.311 & 1016.3 & 0.0232 \\
\hline 4 & 16.922 & 1215.8 & 0.0232 & 20.284 & 1066.0 & 0.0232 & 22.455 & 1044.5 & 0.0230 \\
\hline 5 & 17.233 & 1208.2 & 0.0231 & 20.595 & 1072.4 & 0.0231 & 22.881 & 1065.7 & 0.0229 \\
\hline 6 & 17.299 & 1201.0 & 0.0230 & 20.684 & 1087.8 & 0.0230 & 23.059 & 1082.2 & 0.0228 \\
\hline 7 & 17.273 & 1195.7 & 0.0228 & 20.714 & 1112.1 & 0.0228 & 23.167 & 1096.5 & 0.0227 \\
\hline 8 & 17.217 & 1192.1 & 0.0227 & 20.739 & 1142.1 & 0.0227 & 23.264 & 1109.3 & 0.0226 \\
\hline 9 & 17.168 & 1189.7 & 0.0226 & 20.774 & 1171.4 & 0.0226 & 23.356 & 1118.5 & 0.0225 \\
\hline 10 & 17.155 & 1187.4 & 0.0225 & 20.823 & 1191.4 & 0.0225 & 23.423 & 1119.9 & 0.0223 \\
\hline 11 & 17.186 & 1184.6 & 0.0223 & 20.874 & 1195.6 & 0.0223 & 23.448 & 1112.8 & 0.0222 \\
\hline 12 & 17.241 & 1181.6 & 0.0222 & 20.906 & 1183.3 & 0.0222 & 23.416 & 1098.2 & 0.0221 \\
\hline 13 & 17.283 & 1179.7 & 0.0221 & 20.900 & 1160.0 & 0.0221 & 23.313 & 1077.7 & 0.0220 \\
\hline 14 & 17.245 & 1180.2 & 0.0220 & 20.813 & 1134.3 & 0.0220 & 23.111 & 1053.9 & 0.0219 \\
\hline 15 & 16.990 & 1183.2 & 0.0219 & 20.529 & 1114.0 & 0.0219 & 22.695 & 1027.9 & 0.0218 \\
\hline 16 & 16.201 & 1182.4 & 0.0218 & 19.695 & 1100.3 & 0.0218 & 21.689 & 996.5 & 0.0217 \\
\hline 17 & 14.038 & 1148.9 & 0.0217 & 17.289 & 1074.5 & 0.0217 & 19.010 & 953.4 & 0.0216 \\
\hline 18 & 8.879 & 1005.5 & 0.0216 & 11.174 & 959.2 & 0.0216 & 12.326 & 853.3 & 0.0216 \\
\hline
\end{tabular}


Table 4-22. Burnup and TH Feedback Parameters by Axial Node for Assembly D25a

\begin{tabular}{|c|c|c|c|c|c|c|c|c|c|}
\hline \multirow[b]{2}{*}{$\begin{array}{c}\text { Axial } \\
\text { Node } \\
\end{array}$} & \multicolumn{3}{|c|}{ Datapoint (126.6 Cy4) } & \multicolumn{3}{|c|}{ Statepoint 60 (BOC Cy5) } & \multicolumn{3}{|c|}{ Statepoint 61 (114.4 Cy5) } \\
\hline & $\begin{array}{c}\text { Burnup } \\
\text { (GWd/MTU) }\end{array}$ & $\begin{array}{c}\text { T-Fuel } \\
\left({ }^{\circ} \mathrm{F}\right)\end{array}$ & $\begin{array}{c}\text { Spec. Vol. } \\
\left(\mathrm{ft}^{3} / \mathrm{bm}\right)\end{array}$ & $\begin{array}{c}\text { Burnup } \\
\text { (GWd/MTU) }\end{array}$ & $\begin{array}{c}\text { T-Fuel } \\
\left({ }^{\circ} \mathrm{F}\right) \\
\end{array}$ & $\begin{array}{c}\text { Spec. Vol. } \\
\left(\mathrm{ft}^{3} / \mathrm{lbm}\right)\end{array}$ & \begin{tabular}{|c|} 
Burnup \\
(GWd/MTU)
\end{tabular} & $\begin{array}{c}\text { T-Fuel } \\
\left({ }^{\circ} \mathrm{F}\right)\end{array}$ & $\begin{array}{c}\text { Spec. Vol. } \\
\left(\mathrm{ft}^{3} / \mathrm{lbm}\right)\end{array}$ \\
\hline & 126.6 Cy 4 & $71.2 \mathrm{Cy} 4$ & 71.2 Cy4 & BOC Cy 5 & 203.4 Cy 4 & 203.4 Cy 4 & 114.4 Cy5 & 58.2 Cy5 & 58.2 Cy5 \\
\hline 1 & 1.643 & 918.3 & 0.0244 & 3.947 & 963.6 & 0.0244 & 5.675 & 1031.7 & 0.0238 \\
\hline 2 & 2.826 & 1114.4 & 0.0243 & 6.515 & 1128.7 & 0.0243 & 9.812 & 1255.0 & 0.0237 \\
\hline 3 & 3.550 & 1218.8 & 0.0242 & 7.924 & 1191.2 & 0.0242 & 12.407 & 1308.8 & 0.0235 \\
\hline 4 & 3.938 & 1268.7 & 0.0241 & 8.589 & 1208.7 & 0.0241 & 13.450 & 1307.2 & 0.0234 \\
\hline 5 & 4.129 & 1290.7 & 0.0239 & 8.882 & 1210.2 & 0.0239 & 13.812 & 1290.4 & 0.0232 \\
\hline 6 & 4.205 & 1298.6 & 0.0238 & 9.004 & 1208.0 & 0.0238 & 13.898 & 1268.4 & 0.0231 \\
\hline 7 & 4.212 & 1298.2 & 0.0236 & 9.041 & 1206.0 & 0.0236 & 13.854 & 1245.0 & 0.0229 \\
\hline 8 & 4.180 & 1291.6 & 0.0235 & 9.027 & 1204.1 & 0.0235 & 13.763 & 1229.0 & 0.0228 \\
\hline 9 & 4.142 & 1281.7 & 0.0233 & 8.985 & 1201.5 & 0.0234 & 13.706 & 1231.6 & 0.0227 \\
\hline 10 & 4.135 & 1273.3 & 0.0232 & 8.958 & 1198.9 & 0.0232 & 13.726 & 1249.7 & 0.0225 \\
\hline 11 & 4.167 & 1269.1 & 0.0231 & 8.977 & 1198.0 & 0.0231 & 13.807 & 1272.4 & 0.0224 \\
\hline 12 & 4.220 & 1268.6 & 0.0230 & 9.039 & 1199.6 & 0.0230 & 13.922 & 1292.9 & 0.0223 \\
\hline 13 & 4.256 & 1268.7 & 0.0228 & 9.106 & 1204.1 & 0.0229 & 14.000 & 1302.1 & 0.0222 \\
\hline 14 & 4.218 & 1263.3 & 0.0227 & 9.109 & 1210.0 & 0.0228 & 13.937 & 1294.6 & 0.0220 \\
\hline 15 & 4.041 & 1241.6 & 0.0226 & 8.922 & 1211.6 & 0.0226 & 13.634 & 1278.7 & 0.0219 \\
\hline 16 & 3.653 & 1189.9 & 0.0225 & 8.343 & 1195.6 & 0.0225 & 12.851 & 1255.4 & 0.0218 \\
\hline 17 & 2.926 & 1083.8 & 0.0224 & 6.972 & 1131.8 & 0.0224 & 10.942 & 1193.9 & 0.0217 \\
\hline 18 & 1.676 & 877.0 & 0.0223 & 4.189 & 948.5 & 0.0223 & 6.753 & 1016.2 & 0.0216 \\
\hline
\end{tabular}

\begin{tabular}{|c|c|c|c|c|c|c|c|c|c|}
\hline \multirow[b]{2}{*}{$\begin{array}{l}\text { Axial } \\
\text { Node }\end{array}$} & \multicolumn{3}{|c|}{ Datapoint (206.7 Cy5) } & \multicolumn{3}{|c|}{ Statepoint 62 (BOC Cy6) } & \multicolumn{3}{|c|}{ Statepoint 63 (69.1 Cy6) } \\
\hline & $\begin{array}{c}\text { Burnup } \\
\text { (GWd/MTU) }\end{array}$ & $\begin{array}{l}\text { T-Fuel } \\
\left({ }^{\circ} \mathrm{F}\right)\end{array}$ & $\begin{array}{c}\text { Spec. Vol. } \\
\left(\mathrm{ft}^{3} / \mathrm{bm}\right)\end{array}$ & $\begin{array}{c}\text { Burnup } \\
\text { (GWd/MTU) }\end{array}$ & $\begin{array}{c}\text { T-Fuel } \\
\left({ }^{\circ} \mathrm{F}\right)\end{array}$ & $\begin{array}{c}\text { Spec. Vol. } \\
\left(\mathrm{tt}^{3} / \mathrm{lbm}\right)\end{array}$ & $\begin{array}{c}\text { Burnup } \\
\text { (GWd/MTU) }\end{array}$ & $\begin{array}{l}\text { T-fuel } \\
\left({ }^{\circ} \mathrm{F}\right)\end{array}$ & $\begin{array}{c}\text { Spec. Vol. } \\
\left(\mathrm{ft}^{3} / \mathrm{lbm}\right)\end{array}$ \\
\hline & 206.7 Cy5 & 153.5 Cy5 & $153.5 \mathrm{Cy} 5$ & BOC Cy6 & 252.2 Cy5 & 252.2 Cy5 & 69.1 Cy 6 & 41.5 Cy 6 & 41.5 Cy 6 \\
\hline 1 & 7.136 & 911.4 & 0.0237 & 8.758 & 853.1 & 0.0236 & 9.993 & 913.2 & 0.0233 \\
\hline 2 & 12.848 & 1151.6 & 0.0236 & 15.853 & 1033.1 & 0.0235 & 17.624 & 993.0 & 0.0232 \\
\hline 3 & 15.976 & 1226.2 & 0.0234 & 19.357 & 1069.6 & 0.0234 & 21.359 & 1016.9 & 0.0231 \\
\hline 4 & 17.107 & 1230.5 & 0.0233 & 20.533 & 1073.5 & 0.0233 & 22.683 & 1039.1 & 0.0230 \\
\hline 5 & 17.459 & 1224.3 & 0.0232 & 20.896 & 1082.6 & 0.0232 & 23.151 & 1057.8 & 0.0229 \\
\hline 6 & 17.515 & 1217.5 & 0.0230 & 20.989 & 1103.0 & 0.0231 & 23.328 & 1073.1 & 0.0228 \\
\hline 7 & 17.436 & 1211.0 & 0.0229 & 20.993 & 1137.6 & 0.0230 & 23.401 & 1086.0 & 0.0227 \\
\hline 8 & 17.310 & 1203.9 & 0.0227 & 20.989 & 1185.4 & 0.0228 & 23.456 & 1096.4 & 0.0226 \\
\hline 9 & 17.236 & 1197.8 & 0.0226 & 21.058 & 1236.8 & 0.0227 & 23.564 & 1101.9 & 0.0224 \\
\hline 10 & 17.269 & 1194.7 & 0.0225 & 21.206 & 1271.2 & 0.0225 & 23.717 & 1100.2 & 0.0223 \\
\hline 11 & 17.375 & 1192.6 & 0.0224 & 21.351 & 1278.4 & 0.0224 & 23.831 & 1091.6 & 0.0222 \\
\hline 12 & 17.528 & 1192.4 & 0.0223 & 21.464 & 1257.5 & 0.0222 & 23.883 & 1077.1 & 0.0221 \\
\hline 13 & 17.654 & 1194.9 & 0.0221 & 21.500 & 1217.3 & 0.0221 & 23.833 & 1058.5 & 0.0220 \\
\hline 14 & 17.630 & 1198.2 & 0.0220 & 21.372 & 1171.1 & 0.0220 & 23.603 & 1037.6 & 0.0219 \\
\hline 15 & 17.348 & 1202.2 & 0.0219 & 21.019 & 1136.6 & 0.0219 & 23.129 & 1013.9 & 0.0218 \\
\hline 16 & 16.530 & 1201.7 & 0.0218 & 20.136 & 1116.3 & 0.0218 & 22.082 & 984.2 & 0.0217 \\
\hline 17 & 14.320 & 1167.6 & 0.0217 & 17.668 & 1088.0 & 0.0217 & 19.352 & 943.2 & 0.0216 \\
\hline 18 & 9.057 & 1018.7 & 0.0216 & 11.420 & 969.4 & 0.0216 & 12.551 & 847.0 & 0.0216 \\
\hline
\end{tabular}


Table 4-23. Burnup and TH Feedback Parameters by Axial Node for Assembly D27

\begin{tabular}{|c|c|c|c|c|c|c|c|c|c|}
\hline \multirow{2}{*}{$\begin{array}{c}\text { Axial } \\
\text { Node }\end{array}$} & \multicolumn{3}{|c|}{ Datapoint (126.6 Cy4) } & \multicolumn{2}{c|}{ Statepoint 60 (BOC Cy5) } & \multicolumn{3}{c|}{ Statepoint 61 (114.4 Cy5) } \\
\hline & $\begin{array}{c}\text { Burnup } \\
(\text { GWd/MTU) }\end{array}$ & $\begin{array}{c}\text { T-Fuel } \\
\left({ }^{\circ} \mathrm{F}\right)\end{array}$ & $\begin{array}{c}\text { Spec. Vol. } \\
\left(\mathrm{ft}^{3} / \mathrm{lbm}\right)\end{array}$ & $\begin{array}{c}\text { Burnup } \\
(\mathrm{GWd} / \mathrm{MTU})\end{array}$ & $\begin{array}{c}\text { T-Fuel } \\
\left({ }^{\circ} \mathrm{F}\right)\end{array}$ & $\begin{array}{c}\text { Spec. Vol. } \\
\left(\mathrm{ft}^{3} / \mathrm{lbm}\right)\end{array}$ & $\begin{array}{c}\text { Burnup } \\
(\mathrm{GWd} / \mathrm{MTU})\end{array}$ & $\begin{array}{c}\text { T-Fuel } \\
\left({ }^{\circ} \mathrm{F}\right)\end{array}$ & $\begin{array}{c}\text { Spec. Vol. } \\
\left(\mathrm{ft}^{3} / \mathrm{lbm}\right)\end{array}$ \\
\hline 1 & $126.6 \mathrm{Cy} 4$ & $71.2 \mathrm{Cy} 4$ & $71.2 \mathrm{Cy} 4$ & BOC Cy5 & $203.4 \mathrm{Cy} 4$ & $203.4 \mathrm{Cy} 4$ & 114.4 Cy5 & $58.2 \mathrm{Cy5}$ & $58.2 \mathrm{Cy5}$ \\
\hline 2 & 3.849 & 963.9 & 0.0248 & 4.422 & 1018.6 & 0.0248 & 6.507 & 993.7 & 0.0236 \\
\hline 3 & 4.155 & 1315.8 & 0.0246 & 9.156 & 1262.0 & 0.0245 & 13.238 & 1212.8 & 0.0234 \\
\hline 4 & 4.631 & 1372.3 & 0.0244 & 9.941 & 1278.2 & 0.0244 & 14.359 & 1230.8 & 0.0232 \\
\hline 5 & 4.857 & 1396.4 & 0.0242 & 10.277 & 1278.1 & 0.0242 & 14.834 & 1229.1 & 0.0231 \\
\hline 6 & 4.943 & 1404.7 & 0.0240 & 10.413 & 1275.1 & 0.0240 & 14.993 & 1216.9 & 0.0229 \\
\hline 7 & 4.944 & 1403.5 & 0.0239 & 10.449 & 1272.8 & 0.0239 & 14.968 & 1196.2 & 0.0228 \\
\hline 8 & 4.895 & 1395.0 & 0.0237 & 10.423 & 1271.0 & 0.0237 & 14.828 & 1174.9 & 0.0227 \\
\hline 9 & 4.840 & 1382.4 & 0.0235 & 10.364 & 1268.7 & 0.0235 & 14.672 & 1169.4 & 0.0226 \\
\hline 10 & 4.818 & 1371.0 & 0.0234 & 10.316 & 1266.0 & 0.0234 & 14.607 & 1180.8 & 0.0225 \\
\hline 11 & 4.841 & 1364.0 & 0.0232 & 10.314 & 1264.5 & 0.0233 & 14.660 & 1202.7 & 0.0224 \\
\hline 12 & 4.892 & 1361.3 & 0.0231 & 10.360 & 1265.5 & 0.0231 & 14.809 & 1230.1 & 0.0222 \\
\hline 13 & 4.929 & 1360.7 & 0.0229 & 10.426 & 1269.8 & 0.0230 & 14.978 & 1251.3 & 0.0221 \\
\hline 14 & 4.892 & 1355.5 & 0.0228 & 10.434 & 1276.3 & 0.0228 & 15.017 & 1254.2 & 0.0220 \\
\hline 15 & 4.708 & 1334.0 & 0.0226 & 10.255 & 1279.5 & 0.0227 & 14.739 & 1238.5 & 0.0219 \\
\hline 16 & 4.295 & 1280.8 & 0.0225 & 9.665 & 1265.8 & 0.0226 & 13.863 & 1202.3 & 0.0218 \\
\hline 17 & 3.492 & 1168.8 & 0.0224 & 8.189 & 1201.4 & 0.0224 & 11.743 & 1122.8 & 0.0217 \\
\hline 18 & 2.042 & 940.4 & 0.0223 & 5.024 & 1011.8 & 0.0223 & 7.223 & 950.6 & 0.0216 \\
\hline
\end{tabular}

\begin{tabular}{|c|c|c|c|c|c|c|c|c|c|}
\hline \multirow[b]{2}{*}{$\begin{array}{l}\text { Axial } \\
\text { Node }\end{array}$} & \multicolumn{3}{|c|}{ Datapoint (206.7 Cy5) } & \multicolumn{3}{|c|}{ Statepoint 62 (BOC Cy6) } & \multicolumn{3}{|c|}{ Statepoint 63 (69.1 Cy6) } \\
\hline & $\begin{array}{c}\text { Burnup } \\
\text { (GWd/MTU) }\end{array}$ & $\begin{array}{l}\text { T-Fuel } \\
\left({ }^{\circ} \mathrm{F}\right)\end{array}$ & $\begin{array}{l}\text { Spec. Vol. } \\
\left(\mathrm{tt}^{3} / \mathrm{lbm}\right)\end{array}$ & $\begin{array}{c}\text { Burnup } \\
\text { (GWd/MTU) }\end{array}$ & $\begin{array}{l}\text { T-Fuel } \\
\left({ }^{\circ} \mathrm{F}\right)\end{array}$ & $\begin{array}{l}\text { Spec. Vol. } \\
\left(\mathrm{ft}^{3} / / \mathrm{bm}\right)\end{array}$ & $\begin{array}{c}\text { Burnup } \\
\text { (GWd/MTU) }\end{array}$ & $\begin{array}{c}\text { T-Fuel } \\
\left({ }^{\circ} \mathrm{F}\right)\end{array}$ & $\begin{array}{l}\text { Spec. Vol. } \\
\left(\mathrm{ft}^{3} / \mathrm{lbm}\right)\end{array}$ \\
\hline & 206.7 Cy 5 & 153.5 Cy5 & 153.5 Cy 5 & BOC Cy 6 & 252.2 Cy 5 & 252.2 Cy 5 & 69.1 Cy 6 & 41.5 Cy6 & 41.5 Cy 6 \\
\hline 1 & 8.387 & 989.3 & 0.0236 & 10.344 & 917.1 & 0.0235 & 11.237 & 809.9 & 0.0230 \\
\hline 2 & 13.715 & 1126.3 & 0.0235 & 16.576 & 1015.8 & 0.0235 & 17.974 & 896.7 & 0.0229 \\
\hline 3 & 16.548 & 1175.6 & 0.0233 & 19.737 & 1041.0 & 0.0234 & 21.410 & 935.4 & 0.0228 \\
\hline 4 & 17.810 & 1186.5 & 0.0232 & 21.089 & 1048.2 & 0.0233 & 22.926 & 960.4 & 0.0228 \\
\hline 5 & 18.322 & 1186.4 & 0.0231 & 21.638 & 1058.6 & 0.0231 & 23.579 & 977.3 & 0.0227 \\
\hline 6 & 18.480 & 1183.6 & 0.0229 & 21.848 & 1079.0 & 0.0230 & 23.858 & 988.9 & 0.0226 \\
\hline 7 & 18.430 & 1178.7 & 0.0228 & 21.880 & 1112.3 & 0.0229 & 23.940 & 997.2 & 0.0225 \\
\hline 8 & 18.230 & 1168.5 & 0.0227 & 21.786 & 1157.9 & 0.0228 & 23.881 & 1003.5 & 0.0224 \\
\hline 9 & 18.006 & 1154.8 & 0.0226 & 21.668 & 1206.1 & 0.0226 & 23.783 & 1006.8 & 0.0223 \\
\hline 10 & 17.917 & 1146.8 & 0.0224 & 21.666 & 1238.4 & 0.0225 & 23.779 & 1005.4 & 0.0222 \\
\hline 11 & 17.993 & 1145.0 & 0.0223 & 21.776 & 1245.1 & 0.0224 & 23.870 & 999.6 & 0.0221 \\
\hline 12 & 18.206 & 1148.7 & 0.0222 & 21.967 & 1225.8 & 0.0222 & 24.024 & 990.0 & 0.0220 \\
\hline 13 & 18.479 & 1159.1 & 0.0221 & 22.186 & 1187.6 & 0.0221 & 24.191 & 977.5 & 0.0219 \\
\hline 14 & 18.615 & 1171.0 & 0.0220 & 22.264 & 1146.5 & 0.0220 & 24.200 & 962.6 & 0.0218 \\
\hline 15 & 18.371 & 1176.7 & 0.0219 & 21.965 & 1114.9 & 0.0219 & 23.804 & 944.7 & 0.0218 \\
\hline 16 & 17.410 & 1169.2 & 0.0218 & 20.910 & 1092.6 & 0.0218 & 22.601 & 920.2 & 0.0217 \\
\hline 17 & 14.909 & 1123.7 & 0.0217 & 18.094 & 1059.3 & 0.0217 & 19.528 & 881.5 & 0.0216 \\
\hline 18 & 9.309 & 973.5 & 0.0216 & 11.496 & 938.7 & 0.0216 & 12.421 & 792.1 & 0.0216 \\
\hline
\end{tabular}


Table 4-24. Burnup and TH Feedback Parameters by Axial Node for Assembly D27a

\begin{tabular}{|c|c|c|c|c|c|c|c|c|c|}
\hline \multirow[b]{2}{*}{$\begin{array}{l}\text { Axial } \\
\text { Node }\end{array}$} & \multicolumn{3}{|c|}{ Datapoint (126.6 Cy4) } & \multicolumn{3}{|c|}{ Statepoint 60 (BOC Cy5) } & \multicolumn{3}{|c|}{ Statepoint 61 (114.4 Cy5) } \\
\hline & $\begin{array}{c}\text { Burnup } \\
\text { (GWd/MTU) }\end{array}$ & $\begin{array}{c}\text { T-Fuel } \\
\left({ }^{\circ} \mathrm{F}\right)\end{array}$ & $\begin{array}{c}\text { Spec. Vol. } \\
\left(\mathrm{ft}^{3} / \mathrm{bm}\right)\end{array}$ & $\begin{array}{c}\text { Burnup } \\
\text { (GWd/MTU) }\end{array}$ & $\begin{array}{l}\text { T-Fuel } \\
\left({ }^{\circ} \mathrm{F}\right)\end{array}$ & $\begin{array}{c}\text { Spec. Vol. } \\
\left(\mathrm{ft}^{3} / \mathrm{lbm}\right)\end{array}$ & $\begin{array}{c}\text { Burnup } \\
\text { (GWd/MTU) }\end{array}$ & $\begin{array}{c}\text { T-Fuel } \\
\left({ }^{\circ} \mathrm{F}\right)\end{array}$ & $\begin{array}{c}\text { Spec. Vol. } \\
\left(\mathrm{ft}^{3} / \mathrm{lbm}\right)\end{array}$ \\
\hline & $126.6 \mathrm{Cy} 4$ & 71.2 Cy4 & $71.2 \mathrm{Cy} 4$ & $\mathrm{BOC}$ Cy 5 & 203.4 Cy 4 & $203.4 \mathrm{Cy} 4$ & 114.4 Cy 5 & 58.2 Cy 5 & 58.2 Cy5 \\
\hline 1 & 1.849 & 963.9 & 0.0248 & 4.422 & 1018.6 & 0.0248 & 6.507 & 993.7 & 0.0236 \\
\hline 2 & 3.256 & 1193.5 & 0.0247 & 7.445 & 1196.0 & 0.0247 & 10.831 & 1150.7 & 0.0235 \\
\hline 3 & 4.155 & 1315.8 & 0.0246 & 9.156 & 1262.0 & 0.0245 & 13.238 & 1212.8 & 0.0234 \\
\hline 4 & 4.631 & 1372.3 & 0.0244 & 9.941 & 1278.2 & 0.0244 & 14.359 & 1230.8 & 0.0232 \\
\hline 5 & 4.857 & 1396.4 & 0.0242 & 10.277 & 1278.1 & 0.0242 & 14.834 & 1229.1 & 0.0231 \\
\hline 6 & 4.943 & 1404.7 & 0.0240 & 10.413 & 1275.1 & 0.0240 & 14.993 & 1216.9 & 0.0229 \\
\hline 7 & 4.944 & 1403.5 & 0.0239 & 10.449 & 1272.8 & 0.0239 & 14.968 & 1196.2 & 0.0228 \\
\hline 8 & 4.895 & 1395.0 & 0.0237 & 10.423 & 1271.0 & 0.0237 & 14.828 & 1174.9 & 0.0227 \\
\hline 9 & 4.840 & 1382.4 & 0.0235 & 10.364 & 1268.7 & 0.0235 & 14.672 & 1169.4 & 0.0226 \\
\hline 10 & 4.818 & 1371.0 & 0.0234 & 10.316 & 1266.0 & 0.0234 & 14.607 & 1180.8 & 0.0225 \\
\hline 11 & 4.841 & 1364.0 & 0.0232 & 10.314 & 1264.5 & 0.0233 & 14.660 & 1202.7 & 0.0224 \\
\hline 12 & 4.892 & 1361.3 & 0.0231 & 10.360 & 1265.5 & 0.0231 & 14.809 & 1230.1 & 0.0222 \\
\hline 13 & 4.929 & 1360.7 & 0.0229 & 10.426 & 1269.8 & 0.0230 & 14.978 & 1251.3 & 0.0221 \\
\hline 14 & 4.892 & 1355.5 & 0.0228 & 10.434 & 1276.3 & 0.0228 & 15.017 & 1254.2 & 0.0220 \\
\hline 15 & 4.708 & 1334.0 & 0.0226 & 10.255 & 1279.5 & 0.0227 & 14.739 & 1238.5 & 0.0219 \\
\hline 16 & 4.295 & 1280.8 & 0.0225 & 9.665 & 1265.8 & 0.0226 & 13.863 & 1202.3 & 0.0218 \\
\hline 17 & 3.492 & 1168.8 & 0.0224 & 8.189 & 1201.4 & 0.0224 & 11.743 & 1122.8 & 0.0217 \\
\hline 18 & 2.042 & 940.4 & 0.0223 & 5.024 & 1011.8 & 0.0223 & 7.223 & 950.6 & 0.0216 \\
\hline
\end{tabular}

\begin{tabular}{|c|c|c|c|c|c|c|c|c|c|}
\hline \multirow{2}{*}{$\begin{array}{c}\text { Axial } \\
\text { Node }\end{array}$} & \multicolumn{3}{|c|}{ Datapoint (206.7 Cy5) } & \multicolumn{2}{c|}{ Statepoint 62 (BOC Cy6) } & \multicolumn{3}{c|}{ Statepoint 63 (69.1 Cy6) } \\
\hline & $\begin{array}{c}\text { Burnup } \\
\text { (GWd/MTU) }\end{array}$ & $\begin{array}{c}\text { T-Fuel } \\
\left({ }^{\circ} \mathrm{F}\right)\end{array}$ & $\begin{array}{c}\text { Spec. Vol. } \\
\left(\mathrm{ft}^{3} / \mathrm{lbm}\right)\end{array}$ & $\begin{array}{c}\text { Burnup } \\
(\mathrm{GWd} / \mathrm{MTU})\end{array}$ & $\begin{array}{c}\text { T-Fuel } \\
\left({ }^{\circ} \mathrm{F}\right)\end{array}$ & $\begin{array}{c}\text { Spec. Vol. } \\
\left(\mathrm{ft}^{3} / \mathrm{lbm}\right)\end{array}$ & $\begin{array}{c}\text { Burnup } \\
(\mathrm{GWd} / \mathrm{MTU})\end{array}$ & $\begin{array}{c}\text { T-Fuel } \\
\left({ }^{\circ} \mathrm{F}\right)\end{array}$ & $\begin{array}{c}\text { Spec. Vol. } \\
\left(\mathrm{ft}^{3} / \mathrm{lbm}\right)\end{array}$ \\
\hline 1 & 206.7 Cy5 & 153.5 Cy5 & 153.5 Cy5 & BOC Cy6 & 252.2 Cy5 & 252.2 Cy5 & 69.1 Cy6 & 41.5 Cy6 & 41.5 Cy6 \\
\hline 2 & 8.387 & 989.3 & 0.0236 & 10.351 & 917.1 & 0.0235 & 11.548 & 894.7 & 0.0232 \\
\hline 3 & 13.715 & 1126.3 & 0.0235 & 16.590 & 1015.8 & 0.0235 & 18.342 & 984.5 & 0.0232 \\
\hline 4 & 17.810 & 1186.5 & 0.0232 & 21.114 & 1048.2 & 0.0233 & 23.238 & 1030.6 & 0.0230 \\
\hline 5 & 18.322 & 1186.4 & 0.0231 & 21.666 & 1058.6 & 0.0231 & 23.885 & 1045.7 & 0.0229 \\
\hline 6 & 18.480 & 1183.6 & 0.0229 & 21.878 & 1079.0 & 0.0230 & 24.171 & 1058.5 & 0.0228 \\
\hline 7 & 18.430 & 1178.7 & 0.0228 & 21.913 & 1112.3 & 0.0229 & 24.269 & 1069.8 & 0.0226 \\
\hline 8 & 18.230 & 1168.5 & 0.0227 & 21.822 & 1157.9 & 0.0228 & 24.231 & 1079.6 & 0.0225 \\
\hline 9 & 18.006 & 1154.8 & 0.0226 & 21.710 & 1206.1 & 0.0226 & 24.153 & 1085.6 & 0.0224 \\
\hline 10 & 17.917 & 1146.8 & 0.0224 & 21.714 & 1238.4 & 0.0225 & 24.161 & 1084.7 & 0.0223 \\
\hline 11 & 17.993 & 1145.0 & 0.0223 & 21.830 & 1245.1 & 0.0224 & 24.245 & 1076.2 & 0.0222 \\
\hline 12 & 18.206 & 1148.7 & 0.0222 & 22.023 & 1225.8 & 0.0222 & 24.376 & 1061.2 & 0.0221 \\
\hline 13 & 18.479 & 1159.1 & 0.0221 & 22.241 & 1187.6 & 0.0221 & 24.509 & 1041.2 & 0.0220 \\
\hline 14 & 18.615 & 1171.0 & 0.0220 & 22.315 & 1146.5 & 0.0220 & 24.481 & 1019.1 & 0.0219 \\
\hline 15 & 18.371 & 1176.7 & 0.0219 & 22.010 & 1114.9 & 0.0219 & 24.059 & 995.7 & 0.0218 \\
\hline 16 & 17.410 & 1169.2 & 0.0218 & 20.951 & 1092.6 & 0.0218 & 22.846 & 969.0 & 0.0217 \\
\hline 17 & 14.909 & 1123.7 & 0.0217 & 18.129 & 1059.3 & 0.0217 & 19.779 & 933.1 & 0.0216 \\
\hline 18 & 9.309 & 973.5 & 0.0216 & 11.518 & 938.7 & 0.0216 & 12.633 & 842.8 & 0.0216 \\
\hline
\end{tabular}


Table 4-25. Burnup and TH Feedback Parameters by Axial Node for Assembly D28

\begin{tabular}{|c|c|c|c|c|c|c|c|c|c|}
\hline \multirow{2}{*}{$\begin{array}{c}\text { Axial } \\
\text { Node }\end{array}$} & \multicolumn{2}{|c|}{ Datapoint (126.6 Cy4) } & \multicolumn{2}{c|}{ Statepoint 60 (BOC Cy5) } & \multicolumn{3}{c|}{ Statepoint 61 (114.4 Cy5) } \\
\hline & $\begin{array}{c}\text { Burnup } \\
(\mathrm{GWd} / \mathrm{MTU})\end{array}$ & $\begin{array}{c}\text { T-Fuel } \\
\left({ }^{\circ} \mathrm{F}\right)\end{array}$ & $\begin{array}{c}\text { Spec. Vol. } \\
\left(\mathrm{ft}^{3} / \mathrm{lbm}\right)\end{array}$ & $\begin{array}{c}\text { Burnup } \\
(\mathrm{GWd} / \mathrm{MTU})\end{array}$ & $\begin{array}{c}\text { T-Fuel } \\
\left({ }^{\circ} \mathrm{F}\right)\end{array}$ & $\begin{array}{c}\text { Spec. Vol. } \\
\left(\mathrm{ft}^{3} / \mathrm{lbm}\right)\end{array}$ & $\begin{array}{c}\text { Burnup } \\
(\mathrm{GWd} / \mathrm{MTU})\end{array}$ & $\begin{array}{c}\text { T-Fuel } \\
\left({ }^{\circ} \mathrm{F}\right)\end{array}$ & $\begin{array}{c}\text { Spec. Vol. } \\
\left(\mathrm{ft}^{3} / \mathrm{lbm}\right)\end{array}$ \\
\hline 1 & 1.106 & 810.9 & 0.0237 & 2.693 & 851.7 & 0.0238 & 5.281 & 1099.8 & 0.0238 \\
\hline 2 & 1.946 & 957.2 & 0.0237 & 4.553 & 986.1 & 0.0237 & 8.623 & 1292.4 & 0.0237 \\
\hline 3 & 2.479 & 1041.1 & 0.0236 & 5.612 & 1042.4 & 0.0236 & 10.368 & 1353.2 & 0.0235 \\
\hline 4 & 2.772 & 1082.2 & 0.0235 & 6.122 & 1059.3 & 0.0235 & 11.130 & 1356.7 & 0.0234 \\
\hline 5 & 2.915 & 1100.3 & 0.0234 & 6.346 & 1061.8 & 0.0234 & 11.409 & 1341.6 & 0.0232 \\
\hline 6 & 2.972 & 1106.4 & 0.0233 & 6.436 & 1060.4 & 0.0233 & 11.457 & 1318.8 & 0.0231 \\
\hline 7 & 2.977 & 1105.8 & 0.0232 & 6.461 & 1058.7 & 0.0232 & 11.381 & 1290.5 & 0.0229 \\
\hline 8 & 2.956 & 1101.0 & 0.0231 & 6.451 & 1057.2 & 0.0231 & 11.244 & 1265.2 & 0.0228 \\
\hline 9 & 2.931 & 1093.9 & 0.0230 & 6.425 & 1055.6 & 0.0231 & 11.137 & 1261.5 & 0.0227 \\
\hline 10 & 2.921 & 1087.4 & 0.0229 & 6.405 & 1054.2 & 0.0230 & 11.121 & 1277.2 & 0.0226 \\
\hline 11 & 2.931 & 1083.2 & 0.0228 & 6.405 & 1053.5 & 0.0229 & 11.179 & 1301.9 & 0.0224 \\
\hline 12 & 2.952 & 1081.0 & 0.0227 & 6.426 & 1054.5 & 0.0228 & 11.286 & 1329.4 & 0.0223 \\
\hline 13 & 2.961 & 1079.1 & 0.0227 & 6.449 & 1057.0 & 0.0227 & 11.379 & 1347.2 & 0.0222 \\
\hline 14 & 2.923 & 1073.0 & 0.0226 & 6.427 & 1059.9 & 0.0226 & 11.357 & 1346.5 & 0.0220 \\
\hline 15 & 2.796 & 1055.2 & 0.0225 & 6.279 & 1059.0 & 0.0225 & 11.122 & 1333.8 & 0.0219 \\
\hline 16 & 2.523 & 1014.9 & 0.0224 & 5.853 & 1043.4 & 0.0225 & 10.482 & 1308.6 & 0.0218 \\
\hline 17 & 2.009 & 932.6 & 0.0224 & 4.851 & 986.6 & 0.0224 & 8.900 & 1236.3 & 0.0217 \\
\hline 18 & 1.135 & 781.6 & 0.0223 & 2.866 & 838.2 & 0.0223 & 5.446 & 1033.9 & 0.0216 \\
\hline
\end{tabular}

\begin{tabular}{|c|c|c|c|c|c|c|c|c|c|}
\hline \multirow[b]{2}{*}{$\begin{array}{l}\text { Axial } \\
\text { Node } \\
\end{array}$} & \multicolumn{3}{|c|}{ Datapoint (206.7 Cy5) } & \multicolumn{3}{|c|}{ Statepoint 62 (BOC Cy6) } & \multicolumn{3}{|c|}{ Statepoint 63 (69.1 Cy6) } \\
\hline & $\begin{array}{c}\text { Burnup } \\
\text { (GWd/MTU) }\end{array}$ & $\begin{array}{l}\text { T-Fuel } \\
\left({ }^{\circ} \mathrm{F}\right)\end{array}$ & $\begin{array}{l}\text { Spec. Vol. } \\
\left(\mathrm{ft}^{3} / \mathrm{lbm}\right)\end{array}$ & $\begin{array}{c}\text { Burnup } \\
\text { (GWd/MTU) }\end{array}$ & $\begin{array}{c}\text { T-Fuel } \\
\left({ }^{\circ} \mathrm{F}\right)\end{array}$ & $\begin{array}{l}\text { Spec. Vol. } \\
\left(\mathrm{ft}^{3} / \mathrm{lbm}\right)\end{array}$ & $\begin{array}{c}\text { Burnup } \\
\text { (GWd/MTU) }\end{array}$ & $\begin{array}{c}\text { T-Fuel } \\
\left({ }^{\circ} \mathrm{F}\right)\end{array}$ & $\begin{array}{c}\text { Spec. Vol. } \\
\left(\mathrm{ft}^{3} / \mathrm{lbm}\right)\end{array}$ \\
\hline & 206.7 Cy5 & 153.5 Cy5 & 153.5 Cy5 & BOC Cy 6 & 252.2 Cy 5 & 252.2 Cy 5 & 69.1 Cy6 & 41.5 Cy 6 & 41.5 Cy 6 \\
\hline 1 & 7.473 & 1066.0 & 0.0237 & 9.684 & 967.3 & 0.0237 & 10.837 & 883.7 & 0.0235 \\
\hline 2 & 11.893 & 1218.4 & 0.0236 & 15.052 & 1071.3 & 0.0236 & 16.843 & 996.6 & 0.0234 \\
\hline 3 & 14.036 & 1267.5 & 0.0235 & 17.498 & 1098.9 & 0.0235 & 19.649 & 1055.0 & 0.0233 \\
\hline 4 & 14.886 & 1273.5 & 0.0233 & 18.403 & 1105.7 & 0.0234 & 20.746 & 1093.1 & 0.0232 \\
\hline 5 & 15.159 & 1268.3 & 0.0232 & 18.690 & 1116.4 & 0.0233 & 21.075 & 1099.9 & 0.0230 \\
\hline 6 & 15.179 & 1262.0 & 0.0230 & 18.753 & 1139.3 & 0.0231 & 21.205 & 1107.4 & 0.0229 \\
\hline 7 & 15.061 & 1255.0 & 0.0229 & 18.721 & 1178.0 & 0.0230 & 21.245 & 1120.0 & 0.0228 \\
\hline 8 & 14.861 & 1243.6 & 0.0228 & 18.640 & 1232.1 & 0.0229 & 21.226 & 1131.7 & 0.0227 \\
\hline 9 & 14.691 & 1229.6 & 0.0226 & 18.601 & 1287.2 & 0.0227 & 21.231 & 1139.2 & 0.0226 \\
\hline 10 & 14.661 & 1221.8 & 0.0225 & 18.677 & 1323.4 & 0.0226 & 21.321 & 1139.6 & 0.0224 \\
\hline 11 & 14.739 & 1219.2 & 0.0224 & 18.793 & 1330.9 & 0.0224 & 21.429 & 1134.1 & 0.0223 \\
\hline 12 & 14.898 & 1221.5 & 0.0223 & 18.916 & 1308.6 & 0.0223 & 21.564 & 1127.0 & 0.0222 \\
\hline 13 & 15.081 & 1230.8 & 0.0221 & 19.020 & 1265.2 & 0.0221 & 21.766 & 1140.2 & 0.0221 \\
\hline 14 & 15.143 & 1241.9 & 0.0220 & 18.996 & 1217.1 & 0.0220 & 21.708 & 1143.6 & 0.0220 \\
\hline 15 & 14.952 & 1249.2 & 0.0219 & 18.742 & 1179.8 & 0.0219 & 21.324 & 1120.4 & 0.0218 \\
\hline 16 & 14.272 & 1246.5 & 0.0218 & 17.991 & 1155.8 & 0.0218 & 20.358 & 1082.2 & 0.0217 \\
\hline 17 & 12.361 & 1203.9 & 0.0217 & 15.797 & 1119.3 & 0.0217 & 17.805 & 1023.0 & 0.0217 \\
\hline 18 & 7.780 & 1039.8 & 0.0216 & 10.181 & 988.1 & 0.0216 & 11.489 & 894.8 & 0.0216 \\
\hline
\end{tabular}


Table 4-26. Burnup and TH Feedback Parameters by Axial Node for Assembly E8

\begin{tabular}{|c|c|c|c|c|c|c|c|c|c|}
\hline \multirow[b]{2}{*}{$\begin{array}{l}\text { Axial } \\
\text { Node } \\
\end{array}$} & \multicolumn{3}{|c|}{ Statepoint 61 (114.4 Cy5) } & \multicolumn{3}{|c|}{ Datapoint (206.7 Cy5) } & \multicolumn{3}{|c|}{ Statepoint 62 (BOC Cy6) } \\
\hline & $\begin{array}{c}\text { Burnup } \\
\text { (GWd/MTU) }\end{array}$ & $\begin{array}{c}\text { T-Fuel } \\
\left({ }^{\circ} \mathrm{F}\right)\end{array}$ & $\begin{array}{c}\text { Spec. Vol. } \\
\left(\mathrm{ft}^{3} / \mathrm{lbm}\right)\end{array}$ & $\begin{array}{c}\text { Burnup } \\
\text { (GWd/MTU) }\end{array}$ & $\begin{array}{c}\text { T-Fuel } \\
\left({ }^{\circ} \mathrm{F}\right)\end{array}$ & $\begin{array}{c}\text { Spec. Vol. } \\
\left(\mathrm{ft}^{3} / \mathrm{lbm}\right)\end{array}$ & $\begin{array}{c}\text { Burnup } \\
\text { (GWd/MTU) }\end{array}$ & $\begin{array}{c}\text { T-Fuel } \\
\left({ }^{\circ} \mathrm{F}\right)\end{array}$ & $\begin{array}{c}\text { Spec. Vol. } \\
\left(\mathbb{t}^{3} / \mathrm{lbm}\right)\end{array}$ \\
\hline & 114.4 Cy5 & 58.2 Cy 5 & 58.2 Cy 5 & 206.7 Cy5 & 153.5 Cy 5 & 153.5 Cy5 & BOC Cy 6 & $252.2 \mathrm{Cy} 5$ & $252.2 \mathrm{Cy} 5$ \\
\hline 1 & 1.052 & 841.9 & 0.0229 & 2.117 & 855.1 & 0.0230 & 3.324 & 832.1 & 0.0230 \\
\hline 2 & 1.955 & 1005.5 & 0.0228 & 3.856 & 1019.9 & 0.0229 & 5.889 & 959.4 & 0.0229 \\
\hline 3 & 2.561 & 1091.3 & 0.0228 & 4.902 & 1104.8 & 0.0229 & 7.307 & 1014.8 & 0.0229 \\
\hline 4 & 2.892 & 1126.1 & 0.0227 & 5.408 & 1136.0 & 0.0228 & 7.942 & 1036.4 & 0.0228 \\
\hline 5 & 3.046 & 1136.8 & 0.0226 & 5.625 & 1145.6 & 0.0227 & 8.212 & 1051.1 & 0.0227 \\
\hline 6 & 3.112 & 1137.6 & 0.0225 & 5.716 & 1148.3 & 0.0226 & 8.347 & 1069.1 & 0.0226 \\
\hline 7 & 3.146 & 1136.6 & 0.0224 & 5.765 & 1149.4 & 0.0225 & 8.453 & 1092.2 & 0.0225 \\
\hline 8 & 3.184 & 1140.2 & 0.0224 & 5.826 & 1151.5 & 0.0224 & 8.588 & 1119.2 & 0.0224 \\
\hline 9 & 3.248 & 1151.7 & 0.0223 & 5.933 & 1155.9 & 0.0223 & 8.779 & 1146.1 & 0.0223 \\
\hline 10 & 3.329 & 1168.7 & 0.0222 & 6.065 & 1161.6 & 0.0222 & 8.986 & 1166.1 & 0.0222 \\
\hline 11 & 3.385 & 1183.7 & 0.0221 & 6.162 & 1165.3 & 0.0221 & 9.122 & 1172.8 & 0.0221 \\
\hline 12 & 3.393 & 1191.4 & 0.0220 & 6.195 & 1166.3 & 0.0220 & 9.153 & 1165.6 & 0.0220 \\
\hline 13 & 3.343 & 1188.3 & 0.0219 & 6.150 & 1164.5 & 0.0220 & 9.078 & 1148.2 & 0.0220 \\
\hline 14 & 3.224 & 1171.9 & 0.0218 & 6.009 & 1158.9 & 0.0219 & 8.887 & 1126.1 & 0.0219 \\
\hline 15 & 3.033 & 1141.9 & 0.0218 & 5.758 & 1147.1 & 0.0218 & 8.571 & 1103.4 & 0.0218 \\
\hline 16 & 2.736 & 1090.3 & 0.0217 & 5.311 & 1119.8 & 0.0217 & 8.003 & 1076.4 & 0.0217 \\
\hline 17 & 2.205 & 994.6 & 0.0216 & 4.395 & 1045.3 & 0.0216 & 6.746 & 1019.1 & 0.0216 \\
\hline 18 & 1.265 & 811.5 & 0.0216 & 2.593 & 864.4 & 0.0216 & 4.081 & 867.4 & 0.0216 \\
\hline
\end{tabular}

\begin{tabular}{|c|c|c|c|c|c|c|c|c|c|}
\hline \multirow[b]{2}{*}{$\begin{array}{l}\text { Axial } \\
\text { Node }\end{array}$} & \multicolumn{3}{|c|}{ Statepoint 63 (69.1 Cy6) } & \multicolumn{3}{|c|}{ Datapoint (176.7 Cy6) } & \multicolumn{3}{|c|}{ Datapoint (320.3 Cy6) } \\
\hline & $\begin{array}{c}\text { Burnup } \\
\text { (GWd/MTU) }\end{array}$ & $\begin{array}{c}\text { T-Fuel } \\
\left({ }^{\circ} \mathrm{F}\right)\end{array}$ & $\begin{array}{l}\text { Spec. Vol. } \\
\left(\mathrm{ft}^{3} / \mathrm{lbm}\right)\end{array}$ & $\begin{array}{c}\text { Burnup } \\
\text { (GWd/MTU) }\end{array}$ & $\begin{array}{c}\text { T-Fuel } \\
\left({ }^{\circ} \mathrm{F}\right)\end{array}$ & $\begin{array}{c}\text { Spec. Vol. } \\
\left(\mathrm{ft}^{3} / \mathrm{bm}\right)\end{array}$ & $\begin{array}{c}\text { Burnup } \\
\text { (GWd/MTU) }\end{array}$ & $\begin{array}{l}\text { T-Fuel } \\
\left({ }^{\circ} \mathrm{F}\right)\end{array}$ & $\begin{array}{l}\text { Spec. Vol. } \\
\left(\mathrm{ft}^{3} / \mathrm{bm}\right)\end{array}$ \\
\hline & $69.1 \mathrm{Cy} 6$ & 41.5 Cy6 & 41.5 Cy 6 & 176.7 Cy6 & 128.8 Cy6 & 128.8 Cy 6 & $320.3 \mathrm{Cy} 6$ & 252.5 Cy 6 & 252.5 Cy 6 \\
\hline 1 & 3.805 & 726.6 & 0.0225 & 4.631 & 751.8 & 0.0225 & 5.964 & 783.7 & 0.0225 \\
\hline 2 & 6.753 & 811.1 & 0.0225 & 8.190 & 836.2 & 0.0224 & 10.387 & 861.1 & 0.0224 \\
\hline 3 & 8.426 & 870.4 & 0.0224 & 10.216 & 879.8 & 0.0224 & 12.805 & 892.2 & 0.0224 \\
\hline 4 & 9.222 & 907.7 & 0.0224 & 11.194 & 899.8 & 0.0223 & 13.916 & 898.9 & 0.0223 \\
\hline 5 & 9.590 & 930.6 & 0.0223 & 11.648 & 907.8 & 0.0223 & 14.390 & 895.9 & 0.0223 \\
\hline 6 & 9.784 & 944.1 & 0.0223 & 11.881 & 909.5 & 0.0222 & 14.606 & 890.2 & 0.0222 \\
\hline 7 & 9.927 & 951.7 & 0.0222 & 12.039 & 908.3 & 0.0221 & 14.738 & 884.3 & 0.0221 \\
\hline 8 & 10.083 & 954.9 & 0.0221 & 12.198 & 905.4 & 0.0221 & 14.872 & 878.8 & 0.0221 \\
\hline 9 & 10.281 & 954.4 & 0.0221 & 12.392 & 901.4 & 0.0220 & 15.044 & 873.7 & 0.0220 \\
\hline 10 & 10.485 & 951.2 & 0.0220 & 12.588 & 897.3 & 0.0220 & 15.226 & 869.6 & 0.0220 \\
\hline 11 & 10.611 & 947.1 & 0.0219 & 12.708 & 894.2 & 0.0219 & 15.346 & 867.5 & 0.0219 \\
\hline 12 & 10.628 & 942.8 & 0.0219 & 12.724 & 892.5 & 0.0219 & 15.377 & 867.7 & 0.0219 \\
\hline 13 & 10.531 & 937.7 & 0.0218 & 12.625 & 891.8 & 0.0218 & 15.307 & 870.0 & 0.0218 \\
\hline 14 & 10.301 & 929.7 & 0.0218 & 12.382 & 890.4 & 0.0218 & 15.097 & 873.6 & 0.0218 \\
\hline 15 & 9.915 & 914.6 & 0.0217 & 11.945 & 884.8 & 0.0217 & 14.668 & 875.8 & 0.0217 \\
\hline 16 & 9.217 & 886.0 & 0.0216 & 11.112 & 868.2 & 0.0216 & 13.754 & 870.3 & 0.0217 \\
\hline 17 & 7.729 & 835.3 & 0.0216 & 9.318 & 829.6 & 0.0216 & 11.642 & 842.3 & 0.0216 \\
\hline 18 & 4.661 & 733.6 & 0.0216 & 5.628 & 739.1 & 0.0216 & 7.120 & 761.0 & 0.0216 \\
\hline
\end{tabular}


Table 4-26. Burnup and TH Feedback Parameters by Axial Node for Assembly E8 (Cont.)

\begin{tabular}{|c|c|c|c|c|c|c|c|c|c|}
\hline \multirow{2}{*}{$\begin{array}{c}\text { Axial } \\
\text { Node }\end{array}$} & \multicolumn{2}{|c|}{ Statepoint 64 (BOC Cy7) } & \multicolumn{2}{c|}{ Statepoint 65 (30.0 Cy7) } & \multicolumn{3}{c|}{ Statepoint 66 (108.0 Cy7) } \\
\cline { 2 - 10 } & $\begin{array}{c}\text { Burnup } \\
\text { (GWd/MTU) }\end{array}$ & $\begin{array}{c}\text { T-Fuel } \\
\left({ }^{\circ} \mathrm{F}\right)\end{array}$ & $\begin{array}{c}\text { Spec. Vol. } \\
\left(\mathrm{ft}^{3} / \mathrm{lbm}\right)\end{array}$ & $\begin{array}{c}\text { Burnup } \\
(\mathrm{GWd} / \mathrm{MTU})\end{array}$ & $\begin{array}{c}\text { T-Fuel } \\
\left({ }^{\circ} \mathrm{F}\right)\end{array}$ & $\begin{array}{c}\text { Spec. Vol. } \\
\left(\mathrm{ft}^{3} / \mathrm{lbm}\right)\end{array}$ & $\begin{array}{c}\text { Burnup } \\
(\mathrm{GWd} / \mathrm{MTU})\end{array}$ & $\begin{array}{c}\text { T-Fuel } \\
\left({ }^{\circ} \mathrm{F}\right)\end{array}$ & $\begin{array}{c}\text { Spec. Vol. } \\
\left(\mathrm{ft}^{3} / \mathrm{lbm}\right)\end{array}$ \\
\hline 1 & BOC Cy7 & $376.2 \mathrm{Cy6}$ & 376.2 Cy6 & $30.0 \mathrm{Cy} 7$ & $22.5 \mathrm{Cy} 7$ & $22.5 \mathrm{Cy} 7$ & $108.0 \mathrm{Cy7}$ & $60.4 \mathrm{Cy} 7$ & $60.4 \mathrm{Cy} 7$ \\
\hline 2 & 7.101 & 821.2 & 0.0225 & 7.484 & 850.3 & 0.0229 & 8.528 & 857.2 & 0.0229 \\
\hline 3 & 12.155 & 889.3 & 0.0225 & 12.770 & 938.5 & 0.0228 & 14.425 & 944.9 & 0.0228 \\
\hline 4 & 15.945 & 905.9 & 0.0224 & 16.754 & 1009.3 & 0.0227 & 18.870 & 1006.1 & 0.0227 \\
\hline 5 & 16.400 & 897.7 & 0.0223 & 17.255 & 1025.3 & 0.0226 & 19.464 & 1017.5 & 0.0226 \\
\hline 6 & 16.586 & 889.9 & 0.0222 & 17.472 & 1035.3 & 0.0225 & 19.734 & 1023.5 & 0.0225 \\
\hline 7 & 16.694 & 883.5 & 0.0222 & 17.597 & 1040.4 & 0.0224 & 19.889 & 1025.6 & 0.0224 \\
\hline 8 & 16.808 & 878.3 & 0.0221 & 17.722 & 1041.5 & 0.0224 & 20.025 & 1024.6 & 0.0223 \\
\hline 9 & 16.967 & 873.7 & 0.0221 & 17.882 & 1039.1 & 0.0223 & 20.185 & 1020.9 & 0.0223 \\
\hline 10 & 17.142 & 870.1 & 0.0220 & 18.055 & 1034.6 & 0.0222 & 20.350 & 1016.0 & 0.0222 \\
\hline 11 & 17.263 & 868.1 & 0.0220 & 18.171 & 1029.2 & 0.0221 & 20.456 & 1010.9 & 0.0221 \\
\hline 12 & 17.308 & 868.2 & 0.0219 & 18.206 & 1022.7 & 0.0220 & 20.476 & 1005.4 & 0.0220 \\
\hline 13 & 17.265 & 870.9 & 0.0218 & 18.146 & 1013.8 & 0.0219 & 20.389 & 998.3 & 0.0219 \\
\hline 14 & 17.092 & 875.9 & 0.0218 & 17.946 & 1001.0 & 0.0219 & 20.140 & 988.2 & 0.0219 \\
\hline 15 & 16.698 & 881.9 & 0.0217 & 17.509 & 981.9 & 0.0218 & 19.616 & 972.7 & 0.0218 \\
\hline 16 & 15.774 & 884.0 & 0.0217 & 16.516 & 953.4 & 0.0217 & 18.469 & 948.2 & 0.0217 \\
\hline 17 & 13.487 & 866.5 & 0.0216 & 14.111 & 905.4 & 0.0217 & 15.776 & 904.3 & 0.0217 \\
\hline 18 & 8.365 & 789.4 & 0.0216 & 8.761 & 802.1 & 0.0216 & 9.829 & 802.9 & 0.0216 \\
\hline
\end{tabular}

Table 4-27. Burnup and TH Feedback Parameters by Axial Node for Assembly E15

\begin{tabular}{|c|c|c|c|c|c|c|c|c|c|}
\hline \multirow[b]{2}{*}{$\begin{array}{l}\text { Axial } \\
\text { Node }\end{array}$} & \multicolumn{3}{|c|}{ Statepoint 61 (114.4 Cy5) } & \multicolumn{3}{|c|}{ Datapoint (206.7 Cy5) } & \multicolumn{3}{|c|}{ Statepoint 62 (BOC Cy6) } \\
\hline & \begin{tabular}{|c|} 
Burnup \\
(GWd/MTU)
\end{tabular} & $\begin{array}{c}\text { T-Fuel } \\
\left({ }^{\circ} \mathrm{F}\right)\end{array}$ & $\begin{array}{c}\text { Spec. Vol. } \\
\left(\mathrm{ft}^{3} / \mathrm{lbm}\right)\end{array}$ & \begin{tabular}{|c|} 
Burnup \\
(GWd/MTU)
\end{tabular} & $\begin{array}{l}\text { T-Fuel } \\
\left({ }^{\circ} \mathrm{F}\right)\end{array}$ & $\begin{array}{c}\text { Spec. Vol. } \\
\left(\mathrm{ft}^{3} / \mathrm{lbm}\right)\end{array}$ & $\begin{array}{c}\text { Burnup } \\
\text { (GWd/MTU) }\end{array}$ & $\begin{array}{l}\text { T-Fuel } \\
\left({ }^{\circ} \mathrm{F}\right)\end{array}$ & $\begin{array}{l}\text { Spec. Vol. } \\
\left(\mathrm{ft}^{3} / \mathrm{lbm}\right)\end{array}$ \\
\hline & 114.4 Cy5 & 58.2 Cy5 & 58.2 Cy 5 & 206.7 Cy 5 & 153.5 Cy 5 & 153.5 Cy5 & BOC Cy6 & 252.2 Cy5 & 252.2 Cy 5 \\
\hline 1 & 1.078 & 833.7 & 0.0228 & 2.156 & 857.6 & 0.0229 & 3.361 & 833.4 & 0.0229 \\
\hline 2 & 1.948 & 989.1 & 0.0228 & 3.806 & 1011.0 & 0.0229 & 5.782 & 950.6 & 0.0229 \\
\hline 3 & 2.494 & 1071.5 & 0.0227 & 4.753 & 1088.2 & 0.0228 & 7.072 & 1001.8 & 0.0228 \\
\hline 4 & 2.794 & 1106.2 & 0.0226 & 5.220 & 1118.6 & 0.0227 & 7.665 & 1022.7 & 0.0227 \\
\hline 5 & 2.939 & 1117.4 & 0.0226 & 5.427 & 1128.4 & 0.0226 & 7.926 & 1037.4 & 0.0227 \\
\hline 6 & 3.003 & 1118.3 & 0.0225 & 5.515 & 1131.2 & 0.0226 & 8.058 & 1055.4 & 0.0226 \\
\hline 7 & 3.033 & 1117.0 & 0.0224 & 5.560 & 1132.2 & 0.0225 & 8.159 & 1079.1 & 0.0225 \\
\hline 8 & 3.066 & 1119.9 & 0.0223 & 5.615 & 1134.0 & 0.0224 & 8.287 & 1106.4 & 0.0224 \\
\hline 9 & 3.128 & 1131.6 & 0.0222 & 5.717 & 1138.4 & 0.0223 & 8.475 & 1133.9 & 0.0223 \\
\hline 10 & 3.209 & 1149.3 & 0.0222 & 5.850 & 1144.3 & 0.0222 & 8.686 & 1154.5 & 0.0222 \\
\hline 11 & 3.267 & 1164.8 & 0.0221 & 5.950 & 1148.1 & 0.0221 & 8.825 & 1161.5 & 0.0221 \\
\hline 12 & 3.280 & 1173.2 & 0.0220 & 5.988 & 1149.3 & 0.0220 & 8.861 & 1154.0 & 0.0220 \\
\hline 13 & 3.235 & 1170.9 & 0.0219 & 5.950 & 1147.8 & 0.0219 & 8.792 & 1136.1 & 0.0219 \\
\hline 14 & 3.119 & 1154.8 & 0.0218 & 5.811 & 1142.1 & 0.0219 & 8.600 & 1113.1 & 0.0219 \\
\hline 15 & 2.930 & 1124.1 & 0.0217 & 5.559 & 1129.9 & 0.0218 & 8.277 & 1089.6 & 0.0218 \\
\hline 16 & 2.634 & 1072.1 & 0.0217 & 5.113 & 1102.0 & 0.0217 & 7.706 & 1061.8 & 0.0217 \\
\hline 17 & 2.114 & 977.8 & 0.0216 & 4.213 & 1027.4 & 0.0216 & 6.470 & 1004.1 & 0.0216 \\
\hline 18 & 1.209 & 800.2 & 0.0216 & 2.477 & 851.2 & 0.0216 & 3.900 & 854.9 & 0.0216 \\
\hline
\end{tabular}


Table 4-27. Burnup and TH Feedback Parameters by Axial Node for Assembly E15 (Cont.)

\begin{tabular}{|c|c|c|c|c|c|c|c|c|c|}
\hline \multirow{2}{*}{$\begin{array}{c}\text { Axial } \\
\text { Node }\end{array}$} & \multicolumn{2}{|c|}{ Statepoint 63 (69.1 Cy6) } & \multicolumn{2}{c|}{ Datapoint (176.7 Cy6) } & \multicolumn{3}{c|}{ Datapoint (320.3 Cy6) } \\
\cline { 2 - 10 } & $\begin{array}{c}\text { Burnup } \\
(\mathrm{GWd} / \mathrm{MTU})\end{array}$ & $\begin{array}{c}\text { T-Fuel } \\
\left({ }^{\circ} \mathrm{F}\right)\end{array}$ & $\begin{array}{c}\text { Spec. Vol. } \\
\left(\mathrm{ft}^{3} / \mathrm{lbm}\right)\end{array}$ & $\begin{array}{c}\text { Burnup } \\
(\mathrm{GWd} / \mathrm{MTU})\end{array}$ & $\begin{array}{c}\text { T-Fuel } \\
\left({ }^{\circ} \mathrm{F}\right)\end{array}$ & $\begin{array}{c}\text { Spec. Vol. } \\
\left(\mathrm{ft}^{3} / \mathrm{lbm}\right)\end{array}$ & $\begin{array}{c}\text { Burnup } \\
(\mathrm{GWd} / \mathrm{MTU})\end{array}$ & $\begin{array}{c}\text { T-Fuel } \\
\left({ }^{\circ} \mathrm{F}\right)\end{array}$ & $\begin{array}{c}\text { Spec. Vol. } \\
\left(\mathrm{ft}^{3} / \mathrm{lbm}\right)\end{array}$ \\
\hline 1 & $69.1 \mathrm{Cy6}$ & $41.5 \mathrm{Cy} 6$ & $41.5 \mathrm{Cy6}$ & $176.7 \mathrm{Cy} 6$ & $128.8 \mathrm{Cy} 6$ & $128.8 \mathrm{Cy} 6$ & $320.3 \mathrm{Cy} 6$ & $252.5 \mathrm{Cy} 6$ & $252.5 \mathrm{Cy} 6$ \\
\hline 2 & 4.291 & 882.6 & 0.0233 & 5.841 & 907.8 & 0.0232 & 8.239 & 939.8 & 0.0231 \\
\hline 3 & 7.363 & 1019.2 & 0.0232 & 9.911 & 1028.7 & 0.0231 & 13.645 & 1042.0 & 0.0231 \\
\hline 4 & 9.028 & 1094.4 & 0.0231 & 12.081 & 1086.1 & 0.0230 & 16.345 & 1078.8 & 0.0230 \\
\hline 5 & 10.848 & 1140.9 & 0.0230 & 13.150 & 1110.7 & 0.0229 & 17.574 & 1082.1 & 0.0228 \\
\hline 6 & 10.474 & 1190.4 & 0.0228 & 13.953 & 1121.5 & 0.0227 & 18.359 & 1065.5 & 0.0226 \\
\hline 7 & 10.636 & 1202.3 & 0.0227 & 14.143 & 1119.7 & 0.0226 & 18.508 & 1056.5 & 0.0225 \\
\hline 8 & 10.802 & 1208.4 & 0.0226 & 14.319 & 1115.9 & 0.0225 & 18.649 & 1048.4 & 0.0224 \\
\hline 9 & 11.005 & 1208.7 & 0.0224 & 14.522 & 1110.5 & 0.0224 & 18.822 & 1041.0 & 0.0223 \\
\hline 10 & 11.213 & 1204.5 & 0.0223 & 14.722 & 1104.6 & 0.0223 & 19.005 & 1035.1 & 0.0223 \\
\hline 11 & 11.338 & 1198.4 & 0.0222 & 14.841 & 1100.1 & 0.0222 & 19.129 & 1032.2 & 0.0222 \\
\hline 12 & 11.353 & 1191.8 & 0.0221 & 14.857 & 1097.9 & 0.0221 & 19.176 & 1032.9 & 0.0221 \\
\hline 13 & 11.249 & 1184.1 & 0.0220 & 14.755 & 1097.5 & 0.0220 & 19.130 & 1037.1 & 0.0220 \\
\hline 14 & 10.996 & 1172.5 & 0.0219 & 14.488 & 1096.5 & 0.0219 & 18.925 & 1043.6 & 0.0219 \\
\hline 15 & 10.563 & 1150.2 & 0.0218 & 13.982 & 1089.4 & 0.0218 & 18.449 & 1049.3 & 0.0218 \\
\hline 16 & 9.788 & 1107.6 & 0.0217 & 13.004 & 1066.6 & 0.0217 & 17.371 & 1046.2 & 0.0217 \\
\hline 17 & 8.180 & 1030.1 & 0.0216 & 10.914 & 1009.5 & 0.0216 & 14.810 & 1012.0 & 0.0216 \\
\hline 18 & 4.926 & 867.5 & 0.0216 & 6.626 & 870.2 & 0.0216 & 9.189 & 891.8 & 0.0216 \\
\hline
\end{tabular}

\begin{tabular}{|c|c|c|c|c|c|c|c|c|c|}
\hline \multirow[b]{2}{*}{$\begin{array}{l}\text { Axlal } \\
\text { Node } \\
\end{array}$} & \multicolumn{3}{|c|}{ Statepoint 67 (BOC Cy8) } & \multicolumn{3}{|c|}{ Datapoint (171.1 Cy8) } & \multicolumn{3}{|c|}{ Datapoint (338.1 Cy8) } \\
\hline & $\begin{array}{c}\text { Burnup } \\
\text { (GWd/MTU) }\end{array}$ & $\begin{array}{c}\text { T-Fuel } \\
\left({ }^{\circ} \mathrm{F}\right)\end{array}$ & $\begin{array}{c}\text { Spec. Vol. } \\
\left(\mathrm{ft}^{3} / \mathrm{lbm}\right)\end{array}$ & $\begin{array}{c}\text { Burnup } \\
\text { (GWd/MTU) }\end{array}$ & $\begin{array}{c}\text { T-Fuel } \\
\left({ }^{\circ} \mathbf{F}\right)\end{array}$ & $\begin{array}{c}\text { Spec. Vol. } \\
\left(\mathrm{ft}^{3} / \mathrm{lbm}\right)\end{array}$ & $\begin{array}{c}\text { Burnup } \\
\text { (GWd/MTU) }\end{array}$ & $\begin{array}{c}\text { T-Fuel } \\
\left({ }^{\circ} \mathrm{F}\right)\end{array}$ & $\begin{array}{c}\text { Spec. Vol. } \\
\left(\mathrm{ft}^{3} / \mathrm{lbm}\right)\end{array}$ \\
\hline & BOC Cy8 & 376.2 Cy 6 & 376.2 Cy6 & 171.1 Cy8 & 77.7 Cy 8 & 77.7 Cy 8 & 338.1 Cy 8 & 247.2 Cy 8 & 247.2 Cy8 \\
\hline 1 & 10.162 & 965.5 & 0.0232 & 11.193 & 681.9 & 0.0222 & 12.438 & 704.6 & 0.0222 \\
\hline 2 & 16.485 & 1051.3 & 0.0231 & 18.158 & 721.6 & 0.0222 & 20.074 & 741.3 & 0.0222 \\
\hline 3 & 19.468 & 1067.2 & 0.0230 & 21.493 & 742.6 & 0.0222 & 23.687 & 753.2 & 0.0222 \\
\hline 4 & 20.731 & 1058.5 & 0.0229 & 22.957 & 754.2 & 0.0221 & 25.252 & 755.1 & 0.0221 \\
\hline 5 & 21.229 & 1045.7 & 0.0228 & 23.570 & 760.6 & 0.0221 & 25.890 & 753.5 & 0.0221 \\
\hline 6 & 21.430 & 1034.4 & 0.0227 & 23.837 & 764.0 & 0.0220 & 26.151 & 750.9 & 0.0220 \\
\hline 7 & 21.542 & 1025.5 & 0.0226 & 23.986 & 765.3 & 0.0220 & 26.287 & 748.1 & 0.0220 \\
\hline 8 & 21.656 & 1018.4 & 0.0225 & 24.119 & 765.1 & 0.0220 & 26.405 & 745.4 & 0.0220 \\
\hline 9 & 21.811 & 1012.4 & 0.0224 & 24.279 & 763.8 & 0.0219 & 26.553 & 742.8 & 0.0219 \\
\hline 10 & 21.986 & 1007.5 & 0.0223 & 24.451 & 761.8 & 0.0219 & 26.717 & 740.5 & 0.0219 \\
\hline 11 & 22.115 & 1005.0 & 0.0222 & 24.572 & 759.4 & 0.0219 & 26.836 & 739.0 & 0.0218 \\
\hline 12 & 22.188 & 1005.5 & 0.0221 & 24.629 & 756.8 & 0.0218 & 26.898 & 738.3 & 0.0218 \\
\hline 13 & 22.188 & 1009.5 & 0.0220 & 24.601 & 753.5 & 0.0218 & 26.881 & 738.3 & 0.0218 \\
\hline 14 & 22.046 & 1017.0 & 0.0219 & 24.409 & 748.9 & 0.0218 & 26.697 & 738.6 & 0.0218 \\
\hline 15 & 21.636 & 1026.6 & 0.0218 & 23.907 & 741.9 & 0.0217 & 26.182 & 738.5 & 0.0217 \\
\hline 16 & 20.565 & 1033.6 & 0.0217 & 22.669 & 730.7 & 0.0217 & 24.871 & 736.0 & 0.0217 \\
\hline 17 & 17.774 & 1019.2 & 0.0217 & 19.559 & 7111.2 & 0.0217 & 21.522 & 725.2 & 0.0217 \\
\hline 18 & 11.248 & 916.8 & 0.0216 & 12.385 & 666.6 & 0.0217 & 13.700 & 684.9 & 0.0217 \\
\hline
\end{tabular}


Table 4-27. Burnup and TH Feedback Parameters by Axial Node for Assembly E15 (Cont.)

\begin{tabular}{|c|c|c|c|}
\hline \multirow{2}{*}{$\begin{array}{c}\text { Axial } \\
\text { Node }\end{array}$} & $\begin{array}{c}\text { Burnup } \\
\text { (GWd/MTU) }\end{array}$ & $\begin{array}{c}\text { Statepoint 68 (BOC Cy9) } \\
\text { T-Fuel } \\
\left({ }^{\circ} \mathrm{F} \text { ) }\right.\end{array}$ & $\begin{array}{c}\text { Spec. Vol. } \\
\text { (ft/l/bm) }\end{array}$ \\
\hline & BOC Cy9 & 426.1 Cy8 & 426.1 Cy8 \\
\hline 1 & 13.988 & 731.3 & 0.0223 \\
\hline 2 & 22.329 & 760.5 & 0.0222 \\
\hline 3 & 26.160 & 763.0 & 0.0222 \\
\hline 4 & 27.754 & 757.5 & 0.0221 \\
\hline 5 & 28.362 & 751.4 & 0.0221 \\
\hline 6 & 28.588 & 746.4 & 0.0221 \\
\hline 7 & 28.694 & 742.6 & 0.0220 \\
\hline 8 & 28.792 & 739.5 & 0.0220 \\
\hline 9 & 28.926 & 737.0 & 0.0220 \\
\hline 10 & 29.083 & 734.9 & 0.0219 \\
\hline 11 & 29.205 & 733.6 & 0.0219 \\
\hline 12 & 29.280 & 733.2 & 0.0219 \\
\hline 13 & 29.288 & 734.0 & 0.0218 \\
\hline 14 & 29.140 & 736.1 & 0.0218 \\
\hline 15 & 28.664 & 739.6 & 0.0218 \\
\hline 16 & 27.352 & 743.4 & 0.0217 \\
\hline 17 & 23.832 & 741.3 & 0.0217 \\
\hline 18 & 15.337 & 707.7 & 0.0217 \\
\hline
\end{tabular}

Table 4-28. Burnup and TH Feedback Parameters by Axial Node for Assembly E20

\begin{tabular}{|c|c|c|c|c|c|c|c|c|c|}
\hline \multirow[b]{2}{*}{$\begin{array}{l}\text { Axial } \\
\text { Node }\end{array}$} & \multicolumn{3}{|c|}{ Statepoint 61 (114.4 Cy5) } & \multicolumn{3}{|c|}{ Datapoint (206.7 Cy5) } & \multicolumn{3}{|c|}{ Statepoint $62(\mathrm{BOC}$ Cy6) } \\
\hline & $\begin{array}{c}\text { Burnup } \\
\text { (GWd/MTU) }\end{array}$ & $\begin{array}{c}\text { T-Fuel } \\
\left({ }^{\circ} \mathrm{F}\right)\end{array}$ & $\begin{array}{c}\text { Spec. Vol. } \\
\left(\mathrm{ft}^{3} / \mathrm{lbm}\right)\end{array}$ & $\begin{array}{c}\text { Burnup } \\
\text { (GWd/MTU) }\end{array}$ & $\begin{array}{c}\text { T-Fuel } \\
\left({ }^{\circ} \mathrm{F}\right)\end{array}$ & $\begin{array}{c}\text { Spec. Vol. } \\
\left(\mathrm{ft}^{3} / \mathrm{lbm}\right)\end{array}$ & \begin{tabular}{|c|} 
Burnup \\
(GWd/MTU) \\
\end{tabular} & $\begin{array}{c}\text { T-Fuel } \\
\left({ }^{\circ} \mathrm{F}\right)\end{array}$ & $\begin{array}{r}\text { Spec. Vol } \\
\left(\mathrm{ft}^{3} / \mathrm{lbm}\right)\end{array}$ \\
\hline & 114.4 Cy5 & 58.2 Cy5 & 58.2 Cy 5 & 206.7 Cy5 & 153.5 Cy5 & 153.5 Cy 5 & BOC Cy6 & 252.2 Cy5 & 252.2 Cy5 \\
\hline 1 & 1.783 & 983.9 & 0.0236 & 3.443 & 1008.0 & 0.0236 & 5.207 & 948.4 & 0.0235 \\
\hline 2 & 3.093 & 1190.5 & 0.0235 & 5.813 & 1187.7 & 0.0235 & 8.568 & 1070.6 & 0.0235 \\
\hline 3 & 3.832 & 1294.6 & 0.0233 & 7.042 & 1268.8 & 0.0234 & 10.194 & 1117.7 & 0.0234 \\
\hline 4 & 4.212 & 1336.2 & 0.0232 & 7.615 & 1298.3 & 0.0232 & 10.905 & 1137.0 & 0.0232 \\
\hline 5 & 4.390 & 1347.1 & 0.0231 & 7.863 & 1307.3 & 0.0231 & 11.215 & 1154.0 & 0.0231 \\
\hline 6 & 4.456 & 1343.2 & 0.0230 & 7.951 & 1309.3 & 0.0230 & 11.364 & 1178.6 & 0.0230 \\
\hline 7 & 4.459 & 1333.5 & 0.0228 & 7.959 & 1308.8 & 0.0228 & 11.457 & 1214.3 & 0.0229 \\
\hline 8 & 4.448 & 1327.4 & 0.0227 & 7.949 & 1306.7 & 0.0227 & 11.557 & 1259.5 & 0.0228 \\
\hline 9 & 4.474 & 1335.6 & 0.0226 & 7.991 & 1305.6 & 0.0226 & 11.722 & 1305.9 & 0.0226 \\
\hline 10 & 4.549 & 1357.4 & 0.0225 & 8.108 & 1307.8 & 0.0225 & 11.947 & 1336.1 & 0.0225 \\
\hline 11 & 4.632 & 1383.3 & 0.0223 & 8.243 & 1311.0 & 0.0223 & 12.134 & 1344.7 & 0.0224 \\
\hline 12 & 4.695 & 1405.4 & 0.0222 & 8.358 & 1314.7 & 0.0222 & 12.242 & 1330.8 & 0.0222 \\
\hline 13 & 4.703 & 1414.3 & 0.0221 & 8.415 & 1319.0 & 0.0221 & 12.250 & 1301.0 & 0.0221 \\
\hline 14 & 4.613 & 1403.0 & 0.0220 & 8.344 & 1320.1 & 0.0220 & 12.109 & 1263.4 & 0.0220 \\
\hline 15 & 4.403 & 1369.7 & 0.0219 & 8.096 & 1313.1 & 0.0219 & 11.777 & 1228.3 & 0.0219 \\
\hline 16 & 4.016 & 1306.5 & 0.0217 & 7.547 & 1286.0 & 0.0218 & 11.087 & 1194.2 & 0.0218 \\
\hline 17 & 3.275 & 1183.5 & 0.0217 & 6.325 & 1203.3 & 0.0217 & 9.461 & 1133.3 & 0.0217 \\
\hline 18 & 1.909 & 938.9 & 0.0216 & 3.805 & 989.5 & 0.0216 & 5.847 & 969.4 & 0.0216 \\
\hline
\end{tabular}


Table 4-28. Burnup and TH Feedback Parameters by Axial Node for Assembly E20 (Cont.)

\begin{tabular}{|c|c|c|c|c|c|c|c|c|c|}
\hline \multirow{2}{*}{$\begin{array}{c}\text { Axial } \\
\text { Node }\end{array}$} & \multicolumn{2}{|c|}{ Statepoint 63 (69.1 Cy6) } & \multicolumn{2}{c|}{ Datapoint (176.7 Cy6) } & \multicolumn{3}{c|}{ Datapoint (320.3 Cy6) } \\
\cline { 2 - 10 } & $\begin{array}{c}\text { Burnup } \\
\text { GWd/MTU) }\end{array}$ & $\begin{array}{c}\text { T-Fuel } \\
\left({ }^{\circ} \mathrm{F}\right)\end{array}$ & $\begin{array}{c}\text { Spec. Vol. } \\
\left(\mathrm{ft}^{3} / \mathrm{lbm}\right)\end{array}$ & $\begin{array}{c}\text { Burnup } \\
(\mathrm{GWd} / \mathrm{MTU})\end{array}$ & $\begin{array}{c}\text { T-Fuel } \\
\left({ }^{\circ} \mathrm{F}\right)\end{array}$ & $\begin{array}{c}\text { Spec. Vol. } \\
\left(\mathrm{ft}^{3} / \mathrm{lbm}\right)\end{array}$ & $\begin{array}{c}\text { Burnup } \\
(\mathrm{GWd} / \mathrm{MTU})\end{array}$ & $\begin{array}{c}\text { T-Fuel } \\
\left({ }^{\circ} \mathrm{F}\right)\end{array}$ & $\begin{array}{c}\text { Spec. Vol. } \\
\left(\mathrm{ft}^{3} / \mathrm{lbm}\right)\end{array}$ \\
\hline 1 & $69.1 \mathrm{Cy} 6$ & $41.5 \mathrm{Cy} 6$ & $41.5 \mathrm{Cy} 6$ & $176.7 \mathrm{Cy} 6$ & $128.8 \mathrm{Cy} 6$ & $128.8 \mathrm{Cy} 6$ & $320.3 \mathrm{Cy} 6$ & $252.5 \mathrm{Cy} 6$ & $252.5 \mathrm{Cy} 6$ \\
\hline 2 & 5.707 & 727.7 & 0.0224 & 6.559 & 745.0 & 0.0223 & 7.928 & 774.6 & 0.0224 \\
\hline 3 & 11.203 & 824.9 & 0.0223 & 12.826 & 834.7 & 0.0223 & 15.214 & 853.6 & 0.0223 \\
\hline 4 & 12.017 & 846.6 & 0.0222 & 13.756 & 846.5 & 0.0222 & 16.219 & 855.1 & 0.0222 \\
\hline 5 & 12.390 & 860.1 & 0.0222 & 14.182 & 850.5 & 0.0222 & 16.645 & 850.8 & 0.0222 \\
\hline 6 & 12.579 & 868.4 & 0.0221 & 14.395 & 850.7 & 0.0221 & 16.834 & 845.1 & 0.0221 \\
\hline 7 & 12.698 & 873.2 & 0.0221 & 14.523 & 849.2 & 0.0221 & 16.934 & 839.6 & 0.0221 \\
\hline 8 & 12.811 & 875.3 & 0.0220 & 14.636 & 846.7 & 0.0220 & 17.022 & 834.6 & 0.0220 \\
\hline 9 & 12.979 & 874.3 & 0.0220 & 14.797 & 843.1 & 0.0220 & 17.160 & 830.0 & 0.0220 \\
\hline 10 & 13.196 & 870.7 & 0.0219 & 15.001 & 838.7 & 0.0219 & 17.346 & 825.7 & 0.0219 \\
\hline 11 & 13.367 & 865.5 & 0.0219 & 15.158 & 834.6 & 0.0219 & 17.493 & 822.6 & 0.0219 \\
\hline 12 & 13.453 & 859.5 & 0.0218 & 15.232 & 831.2 & 0.0218 & 17.570 & 821.3 & 0.0218 \\
\hline 13 & 13.435 & 852.8 & 0.0218 & 15.200 & 828.6 & 0.0218 & 17.553 & 821.9 & 0.0218 \\
\hline 14 & 13.257 & 844.6 & 0.0217 & 15.003 & 825.9 & 0.0217 & 17.377 & 824.0 & 0.0217 \\
\hline 15 & 12.870 & 832.6 & 0.0217 & 14.574 & 821.1 & 0.0217 & 16.957 & 826.0 & 0.0217 \\
\hline 16 & 12.087 & 812.4 & 0.0216 & 13.692 & 809.1 & 0.0216 & 16.019 & 823.2 & 0.0216 \\
\hline 17 & 10.291 & 776.0 & 0.0216 & 11.665 & 779.8 & 0.0216 & 13.745 & 803.3 & 0.0216 \\
\hline 18 & 6.356 & 705.7 & 0.0216 & 7.222 & 713.2 & 0.0216 & 8.596 & 737.1 & 0.0216 \\
\hline
\end{tabular}

\begin{tabular}{|c|c|c|c|c|c|c|c|c|c|}
\hline \multirow[b]{2}{*}{$\begin{array}{l}\text { Axial } \\
\text { Node }\end{array}$} & \multicolumn{3}{|c|}{ Statepoint 64 (BOC Cy7) } & \multicolumn{3}{|c|}{ Statepoint 65 (30.0 Cy7) } & \multicolumn{3}{|c|}{ Statepoint 66 (108.0 Cy7) } \\
\hline & $\begin{array}{c}\text { Burnup } \\
\text { (GWd/MTU) }\end{array}$ & $\begin{array}{c}\text { T-Fuel } \\
\left({ }^{\circ} \mathrm{F}\right)\end{array}$ & $\begin{array}{c}\text { Spec. Vol. } \\
\left(\mathrm{ft}^{3} / \mathrm{lbm}\right)\end{array}$ & $\begin{array}{c}\text { Burnup } \\
\text { (GWd/MTU) }\end{array}$ & $\begin{array}{l}\text { T-Fuel } \\
\left({ }^{\circ} \mathrm{F}\right)\end{array}$ & $\begin{array}{l}\text { Spec. Vol. } \\
\left(\mathrm{ft}^{3} / \mathrm{lbm}\right)\end{array}$ & $\begin{array}{c}\text { Burnup } \\
\text { (GWd/MTU) }\end{array}$ & $\begin{array}{c}\text { T-Fuel } \\
\left({ }^{\circ} \mathrm{F}\right)\end{array}$ & $\begin{array}{c}\text { Spec. Vol. } \\
\left(\mathrm{ft}^{3} / \mathrm{lbm}\right)\end{array}$ \\
\hline & BOC Cy 7 & 376.2 Cy 6 & 376.2 Cy 6 & 30.0 Cy 7 & 22.5 Cy 7 & 22.5 Cy 7 & 108.0 Cy 7 & 60.4 Cy? & $60.4 \mathrm{Cy} 7$ \\
\hline 1 & 9.082 & 806.7 & 0.0225 & 9.266 & 692.6 & 0.0222 & 9.774 & 697.3 & 0.0222 \\
\hline 2 & 14.587 & 862.5 & 0.0224 & 14.880 & 734.0 & 0.0221 & 15.679 & 738.7 & 0.0221 \\
\hline 3 & 17.076 & 873.8 & 0.0224 & 17.423 & 754.0 & 0.0221 & 18.359 & 757.3 & 0.0221 \\
\hline 4 & 18.095 & 868.4 & 0.0223 & 18.474 & 764.8 & 0.0221 & 19.478 & 766.2 & 0.0221 \\
\hline 5 & 18.495 & 860.2 & 0.0222 & 18.893 & 771.5 & 0.0220 & 19.936 & 771.0 & 0.0220 \\
\hline 6 & 18.652 & 852.9 & 0.0222 & 19.062 & 775.4 & 0.0220 & 20.127 & 773.2 & 0.0220 \\
\hline 7 & 18.727 & 847.0 & 0.0221 & 19.144 & 777.2 & 0.0220 & 20.218 & 773.6 & 0.0220 \\
\hline 8 & 18.796 & 842.3 & 0.0221 & 19.216 & 777.1 & 0.0219 & 20.292 & 772.5 & 0.0219 \\
\hline 9 & 18.920 & 838.1 & 0.0220 & 19.340 & 775.3 & 0.0219 & 20.411 & 770.2 & 0.0219 \\
\hline 10 & 19.095 & 834.2 & 0.0220 & 19.512 & 772.3 & 0.0219 & 20.575 & 767.0 & 0.0219 \\
\hline 11 & 19.239 & 831.5 & 0.0219 & 19.651 & 768.7 & 0.0218 & 20.705 & 763.6 & 0.0218 \\
\hline 12 & 19.322 & 830.6 & 0.0219 & 19.728 & 764.7 & 0.0218 & 20.770 & 760.0 & 0.0218 \\
\hline 13 & 19.324 & 831.8 & 0.0218 & 19.720 & 759.7 & 0.0218 & 20.747 & 755.8 & 0.0218 \\
\hline 14 & 19.179 & 835.5 & 0.0218 & 19.562 & 753.2 & 0.0217 & 20.564 & 750.5 & 0.0217 \\
\hline 15 & 18.792 & 840.9 & 0.0217 & 19.156 & 744.3 & 0.0217 & 20.117 & 743.1 & 0.0217 \\
\hline 16 & 17.854 & 844.4 & 0.0217 & 18.188 & 731.5 & 0.0217 & 19.079 & 731.9 & 0.0217 \\
\hline 17 & 15.443 & 833.5 & 0.0216 & 15.724 & 710.5 & 0.0216 & 16.484 & 712.2 & 0.0216 \\
\hline 18 & 9.770 & 767.9 & 0.0216 & 9.948 & 665.1 & 0.0216 & 10.435 & 667.2 & 0.0216 \\
\hline
\end{tabular}


Table 4-29. Burnup and TH Feedback Parameters by Axial Node for Assembly E20a

\begin{tabular}{|c|c|c|c|c|c|c|c|c|c|}
\hline \multirow[b]{2}{*}{$\begin{array}{l}\text { Axial } \\
\text { Node }\end{array}$} & \multicolumn{3}{|c|}{ Statepoint 61 (114.4 Cy5) } & \multicolumn{3}{|c|}{ Datapoint (206.7 Cy5) } & \multicolumn{3}{|c|}{ Statepoint 62 (BOC Cy6) } \\
\hline & $\begin{array}{c}\text { Burnup } \\
\text { (GWd/MTU) }\end{array}$ & $\begin{array}{c}\text { T-Fuel } \\
\left({ }^{\circ} \mathrm{F}\right)\end{array}$ & $\begin{array}{l}\text { Spec. Vol. } \\
\left(\mathrm{ft}^{3} / \mathrm{lbm}\right)\end{array}$ & $\begin{array}{c}\text { Burnup } \\
\text { (GWd/MTU) }\end{array}$ & $\begin{array}{c}\text { T-Fuel } \\
\left({ }^{\circ} \mathrm{F}\right)\end{array}$ & $\begin{array}{c}\text { Spec. Vol. } \\
\left(\mathrm{ft}^{3} / \mathrm{lbm}\right)\end{array}$ & $\begin{array}{c}\text { Burnup } \\
\text { (GWd/MTU) }\end{array}$ & $\begin{array}{l}\text { T-Fuel } \\
\left({ }^{\circ} \mathrm{F}\right)\end{array}$ & $\begin{array}{c}\text { Spec. Vol. } \\
\left(\mathrm{ft}^{3} / \mathrm{lbm}\right)\end{array}$ \\
\hline & 114.4 Cy 5 & 58.2 Cy 5 & 58.2 Cy5 & 206.7 Cy5 & 153.5 Cy 5 & 153.5 Cy5 & BOC Cy6 & 252.2 Cy 5 & 252.2 Cy 5 \\
\hline 1 & 1.783 & 983.9 & 0.0236 & 3.443 & 1008.0 & 0.0236 & 5.207 & 948.4 & 0.0235 \\
\hline 2 & 3.093 & 1190.5 & 0.0235 & 5.813 & 1187.7 & 0.0235 & 8.568 & 1070.6 & 0.0235 \\
\hline 3 & 3.832 & 1294.6 & 0.0233 & 7.042 & 1268.8 & 0.0234 & 10.194 & 1117.7 & 0.0234 \\
\hline 4 & 4.212 & 1336.2 & 0.0232 & 7.615 & 1298.3 & 0.0232 & 10.905 & 1137.0 & 0.0232 \\
\hline 5 & 4.390 & 1347.1 & 0.0231 & 7.863 & 1307.3 & 0.0231 & 11.215 & 1154.0 & 0.0231 \\
\hline 6 & 4.456 & 1343.2 & 0.0230 & 7.951 & 1309.3 & 0.0230 & 11.364 & 1178.6 & 0.0230 \\
\hline 7 & 4.459 & 1333.5 & 0.0228 & 7.959 & 1308.8 & 0.0228 & 11.457 & 1214.3 & 0.0229 \\
\hline 8 & 4.448 & 1327.4 & 0.0227 & 7.949 & 1306.7 & 0.0227 & 11.557 & 1259.5 & 0.0228 \\
\hline 9 & 4.474 & 1335.6 & 0.0226 & 7.991 & 1305.6 & 0.0226 & 11.722 & 1305.9 & 0.0226 \\
\hline 10 & 4.549 & 1357.4 & 0.0225 & 8.108 & 1307.8 & 0.0225 & 11.947 & 1336.1 & 0.0225 \\
\hline 11 & 4.632 & 1383.3 & 0.0223 & 8.243 & 1311.0 & 0.0223 & 12.134 & 1344.7 & 0.0224 \\
\hline 12 & 4.695 & 1405.4 & 0.0222 & 8.358 & 1314.7 & 0.0222 & 12.242 & 1330.8 & 0.0222 \\
\hline 13 & 4.703 & 1414.3 & 0.0221 & 8.415 & 1319.0 & 0.0221 & 12.250 & 1301.0 & 0.0221 \\
\hline 14 & 4.613 & 1403.0 & 0.0220 & 8.344 & 1320.1 & 0.0220 & 12.109 & 1263.4 & 0.0220 \\
\hline 15 & 4.403 & 1369.7 & 0.0219 & 8.096 & 1313.1 & 0.0219 & 11.777 & 1228.3 & 0.0219 \\
\hline 16 & 4.016 & 1306.5 & 0.0217 & 7.547 & 1286.0 & 0.0218 & 11.087 & 1194.2 & 0.0218 \\
\hline 17 & 3.275 & 1183.5 & 0.0217 & 6.325 & 1203.3 & 0.0217 & 9.461 & 1133.3 & 0.0217 \\
\hline 18 & 1.909 & 938.9 & 0.0216 & 3.805 & 989.5 & 0.0216 & 5.847 & 969.4 & 0.0216 \\
\hline
\end{tabular}

\begin{tabular}{|c|c|c|c|c|c|c|c|c|c|}
\hline \multirow[b]{2}{*}{$\begin{array}{l}\text { Axial } \\
\text { Node }\end{array}$} & \multicolumn{3}{|c|}{ Statepoint 63 (69.1 Cy6) } & \multicolumn{3}{|c|}{ Datapoint (176.7 Cy6) } & \multicolumn{3}{|c|}{ Datapoint (320.3 Cy6) } \\
\hline & $\begin{array}{c}\text { Burnup } \\
\text { (GWd/MTU) }\end{array}$ & $\begin{array}{c}\text { T-Fuel } \\
\left({ }^{\circ} \mathrm{F}\right)\end{array}$ & $\begin{array}{c}\text { Spec. Vol. } \\
\left(\mathrm{ft}^{3} / \mathrm{bm}\right)\end{array}$ & $\begin{array}{c}\text { Burnup } \\
\text { (GWd/MTU) }\end{array}$ & $\begin{array}{c}\text { T-Fuel } \\
\left({ }^{\circ} \mathrm{F}\right)\end{array}$ & $\begin{array}{c}\text { Spec. Vol. } \\
\left(\mathrm{tt}^{3} / \mathrm{bm}\right)\end{array}$ & $\begin{array}{c}\text { Burnup } \\
\text { (GWd/MTU) }\end{array}$ & $\begin{array}{c}\text { T-Fuel } \\
\left({ }^{\circ} \mathrm{F}\right)\end{array}$ & $\begin{array}{c}\text { Spec. Vol. } \\
\left(\mathrm{ft}^{3} / \mathrm{lbm}\right)\end{array}$ \\
\hline & 69.1 Cy 6 & 41.5 Cy 6 & 41.5 Cy6 & 176.7 Cy6 & 128.8 Cy 6 & 128.8 Cy 6 & 320.3 Cy 6 & 252.5 Cy 6 & $252.5 \mathrm{Cy} 6$ \\
\hline 1 & 5.707 & 727.7 & 0.0224 & 6.559 & 745.0 & 0.0223 & 7.928 & 774.6 & 0.0224 \\
\hline 2 & 9.398 & 789.8 & 0.0223 & 10.774 & 804.9 & 0.0223 & 12.885 & 833.0 & 0.0223 \\
\hline 3 & 11.203 & 824.9 & 0.0223 & 12.826 & 834.7 & 0.0223 & 15.214 & 853.6 & 0.0223 \\
\hline 4 & 12.017 & 846.6 & 0.0222 & 13.756 & 846.5 & 0.0222 & 16.219 & 855.1 & 0.0222 \\
\hline 5 & 12.390 & 860.1 & 0.0222 & 14.182 & 850.5 & 0.0222 & 16.645 & 850.8 & 0.0222 \\
\hline 6 & 12.579 & 868.4 & 0.0221 & 14.395 & 850.7 & 0.0221 & 16.834 & 845.1 & 0.0221 \\
\hline 7 & 12.698 & 873.2 & 0.0221 & 14.523 & 849.2 & 0.0221 & 16.934 & 839.6 & 0.0221 \\
\hline 8 & 12.811 & 875.3 & 0.0220 & 14.636 & 846.7 & 0.0220 & 17.022 & 834.6 & 0.0220 \\
\hline 9 & 12.979 & 874.3 & 0.0220 & 14.797 & 843.1 & 0.0220 & 17.160 & 830.0 & 0.0220 \\
\hline 10 & 13.196 & 870.7 & 0.0219 & 15.001 & 838.7 & 0.0219 & 17.346 & 825.7 & 0.0219 \\
\hline 11 & 13.367 & 865.5 & 0.0219 & 15.158 & 834.6 & 0.0219 & 17.493 & 822.6 & 0.0219 \\
\hline 12 & 13.453 & 859.5 & 0.0218 & 15.232 & 831.2 & 0.0218 & 17.570 & 821.3 & 0.0218 \\
\hline 13 & 13.435 & 852.8 & 0.0218 & 15.200 & 828.6 & 0.0218 & 17.553 & 821.9 & 0.0218 \\
\hline 14 & 13.257 & 844.6 & 0.0217 & 15.003 & 825.9 & 0.0217 & 17.377 & 824.0 & 0.0217 \\
\hline 15 & 12.870 & 832.6 & 0.0217 & 14.574 & 821.1 & 0.0217 & 16.957 & 826.0 & 0.0217 \\
\hline 16 & 12.087 & 812.4 & 0.0216 & 13.692 & 809.1 & 0.0216 & 16.019 & 823.2 & 0.0216 \\
\hline 17 & 10.291 & 776.0 & 0.0216 & 11.665 & 779.8 & 0.0216 & 13.745 & 803.3 & 0.0216 \\
\hline 18 & 6.356 & 705.7 & 0.0216 & 7.222 & 713.2 & 0.0216 & 8.596 & 737.1 & 0.0216 \\
\hline
\end{tabular}


Table 4-29. Burnup and TH Feedback Parameters by Axial Node for Assembly E20a (Cont.)

\begin{tabular}{|c|c|c|c|c|c|c|c|c|c|}
\hline \multirow[b]{2}{*}{$\begin{array}{l}\text { Axial } \\
\text { Node }\end{array}$} & \multicolumn{3}{|c|}{ Statepoint 64 (BOC Cy7) } & \multicolumn{3}{|c|}{ Statepoint 65 (30.0 Cy7) } & \multicolumn{3}{|c|}{ Statepoint $66(108.0 \mathrm{Cy} 7)$} \\
\hline & $\begin{array}{c}\text { Burnup } \\
\text { (GWd/MTU) }\end{array}$ & $\begin{array}{c}\text { T-Fuel } \\
\left({ }^{\circ} \mathrm{F}\right)\end{array}$ & $\begin{array}{c}\text { Spec. Vol. } \\
\left(\mathrm{ft}^{3} / \mathrm{lbm}\right)\end{array}$ & $\begin{array}{c}\text { Burnup } \\
\text { (GWd/MTU) }\end{array}$ & $\begin{array}{c}\text { T-Fuel } \\
\left({ }^{\circ} \mathrm{F}\right)\end{array}$ & $\begin{array}{c}\text { Spec. Vol. } \\
\left(\mathrm{ft}^{3} / \mathrm{lbm}\right)\end{array}$ & $\begin{array}{c}\text { Burnup } \\
\text { (GWd/MTU) }\end{array}$ & $\begin{array}{c}\text { T-Fuel } \\
\left({ }^{\circ} \mathrm{F}\right)\end{array}$ & $\begin{array}{l}\text { Spec. Vol } \\
\left(\mathrm{ft}^{3} / \mathrm{lbm}\right)\end{array}$ \\
\hline & BOC Cy7 & 376.2 Cy 6 & 376.2 Cy6 & $30.0 \mathrm{Cy} 7$ & $22.5 \mathrm{Cy} 7$ & $22.5 \mathrm{Cy} 7$ & $108.0 \mathrm{Cy} 7$ & $60.4 \mathrm{Cy} 7$ & $60.4 \mathrm{Cy} 7$ \\
\hline 1 & 9.074 & 806.7 & 0.0225 & 9.612 & 947.5 & 0.0234 & 11.106 & 959.9 & 0.0235 \\
\hline 2 & 14.574 & 862.5 & 0.0224 & 15.407 & 1054.3 & 0.0233 & 17.709 & 1068.2 & 0.0234 \\
\hline 3 & 17.061 & 873.8 & 0.0224 & 18.047 & 1107.9 & 0.0232 & 20.753 & 1119.5 & 0.0233 \\
\hline 4 & 18.081 & 868.4 & 0.0223 & 19.159 & 1140.7 & 0.0231 & 22.098 & 1149.4 & 0.0232 \\
\hline 5 & 18.481 & 860.2 & 0.0222 & 19.621 & 1162.5 & 0.0230 & 22.701 & 1168.8 & 0.0230 \\
\hline 6 & 18.639 & 852.9 & 0.0222 & 19.820 & 1176.3 & 0.0229 & 22.983 & 1179.7 & 0.0229 \\
\hline 7 & 18.715 & 847.0 & 0.0221 & 19.920 & 1183.5 & 0.0227 & 23.130 & 1184.3 & 0.0228 \\
\hline 8 & 18.785 & 842.3 & 0.0221 & 20.003 & 1185.2 & 0.0226 & 23.233 & 1184.2 & 0.0226 \\
\hline 9 & 18.910 & 838.1 & 0.0220 & 20.130 & 1181.7 & 0.0225 & 23.359 & 1180.0 & 0.0225 \\
\hline 10 & 19.087 & 834.2 & 0.0220 & 20.299 & 1173.9 & 0.0224 & 23.512 & 1172.5 & 0.0224 \\
\hline 11 & 19.231 & 831.5 & 0.0219 & 20.429 & 1164.0 & 0.0223 & 23.614 & 1163.3 & 0.0223 \\
\hline 12 & 19.313 & 830.6 & 0.0219 & 20.490 & 1151.1 & 0.0222 & 23.633 & 1152.0 & 0.0222 \\
\hline 13 & 19.315 & 831.8 & 0.0218 & 20.459 & 1134.3 & 0.0220 & 23.540 & 1137.6 & 0.0221 \\
\hline 14 & 19.169 & 835.5 & 0.0218 & 20.269 & 1112.4 & 0.0219 & 23.257 & 1118.7 & 0.0220 \\
\hline 15 & 18.782 & 840.9 & 0.0217 & 19.817 & 1084.0 & 0.0218 & 22.661 & 1092.3 & 0.0219 \\
\hline 16 & 17.845 & 844.4 & 0.0217 & 18.785 & 1045.4 & 0.0218 & 21.397 & 1054.6 & 0.0218 \\
\hline 17 & 15.434 & 833.5 & 0.0216 & 16.227 & 988.6 & 0.0217 & 18.447 & 1000.3 & 0.0217 \\
\hline 18 & 9.764 & 767.9 & 0.0216 & 10.278 & 866.6 & 0.0216 & 11.725 & 878.4 & 0.0216 \\
\hline
\end{tabular}

Table 4-30. Burnup and TH Feedback Parameters by Axial Node for Assembly E20b

\begin{tabular}{|c|c|c|c|c|c|c|c|c|c|}
\hline \multirow[b]{2}{*}{$\begin{array}{l}\text { Axlal } \\
\text { Node }\end{array}$} & \multicolumn{3}{|c|}{ Statepoint 61 (114.4 Cy5) } & \multicolumn{3}{|c|}{ Datapoint (206.7 Cy5) } & \multicolumn{3}{|c|}{ Statepoint 62 (BOC Cy6) } \\
\hline & $\begin{array}{c}\text { Burnup } \\
\text { (GWd/MTU) }\end{array}$ & $\begin{array}{c}\text { T-Fuel } \\
\left({ }^{\circ} \mathrm{F}\right)\end{array}$ & $\begin{array}{c}\text { Spec. Vol. } \\
\left(\mathrm{ft}^{3} / \mathrm{lbm}\right)\end{array}$ & $\begin{array}{c}\text { Burnup } \\
\text { (GWd/MTU) }\end{array}$ & $\begin{array}{c}\text { T-Fuel } \\
\left({ }^{\circ} \mathrm{F}\right)\end{array}$ & $\begin{array}{c}\text { Spec. Vol. } \\
\left(\mathrm{ft}^{3} / \mathrm{lbm}\right)\end{array}$ & $\begin{array}{c}\text { Burnup } \\
\text { (GWd/MTU) }\end{array}$ & $\begin{array}{l}\text { T-Fuel } \\
\left({ }^{\circ} \mathrm{F}\right)\end{array}$ & $\begin{array}{l}\text { Spec. Vol. } \\
\left(\mathrm{tt}^{3} / \mathrm{bm}\right)\end{array}$ \\
\hline & 114.4 Cy5 & 58.2 Cy5 & 58.2 Cy5 & 206.7 Cy5 & $153.5 \mathrm{Cy} 5$ & $153.5 \mathrm{Cy} 5$ & BOC Cy 6 & 252.2 Cy5 & 252.2 Cy 5 \\
\hline 1 & 1.783 & 983.9 & 0.0236 & 3.443 & 1008.0 & 0.0236 & 5.207 & 948.4 & 0.0235 \\
\hline 2 & 3.093 & 1190.5 & 0.0235 & 5.813 & 1187.7 & 0.0235 & 8.568 & 1070.6 & 0.0235 \\
\hline 3 & 3.832 & 1294.6 & 0.0233 & 7.042 & 1268.8 & 0.0234 & 10.194 & 1117.7 & 0.0234 \\
\hline 4 & 4.212 & 1336.2 & 0.0232 & 7.615 & 1298.3 & 0.0232 & 10.905 & 1137.0 & 0.0232 \\
\hline 5 & 4.390 & 1347.1 & 0.0231 & 7.863 & 1307.3 & 0.0231 & 11.215 & 1154.0 & 0.0231 \\
\hline 6 & 4.456 & 1343.2 & 0.0230 & 7.951 & 1309.3 & 0.0230 & 11.364 & 1178.6 & 0.0230 \\
\hline 7 & 4.459 & 1333.5 & 0.0228 & 7.959 & 1308.8 & 0.0228 & 11.457 & 1214.3 & 0.0229 \\
\hline 8 & 4.448 & 1327.4 & 0.0227 & 7.949 & 1306.7 & 0.0227 & 11.557 & 1259.5 & 0.0228 \\
\hline 9 & 4.474 & 1335.6 & 0.0226 & 7.991 & 1305.6 & 0.0226 & 11.722 & 1305.9 & 0.0226 \\
\hline 10 & 4.549 & 1357.4 & 0.0225 & 8.108 & 1307.8 & 0.0225 & 11.947 & 1336.1 & 0.0225 \\
\hline 11 & 4.632 & 1383.3 & 0.0223 & 8.243 & 1311.0 & 0.0223 & 12.134 & 1344.7 & 0.0224 \\
\hline 12 & 4.695 & 1405.4 & 0.0222 & 8.358 & 1314.7 & 0.0222 & 12.242 & 1330.8 & 0.0222 \\
\hline 13 & 4.703 & 1414.3 & 0.0221 & 8.415 & 1319.0 & 0.0221 & 12.250 & 1301.0 & 0.0221 \\
\hline 14 & 4.613 & 1403.0 & 0.0220 & 8.344 & 1320.1 & 0.0220 & 12.109 & 1263.4 & 0.0220 \\
\hline 15 & 4.403 & 1369.7 & 0.0219 & 8.096 & 1313.1 & 0.0219 & 11.777 & 1228.3 & 0.0219 \\
\hline 16 & 4.016 & 1306.5 & 0.0217 & 7.547 & 1286.0 & 0.0218 & 11.087 & 1194.2 & 0.0218 \\
\hline 17 & 3.275 & 1183.5 & 0.0217 & 6.325 & 1203.3 & 0.0217 & 9.461 & 1133.3 & 0.0217 \\
\hline 18 & 1.909 & 938.9 & 0.0216 & 3.805 & 989.5 & 0.0216 & 5.847 & 969.4 & 0.0216 \\
\hline
\end{tabular}


Table 4-30. Burnup and TH Feedback Parameters by Axial Node for Assembly E20b (Cont.)

\begin{tabular}{|c|c|c|c|c|c|c|c|c|c|}
\hline \multirow{2}{*}{$\begin{array}{c}\text { Axial } \\
\text { Node }\end{array}$} & \multicolumn{2}{|c|}{ Statepoint 63 (69.1 Cy6) } & \multicolumn{2}{c|}{ Datapoint (176.7 Cy6) } & \multicolumn{3}{c|}{ Datapolnt (320.3 Cy6) } \\
\cline { 2 - 10 } & $\begin{array}{c}\text { Burnup } \\
(\mathrm{GWd} / \mathrm{MTU})\end{array}$ & $\begin{array}{c}\text { T-Fuel } \\
\left({ }^{\circ} \mathrm{F}\right)\end{array}$ & $\begin{array}{c}\text { Spec. Vol. } \\
\left(\mathrm{ft}^{3} / \mathrm{lbm}\right)\end{array}$ & $\begin{array}{c}\text { Burnup } \\
(\mathrm{GWd} / \mathrm{MTU})\end{array}$ & $\begin{array}{c}\text { T-Fuel } \\
\left({ }^{\circ} \mathrm{F}\right)\end{array}$ & $\begin{array}{c}\text { Spec. Vol. } \\
\left(\mathrm{ft}^{3} / \mathrm{lbm}\right)\end{array}$ & $\begin{array}{c}\text { Burnup } \\
(\mathrm{GWd} / \mathrm{MTU})\end{array}$ & $\begin{array}{c}\text { T-Fuel } \\
\left({ }^{\circ} \mathrm{F}\right)\end{array}$ & $\begin{array}{c}\text { Spec. Vol. } \\
\left(\mathrm{ft}^{3} / \mathrm{lbm}\right)\end{array}$ \\
\hline 1 & $69.1 \mathrm{Cy} 6$ & $41.5 \mathrm{Cy} 6$ & $41.5 \mathrm{Cy} 6$ & $176.7 \mathrm{Cy} 6$ & $128.8 \mathrm{Cy} 6$ & $128.8 \mathrm{Cy} 6$ & 320.3 Cy6 & $252.5 \mathrm{Cy} 6$ & $252.5 \mathrm{Cy} 6$ \\
\hline 2 & 5.707 & 727.7 & 0.0224 & 6.559 & 745.0 & 0.0223 & 7.928 & 774.6 & 0.0224 \\
\hline 3 & 11.203 & 824.9 & 0.0223 & 12.826 & 834.7 & 0.0223 & 15.214 & 853.6 & 0.0223 \\
\hline 4 & 12.017 & 846.6 & 0.0222 & 13.756 & 846.5 & 0.0222 & 16.219 & 855.1 & 0.0222 \\
\hline 5 & 12.390 & 860.1 & 0.0222 & 14.182 & 850.5 & 0.0222 & 16.645 & 850.8 & 0.0222 \\
\hline 6 & 12.579 & 868.4 & 0.0221 & 14.395 & 850.7 & 0.0221 & 16.834 & 845.1 & 0.0221 \\
\hline 7 & 12.698 & 873.2 & 0.0221 & 14.523 & 849.2 & 0.0221 & 16.934 & 839.6 & 0.0221 \\
\hline 8 & 12.811 & 875.3 & 0.0220 & 14.636 & 846.7 & 0.0220 & 17.022 & 834.6 & 0.0220 \\
\hline 9 & 12.979 & 874.3 & 0.0220 & 14.797 & 843.1 & 0.0220 & 17.160 & 830.0 & 0.0220 \\
\hline 10 & 13.196 & 870.7 & 0.0219 & 15.001 & 838.7 & 0.0219 & 17.346 & 825.7 & 0.0219 \\
\hline 11 & 13.367 & 865.5 & 0.0219 & 15.158 & 834.6 & 0.0219 & 17.493 & 822.6 & 0.0219 \\
\hline 12 & 13.453 & 859.5 & 0.0218 & 15.232 & 831.2 & 0.0218 & 17.570 & 821.3 & 0.0218 \\
\hline 13 & 13.435 & 852.8 & 0.0218 & 15.200 & 828.6 & 0.0218 & 17.553 & 821.9 & 0.0218 \\
\hline 14 & 13.257 & 844.6 & 0.0217 & 15.003 & 825.9 & 0.0217 & 17.377 & 824.0 & 0.0217 \\
\hline 15 & 12.870 & 832.6 & 0.0217 & 14.574 & 821.1 & 0.0217 & 16.957 & 826.0 & 0.0217 \\
\hline 16 & 12.087 & 812.4 & 0.0216 & 13.692 & 809.1 & 0.0216 & 16.019 & 823.2 & 0.0216 \\
\hline 17 & 10.291 & 776.0 & 0.0216 & 11.665 & 779.8 & 0.0216 & 13.745 & 803.3 & 0.0216 \\
\hline 18 & 6.356 & 705.7 & 0.0216 & 7.222 & 713.2 & 0.0216 & 8.596 & 737.1 & 0.0216 \\
\hline
\end{tabular}

\begin{tabular}{|c|c|c|c|}
\hline \multirow{2}{*}{$\begin{array}{c}\text { Axial } \\
\text { Node }\end{array}$} & $\begin{array}{c}\text { Burnup } \\
\text { (GWd/MTU) }\end{array}$ & $\begin{array}{c}\text { Statepoint 67 (BOC Cy8) } \\
\text { T-Fuel } \\
\text { ( } \mathrm{F} \text { ) }\end{array}$ & $\begin{array}{c}\text { Spec. Vol. } \\
\text { (ft/1/bm) }\end{array}$ \\
\hline 1 & BOC Cy8 & 376.2 Cy6 & 376.2 Cy6 \\
\hline 2 & 9.074 & 806.7 & 0.0225 \\
\hline 3 & 14.574 & 862.5 & 0.0224 \\
\hline 4 & 17.061 & 873.8 & 0.0224 \\
\hline 5 & 18.081 & 868.4 & 0.0223 \\
\hline 6 & 18.481 & 860.2 & 0.0222 \\
\hline 7 & 18.639 & 852.9 & 0.0222 \\
\hline 8 & 18.715 & 847.0 & 0.0221 \\
\hline 9 & 18.785 & 842.3 & 0.0221 \\
\hline 10 & 18.910 & 838.1 & 0.0220 \\
\hline 11 & 19.087 & 834.2 & 0.0220 \\
\hline 12 & 19.231 & 831.5 & 0.0219 \\
\hline 13 & 19.313 & 830.6 & 0.0219 \\
\hline 14 & 19.315 & 831.8 & 0.0218 \\
\hline 15 & 19.169 & 835.5 & 0.0218 \\
\hline 16 & 18.782 & 840.9 & 0.0217 \\
\hline 17 & 17.845 & 844.4 & 0.0217 \\
\hline 18 & 15.434 & 833.5 & 0.0216 \\
\hline
\end{tabular}


Table 4-31. Burnup and TH Feedback Parameters by Axial Node for Assembly E20c

\begin{tabular}{|c|c|c|c|c|c|c|c|c|c|}
\hline \multirow{2}{*}{$\begin{array}{c}\text { Axial } \\
\text { Node }\end{array}$} & \multicolumn{2}{|c|}{ Statepoint 61 (114.4 Cy5) } & \multicolumn{2}{c|}{ Datapoint (206.7 Cy5) } & \multicolumn{3}{c|}{ Statepoint 62 (BOC Cy6) } \\
\cline { 2 - 10 } & $\begin{array}{c}\text { Burnup } \\
(\mathrm{GWd} / \mathrm{MTU})\end{array}$ & $\begin{array}{c}\text { T-Fuel } \\
\left({ }^{\circ} \mathrm{F}\right)\end{array}$ & $\begin{array}{c}\text { Spec. Vol. } \\
\left(\mathrm{ft}^{3} / \mathrm{lbm}\right)\end{array}$ & $\begin{array}{c}\text { Burnup } \\
(\mathrm{GWd} / \mathrm{MTU})\end{array}$ & $\begin{array}{c}\text { T-Fuel } \\
\left({ }^{\circ} \mathrm{F}\right)\end{array}$ & $\begin{array}{c}\text { Spec. Vol. } \\
\left(\mathrm{ft}^{3} / \mathrm{lbm}\right)\end{array}$ & $\begin{array}{c}\text { Burnup } \\
(\mathrm{GWd} / \mathrm{MTU})\end{array}$ & $\begin{array}{c}\text { T-Fuel } \\
\left({ }^{\circ} \mathrm{F}\right)\end{array}$ & $\begin{array}{c}\text { Spec. Vol. } \\
\left(\mathrm{ft}^{3} / \mathrm{lbm}\right)\end{array}$ \\
\hline 1 & $114.4 \mathrm{Cy} 5$ & 58.2 Cy5 & 58.2 Cy5 & 206.7 Cy5 & 153.5 Cy5 & 153.5 Cy5 & BOC Cy6 & 252.2 Cy5 & 252.2 Cy5 \\
\hline 2 & 1.783 & 983.9 & 0.0236 & 3.443 & 1008.0 & 0.0236 & 5.207 & 948.4 & 0.0235 \\
\hline 3 & 3.093 & 1190.5 & 0.0235 & 5.813 & 1187.7 & 0.0235 & 8.568 & 1070.6 & 0.0235 \\
\hline 4 & 4.212 & 1336.2 & 0.0232 & 7.615 & 1298.3 & 0.0232 & 10.905 & 1137.0 & 0.0232 \\
\hline 5 & 4.390 & 1347.1 & 0.0231 & 7.863 & 1307.3 & 0.0231 & 11.215 & 1154.0 & 0.0231 \\
\hline 6 & 4.456 & 1343.2 & 0.0230 & 7.951 & 1309.3 & 0.0230 & 11.364 & 1178.6 & 0.0230 \\
\hline 7 & 4.459 & 1333.5 & 0.0228 & 7.959 & 1308.8 & 0.0228 & 11.457 & 1214.3 & 0.0229 \\
\hline 8 & 4.448 & 1327.4 & 0.0227 & 7.949 & 1306.7 & 0.0227 & 11.557 & 1259.5 & 0.0228 \\
\hline 9 & 4.474 & 1335.6 & 0.0226 & 7.991 & 1305.6 & 0.0226 & 11.722 & 1305.9 & 0.0226 \\
\hline 10 & 4.549 & 1357.4 & 0.0225 & 8.108 & 1307.8 & 0.0225 & 11.947 & 1336.1 & 0.0225 \\
\hline 11 & 4.632 & 1383.3 & 0.0223 & 8.243 & 1311.0 & 0.0223 & 12.134 & 1344.7 & 0.0224 \\
\hline 12 & 4.695 & 1405.4 & 0.0222 & 8.358 & 1314.7 & 0.0222 & 12.242 & 1330.8 & 0.0222 \\
\hline 13 & 4.703 & 1414.3 & 0.0221 & 8.415 & 1319.0 & 0.0221 & 12.250 & 1301.0 & 0.0221 \\
\hline 14 & 4.613 & 1403.0 & 0.0220 & 8.344 & 1320.1 & 0.0220 & 12.109 & 1263.4 & 0.0220 \\
\hline 15 & 4.403 & 1369.7 & 0.0219 & 8.096 & 1313.1 & 0.0219 & 11.777 & 1228.3 & 0.0219 \\
\hline 16 & 4.016 & 1306.5 & 0.0217 & 7.547 & 1286.0 & 0.0218 & 11.087 & 1194.2 & 0.0218 \\
\hline 17 & 3.275 & 1183.5 & 0.0217 & 6.325 & 1203.3 & 0.0217 & 9.461 & 1133.3 & 0.0217 \\
\hline 18 & 1.909 & 938.9 & 0.0216 & 3.805 & 989.5 & 0.0216 & 5.847 & 969.4 & 0.0216 \\
\hline
\end{tabular}

\begin{tabular}{|c|c|c|c|c|c|c|c|c|c|}
\hline \multirow[b]{2}{*}{$\begin{array}{l}\text { Axial } \\
\text { Node }\end{array}$} & \multicolumn{3}{|c|}{ Statepoint 63 (69.1 Cy6) } & \multicolumn{3}{|c|}{ Datapoint (176.7 Cy6) } & \multicolumn{3}{|c|}{ Datapoint (320.3 Cy6) } \\
\hline & $\begin{array}{c}\text { Burnup } \\
\text { (GWd/MTU) }\end{array}$ & $\begin{array}{c}\text { T-Fuel } \\
\left({ }^{\circ} \mathrm{F}\right)\end{array}$ & $\begin{array}{c}\text { Spec. Vol. } \\
\left(\mathrm{ft}^{3} / \mathrm{lbm}\right)\end{array}$ & $\begin{array}{c}\text { Burnup } \\
\text { (GWd/MTU) }\end{array}$ & $\begin{array}{c}\text { T-Fuel } \\
\left({ }^{\circ} \mathrm{F}\right)\end{array}$ & $\begin{array}{c}\text { Spec. Vol. } \\
\left(\mathrm{ft}^{3} / \mathrm{lbm}\right)\end{array}$ & $\begin{array}{c}\text { Burnup } \\
\text { (GWd/MTU) }\end{array}$ & $\begin{array}{c}\text { T-Fuel } \\
\left({ }^{\circ} \mathrm{F}\right)\end{array}$ & $\begin{array}{c}\text { Spec. Vol. } \\
\left(\mathrm{tt}^{3} / \mathrm{lbm}\right)\end{array}$ \\
\hline & 69.1 Cy6 & 41.5 Cy6 & 41.5 Cy 6 & 176.7 Cy 6 & $128.8 \mathrm{Cy} 6$ & 128.8 Cy 6 & 320.3 Cy 6 & 252.5 Cy6 & 252.5 Cy 6 \\
\hline 1 & 5.707 & 727.7 & 0.0224 & 6.559 & 745.0 & 0.0223 & 7.928 & 774.6 & 0.0224 \\
\hline 2 & 9.398 & 789.8 & 0.0223 & 10.774 & 804.9 & 0.0223 & 12.885 & 833.0 & 0.0223 \\
\hline 3 & 11.203 & 824.9 & 0.0223 & 12.826 & 834.7 & 0.0223 & 15.214 & 853.6 & 0.0223 \\
\hline 4 & 12.017 & 846.6 & 0.0222 & 13.756 & 846.5 & 0.0222 & 16.219 & 855.1 & 0.0222 \\
\hline 5 & 12.390 & 860.1 & 0.0222 & 14.182 & 850.5 & 0.0222 & 16.645 & 850.8 & 0.0222 \\
\hline 6 & 12.579 & 868.4 & 0.0221 & 14.395 & 850.7 & 0.0221 & 16.834 & 845.1 & 0.0221 \\
\hline 7 & 12.698 & 873.2 & 0.0221 & 14.523 & 849.2 & 0.0221 & 16.934 & 839.6 & 0.0221 \\
\hline 8 & 12.811 & 875.3 & 0.0220 & 14.636 & 846.7 & 0.0220 & 17.022 & 834.6 & 0.0220 \\
\hline 9 & 12.979 & 874.3 & 0.0220 & 14.797 & 843.1 & 0.0220 & 17.160 & 830.0 & 0.0220 \\
\hline 10 & 13.196 & 870.7 & 0.0219 & 15.001 & 838.7 & 0.0219 & 17.346 & 825.7 & 0.0219 \\
\hline 11 & 13.367 & 865.5 & 0.0219 & 15.158 & 834.6 & 0.0219 & 17.493 & 822.6 & 0.0219 \\
\hline 12 & 13.453 & 859.5 & 0.0218 & 15.232 & 831.2 & 0.0218 & 17.570 & 821.3 & 0.0218 \\
\hline 13 & 13.435 & 852.8 & 0.0218 & 15.200 & 828.6 & 0.0218 & 17.553 & 821.9 & 0.0218 \\
\hline 14 & 13.257 & 844.6 & 0.0217 & 15.003 & 825.9 & 0.0217 & 17.377 & 824.0 & 0.0217 \\
\hline 15 & 12.870 & 832.6 & 0.0217 & 14.574 & 821.1 & 0.0217 & 16.957 & 826.0 & 0.0217 \\
\hline 16 & 12.087 & 812.4 & 0.0216 & 13.692 & 809.1 & 0.0216 & 16.019 & 823.2 & 0.0216 \\
\hline 17 & 10.291 & 776.0 & 0.0216 & 11.665 & 779.8 & 0.0216 & 13.745 & 803.3 & 0.0216 \\
\hline 18 & 6.356 & 705.7 & 0.0216 & 7.222 & 713.2 & 0.0216 & 8.596 & 737.1 & 0.0216 \\
\hline
\end{tabular}


Table 4-31. Burnup and TH Feedback Parameters by Axial Node for Assembly E20c (Cont.)

\begin{tabular}{|c|c|c|c|}
\hline \multirow{3}{*}{$\begin{array}{c}\text { Axial } \\
\text { Node }\end{array}$} & $\begin{array}{c}\text { Burnup } \\
\text { (GWd/MTU) }\end{array}$ & $\begin{array}{c}\text { Statepoint 68 (BOC Cy9) } \\
\text { T-Fuel } \\
\left({ }^{\circ} \mathrm{F} \text { ) }\right.\end{array}$ & $\begin{array}{c}\text { Spec. Vol. } \\
\text { (ft'/1bm) }\end{array}$ \\
\hline & BOC Cy9 & 376.2 Cy6 & 376.2 Cy6 \\
\hline 1 & 9.074 & 806.7 & 0.0225 \\
\hline 2 & 14.574 & 862.5 & 0.0224 \\
\hline 3 & 17.061 & 873.8 & 0.0224 \\
\hline 4 & 18.081 & 868.4 & 0.0223 \\
\hline 5 & 18.481 & 860.2 & 0.0222 \\
\hline 6 & 18.639 & 852.9 & 0.0222 \\
\hline 7 & 18.715 & 847.0 & 0.0221 \\
\hline 8 & 18.785 & 842.3 & 0.0221 \\
\hline 9 & 18.910 & 838.1 & 0.0220 \\
\hline 10 & 19.087 & 834.2 & 0.0220 \\
\hline 11 & 19.231 & 831.5 & 0.0219 \\
\hline 12 & 19.313 & 830.6 & 0.0219 \\
\hline 13 & 19.315 & 831.8 & 0.0218 \\
\hline 14 & 19.169 & 835.5 & 0.0218 \\
\hline 15 & 18.782 & 840.9 & 0.0217 \\
\hline 16 & 17.845 & 844.4 & 0.0217 \\
\hline 17 & 15.434 & 833.5 & 0.0216 \\
\hline 18 & 9.764 & 767.9 & 0.0216 \\
\hline
\end{tabular}

Table 4-32. Burnup and TH Feedback Parameters by Axial Node for Assembly E21

\begin{tabular}{|c|c|c|c|c|c|c|c|c|c|}
\hline \multirow[b]{2}{*}{$\begin{array}{l}\text { Axial } \\
\text { Node }\end{array}$} & \multicolumn{3}{|c|}{ Statepoint 61 (114.4 Cy5) } & \multicolumn{3}{|c|}{ Datapoint (206.7 Cy5) } & \multicolumn{3}{|c|}{ Statepoint 62 (BOC Cy6) } \\
\hline & $\begin{array}{c}\text { Burnup } \\
\text { (GWd/MTU) }\end{array}$ & $\begin{array}{l}\text { T-Fuel } \\
\left({ }^{\circ} \mathrm{F}\right)\end{array}$ & $\begin{array}{c}\text { Spec. Vol. } \\
\left(\mathrm{ft}^{3} / \mathrm{lbm}\right)\end{array}$ & $\begin{array}{c}\text { Burnup } \\
\text { (GWd/MTU) }\end{array}$ & $\begin{array}{c}\text { T-Fuel } \\
\left({ }^{\circ} \mathrm{F}\right)\end{array}$ & $\begin{array}{c}\text { Spec. Vol. } \\
\left(\mathrm{ft}^{3} / \mathrm{lbm}\right)\end{array}$ & $\begin{array}{c}\text { Burnup } \\
\text { (GWd/MTU) }\end{array}$ & $\begin{array}{l}\text { T-Fuel } \\
\left({ }^{\circ} \mathrm{F}\right)\end{array}$ & $\begin{array}{c}\text { Spec. Vol. } \\
\left(\mathrm{ft}^{3} / \mathrm{lbm}\right)\end{array}$ \\
\hline & 114.4 Cy5 & 58.2 Cy5 & 58.2 Cy5 & 206.7 Cy 5 & 153.5 Cy 5 & 153.5 Cy 5 & BOC Cy6 & 252.2 Cy 5 & 252.2 Cy 5 \\
\hline 1 & 0.929 & 785.8 & 0.0226 & 1.842 & 812.8 & 0.0227 & 2.852 & 792.1 & 0.0227 \\
\hline 2 & 1.673 & 917.4 & 0.0226 & 3.238 & 943.5 & 0.0227 & 4.895 & 892.4 & 0.0226 \\
\hline 3 & 2.123 & 991.4 & 0.0225 & 4.019 & 1009.3 & 0.0226 & 5.962 & 938.0 & 0.0226 \\
\hline 4 & 2.369 & 1023.2 & 0.0225 & 4.406 & 1035.4 & 0.0225 & 6.459 & 957.4 & 0.0225 \\
\hline 5 & 2.490 & 1033.6 & 0.0224 & 4.581 & 1044.6 & 0.0224 & 6.682 & 971.0 & 0.0225 \\
\hline 6 & 2.542 & 1034.3 & 0.0223 & 4.653 & 1047.2 & 0.0224 & 6.793 & 987.1 & 0.0224 \\
\hline 7 & 2.561 & 1032.0 & 0.0223 & 4.681 & 1047.8 & 0.0223 & 6.870 & 1008.5 & 0.0223 \\
\hline 8 & 2.576 & 1032.4 & 0.0222 & 4.708 & 1048.2 & 0.0222 & 6.957 & 1034.1 & 0.0223 \\
\hline 9 & 2.608 & 1039.4 & 0.0221 & 4.761 & 1050.0 & 0.0222 & 7.078 & 1059.3 & 0.0222 \\
\hline 10 & 2.657 & 1052.1 & 0.0221 & 4.840 & 1053.0 & 0.0221 & 7.214 & 1077.3 & 0.0221 \\
\hline 11 & 2.699 & 1065.4 & 0.0220 & 4.912 & 1055.5 & 0.0220 & 7.314 & 1083.0 & 0.0220 \\
\hline 12 & 2.717 & 1074.4 & 0.0219 & 4.954 & 1057.0 & 0.0219 & 7.354 & 1075.9 & 0.0220 \\
\hline 13 & 2.696 & 1075.2 & 0.0219 & 4.947 & 1057.2 & 0.0219 & 7.321 & 1059.2 & 0.0219 \\
\hline 14 & 2.620 & 1064.2 & 0.0218 & 4.864 & 1054.3 & 0.0218 & 7.198 & 1038.3 & 0.0218 \\
\hline 15 & 2.475 & 1039.5 & 0.0217 & 4.675 & 1044.8 & 0.0217 & 6.952 & 1016.3 & 0.0217 \\
\hline 16 & 2.222 & 994.5 & 0.0217 & 4.294 & 1019.0 & 0.0217 & 6.459 & 989.4 & 0.0217 \\
\hline 17 & 1.765 & 909.7 & 0.0216 & 3.503 & 952.1 & 0.0216 & 5.371 & 935.7 & 0.0216 \\
\hline 18 & 0.991 & 756.4 & 0.0216 & 2.023 & 797.6 & 0.0216 & 3.181 & 801.7 & 0.0216 \\
\hline
\end{tabular}


Table 4-32. Burnup and TH Feedback Parameters by Axial Node for Assembly E21 (Cont.)

\begin{tabular}{|c|c|c|c|c|c|c|c|c|c|}
\hline \multirow{2}{*}{$\begin{array}{c}\text { Axial } \\
\text { Node }\end{array}$} & \multicolumn{2}{|c|}{ Statepoint 63 (69.1 Cy6) } & \multicolumn{2}{c|}{ Datapoint (176.7 Cy6) } & \multicolumn{3}{c|}{ Datapoint (320.3 Cy6) } \\
\cline { 2 - 10 } & $\begin{array}{c}\text { Burnup } \\
\text { GWd/MTU) }\end{array}$ & $\begin{array}{c}\text { T-Fuel } \\
\left({ }^{\circ} \mathrm{F}\right)\end{array}$ & $\begin{array}{c}\text { Spec. Vol. } \\
\left(\mathrm{ft}^{3} / \mathrm{lbm}\right)\end{array}$ & $\begin{array}{c}\text { Burnup } \\
(\mathrm{GWd} / \mathrm{MTU})\end{array}$ & $\begin{array}{c}\text { T-Fuel } \\
\left({ }^{\circ} \mathrm{F}\right)\end{array}$ & $\begin{array}{c}\text { Spec. Vol. } \\
\left(\mathrm{ft}^{3} / \mathrm{lbm}\right)\end{array}$ & $\begin{array}{c}\text { Burnup } \\
(\mathrm{GWd} / \mathrm{MTU})\end{array}$ & $\begin{array}{c}\text { T-Fuel } \\
\left({ }^{\circ} \mathrm{F}\right)\end{array}$ & $\begin{array}{c}\text { Spec. Vol. } \\
\left(\mathrm{ft}^{3} / \mathrm{lbm}\right)\end{array}$ \\
\hline 1 & $69.1 \mathrm{Cy6}$ & $41.5 \mathrm{Cy} 6$ & $41.5 \mathrm{Cy} 6$ & $176.7 \mathrm{Cy} 6$ & $128.8 \mathrm{Cy} 6$ & $128.8 \mathrm{Cy} 6$ & $320.3 \mathrm{Cy} 6$ & $252.5 \mathrm{Cy} 6$ & $252.5 \mathrm{Cy} 6$ \\
\hline 2 & 3.314 & 722.3 & 0.0224 & 4.098 & 742.1 & 0.0224 & 5.353 & 771.2 & 0.0224 \\
\hline 3 & 7.714 & 805.2 & 0.0224 & 7.061 & 823.2 & 0.0224 & 9.105 & 845.6 & 0.0224 \\
\hline 4 & 7.647 & 893.1 & 0.0223 & 9.466 & 883.6 & 0.0223 & 11.973 & 881.6 & 0.0223 \\
\hline 5 & 7.958 & 913.8 & 0.0223 & 9.853 & 891.2 & 0.0222 & 12.379 & 878.6 & 0.0222 \\
\hline 6 & 8.123 & 926.4 & 0.0222 & 10.053 & 892.9 & 0.0222 & 12.563 & 873.1 & 0.0221 \\
\hline 7 & 8.234 & 933.7 & 0.0221 & 10.179 & 891.9 & 0.0221 & 12.665 & 867.6 & 0.0221 \\
\hline 8 & 8.341 & 937.3 & 0.0221 & 10.290 & 889.6 & 0.0220 & 12.754 & 862.7 & 0.0220 \\
\hline 9 & 8.470 & 937.7 & 0.0220 & 10.417 & 886.5 & 0.0220 & 12.863 & 858.3 & 0.0220 \\
\hline 10 & 8.605 & 935.6 & 0.0220 & 10.547 & 883.2 & 0.0219 & 12.984 & 854.9 & 0.0219 \\
\hline 11 & 8.698 & 932.2 & 0.0219 & 10.636 & 880.5 & 0.0219 & 13.074 & 853.2 & 0.0219 \\
\hline 12 & 8.724 & 928.0 & 0.0219 & 10.661 & 878.9 & 0.0218 & 13.114 & 853.3 & 0.0218 \\
\hline 13 & 8.671 & 922.7 & 0.0218 & 10.605 & 878.0 & 0.0218 & 13.083 & 855.3 & 0.0218 \\
\hline 14 & 8.510 & 914.2 & 0.0217 & 10.430 & 876.2 & 0.0217 & 12.936 & 858.4 & 0.0217 \\
\hline 15 & 8.197 & 899.0 & 0.0217 & 10.068 & 870.2 & 0.0217 & 12.578 & 860.3 & 0.0217 \\
\hline 16 & 7.582 & 871.4 & 0.0216 & 9.325 & 853.4 & 0.0216 & 11.756 & 855.3 & 0.0216 \\
\hline 17 & 6.275 & 819.8 & 0.0216 & 7.729 & 814.6 & 0.0216 & 9.858 & 827.9 & 0.0216 \\
\hline 18 & 3.705 & 719.2 & 0.0216 & 4.578 & 724.4 & 0.0216 & 5.928 & 745.9 & 0.0216 \\
\hline
\end{tabular}

\begin{tabular}{|c|c|c|c|c|c|c|c|c|c|}
\hline \multirow[b]{2}{*}{$\begin{array}{l}\text { Axial } \\
\text { Node }\end{array}$} & \multicolumn{3}{|c|}{ Statepoint 64 (BOC Cy7) } & \multicolumn{3}{|c|}{ Statepoint $65(30.0 \mathrm{Cy} 7)$} & \multicolumn{3}{|c|}{ Statepoint 66 (108.0 Cy7) } \\
\hline & $\begin{array}{c}\text { Burnup } \\
\text { (GWd/MTU) }\end{array}$ & $\begin{array}{c}\text { T-Fuel } \\
\left({ }^{\circ} \mathrm{F}\right)\end{array}$ & $\begin{array}{c}\text { Spec. Vol. } \\
\left(\mathrm{ft}^{3} / \mathrm{lbm}\right)\end{array}$ & $\begin{array}{c}\text { Burnup } \\
\text { (GWd/MTU) }\end{array}$ & $\begin{array}{l}\text { T-Fuel } \\
\left({ }^{\circ} \mathrm{F}\right)\end{array}$ & $\begin{array}{c}\text { Spec. Vol. } \\
\left(\mathrm{ft}^{3} / \mathrm{lbm}\right)\end{array}$ & $\begin{array}{c}\text { Burnup } \\
\text { (GWd/MTU) }\end{array}$ & $\begin{array}{c}\text { T-Fuel } \\
\left({ }^{\circ} \mathrm{F}\right)\end{array}$ & $\begin{array}{c}\text { Spec. Vol } \\
\left(\mathrm{tt}^{3} / \mathrm{lbm}\right)\end{array}$ \\
\hline & BOC Cy7 & 376.2 Cy 6 & 376.2 Cy 6 & $30.0 \mathrm{Cy} 7$ & $22.5 \mathrm{Cy} 7$ & 22.5 Cy7 & $108.0 \mathrm{Cy} 7$ & $60.4 \mathrm{Cy} 7$ & 60.4 Cy 7 \\
\hline 1 & 6.412 & 804.1 & 0.0225 & 6.840 & 891.5 & 0.0233 & 7.995 & 897.4 & 0.0232 \\
\hline 2 & 10.740 & 871.9 & 0.0224 & 11.457 & 1010.7 & 0.0232 & 13.358 & 1013.3 & 0.0231 \\
\hline 3 & 12.895 & 890.5 & 0.0224 & 13.784 & 1081.0 & 0.0231 & 16.097 & 1076.4 & 0.0231 \\
\hline 4 & 13.847 & 888.4 & 0.0223 & 14.842 & 1122.5 & 0.0230 & 17.378 & 1108.5 & 0.0230 \\
\hline 5 & 14.236 & 881.0 & 0.0222 & 15.297 & 1147.8 & 0.0229 & 17.958 & 1126.0 & 0.0228 \\
\hline 6 & 14.393 & 873.9 & 0.0222 & 15.496 & 1163.7 & 0.0228 & 18.231 & 1135.5 & 0.0227 \\
\hline 7 & 14.473 & 868.1 & 0.0221 & 15.604 & 1173.0 & 0.0227 & 18.381 & 1139.8 & 0.0226 \\
\hline 8 & 14.546 & 863.5 & 0.0221 & 15.693 & 1177.2 & 0.0226 & 18.493 & 1140.4 & 0.0225 \\
\hline 9 & 14.645 & 859.8 & 0.0220 & 15.801 & 1177.6 & 0.0225 & 18.610 & 1138.5 & 0.0224 \\
\hline 10 & 14.760 & 856.9 & 0.0220 & 15.919 & 1175.6 & 0.0223 & 18.733 & 1135.3 & 0.0223 \\
\hline 11 & 14.853 & 855.3 & 0.0219 & 16.012 & 1173.1 & 0.0222 & 18.831 & 1132.4 & 0.0222 \\
\hline 12 & 14.906 & 855.5 & 0.0219 & 16.061 & 1169.2 & 0.0221 & 18.880 & 1129.1 & 0.0221 \\
\hline 13 & 14.899 & 857.7 & 0.0218 & 16.039 & 1159.9 & 0.0220 & 18.838 & 1122.4 & 0.0220 \\
\hline 14 & 14.784 & 862.0 & 0.0218 & 15.891 & 1142.6 & 0.0219 & 18.631 & 1109.6 & 0.0219 \\
\hline 15 & 14.456 & 867.0 & 0.0217 & 15.503 & 1114.9 & 0.0218 & 18.130 & 1088.0 & 0.0218 \\
\hline 16 & 13.622 & 868.5 & 0.0217 & 14.570 & 1071.8 & 0.0218 & 16.989 & 1052.6 & 0.0218 \\
\hline 17 & 11.553 & 851.2 & 0.0216 & 12.336 & 1001.6 & 0.0217 & 14.365 & 989.9 & 0.0217 \\
\hline 18 & 7.058 & 774.1 & 0.0216 & 7.539 & 862.1 & 0.0216 & 8.806 & 858.0 & 0.0216 \\
\hline
\end{tabular}


Table 4-33. Burnup and TH Feedback Parameters by Axial Node for Assembly E25

\begin{tabular}{|c|c|c|c|c|c|c|c|c|c|}
\hline \multirow[b]{2}{*}{$\begin{array}{l}\text { Axial } \\
\text { Node }\end{array}$} & \multicolumn{3}{|c|}{ Statepoint 61 (114.4 Cy5) } & \multicolumn{3}{|c|}{ Datapoint (206.7 Cy5) } & \multicolumn{3}{|c|}{ Statepoint 62 (BOC Cy6) } \\
\hline & $\begin{array}{c}\text { Burnup } \\
\text { (GWd/MTU) }\end{array}$ & $\begin{array}{l}\text { T-Fuel } \\
\left({ }^{\circ} \mathrm{F}\right)\end{array}$ & $\begin{array}{l}\text { Spec. Vol. } \\
\left(\mathrm{ft}^{3} / \mathrm{lbm}\right)\end{array}$ & $\begin{array}{c}\text { Burnup } \\
\text { (GWd/MTU) }\end{array}$ & $\begin{array}{l}\text { T-Fuel } \\
\left({ }^{\circ} \mathrm{F}\right)\end{array}$ & $\begin{array}{l}\text { Spec. Vol. } \\
\left(\mathrm{ft}^{3} / \mathrm{lbm}\right)\end{array}$ & $\begin{array}{c}\text { Burnup } \\
\text { (GWd/MTU) }\end{array}$ & $\begin{array}{c}\text { T-Fuel } \\
\left({ }^{\circ} \mathrm{F}\right)\end{array}$ & $\begin{array}{c}\text { Spec. Vol. } \\
\left(\mathrm{ft}^{3} / \mathrm{lbm}\right)\end{array}$ \\
\hline & $114.4 \mathrm{Cy} 5$ & 58.2 Cy 5 & 58.2 Cy5 & $206.7 \mathrm{Cy} 5$ & 153.5 Cy 5 & 153.5 Cy 5 & BOC Cy6 & 252.2 Cy 5 & 252.2 Cy5 \\
\hline 1 & 1.534 & 925.4 & 0.0233 & 2.959 & 947.5 & 0.0233 & 4.473 & 897.5 & 0.0233 \\
\hline 2 & 2.715 & 1118.9 & 0.0232 & 5.093 & 1119.0 & 0.0232 & 7.500 & 1016.3 & 0.0232 \\
\hline 3 & 3.404 & 1219.2 & 0.0231 & 6.236 & 1197.3 & 0.0231 & 9.013 & 1063.7 & 0.0231 \\
\hline 4 & 3.766 & 1260.3 & 0.0230 & 6.783 & 1226.0 & 0.0230 & 9.691 & 1083.0 & 0.0230 \\
\hline 5 & 3.939 & 1271.9 & 0.0229 & 7.022 & 1235.0 & 0.0229 & 9.989 & 1098.9 & 0.0229 \\
\hline 6 & 4.003 & 1269.1 & 0.0228 & 7.106 & 1236.9 & 0.0228 & 10.129 & 1121.5 & 0.0228 \\
\hline 7 & 4.003 & 1260.1 & 0.0227 & 7.108 & 1235.9 & 0.0227 & 10.207 & 1153.8 & 0.0227 \\
\hline 8 & 3.978 & 1252.8 & 0.0226 & 7.076 & 1232.7 & 0.0226 & 10.266 & 1193.8 & 0.0226 \\
\hline 9 & 3.966 & 1255.0 & 0.0225 & 7.060 & 1228.8 & 0.0225 & 10.344 & 1233.5 & 0.0225 \\
\hline 10 & 3.989 & 1268.1 & 0.0224 & 7.095 & 1226.7 & 0.0223 & 10.453 & 1260.3 & 0.0224 \\
\hline 11 & 4.039 & 1287.6 & 0.0222 & 7.176 & 1227.2 & 0.0222 & 10.568 & 1267.2 & 0.0223 \\
\hline 12 & 4.096 & 1307.4 & 0.0221 & 7.279 & 1230.5 & 0.0221 & 10.664 & 1254.1 & 0.0221 \\
\hline 13 & 4.128 & 1319.4 & 0.0220 & 7.364 & 1236.0 & 0.0220 & 10.713 & 1226.9 & 0.0220 \\
\hline 14 & 4.092 & 1316.1 & 0.0219 & 7.367 & 1240.5 & 0.0219 & 10.669 & 1194.1 & 0.0219 \\
\hline 15 & 3.942 & 1291.9 & 0.0218 & 7.206 & 1237.7 & 0.0218 & 10.449 & 1164.1 & 0.0218 \\
\hline 16 & 3.601 & 1235.8 & 0.0217 & 6.725 & 1213.6 & 0.0217 & 9.842 & 1133.0 & 0.0217 \\
\hline 17 & 2.909 & 1119.5 & 0.0216 & 5.586 & 1135.3 & 0.0216 & 8.326 & 1074.7 & 0.0216 \\
\hline 18 & 1.663 & 889.5 & 0.0216 & 3.296 & 931.6 & 0.0216 & 5.048 & 915.4 & 0.0216 \\
\hline
\end{tabular}

\begin{tabular}{|c|c|c|c|}
\hline \multirow{2}{*}{$\begin{array}{c}\text { Axial } \\
\text { Node }\end{array}$} & $\begin{array}{c}\text { Burnup } \\
\text { (GWd/MTU) }\end{array}$ & $\begin{array}{c}\text { Statepoint 63 (69.1 Cy6) } \\
\text { T-Fuel } \\
\text { ('F) }\end{array}$ & $\begin{array}{c}\text { Spec. Vol. } \\
\text { (tt/l/bm) }\end{array}$ \\
\hline & 69.1 Cy6 & 41.5 Cy6 & 41.5 Cy6 \\
\hline 1 & 5.657 & 955.4 & 0.0238 \\
\hline 2 & 9.471 & 1098.1 & 0.0237 \\
\hline 3 & 11.444 & 1190.8 & 0.0236 \\
\hline 4 & 12.402 & 1250.1 & 0.0235 \\
\hline 5 & 12.871 & 1286.4 & 0.0233 \\
\hline 6 & 13.125 & 1309.8 & 0.0232 \\
\hline 7 & 13.288 & 1325.4 & 0.0230 \\
\hline 8 & 13.412 & 1335.5 & 0.0229 \\
\hline 9 & 13.530 & 1340.6 & 0.0227 \\
\hline 10 & 13.650 & 1339.6 & 0.0226 \\
\hline 11 & 13.751 & 1333.5 & 0.0224 \\
\hline 12 & 13.813 & 1324.0 & 0.0223 \\
\hline 13 & 13.809 & 1311.7 & 0.0221 \\
\hline 14 & 13.677 & 1294.8 & 0.0220 \\
\hline 15 & 13.311 & 1266.5 & 0.0219 \\
\hline 16 & 12.462 & 1217.5 & 0.0218 \\
\hline 17 & 10.520 & 1131.4 & 0.0217 \\
\hline 18 & 6.419 & 958.8 & 0.0216 \\
\hline
\end{tabular}


Table 4-34. Burnup and TH Feedback Parameters by Axial Node for Assembly E27

\begin{tabular}{|c|c|c|c|c|c|c|c|c|c|}
\hline \multirow[b]{2}{*}{$\begin{array}{l}\text { Axial } \\
\text { Node }\end{array}$} & \multicolumn{3}{|c|}{ Statepoint 61 (114.4 Cy5) } & \multicolumn{3}{|c|}{ Datapoint (206.7 Cy5) } & \multicolumn{3}{|c|}{ Statepoint 62 (BOC Cy6) } \\
\hline & $\begin{array}{c}\text { Burnup } \\
\text { (GWd/MTU) }\end{array}$ & $\begin{array}{c}\text { T-Fuel } \\
\left({ }^{\circ} \mathrm{F}\right)\end{array}$ & $\begin{array}{l}\text { Spec. Vol. } \\
\left(\mathrm{ft}^{3} / \mathrm{lbm}\right)\end{array}$ & $\begin{array}{c}\text { Burnup } \\
\text { (GWd/MTU) }\end{array}$ & $\begin{array}{l}\text { T-Fuel } \\
\left({ }^{\circ} \mathrm{F}\right)\end{array}$ & $\begin{array}{c}\text { Spec. Vol. } \\
\left(\mathrm{ft}^{3} / \mathrm{lbm}\right)\end{array}$ & $\begin{array}{c}\text { Burnup } \\
\text { (GWd/MTU) }\end{array}$ & $\begin{array}{c}\text { T-Fuel } \\
\left({ }^{\circ} \mathrm{F}\right)\end{array}$ & $\begin{array}{c}\text { Spec. Vol. } \\
\left(\mathrm{ft}^{3} / \mathrm{lbm}\right)\end{array}$ \\
\hline & 114.4 Cy 5 & 58.2 Cy 5 & 58.2 Cy5 & 206.7 Cy5 & 153.5 Cy 5 & 153.5 Cy 5 & BOC Cy 6 & 252.2 Cy 5 & 252.2 Cy 5 \\
\hline 1 & 1.712 & 991.0 & 0.0236 & 3.301 & 989.3 & 0.0236 & 4.995 & 929.4 & 0.0235 \\
\hline 2 & 3.056 & 1205.5 & 0.0235 & 5.747 & 1179.5 & 0.0235 & 8.457 & 1059.1 & 0.0234 \\
\hline 3 & 3.885 & 1312.3 & 0.0234 & 7.100 & 1266.9 & 0.0234 & 10.221 & 1108.8 & 0.0233 \\
\hline 4 & 4.316 & 1352.3 & 0.0232 & 7.724 & 1296.3 & 0.0232 & 10.975 & 1127.6 & 0.0232 \\
\hline 5 & 4.509 & 1361.5 & 0.0231 & 7.980 & 1304.2 & 0.0231 & 11.287 & 1144.7 & 0.0231 \\
\hline 6 & 4.571 & 1355.6 & 0.0230 & 8.058 & 1305.3 & 0.0230 & 11.426 & 1170.4 & 0.0230 \\
\hline 7 & 4.557 & 1342.8 & 0.0228 & 8.040 & 1303.3 & 0.0228 & 11.493 & 1208.0 & 0.0229 \\
\hline 8 & 4.510 & 1331.8 & 0.0227 & 7.975 & 1298.4 & 0.0227 & 11.534 & 1254.7 & 0.0228 \\
\hline 9 & 4.473 & 1331.3 & 0.0226 & 7.921 & 1292.2 & 0.0226 & 11.587 & 1300.3 & 0.0226 \\
\hline 10 & 4.477 & 1343.7 & 0.0225 & 7.927 & 1287.9 & 0.0225 & 11.675 & 1329.9 & 0.0225 \\
\hline 11 & 4.523 & 1365.1 & 0.0224 & 8.001 & 1287.1 & 0.0223 & 11.783 & 1337.0 & 0.0223 \\
\hline 12 & 4.591 & 1388.5 & 0.0222 & 8.120 & 1290.6 & 0.0222 & 11.889 & 1321.6 & 0.0222 \\
\hline 13 & 4.643 & 1403.7 & 0.0221 & 8.239 & 1297.7 & 0.0221 & 11.964 & 1290.2 & 0.0221 \\
\hline 14 & 4.631 & 1403.8 & 0.0220 & 8.285 & 1305.0 & 0.0220 & 11.958 & 1252.9 & 0.0220 \\
\hline 15 & 4.498 & 1383.2 & 0.0219 & 8.159 & 1305.3 & 0.0219 & 11.773 & 1218.8 & 0.0219 \\
\hline 16 & 4.155 & 1327.7 & 0.0218 & 7.690 & 1284.2 & 0.0218 & 11.183 & 1185.7 & 0.0217 \\
\hline 17 & 3.413 & 1205.5 & 0.0217 & 6.488 & 1205.8 & 0.0217 & 9.597 & 1126.8 & 0.0217 \\
\hline 18 & 1.996 & 955.5 & 0.0216 & 3.917 & 993.6 & 0.0216 & 5.950 & 965.9 & 0.0216 \\
\hline
\end{tabular}

\begin{tabular}{|c|c|c|c|c|c|c|c|c|c|}
\hline \multirow[b]{2}{*}{$\begin{array}{l}\text { Axial } \\
\text { Node }\end{array}$} & \multicolumn{3}{|c|}{ Statepoint 63 (69.1 Cy6) } & \multicolumn{3}{|c|}{ Datapoint (176.7 Cy6) } & \multicolumn{3}{|c|}{ Datapoint (320.3 Cy6) } \\
\hline & $\begin{array}{c}\text { Burnup } \\
\text { (GWd/MTU) }\end{array}$ & $\begin{array}{l}\text { T-Fuel } \\
\left({ }^{\circ} \mathrm{F}\right)\end{array}$ & $\begin{array}{c}\text { Spec. Vol. } \\
\left(\mathrm{ft}^{3} / \mathrm{lbm}\right)\end{array}$ & $\begin{array}{c}\text { Burnup } \\
\text { (GWd/MTU) }\end{array}$ & $\begin{array}{l}\text { T-Fuel } \\
\left({ }^{\circ} \mathrm{F}\right)\end{array}$ & $\begin{array}{l}\text { Spec. Vol. } \\
\left(\mathrm{ft}^{3} / / \mathrm{bm}\right)\end{array}$ & $\begin{array}{c}\text { Burnup } \\
\text { (GWd/MTU) }\end{array}$ & $\begin{array}{c}\text { T-Fuel } \\
\left({ }^{\circ} \mathrm{F}\right)\end{array}$ & $\begin{array}{c}\text { Spec. Vol. } \\
\left(\mathrm{ft}^{3} / / \mathrm{bm}\right)\end{array}$ \\
\hline & $69.1 \mathrm{Cy} 6$ & 41.5 Cy 6 & 41.5 Cy 6 & 176.7 Cy6 & 128.8 Cy6 & $128.8 \mathrm{Cy} 6$ & $320.3 \mathrm{Cy} 6$ & 252.5 Cy 6 & $252.5 \mathrm{Cy} 6$ \\
\hline 1 & 5.401 & 697.4 & 0.0222 & 6.093 & 711.8 & 0.0222 & 7.208 & 737.4 & 0.0222 \\
\hline 2 & 9.141 & 750.5 & 0.0222 & 10.272 & 762.9 & 0.0222 & 12.010 & 787.1 & 0.0222 \\
\hline 3 & 11.057 & 780.2 & 0.0222 & 12.398 & 787.9 & 0.0221 & 14.374 & 805.3 & 0.0222 \\
\hline 4 & 11.900 & 798.6 & 0.0221 & 13.341 & 798.5 & 0.0221 & 15.385 & 807.3 & 0.0221 \\
\hline 5 & 12.267 & 810.4 & 0.0221 & 13.755 & 802.1 & 0.0221 & 15.801 & 804.0 & 0.0221 \\
\hline 6 & 12.440 & 817.7 & 0.0220 & 13.948 & 802.5 & 0.0220 & 15.975 & 799.3 & 0.0220 \\
\hline 7 & 12.529 & 822.0 & 0.0220 & 14.044 & 801.3 & 0.0220 & 16.047 & 794.8 & 0.0220 \\
\hline 8 & 12.582 & 824.0 & 0.0219 & 14.097 & 799.4 & 0.0219 & 16.079 & 790.8 & 0.0219 \\
\hline 9 & 12.637 & 823.8 & 0.0219 & 14.147 & 796.9 & 0.0219 & 16.111 & 787.4 & 0.0219 \\
\hline 10 & 12.720 & 821.6 & 0.0219 & 14.221 & 794.0 & 0.0218 & 16.172 & 784.5 & 0.0219 \\
\hline 11 & 12.817 & 818.0 & 0.0218 & 14.309 & 791.1 & 0.0218 & 16.255 & 782.4 & 0.0218 \\
\hline 12 & 12.908 & 813.4 & 0.0218 & 14.391 & 788.6 & 0.0218 & 16.342 & 781.6 & 0.0218 \\
\hline 13 & 12.962 & 807.8 & 0.0217 & 14.437 & 786.4 & 0.0217 & 16.401 & 781.9 & 0.0217 \\
\hline 14 & 12.925 & 800.4 & 0.0217 & 14.384 & 783.7 & 0.0217 & 16.364 & 783.1 & 0.0217 \\
\hline 15 & 12.692 & 789.3 & 0.0217 & 14.113 & 778.7 & 0.0217 & 16.096 & 783.7 & 0.0217 \\
\hline 16 & 12.019 & 770.8 & 0.0216 & 13.351 & 767.3 & 0.0216 & 15.279 & 779.8 & 0.0216 \\
\hline 17 & 10.284 & 738.5 & 0.0216 & 11.414 & 740.9 & 0.0216 & 13.121 & 760.9 & 0.0216 \\
\hline 18 & 6.364 & 678.0 & 0.0216 & 7.064 & 683.8 & 0.0216 & 8.174 & 703.9 & 0.0216 \\
\hline
\end{tabular}


Table 4-34. Burnup and TH Feedback Parameters by Axial Node for Assembly E27 (Cont.)

\begin{tabular}{|c|c|c|c|c|c|c|c|c|c|}
\hline \multirow{2}{*}{$\begin{array}{c}\text { Axial } \\
\text { Node }\end{array}$} & \multicolumn{2}{|c|}{ Statepoint 64 (BOC Cy7) } & \multicolumn{2}{c|}{ Statepoint 65 (30.0 Cy7) } & \multicolumn{3}{c|}{ Statepoint 66 (108.0 Cy7) } \\
\cline { 2 - 10 } & $\begin{array}{c}\text { Burnup } \\
\text { GWd/MTU) }\end{array}$ & $\begin{array}{c}\text { T-Fuel } \\
\left({ }^{\circ} \mathrm{F}\right)\end{array}$ & $\begin{array}{c}\text { Spec. Vol. } \\
\left(\mathrm{ft}^{3} / \mathrm{lbm}\right)\end{array}$ & $\begin{array}{c}\text { Burnup } \\
(\mathrm{GWd} / \mathrm{MTU})\end{array}$ & $\begin{array}{c}\text { T-Fuel } \\
\left({ }^{\circ} \mathrm{F}\right)\end{array}$ & $\begin{array}{c}\text { Spec. Vol. } \\
\left(\mathrm{ft}^{3} / \mathrm{lbm}\right)\end{array}$ & $\begin{array}{c}\text { Burnup } \\
(\mathrm{GWd} / \mathrm{MTU})\end{array}$ & $\begin{array}{c}\text { T-Fuel } \\
\left({ }^{\circ} \mathrm{F}\right)\end{array}$ & $\begin{array}{c}\text { Spec. Vol. } \\
\left(\mathrm{ft}^{3} / \mathrm{lbm}\right)\end{array}$ \\
\hline 1 & 8.155 & 767.0 & 0.0223 & 8.625 & 907.1 & 0.0233 & 9.903 & 915.4 & 0.0233 \\
\hline 2 & 13.424 & 815.4 & 0.0223 & 14.180 & 1014.5 & 0.0232 & 16.213 & 1022.4 & 0.0232 \\
\hline 3 & 15.929 & 826.5 & 0.0222 & 16.846 & 1074.1 & 0.0231 & 19.276 & 1076.0 & 0.0231 \\
\hline 4 & 16.955 & 822.6 & 0.0222 & 17.970 & 1108.8 & 0.0230 & 20.619 & 1104.6 & 0.0230 \\
\hline 5 & 17.350 & 815.8 & 0.0221 & 18.426 & 1130.9 & 0.0229 & 21.199 & 1120.5 & 0.0229 \\
\hline 6 & 17.498 & 809.5 & 0.0221 & 18.613 & 1145.0 & 0.0228 & 21.460 & 1129.3 & 0.0228 \\
\hline 7 & 17.549 & 804.6 & 0.0220 & 18.690 & 1153.3 & 0.0227 & 21.580 & 1133.5 & 0.0227 \\
\hline 8 & 17.565 & 800.8 & 0.0220 & 18.722 & 1157.3 & 0.0226 & 21.635 & 1134.5 & 0.0226 \\
\hline 9 & 17.586 & 797.6 & 0.0219 & 18.750 & 1157.8 & 0.0225 & 21.674 & 1133.1 & 0.0225 \\
\hline 10 & 17.640 & 795.1 & 0.0219 & 18.806 & 1155.8 & 0.0224 & 21.732 & 1130.1 & 0.0223 \\
\hline 11 & 17.721 & 793.2 & 0.0219 & 18.885 & 1152.3 & 0.0223 & 21.812 & 1126.3 & 0.0222 \\
\hline 12 & 17.814 & 792.6 & 0.0218 & 18.971 & 1146.5 & 0.0221 & 21.891 & 1121.3 & 0.0221 \\
\hline 13 & 17.889 & 793.4 & 0.0218 & 19.027 & 1135.1 & 0.0220 & 21.919 & 1112.3 & 0.0220 \\
\hline 14 & 17.876 & 795.9 & 0.0217 & 18.979 & 1116.2 & 0.0219 & 21.802 & 1097.3 & 0.0219 \\
\hline 15 & 17.632 & 799.5 & 0.0217 & 18.675 & 1088.7 & 0.0218 & 21.377 & 1074.8 & 0.0218 \\
\hline 16 & 16.808 & 800.9 & 0.0216 & 17.759 & 1049.6 & 0.0218 & 20.252 & 1041.1 & 0.0218 \\
\hline 17 & 14.523 & 788.7 & 0.0216 & 15.322 & 990.4 & 0.0217 & 17.443 & 987.0 & 0.0217 \\
\hline 18 & 9.128 & 730.8 & 0.0216 & 9.638 & 865.4 & 0.0216 & 11.009 & 865.1 & 0.0216 \\
\hline
\end{tabular}

Table 4-35. Burnup and TH Feedback Parameters by Axial Node for Assembly E28

\begin{tabular}{|c|c|c|c|c|c|c|c|c|c|}
\hline \multirow[b]{2}{*}{$\begin{array}{l}\text { Axial } \\
\text { Node }\end{array}$} & \multicolumn{3}{|c|}{ Statepoint 61 (114.4 Cy5) } & \multicolumn{3}{|c|}{ Datapoint (206.7 Cy5) } & \multicolumn{3}{|c|}{ Statepoint 62 (BOC Cy6) } \\
\hline & $\begin{array}{c}\text { Burnup } \\
\text { (GWd/MTU) }\end{array}$ & $\begin{array}{c}\text { T-Fuel } \\
\left({ }^{\circ} \mathrm{F}\right)\end{array}$ & $\begin{array}{l}\text { Spec. Vol. } \\
\left(\mathrm{ft}^{3} / \mathrm{lbm}\right)\end{array}$ & $\begin{array}{c}\text { Burnup } \\
\text { (GWd/MTU) }\end{array}$ & $\begin{array}{c}\text { T-Fuel } \\
\left({ }^{\circ} \mathrm{F}\right)\end{array}$ & $\begin{array}{c}\text { Spec. Vol. } \\
\left(\mathrm{ft}^{3} / \mathrm{lbm}\right)\end{array}$ & $\begin{array}{c}\text { Burnup } \\
\text { (GWd/MTU) }\end{array}$ & $\begin{array}{c}\text { T-Fuel } \\
\left({ }^{\circ} \mathrm{F}\right)\end{array}$ & $\begin{array}{c}\text { Spec. Vol. } \\
\left(\mathrm{ft}^{3} / \mathrm{lbm}\right)\end{array}$ \\
\hline & 114.4 Cy5 & 58.2 Cy5 & 58.2 Cy5 & 206.7 Cy5 & 153.5 Cy5 & 153.5 Cy5 & BOC Cy6 & 252.2 Cy5 & 252.2 Cy 5 \\
\hline 1 & 1.035 & 811.6 & 0.0227 & 2.017 & 830.2 & 0.0228 & 3.077 & 801.0 & 0.0227 \\
\hline 2 & 1.867 & 955.9 & 0.0227 & 3.543 & 966.4 & 0.0227 & 5.271 & 901.9 & 0.0227 \\
\hline 3 & 2.372 & 1035.8 & 0.0226 & 4.393 & 1033.9 & 0.0226 & 6.408 & 945.5 & 0.0226 \\
\hline 4 & 2.645 & 1068.9 & 0.0225 & 4.808 & 1059.5 & 0.0226 & 6.927 & 963.9 & 0.0226 \\
\hline 5 & 2.776 & 1078.8 & 0.0225 & 4.989 & 1067.5 & 0.0225 & 7.154 & 977.9 & 0.0225 \\
\hline 6 & 2.824 & 1077.3 & 0.0224 & 5.052 & 1069.1 & 0.0224 & 7.257 & 995.9 & 0.0224 \\
\hline 7 & 2.826 & 1071.1 & 0.0223 & 5.054 & 1068.1 & 0.0223 & 7.312 & 1020.5 & 0.0224 \\
\hline 8 & 2.808 & 1066.0 & 0.0222 & 5.031 & 1065.5 & 0.0223 & 7.351 & 1049.4 & 0.0223 \\
\hline 9 & 2.795 & 1066.6 & 0.0222 & 5.013 & 1062.4 & 0.0222 & 7.396 & 1077.3 & 0.0222 \\
\hline 10 & 2.800 & 1074.1 & 0.0221 & 5.023 & 1060.2 & 0.0221 & 7.452 & 1096.5 & 0.0221 \\
\hline 11 & 2.825 & 1086.1 & 0.0220 & 5.064 & 1059.9 & 0.0220 & 7.513 & 1101.6 & 0.0220 \\
\hline 12 & 2.856 & 1098.5 & 0.0220 & 5.124 & 1061.8 & 0.0220 & 7.566 & 1092.0 & 0.0220 \\
\hline 13 & 2.874 & 1106.3 & 0.0219 & 5.175 & 1065.3 & 0.0219 & 7.592 & 1072.0 & 0.0219 \\
\hline 14 & 2.850 & 1104.1 & 0.0218 & 5.177 & 1068.0 & 0.0218 & 7.561 & 1048.5 & 0.0218 \\
\hline 15 & 2.747 & 1086.8 & 0.0217 & 5.062 & 1064.7 & 0.0217 & 7.402 & 1025.9 & 0.0217 \\
\hline 16 & 2.503 & 1044.1 & 0.0217 & 4.709 & 1043.9 & 0.0217 & 6.948 & 999.7 & 0.0217 \\
\hline 17 & 2.005 & 953.1 & 0.0216 & 3.875 & 977.6 & 0.0216 & 5.821 & 946.8 & 0.0216 \\
\hline 18 & 1.130 & 783.6 & 0.0216 & 2.250 & 817.1 & 0.0216 & 3.467 & 812.7 & 0.0216 \\
\hline
\end{tabular}


Table 4-35. Burnup and TH Feedback Parameters by Axial Node for Assembly E28 (Cont.)

\begin{tabular}{|c|c|c|c|c|c|c|c|c|c|}
\hline \multirow[b]{2}{*}{$\begin{array}{l}\text { Axial } \\
\text { Node }\end{array}$} & \multicolumn{3}{|c|}{ Statepoint $63(69.1$ Cy6) } & \multicolumn{3}{|c|}{ Datapoint (176.7 Cy6) } & \multicolumn{3}{|c|}{ Datapoint (320.3 Cy6) } \\
\hline & $\begin{array}{c}\text { Burnup } \\
\text { (GWd/MTU) }\end{array}$ & $\begin{array}{c}\text { T-Fuel } \\
\left({ }^{\circ} \mathrm{F}\right)\end{array}$ & $\begin{array}{c}\text { Spec. Vol. } \\
\left(\mathrm{ft}^{3} / \mathrm{lbm}\right)\end{array}$ & $\begin{array}{c}\text { Burnup } \\
\text { (GWd/MTU) }\end{array}$ & $\begin{array}{c}\text { T-Fuel } \\
\left({ }^{\circ} \mathrm{F}\right)\end{array}$ & $\begin{array}{c}\text { Spec. Vol. } \\
\left(\mathrm{ft}^{3} / \mathrm{lbm}\right)\end{array}$ & $\begin{array}{c}\text { Burnup } \\
\text { (GWd/MTU) }\end{array}$ & $\begin{array}{c}\text { T-Fuel } \\
\left({ }^{\circ} \mathrm{F}\right)\end{array}$ & $\begin{array}{c}\text { Spec. Vol. } \\
\left(\mathrm{tt}^{3} / \mathrm{lbm}\right)\end{array}$ \\
\hline & $69.1 \mathrm{Cy} 6$ & 41.5 Cy 6 & 41.5 Cy 6 & 176.7 Cy6 & 128.8 Cy 6 & 128.8 Cy 6 & 320.3 Cy 6 & 252.5 Cy6 & $252.5 \mathrm{Cy} 6$ \\
\hline 1 & 4.168 & 936.5 & 0.0238 & 5.987 & 970.8 & 0.0236 & 8.784 & 1001.7 & 0.0235 \\
\hline 2 & 7.151 & 1100.5 & 0.0237 & 10.183 & 1116.7 & 0.0235 & 14.587 & 1123.6 & 0.0234 \\
\hline 3 & 8.778 & 1204.0 & 0.0236 & 12.466 & 1193.6 & 0.0234 & 17.549 & 1170.1 & 0.0233 \\
\hline 4 & 9.599 & 1271.5 & 0.0234 & 13.621 & 1228.3 & 0.0233 & 18.918 & 1174.4 & 0.0232 \\
\hline 5 & 10.011 & 1312.7 & 0.0233 & 14.190 & 1240.5 & 0.0231 & 19.509 & 1165.6 & 0.0230 \\
\hline 6 & 10.236 & 1338.6 & 0.0231 & 14.491 & 1243.4 & 0.0230 & 19.776 & 1154.7 & 0.0229 \\
\hline 7 & 10.376 & 1354.2 & 0.0230 & 14.676 & 1242.8 & 0.0229 & 19.922 & 1145.0 & 0.0228 \\
\hline 8 & 10.475 & 1364.5 & 0.0228 & 14.805 & 1241.0 & 0.0227 & 20.022 & 1137.3 & 0.0227 \\
\hline 9 & 10.556 & 1369.8 & 0.0227 & 14.905 & 1238.4 & 0.0226 & 20.105 & 1131.5 & 0.0225 \\
\hline 10 & 10.626 & 1370.2 & 0.0225 & 14.986 & 1235.4 & 0.0225 & 20.185 & 1127.5 & 0.0224 \\
\hline 11 & 10.684 & 1366.8 & 0.0224 & 15.053 & 1232.7 & 0.0223 & 20.273 & 1126.0 & 0.0223 \\
\hline 12 & 10.722 & 1360.9 & 0.0223 & 15.104 & 1231.3 & 0.0222 & 20.375 & 1127.8 & 0.0222 \\
\hline 13 & 10.714 & 1352.5 & 0.0221 & 15.111 & 1231.2 & 0.0221 & 20.460 & 1132.7 & 0.0221 \\
\hline 14 & 10.608 & 1337.7 & 0.0220 & 14.988 & 1229.0 & 0.0220 & 20.412 & 1138.7 & 0.0220 \\
\hline 15 & 10.303 & 1309.8 & 0.0219 & 14.588 & 1218.6 & 0.0219 & 20.041 & 1143.6 & 0.0219 \\
\hline 16 & 9.586 & 1254.7 & 0.0218 & 13.613 & 1189.5 & 0.0218 & 18.942 & 1140.1 & 0.0218 \\
\hline 17 & 7.987 & 1152.9 & 0.0217 & 11.414 & 1117.9 & 0.0217 & 16.182 & 1102.2 & 0.0217 \\
\hline 18 & 4.771 & 953.4 & 0.0216 & 6.911 & 950.5 & 0.0216 & 10.070 & 962.0 & 0.0216 \\
\hline
\end{tabular}

\begin{tabular}{|c|c|c|c|}
\hline \multirow[b]{2}{*}{$\begin{array}{l}\text { Axial } \\
\text { Node }\end{array}$} & \multicolumn{3}{|c|}{ Statepoint 68 (BOC Cy9) } \\
\hline & $\begin{array}{c}\text { Burnup } \\
\text { (GWd/MTU) }\end{array}$ & $\begin{array}{l}\text { T-Fuel } \\
\left({ }^{\circ} \mathrm{F}\right)\end{array}$ & $\begin{array}{c}\text { Spec. Vol. } \\
\left(\mathrm{ft}^{3} / \mathrm{lbm}\right)\end{array}$ \\
\hline & BOC Cy9 & 376.2 Cy6 & 376.2 Cy6 \\
\hline 1 & 11.003 & 1023.1 & 0.0235 \\
\hline 2 & 17.883 & 1119.7 & 0.0234 \\
\hline 3 & 21.195 & 1137.7 & 0.0233 \\
\hline 4 & 22.607 & 1127.5 & 0.0231 \\
\hline 5 & 23.151 & 1112.3 & 0.0230 \\
\hline 6 & 23.363 & 1099.4 & 0.0229 \\
\hline 7 & 23.469 & 1089.8 & 0.0228 \\
\hline 8 & 23.545 & 1082.9 & 0.0226 \\
\hline 9 & 23.617 & 1077.9 & 0.0225 \\
\hline 10 & 23.698 & 1074.5 & 0.0224 \\
\hline 11 & 23.799 & 1072.8 & 0.0223 \\
\hline 12 & 23.936 & 1073.7 & 0.0222 \\
\hline 13 & 24.079 & 1077.8 & 0.0221 \\
\hline 14 & 24.103 & 1084.8 & 0.0220 \\
\hline 15 & 23.805 & 1094.1 & 0.0219 \\
\hline 16 & 22.716 & 1101.5 & 0.0218 \\
\hline 17 & 19.695 & 1086.7 & 0.0217 \\
\hline 18 & 12.535 & 976.9 & 0.0216 \\
\hline
\end{tabular}


Table 4-36. Burnup and TH Feedback Parameters by Axial Node for Assembly F2

\begin{tabular}{|c|c|c|c|c|c|c|c|c|c|}
\hline \multirow[b]{2}{*}{$\begin{array}{l}\text { Axial } \\
\text { Node }\end{array}$} & \multicolumn{3}{|c|}{ Statepoint 63 (69.1 Cy6) } & \multicolumn{3}{|c|}{ Datapoint (176.7 Cy6) } & \multicolumn{3}{|c|}{ Datapoint (320.3 Cy6) } \\
\hline & $\begin{array}{c}\text { Burnup } \\
\text { (GWd/MTU) }\end{array}$ & $\begin{array}{c}\text { T-Fuel } \\
\left({ }^{\circ} \mathrm{F}\right)\end{array}$ & $\begin{array}{l}\text { Spec. Vol. } \\
\left(\mathrm{ft}^{3} / \mathrm{bm}\right)\end{array}$ & $\begin{array}{c}\text { Burnup } \\
\text { (GWd/MTU) }\end{array}$ & $\begin{array}{c}\text { T-Fuel } \\
\left({ }^{\circ} \mathrm{F}\right)\end{array}$ & $\begin{array}{l}\text { Spec. Vol. } \\
\left(\mathrm{ft}^{3} / \mathrm{lbm}\right)\end{array}$ & $\begin{array}{c}\text { Burnup } \\
\text { (GWd/MTU) }\end{array}$ & $\begin{array}{c}\text { T-Fuel } \\
\left({ }^{\circ} \mathrm{F}\right)\end{array}$ & $\begin{array}{c}\text { Spec. Vol. } \\
\left(\mathrm{ft}^{3} / \mathrm{bm}\right)\end{array}$ \\
\hline & 69.1 Cy 6 & $41.5 \mathrm{Cy} 6$ & 41.5 Cy 6 & $176.7 \mathrm{Cy} 6$ & 128.8 Cy 6 & $128.8 \mathrm{Cy} 6$ & 320.3 Cy 6 & 252.5 Cy 6 & 252.5 Cy 6 \\
\hline 1 & 1.271 & 1022.7 & 0.0236 & 3.487 & 1082.9 & 0.0238 & 6.921 & 1118.9 & 0.0240 \\
\hline 2 & 1.889 & 1178.5 & 0.0235 & 5.232 & 1244.7 & 0.0237 & 10.368 & 1270.0 & 0.0238 \\
\hline 3 & 2.224 & 1269.8 & 0.0234 & 6.149 & 1333.1 & 0.0236 & 12.017 & 1334.8 & 0.0237 \\
\hline 4 & 2.422 & 1324.6 & 0.0233 & 6.652 & 1375.1 & 0.0234 & 12.781 & 1350.2 & 0.0235 \\
\hline 5 & 2.555 & 1361.4 & 0.0231 & 6.958 & 1395.8 & 0.0233 & 13.157 & 1348.3 & 0.0233 \\
\hline 6 & 2.656 & 1388.9 & 0.0230 & 7.170 & 1406.7 & 0.0231 & 13.371 & 1340.5 & 0.0232 \\
\hline 7 & 2.740 & 1411.2 & 0.0229 & 7.338 & 1413.3 & 0.0230 & 13.526 & 1331.9 & 0.0230 \\
\hline 8 & 2.810 & 1429.0 & 0.0227 & 7.476 & 1417.8 & 0.0228 & 13.657 & 1324.7 & 0.0229 \\
\hline 9 & 2.857 & 1440.4 & 0.0226 & 7.573 & 1419.1 & 0.0227 & 13.757 & 1319.6 & 0.0227 \\
\hline 10 & 2.870 & 1442.6 & 0.0224 & 7.608 & 1417.9 & 0.0225 & 13.804 & 1316.7 & 0.0226 \\
\hline 11 & 2.843 & 1434.5 & 0.0223 & 7.572 & 1413.9 & 0.0224 & 13.792 & 1316.6 & 0.0225 \\
\hline 12 & 2.781 & 1416.8 & 0.0222 & 7.471 & 1407.5 & 0.0223 & 13.730 & 1319.4 & 0.0223 \\
\hline 13 & 2.689 & 1391.2 & 0.0221 & 7.312 & 1398.0 & 0.0221 & 13.622 & 1324.0 & 0.0222 \\
\hline 14 & 2.573 & 1358.8 & 0.0219 & 7.098 & 1383.4 & 0.0220 & 13.457 & 1328.9 & 0.0221 \\
\hline 15 & 2.426 & 1317.4 & 0.0218 & 6.794 & 1359.9 & 0.0219 & 13.150 & 1330.7 & 0.0219 \\
\hline 16 & 2.215 & 1257.4 & 0.0217 & 6.290 & 1314.5 & 0.0218 & 12.454 & 1319.1 & 0.0218 \\
\hline 17 & 1.874 & 1159.1 & 0.0217 & 5.365 & 1221.1 & 0.0217 & 10.840 & 1254.6 & 0.0217 \\
\hline 18 & 1.299 & 985.2 & 0.0216 & 3.696 & 1038.2 & 0.0216 & 7.502 & 1079.0 & 0.0216 \\
\hline
\end{tabular}

\begin{tabular}{|c|c|c|c|c|c|c|c|c|c|}
\hline \multirow[b]{2}{*}{$\begin{array}{l}\text { Axial } \\
\text { Node }\end{array}$} & \multicolumn{3}{|c|}{ Statepoint 64 (BOC Cy7) } & \multicolumn{3}{|c|}{ Statepoint 65 (30.0 Cy7) } & \multicolumn{3}{|c|}{ Statepoint 66 (108.0 Cy7) } \\
\hline & $\begin{array}{c}\text { Burnup } \\
\text { (GWd/MTU) }\end{array}$ & $\begin{array}{l}\text { T-Fuel } \\
\left({ }^{\circ} \mathrm{F}\right)\end{array}$ & $\begin{array}{c}\text { Spec. Vol. } \\
\left(\mathrm{ft}^{3} / \mathrm{lbm}\right)\end{array}$ & $\begin{array}{c}\text { Burnup } \\
\text { (GWd/MTU) }\end{array}$ & $\begin{array}{c}\text { T-Fuel } \\
\left({ }^{\circ} \mathrm{F}\right)\end{array}$ & $\begin{array}{c}\text { Spec. Vol. } \\
\left(\mathrm{ft}^{3} / \mathrm{lbm}\right)\end{array}$ & $\begin{array}{c}\text { Burnup } \\
\text { (GWd/MTU) }\end{array}$ & $\begin{array}{l}\text { T-Fuel } \\
\left({ }^{\circ} \mathrm{F}\right)\end{array}$ & $\begin{array}{c}\text { Spec. Vol. } \\
\left(\mathrm{ft}^{3} / \mathrm{lbm}\right)\end{array}$ \\
\hline & BOC Cy7 & 376.2 Cy 6 & 376.2 Cy 6 & 30.0 Cy 7 & $22.5 \mathrm{Cy} 7$ & 22.5 Cy7 & $108.0 \mathrm{Cy} 7$ & 60.4 Cy 7 & $60.4 \mathrm{Cy} 7$ \\
\hline 1 & 9.527 & 1111.9 & 0.0239 & 9.817 & 785.4 & 0.0234 & 10.612 & 793.4 & 0.0233 \\
\hline 2 & 14.150 & 1235.5 & 0.0237 & 14.788 & 950.4 & 0.0233 & 16.541 & 974.8 & 0.0232 \\
\hline 3 & 16.194 & 1273.0 & 0.0236 & 17.116 & 1074.6 & 0.0232 & 19.517 & 1070.8 & 0.0232 \\
\hline 4 & 17.025 & 1269.1 & 0.0234 & 18.084 & 1128.4 & 0.0231 & 20.770 & 1110.3 & 0.0230 \\
\hline 5 & 17.370 & 1255.5 & 0.0233 & 18.505 & 1156.7 & 0.0230 & 21.336 & 1129.1 & 0.0229 \\
\hline 6 & 17.533 & 1241.7 & 0.0231 & 18.715 & 1173.1 & 0.0229 & 21.626 & 1138.2 & 0.0228 \\
\hline 7 & 17.644 & 1230.0 & 0.0230 & 18.856 & 1181.7 & 0.0228 & 21.809 & 1141.6 & 0.0227 \\
\hline 8 & 17.747 & 1221.0 & 0.0228 & 18.976 & 1185.1 & 0.0226 & 21.952 & 1141.5 & 0.0226 \\
\hline 9 & 17.835 & 1214.6 & 0.0227 & 19.073 & 1185.3 & 0.0225 & 22.058 & 1139.6 & 0.0225 \\
\hline 10 & 17.884 & 1211.0 & 0.0226 & 19.127 & 1184.4 & 0.0224 & 22.119 & 1137.3 & 0.0224 \\
\hline 11 & 17.892 & 1210.5 & 0.0224 & 19.136 & 1182.8 & 0.0223 & 22.134 & 1135.2 & 0.0223 \\
\hline 12 & 17.869 & 1213.4 & 0.0223 & 19.108 & 1179.1 & 0.0222 & 22.105 & 1132.1 & 0.0221 \\
\hline 13 & 17.824 & 1220.1 & 0.0222 & 19.046 & 1169.5 & 0.0221 & 22.019 & 1125.0 & 0.0220 \\
\hline 14 & 17.742 & 1230.5 & 0.0221 & 18.925 & 1150.8 & 0.0220 & 21.833 & 1111.0 & 0.0219 \\
\hline 15 & 17.515 & 1242.5 & 0.0219 & 18.631 & 1120.1 & 0.0219 & 21.410 & 1087.4 & 0.0218 \\
\hline 16 & 16.814 & 1247.5 & 0.0218 & 17.818 & 1073.1 & 0.0218 & 20.365 & 1049.6 & 0.0218 \\
\hline 17 & 14.869 & 1214.5 & 0.0217 & 15.687 & 996.0 & 0.0217 & 17.801 & 983.1 & 0.0217 \\
\hline 18 & 10.402 & 1068.9 & 0.0216 & 10.891 & 843.4 & 0.0216 & 12.179 & 840.5 & 0.0216 \\
\hline
\end{tabular}


Table 4-37. Burnup and TH Feedback Parameters by Axial Node for Assembly F4

\begin{tabular}{|c|c|c|c|c|c|c|c|c|c|}
\hline \multirow[b]{2}{*}{$\begin{array}{l}\text { Axial } \\
\text { Node }\end{array}$} & \multicolumn{3}{|c|}{ Statepoint 63 (69.1 Cy6) } & \multicolumn{3}{|c|}{ Datapoint (176.7 Cy6) } & \multicolumn{3}{|c|}{ Datapoint (320.3 Cy6) } \\
\hline & $\begin{array}{c}\text { Burnup } \\
\text { (GWd/MTU) }\end{array}$ & $\begin{array}{c}\text { T-Fuel } \\
\left({ }^{\circ} \mathrm{F}\right)\end{array}$ & $\begin{array}{c}\text { Spec. Vol. } \\
\left(\mathrm{ft}^{3} / \mathrm{lbm}\right)\end{array}$ & $\begin{array}{c}\text { Burnup } \\
\text { (GWd/MTU) }\end{array}$ & $\begin{array}{c}\text { T-Fuel } \\
\left({ }^{\circ} \mathrm{F}\right)\end{array}$ & $\begin{array}{c}\text { Spec. Vol. } \\
\left(\mathrm{ft}^{3} / \mathrm{lbm}\right)\end{array}$ & $\begin{array}{c}\text { Burnup } \\
\text { (GWd/MTU) }\end{array}$ & $\begin{array}{c}\text { T-Fuel } \\
\left({ }^{\circ} \mathrm{F}\right)\end{array}$ & $\begin{array}{c}\text { Spec. Vol. } \\
\left(\mathrm{ft}^{3} / \mathrm{lbm}\right)\end{array}$ \\
\hline & 69.1 Cy 6 & 41.5 Cy6 & 41.5 Cy 6 & 176.7 Cy6 & 128.8 Cy6 & 128.8 Cy6 & 320.3 Cy 6 & 252.5 Cy 6 & 252.5 Cy 6 \\
\hline 1 & 1.141 & 971.5 & 0.0237 & 3.134 & 1038.3 & 0.0238 & 6.258 & 1084.9 & 0.0239 \\
\hline 2 & 1.784 & 1136.8 & 0.0236 & 4.933 & 1213.1 & 0.0237 & 9.805 & 1246.3 & 0.0238 \\
\hline 3 & 2.210 & 1257.1 & 0.0235 & 6.054 & 1318.8 & 0.0236 & 11.768 & 1319.7 & 0.0236 \\
\hline 4 & 2.469 & 1332.7 & 0.0234 & 6.679 & 1369.2 & 0.0235 & 12.690 & 1335.9 & 0.0235 \\
\hline 5 & 2.629 & 1378.2 & 0.0232 & 7.029 & 1391.7 & 0.0233 & 13.111 & 1332.7 & 0.0233 \\
\hline 6 & 2.745 & 1409.9 & 0.0231 & 7.262 & 1402.8 & 0.0232 & 13.342 & 1324.3 & 0.0232 \\
\hline 7 & 2.851 & 1437.6 & 0.0229 & 7.469 & 1411.4 & 0.0230 & 13.548 & 1316.6 & 0.0230 \\
\hline 8 & 2.963 & 1466.2 & 0.0228 & 7.696 & 1420.6 & 0.0229 & 13.807 & 1312.4 & 0.0229 \\
\hline 9 & 3.061 & 1487.3 & 0.0226 & 7.903 & 1427.9 & 0.0227 & 14.064 & 1310.9 & 0.0227 \\
\hline 10 & 3.103 & 1494.4 & 0.0225 & 7.997 & 1429.7 & 0.0226 & 14.193 & 1309.5 & 0.0226 \\
\hline 11 & 3.084 & 1488.4 & 0.0224 & 7.975 & 1426.2 & 0.0224 & 14.194 & 1309.0 & 0.0224 \\
\hline 12 & 3.010 & 1470.7 & 0.0222 & 7.850 & 1418.1 & 0.0223 & 14.088 & 1309.9 & 0.0223 \\
\hline 13 & 2.888 & 1439.8 & 0.0221 & 7.627 & 1405.6 & 0.0221 & 13.877 & 1311.9 & 0.0222 \\
\hline 14 & 2.741 & 1400.1 & 0.0220 & 7.350 & 1388.7 & 0.0220 & 13.613 & 1315.0 & 0.0220 \\
\hline 15 & 2.574 & 1354.6 & 0.0219 & 7.015 & 1364.1 & 0.0219 & 13.261 & 1316.2 & 0.0219 \\
\hline 16 & 2.344 & 1290.3 & 0.0217 & 6.484 & 1318.7 & 0.0218 & 12.544 & 1302.2 & 0.0218 \\
\hline 17 & 1.973 & 1184.6 & 0.0217 & 5.510 & 1224.1 & 0.0217 & 10.893 & 1240.0 & 0.0217 \\
\hline 18 & 1.361 & 1003.2 & 0.0216 & 3.777 & 1038.7 & 0.0216 & 7.508 & 1067.9 & 0.0216 \\
\hline
\end{tabular}

\begin{tabular}{|c|c|c|c|c|c|c|c|c|c|}
\hline \multirow[b]{2}{*}{$\begin{array}{l}\text { Axial } \\
\text { Node }\end{array}$} & \multicolumn{3}{|c|}{ Statepoint 64 (BOC Cy7) } & \multicolumn{3}{|c|}{ Statepoint 65 (30.0 Cy7) } & \multicolumn{3}{|c|}{ Statepoint 66 (108.0 Cyn) } \\
\hline & $\begin{array}{c}\text { Burnup } \\
\text { (GWd/MTU) }\end{array}$ & $\begin{array}{c}\text { T-Fuel } \\
\left({ }^{\circ} \mathrm{F}\right)\end{array}$ & $\begin{array}{l}\text { Spec. Vol. } \\
\left(\mathrm{ft}^{3} / \mathrm{lbm}\right)\end{array}$ & $\begin{array}{c}\text { Burnup } \\
\text { (GWd/MTU) }\end{array}$ & $\begin{array}{l}\text { T-Fuel } \\
\left({ }^{\circ} \mathrm{F}\right)\end{array}$ & $\begin{array}{c}\text { Spec. Vol. } \\
\left(\mathrm{ft}^{3} / \mathrm{lbm}\right)\end{array}$ & $\begin{array}{c}\text { Burnup } \\
\text { (GWd/MTU) }\end{array}$ & $\begin{array}{l}\text { T-Fuel } \\
\left({ }^{\circ} \mathrm{F}\right)\end{array}$ & $\begin{array}{c}\text { Spec. Vol. } \\
\left(\mathrm{tt}^{3} / \mathrm{lbm}\right)\end{array}$ \\
\hline & BOC Cy? & 376.2 Cy 6 & 376.2 Cy 6 & $30.0 \mathrm{Cy} 7$ & $22.5 \mathrm{Cy} 7$ & $22.5 \mathrm{Cy} 7$ & 108.0 Cy 7 & $60.4 \mathrm{Cy} 7$ & $60.4 \mathrm{Cy} 7$ \\
\hline 1 & 8.700 & 1096.7 & 0.0238 & 9.035 & 821.7 & 0.0235 & 9.971 & 835.0 & 0.0235 \\
\hline 2 & 13.468 & 1226.2 & 0.0237 & 14.167 & 992.1 & 0.0234 & 16.144 & 1028.3 & 0.0234 \\
\hline 3 & 15.874 & 1266.6 & 0.0235 & 16.856 & 1114.2 & 0.0233 & 19.506 & 1123.5 & 0.0233 \\
\hline 4 & 16.869 & 1260.9 & 0.0234 & 17.980 & 1163.7 & 0.0232 & 20.919 & 1161.2 & 0.0232 \\
\hline 5 & 17.252 & 1245.6 & 0.0232 & 18.433 & 1189.9 & 0.0231 & 21.522 & 1180.1 & 0.0231 \\
\hline 6 & 17.430 & 1231.1 & 0.0231 & 18.655 & 1205.1 & 0.0229 & 21.827 & 1189.5 & 0.0229 \\
\hline 7 & 17.599 & 1219.8 & 0.0229 & 18.850 & 1211.7 & 0.0228 & 22.064 & 1192.2 & 0.0228 \\
\hline 8 & 17.847 & 1212.0 & 0.0228 & 19.110 & 1211.1 & 0.0227 & 22.340 & 1189.8 & 0.0227 \\
\hline 9 & 18.113 & 1206.9 & 0.0227 & 19.379 & 1206.6 & 0.0226 & 22.608 & 1184.6 & 0.0225 \\
\hline 10 & 18.252 & 1203.5 & 0.0225 & 19.517 & 1202.0 & 0.0224 & 22.743 & 1179.8 & 0.0224 \\
\hline 11 & 18.270 & 1202.3 & 0.0224 & 19.531 & 1198.2 & 0.0223 & 22.755 & 1175.8 & 0.0223 \\
\hline 12 & 18.191 & 1204.0 & 0.0223 & 19.444 & 1193.2 & 0.0222 & 22.660 & 1171.5 & 0.0222 \\
\hline 13 & 18.022 & 1208.8 & 0.0222 & 19.255 & 1183.8 & 0.0221 & 22.439 & 1163.7 & 0.0221 \\
\hline 14 & 17.819 & 1217.3 & 0.0220 & 19.013 & 1165.4 & 0.0220 & 22.123 & 1148.5 & 0.0220 \\
\hline 15 & 17.539 & 1228.5 & 0.0219 & 18.667 & 1134.9 & 0.0219 & 21.636 & 1122.7 & 0.0219 \\
\hline 16 & 16.819 & 1233.9 & 0.0218 & 17.841 & 1089.4 & 0.0218 & 20.568 & 1083.5 & 0.0218 \\
\hline 17 & 14.846 & 1202.5 & 0.0217 & 15.697 & 1018.5 & 0.0217 & 17.993 & 1018.4 & 0.0217 \\
\hline 18 & 10.350 & 1059.6 & 0.0216 & 10.880 & 870.2 & 0.0216 & 12.325 & 874.6 & 0.0216 \\
\hline
\end{tabular}


Table 4-38. Burnup and TH Feedback Parameters by Axial Node for Assembly F6

\begin{tabular}{|c|c|c|c|c|c|c|c|c|c|}
\hline \multirow[b]{2}{*}{$\begin{array}{l}\text { Axial } \\
\text { Node }\end{array}$} & \multicolumn{3}{|c|}{ Statepoint 63 (69.1 Cy6) } & \multicolumn{3}{|c|}{ Datapoint (176.7 Cy6) } & \multicolumn{3}{|c|}{ Datapoint (320.3 Cy6) } \\
\hline & $\begin{array}{c}\text { Burnup } \\
\text { (GWd/MTU) }\end{array}$ & $\begin{array}{c}\text { T-Fuel } \\
\left({ }^{\circ} \mathrm{F}\right) \\
\end{array}$ & $\begin{array}{c}\text { Spec. Vol. } \\
\left(\mathrm{ft}^{3} / \mathrm{lbm}\right)\end{array}$ & $\begin{array}{c}\text { Burnup } \\
\text { (GWd/MTU) }\end{array}$ & $\begin{array}{c}\text { T-Fuel } \\
\left({ }^{\circ} \mathrm{F}\right) \\
\end{array}$ & $\begin{array}{c}\text { Spec. Vol. } \\
\left(\mathrm{ft}^{3} / \mathrm{lbm}\right)\end{array}$ & $\begin{array}{c}\text { Burnup } \\
\text { (GWd/MTU) }\end{array}$ & $\begin{array}{c}\text { T-Fuel } \\
\left({ }^{\circ} \mathrm{F}\right) \\
\end{array}$ & $\begin{array}{c}\text { Spec. Vol. } \\
\left(\mathrm{ft}^{3} / \mathrm{lbm}\right)\end{array}$ \\
\hline & 69.1 Cy6 & 41.5 Cy6 & 41.5 Cy 6 & 176.7 Cy6 & 128.8 Cy 6 & 128.8 Cy6 & 320.3 Cy6 & 252.5 Cy 6 & 252.5 Cy 6 \\
\hline 1 & 1.122 & 963.6 & 0.0238 & 3.025 & 1016.6 & 0.0238 & 5.974 & 1060.9 & 0.0238 \\
\hline 2 & 1.838 & 1147.2 & 0.0237 & 4.952 & 1202.1 & 0.0237 & 9.646 & 1221.7 & 0.0236 \\
\hline 3 & 2.330 & 1283.0 & 0.0236 & 6.180 & 1311.4 & 0.0236 & 11.698 & 1290.9 & 0.0235 \\
\hline 4 & 2.633 & 1368.8 & 0.0234 & 6.863 & 1362.1 & 0.0234 & 12.659 & 1304.2 & 0.0234 \\
\hline 5 & 2.816 & 1419.4 & 0.0233 & 7.233 & 1382.8 & 0.0233 & 13.081 & 1299.1 & 0.0232 \\
\hline 6 & 2.937 & 1451.7 & 0.0231 & 7.454 & 1390.5 & 0.0231 & 13.281 & 1289.2 & 0.0231 \\
\hline 7 & 3.027 & 1473.5 & 0.0230 & 7.609 & 1393.3 & 0.0230 & 13.405 & 1279.6 & 0.0229 \\
\hline 8 & 3.096 & 1488.9 & 0.0228 & 7.728 & 1394.1 & 0.0228 & 13.505 & 1271.9 & 0.0228 \\
\hline 9 & 3.142 & 1497.5 & 0.0227 & 7.809 & 1393.8 & 0.0227 & 13.580 & 1266.4 & 0.0227 \\
\hline 10 & 3.157 & 1499.3 & 0.0225 & 7.843 & 1392.0 & 0.0225 & 13.619 & 1263.1 & 0.0225 \\
\hline 11 & 3.143 & 1494.8 & 0.0224 & 7.831 & 1389.1 & 0.0224 & 13.628 & 1262.3 & 0.0224 \\
\hline 12 & 3.104 & 1484.8 & 0.0223 & 7.783 & 1385.8 & 0.0223 & 13.623 & 1264.6 & 0.0223 \\
\hline 13 & 3.039 & 1469.2 & 0.0221 & 7.695 & 1381.7 & 0.0221 & 13.596 & 1269.7 & 0.0221 \\
\hline 14 & 2.941 & 1445.4 & 0.0220 & 7.543 & 1374.7 & 0.0220 & 13.507 & 1276.2 & 0.0220 \\
\hline 15 & 2.788 & 1406.1 & 0.0219 & 7.264 & 1357.7 & 0.0219 & 13.247 & 1279.7 & 0.0219 \\
\hline 16 & 2.534 & 1337.0 & 0.0218 & 6.718 & 1315.2 & 0.0218 & 12.541 & 1268.1 & 0.0218 \\
\hline 17 & 2.099 & 1215.7 & 0.0217 & 5.649 & 1218.6 & 0.0217 & 10.819 & 1210.8 & 0.0217 \\
\hline 18 & 1.392 & 1010.7 & 0.0216 & 3.750 & 1024.8 & 0.0216 & 7.288 & 1044.1 & 0.0216 \\
\hline
\end{tabular}

\begin{tabular}{|c|c|c|c|c|c|c|c|c|c|}
\hline \multirow[b]{2}{*}{$\begin{array}{l}\text { Axlal } \\
\text { Node }\end{array}$} & \multicolumn{3}{|c|}{ Statepoint 64 (BOC Cy7) } & \multicolumn{3}{|c|}{ Statepoint $65(30.0 \mathrm{Cy} 7)$} & \multicolumn{3}{|c|}{ Statepoint 66 (108.0 Cy7) } \\
\hline & $\begin{array}{c}\text { Burnup } \\
\text { (GWd/MTU) }\end{array}$ & $\begin{array}{c}\text { T-Fuel } \\
\left({ }^{\circ} \mathrm{F}\right)\end{array}$ & $\begin{array}{c}\text { Spec. Vol. } \\
\left(\mathrm{ft}^{3} / / \mathrm{bm}\right)\end{array}$ & $\begin{array}{c}\text { Burnup } \\
\text { (GWd/MTU) }\end{array}$ & $\begin{array}{l}\text { T-Fuel } \\
\left({ }^{\circ} \mathrm{F}\right)\end{array}$ & $\begin{array}{c}\text { Spec. Vol. } \\
\left(\mathrm{ft}^{3} / \mathrm{lbm}\right)\end{array}$ & $\begin{array}{c}\text { Burnup } \\
\text { (GWd/MTU) }\end{array}$ & $\begin{array}{c}\text { T-Fuel } \\
\left({ }^{\circ} \mathrm{F}\right)\end{array}$ & $\begin{array}{c}\text { Spec. Vol. } \\
\left(\mathrm{ft}^{3} / \mathrm{lbm}\right)\end{array}$ \\
\hline & BOC Cy7 & 376.2 Cy 6 & 376.2 Cy 6 & $30.0 \mathrm{Cy} 7$ & $22.5 \mathrm{Cy} 7$ & 22.5 Cy 7 & $108.0 \mathrm{Cy} 7$ & 60.4 Cy 7 & 60.4 Cy7 \\
\hline 1 & 8.312 & 1080.9 & 0.0237 & 8.863 & 966.0 & 0.0236 & 10.389 & 977.2 & 0.0236 \\
\hline 2 & 13.192 & 1208.7 & 0.0236 & 14.066 & 1089.4 & 0.0235 & 16.471 & 1102.0 & 0.0235 \\
\hline 3 & 15.675 & 1246.9 & 0.0235 & 16.718 & 1152.3 & 0.0234 & 19.566 & 1161.2 & 0.0234 \\
\hline 4 & 16.700 & 1240.0 & 0.0233 & 17.848 & 1190.3 & 0.0232 & 20.951 & 1193.9 & 0.0233 \\
\hline 5 & 17.080 & 1224.6 & 0.0232 & 18.297 & 1215.8 & 0.0231 & 21.553 & 1214.0 & 0.0231 \\
\hline 6 & 17.226 & 1210.4 & 0.0230 & 18.487 & 1231.8 & 0.0230 & 21.834 & 1225.6 & 0.0230 \\
\hline 7 & 17.308 & 1199.6 & 0.0229 & 18.596 & 1240.2 & 0.0228 & 21.994 & 1230.3 & 0.0229 \\
\hline 8 & 17.383 & 1191.7 & 0.0228 & 18.686 & 1242.5 & 0.0227 & 22.106 & 1230.1 & 0.0227 \\
\hline 9 & 17.449 & 1186.3 & 0.0226 & 18.755 & 1240.6 & 0.0226 & 22.180 & 1226.9 & 0.0226 \\
\hline 10 & 17.490 & 1183.0 & 0.0225 & 18.793 & 1235.9 & 0.0224 & 22.211 & 1221.9 & 0.0225 \\
\hline 11 & 17.515 & 1181.9 & 0.0224 & 18.808 & 1228.3 & 0.0223 & 22.208 & 1215.0 & 0.0223 \\
\hline 12 & 17.543 & 1183.6 & 0.0223 & 18.816 & 1216.3 & 0.0222 & 22.180 & 1204.9 & 0.0222 \\
\hline 13 & 17.573 & 1188.5 & 0.0221 & 18.812 & 1198.1 & 0.0221 & 22.113 & 1189.9 & 0.0221 \\
\hline 14 & 17.560 & 1197.1 & 0.0220 & 18.749 & 1172.3 & 0.0220 & 21.947 & 1168.3 & 0.0220 \\
\hline 15 & 17.376 & 1208.0 & 0.0219 & 18.491 & 1137.0 & 0.0219 & 21.527 & 1137.4 & 0.0219 \\
\hline 16 & 16.672 & 1213.7 & 0.0218 & 17.680 & 1089.3 & 0.0218 & 20.455 & 1094.5 & 0.0218 \\
\hline 17 & 14.642 & 1184.3 & 0.0217 & 15.480 & 1018.4 & 0.0217 & 17.812 & 1027.4 & 0.0217 \\
\hline 18 & 10.025 & 1046.2 & 0.0216 & 10.550 & 871.9 & 0.0216 & 12.022 & 882.3 & 0.0216 \\
\hline
\end{tabular}


Table 4-39. Burnup and TH Feedback Parameters by Axial Node for Assembly F10

\begin{tabular}{|c|c|c|c|c|c|c|c|c|c|}
\hline \multirow[b]{2}{*}{$\begin{array}{l}\text { Axial } \\
\text { Node }\end{array}$} & \multicolumn{3}{|c|}{ Statepoint 63 (69.1 Cy6) } & \multicolumn{3}{|c|}{ Datapoint (176.7 Cy6) } & \multicolumn{3}{|c|}{ Datapoint (320.3 Cy6) } \\
\hline & \begin{tabular}{|c|} 
Burnup \\
(GWd/MTU)
\end{tabular} & $\begin{array}{c}\text { T-Fuel } \\
\left({ }^{\circ} \mathrm{F}\right)\end{array}$ & $\begin{array}{c}\text { Spec. Vol. } \\
\left(\mathrm{ft}^{3} / \mathrm{lbm}\right)\end{array}$ & \begin{tabular}{|c|} 
Burnup \\
(GWd/MTU)
\end{tabular} & $\begin{array}{c}\text { T-Fuel } \\
\left({ }^{\circ} \mathrm{F}\right)\end{array}$ & $\begin{array}{c}\text { Spec. Vol. } \\
\left(\mathrm{ft}^{3} / \mathrm{lbm}\right)\end{array}$ & $\begin{array}{c}\text { Burnup } \\
\text { (GWd/MTU) }\end{array}$ & $\begin{array}{c}\text { T-Fuel } \\
\left({ }^{\circ} \mathrm{F}\right)\end{array}$ & $\begin{array}{c}\text { Spec. Vol. } \\
\left(\mathrm{ft}^{3} / \mathrm{lbm}\right)\end{array}$ \\
\hline & 69.1 Cy6 & 41.5 Cy 6 & 41.5 Cy 6 & 176.7 Cy 6 & 128.8 Cy 6 & 128.8 Cy 6 & 320.3 Cy 6 & 252.5 Cy 6 & 252.5 Cy 6 \\
\hline 1 & 1.237 & 1009.3 & 0.0236 & 3.388 & 1070.1 & 0.0238 & 6.727 & 1107.7 & 0.0239 \\
\hline 2 & 1.855 & 1166.5 & 0.0235 & 5.124 & 1232.0 & 0.0237 & 10.147 & 1258.4 & 0.0238 \\
\hline 3 & 2.207 & 1262.9 & 0.0234 & 6.075 & 1323.0 & 0.0236 & 11.838 & 1323.6 & 0.0236 \\
\hline 4 & 2.421 & 1322.6 & 0.0233 & 6.607 & 1366.7 & 0.0234 & 12.634 & 1338.8 & 0.0235 \\
\hline 5 & 2.563 & 1362.1 & 0.0232 & 6.924 & 1387.6 & 0.0233 & 13.017 & 1335.7 & 0.0233 \\
\hline 6 & 2.670 & 1391.1 & 0.0230 & 7.142 & 1398.3 & 0.0231 & 13.235 & 1327.6 & 0.0232 \\
\hline 7 & 2.764 & 1416.0 & 0.0229 & 7.329 & 1405.9 & 0.0230 & 13.414 & 1319.4 & 0.0230 \\
\hline 8 & 2.857 & 1439.6 & 0.0227 & 7.514 & 1413.6 & 0.0228 & 13.612 & 1313.9 & 0.0229 \\
\hline 9 & 2.931 & 1457.8 & 0.0226 & 7.669 & 1418.6 & 0.0227 & 13.794 & 1310.6 & 0.0227 \\
\hline 10 & 2.958 & 1462.9 & 0.0225 & 7.735 & 1418.9 & 0.0225 & 13.882 & 1308.4 & 0.0226 \\
\hline 11 & 2.936 & 1455.9 & 0.0223 & 7.705 & 1415.1 & 0.0224 & 13.875 & 1307.9 & 0.0224 \\
\hline 12 & 2.869 & 1437.3 & 0.0222 & 7.591 & 1407.8 & 0.0223 & 13.789 & 1309.7 & 0.0223 \\
\hline 13 & 2.762 & 1407.9 & 0.0221 & 7.400 & 1396.1 & 0.0221 & 13.627 & 1313.2 & 0.0222 \\
\hline 14 & 2.631 & 1372.1 & 0.0220 & 7.156 & 1379.7 & 0.0220 & 13.413 & 1317.8 & 0.0220 \\
\hline 15 & 2.476 & 1329.2 & 0.0218 & 6.840 & 1356.0 & 0.0219 & 13.088 & 1319.4 & 0.0219 \\
\hline 16 & 2.259 & 1268.0 & 0.0217 & 6.330 & 1311.0 & 0.0218 & 12.391 & 1305.8 & 0.0218 \\
\hline 17 & 1.907 & 1167.1 & 0.0217 & 5.391 & 1217.8 & 0.0217 & 10.775 & 1242.8 & 0.0217 \\
\hline 18 & 1.320 & 990.7 & 0.0216 & 3.706 & 1035.0 & 0.0216 & 7.441 & 1069.8 & 0.0216 \\
\hline
\end{tabular}

\begin{tabular}{|c|c|c|c|c|c|c|c|c|c|}
\hline \multirow[b]{2}{*}{$\begin{array}{l}\text { Axial } \\
\text { Node }\end{array}$} & \multicolumn{3}{|c|}{ Statepoint 64 (BOC Cy7) } & \multicolumn{3}{|c|}{ Statepoint $65(30.0 \mathrm{Cy} 7)$} & \multicolumn{3}{|c|}{ Statepoint 66 (108.0 Cy7) } \\
\hline & $\begin{array}{c}\text { Burnup } \\
\text { (GWd/MTU) }\end{array}$ & $\begin{array}{l}\text { T-Fuel } \\
\left({ }^{\circ} \mathrm{F}\right)\end{array}$ & $\begin{array}{l}\text { Spec. Vol. } \\
\left(\mathrm{ft}^{3} / \mathrm{lbm}\right)\end{array}$ & $\begin{array}{c}\text { Burnup } \\
\text { (GWd/MTU) }\end{array}$ & $\begin{array}{c}\text { T-Fuel } \\
\left({ }^{\circ} \mathrm{F}\right)\end{array}$ & $\begin{array}{l}\text { Spec. Vol. } \\
\left(\mathrm{ft}^{3} / \mathrm{lbm}\right)\end{array}$ & $\begin{array}{c}\text { Burnup } \\
\text { (GWd/MTU) }\end{array}$ & $\begin{array}{c}\text { T-Fuel } \\
\left({ }^{\circ} \mathrm{F}\right)\end{array}$ & $\begin{array}{c}\text { Spec. Vol. } \\
\left(\mathrm{ft}^{3} / \mathrm{lbm}\right)\end{array}$ \\
\hline & BOC Cy? & 376.2 Cy6 & 376.2 Cy 6 & $30.0 \mathrm{Cy} 7$ & 22.5 Cy? & 22.5 Cy7 & $108.0 \mathrm{Cy} 7$ & $60.4 \mathrm{Cy} 7$ & 60.4 Cy7 \\
\hline 1 & 9.281 & 1105.7 & 0.0238 & 9.465 & 693.2 & 0.02 .22 & 9.972 & 697.5 & 0.0222 \\
\hline 2 & 13.869 & 1228.9 & 0.0237 & 14.172 & 743.0 & 0.0222 & 14.996 & 747.8 & 0.0222 \\
\hline 3 & 15.957 & 1266.4 & 0.0235 & 16.322 & 768.7 & 0.0221 & 17.301 & 771.9 & 0.0221 \\
\hline 4 & 16.818 & 1261.7 & 0.0234 & 17.218 & 782.6 & 0.0221 & 18.277 & 783.5 & 0.0221 \\
\hline 5 & 17.166 & 1247.4 & 0.0232 & 17.587 & 790.8 & 0.0221 & 18.691 & 789.5 & 0.0221 \\
\hline 6 & 17.330 & 1233.2 & 0.0231 & 17.765 & 795.5 & 0.0220 & 18.892 & 792.2 & 0.0220 \\
\hline 7 & 17.469 & 1221.7 & 0.0229 & 17.912 & 797.2 & 0.0220 & 19.048 & 792.4 & 0.0220 \\
\hline 8 & 17.648 & 1213.3 & 0.0228 & 18.093 & 796.6 & 0.0220 & 19.231 & 790.7 & 0.0220 \\
\hline 9 & 17.828 & 1207.5 & 0.0227 & 18.273 & 794.5 & 0.0219 & 19.406 & 788.0 & 0.0219 \\
\hline 10 & 17.921 & 1203.8 & 0.0225 & 18.364 & 792.0 & 0.0219 & 19.491 & 785.3 & 0.0219 \\
\hline 11 & 17.931 & 1202.9 & 0.0224 & 18.370 & 789.3 & 0.0218 & 19.490 & 782.7 & 0.0218 \\
\hline 12 & 17.877 & 1205.1 & 0.0223 & 18.311 & 786.0 & 0.0218 & 19.421 & 779.9 & 0.0218 \\
\hline 13 & 17.767 & 1210.7 & 0.0222 & 18.191 & 781.2 & 0.0218 & 19.286 & 776.0 & 0.0218 \\
\hline 14 & 17.624 & 1220.1 & 0.0220 & 18.034 & 773.9 & 0.0217 & 19.102 & 770.1 & 0.0217 \\
\hline 15 & 17.375 & 1231.7 & 0.0219 & 17.762 & 762.8 & 0.0217 & 18.783 & 760.8 & 0.0217 \\
\hline 16 & 16.674 & 1236.8 & 0.0218 & 17.026 & 746.4 & 0.0217 & 17.966 & 746.3 & 0.0217 \\
\hline 17 & 14.733 & 1204.6 & 0.0217 & 15.025 & 719.3 & 0.0216 & 15.813 & 720.8 & 0.0216 \\
\hline 18 & 10.287 & 1061.0 & 0.0216 & 10.464 & 663.7 & 0.0216 & 10.949 & 665.7 & 0.0216 \\
\hline
\end{tabular}


Table 4-40. Burnup and TH Feedback Parameters by Axial Node for Assembly F12

\begin{tabular}{|c|c|c|c|c|c|c|c|c|c|}
\hline \multirow[b]{2}{*}{$\begin{array}{l}\text { Axial } \\
\text { Node }\end{array}$} & \multicolumn{3}{|c|}{ Statepoint 63 (69.1 Cy6) } & \multicolumn{3}{|c|}{ Datapoint (176.7 Cy6) } & \multicolumn{3}{|c|}{ Datapoint (320.3 Cy6) } \\
\hline & $\begin{array}{c}\text { Burnup } \\
\text { (GWd/MTU) }\end{array}$ & $\begin{array}{c}\text { T-Fuel } \\
\left({ }^{\circ} \mathrm{F}\right)\end{array}$ & $\begin{array}{c}\text { Spec. Vol. } \\
\left(\mathrm{ft}^{3} / \mathrm{lbm}\right)\end{array}$ & $\begin{array}{c}\text { Burnup } \\
\text { (GWd/MTU) }\end{array}$ & $\begin{array}{c}\text { T-Fuel } \\
\left({ }^{\circ} \mathrm{F}\right)\end{array}$ & $\begin{array}{c}\text { Spec. Vol. } \\
\left(\mathrm{ft}^{3} / \mathrm{lbm}\right)\end{array}$ & $\begin{array}{c}\text { Burnup } \\
\text { (GWd/MTU) }\end{array}$ & $\begin{array}{c}\text { T-Fuel } \\
\left({ }^{\circ} \mathrm{F}\right)\end{array}$ & $\begin{array}{c}\text { Spec. Vol. } \\
\left(\mathrm{ft}^{3} / \mathrm{lbm}\right)\end{array}$ \\
\hline & 69.1 Cy6 & 41.5 Cy 6 & 41.5 Cy 6 & 176.7 Cy6 & 128.8 Cy6 & 128.8 Cy6 & 320.3 Cy 6 & 252.5 Cy6 & 252.5 Cy6 \\
\hline 1 & 1.170 & 980.1 & 0.0238 & 3.174 & 1040.0 & 0.0239 & 6.282 & 1082.2 & 0.0239 \\
\hline 2 & 1.862 & 1155.8 & 0.0237 & 5.073 & 1221.4 & 0.0238 & 9.961 & 1245.7 & 0.0238 \\
\hline 3 & 2.338 & 1288.6 & 0.0236 & 6.290 & 1332.4 & 0.0237 & 12.039 & 1318.9 & 0.0236 \\
\hline 4 & 2.626 & 1372.1 & 0.0235 & 6.961 & 1384.3 & 0.0235 & 12.997 & 1333.1 & 0.0235 \\
\hline 5 & 2.792 & 1419.0 & 0.0233 & 7.305 & 1404.1 & 0.0233 & 13.379 & 1327.1 & 0.0233 \\
\hline 6 & 2.909 & 1449.6 & 0.0232 & 7.521 & 1411.8 & 0.0232 & 13.574 & 1316.8 & 0.0232 \\
\hline 7 & 3.009 & 1474.5 & 0.0230 & 7.703 & 1416.6 & 0.0230 & 13.740 & 1307.8 & 0.0230 \\
\hline 8 & 3.102 & 1494.7 & 0.0229 & 7.881 & 1421.1 & 0.0229 & 13.924 & 1301.8 & 0.0229 \\
\hline 9 & 3.177 & 1509.3 & 0.0227 & 8.031 & 1424.6 & 0.0227 & 14.098 & 1298.3 & 0.0227 \\
\hline 10 & 3.208 & 1514.4 & 0.0226 & 8.102 & 1424.7 & 0.0226 & 14.191 & 1296.1 & 0.0226 \\
\hline 11 & 3.199 & 1510.0 & 0.0224 & 8.098 & 1422.1 & 0.0224 & 14.216 & 1295.9 & 0.0224 \\
\hline 12 & 3.159 & 1498.7 & 0.0223 & 8.047 & 1418.1 & 0.0223 & 14.219 & 1299.5 & 0.0223 \\
\hline 13 & 3.092 & 1482.5 & 0.0221 & 7.955 & 1413.8 & 0.0222 & 14.210 & 1307.1 & 0.0222 \\
\hline 14 & 2.983 & 1458.7 & 0.0220 & 7.782 & 1406.3 & 0.0220 & 14.096 & 1313.2 & 0.0220 \\
\hline 15 & 2.821 & 1418.0 & 0.0219 & 7.477 & 1389.2 & 0.0219 & 13.798 & 1315.6 & 0.0219 \\
\hline 16 & 2.566 & 1348.2 & 0.0218 & 6.912 & 1344.0 & 0.0218 & 13.055 & 1302.9 & 0.0218 \\
\hline 17 & 2.141 & 1229.1 & 0.0217 & 5.839 & 1244.8 & 0.0217 & 11.294 & 1241.4 & 0.0217 \\
\hline 18 & 1.457 & 1032.0 & 0.0216 & 3.958 & 1050.2 & 0.0216 & 7.720 & 1068.4 & 0.0216 \\
\hline
\end{tabular}

\begin{tabular}{|c|c|c|c|c|c|c|c|c|c|}
\hline \multirow[b]{2}{*}{$\begin{array}{l}\text { Axial } \\
\text { Node }\end{array}$} & \multicolumn{3}{|c|}{ Statepoint 64 (BOC Cy7) } & \multicolumn{3}{|c|}{ Statepoint 65 ( 30.0 Cy7) } & \multicolumn{3}{|c|}{ Statepoint $66(108.0 \mathrm{Cy})$} \\
\hline & $\begin{array}{c}\text { Burnup } \\
\text { (GWd/MTU) }\end{array}$ & $\begin{array}{c}\text { T-Fuel } \\
\left({ }^{\circ} \mathrm{F}\right)\end{array}$ & $\begin{array}{c}\text { Spec. Vol. } \\
\left(\mathrm{ft}^{3} / \mathrm{lbm}\right)\end{array}$ & $\begin{array}{c}\text { Burnup } \\
\text { (GWd/MTU) }\end{array}$ & $\begin{array}{l}\text { T-Fuel } \\
\left({ }^{\circ} \mathrm{F}\right)\end{array}$ & $\begin{array}{c}\text { Spec. Vol. } \\
\left(\mathrm{ft}^{3} / \mathrm{lbm}\right)\end{array}$ & \begin{tabular}{c|} 
Burnup \\
(GWd/MTU)
\end{tabular} & $\begin{array}{l}\text { T-Fuel } \\
\left({ }^{\circ} \mathrm{F}\right)\end{array}$ & $\begin{array}{c}\text { Spec. Vol. } \\
\left(\mathrm{ft}^{3} / \mathrm{bm}\right)\end{array}$ \\
\hline & BOC Cy7 & 376.2 Cy 6 & 376.2 Cy6 & 30.0 Cy 7 & $22.5 \mathrm{Cy} 7$ & $22.5 \mathrm{Cy} 7$ & $108.0 \mathrm{Cy} 7$ & $60.4 \mathrm{Cy} 7$ & 60.4 Cy 7 \\
\hline 1 & 8.720 & 1096.2 & 0.0238 & 8.861 & 663.1 & 0.0221 & 9.248 & 666.5 & 0.0221 \\
\hline 2 & 13.637 & 1227.2 & 0.0237 & 13.869 & 700.6 & 0.0220 & 14.500 & 704.4 & 0.0220 \\
\hline 3 & 16.162 & 1266.5 & 0.0235 & 16.441 & 719.3 & 0.0220 & 17.191 & 721.9 & 0.0220 \\
\hline 4 & 17.183 & 1258.8 & 0.0234 & 17.490 & 729.6 & 0.0220 & 18.301 & 730.5 & 0.0220 \\
\hline 5 & 17.508 & 1241.1 & 0.0232 & 17.831 & 736.3 & 0.0220 & 18.677 & 735.4 & 0.0220 \\
\hline 6 & 17.640 & 1225.7 & 0.0231 & 17.975 & 740.1 & 0.0219 & 18.840 & 737.7 & 0.0219 \\
\hline 7 & 17.765 & 1214.4 & 0.0229 & 18.106 & 741.6 & 0.0219 & 18.978 & 738.0 & 0.0219 \\
\hline 8 & 17.933 & 1206.5 & 0.0228 & 18.276 & 741.2 & 0.0219 & 19.149 & 736.7 & 0.0219 \\
\hline 9 & 18.106 & 1201.2 & 0.0227 & 18.449 & 739.7 & 0.0218 & 19.319 & 734.7 & 0.0218 \\
\hline 10 & 18.206 & 1197.8 & 0.0225 & 18.547 & 737.8 & 0.0218 & 19.413 & 732.6 & 0.0218 \\
\hline 11 & 18.249 & 1196.6 & 0.0224 & 18.587 & 735.7 & 0.0218 & 19.448 & 730.5 & 0.0218 \\
\hline 12 & 18.293 & 1199.0 & 0.0223 & 18.627 & 732.6 & 0.0218 & 19.479 & 727.8 & 0.0218 \\
\hline 13 & 18.358 & 1206.2 & 0.0222 & 18.683 & 727.8 & 0.0217 & 19.520 & 723.7 & 0.0217 \\
\hline 14 & 18.325 & 1215.6 & 0.0221 & 18.638 & 721.3 & 0.0217 & 19.452 & 718.4 & 0.0217 \\
\hline 15 & 18.105 & 1227.1 & 0.0219 & 18.400 & 712.3 & 0.0217 & 19.176 & 710.8 & 0.0217 \\
\hline 16 & 17.364 & 1233.3 & 0.0218 & 17.631 & 699.6 & 0.0216 & 18.342 & 699.5 & 0.0216 \\
\hline 17 & 15.282 & 1203.8 & 0.0217 & 15.501 & 678.9 & 0.0216 & 16.095 & 680.0 & 0.0216 \\
\hline 18 & 10.584 & 1060.7 & 0.0216 & 10.716 & 637.0 & 0.0216 & 11.079 & 638.5 & 0.0216 \\
\hline
\end{tabular}


Table 4-40. Burnup and TH Feedback Parameters by Axial Node for Assembly F12 (Cont.)

\begin{tabular}{|c|c|c|c|c|c|c|c|c|c|}
\hline \multirow[b]{2}{*}{$\begin{array}{l}\text { Axial } \\
\text { Node }\end{array}$} & \multicolumn{3}{|c|}{ Datapoint (227.4 Cy7) } & \multicolumn{3}{|c|}{ Datapoint (367.3 Cy7) } & \multicolumn{3}{|c|}{ Statepoint 67 (BOC CyB) } \\
\hline & $\begin{array}{c}\text { Burnup } \\
\text { (GWd/MTU) }\end{array}$ & $\begin{array}{l}\text { T-Fuel } \\
\left({ }^{\circ} \mathrm{F}\right)\end{array}$ & $\begin{array}{c}\text { Spec. Vol. } \\
\left(\mathrm{ft}^{3} / \mathrm{bm}\right)\end{array}$ & $\begin{array}{c}\text { Burnup } \\
\text { (GWd/MTU) }\end{array}$ & $\begin{array}{l}\text { T-Fuel } \\
\left({ }^{\circ} \mathrm{F}\right)\end{array}$ & $\begin{array}{c}\text { Spec. Vol. } \\
\left(\mathrm{ft}^{3} / \mathrm{bm}\right)\end{array}$ & \begin{tabular}{|c|} 
Burnup \\
(GWd/MTU) \\
\end{tabular} & $\begin{array}{l}\text { T-Fuel } \\
\left({ }^{\circ} \mathrm{F}\right)\end{array}$ & $\begin{array}{c}\text { Spec. Vol. } \\
\left(\mathrm{ft}^{3} / \mathrm{lbm}\right)\end{array}$ \\
\hline & $227.4 \mathrm{Cy} 7$ & $164.6 \mathrm{Cy} 7$ & $164.6 \mathrm{Cy} 7$ & 367.3 Cy7 & 297.3 Cy7 & 297.3 Cy 7 & BOC Cy8 & 430.3 Cy7 & $430.3 \mathrm{Cy} 7$ \\
\hline 1 & 9.950 & 683.9 & 0.0221 & 10.977 & 712.0 & 0.0222 & 11.952 & 741.1 & 0.0222 \\
\hline 2 & 15.610 & 723.4 & 0.0221 & 17.165 & 751.9 & 0.0221 & 18.583 & 777.9 & 0.0222 \\
\hline 3 & 18.469 & 737.2 & 0.0220 & 20.200 & 760.8 & 0.0221 & 21.734 & 781.4 & 0.0222 \\
\hline 4 & 19.643 & 741.0 & 0.0220 & 21.414 & 759.7 & 0.0221 & 22.953 & 776.6 & 0.0221 \\
\hline 5 & 20.042 & 741.5 & 0.0220 & 21.810 & 756.7 & 0.0220 & 23.329 & 771.8 & 0.0221 \\
\hline 6 & 20.206 & 740.1 & 0.0219 & 21.957 & 753.2 & 0.0220 & 23.456 & 767.9 & 0.0220 \\
\hline 7 & 20.337 & 737.7 & 0.0219 & 22.069 & 749.6 & 0.0220 & 23.549 & 764.7 & 0.0220 \\
\hline 8 & 20.496 & 734.7 & 0.0219 & 22.211 & 746.2 & 0.0219 & 23.676 & 761.9 & 0.0220 \\
\hline 9 & 20.654 & 731.9 & 0.0219 & 22.356 & 743.3 & 0.0219 & 23.808 & 759.6 & 0.0219 \\
\hline 10 & 20.740 & 729.8 & 0.0218 & 22.435 & 741.4 & 0.0219 & 23.879 & 758.1 & 0.0219 \\
\hline 11 & 20.770 & 728.4 & 0.0218 & 22.467 & 740.5 & 0.0218 & 23.906 & 757.3 & 0.0219 \\
\hline 12 & 20.799 & 727.3 & 0.0218 & 22.504 & 740.1 & 0.0218 & 23.941 & 757.0 & 0.0218 \\
\hline 13 & 20.837 & 726.1 & 0.0217 & 22.554 & 740.3 & 0.0218 & 23.994 & 757.3 & 0.0218 \\
\hline 14 & 20.760 & 724.5 & 0.0217 & 22.493 & 741.2 & 0.0217 & 23.944 & 758.9 & 0.0218 \\
\hline 15 & 20.459 & 721.6 & 0.0217 & 22.199 & 742.1 & 0.0217 & 23.663 & 761.7 & 0.0217 \\
\hline 16 & 19.560 & 715.2 & 0.0217 & 21.266 & 740.7 & 0.0217 & 22.723 & 764.1 & 0.0217 \\
\hline 17 & 17.147 & 699.1 & 0.0216 & 18.681 & 729.3 & 0.0216 & 20.026 & 757.7 & 0.0216 \\
\hline 18 & 11.743 & 655.1 & 0.0216 & 12.758 & 682.6 & 0.0216 & 13.683 & 711.5 & 0.0216 \\
\hline
\end{tabular}

\begin{tabular}{|c|c|c|c|c|c|c|c|c|c|}
\hline \multirow[b]{2}{*}{$\begin{array}{l}\text { Axial } \\
\text { Node }\end{array}$} & \multicolumn{3}{|c|}{ Datapoint (171.1 Cy8) } & \multicolumn{3}{|c|}{ Datapoint (338.1 Cy8) } & \multicolumn{3}{|c|}{ Statepoint 68 (BOC Cy9) } \\
\hline & $\begin{array}{c}\text { Burnup } \\
\text { (GWd/MTU) }\end{array}$ & $\begin{array}{c}\text { T-Fuel } \\
\left({ }^{\circ} \mathrm{F}\right)\end{array}$ & $\begin{array}{c}\text { Spec. Vol. } \\
\left(\mathrm{ft}^{3} / \mathrm{bm}\right)\end{array}$ & $\begin{array}{c}\text { Burnup } \\
\text { (GWd/MTU) }\end{array}$ & $\begin{array}{c}\text { T-Fuel } \\
\left({ }^{\circ} \mathrm{F}\right)\end{array}$ & $\begin{array}{c}\text { Spec. Vol. } \\
\left(\mathrm{ft}^{3} / / \mathrm{bm}\right)\end{array}$ & $\begin{array}{c}\text { Burnup } \\
\text { (GWd/MTU) }\end{array}$ & $\begin{array}{l}\text { T-Fuel } \\
\left({ }^{\circ} \mathrm{F}\right)\end{array}$ & $\begin{array}{c}\text { Spec. Vol. } \\
\left(\mathrm{ft}^{3} / \mathrm{lbm}\right)\end{array}$ \\
\hline & 171.1 Cy8 & 77.7 Cy8 & 77.7 Cy8 & 338.1 Cy8 & 247.2 Cy8 & 247.2 Cy 8 & BOC Cy9 & $426.1 \mathrm{Cy} 8$ & 426.1 Cy8 \\
\hline 1 & 13.044 & 682.6 & 0.0222 & 14.360 & 706.0 & 0.0222 & 16.010 & 733.8 & 0.0223 \\
\hline 2 & 20.292 & 716.0 & 0.0222 & 22.257 & 736.2 & 0.0222 & 24.599 & 757.1 & 0.0222 \\
\hline 3 & 23.735 & 730.2 & 0.0221 & 25.934 & 742.9 & 0.0221 & 28.454 & 756.3 & 0.0222 \\
\hline 4 & 25.116 & 738.0 & 0.0221 & 27.390 & 742.8 & 0.0221 & 29.918 & 752.3 & 0.0222 \\
\hline 5 & 25.590 & 743.3 & 0.0221 & 27.881 & 741.2 & 0.0221 & 30.377 & 747.5 & 0.0221 \\
\hline 6 & 25.774 & 746.1 & 0.0220 & 28.059 & 739.0 & 0.0220 & 30.518 & 743.2 & 0.0221 \\
\hline 7 & 25.898 & 747.0 & 0.0220 & 28.168 & 736.3 & 0.0220 & 30.597 & 739.6 & 0.0220 \\
\hline 8 & 26.038 & 746.3 & 0.0220 & 28.291 & 733.6 & 0.0220 & 30.697 & 736.6 & 0.0220 \\
\hline 9 & 26.172 & 744.8 & 0.0219 & 28.411 & 731.2 & 0.0219 & 30.803 & 734.3 & 0.0220 \\
\hline 10 & 26.237 & 743.0 & 0.0219 & 28.469 & 729.4 & 0.0219 & 30.855 & 732.7 & 0.0219 \\
\hline 11 & 26.251 & 740.8 & 0.0219 & 28.480 & 728.1 & 0.0219 & 30.869 & 731.7 & 0.0219 \\
\hline 12 & 26.262 & 737.7 & 0.0218 & 28.490 & 727.1 & 0.0218 & 30.889 & 731.3 & 0.0219 \\
\hline 13 & 26.273 & 733.3 & 0.0218 & 28.501 & 726.1 & 0.0218 & 30.917 & 731.5 & 0.0218 \\
\hline 14 & 26.162 & 727.8 & 0.0218 & 28.386 & 725.3 & 0.0218 & 30.831 & 732.7 & 0.0218 \\
\hline 15 & 25.786 & 720.5 & 0.0217 & 27.991 & 723.9 & 0.0217 & 30.467 & 734.6 & 0.0218 \\
\hline 16 & 24.687 & 710.4 & 0.0217 & 26.818 & 721.1 & 0.0217 & 29.290 & 735.6 & 0.0217 \\
\hline 17 & 21.699 & 693.9 & 0.0217 & 23.601 & 711.7 & 0.0217 & 25.902 & 731.8 & 0.0217 \\
\hline 18 & 14.752 & 655.4 & 0.0216 & 16.026 & 675.1 & 0.0217 & 17.650 & 700.0 & 0.0217 \\
\hline
\end{tabular}


Table 4-41. Burnup and TH Feedback Parameters by Axial Node for Assembly F14

\begin{tabular}{|c|c|c|c|c|c|c|c|c|c|}
\hline \multirow[b]{2}{*}{$\begin{array}{l}\text { Axial } \\
\text { Node }\end{array}$} & \multicolumn{3}{|c|}{ Statepoint 63 (69.1 Cy6) } & \multicolumn{3}{|c|}{ Datapoint (176.7 Cy6) } & \multicolumn{3}{|c|}{ Datapoint (320.3 Cy6) } \\
\hline & $\begin{array}{c}\text { Burnup } \\
\text { (GWd/MTU) }\end{array}$ & $\begin{array}{c}\text { T-Fuel } \\
\left({ }^{\circ} \mathrm{F}\right)\end{array}$ & $\begin{array}{l}\text { Spec. Vol. } \\
\left(\mathrm{ft}^{3} / \mathrm{lbm}\right)\end{array}$ & $\begin{array}{c}\text { Burnup } \\
\text { (GWd/MTU) }\end{array}$ & $\begin{array}{c}\text { T-Fuel } \\
\left({ }^{\circ} \mathrm{F}\right)\end{array}$ & $\begin{array}{c}\text { Spec. Vol. } \\
\left(\mathrm{ft}^{3} / \mathrm{lbm}\right)\end{array}$ & $\begin{array}{c}\text { Burnup } \\
\text { (GWd/MTU) } \\
\end{array}$ & $\begin{array}{c}\text { T-Fuel } \\
\left({ }^{\circ} \mathrm{F}\right)\end{array}$ & $\begin{array}{c}\text { Spec. Vol. } \\
\left(\mathrm{ft}^{3} / \mathrm{lbm}\right)\end{array}$ \\
\hline & $69.1 \mathrm{Cy} 6$ & $41.5 \mathrm{Cy} 6$ & 41.5 Cy 6 & 176.7 Cy 6 & 128.8 Cy 6 & 128.8 Cy6 & 320.3 Cy 6 & 252.5 Cy6 & 252.5 Cy 6 \\
\hline 1 & 0.979 & 918.3 & 0.0234 & 2.621 & 953.8 & 0.0233 & 5.171 & 996.2 & 0.0233 \\
\hline 2 & 1.659 & 1101.6 & 0.0233 & 4.380 & 1127.2 & 0.0233 & 8.407 & 1139.0 & 0.0232 \\
\hline 3 & 2.069 & 1211.2 & 0.0233 & 5.366 & 1213.1 & 0.0232 & 10.015 & 1193.2 & 0.0231 \\
\hline 4 & 2.317 & 1277.4 & 0.0231 & 5.903 & 1251.2 & 0.0230 & 10.752 & 1203.1 & 0.0230 \\
\hline 5 & 2.472 & 1318.5 & 0.0230 & 6.199 & 1266.2 & 0.0229 & 11.078 & 1197.4 & 0.0229 \\
\hline 6 & 2.573 & 1344.8 & 0.0229 & 6.371 & 1270.6 & 0.0228 & 11.224 & 1187.6 & 0.0228 \\
\hline 7 & 2.643 & 1362.4 & 0.0228 & 6.480 & 1270.8 & 0.0227 & 11.297 & 1178.3 & 0.0227 \\
\hline 8 & 2.690 & 1373.8 & 0.0226 & 6.551 & 1269.3 & 0.0226 & 11.339 & 1170.8 & 0.0225 \\
\hline 9 & 2.716 & 1379.6 & 0.0225 & 6.589 & 1266.9 & 0.0225 & 11.359 & 1165.1 & 0.0224 \\
\hline 10 & 2.720 & 1379.8 & 0.0224 & 6.596 & 1264.0 & 0.0223 & 11.361 & 1161.6 & 0.0223 \\
\hline 11 & 2.707 & 1375.3 & 0.0223 & 6.581 & 1261.0 & 0.0222 & 11.358 & 1160.4 & 0.0222 \\
\hline 12 & 2.677 & 1366.9 & 0.0222 & 6.548 & 1258.6 & 0.0221 & 11.358 & 1162.1 & 0.0221 \\
\hline 13 & 2.630 & 1354.2 & 0.0220 & 6.490 & 1256.2 & 0.0220 & 11.353 & 1166.4 & 0.0220 \\
\hline 14 & 2.553 & 1334.3 & 0.0219 & 6.381 & 1251.4 & 0.0219 & 11.298 & 1172.1 & 0.0219 \\
\hline 15 & 2.425 & 1300.5 & 0.0218 & 6.157 & 1238.0 & 0.0218 & 11.091 & 1175.8 & 0.0218 \\
\hline 16 & 2.201 & 1239.9 & 0.0217 & 5.693 & 1203.1 & 0.0217 & 10.496 & 1166.6 & 0.0217 \\
\hline 17 & 1.799 & 1128.4 & 0.0216 & 4.745 & 1119.1 & 0.0216 & 9.000 & 1116.2 & 0.0217 \\
\hline 18 & 1.116 & 920.8 & 0.0216 & 2.989 & 932.7 & 0.0216 & 5.836 & 965.1 & 0.0216 \\
\hline
\end{tabular}

\begin{tabular}{|c|c|c|c|c|c|c|c|c|c|}
\hline \multirow[b]{2}{*}{$\begin{array}{l}\text { Axial } \\
\text { Node } \\
\end{array}$} & \multicolumn{3}{|c|}{ Statepoint 64 (BOC Cy7) } & \multicolumn{3}{|c|}{ Statepoint 65 (30.0 Cy7) } & \multicolumn{3}{|c|}{ Statepoint 66 (108.0 Cy7) } \\
\hline & $\begin{array}{c}\text { Burnup } \\
\text { (GWd/MTU) }\end{array}$ & $\begin{array}{c}\text { T-Fuel } \\
\left({ }^{\circ} \mathrm{F}\right)\end{array}$ & $\begin{array}{c}\text { Spec. Vol. } \\
\left(\mathrm{ft}^{3} / \mathrm{lbm}\right)\end{array}$ & $\begin{array}{c}\text { Burnup } \\
\text { (GWd/MTU) }\end{array}$ & $\begin{array}{c}\text { T-Fuel } \\
\left({ }^{\circ} \mathrm{F}\right)\end{array}$ & $\begin{array}{c}\text { Spec. Vol. } \\
\left(\mathrm{tt}^{3} / \mathrm{lbm}\right)\end{array}$ & $\begin{array}{c}\text { Burnup } \\
\text { (GWd/MTU) }\end{array}$ & $\begin{array}{c}\text { T-Fuel } \\
\left({ }^{\circ} \mathrm{F}\right)\end{array}$ & $\begin{array}{c}\text { Spec. Vol. } \\
\left(\mathrm{ft}^{3} / \mathrm{lbm}\right)\end{array}$ \\
\hline & BOC Cy 7 & 376.2 Cy6 & 376.2 Cy 6 & 30.0 Cy7 & $22.5 \mathrm{Cy} 7$ & $22.5 \mathrm{Cy} 7$ & $108.0 \mathrm{Cy} 7$ & $60.4 \mathrm{Cy} 7$ & $60.4 \mathrm{Cy} 7$ \\
\hline 1 & 7.211 & 1023.7 & 0.0234 & 7.764 & 980.3 & 0.0237 & 9.294 & 992.3 & 0.0237 \\
\hline 2 & 11.467 & 1133.5 & 0.0233 & 12.355 & 1111.6 & 0.0236 & 14.791 & 1125.0 & 0.0236 \\
\hline 3 & 13.414 & 1167.2 & 0.0231 & 14.487 & 1189.9 & 0.0234 & 17.404 & 1198.8 & 0.0235 \\
\hline 4 & 14.204 & 1164.0 & 0.0230 & 15.391 & 1237.6 & 0.0233 & 18.587 & 1239.9 & 0.0233 \\
\hline 5 & 14.496 & 1151.9 & 0.0229 & 15.759 & 1268.2 & 0.0232 & 19.120 & 1263.9 & 0.0232 \\
\hline 6 & 14.595 & 1140.1 & 0.0228 & 15.907 & 1286.7 & 0.0230 & 19.366 & 1276.2 & 0.0231 \\
\hline 7 & 14.632 & 1130.9 & 0.0227 & 15.974 & 1296.1 & 0.0229 & 19.491 & 1281.6 & 0.0229 \\
\hline 8 & 14.652 & 1124.3 & 0.0226 & 16.011 & 1299.8 & 0.0228 & 19.556 & 1282.6 & 0.0228 \\
\hline 9 & 14.661 & 1119.7 & 0.0225 & 16.027 & 1299.7 & 0.0226 & 19.584 & 1280.9 & 0.0226 \\
\hline 10 & 14.663 & 1116.8 & 0.0224 & 16.029 & 1296.7 & 0.0225 & 19.586 & 1277.2 & 0.0225 \\
\hline 11 & 14.672 & 1115.9 & 0.0223 & 16.029 & 1290.5 & 0.0224 & 19.574 & 1271.4 & 0.0224 \\
\hline 12 & 14.700 & 1117.3 & 0.0222 & 16.039 & 1279.8 & 0.0222 & 19.553 & 1262.3 & 0.0222 \\
\hline 13 & 14.741 & 1121.6 & 0.0221 & 16.048 & 1262.8 & 0.0221 & 19.502 & 1248.2 & 0.0221 \\
\hline 14 & 14.750 & 1128.9 & 0.0220 & 16.006 & 1235.7 & 0.0220 & 19.356 & 1226.5 & 0.0220 \\
\hline 15 & 14.607 & 1137.9 & 0.0219 & 15.785 & 1196.6 & 0.0219 & 18.966 & 1193.3 & 0.0219 \\
\hline 16 & 14.006 & 1140.6 & 0.0218 & 15.069 & 1142.0 & 0.0218 & 17.976 & 1144.9 & 0.0218 \\
\hline 17 & 12.234 & 1109.9 & 0.0217 & 13.116 & 1059.7 & 0.0217 & 15.552 & 1067.3 & 0.0217 \\
\hline 18 & 8.121 & 988.9 & 0.0216 & 8.672 & 902.7 & 0.0216 & 10.205 & 909.4 & 0.0216 \\
\hline
\end{tabular}


Table 4-42. Burnup and TH Feedback Parameters by Axial Node for Assembly F14a

\begin{tabular}{|c|c|c|c|c|c|c|c|c|c|}
\hline \multirow{2}{*}{$\begin{array}{c}\text { Axial } \\
\text { Node }\end{array}$} & \multicolumn{2}{|c|}{ Statepoint 63 (69.1 Cy6) } & \multicolumn{2}{c|}{ Datapoint (176.7 Cy6) } & \multicolumn{3}{c|}{ Datapoint (320.3 Cy6) } \\
\cline { 2 - 10 } & $\begin{array}{c}\text { Burnup } \\
\text { (GW/MTU) }\end{array}$ & $\begin{array}{c}\text { T-Fuel } \\
\left({ }^{\circ} \mathrm{F}\right)\end{array}$ & $\begin{array}{c}\text { Spec. Vol. } \\
\left(\mathrm{ft}^{3} / \mathrm{lbm}\right)\end{array}$ & $\begin{array}{c}\text { Burnup } \\
(\mathrm{GWd} / \mathrm{MTU})\end{array}$ & $\begin{array}{c}\text { T-Fuel } \\
\left({ }^{\circ} \mathrm{F}\right)\end{array}$ & $\begin{array}{c}\text { Spec. Vol. } \\
\left(\mathrm{ft}^{3} / \mathrm{lbm}\right)\end{array}$ & $\begin{array}{c}\text { Burnup } \\
(\mathrm{GWd} / \mathrm{MTU})\end{array}$ & $\begin{array}{c}\text { T-Fuel } \\
\left({ }^{\circ} \mathrm{F}\right)\end{array}$ & $\begin{array}{c}\text { Spec. Vol. } \\
\left(\mathrm{ft}^{3} / \mathrm{lbm}\right)\end{array}$ \\
\hline 1 & $69.1 \mathrm{Cy6}$ & $41.5 \mathrm{Cy} 6$ & $41.5 \mathrm{Cy} 6$ & $176.7 \mathrm{Cy} 6$ & $128.8 \mathrm{Cy} 6$ & $128.8 \mathrm{Cy} 6$ & $320.3 \mathrm{Cy} 6$ & $252.5 \mathrm{Cy6}$ & $252.5 \mathrm{Cy} 6$ \\
\hline 2 & 0.979 & 918.3 & 0.0234 & 2.621 & 953.8 & 0.0233 & 5.171 & 996.2 & 0.0233 \\
\hline 3 & 1.659 & 1101.6 & 0.0233 & 4.380 & 1127.2 & 0.0233 & 8.407 & 1139.0 & 0.0232 \\
\hline 4 & 2.069 & 1211.2 & 0.0233 & 5.366 & 1213.1 & 0.0232 & 10.015 & 1193.2 & 0.0231 \\
\hline 5 & 2.317 & 1277.4 & 0.0231 & 5.903 & 1251.2 & 0.0230 & 10.752 & 1203.1 & 0.0230 \\
\hline 6 & 2.473 & 1318.5 & 0.0230 & 6.199 & 1266.2 & 0.0229 & 11.078 & 1197.4 & 0.0229 \\
\hline 7 & 2.643 & 1362.4 & 0.0228 & 6.480 & 1270.8 & 0.0227 & 11.297 & 1178.3 & 0.0227 \\
\hline 8 & 2.690 & 1373.8 & 0.0226 & 6.551 & 1269.3 & 0.0226 & 11.339 & 1170.8 & 0.0225 \\
\hline 9 & 2.716 & 1379.6 & 0.0225 & 6.589 & 1266.9 & 0.0225 & 11.359 & 1165.1 & 0.0224 \\
\hline 10 & 2.720 & 1379.8 & 0.0224 & 6.596 & 1264.0 & 0.0223 & 11.361 & 1161.6 & 0.0223 \\
\hline 11 & 2.707 & 1375.3 & 0.0223 & 6.581 & 1261.0 & 0.0222 & 11.358 & 1160.4 & 0.0222 \\
\hline 12 & 2.677 & 1366.9 & 0.0222 & 6.548 & 1258.6 & 0.0221 & 11.358 & 1162.1 & 0.0221 \\
\hline 13 & 2.630 & 1354.2 & 0.0220 & 6.490 & 1256.2 & 0.0220 & 11.353 & 1166.4 & 0.0220 \\
\hline 14 & 2.553 & 1334.3 & 0.0219 & 6.381 & 1251.4 & 0.0219 & 11.298 & 1172.1 & 0.0219 \\
\hline 15 & 2.425 & 1300.5 & 0.0218 & 6.157 & 1238.0 & 0.0218 & 11.091 & 1175.8 & 0.0218 \\
\hline 16 & 2.201 & 1239.9 & 0.0217 & 5.693 & 1203.1 & 0.0217 & 10.496 & 1166.6 & 0.0217 \\
\hline 17 & 1.799 & 1128.4 & 0.0216 & 4.745 & 1119.1 & 0.0216 & 9.000 & 1116.2 & 0.0217 \\
\hline 18 & 1.116 & 920.8 & 0.0216 & 2.989 & 932.7 & 0.0216 & 5.836 & 965.1 & 0.0216 \\
\hline
\end{tabular}

\begin{tabular}{|c|c|c|c|c|c|c|c|c|c|}
\hline \multirow[b]{2}{*}{$\begin{array}{l}\text { Axial } \\
\text { Node }\end{array}$} & \multicolumn{3}{|c|}{ Statepoint 64 (BOC Cy7) } & \multicolumn{3}{|c|}{ Statepoint 65 (30.0 Cy7) } & \multicolumn{3}{|c|}{ Statepoint $66(108.0 \mathrm{Cy})$} \\
\hline & $\begin{array}{c}\text { Burnup } \\
\text { (GWd/MTU) }\end{array}$ & $\begin{array}{l}\text { T-Fuel } \\
\left({ }^{\circ} \mathrm{F}\right)\end{array}$ & $\begin{array}{c}\text { Spec. Vol. } \\
\left(\mathrm{ft}^{3} / \mathrm{lbm}\right)\end{array}$ & $\begin{array}{c}\text { Burnup } \\
\text { (GWd/MTU) }\end{array}$ & $\begin{array}{l}\text { T-Fuel } \\
\left({ }^{\circ} \mathrm{F}\right)\end{array}$ & $\begin{array}{c}\text { Spec. Vol. } \\
\left(\mathrm{ft}^{3} / \mathrm{lbm}\right)\end{array}$ & $\begin{array}{c}\text { Burnup } \\
\text { (GWd/MTU) }\end{array}$ & $\begin{array}{c}\text { T-Fuel } \\
\left({ }^{\circ} \mathrm{F}\right)\end{array}$ & $\begin{array}{c}\text { Spec. Vol. } \\
\left(\mathrm{ft}^{3} / \mathrm{lbm}\right)\end{array}$ \\
\hline & BOC Cy 7 & 376.2 Cy 6 & 376.2 Cy 6 & 30.0 Cy 7 & 22.5 Cy 7 & $22.5 \mathrm{Cy} 7$ & $108.0 \mathrm{Cy} 7$ & $60.4 \mathrm{Cy} 7$ & $60.4 \mathrm{Cy} 7$ \\
\hline 1 & 7.190 & 1023.7 & 0.0234 & 7.708 & 955.5 & 0.0237 & 9.121 & 963.4 & 0.0236 \\
\hline 2 & 11.432 & 1133.5 & 0.0233 & 12.294 & 1093.7 & 0.0236 & 14.607 & 1100.1 & 0.0235 \\
\hline 3 & 13.372 & 1167.2 & 0.0231 & 14.437 & 1179.7 & 0.0235 & 17.245 & 1177.1 & 0.0234 \\
\hline 4 & 14.159 & 1164.0 & 0.0230 & 15.347 & 1229.1 & 0.0233 & 18.423 & 1216.2 & 0.0233 \\
\hline 5 & 14.450 & 1151.9 & 0.0229 & 15.714 & 1259.6 & 0.0232 & 18.941 & 1237.5 & 0.0232 \\
\hline 6 & 14.550 & 1140.1 & 0.0228 & 15.863 & 1278.9 & 0.0231 & 19.180 & 1249.4 & 0.0230 \\
\hline 7 & 14.588 & 1130.9 & 0.0227 & 15.933 & 1289.2 & 0.0229 & 19.303 & 1254.7 & 0.0229 \\
\hline 8 & 14.609 & 1124.3 & 0.0226 & 15.974 & 1293.9 & 0.0228 & 19.373 & 1256.0 & 0.0228 \\
\hline 9 & 14.621 & 1119.7 & 0.0225 & 15.997 & 1295.4 & 0.0226 & 19.412 & 1255.0 & 0.0226 \\
\hline 10 & 14.624 & 1116.8 & 0.0224 & 16.006 & 1295.3 & 0.0225 & 19.433 & 1253.5 & 0.0225 \\
\hline 11 & 14.632 & 1115.9 & 0.0223 & 16.018 & 1295.0 & 0.0224 & 19.459 & 1252.4 & 0.0224 \\
\hline 12 & 14.659 & 1117.3 & 0.0222 & 16.044 & 1292.7 & 0.0222 & 19.497 & 1250.4 & 0.0222 \\
\hline 13 & 14.698 & 1121.6 & 0.0221 & 16.067 & 1282.4 & 0.0221 & 19.496 & 1242.3 & 0.0221 \\
\hline 14 & 14.704 & 1128.9 & 0.0220 & 16.030 & 1260.5 & 0.0220 & 19.380 & 1225.0 & 0.0220 \\
\hline 15 & 14.559 & 1137.9 & 0.0219 & 15.808 & 1223.0 & 0.0219 & 19.002 & 1194.8 & 0.0219 \\
\hline 16 & 13.959 & 1140.6 & 0.0218 & 15.084 & 1165.7 & 0.0218 & 18.008 & 1147.4 & 0.0218 \\
\hline 17 & 12.191 & 1109.9 & 0.0217 & 13.113 & 1075.1 & 0.0217 & 15.549 & 1066.9 & 0.0217 \\
\hline 18 & 8.091 & 988.9 & 0.0216 & 8.653 & 906.1 & 0.0216 & 10.161 & 903.5 & 0.0216 \\
\hline
\end{tabular}


Table 4-43. Burnup and TH Feedback Parameters by Axial Node for Assembly F17

\begin{tabular}{|c|c|c|c|c|c|c|c|c|c|}
\hline \multirow[b]{2}{*}{$\begin{array}{l}\text { Axial } \\
\text { Node }\end{array}$} & \multicolumn{3}{|c|}{ Statepoint 63 (69.1 Cy6) } & \multicolumn{3}{|c|}{ Datapoint (176.7 Cy6) } & \multicolumn{3}{|c|}{ Datapoint (320.3 Cy6) } \\
\hline & $\begin{array}{c}\text { Burnup } \\
\text { (GWd/MTU) }\end{array}$ & $\begin{array}{c}\text { T-Fuel } \\
\left({ }^{\circ} \mathrm{F}\right)\end{array}$ & $\begin{array}{c}\text { Spec. Vol. } \\
\left(\mathrm{ft}^{3} / \mathrm{lbm}\right)\end{array}$ & $\begin{array}{c}\text { Burnup } \\
\text { (GWd/MTU) }\end{array}$ & $\begin{array}{l}\text { T-Fuel } \\
\left({ }^{\circ} \mathrm{F}\right)\end{array}$ & $\begin{array}{c}\text { Spec. Vol. } \\
\left(\mathrm{ft}^{3} / \mathrm{lbm}\right)\end{array}$ & $\begin{array}{c}\text { Burnup } \\
\text { (GWd/MTU) }\end{array}$ & $\begin{array}{c}\text { T-Fuel } \\
\left({ }^{\circ} \mathrm{F}\right) \\
\end{array}$ & $\begin{array}{l}\text { Spec. Vol } \\
\left(\mathrm{ft}^{3} / \mathrm{lbm}\right)\end{array}$ \\
\hline & 69.1 Cy6 & $41.5 \mathrm{Cy} 6$ & 41.5 Cy 6 & 176.7 Cy 6 & $128.8 \mathrm{Cy} 6$ & 128.8 Cy 6 & 320.3 Cy 6 & 252.5 Cy 6 & 252.5 Cy 6 \\
\hline 1 & 1.261 & 1015.0 & 0.0238 & 3.418 & 1071.4 & 0.0239 & 6.744 & 1106.3 & 0.0239 \\
\hline 2 & 1.926 & 1182.5 & 0.0237 & 5.250 & 1239.8 & 0.0238 & 10.291 & 1258.9 & 0.0238 \\
\hline 3 & 2.332 & 1293.1 & 0.0236 & 6.310 & 1337.7 & 0.0236 & 12.123 & 1325.2 & 0.0236 \\
\hline 4 & 2.578 & 1362.5 & 0.0234 & 6.898 & 1384.1 & 0.0235 & 12.970 & 1338.6 & 0.0235 \\
\hline 5 & 2.727 & 1403.9 & 0.0233 & 7.210 & 1402.3 & 0.0233 & 13.315 & 1332.7 & 0.0233 \\
\hline 6 & 2.835 & 1432.3 & 0.0231 & 7.414 & 1409.7 & 0.0232 & 13.498 & 1322.5 & 0.0232 \\
\hline 7 & 2.931 & 1456.8 & 0.0230 & 7.592 & 1415.1 & 0.0230 & 13.661 & 1313.8 & 0.0230 \\
\hline 8 & 3.023 & 1479.0 & 0.0228 & 7.769 & 1420.0 & 0.0229 & 13.845 & 1307.8 & 0.0229 \\
\hline 9 & 3.097 & 1493.3 & 0.0227 & 7.917 & 1423.6 & 0.0227 & 14.018 & 1304.3 & 0.0227 \\
\hline 10 & 3.127 & 1498.1 & 0.0225 & 7.987 & 1423.7 & 0.0226 & 14.110 & 1302.0 & 0.0226 \\
\hline 11 & 3.118 & 1493.5 & 0.0224 & 7.982 & 1420.9 & 0.0224 & 14.135 & 1302.0 & 0.0225 \\
\hline 12 & 3.080 & 1482.2 & 0.0223 & 7.932 & 1417.0 & 0.0223 & 14.143 & 1306.1 & 0.0223 \\
\hline 13 & 3.016 & 1466.5 & 0.0221 & 7.846 & 1412.8 & 0.0222 & 14.145 & 1313.9 & 0.0222 \\
\hline 14 & 2.911 & 1442.2 & 0.0220 & 7.678 & 1405.4 & 0.0220 & 14.040 & 1319.5 & 0.0221 \\
\hline 15 & 2.755 & 1401.2 & 0.0219 & 7.381 & 1387.9 & 0.0219 & 13.749 & 1321.9 & 0.0219 \\
\hline 16 & 2.511 & 1334.2 & 0.0218 & 6.833 & 1342.9 & 0.0218 & 13.022 & 1309.9 & 0.0218 \\
\hline 17 & 2.107 & 1220.3 & 0.0217 & 5.797 & 1245.3 & 0.0217 & 11.298 & 1248.0 & 0.0217 \\
\hline 18 & 1.445 & 1029.1 & 0.0216 & 3.958 & 1053.1 & 0.0216 & 7.770 & 1074.7 & 0.0216 \\
\hline
\end{tabular}

\begin{tabular}{|c|c|c|c|c|c|c|c|c|c|}
\hline \multirow[b]{2}{*}{$\begin{array}{l}\text { Axial } \\
\text { Node }\end{array}$} & \multicolumn{3}{|c|}{ Statepoint 64 (BOC Cy7) } & \multicolumn{3}{|c|}{ Statepoint 65 (30.0 Cyn) } & \multicolumn{3}{|c|}{ Statepoint 66 (108.0 Cy7) } \\
\hline & \begin{tabular}{|c|} 
Burnup \\
(GWd/MTU)
\end{tabular} & $\begin{array}{l}\text { T-Fuel } \\
\left({ }^{\circ} \mathrm{F}\right)\end{array}$ & $\begin{array}{l}\text { Spec. Vol. } \\
\left(\mathrm{tt}^{3} / \mathrm{lbm}\right)\end{array}$ & $\begin{array}{c}\text { Burnup } \\
\text { (GWd/MTU) }\end{array}$ & $\begin{array}{l}\text { T-Fuel } \\
\left({ }^{\circ} \mathrm{F}\right)\end{array}$ & $\begin{array}{c}\text { Spec. Vol. } \\
\left(\mathrm{ft}^{3} / \mathrm{lbm}\right)\end{array}$ & $\begin{array}{c}\text { Burnup } \\
\text { (GWd/MTU) }\end{array}$ & $\begin{array}{c}\text { T-Fuel } \\
\left({ }^{\circ} \mathrm{F}\right)\end{array}$ & $\begin{array}{c}\text { Spec. Vol. } \\
\left(\mathrm{ft}^{3} / \mathrm{lbm}\right)\end{array}$ \\
\hline & BOC Cy7 & 376.2 Cy6 & 376.2 Cy 6 & 30.0 Cy7 & $22.5 \mathrm{Cy} 7$ & 22.5 Cy 7 & $108.0 \mathrm{Cy} 7$ & $60.4 \mathrm{Cy} 7$ & 60.4 Cy 7 \\
\hline 1 & 9.304 & 1108.2 & 0.0238 & 9.487 & 695.1 & 0.0223 & 9.986 & 699.7 & 0.0223 \\
\hline 2 & 14.038 & 1232.9 & 0.0237 & 14.360 & 755.4 & 0.0223 & 15.226 & 760.4 & 0.0223 \\
\hline 3 & 16.280 & 1270.0 & 0.0236 & 16.694 & 795.0 & 0.0223 & 17.783 & 796.1 & 0.0222 \\
\hline 4 & 17.184 & 1263.1 & 0.0234 & 17.656 & 818.8 & 0.0222 & 18.871 & 815.2 & 0.0222 \\
\hline 5 & 17.470 & 1245.7 & 0.0232 & 17.978 & 833.1 & 0.0222 & 19.261 & 825.4 & 0.0222 \\
\hline 6 & 17.590 & 1230.4 & 0.0231 & 18.119 & 841.1 & 0.0221 & 19.439 & 830.2 & 0.0221 \\
\hline 7 & 17.711 & 1218.9 & 0.0230 & 18.252 & 844.6 & 0.0221 & 19.589 & 831.5 & 0.0221 \\
\hline 8 & 17.877 & 1210.8 & 0.0228 & 18.425 & 845.1 & 0.0220 & 19.768 & 830.4 & 0.0220 \\
\hline 9 & 18.048 & 1205.2 & 0.0227 & 18.598 & 844.0 & 0.0220 & 19.941 & 828.2 & 0.0220 \\
\hline 10 & 18.146 & 1201.7 & 0.0226 & 18.695 & 842.2 & 0.0219 & 20.036 & 826.0 & 0.0219 \\
\hline 11 & 18.189 & 1200.6 & 0.0224 & 18.736 & 839.9 & 0.0219 & 20.074 & 823.6 & 0.0219 \\
\hline 12 & 18.241 & 1203.2 & 0.0223 & 18.782 & 835.7 & 0.0218 & 20.110 & 820.1 & 0.0218 \\
\hline 13 & 18.321 & 1210.8 & 0.0222 & 18.850 & 828.6 & 0.0218 & 20.157 & 814.3 & 0.0218 \\
\hline 14 & 18.301 & 1220.7 & 0.0221 & 18.810 & 818.3 & 0.0218 & 20.081 & 806.3 & 0.0217 \\
\hline 15 & 18.090 & 1232.3 & 0.0219 & 18.568 & 803.5 & 0.0217 & 19.777 & 794.3 & 0.0217 \\
\hline 16 & 17.363 & 1238.4 & 0.0218 & 17.792 & 781.5 & 0.0217 & 18.894 & 775.6 & 0.0217 \\
\hline 17 & 15.318 & 1208.4 & 0.0217 & 15.665 & 746.0 & 0.0216 & 16.573 & 743.5 & 0.0216 \\
\hline 18 & 10.665 & 1064.9 & 0.0216 & 10.871 & 679.0 & 0.0216 & 11.419 & 679.0 & 0.0216 \\
\hline
\end{tabular}


Table 4-44. Burnup and TH Feedback Parameters by Axial Node for Assembly F17a

\begin{tabular}{|c|c|c|c|c|c|c|c|c|c|}
\hline \multirow{2}{*}{$\begin{array}{c}\text { Axial } \\
\text { Node }\end{array}$} & \multicolumn{2}{|c|}{ Statepoint 63 (69.1 Cy6) } & \multicolumn{2}{c|}{ Datapoint (176.7 Cy6) } & \multicolumn{3}{c|}{ Datapoint (320.3 Cy6) } \\
\cline { 2 - 10 } & $\begin{array}{c}\text { Burnup } \\
(\mathrm{GWd} / \mathrm{MTU})\end{array}$ & $\begin{array}{c}\text { T-Fuel } \\
\left({ }^{\circ} \mathrm{F}\right)\end{array}$ & $\begin{array}{c}\text { Spec. Vol. } \\
\left(\mathrm{ft}^{3} / \mathrm{lbm}\right)\end{array}$ & $\begin{array}{c}\text { Burnup } \\
(\mathrm{GWd} / \mathrm{MTU})\end{array}$ & $\begin{array}{c}\text { T-Fuel } \\
\left({ }^{\circ} \mathrm{F}\right)\end{array}$ & $\begin{array}{c}\text { Spec. Vol. } \\
\left(\mathrm{ft}^{3} / \mathrm{lbm}\right)\end{array}$ & $\begin{array}{c}\text { Burnup } \\
(\mathrm{GWd} / \mathrm{MTU})\end{array}$ & $\begin{array}{c}\text { T-Fuel } \\
\left({ }^{\circ} \mathrm{F}\right)\end{array}$ & $\begin{array}{c}\text { Spec. Vol. } \\
\left(\mathrm{ft}^{3} / \mathrm{lbm}\right)\end{array}$ \\
\hline 1 & $69.1 \mathrm{Cy6}$ & $41.5 \mathrm{Cy} 6$ & $41.5 \mathrm{Cy} 6$ & $176.7 \mathrm{Cy} 6$ & $128.8 \mathrm{Cy} 6$ & $128.8 \mathrm{Cy} 6$ & 320.3 Cy6 & $252.5 \mathrm{Cy} 6$ & $252.5 \mathrm{Cy} 6$ \\
\hline 2 & 1.261 & 1015.0 & 0.0238 & 3.418 & 1071.4 & 0.0239 & 6.744 & 1106.3 & 0.0239 \\
\hline 3 & 1.926 & 1182.5 & 0.0237 & 5.250 & 1239.8 & 0.0238 & 10.291 & 1258.9 & 0.0238 \\
\hline 4 & 2.332 & 1293.1 & 0.0236 & 6.310 & 1337.7 & 0.0236 & 12.123 & 1325.2 & 0.0236 \\
\hline 5 & 2.727 & 1403.9 & 0.0233 & 7.210 & 1402.3 & 0.0233 & 13.315 & 1332.7 & 0.0233 \\
\hline 6 & 2.835 & 1432.3 & 0.0231 & 7.414 & 1409.7 & 0.0232 & 13.498 & 1322.5 & 0.0232 \\
\hline 7 & 2.931 & 1456.8 & 0.0230 & 7.592 & 1415.1 & 0.0230 & 13.661 & 1313.8 & 0.0230 \\
\hline 8 & 3.023 & 1479.0 & 0.0228 & 7.769 & 1420.0 & 0.0229 & 13.845 & 1307.8 & 0.0229 \\
\hline 9 & 3.097 & 1493.3 & 0.0227 & 7.917 & 1423.6 & 0.0227 & 14.018 & 1304.3 & 0.0227 \\
\hline 10 & 3.127 & 1498.1 & 0.0225 & 7.987 & 1423.7 & 0.0226 & 14.110 & 1302.0 & 0.0226 \\
\hline 11 & 3.118 & 1493.5 & 0.0224 & 7.982 & 1420.9 & 0.0224 & 14.135 & 1302.0 & 0.0225 \\
\hline 12 & 3.080 & 1482.2 & 0.0223 & 7.932 & 1417.0 & 0.0223 & 14.143 & 1306.1 & 0.0223 \\
\hline 13 & 3.016 & 1466.5 & 0.0221 & 7.846 & 1412.8 & 0.0222 & 14.145 & 1313.9 & 0.0222 \\
\hline 14 & 2.911 & 1442.2 & 0.0220 & 7.678 & 1405.4 & 0.0220 & 14.040 & 1319.5 & 0.0221 \\
\hline 15 & 2.755 & 1401.2 & 0.0219 & 7.381 & 1387.9 & 0.0219 & 13.749 & 1321.9 & 0.0219 \\
\hline 16 & 2.511 & 1334.2 & 0.0218 & 6.833 & 1342.9 & 0.0218 & 13.022 & 1309.9 & 0.0218 \\
\hline 17 & 2.107 & 1220.3 & 0.0217 & 5.797 & 1245.3 & 0.0217 & 11.298 & 1248.0 & 0.0217 \\
\hline 18 & 1.445 & 1029.1 & 0.0216 & 3.958 & 1053.1 & 0.0216 & 7.770 & 1074.7 & 0.0216 \\
\hline
\end{tabular}

\begin{tabular}{|c|c|c|c|c|c|c|c|c|c|}
\hline \multirow[b]{2}{*}{$\begin{array}{l}\text { Axial } \\
\text { Node } \\
\end{array}$} & \multicolumn{3}{|c|}{ Statepoint 64 (BOC Cy7) } & \multicolumn{3}{|c|}{ Statepoint $65(30.0 \mathrm{Cy} 7)$} & \multicolumn{3}{|c|}{ Statepoint $66(108.0 \mathrm{Cy} 7)$} \\
\hline & $\begin{array}{c}\text { Burnup } \\
\text { (GWd/MTU) }\end{array}$ & $\begin{array}{c}\text { T-Fuel } \\
\left({ }^{\circ} \mathrm{F}\right) \\
\end{array}$ & $\begin{array}{c}\text { Spec. Vol. } \\
\left(\mathrm{ft}^{3} / / \mathrm{bm}\right)\end{array}$ & $\begin{array}{c}\text { Burnup } \\
\text { (GWd/MTU) }\end{array}$ & $\begin{array}{c}\text { T-Fuel } \\
\left({ }^{\circ} \mathrm{F}\right)\end{array}$ & $\begin{array}{c}\text { Spec. Vol. } \\
\left(\mathrm{tt}^{3} / \mathrm{lbm}\right)\end{array}$ & $\begin{array}{c}\text { Burnup } \\
\text { (GWd/MTU) }\end{array}$ & $\begin{array}{c}\text { T-Fuel } \\
\left({ }^{\circ} \mathrm{F}\right)\end{array}$ & $\begin{array}{c}\text { Spec. Vol } \\
\left(\mathrm{ft}^{3} / \mathrm{lbm}\right)\end{array}$ \\
\hline & BOC Cy7 & 376.2 Cy 6 & 376.2 Cy 6 & $30.0 \mathrm{Cy} 7$ & 22.5 Cy 7 & 22.5 Cy7 & $108.0 \mathrm{Cy} 7$ & 60.4 Cy7 & 60.4 Cy 7 \\
\hline 1 & 9.312 & 1108.2 & 0.0238 & 9.861 & 955.7 & 0.0236 & 11.373 & 967.1 & 0.0237 \\
\hline 2 & 14.053 & 1232.9 & 0.0237 & 14.939 & 1088.4 & 0.0236 & 17.353 & 1098.0 & 0.0236 \\
\hline 3 & 16.300 & 1270.0 & 0.0236 & 17.370 & 1158.8 & 0.0234 & 20.255 & 1162.9 & 0.0234 \\
\hline 4 & 17.206 & 1263.1 & 0.0234 & 18.390 & 1201.1 & 0.0233 & 21.544 & 1198.3 & 0.0233 \\
\hline 5 & 17.494 & 1245.7 & 0.0232 & 18.752 & 1229.5 & 0.0232 & 22.066 & 1220.1 & 0.0232 \\
\hline 6 & 17.614 & 1230.4 & 0.0231 & 18.920 & 1246.8 & 0.0230 & 22.328 & 1232.4 & 0.0230 \\
\hline 7 & 17.735 & 1218.9 & 0.0230 & 19.070 & 1254.3 & 0.0229 & 22.527 & 1236.5 & 0.0229 \\
\hline 8 & 17.901 & 1210.8 & 0.0228 & 19.251 & 1255.2 & 0.0228 & 22.729 & 1235.5 & 0.0228 \\
\hline 9 & 18.072 & 1205.2 & 0.0227 & 19.427 & 1252.3 & 0.0226 & 22.910 & 1231.6 & 0.0226 \\
\hline 10 & 18.170 & 1201.7 & 0.0226 & 19.524 & 1248.3 & 0.0225 & 23.006 & 1227.2 & 0.0225 \\
\hline 11 & 18.215 & 1200.6 & 0.0224 & 19.564 & 1243.2 & 0.0224 & 23.040 & 1222.3 & 0.0224 \\
\hline 12 & 18.269 & 1203.2 & 0.0223 & 19.605 & 1234.6 & 0.0222 & 23.061 & 1214.9 & 0.0222 \\
\hline 13 & 18.351 & 1210.8 & 0.0222 & 19.658 & 1218.8 & 0.0221 & 23.063 & 1201.9 & 0.0221 \\
\hline 14 & 18.332 & 1220.7 & 0.0221 & 19.591 & 1194.3 & 0.0220 & 22.901 & 1181.5 & 0.0220 \\
\hline 15 & 18.121 & 1232.3 & 0.0219 & 19.304 & 1159.0 & 0.0219 & 22.452 & 1150.7 & 0.0219 \\
\hline 16 & 17.391 & 1238.4 & 0.0218 & 18.459 & 1109.4 & 0.0218 & 21.338 & 1105.9 & 0.0218 \\
\hline 17 & 15.339 & 1208.4 & 0.0217 & 16.223 & 1034.2 & 0.0217 & 18.635 & 1036.7 & 0.0217 \\
\hline 18 & 10.677 & 1064.9 & 0.0216 & 11.226 & 881.1 & 0.0216 & 12.737 & 887.6 & 0.0216 \\
\hline
\end{tabular}


Table 4-45. Burnup and TH Feedback Parameters by Axial Node for Assembly F19

\begin{tabular}{|c|c|c|c|c|c|c|c|c|c|}
\hline \multirow[b]{2}{*}{$\begin{array}{l}\text { Axial } \\
\text { Node }\end{array}$} & \multicolumn{3}{|c|}{ Statepoint 63 (69.1 Cy6) } & \multicolumn{3}{|c|}{ Datapoint (176.7 Cy6) } & \multicolumn{3}{|c|}{ Datapoint (320.3 Cy6) } \\
\hline & \begin{tabular}{|c|} 
Burnup \\
(GWd/MTU)
\end{tabular} & $\begin{array}{c}\text { T-Fuel } \\
\left({ }^{\circ} \mathrm{F}\right)\end{array}$ & $\begin{array}{c}\text { Spec. Vol. } \\
\left(\mathrm{ft}^{3} / \mathrm{lbm}\right)\end{array}$ & $\begin{array}{c}\text { Burnup } \\
\text { (GWd/MTU) }\end{array}$ & $\begin{array}{c}\text { T-Fuel } \\
\left({ }^{\circ} \mathrm{F}\right)\end{array}$ & $\begin{array}{l}\text { Spec. Vol. } \\
\left(\mathrm{ft}^{3} / \mathrm{lbm}\right)\end{array}$ & $\begin{array}{c}\text { Burnup } \\
\text { (GWd/MTU) }\end{array}$ & $\begin{array}{l}\text { T-Fuel } \\
\left({ }^{\circ} \mathrm{F}\right)\end{array}$ & $\begin{array}{l}\text { Spec. Vol } \\
\left(\mathrm{ft}^{3} / \mathrm{lbm}\right)\end{array}$ \\
\hline & 69.1 Cy6 & 41.5 Cy6 & 41.5 Cy 6 & 176.7 Cy6 & 128.8 Cy 6 & 128.8 Cy6 & 320.3 Cy 6 & 252.5 Cy 6 & 252.5 Cy 6 \\
\hline 1 & 1.190 & 990.6 & 0.0238 & 3.177 & 1033.4 & 0.0238 & 6.220 & 1069.6 & 0.0237 \\
\hline 2 & 1.923 & 1178.0 & 0.0238 & 5.118 & 1213.7 & 0.0237 & 9.858 & 1221.7 & 0.0236 \\
\hline 3 & 2.402 & 1305.5 & 0.0236 & 6.300 & 1317.0 & 0.0236 & 11.809 & 1285.9 & 0.0235 \\
\hline 4 & 2.700 & 1386.4 & 0.0235 & 6.963 & 1365.1 & 0.0234 & 12.726 & 1297.7 & 0.0233 \\
\hline 5 & 2.878 & 1434.5 & 0.0234 & 7.310 & 1382.8 & 0.0233 & 13.099 & 1290.8 & 0.0232 \\
\hline 6 & 2.998 & 1465.1 & 0.0232 & 7.516 & 1388.0 & 0.0231 & 13.275 & 1280.2 & 0.0231 \\
\hline 7 & 3.087 & 1485.7 & 0.0230 & 7.663 & 1389.5 & 0.0230 & 13.389 & 1270.6 & 0.0229 \\
\hline 8 & 3.153 & 1498.7 & 0.0229 & 7.772 & 1389.7 & 0.0228 & 13.475 & 1262.9 & 0.0228 \\
\hline 9 & 3.195 & 1506.4 & 0.0227 & 7.845 & 1389.0 & 0.0227 & 13.539 & 1257.4 & 0.0226 \\
\hline 10 & 3.213 & 1508.6 & 0.0226 & 7.882 & 1387.4 & 0.0225 & 13.583 & 1254.2 & 0.0225 \\
\hline 11 & 3.211 & 1506.1 & 0.0224 & 7.893 & 1385.8 & 0.0224 & 13.624 & 1254.3 & 0.0224 \\
\hline 12 & 3.199 & 1500.8 & 0.0223 & 7.901 & 1385.8 & 0.0223 & 13.703 & 1259.0 & 0.0223 \\
\hline 13 & 3.172 & 1493.8 & 0.0221 & 7.903 & 1387.7 & 0.0221 & 13.819 & 1268.7 & 0.0221 \\
\hline 14 & 3.097 & 1478.6 & 0.0220 & 7.814 & 1386.0 & 0.0220 & 13.820 & 1277.0 & 0.0220 \\
\hline 15 & 2.943 & 1443.9 & 0.0219 & 7.546 & 1371.9 & 0.0219 & 13.575 & 1280.9 & 0.0219 \\
\hline 16 & 2.666 & 1370.2 & 0.0218 & 6.962 & 1328.4 & 0.0218 & 12.826 & 1268.3 & 0.0218 \\
\hline 17 & 2.186 & 1237.9 & 0.0217 & 5.807 & 1227.2 & 0.0217 & 10.996 & 1209.6 & 0.0217 \\
\hline 18 & 1.428 & 1020.8 & 0.0216 & 3.806 & 1027.2 & 0.0216 & 7.332 & 1041.5 & 0.0216 \\
\hline
\end{tabular}

\begin{tabular}{|c|c|c|c|c|c|c|c|c|c|}
\hline \multirow[b]{2}{*}{$\begin{array}{l}\text { Axial } \\
\text { Node }\end{array}$} & \multicolumn{3}{|c|}{ Statepoint 64 (BOC Cy7) } & \multicolumn{3}{|c|}{ Statepoint 65 (30.0 Cy7) } & \multicolumn{3}{|c|}{ Statepoint 66 (108.0 Cy7) } \\
\hline & $\begin{array}{c}\text { Burnup } \\
\text { (GWd/MTU) } \\
\end{array}$ & $\begin{array}{c}\text { T-Fuel } \\
\left({ }^{\circ} \mathrm{F}\right)\end{array}$ & $\begin{array}{c}\text { Spec. Vol. } \\
\left(\mathrm{ft}^{3} / \mathrm{bm}\right)\end{array}$ & $\begin{array}{c}\text { Burnup } \\
\text { (GWd/MTU) }\end{array}$ & $\begin{array}{c}\text { T-Fuel } \\
\left({ }^{\circ} \mathrm{F}\right)\end{array}$ & $\begin{array}{c}\text { Spec. Vol. } \\
\left(\mathrm{tt}^{3} / \mathrm{lbm}\right)\end{array}$ & $\begin{array}{c}\text { Burnup } \\
\text { (GWd/MTU) }\end{array}$ & $\begin{array}{c}\text { T-Fuel } \\
\left({ }^{\circ} \mathrm{F}\right)\end{array}$ & $\begin{array}{c}\text { Spec. Vol } \\
\left(\mathrm{tt}^{3} / \mathrm{lbm}\right)\end{array}$ \\
\hline & BOC Cy7 & 376.2 Cy 6 & 376.2 Cy 6 & $30.0 \mathrm{Cy} 7$ & $22.5 \mathrm{Cy} 7$ & $22.5 \mathrm{Cy} 7$ & $108.0 \mathrm{Cy} 7$ & $60.4 \mathrm{Cy} 7$ & $60.4 \mathrm{Cy} 7$ \\
\hline 1 & 8.599 & 1081.1 & 0.0237 & 8.768 & 686.2 & 0.0222 & 9.230 & 689.7 & 0.0222 \\
\hline 2 & 13.405 & 1204.4 & 0.0236 & 13.696 & 737.5 & 0.0222 & 14.477 & 740.9 & 0.0222 \\
\hline 3 & 15.763 & 1241.5 & 0.0234 & 16.128 & 768.7 & 0.0222 & 17.088 & 769.4 & 0.0222 \\
\hline 4 & 16.740 & 1235.1 & 0.0233 & 17.152 & 787.3 & 0.0221 & 18.212 & 784.5 & 0.0221 \\
\hline 5 & 17.058 & 1218.5 & 0.0232 & 17.499 & 798.9 & 0.0221 & 18.616 & 793.0 & 0.0221 \\
\hline 6 & 17.175 & 1204.1 & 0.0230 & 17.634 & 805.6 & 0.0221 & 18.782 & 797.1 & 0.0220 \\
\hline 7 & 17.248 & 1193.6 & 0.0229 & 17.718 & 808.9 & 0.0220 & 18.881 & 798.5 & 0.0220 \\
\hline 8 & 17.312 & 1186.1 & 0.0228 & 17.787 & 810.0 & 0.0220 & 18.956 & 798.2 & 0.0220 \\
\hline 9 & 17.367 & 1180.9 & 0.0226 & 17.844 & 809.7 & 0.0219 & 19.015 & 797.0 & 0.0219 \\
\hline 10 & 17.413 & 1177.8 & 0.0225 & 17.891 & 808.4 & 0.0219 & 19.061 & 795.3 & 0.0219 \\
\hline 11 & 17.474 & 1176.9 & 0.0224 & 17.949 & 806.2 & 0.0219 & 19.115 & 793.0 & 0.0218 \\
\hline 12 & 17.600 & 1179.5 & 0.0223 & 18.069 & 802.0 & 0.0218 & 19.226 & 789.4 & 0.0218 \\
\hline 13 & 17.799 & 1186.8 & 0.0221 & 18.257 & 795.0 & 0.0218 & 19.394 & 783.6 & 0.0218 \\
\hline 14 & 17.888 & 1196.0 & 0.0220 & 18.328 & 785.4 & 0.0217 & 19.432 & 775.9 & 0.0217 \\
\hline 15 & 17.721 & 1206.9 & 0.0219 & 18.135 & 772.2 & 0.0217 & 19.185 & 765.0 & 0.0217 \\
\hline 16 & 16.970 & 1212.5 & 0.0218 & 17.341 & 753.1 & 0.0217 & 18.299 & 748.7 & 0.0217 \\
\hline 17 & 14.822 & 1183.1 & 0.0217 & 15.124 & 722.5 & 0.0216 & 15.913 & 720.8 & 0.0216 \\
\hline 18 & 10.057 & 1043.9 & 0.0216 & 10.235 & 663.8 & 0.0216 & 10.710 & 664.0 & 0.0216 \\
\hline
\end{tabular}


Table 4-45. Burnup and TH Feedback Parameters by Axial Node for Assembly F19 (Cont.)

\begin{tabular}{|c|c|c|c|c|c|c|c|c|c|}
\hline \multirow[b]{2}{*}{$\begin{array}{l}\text { Axial } \\
\text { Node }\end{array}$} & \multicolumn{3}{|c|}{ Datapoint (227.4 Cy7) } & \multicolumn{3}{|c|}{ Datapoint (367.3 Cy7) } & \multicolumn{3}{|c|}{ Statepoint 67 (BOC Cy8) } \\
\hline & $\begin{array}{c}\text { Burnup } \\
\text { (GWd/MTU) }\end{array}$ & $\begin{array}{c}\text { T-Fuel } \\
\left({ }^{\circ} \mathrm{F}\right)\end{array}$ & $\begin{array}{l}\text { Spec. Vol. } \\
\left(\mathrm{ft}^{3} / \mathrm{lbm}\right)\end{array}$ & $\begin{array}{c}\text { Burnup } \\
\text { (GWd/MTU) }\end{array}$ & $\begin{array}{c}\text { T-Fuel } \\
\left({ }^{\circ} \mathrm{F}\right)\end{array}$ & $\begin{array}{c}\text { Spec. Vol. } \\
\left(\mathrm{ft}^{3} / \mathrm{lbm}\right)\end{array}$ & $\begin{array}{c}\text { Burnup } \\
\text { (GWd/MTU) }\end{array}$ & $\begin{array}{c}\text { T-Fuel } \\
\left({ }^{\circ} \mathrm{F}\right)\end{array}$ & $\begin{array}{l}\text { Spec. Vol } \\
\left(\mathrm{ft}^{3} / \mathrm{lbm}\right)\end{array}$ \\
\hline & 227.4 Cy 7 & 164.6 Cy 7 & $164.6 \mathrm{Cy} 7$ & 367.3 Cy 7 & 297.3 Cy 7 & 297.3 Cy7 & BOC Cy 8 & 430.3 Cy 7 & $430.3 \mathrm{Cy} 7$ \\
\hline 1 & 10.046 & 705.7 & 0.0222 & 11.198 & 731.9 & 0.0223 & 12.282 & 759.3 & 0.0223 \\
\hline 2 & 15.807 & 756.7 & 0.0222 & 17.595 & 780.6 & 0.0222 & 19.197 & 802.1 & 0.0223 \\
\hline 3 & 18.664 & 778.6 & 0.0222 & 20.700 & 795.0 & 0.0222 & 22.460 & 809.5 & 0.0223 \\
\hline 4 & 19.892 & 785.9 & 0.0221 & 22.002 & 795.9 & 0.0222 & 23.784 & 805.9 & 0.0222 \\
\hline 5 & 20.335 & 787.5 & 0.0221 & 22.452 & 793.1 & 0.0221 & 24.217 & 801.2 & 0.0222 \\
\hline 6 & 20.508 & 786.4 & 0.0220 & 22.611 & 789.4 & 0.0221 & 24.355 & 797.2 & 0.0221 \\
\hline 7 & 20.602 & 784.0 & 0.0220 & 22.687 & 785.8 & 0.0220 & 24.415 & 794.2 & 0.0221 \\
\hline 8 & 20.667 & 781.3 & 0.0220 & 22.738 & 782.8 & 0.0220 & 24.454 & 792.1 & 0.0220 \\
\hline 9 & 20.717 & 778.9 & 0.0219 & 22.779 & 780.5 & 0.0220 & 24.487 & 790.5 & 0.0220 \\
\hline 10 & 20.757 & 776.9 & 0.0219 & 22.816 & 778.8 & 0.0219 & 24.517 & 789.5 & 0.0220 \\
\hline 11 & 20.809 & 775.5 & 0.0218 & 22.872 & 777.8 & 0.0219 & 24.568 & 788.7 & 0.0219 \\
\hline 12 & 20.918 & 774.0 & 0.0218 & 22.991 & 777.1 & 0.0218 & 24.684 & 788.0 & 0.0219 \\
\hline 13 & 21.082 & 772.0 & 0.0218 & 23.169 & 776.6 & 0.0218 & 24.862 & 787.5 & 0.0218 \\
\hline 14 & 21.110 & 769.8 & 0.0217 & 23.215 & 777.2 & 0.0218 & 24.919 & 788.7 & 0.0218 \\
\hline 15 & 20.831 & 766.0 & 0.0217 & 22.945 & 778.3 & 0.0217 & 24.664 & 791.8 & 0.0217 \\
\hline 16 & 19.857 & 757.7 & 0.0217 & 21.929 & 776.9 & 0.0217 & 23.638 & 794.8 & 0.0217 \\
\hline 17 & 17.251 & 736.9 & 0.0216 & 19.111 & 763.7 & 0.0216 & 20.690 & 788.5 & 0.0217 \\
\hline 18 & 11.548 & 680.5 & 0.0216 & 12.775 & 708.3 & 0.0216 & 13.863 & 737.1 & 0.0216 \\
\hline
\end{tabular}

Table 4-46. Burnup and TH Feedback Parameters by Axial Node for Assembly F23

\begin{tabular}{|c|c|c|c|c|c|c|c|c|c|}
\hline \multirow[b]{2}{*}{$\begin{array}{l}\text { Axial } \\
\text { Node }\end{array}$} & \multicolumn{3}{|c|}{ Statepoint 63 (69.1 Cy6) } & \multicolumn{3}{|c|}{ Datapoint (176.7 Cy6) } & \multicolumn{3}{|c|}{ Datapoint (320.3 Cy6) } \\
\hline & \begin{tabular}{|c|} 
Burnup \\
(GWd/MTU)
\end{tabular} & $\begin{array}{c}\text { T-Fuel } \\
\left({ }^{\circ} \mathrm{F}\right)\end{array}$ & $\begin{array}{c}\text { Spec. Vol. } \\
\left(\mathrm{ft}^{3} / \mathrm{lbm}\right)\end{array}$ & $\begin{array}{c}\text { Burnup } \\
\text { (GWd/MTU) }\end{array}$ & $\begin{array}{l}\text { T-Fuel } \\
\left({ }^{\circ} \mathrm{F}\right)\end{array}$ & $\begin{array}{c}\text { Spec. Vol. } \\
\left(\mathrm{ft}^{3} / \mathrm{bm}\right)\end{array}$ & $\begin{array}{c}\text { Burnup } \\
\text { (GWd/MTU) }\end{array}$ & $\begin{array}{l}\text { T-Fuel } \\
\left({ }^{\circ} \mathrm{F}\right)\end{array}$ & $\begin{array}{c}\text { Spec. Vol. } \\
\left(\mathrm{ft}^{3} / \mathrm{lbm}\right)\end{array}$ \\
\hline & 69.1 Cy 6 & 41.5 Cy 6 & 41.5 Cy 6 & 176.7 Cy 6 & 128.8 Cy6 & 128.8 Cy 6 & 320.3 Cy6 & 252.5 Cy 6 & 252.5 Cy 6 \\
\hline 1 & 1.134 & 967.6 & 0.0238 & 3.055 & 1021.1 & 0.0238 & 6.025 & 1063.0 & 0.0238 \\
\hline 2 & 1.855 & 1152.2 & 0.0237 & 4.996 & 1206.8 & 0.0237 & 9.711 & 1223.6 & 0.0237 \\
\hline 3 & 2.357 & 1290.4 & 0.0236 & 6.245 & 1317.6 & 0.0236 & 11.791 & 1293.1 & 0.0235 \\
\hline 4 & 2.666 & 1378.3 & 0.0235 & 6.938 & 1368.9 & 0.0234 & 12.756 & 1305.3 & 0.0234 \\
\hline 5 & 2.845 & 1427.8 & 0.0233 & 7.291 & 1387.5 & 0.0233 & 13.138 & 1298.5 & 0.0232 \\
\hline 6 & 2.964 & 1458.7 & 0.0232 & 7.499 & 1393.1 & 0.0231 & 13.317 & 1287.9 & 0.0231 \\
\hline 7 & 3.054 & 1480.1 & 0.0230 & 7.649 & 1395.0 & 0.0230 & 13.435 & 1278.3 & 0.0229 \\
\hline 8 & 3.121 & 1494.0 & 0.0229 & 7.762 & 1395.7 & 0.0228 & 13.528 & 1270.6 & 0.0228 \\
\hline 9 & 3.165 & 1502.0 & 0.0227 & 7.840 & 1395.3 & 0.0227 & 13.598 & 1265.2 & 0.0227 \\
\hline 10 & 3.184 & 1504.2 & 0.0226 & 7.877 & 1393.8 & 0.0226 & 13.643 & 1262.0 & 0.0225 \\
\hline 11 & 3.182 & 1501.6 & 0.0224 & 7.888 & 1392.1 & 0.0224 & 13.684 & 1262.0 & 0.0224 \\
\hline 12 & 3.171 & 1496.3 & 0.0223 & 7.898 & 1392.0 & 0.0223 & 13.766 & 1266.9 & 0.0223 \\
\hline 13 & 3.147 & 1489.6 & 0.0221 & 7.904 & 1394.0 & 0.0221 & 13.888 & 1276.8 & 0.0222 \\
\hline 14 & 3.073 & 1474.7 & 0.0220 & 7.817 & 1392.3 & 0.0220 & 13.893 & 1285.2 & 0.0220 \\
\hline 15 & 2.922 & 1440.0 & 0.0219 & 7.550 & 1377.9 & 0.0219 & 13.649 & 1289.0 & 0.0219 \\
\hline 16 & 2.651 & 1367.5 & 0.0218 & 6.974 & 1334.3 & 0.0218 & 12.906 & 1276.0 & 0.0218 \\
\hline 17 & 2.187 & 1238.8 & 0.0217 & 5.843 & 1234.0 & 0.0217 & 11.104 & 1217.5 & 0.0217 \\
\hline 18 & 1.444 & 1026.6 & 0.0216 & 3.867 & 1035.2 & 0.0216 & 7.462 & 1049.1 & 0.0216 \\
\hline
\end{tabular}


Table 4-46. Burnup and TH Feedback Parameters by Axial Node for Assembly F23 (Cont.)

\begin{tabular}{|c|c|c|c|c|c|c|c|c|c|}
\hline \multirow[b]{2}{*}{$\begin{array}{l}\text { Axial } \\
\text { Node }\end{array}$} & \multicolumn{3}{|c|}{ Statepoint 64 (BOC Cy7) } & \multicolumn{3}{|c|}{ Statepoint 65 (30.0 Cy7) } & \multicolumn{3}{|c|}{ Statepoint 66 (108.0 Cy7) } \\
\hline & $\begin{array}{c}\text { Burnup } \\
\text { (GWd/MTU) }\end{array}$ & $\begin{array}{c}\text { T-Fuel } \\
\left({ }^{\circ} \mathrm{F}\right)\end{array}$ & $\begin{array}{l}\text { Spec. Vol. } \\
\left(\mathrm{tt}^{3} / \mathrm{lbm}\right)\end{array}$ & \begin{tabular}{|c|} 
Burnup \\
(GWd/MTU)
\end{tabular} & $\begin{array}{c}\text { T-Fuel } \\
\left({ }^{\circ} F\right)\end{array}$ & $\begin{array}{l}\text { Spec. Vol. } \\
\left(\mathrm{ft}^{3} / \mathrm{lbm}\right)\end{array}$ & $\begin{array}{c}\text { Burnup } \\
\text { (GWd/MTU) }\end{array}$ & $\begin{array}{c}\text { T-Fuel } \\
\left({ }^{\circ} \mathrm{F}\right)\end{array}$ & $\begin{array}{c}\text { Spec. Vol. } \\
\left(\mathrm{ft}^{3} / \mathrm{lbm}\right)\end{array}$ \\
\hline & BOC Cy 7 & 376.2 Cy 6 & 376.2 Cy6 & $30.0 \mathrm{Cy} 7$ & 22.5 Cy 7 & $22.5 \mathrm{Cy} 7$ & $108.0 \mathrm{Cy} 7$ & $60.4 \mathrm{Cy} 7$ & $60.4 \mathrm{Cy} 7$ \\
\hline 1 & 8.370 & 1081.4 & 0.0237 & 8.870 & 927.1 & 0.0234 & 10.230 & 934.6 & 0.0234 \\
\hline 2 & 13.263 & 1209.1 & 0.0236 & 14.078 & 1049.8 & 0.0233 & 16.268 & 1056.3 & 0.0233 \\
\hline 3 & 15.779 & 1247.8 & 0.0235 & 16.765 & 1112.1 & 0.0232 & 19.372 & 1112.2 & 0.0232 \\
\hline 4 & 16.805 & 1240.4 & 0.0233 & 17.860 & 1124.6 & 0.0231 & 20.579 & 1120.3 & 0.0231 \\
\hline 5 & 17.130 & 1223.1 & 0.0232 & 18.224 & 1144.3 & 0.0230 & 21.045 & 1131.9 & 0.0230 \\
\hline 6 & 17.248 & 1208.3 & 0.0230 & 18.380 & 1158.9 & 0.0229 & 21.274 & 1140.6 & 0.0229 \\
\hline 7 & 17.325 & 1197.5 & 0.0229 & 18.483 & 1166.9 & 0.0228 & 21.419 & 1144.3 & 0.0228 \\
\hline 8 & 17.394 & 1189.7 & 0.0228 & 18.567 & 1170.1 & 0.0227 & 21.524 & 1144.2 & 0.0226 \\
\hline 9 & 17.454 & 1184.4 & 0.0226 & 18.634 & 1170.2 & 0.0225 & 21.600 & 1142.3 & 0.0225 \\
\hline 10 & 17.501 & 1181.1 & 0.0225 & 18.684 & 1169.0 & 0.0224 & 21.657 & 1139.8 & 0.0224 \\
\hline 11 & 17.561 & 1180.0 & 0.0224 & 18.750 & 1171.0 & 0.0223 & 21.749 & 1140.5 & 0.0223 \\
\hline 12 & 17.690 & 1182.5 & 0.0223 & 18.927 & 1202.3 & 0.0222 & 22.098 & 1166.1 & 0.0222 \\
\hline 13 & 17.896 & 1189.9 & 0.0222 & 19.162 & 1199.4 & 0.0221 & 22.368 & 1167.8 & 0.0221 \\
\hline 14 & 17.989 & 1199.2 & 0.0220 & 19.219 & 1177.3 & 0.0220 & 22.351 & 1150.8 & 0.0220 \\
\hline 15 & 17.824 & 1210.0 & 0.0219 & 18.984 & 1144.0 & 0.0219 & 21.972 & 1123.2 & 0.0219 \\
\hline 16 & 17.081 & 1215.8 & 0.0218 & 18.130 & 1097.1 & 0.0218 & 20.872 & 1083.5 & 0.0218 \\
\hline 17 & 14.965 & 1186.8 & 0.0217 & 15.833 & 1024.4 & 0.0217 & 18.136 & 1018.7 & 0.0217 \\
\hline 18 & 10.224 & 1048.0 & 0.0216 & 10.761 & 873.5 & 0.0216 & 12.203 & 874.7 & 0.0216 \\
\hline
\end{tabular}

Table 4-47. Burnup and TH Feedback Parameters by Axial Node for Assembly F25

\begin{tabular}{|c|c|c|c|c|c|c|c|c|c|}
\hline \multirow[b]{2}{*}{$\begin{array}{l}\text { Axlal } \\
\text { Node }\end{array}$} & \multicolumn{3}{|c|}{ Statepoint 63 (69.1 Cy6) } & \multicolumn{3}{|c|}{ Datapoint (176.7 Cy6) } & \multicolumn{3}{|c|}{ Datapoint (320.3 Cy6) } \\
\hline & $\begin{array}{c}\text { Burnup } \\
\text { (GWd/MTU) }\end{array}$ & $\begin{array}{l}\text { T-Fuel } \\
\left({ }^{\circ} \mathrm{F}\right)\end{array}$ & $\begin{array}{c}\text { Spec. Vol. } \\
\left(\mathrm{ft}^{3} / \mathrm{lbm}\right)\end{array}$ & $\begin{array}{c}\text { Burnup } \\
\text { (GWd/MTU) }\end{array}$ & $\begin{array}{c}\text { T-Fuel } \\
\left({ }^{\circ} \mathrm{F}\right)\end{array}$ & $\begin{array}{l}\text { Spec. Vol. } \\
\left(\mathrm{ft}^{3} / \mathrm{lbm}\right)\end{array}$ & $\begin{array}{c}\text { Burnup } \\
\text { (GWd/MTU) }\end{array}$ & $\begin{array}{c}\text { T-Fuel } \\
\left({ }^{\circ} \mathrm{F}\right)\end{array}$ & $\begin{array}{c}\text { Spec. Vol. } \\
\left(\mathrm{ft}^{3} / \mathrm{lbm}\right)\end{array}$ \\
\hline & 69.1 Cy6 & $41.5 \mathrm{Cy} 6$ & 41.5 Cy6 & 176.7 Cy 6 & $128.8 \mathrm{Cy} 6$ & $128.8 \mathrm{Cy} 6$ & 320.3 Cy 6 & 252.5 Cy 6 & 252.5 Cy 6 \\
\hline 1 & 0.755 & 838.5 & 0.0231 & 2.021 & 867.6 & 0.0230 & 4.008 & 906.8 & 0.0230 \\
\hline 2 & 1.350 & 1002.1 & 0.0230 & 3.538 & 1024.4 & 0.0229 & 6.778 & 1043.4 & 0.0229 \\
\hline 3 & 1.722 & 1106.7 & 0.0230 & 4.417 & 1106.2 & 0.0228 & 8.203 & 1096.0 & 0.0228 \\
\hline 4 & 1.952 & 1170.1 & 0.0229 & 4.905 & 1142.4 & 0.0228 & 8.877 & 1107.2 & 0.0227 \\
\hline 5 & 2.095 & 1209.0 & 0.0228 & 5.174 & 1156.8 & 0.0227 & 9.177 & 1103.3 & 0.0226 \\
\hline 6 & 2.186 & 1232.6 & 0.0227 & 5.325 & 1160.9 & 0.0226 & 9.307 & 1095.3 & 0.0225 \\
\hline 7 & 2.247 & 1247.6 & 0.0226 & 5.415 & 1160.7 & 0.0225 & 9.367 & 1087.3 & 0.0224 \\
\hline 8 & 2.285 & 1256.8 & 0.0225 & 5.469 & 1158.9 & 0.0224 & 9.395 & 1080.7 & 0.0224 \\
\hline 9 & 2.307 & 1261.4 & 0.0224 & 5.498 & 1156.5 & 0.0223 & 9.406 & 1075.8 & 0.0223 \\
\hline 10 & 2.313 & 1262.0 & 0.0223 & 5.507 & 1154.0 & 0.0222 & 9.411 & 1072.8 & 0.0222 \\
\hline 11 & 2.307 & 1259.6 & 0.0222 & 5.505 & 1152.1 & 0.0221 & 9.422 & 1072.0 & 0.0221 \\
\hline 12 & 2.291 & 1254.6 & 0.0221 & 5.494 & 1151.1 & 0.0220 & 9.443 & 1073.8 & 0.0220 \\
\hline 13 & 2.260 & 1246.2 & 0.0220 & 5.464 & 1150.4 & 0.0219 & 9.461 & 1077.7 & 0.0219 \\
\hline 14 & 2.199 & 1230.7 & 0.0219 & 5.382 & 1147.0 & 0.0219 & 9.426 & 1082.6 & 0.0219 \\
\hline 15 & 2.085 & 1201.0 & 0.0218 & 5.187 & 1135.0 & 0.0218 & 9.239 & 1084.8 & 0.0218 \\
\hline 16 & 1.878 & 1144.1 & 0.0217 & 4.765 & 1102.4 & 0.0217 & 8.690 & 1074.5 & 0.0217 \\
\hline 17 & 1.502 & 1036.0 & 0.0216 & 3.898 & 1022.2 & 0.0216 & 7.327 & 1024.9 & 0.0216 \\
\hline 18 & 0.856 & 835.2 & 0.0216 & 2.271 & 843.0 & 0.0216 & 4.422 & 872.1 & 0.0216 \\
\hline
\end{tabular}


Table 4-47. Burnup and TH Feedback Parameters by Axial Node for Assembly F25 (Cont.)

\begin{tabular}{|c|c|c|c|c|c|c|c|c|c|}
\hline \multirow[b]{2}{*}{$\begin{array}{l}\text { Axial } \\
\text { Node }\end{array}$} & \multicolumn{3}{|c|}{ Statepoint 64 (BOC Cy7) } & \multicolumn{3}{|c|}{ Statepoint $65(30.0$ Cy 7$)$} & \multicolumn{3}{|c|}{ Statepoint 66 (108.0 Cy7) } \\
\hline & \begin{tabular}{|c|} 
Burnup \\
(GWd/MTU)
\end{tabular} & $\begin{array}{c}\text { T-Fuel } \\
\left({ }^{\circ} \mathrm{F}\right)\end{array}$ & $\begin{array}{l}\text { Spec. Vol. } \\
\left(\mathrm{ft}^{3} / \mathrm{lbm}\right)\end{array}$ & $\begin{array}{c}\text { Burnup } \\
\text { (GWd/MTU) }\end{array}$ & $\begin{array}{l}\text { T-Fuel } \\
\left({ }^{\circ} \mathrm{F}\right)\end{array}$ & $\begin{array}{c}\text { Spec. Vol. } \\
\left(\mathrm{ft}^{3} / \mathrm{lbm}\right)\end{array}$ & $\begin{array}{c}\text { Burnup } \\
\text { (GWd/MTU) }\end{array}$ & $\begin{array}{c}\text { T-Fuel } \\
\left({ }^{\circ} \mathrm{F}\right)\end{array}$ & $\begin{array}{c}\text { Spec. Vol. } \\
\left(\mathrm{ft}^{3} / \mathrm{lbm}\right)\end{array}$ \\
\hline & BOC Cy7 & 376.2 Cy6 & 376.2 Cy 6 & $30.0 \mathrm{Cy} 7$ & $22.5 \mathrm{Cy} 7$ & $22.5 \mathrm{Cy} 7$ & $108.0 \mathrm{Cy} 7$ & $60.4 \mathrm{Cy} 7$ & $60.4 \mathrm{Cy} 7$ \\
\hline 1 & 5.630 & 943.2 & 0.0230 & 6.174 & 990.3 & 0.0237 & 7.672 & 1002.8 & 0.0237 \\
\hline 2 & 9.281 & 1052.1 & 0.0229 & 10.173 & 1135.3 & 0.0236 & 12.605 & 1145.8 & 0.0236 \\
\hline 3 & 11.013 & 1084.9 & 0.0228 & 12.107 & 1224.2 & 0.0235 & 15.050 & 1229.8 & 0.0235 \\
\hline 4 & 11.745 & 1083.5 & 0.0227 & 12.961 & 1277.3 & 0.0234 & 16.191 & 1274.9 & 0.0234 \\
\hline 5 & 12.022 & 1073.4 & 0.0227 & 13.316 & 1309.6 & 0.0232 & 16.712 & 1299.0 & 0.0232 \\
\hline 6 & 12.115 & 1063.3 & 0.0226 & 13.460 & 1328.2 & 0.0231 & 16.955 & 1311.3 & 0.0231 \\
\hline 7 & 12.145 & 1055.4 & 0.0225 & 13.522 & 1338.4 & 0.0230 & 17.075 & 1317.1 & 0.0230 \\
\hline 8 & 12.154 & 1049.8 & 0.0224 & 13.550 & 1343.0 & 0.0228 & 17.134 & 1318.5 & 0.0228 \\
\hline 9 & 12.156 & 1045.8 & 0.0223 & 13.562 & 1343.9 & 0.0227 & 17.162 & 1317.4 & 0.0227 \\
\hline 10 & 12.161 & 1043.5 & 0.0222 & 13.570 & 1342.4 & 0.0225 & 17.177 & 1314.9 & 0.0225 \\
\hline 11 & 12.183 & 1042.8 & 0.0221 & 13.589 & 1338.9 & 0.0224 & 17.197 & 1311.2 & 0.0224 \\
\hline 12 & 12.229 & 1044.1 & 0.0220 & 13.625 & 1332.1 & 0.0223 & 17.224 & 1305.2 & 0.0223 \\
\hline 13 & 12.287 & 1047.8 & 0.0220 & 13.658 & 1317.9 & 0.0221 & 17.214 & 1293.7 & 0.0221 \\
\hline 14 & 12.304 & 1053.9 & 0.0219 & 13.628 & 1293.3 & 0.0220 & 17.089 & 1273.9 & 0.0220 \\
\hline 15 & 12.164 & 1060.9 & 0.0218 & 13.409 & 1255.5 & 0.0219 & 16.705 & 1242.4 & 0.0219 \\
\hline 16 & 11.595 & 1061.7 & 0.0217 & 12.720 & 1196.4 & 0.0218 & 15.737 & 1190.9 & 0.0218 \\
\hline 17 & 9.968 & 1030.5 & 0.0216 & 10.899 & 1105.8 & 0.0217 & 13.426 & 1105.6 & 0.0217 \\
\hline 18 & 6.176 & 904.2 & 0.0216 & 6.755 & 938.3 & 0.0216 & 8.343 & 941.6 & 0.0216 \\
\hline
\end{tabular}

Table 4-48. Burnup and TH Feedback Parameters by Axial Node for Assembly F27

\begin{tabular}{|c|c|c|c|c|c|c|c|c|c|}
\hline \multirow[b]{2}{*}{$\begin{array}{l}\text { Axial } \\
\text { Node }\end{array}$} & \multicolumn{3}{|c|}{ Statepoint $63(69.1 \mathrm{Cy} 6)$} & \multicolumn{3}{|c|}{ Datapoint (176.7 Cy6) } & \multicolumn{3}{|c|}{ Datapoint (320.3 Cy6) } \\
\hline & \begin{tabular}{|c|} 
Burnup \\
(GWd/MTU)
\end{tabular} & $\begin{array}{l}\text { T-Fuel } \\
\left({ }^{\circ} \mathrm{F}\right)\end{array}$ & $\begin{array}{c}\text { Spec. Vol. } \\
\left(\mathrm{ft}^{3} / \mathrm{lbm}\right)\end{array}$ & \begin{tabular}{c|} 
Burnup \\
(GWd/MTU) \\
\end{tabular} & $\begin{array}{l}\text { T-Fuel } \\
\left({ }^{\circ} \mathrm{F}\right)\end{array}$ & $\begin{array}{c}\text { Spec. Vol. } \\
\left(\mathrm{ft}^{3} / \mathrm{lbm}\right)\end{array}$ & $\begin{array}{c}\text { Burnup } \\
\text { (GWd/MTU) }\end{array}$ & $\begin{array}{c}\text { T-Fuel } \\
\left({ }^{\circ} \mathrm{F}\right)\end{array}$ & $\begin{array}{c}\text { Spec. Vol. } \\
\left(\mathrm{ft}^{3} / \mathrm{lbm}\right)\end{array}$ \\
\hline & 69.1 Cy 6 & 41.5 Cy6 & 41.5 Cy6 & 176.7 Cy6 & 128.8 Cy 6 & 128.8 Cy 6 & 320.3 Cy 6 & 252.5 Cy 6 & 252.5 Cy6 \\
\hline 1 & 0.816 & 856.4 & 0.0234 & 2.198 & 898.4 & 0.0233 & 4.375 & 942.8 & 0.0232 \\
\hline 2 & 1.473 & 1033.3 & 0.0233 & 3.910 & 1077.6 & 0.0232 & 7.553 & 1100.1 & 0.0231 \\
\hline 3 & 1.940 & 1167.1 & 0.0232 & 5.024 & 1177.6 & 0.0231 & 9.370 & 1162.4 & 0.0230 \\
\hline 4 & 2.233 & 1249.3 & 0.0231 & 5.650 & 1222.6 & 0.0230 & 10.237 & 1175.1 & 0.0229 \\
\hline 5 & 2.409 & 1297.4 & 0.0230 & 5.986 & 1240.3 & 0.0229 & 10.620 & 1170.5 & 0.0228 \\
\hline 6 & 2.520 & 1326.6 & 0.0229 & 6.175 & 1245.8 & 0.0228 & 10.791 & 1161.5 & 0.0227 \\
\hline 7 & 2.594 & 1345.4 & 0.0227 & 6.290 & 1246.2 & 0.0226 & 10.875 & 1152.8 & 0.0226 \\
\hline 8 & 2.643 & 1357.2 & 0.0226 & 6.363 & 1244.8 & 0.0225 & 10.922 & 1145.7 & 0.0225 \\
\hline 9 & 2.671 & 1363.5 & 0.0225 & 6.404 & 1242.6 & 0.0224 & 10.948 & 1140.5 & 0.0224 \\
\hline 10 & 2.681 & 1364.7 & 0.0224 & 6.421 & 1240.1 & 0.0223 & 10.963 & 1137.4 & 0.0223 \\
\hline 11 & 2.676 & 1362.3 & 0.0223 & 6.422 & 1238.3 & 0.0222 & 10.981 & 1136.7 & 0.0222 \\
\hline 12 & 2.659 & 1357.0 & 0.0221 & 6.412 & 1237.4 & 0.0221 & 11.010 & 1138.7 & 0.0221 \\
\hline 13 & 2.623 & 1347.8 & 0.0220 & 6.380 & 1236.8 & 0.0220 & 11.034 & 1143.3 & 0.0220 \\
\hline 14 & 2.554 & 1330.0 & 0.0219 & 6.287 & 1233.3 & 0.0219 & 10.998 & 1148.9 & 0.0219 \\
\hline 15 & 2.423 & 1296.0 & 0.0218 & 6.062 & 1220.3 & 0.0218 & 10.786 & 1151.9 & 0.0218 \\
\hline 16 & 2.184 & 1232.0 & 0.0217 & 5.573 & 1184.3 & 0.0217 & 10.155 & 1141.8 & 0.0217 \\
\hline 17 & 1.759 & 1114.5 & 0.0216 & 4.587 & 1098.1 & 0.0216 & 8.613 & 1090.6 & 0.0216 \\
\hline 18 & 1.070 & 904.5 & 0.0216 & 2.839 & 911.8 & 0.0216 & 5.499 & 940.1 & 0.0216 \\
\hline
\end{tabular}


Table 4-48. Burnup and TH Feedback Parameters by Axial Node for Assembly F27 (Cont.)

\begin{tabular}{|c|c|c|c|c|c|c|c|c|c|}
\hline \multirow{2}{*}{$\begin{array}{c}\text { Axial } \\
\text { Node }\end{array}$} & \multicolumn{2}{|c|}{ Statepoint 64 (BOC Cy7) } & \multicolumn{2}{c|}{ Statepoint 65 (30.0 Cy7) } & \multicolumn{2}{c|}{ Statepoint 66 (108.0 Cy7) } \\
\hline & $\begin{array}{c}\text { Burnup } \\
\text { (WdMTU) }\end{array}$ & $\begin{array}{c}\text { T-Fuel } \\
\left({ }^{\circ} \mathrm{F}\right)\end{array}$ & $\begin{array}{c}\text { Spec. Vol. } \\
\left(\mathrm{ft}^{3} / / \mathrm{bm}\right)\end{array}$ & $\begin{array}{c}\text { Burnup } \\
(\mathrm{GWd} / \mathrm{MTU})\end{array}$ & $\begin{array}{c}\text { T-Fuel } \\
\left({ }^{\circ} \mathrm{F}\right)\end{array}$ & $\begin{array}{c}\text { Spec. Vol. } \\
\left(\mathrm{ft}^{3} / \mathrm{lbm}\right)\end{array}$ & $\begin{array}{c}\text { Burnup } \\
(\mathrm{GWd} / \mathrm{MTU})\end{array}$ & $\begin{array}{c}\text { T-Fuel } \\
\left({ }^{\circ} \mathrm{F}\right)\end{array}$ & $\begin{array}{c}\text { Spec. Vol. } \\
\left(\mathrm{ft}^{3} / \mathrm{lbm}\right)\end{array}$ \\
\hline 1 & 6.165 & 986.0 & 0.0232 & 6.520 & 836.6 & 0.0229 & 7.481 & 842.0 & 0.0228 \\
\hline 2 & 10.370 & 1104.5 & 0.0232 & 10.954 & 930.5 & 0.0228 & 12.514 & 933.7 & 0.0228 \\
\hline 3 & 12.570 & 1141.3 & 0.0231 & 13.279 & 982.6 & 0.0227 & 15.144 & 982.2 & 0.0227 \\
\hline 4 & 13.508 & 1138.8 & 0.0229 & 14.292 & 1012.7 & 0.0227 & 16.322 & 1006.6 & 0.0226 \\
\hline 5 & 13.866 & 1127.5 & 0.0228 & 14.698 & 1031.5 & 0.0226 & 16.823 & 1020.2 & 0.0226 \\
\hline 6 & 13.996 & 1116.4 & 0.0227 & 14.859 & 1043.4 & 0.0225 & 17.040 & 1027.5 & 0.0225 \\
\hline 7 & 14.048 & 1107.8 & 0.0226 & 14.931 & 1050.2 & 0.0224 & 17.143 & 1030.8 & 0.0224 \\
\hline 8 & 14.075 & 1101.7 & 0.0225 & 14.970 & 1053.3 & 0.0223 & 17.199 & 1031.3 & 0.0223 \\
\hline 9 & 14.093 & 1097.5 & 0.0224 & 14.994 & 1053.8 & 0.0223 & 17.229 & 1030.2 & 0.0222 \\
\hline 10 & 14.109 & 1094.9 & 0.0223 & 15.011 & 1052.6 & 0.0222 & 17.248 & 1028.0 & 0.0222 \\
\hline 11 & 14.141 & 1094.2 & 0.0222 & 15.041 & 1049.7 & 0.0221 & 17.276 & 1025.1 & 0.0221 \\
\hline 12 & 14.199 & 1095.6 & 0.0221 & 15.092 & 1044.2 & 0.0220 & 17.317 & 1020.4 & 0.0220 \\
\hline 13 & 14.270 & 1099.7 & 0.0220 & 15.146 & 1034.1 & 0.0219 & 17.344 & 1012.4 & 0.0219 \\
\hline 14 & 14.294 & 1106.6 & 0.0219 & 15.141 & 1017.8 & 0.0219 & 17.285 & 999.6 & 0.0219 \\
\hline 15 & 14.138 & 1114.6 & 0.0218 & 14.937 & 993.7 & 0.0218 & 16.985 & 979.9 & 0.0218 \\
\hline 16 & 13.491 & 1115.8 & 0.0217 & 14.214 & 958.1 & 0.0217 & 16.096 & 949.5 & 0.0217 \\
\hline 17 & 11.665 & 1084.6 & 0.0217 & 12.262 & 899.8 & 0.0217 & 13.837 & 896.4 & 0.0217 \\
\hline 18 & 7.631 & 965.3 & 0.0216 & 7.995 & 787.5 & 0.0216 & 8.970 & 787.0 & 0.0216 \\
\hline
\end{tabular}

Table 4-49. Burnup and TH Feedback Parameters by Axial Node for Assembly G2

\begin{tabular}{|c|c|c|c|c|c|c|c|c|c|}
\hline \multirow[b]{2}{*}{$\begin{array}{l}\text { Axial } \\
\text { Node }\end{array}$} & \multicolumn{3}{|c|}{ Statepoint 65 (30.0 Cy7) } & \multicolumn{3}{|c|}{ Statepoint 66 (108.0 Cy7) } & \multicolumn{3}{|c|}{ Datapoint (227.4 Cym) } \\
\hline & $\begin{array}{c}\text { Burnup } \\
\text { (GWd/MTU) }\end{array}$ & $\begin{array}{c}\text { T-Fuel } \\
\left({ }^{\circ} \mathrm{F}\right)\end{array}$ & $\begin{array}{c}\text { Spec. Vol. } \\
\left(\mathrm{ft}^{3} / \mathrm{lbm}\right)\end{array}$ & $\begin{array}{c}\text { Burnup } \\
\text { (GWd/MTU) }\end{array}$ & $\begin{array}{c}\text { T-Fuel } \\
\left({ }^{\circ} \mathrm{F}\right)\end{array}$ & $\begin{array}{c}\text { Spec. Vol. } \\
\left(\mathrm{ft}^{3} / \mathrm{lbm}\right)\end{array}$ & $\begin{array}{c}\text { Burnup } \\
\text { (GWd/MTU) }\end{array}$ & $\begin{array}{l}\text { T-Fuel } \\
\left({ }^{\circ} \mathrm{F}\right)\end{array}$ & $\begin{array}{c}\text { Spec. Vol. } \\
\left(\mathrm{ft}^{3} / \mathrm{lbm}\right)\end{array}$ \\
\hline & $30.0 \mathrm{Cy} 7$ & $22.5 \mathrm{Cy} 7$ & $22.5 \mathrm{Cy} 7$ & $108.0 \mathrm{Cy} 7$ & $60.4 \mathrm{Cy} 7$ & 60.4 Cy7 & $227.4 \mathrm{Cy} 7$ & 164.6 Cy7 & 164.6 Cy 7 \\
\hline 1 & 0.580 & 1064.2 & 0.0237 & 2.188 & 1086.5 & 0.0238 & 4.936 & 1117.3 & 0.0239 \\
\hline 2 & 0.893 & 1249.6 & 0.0236 & 3.399 & 1276.2 & 0.0237 & 7.662 & 1298.9 & 0.0238 \\
\hline 3 & 1.086 & 1372.6 & 0.0235 & 4.125 & 1398.5 & 0.0236 & 9.161 & 1394.6 & 0.0237 \\
\hline 4 & 1.207 & 1447.6 & 0.0234 & 4.562 & 1468.4 & 0.0235 & 9.953 & 1427.2 & 0.0235 \\
\hline 5 & 1.285 & 1493.5 & 0.0232 & 4.829 & 1502.1 & 0.0233 & 10.366 & 1434.5 & 0.0233 \\
\hline 6 & 1.336 & 1517.6 & 0.0231 & 4.993 & 1519.8 & 0.0232 & 10.581 & 1432.9 & 0.0232 \\
\hline 7 & 1.368 & 1530.6 & 0.0229 & 5.091 & 1528.4 & 0.0230 & 10.687 & 1428.0 & 0.0230 \\
\hline 8 & 1.385 & 1536.4 & 0.0228 & 5.143 & 1531.1 & 0.0228 & 10.733 & 1422.6 & 0.0229 \\
\hline 9 & 1.393 & 1536.9 & 0.0227 & 5.163 & 1529.8 & 0.0227 & 10.746 & 1418.1 & 0.0227 \\
\hline 10 & 1.391 & 1533.2 & 0.0225 & 5.160 & 1525.6 & 0.0226 & 10.742 & 1415.0 & 0.0226 \\
\hline 11 & 1.381 & 1525.4 & 0.0224 & 5.133 & 1518.6 & 0.0224 & 10.723 & 1413.5 & 0.0225 \\
\hline 12 & 1.361 & 1512.4 & 0.0222 & 5.076 & 1507.8 & 0.0223 & 10.679 & 1413.3 & 0.0223 \\
\hline 13 & 1.327 & 1492.3 & 0.0221 & 4.975 & 1491.4 & 0.0221 & 10.585 & 1413.6 & 0.0222 \\
\hline 14 & 1.274 & 1460.7 & 0.0220 & 4.809 & 1466.2 & 0.0220 & 10.395 & 1411.6 & 0.0221 \\
\hline 15 & 1.195 & 1408.2 & 0.0219 & 4.543 & 1424.8 & 0.0219 & 10.017 & 1401.8 & 0.0219 \\
\hline 16 & 1.073 & 1327.2 & 0.0218 & 4.108 & 1349.1 & 0.0218 & 9.265 & 1370.5 & 0.0218 \\
\hline 17 & 0.882 & 1199.7 & 0.0217 & 3.388 & 1222.7 & 0.0217 & 7.796 & 1272.6 & 0.0217 \\
\hline 18 & 0.591 & 1000.7 & 0.0216 & 2.253 & 1019.2 & 0.0216 & 5.205 & 1070.3 & 0.0216 \\
\hline
\end{tabular}


Table 4-49. Burnup and TH Feedback Parameters by Axial Node for Assembly G2 (Cont.)

\begin{tabular}{|c|c|c|c|c|c|c|}
\hline \multirow[b]{2}{*}{$\begin{array}{l}\text { Axial } \\
\text { Node }\end{array}$} & \multicolumn{3}{|c|}{ Datapoint (367.3 Cy7) } & \multicolumn{3}{|c|}{ Statepoint 67 (BOC Cy8) } \\
\hline & $\begin{array}{c}\text { Burnup } \\
\text { (GWd/MTU) }\end{array}$ & $\begin{array}{c}\text { T-Fuel } \\
\left({ }^{\circ} \mathrm{F}\right)\end{array}$ & $\begin{array}{c}\text { Spec. Vol. } \\
\left(\mathrm{ft}^{3} / / \mathrm{bm}\right)\end{array}$ & $\begin{array}{c}\text { Burnup } \\
\text { (GWd/MTU) }\end{array}$ & $\begin{array}{l}\text { T-Fuel } \\
\left({ }^{\circ} \mathrm{F}\right)\end{array}$ & $\begin{array}{c}\text { Spec. Vol. } \\
\left(\mathrm{ft}^{3} / \mathrm{lbm}\right)\end{array}$ \\
\hline & $367.3 \mathrm{Cy} 7$ & 297.3 Cy7 & $297.3 \mathrm{Cy} 7$ & BOC Cy 8 & $430.3 \mathrm{Cy} 7$ & 430.3 Cy 7 \\
\hline 1 & 8.394 & 1111.5 & 0.0238 & 11.195 & 1080.5 & 0.0236 \\
\hline 2 & 12.869 & 1254.5 & 0.0237 & 16.909 & 1190.8 & 0.0235 \\
\hline 3 & 15.049 & 1310.6 & 0.0235 & 19.452 & 1211.7 & 0.0234 \\
\hline 4 & 16.020 & 1315.6 & 0.0234 & 20.444 & 1201.0 & 0.0232 \\
\hline 5 & 16.432 & 1305.5 & 0.0232 & 20.794 & 1186.9 & 0.0231 \\
\hline 6 & 16.597 & 1293.2 & 0.0231 & 20.896 & 1175.9 & 0.0230 \\
\hline 7 & 16.655 & 1282.8 & 0.0229 & 20.908 & 1168.7 & 0.0229 \\
\hline 8 & 16.669 & 1275.1 & 0.0228 & 20.893 & 1164.3 & 0.0227 \\
\hline 9 & 16.669 & 1270.0 & 0.0227 & 20.877 & 1161.7 & 0.0226 \\
\hline 10 & 16.672 & 1267.3 & 0.0226 & 20.871 & 1160.2 & 0.0225 \\
\hline 11 & 16.681 & 1267.2 & 0.0224 & 20.879 & 1159.7 & 0.0224 \\
\hline 12 & 16.690 & 1270.0 & 0.0223 & 20.898 & 1160.6 & 0.0223 \\
\hline 13 & 16.674 & 1276.2 & 0.0222 & 20.911 & 1164.2 & 0.0222 \\
\hline 14 & 16.576 & 1285.2 & 0.0221 & 20.866 & 1171.6 & 0.0221 \\
\hline 15 & 16.263 & 1293.2 & 0.0220 & 20.621 & 1183.3 & 0.0219 \\
\hline 16 & 15.410 & 1291.5 & 0.0218 & 19.779 & 1194.2 & 0.0218 \\
\hline 17 & 13.327 & 1238.9 & 0.0217 & 17.401 & 1177.6 & 0.0217 \\
\hline 18 & 9.046 & 1074.0 & 0.0216 & 12.003 & 1052.1 & 0.0216 \\
\hline
\end{tabular}

Table 4-50. Burnup and TH Feedback Parameters by Axial Node for Assembly G4

\begin{tabular}{|c|c|c|c|c|c|c|c|c|c|}
\hline \multirow[b]{2}{*}{$\begin{array}{l}\text { Axial } \\
\text { Node }\end{array}$} & \multicolumn{3}{|c|}{ Statepoint 65 (30.0 Cy7) } & \multicolumn{3}{|c|}{ Statepolnt 66 (108.0 Cy7) } & \multicolumn{3}{|c|}{ Datapoint (227.4 Cy7) } \\
\hline & $\begin{array}{c}\text { Burnup } \\
\text { (GWd/MTU) }\end{array}$ & $\begin{array}{l}\text { T-Fuel } \\
\left({ }^{\circ} \mathrm{F}\right)\end{array}$ & $\begin{array}{c}\text { Spec. Vol. } \\
\left(\mathrm{ft}^{3} / \mathrm{lbm}\right)\end{array}$ & $\begin{array}{c}\text { Burnup } \\
\text { (GWd/MTU) }\end{array}$ & $\begin{array}{c}\text { T-Fuel } \\
\left({ }^{\circ} \mathrm{F}\right)\end{array}$ & $\begin{array}{c}\text { Spec. Vol. } \\
\left(\mathrm{ft}^{3} / / \mathrm{bm}\right)\end{array}$ & $\begin{array}{c}\text { Burnup } \\
\text { (GWd/MTU) } \\
\end{array}$ & $\begin{array}{c}\text { T-Fuel } \\
\left({ }^{\circ} \mathrm{F}\right)\end{array}$ & $\begin{array}{c}\text { Spec. Vol. } \\
\left(\mathrm{ft}^{3} / \mathrm{lbm}\right)\end{array}$ \\
\hline & $30.0 \mathrm{Cy} 7$ & $22.5 \mathrm{Cy} 7$ & 22.5 Cy7 & $108.0 \mathrm{Cy} 7$ & 60.4 Cy 7 & $60.4 \mathrm{Cy} 7$ & $227.4 \mathrm{Cy} 7$ & $164.6 \mathrm{Cy} 7$ & $164.6 \mathrm{Cy} 7$ \\
\hline 1 & 0.526 & 1021.4 & 0.0237 & 1.985 & 1045.2 & 0.0238 & 4.500 & 1081.8 & 0.0238 \\
\hline 2 & 0.843 & 1215.4 & 0.0236 & 3.205 & 1245.3 & 0.0237 & 7.246 & 1270.2 & 0.0237 \\
\hline 3 & 1.062 & 1354.2 & 0.0235 & 4.011 & 1379.0 & 0.0236 & 8.900 & 1375.4 & 0.0236 \\
\hline 4 & 1.198 & 1437.8 & 0.0234 & 4.487 & 1453.2 & 0.0234 & 9.759 & 1414.1 & 0.0235 \\
\hline 5 & 1.281 & 1486.6 & 0.0232 & 4.762 & 1489.7 & 0.0233 & 10.181 & 1421.2 & 0.0233 \\
\hline 6 & 1.333 & 1512.3 & 0.0231 & 4.927 & 1507.0 & 0.0231 & 10.391 & 1419.1 & 0.0232 \\
\hline 7 & 1.366 & 1525.7 & 0.0230 & 5.024 & 1515.3 & 0.0230 & 10.493 & 1414.0 & 0.0230 \\
\hline 8 & 1.385 & 1532.1 & 0.0228 & 5.079 & 1518.1 & 0.0228 & 10.539 & 1408.5 & 0.0229 \\
\hline 9 & 1.394 & 1533.8 & 0.0227 & 5.105 & 1517.6 & 0.0227 & 10.559 & 1403.9 & 0.0227 \\
\hline 10 & 1.397 & 1532.4 & 0.0225 & 5.114 & 1515.0 & 0.0226 & 10.570 & 1401.0 & 0.0226 \\
\hline 11 & 1.393 & 1528.2 & 0.0224 & 5.109 & 1510.9 & 0.0224 & 10.580 & 1399.9 & 0.0224 \\
\hline 12 & 1.381 & 1519.6 & 0.0223 & 5.079 & 1503.8 & 0.0223 & 10.576 & 1400.7 & 0.0223 \\
\hline 13 & 1.354 & 1503.3 & 0.0221 & 5.004 & 1490.7 & 0.0221 & 10.524 & 1402.1 & 0.0222 \\
\hline 14 & 1.306 & 1475.3 & 0.0220 & 4.855 & 1468.1 & 0.0220 & 10.366 & 1401.4 & 0.0221 \\
\hline 15 & 1.227 & 1424.7 & 0.0219 & 4.596 & 1428.9 & 0.0219 & 10.008 & 1392.7 & 0.0219 \\
\hline 16 & 1.102 & 1342.0 & 0.0218 & 4.158 & 1353.4 & 0.0218 & 9.264 & 1361.5 & 0.0218 \\
\hline 17 & 0.903 & 1210.0 & 0.0217 & 3.421 & 1225.3 & 0.0217 & 7.784 & 1264.8 & 0.0217 \\
\hline 18 & 0.602 & 1006.0 & 0.0216 & 2.264 & 1019.0 & 0.0216 & 5.176 & 1063.7 & 0.0216 \\
\hline
\end{tabular}


Table 4-50. Burnup and TH Feedback Parameters by Axial Node for Assembly G4 (Cont.)

\begin{tabular}{|c|c|c|c|c|c|c|}
\hline \multirow{2}{*}{$\begin{array}{c}\text { Axial } \\
\text { Node }\end{array}$} & $\begin{array}{c}\text { Burnup } \\
\text { (GWd/MTU) }\end{array}$ & $\begin{array}{c}\text { T-Fuel } \\
{ }^{\circ} \mathrm{F}\end{array}$ & $\begin{array}{c}\text { Spec. Vol. } \\
\mathrm{ft}^{3} / \mathrm{lbm}\end{array}$ & $\begin{array}{c}\text { Burnup } \\
\text { (GWd/MTU) }\end{array}$ & $\begin{array}{c}\text { T-Fuel } \\
{ }^{\circ} \mathrm{F}\end{array}$ & $\begin{array}{c}\text { Spec. Vol. } \\
\mathrm{ft}^{3} / \mathrm{bm}\end{array}$ \\
\hline & $367.3 \mathrm{Cy7}$ & $297.3 \mathrm{Cy} 7$ & $297.3 \mathrm{Cy} 7$ & $\mathrm{BOC}$ Cy8 & $430.3 \mathrm{Cy} 7$ & $430.3 \mathrm{Cy} 7$ \\
\hline 1 & 7.700 & 1085.4 & 0.0237 & 10.383 & 1064.5 & 0.0236 \\
\hline 2 & 12.220 & 1236.3 & 0.0236 & 16.200 & 1183.3 & 0.0235 \\
\hline 3 & 14.664 & 1302.1 & 0.0235 & 19.068 & 1212.9 & 0.0234 \\
\hline 4 & 15.752 & 1311.1 & 0.0234 & 20.199 & 1204.5 & 0.0232 \\
\hline 5 & 16.186 & 1302.1 & 0.0232 & 20.578 & 1191.0 & 0.0231 \\
\hline 6 & 16.350 & 1290.2 & 0.0231 & 20.684 & 1180.4 & 0.0230 \\
\hline 7 & 16.407 & 1280.0 & 0.0229 & 20.698 & 1173.4 & 0.0229 \\
\hline 8 & 16.422 & 1272.5 & 0.0228 & 20.687 & 1169.2 & 0.0227 \\
\hline 9 & 16.430 & 1267.5 & 0.0227 & 20.678 & 1166.6 & 0.0226 \\
\hline 10 & 16.449 & 1264.8 & 0.0225 & 20.687 & 1165.0 & 0.0225 \\
\hline 11 & 16.489 & 1264.5 & 0.0224 & 20.723 & 1164.3 & 0.0224 \\
\hline 12 & 16.541 & 1267.2 & 0.0223 & 20.781 & 1165.0 & 0.0223 \\
\hline 13 & 16.572 & 1273.5 & 0.0222 & 20.837 & 1168.3 & 0.0222 \\
\hline 14 & 16.510 & 1282.8 & 0.0221 & 20.824 & 1175.3 & 0.0221 \\
\hline 15 & 16.218 & 1290.7 & 0.0219 & 20.594 & 1186.5 & 0.0219 \\
\hline 16 & 15.376 & 1288.8 & 0.0218 & 19.760 & 1197.1 & 0.0218 \\
\hline 17 & 13.287 & 1236.2 & 0.0217 & 17.376 & 1180.6 & 0.0217 \\
\hline 18 & 8.991 & 1071.6 & 0.0216 & 11.955 & 1054.1 & 0.0216 \\
\hline
\end{tabular}

Table 4-51. Burnup and TH Feedback Parameters by Axial Node for Assembly G6

\begin{tabular}{|c|c|c|c|c|c|c|c|c|c|}
\hline \multirow[b]{2}{*}{$\begin{array}{l}\text { Axial } \\
\text { Node }\end{array}$} & \multicolumn{3}{|c|}{ Statepoint 65 (30.0 Cy7) } & \multicolumn{3}{|c|}{ Statepoint 66 (108.0 Cy7) } & \multicolumn{3}{|c|}{ Datapoint (227.4 Cy7) } \\
\hline & \begin{tabular}{|c|} 
Burnup \\
(GWd/MTU) \\
\end{tabular} & $\begin{array}{c}\text { T-Fuel } \\
\left({ }^{\circ} \mathrm{F}\right)\end{array}$ & $\begin{array}{c}\text { Spec. Vol. } \\
\left(\mathrm{ft}^{3} / \mathrm{lbm}\right)\end{array}$ & \begin{tabular}{|c|} 
Burnup \\
(GWd/MTU)
\end{tabular} & $\begin{array}{c}\text { T-Fuel } \\
\left({ }^{\circ} \mathrm{F}\right)\end{array}$ & $\begin{array}{l}\text { Spec. Vol. } \\
\left(\mathrm{ft}^{3} / \mathrm{lbm}\right)\end{array}$ & $\begin{array}{c}\text { Burnup } \\
\text { (GWd/MTU) } \\
\end{array}$ & $\begin{array}{c}\text { T-Fuel } \\
\left({ }^{\circ} \mathrm{F}\right)\end{array}$ & $\begin{array}{c}\text { Spec. Vol. } \\
\left(\mathrm{ft}^{3} / \mathrm{lbm}\right)\end{array}$ \\
\hline & 30.0 Cy 7 & $22.5 \mathrm{Cy} 7$ & $22.5 \mathrm{Cy} 7$ & $108.0 \mathrm{Cy} 7$ & 60.4 Cy 7 & $60.4 \mathrm{Cy} 7$ & 227.4 Cy7 & $164.6 \mathrm{Cy} 7$ & 164.6 Cy7 \\
\hline 1 & 0.535 & 1003.9 & 0.0237 & 1.997 & 1022.0 & 0.0237 & 4.540 & 1064.1 & 0.0238 \\
\hline 2 & 0.836 & 1206.5 & 0.0236 & 3.124 & 1227.5 & 0.0236 & 7.058 & 1257.5 & 0.0237 \\
\hline 3 & 1.053 & 1341.8 & 0.0235 & 3.897 & 1354.1 & 0.0235 & 8.643 & 1358.7 & 0.0236 \\
\hline 4 & 1.187 & 1422.6 & 0.0234 & 4.350 & 1423.2 & 0.0234 & 9.465 & 1397.7 & 0.0234 \\
\hline 5 & 1.268 & 1469.2 & 0.0232 & 4.608 & 1458.8 & 0.0232 & 9.866 & 1406.9 & 0.0233 \\
\hline 6 & 1.319 & 1495.0 & 0.0231 & 4.763 & 1476.4 & 0.0231 & 10.068 & 1406.0 & 0.0231 \\
\hline 7 & 1.352 & 1509.6 & 0.0229 & 4.857 & 1484.9 & 0.0229 & 10.170 & 1401.7 & 0.0230 \\
\hline 8 & 1.372 & 1516.3 & 0.0228 & 4.910 & 1487.6 & 0.0228 & 10.220 & 1396.8 & 0.0228 \\
\hline 9 & 1.383 & 1518.4 & 0.0227 & 4.938 & 1487.1 & 0.0227 & 10.244 & 1392.7 & 0.0227 \\
\hline 10 & 1.386 & 1517.7 & 0.0225 & 4.950 & 1485.1 & 0.0225 & 10.260 & 1390.1 & 0.0226 \\
\hline 11 & 1.385 & 1514.7 & 0.0224 & 4.952 & 1481.9 & 0.0224 & 10.280 & 1389.5 & 0.0224 \\
\hline 12 & 1.377 & 1508.3 & 0.0223 & 4.935 & 1476.7 & 0.0223 & 10.295 & 1390.7 & 0.0223 \\
\hline 13 & 1.355 & 1495.0 & 0.0221 & 4.880 & 1466.5 & 0.0221 & 10.271 & 1392.6 & 0.0222 \\
\hline 14 & 1.314 & 1469.4 & 0.0220 & 4.757 & 1446.5 & 0.0220 & 10.152 & 1392.5 & 0.0221 \\
\hline 15 & 1.242 & 1424.8 & 0.0219 & 4.531 & 1410.4 & 0.0219 & 9.843 & 1384.7 & 0.0219 \\
\hline 16 & 1.123 & 1347.6 & 0.0218 & 4.129 & 1342.1 & 0.0218 & 9.160 & 1353.4 & 0.0218 \\
\hline 17 & 0.929 & 1221.1 & 0.0217 & 3.435 & 1223.0 & 0.0217 & 7.772 & 1262.4 & 0.0217 \\
\hline 18 & 0.631 & 1024.3 & 0.0216 & 2.328 & 1028.6 & 0.0216 & 5.306 & 1074.1 & 0.0216 \\
\hline
\end{tabular}


Table 4-51. Burnup and TH Feedback Parameters by Axial Node for Assembly G6 (Cont.)

\begin{tabular}{|c|c|c|c|c|c|c|}
\hline \multirow[b]{2}{*}{$\begin{array}{l}\text { Axial } \\
\text { Node }\end{array}$} & \multicolumn{3}{|c|}{ Datapoint (367.3 Cy7) } & \multicolumn{3}{|c|}{ Statepoint 67 (BOC Cy8) } \\
\hline & $\begin{array}{c}\text { Burnup } \\
\text { (GWd/MTU) }\end{array}$ & $\begin{array}{c}\text { T-Fuel } \\
{ }^{\circ} \mathrm{F}\end{array}$ & $\begin{array}{c}\text { Spec. Vol. } \\
\mathrm{ft}^{3} / \mathrm{lbm}\end{array}$ & $\begin{array}{c}\text { Burnup } \\
\text { (GWd/MTU) }\end{array}$ & $\begin{array}{c}\text { T-Fuel } \\
{ }^{\circ} \mathrm{F}\end{array}$ & $\begin{array}{c}\text { Spec. Vol. } \\
\mathrm{ft}^{3} / \mathrm{lbm}\end{array}$ \\
\hline & 367.3 Cy7 & 297.3 Cy 7 & 297.3 Cy7 & BOC Сy8 & $430.3 \mathrm{Cy} 7$ & 430.3 Cy 7 \\
\hline 1 & 7.896 & 1086.1 & 0.0239 & 10.819 & 1080.5 & 0.0238 \\
\hline 2 & 12.103 & 1254.4 & 0.0237 & 16.323 & 1225.3 & 0.0237 \\
\hline 3 & 14.512 & 1325.0 & 0.0236 & 19.208 & 1260.8 & 0.0236 \\
\hline 4 & 15.600 & 1337.0 & 0.0235 & 20.369 & 1255.2 & 0.0234 \\
\hline 5 & 16.040 & 1330.9 & 0.0233 & 20.772 & 1243.3 & 0.0233 \\
\hline 6 & 16.218 & 1321.7 & 0.0232 & 20.903 & 1233.8 & 0.0231 \\
\hline 7 & 16.291 & 1313.7 & 0.0230 & 20.945 & 1227.9 & 0.0230 \\
\hline 8 & 16.325 & 1307.7 & 0.0229 & 20.962 & 1224.7 & 0.0229 \\
\hline 9 & 16.348 & 1303.9 & 0.0227 & 20.978 & 1223.1 & 0.0227 \\
\hline 10 & 16.382 & 1302.0 & 0.0226 & 21.010 & 1222.4 & 0.0226 \\
\hline 11 & 16.441 & 1302.3 & 0.0225 & 21.073 & 1222.5 & 0.0225 \\
\hline 12 & 16.524 & 1305.1 & 0.0223 & 21.170 & 1223.9 & 0.0224 \\
\hline 13 & 16.596 & 1310.7 & 0.0222 & 21.277 & 1227.7 & 0.0222 \\
\hline 14 & 16.582 & 1318.6 & 0.0221 & 21.322 & 1234.9 & 0.0221 \\
\hline 15 & 16.341 & 1325.8 & 0.0220 & 21.151 & 1245.9 & 0.0220 \\
\hline 16 & 15.549 & 1321.8 & 0.0218 & 20.361 & 1255.6 & 0.0219 \\
\hline 17 & 13.530 & 1271.5 & 0.0217 & 18.013 & 1239.2 & 0.0217 \\
\hline 18 & 9.379 & 1104.5 & 0.0216 & 12.664 & 1105.0 & 0.0216 \\
\hline
\end{tabular}

Table 4-52. Burnup and TH Feedback Parameters by Axial Node for Assembly G10

\begin{tabular}{|c|c|c|c|c|c|c|c|c|c|}
\hline \multirow[b]{2}{*}{$\begin{array}{l}\text { Axial } \\
\text { Node } \\
\end{array}$} & \multicolumn{3}{|c|}{ Statepoint 65 ( 30.0 Cy7) } & \multicolumn{3}{|c|}{ Statepoint 66 (108.0 Cy7) } & \multicolumn{3}{|c|}{ Datapoint (227.4 Cy7) } \\
\hline & $\begin{array}{c}\text { Burnup } \\
\text { (GWd/MTU) }\end{array}$ & $\begin{array}{l}\text { T-Fuel } \\
\left.\text { ( }{ }^{\circ} \mathrm{F}\right)\end{array}$ & $\begin{array}{c}\text { Spec. Vol. } \\
\left(\mathrm{ft}^{3} / / \mathrm{bm}\right)\end{array}$ & $\begin{array}{c}\text { Burnup } \\
\text { (GWd/MTU) }\end{array}$ & $\begin{array}{l}\text { T-Fuel } \\
\text { ('F) }\end{array}$ & $\begin{array}{c}\text { Spec. Vol. } \\
\left(\mathrm{ft}^{3} / / \mathrm{bm}\right)\end{array}$ & $\begin{array}{c}\text { Burnup } \\
\text { (GWd/MTU) }\end{array}$ & $\begin{array}{c}\text { T-Fuel } \\
\left({ }^{\circ} \mathrm{F}\right)\end{array}$ & $\begin{array}{c}\text { Spec. Vol } \\
\left(\mathrm{ft}^{3} / \mathrm{lbm}\right)\end{array}$ \\
\hline & $30.0 \mathrm{Cy} 7$ & $22.5 \mathrm{Cy} 7$ & 22.5 Cy 7 & $108.0 \mathrm{Cy} 7$ & 60.4 Cy 7 & $60.4 \mathrm{Cy} 7$ & 227.4 Cy7 & $164.6 \mathrm{Cy} 7$ & $164.6 \mathrm{Cy} 7$ \\
\hline 1 & 0.571 & 1058.1 & 0.0238 & 2.150 & 1079.7 & 0.0239 & 4.847 & 1109.6 & 0.0239 \\
\hline 2 & 0.900 & 1254.1 & 0.0237 & 3.411 & 1278.9 & 0.0238 & 7.656 & 1296.1 & 0.0238 \\
\hline 3 & 1.105 & 1384.6 & 0.0236 & 4.174 & 1406.8 & 0.0237 & 9.209 & 1393.1 & 0.0237 \\
\hline 4 & 1.235 & 1464.3 & 0.0234 & 4.633 & 1478.8 & 0.0235 & 10.024 & 1425.4 & 0.0235 \\
\hline 5 & 1.318 & 1510.9 & 0.0233 & 4.910 & 1512.0 & 0.0233 & 10.445 & 1431.9 & 0.0233 \\
\hline 6 & 1.371 & 1535.3 & 0.0231 & 5.081 & 1530.0 & 0.0232 & 10.661 & 1429.5 & 0.0232 \\
\hline 7 & 1.405 & 1549.1 & 0.0230 & 5.182 & 1538.8 & 0.0230 & 10.768 & 1424.1 & 0.0230 \\
\hline 8 & 1.425 & 1555.7 & 0.0228 & 5.239 & 1541.8 & 0.0229 & 10.817 & 1418.5 & 0.0229 \\
\hline 9 & 1.434 & 1557.3 & 0.0227 & 5.265 & 1541.2 & 0.0227 & 10.837 & 1413.9 & 0.0227 \\
\hline 10 & 1.436 & 1555.3 & 0.0225 & 5.272 & 1538.3 & 0.0226 & 10.846 & 1410.9 & 0.0226 \\
\hline 11 & 1.430 & 1549.9 & 0.0224 & 5.260 & 1533.1 & 0.0224 & 10.847 & 1409.8 & 0.0225 \\
\hline 12 & 1.414 & 1539.3 & 0.0223 & 5.219 & 1524.5 & 0.0223 & 10.827 & 1410.3 & 0.0223 \\
\hline 13 & 1.383 & 1520.7 & 0.0221 & 5.130 & 1509.6 & 0.0222 & 10.755 & 1411.4 & 0.0222 \\
\hline 14 & 1.331 & 1490.9 & 0.0220 & 4.968 & 1485.3 & 0.0220 & 10.578 & 1410.5 & 0.0221 \\
\hline 15 & 1.249 & 1440.9 & 0.0219 & 4.697 & 1446.0 & 0.0219 & 10.205 & 1401.9 & 0.0219 \\
\hline 16 & 1.120 & 1355.7 & 0.0218 & 4.245 & 1369.7 & 0.0218 & 9.442 & 1372.4 & 0.0218 \\
\hline 17 & 0.915 & 1219.8 & 0.0217 & 3.485 & 1237.5 & 0.0217 & 7.924 & 1274.9 & 0.0217 \\
\hline 18 & 0.601 & 1007.1 & 0.0216 & 2.275 & 1022.3 & 0.0216 & 5.211 & 1067.2 & 0.0216 \\
\hline
\end{tabular}


Table 4-52. Burnup and TH Feedback Parameters by Axial Node for Assembly G10 (Cont.)

\begin{tabular}{|c|c|c|c|c|c|c|}
\hline \multirow[b]{2}{*}{$\begin{array}{l}\text { Axial } \\
\text { Node }\end{array}$} & \multicolumn{3}{|c|}{ Datapoint (367.3 Cy7) } & \multicolumn{3}{|c|}{ Statepoint 67 (BOC Cy8) } \\
\hline & $\begin{array}{c}\text { Burnup } \\
\text { (GWd/MTU) }\end{array}$ & $\begin{array}{l}\text { T-Fuel } \\
{ }^{\circ} \mathrm{F}\end{array}$ & $\begin{array}{c}\text { Spec. Vol. } \\
\mathrm{tt}^{3} / \mathrm{lbm}\end{array}$ & $\begin{array}{c}\text { Burnup } \\
\text { (GWd/MTU) }\end{array}$ & $\begin{array}{l}\text { T-Fuel } \\
{ }^{\circ} \mathrm{F}\end{array}$ & $\begin{array}{c}\text { Spec. Vol. } \\
\mathrm{ft}^{3} / \mathrm{lbm}\end{array}$ \\
\hline & 367.3 Cy 7 & 297.3 Cy 7 & 297.3 Cy7 & BOC Cy8 & $430.3 \mathrm{Cy} 7$ & $430.3 \mathrm{Cy} 7$ \\
\hline 1 & 8.246 & 1105.2 & 0.0238 & 11.024 & 1077.1 & 0.0236 \\
\hline 2 & 12.813 & 1249.0 & 0.0236 & 16.837 & 1186.9 & 0.0235 \\
\hline 3 & 15.055 & 1305.8 & 0.0235 & 19.445 & 1208.1 & 0.0234 \\
\hline 4 & 16.054 & 1310.8 & 0.0234 & 20.469 & 1197.6 & 0.0232 \\
\hline 5 & 16.475 & 1300.6 & 0.0232 & 20.831 & 1183.6 & 0.0231 \\
\hline 6 & 16.642 & 1288.1 & 0.0231 & 20.936 & 1172.7 & 0.0230 \\
\hline 7 & 16.699 & 1277.6 & 0.0229 & 20.950 & 1165.6 & 0.0228 \\
\hline 8 & 16.715 & 1269.9 & 0.0228 & 20.938 & 1161.2 & 0.0227 \\
\hline 9 & 16.722 & 1264.7 & 0.0227 & 20.927 & 1158.5 & 0.0226 \\
\hline 10 & 16.738 & 1261.9 & 0.0225 & 20.933 & 1156.8 & 0.0225 \\
\hline 11 & 16.768 & 1261.5 & 0.0224 & 20.959 & 1156.1 & 0.0224 \\
\hline 12 & 16.802 & 1264.1 & 0.0223 & 20.999 & 1156.7 & 0.0223 \\
\hline 13 & 16.808 & 1270.1 & 0.0222 & 21.029 & 1159.9 & 0.0222 \\
\hline 14 & 16.725 & 1279.3 & 0.0221 & 20.995 & 1166.9 & 0.0221 \\
\hline 15 & 16.417 & 1287.7 & 0.0219 & 20.751 & 1178.2 & 0.0219 \\
\hline 16 & 15.559 & 1286.2 & 0.0218 & 19.902 & 1189.0 & 0.0218 \\
\hline 17 & 13.435 & 1234.8 & 0.0217 & 17.488 & 1173.2 & 0.0217 \\
\hline 18 & 9.020 & 1070.1 & 0.0216 & 11.959 & 1049.4 & 0.0216 \\
\hline
\end{tabular}

Table 4-53. Burnup and TH Feedback Parameters by Axial Node for Assembly G12

\begin{tabular}{|c|c|c|c|c|c|c|c|c|c|}
\hline \multirow[b]{2}{*}{$\begin{array}{l}\text { Axial } \\
\text { Node }\end{array}$} & \multicolumn{3}{|c|}{ Statepoint 65 (30.0 Cy7) } & \multicolumn{3}{|c|}{ Statepoint 66 (108.0 Cy7) } & \multicolumn{3}{|c|}{ Datapoint (227.4 Cy7) } \\
\hline & $\begin{array}{c}\text { Burnup } \\
\text { (GWd/MTU) }\end{array}$ & $\begin{array}{c}\text { T-Fuel } \\
\left({ }^{\circ} \mathrm{F}\right)\end{array}$ & $\begin{array}{c}\text { Spec. Vol. } \\
\left(\mathrm{ft}^{3} / \mathrm{lbm}\right)\end{array}$ & $\begin{array}{c}\text { Burnup } \\
\text { (GWd/MTU) }\end{array}$ & $\begin{array}{c}\text { T-Fuel } \\
\left({ }^{\circ} \mathrm{F}\right) \\
\end{array}$ & $\begin{array}{c}\text { Spec. Vol. } \\
\left(\mathrm{ft}^{3} / \mathrm{lbm}\right)\end{array}$ & $\begin{array}{c}\text { Burnup } \\
\text { (GWd/MTU) }\end{array}$ & $\begin{array}{c}\text { T-Fuel } \\
\left({ }^{\circ} \mathrm{F}\right)\end{array}$ & $\begin{array}{c}\text { Spec. Vol. } \\
\left(\mathrm{ft}^{3} / \mathrm{lbm}\right)\end{array}$ \\
\hline & $30.0 \mathrm{Cy} 7$ & $22.5 \mathrm{Cy} 7$ & 22.5 Cy 7 & $108.0 \mathrm{Cy} 7$ & 60.4 Cy 7 & $60.4 \mathrm{Cy} 7$ & 227.4 Cy7 & $164.6 \mathrm{Cy} 7$ & 164.6 Cy? \\
\hline 1 & 0.508 & 1005.8 & 0.0237 & 1.904 & 1026.4 & 0.0238 & 4.319 & 1064.3 & 0.0238 \\
\hline 2 & 0.847 & 1214.4 & 0.0236 & 3.181 & 1238.2 & 0.0237 & 7.141 & 1257.1 & 0.0237 \\
\hline 3 & 1.070 & 1354.5 & 0.0235 & 3.985 & 1370.1 & $\underline{0.0236}$ & 8.764 & 1357.7 & 0.0235 \\
\hline 4 & 1.203 & 1434.1 & 0.0234 & 4.433 & 1438.4 & 0.0234 & 9.557 & 1393.3 & 0.0234 \\
\hline 5 & 1.282 & 1480.4 & 0.0233 & 4.687 & 1473.0 & 0.0233 & 9.925 & 1399.0 & 0.0232 \\
\hline 6 & 1.333 & 1506.3 & 0.0231 & 4.843 & 1489.8 & 0.0231 & 10.110 & 1395.9 & 0.0231 \\
\hline 7 & 1.366 & 1519.9 & 0.0230 & 4.938 & 1497.6 & 0.0230 & 10.205 & 1390.6 & 0.0230 \\
\hline 8 & 1.386 & 1526.6 & 0.0228 & 4.992 & 1500.3 & 0.0228 & 10.250 & 1385.0 & 0.0228 \\
\hline 9 & 1.396 & 1528.9 & 0.0227 & 5.021 & 1500.0 & 0.0227 & 10.272 & 1380.4 & 0.0227 \\
\hline 10 & 1.401 & 1528.9 & 0.0225 & 5.037 & 1498.5 & 0.0225 & 10.292 & 1377.7 & 0.0226 \\
\hline 11 & 1.403 & 1528.7 & 0.0224 & 5.055 & 1497.4 & 0.0224 & 10.329 & 1377.4 & 0.0224 \\
\hline 12 & 1.403 & 1527.5 & 0.0223 & 5.070 & 1496.5 & 0.0223 & 10.393 & 1380.4 & 0.0223 \\
\hline 13 & 1.389 & 1516.5 & 0.0221 & 5.033 & 1488.4 & 0.0221 & 10.417 & 1385.1 & 0.0222 \\
\hline 14 & 1.346 & 1491.2 & 0.0220 & 4.905 & 1468.7 & 0.0220 & 10.304 & 1386.4 & 0.0221 \\
\hline 15 & 1.269 & 1445.0 & 0.0219 & 4.660 & 1431.9 & 0.0219 & 9.978 & 1379.5 & 0.0219 \\
\hline 16 & 1.142 & 1362.3 & 0.0218 & 4.230 & 1359.8 & 0.0218 & 9.270 & 1349.2 & 0.0218 \\
\hline 17 & 0.934 & 1225.9 & 0.0217 & 3.480 & 1231.2 & 0.0217 & 7.806 & 1257.3 & 0.0217 \\
\hline 18 & 0.601 & 1002.6 & 0.0216 & 2.233 & 1010.5 & 0.0216 & 5.073 & 1052.9 & 0.0216 \\
\hline
\end{tabular}


Table 4-53. Burnup and TH Feedback Parameters by Axial Node for Assembly G12 (Cont.)

\begin{tabular}{|c|c|c|c|c|c|c|}
\hline \multirow[b]{2}{*}{$\begin{array}{l}\text { Axial } \\
\text { Node }\end{array}$} & \multicolumn{3}{|c|}{ Datapoint (367.3 Cy7) } & \multicolumn{3}{|c|}{ Statepoint 67 (BOC CyB) } \\
\hline & $\begin{array}{c}\text { Burnup } \\
\text { (GWd/MTU) }\end{array}$ & $\begin{array}{c}\text { T-Fuel } \\
{ }^{\circ} \mathrm{F}\end{array}$ & $\begin{array}{c}\text { Spec. Vol. } \\
\mathrm{ft}^{3} / \mathrm{bm}\end{array}$ & $\begin{array}{c}\text { Burnup } \\
\text { (GWd/MTU) }\end{array}$ & $\begin{array}{c}\text { T-Fuel } \\
{ }^{\circ} \mathrm{F} \\
\end{array}$ & $\begin{array}{c}\text { Spec. Vol } \\
\mathrm{tt}^{3} / \mathrm{lbm}\end{array}$ \\
\hline & 367.3 Cy7 & $297.3 \mathrm{Cy} 7$ & 297.3 Cy7 & BOC Cy 8 & $430.3 \mathrm{Cy} 7$ & $430.3 \mathrm{Cy} 7$ \\
\hline 1 & 7.440 & 1077.4 & 0.0237 & 10.111 & 1065.1 & 0.0236 \\
\hline 2 & 12.033 & 1228.2 & 0.0236 & 16.003 & 1182.8 & 0.0235 \\
\hline 3 & 14.423 & 1291.9 & 0.0235 & 18.819 & 1213.3 & 0.0234 \\
\hline 4 & 15.430 & 1300.4 & 0.0233 & 19.872 & 1205.5 & 0.0232 \\
\hline 5 & 15.792 & 1290.3 & 0.0232 & 20.178 & 1191.7 & 0.0231 \\
\hline 6 & 15.927 & 1278.4 & 0.0230 & 20.257 & 1181.3 & 0.0230 \\
\hline 7 & 15.980 & 1268.9 & 0.0229 & 20.272 & 1174.8 & 0.0229 \\
\hline 8 & 15.998 & 1261.9 & 0.0228 & 20.268 & 1171.0 & 0.0227 \\
\hline 9 & 16.010 & 1257.2 & 0.0227 & 20.266 & 1168.7 & 0.0226 \\
\hline 10 & 16.039 & 1254.7 & 0.0225 & 20.286 & 1167.3 & 0.0225 \\
\hline 11 & 16.108 & 1254.3 & 0.0224 & 20.350 & 1166.8 & 0.0224 \\
\hline 12 & 16.241 & 1257.8 & 0.0223 & 20.492 & 1168.3 & 0.0223 \\
\hline 13 & 16.383 & 1267.0 & 0.0222 & 20.670 & 1173.3 & 0.0222 \\
\hline 14 & 16.382 & 1277.4 & 0.0221 & 20.722 & 1180.9 & 0.0221 \\
\hline 15 & 16.125 & 1286.0 & 0.0219 & 20.526 & 1191.9 & 0.0219 \\
\hline 16 & 15.327 & 1283.3 & 0.0218 & 19.734 & 1201.8 & 0.0218 \\
\hline 17 & 13.278 & 1232.8 & 0.0217 & 17.392 & 1185.1 & 0.0217 \\
\hline 18 & 8.854 & 1069.5 & 0.0216 & 11.842 & 1059.7 & 0.0216 \\
\hline
\end{tabular}

Table 4-54. Burnup and TH Feedback Parameters by Axial Node for Assembly G12a

\begin{tabular}{|c|c|c|c|c|c|c|c|c|c|}
\hline \multirow[b]{2}{*}{$\begin{array}{l}\text { Axlal } \\
\text { Node }\end{array}$} & \multicolumn{3}{|c|}{ Statepoint 65 (30.0 Cy7) } & \multicolumn{3}{|c|}{ Statepoint $66(108.0 \mathrm{Cy} 7)$} & \multicolumn{3}{|c|}{ Datapoint (227.4 Cy7) } \\
\hline & $\begin{array}{c}\text { Burnup } \\
\text { (GWd/MTU) }\end{array}$ & $\begin{array}{l}\text { T-Fuel } \\
{ }^{\circ} \mathrm{F}\end{array}$ & $\begin{array}{l}\text { Spec. Vol. } \\
\mathrm{ft}^{3} / \mathrm{lbm}\end{array}$ & $\begin{array}{c}\text { Burnup } \\
\text { (GWd/MTU) }\end{array}$ & $\begin{array}{l}\text { T-Fuel } \\
{ }^{\circ} \mathrm{F}\end{array}$ & $\begin{array}{l}\text { Spec. Vol. } \\
\mathrm{ft}^{3} / \mathrm{lbm}\end{array}$ & $\begin{array}{c}\text { Burnup } \\
\text { (GWd/MTU) }\end{array}$ & $\begin{array}{c}\text { T-Fuel } \\
\text { of }\end{array}$ & $\begin{array}{l}\text { Spec. Vol. } \\
\mathrm{ft}^{3} / \mathrm{bm}\end{array}$ \\
\hline & 30.0 Cy7 & 22.5 Cy 7 & 22.5 Cy 7 & $108.0 \mathrm{Cy} 7$ & $60.4 \mathrm{Cy} 7$ & 60.4 Cy7 & 227.4 Cy7 & $164.6 \mathrm{Cy} 7$ & $164.6 \mathrm{Cy} 7$ \\
\hline 1 & 0.508 & 1005.8 & 0.0237 & 1.904 & 1026.4 & 0.0238 & 4.319 & 1064.3 & 0.0238 \\
\hline 2 & 0.847 & 1214.4 & 0.0236 & 3.181 & 1238.2 & 0.0237 & 7.141 & 1257.1 & 0.0237 \\
\hline 3 & 1.070 & 1354.5 & 0.0235 & 3.985 & 1370.1 & 0.0236 & 8.764 & 1357.7 & 0.0235 \\
\hline 4 & 1.203 & 1434.1 & 0.0234 & 4.433 & 1438.4 & 0.0234 & 9.557 & 1393.3 & 0.0234 \\
\hline 5 & 1.282 & 1480.4 & 0.0233 & 4.687 & 1473.0 & 0.0233 & 9.925 & 1399.0 & 0.0232 \\
\hline 6 & 1.333 & 1506.3 & 0.0231 & 4.843 & 1489.8 & 0.0231 & 10.110 & 1395.9 & 0.0231 \\
\hline 7 & 1.366 & 1519.9 & 0.0230 & 4.938 & 1497.6 & 0.0230 & 10.205 & 1390.6 & 0.0230 \\
\hline 8 & 1.386 & 1526.6 & 0.0228 & 4.992 & 1500.3 & 0.0228 & 10.250 & 1385.0 & 0.0228 \\
\hline 9 & 1.396 & 1528.9 & 0.0227 & 5.021 & 1500.0 & 0.0227 & 10.272 & 1380.4 & 0.0227 \\
\hline 10 & 1.401 & 1528.9 & 0.0225 & 5.037 & 1498.5 & 0.0225 & 10.292 & 1377.7 & 0.0226 \\
\hline 11 & 1.403 & 1528.7 & 0.0224 & 5.055 & 1497.4 & 0.0224 & 10.329 & 1377.4 & 0.0224 \\
\hline 12 & 1.403 & 1527.5 & 0.0223 & 5.070 & 1496.5 & 0.0223 & 10.393 & 1380.4 & 0.0223 \\
\hline 13 & 1.389 & 1516.5 & 0.0221 & 5.033 & 1488.4 & 0.0221 & 10.417 & 1385.1 & 0.0222 \\
\hline 14 & 1.346 & 1491.2 & 0.0220 & 4.905 & 1468.7 & 0.0220 & 10.304 & 1386.4 & 0.0221 \\
\hline 15 & 1.269 & 1445.0 & 0.0219 & 4.660 & 1431.9 & 0.0219 & 9.978 & 1379.5 & 0.0219 \\
\hline 16 & 1.142 & 1362.3 & 0.0218 & 4.230 & 1359.8 & 0.0218 & 9.270 & 1349.2 & 0.0218 \\
\hline 17 & 0.934 & 1225.9 & 0.0217 & 3.480 & 1231.2 & 0.0217 & 7.806 & 1257.3 & 0.0217 \\
\hline 18 & 0.601 & 1002.6 & 0.0216 & 2.233 & 1010.5 & 0.0216 & 5.073 & 1052.9 & 0.0216 \\
\hline
\end{tabular}


Table 4-54. Burnup and TH Feedback Parameters by Axial Node for Assembly G12a (Cont.)

\begin{tabular}{|c|c|c|c|c|c|c|}
\hline \multirow[b]{2}{*}{$\begin{array}{l}\text { Axial } \\
\text { Node }\end{array}$} & \multicolumn{3}{|c|}{ Datapoint (367.3 Cy7) } & \multicolumn{3}{|c|}{ Statepoint 67 (BOC Cy8) } \\
\hline & $\begin{array}{c}\text { Burnup } \\
\text { (GWd/MTU) }\end{array}$ & $\begin{array}{c}\text { T-Fuel } \\
{ }^{\circ} \mathbf{F} \\
\end{array}$ & $\begin{array}{c}\text { Spec. Vol. } \\
\mathrm{ft}^{3} / \mathrm{lbm}\end{array}$ & $\begin{array}{c}\text { Burnup } \\
\text { (GWd/MTU) }\end{array}$ & $\begin{array}{c}\text { T-Fuel } \\
{ }^{\circ} \mathrm{F} \\
\end{array}$ & $\begin{array}{c}\text { Spec. Vol. } \\
\mathrm{ft}^{3} / \mathrm{lbm}\end{array}$ \\
\hline & $367.3 \mathrm{Cy} 7$ & 297.3 Cy 7 & 297.3 Cy 7 & BOC Cy8 & 430.3 Cy7 & 430.3 Cy 7 \\
\hline 1 & 7.440 & 1077.4 & 0.0237 & 10.097 & 1065.1 & 0.0236 \\
\hline 2 & 12.033 & 1228.2 & 0.0236 & 15.981 & 1182.8 & 0.0235 \\
\hline 3 & 14.423 & 1291.9 & 0.0235 & 18.792 & 1213.3 & 0.0234 \\
\hline 4 & 15.430 & 1300.4 & 0.0233 & 19.842 & 1205.5 & 0.0232 \\
\hline 5 & 15.792 & 1290.3 & 0.0232 & 20.147 & 1191.7 & 0.0231 \\
\hline 6 & 15.927 & 1278.4 & 0.0230 & 20.226 & 1181.3 & 0.0230 \\
\hline 7 & 15.980 & 1268.9 & 0.0229 & 20.240 & 1174.8 & 0.0229 \\
\hline 8 & 15.998 & 1261.9 & 0.0228 & 20.235 & 1171.0 & 0.0227 \\
\hline 9 & 16.010 & 1257.2 & 0.0227 & 20.234 & 1168.7 & 0.0226 \\
\hline 10 & 16.039 & 1254.7 & 0.0225 & 20.253 & 1167.3 & 0.0225 \\
\hline 11 & 16.108 & 1254.3 & 0.0224 & 20.317 & 1166.8 & 0.0224 \\
\hline 12 & 16.241 & 1257.8 & 0.0223 & 20.458 & 1168.3 & 0.0223 \\
\hline 13 & 16.383 & 1267.0 & 0.0222 & 20.636 & 1173.3 & 0.0222 \\
\hline 14 & 16.382 & 1277.4 & 0.0221 & 20.686 & 1180.9 & 0.0221 \\
\hline 15 & 16.125 & 1286.0 & 0.0219 & 20.491 & 1191.9 & 0.0219 \\
\hline 16 & 15.327 & 1283.3 & 0.0218 & 19.700 & 1201.8 & 0.0218 \\
\hline 17 & 13.278 & 1232.8 & 0.0217 & 17.362 & 1185.1 & 0.0217 \\
\hline 18 & 8.854 & 1069.5 & 0.0216 & 11.822 & 1059.7 & 0.0216 \\
\hline
\end{tabular}

Table 4-55. Burnup and TH Feedback Parameters by Axial Node for Assembly G14

\begin{tabular}{|c|c|c|c|c|c|c|c|c|c|}
\hline \multirow[b]{2}{*}{$\begin{array}{l}\text { Axial } \\
\text { Node }\end{array}$} & \multicolumn{3}{|c|}{ Statepoint 65 (30.0 Cy7) } & \multicolumn{3}{|c|}{ Statepoint 66 (108.0 Cy7) } & \multicolumn{3}{|c|}{ Datapoint (227.4 Cy7) } \\
\hline & \begin{tabular}{|c|} 
Burnup \\
(GWd/MTU)
\end{tabular} & $\begin{array}{l}\text { T-Fuel } \\
{ }^{\circ} \mathrm{F}\end{array}$ & $\begin{array}{l}\text { Spec. Vol. } \\
\mathrm{ft}^{3} / \mathrm{lbm}\end{array}$ & $\begin{array}{c}\text { Burnup } \\
\text { (GWd/MTU) }\end{array}$ & $\begin{array}{l}\text { TrFuel } \\
{ }^{\circ} \mathrm{F}\end{array}$ & $\begin{array}{l}\text { Spec. Vol. } \\
\mathrm{ft}^{3} / \mathrm{lbm}\end{array}$ & $\begin{array}{c}\text { Burnup } \\
\text { (GWd/MTU) }\end{array}$ & $\begin{array}{l}\text { T-Fuel } \\
{ }^{\circ} \mathrm{F}\end{array}$ & $\begin{array}{l}\text { Spec. Vol. } \\
\mathrm{ft}^{3} / \mathrm{lbm}\end{array}$ \\
\hline & 30.0 Cy 7 & 22.5 Cy 7 & $22.5 \mathrm{Cy} 7$ & 108.0 Cy 7 & $60.4 \mathrm{Cy} 7$ & 60.4 Cy 7 & 227.4 Cy7 & $164.6 \mathrm{Cy} 7$ & $164.6 \mathrm{Cy} 7$ \\
\hline 1 & 0.455 & 937.7 & 0.0233 & 1.686 & 948.7 & 0.0233 & 3.843 & 990.0 & 0.0234 \\
\hline 2 & 0.748 & 1141.3 & 0.0233 & 2.757 & 1149.9 & 0.0233 & 6.171 & 1175.5 & 0.0233 \\
\hline 3 & 0.926 & 1251.2 & 0.0232 & 3.377 & 1252.1 & 0.0232 & 7.408 & 1253.5 & 0.0232 \\
\hline 4 & 1.033 & 1314.6 & 0.0231 & 3.729 & 1306.3 & 0.0231 & 8.025 & 1282.5 & 0.0231 \\
\hline 5 & 1.100 & 1352.6 & 0.0230 & 3.935 & 1335.3 & 0.0229 & 8.334 & 1290.0 & 0.0230 \\
\hline 6 & 1.144 & 1375.7 & 0.0228 & 4.060 & 1350.9 & 0.0228 & 8.490 & 1288.8 & 0.0228 \\
\hline 7 & 1.172 & 1389.4 & 0.0227 & 4.136 & 1358.6 & 0.0227 & 8.569 & 1284.7 & 0.0227 \\
\hline 8 & 1.189 & 1396.4 & 0.0226 & 4.179 & 1361.4 & 0.0226 & 8.605 & 1280.1 & 0.0226 \\
\hline 9 & 1.198 & 1398.9 & 0.0225 & 4.200 & 1361.1 & 0.0225 & 8.620 & 1276.1 & 0.0225 \\
\hline 10 & 1.200 & 1398.1 & 0.0224 & 4.208 & 1359.1 & 0.0224 & 8.627 & 1273.5 & 0.0224 \\
\hline 11 & 1.198 & 1394.8 & 0.0223 & 4.205 & 1355.7 & 0.0223 & 8.635 & 1272.8 & 0.0223 \\
\hline 12 & 1.189 & 1387.9 & 0.0222 & 4.186 & 1350.2 & 0.0221 & 8.637 & 1273.9 & 0.0222 \\
\hline 13 & 1.170 & 1374.1 & 0.0220 & 4.136 & 1339.9 & 0.0220 & 8.610 & 1275.7 & 0.0221 \\
\hline 14 & 1.134 & 1349.9 & 0.0219 & 4.033 & 1321.4 & 0.0219 & 8.507 & 1275.5 & 0.0220 \\
\hline 15 & 1.072 & 1310.0 & 0.0218 & 3.845 & 1289.1 & 0.0218 & 8.255 & 1267.5 & 0.0219 \\
\hline 16 & 0.969 & 1243.9 & 0.0218 & 3.508 & 1231.9 & 0.0218 & 7.695 & 1238.9 & 0.0218 \\
\hline 17 & 0.792 & 1129.7 & 0.0217 & 2.893 & 1126.8 & 0.0217 & 6.495 & 1160.8 & 0.0217 \\
\hline 18 & 0.493 & 922.9 & 0.0216 & 1.815 & 926.2 & 0.0216 & 4.159 & 976.9 & 0.0216 \\
\hline
\end{tabular}


Table 4-55. Burnup and TH Feedback Parameters by Axial Node for Assembly G14 (Cont.)

\begin{tabular}{|c|c|c|c|c|c|c|}
\hline \multirow[b]{2}{*}{$\begin{array}{l}\text { Axial } \\
\text { Node }\end{array}$} & \multicolumn{3}{|c|}{ Datapoint (367.3 Cy7) } & \multicolumn{3}{|c|}{ Statepoint 67 (BOC CyB) } \\
\hline & $\begin{array}{c}\text { Burnup } \\
\text { (GWd/MTU) }\end{array}$ & $\begin{array}{c}\text { T-Fuel } \\
{ }^{\circ} \mathbf{F} \\
\end{array}$ & $\begin{array}{c}\text { Spec. Vol. } \\
\mathrm{ft}^{3} / \mathrm{lbm}\end{array}$ & $\begin{array}{c}\text { Burnup } \\
\text { (GWd/MTU) }\end{array}$ & $\begin{array}{c}\text { T-Fuel } \\
{ }^{\circ} \mathrm{F} \\
\end{array}$ & $\begin{array}{c}\text { Spec. Vol } \\
\mathrm{tt}^{3} / \mathrm{lbm}\end{array}$ \\
\hline & 367.3 Cy & $297.3 \mathrm{Cy} 7$ & 297.3 Cy7 & BOC Cy 8 & 430.3 Cy 7 & 430.3 Cy7 \\
\hline 1 & 6.781 & 1031.1 & 0.0235 & 9.372 & 1044.1 & 0.0235 \\
\hline 2 & 10.598 & 1183.3 & 0.0234 & 14.314 & 1169.0 & 0.0234 \\
\hline 3 & 12.437 & 1233.9 & 0.0232 & 16.518 & 1198.9 & 0.0233 \\
\hline 4 & 13.232 & 1242.2 & 0.0231 & 17.365 & 1195.3 & 0.0232 \\
\hline 5 & 13.562 & 1236.5 & 0.0230 & 17.663 & 1185.7 & 0.0230 \\
\hline 6 & 13.695 & 1228.3 & 0.0229 & 17.757 & 1177.8 & 0.0229 \\
\hline 7 & 13.747 & 1221.0 & 0.0228 & 17.784 & 1172.8 & 0.0228 \\
\hline 8 & 13.768 & 1215.6 & 0.0227 & 17.789 & 1170.0 & 0.0227 \\
\hline 9 & 13.780 & 1212.1 & 0.0225 & 17.795 & 1168.7 & 0.0226 \\
\hline 10 & 13.800 & 1210.4 & 0.0224 & 17.813 & 1168.2 & 0.0225 \\
\hline 11 & 13.838 & 1210.7 & 0.0223 & 17.854 & 1168.7 & 0.0224 \\
\hline 12 & 13.893 & 1213.7 & 0.0222 & 17.922 & 1170.5 & 0.0223 \\
\hline 13 & 13.939 & 1219.6 & 0.0221 & 17.998 & 1174.6 & 0.0221 \\
\hline 14 & 13.921 & 1227.5 & 0.0220 & 18.028 & 1182.0 & 0.0220 \\
\hline 15 & 13.721 & 1234.2 & 0.0219 & 17.886 & 1192.1 & 0.0219 \\
\hline 16 & 13.070 & 1229.1 & 0.0218 & 17.229 & 1198.7 & 0.0218 \\
\hline 17 & 11.336 & 1180.9 & 0.0217 & 15.199 & 1174.7 & 0.0217 \\
\hline 18 & 7.486 & 1028.4 & 0.0216 & 10.275 & 1047.3 & 0.0216 \\
\hline
\end{tabular}

Table 4-56. Burnup and TH Feedback Parameters by Axial Node for Assembly G17

\begin{tabular}{|c|c|c|c|c|c|c|c|c|c|}
\hline \multirow[b]{2}{*}{$\begin{array}{l}\text { Axial } \\
\text { Node }\end{array}$} & \multicolumn{3}{|c|}{ Statepoint 65 (30.0 Cy7) } & \multicolumn{3}{|c|}{ Statepoint 66 (108.0 Cyn } & \multicolumn{3}{|c|}{ Datapoint (227.4 Cy7) } \\
\hline & $\begin{array}{c}\text { Burnup } \\
\text { (GWd/MTU) } \\
\end{array}$ & $\begin{array}{c}\text { T-Fuel } \\
{ }^{\circ} \mathbf{F}\end{array}$ & $\begin{array}{c}\text { Spec. Vol. } \\
\mathrm{ft}^{3} / \mathrm{lbm}\end{array}$ & $\begin{array}{c}\text { Burnup } \\
\text { (GWd/MTU) }\end{array}$ & $\begin{array}{c}\text { T-Fuel } \\
{ }^{\circ} \mathrm{F} \\
\end{array}$ & $\begin{array}{c}\text { Spec. Vol. } \\
\mathrm{ft}^{3} / \mathrm{bm}\end{array}$ & $\begin{array}{c}\text { Burnup } \\
\text { (GWd/MTU) }\end{array}$ & $\begin{array}{c}\text { T-Fuel } \\
{ }^{\circ} \mathbf{F} \\
\end{array}$ & $\begin{array}{c}\text { Spec. Vol. } \\
\mathrm{ft}^{3} / \mathrm{lbm}\end{array}$ \\
\hline & $30.0 \mathrm{Cy} 7$ & 22.5 Cy7 & $22.5 \mathrm{Cy} 7$ & $108.0 \mathrm{Cy} 7$ & $60.4 \mathrm{Cy} 7$ & 60.4 Cy7 & $227.4 \mathrm{Cy} 7$ & $164.6 \mathrm{Cy} 7$ & $164.6 \mathrm{Cy} 7$ \\
\hline 1 & 0.557 & 1045.3 & 0.0238 & 2.082 & 1063.9 & 0.0238 & 4.685 & 1093.9 & 0.0238 \\
\hline 2 & 0.889 & 1243.6 & 0.0237 & 3.337 & 1263.8 & 0.0237 & 7.460 & 1278.0 & 0.0237 \\
\hline 3 & 1.100 & 1375.8 & 0.0236 & 4.103 & 1390.9 & 0.0236 & 9.006 & 1373.6 & 0.0236 \\
\hline 4 & 1.228 & 1453.3 & 0.0234 & 4.543 & 1459.2 & 0.0235 & 9.779 & 1406.9 & 0.0234 \\
\hline 5 & 1.308 & 1499.6 & 0.0233 & 4.803 & 1492.3 & 0.0233 & 10.151 & 1411.0 & 0.0233 \\
\hline 6 & 1.361 & 1524.3 & 0.0232 & 4.965 & 1509.0 & 0.0232 & 10.342 & 1407.2 & 0.0231 \\
\hline 7 & 1.395 & 1538.4 & 0.0230 & 5.065 & 1517.3 & 0.0230 & 10.441 & 1401.5 & 0.0230 \\
\hline 8 & 1.416 & 1545.6 & 0.0229 & 5.123 & 1520.4 & 0.0229 & 10.489 & 1395.9 & 0.0228 \\
\hline 9 & 1.428 & 1548.5 & 0.0227 & 5.155 & 1520.4 & 0.0227 & 10.515 & 1391.3 & 0.0227 \\
\hline 10 & 1.434 & 1549.0 & 0.0226 & 5.176 & 1519.2 & 0.0226 & 10.539 & 1388.5 & 0.0226 \\
\hline 11 & 1.437 & 1549.2 & 0.0224 & 5.196 & 1518.5 & 0.0224 & 10.582 & 1387.9 & 0.0224 \\
\hline 12 & 1.437 & 1548.2 & 0.0223 & 5.213 & 1517.6 & 0.0223 & 10.648 & 1390.7 & 0.0223 \\
\hline 13 & 1.422 & 1536.5 & 0.0221 & 5.173 & 1509.1 & 0.0222 & 10.671 & 1395.6 & 0.0222 \\
\hline 14 & 1.377 & 1509.6 & 0.0220 & 5.036 & 1488.1 & 0.0220 & 10.547 & 1397.1 & 0.0221 \\
\hline 15 & 1.295 & 1463.9 & 0.0219 & 4.773 & 1450.6 & 0.0219 & 10.199 & 1390.1 & 0.0219 \\
\hline 16 & 1.161 & 1376.4 & 0.0218 & 4.316 & 1375.9 & 0.0218 & 9.446 & 1360.9 & 0.0218 \\
\hline 17 & 0.944 & 1234.3 & 0.0217 & 3.533 & 1241.5 & 0.0217 & 7.917 & 1265.0 & 0.0217 \\
\hline 18 & 0.615 & 1014.4 & 0.0216 & 2.292 & 1022.8 & 0.0216 & 5.184 & 1059.9 & 0.0216 \\
\hline
\end{tabular}


Table 4-56. Burnup and TH Feedback Parameters by Axial Node for Assembly G17 (Cont.)

\begin{tabular}{|c|c|c|c|c|c|c|}
\hline \multirow[b]{2}{*}{$\begin{array}{l}\text { Axial } \\
\text { Node }\end{array}$} & \multicolumn{3}{|c|}{ Datapoint (367.3 Cy7) } & \multicolumn{3}{|c|}{ Statepoint 67 (BOC Cy8) } \\
\hline & $\begin{array}{c}\text { Burnup } \\
\text { (GWd/MTU) }\end{array}$ & $\begin{array}{c}\text { T-Fuel } \\
{ }^{\circ} \mathrm{F} \\
\end{array}$ & $\begin{array}{c}\text { Spec. Vol. } \\
\mathrm{ft}^{3} / \mathrm{lbm}\end{array}$ & $\begin{array}{l}\text { Burnup } \\
\text { (GWd/MTU) }\end{array}$ & $\begin{array}{c}\text { T-Fuel } \\
{ }^{\circ} \mathrm{F}\end{array}$ & $\begin{array}{c}\text { Spec. Vol. } \\
\mathrm{ft}^{3} / \mathrm{lbm}\end{array}$ \\
\hline & 367.3 Cy7 & 297.3 Cy 7 & 297.3 Cy 7 & BOC Cy 8 & $430.3 \mathrm{Cy} 7$ & $430.3 \mathrm{Cy} 7$ \\
\hline 1 & 7.997 & 1096.3 & 0.0237 & 10.760 & 1075.7 & 0.0236 \\
\hline 2 & 12.510 & 1239.1 & 0.0236 & 16.527 & 1186.7 & 0.0235 \\
\hline 3 & 14.748 & 1297.1 & 0.0235 & 19.144 & 1209.6 & 0.0234 \\
\hline 4 & 15.701 & 1302.3 & 0.0233 & 20.127 & 1199.5 & 0.0232 \\
\hline 5 & 16.053 & 1290.7 & 0.0232 & 20.419 & 1184.9 & 0.0231 \\
\hline 6 & 16.186 & 1277.8 & 0.0230 & 20.493 & 1174.0 & 0.0230 \\
\hline 7 & 16.237 & 1267.6 & 0.0229 & 20.504 & 1167.1 & 0.0228 \\
\hline 8 & 16.255 & 1260.2 & 0.0228 & 20.497 & 1163.0 & 0.0227 \\
\hline 9 & 16.269 & 1255.2 & 0.0227 & 20.495 & 1160.5 & 0.0226 \\
\hline 10 & 16.301 & 1252.3 & 0.0225 & 20.516 & 1158.9 & 0.0225 \\
\hline 11 & 16.373 & 1251.7 & 0.0224 & 20.582 & 1158.2 & 0.0224 \\
\hline 12 & 16.509 & 1255.1 & 0.0223 & 20.724 & 1159.5 & 0.0223 \\
\hline 13 & 16.650 & 1264.2 & 0.0222 & 20.898 & 1164.5 & 0.0222 \\
\hline 14 & 16.640 & 1274.8 & 0.0221 & 20.939 & 1172.0 & 0.0221 \\
\hline 15 & 16.361 & 1283.8 & 0.0219 & 20.721 & 1183.1 & 0.0219 \\
\hline 16 & 15.517 & 1281.8 & 0.0218 & 19.884 & 1193.6 & 0.0218 \\
\hline 17 & 13.389 & 1230.9 & 0.0217 & 17.464 & 1177.8 & 0.0217 \\
\hline 18 & 8.965 & 1067.5 & 0.0216 & 11.920 & 1053.2 & 0.0216 \\
\hline
\end{tabular}

Table 4-57. Burnup and TH Feedback Parameters by Axial Node for Assembly G19

\begin{tabular}{|c|c|c|c|c|c|c|c|c|c|}
\hline \multirow[b]{2}{*}{$\begin{array}{l}\text { Axlal } \\
\text { Node }\end{array}$} & \multicolumn{3}{|c|}{ Statepoint 65 (30.0 Cy7) } & \multicolumn{3}{|c|}{ Statepoint 66 (108.0 Cy7) } & \multicolumn{3}{|c|}{ Datapoint (227.4 Cy7) } \\
\hline & $\begin{array}{c}\text { Burnup } \\
\text { (GWd/MTU) }\end{array}$ & $\begin{array}{c}\text { T-Fuel } \\
{ }^{\circ} \mathrm{F}\end{array}$ & $\begin{array}{c}\text { Spec. Vol. } \\
\mathrm{ft}^{3} / \mathrm{lbm}\end{array}$ & $\begin{array}{c}\text { Burnup } \\
\text { (GWd/MTU) }\end{array}$ & $\begin{array}{c}\text { T-Fuel } \\
{ }^{\circ} \mathrm{F}\end{array}$ & $\begin{array}{c}\text { Spec. Vol. } \\
\mathrm{ft}^{3} / \mathrm{lbm}\end{array}$ & $\begin{array}{c}\text { Burnup } \\
\text { (GWd/MTU) }\end{array}$ & $\begin{array}{c}\text { T-Fuel } \\
{ }^{\circ} \mathrm{F}\end{array}$ & $\begin{array}{c}\text { Spec. Vol. } \\
\mathrm{ft}^{3} / \mathrm{lbm}\end{array}$ \\
\hline & $30.0 \mathrm{Cy} 7$ & $22.5 \mathrm{Cy} 7$ & $22.5 \mathrm{Cy} 7$ & $108.0 \mathrm{Cy} 7$ & $60.4 \mathrm{Cy} 7$ & $60.4 \mathrm{Cy} 7$ & $227.4 \mathrm{Cy} 7$ & 164.6 Cy7 & $164.6 \mathrm{Cy} 7$ \\
\hline 1 & 0.552 & 1014.8 & 0.0237 & 2.043 & 1027.6 & 0.0237 & 4.612 & 1064.4 & 0.0237 \\
\hline 2 & 0.846 & 1210.5 & 0.0236 & 3.129 & 1222.3 & 0.0236 & 7.011 & 1245.9 & 0.0236 \\
\hline 3 & 1.044 & 1332.8 & 0.0235 & 3.829 & 1337.3 & 0.0235 & 8.432 & 1335.9 & 0.0235 \\
\hline 4 & 1.166 & 1404.4 & 0.0233 & 4.230 & 1398.6 & 0.0233 & 9.151 & 1369.9 & 0.0233 \\
\hline 5 & 1.242 & 1448.0 & 0.0232 & 4.467 & 1431.8 & 0.0232 & 9.496 & 1377.0 & 0.0232 \\
\hline 6 & 1.292 & 1475.4 & 0.0231 & 4.616 & 1450.1 & 0.0230 & 9.678 & 1375.0 & 0.0231 \\
\hline 7 & 1.326 & 1490.6 & 0.0229 & 4.709 & 1458.9 & 0.0229 & 9.778 & 1370.6 & 0.0229 \\
\hline 8 & 1.347 & 1499.0 & 0.0228 & 4.767 & 1462.7 & 0.0228 & 9.832 & 1366.0 & 0.0228 \\
\hline 9 & 1.360 & 1502.6 & 0.0226 & 4.800 & 1463.4 & 0.0226 & 9.864 & 1362.2 & 0.0227 \\
\hline 10 & 1.367 & 1503.8 & 0.0225 & 4.822 & 1462.8 & 0.0225 & 9.893 & 1360.0 & 0.0225 \\
\hline 11 & 1.371 & 1504.9 & 0.0224 & 4.846 & 1462.8 & 0.0224 & 9.941 & 1360.4 & 0.0224 \\
\hline 12 & 1.374 & 1505.4 & 0.0223 & 4.871 & 1463.4 & 0.0223 & 10.018 & 1364.4 & 0.0223 \\
\hline 13 & 1.362 & 1495.8 & 0.0221 & 4.844 & 1456.7 & 0.0221 & 10.058 & 1370.1 & 0.0222 \\
\hline 14 & 1.323 & 1470.9 & 0.0220 & 4.730 & 1437.5 & 0.0220 & 9.961 & 1371.6 & 0.0220 \\
\hline 15 & 1.250 & 1426.0 & 0.0219 & 4.501 & 1401.2 & 0.0219 & 9.655 & 1363.3 & 0.0219 \\
\hline 16 & 1.126 & 1346.2 & 0.0218 & 4.092 & 1332.2 & 0.0218 & 8.968 & 1330.7 & 0.0218 \\
\hline 17 & 0.924 & 1215.7 & 0.0217 & 3.383 & 1211.7 & 0.0217 & 7.573 & 1240.9 & 0.0217 \\
\hline 18 & 0.621 & 1015.5 & 0.0216 & 2.272 & 1016.5 & 0.0216 & 5.134 & 1056.8 & 0.0216 \\
\hline
\end{tabular}


Table 4-57. Burnup and TH Feedback Parameters by Axial Node for Assembly G19 (Cont.)

\begin{tabular}{|c|c|c|c|c|c|c|}
\hline \multirow[b]{2}{*}{$\begin{array}{l}\text { Axial } \\
\text { Node }\end{array}$} & \multicolumn{3}{|c|}{ Datapoint (367.3 Cy7) } & \multicolumn{3}{|c|}{ Statepoint 67 (BOC Cy8) } \\
\hline & $\begin{array}{c}\text { Burnup } \\
\text { (GWd/MTU) }\end{array}$ & $\begin{array}{c}\text { T-Fuel } \\
\quad{ }^{\circ} \mathrm{F} \\
\end{array}$ & $\begin{array}{c}\text { Spec. Vol. } \\
\mathrm{ft}^{3} / \mathrm{lbm}\end{array}$ & $\begin{array}{c}\text { Burnup } \\
\text { (GWd/MTU) }\end{array}$ & $\begin{array}{c}\text { T-Fuel } \\
{ }^{\circ} \mathrm{F} \\
\end{array}$ & $\begin{array}{c}\text { Spec. Vol. } \\
\mathrm{ft}^{3} / / \mathrm{bm}\end{array}$ \\
\hline & $367.3 \mathrm{Cy}$ & 297.3 Cy7 & 297.3 Cy? & BOC Cy 8 & $430.3 \mathrm{Cy} 7$ & $430.3 \mathrm{Cy} 7$ \\
\hline 1 & 8.003 & 1086.6 & 0.0238 & 10.936 & 1082.9 & 0.0238 \\
\hline 2 & 12.001 & 1244.5 & 0.0237 & 16.163 & 1220.0 & 0.0237 \\
\hline 3 & 14.135 & 1305.7 & 0.0235 & 18.724 & 1250.1 & 0.0235 \\
\hline 4 & 15.069 & 1316.1 & 0.0234 & 19.719 & 1243.5 & 0.0234 \\
\hline 5 & 15.420 & 1308.0 & 0.0232 & 20.028 & 1230.8 & 0.0232 \\
\hline 6 & 15.566 & 1297.8 & 0.0231 & 20.129 & 1221.3 & 0.0231 \\
\hline 7 & 15.637 & 1289.5 & 0.0230 & 20.172 & 1215.6 & 0.0230 \\
\hline 8 & 15.677 & 1283.7 & 0.0228 & 20.198 & 1212.7 & 0.0228 \\
\hline 9 & 15.710 & 1280.1 & 0.0227 & 20.226 & 1211.2 & 0.0227 \\
\hline 10 & 15.757 & 1278.4 & 0.0226 & 20.271 & 1210.7 & 0.0226 \\
\hline 11 & 15.845 & 1278.9 & 0.0224 & 20.361 & 1211.0 & 0.0225 \\
\hline 12 & 16.002 & 1283.2 & 0.0223 & 20.535 & 1213.3 & 0.0223 \\
\hline 13 & 16.172 & 1292.6 & 0.0222 & 20.749 & 1219.3 & 0.0222 \\
\hline 14 & 16.192 & 1301.9 & 0.0221 & 20.829 & 1227.3 & 0.0221 \\
\hline 15 & 15.951 & 1309.1 & 0.0220 & 20.654 & 1238.2 & 0.0220 \\
\hline 16 & 15.151 & 1304.4 & 0.0218 & 19.852 & 1247.5 & 0.0219 \\
\hline 17 & 13.133 & 1250.7 & 0.0217 & 17.502 & 1228.8 & 0.0217 \\
\hline 18 & 9.053 & 1088.2 & 0.0216 & 12.243 & 1093.6 & 0.0216 \\
\hline
\end{tabular}

Table 4-58. Burnup and TH Feedback Parameters by Axial Node for Assembly G23

\begin{tabular}{|c|c|c|c|c|c|c|c|c|c|}
\hline \multirow[b]{2}{*}{$\begin{array}{l}\text { Axial } \\
\text { Node }\end{array}$} & \multicolumn{3}{|c|}{ Statepoint 65 (30.0 Cy7) } & \multicolumn{3}{|c|}{ Statepoint 66 (108.0 Cy7) } & \multicolumn{3}{|c|}{ Datapoint (227.4 Cyn) } \\
\hline & $\begin{array}{c}\text { Burnup } \\
\text { (GWd/MTU) }\end{array}$ & $\begin{array}{c}\text { T-Fuel } \\
{ }^{\circ} \mathrm{F}\end{array}$ & $\begin{array}{c}\text { Spec. Vol. } \\
\mathrm{ft}^{3} / \mathrm{lbm}\end{array}$ & $\begin{array}{c}\text { Burnup } \\
\text { (GWd/MTU) } \\
\end{array}$ & $\begin{array}{l}\text { T-Fuel } \\
{ }^{\circ} \mathrm{F} \\
\end{array}$ & $\begin{array}{c}\text { Spec. Vol. } \\
\mathrm{ft}^{3} / \mathrm{lbm}\end{array}$ & $\begin{array}{c}\text { Burnup } \\
\text { (GWd/MTU) }\end{array}$ & $\begin{array}{c}\text { T-Fuel } \\
{ }^{\circ} \mathrm{F}\end{array}$ & $\begin{array}{c}\text { Spec. Vol. } \\
\mathrm{ft}^{3} / \mathrm{lbm}\end{array}$ \\
\hline & 30.0 Cy 7 & $22.5 \mathrm{Cy} 7$ & 22.5 Cy7 & 108.0 Cy7 & $60.4 \mathrm{Cy} 7$ & 60.4 Cy7 & $227.4 \mathrm{Cy} 7$ & 164.6 Cy7 & 164.6 Cy7 \\
\hline 1 & 0.487 & 989.6 & 0.0238 & 1.811 & 1004.7 & 0.0237 & 4.079 & 1035.7 & 0.0236 \\
\hline 2 & 0.851 & 1214.6 & 0.0237 & 3.144 & 1227.4 & 0.0237 & 6.934 & 1229.2 & 0.0235 \\
\hline 3 & 1.093 & 1363.5 & 0.0236 & 3.978 & 1362.0 & 0.0235 & 8.555 & 1324.9 & 0.0234 \\
\hline 4 & 1.237 & 1447.9 & 0.0235 & 4.439 & 1430.2 & 0.0234 & 9.337 & 1357.1 & 0.0233 \\
\hline 5 & 1.323 & 1496.1 & 0.0233 & 4.699 & 1464.6 & 0.0233 & 9.694 & 1361.7 & 0.0232 \\
\hline 6 & 1.379 & 1522.2 & 0.0232 & 4.858 & 1481.6 & 0.0231 & 9.873 & 1357.7 & 0.0230 \\
\hline 7 & 1.416 & 1537.0 & 0.0230 & 4.957 & 1489.2 & 0.0230 & 9.967 & 1351.7 & 0.0229 \\
\hline 8 & 1.440 & 1545.1 & 0.0229 & 5.017 & 1492.1 & 0.0228 & 10.017 & 1345.8 & 0.0228 \\
\hline 9 & 1.454 & 1549.1 & 0.0227 & 5.054 & 1492.5 & 0.0227 & 10.046 & 1341.2 & 0.0226 \\
\hline 10 & 1.463 & 1550.9 & 0.0226 & 5.080 & 1492.0 & 0.0225 & 10.076 & 1338.5 & 0.0225 \\
\hline 11 & 1.470 & 1553.0 & 0.0224 & 5.111 & 1492.6 & 0.0224 & 10.128 & 1338.7 & 0.0224 \\
\hline 12 & 1.475 & 1554.4 & 0.0223 & 5.142 & 1493.6 & 0.0223 & 10.210 & 1343.0 & 0.0223 \\
\hline 13 & 1.464 & 1545.2 & 0.0222 & 5.118 & 1487.6 & 0.0221 & 10.254 & 1349.7 & 0.0221 \\
\hline 14 & 1.422 & 1520.5 & 0.0220 & 5.000 & 1469.8 & 0.0220 & 10.160 & 1352.5 & 0.0220 \\
\hline 15 & 1.340 & 1476.4 & 0.0219 & 4.755 & 1434.8 & 0.0219 & 9.850 & 1346.1 & 0.0219 \\
\hline 16 & 1.201 & 1391.2 & 0.0218 & 4.312 & 1363.5 & 0.0218 & 9.150 & 1316.2 & 0.0218 \\
\hline 17 & 0.967 & 1241.6 & 0.0217 & 3.513 & 1230.1 & 0.0217 & 7.662 & 1229.1 & 0.0217 \\
\hline 18 & 0.592 & 994.0 & 0.0216 & 2.165 & 994.3 & 0.0216 & 4.841 & 1027.9 & 0.0216 \\
\hline
\end{tabular}


Table 4-58. Burnup and TH Feedback Parameters by Axial Node for Assembly G23 (Cont.)

\begin{tabular}{|c|c|c|c|c|c|c|}
\hline \multirow{2}{*}{$\begin{array}{c}\text { Axial } \\
\text { Node }\end{array}$} & $\begin{array}{c}\text { Burnup } \\
\text { (GWd/MTU) }\end{array}$ & $\begin{array}{c}\text { T-Fuel } \\
{ }^{\circ} \mathrm{F}\end{array}$ & $\begin{array}{c}\text { Spec. Vol. } \\
\mathrm{ft}^{3} / \mathrm{lbm}\end{array}$ & $\begin{array}{c}\text { Burnup } \\
\text { (GWd/MTU) }\end{array}$ & $\begin{array}{c}\text { T-Fuel } \\
{ }^{\circ} \mathrm{F}\end{array}$ & $\begin{array}{c}\text { Spec. Vol. } \\
\mathrm{ft}^{3} / \mathrm{lbm}\end{array}$ \\
\hline 1 & $367.3 \mathrm{Cy} 7$ & $297.3 \mathrm{Cy}$ & $297.3 \mathrm{Cy}$ & BOC Cy8 & 430.3 Cy7 & $430.3 \mathrm{Cy} 7$ \\
\hline 2 & 7.022 & 1054.5 & 0.0236 & 9.578 & 1050.6 & 0.0235 \\
\hline 3 & 11.584 & 1200.4 & 0.0235 & 15.400 & 1164.6 & 0.0234 \\
\hline 4 & 13.935 & 1260.1 & 0.0234 & 18.169 & 1196.1 & 0.0233 \\
\hline 5 & 14.918 & 1268.3 & 0.0232 & 19.203 & 1189.9 & 0.0232 \\
\hline 6 & 15.265 & 1258.5 & 0.0231 & 19.501 & 1177.1 & 0.0231 \\
\hline 7 & 15.395 & 1247.1 & 0.0230 & 19.580 & 1167.3 & 0.0229 \\
\hline 8 & 15.449 & 1238.0 & 0.0228 & 19.601 & 1161.3 & 0.0228 \\
\hline 9 & 15.474 & 1231.4 & 0.0227 & 19.606 & 1157.7 & 0.0227 \\
\hline 10 & 15.495 & 1226.9 & 0.0226 & 19.616 & 1155.7 & 0.0226 \\
\hline 11 & 15.534 & 1224.4 & 0.0225 & 19.646 & 1154.5 & 0.0225 \\
\hline 12 & 15.615 & 1223.9 & 0.0224 & 19.722 & 1154.1 & 0.0224 \\
\hline 13 & 15.765 & 1227.2 & 0.0223 & 19.880 & 1155.7 & 0.0223 \\
\hline 14 & 15.925 & 1236.2 & 0.0222 & 20.074 & 1160.8 & 0.0222 \\
\hline 15 & 15.939 & 1246.1 & 0.0220 & 20.137 & 1168.3 & 0.0220 \\
\hline 16 & 15.696 & 1254.5 & 0.0219 & 19.951 & 1178.7 & 0.0219 \\
\hline 17 & 14.914 & 1251.5 & 0.0218 & 19.171 & 1187.8 & 0.0218 \\
\hline 18 & 12.869 & 1203.2 & 0.0217 & 16.840 & 1170.6 & 0.0217 \\
\hline
\end{tabular}

Table 4-59. Burnup and TH Feedback Parameters by Axial Node for Assembly G25

\begin{tabular}{|c|c|c|c|c|c|c|c|c|c|}
\hline \multirow[b]{2}{*}{$\begin{array}{l}\text { Axial } \\
\text { Node }\end{array}$} & \multicolumn{3}{|c|}{ Statepoint 65 (30.0 Cy7) } & \multicolumn{3}{|c|}{ Statepoint 66 (108.0 Cy7) } & \multicolumn{3}{|c|}{ Datapoint (227.4 Cy7) } \\
\hline & $\begin{array}{c}\text { Burnup } \\
\text { (GWd/MTU) }\end{array}$ & $\begin{array}{c}\text { T-Fuel } \\
{ }^{\circ} \mathrm{F}\end{array}$ & $\begin{array}{c}\text { Spec. Vol. } \\
\mathrm{ft}^{3} / \mathrm{lbm}\end{array}$ & $\begin{array}{c}\text { Burnup } \\
\text { (GWd/MTU) } \\
\end{array}$ & $\begin{array}{c}\text { T-Fuel } \\
{ }^{\circ} \mathrm{F} \\
\end{array}$ & $\begin{array}{c}\text { Spec. Vol. } \\
\mathrm{ft}^{3} / \mathrm{lbm}\end{array}$ & $\begin{array}{c}\text { Burnup } \\
\text { (GWd/MTU) }\end{array}$ & $\begin{array}{c}\text { T-Fuel } \\
{ }^{\circ} \mathbf{F} \\
\end{array}$ & $\begin{array}{c}\text { Spec. Vol. } \\
\mathrm{ft}^{3} / \mathrm{lbm}\end{array}$ \\
\hline & $30.0 \mathrm{Cy} 7$ & $22.5 \mathrm{Cy} 7$ & $22.5 \mathrm{Cy} 7$ & $108.0 \mathrm{Cy} 7$ & 60.4 Cy7 & $60.4 \mathrm{Cy} 7$ & 227.4 Cy7 & $164.6 \mathrm{Cy} 7$ & $164.6 \mathrm{Cy} 7$ \\
\hline 1 & 0.341 & 845.8 & 0.0230 & 1.259 & 852.9 & 0.0229 & 2.870 & 886.1 & 0.0229 \\
\hline 2 & 0.590 & 1022.7 & 0.0229 & 2.156 & 1028.0 & 0.0229 & 4.797 & 1051.7 & 0.0229 \\
\hline 3 & 0.747 & 1123.7 & 0.0229 & 2.690 & 1121.6 & 0.0228 & 5.848 & 1123.2 & 0.0228 \\
\hline 4 & 0.843 & 1181.6 & 0.0228 & 2.996 & 1170.0 & 0.0227 & 6.379 & 1149.9 & 0.0227 \\
\hline 5 & 0.903 & 1216.0 & 0.0227 & 3.175 & 1195.9 & 0.0226 & 6.643 & 1156.9 & 0.0226 \\
\hline 6 & 0.941 & 1236.3 & 0.0226 & 3.282 & 1209.1 & 0.0226 & 6.772 & 1155.8 & 0.0225 \\
\hline 7 & 0.966 & 1248.2 & 0.0225 & 3.345 & 1215.5 & 0.0225 & 6.835 & 1152.2 & 0.0225 \\
\hline 8 & 0.981 & 1254.5 & 0.0224 & 3.382 & 1217.9 & 0.0224 & 6.864 & 1148.2 & 0.0224 \\
\hline 9 & 0.989 & 1257.1 & 0.0223 & 3.401 & 1217.8 & 0.0223 & 6.877 & 1144.8 & 0.0223 \\
\hline 10 & 0.992 & 1257.2 & 0.0222 & 3.410 & 1216.5 & 0.0222 & 6.885 & 1142.6 & 0.0222 \\
\hline 11 & 0.992 & 1255.3 & 0.0221 & 3.411 & 1214.2 & 0.0221 & 6.893 & 1141.8 & 0.0221 \\
\hline 12 & 0.985 & 1250.0 & 0.0220 & 3.398 & 1210.1 & 0.0220 & 6.896 & 1142.4 & 0.0220 \\
\hline 13 & 0.969 & 1238.6 & 0.0220 & 3.356 & 1201.7 & 0.0220 & 6.870 & 1143.5 & 0.0220 \\
\hline 14 & 0.938 & 1217.9 & 0.0219 & 3.269 & 1186.0 & 0.0219 & 6.780 & 1142.7 & 0.0219 \\
\hline 15 & 0.883 & 1182.8 & 0.0218 & 3.107 & 1157.9 & 0.0218 & 6.561 & 1135.1 & 0.0218 \\
\hline 16 & 0.791 & 1123.4 & 0.0217 & 2.816 & 1107.4 & 0.0217 & 6.080 & 1109.0 & 0.0217 \\
\hline 17 & 0.633 & 1018.0 & 0.0217 & 2.280 & 1011.3 & 0.0217 & 5.051 & 1037.8 & 0.0217 \\
\hline 18 & 0.363 & 826.1 & 0.0216 & 1.322 & 826.2 & 0.0216 & 3.006 & 863.1 & 0.0216 \\
\hline
\end{tabular}


Table 4-59. Burnup and TH Feedback Parameters by Axial Node for Assembly G25 (Cont.)

\begin{tabular}{|c|c|c|c|c|c|c|}
\hline \multirow{2}{*}{$\begin{array}{c}\text { Axial } \\
\text { Node }\end{array}$} & $\begin{array}{c}\text { Burnup } \\
\text { (GWd/MTU) }\end{array}$ & $\begin{array}{c}\text { T-Fuel } \\
{ }^{\circ} \mathrm{F}\end{array}$ & $\begin{array}{c}\text { Spec. Vol. } \\
\mathrm{ft}^{3} / \mathrm{lbm}\end{array}$ & $\begin{array}{c}\text { Burnup } \\
(\text { GWd/MTU) }\end{array}$ & $\begin{array}{c}\text { T-Fuel } \\
{ }^{\circ} \mathrm{F}\end{array}$ & $\begin{array}{c}\text { Spec. Vol. } \\
\mathrm{f}^{3} / \mathrm{lbm}\end{array}$ \\
\hline 1 & $367.3 \mathrm{Cy} 7$ & $297.3 \mathrm{Cy} 7$ & $297.3 \mathrm{Cy} 7$ & BOC Cy8 & 430.3 Cy7 & $430.3 \mathrm{Cy} 7$ \\
\hline 2 & 5.099 & 929.9 & 0.0230 & 7.123 & 959.7 & 0.0231 \\
\hline 3 & 8.257 & 1073.0 & 0.0229 & 11.240 & 1076.6 & 0.0230 \\
\hline 4 & 9.822 & 1119.5 & 0.0229 & 13.129 & 1106.4 & 0.0229 \\
\hline 5 & 10.511 & 1127.9 & 0.0228 & 13.875 & 1105.9 & 0.0228 \\
\hline 6 & 10.796 & 1123.9 & 0.0227 & 14.140 & 1098.7 & 0.0227 \\
\hline 7 & 10.907 & 1117.0 & 0.0226 & 14.220 & 1092.2 & 0.0227 \\
\hline 8 & 10.948 & 1110.9 & 0.0225 & 14.240 & 1088.0 & 0.0226 \\
\hline 9 & 10.963 & 1106.3 & 0.0224 & 14.242 & 1085.6 & 0.0225 \\
\hline 10 & 10.971 & 1103.2 & 0.0223 & 14.245 & 1084.5 & 0.0224 \\
\hline 11 & 10.987 & 1101.6 & 0.0222 & 14.258 & 1084.2 & 0.0223 \\
\hline 12 & 11.017 & 1101.7 & 0.0222 & 14.288 & 1084.6 & 0.0222 \\
\hline 13 & 11.058 & 1103.7 & 0.0221 & 14.336 & 1086.1 & 0.0221 \\
\hline 14 & 11.086 & 1108.0 & 0.0220 & 14.383 & 1089.5 & 0.0220 \\
\hline 15 & 11.056 & 1113.8 & 0.0219 & 14.387 & 1095.4 & 0.0219 \\
\hline 16 & 10.869 & 1118.3 & 0.0218 & 14.239 & 1103.2 & 0.0219 \\
\hline 17 & 10.297 & 1111.9 & 0.0218 & 13.647 & 1106.3 & 0.0218 \\
\hline 18 & 8.801 & 1067.7 & 0.0217 & 11.873 & 1079.4 & 0.0217 \\
\hline
\end{tabular}

Table 4-60. Burnup and TH Feedback Parameters by Axial Node for Assembly G25a

\begin{tabular}{|c|c|c|c|c|c|c|c|c|c|}
\hline \multirow[b]{2}{*}{$\begin{array}{l}\text { Axial } \\
\text { Node }\end{array}$} & \multicolumn{3}{|c|}{ Statepoint 65 (30.0 Cy7) } & \multicolumn{3}{|c|}{ Statepoint 66 (108.0 Cy7) } & \multicolumn{3}{|c|}{ Datapoint (227.4 Cy7) } \\
\hline & $\begin{array}{c}\text { Burnup } \\
\text { (GWd/MTU) }\end{array}$ & $\begin{array}{l}\text { T-Fuel } \\
{ }^{\circ} \mathbf{F}\end{array}$ & $\begin{array}{c}\text { Spec. Vol. } \\
\mathrm{ft}^{3} / \mathrm{lbm}\end{array}$ & \begin{tabular}{|c|} 
Burnup \\
(GWd/MTU)
\end{tabular} & $\begin{array}{l}\text { T-Fuel } \\
{ }^{\circ} \mathbf{F} \\
\end{array}$ & $\begin{array}{c}\text { Spec. Vol. } \\
\mathrm{ft}^{3} / \mathrm{lbm}\end{array}$ & \begin{tabular}{|c|} 
Burnup \\
(GWd/MTU)
\end{tabular} & $\begin{array}{l}\text { T-Fuel } \\
{ }^{\circ} \mathrm{F} \\
\end{array}$ & $\begin{array}{c}\text { Spec. Vol } \\
\mathrm{ft}^{3} / \mathrm{lbm}\end{array}$ \\
\hline & 30.0 Cy7 & $22.5 \mathrm{Cy} 7$ & $22.5 \mathrm{Cy} 7$ & $108.0 \mathrm{Cy} 7$ & 60.4 Cy? & 60.4 Cy 7 & $227.4 \mathrm{Cy} 7$ & 164.6 Cy7 & 164.6 Cy7 \\
\hline 1 & 0.341 & 845.8 & 0.0230 & 1.259 & 852.9 & 0.0229 & 2.870 & 886.1 & 0.0229 \\
\hline 2 & 0.590 & 1022.7 & 0.0229 & 2.156 & 1028.0 & 0.0229 & 4.797 & 1051.7 & 0.0229 \\
\hline 3 & 0.747 & 1123.7 & 0.0229 & 2.690 & 1121.6 & 0.0228 & 5.848 & 1123.2 & 0.0228 \\
\hline 4 & 0.843 & 1181.6 & 0.0228 & 2.996 & 1170.0 & 0.0227 & 6.379 & 1149.9 & 0.0227 \\
\hline 5 & 0.903 & 1216.0 & 0.0227 & 3.175 & 1195.9 & 0.0226 & 6.643 & 1156.9 & 0.0226 \\
\hline 6 & 0.941 & 1236.3 & 0.0226 & 3.282 & 1209.1 & 0.0226 & 6.772 & 1155.8 & 0.0225 \\
\hline 7 & 0.966 & 1248.2 & 0.0225 & 3.345 & 1215.5 & 0.0225 & 6.835 & 1152.2 & 0.0225 \\
\hline 8 & 0.981 & 1254.5 & 0.0224 & 3.382 & 1217.9 & 0.0224 & 6.864 & 1148.2 & 0.0224 \\
\hline 9 & 0.989 & 1257.1 & 0.0223 & 3.401 & 1217.8 & 0.0223 & 6.877 & 1144.8 & 0.0223 \\
\hline 10 & 0.992 & 1257.2 & 0.0222 & 3.410 & 1216.5 & 0.0222 & 6.885 & 1142.6 & 0.0222 \\
\hline 11 & 0.992 & 1255.3 & 0.0221 & 3.411 & 1214.2 & 0.0221 & 6.893 & 1141.8 & 0.0221 \\
\hline 12 & 0.985 & 1250.0 & 0.0220 & 3.398 & 1210.1 & 0.0220 & 6.896 & 1142.4 & 0.0220 \\
\hline 13 & 0.969 & 1238.6 & 0.0220 & 3.356 & 1201.7 & 0.0220 & 6.870 & 1143.5 & 0.0220 \\
\hline 14 & 0.938 & 1217.9 & 0.0219 & 3.269 & 1186.0 & 0.0219 & 6.780 & 1142.7 & 0.0219 \\
\hline 15 & 0.883 & 1182.8 & 0.0218 & 3.107 & 1157.9 & 0.0218 & 6.561 & 1135.1 & 0.0218 \\
\hline 16 & 0.791 & 1123.4 & 0.0217 & 2.816 & 1107.4 & 0.0217 & 6.080 & 1109.0 & 0.0217 \\
\hline 17 & 0.633 & 1018.0 & 0.0217 & 2.280 & 1011.3 & 0.0217 & 5.051 & 1037.8 & 0.0217 \\
\hline 18 & 0.363 & 826.1 & 0.0216 & 1.322 & 826.2 & 0.0216 & 3.006 & 863.1 & 0.0216 \\
\hline
\end{tabular}


Table 4-60. Burnup and TH Feedback Parameters by Axial Node for Assembly G25a (Cont.)

\begin{tabular}{|c|c|c|c|c|c|c|}
\hline \multirow[b]{2}{*}{$\begin{array}{l}\text { Axial } \\
\text { Node }\end{array}$} & \multicolumn{3}{|c|}{ Datapoint (367.3 Cy7) } & \multicolumn{3}{|c|}{ Statepoint 67 (BOC Cy8) } \\
\hline & $\begin{array}{c}\text { Burnup } \\
\text { (GWd/MTU) }\end{array}$ & $\begin{array}{c}\text { T-Fuel } \\
{ }^{\circ} \mathrm{F}\end{array}$ & $\begin{array}{c}\text { Spec. Vol. } \\
\mathrm{ft}^{3} / \mathrm{lbm}\end{array}$ & $\begin{array}{c}\text { Burnup } \\
\text { (GWd/MTU) }\end{array}$ & $\begin{array}{l}\text { T-Fuel } \\
{ }^{\circ} \mathrm{F}\end{array}$ & $\begin{array}{c}\text { Spec. Vol. } \\
\mathrm{ft}^{3} / \mathrm{lbm}\end{array}$ \\
\hline & $367.3 \mathrm{Cy} 7$ & 297.3 Cy7 & 297.3 Cy7 & BOC Cy 8 & 430.3 Cy 7 & 430.3 Cy 7 \\
\hline 1 & 5.099 & 929.9 & 0.0230 & 7.110 & 959.7 & 0.0231 \\
\hline 2 & 8.257 & 1073.0 & 0.0229 & 11.220 & 1076.6 & 0.0230 \\
\hline 3 & 9.822 & 1119.5 & 0.0229 & 13.106 & 1106.4 & 0.0229 \\
\hline 4 & 10.511 & 1127.9 & 0.0228 & 13.850 & 1105.9 & 0.0228 \\
\hline 5 & 10.796 & 1123.9 & 0.0227 & 14.114 & 1098.7 & 0.0227 \\
\hline 6 & 10.907 & 1117.0 & 0.0226 & 14.195 & 1092.2 & 0.0227 \\
\hline 7 & 10.948 & 1110.9 & 0.0225 & 14.214 & 1088.0 & 0.0226 \\
\hline 8 & 10.963 & 1106.3 & 0.0224 & 14.216 & 1085.6 & 0.0225 \\
\hline 9 & 10.971 & 1103.2 & 0.0223 & 14.219 & 1084.5 & 0.0224 \\
\hline 10 & 10.987 & 1101.6 & 0.0222 & 14.232 & 1084.2 & 0.0223 \\
\hline 11 & 11.017 & 1101.7 & 0.0222 & 14.262 & 1084.6 & 0.0222 \\
\hline 12 & 11.058 & 1103.7 & 0.0221 & 14.309 & 1086.1 & 0.0221 \\
\hline 13 & 11.086 & 1108.0 & 0.0220 & 14.356 & 1089.5 & 0.0220 \\
\hline 14 & 11.056 & 1113.8 & 0.0219 & 14.359 & 1095.4 & 0.0219 \\
\hline 15 & 10.869 & 1118.3 & 0.0218 & 14.211 & 1103.2 & 0.0219 \\
\hline 16 & 10.297 & 1111.9 & 0.0218 & 13.620 & 1106.3 & 0.0218 \\
\hline 17 & 8.801 & 1067.7 & 0.0217 & 11.849 & 1079.4 & 0.0217 \\
\hline 18 & 5.423 & 915.4 & 0.0216 & 7.488 & 950.9 & 0.0216 \\
\hline
\end{tabular}

Table 4-61. Burnup and TH Feedback Parameters by Axial Node for Assembly G27

\begin{tabular}{|c|c|c|c|c|c|c|c|c|c|}
\hline \multirow[b]{2}{*}{$\begin{array}{l}\text { Axial } \\
\text { Node }\end{array}$} & \multicolumn{3}{|c|}{ Statepoint 65 (30.0 Cy7) } & \multicolumn{3}{|c|}{ Statepoint 66 (108.0 Cy7) } & \multicolumn{3}{|c|}{ Datapoint (227.4 Cy7) } \\
\hline & $\begin{array}{c}\text { Burnup } \\
\text { (GWd/MTU) }\end{array}$ & $\begin{array}{c}\text { T-Fuel } \\
{ }^{\circ} \mathrm{F} \\
\end{array}$ & $\begin{array}{c}\text { Spec. Vol. } \\
\mathrm{ft}^{3} / \mathrm{lbm}\end{array}$ & $\begin{array}{c}\text { Burnup } \\
\text { (GWd/MTU) }\end{array}$ & $\begin{array}{c}\text { T-Fuel } \\
{ }^{\circ} \mathrm{F} \\
\end{array}$ & $\begin{array}{c}\text { Spec. Vol. } \\
\mathrm{ft}^{3} / \mathrm{lbm}\end{array}$ & $\begin{array}{c}\text { Burnup } \\
\text { (GWd/MTU) }\end{array}$ & $\begin{array}{c}\text { T-Fuel } \\
{ }^{\circ} \mathrm{F} \\
\end{array}$ & $\begin{array}{c}\text { Spec. Vol. } \\
\mathrm{ft}^{3} / \mathrm{lbm}\end{array}$ \\
\hline & $30.0 \mathrm{Cy} 7$ & 22.5 Cy 7 & 22.5 Cy 7 & $108.0 \mathrm{Cy} 7$ & 60.4 Cy7 & 60.4 Cy 7 & 227.4 Cy 7 & 164.6 Cy7 & 164.6 Cy7 \\
\hline 1 & 0.379 & 881.0 & 0.0233 & 1.403 & 891.7 & 0.0233 & 3.183 & 922.5 & 0.0232 \\
\hline 2 & 0.671 & 1087.6 & 0.0233 & 2.463 & 1098.5 & 0.0232 & 5.459 & 1114.0 & 0.0231 \\
\hline 3 & 0.883 & 1220.8 & 0.0232 & 3.187 & 1218.3 & 0.0231 & 6.874 & 1202.7 & 0.0231 \\
\hline 4 & 1.016 & 1299.4 & 0.0231 & 3.609 & 1281.6 & 0.0230 & 7.600 & 1235.8 & 0.0230 \\
\hline 5 & 1.096 & 1344.6 & 0.0230 & 3.846 & 1314.5 & 0.0229 & 7.948 & 1244.1 & 0.0228 \\
\hline 6 & 1.146 & 1371.3 & 0.0228 & 3.985 & 1331.5 & 0.0228 & 8.118 & 1242.8 & 0.0227 \\
\hline 7 & 1.178 & 1386.0 & 0.0227 & 4.068 & 1339.8 & 0.0227 & 8.201 & 1238.5 & 0.0226 \\
\hline 8 & 1.198 & 1394.0 & 0.0226 & 4.116 & 1343.2 & 0.0226 & 8.241 & 1233.7 & 0.0225 \\
\hline 9 & 1.210 & 1397.8 & 0.0225 & 4.144 & 1343.6 & 0.0225 & 8.263 & 1229.8 & 0.0224 \\
\hline 10 & 1.216 & 1398.9 & 0.0224 & 4.161 & 1342.7 & 0.0223 & 8.281 & 1227.4 & 0.0223 \\
\hline 11 & 1.217 & 1397.7 & 0.0223 & 4.168 & 1340.8 & 0.0222 & 8.300 & 1226.9 & 0.0222 \\
\hline 12 & 1.211 & 1392.5 & 0.0222 & 4.158 & 1336.8 & 0.0221 & 8.313 & 1228.1 & 0.0221 \\
\hline 13 & 1.193 & 1379.9 & 0.0220 & 4.113 & 1327.8 & 0.0220 & 8.290 & 1230.1 & 0.0220 \\
\hline 14 & 1.155 & 1356.0 & 0.0219 & 4.008 & 1309.8 & 0.0219 & 8.188 & 1230.1 & 0.0219 \\
\hline 15 & 1.087 & 1313.9 & 0.0218 & 3.810 & 1276.6 & 0.0218 & 7.927 & 1222.7 & 0.0219 \\
\hline 16 & 0.972 & 1241.3 & 0.0218 & 3.447 & 1216.3 & 0.0218 & 7.342 & 1194.8 & 0.0218 \\
\hline 17 & 0.778 & 1116.8 & 0.0217 & 2.792 & 1105.1 & 0.0217 & 6.108 & 1118.0 & 0.0217 \\
\hline 18 & 0.471 & 905.0 & 0.0216 & 1.707 & 903.0 & 0.0216 & 3.829 & 938.4 & 0.0216 \\
\hline
\end{tabular}


Table 4-61. Burnup and TH Feedback Parameters by Axial Node for Assembly G27 (Cont.)

\begin{tabular}{|c|c|c|c|c|c|c|}
\hline \multirow{2}{*}{$\begin{array}{c}\text { Axial } \\
\text { Node }\end{array}$} & $\begin{array}{c}\text { Burnup } \\
\text { (GWd/MTU) }\end{array}$ & $\begin{array}{c}\text { T-Fuel } \\
{ }^{\circ} \mathrm{F}\end{array}$ & $\begin{array}{c}\text { Spec. Vol. } \\
\mathrm{ft}^{3} / \mathrm{lbm}\end{array}$ & $\begin{array}{c}\text { Burnup } \\
\text { (GWd/MTU) }\end{array}$ & $\begin{array}{c}\text { T-Fuel } \\
{ }^{\circ} \mathrm{F}\end{array}$ & $\begin{array}{c}\text { Spec. Vol. } \\
\mathrm{ft}^{3} / \mathrm{bmm}\end{array}$ \\
\hline & $367.3 \mathrm{Cy7}$ & $297.3 \mathrm{Cy} 7$ & $297.3 \mathrm{Cy} 7$ & BOC Cy8 & 430.3 Cy7 & $430.3 \mathrm{Cy} 7$ \\
\hline 1 & 5.581 & 959.5 & 0.0232 & 7.765 & 981.6 & 0.0233 \\
\hline 2 & 9.276 & 1117.8 & 0.0232 & 12.556 & 1108.2 & 0.0232 \\
\hline 3 & 11.376 & 1175.6 & 0.0231 & 15.069 & 1148.2 & 0.0231 \\
\hline 4 & 12.326 & 1187.4 & 0.0230 & 16.095 & 1149.1 & 0.0230 \\
\hline 5 & 12.711 & 1183.5 & 0.0229 & 16.458 & 1141.2 & 0.0229 \\
\hline 6 & 12.861 & 1175.9 & 0.0228 & 16.575 & 1134.0 & 0.0228 \\
\hline 7 & 12.919 & 1168.9 & 0.0226 & 16.609 & 1129.3 & 0.0227 \\
\hline 8 & 12.942 & 1163.7 & 0.0225 & 16.619 & 1126.7 & 0.0226 \\
\hline 9 & 12.960 & 1160.2 & 0.0224 & 16.630 & 1125.5 & 0.0225 \\
\hline 10 & 12.988 & 1158.4 & 0.0224 & 16.655 & 1125.0 & 0.0224 \\
\hline 11 & 13.034 & 1158.4 & 0.0223 & 16.702 & 1125.4 & 0.0223 \\
\hline 12 & 13.092 & 1160.8 & 0.0222 & 16.769 & 1126.9 & 0.0222 \\
\hline 13 & 13.135 & 1165.8 & 0.0221 & 16.833 & 1130.5 & 0.0221 \\
\hline 14 & 13.105 & 1172.7 & 0.0220 & 16.843 & 1137.0 & 0.0220 \\
\hline 15 & 12.887 & 1178.4 & 0.0219 & 16.672 & 1145.9 & 0.0219 \\
\hline 16 & 12.208 & 1172.5 & 0.0218 & 15.979 & 1150.9 & 0.0218 \\
\hline 17 & 10.464 & 1127.6 & 0.0217 & 13.948 & 1125.9 & 0.0217 \\
\hline 18 & 6.789 & 983.5 & 0.0216 & 9.280 & 1007.2 & 0.0216 \\
\hline
\end{tabular}

Table 4-62. Burnup and TH Feedback Parameters by Axial Node for Assembly G27a

\begin{tabular}{|c|c|c|c|c|c|c|c|c|c|}
\hline \multirow[b]{2}{*}{$\begin{array}{l}\text { Axial } \\
\text { Node }\end{array}$} & \multicolumn{3}{|c|}{ Statepoint 65 (30.0 Cy7) } & \multicolumn{3}{|c|}{ Statepoint 66 (108.0 Cy7) } & \multicolumn{3}{|c|}{ Datapoint (227.4 Cy7) } \\
\hline & \begin{tabular}{|c|} 
Burnup \\
(GWd/MTU)
\end{tabular} & $\begin{array}{l}\text { T-Fuel } \\
\quad{ }^{\circ} \mathbf{F}\end{array}$ & $\begin{array}{c}\text { Spec. Vol. } \\
\mathrm{ft}^{3} / \mathrm{lbm}\end{array}$ & \begin{tabular}{|c|} 
Burnup \\
(GWd/MTU)
\end{tabular} & $\begin{array}{l}\text { T-Fuel } \\
{ }^{\circ} \mathrm{F}\end{array}$ & $\begin{array}{c}\text { Spec. Vol. } \\
\mathrm{ft}^{3} / \mathrm{lbm}\end{array}$ & $\begin{array}{c}\text { Burnup } \\
\text { (GWd/MTU) }\end{array}$ & $\begin{array}{l}\text { T-Fuel } \\
{ }^{\circ} \mathbf{F}\end{array}$ & $\begin{array}{c}\text { Spec. Vol. } \\
\mathrm{ft}^{3} / \mathrm{lbm}\end{array}$ \\
\hline & $30.0 \mathrm{Cy} 7$ & $22.5 \mathrm{Cy} 7$ & $22.5 \mathrm{Cy} 7$ & $108.0 \mathrm{Cy} 7$ & 60.4 Cy? & $60.4 \mathrm{Cy} 7$ & 227.4 Cy 7 & $164.6 \mathrm{Cy} 7$ & $164.6 \mathrm{Cy} 7$ \\
\hline 1 & 0.379 & 881.0 & 0.0233 & 1.403 & 891.7 & 0.0233 & 3.183 & 922.5 & 0.0232 \\
\hline 2 & 0.671 & 1087.6 & 0.0233 & 2.463 & 1098.5 & 0.0232 & 5.459 & 1114.0 & 0.0231 \\
\hline 3 & 0.883 & 1220.8 & 0.0232 & 3.187 & 1218.3 & 0.0231 & 6.874 & 1202.7 & 0.0231 \\
\hline 4 & 1.016 & 1299.4 & 0.0231 & 3.609 & 1281.6 & 0.0230 & 7.600 & 1235.8 & 0.0230 \\
\hline 5 & 1.096 & 1344.6 & 0.0230 & 3.846 & 1314.5 & 0.0229 & 7.948 & 1244.1 & 0.0228 \\
\hline 6 & 1.146 & 1371.3 & 0.0228 & 3.985 & 1331.5 & 0.0228 & 8.118 & 1242.8 & 0.0227 \\
\hline 7 & 1.178 & 1386.0 & 0.0227 & 4.068 & 1339.8 & 0.0227 & 8.201 & 1238.5 & 0.0226 \\
\hline 8 & 1.198 & 1394.0 & 0.0226 & 4.116 & 1343.2 & 0.0226 & 8.241 & 1233.7 & 0.0225 \\
\hline 9 & 1.210 & 1397.8 & 0.0225 & 4.144 & 1343.6 & 0.0225 & 8.263 & 1229.8 & 0.0224 \\
\hline 10 & 1.216 & 1398.9 & 0.0224 & 4.161 & 1342.7 & 0.0223 & 8.281 & 1227.4 & 0.0223 \\
\hline 11 & 1.217 & 1397.7 & 0.0223 & 4.168 & 1340.8 & 0.0222 & 8.300 & 1226.9 & 0.0222 \\
\hline 12 & 1.211 & 1392.5 & 0.0222 & 4.158 & 1336.8 & 0.0221 & 8.313 & 1228.1 & 0.0221 \\
\hline 13 & 1.193 & 1379.9 & 0.0220 & 4.113 & 1327.8 & 0.0220 & 8.290 & 1230.1 & 0.0220 \\
\hline 14 & 1.155 & 1356.0 & 0.0219 & 4.008 & 1309.8 & 0.0219 & 8.188 & 1230.1 & 0.0219 \\
\hline 15 & 1.087 & 1313.9 & 0.0218 & 3.810 & 1276.6 & 0.0218 & 7.927 & 1222.7 & 0.0219 \\
\hline 16 & 0.972 & 1241.3 & 0.0218 & 3.447 & 1216.3 & 0.0218 & 7.342 & 1194.8 & 0.0218 \\
\hline 17 & 0.778 & 1116.8 & 0.0217 & 2.792 & 1105.1 & 0.0217 & 6.108 & 1118.0 & 0.0217 \\
\hline 18 & 0.471 & 905.0 & 0.0216 & 1.707 & 903.0 & 0.0216 & 3.829 & 938.1 & 0.0216 \\
\hline
\end{tabular}


Table 4-62. Burnup and TH Feedback Parameters by Axial Node for Assembly G27a (Cont.)

\begin{tabular}{|c|c|c|c|c|c|c|}
\hline \multirow[b]{2}{*}{$\begin{array}{l}\text { Axial } \\
\text { Node }\end{array}$} & \multicolumn{3}{|c|}{ Datapoint (367.3 Cy7) } & \multicolumn{3}{|c|}{ Statepoint 67 (BOC Cy8) } \\
\hline & $\begin{array}{c}\text { Burnup } \\
\text { (GWd/MTU) }\end{array}$ & $\begin{array}{l}\text { T-Fuel } \\
{ }^{\circ} \mathrm{F}\end{array}$ & $\begin{array}{l}\text { Spec. Vol. } \\
\mathrm{ft}^{3} / \mathrm{bm}\end{array}$ & $\begin{array}{c}\text { Burnup } \\
\text { (GWd/MTU) }\end{array}$ & $\begin{array}{c}\text { T-Fuel } \\
{ }^{\circ} \mathrm{F}\end{array}$ & $\begin{array}{c}\text { Spec. Vol. } \\
t^{3} / \mathrm{lbm}\end{array}$ \\
\hline & 367.3 Cy7 & 297.3 Cy7 & 297.3 Cy7 & BOC Cy8 & 430.3 Cy7 & $430.3 \mathrm{Cy} 7$ \\
\hline 1 & 5.581 & 959.5 & 0.0232 & 7.760 & 981.6 & 0.0233 \\
\hline 2 & 9.276 & 1117.8 & 0.0232 & 12.548 & 1108.2 & 0.0232 \\
\hline 3 & 11.376 & 1175.6 & 0.0231 & 15.059 & 1148.2 & 0.0231 \\
\hline 4 & 12.326 & 1187.4 & 0.0230 & 16.085 & 1149.1 & 0.0230 \\
\hline 5 & 12.711 & 1183.5 & 0.0229 & 16.448 & 1141.2 & 0.0229 \\
\hline 6 & 12.861 & 1175.9 & 0.0228 & 16.565 & 1134.0 & 0.0228 \\
\hline 7 & 12.919 & 1168.9 & 0.0226 & 16.598 & 1129.3 & 0.0227 \\
\hline 8 & 12.942 & 1163.7 & 0.0225 & 16.608 & 1126.7 & 0.0226 \\
\hline 9 & 12.960 & 1160.2 & 0.0224 & 16.618 & 1125.5 & 0.0225 \\
\hline 10 & 12.988 & 1158.4 & 0.0224 & 16.643 & 1125.0 & 0.0224 \\
\hline 11 & 13.034 & 1158.4 & 0.0223 & 16.689 & 1125.4 & 0.0223 \\
\hline 12 & 13.092 & 1160.8 & 0.0222 & 16.755 & 1126.9 & 0.0222 \\
\hline 13 & 13.135 & 1165.8 & 0.0221 & 16.818 & 1130.5 & 0.0221 \\
\hline 14 & 13.105 & 1172.7 & 0.0220 & 16.828 & 1137.0 & 0.0220 \\
\hline 15 & 12.887 & 1178.4 & 0.0219 & 16.657 & 1145.9 & 0.0219 \\
\hline 16 & 12.208 & 1172.5 & 0.0218 & 15.965 & 1150.9 & 0.0218 \\
\hline 17 & 10.464 & 1127.6 & 0.0217 & 13.936 & 1125.9 & 0.0217 \\
\hline 18 & 6.789 & 983.5 & 0.0216 & 9.272 & 1007.2 & 0.0216 \\
\hline
\end{tabular}

Table 4-63. Burnup and TH Feedback Parameters by Axial Node for Assembly H6

\begin{tabular}{|c|c|c|c|c|c|c|c|c|c|}
\hline \multirow[b]{2}{*}{$\begin{array}{l}\text { Axial } \\
\text { Node } \\
\end{array}$} & \multicolumn{3}{|c|}{ Datapoint (171.1 Cy8) } & \multicolumn{3}{|c|}{ Datapoint (338.1 Cy8) } & \multicolumn{3}{|c|}{ Statepoint 68 (BOC Cy9) } \\
\hline & $\begin{array}{c}\text { Burnup } \\
\text { (GWd/MTU) }\end{array}$ & $\begin{array}{c}\text { T-Fuel } \\
{ }^{\circ} \mathrm{F} \\
\end{array}$ & $\begin{array}{c}\text { Spec. Vol. } \\
\mathrm{ft}^{3} / \mathrm{lbm}\end{array}$ & $\begin{array}{c}\text { Burnup } \\
\text { (GWd/MTU) }\end{array}$ & $\begin{array}{l}\text { T-Fuel } \\
{ }^{\circ} \mathrm{F}\end{array}$ & $\begin{array}{c}\text { Spec. Vol. } \\
\mathrm{ft}^{3} / \mathrm{lbm}\end{array}$ & $\begin{array}{c}\text { Burnup } \\
\text { (GWd/MTU) }\end{array}$ & $\begin{array}{c}\text { T-Fuel } \\
{ }^{\circ} \mathrm{F}\end{array}$ & $\begin{array}{c}\text { Spec. Vol. } \\
\mathrm{ft}^{3} / \mathrm{lbm}\end{array}$ \\
\hline & 171.1 Cy8 & 77.7 Cy8 & 77.7 Cy8 & $338.1 \mathrm{Cy} 8$ & 247.2 Cy 8 & 247.2 Cy8 & BOC Cy9 & 426.1 Cy 8 & 426.1 Cy 8 \\
\hline 1 & 3.333 & 1010.5 & 0.0237 & 7.135 & 1046.6 & 0.0237 & 11.487 & 1044.1 & 0.0237 \\
\hline 2 & 5.069 & 1195.1 & 0.0236 & 10.783 & 1207.6 & 0.0236 & 17.119 & 1184.1 & 0.0236 \\
\hline 3 & 6.265 & 1311.9 & 0.0235 & 13.018 & 1283.0 & 0.0235 & 20.170 & 1219.4 & 0.0234 \\
\hline 4 & 6.965 & 1377.5 & 0.0233 & 14.142 & 1307.2 & 0.0233 & 21.463 & 1212.7 & 0.0233 \\
\hline 5 & 7.357 & 1412.2 & 0.0232 & 14.670 & 1310.9 & 0.0232 & 21.952 & 1198.3 & 0.0232 \\
\hline 6 & 7.590 & 1431.0 & 0.0231 & 14.932 & 1306.6 & 0.0231 & 22.142 & 1185.6 & 0.0230 \\
\hline 7 & 7.737 & 1441.1 & 0.0229 & 15.076 & 1300.6 & 0.0229 & 22.232 & 1176.3 & 0.0229 \\
\hline 8 & 7.831 & 1445.4 & 0.0228 & 15.165 & 1295.2 & 0.0228 & 22.296 & 1169.9 & 0.0228 \\
\hline 9 & 7.888 & 1446.3 & 0.0227 & 15.228 & 1291.2 & 0.0227 & 22.357 & 1165.9 & 0.0227 \\
\hline 10 & 7.917 & 1444.6 & 0.0225 & 15.275 & 1289.0 & 0.0226 & 22.425 & 1163.8 & 0.0225 \\
\hline 11 & 7.918 & 1440.8 & 0.0224 & 15.309 & 1288.7 & 0.0224 & 22.503 & 1163.4 & 0.0224 \\
\hline 12 & 7.886 & 1434.2 & 0.0223 & 15.324 & 1290.2 & 0.0223 & 22.586 & 1165.1 & 0.0223 \\
\hline 13 & 7.805 & 1423.3 & 0.0222 & 15.294 & 1293.1 & 0.0222 & 22.654 & 1169.7 & 0.0222 \\
\hline 14 & 7.641 & 1403.9 & 0.0220 & 15.160 & 1295.3 & 0.0221 & 22.648 & 1177.7 & 0.0221 \\
\hline 15 & 7.333 & 1370.4 & 0.0219 & 14.799 & 1291.8 & 0.0220 & 22.410 & 1188.6 & 0.0220 \\
\hline 16 & 6.762 & 1311.4 & 0.0218 & 13.939 & 1269.6 & 0.0219 & 21.531 & 1195.6 & 0.0219 \\
\hline 17 & 5.722 & 1206.2 & 0.0217 & 12.040 & 1204.1 & 0.0218 & 19.049 & 1170.5 & 0.0218 \\
\hline 18 & 3.976 & 1029.1 & 0.0217 & 8.408 & 1047.4 & 0.0217 & 13.495 & 1040.8 & 0.0217 \\
\hline
\end{tabular}


Table 4-63. Burnup and TH Feedback Parameters by Axial Node for Assembly H6 (Cont.)

\begin{tabular}{|c|c|c|c|c|c|c|c|c|c|}
\hline \multirow[b]{2}{*}{$\begin{array}{l}\text { Axial } \\
\text { Node }\end{array}$} & \multicolumn{3}{|c|}{ Datapoint (214.0 Cy9) } & \multicolumn{3}{|c|}{ Datapoint (425.0 Cy9) } & \multicolumn{3}{|c|}{ Statepoint 73 (BOC Cy11) } \\
\hline & $\begin{array}{c}\text { Burnup } \\
\text { (GWd/MTU) }\end{array}$ & $\begin{array}{l}\text { T-Fuel } \\
{ }^{\circ} \mathrm{F}\end{array}$ & $\begin{array}{l}\text { Spec. Vol. } \\
\mathrm{ft}^{3} / \mathrm{lbm}\end{array}$ & \begin{tabular}{|c|} 
Burnup \\
(GWd/MTU)
\end{tabular} & $\begin{array}{l}\text { T-Fuel } \\
{ }^{\circ} \mathrm{F}\end{array}$ & $\begin{array}{l}\text { Spec. Vol. } \\
\mathrm{ft}^{3} / \mathrm{lbm}\end{array}$ & $\begin{array}{c}\text { Burnup } \\
\text { (GWd/MTU) }\end{array}$ & $\begin{array}{c}\text { T-Fuel } \\
{ }^{\circ} \mathrm{F}\end{array}$ & $\begin{array}{c}\text { Spec. Vol. } \\
\mathrm{ft}^{3} / \mathrm{lbm}\end{array}$ \\
\hline & $214.0 \mathrm{Cyg}$ & 109.3 Cy9 & 109.3 Cy9 & 425.0 Cy9 & 328.7 Cy9 & 328.7 Cy 9 & BOC Cy11 & 549.2 Cy9 & 549.2 Cy 9 \\
\hline 1 & 14.371 & 826.0 & 0.0228 & 17.898 & 864.2 & 0.0229 & 21.673 & 902.8 & 0.0230 \\
\hline 2 & 21.589 & 914.1 & 0.0228 & 26.733 & 933.3 & 0.0228 & 31.738 & 949.7 & 0.0229 \\
\hline 3 & 25.408 & 944.1 & 0.0227 & 31.124 & 943.4 & 0.0228 & 37.146 & 957.4 & 0.0228 \\
\hline 4 & 27.103 & 957.5 & 0.0226 & 32.975 & 942.5 & 0.0227 & 39.188 & 948.9 & 0.0227 \\
\hline 5 & 27.809 & 963.6 & 0.0226 & 33.690 & 937.2 & 0.0226 & 39.875 & 938.8 & 0.0227 \\
\hline 6 & 28.111 & 965.1 & 0.0225 & 33.959 & 931.1 & 0.0225 & 40.078 & 931.1 & 0.0226 \\
\hline 7 & 28.252 & 964.0 & 0.0224 & 34.063 & 925.9 & 0.0224 & 40.129 & 925.9 & 0.0225 \\
\hline 8 & 28.331 & 961.4 & 0.0223 & 34.115 & 921.7 & 0.0224 & 40.145 & 922.4 & 0.0224 \\
\hline 9 & 28.383 & 957.9 & 0.0222 & 34.154 & 918.8 & 0.0223 & 40.161 & 920.0 & 0.0223 \\
\hline 10 & 28.426 & 953.8 & 0.0222 & 34.197 & 916.9 & 0.0222 & 40.190 & 918.3 & 0.0222 \\
\hline 11 & 28.464 & 949.3 & 0.0221 & 34.250 & 916.0 & 0.0221 & 40.237 & 917.2 & 0.0222 \\
\hline 12 & 28.488 & 944.1 & 0.0220 & 34.305 & 916.1 & 0.0220 & 40.296 & 917.0 & 0.0221 \\
\hline 13 & 28.470 & 937.6 & 0.0219 & 34.327 & 917.3 & 0.0220 & 40.349 & 918.4 & 0.0220 \\
\hline 14 & 28.330 & 928.9 & 0.0219 & 34.230 & 919.1 & 0.0219 & 40.320 & 922.2 & 0.0219 \\
\hline 15 & 27.878 & 916.6 & 0.0218 & 33.790 & 920.7 & 0.0218 & 39.979 & 928.8 & 0.0219 \\
\hline 16 & 26.628 & 897.9 & 0.0217 & 32.424 & 917.7 & 0.0218 & 38.659 & 935.0 & 0.0218 \\
\hline 17 & 23.432 & 864.2 & 0.0217 & 28.697 & 900.9 & 0.0217 & 34.562 & 923.7 & 0.0217 \\
\hline 18 & 16.308 & 777.1 & 0.0216 & 19.897 & 826.3 & 0.0216 & 24.150 & 864.6 & 0.0216 \\
\hline
\end{tabular}

\begin{tabular}{|c|c|c|c|c|c|c|c|c|c|}
\hline \multirow[b]{2}{*}{$\begin{array}{l}\text { Axial } \\
\text { Node }\end{array}$} & \multicolumn{3}{|c|}{ Datapoint (208.7 Cy11) } & \multicolumn{3}{|c|}{ Datapoint (417.8 Cy11) } & \multicolumn{3}{|c|}{ Statepoint 74 (615.0 Cy11) } \\
\hline & $\begin{array}{c}\text { Burnup } \\
\text { (GWd/MTU) }\end{array}$ & $\begin{array}{c}\text { T-Fuel } \\
{ }^{\circ} \mathrm{F}\end{array}$ & $\begin{array}{c}\begin{array}{c}\text { Spec. Vol. } \\
\mathrm{ft}^{3} / \mathrm{lbm}\end{array} \\
\end{array}$ & $\begin{array}{c}\text { Burnup } \\
\text { (GWd/MTU) }\end{array}$ & $\begin{array}{c}\text { T-Fuel } \\
{ }^{\circ} \mathrm{F}\end{array}$ & \begin{tabular}{|c} 
Spec. Vol. \\
$\mathrm{ft}^{3} / \mathrm{lbm}$
\end{tabular} & $\begin{array}{c}\text { Burnup } \\
\text { (GWd/MTU) }\end{array}$ & $\begin{array}{l}\text { T-Fuel } \\
{ }^{\circ} \mathrm{F}\end{array}$ & $\begin{array}{c}\text { Spec. Vol. } \\
\mathrm{ft}^{3} / \mathrm{lbm}\end{array}$ \\
\hline & 208.7 Cy 11 & 103.8 Cy 11 & $103.8 \mathrm{Cy} 11$ & 417.8 Cy 11 & 312.8 Cy11 & 312.8 Cy11 & $615.0 \mathrm{Cy} 11$ & 515.7 Cy 11 & 515.7 Cy11 \\
\hline 1 & 22.971 & 666.1 & 0.0222 & 24.528 & 679.9 & 0.0222 & 26.295 & 698.1 & 0.0222 \\
\hline 2 & 33.806 & 697.5 & 0.0222 & 36.140 & 710.0 & 0.0222 & 38.645 & 727.5 & 0.0222 \\
\hline 3 & 39.649 & 717.9 & 0.0221 & 42.326 & 724.3 & 0.0221 & 45.083 & 736.3 & 0.0222 \\
\hline 4 & 41.908 & 727.4 & 0.0221 & 44.695 & 727.7 & 0.0221 & 47.485 & 735.2 & 0.0221 \\
\hline 5 & 42.705 & 731.7 & 0.0221 & 45.515 & 727.4 & 0.0221 & 48.282 & 732.1 & 0.0221 \\
\hline 6 & 42.967 & 733.5 & 0.0220 & 45.772 & 726.0 & 0.0220 & 48.508 & 729.2 & 0.0220 \\
\hline 7 & 43.047 & 733.8 & 0.0220 & 45.842 & 724.4 & 0.0220 & 48.555 & 726.8 & 0.0220 \\
\hline 8 & 43.076 & 733.3 & 0.0220 & 45.859 & 722.8 & 0.0220 & 48.558 & 724.9 & 0.0220 \\
\hline 9 & 43.094 & 732.2 & 0.0219 & 45.870 & 721.4 & 0.0219 & 48.560 & 723.4 & 0.0219 \\
\hline 10 & 43.118 & 730.8 & 0.0219 & 45.890 & 720.2 & 0.0219 & 48.577 & 722.2 & 0.0219 \\
\hline 11 & 43.152 & 729.0 & 0.0218 & 45.925 & 719.2 & 0.0218 & 48.614 & 721.3 & 0.0219 \\
\hline 12 & 43.192 & 726.8 & 0.0218 & 45.969 & 718.4 & 0.0218 & 48.665 & 720.6 & 0.0218 \\
\hline 13 & 43.211 & 723.9 & 0.0218 & 45.995 & 717.8 & 0.0218 & 48.706 & 720.5 & 0.0218 \\
\hline 14 & 43.126 & 719.9 & 0.0217 & 45.912 & 717.0 & 0.0217 & 48.645 & 720.8 & 0.0218 \\
\hline 15 & 42.691 & 714.0 & 0.0217 & 45.460 & 715.5 & 0.0217 & 48.218 & 721.5 & 0.0217 \\
\hline 16 & 41.201 & 704.6 & 0.0217 & 43.899 & 711.9 & 0.0217 & 46.648 & 721.4 & 0.0217 \\
\hline 17 & 36.760 & 687.1 & 0.0216 & 39.205 & 700.3 & 0.0216 & 41.786 & 714.6 & 0.0216 \\
\hline 18 & 25.591 & 654.3 & 0.0216 & 27.285 & 668.2 & 0.0216 & 29.167 & 682.5 & 0.0216 \\
\hline
\end{tabular}


Table 4-64. Burnup and TH Feedback Parameters by Axial Node for Assembly H1O

\begin{tabular}{|c|c|c|c|c|c|c|c|c|c|}
\hline \multirow[b]{2}{*}{$\begin{array}{l}\text { Axial } \\
\text { Node }\end{array}$} & \multicolumn{3}{|c|}{ Datapoint (171.1 Cyв) } & \multicolumn{3}{|c|}{ Datapoint (338.1 Cy8) } & \multicolumn{3}{|c|}{ Statepoint 68 (BOC Cy9) } \\
\hline & $\begin{array}{c}\text { Burnup } \\
\text { (GWd/MTU) }\end{array}$ & $\begin{array}{c}\text { T-Fuel } \\
{ }^{\circ} \mathrm{F} \\
\end{array}$ & $\begin{array}{c}\text { Spec. Vol. } \\
\mathrm{ft}^{3} / \mathrm{lbm}\end{array}$ & $\begin{array}{c}\text { Burnup } \\
\text { (GWd/MTU) }\end{array}$ & $\begin{array}{c}\text { T-Fuel } \\
{ }^{\circ} \mathbf{F}\end{array}$ & $\begin{array}{c}\text { Spec. Vol. } \\
\mathrm{ft}^{3} / \mathrm{lbm}\end{array}$ & $\begin{array}{c}\text { Burnup } \\
\text { (GWd/MTU) }\end{array}$ & $\begin{array}{c}\text { T-Fuel } \\
{ }^{\circ} \mathrm{F}\end{array}$ & $\begin{array}{c}\text { Spec. Vol. } \\
\mathrm{ft}^{3} / \mathrm{lbm}\end{array}$ \\
\hline & 171.1 Cy 8 & 77.7 Су8 & 77.7 Cy 8 & 338.1 Cy 8 & 247.2 Cy8 & 247.2 Cy8 & BOC Cy 9 & $426.1 \mathrm{Cy} 8$ & 426.1 Cy 8 \\
\hline 1 & 3.784 & 1061.9 & 0.0237 & 7.877 & 1068.7 & 0.0237 & 12.434 & 1050.2 & 0.0236 \\
\hline 2 & 5.627 & 1248.6 & 0.0236 & 11.645 & 1225.7 & 0.0236 & 18.098 & 1178.5 & 0.0235 \\
\hline 3 & 6.676 & 1349.8 & 0.0235 & 13.570 & 1287.7 & 0.0235 & 20.667 & 1204.6 & 0.0234 \\
\hline 4 & 7.241 & 1402.0 & 0.0233 & 14.454 & 1306.1 & 0.0233 & 21.652 & 1197.3 & 0.0233 \\
\hline 5 & 7.553 & 1428.8 & 0.0232 & 14.855 & 1306.7 & 0.0232 & 21.999 & 1184.3 & 0.0231 \\
\hline 6 & 7.734 & 1442.6 & 0.0231 & 15.043 & 1301.2 & 0.0231 & 22.115 & 1172.9 & 0.0230 \\
\hline 7 & 7.843 & 1448.6 & 0.0229 & 15.138 & 1294.7 & 0.0229 & 22.159 & 1164.6 & 0.0229 \\
\hline 8 & 7.906 & 1449.8 & 0.0228 & 15.188 & 1289.1 & 0.0228 & 22.186 & 1159.0 & 0.0228 \\
\hline 9 & 7.934 & 1448.2 & 0.0227 & 15.215 & 1285.1 & 0.0227 & 22.213 & 1155.6 & 0.0226 \\
\hline 10 & 7.934 & 1444.3 & 0.0225 & 15.225 & 1282.7 & 0.0225 & 22.245 & 1154.0 & 0.0225 \\
\hline 11 & 7.904 & 1438.2 & 0.0224 & 15.220 & 1282.1 & 0.0224 & 22.282 & 1154.1 & 0.0224 \\
\hline 12 & 7.842 & 1429.0 & 0.0223 & 15.194 & 1283.2 & 0.0223 & 22.322 & 1156.3 & 0.0223 \\
\hline 13 & 7.735 & 1415.3 & 0.0222 & 15.129 & 1285.5 & 0.0222 & 22.351 & 1161.0 & 0.0222 \\
\hline 14 & 7.559 & 1394.6 & 0.0220 & 14.976 & 1287.3 & 0.0221 & 22.320 & 1168.9 & 0.0221 \\
\hline 15 & 7.260 & 1362.0 & 0.0219 & 14.629 & 1284.0 & 0.0220 & 22.091 & 1179.2 & 0.0220 \\
\hline 16 & 6.718 & 1305.8 & 0.0218 & 13.817 & 1263.3 & 0.0219 & 21.265 & 1185.6 & 0.0219 \\
\hline 17 & 5.697 & 1202.8 & 0.0217 & 11.961 & 1199.9 & 0.0218 & 18.852 & 1161.4 & 0.0218 \\
\hline 18 & 3.906 & 1020.6 & 0.0217 & 8.259 & 1041.1 & 0.0217 & 13.254 & 1033.8 & 0.0217 \\
\hline
\end{tabular}

Table 4-65. Burnup and TH Feedback Parameters by Axial Node for Assembly H10a

\begin{tabular}{|c|c|c|c|c|c|c|c|c|c|}
\hline \multirow[b]{2}{*}{$\begin{array}{l}\text { Axial } \\
\text { Node } \\
\end{array}$} & \multicolumn{3}{|c|}{ Datapoint (171.1 Cy8) } & \multicolumn{3}{|c|}{ Datapoint (338.1 Cy8) } & \multicolumn{3}{|c|}{ Statepoint 68 (BOC Cy9) } \\
\hline & \begin{tabular}{|c|} 
Burnup \\
(GWd/MTU)
\end{tabular} & $\begin{array}{c}\text { T-Fuel } \\
{ }^{\circ} \mathrm{F}\end{array}$ & $\begin{array}{c}\text { Spec. Vol. } \\
\mathrm{ft}^{3} / \mathrm{lbm}\end{array}$ & $\begin{array}{c}\text { Burnup } \\
\text { (GWd/MTU) }\end{array}$ & $\begin{array}{c}\text { T-Fuel } \\
{ }^{\circ} \mathrm{F}\end{array}$ & $\begin{array}{c}\text { Spec. Vol. } \\
\mathrm{ft}^{3} / \mathrm{lbm}\end{array}$ & $\begin{array}{c}\text { Burnup } \\
\text { (GWd/MTU) }\end{array}$ & $\begin{array}{l}\text { T-Fuel } \\
{ }^{\circ} \mathrm{F}\end{array}$ & $\begin{array}{c}\text { Spec. Vol. } \\
\mathrm{ft}^{3} / \mathrm{bm}\end{array}$ \\
\hline & 171.1 Cy 8 & 77.7 Cy8 & 77.7 Cy 8 & 338.1 Cy 8 & 247.2 Cy8 & 247.2 Cy 8 & BOC Cy 9 & 426.1 Cy 8 & 426.1 Cy 8 \\
\hline 1 & 3.784 & 1061.9 & 0.0237 & 7.877 & 1068.7 & 0.0237 & 12.430 & 1050.2 & 0.0236 \\
\hline 2 & 5.627 & 1248.6 & 0.0236 & 11.645 & 1225.7 & 0.0236 & 18.082 & 1178.5 & 0.0235 \\
\hline 3 & 6.676 & 1349.8 & 0.0235 & 13.570 & 1287.7 & 0.0235 & 20.638 & 1204.6 & 0.0234 \\
\hline 4 & 7.241 & 1402.0 & 0.0233 & 14.454 & 1306.1 & 0.0233 & 21.615 & 1197.3 & 0.0233 \\
\hline 5 & 7.553 & 1428.8 & 0.0232 & 14.855 & 1306.7 & 0.0232 & 21.958 & 1184.3 & 0.0231 \\
\hline 6 & 7.734 & 1442.6 & 0.0231 & 15.043 & 1301.2 & 0.0231 & 22.072 & 1172.9 & 0.0230 \\
\hline 7 & 7.843 & 1448.6 & 0.0229 & 15.138 & 1294.7 & 0.0229 & 22.114 & 1164.6 & 0.0229 \\
\hline 8 & 7.906 & 1449.8 & 0.0228 & 15.188 & 1289.1 & 0.0228 & 22.141 & 1159.0 & 0.0228 \\
\hline 9 & 7.934 & 1448.2 & 0.0227 & 15.215 & 1285.1 & 0.0227 & 22.168 & 1155.6 & 0.0226 \\
\hline 10 & 7.934 & 1444.3 & 0.0225 & 15.225 & 1282.7 & 0.0225 & 22.199 & 1154.0 & 0.0225 \\
\hline 11 & 7.904 & 1438.2 & 0.0224 & 15.220 & 1282.1 & 0.0224 & 22.237 & 1154.1 & 0.0224 \\
\hline 12 & 7.842 & 1429.0 & 0.0223 & 15.194 & 1283.2 & 0.0223 & 22.279 & 1156.3 & 0.0223 \\
\hline 13 & 7.735 & 1415.3 & 0.0222 & 15.129 & 1285.5 & 0.0222 & 22.311 & 1161.0 & 0.0222 \\
\hline 14 & 7.559 & 1394.6 & 0.0220 & 14.976 & 1287.3 & 0.0221 & 22.282 & 1168.9 & 0.0221 \\
\hline 15 & 7.260 & 1362.0 & 0.0219 & 14.629 & 1284.0 & 0.0220 & 22.054 & 1179.2 & 0.0220 \\
\hline 16 & 6.718 & 1305.8 & 0.0218 & 13.817 & 1263.3 & 0.0219 & 21.229 & 1185.6 & 0.0219 \\
\hline 17 & 5.697 & 1202.8 & 0.0217 & 11.961 & 1199.9 & 0.0218 & 18.822 & 1161.4 & 0.0218 \\
\hline 18 & 3.906 & 1020.6 & 0.0217 & 8.259 & 1041.1 & 0.0217 & 13.235 & 1033.8 & 0.0217 \\
\hline
\end{tabular}


Table 4-65. Burnup and TH Feedback Parameters by Axial Node for Assembly H10a (Cont.)

\begin{tabular}{|c|c|c|c|c|c|c|c|c|c|}
\hline \multirow[b]{2}{*}{$\begin{array}{l}\text { Axial } \\
\text { Node } \\
\end{array}$} & \multicolumn{3}{|c|}{ Datapoint (214.0 Cy9) } & \multicolumn{3}{|c|}{ Datapoint (425.0 Cy9) } & \multicolumn{3}{|c|}{ Statepoint 69 (BOC Cy10) } \\
\hline & \begin{tabular}{|c|} 
Burnup \\
(GWd/MTU)
\end{tabular} & $\begin{array}{c}\text { T-Fuel } \\
{ }^{\circ} \mathrm{F}\end{array}$ & $\begin{array}{c}\text { Spec. Vol. } \\
\mathrm{ft}^{3} / \mathrm{lbm}\end{array}$ & $\begin{array}{c}\text { Burnup } \\
\text { (GWd/MTU) }\end{array}$ & $\begin{array}{c}\text { T-Fuel } \\
{ }^{\circ} \mathrm{F}\end{array}$ & $\begin{array}{c}\text { Spec. Vol. } \\
\mathrm{ft}^{3} / \mathrm{lbm}\end{array}$ & $\begin{array}{c}\text { Burnup } \\
\text { (GWd/MTU) }\end{array}$ & $\begin{array}{l}\text { T-Fuel } \\
{ }^{\circ} \mathrm{F}\end{array}$ & $\begin{array}{c}\text { Spec. Vol. } \\
\mathrm{ft}^{3} / \mathrm{lbm}\end{array}$ \\
\hline & 214.0 Cy9 & 109.3 Cy9 & 109.3 Cy9 & 425.0 Cy 9 & 328.7 Cy9 & 328.7 Cy9 & BOC Cy 10 & 549.2 Су9 & 549.2 Cy9 \\
\hline 1 & 13.884 & 697.1 & 0.0223 & 15.687 & 721.2 & 0.0223 & 17.551 & 759.3 & 0.0224 \\
\hline 2 & 20.526 & 755.8 & 0.0223 & 23.328 & 771.3 & 0.0223 & 25.980 & 799.1 & 0.0224 \\
\hline 3 & 23.692 & 784.9 & 0.0223 & 26.949 & 786.5 & 0.0223 & 30.497 & 805.4 & 0.0223 \\
\hline 4 & 25.006 & 798.7 & 0.0222 & 28.420 & 788.6 & 0.0222 & 32.203 & 802.6 & 0.0223 \\
\hline 5 & 25.517 & 804.2 & 0.0222 & 28.959 & 786.2 & 0.0222 & 32.764 & 797.8 & 0.0222 \\
\hline 6 & 25.714 & 805.7 & 0.0221 & 29.142 & 782.8 & 0.0221 & 32.917 & 793.5 & 0.0222 \\
\hline 7 & 25.795 & 805.2 & 0.0221 & 29.202 & 779.8 & 0.0221 & 32.946 & 790.3 & 0.0221 \\
\hline 8 & 25.836 & 803.8 & 0.0220 & 29.226 & 777.3 & 0.0220 & 32.948 & 788.1 & 0.0221 \\
\hline 9 & 25.864 & 802.0 & 0.0220 & 29.246 & 775.5 & 0.0220 & 32.952 & 786.5 & 0.0220 \\
\hline 10 & 25.887 & 800.0 & 0.0219 & 29.270 & 774.3 & 0.0219 & 32.966 & 785.4 & 0.0220 \\
\hline 11 & 25.910 & 797.9 & 0.0219 & 29.302 & 773.7 & 0.0219 & 32.992 & 784.5 & 0.0219 \\
\hline 12 & 25.928 & 795.5 & 0.0218 & 29.339 & 773.7 & 0.0219 & 33.029 & 784.3 & 0.0219 \\
\hline 13 & 25.917 & 792.5 & 0.0218 & 29.354 & 774.4 & 0.0218 & 33.059 & 785.0 & 0.0218 \\
\hline 14 & 25.813 & 787.9 & 0.0218 & 29.273 & 775.5 & 0.0218 & 33.015 & 787.0 & 0.0218 \\
\hline 15 & 25.448 & 780.6 & 0.0217 & 28.910 & 776.5 & 0.0217 & 32.702 & 790.3 & 0.0218 \\
\hline 16 & 24.372 & 768.1 & 0.0217 & 27.751 & 775.5 & 0.0217 & 31.545 & 793.2 & 0.0217 \\
\hline 17 & 21.463 & 743.6 & 0.0216 & 24.497 & 764.8 & 0.0217 & 28.006 & 790.5 & 0.0217 \\
\hline 18 & 14.857 & 685.1 & 0.0216 & 16.871 & 714.4 & 0.0216 & 19.337 & 751.3 & 0.0216 \\
\hline
\end{tabular}

\begin{tabular}{|c|c|c|c|c|c|c|c|c|c|}
\hline \multirow[b]{2}{*}{$\begin{array}{l}\text { Axial } \\
\text { Node }\end{array}$} & \multicolumn{3}{|c|}{ Statepoint 70 (27.0 Cy10) } & \multicolumn{3}{|c|}{ Statepoint 71 (143.0 Cy10) } & \multicolumn{3}{|c|}{ Statepoint $72(209.0$ Cy10) } \\
\hline & $\begin{array}{c}\text { Burnup } \\
\text { (GWd/MTU) }\end{array}$ & $\begin{array}{c}\text { T-Fuel } \\
{ }^{\circ} \mathrm{F} \\
\end{array}$ & $\begin{array}{c}\text { Spec. Vol. } \\
\mathrm{ft}^{3} / \mathrm{lbm}\end{array}$ & $\begin{array}{c}\text { Burnup } \\
\text { (GWd/MTU) }\end{array}$ & $\begin{array}{l}\text { T-Fuel } \\
{ }^{\circ} \mathrm{F}\end{array}$ & $\begin{array}{l}\text { Spec. Vol. } \\
\mathrm{ft}^{3} / \mathrm{lbm}\end{array}$ & $\begin{array}{c}\text { Burnup } \\
\text { (GWd/MTU) }\end{array}$ & $\begin{array}{c}\text { T-Fuel } \\
{ }^{\circ} \mathrm{F}\end{array}$ & $\begin{array}{c}\text { Spec. Vol. } \\
\mathrm{ft}^{3} / \mathrm{lbm}\end{array}$ \\
\hline & $27.0 \mathrm{Cy} 10$ & 18.0 Cy 10 & 18.0 Cy 10 & $143.0 \mathrm{Cy} 10$ & 82.9 Cy 10 & 82.9 Cy 10 & $209.0 \mathrm{Cy} 10$ & 176.7 Cy10 & 176.7 Cy 10 \\
\hline 1 & 17.845 & 787.1 & 0.0230 & 19.097 & 766.5 & 0.0230 & 19.880 & 777.0 & 0.0230 \\
\hline 2 & 26.522 & 895.7 & 0.0229 & 28.794 & 874.4 & 0.0229 & 30.253 & 874.4 & 0.0230 \\
\hline 3 & 31.199 & 932.4 & 0.0228 & 34.247 & 930.0 & 0.0229 & 36.084 & 939.5 & 0.0229 \\
\hline 4 & 32.974 & 956.1 & 0.0228 & 36.325 & 955.6 & 0.0228 & 38.294 & 959.9 & 0.0228 \\
\hline 5 & 33.575 & 969.7 & 0.0227 & 37.078 & 968.0 & 0.0227 & 39.104 & 967.3 & 0.0227 \\
\hline 6 & 33.753 & 977.6 & 0.0226 & 37.345 & 974.3 & 0.0226 & 39.396 & 969.6 & 0.0226 \\
\hline 7 & 33.797 & 981.2 & 0.0225 & 37.438 & 976.6 & 0.0225 & 39.501 & 969.1 & 0.0225 \\
\hline 8 & 33.807 & 981.5 & 0.0224 & 37.472 & 976.2 & 0.0224 & 39.537 & 967.3 & 0.0224 \\
\hline 9 & 33.812 & 979.0 & 0.0223 & 37.481 & 973.7 & 0.0223 & 39.545 & 964.7 & 0.0223 \\
\hline 10 & 33.821 & 974.1 & 0.0222 & 37.477 & 969.5 & 0.0222 & 39.538 & 961.7 & 0.0222 \\
\hline 11 & 33.837 & 966.6 & 0.0221 & 37.465 & 963.5 & 0.0221 & 39.519 & 958.2 & 0.0222 \\
\hline 12 & 33.858 & 956.5 & 0.0220 & 37.439 & 955.6 & 0.0220 & 39.484 & 954.0 & 0.0221 \\
\hline 13 & 33.866 & 943.4 & 0.0220 & 37.378 & 945.3 & 0.0220 & 39.407 & 948.6 & 0.0220 \\
\hline 14 & 33.791 & 926.8 & 0.0219 & 37.207 & 932.0 & 0.0219 & 39.208 & 941.2 & 0.0219 \\
\hline 15 & 33.438 & 906.1 & 0.0218 & 36.716 & 914.5 & 0.0218 & 38.667 & 930.2 & 0.0218 \\
\hline 16 & 32.227 & 879.8 & 0.0217 & 35.299 & 890.7 & 0.0217 & 37.159 & 912.4 & 0.0218 \\
\hline 17 & 28.603 & 847.3 & 0.0217 & 31.315 & 853.2 & 0.0217 & 32.983 & 878.2 & 0.0217 \\
\hline 18 & 19.743 & 788.2 & 0.0216 & 21.605 & 795.9 & 0.0216 & 22.766 & 814.6 & 0.0216 \\
\hline
\end{tabular}


Table 4-66. Burnup and TH Feedback Parameters by Axial Node for Assembly H10b

\begin{tabular}{|c|c|c|c|c|c|c|c|c|c|}
\hline \multirow[b]{2}{*}{$\begin{array}{l}\text { Axial } \\
\text { Node }\end{array}$} & \multicolumn{3}{|c|}{ Datapoint (171.1 Cy8) } & \multicolumn{3}{|c|}{ Datapoint (338.1 Cy8) } & \multicolumn{3}{|c|}{ Statepoint 68 (BOC Cy9) } \\
\hline & $\begin{array}{c}\text { Burnup } \\
\text { (GWd/MTU) }\end{array}$ & $\begin{array}{c}\text { T-Fuel } \\
{ }^{\circ} \mathrm{F}\end{array}$ & $\begin{array}{c}\text { Spec. Vol. } \\
\mathrm{ft}^{3} / \mathrm{lbm}\end{array}$ & \begin{tabular}{|c|} 
Burnup \\
(GWd/MTU) \\
\end{tabular} & $\begin{array}{c}\text { T-Fuel } \\
{ }^{\circ} \mathrm{F}\end{array}$ & $\begin{array}{c}\text { Spec. Vol. } \\
\mathrm{ft}^{3} / \mathrm{lbm}\end{array}$ & $\begin{array}{c}\text { Burnup } \\
\text { (GWd/MTU) }\end{array}$ & $\begin{array}{l}\text { T-Fuel } \\
{ }^{\circ} \mathrm{F} \\
\end{array}$ & $\begin{array}{c}\text { Spec. Vol. } \\
\mathrm{ft}^{3} / \mathrm{bm}\end{array}$ \\
\hline & $171.1 \mathrm{Cy} 8$ & 77.7 Cy8 & 77.7 Cy8 & 338.1 Cy 8 & 247.2 Cy 8 & 247.2 Cy8 & BOC Cyg & 426.1 Cy 8 & 426.1 Cy 8 \\
\hline 1 & 3.784 & 1061.9 & 0.0237 & 7.877 & 1068.7 & 0.0237 & 12.430 & 1050.2 & 0.0236 \\
\hline 2 & 5.627 & 1248.6 & 0.0236 & 11.645 & 1225.7 & 0.0236 & 18.082 & 1178.5 & 0.0235 \\
\hline 3 & 6.676 & 1349.8 & 0.0235 & 13.570 & 1287.7 & 0.0235 & 20.638 & 1204.6 & 0.0234 \\
\hline 4 & 7.241 & 1402.0 & 0.0233 & 14.454 & 1306.1 & 0.0233 & 21.615 & 1197.3 & 0.0233 \\
\hline 5 & 7.553 & 1428.8 & 0.0232 & 14.855 & 1306.7 & 0.0232 & 21.958 & 1184.3 & 0.0231 \\
\hline 6 & 7.734 & 1442.6 & 0.0231 & 15.043 & 1301.2 & 0.0231 & 22.072 & 1172.9 & 0.0230 \\
\hline 7 & 7.843 & 1448.6 & 0.0229 & 15.138 & 1294.7 & 0.0229 & 22.114 & 1164.6 & 0.0229 \\
\hline 8 & 7.906 & 1449.8 & 0.0228 & 15.188 & 1289.1 & 0.0228 & 22.141 & 1159.0 & 0.0228 \\
\hline 9 & 7.934 & 1448.2 & 0.0227 & 15.215 & 1285.1 & 0.0227 & 22.168 & 1155.6 & 0.0226 \\
\hline 10 & 7.934 & 1444.3 & 0.0225 & 15.225 & 1282.7 & 0.0225 & 22.199 & 1154.0 & 0.0225 \\
\hline 11 & 7.904 & 1438.2 & 0.0224 & 15.220 & 1282.1 & 0.0224 & 22.237 & 1154.1 & 0.0224 \\
\hline 12 & 7.842 & 1429.0 & 0.0223 & 15.194 & 1283.2 & 0.0223 & 22.279 & 1156.3 & 0.0223 \\
\hline 13 & 7.735 & 1415.3 & 0.0222 & 15.129 & 1285.5 & 0.0222 & 22.311 & 1161.0 & 0.0222 \\
\hline 14 & 7.559 & 1394.6 & 0.0220 & 14.976 & 1287.3 & 0.0221 & 22.282 & 1168.9 & 0.0221 \\
\hline 15 & 7.260 & 1362.0 & 0.0219 & 14.629 & 1284.0 & 0.0220 & 22.054 & 1179.2 & 0.0220 \\
\hline 16 & 6.718 & 1305.8 & 0.0218 & 13.817 & 1263.3 & 0.0219 & 21.229 & 1185.6 & 0.0219 \\
\hline 17 & 5.697 & 1202.8 & 0.0217 & 11.961 & 1199.9 & 0.0218 & 18.822 & 1161.4 & 0.0218 \\
\hline 18 & 3.906 & 1020.6 & 0.0217 & 8.259 & 1041.1 & 0.0217 & 13.235 & 1033.8 & 0.0217 \\
\hline
\end{tabular}

\begin{tabular}{|c|c|c|c|c|c|c|c|c|c|}
\hline \multirow[b]{2}{*}{$\begin{array}{l}\text { Axial } \\
\text { Node }\end{array}$} & \multicolumn{3}{|c|}{ Datapoint (214.0 Cy9) } & \multicolumn{3}{|c|}{ Datapoint (425.0 Cy9) } & \multicolumn{3}{|c|}{ Statepoint 73 (BOC Cy11) } \\
\hline & $\begin{array}{c}\text { Burnup } \\
\text { (GWd/MTU) }\end{array}$ & $\begin{array}{c}\begin{array}{c}\text { T-Fuel } \\
{ }^{\circ} \mathrm{F} \\
\end{array} \\
\end{array}$ & $\begin{array}{c}\begin{array}{c}\text { Spec. Vol. } \\
\mathrm{tt}^{3} / \mathrm{lbm}\end{array} \\
\end{array}$ & $\begin{array}{c}\text { Burnup } \\
\text { (GWd/MTU) } \\
\end{array}$ & $\begin{array}{c}\text { T-Fuel } \\
{ }^{\circ} \mathrm{F} \\
\end{array}$ & $\begin{array}{c}\text { Spec. Vol. } \\
\mathrm{ft}^{3} / \mathrm{bm}\end{array}$ & $\begin{array}{c}\text { Burnup } \\
\text { (GWd/MTU) }\end{array}$ & $\begin{array}{c}\text { T-Fuel } \\
{ }^{\circ} \mathbf{F} \\
\end{array}$ & $\begin{array}{c}\text { Spec. Vol. } \\
\mathrm{ft}^{3} / \mathrm{lbm}\end{array}$ \\
\hline & 214.0 Cy 9 & 109.3 Cy9 & 109.3 Cy9 & 425.0 Cy 9 & 328.7 Cy 9 & 328.7 Cy9 & BOC Cy11 & 549.2 Cy9 & 549.2 Cy 9 \\
\hline 1 & 13.884 & 697.1 & 0.0223 & 15.687 & 721.2 & 0.0223 & 17.551 & 759.3 & 0.0224 \\
\hline 2 & 20.526 & 755.8 & 0.0223 & 23.328 & 771.3 & 0.0223 & 25.980 & 799.1 & 0.0224 \\
\hline 3 & 23.692 & 784.9 & 0.0223 & 26.949 & 786.5 & 0.0223 & 30.497 & 805.4 & 0.0223 \\
\hline 4 & 25.006 & 798.7 & 0.0222 & 28.420 & 788.6 & 0.0222 & 32.203 & 802.6 & 0.0223 \\
\hline 5 & 25.517 & 804.2 & 0.0222 & 28.959 & 786.2 & 0.0222 & 32.764 & 797.8 & 0.0222 \\
\hline 6 & 25.714 & 805.7 & 0.0221 & 29.142 & 782.8 & 0.0221 & 32.917 & 793.5 & 0.0222 \\
\hline 7 & 25.795 & 805.2 & 0.0221 & 29.202 & 779.8 & 0.0221 & 32.946 & 790.3 & 0.0221 \\
\hline 8 & 25.836 & 803.8 & 0.0220 & 29.226 & 777.3 & 0.0220 & 32.948 & 788.1 & 0.0221 \\
\hline 9 & 25.864 & 802.0 & 0.0220 & 29.246 & 775.5 & 0.0220 & 32.952 & 786.5 & 0.0220 \\
\hline 10 & 25.887 & 800.0 & 0.0219 & 29.270 & 774.3 & 0.0219 & 32.966 & 785.4 & 0.0220 \\
\hline 11 & 25.910 & 797.9 & 0.0219 & 29.302 & 773.7 & 0.0219 & 32.992 & 784.5 & 0.0219 \\
\hline 12 & 25.928 & 795.5 & 0.0218 & 29.339 & 773.7 & 0.0219 & 33.029 & 784.3 & 0.0219 \\
\hline 13 & 25.917 & 792.5 & 0.0218 & 29.354 & 774.4 & 0.0218 & 33.059 & 785.0 & 0.0218 \\
\hline 14 & 25.813 & 787.9 & 0.0218 & 29.273 & 775.5 & 0.0218 & 33.015 & 787.0 & 0.0218 \\
\hline 15 & 25.448 & 780.6 & 0.0217 & 28.910 & 776.5 & 0.0217 & 32.702 & 790.3 & 0.0218 \\
\hline 16 & 24.372 & 768.1 & 0.0217 & 27.751 & 775.5 & 0.0217 & 31.545 & 793.2 & 0.0217 \\
\hline 17 & 21.463 & 743.6 & 0.0216 & 24.497 & 764.8 & 0.0217 & 28.006 & 790.5 & 0.0217 \\
\hline 18 & 14.857 & 685.1 & 0.0216 & 16.871 & 714.4 & 0.0216 & 19.337 & 751.3 & 0.0216 \\
\hline
\end{tabular}


Table 4-66. Burnup and TH Feedback Parameters by Axial Node for Assembly H10b (Cont.)

\begin{tabular}{|c|c|c|c|c|c|c|c|c|c|}
\hline \multirow{2}{*}{$\begin{array}{c}\text { Axial } \\
\text { Node }\end{array}$} & $\begin{array}{c}\text { Burnup } \\
(\text { GWd/MTU) }\end{array}$ & $\begin{array}{c}\text { T-Fuel } \\
{ }^{\circ} \mathbf{F}\end{array}$ & $\begin{array}{c}\text { Spec. Vol. } \\
\mathbf{f t}^{3} / \mathbf{l b m}\end{array}$ & $\begin{array}{c}\text { Burnup } \\
\text { (GWd/MTU) }\end{array}$ & $\begin{array}{c}\text { T-Fuel } \\
{ }^{\circ} \mathbf{F}\end{array}$ & $\begin{array}{c}\text { Spec. Vol. } \\
\mathbf{f t}^{3} / \mathrm{lbm}\end{array}$ & $\begin{array}{c}\text { Burnup } \\
\text { (GWd/MTU) }\end{array}$ & $\begin{array}{c}\text { T-Fuel } \\
{ }^{\circ} \mathbf{F}\end{array}$ & $\begin{array}{c}\text { Spec. Vol. } \\
\mathbf{f t}^{3} / \mathrm{lbm}\end{array}$ \\
\hline & 208.7 Cy11 & 103.8 Cy11 & 103.8 Cy11 & 417.8 Cy11 & 312.8 Cy11 & 312.8 Cy11 & 615.0 Cy11 & 515.7 Cy11 & 515.7 Cy11 \\
\hline 1 & 20.220 & 795.6 & 0.0231 & 23.190 & 804.8 & 0.0231 & 26.283 & 813.7 & 0.0231 \\
\hline 2 & 30.914 & 907.0 & 0.0231 & 36.201 & 906.2 & 0.0231 & 41.462 & 917.7 & 0.0230 \\
\hline 3 & 36.732 & 972.6 & 0.0230 & 43.148 & 968.7 & 0.0230 & 49.255 & 959.1 & 0.0229 \\
\hline 4 & 38.900 & 995.4 & 0.0229 & 45.604 & 981.5 & 0.0229 & 51.809 & 958.9 & 0.0228 \\
\hline 5 & 39.676 & 1004.8 & 0.0228 & 46.452 & 982.5 & 0.0228 & 52.607 & 952.0 & 0.0227 \\
\hline 6 & 39.940 & 1008.4 & 0.0227 & 46.723 & 980.6 & 0.0227 & 52.817 & 945.4 & 0.0227 \\
\hline 7 & 40.021 & 1008.7 & 0.0226 & 46.793 & 977.7 & 0.0226 & 52.840 & 940.2 & 0.0226 \\
\hline 8 & 40.035 & 1006.5 & 0.0225 & 46.793 & 974.7 & 0.0225 & 52.813 & 936.3 & 0.0225 \\
\hline 9 & 40.022 & 1002.8 & 0.0224 & 46.772 & 972.0 & 0.0224 & 52.780 & 933.4 & 0.0224 \\
\hline 10 & 39.996 & 997.8 & 0.0223 & 46.742 & 969.6 & 0.0223 & 52.752 & 931.4 & 0.0223 \\
\hline 11 & 39.961 & 991.5 & 0.0222 & 46.708 & 967.5 & 0.0222 & 52.731 & 930.1 & 0.0222 \\
\hline 12 & 39.914 & 983.9 & 0.0221 & 46.665 & 965.5 & 0.0221 & 52.714 & 929.7 & 0.0221 \\
\hline 13 & 39.834 & 974.7 & 0.0220 & 46.588 & 963.6 & 0.0220 & 52.679 & 930.3 & 0.0220 \\
\hline 14 & 39.647 & 963.7 & 0.0219 & 46.397 & 961.3 & 0.0219 & 52.547 & 932.2 & 0.0219 \\
\hline 15 & 39.138 & 949.8 & 0.0218 & 45.847 & 957.4 & 0.0219 & 52.060 & 935.1 & 0.0219 \\
\hline 16 & 37.673 & 930.3 & 0.0218 & 44.227 & 948.3 & 0.0218 & 50.439 & 936.0 & 0.0218 \\
\hline 17 & 33.519 & 895.1 & 0.0217 & 39.551 & 921.1 & 0.0217 & 45.428 & 921.4 & 0.0217 \\
\hline 18 & 23.199 & 831.0 & 0.0216 & 27.546 & 845.6 & 0.0216 & 31.951 & 841.9 & 0.0216 \\
\hline
\end{tabular}

Table 4-67. Burnup and TH Feedback Parameters by Axial Node for Assembly H12

\begin{tabular}{|c|c|c|c|c|c|c|c|c|c|}
\hline \multirow[b]{2}{*}{$\begin{array}{l}\text { Axial } \\
\text { Node }\end{array}$} & \multicolumn{3}{|c|}{ Datapoint (171.1 Cy8) } & \multicolumn{3}{|c|}{ Datapoint (338.1 Cy8) } & \multicolumn{3}{|c|}{ Statepoint 68 (BOC Cy9) } \\
\hline & $\begin{array}{c}\text { Burnup } \\
\text { (GWd/MTU) }\end{array}$ & $\begin{array}{l}\text { T-Fuel } \\
{ }^{\circ} \mathbf{F}\end{array}$ & $\begin{array}{c}\text { Spec. Vol. } \\
\mathrm{ft}^{3} / \mathrm{lbm}\end{array}$ & $\begin{array}{c}\text { Burnup } \\
\text { (GWd/MTU) }\end{array}$ & $\begin{array}{l}\text { T-Fuel } \\
{ }^{\circ} \mathrm{F}\end{array}$ & $\begin{array}{c}\text { Spec. Vol. } \\
\mathrm{ft}^{3 / / \mathrm{bm}}\end{array}$ & $\begin{array}{c}\text { Burnup } \\
\text { (GWd/MTU) }\end{array}$ & $\begin{array}{l}\text { T-Fuel } \\
{ }^{\circ} \mathrm{F}\end{array}$ & $\begin{array}{l}\text { Spec. Vol. } \\
\mathrm{ft}^{3} / \mathrm{lbm}\end{array}$ \\
\hline & 171.1 Cy 8 & 77.7 Cy 8 & 77.7 Cy8 & $338.1 \mathrm{Cy} 8$ & 247.2 Cy 8 & 247.2 Cy 8 & BOC Cy 9 & 426.1 Cy 8 & $426.1 \mathrm{Cy} 8$ \\
\hline 1 & 3.370 & 1014.2 & 0.0236 & 7.212 & 1050.4 & 0.0237 & 11.597 & 1046.5 & 0.0237 \\
\hline 2 & 5.088 & 1196.4 & 0.0235 & 10.849 & 1212.2 & 0.0236 & 17.235 & 1188.0 & 0.0236 \\
\hline 3 & 6.256 & 1310.5 & 0.0234 & 13.055 & 1288.0 & 0.0235 & 20.263 & 1223.9 & 0.0234 \\
\hline 4 & 6.918 & 1372.7 & 0.0233 & 14.127 & 1311.7 & 0.0234 & 21.496 & 1216.9 & 0.0233 \\
\hline 5 & 7.257 & 1402.3 & 0.0232 & 14.574 & 1313.4 & 0.0232 & 21.883 & 1201.8 & 0.0232 \\
\hline 6 & 7.456 & 1418.0 & 0.0230 & 14.789 & 1308.2 & 0.0231 & 22.018 & 1189.0 & 0.0230 \\
\hline 7 & 7.584 & 1426.7 & 0.0229 & 14.911 & 1302.1 & 0.0229 & 22.085 & 1179.7 & 0.0229 \\
\hline 8 & 7.665 & 1430.5 & 0.0228 & 14.985 & 1296.8 & 0.0228 & 22.134 & 1173.6 & 0.0228 \\
\hline 9 & 7.711 & 1430.9 & 0.0226 & 15.035 & 1292.9 & 0.0227 & 22.183 & 1169.7 & 0.0227 \\
\hline 10 & 7.731 & 1428.8 & 0.0225 & 15.073 & 1290.7 & 0.0226 & 22.243 & 1167.8 & 0.0226 \\
\hline 11 & 7.730 & 1424.6 & 0.0224 & 15.109 & 1290.6 & 0.0224 & 22.328 & 1167.8 & 0.0224 \\
\hline 12 & 7.721 & 1419.7 & 0.0223 & 15.169 & 1293.5 & 0.0223 & 22.478 & 1170.7 & 0.0223 \\
\hline 13 & 7.693 & 1413.0 & 0.0222 & 15.235 & 1299.9 & 0.0222 & 22.681 & 1177.0 & 0.0222 \\
\hline 14 & 7.557 & 1396.1 & 0.0220 & 15.145 & 1303.4 & 0.0221 & 22.732 & 1185.6 & 0.0221 \\
\hline 15 & 7.257 & 1363.3 & 0.0219 & 14.788 & 1299.4 & 0.0220 & 22.498 & 1196.4 & 0.0220 \\
\hline 16 & 6.696 & 1305.1 & 0.0218 & 13.926 & 1276.0 & 0.0219 & 21.607 & 1202.8 & 0.0219 \\
\hline 17 & 5.680 & 1202.0 & 0.0217 & 12.037 & 1208.9 & 0.0218 & 19.116 & 1176.3 & 0.0218 \\
\hline 18 & 3.968 & 1028.1 & 0.0217 & 8.434 & 1051.1 & 0.0217 & 13.568 & 1044.9 & 0.0217 \\
\hline
\end{tabular}


Table 4-67. Burnup and TH Feedback Parameters by Axial Node for Assembly H12 (Cont.)

\begin{tabular}{|c|c|c|c|c|c|c|c|c|c|}
\hline \multirow[b]{2}{*}{$\begin{array}{l}\text { Axial } \\
\text { Node }\end{array}$} & \multicolumn{3}{|c|}{ Datapoint (214.0 Cy9) } & \multicolumn{3}{|c|}{ Datapoint (425.0 Cy9) } & \multicolumn{3}{|c|}{ Statepoint 69 (BOC Cy10) } \\
\hline & \begin{tabular}{|c|} 
Burnup \\
(GWd/MTU)
\end{tabular} & $\begin{array}{c}\text { T-Fuel } \\
{ }^{\circ} \mathrm{F} \\
\end{array}$ & $\begin{array}{c}\text { Spec. Vol. } \\
\mathrm{ft}^{3} / \mathrm{lbm}\end{array}$ & $\begin{array}{c}\text { Burnup } \\
\text { (GWd/MTU) }\end{array}$ & $\begin{array}{l}\text { T-Fuel } \\
{ }^{\circ} \mathrm{F} \\
\end{array}$ & $\begin{array}{c}\text { Spec. Vol. } \\
\mathrm{ft}^{3} / \mathrm{lbm}\end{array}$ & $\begin{array}{c}\text { Burnup } \\
\text { (GWd/MTU) }\end{array}$ & $\begin{array}{c}\text { T-Fuel } \\
{ }^{\circ} \mathrm{F} \\
\end{array}$ & $\begin{array}{c}\text { Spec. Vol. } \\
\mathrm{ft}^{3} / \mathrm{lbm}\end{array}$ \\
\hline & 214.0 Cy 9 & 109.3 Cy9 & 109.3 Cy9 & 425.0 Cy 9 & 328.7 Cy 9 & 328.7 Cy 9 & BOC Cy 10 & 549.2 Cy 9 & 549.2 Cy9 \\
\hline 1 & 14.442 & 837.8 & 0.0234 & 17.739 & 855.6 & 0.0234 & 20.937 & 870.8 & 0.0233 \\
\hline 2 & 22.468 & 996.3 & 0.0234 & 28.291 & 981.5 & 0.0233 & 33.320 & 967.1 & 0.0232 \\
\hline 3 & 27.572 & 1088.4 & 0.0233 & 34.968 & 1046.2 & 0.0232 & 42.052 & 1024.3 & 0.0231 \\
\hline 4 & 29.547 & 1116.1 & 0.0231 & 37.279 & 1057.3 & 0.0231 & 44.839 & 1019.9 & 0.0230 \\
\hline 5 & 30.251 & 1125.8 & 0.0230 & 38.009 & 1052.9 & 0.0229 & 45.531 & 1008.5 & 0.0229 \\
\hline 6 & 30.544 & 1128.1 & 0.0229 & 38.258 & 1045.9 & 0.0228 & 45.696 & 999.4 & 0.0228 \\
\hline 7 & 30.685 & 1126.8 & 0.0228 & 38.351 & 1039.4 & 0.0227 & 45.720 & 993.1 & 0.0227 \\
\hline 8 & 30.758 & 1123.5 & 0.0226 & 38.392 & 1034.4 & 0.0226 & 45.716 & 988.9 & 0.0226 \\
\hline 9 & 30.800 & 1119.2 & 0.0225 & 38.420 & 1030.8 & 0.0225 & 45.715 & 986.0 & 0.0225 \\
\hline 10 & 30.830 & 1114.1 & 0.0224 & 38.454 & 1028.7 & 0.0224 & 45.731 & 983.8 & 0.0224 \\
\hline 11 & 30.864 & 1108.3 & 0.0223 & 38.514 & 1027.8 & 0.0223 & 45.777 & 982.3 & 0.0223 \\
\hline 12 & 30.934 & 1101.1 & 0.0222 & 38.626 & 1028.1 & 0.0222 & 45.885 & 981.6 & 0.0222 \\
\hline 13 & 31.016 & 1091.6 & 0.0221 & 38.762 & 1029.4 & 0.0221 & 46.043 & 982.5 & 0.0221 \\
\hline 14 & 30.893 & 1080.3 & 0.0220 & 38.701 & 1032.1 & 0.0220 & 46.058 & 986.6 & 0.0220 \\
\hline 15 & 30.382 & 1065.0 & 0.0219 & 38.223 & 1034.3 & 0.0219 & 45.704 & 994.5 & 0.0219 \\
\hline 16 & 29.014 & 1041.2 & 0.0218 & 36.735 & 1029.6 & 0.0218 & 44.295 & 1003.2 & 0.0218 \\
\hline 17 & 25.567 & 996.1 & 0.0217 & 32.649 & 999.2 & 0.0217 & 39.816 & 994.1 & 0.0217 \\
\hline 18 & 17.782 & 880.4 & 0.0216 & 22.702 & 908.6 & 0.0216 & 28.007 & 914.8 & 0.0216 \\
\hline
\end{tabular}

\begin{tabular}{|c|c|c|c|c|c|c|c|c|c|}
\hline \multirow[b]{2}{*}{$\begin{array}{l}\text { Axial } \\
\text { Node } \\
\end{array}$} & \multicolumn{3}{|c|}{ Statepoint 70 (27.0 Cy10) } & \multicolumn{3}{|c|}{ Statepoint $71(143.0 \mathrm{Cy} 10)$} & \multicolumn{3}{|c|}{ Statepoint 72 (209.0 Cy10) } \\
\hline & $\begin{array}{c}\text { Burnup } \\
\text { (GWd/MTU) }\end{array}$ & $\begin{array}{c}\text { T-Fuel } \\
{ }^{\circ} \mathbf{F}\end{array}$ & $\begin{array}{c}\text { Spec. Vol. } \\
\mathrm{ft}^{3} / \mathrm{lbm}\end{array}$ & $\begin{array}{c}\text { Burnup } \\
\text { (GWd/MTU) }\end{array}$ & $\begin{array}{l}\text { T-Fuel } \\
{ }^{\circ} \mathrm{F}\end{array}$ & $\begin{array}{c}\text { Spec. Vol. } \\
\mathrm{ft}^{3} / \mathrm{lbm}\end{array}$ & $\begin{array}{c}\text { Burnup } \\
\text { (GWd/MTU) }\end{array}$ & $\begin{array}{l}\text { T-Fuel } \\
{ }^{\circ} \mathrm{F}\end{array}$ & $\begin{array}{c}\text { Spec. Vol. } \\
\mathrm{ft}^{3} / \mathrm{lbm}\end{array}$ \\
\hline & $27.0 \mathrm{Cy} 10$ & $18.0 \mathrm{Cy} 10$ & $18.0 \mathrm{Cy} 10$ & 143.0 Cy 10 & 82.9 Cy 10 & 82.9 Cy 10 & 209.0 Cy 10 & 176.7 Cy 10 & 176.7 Cy 10 \\
\hline 1 & 21.071 & 646.0 & 0.0220 & 21.693 & 650.4 & 0.0220 & 22.092 & 659.6 & 0.0220 \\
\hline 2 & 33.515 & 660.8 & 0.0220 & 34.412 & 666.0 & 0.0220 & 34.978 & 675.5 & 0.0220 \\
\hline 3 & 42.267 & 664.5 & 0.0220 & 43.250 & 669.6 & 0.0220 & 43.859 & 677.5 & 0.0220 \\
\hline 4 & 45.068 & 667.9 & 0.0219 & 46.108 & 672.7 & 0.0219 & 46.738 & 678.6 & 0.0220 \\
\hline 5 & 45.772 & 671.3 & 0.0219 & 46.855 & 675.6 & 0.0219 & 47.499 & 679.7 & 0.0219 \\
\hline 6 & 45.945 & 673.4 & 0.0219 & 47.057 & 677.3 & 0.0219 & 47.707 & 679.8 & 0.0219 \\
\hline 7 & 45.974 & 674.5 & 0.0219 & 47.103 & 677.9 & 0.0219 & 47.754 & 679.2 & 0.0219 \\
\hline 8 & 45.972 & 674.6 & 0.0218 & 47.109 & 677.8 & 0.0218 & 47.759 & 678.3 & 0.0219 \\
\hline 9 & 45.973 & 673.9 & 0.0218 & 47.110 & 677.0 & 0.0218 & 47.759 & 677.2 & 0.0218 \\
\hline 10 & 45.987 & 672.6 & 0.0218 & 47.121 & 675.7 & 0.0218 & 47.768 & 676.0 & 0.0218 \\
\hline 11 & 46.030 & 670.6 & 0.0218 & 47.155 & 674.0 & 0.0218 & 47.798 & 674.7 & 0.0218 \\
\hline 12 & 46.133 & 667.7 & 0.0217 & 47.241 & 671.5 & 0.0217 & 47.880 & 673.2 & 0.0217 \\
\hline 13 & 46.283 & 663.8 & 0.0217 & 47.366 & 668.2 & 0.0217 & 47.999 & 671.2 & 0.0217 \\
\hline 14 & 46.288 & 658.9 & 0.0217 & 47.337 & 664.0 & 0.0217 & 47.959 & 668.7 & 0.0217 \\
\hline 15 & 45.921 & 652.9 & 0.0217 & 46.920 & 658.7 & 0.0217 & 47.525 & 665.4 & 0.0217 \\
\hline 16 & 44.494 & 645.5 & 0.0216 & 45.421 & 651.6 & 0.0216 & 45.995 & 660.2 & 0.0217 \\
\hline 17 & 39.987 & 634.9 & 0.0216 & 40.792 & 641.0 & 0.0216 & 41.300 & 650.5 & 0.0216 \\
\hline 18 & 28.122 & 614.8 & 0.0216 & 28.669 & 619.4 & 0.0216 & 29.020 & 627.2 & 0.0216 \\
\hline
\end{tabular}


Table 4-68. Burnup and TH Feedback Parameters by Axial Node for Assembly H12a

\begin{tabular}{|c|c|c|c|c|c|c|c|c|c|}
\hline \multirow[b]{2}{*}{$\begin{array}{l}\text { Axial } \\
\text { Node } \\
\end{array}$} & \multicolumn{3}{|c|}{ Datapoint (171.1 CyB) } & \multicolumn{3}{|c|}{ Datapoint (338.1 Cy8) } & \multicolumn{3}{|c|}{ Statepoint 68 (BOC Cy9) } \\
\hline & $\begin{array}{c}\text { Burnup } \\
\text { (GWd/MTU) }\end{array}$ & $\begin{array}{c}\text { T-Fuel } \\
{ }^{\circ} \mathrm{F} \\
\end{array}$ & $\begin{array}{c}\text { Spec. Vol. } \\
\mathrm{ft}^{3} / \mathrm{lbm}\end{array}$ & $\begin{array}{c}\text { Burnup } \\
\text { (GWd/MTU) }\end{array}$ & $\begin{array}{l}\text { T-Fuel } \\
{ }^{\circ} \mathrm{F}\end{array}$ & $\begin{array}{c}\text { Spec. Vol. } \\
\mathrm{ft}^{3} / / \mathrm{bm}\end{array}$ & $\begin{array}{c}\text { Burnup } \\
\text { (GWd/MTU) }\end{array}$ & $\begin{array}{c}\text { T-Fuel } \\
{ }^{\circ} \mathrm{F}\end{array}$ & $\begin{array}{c}\text { Spec. Vol. } \\
\mathrm{tt}^{3} / \mathrm{lbm}\end{array}$ \\
\hline & 171.1 Cy8 & 77.7 Cy8 & 77.7 Cy8 & 338.1 Cy8 & 247.2 Cy 8 & 247.2 Сy8 & BOC Cy9 & $426.1 \mathrm{Cy} 8$ & 426.1 Cy 8 \\
\hline 1 & 3.370 & 1014.2 & 0.0236 & 7.212 & 1050.4 & 0.0237 & 11.596 & 1046.5 & 0.0237 \\
\hline 2 & 5.088 & 1196.4 & 0.0235 & 10.849 & 1212.2 & 0.0236 & 17.234 & 1188.0 & 0.0236 \\
\hline 3 & 6.256 & 1310.5 & 0.0234 & 13.055 & 1288.0 & 0.0235 & 20.261 & 1223.9 & 0.0234 \\
\hline 4 & 6.918 & 1372.7 & 0.0233 & 14.127 & 1311.7 & 0.0234 & 21.493 & 1216.9 & 0.0233 \\
\hline 5 & 7.257 & 1402.3 & 0.0232 & 14.574 & 1313.4 & 0.0232 & 21.880 & 1201.8 & 0.0232 \\
\hline 6 & 7.456 & 1418.0 & 0.0230 & 14.789 & 1308.2 & 0.0231 & 22.015 & 1189.0 & 0.0230 \\
\hline 7 & 7.584 & 1426.7 & 0.0229 & 14.911 & 1302.1 & 0.0229 & 22.082 & 1179.7 & 0.0229 \\
\hline 8 & 7.665 & 1430.5 & 0.0228 & 14.985 & 1296.8 & 0.0228 & 22.130 & 1173.6 & 0.0228 \\
\hline 9 & 7.711 & 1430.9 & 0.0226 & 15.035 & 1292.9 & 0.0227 & 22.180 & 1169.7 & 0.0227 \\
\hline 10 & 7.731 & 1428.8 & 0.0225 & 15.073 & 1290.7 & 0.0226 & 22.240 & 1167.8 & 0.0226 \\
\hline 11 & 7.730 & 1424.6 & 0.0224 & 15.109 & 1290.6 & 0.0224 & 22.326 & 1167.8 & 0.0224 \\
\hline 12 & 7.721 & 1419.7 & 0.0223 & 15.169 & 1293.5 & 0.0223 & 22.476 & 1170.7 & 0.0223 \\
\hline 13 & 7.693 & 1413.0 & 0.0222 & 15.235 & 1299.9 & 0.0222 & 22.680 & 1177.0 & 0.0222 \\
\hline 14 & 7.557 & 1396.1 & 0.0220 & 15.145 & 1303.4 & 0.0221 & 22.732 & 1185.6 & 0.0221 \\
\hline 15 & 7.257 & 1363.3 & 0.0219 & 14.788 & 1299.4 & 0.0220 & 22.498 & 1196.4 & 0.0220 \\
\hline 16 & 6.696 & 1305.1 & 0.0218 & 13.926 & 1276.0 & 0.0219 & 21.607 & 1202.8 & 0.0219 \\
\hline 17 & 5.680 & 1202.0 & 0.0217 & 12.037 & 1208.9 & 0.0218 & 19.116 & 1176.3 & 0.0218 \\
\hline 18 & 3.968 & 1028.1 & 0.0217 & 8.434 & 1051.1 & 0.0217 & 13.567 & 1044.9 & 0.0217 \\
\hline
\end{tabular}

Table 4-69. Burnup and TH Feedback Parameters by Axial Node for Assembly H14

\begin{tabular}{|c|c|c|c|c|c|c|c|c|c|}
\hline \multirow[b]{2}{*}{$\begin{array}{l}\text { Axlal } \\
\text { Node }\end{array}$} & \multicolumn{3}{|c|}{ Datapoint (171.1 Cy8) } & \multicolumn{3}{|c|}{ Datapoint (338.1 Cy8) } & \multicolumn{3}{|c|}{ Statepoint 68 (BOC Cy9) } \\
\hline & $\begin{array}{c}\text { Burnup } \\
\text { (GWd/MTU) }\end{array}$ & $\begin{array}{c}\text { T-Fuel } \\
{ }^{\circ} \mathrm{F} \\
\end{array}$ & $\begin{array}{c}\text { Spec. Vol. } \\
\mathrm{ft}^{3} / \mathrm{lbm}\end{array}$ & $\begin{array}{c}\text { Burnup } \\
\text { (GWd/MTU) }\end{array}$ & $\begin{array}{l}\text { T-Fuel } \\
{ }^{\circ} \mathrm{F} \\
\end{array}$ & $\begin{array}{c}\text { Spec. Vol. } \\
\mathrm{ft}^{3} / \mathrm{lbm}\end{array}$ & $\begin{array}{c}\text { Burnup } \\
\text { (GWd/MTU) }\end{array}$ & $\begin{array}{l}\text { T-Fuel } \\
{ }^{\circ} \mathrm{F} \\
\end{array}$ & $\begin{array}{c}\text { Spec. Vol. } \\
\mathrm{ft}^{3} / \mathrm{bm}\end{array}$ \\
\hline & 171.1 Cy8 & 77.7 Cy8 & 77.7 Cy 8 & 338.1 Cy 8 & 247.2 Cy8 & 247.2 Cy8 & BOC Cy 9 & 426.1 Cy8 & $426.1 \mathrm{Cy} 8$ \\
\hline 1 & 2.878 & 950.8 & 0.0234 & 6.176 & 987.4 & 0.0234 & 10.019 & 999.3 & 0.0233 \\
\hline 2 & 4.594 & 1140.6 & 0.0233 & 9.650 & 1142.7 & 0.0233 & 15.222 & 1117.9 & 0.0232 \\
\hline 3 & 5.613 & 1241.4 & 0.0232 & 11.480 & 1202.1 & 0.0232 & 17.654 & 1147.0 & 0.0231 \\
\hline 4 & 6.198 & 1297.0 & 0.0231 & 12.374 & 1218.6 & 0.0231 & 18.655 & 1142.1 & 0.0230 \\
\hline 5 & 6.541 & 1327.7 & 0.0230 & 12.811 & 1219.2 & 0.0229 & 19.049 & 1130.0 & 0.0229 \\
\hline 6 & 6.749 & 1344.6 & 0.0229 & 13.032 & 1214.0 & 0.0228 & 19.203 & 1118.9 & 0.0228 \\
\hline 7 & 6.879 & 1353.7 & 0.0228 & 13.152 & 1207.8 & 0.0227 & 19.273 & 1110.5 & 0.0227 \\
\hline 8 & 6.963 & 1358.1 & 0.0227 & 13.224 & 1202.4 & 0.0226 & 19.318 & 1104.8 & 0.0226 \\
\hline 9 & 7.014 & 1359.4 & 0.0225 & 13.274 & 1198.4 & 0.0225 & 19.363 & 1101.1 & 0.0225 \\
\hline 10 & 7.041 & 1358.2 & 0.0224 & 13.312 & 1196.1 & 0.0224 & 19.415 & 1099.0 & 0.0224 \\
\hline 11 & 7.045 & 1354.9 & 0.0223 & 13.341 & 1195.5 & 0.0223 & 19.477 & 1098.6 & 0.0223 \\
\hline 12 & 7.019 & 1349.0 & 0.0222 & 13.352 & 1196.6 & 0.0222 & 19.543 & 1100.0 & 0.0222 \\
\hline 13 & 6.948 & 1338.8 & 0.0221 & 13.324 & 1199.0 & 0.0221 & 19.596 & 1103.8 & 0.0221 \\
\hline 14 & 6.801 & 1321.3 & 0.0220 & 13.206 & 1201.3 & 0.0220 & 19.582 & 1110.7 & 0.0220 \\
\hline 15 & 6.521 & 1290.8 & 0.0219 & 12.888 & 1199.4 & 0.0219 & 19.366 & 1119.6 & 0.0219 \\
\hline 16 & 5.993 & 1235.7 & 0.0218 & 12.121 & 1182.6 & 0.0218 & 18.579 & 1124.1 & 0.0218 \\
\hline 17 & 4.990 & 1133.4 & 0.0217 & 10.367 & 1125.1 & 0.0217 & 16.320 & 1098.6 & 0.0218 \\
\hline 18 & 3.211 & 941.1 & 0.0217 & 6.822 & 972.4 & 0.0217 & 11.069 & 981.5 & 0.0217 \\
\hline
\end{tabular}


Table 4-69. Burnup and TH Feedback Parameters by Axial Node for Assembly H14 (Cont.)

\begin{tabular}{|c|c|c|c|c|c|c|c|c|c|}
\hline \multirow[b]{2}{*}{$\begin{array}{l}\text { Axial } \\
\text { Node }\end{array}$} & \multicolumn{3}{|c|}{ Datapoint (214.0 Cy9) } & \multicolumn{3}{|c|}{ Datapoint ( 425.0 Cy9) } & \multicolumn{3}{|c|}{ Statepoint 73 (BOC Cy11) } \\
\hline & $\begin{array}{c}\text { Burnup } \\
\text { (GWd/MTU) }\end{array}$ & $\begin{array}{c}\text { T-Fuel } \\
{ }^{\circ} \mathrm{F} \\
\end{array}$ & $\begin{array}{c}\text { Spec. Vol. } \\
\mathrm{ft}^{3} / \mathrm{lbm}\end{array}$ & $\begin{array}{c}\text { Burnup } \\
\text { (GWd/MTU) }\end{array}$ & $\begin{array}{c}\text { T-Fuel } \\
{ }^{\circ} \mathrm{F}\end{array}$ & $\begin{array}{c}\text { Spec. Vol. } \\
\mathrm{ft}^{3} / \mathrm{lbm}\end{array}$ & $\begin{array}{c}\text { Burnup } \\
\text { (GWd/MTU) }\end{array}$ & $\begin{array}{c}\text { T-Fuel } \\
{ }^{\circ} \mathrm{F} \\
\end{array}$ & $\begin{array}{c}\text { Spec. Vol. } \\
\mathrm{tt}^{3} / \mathrm{lbm}\end{array}$ \\
\hline & 214.0 Cy9 & 109.3 Cy9 & 109.3 Сy9 & 425.0 Cy9 & 328.7 Cy 9 & 328.7 Cy 9 & BOC Cy11 & 549.2 Cy 9 & 549.2 Cy9 \\
\hline 1 & 13.507 & 888.4 & 0.0233 & 17.585 & 915.4 & 0.0232 & 21.873 & 944.1 & 0.0233 \\
\hline 2 & 20.725 & 1003.3 & 0.0232 & 26.730 & 998.8 & 0.0231 & 32.513 & 1002.1 & 0.0232 \\
\hline 3 & 24.278 & 1053.3 & 0.0231 & 31.082 & 1022.7 & 0.0230 & 38.093 & 1019.2 & 0.0231 \\
\hline 4 & 25.888 & 1077.4 & 0.0230 & 32.948 & 1027.0 & 0.0229 & 40.213 & 1013.2 & 0.0230 \\
\hline 5 & 26.595 & 1087.2 & 0.0229 & 33.688 & 1022.2 & 0.0228 & 40.939 & 1002.8 & 0.0229 \\
\hline 6 & 26.914 & 1090.1 & 0.0228 & 33.978 & 1015.7 & 0.0227 & 41.162 & 994.4 & 0.0227 \\
\hline 7 & 27.072 & 1089.7 & 0.0227 & 34.103 & 1010.1 & 0.0226 & 41.235 & 988.8 & 0.0226 \\
\hline 8 & 27.162 & 1087.6 & 0.0225 & 34.172 & 1005.9 & 0.0225 & 41.272 & 985.3 & 0.0226 \\
\hline 9 & 27.223 & 1084.6 & 0.0224 & 34.229 & 1003.0 & 0.0224 & 41.312 & 982.9 & 0.0225 \\
\hline 10 & 27.273 & 1081.3 & 0.0223 & 34.295 & 1001.4 & 0.0223 & 41.368 & 981.3 & 0.0224 \\
\hline 11 & 27.321 & 1077.9 & 0.0222 & 34.382 & 1001.3 & 0.0222 & 41.453 & 980.5 & 0.0223 \\
\hline 12 & 27.366 & 1074.7 & 0.0221 & 34.491 & 1003.1 & 0.0222 & 41.575 & 981.0 & 0.0222 \\
\hline 13 & 27.368 & 1070.6 & 0.0220 & 34.578 & 1006.5 & 0.0221 & 41.704 & 983.6 & 0.0221 \\
\hline 14 & 27.222 & 1062.5 & 0.0219 & 34.509 & 1010.4 & 0.0220 & 41.719 & 988.6 & 0.0220 \\
\hline 15 & 26.736 & 1047.7 & 0.0219 & 34.047 & 1012.9 & 0.0219 & 41.374 & 996.5 & 0.0219 \\
\hline 16 & 25.441 & 1021.7 & 0.0218 & 32.605 & 1008.7 & 0.0218 & 39.983 & 1003.7 & 0.0218 \\
\hline 17 & 22.190 & 974.4 & 0.0217 & 28.699 & 985.7 & 0.0217 & 35.643 & 990.8 & 0.0217 \\
\hline 18 & 14.825 & 856.8 & 0.0216 & 19.299 & 896.6 & 0.0216 & 24.384 & 921.9 & 0.0216 \\
\hline
\end{tabular}

\begin{tabular}{|c|c|c|c|c|c|c|c|c|c|}
\hline \multirow[b]{2}{*}{$\begin{array}{l}\text { Axial } \\
\text { Node }\end{array}$} & \multicolumn{3}{|c|}{ Datapoint (208.7 Cy11) } & \multicolumn{3}{|c|}{ Datapoint (417.8 Cy11) } & \multicolumn{3}{|c|}{ Statepoint 74 (615.0 Cy11) } \\
\hline & $\begin{array}{c}\text { Burnup } \\
\text { (GWd/MTU) }\end{array}$ & $\begin{array}{l}\text { T-Fuel } \\
{ }^{\circ} \mathrm{F}\end{array}$ & $\begin{array}{c}\text { Spec. Vol. } \\
\mathrm{ft}^{3} / / \mathrm{bm}\end{array}$ & $\begin{array}{c}\text { Burnup } \\
\text { (GWd/MTU) }\end{array}$ & $\begin{array}{l}\text { TrFuel } \\
{ }^{\circ} \mathbf{F}\end{array}$ & $\begin{array}{l}\text { Spec. Vol. } \\
\mathrm{ft}^{3} / \mathrm{bmm}\end{array}$ & $\begin{array}{c}\text { Burnup } \\
\text { (GWd/MTU) }\end{array}$ & $\begin{array}{l}\text { T-Fuel } \\
{ }^{\circ} \mathrm{F}\end{array}$ & \begin{tabular}{|c} 
Spec. Vol. \\
$\mathrm{tt}^{3} / \mathrm{lbm}$
\end{tabular} \\
\hline & 208.7 Cy 11 & 103.8 Cy 11 & 103.8 Cy 11 & 417.8 Cy 11 & 312.8 Cy 11 & 312.8 Cy 11 & 615.0 Cy 11 & 515.7 Cy 11 & 515.7 Cy 11 \\
\hline 1 & 22.809 & 635.0 & 0.0220 & 23.993 & 650.9 & 0.0220 & 25.345 & 665.9 & 0.0220 \\
\hline 2 & 33.922 & 652.7 & 0.0220 & 35.620 & 668.9 & 0.0220 & 37.463 & 683.1 & 0.0220 \\
\hline 3 & 39.728 & 662.8 & 0.0219 & 41.610 & 675.5 & 0.0220 & 43.579 & 686.2 & 0.0220 \\
\hline 4 & 41.956 & 667.3 & 0.0219 & 43.883 & 676.0 & 0.0219 & 45.849 & 683.8 & 0.0220 \\
\hline 5 & 42.740 & 669.7 & 0.0219 & 44.671 & 675.1 & 0.0219 & 46.609 & 681.0 & 0.0219 \\
\hline 6 & 42.996 & 670.7 & 0.0219 & 44.919 & 673.8 & 0.0219 & 46.831 & 678.6 & 0.0219 \\
\hline 7 & 43.084 & 670.9 & 0.0218 & 44.997 & 672.4 & 0.0219 & 46.889 & 676.8 & 0.0219 \\
\hline 8 & 43.127 & 670.4 & 0.0218 & 45.029 & 671.2 & 0.0218 & 46.909 & 675.3 & 0.0219 \\
\hline 9 & 43.164 & 669.6 & 0.0218 & 45.059 & 670.1 & 0.0218 & 46.931 & 674.1 & 0.0218 \\
\hline 10 & 43.212 & 668.4 & 0.0218 & 45.102 & 869.1 & 0.0218 & 46.969 & 673.1 & 0.0218 \\
\hline 11 & 43.283 & 666.9 & 0.0218 & 45.171 & 668.3 & 0.0218 & 47.036 & 672.3 & 0.0218 \\
\hline 12 & 43.386 & 665.0 & 0.0217 & 45.272 & 667.5 & 0.0217 & 47.140 & 671.6 & 0.0218 \\
\hline 13 & 43.486 & 662.6 & 0.0217 & 45.373 & 666.8 & 0.0217 & 47.248 & 671.3 & 0.0217 \\
\hline 14 & 43.459 & 659.5 & 0.0217 & 45.344 & 666.0 & 0.0217 & 47.232 & 671.4 & 0.0217 \\
\hline 15 & 43.050 & 655.3 & 0.0217 & 44.922 & 664.9 & 0.0217 & 46.826 & 671.8 & 0.0217 \\
\hline 16 & 41.553 & 649.2 & 0.0216 & 43.378 & 662.6 & 0.0217 & 45.280 & 671.9 & 0.0217 \\
\hline 17 & 37.011 & 638.8 & 0.0216 & 38.673 & 655.3 & 0.0216 & 40.467 & 667.6 & 0.0216 \\
\hline 18 & 25.305 & 620.2 & 0.0216 & 26.478 & 635.5 & 0.0216 & 27.806 & 648.1 & 0.0216 \\
\hline
\end{tabular}


Table 4-70. Burnup and TH Feedback Parameters by Axial Node for Assembly $\mathrm{H} 17$

\begin{tabular}{|c|c|c|c|c|c|c|c|c|c|}
\hline \multirow[b]{2}{*}{$\begin{array}{l}\text { Axial } \\
\text { Node }\end{array}$} & \multicolumn{3}{|c|}{ Datapoint (171.1 Cy8) } & \multicolumn{3}{|c|}{ Datapoint (338.1 Cy8) } & \multicolumn{3}{|c|}{ Statepoint 68 (BOC Cy9) } \\
\hline & \begin{tabular}{|c|} 
Burnup \\
(GWd/MTU)
\end{tabular} & $\begin{array}{l}\text { T-Fuel } \\
{ }^{\circ} \mathrm{F} \\
\end{array}$ & $\begin{array}{c}\text { Spec. Vol. } \\
\mathrm{ft}^{3} / \mathrm{lbm}\end{array}$ & $\begin{array}{c}\text { Burnup } \\
\text { (GWd/MTU) }\end{array}$ & $\begin{array}{l}\text { T-Fuel } \\
{ }^{\circ} \mathrm{F}\end{array}$ & $\begin{array}{c}\text { Spec. Vol. } \\
\mathrm{ft}^{3} / \mathrm{lbm}\end{array}$ & $\begin{array}{c}\text { Burnup } \\
\text { (GWd/MTU) }\end{array}$ & $\begin{array}{c}\text { T-Fuel } \\
{ }^{\circ} \mathrm{F} \\
\end{array}$ & $\begin{array}{c}\text { Spec. Vol. } \\
\mathrm{ft}^{3} / \mathrm{lbm}\end{array}$ \\
\hline & 171.1 Cy 8 & 77.7 Cy8 & 77.7 Cy8 & 338.1 Cy8 & 247.2 Cy 8 & 247.2 Cy 8 & BOC Cy9 & 426.1 Cy 8 & 426.1 Cy 8 \\
\hline 1 & 3.583 & 1037.3 & 0.0236 & 7.617 & 1066.2 & 0.0237 & 12.197 & 1057.4 & 0.0237 \\
\hline 2 & 5.243 & 1209.5 & 0.0235 & 11.131 & 1220.5 & 0.0236 & 17.646 & 1192.6 & 0.0236 \\
\hline 3 & 6.249 & 1307.8 & 0.0234 & 13.054 & 1287.9 & 0.0235 & 20.288 & 1224.5 & 0.0234 \\
\hline 4 & 6.817 & 1361.2 & 0.0233 & 13.981 & 1308.6 & 0.0233 & 21.343 & 1217.9 & 0.0233 \\
\hline 5 & 7.114 & 1387.1 & 0.0231 & 14.371 & 1309.6 & 0.0232 & 21.668 & 1203.4 & 0.0232 \\
\hline 6 & 7.291 & 1400.8 & 0.0230 & 14.559 & 1304.3 & 0.0231 & 21.776 & 1190.9 & 0.0230 \\
\hline 7 & 7.405 & 1408.2 & 0.0229 & 14.665 & 1298.4 & 0.0229 & 21.830 & 1182.0 & 0.0229 \\
\hline 8 & 7.476 & 1411.3 & 0.0227 & 14.730 & 1293.2 & 0.0228 & 21.870 & 1176.1 & 0.0228 \\
\hline 9 & 7.515 & 1411.2 & 0.0226 & 14.772 & 1289.4 & 0.0227 & 21.913 & 1172.4 & 0.0227 \\
\hline 10 & 7.529 & 1408.7 & 0.0225 & 14.803 & 1287.3 & 0.0226 & 21.968 & 1170.7 & 0.0226 \\
\hline 11 & 7.525 & 1404.3 & 0.0224 & 14.836 & 1287.3 & 0.0224 & 22.052 & 1171.0 & 0.0224 \\
\hline 12 & 7.517 & 1399.4 & 0.0223 & 14.899 & 1290.4 & 0.0223 & 22.208 & 1174.1 & 0.0223 \\
\hline 13 & 7.495 & 1393.3 & 0.0221 & 14.974 & 1297.0 & 0.0222 & 22.423 & 1180.6 & 0.0222 \\
\hline 14 & 7.366 & 1377.1 & 0.0220 & 14.890 & 1300.3 & 0.0221 & 22.482 & 1189.2 & 0.0221 \\
\hline 15 & 7.076 & 1345.2 & 0.0219 & 14.539 & 1295.7 & 0.0220 & 22.250 & 1199.8 & 0.0220 \\
\hline 16 & 6.534 & 1288.7 & 0.0218 & 13.692 & 1272.3 & 0.0219 & 21.365 & 1205.4 & 0.0219 \\
\hline 17 & 5.556 & 1189.3 & 0.0217 & 11.848 & 1205.0 & 0.0218 & 18.908 & 1177.3 & 0.0218 \\
\hline 18 & 3.900 & 1020.3 & 0.0217 & 8.327 & 1048.4 & 0.0217 & 13.446 & 1044.6 & 0.0217 \\
\hline
\end{tabular}

Table 4-71. Burnup and TH Feedback Parameters by Axial Node for Assembly H19

\begin{tabular}{|c|c|c|c|c|c|c|c|c|c|}
\hline \multirow[b]{2}{*}{$\begin{array}{l}\text { Axial } \\
\text { Node }\end{array}$} & \multicolumn{3}{|c|}{ Datapoint (171.1 Cy8) } & \multicolumn{3}{|c|}{ Datapoint (338.1 Cy8) } & \multicolumn{3}{|c|}{ Statepoint 68 (BOC Cy9) } \\
\hline & $\begin{array}{c}\text { Burnup } \\
\text { (GWd/MTU) }\end{array}$ & $\begin{array}{c}\text { T-Fuel } \\
{ }^{\circ} \mathrm{F}\end{array}$ & $\begin{array}{c}\text { Spec. Vol. } \\
\mathrm{ft}^{3} / \mathrm{lbm}\end{array}$ & $\begin{array}{c}\text { Burnup } \\
\text { (GWd/MTU) }\end{array}$ & $\begin{array}{c}\text { T-Fuel } \\
{ }^{\circ} \mathrm{F} \\
\end{array}$ & $\begin{array}{c}\text { Spec. Vol. } \\
\mathrm{ft}^{3} / \mathrm{lbm}\end{array}$ & $\begin{array}{c}\text { Burnup } \\
\text { (GWd/MTU) }\end{array}$ & $\begin{array}{c}\text { T-Fuel } \\
{ }^{\circ} \mathrm{F}\end{array}$ & $\begin{array}{c}\text { Spec. Vol. } \\
\mathrm{ft}^{3} / \mathrm{lbm}\end{array}$ \\
\hline & 171.1 Cy 8 & 77.7 Cy 8 & 77.7 Cy8 & 338.1 Cy8 & 247.2 Cy 8 & 247.2 Cy8 & BOC Cy9 & 426.1 Cy 8 & 426.1 Cy 8 \\
\hline 1 & 3.413 & 1019.0 & 0.0237 & 7.286 & 1051.9 & 0.0237 & 11.724 & 1048.4 & 0.0236 \\
\hline 2 & 5.132 & 1200.1 & 0.0236 & 10.868 & 1207.0 & 0.0236 & 17.229 & 1182.1 & 0.0235 \\
\hline 3 & 6.257 & 1310.3 & 0.0235 & 12.954 & 1277.0 & 0.0235 & 20.053 & 1214.4 & 0.0234 \\
\hline 4 & 6.919 & 1372.6 & 0.0233 & 14.003 & 1298.9 & 0.0233 & 21.241 & 1207.4 & 0.0233 \\
\hline 5 & 7.282 & 1404.4 & 0.0232 & 14.472 & 1300.2 & 0.0232 & 21.648 & 1192.3 & 0.0231 \\
\hline 6 & 7.504 & 1422.1 & 0.0231 & 14.712 & 1294.9 & 0.0231 & 21.809 & 1179.4 & 0.0230 \\
\hline 7 & 7.652 & 1432.3 & 0.0229 & 14.856 & 1288.8 & 0.0229 & 21.899 & 1170.1 & 0.0229 \\
\hline 8 & 7.751 & 1437.3 & 0.0228 & 14.951 & 1283.5 & 0.0228 & 21.969 & 1163.7 & 0.0228 \\
\hline 9 & 7.815 & 1439.0 & 0.0227 & 15.022 & 1279.7 & 0.0227 & 22.039 & 1159.7 & 0.0227 \\
\hline 10 & 7.853 & 1438.4 & 0.0225 & 15.082 & 1277.7 & 0.0225 & 22.122 & 1157.6 & 0.0225 \\
\hline 11 & 7.873 & 1436.1 & 0.0224 & 15.145 & 1277.9 & 0.0224 & 22.234 & 1157.4 & 0.0224 \\
\hline 12 & 7.886 & 1433.1 & 0.0223 & 15.235 & 1281.3 & 0.0223 & 22.415 & 1160.0 & 0.0223 \\
\hline 13 & 7.878 & 1428.5 & 0.0222 & 15.331 & 1288.2 & 0.0222 & 22.649 & 1166.1 & 0.0222 \\
\hline 14 & 7.751 & 1413.4 & 0.0220 & 15.261 & 1292.6 & 0.0221 & 22.721 & 1174.5 & 0.0221 \\
\hline 15 & 7.443 & 1380.2 & 0.0219 & 14.904 & 1289.5 & 0.0220 & 22.487 & 1185.2 & 0.0220 \\
\hline 16 & 6.841 & 1318.5 & 0.0218 & 14.001 & 1266.6 & 0.0219 & 21.557 & 1192.0 & 0.0219 \\
\hline 17 & 5.742 & 1207.8 & 0.0217 & 12.017 & 1199.6 & 0.0218 & 18.973 & 1166.4 & 0.0218 \\
\hline 18 & 3.945 & 1025.4 & 0.0217 & 8.315 & 1041.9 & 0.0217 & 13.339 & 1035.9 & 0.0217 \\
\hline
\end{tabular}


Table 4-71. Burnup and TH Feedback Parameters by Axial Node for Assembly H19 (Cont.)

\begin{tabular}{|c|c|c|c|c|c|c|c|c|c|}
\hline \multirow[b]{2}{*}{$\begin{array}{l}\text { Axial } \\
\text { Node }\end{array}$} & \multicolumn{3}{|c|}{ Datapoint (214.0 Cy9) } & \multicolumn{3}{|c|}{ Datapoint (425.0 Cy9) } & \multicolumn{3}{|c|}{ Statepoint 69 (BOC Cy10) } \\
\hline & \begin{tabular}{|c|} 
Burnup \\
(GWd/MTU) \\
\end{tabular} & $\begin{array}{l}\text { T-Fuel } \\
{ }^{\circ} \mathrm{F}\end{array}$ & $\begin{array}{c}\text { Spec. Vol. } \\
\mathrm{ft}^{3} / \mathrm{lbm}\end{array}$ & $\begin{array}{c}\text { Burnup } \\
\text { (GWd/MTU) }\end{array}$ & $\begin{array}{c}\text { T-Fuel } \\
{ }^{\circ} \mathrm{F}\end{array}$ & $\begin{array}{c}\text { Spec. Vol. } \\
\mathrm{ft}^{3} / \mathrm{lbm}\end{array}$ & $\begin{array}{c}\text { Burnup } \\
\text { (GWd/MTU) }\end{array}$ & $\begin{array}{c}\text { T-Fuel } \\
{ }^{\circ} \mathrm{F} \\
\end{array}$ & $\begin{array}{c}\text { Spec. Vol. } \\
\mathrm{ft}^{3} / \mathrm{bm}\end{array}$ \\
\hline & 214.0 Cy9 & 109.3 Cy9 & 109.3 Cy 9 & $425.0 \mathrm{Cy} 9$ & 328.7 Cy9 & 328.7 Cy 9 & BOC Cy 10 & 549.2 Cy9 & 549.2 Cy 9 \\
\hline 1 & 15.565 & 911.0 & 0.0234 & 20.029 & 934.5 & 0.0234 & 24.631 & 952.3 & 0.0233 \\
\hline 2 & 23.188 & 1022.8 & 0.0233 & 29.706 & 1018.4 & 0.0233 & 35.822 & 1019.0 & 0.0232 \\
\hline 3 & 27.121 & 1070.8 & 0.0232 & 34.445 & 1043.1 & 0.0232 & 41.799 & 1034.7 & 0.0231 \\
\hline 4 & 28.900 & 1093.3 & 0.0231 & 36.465 & 1049.5 & 0.0230 & 44.063 & 1025.8 & 0.0230 \\
\hline 5 & 29.615 & 1103.0 & 0.0229 & 37.207 & 1045.2 & 0.0229 & 44.778 & 1014.3 & 0.0229 \\
\hline 6 & 29.936 & 1105.8 & 0.0228 & 37.491 & 1038.6 & 0.0228 & 44.983 & 1005.2 & 0.0228 \\
\hline 7 & 30.107 & 1104.9 & 0.0227 & 37.622 & 1032.6 & 0.0227 & 45.051 & 999.1 & 0.0227 \\
\hline 8 & 30.209 & 1102.1 & 0.0226 & 37.697 & 1027.9 & 0.0226 & 45.087 & 995.2 & 0.0226 \\
\hline 9 & 30.281 & 1098.1 & 0.0225 & 37.760 & 1024.7 & 0.0225 & 45.127 & 992.4 & 0.0225 \\
\hline 10 & 30.343 & 1093.6 & 0.0224 & 37.833 & 1022.7 & 0.0224 & 45.185 & 990.5 & 0.0224 \\
\hline 11 & 30.417 & 1088.4 & 0.0223 & 37.938 & 1022.1 & 0.0223 & 45.281 & 989.1 & 0.0223 \\
\hline 12 & 30.539 & 1082.1 & 0.0222 & 38.111 & 1022.9 & 0.0222 & 45.455 & 988.8 & 0.0222 \\
\hline 13 & 30.677 & 1073.8 & 0.0221 & 38.313 & 1024.7 & 0.0221 & 45.688 & 990.1 & 0.0221 \\
\hline 14 & 30.586 & 1063.1 & 0.0220 & 38.290 & 1027.6 & 0.0220 & 45.746 & 994.6 & 0.0220 \\
\hline 15 & 30.072 & 1047.4 & 0.0219 & 37.801 & 1029.4 & 0.0219 & 45.382 & 1002.7 & 0.0219 \\
\hline 16 & 28.646 & 1022.5 & 0.0218 & 36.236 & 1023.6 & 0.0218 & 43.886 & 1011.1 & 0.0218 \\
\hline 17 & 25.104 & 977.3 & 0.0217 & 32.032 & 994.0 & 0.0217 & 39.257 & 1000.5 & 0.0217 \\
\hline 18 & 17.324 & 864.9 & 0.0216 & 22.106 & 903.2 & 0.0216 & 27.420 & 919.8 & 0.0216 \\
\hline
\end{tabular}

\begin{tabular}{|c|c|c|c|c|c|c|c|c|c|}
\hline \multirow[b]{2}{*}{$\begin{array}{l}\text { Axial } \\
\text { Noode }\end{array}$} & \multicolumn{3}{|c|}{ Statepoint 70 (27.0 Cy10) } & \multicolumn{3}{|c|}{ Statepoint $71(143.0$ Cy10) } & \multicolumn{3}{|c|}{ Statepoint $72(209.0$ Cy10) } \\
\hline & $\begin{array}{c}\text { Burnup } \\
\text { (GWd/MTU) }\end{array}$ & $\begin{array}{c}\text { T-Fuel } \\
{ }^{\circ} \mathrm{F}\end{array}$ & $\begin{array}{c}\text { Spec. Vol. } \\
\mathrm{ft}^{3} / \mathrm{lbm}\end{array}$ & $\begin{array}{c}\text { Burnup } \\
\text { (GWd/MTU) }\end{array}$ & $\begin{array}{c}\text { T-Fuel } \\
{ }^{\circ} \mathrm{F} \\
\end{array}$ & $\begin{array}{c}\text { Spec. Vol. } \\
\mathrm{ft}^{3} / \mathrm{lbm}\end{array}$ & $\begin{array}{c}\text { Burnup } \\
\text { (GWd/MTU) }\end{array}$ & $\begin{array}{c}\text { T-Fuel } \\
{ }^{\circ} \mathrm{F}\end{array}$ & $\begin{array}{c}\text { Spec. Vol. } \\
\mathrm{ft}^{3} / \mathrm{lbm}\end{array}$ \\
\hline & 27.0 Cy 10 & 18.0 Cy 10 & $18.0 \mathrm{Cy} 10$ & $143.0 \mathrm{Cy} 10$ & 82.9 Cy 10 & 82.9 Cy 10 & 209.0 Cy 10 & 176.7 Cy 10 & 176.7 Cy 10 \\
\hline 1 & 24.758 & 637.8 & 0.0220 & 25.341 & 641.5 & 0.0220 & 25.715 & 649.3 & 0.0220 \\
\hline 2 & 36.014 & 659.0 & 0.0220 & 36.893 & 663.6 & 0.0220 & 37.445 & 672.2 & 0.0220 \\
\hline 3 & 42.025 & 670.3 & 0.0220 & 43.052 & 674.9 & 0.0220 & 43.682 & 681.9 & 0.0220 \\
\hline 4 & 44.310 & 676.4 & 0.0220 & 45.417 & 680.6 & 0.0220 & 46.082 & 685.5 & 0.0220 \\
\hline 5 & 45.039 & 680.6 & 0.0219 & 46.198 & 684.3 & 0.0219 & 46.879 & 687.0 & 0.0220 \\
\hline 6 & 45.253 & 683.2 & 0.0219 & 46.444 & 686.2 & 0.0219 & 47.132 & 687.2 & 0.0219 \\
\hline 7 & 45.326 & 684.4 & 0.0219 & 46.536 & 686.9 & 0.0219 & 47.226 & 686.5 & 0.0219 \\
\hline 8 & 45.366 & 684.5 & 0.0218 & 46.584 & 686.7 & 0.0219 & 47.274 & 685.5 & 0.0219 \\
\hline 9 & 45.406 & 683.9 & 0.0218 & 46.627 & 685.9 & 0.0218 & 47.315 & 684.4 & 0.0218 \\
\hline 10 & 45.463 & 682.5 & 0.0218 & 46.681 & 684.6 & 0.0218 & 47.366 & 683.1 & 0.0218 \\
\hline 11 & 45.557 & 680.3 & 0.0218 & 46.764 & 682.7 & 0.0218 & 47.447 & 681.7 & 0.0218 \\
\hline 12 & 45.725 & 677.1 & 0.0217 & 46.915 & 679.9 & 0.0217 & 47.592 & 680.0 & 0.0218 \\
\hline 13 & 45.949 & 672.6 & 0.0217 & 47.110 & 676.2 & 0.0217 & 47.779 & 677.7 & 0.0217 \\
\hline 14 & 45.996 & 667.2 & 0.0217 & 47.119 & 671.7 & 0.0217 & 47.778 & 675.1 & 0.0217 \\
\hline 15 & 45.617 & 660.6 & 0.0217 & 46.687 & 665.9 & 0.0217 & 47.327 & 671.6 & 0.0217 \\
\hline 16 & 44.100 & 652.3 & 0.0216 & 45.090 & 658.1 & 0.0216 & 45.696 & 666.0 & 0.0217 \\
\hline 17 & 39.440 & 640.2 & 0.0216 & 40.293 & 646.1 & 0.0216 & 40.826 & 655.3 & 0.0216 \\
\hline 18 & 27.541 & 618.6 & 0.0216 & 28.115 & 623.0 & 0.0216 & 28.480 & 630.8 & 0.0216 \\
\hline
\end{tabular}


Table 4-72. Burnup and TH Feedback Parameters by Axial Node for Assembly $\mathrm{H} 2 \mathrm{O}$

\begin{tabular}{|c|c|c|c|c|c|c|c|c|c|}
\hline \multirow[b]{2}{*}{$\begin{array}{l}\text { Axial } \\
\text { Node }\end{array}$} & \multicolumn{3}{|c|}{ Datapoint (171.1 Cy8) } & \multicolumn{3}{|c|}{ Datapoint (338.1 Cy8) } & \multicolumn{3}{|c|}{ Statepoint 68 (BOC Cy9) } \\
\hline & $\begin{array}{c}\text { Burnup } \\
\text { (GWd/MTU) }\end{array}$ & $\begin{array}{c}\text { T-Fuel } \\
{ }^{\circ} \mathrm{F} \\
\end{array}$ & $\begin{array}{c}\text { Spec. Vol. } \\
\mathrm{ft}^{3} / \mathrm{lbm}\end{array}$ & $\begin{array}{c}\text { Burnup } \\
\text { (GWd/MTU) }\end{array}$ & $\begin{array}{c}\text { T-Fuel } \\
{ }^{\circ} \mathrm{F}\end{array}$ & $\begin{array}{c}\text { Spec. Vol. } \\
\mathrm{ft}^{3} / \mathrm{lbm}\end{array}$ & $\begin{array}{c}\text { Burnup } \\
\text { (GWd/MTU) }\end{array}$ & $\begin{array}{c}\text { T-Fuel } \\
{ }^{\circ} \mathbf{F} \\
\end{array}$ & $\begin{array}{c}\text { Spec. Vol. } \\
\mathrm{t}^{3} / \mathrm{lbm}\end{array}$ \\
\hline & 171.1 Cy 8 & 77.7 Cy 8 & 77.7 Cy8 & 338.1 Cy 8 & 247.2 Cy 8 & 247.2 Cy8 & BOC Cy9 & 426.1 Cy 8 & $426.1 \mathrm{Cy} 8$ \\
\hline 1 & 2.453 & 913.2 & 0.0232 & 5.288 & 952.0 & 0.0232 & 8.627 & 973.7 & 0.0231 \\
\hline 2 & 4.205 & 1094.9 & 0.0232 & 8.768 & 1094.8 & 0.0231 & 13.799 & 1072.6 & 0.0231 \\
\hline 3 & 5.200 & 1195.1 & 0.0231 & 10.524 & 1151.0 & 0.0230 & 16.124 & 1102.1 & 0.0230 \\
\hline 4 & 5.766 & 1248.9 & 0.0230 & 11.378 & 1166.7 & 0.0229 & 17.083 & 1098.8 & 0.0229 \\
\hline 5 & 6.092 & 1278.0 & 0.0229 & 11.789 & 1166.4 & 0.0228 & 17.454 & 1088.0 & 0.0228 \\
\hline 6 & 6.287 & 1293.6 & 0.0228 & 11.991 & 1161.1 & 0.0227 & 17.594 & 1077.8 & 0.0227 \\
\hline 7 & 6.408 & 1301.9 & 0.0227 & 12.098 & 1155.1 & 0.0226 & 17.655 & 1070.0 & 0.0226 \\
\hline 8 & 6.486 & 1305.8 & 0.0226 & 12.164 & 1149.8 & 0.0225 & 17.694 & 1064.6 & 0.0225 \\
\hline 9 & 6.535 & 1307.0 & 0.0225 & 12.209 & 1146.0 & 0.0224 & 17.734 & 1061.2 & 0.0224 \\
\hline 10 & 6.562 & 1306.2 & 0.0224 & 12.246 & 1143.8 & 0.0223 & 17.782 & 1059.2 & 0.0223 \\
\hline 11 & 6.571 & 1303.6 & 0.0223 & 12.278 & 1143.2 & 0.0222 & 17.843 & 1058.8 & 0.0222 \\
\hline 12 & 6.557 & 1299.0 & 0.0222 & 12.302 & 1144.4 & 0.0221 & 17.917 & 1060.1 & 0.0222 \\
\hline 13 & 6.503 & 1290.7 & 0.0221 & 12.293 & 1147.0 & 0.0221 & 17.982 & 1063.6 & 0.0221 \\
\hline 14 & 6.374 & 1275.3 & 0.0220 & 12.193 & 1149.3 & 0.0220 & 17.977 & 1069.8 & 0.0220 \\
\hline 15 & 6.108 & 1246.6 & 0.0219 & 11.892 & 1147.7 & 0.0219 & 17.764 & 1077.6 & 0.0219 \\
\hline 16 & 5.593 & 1193.4 & 0.0218 & 11.151 & 1132.4 & 0.0218 & 16.994 & 1080.3 & 0.0218 \\
\hline 17 & 4.597 & 1091.6 & 0.0217 & 9.442 & 1077.6 & 0.0217 & 14.796 & 1053.3 & 0.0217 \\
\hline 18 & 2.722 & 883.2 & 0.0217 & 5.788 & 915.5 & 0.0217 & 9.421 & 933.7 & 0.0217 \\
\hline
\end{tabular}

Table 4-73. Burnup and TH Feedback Parameters by Axial Node for Assembly H23

\begin{tabular}{|c|c|c|c|c|c|c|c|c|c|}
\hline \multirow[b]{2}{*}{$\begin{array}{l}\text { Axial } \\
\text { Node }\end{array}$} & \multicolumn{3}{|c|}{ Datapoint (171.1 Cy8) } & \multicolumn{3}{|c|}{ Datapoint (338.1 Cy8) } & \multicolumn{3}{|c|}{ Statepoint $6 B$ (BOC Cy9) } \\
\hline & $\begin{array}{c}\text { Burnup } \\
\text { (GWd/MTU) }\end{array}$ & $\begin{array}{l}\text { T-Fuel } \\
{ }^{\circ} \mathbf{F} \\
\end{array}$ & $\begin{array}{c}\text { Spec. Vol. } \\
\mathrm{ft}^{3} / \mathrm{lbm}\end{array}$ & $\begin{array}{c}\text { Burnup } \\
\text { (GWd/MTU) }\end{array}$ & $\begin{array}{c}\text { T-Fuel } \\
{ }^{\circ} \mathrm{F}\end{array}$ & $\begin{array}{c}\text { Spec. Vol. } \\
\mathrm{ft}^{3} / \mathrm{bm}\end{array}$ & $\begin{array}{c}\text { Burnup } \\
\text { (GWd/MTU) }\end{array}$ & $\begin{array}{l}\text { T-Fuel } \\
{ }^{\circ} \mathrm{F}\end{array}$ & $\begin{array}{c}\text { Spec. Vol. } \\
\mathrm{ft}^{3} / \mathrm{lbm}\end{array}$ \\
\hline & 171.1 Cy 8 & 77.7 Cy 8 & 77.7 Cy8 & 338.1 Cy 8 & 247.2 Cy 8 & 247.2 Cy8 & BOC Cy9 & 426.1 Cy 8 & 426.1 Cy 8 \\
\hline 1 & 3.290 & 1005.2 & 0.0237 & 7.076 & 1045.7 & 0.0237 & 11.430 & 1046.2 & 0.0237 \\
\hline 2 & 5.005 & 1188.4 & 0.0236 & 10.706 & 1208.0 & 0.0236 & 17.066 & 1188.3 & 0.0236 \\
\hline 3 & 6.200 & 1305.1 & 0.0235 & 12.954 & 1285.0 & 0.0235 & 20.150 & 1224.9 & 0.0234 \\
\hline 4 & 6.894 & 1370.1 & 0.0233 & 14.070 & 1309.1 & 0.0234 & 21.432 & 1217.6 & 0.0233 \\
\hline 5 & 7.255 & 1401.7 & 0.0232 & 14.545 & 1310.9 & 0.0232 & 21.847 & 1201.9 & 0.0232 \\
\hline 6 & 7.471 & 1418.8 & 0.0231 & 14.780 & 1305.7 & 0.0231 & 22.001 & 1188.6 & 0.0230 \\
\hline 7 & 7.614 & 1428.6 & 0.0229 & 14.921 & 1299.6 & 0.0229 & 22.086 & 1179.1 & 0.0229 \\
\hline 8 & 7.710 & 1433.8 & 0.0228 & 15.013 & 1294.4 & 0.0228 & 22.153 & 1172.7 & 0.0228 \\
\hline 9 & 7.772 & 1435.3 & 0.0227 & 15.083 & 1290.6 & 0.0227 & 22.221 & 1168.6 & 0.0227 \\
\hline 10 & 7.809 & 1434.4 & 0.0225 & 15.142 & 1288.6 & 0.0226 & 22.304 & 1166.4 & 0.0226 \\
\hline 11 & 7.830 & 1432.1 & 0.0224 & 15.207 & 1288.8 & 0.0224 & 22.419 & 1166.2 & 0.0224 \\
\hline 12 & 7.846 & 1429.4 & 0.0223 & 15.304 & 1292.3 & 0.0223 & 22.608 & 1168.8 & 0.0223 \\
\hline 13 & 7.845 & 1425.5 & 0.0222 & 15.409 & 1299.4 & 0.0222 & 22.854 & 1174.8 & 0.0222 \\
\hline 14 & 7.726 & 1410.8 & 0.0220 & 15.348 & 1303.7 & 0.0221 & 22.936 & 1183.2 & 0.0221 \\
\hline 15 & 7.429 & 1378.6 & 0.0219 & 15.001 & 1300.4 & 0.0220 & 22.714 & 1193.9 & 0.0220 \\
\hline 16 & 6.854 & 1319.4 & 0.0218 & 14.126 & 1277.3 & 0.0219 & 21.815 & 1200.7 & 0.0219 \\
\hline 17 & 5.805 & 1213.7 & 0.0217 & 12.203 & 1210.4 & 0.0218 & 19.295 & 1175.3 & 0.0218 \\
\hline 18 & 4.045 & 1035.9 & 0.0217 & 8.534 & 1052.2 & 0.0217 & 13.679 & 1044.9 & 0.0217 \\
\hline
\end{tabular}


Table 4-73. Burnup and TH Feedback Parameters by Axial Node for Assembly H23 (Cont.)

\begin{tabular}{|c|c|c|c|c|c|c|c|c|c|}
\hline \multirow[b]{2}{*}{$\begin{array}{l}\text { Axial } \\
\text { Node }\end{array}$} & \multicolumn{3}{|c|}{ Datapoint (214.0 Cy9) } & \multicolumn{3}{|c|}{ Datapoint (425.0 Cy9) } & \multicolumn{3}{|c|}{ Statepoint 69 (BOC Cy10) } \\
\hline & \begin{tabular}{|c|} 
Burnup \\
(GWd/MTU)
\end{tabular} & $\begin{array}{c}\text { T-Fuel } \\
{ }^{\circ} \mathrm{F}\end{array}$ & $\begin{array}{c}\text { Spec. Vol. } \\
\mathrm{ft}^{3} / \mathrm{lbm}\end{array}$ & $\begin{array}{c}\text { Burnup } \\
\text { (GWd/MTU) }\end{array}$ & $\begin{array}{c}\text { T-Fuel } \\
{ }^{\circ} \mathrm{F}\end{array}$ & $\begin{array}{c}\text { Spec. Vol. } \\
\mathrm{ft}^{3} / \mathrm{lbm}\end{array}$ & $\begin{array}{c}\text { Burnup } \\
\text { (GWd/MTU) }\end{array}$ & $\begin{array}{c}\text { T-Fuel } \\
{ }^{\circ} \mathrm{F}\end{array}$ & $\begin{array}{c}\text { Spec. Vol. } \\
\mathrm{ft}^{3} / \mathrm{lbm}\end{array}$ \\
\hline & 214.0 Cy 9 & 109.3 Сy9 & 109.3 Cy9 & 425.0 Cy 9 & 328.7 Cy9 & 328.7 Cy 9 & BOC Cy 10 & 549.2 Cy9 & 549.2 Cy9 \\
\hline 1 & 15.591 & 936.1 & 0.0234 & 20.293 & 950.4 & 0.0234 & 25.092 & 959.5 & 0.0233 \\
\hline 2 & 23.359 & 1043.8 & 0.0233 & 30.112 & 1031.7 & 0.0233 & 36.398 & 1025.8 & 0.0232 \\
\hline 3 & 27.468 & 1084.5 & 0.0232 & 34.975 & 1054.0 & 0.0232 & 42.493 & 1038.4 & 0.0231 \\
\hline 4 & 29.187 & 1097.2 & 0.0231 & 36.813 & 1053.7 & 0.0230 & 44.551 & 1023.4 & 0.0230 \\
\hline 5 & 29.608 & 1088.4 & 0.0230 & 37.035 & 1036.0 & 0.0229 & 44.614 & 1000.3 & 0.0229 \\
\hline 6 & 29.872 & 1088.3 & 0.0228 & 37.235 & 1027.9 & 0.0228 & 44.688 & 989.8 & 0.0228 \\
\hline 7 & 30.026 & 1087.0 & 0.0227 & 37.347 & 1021.9 & 0.0227 & 44.739 & 983.6 & 0.0227 \\
\hline 8 & 30.120 & 1083.9 & 0.0226 & 37.412 & 1017.3 & 0.0226 & 44.764 & 979.6 & 0.0226 \\
\hline 9 & 30.186 & 1080.0 & 0.0225 & 37.469 & 1014.0 & 0.0225 & 44.794 & 976.8 & 0.0225 \\
\hline 10 & 30.248 & 1075.4 & 0.0224 & 37.541 & 1012.2 & 0.0224 & 44.848 & 974.7 & 0.0224 \\
\hline 11 & 30.339 & 1071.1 & 0.0223 & 37.672 & 1012.1 & 0.0223 & 44.970 & 973.7 & 0.0223 \\
\hline 12 & 30.595 & 1071.5 & 0.0222 & 38.089 & 1018.4 & 0.0222 & 45.421 & 977.9 & 0.0222 \\
\hline 13 & 31.224 & 1092.0 & 0.0221 & 39.162 & 1042.8 & 0.0221 & 46.732 & 997.8 & 0.0221 \\
\hline 14 & 31.207 & 1084.8 & 0.0220 & 39.260 & 1048.5 & 0.0220 & 46.948 & 1004.7 & 0.0220 \\
\hline 15 & 30.704 & 1069.3 & 0.0219 & 38.783 & 1050.2 & 0.0219 & 46.597 & 1012.9 & 0.0219 \\
\hline 16 & 29.304 & 1044.3 & 0.0218 & 37.233 & 1043.6 & 0.0218 & 45.113 & 1021.4 & 0.0218 \\
\hline 17 & 25.821 & 999.2 & 0.0217 & 33.072 & 1009.3 & 0.0217 & 40.512 & 1010.4 & 0.0217 \\
\hline 18 & 17.984 & 885.9 & 0.0216 & 23.036 & 916.6 & 0.0216 & 28.528 & 924.8 & 0.0216 \\
\hline
\end{tabular}

\begin{tabular}{|c|c|c|c|c|c|c|c|c|c|}
\hline \multirow[b]{2}{*}{$\begin{array}{l}\text { Axial } \\
\text { Node }\end{array}$} & \multicolumn{3}{|c|}{ Statepoint $70(27.0$ Cy10) } & \multicolumn{3}{|c|}{ Statepoint $71(143.0$ Cy10) } & \multicolumn{3}{|c|}{ Statepoint 72 (209.0 Cy10) } \\
\hline & $\begin{array}{c}\text { Burnup } \\
\text { (GWd/MTU) }\end{array}$ & $\begin{array}{c}\text { T-Fuel } \\
{ }^{\circ} \mathrm{F} \\
\end{array}$ & $\begin{array}{c}\text { Spec. Vol. } \\
\mathrm{ft}^{3} / \mathrm{lbm}\end{array}$ & $\begin{array}{c}\text { Burnup } \\
\text { (GWd/MTU) }\end{array}$ & $\begin{array}{l}\text { T-Fuel } \\
{ }^{\circ} \mathrm{F}\end{array}$ & $\begin{array}{l}\text { Spec. Vol. } \\
\mathrm{tt}^{3} / \mathrm{lbm}\end{array}$ & $\begin{array}{c}\text { Burnup } \\
\text { (GWd/MTU) }\end{array}$ & $\begin{array}{l}\text { T-Fuel } \\
{ }^{\circ} \mathrm{F}\end{array}$ & $\begin{array}{c}\text { Spec. Vol. } \\
\mathrm{ft}^{3} / \mathrm{bm}\end{array}$ \\
\hline & 27.0 Cy 10 & 18.0 Cy 10 & 18.0 Cy 10 & 143.0 Cy 10 & 82.9 Cy 10 & 82.9 Cy 10 & 209.0 Cy 10 & 176.7 Cy 10 & 176.7 Cy 10 \\
\hline 1 & 25.199 & 626.1 & 0.0220 & 25.689 & 628.6 & 0.0220 & 26.000 & 634.3 & 0.0220 \\
\hline 2 & 36.567 & 646.7 & 0.0220 & 37.326 & 649.5 & 0.0220 & 37.797 & 655.6 & 0.0220 \\
\hline 3 & 42.696 & 658.4 & 0.0219 & 43.601 & 660.9 & 0.0220 & 44.147 & 665.1 & 0.0220 \\
\hline 4 & 44.776 & 665.7 & 0.0219 & 45.767 & 667.5 & 0.0219 & 46.350 & 669.5 & 0.0219 \\
\hline 5 & 44.858 & 671.9 & 0.0219 & 45.915 & 673.0 & 0.0219 & 46.522 & 672.9 & 0.0219 \\
\hline 6 & 44.941 & 675.0 & 0.0219 & 46.032 & 675.3 & 0.0219 & 46.648 & 673.4 & 0.0219 \\
\hline 7 & 44.998 & 676.2 & 0.0219 & 46.107 & 676.0 & 0.0219 & 46.724 & 672.9 & 0.0219 \\
\hline 8 & 45.026 & 676.5 & 0.0218 & 46.143 & 675.8 & 0.0218 & 46.759 & 672.0 & 0.0218 \\
\hline 9 & 45.057 & 676.0 & 0.0218 & 46.177 & 675.1 & 0.0218 & 46.792 & 671.0 & 0.0218 \\
\hline 10 & 45.110 & 674.8 & 0.0218 & 46.227 & 674.0 & 0.0218 & 46.840 & 669.9 & 0.0218 \\
\hline 11 & 45.229 & 672.7 & 0.0218 & 46.337 & 672.2 & 0.0218 & 46.948 & 668.6 & 0.0218 \\
\hline 12 & 45.674 & 668.8 & 0.0217 & 46.759 & 668.9 & 0.0217 & 47.361 & 666.3 & 0.0217 \\
\hline 13 & 46.970 & 661.3 & 0.0217 & 48.002 & 662.3 & 0.0217 & 48.583 & 661.2 & 0.0217 \\
\hline 14 & 47.175 & 656.0 & 0.0217 & 48.169 & 657.9 & 0.0217 & 48.739 & 658.6 & 0.0217 \\
\hline 15 & 46.810 & 649.9 & 0.0217 & 47.756 & 652.7 & 0.0217 & 48.309 & 655.5 & 0.0217 \\
\hline 16 & 45.306 & 641.8 & 0.0216 & 46.176 & 645.4 & 0.0216 & 46.698 & 650.3 & 0.0216 \\
\hline 17 & 40.673 & 629.8 & 0.0216 & 41.412 & 633.8 & 0.0216 & 41.866 & 640.3 & 0.0216 \\
\hline 18 & 28.632 & 608.4 & 0.0216 & 29.114 & 611.5 & 0.0216 & 29.418 & 617.3 & 0.0216 \\
\hline
\end{tabular}


Table 4-74. Burnup and TH Feedback Parameters by Axial Node for Assembly H25

\begin{tabular}{|c|c|c|c|c|c|c|c|c|c|}
\hline \multirow[b]{2}{*}{$\begin{array}{l}\text { Axial } \\
\text { Node }\end{array}$} & \multicolumn{3}{|c|}{ Datapoint (171.1 Cy8) } & \multicolumn{3}{|c|}{ Datapoint (338.1 Cy8) } & \multicolumn{3}{|c|}{ Statepoint 68 (BOC Cy9) } \\
\hline & $\begin{array}{c}\text { Burnup } \\
\text { (GWd/MTU) }\end{array}$ & $\begin{array}{l}\text { T-Fuel } \\
{ }^{\circ} \mathrm{F}\end{array}$ & $\begin{array}{l}\text { Spec. Vol. } \\
\mathrm{ft}^{3} / \mathrm{lbm}\end{array}$ & $\begin{array}{c}\text { Burnup } \\
\text { (GWd/MTU) }\end{array}$ & $\begin{array}{l}\text { T-Fuel } \\
{ }^{\circ} \mathrm{F}\end{array}$ & $\begin{array}{l}\text { Spec. Vol. } \\
\mathrm{ft}^{3} / \mathrm{lbm}\end{array}$ & $\begin{array}{c}\text { Burnup } \\
\text { (GWd/MTU) }\end{array}$ & $\begin{array}{l}\text { T-Fuel } \\
{ }^{\circ} \mathrm{F}\end{array}$ & $\begin{array}{l}\text { Spec. Vol. } \\
\mathrm{ft}^{3} / \mathrm{lbm}\end{array}$ \\
\hline & 171.1 Cy 8 & 77.7 Cy8 & 77.7 Cy8 & 338.1 Cy 8 & 247.2 Cy 8 & 247.2 Cy 8 & BOC Cy9 & 426.1 Cy 8 & 426.1 Cy 8 \\
\hline 1 & 2.163 & 857.9 & 0.0230 & 4.687 & 895.4 & 0.0230 & 7.708 & 923.8 & 0.0230 \\
\hline 2 & 3.679 & 1032.7 & 0.0230 & 7.704 & 1042.9 & 0.0229 & 12.209 & 1034.4 & 0.0229 \\
\hline 3 & 4.578 & 1126.9 & 0.0229 & 9.294 & 1097.9 & 0.0228 & 14.328 & 1062.6 & 0.0228 \\
\hline 4 & 5.092 & 1177.4 & 0.0228 & 10.073 & 1113.7 & 0.0228 & 15.211 & 1060.1 & 0.0227 \\
\hline 5 & 5.387 & 1204.7 & 0.0227 & 10.445 & 1114.0 & 0.0227 & 15.551 & 1050.2 & 0.0227 \\
\hline 6 & 5.561 & 1218.8 & 0.0226 & 10.625 & 1109.1 & 0.0226 & 15.676 & 1040.6 & 0.0226 \\
\hline 7 & 5.668 & 1226.2 & 0.0226 & 10.719 & 1103.5 & 0.0225 & 15.726 & 1033.3 & 0.0225 \\
\hline 8 & 5.736 & 1229.7 & 0.0225 & 10.775 & 1098.6 & 0.0224 & 15.757 & 1028.3 & 0.0224 \\
\hline 9 & 5.778 & 1230.6 & 0.0224 & 10.812 & 1095.0 & 0.0223 & 15.787 & 1025.0 & 0.0223 \\
\hline 10 & 5.802 & 1229.8 & 0.0223 & 10.842 & 1092.8 & 0.0222 & 15.826 & 1023.1 & 0.0223 \\
\hline 11 & 5.810 & 1227.5 & 0.0222 & 10.869 & 1092.1 & 0.0222 & 15.878 & 1022.7 & 0.0222 \\
\hline 12 & 5.799 & 1223.5 & 0.0221 & 10.890 & 1093.2 & 0.0221 & 15.944 & 1023.9 & 0.0221 \\
\hline 13 & 5.753 & 1216.3 & 0.0220 & 10.883 & 1095.4 & 0.0220 & 16.002 & 1027.2 & 0.0220 \\
\hline 14 & 5.640 & 1202.6 & 0.0219 & 10.796 & 1097.5 & 0.0219 & 15.998 & 1032.8 & 0.0220 \\
\hline 15 & 5.407 & 1176.9 & 0.0218 & 10.530 & 1095.7 & 0.0219 & 15.809 & 1039.8 & 0.0219 \\
\hline 16 & 4.952 & 1128.4 & 0.0218 & 9.874 & 1081.0 & 0.0218 & 15.119 & 1041.8 & 0.0218 \\
\hline 17 & 4.063 & 1033.8 & 0.0217 & 8.342 & 1028.5 & 0.0217 & 13.133 & 1015.5 & 0.0217 \\
\hline 18 & 2.386 & 844.2 & 0.0217 & 5.073 & 875.3 & 0.0217 & 8.295 & 899.8 & 0.0217 \\
\hline
\end{tabular}

Table 4-75. Burnup and TH Feedback Parameters by Axial Node for Assembly $\mathrm{H} 25 \mathrm{a}$

\begin{tabular}{|c|c|c|c|c|c|c|c|c|c|}
\hline \multirow[b]{2}{*}{$\begin{array}{l}\text { Axial } \\
\text { Node }\end{array}$} & \multicolumn{3}{|c|}{ Datapoint (171.1 Cy8) } & \multicolumn{3}{|c|}{ Datapoint (338.1 Cy8) } & \multicolumn{3}{|c|}{ Statepoint 68 (BOC Cy9) } \\
\hline & \begin{tabular}{|c|} 
Burnup \\
(GWd/MTU)
\end{tabular} & $\begin{array}{c}\text { T-Fuel } \\
{ }^{\circ} \mathrm{F} \\
\end{array}$ & $\begin{array}{c}\text { Spec. Vol. } \\
\mathrm{ft}^{3} / \mathrm{lbm}\end{array}$ & \begin{tabular}{|c|} 
Burnup \\
(GWd/MTU) \\
\end{tabular} & $\begin{array}{c}\text { T-Fuel } \\
{ }^{\circ} \mathrm{F} \\
\end{array}$ & $\begin{array}{c}\text { Spec. Vol. } \\
\mathrm{ft}^{3} / \mathrm{lbm}\end{array}$ & $\begin{array}{c}\text { Burnup } \\
\text { (GWd/MTU) }\end{array}$ & $\begin{array}{c}\text { T-Fuel } \\
{ }^{\circ} \mathrm{F} \\
\end{array}$ & $\begin{array}{c}\text { Spec. Vol. } \\
\mathrm{ft}^{3} / \mathrm{lbm}\end{array}$ \\
\hline & 171.1 Cy8 & 77.7 Cy8 & 77.7 Cy8 & 338.1 Cy 8 & 247.2 Cy8 & 247.2 Cy8 & BOC Cy 9 & 426.1 Cy8 & $426.1 \mathrm{Cy} 8$ \\
\hline 1 & 2.163 & 857.9 & 0.0230 & 4.687 & 895.4 & 0.0230 & 7.708 & 923.8 & 0.0230 \\
\hline 2 & 3.679 & 1032.7 & 0.0230 & 7.704 & 1042.9 & 0.0229 & 12.210 & 1034.4 & 0.0229 \\
\hline 3 & 4.578 & 1126.9 & 0.0229 & 9.294 & 1097.9 & 0.0228 & 14.332 & 1062.6 & 0.0228 \\
\hline 4 & 5.092 & 1177.4 & 0.0228 & 10.073 & 1113.7 & 0.0228 & 15.216 & 1060.1 & 0.0227 \\
\hline 5 & 5.387 & 1204.7 & 0.0227 & 10.445 & 1114.0 & 0.0227 & 15.557 & 1050.2 & 0.0227 \\
\hline 6 & 5.561 & 1218.8 & 0.0226 & 10.625 & 1109.1 & 0.0226 & 15.682 & 1040.6 & 0.0226 \\
\hline 7 & 5.668 & 1226.2 & 0.0226 & 10.719 & 1103.5 & 0.0225 & 15.732 & 1033.3 & 0.0225 \\
\hline 8 & 5.736 & 1229.7 & 0.0225 & 10.775 & 1098.6 & 0.0224 & 15.763 & 1028.3 & 0.0224 \\
\hline 9 & 5.778 & 1230.6 & 0.0224 & 10.812 & 1095.0 & 0.0223 & 15.794 & 1025.0 & 0.0223 \\
\hline 10 & 5.802 & 1229.8 & 0.0223 & 10.842 & 1092.8 & 0.0222 & 15.833 & 1023.1 & 0.0223 \\
\hline 11 & 5.810 & 1227.5 & 0.0222 & 10.869 & 1092.1 & 0.0222 & 15.886 & 1022.7 & 0.0222 \\
\hline 12 & 5.799 & 1223.5 & 0.0221 & 10.890 & 1093.2 & 0.0221 & 15.952 & 1023.9 & 0.0221 \\
\hline 13 & 5.753 & 1216.3 & 0.0220 & 10.883 & 1095.4 & 0.0220 & 16.011 & 1027.2 & 0.0220 \\
\hline 14 & 5.640 & 1202.6 & 0.0219 & 10.796 & 1097.5 & 0.0219 & 16.008 & 1032.8 & 0.0220 \\
\hline 15 & 5.407 & 1176.9 & 0.0218 & 10.530 & 1095.7 & 0.0219 & 15.819 & 1039.8 & 0.0219 \\
\hline 16 & 4.952 & 1128.4 & 0.0218 & 9.874 & 1081.0 & 0.0218 & 15.128 & 1041.8 & 0.0218 \\
\hline 17 & 4.063 & 1033.8 & 0.0217 & 8.342 & 1028.5 & 0.0217 & 13.141 & 1015.5 & 0.0217 \\
\hline 18 & 2.386 & 844.2 & 0.0217 & 5.073 & 875.3 & 0.0217 & 8.299 & 899.8 & 0.0217 \\
\hline
\end{tabular}


Table 4-76. Burnup and TH Feedback Parameters by Axial Node for Assembly H27

\begin{tabular}{|c|c|c|c|c|c|c|c|c|c|}
\hline \multirow[b]{2}{*}{$\begin{array}{l}\text { Axial } \\
\text { Node }\end{array}$} & \multicolumn{3}{|c|}{ Datapoint (171.1 Cy8) } & \multicolumn{3}{|c|}{ Datapoint (338.1 Cy8) } & \multicolumn{3}{|c|}{ Statepoint 68 (BOC Cy9) } \\
\hline & $\begin{array}{c}\text { Burnup } \\
\text { (GWd/MTU) }\end{array}$ & $\begin{array}{l}\text { T-Fuel } \\
{ }^{\circ} \mathrm{F}\end{array}$ & $\begin{array}{c}\text { Spec. Vol. } \\
\mathrm{ft}^{3} / / \mathrm{bm}\end{array}$ & $\begin{array}{c}\text { Burnup } \\
\text { (GWd/MTU) }\end{array}$ & $\begin{array}{c}\text { T-Fuel } \\
{ }^{\circ} \mathrm{F}\end{array}$ & $\begin{array}{c}\text { Spec. Vol. } \\
\mathrm{ft}^{3} / \mathrm{bm}\end{array}$ & $\begin{array}{c}\text { Burnup } \\
\text { (GWd/MTU) }\end{array}$ & $\begin{array}{c}\text { T-Fuel } \\
{ }^{\circ} \mathrm{F}\end{array}$ & $\begin{array}{c}\text { Spec. Vol. } \\
\mathrm{ft}^{3} / / \mathrm{bm}\end{array}$ \\
\hline & 171.1 Cy 8 & 77.7 Cy 8 & 77.7 Cy 8 & 338.1 Cy 8 & 247.2 Cy8 & 247.2 Cy 8 & BOC Cy 9 & 426.1 Cy 8 & $426.1 \mathrm{Cy} 8$ \\
\hline 1 & 2.392 & 891.7 & 0.0233 & 5.214 & 936.1 & 0.0232 & 8.556 & 961.4 & 0.0232 \\
\hline 2 & 4.010 & 1078.2 & 0.0232 & 8.540 & 1099.7 & 0.0232 & 13.604 & 1086.8 & 0.0231 \\
\hline 3 & 5.129 & 1191.5 & 0.0231 & 10.568 & 1168.0 & 0.0231 & 16.341 & 1122.9 & 0.0230 \\
\hline 4 & 5.783 & 1253.7 & 0.0230 & 11.583 & 1188.2 & 0.0230 & 17.510 & 1119.6 & 0.0229 \\
\hline 5 & 6.144 & 1286.1 & 0.0229 & 12.052 & 1189.3 & 0.0229 & 17.949 & 1107.9 & 0.0228 \\
\hline 6 & 6.355 & 1303.5 & 0.0228 & 12.280 & 1184.4 & 0.0228 & 18.116 & 1097.0 & 0.0227 \\
\hline 7 & 6.487 & 1312.9 & 0.0227 & 12.404 & 1178.5 & 0.0227 & 18.193 & 1088.9 & 0.0226 \\
\hline 8 & 6.573 & 1317.7 & 0.0226 & 12.482 & 1173.3 & 0.0226 & 18.246 & 1083.3 & 0.0225 \\
\hline 9 & 6.630 & 1319.7 & 0.0225 & 12.539 & 1169.5 & 0.0225 & 18.298 & 1079.6 & 0.0225 \\
\hline 10 & 6.664 & 1319.5 & 0.0224 & 12.586 & 1167.3 & 0.0224 & 18.358 & 1077.6 & 0.0224 \\
\hline 11 & 6.681 & 1317.6 & 0.0223 & 12.630 & 1166.9 & 0.0223 & 18.433 & 1077.0 & 0.0223 \\
\hline 12 & 6.675 & 1313.6 & 0.0222 & 12.666 & 1168.3 & 0.0222 & 18.523 & 1078.4 & 0.0222 \\
\hline 13 & 6.629 & 1305.9 & 0.0221 & 12.670 & 1171.2 & 0.0221 & 18.606 & 1082.0 & 0.0221 \\
\hline 14 & 6.508 & 1290.9 & 0.0220 & 12.583 & 1173.9 & 0.0220 & 18.620 & 1088.5 & 0.0220 \\
\hline 15 & 6.252 & 1262.9 & 0.0219 & 12.295 & 1172.5 & 0.0219 & 18.428 & 1096.7 & 0.0219 \\
\hline 16 & 5.752 & 1210.8 & 0.0218 & 11.571 & 1156.7 & 0.0218 & 17.682 & 1100.3 & 0.0218 \\
\hline 17 & 4.794 & 1112.6 & 0.0217 & 9.899 & 1101.6 & 0.0217 & 15.530 & 1074.6 & 0.0217 \\
\hline 18 & 3.087 & 926.3 & 0.0217 & 6.516 & 953.5 & 0.0217 & 10.528 & 962.6 & 0.0217 \\
\hline
\end{tabular}

Table 4-77. Burnup and TH Feedback Parameters by Axial Node for Assembly H27a

\begin{tabular}{|c|c|c|c|c|c|c|c|c|c|}
\hline \multirow[b]{2}{*}{$\begin{array}{l}\text { Axial } \\
\text { Node }\end{array}$} & \multicolumn{3}{|c|}{ Datapoint (171.1 Cy8) } & \multicolumn{3}{|c|}{ Datapoint (338.1 CyB) } & \multicolumn{3}{|c|}{ Statepoint 68 (BOC Cy9) } \\
\hline & $\begin{array}{c}\text { Burnup } \\
\text { (GWd/MTU) }\end{array}$ & $\begin{array}{c}\text { T-Fuel } \\
{ }^{\circ} \mathrm{F}\end{array}$ & $\begin{array}{c}\text { Spec. Vol. } \\
\mathrm{ft}^{3} / / \mathrm{bm}\end{array}$ & $\begin{array}{c}\text { Burnup } \\
\text { (GWd/MTU) } \\
\end{array}$ & $\begin{array}{c}\text { T-Fuel } \\
{ }^{\circ} \mathrm{F} \\
\end{array}$ & $\begin{array}{c}\text { Spec. Vol. } \\
\mathrm{ft}^{3} / \mathrm{lbm}\end{array}$ & $\begin{array}{c}\text { Burnup } \\
\text { (GWd/MTU) }\end{array}$ & $\begin{array}{c}\text { T-Fuel } \\
{ }^{\circ} \mathrm{F} \\
\end{array}$ & $\begin{array}{c}\text { Spec. Vol. } \\
\mathrm{ft}^{3} / \mathrm{lbm}\end{array}$ \\
\hline & 171.1 Cy8 & 77.7 Cy 8 & 77.7 Cy8 & 338.1 Cy8 & 247.2 Cy8 & 247.2 Cy 8 & BOC Cy 9 & 426.1 Cy8 & 426.1 Cy 8 \\
\hline 1 & 2.392 & 891.7 & 0.0233 & 5.214 & 936.1 & 0.0232 & 8.543 & 961.4 & 0.0232 \\
\hline 2 & 4.010 & 1078.2 & 0.0232 & 8.540 & 1099.7 & 0.0232 & 13.583 & 1086.8 & 0.0231 \\
\hline 3 & 5.129 & 1191.5 & 0.0231 & 10.568 & 1168.0 & 0.0231 & 16.318 & 1122.9 & 0.0230 \\
\hline 4 & 5.783 & 1253.7 & 0.0230 & 11.583 & 1188.2 & 0.0230 & 17.487 & 1119.6 & 0.0229 \\
\hline 5 & 6.144 & 1286.1 & 0.0229 & 12.052 & 1189.3 & 0.0229 & 17.926 & 1107.9 & 0.0228 \\
\hline 6 & 6.355 & 1303.5 & 0.0228 & 12.280 & 1184,4 & 0.0228 & 18.092 & 1097.0 & 0.0227 \\
\hline 7 & 6.487 & 1312.9 & 0.0227 & 12.404 & 1178.5 & 0.0227 & 18.169 & 1088.9 & 0.0226 \\
\hline 8 & 6.573 & 1317.7 & 0.0226 & 12.482 & 1173.3 & 0.0226 & 18.221 & 1083.3 & 0.0225 \\
\hline 9 & 6.630 & 1319.7 & 0.0225 & 12.539 & 1169.5 & 0.0225 & 18.272 & 1079.6 & 0.0225 \\
\hline 10 & 6.664 & 1319.5 & 0.0224 & 12.586 & 1167.3 & 0.0224 & 18.332 & 1077.6 & 0.0224 \\
\hline 11 & 6.681 & 1317.6 & 0.0223 & 12.630 & 1166.9 & 0.0223 & 18.407 & 1077.0 & 0.0223 \\
\hline 12 & 6.675 & 1313.6 & 0.0222 & 12.666 & 1168.3 & 0.0222 & 18.498 & 1078.4 & 0.0222 \\
\hline 13 & 6.629 & 1305.9 & 0.0221 & 12.670 & 1171.2 & 0.0221 & 18.581 & 1082.0 & 0.0221 \\
\hline 14 & 6.508 & 1290.9 & 0.0220 & 12.583 & 1173.9 & 0.0220 & 18.595 & 1088.5 & 0.0220 \\
\hline 15 & 6.252 & 1262.9 & 0.0219 & 12.295 & 1172.5 & 0.0219 & 18.403 & 1096.7 & 0.0219 \\
\hline 16 & 5.752 & 1210.8 & 0.0218 & 11.571 & 1156.7 & 0.0218 & 17.658 & 1100.3 & 0.0218 \\
\hline 17 & 4.794 & 1112.6 & 0.0217 & 9.899 & 1101.6 & 0.0217 & 15.510 & 1074.6 & 0.0217 \\
\hline 18 & 3.087 & 926.3 & 0.0217 & 6.516 & 953.5 & 0.0217 & 10.517 & 962.6 & 0.0217 \\
\hline
\end{tabular}


Table 4-78. Burnup and TH Feedback Parameters by Axial Node for Assembly J2

\begin{tabular}{|c|c|c|c|c|c|c|c|c|c|}
\hline \multirow{2}{*}{$\begin{array}{c}\text { Axial } \\
\text { Node }\end{array}$} & \multicolumn{2}{|c|}{ Datapoint (214.0 Cy9) } & \multicolumn{2}{c|}{ Datapoint (425.0 Cy9) } & \multicolumn{3}{c|}{ Statepoint 69 (BOC Cy10) } \\
\hline & $\begin{array}{c}\text { Burnup } \\
\text { (GWd/MTU) }\end{array}$ & $\begin{array}{c}\text { T-Fuel } \\
{ }^{\circ} \mathrm{F}\end{array}$ & $\begin{array}{c}\text { Spec. Vol. } \\
\mathrm{ft}^{3} / \mathrm{lbm}\end{array}$ & $\begin{array}{c}\text { Burnup } \\
\text { (GWd/MTU) }\end{array}$ & $\begin{array}{c}\text { T-Fuel } \\
{ }^{\circ} \mathrm{F}\end{array}$ & $\begin{array}{c}\text { Spec. Vol. } \\
\mathrm{ft}^{3} / \mathrm{lbm}\end{array}$ & $\begin{array}{c}\text { Burnup } \\
\text { (GWd/MTU) }\end{array}$ & $\begin{array}{c}\text { T-Fuel } \\
{ }^{\circ} \mathrm{F}\end{array}$ & $\begin{array}{c}\text { Spec. Vol. } \\
\mathrm{ft}^{3} / \mathrm{lbm}\end{array}$ \\
\hline 1 & $214.0 \mathrm{Cy9}$ & 109.3 Cy9 & 109.3 Cy9 & 425.0 Cy9 & 328.7 Cy9 & 328.7 Cy9 & BOC Cy10 & 549.2 Cy9 & 549.2 Cy9 \\
\hline 2 & 7.166 & 1129.0 & 0.0239 & 10.651 & 1091.5 & 0.0239 & 16.165 & 1061.5 & 0.0236 \\
\hline 3 & 8.905 & 1427.7 & 0.0237 & 17.932 & 1301.8 & 0.0236 & 26.560 & 1173.8 & 0.0234 \\
\hline 4 & 9.617 & 1469.9 & 0.0235 & 18.981 & 1308.5 & 0.0234 & 27.753 & 1162.1 & 0.0233 \\
\hline 5 & 9.992 & 1486.2 & 0.0234 & 19.412 & 1301.6 & 0.0233 & 28.095 & 1148.1 & 0.0231 \\
\hline 6 & 10.192 & 1491.8 & 0.0232 & 19.584 & 1292.9 & 0.0231 & 28.159 & 1137.5 & 0.0230 \\
\hline 7 & 10.295 & 1492.0 & 0.0230 & 19.648 & 1285.4 & 0.0230 & 28.147 & 1130.7 & 0.0229 \\
\hline 8 & 10.338 & 1489.5 & 0.0229 & 19.668 & 1280.1 & 0.0229 & 28.124 & 1126.5 & 0.0227 \\
\hline 9 & 10.338 & 1485.5 & 0.0227 & 19.669 & 1277.0 & 0.0227 & 28.103 & 1124.0 & 0.0226 \\
\hline 10 & 10.306 & 1480.4 & 0.0226 & 19.661 & 1276.1 & 0.0226 & 28.090 & 1122.5 & 0.0225 \\
\hline 11 & 10.245 & 1474.6 & 0.0225 & 19.649 & 1277.1 & 0.0225 & 28.087 & 1122.0 & 0.0224 \\
\hline 12 & 10.153 & 1467.7 & 0.0223 & 19.630 & 1280.2 & 0.0223 & 28.098 & 1122.8 & 0.0223 \\
\hline 13 & 10.015 & 1458.6 & 0.0222 & 19.587 & 1285.5 & 0.0222 & 28.120 & 1126.0 & 0.0222 \\
\hline 14 & 9.804 & 1445.4 & 0.0221 & 19.469 & 1292.3 & 0.0221 & 28.120 & 1132.7 & 0.0221 \\
\hline 15 & 9.450 & 1423.2 & 0.0219 & 19.142 & 1297.8 & 0.0220 & 27.947 & 1143.6 & 0.0220 \\
\hline 16 & 8.794 & 1375.7 & 0.0218 & 18.249 & 1292.4 & 0.0218 & 27.115 & 1154.4 & 0.0218 \\
\hline 17 & 7.534 & 1274.0 & 0.0217 & 16.008 & 1241.5 & 0.0217 & 24.335 & 1141.1 & 0.0217 \\
\hline 18 & 5.261 & 1082.4 & 0.0216 & 11.242 & 1078.8 & 0.0216 & 17.415 & 1045.1 & 0.0216 \\
\hline
\end{tabular}

\begin{tabular}{|c|c|c|c|c|c|c|c|c|c|}
\hline \multirow[b]{2}{*}{$\begin{array}{l}\text { Axial } \\
\text { Node }\end{array}$} & \multicolumn{3}{|c|}{ Statepoint 70 (27.0 Cy10) } & \multicolumn{3}{|c|}{ Statepoint 71 (143.0 Cy10) } & \multicolumn{3}{|c|}{ Statepoint 72 (209.0 Cy10) } \\
\hline & $\begin{array}{c}\text { Burnup } \\
\text { (GWd/MTU) }\end{array}$ & $\begin{array}{l}\text { T-Fuel } \\
{ }^{\circ} \mathrm{F}\end{array}$ & $\begin{array}{c}\text { Spec. Vol. } \\
\mathrm{ft}^{3} / \mathrm{lbm}\end{array}$ & \begin{tabular}{|c|} 
Burnup \\
(GWd/MTU)
\end{tabular} & $\begin{array}{l}\text { T-Fuel } \\
{ }^{\circ} \mathrm{F}\end{array}$ & $\begin{array}{c}\text { Spec. Vol. } \\
\mathrm{ft}^{3} / \mathrm{lbm}\end{array}$ & \begin{tabular}{|c|} 
Burnup \\
(GWd/MTU)
\end{tabular} & $\begin{array}{l}\text { T-Fuel } \\
{ }^{\circ} \mathrm{F}\end{array}$ & $\begin{array}{l}\text { Spec. Vol. } \\
\mathrm{ft}^{3} / \mathrm{lbm}\end{array}$ \\
\hline & 27.0 Cy 10 & 18.0 Cy 10 & $18.0 \mathrm{Cy} 10$ & 143.0 Cy 10 & 82.9 Cy 10 & $82.9 \mathrm{Cy} 10$ & 209.0 Cy 10 & 176.7 Cy 10 & $176.7 \mathrm{Cy} 10$ \\
\hline 1 & 16.587 & 859.9 & 0.0232 & 18.454 & 857.2 & 0.0232 & 19.604 & 867.1 & 0.0232 \\
\hline 2 & 23.711 & 952.1 & 0.0231 & 26.571 & 944.3 & 0.0231 & 28.300 & 946.2 & 0.0231 \\
\hline 3 & 27.344 & 994.9 & 0.0230 & 30.753 & 984.4 & 0.0230 & 32.772 & 985.7 & 0.0230 \\
\hline 4 & 28.612 & 1019.9 & 0.0229 & 32.319 & 1008.5 & 0.0229 & 34.471 & 1007.1 & 0.0229 \\
\hline 5 & 29.000 & 1035.7 & 0.0228 & 32.878 & 1023.6 & 0.0228 & 35.092 & 1016.0 & 0.0228 \\
\hline 6 & 29.093 & 1045.2 & 0.0227 & 33.072 & 1031.3 & 0.0227 & 35.315 & 1018.8 & 0.0227 \\
\hline 7 & 29.100 & 1049.9 & 0.0226 & 33.135 & 1034.4 & 0.0226 & 35.391 & 1018.5 & 0.0226 \\
\hline 8 & 29.085 & 1050.9 & 0.0225 & 33.150 & 1034.3 & 0.0225 & 35.409 & 1016.7 & 0.0225 \\
\hline 9 & 29.067 & 1048.7 & 0.0224 & 33.139 & 1032.0 & 0.0224 & 35.398 & 1014.2 & 0.0224 \\
\hline 10 & 29.050 & 1043.8 & 0.0223 & 33.112 & 1027.8 & 0.0223 & 35.369 & 1011.2 & 0.0223 \\
\hline 11 & 29.037 & 1036.1 & 0.0222 & 33.072 & 1021.7 & 0.0222 & 35.325 & 1007.9 & 0.0222 \\
\hline 12 & 29.031 & 1025.2 & 0.0221 & 33.020 & 1013.5 & 0.0221 & 35.265 & 1003.9 & 0.0221 \\
\hline 13 & 29.029 & 1010.7 & 0.0220 & 32.946 & 1002.4 & 0.0220 & 35.175 & 998.4 & 0.0220 \\
\hline 14 & 28.994 & 991.9 & 0.0219 & 32.805 & 987.5 & 0.0219 & 35.005 & 990.5 & 0.0219 \\
\hline 15 & 28.775 & 968.2 & 0.0218 & 32.429 & 967.1 & 0.0218 & 34.575 & 977.8 & 0.0218 \\
\hline 16 & 27.879 & 939.1 & 0.0217 & 31.290 & 940.0 & 0.0218 & 33.330 & 956.4 & 0.0218 \\
\hline 17 & 24.995 & 900.5 & 0.0217 & 27.974 & 904.7 & 0.0217 & 29.785 & 918.0 & 0.0217 \\
\hline 18 & 17.850 & 812.9 & 0.0216 & 19.836 & 820.0 & 0.0216 & 21.065 & 837.8 & 0.0216 \\
\hline
\end{tabular}


Table 4-79. Burnup and TH Feedback Parameters by Axial Node for Assembly J10

\begin{tabular}{|c|c|c|c|c|c|c|c|c|c|}
\hline \multirow[b]{2}{*}{$\begin{array}{l}\text { Axial } \\
\text { Node }\end{array}$} & \multicolumn{3}{|c|}{ Datapoint (214.0 Cy9) } & \multicolumn{3}{|c|}{ Datapoint (425.0 Cy9) } & \multicolumn{3}{|c|}{ Statepoint 69 (BOC Cy10) } \\
\hline & \begin{tabular}{|c|} 
Burnup \\
(GWd/MTU)
\end{tabular} & $\begin{array}{l}\text { T-Fuel } \\
{ }^{\circ} \mathrm{F}\end{array}$ & $\begin{array}{l}\text { Spec. Vol. } \\
\mathrm{ft}^{3} / \mathrm{lbm}\end{array}$ & $\begin{array}{c}\text { Burnup } \\
\text { (GWd/MTU) }\end{array}$ & $\begin{array}{l}\text { T-Fuel } \\
{ }^{\circ} \mathrm{F}\end{array}$ & $\begin{array}{c}\text { Spec. Vol. } \\
\mathrm{tt}^{3} / \mathrm{bm}\end{array}$ & $\begin{array}{c}\text { Burnup } \\
\text { (GWd/MTU) }\end{array}$ & $\begin{array}{c}\text { T-Fuel } \\
{ }^{\circ} \mathrm{F} \\
\end{array}$ & $\begin{array}{c}\text { Spec. Vol } \\
\mathrm{ft}^{3} / \mathrm{lbm}\end{array}$ \\
\hline & 214.0 Cy 9 & 109.3 Cy9 & 109.3 Cy9 & 425.0 Cy9 & 328.7 Cy9 & 328.7 Cy9 & BOC Cy 10 & 549.2 Cy 9 & 549.2 Cy9 \\
\hline 1 & 5.090 & 1123.2 & 0.0239 & 10.538 & 1090.2 & 0.0239 & 16.088 & 1064.7 & 0.0237 \\
\hline 2 & 7.486 & 1319.9 & 0.0238 & 15.401 & 1245.0 & 0.0237 & 22.996 & 1162.2 & 0.0235 \\
\hline 3 & 8.862 & 1423.3 & 0.0237 & 17.854 & 1300.6 & 0.0236 & 26.552 & 1178.6 & 0.0234 \\
\hline 4 & 9.592 & 1466.5 & 0.0235 & 18.926 & 1307.8 & 0.0234 & 27.782 & 1167.1 & 0.0233 \\
\hline 5 & 9.976 & 1482.7 & 0.0234 & 19.371 & 1301.3 & 0.0233 & 28.146 & 1153.1 & 0.0231 \\
\hline 6 & 10.182 & 1488.1 & 0.0232 & 19.551 & 1292.5 & 0.0231 & 28.223 & 1142.5 & 0.0230 \\
\hline 7 & 10.290 & 1488.4 & 0.0230 & 19.624 & 1285.1 & 0.0230 & 28.223 & 1135.6 & 0.0229 \\
\hline 8 & 10.339 & 1486.1 & 0.0229 & 19.653 & 1279.8 & 0.0229 & 28.211 & 1131.4 & 0.0228 \\
\hline 9 & 10.349 & 1482.5 & 0.0227 & 19.666 & 1276.8 & 0.0227 & 28.204 & 1128.8 & 0.0226 \\
\hline 10 & 10.329 & 1478.1 & 0.0226 & 19.674 & 1275.9 & 0.0226 & 28.207 & 1127.2 & 0.0225 \\
\hline 11 & 10.282 & 1473.0 & 0.0225 & 19.679 & 1277.0 & 0.0225 & 28.219 & 1126.6 & 0.0224 \\
\hline 12 & 10.203 & 1466.9 & 0.0223 & 19.677 & 1280.2 & 0.0223 & 28.241 & 1127.3 & 0.0223 \\
\hline 13 & 10.077 & 1458.7 & 0.0222 & 19.646 & 1285.3 & 0.0222 & 28.268 & 1130.4 & 0.0222 \\
\hline 14 & 9.873 & 1446.1 & 0.0221 & 19.536 & 1291.9 & 0.0221 & 28.268 & 1137.1 & 0.0221 \\
\hline 15 & 9.522 & 1424.4 & 0.0219 & 19.214 & 1297.3 & 0.0220 & 28.096 & 1148.0 & 0.0220 \\
\hline 16 & 8.865 & 1378.1 & 0.0218 & 18.325 & 1292.0 & 0.0218 & 27.268 & 1158.7 & 0.0218 \\
\hline 17 & 7.585 & 1275.9 & 0.0217 & 16.067 & 1241.7 & 0.0217 & 24.466 & 1145.4 & 0.0217 \\
\hline 18 & 5.272 & 1082.2 & 0.0216 & 11.254 & 1079.0 & 0.0216 & 17.479 & 1048.8 & 0.0216 \\
\hline
\end{tabular}

\begin{tabular}{|c|c|c|c|c|c|c|c|c|c|}
\hline \multirow[b]{2}{*}{$\begin{array}{l}\text { Axial } \\
\text { Node }\end{array}$} & \multicolumn{3}{|c|}{ Statepoint 70 (27.0 Cy10) } & \multicolumn{3}{|c|}{ Statepoint 71 (143.0 Cy10) } & \multicolumn{3}{|c|}{ Statepoint 72 (209.0 Cy10) } \\
\hline & \begin{tabular}{|c|} 
Burnup \\
(GWd/MTU)
\end{tabular} & $\begin{array}{c}\text { T-Fuel } \\
\quad{ }^{\circ} \mathrm{F} \\
\end{array}$ & $\begin{array}{c}\text { Spec. Vol. } \\
\mathrm{ft}^{3} / \mathrm{lbm}\end{array}$ & $\begin{array}{c}\text { Burnup } \\
\text { (GWd/MTU) }\end{array}$ & $\begin{array}{c}\text { T-Fuel } \\
{ }^{\circ} \mathrm{F}\end{array}$ & $\begin{array}{c}\text { Spec. Vol. } \\
\mathrm{ft}^{3} / \mathrm{lbm}\end{array}$ & $\begin{array}{c}\text { Burnup } \\
\text { (GWd/MTU) }\end{array}$ & $\begin{array}{c}\text { T-Fuel } \\
{ }^{\circ} \mathbf{F}\end{array}$ & $\begin{array}{c}\text { Spec. Vol. } \\
\mathrm{ft}^{3} / \mathrm{bmm}\end{array}$ \\
\hline & 27.0 Cy 10 & 18.0 Cy 10 & 18.0 Cy 10 & 143.0 Cy 10 & 82.9 Cy 10 & 82.9 Cy 10 & 209.0 Cy 10 & 176.7 Cy 10 & 176.7 Cy 10 \\
\hline 1 & 16.239 & 670.0 & 0.0222 & 16.901 & 669.0 & 0.0222 & 17.313 & 674.1 & 0.0222 \\
\hline 2 & 23.250 & 714.0 & 0.0222 & 24.347 & 710.7 & 0.0222 & 25.009 & 713.0 & 0.0222 \\
\hline 3 & 26.872 & 735.9 & 0.0222 & 28.234 & 730.2 & 0.0221 & 29.028 & 727.5 & 0.0221 \\
\hline 4 & 28.142 & 748.2 & 0.0221 & 29.648 & 740.2 & 0.0221 & 30.502 & 734.3 & 0.0221 \\
\hline 5 & 28.529 & 756.0 & 0.0221 & 30.118 & 746.0 & 0.0221 & 30.997 & 737.3 & 0.0221 \\
\hline 6 & 28.623 & 760.9 & 0.0221 & 30.258 & 749.1 & 0.0220 & 31.146 & 737.8 & 0.0220 \\
\hline 7 & 28.632 & 763.6 & 0.0220 & 30.294 & 750.3 & 0.0220 & 31.185 & 737.2 & 0.0220 \\
\hline 8 & 28.626 & 764.7 & 0.0220 & 30.302 & 750.3 & 0.0220 & 31.193 & 736.0 & 0.0219 \\
\hline 9 & 28.622 & 764.4 & 0.0219 & 30.304 & 749.5 & 0.0219 & 31.193 & 734.7 & 0.0219 \\
\hline 10 & 28.624 & 762.9 & 0.0219 & 30.305 & 748.1 & 0.0219 & 31.193 & 733.5 & 0.0219 \\
\hline 11 & 28.633 & 760.3 & 0.0218 & 30.307 & 746.1 & 0.0218 & 31.194 & 732.2 & 0.0218 \\
\hline 12 & 28.650 & 756.4 & 0.0218 & 30.309 & 743.3 & 0.0218 & 31.194 & 730.8 & 0.0218 \\
\hline 13 & 28.667 & 750.6 & 0.0218 & 30.300 & 739.3 & 0.0218 & 31.182 & 729.1 & 0.0218 \\
\hline 14 & 28.652 & 742.5 & 0.0217 & 30.241 & 733.6 & 0.0217 & 31.112 & 726.3 & 0.0217 \\
\hline 15 & 28.456 & 731.5 & 0.0217 & 29.972 & 725.3 & 0.0217 & 30.820 & 721.5 & 0.0217 \\
\hline 16 & 27.592 & 716.7 & 0.0217 & 28.984 & 713.6 & 0.0217 & 29.782 & 713.1 & 0.0217 \\
\hline 17 & 24.731 & 694.9 & 0.0216 & 25.893 & 695.0 & 0.0216 & 26.579 & 698.8 & 0.0216 \\
\hline 18 & 17.638 & 651.4 & 0.0216 & 18.351 & 653.6 & 0.0216 & 18.785 & 660.3 & 0.0216 \\
\hline
\end{tabular}


Table 4-79. Burnup and TH Feedback Parameters by Axial Node for Assembly J10 (Cont.)

\begin{tabular}{|c|c|c|c|c|c|c|c|c|c|}
\hline \multirow{2}{*}{$\begin{array}{c}\text { Axial } \\
\text { Node }\end{array}$} & \multicolumn{2}{|c|}{ Datapoint (430.4 Cy10) } & \multicolumn{2}{c|}{ Statepoint 73 (BOC Cy11) } & \multicolumn{3}{c|}{ Datapoint (208.7 Cy11) } \\
\cline { 2 - 10 } & $\begin{array}{c}\text { Burnup } \\
\text { (GWd/MTU) }\end{array}$ & $\begin{array}{c}\text { T-Fuel } \\
{ }^{\circ} \mathrm{F}\end{array}$ & $\begin{array}{c}\text { Spec. Vol. } \\
\mathrm{ft}^{3} / \mathrm{lbm}\end{array}$ & $\begin{array}{c}\text { Burnup } \\
\text { (GWd/MTU) }\end{array}$ & $\begin{array}{c}\text { T-Fuel } \\
{ }^{\circ} \mathrm{F}\end{array}$ & $\begin{array}{c}\text { Spec. Vol. } \\
\mathrm{ft}^{3} / \mathrm{lbm}\end{array}$ & $\begin{array}{c}\text { Burnup } \\
\text { (GWd/MTU) }\end{array}$ & $\begin{array}{c}\text { T-Fuel } \\
{ }^{\circ} \mathrm{F}\end{array}$ & $\begin{array}{c}\text { Spec. Vol. } \\
\mathrm{ft}^{3} / \mathrm{lbm}\end{array}$ \\
\hline 1 & 430.4 Cy10 & $319.5 \mathrm{Cy} 10$ & 319.5 Cy10 & BOC Cy11 & $549.3 \mathrm{Cy} 10$ & $549.3 \mathrm{Cy} 10$ & 208.7 Cy11 & $103.8 \mathrm{Cy} 11$ & $103.8 \mathrm{Cy} 11$ \\
\hline 2 & 18.923 & 688.6 & 0.0222 & 21.036 & 715.4 & 0.0223 & 22.236 & 657.9 & 0.0221 \\
\hline 3 & 37.474 & 724.0 & 0.0222 & 30.498 & 744.7 & 0.0222 & 32.307 & 681.7 & 0.0221 \\
\hline 4 & 33.447 & 736.7 & 0.0221 & 36.793 & 751.3 & 0.0221 & 39.048 & 701.8 & 0.0220 \\
\hline 5 & 33.956 & 735.6 & 0.0221 & 37.262 & 747.6 & 0.0221 & 39.598 & 705.4 & 0.0220 \\
\hline 6 & 34.090 & 733.4 & 0.0220 & 37.354 & 744.1 & 0.0221 & 39.736 & 707.2 & 0.0220 \\
\hline 7 & 34.108 & 731.1 & 0.0220 & 37.342 & 741.4 & 0.0220 & 39.748 & 707.7 & 0.0219 \\
\hline 8 & 34.098 & 729.1 & 0.0219 & 37.315 & 739.3 & 0.0220 & 39.729 & 707.4 & 0.0219 \\
\hline 9 & 34.087 & 727.4 & 0.0219 & 37.295 & 737.7 & 0.0219 & 39.709 & 706.5 & 0.0219 \\
\hline 10 & 34.083 & 726.2 & 0.0219 & 37.289 & 736.5 & 0.0219 & 39.694 & 705.1 & 0.0218 \\
\hline 11 & 34.086 & 725.4 & 0.0218 & 37.297 & 735.5 & 0.0219 & 39.686 & 703.3 & 0.0218 \\
\hline 12 & 34.093 & 724.9 & 0.0218 & 37.316 & 735.0 & 0.0218 & 39.682 & 700.9 & 0.0218 \\
\hline 13 & 34.092 & 724.6 & 0.0218 & 37.338 & 735.1 & 0.0218 & 39.667 & 697.9 & 0.0217 \\
\hline 14 & 34.031 & 724.2 & 0.0217 & 37.312 & 735.9 & 0.0218 & 39.587 & 693.7 & 0.0217 \\
\hline 15 & 33.726 & 722.8 & 0.0217 & 37.049 & 737.2 & 0.0217 & 39.238 & 687.9 & 0.0217 \\
\hline 16 & 32.604 & 718.5 & 0.0217 & 35.929 & 737.4 & 0.0217 & 37.978 & 679.4 & 0.0217 \\
\hline 17 & 29.100 & 706.9 & 0.0216 & 32.214 & 729.4 & 0.0216 & 33.995 & 665.1 & 0.0216 \\
\hline 18 & 20.457 & 672.8 & 0.0216 & 22.668 & 699.3 & 0.0216 & 23.848 & 639.9 & 0.0216 \\
\hline
\end{tabular}

\begin{tabular}{|c|c|c|c|c|c|c|}
\hline \multirow{2}{*}{$\begin{array}{c}\text { Axial } \\
\text { Node }\end{array}$} & $\begin{array}{c}\text { Burnup } \\
\text { (GWd/MTU) }\end{array}$ & $\begin{array}{c}\text { T-Fuel } \\
\text { oF }\end{array}$ & $\begin{array}{c}\text { Spec. Vol. } \\
\mathrm{ft}^{3} / \mathrm{lbm}\end{array}$ & $\begin{array}{c}\text { Burnup } \\
\text { (GWd/MTU) }\end{array}$ & $\begin{array}{c}\text { S-Fuel } \\
{ }^{\mathbf{F}} \mathbf{F}\end{array}$ & $\begin{array}{c}\text { Spec. Vol. } \\
\mathrm{t}^{3} / \mathrm{lbm}\end{array}$ \\
\hline & 417.8 Cy11 & 312.8 Cy11 & 312.8 Cy11 & $615.0 \mathrm{Cy} 11$ & 515.7 Cy11 & 515.7 Cy11 \\
\hline 1 & 23.759 & 678.5 & 0.0221 & 25.475 & 695.4 & 0.0222 \\
\hline 2 & 34.502 & 702.5 & 0.0221 & 36.850 & 718.1 & 0.0221 \\
\hline 3 & 39.741 & 712.5 & 0.0221 & 42.260 & 723.4 & 0.0221 \\
\hline 4 & 41.561 & 714.0 & 0.0220 & 44.081 & 721.0 & 0.0221 \\
\hline 5 & 42.121 & 713.2 & 0.0220 & 44.609 & 717.8 & 0.0220 \\
\hline 6 & 42.252 & 711.8 & 0.0220 & 44.710 & 715.0 & 0.0220 \\
\hline 7 & 42.254 & 710.3 & 0.0219 & 44.690 & 712.9 & 0.0220 \\
\hline 8 & 42.226 & 708.9 & 0.0219 & 44.648 & 711.1 & 0.0219 \\
\hline 9 & 42.198 & 707.7 & 0.0219 & 44.612 & 709.8 & 0.0219 \\
\hline 10 & 42.179 & 706.6 & 0.0219 & 44.590 & 708.7 & 0.0219 \\
\hline 11 & 42.171 & 705.7 & 0.0218 & 44.582 & 707.8 & 0.0218 \\
\hline 12 & 42.168 & 704.9 & 0.0218 & 44.585 & 707.3 & 0.0218 \\
\hline 13 & 42.156 & 704.2 & 0.0218 & 44.585 & 707.1 & 0.0218 \\
\hline 14 & 42.075 & 703.3 & 0.0217 & 44.524 & 707.4 & 0.0217 \\
\hline 15 & 41.711 & 701.9 & 0.0217 & 44.182 & 708.0 & 0.0217 \\
\hline 16 & 40.388 & 698.6 & 0.0217 & 42.858 & 708.0 & 0.0217 \\
\hline 17 & 36.184 & 687.9 & 0.0216 & 38.510 & 701.8 & 0.0216 \\
\hline 18 & 25.363 & 660.5 & 0.0216 & 27.058 & 675.1 & 0.0216 \\
\hline
\end{tabular}


Table 4-80. Burnup and TH Feedback Parameters by Axial Node for Assembly $\mathrm{J} 12$

\begin{tabular}{|c|c|c|c|c|c|c|c|c|c|}
\hline \multirow{2}{*}{$\begin{array}{c}\text { Axial } \\
\text { Node }\end{array}$} & \multicolumn{3}{|c|}{ Datapoint (214.0 Cy9) } & \multicolumn{2}{c|}{ Datapoint (425.0 Cy9) } & \multicolumn{3}{c|}{ Statepoint 69 (BOC Cy10) } \\
\cline { 2 - 10 } & $\begin{array}{c}\text { Burnup } \\
\text { (GWd/MTU) }\end{array}$ & $\begin{array}{c}\text { T-Fuel } \\
{ }^{\circ} \mathrm{F}\end{array}$ & $\begin{array}{c}\text { Spec. Vol. } \\
\mathrm{ft}^{3} / \mathrm{lbm}\end{array}$ & $\begin{array}{c}\text { Burnup } \\
\text { (GWd/MTU) }\end{array}$ & $\begin{array}{c}\text { T-Fuel } \\
{ }^{\circ} \mathrm{F}\end{array}$ & $\begin{array}{c}\text { Spec. Vol. } \\
\mathrm{ft}^{3} / \mathrm{lbm}\end{array}$ & $\begin{array}{c}\text { Burnup } \\
\text { (GWd/MTU) }\end{array}$ & $\begin{array}{c}\text { T-Fuel } \\
{ }^{\circ} \mathrm{F}\end{array}$ & $\begin{array}{c}\text { Spec. Vol. } \\
\mathrm{ft}^{3} / \mathrm{lbm}\end{array}$ \\
\hline 1 & 214.0 Cy9 & 109.3 Cy9 & 109.3 Cy9 & 425.0 Cy9 & 328.7 Cy9 & 328.7 Cy9 & BOC Cy10 & 549.2 Cy9 & 549.2 Cy9 \\
\hline 2 & 4.532 & 1078.3 & 0.0238 & 9.644 & 1073.6 & 0.0238 & 15.116 & 1066.1 & 0.0238 \\
\hline 3 & 6.872 & 1275.7 & 0.0237 & 14.455 & 1230.4 & 0.0237 & 22.078 & 1176.3 & 0.0236 \\
\hline 4 & 9.412 & 1390.6 & 0.0236 & 17.238 & 1298.1 & 0.0236 & 26.155 & 1200.4 & 0.0235 \\
\hline 5 & 9.617 & 1459.1 & 0.0233 & 18.921 & 1303.9 & 0.0233 & 28.042 & 1176.8 & 0.0232 \\
\hline 6 & 9.821 & 1464.2 & 0.0232 & 19.100 & 1295.5 & 0.0231 & 28.125 & 1165.9 & 0.0231 \\
\hline 7 & 9.933 & 1464.6 & 0.0230 & 19.181 & 1288.3 & 0.0230 & 28.142 & 1159.0 & 0.0229 \\
\hline 8 & 9.988 & 1462.6 & 0.0229 & 19.222 & 1283.2 & 0.0229 & 28.149 & 1155.0 & 0.0228 \\
\hline 9 & 10.007 & 1459.4 & 0.0227 & 19.247 & 1280.4 & 0.0227 & 28.161 & 1152.6 & 0.0227 \\
\hline 10 & 9.999 & 1455.7 & 0.0226 & 19.273 & 1279.7 & 0.0226 & 28.184 & 1151.2 & 0.0226 \\
\hline 11 & 9.976 & 1451.9 & 0.0224 & 19.313 & 1280.9 & 0.0225 & 28.232 & 1150.8 & 0.0224 \\
\hline 12 & 9.949 & 1448.7 & 0.0223 & 19.392 & 1284.7 & 0.0223 & 28.341 & 1152.4 & 0.0223 \\
\hline 13 & 9.901 & 1445.0 & 0.0222 & 19.487 & 1291.6 & 0.0222 & 28.511 & 1157.4 & 0.0222 \\
\hline 14 & 9.736 & 1434.9 & 0.0220 & 19.437 & 1298.7 & 0.0221 & 28.578 & 1165.2 & 0.0221 \\
\hline 15 & 9.395 & 1414.1 & 0.0219 & 19.126 & 1303.8 & 0.0220 & 28.421 & 1176.7 & 0.0220 \\
\hline 16 & 8.738 & 1365.9 & 0.0218 & 18.232 & 1297.9 & 0.0218 & 27.582 & 1187.5 & 0.0219 \\
\hline 17 & 7.463 & 1264.6 & 0.0217 & 15.974 & 1247.4 & 0.0217 & 24.744 & 1172.2 & 0.0217 \\
\hline 18 & 5.181 & 1074.1 & 0.0216 & 11.211 & 1085.6 & 0.0216 & 17.724 & 1071.2 & 0.0216 \\
\hline
\end{tabular}

\begin{tabular}{|c|c|c|c|c|c|c|c|c|c|}
\hline \multirow[b]{2}{*}{$\begin{array}{l}\text { Axial } \\
\text { Node }\end{array}$} & \multicolumn{3}{|c|}{ Statepoint 70 (27.0 Cy10) } & \multicolumn{3}{|c|}{ Statepoint 71 (143.0 Cy10) } & \multicolumn{3}{|c|}{ Statepoint $72(209.0$ Cy10) } \\
\hline & $\begin{array}{c}\text { Burnup } \\
\text { (GWd/MTU) }\end{array}$ & $\begin{array}{c}\text { T-Fuel } \\
{ }^{\circ} \mathrm{F} \\
\end{array}$ & $\begin{array}{c}\text { Spec. Vol. } \\
\mathrm{ft}^{3} / \mathrm{lbm}\end{array}$ & $\begin{array}{c}\text { Burnup } \\
\text { (GWd/MTU) }\end{array}$ & $\begin{array}{c}\text { T-Fuel } \\
{ }^{\circ} \mathrm{F} \\
\end{array}$ & $\begin{array}{c}\text { Spec. Vol. } \\
\mathrm{ft}^{3} / \mathrm{lbm}\end{array}$ & $\begin{array}{c}\text { Burnup } \\
\text { (GWd/MTU) }\end{array}$ & $\begin{array}{l}\text { T-Fuel } \\
{ }^{\circ} \mathrm{F} \\
\end{array}$ & $\begin{array}{c}\text { Spec. Vol. } \\
\mathrm{ft}^{3} / \mathrm{lbm}\end{array}$ \\
\hline & 27.0 Cy 10 & $18.0 \mathrm{Cy} 10$ & 18.0 Cy 10 & 143.0 Cy 10 & $82.9 \mathrm{Cy} 10$ & 82.9 Cy 10 & 209.0 Cy 10 & 176.7 Cy 10 & 176.7 Cy 10 \\
\hline 1 & 15.589 & 901.5 & 0.0234 & 17.661 & 895.4 & 0.0234 & 18.930 & 901.8 & 0.0234 \\
\hline 2 & 22.811 & 1005.9 & 0.0233 & 25.990 & 993.2 & 0.0233 & 27.893 & 989.3 & 0.0233 \\
\hline 3 & 27.035 & 1051.8 & 0.0232 & 30.818 & 1035.2 & 0.0232 & 33.031 & 1028.4 & 0.0232 \\
\hline 4 & 28.617 & 1077.7 & 0.0231 & 32.724 & 1060.1 & 0.0231 & 35.072 & 1048.8 & 0.0231 \\
\hline 5 & 29.057 & 1094.8 & 0.0230 & 33.346 & 1075.8 & 0.0230 & 35.752 & 1056.3 & 0.0230 \\
\hline 6 & 29.175 & 1105.8 & 0.0229 & 33.573 & 1083.9 & 0.0229 & 36.004 & 1058.1 & 0.0228 \\
\hline 7 & 29.215 & 1112.2 & 0.0228 & 33.683 & 1087.8 & 0.0227 & 36.126 & 1057.3 & 0.0227 \\
\hline 8 & 29.235 & 1114.8 & 0.0226 & 33.744 & 1088.6 & 0.0226 & 36.192 & 1055.3 & 0.0226 \\
\hline 9 & 29.254 & 1114.1 & 0.0225 & 33.782 & 1087.1 & 0.0225 & 36.233 & 1052.9 & 0.0225 \\
\hline 10 & 29.278 & 1110.6 & 0.0224 & 33.810 & 1084.0 & 0.0224 & 36.262 & 1050.4 & 0.0224 \\
\hline 11 & 29.321 & 1104.7 & 0.0223 & 33.844 & 1079.5 & 0.0223 & 36.300 & 1048.3 & 0.0223 \\
\hline 12 & 29.420 & 1096.4 & 0.0222 & 33.924 & 1074.0 & 0.0222 & 36.387 & 1046.6 & 0.0222 \\
\hline 13 & 29.573 & 1084.1 & 0.0221 & 34.037 & 1065.9 & 0.0221 & 36.501 & 1044.1 & 0.0221 \\
\hline 14 & 29.607 & 1065.3 & 0.0220 & 33.979 & 1052.3 & 0.0220 & 36.426 & 1038.1 & 0.0220 \\
\hline 15 & 29.397 & 1038.1 & 0.0219 & 33.598 & 1030.1 & 0.0219 & 35.992 & 1025.8 & 0.0219 \\
\hline 16 & 28.480 & 1002.5 & 0.0218 & 32.397 & 996.3 & 0.0218 & 34.675 & 1002.6 & 0.0218 \\
\hline 17 & 25.515 & 954.5 & 0.0217 & 28.927 & 952.3 & 0.0217 & 30.949 & 955.7 & 0.0217 \\
\hline 18 & 18.231 & 852.0 & 0.0216 & 20.504 & 855.1 & 0.0216 & 21.879 & 866.6 & 0.0216 \\
\hline
\end{tabular}


Table 4-81. Burnup and TH Feedback Parameters by Axial Node for Assembly J12a

\begin{tabular}{|c|c|c|c|c|c|c|c|c|c|}
\hline \multirow[b]{2}{*}{$\begin{array}{l}\text { Axial } \\
\text { Node }\end{array}$} & \multicolumn{3}{|c|}{ Datapoint (214.0 Cy9) } & \multicolumn{3}{|c|}{ Datapoint (425.0 Cy9) } & \multicolumn{3}{|c|}{ Statepoint 69 (BOC Cy10) } \\
\hline & $\begin{array}{c}\text { Burnup } \\
\text { (GWd/MTU) }\end{array}$ & $\begin{array}{c}\text { T-Fuel } \\
{ }^{\circ} \mathbf{F} \\
\end{array}$ & $\begin{array}{c}\text { Spec. Vol. } \\
\mathrm{ft}^{3} / \mathrm{lbm}\end{array}$ & $\begin{array}{c}\text { Burnup } \\
\text { (GWd/MTU) }\end{array}$ & $\begin{array}{c}\text { T-Fuel } \\
{ }^{\circ} \mathrm{F} \\
\end{array}$ & $\begin{array}{c}\text { Spec. Vol. } \\
\mathrm{ft}^{3} / \mathrm{lbm}\end{array}$ & $\begin{array}{c}\text { Burnup } \\
\text { (GWd/MTU) }\end{array}$ & $\begin{array}{c}\text { T-Fuel } \\
{ }^{\circ} \mathrm{F}\end{array}$ & $\begin{array}{c}\text { Spec. Vol. } \\
\mathrm{ft}^{3} / \mathrm{lbm}\end{array}$ \\
\hline & 214.0 Cy 9 & 109.3 Cy9 & 109.3 Cy9 & 425.0 Cy9 & 328.7 Cy9 & 328.7 Cy9 & BOC Cy 10 & 549.2 Cy9 & 549.2 Cy 9 \\
\hline 1 & 4.532 & 1078.3 & 0.0238 & 9.644 & 1073.6 & 0.0238 & 15.071 & 1066.1 & 0.0238 \\
\hline 2 & 6.872 & 1275.7 & 0.0237 & 14.455 & 1230.4 & 0.0237 & 21.997 & 1176.3 & 0.0236 \\
\hline 3 & 8.412 & 1390.6 & 0.0236 & 17.238 & 1298.1 & 0.0236 & 26.040 & 1200.4 & 0.0235 \\
\hline 4 & 9.230 & 1443.3 & 0.0235 & 18.472 & 1310.4 & 0.0234 & 27.518 & 1191.4 & 0.0233 \\
\hline 5 & 9.617 & 1459.1 & 0.0233 & 18.921 & 1303.9 & 0.0233 & 27.894 & 1176.8 & 0.0232 \\
\hline 6 & 9.821 & 1464.2 & 0.0232 & 19.100 & 1295.5 & 0.0231 & 27.970 & 1165.9 & 0.0231 \\
\hline 7 & 9.933 & 1464.6 & 0.0230 & 19.181 & 1288.3 & 0.0230 & 27.984 & 1159.0 & 0.0229 \\
\hline 8 & 9.988 & 1462.6 & 0.0229 & 19.222 & 1283.2 & 0.0229 & 27.988 & 1155.0 & 0.0228 \\
\hline 9 & 10.007 & 1459.4 & 0.0227 & 19.247 & 1280.4 & 0.0227 & 27.997 & 1152.6 & 0.0227 \\
\hline 10 & 9.999 & 1455.7 & 0.0226 & 19.273 & 1279.7 & 0.0226 & 28.019 & 1151.2 & 0.0226 \\
\hline 11 & 9.976 & 1451.9 & 0.0224 & 19.313 & 1280.9 & 0.0225 & 28.066 & 1150.8 & 0.0224 \\
\hline 12 & 9.949 & 1448.7 & 0.0223 & 19.392 & 1284.7 & 0.0223 & 28.176 & 1152.4 & 0.0223 \\
\hline 13 & 9.901 & 1445.0 & 0.0222 & 19.487 & 1291.6 & 0.0222 & 28.351 & 1157.4 & 0.0222 \\
\hline 14 & 9.736 & 1434.9 & 0.0220 & 19.437 & 1298.7 & 0.0221 & 28.421 & 1165.2 & 0.0221 \\
\hline 15 & 9.395 & 1414.1 & 0.0219 & 19.126 & 1303.8 & 0.0220 & 28.265 & 1176.7 & 0.0220 \\
\hline 16 & 8.738 & 1365.9 & 0.0218 & 18.232 & 1297.9 & 0.0218 & 27.432 & 1187.5 & 0.0219 \\
\hline 17 & 7.463 & 1264.6 & 0.0217 & 15.974 & 1247.4 & 0.0217 & 24.613 & 1172.2 & 0.0217 \\
\hline 18 & 5.181 & 1074.1 & 0.0216 & 11.211 & 1085.6 & 0.0216 & 17.638 & 1071.2 & 0.0216 \\
\hline
\end{tabular}

\begin{tabular}{|c|c|c|c|c|c|c|c|c|c|}
\hline \multirow[b]{2}{*}{$\begin{array}{l}\text { Axial } \\
\text { Node }\end{array}$} & \multicolumn{3}{|c|}{ Statepoint 70 (27.0 Cy10) } & \multicolumn{3}{|c|}{ Statepoint 71 (143.0 Cy10) } & \multicolumn{3}{|c|}{ Statepoint 72 (209.0 Cy10) } \\
\hline & \begin{tabular}{|c|} 
Burnup \\
(GWd/MTU)
\end{tabular} & $\begin{array}{c}\text { T-Fuel } \\
{ }^{\circ} \mathrm{F}\end{array}$ & $\begin{array}{c}\text { Spec. Vol. } \\
\mathrm{ft}^{3} / \mathrm{lbm}\end{array}$ & $\begin{array}{c}\text { Burnup } \\
\text { (GWd/MTU) }\end{array}$ & $\begin{array}{c}\text { T-Fuel } \\
{ }^{\circ} \mathrm{F}\end{array}$ & $\begin{array}{c}\text { Spec. Vol. } \\
\mathrm{ft}^{3} / \mathrm{lbm}\end{array}$ & $\begin{array}{c}\text { Burnup } \\
\text { (GWd/MTU) }\end{array}$ & $\begin{array}{c}\text { T-Fuel } \\
{ }^{\circ} \mathrm{F}\end{array}$ & $\begin{array}{c}\text { Spec. Vol. } \\
\mathrm{ft}^{3} / \mathrm{lbm}\end{array}$ \\
\hline & 27.0 Cy 10 & 18.0 Cy 10 & 18.0 Cy 10 & 143.0 Cy 10 & 82.9 Cy 10 & 82.9 Cy 10 & 209.0 Cy10 & 176.7 Cy 10 & 176.7 Cy 10 \\
\hline 1 & 15.554 & 904.5 & 0.0234 & 17.686 & 901.7 & 0.0234 & 18.988 & 909.3 & 0.0234 \\
\hline 2 & 22.737 & 1006.6 & 0.0233 & 25.968 & 997.4 & 0.0233 & 27.900 & 995.2 & 0.0233 \\
\hline 3 & 26.917 & 1049.2 & 0.0232 & 30.713 & 1035.6 & 0.0232 & 32.939 & 1031.2 & 0.0232 \\
\hline 4 & 28.472 & 1073.6 & 0.0231 & 32.568 & 1058.2 & 0.0231 & 34.923 & 1050.7 & 0.0231 \\
\hline 5 & 28.898 & 1090.4 & 0.0230 & 33.173 & 1073.9 & 0.0230 & 35.589 & 1058.9 & 0.0230 \\
\hline 6 & 29.007 & 1101.0 & 0.0229 & 33.391 & 1082.1 & 0.0228 & 35.836 & 1061.3 & 0.0228 \\
\hline 7 & 29.042 & 1106.5 & 0.0227 & 33.490 & 1085.6 & 0.0227 & 35.948 & 1060.7 & 0.0227 \\
\hline 8 & 29.057 & 1108.1 & 0.0226 & 33.541 & 1085.9 & 0.0226 & 36.003 & 1058.7 & 0.0226 \\
\hline 9 & 29.071 & 1106.3 & 0.0225 & 33.569 & 1083.8 & 0.0225 & 36.032 & 1056.1 & 0.0225 \\
\hline 10 & 29.091 & 1101.7 & 0.0224 & 33.584 & 1079.8 & 0.0224 & 36.047 & 1053.2 & 0.0224 \\
\hline 11 & 29.129 & 1094.1 & 0.0223 & 33.602 & 1074.1 & 0.0223 & 36.064 & 1050.2 & 0.0223 \\
\hline 12 & 29.224 & 1083.0 & 0.0222 & 33.657 & 1066.1 & 0.0222 & 36.115 & 1046.5 & 0.0222 \\
\hline 13 & 29.374 & 1067.3 & 0.0220 & 33.739 & 1054.8 & 0.0221 & 36.185 & 1041.3 & 0.0221 \\
\hline 14 & 29.409 & 1047.1 & 0.0219 & 33.666 & 1039.4 & 0.0220 & 36.086 & 1033.6 & 0.0220 \\
\hline 15 & 29.202 & 1021.0 & 0.0219 & 33.291 & 1017.5 & 0.0219 & 35.656 & 1020.7 & 0.0219 \\
\hline 16 & 28.296 & 988.0 & 0.0218 & 32.117 & 985.6 & 0.0218 & 34.368 & 998.0 & 0.0218 \\
\hline 17 & 25.359 & 943.3 & 0.0217 & 28.697 & 944.6 & 0.0217 & 30.698 & 952.8 & 0.0217 \\
\hline 18 & 18.130 & 844.5 & 0.0216 & 20.357 & 849.8 & 0.0216 & 21.719 & 864.6 & 0.0216 \\
\hline
\end{tabular}


Table 4-82. Burnup and TH Feedback Parameters by Axial Node for Assembly J14

\begin{tabular}{|c|c|c|c|c|c|c|c|c|c|}
\hline \multirow[b]{2}{*}{$\begin{array}{l}\text { Axial } \\
\text { Node }\end{array}$} & \multicolumn{3}{|c|}{ Datapoint (214.0 Cy9) } & \multicolumn{3}{|c|}{ Datapoint (425.0 Cy9) } & \multicolumn{3}{|c|}{ Statepoint 69 (BOC Cy10) } \\
\hline & $\begin{array}{c}\text { Burnup } \\
\text { (GWd/MTU) }\end{array}$ & $\begin{array}{l}\text { T-Fuel } \\
{ }^{\circ} \mathrm{F}\end{array}$ & $\begin{array}{c}\text { Spec. Vol. } \\
\mathrm{ft}^{3} / \mathrm{lbm}\end{array}$ & $\begin{array}{c}\text { Burnup } \\
\text { (GWd/MTU) }\end{array}$ & $\begin{array}{l}\text { T-Fuel } \\
{ }^{\circ} \mathrm{F}\end{array}$ & $\begin{array}{c}\text { Spec. Vol. } \\
\mathrm{ft}^{3} / \mathrm{lbm}\end{array}$ & $\begin{array}{c}\text { Burnup } \\
\text { (GWd/MTU) }\end{array}$ & $\begin{array}{l}\text { T-Fuel } \\
{ }^{\circ} \mathrm{F}\end{array}$ & $\begin{array}{c}\text { Spec. Vol. } \\
\mathrm{tt}^{3} / \mathrm{lbm}\end{array}$ \\
\hline & 214.0 Cy 9 & 109.3 Cy 9 & 109.3 Cy9 & 425.0 Cy 9 & 328.7 Cy 9 & 328.7 Cy9 & BOC Cy 10 & 549.2 Cy9 & 549.2 Cy9 \\
\hline 1 & 3.368 & 950.4 & 0.0232 & 7.431 & 990.8 & 0.0233 & 12.069 & 1016.5 & 0.0234 \\
\hline 2 & 5.285 & 1128.6 & 0.0232 & 11.385 & 1128.6 & 0.0232 & 17.899 & 1124.8 & 0.0233 \\
\hline 3 & 6.409 & 1217.5 & 0.0231 & 13.408 & 1178.4 & 0.0231 & 20.939 & 1142.3 & 0.0232 \\
\hline 4 & 7.026 & 1260.9 & 0.0230 & 14.329 & 1188.3 & 0.0230 & 22.042 & 1134.3 & 0.0231 \\
\hline 5 & 7.357 & 1280.3 & 0.0229 & 14.725 & 1184.9 & 0.0229 & 22.399 & 1123.1 & 0.0229 \\
\hline 6 & 7.535 & 1287.8 & 0.0227 & 14.892 & 1178.4 & 0.0228 & 22.496 & 1114.4 & 0.0228 \\
\hline 7 & 7.628 & 1289.6 & 0.0226 & 14.962 & 1172.5 & 0.0227 & 22.516 & 1108.8 & 0.0227 \\
\hline 8 & 7.673 & 1288.5 & 0.0225 & 14.993 & 1168.3 & 0.0226 & 22.522 & 1105.6 & 0.0226 \\
\hline 9 & 7.688 & 1286.1 & 0.0224 & 15.012 & 1165.9 & 0.0225 & 22.533 & 1103.8 & 0.0225 \\
\hline 10 & 7.683 & 1283.1 & 0.0223 & 15.031 & 1165.3 & 0.0224 & 22.554 & 1102.9 & 0.0224 \\
\hline 11 & 7.662 & 1279.7 & 0.0222 & 15.054 & 1166.5 & 0.0223 & 22.588 & 1102.8 & 0.0223 \\
\hline 12 & 7.619 & 1275.3 & 0.0221 & 15.075 & 1169.6 & 0.0222 & 22.636 & 1104.1 & 0.0222 \\
\hline 13 & 7.538 & 1268.7 & 0.0220 & 15.071 & 1174.6 & 0.0221 & 22.688 & 1107.4 & 0.0221 \\
\hline 14 & 7.384 & 1256.9 & 0.0219 & 14.990 & 1180.6 & 0.0220 & 22.705 & 1114.1 & 0.0220 \\
\hline 15 & 7.095 & 1234.6 & 0.0218 & 14.711 & 1184.2 & 0.0219 & 22.549 & 1124.1 & 0.0219 \\
\hline 16 & 6.541 & 1190.3 & 0.0218 & 13.944 & 1175.5 & 0.0218 & 21.798 & 1132.4 & 0.0218 \\
\hline 17 & 5.475 & 1101.7 & 0.0217 & 12.049 & 1126.0 & 0.0217 & 19.352 & 1118.2 & 0.0217 \\
\hline 18 & 3.586 & 926.6 & 0.0216 & 8.094 & 985.5 & 0.0216 & 13.414 & 1011.5 & 0.0216 \\
\hline
\end{tabular}

\begin{tabular}{|c|c|c|c|c|c|c|c|c|c|}
\hline \multirow[b]{2}{*}{$\begin{array}{l}\text { Axial } \\
\text { Node }\end{array}$} & \multicolumn{3}{|c|}{ Statepoint 70 (27.0 Cy10) } & \multicolumn{3}{|c|}{ Statepoint 71 (143.0 Cy10) } & \multicolumn{3}{|c|}{ Statepoint 72 (209.0 Cy10) } \\
\hline & $\begin{array}{c}\text { Burnup } \\
\text { (GWd/MTU) }\end{array}$ & $\begin{array}{c}\text { T-Fuel } \\
{ }^{\circ} \mathrm{F}\end{array}$ & $\begin{array}{c}\text { Spec. Vol. } \\
\mathrm{ft}^{3} / \mathrm{lbm}\end{array}$ & $\begin{array}{c}\text { Burnup } \\
\text { (GWd/MTU) }\end{array}$ & $\begin{array}{c}\text { T-Fuel } \\
{ }^{\circ} \mathrm{F} \\
\end{array}$ & $\begin{array}{c}\text { Spec. Vol. } \\
\mathrm{ft}^{3} / \mathrm{lbm}\end{array}$ & $\begin{array}{c}\text { Burnup } \\
\text { (GWd/MTU) }\end{array}$ & $\begin{array}{c}\text { T-Fuel } \\
{ }^{\circ} \mathrm{F}\end{array}$ & $\begin{array}{c}\text { Spec. Vol. } \\
\mathrm{ft}^{3} / \mathrm{lbm}\end{array}$ \\
\hline & 27.0 Cy 10 & 18.0 Cy 10 & 18.0 Cy 10 & 143.0 Cy 10 & 82.9 Cy 10 & 82.9 Cy 10 & 209.0 Cy 10 & 176.7 Cy 10 & 176.7 Cy 10 \\
\hline 1 & 12.521 & 903.6 & 0.0235 & 14.540 & 904.5 & 0.0235 & 15.801 & 919.2 & 0.0235 \\
\hline 2 & 18.608 & 1020.5 & 0.0234 & 21.752 & 1017.2 & 0.0234 & 23.681 & 1023.6 & 0.0234 \\
\hline 3 & 21.807 & 1079.8 & 0.0233 & 25.637 & 1076.2 & 0.0233 & 27.934 & 1073.7 & 0.0233 \\
\hline 4 & 23.006 & 1115.7 & 0.0232 & 27.230 & 1110.7 & 0.0232 & 29.701 & 1097.5 & 0.0232 \\
\hline 5 & 23.423 & 1138.4 & 0.0230 & 27.875 & 1130.4 & 0.0231 & 30.426 & 1108.1 & 0.0231 \\
\hline 6 & 23.559 & 1152.9 & 0.0229 & 28.154 & 1141.7 & 0.0229 & 30.742 & 1111.6 & 0.0229 \\
\hline 7 & 23.607 & 1161.8 & 0.0228 & 28.293 & 1148.0 & 0.0228 & 30.901 & 1111.9 & 0.0228 \\
\hline 8 & 23.631 & 1166.3 & 0.0227 & 28.375 & 1150.7 & 0.0227 & 30.995 & 1110.7 & 0.0227 \\
\hline 9 & 23.650 & 1167.1 & 0.0225 & 28.430 & 1150.7 & 0.0226 & 31.057 & 1109.0 & 0.0226 \\
\hline 10 & 23.674 & 1164.7 & 0.0224 & 28.468 & 1148.7 & 0.0224 & 31.103 & 1107.3 & 0.0225 \\
\hline 11 & 23.705 & 1159.4 & 0.0223 & 28.498 & 1144.9 & 0.0223 & 31.141 & 1105.9 & 0.0223 \\
\hline 12 & 23.743 & 1151.2 & 0.0222 & 28.519 & 1139.3 & 0.0222 & 31.171 & 1104.9 & 0.0222 \\
\hline 13 & 23.776 & 1138.6 & 0.0221 & 28.507 & 1130.6 & 0.0221 & 31.164 & 1103.0 & 0.0221 \\
\hline 14 & 23.758 & 1118.5 & 0.0220 & 28.387 & 1115.5 & 0.0220 & 31.027 & 1097.6 & 0.0220 \\
\hline 15 & 23.547 & 1089.0 & 0.0219 & 27.985 & 1091.5 & 0.0219 & 30.569 & 1085.3 & 0.0219 \\
\hline 16 & 22.711 & 1048.6 & 0.0218 & 26.825 & 1055.8 & 0.0218 & 29.275 & 1060.7 & 0.0218 \\
\hline 17 & 20.130 & 992.7 & 0.0217 & 23.666 & 1001.0 & 0.0217 & 25.816 & 1015.4 & 0.0217 \\
\hline 18 & 13.923 & 875.0 & 0.0216 & 16.252 & 885.9 & 0.0216 & 17.689 & 905.1 & 0.0216 \\
\hline
\end{tabular}


Table 4-83. Burnup and TH Feedback Parameters by Axial Node for Assembly J17

\begin{tabular}{|c|c|c|c|c|c|c|c|c|c|}
\hline \multirow[b]{2}{*}{$\begin{array}{l}\text { Axial } \\
\text { Node } \\
\end{array}$} & \multicolumn{3}{|c|}{ Datapoint (214.0 Cy9) } & \multicolumn{3}{|c|}{ Datapoint (425.0 Cy9) } & \multicolumn{3}{|c|}{ Statepoint 69 (BOC Cy10) } \\
\hline & \begin{tabular}{|c|} 
Burnup \\
(GWd/MTU)
\end{tabular} & $\begin{array}{c}\text { T-Fuel } \\
{ }^{\circ} \mathrm{F} \\
\end{array}$ & $\begin{array}{c}\text { Spec. Vol. } \\
\mathrm{ft}^{3} / \mathrm{lbm}\end{array}$ & $\begin{array}{c}\text { Burnup } \\
\text { (GWd/MTU) }\end{array}$ & $\begin{array}{c}\text { T-Fuel } \\
{ }^{\circ} \mathrm{F} \\
\end{array}$ & $\begin{array}{c}\text { Spec. Vol. } \\
\mathrm{ft}^{3} / \mathrm{lbm}\end{array}$ & $\begin{array}{c}\text { Burnup } \\
\text { (GWd/MTU) }\end{array}$ & $\begin{array}{c}\text { T-Fuel } \\
{ }^{\circ} \mathrm{F}\end{array}$ & $\begin{array}{c}\text { Spec. Vol. } \\
\mathrm{ft}^{3} / \mathrm{lbm}\end{array}$ \\
\hline & 214.0 Cy 9 & 109.3 Cy9 & 109.3 Cy9 & 425.0 Cy9 & 328.7 Cy9 & 328.7 Cy9 & BOC Cy 10 & 549.2 Cy9 & 549.2 Cy 9 \\
\hline 1 & 4.992 & 1115.7 & 0.0239 & 10.492 & 1098.1 & 0.0239 & 16.297 & 1083.8 & 0.0238 \\
\hline 2 & 7.252 & 1301.9 & 0.0238 & 15.161 & 1251.0 & 0.0238 & 23.098 & 1187.7 & 0.0237 \\
\hline 3 & 8.603 & 1403.2 & 0.0236 & 17.634 & 1311.5 & 0.0236 & 26.765 & 1209.4 & 0.0235 \\
\hline 4 & 9.329 & 1449.4 & 0.0235 & 18.737 & 1320.6 & 0.0235 & 28.082 & 1198.9 & 0.0234 \\
\hline 5 & 9.691 & 1464.0 & 0.0233 & 19.161 & 1314.6 & 0.0233 & 28.438 & 1183.9 & 0.0232 \\
\hline 6 & 9.888 & 1468.7 & 0.0232 & 19.335 & 1306.4 & 0.0232 & 28.516 & 1172.7 & 0.0231 \\
\hline 7 & 9.998 & 1469.0 & 0.0230 & 19.417 & 1299.5 & 0.0230 & 28.532 & 1165.8 & 0.0230 \\
\hline 8 & 10.052 & 1467.0 & 0.0229 & 19.459 & 1294.7 & 0.0229 & 28.540 & 1161.6 & 0.0228 \\
\hline 9 & 10.071 & 1463.8 & 0.0227 & 19.487 & 1291.9 & 0.0228 & 28.555 & 1159.2 & 0.0227 \\
\hline 10 & 10.065 & 1460.1 & 0.0226 & 19.517 & 1291.1 & 0.0226 & 28.583 & 1157.8 & 0.0226 \\
\hline 11 & 10.046 & 1456.6 & 0.0224 & 19.566 & 1292.5 & 0.0225 & 28.641 & 1157.3 & 0.0225 \\
\hline 12 & 10.028 & 1453.8 & 0.0223 & 19.662 & 1296.7 & 0.0224 & 28.768 & 1159.0 & 0.0223 \\
\hline 13 & 9.989 & 1450.6 & 0.0222 & 19.776 & 1303.9 & 0.0222 & 28.959 & 1164.1 & 0.0222 \\
\hline 14 & 9.831 & 1440.7 & 0.0221 & 19.735 & 1311.1 & 0.0221 & 29.037 & 1172.0 & 0.0221 \\
\hline 15 & 9.490 & 1419.9 & 0.0219 & 19.415 & 1315.7 & 0.0220 & 28.872 & 1183.8 & 0.0220 \\
\hline 16 & 8.831 & 1373.0 & 0.0218 & 18.495 & 1308.1 & 0.0219 & 27.998 & 1194.5 & 0.0219 \\
\hline 17 & 7.576 & 1273.4 & 0.0217 & 16.229 & 1257.2 & 0.0217 & 25.123 & 1177.8 & 0.0217 \\
\hline 18 & 5.361 & 1088.9 & 0.0216 & 11.550 & 1095.2 & 0.0216 & 18.158 & 1074.0 & 0.0216 \\
\hline
\end{tabular}

\begin{tabular}{|c|c|c|c|c|c|c|c|c|c|}
\hline \multirow[b]{2}{*}{$\begin{array}{l}\text { Axial } \\
\text { Node }\end{array}$} & \multicolumn{3}{|c|}{ Statepoint 70 (27.0 Cy10) } & \multicolumn{3}{|c|}{ Statepoint 71 (143.0 Cy10) } & \multicolumn{3}{|c|}{ Statepoint 72 (209.0 Cy10) } \\
\hline & $\begin{array}{c}\text { Burnup } \\
\text { (GWd/MTU) }\end{array}$ & $\begin{array}{c}\text { T-Fuel } \\
{ }^{\circ} \mathrm{F}\end{array}$ & $\begin{array}{c}\text { Spec. Vol. } \\
\mathrm{ft}^{3} / \mathrm{lbm}\end{array}$ & \begin{tabular}{|c|} 
Burnup \\
(GWd/MTU)
\end{tabular} & $\begin{array}{l}\text { T-Fuel } \\
{ }^{\circ} \mathrm{F}\end{array}$ & $\begin{array}{c}\text { Spec. Vol. } \\
\mathrm{ft}^{3} / \mathrm{lbm}\end{array}$ & \begin{tabular}{|c|} 
Burnup \\
(GWd/MTU)
\end{tabular} & $\begin{array}{l}\text { T-Fuel } \\
{ }^{\circ} \mathrm{F}\end{array}$ & $\begin{array}{c}\text { Spec. Vol. } \\
\mathrm{ft}^{3} / \mathrm{lbm}\end{array}$ \\
\hline & 27.0 Cy 10 & $18.0 \mathrm{Cy} 10$ & $18.0 \mathrm{Cy} 10$ & 143.0 Cy 10 & 82.9 Cy 10 & 82.9 Cy 10 & 209.0 Cy 10 & 176.7 Cy 10 & 176.7 Cy 10 \\
\hline 1 & 16.473 & 690.5 & 0.0224 & 17.231 & 686.0 & 0.0223 & 17.701 & 690.1 & 0.0223 \\
\hline 2 & 23.399 & 744.7 & 0.0223 & 24.675 & 736.8 & 0.0223 & 25.442 & 736.6 & 0.0223 \\
\hline 3 & 27.148 & 771.3 & 0.0223 & 28.756 & 760.8 & 0.0223 & 29.687 & 754.7 & 0.0222 \\
\hline 4 & 28.514 & 786.1 & 0.0222 & 30.302 & 772.9 & 0.0222 & 31.304 & 763.8 & 0.0222 \\
\hline 5 & 28.900 & 795.6 & 0.0222 & 30.787 & 780.2 & 0.0222 & 31.818 & 767.4 & 0.0221 \\
\hline 6 & 28.996 & 801.5 & 0.0221 & 30.939 & 784.0 & 0.0221 & 31.981 & 768.0 & 0.0221 \\
\hline 7 & 29.025 & 804.7 & 0.0221 & 30.999 & 785.4 & 0.0221 & 32.044 & 767.1 & 0.0220 \\
\hline 8 & 29.040 & 805.9 & 0.0220 & 31.030 & 785.4 & 0.0220 & 32.075 & 765.8 & 0.0220 \\
\hline 9 & 29.058 & 805.5 & 0.0220 & 31.055 & 784.4 & 0.0220 & 32.098 & 764.2 & 0.0220 \\
\hline 10 & 29.086 & 803.8 & 0.0219 & 31.082 & 782.6 & 0.0219 & 32.124 & 762.7 & 0.0219 \\
\hline 11 & 29.141 & 800.5 & 0.0219 & 31.129 & 780.1 & 0.0219 & 32.169 & 761.1 & 0.0219 \\
\hline 12 & 29.261 & 795.3 & 0.0218 & 31.230 & 776.5 & 0.0218 & 32.267 & 759.3 & 0.0218 \\
\hline 13 & 29.440 & 787.7 & 0.0218 & 31.376 & 771.3 & 0.0218 & 32.408 & 756.9 & 0.0218 \\
\hline 14 & 29.499 & 777.7 & 0.0218 & 31.385 & 764.4 & 0.0218 & 32.404 & 753.6 & 0.0218 \\
\hline 15 & 29.306 & 764.6 & 0.0217 & 31.108 & 754.3 & 0.0217 & 32.102 & 748.0 & 0.0217 \\
\hline 16 & 28.391 & 747.1 & 0.0217 & 30.050 & 740.2 & 0.0217 & 30.990 & 737.9 & 0.0217 \\
\hline 17 & 25.445 & 722.1 & 0.0216 & 26.837 & 719.7 & 0.0216 & 27.649 & 721.4 & 0.0216 \\
\hline 18 & 18.352 & 670.4 & 0.0216 & 19.211 & 671.6 & 0.0216 & 19.729 & 677.9 & 0.0216 \\
\hline
\end{tabular}


Table 4-83. Burnup and TH Feedback Parameters by Axial Node for Assembly J17 (Cont.)

\begin{tabular}{|c|c|c|c|c|c|c|c|c|c|}
\hline \multirow[b]{2}{*}{$\begin{array}{l}\text { Axial } \\
\text { Node } \\
\end{array}$} & \multicolumn{3}{|c|}{ Datapoint (430.4 Cy10) } & \multicolumn{3}{|c|}{ Statepoint 73 (BOC Cy11) } & \multicolumn{3}{|c|}{ Datapoint (208.7 Cy11) } \\
\hline & $\begin{array}{c}\text { Burnup } \\
\text { (GWd/MTU) }\end{array}$ & $\begin{array}{l}\text { T-Fuel } \\
{ }^{\circ} \mathrm{F} \\
\end{array}$ & $\begin{array}{c}\text { Spec. Vol. } \\
\mathrm{ft}^{3} / \mathrm{lbm}\end{array}$ & $\begin{array}{c}\text { Burnup } \\
\text { (GWd/MTU) }\end{array}$ & $\begin{array}{l}\text { T-Fuel } \\
{ }^{\circ} \mathrm{F}\end{array}$ & $\begin{array}{c}\text { Spec. Vol. } \\
\mathrm{ft}^{3} / \mathrm{lbm}\end{array}$ & $\begin{array}{c}\text { Burnup } \\
\text { (GWd/MTU) }\end{array}$ & $\begin{array}{l}\text { T-Fuel } \\
{ }^{\circ} \mathrm{F}\end{array}$ & $\begin{array}{c}\text { Spec. Vol. } \\
\mathrm{ft}^{3} / \mathrm{lbm}\end{array}$ \\
\hline & 430.4 Cy 10 & 319.5 Cy 10 & 319.5 Cy 10 & BOC Cy 11 & 549.3 Cy 10 & 549.3 Cy 10 & 208.7 Cy 11 & 103.8 Cy 11 & $103.8 \mathrm{Cy} 11$ \\
\hline 1 & 19.538 & 706.5 & 0.0223 & 21.931 & 734.2 & 0.0224 & 23.181 & 660.0 & 0.0221 \\
\hline 2 & 28.292 & 748.4 & 0.0223 & 31.754 & 768.4 & 0.0223 & 33.615 & 683.5 & 0.0221 \\
\hline 3 & 32.991 & 761.7 & 0.0222 & 36.814 & 780.7 & 0.0223 & 38.955 & 695.8 & 0.0220 \\
\hline 4 & 34.737 & 765.1 & 0.0222 & 38.591 & 779.3 & 0.0222 & 40.865 & 701.6 & 0.0220 \\
\hline 5 & 35.266 & 763.8 & 0.0221 & 39.074 & 774.9 & 0.0222 & 41.426 & 704.9 & 0.0220 \\
\hline 6 & 35.410 & 761.2 & 0.0221 & 39.171 & 770.9 & 0.0221 & 41.566 & 706.6 & 0.0220 \\
\hline 7 & 35.449 & 758.5 & 0.0220 & 39.176 & 767.8 & 0.0221 & 41.593 & 707.0 & 0.0219 \\
\hline 8 & 35.460 & 756.1 & 0.0220 & 39.167 & 765.4 & 0.0220 & 41.592 & 706.5 & 0.0219 \\
\hline 9 & 35.470 & 754.2 & 0.0220 & 39.167 & 763.5 & 0.0220 & 41.589 & 705.5 & 0.0219 \\
\hline 10 & 35.491 & 752.7 & 0.0219 & 39.186 & 762.0 & 0.0220 & 41.597 & 703.9 & 0.0218 \\
\hline 11 & 35.537 & 751.7 & 0.0219 & 39.237 & 760.9 & 0.0219 & 41.629 & 701.9 & 0.0218 \\
\hline 12 & 35.642 & 750.9 & 0.0218 & 39.354 & 760.1 & 0.0219 & 41.716 & 699.2 & 0.0218 \\
\hline 13 & 35.792 & 750.3 & 0.0218 & 39.525 & 759.8 & 0.0218 & 41.843 & 695.6 & 0.0217 \\
\hline 14 & 35.797 & 749.7 & 0.0218 & 39.570 & 760.7 & 0.0218 & 41.830 & 691.2 & 0.0217 \\
\hline 15 & 35.483 & 748.3 & 0.0217 & 39.306 & 762.5 & 0.0217 & 41.481 & 685.5 & 0.0217 \\
\hline 16 & 34.282 & 743.4 & 0.0217 & 38.117 & 763.3 & 0.0217 & 40.160 & 677.6 & 0.0217 \\
\hline 17 & 30.608 & 729.2 & 0.0216 & 34.221 & 754.4 & 0.0217 & 36.011 & 664.3 & 0.0216 \\
\hline 18 & 21.708 & 691.2 & 0.0216 & 24.298 & 719.3 & 0.0216 & 25.499 & 638.9 & 0.0216 \\
\hline
\end{tabular}

\begin{tabular}{|c|c|c|c|c|c|c|}
\hline \multirow[b]{2}{*}{$\begin{array}{l}\text { Axial } \\
\text { Node }\end{array}$} & \multicolumn{3}{|c|}{ Datapoint (417.8 Cy11) } & \multicolumn{3}{|c|}{ Statepoint 74 (615.0 Cy11) } \\
\hline & $\begin{array}{c}\text { Burnup } \\
\text { (GWd/MTU) }\end{array}$ & $\begin{array}{l}\text { T-Fuel } \\
{ }^{\circ} \mathrm{F}\end{array}$ & $\begin{array}{c}\text { Spec. Vol. } \\
\mathrm{ft}^{3} / \mathrm{bm}\end{array}$ & $\begin{array}{c}\text { Burnup } \\
\text { (GWd/MTU) }\end{array}$ & $\begin{array}{l}\text { T-Fuel } \\
{ }^{\circ} \mathrm{F}\end{array}$ & $\begin{array}{c}\text { Spec. Vol. } \\
\mathrm{ft}^{3} / \mathrm{lbm}\end{array}$ \\
\hline & 417.8 Cy 11 & $312.8 \mathrm{Cy} 11$ & $312.8 \mathrm{Cy} 11$ & 615.0 Cy 11 & 515.7 Cy11 & 515.7 Cy 11 \\
\hline 1 & 24.774 & 681.6 & 0.0221 & 26.574 & 699.1 & 0.0222 \\
\hline 2 & 35.882 & 705.9 & 0.0221 & 38.315 & 722.5 & 0.0222 \\
\hline 3 & 41.457 & 714.5 & 0.0221 & 44.045 & 726.3 & 0.0221 \\
\hline 4 & 43.422 & 715.2 & 0.0221 & 45.999 & 723.1 & 0.0221 \\
\hline 5 & 43.989 & 714.2 & 0.0220 & 46.530 & 719.6 & 0.0220 \\
\hline 6 & 44.121 & 712.7 & 0.0220 & 46.630 & 716.7 & 0.0220 \\
\hline 7 & 44.136 & 711.1 & 0.0220 & 46.621 & 714.4 & 0.0220 \\
\hline 8 & 44.123 & 709.6 & 0.0219 & 46.593 & 712.6 & 0.0219 \\
\hline 9 & 44.112 & 708.2 & 0.0219 & 46.572 & 711.1 & 0.0219 \\
\hline 10 & 44.113 & 707.0 & 0.0219 & 46.568 & 709.9 & 0.0219 \\
\hline 11 & 44.142 & 705.9 & 0.0218 & 46.596 & 708.9 & 0.0218 \\
\hline 12 & 44.225 & 704.7 & 0.0218 & 46.681 & 708.0 & 0.0218 \\
\hline 13 & 44.348 & 703.5 & 0.0218 & 46.809 & 707.3 & 0.0218 \\
\hline 14 & 44.330 & 702.4 & 0.0217 & 46.808 & 707.4 & 0.0217 \\
\hline 15 & 43.966 & 701.0 & 0.0217 & 46.468 & 708.0 & 0.0217 \\
\hline 16 & 42.588 & 698.0 & 0.0217 & 45.093 & 708.4 & 0.0217 \\
\hline 17 & 38.229 & 688.2 & 0.0216 & 40.602 & 703.0 & 0.0216 \\
\hline 18 & 27.051 & 659.8 & 0.0216 & 28.797 & 674.6 & 0.0216 \\
\hline
\end{tabular}


Table 4-84. Burnup and TH Feedback Parameters by Axial Node for Assembly J17a

\begin{tabular}{|c|c|c|c|c|c|c|c|c|c|}
\hline \multirow{2}{*}{$\begin{array}{c}\text { Axial } \\
\text { Node }\end{array}$} & $\begin{array}{c}\text { Datapoint (214.0 Cy9) } \\
\text { Burnup } \\
(\text { GWd/MTU) }\end{array}$ & $\begin{array}{c}\text { T-Fuel } \\
{ }^{\circ} \mathbf{F}\end{array}$ & $\begin{array}{c}\text { Spec. Vol. } \\
\mathrm{ft}^{3} / \mathrm{lbm}\end{array}$ & $\begin{array}{c}\text { Burnup } \\
\text { (GWd/MTU) }\end{array}$ & $\begin{array}{c}\text { T-Fuel } \\
{ }^{\circ} \mathbf{F}\end{array}$ & $\begin{array}{c}\text { Spec. Vol. } \\
\mathrm{ft}^{3} / / \mathrm{bm}\end{array}$ & $\begin{array}{c}\text { Burnup } \\
\text { (GWd/MTU) }\end{array}$ & $\begin{array}{c}\text { T-Fuel } \\
{ }^{\circ} \mathrm{F}\end{array}$ & $\begin{array}{c}\text { Spec. Vol. } \\
\mathrm{ft}^{3} / \mathrm{lbm}\end{array}$ \\
\hline & 214.0 Cy9 & 109.3 Cy9 & 109.3 Cy9 & 425.0 Cy9 & 328.7 Cy9 & 328.7 Cy9 & BOC Cy10 & 549.2 Cy9 & 549.2 Cy9 \\
\hline 1 & 4.992 & 1115.7 & 0.0239 & 10.492 & 1098.1 & 0.0239 & 16.268 & 1083.8 & 0.0238 \\
\hline 2 & 7.252 & 1301.9 & 0.0238 & 15.161 & 1251.0 & 0.0238 & 23.050 & 1187.7 & 0.0237 \\
\hline 3 & 8.603 & 1403.2 & 0.0236 & 17.634 & 1311.5 & 0.0236 & 26.699 & 1209.4 & 0.0235 \\
\hline 4 & 9.329 & 1449.4 & 0.0235 & 18.737 & 1320.6 & 0.0235 & 28.006 & 1198.9 & 0.0234 \\
\hline 5 & 9.691 & 1464.0 & 0.0233 & 19.161 & 1314.6 & 0.0233 & 28.355 & 1183.9 & 0.0232 \\
\hline 6 & 9.888 & 1468.7 & 0.0232 & 19.335 & 1306.4 & 0.0232 & 28.429 & 1172.7 & 0.0231 \\
\hline 7 & 9.998 & 1469.0 & 0.0230 & 19.417 & 1299.5 & 0.0230 & 28.444 & 1165.8 & 0.0230 \\
\hline 8 & 10.052 & 1467.0 & 0.0229 & 19.459 & 1294.7 & 0.0229 & 28.450 & 1161.6 & 0.0228 \\
\hline 9 & 10.071 & 1463.8 & 0.0227 & 19.487 & 1291.9 & 0.0228 & 28.463 & 1159.2 & 0.0227 \\
\hline 10 & 10.065 & 1460.1 & 0.0226 & 19.517 & 1291.1 & 0.0226 & 28.490 & 1157.8 & 0.0226 \\
\hline 11 & 10.046 & 1456.6 & 0.0224 & 19.566 & 1292.5 & 0.0225 & 28.547 & 1157.3 & 0.0225 \\
\hline 12 & 10.028 & 1453.8 & 0.0223 & 19.662 & 1296.7 & 0.0224 & 28.675 & 1159.0 & 0.0223 \\
\hline 13 & 9.989 & 1450.6 & 0.0222 & 19.776 & 1303.9 & 0.0222 & 28.868 & 1164.1 & 0.0222 \\
\hline 14 & 9.831 & 1440.7 & 0.0221 & 19.735 & 1311.1 & 0.0221 & 28.948 & 1172.0 & 0.0221 \\
\hline 15 & 9.490 & 1419.9 & 0.0219 & 19.415 & 1315.7 & 0.0220 & 28.783 & 1183.8 & 0.0220 \\
\hline 16 & 8.831 & 1373.0 & 0.0218 & 18.495 & 1308.1 & 0.0219 & 27.912 & 1194.5 & 0.0219 \\
\hline 17 & 7.576 & 1273.4 & 0.0217 & 16.229 & 1257.2 & 0.0217 & 25.047 & 1177.8 & 0.0217 \\
\hline 18 & 5.361 & 1088.9 & 0.0216 & 11.550 & 1095.2 & 0.0216 & 18.107 & 1074.0 & 0.0216 \\
\hline
\end{tabular}

\begin{tabular}{|c|c|c|c|c|c|c|c|c|c|}
\hline \multirow[b]{2}{*}{$\begin{array}{l}\text { Axial } \\
\text { Node }\end{array}$} & \multicolumn{3}{|c|}{ Statepoint $70(27.0$ Cy10) } & \multicolumn{3}{|c|}{ Statepoint 71 (143.0 Cy10) } & \multicolumn{3}{|c|}{ Statepoint 72 (209.0 Cy10) } \\
\hline & $\begin{array}{c}\text { Burnup } \\
\text { (GWd/MTU) }\end{array}$ & $\begin{array}{c}\text { T-Fuel } \\
{ }^{\circ} \mathrm{F} \\
\end{array}$ & $\begin{array}{c}\text { Spec. Vol. } \\
\mathrm{ft}^{3} / \mathrm{lbm}\end{array}$ & $\begin{array}{c}\text { Burnup } \\
\text { (GWd/MTU) }\end{array}$ & $\begin{array}{l}\text { T-Fuel } \\
{ }^{\circ} \mathrm{F}\end{array}$ & $\begin{array}{c}\text { Spec. Vol. } \\
\mathrm{ft}^{3} / \mathrm{lbm}\end{array}$ & $\begin{array}{c}\text { Burnup } \\
\text { (GWd/MTU) }\end{array}$ & $\begin{array}{c}\text { T-Fuel } \\
{ }^{\circ} \mathrm{F}\end{array}$ & $\begin{array}{c}\text { Spec. Vol. } \\
\mathrm{ft}^{3} / / \mathrm{bm}\end{array}$ \\
\hline & 27.0 Cy 10 & $18.0 \mathrm{Cy} 10$ & 18.0 Cy 10 & 143.0 Cy 10 & 82.9 Cy 10 & 82.9 Cy 10 & 209.0 Cy 10 & 176.7 Cy10 & 176.7 Cy 10 \\
\hline 1 & 16.710 & 872.3 & 0.0232 & 18.665 & 869.6 & 0.0232 & 19.867 & 878.7 & 0.0232 \\
\hline 2 & 23.734 & 969.4 & 0.0232 & 26.726 & 960.6 & 0.0232 & 28.525 & 960.6 & 0.0232 \\
\hline 3 & 27.517 & 1012.2 & 0.0231 & 31.057 & 999.5 & 0.0231 & 33.143 & 999.4 & 0.0231 \\
\hline 4 & 28.897 & 1035.9 & 0.0230 & 32.727 & 1023.3 & 0.0230 & 34.938 & 1018.9 & 0.0230 \\
\hline 5 & 29.293 & 1051.6 & 0.0229 & 33.292 & 1038.0 & 0.0229 & 35.562 & 1027.0 & 0.0229 \\
\hline 6 & 29.396 & 1061.0 & 0.0227 & 33.496 & 1045.5 & 0.0227 & 35.793 & 1029.2 & 0.0227 \\
\hline 7 & 29.429 & 1065.6 & 0.0226 & 33.586 & 1048.3 & 0.0226 & 35.893 & 1028.5 & 0.0226 \\
\hline 8 & 29.445 & 1066.2 & 0.0225 & 33.629 & 1048.0 & 0.0225 & 35.939 & 1026.3 & 0.0225 \\
\hline 9 & 29.460 & 1063.7 & 0.0224 & 33.651 & 1045.3 & 0.0224 & 35.960 & 1023.3 & 0.0224 \\
\hline 10 & 29.482 & 1058.2 & 0.0223 & 33.662 & 1040.8 & 0.0223 & 35.967 & 1020.0 & 0.0223 \\
\hline 11 & 29.529 & 1049.7 & 0.0222 & 33.679 & 1034.2 & 0.0222 & 35.978 & 1016.2 & 0.0222 \\
\hline 12 & 29.638 & 1037.1 & 0.0221 & 33.735 & 1024.9 & 0.0221 & 36.023 & 1011.3 & 0.0221 \\
\hline 13 & 29.804 & 1020.2 & 0.0220 & 33.820 & 1012.4 & 0.0220 & 36.089 & 1004.6 & 0.0220 \\
\hline 14 & 29.849 & 1000.1 & 0.0219 & 33.753 & 996.7 & 0.0219 & 35.992 & 996.2 & 0.0219 \\
\hline 15 & 29.637 & 976.0 & 0.0218 & 33.384 & 976.3 & 0.0218 & 35.570 & 983.9 & 0.0219 \\
\hline 16 & 28.701 & 947.3 & 0.0218 & 32.209 & 947.7 & 0.0218 & 34.293 & 963.5 & 0.0218 \\
\hline 17 & 25.729 & 908.1 & 0.0217 & 28.798 & 910.8 & 0.0217 & 30.653 & 922.2 & 0.0217 \\
\hline 18 & 18.553 & 816.9 & 0.0216 & 20.593 & 823.6 & 0.0216 & 21.851 & 840.5 & 0.0216 \\
\hline
\end{tabular}


Table 4-85. Burnup and TH Feedback Parameters by Axial Node for Assembly J19

\begin{tabular}{|c|c|c|c|c|c|c|c|c|c|}
\hline \multirow[b]{2}{*}{$\begin{array}{l}\text { Axial } \\
\text { Node }\end{array}$} & \multicolumn{3}{|c|}{ Datapoint (214.0 Cy9) } & \multicolumn{3}{|c|}{ Datapoint (425.0 Cy9) } & \multicolumn{3}{|c|}{ Statepoint 69 (BOC Cy10) } \\
\hline & $\begin{array}{c}\text { Burnup } \\
\text { (GWd/MTU) } \\
\end{array}$ & $\begin{array}{c}\text { T-Fuel } \\
{ }^{\circ} \mathrm{F} \\
\end{array}$ & $\begin{array}{c}\text { Spec. Vol. } \\
\mathrm{ft}^{3} / \mathrm{bm}\end{array}$ & $\begin{array}{c}\text { Burnup } \\
\text { (GWd/MTU) }\end{array}$ & $\begin{array}{l}\text { T-Fuel } \\
{ }^{\circ} \mathrm{F}\end{array}$ & $\begin{array}{c}\text { Spec. Vol. } \\
\mathrm{ft}^{3} / \mathrm{lbm}\end{array}$ & $\begin{array}{c}\text { Burnup } \\
\text { (GWd/MTU) }\end{array}$ & $\begin{array}{c}\text { T-Fuel } \\
{ }^{\circ} \mathrm{F}\end{array}$ & $\begin{array}{c}\text { Spec. Vol. } \\
\mathrm{ft}^{3} / \mathrm{lbm}\end{array}$ \\
\hline & 214.0 Cy9 & 109.3 Сy9 & 109.3 Cy9 & 425.0 Cy9 & 328.7 Cy9 & 328.7 Cy9 & BOC Cy 10 & 549.2 Cy9 & 549.2 Cy9 \\
\hline 1 & 4.343 & 1055.4 & 0.0237 & 9.346 & 1066.3 & 0.0237 & 14.873 & 1073.1 & 0.0238 \\
\hline 2 & 6.463 & 1234.9 & 0.0236 & 13.735 & 1210.3 & 0.0236 & 21.370 & 1184.8 & 0.0236 \\
\hline 3 & 7.812 & 1337.8 & 0.0235 & 16.190 & 1273.2 & 0.0235 & 25.025 & 1204.9 & 0.0235 \\
\hline 4 & 8.563 & 1388.2 & 0.0233 & 17.332 & 1286.9 & 0.0234 & 26.419 & 1196.7 & 0.0234 \\
\hline 5 & 8.945 & 1408.8 & 0.0232 & 17.787 & 1282.5 & 0.0232 & 26.832 & 1183.0 & 0.0232 \\
\hline 6 & 9.154 & 1416.7 & 0.0230 & 17.987 & 1274.9 & 0.0231 & 26.951 & 1172.7 & 0.0231 \\
\hline 7 & 9.276 & 1418.9 & 0.0229 & 18.088 & 1268.4 & 0.0229 & 27.001 & 1166.4 & 0.0229 \\
\hline 8 & 9.342 & 1418.1 & 0.0228 & 18.149 & 1263.9 & 0.0228 & 27.037 & 1162.8 & 0.0228 \\
\hline 9 & 9.374 & 1416.0 & 0.0226 & 18.195 & 1261.6 & 0.0227 & 27.079 & 1160.8 & 0.0227 \\
\hline 10 & 9.384 & 1413.4 & 0.0225 & 18.244 & 1261.4 & 0.0226 & 27.135 & 1159.8 & 0.0226 \\
\hline 11 & 9.383 & 1411.0 & 0.0224 & 18.314 & 1263.5 & 0.0224 & 27.220 & 1159.7 & 0.0224 \\
\hline 12 & 9.384 & 1409.6 & 0.0223 & 18.430 & 1269.0 & 0.0223 & 27.376 & 1161.7 & 0.0223 \\
\hline 13 & 9.365 & 1407.8 & 0.0221 & 18.565 & 1277.1 & 0.0222 & 27.596 & 1167.1 & 0.0222 \\
\hline 14 & 9.220 & 1397.9 & 0.0220 & 18.535 & 1284.5 & 0.0221 & 27.690 & 1175.2 & 0.0221 \\
\hline 15 & 8.879 & 1373.1 & 0.0219 & 18.211 & 1288.7 & 0.0220 & 27.515 & 1186.9 & 0.0220 \\
\hline 16 & 8.202 & 1321.6 & 0.0218 & 17.265 & 1279.8 & 0.0218 & 26.598 & 1196.7 & 0.0219 \\
\hline 17 & 6.931 & 1220.3 & 0.0217 & 14.994 & 1222.0 & 0.0217 & 23.690 & 1178.4 & 0.0217 \\
\hline 18 & 4.807 & 1042.0 & 0.0216 & 10.532 & 1068.1 & 0.0216 & 16.950 & 1072.3 & 0.0216 \\
\hline
\end{tabular}

\begin{tabular}{|c|c|c|c|c|c|c|c|c|c|}
\hline \multirow[b]{2}{*}{$\begin{array}{l}\text { Axial } \\
\text { Node }\end{array}$} & \multicolumn{3}{|c|}{ Statepoint 70 (27.0 Cy10) } & \multicolumn{3}{|c|}{ Statepoint 71 (143.0 Cy10) } & \multicolumn{3}{|c|}{ Statepoint 72 (209.0 Cy10) } \\
\hline & $\begin{array}{c}\text { Burnup } \\
\text { (GWd/MTU) }\end{array}$ & $\begin{array}{c}\text { T-Fuel } \\
{ }^{\circ} \mathrm{F}\end{array}$ & $\begin{array}{c}\text { Spec. Vol. } \\
\mathrm{ft}^{3} / \mathrm{lbm}\end{array}$ & $\begin{array}{c}\text { Burnup } \\
\text { (GWd/MTU) }\end{array}$ & $\begin{array}{c}\text { T-Fuel } \\
{ }^{\circ} \mathrm{F}\end{array}$ & $\begin{array}{c}\text { Spec. Vol. } \\
\mathrm{ft}^{3} / \mathrm{lbm}\end{array}$ & $\begin{array}{c}\text { Burnup } \\
\text { (GWd/MTU) }\end{array}$ & $\begin{array}{c}\text { T-Fuel } \\
{ }^{\circ} \mathrm{F}\end{array}$ & $\begin{array}{c}\text { Spec. Vol. } \\
\mathrm{ft}^{3} / \mathrm{lbm}\end{array}$ \\
\hline & 27.0 Cy 10 & 18.0 Cy 10 & 18.0 Cy 10 & 143.0 Cy 10 & 82.9 Cy 10 & 82.9 Cy 10 & 209.0 Cy 10 & 176.7 Cy 10 & 176.7 Cy 10 \\
\hline 1 & 15.328 & 889.8 & 0.0233 & 17.342 & 886.6 & 0.0233 & 18.584 & 896.4 & 0.0233 \\
\hline 2 & 22.077 & 994.0 & 0.0232 & 25.169 & 985.3 & 0.0232 & 27.039 & 986.4 & 0.0232 \\
\hline 3 & 25.871 & 1039.7 & 0.0231 & 29.550 & 1028.5 & 0.0231 & 31.727 & 1022.9 & 0.0231 \\
\hline 4 & 27.341 & 1063.6 & 0.0230 & 31.325 & 1050.1 & 0.0230 & 33.634 & 1043.3 & 0.0230 \\
\hline 5 & 27.800 & 1078.6 & 0.0229 & 31.956 & 1062.6 & 0.0229 & 34.322 & 1050.8 & 0.0229 \\
\hline 6 & 27.950 & 1087.8 & 0.0228 & 32.208 & 1069.5 & 0.0228 & 34.599 & 1052.6 & 0.0228 \\
\hline 7 & 28.019 & 1092.6 & 0.0227 & 32.338 & 1072.5 & 0.0227 & 34.739 & 1051.6 & 0.0227 \\
\hline 8 & 28.066 & 1093.6 & 0.0226 & 32.418 & 1072.4 & 0.0226 & 34.822 & 1049.2 & 0.0226 \\
\hline 9 & 28.111 & 1091.3 & 0.0225 & 32.474 & 1070.0 & 0.0225 & 34.878 & 1046.3 & 0.0225 \\
\hline 10 & 28.164 & 1086.2 & 0.0223 & 32.520 & 1065.7 & 0.0224 & 34.922 & 1043.1 & 0.0224 \\
\hline 11 & 28.241 & 1078.4 & 0.0222 & 32.575 & 1059.7 & 0.0222 & 34.975 & 1039.8 & 0.0223 \\
\hline 12 & 28.381 & 1067.3 & 0.0221 & 32.676 & 1051.7 & 0.0221 & 35.072 & 1036.1 & 0.0222 \\
\hline 13 & 28.578 & 1052.0 & 0.0220 & 32.808 & 1040.9 & 0.0220 & 35.194 & 1031.2 & 0.0221 \\
\hline 14 & 28.638 & 1032.5 & 0.0219 & 32.765 & 1025.9 & 0.0219 & 35.126 & 1023.7 & 0.0220 \\
\hline 15 & 28.415 & 1007.7 & 0.0218 & 32.381 & 1004.9 & 0.0219 & 34.689 & 1011.5 & 0.0219 \\
\hline 16 & 27.431 & 977.6 & 0.0218 & 31.143 & 977.9 & 0.0218 & 33.346 & 990.4 & 0.0218 \\
\hline 17 & 24.412 & 936.2 & 0.0217 & 27.662 & 940.1 & 0.0217 & 29.625 & 951.3 & 0.0217 \\
\hline 18 & 17.425 & 838.4 & 0.0216 & 19.591 & 845.5 & 0.0216 & 20.922 & 861.9 & 0.0216 \\
\hline
\end{tabular}


Table 4-86. Burnup and TH Feedback Parameters by Axial Node for Assembly J20

\begin{tabular}{|c|c|c|c|c|c|c|c|c|c|}
\hline \multirow{2}{*}{$\begin{array}{c}\text { Axial } \\
\text { Node }\end{array}$} & \multicolumn{3}{|c|}{ Datapoint (214.0 Cy9) } & \multicolumn{2}{c|}{ Datapoint (425.0 Cy9) } & \multicolumn{3}{c|}{ Statepoint 69 (BOC Cy10) } \\
\cline { 2 - 10 } & $\begin{array}{c}\text { Burnup } \\
\text { (GWd/MTU) }\end{array}$ & $\begin{array}{c}\text { T-Fuel } \\
{ }^{\circ} \mathrm{F}\end{array}$ & $\begin{array}{c}\text { Spec. Vol. } \\
\mathrm{ft}^{3} / \mathrm{lbm}\end{array}$ & $\begin{array}{c}\text { Burnup } \\
\text { (GWd/MTU) }\end{array}$ & $\begin{array}{c}\text { T-Fuel } \\
{ }^{\circ} \mathrm{F}\end{array}$ & $\begin{array}{c}\text { Spec. Vol. } \\
\mathrm{ft}^{3} / \mathrm{lbm}\end{array}$ & $\begin{array}{c}\text { Burnup } \\
\text { (GWd/MTU) }\end{array}$ & $\begin{array}{c}\text { T-Fuel } \\
{ }^{\circ} \mathrm{F}\end{array}$ & $\begin{array}{c}\text { Spec. Vol. } \\
\mathrm{ft}^{3} / \mathrm{lbm}\end{array}$ \\
\hline 1 & 214.0 Cy9 & 109.3 Cy9 & 109.3 Cy9 & 425.0 Cy9 & 328.7 Cy9 & 328.7 Cy9 & BOC Cy10 & 549.2 Cy9 & 549.2 Cy9 \\
\hline 2 & 3.303 & 944.8 & 0.0233 & 7.247 & 980.0 & 0.0233 & 11.706 & 1006.4 & 0.0234 \\
\hline 3 & 6.503 & 1145.6 & 0.0232 & 11.586 & 1122.2 & 0.0232 & 17.948 & 1112.9 & 0.0233 \\
\hline 4 & 7.421 & 1286.9 & 0.0230 & 14.714 & 1180.7 & 0.0230 & 22.308 & 1122.7 & 0.0230 \\
\hline 5 & 7.784 & 1307.1 & 0.0229 & 15.143 & 1177.1 & 0.0229 & 22.708 & 1111.8 & 0.0229 \\
\hline 6 & 7.981 & 1314.7 & 0.0228 & 15.328 & 1170.6 & 0.0228 & 22.831 & 1103.2 & 0.0228 \\
\hline 7 & 8.089 & 1316.6 & 0.0227 & 15.415 & 1164.8 & 0.0227 & 22.872 & 1097.8 & 0.0227 \\
\hline 8 & 8.148 & 1315.9 & 0.0226 & 15.463 & 1160.8 & 0.0226 & 22.899 & 1094.6 & 0.0226 \\
\hline 9 & 8.177 & 1314.1 & 0.0225 & 15.500 & 1158.5 & 0.0225 & 22.929 & 1092.8 & 0.0225 \\
\hline 10 & 8.186 & 1311.9 & 0.0224 & 15.538 & 1158.1 & 0.0224 & 22.971 & 1091.9 & 0.0224 \\
\hline 11 & 8.182 & 1309.6 & 0.0223 & 15.584 & 1159.5 & 0.0223 & 23.029 & 1091.7 & 0.0223 \\
\hline 12 & 8.158 & 1307.0 & 0.0222 & 15.634 & 1162.9 & 0.0222 & 23.106 & 1092.8 & 0.0222 \\
\hline 13 & 8.096 & 1302.2 & 0.0220 & 15.661 & 1168.2 & 0.0221 & 23.189 & 1096.0 & 0.0221 \\
\hline 14 & 7.950 & 1292.0 & 0.0219 & 15.598 & 1174.4 & 0.0220 & 23.222 & 1102.4 & 0.0220 \\
\hline 15 & 7.644 & 1269.9 & 0.0219 & 15.310 & 1178.4 & 0.0219 & 23.054 & 1112.2 & 0.0219 \\
\hline 16 & 7.035 & 1223.6 & 0.0218 & 14.492 & 1170.3 & 0.0218 & 22.249 & 1120.5 & 0.0218 \\
\hline 17 & 5.826 & 1126.8 & 0.0217 & 12.438 & 1122.3 & 0.0217 & 19.630 & 1105.7 & 0.0217 \\
\hline 18 & 3.479 & 914.6 & 0.0216 & 7.762 & 966.2 & 0.0216 & 12.781 & 991.7 & 0.0216 \\
\hline
\end{tabular}

\begin{tabular}{|c|c|c|c|c|c|c|c|c|c|}
\hline \multirow[b]{2}{*}{$\begin{array}{l}\text { Axial } \\
\text { Node }\end{array}$} & \multicolumn{3}{|c|}{ Statepoint 70 (27.0 Cy10) } & \multicolumn{3}{|c|}{ Statepoint 71 (143.0 Cy10) } & \multicolumn{3}{|c|}{ Statepoint 72 (209.0 Cy10) } \\
\hline & $\begin{array}{c}\text { Burnup } \\
\text { (GWd/MTU) }\end{array}$ & $\begin{array}{c}\text { T-Fuel } \\
{ }^{\circ} \mathrm{F} \\
\end{array}$ & $\begin{array}{c}\text { Spec. Vol. } \\
\mathrm{ft}^{3} / \mathrm{lbm}\end{array}$ & $\begin{array}{c}\text { Burnup } \\
\text { (GWd/MTU) }\end{array}$ & $\begin{array}{c}\text { T-Fuel } \\
{ }^{\circ} \mathrm{F} \\
\end{array}$ & $\begin{array}{c}\text { Spec. Vol. } \\
\mathrm{ft}^{3} / \mathrm{lbm}\end{array}$ & $\begin{array}{c}\text { Burnup } \\
\text { (GWd/MTU) }\end{array}$ & $\begin{array}{c}\text { T-Fuel } \\
{ }^{\circ} \mathrm{F} \\
\end{array}$ & $\begin{array}{c}\text { Spec. Vol. } \\
\mathrm{ft}^{3} / \mathrm{lbm}\end{array}$ \\
\hline & 27.0 Cy 10 & $18.0 \mathrm{Cy} 10$ & 18.0 Cy 10 & $143.0 \mathrm{Cy} 10$ & 82.9 Cy 10 & 82.9 Cy 10 & 209.0 Cy10 & 176.7 Cy 10 & 176.7 Cy 10 \\
\hline 1 & 12.156 & 899.1 & 0.0233 & 14.147 & 897.7 & 0.0233 & 15.376 & 909.0 & 0.0233 \\
\hline 2 & 18.638 & 1005.1 & 0.0232 & 21.670 & 998.4 & 0.0232 & 23.509 & 1000.4 & 0.0232 \\
\hline 3 & 21.961 & 1053.7 & 0.0231 & 25.582 & 1045.6 & 0.0231 & 27.732 & 1039.4 & 0.0231 \\
\hline 4 & 23.207 & 1075.9 & 0.0230 & 27.115 & 1067.7 & 0.0230 & 29.380 & 1052.4 & 0.0230 \\
\hline 5 & 23.621 & 1074.8 & 0.0229 & 27.560 & 1064.2 & 0.0229 & 29.811 & 1044.8 & 0.0229 \\
\hline 6 & 23.770 & 1083.0 & 0.0228 & 27.798 & 1069.7 & 0.0228 & 30.067 & 1044.8 & 0.0228 \\
\hline 7 & 23.832 & 1088.8 & 0.0227 & 27.925 & 1073.3 & 0.0227 & 30.206 & 1044.0 & 0.0227 \\
\hline 8 & 23.871 & 1091.1 & 0.0226 & 28.003 & 1074.0 & 0.0226 & 30.289 & 1042.1 & 0.0226 \\
\hline 9 & 23.908 & 1090.3 & 0.0225 & 28.059 & 1072.7 & 0.0225 & 30.347 & 1039.7 & 0.0225 \\
\hline 10 & 23.950 & 1087.0 & 0.0224 & 28.104 & 1069.6 & 0.0224 & 30.395 & 1037.4 & 0.0224 \\
\hline 11 & 24.004 & 1081.9 & 0.0223 & 28.152 & 1065.5 & 0.0223 & 30.449 & 1035.7 & 0.0223 \\
\hline 12 & 24.084 & 1080.8 & 0.0222 & 28.252 & 1063.9 & 0.0222 & 30.584 & 1039.2 & 0.0222 \\
\hline 13 & 24.211 & 1100.2 & 0.0221 & 28.592 & 1085.8 & 0.0221 & 31.052 & 1062.1 & 0.0221 \\
\hline 14 & 24.219 & 1085.2 & 0.0220 & 28.541 & 1075.5 & 0.0220 & 30.998 & 1059.3 & 0.0220 \\
\hline 15 & 24.000 & 1058.3 & 0.0219 & 28.152 & 1054.1 & 0.0219 & 30.556 & 1047.7 & 0.0219 \\
\hline 16 & 23.119 & 1022.6 & 0.0218 & 26.984 & 1023.3 & 0.0218 & 29.269 & 1025.7 & 0.0218 \\
\hline 17 & 20.379 & 975.7 & 0.0217 & 23.744 & 978.5 & 0.0217 & 25.769 & 988.4 & 0.0217 \\
\hline 18 & 13.285 & 875.5 & 0.0216 & 15.566 & 882.4 & 0.0216 & 16.959 & 8987 & 0.0216 \\
\hline
\end{tabular}


Table 4-87. Burnup and TH Feedback Parameters by Axial Node for Assembly J23

\begin{tabular}{|c|c|c|c|c|c|c|c|c|c|}
\hline \multirow[b]{2}{*}{$\begin{array}{l}\text { Axial } \\
\text { Node }\end{array}$} & \multicolumn{3}{|c|}{ Datapoint (214.0 Cy9) } & \multicolumn{3}{|c|}{ Datapoint (425.0 Cy9) } & \multicolumn{3}{|c|}{ Statepoint 69 (BOC Cy10) } \\
\hline & $\begin{array}{c}\text { Burnup } \\
\text { (GWd/MTU) }\end{array}$ & $\begin{array}{c}\text { T-Fuel } \\
{ }^{\circ} \mathbf{F}\end{array}$ & $\begin{array}{c}\text { Spec. Vol. } \\
\mathrm{ft}^{3} / \mathrm{lbm}\end{array}$ & $\begin{array}{c}\text { Burnup } \\
\text { (GWd/MTU) }\end{array}$ & $\begin{array}{l}\text { T-Fuel } \\
{ }^{\circ} \mathrm{F}\end{array}$ & $\begin{array}{c}\text { Spec. Vol. } \\
\mathrm{ft}^{3} / \mathrm{lbm}\end{array}$ & $\begin{array}{c}\text { Burnup } \\
\text { (GWd/MTU) }\end{array}$ & $\begin{array}{c}\text { T-Fuel } \\
{ }^{\circ} \mathrm{F}\end{array}$ & $\begin{array}{c}\text { Spec. Vol. } \\
\mathrm{ft}^{3} / \mathrm{lbm}\end{array}$ \\
\hline & 214.0 Cy 9 & 109.3 Cy9 & 109.3 Cy9 & 425.0 Cy9 & 328.7 Cy9 & 328.7 Cy9 & BOC Cy 10 & 549.2 Cy9 & 549.2 Cy9 \\
\hline 1 & 4.294 & 1053.3 & 0.0237 & 9.215 & 1061.3 & 0.0238 & 14.627 & 1064.2 & 0.0238 \\
\hline 2 & 6.446 & 1237.8 & 0.0236 & 13.695 & 1210.1 & 0.0237 & 21.260 & 1180.8 & 0.0237 \\
\hline 3 & 7.919 & 1348.4 & 0.0235 & 16.404 & 1280.9 & 0.0235 & 25.297 & 1207.3 & 0.0235 \\
\hline 4 & 8.731 & 1401.2 & 0.0234 & 17.660 & 1297.5 & 0.0234 & 26.866 & 1200.7 & 0.0234 \\
\hline 5 & 9.119 & 1421.4 & 0.0232 & 18.130 & 1293.3 & 0.0233 & 27.301 & 1186.9 & 0.0232 \\
\hline 6 & 9.325 & 1428.1 & 0.0231 & 18.324 & 1285.5 & 0.0231 & 27.412 & 1176.3 & 0.0231 \\
\hline 7 & 9.443 & 1429.3 & 0.0229 & 18.420 & 1278.8 & 0.0230 & 27.452 & 1169.7 & 0.0230 \\
\hline 8 & 9.507 & 1428.0 & 0.0228 & 18.475 & 1274.2 & 0.0228 & 27.480 & 1165.8 & 0.0228 \\
\hline 9 & 9.537 & 1425.5 & 0.0227 & 18.517 & 1271.7 & 0.0227 & 27.514 & 1163.5 & 0.0227 \\
\hline 10 & 9.546 & 1422.7 & 0.0225 & 18.564 & 1271.3 & 0.0226 & 27.563 & 1162.3 & 0.0226 \\
\hline 11 & 9.548 & 1420.3 & 0.0224 & 18.637 & 1273.3 & 0.0225 & 27.647 & 1162.0 & 0.0225 \\
\hline 12 & 9.557 & 1419.2 & 0.0223 & 18.765 & 1278.3 & 0.0223 & 27.809 & 1163.8 & 0.0223 \\
\hline 13 & 9.548 & 1417.9 & 0.0222 & 18.916 & 1285.9 & 0.0222 & 28.040 & 1169.0 & 0.0222 \\
\hline 14 & 9.411 & 1409.2 & 0.0220 & 18.898 & 1293.0 & 0.0221 & 28.143 & 1177.0 & 0.0221 \\
\hline 15 & 9.078 & 1386.4 & 0.0219 & 18.583 & 1297.1 & 0.0220 & 27.978 & 1188.7 & 0.0220 \\
\hline 16 & 8.418 & 1336.6 & 0.0218 & 17.660 & 1288.8 & 0.0218 & 27.090 & 1198.8 & 0.0219 \\
\hline 17 & 7.172 & 1238.2 & 0.0217 & 15.429 & 1233.7 & 0.0217 & 24.238 & 1180.9 & 0.0217 \\
\hline 18 & 5.037 & 1060.3 & 0.0216 & 10.938 & 1078.5 & 0.0216 & 17.470 & 1076.0 & 0.0216 \\
\hline
\end{tabular}

\begin{tabular}{|c|c|c|c|c|c|c|c|c|c|}
\hline \multirow[b]{2}{*}{$\begin{array}{l}\text { Axial } \\
\text { Node }\end{array}$} & \multicolumn{3}{|c|}{ Statepoint 70 (27.0 Cy10) } & \multicolumn{3}{|c|}{ Statepoint 71 (143.0 Cy10) } & \multicolumn{3}{|c|}{ Statepoint 72 (209.0 Cy10) } \\
\hline & \begin{tabular}{|c|} 
Burnup \\
(GWd/MTU)
\end{tabular} & $\begin{array}{l}\text { T-Fuel } \\
{ }^{\circ} \mathrm{F}\end{array}$ & $\begin{array}{c}\text { Spec. Vol. } \\
\mathrm{ft}^{3} / \mathrm{lbm}\end{array}$ & $\begin{array}{c}\text { Burnup } \\
\text { (GWd/MTU) }\end{array}$ & $\begin{array}{c}\text { T-Fuel } \\
{ }^{\circ} \mathrm{F}\end{array}$ & $\begin{array}{c}\text { Spec. Vol. } \\
\text { ft } \mathrm{t}^{3} / \mathrm{lbm}\end{array}$ & $\begin{array}{c}\text { Burnup } \\
\text { (GWd/MTU) }\end{array}$ & $\begin{array}{c}\text { T-Fuel } \\
{ }^{\circ} \mathrm{F} \\
\end{array}$ & \begin{tabular}{|c|} 
Spec. Vol. \\
$\mathrm{ft}^{3} / \mathrm{lbm}$
\end{tabular} \\
\hline & 27.0 Cy 10 & $18.0 \mathrm{Cy} 10$ & $18.0 \mathrm{Cy} 10$ & 143.0 Cy 10 & 82.9 Cy 10 & 82.9 Cy 10 & $209.0 \mathrm{Cy} 10$ & 176.7 Cy 10 & 176.7 Cy 10 \\
\hline 1 & 15.028 & 855.7 & 0.0233 & 16.781 & 850.2 & 0.0232 & 17.860 & 857.5 & 0.0232 \\
\hline 2 & 21.908 & 961.2 & 0.0232 & 24.700 & 948.5 & 0.0232 & 26.374 & 944.9 & 0.0231 \\
\hline 3 & 26.096 & 1011.2 & 0.0231 & 29.500 & 993.2 & 0.0231 & 31.480 & 979.3 & 0.0230 \\
\hline 4 & 27.755 & 1040.3 & 0.0230 & 31.494 & 1017.3 & 0.0230 & 33.609 & 1000.2 & 0.0229 \\
\hline 5 & 28.246 & 1060.0 & 0.0229 & 32.179 & 1033.1 & 0.0229 & 34.353 & 1008.1 & 0.0228 \\
\hline 6 & 28.394 & 1072.8 & 0.0228 & 32.444 & 1041.9 & 0.0228 & 34.644 & 1010.1 & 0.0227 \\
\hline 7 & 28.460 & 1080.4 & 0.0227 & 32.583 & 1046.3 & 0.0226 & 34.793 & 1009.4 & 0.0226 \\
\hline 8 & 28.504 & 1084.0 & 0.0226 & 32.671 & 1047.7 & 0.0225 & 34.886 & 1007.5 & 0.0225 \\
\hline 9 & 28.546 & 1084.2 & 0.0225 & 32.737 & 1046.9 & 0.0224 & 34.955 & 1005.3 & 0.0224 \\
\hline 10 & 28.598 & 1081.6 & 0.0223 & 32.796 & 1044.4 & 0.0223 & 35.016 & 1003.1 & 0.0223 \\
\hline 11 & 28.677 & 1076.1 & 0.0222 & 32.870 & 1040.5 & 0.0222 & 35.093 & 1001.0 & 0.0222 \\
\hline 12 & 28.829 & 1067.1 & 0.0221 & 32.999 & 1034.6 & 0.0221 & 35.225 & 998.9 & 0.0221 \\
\hline 13 & 29.040 & 1053.4 & 0.0220 & 33.162 & 1025.8 & 0.0220 & 35.387 & 995.7 & 0.0220 \\
\hline 14 & 29.109 & 1033.9 & 0.0219 & 33.137 & 1012.2 & 0.0219 & 35.344 & 990.0 & 0.0219 \\
\hline 15 & 28.890 & 1006.5 & 0.0218 & 32.750 & 990.6 & 0.0218 & 34.909 & 978.9 & 0.0218 \\
\hline 16 & 27.920 & 969.6 & 0.0218 & 31.493 & 958.2 & 0.0218 & 33.541 & 956.8 & 0.0218 \\
\hline 17 & 24.934 & 916.9 & 0.0217 & 27.980 & 912.8 & 0.0217 & 29.772 & 913.4 & 0.0217 \\
\hline 18 & 17.908 & 812.8 & 0.0216 & 19.862 & 815.5 & 0.0216 & 21.040 & 826.2 & 0.0216 \\
\hline
\end{tabular}


Table 4-88. Burnup and TH Feedback Parameters by Axial Node for Assembly J25

\begin{tabular}{|c|c|c|c|c|c|c|c|c|c|}
\hline \multirow[b]{2}{*}{$\begin{array}{l}\text { Axial } \\
\text { Node }\end{array}$} & \multicolumn{3}{|c|}{ Datapoint (214.0 Cy9) } & \multicolumn{3}{|c|}{ Datapoint (425.0 Cy9) } & \multicolumn{3}{|c|}{ Statepoint 69 (BOC Cy10) } \\
\hline & $\begin{array}{c}\text { Burnup } \\
\text { (GWd/MTU) }\end{array}$ & $\begin{array}{l}\text { T-Fuel } \\
{ }^{\circ} \mathrm{F}\end{array}$ & $\begin{array}{c}\text { Spec. Vol. } \\
\mathrm{ft}^{3} / \mathrm{lbm}\end{array}$ & $\begin{array}{c}\text { Burnup } \\
\text { (GWd/MTU) }\end{array}$ & $\begin{array}{l}\text { T-Fuel } \\
{ }^{\circ} \mathrm{F}\end{array}$ & $\begin{array}{c}\text { Spec. Vol. } \\
\mathrm{ft}^{3} / \mathrm{lbm}\end{array}$ & $\begin{array}{c}\text { Burnup } \\
\text { (GWd/MTU) }\end{array}$ & $\begin{array}{l}\text { T-Fuel } \\
{ }^{\circ} \mathrm{F} \\
\end{array}$ & $\begin{array}{c}\text { Spec. Vol. } \\
\mathrm{ft}^{3} / \mathrm{lbm}\end{array}$ \\
\hline & 214.0 Cy9 & 109.3 Cy9 & 109.3 Cy 9 & 425.0 Cy9 & 328.7 Cy 9 & 328.7 Cy9 & BOC Cy 10 & 549.2 Cy 9 & 549.2 Cy 9 \\
\hline 1 & 2.676 & 874.3 & 0.0230 & 5.904 & 912.2 & 0.0230 & 9.644 & 951.8 & 0.0231 \\
\hline 2 & 4.549 & 1051.9 & 0.0229 & 9.611 & 1047.3 & 0.0229 & 15.039 & 1054.2 & 0.0230 \\
\hline 3 & 5.632 & 1140.2 & 0.0229 & 11.487 & 1092.3 & 0.0228 & 17.846 & 1074.9 & 0.0229 \\
\hline 4 & 6.220 & 1181.6 & 0.0228 & 12.344 & 1102.0 & 0.0228 & 18.892 & 1069.5 & 0.0228 \\
\hline 5 & 6.532 & 1199.4 & 0.0227 & 12.713 & 1099.2 & 0.0227 & 19.243 & 1060.3 & 0.0227 \\
\hline 6 & 6.698 & 1205.7 & 0.0226 & 12.867 & 1093.4 & 0.0226 & 19.342 & 1052.8 & 0.0226 \\
\hline 7 & 6.786 & 1207.0 & 0.0225 & 12.933 & 1088.2 & 0.0225 & 19.367 & 1047.8 & 0.0225 \\
\hline 8 & 6.832 & 1206.0 & 0.0224 & 12.967 & 1084.4 & 0.0224 & 19.378 & 1044.9 & 0.0225 \\
\hline 9 & 6.854 & 1204.2 & 0.0223 & 12.991 & 1082.2 & 0.0223 & 19.393 & 1043.2 & 0.0224 \\
\hline 10 & 6.859 & 1202.1 & 0.0222 & 13.016 & 1081.6 & 0.0222 & 19.418 & 1042.3 & 0.0223 \\
\hline 11 & 6.853 & 1200.0 & 0.0221 & 13.047 & 1082.5 & 0.0222 & 19.456 & 1042.1 & 0.0222 \\
\hline 12 & 6.832 & 1197.5 & 0.0221 & 13.082 & 1085.1 & 0.0221 & 19.510 & 1042.9 & 0.0221 \\
\hline 13 & 6.777 & 1193.3 & 0.0220 & 13.096 & 1089.3 & 0.0220 & 19.568 & 1045.7 & 0.0220 \\
\hline 14 & 6.651 & 1184.3 & 0.0219 & 13.035 & 1094.3 & 0.0219 & 19.583 & 1051.3 & 0.0219 \\
\hline 15 & 6.392 & 1165.1 & 0.0218 & 12.783 & 1097.3 & 0.0218 & 19.425 & 1059.5 & 0.0219 \\
\hline 16 & 5.873 & 1124.2 & 0.0217 & 12.081 & 1089.2 & 0.0218 & 18.716 & 1065.3 & 0.0218 \\
\hline 17 & 4.830 & 1036.6 & 0.0217 & 10.306 & 1045.0 & 0.0217 & 16.421 & 1048.2 & 0.0217 \\
\hline 18 & 2.834 & 849.3 & 0.0216 & 6.329 & 900.7 & 0.0216 & 10.528 & 938.2 & 0.0216 \\
\hline
\end{tabular}

\begin{tabular}{|c|c|c|c|c|c|c|c|c|c|}
\hline \multirow[b]{2}{*}{$\begin{array}{l}\text { Axlal } \\
\text { Node }\end{array}$} & \multicolumn{3}{|c|}{ Statepoint $70(27.0$ Cy10) } & \multicolumn{3}{|c|}{ Statepoint 71 (143.0 Cy10) } & \multicolumn{3}{|c|}{ Statepoint 72 (209.0 Cy10) } \\
\hline & $\begin{array}{c}\text { Burnup } \\
\text { (GWd/MTU) }\end{array}$ & $\begin{array}{c}\text { T-Fuel } \\
{ }^{\circ} \mathbf{F} \\
\end{array}$ & $\begin{array}{c}\text { Spec. Vol. } \\
\mathrm{ft}^{3} / / \mathrm{bm}\end{array}$ & \begin{tabular}{|c|} 
Burnup \\
(GWd/MTU)
\end{tabular} & $\begin{array}{c}\text { T-Fuel } \\
{ }^{\circ} \mathrm{F} \\
\end{array}$ & $\begin{array}{c}\text { Spec. Vol. } \\
\mathrm{ft}^{3} / \mathrm{lbm}\end{array}$ & $\begin{array}{c}\text { Burnup } \\
\text { (GWd/MTU) }\end{array}$ & $\begin{array}{c}\text { T-Fuel } \\
{ }^{\circ} \mathbf{F} \\
\end{array}$ & \begin{tabular}{|c} 
Spec. Vol. \\
$\mathrm{ft}^{3} / \mathrm{lbm}$
\end{tabular} \\
\hline & 27.0 Cy 10 & 18.0 Cy 10 & 18.0 Cy 10 & 143.0 Cy 10 & 82.9 Cy 10 & 82.9 Cy 10 & 209.0 Cy 10 & 176.7 Cy 10 & 176.7 Cy 10 \\
\hline 1 & 9.999 & 839.3 & 0.0230 & 11.606 & 844.4 & 0.0230 & 12.620 & 861.0 & 0.0231 \\
\hline 2 & 15.598 & 938.7 & 0.0229 & 18.107 & 942.4 & 0.0230 & 19.659 & 954.6 & 0.0230 \\
\hline 3 & 18.525 & 986.8 & 0.0229 & 21.553 & 988.9 & 0.0229 & 23.384 & 992.3 & 0.0229 \\
\hline 4 & 19.643 & 1014.9 & 0.0228 & 22.965 & 1014.8 & 0.0228 & 24.925 & 1008.5 & 0.0228 \\
\hline 5 & 20.041 & 1033.3 & 0.0227 & 23.542 & 1030.4 & 0.0227 & 25.564 & 1015.3 & 0.0227 \\
\hline 6 & 20.172 & 1045.3 & 0.0226 & 23.785 & 1039.7 & 0.0226 & 25.837 & 1017.5 & 0.0226 \\
\hline 7 & 20.218 & 1052.5 & 0.0225 & 23.903 & 1044.7 & 0.0225 & 25.969 & 1017.3 & 0.0225 \\
\hline 8 & 20.242 & 1056.0 & 0.0224 & 23.971 & 1046.7 & 0.0224 & 26.045 & 1016.0 & 0.0224 \\
\hline 9 & 20.264 & 1056.3 & 0.0223 & 24.018 & 1046.4 & 0.0223 & 26.096 & 1014.2 & 0.0224 \\
\hline 10 & 20.290 & 1053.9 & 0.0222 & 24.052 & 1044.3 & 0.0222 & 26.134 & 1012.4 & 0.0223 \\
\hline 11 & 20.324 & 1048.7 & 0.0221 & 24.078 & 1040.5 & 0.0222 & 26.164 & 1010.6 & 0.0222 \\
\hline 12 & 20.366 & 1040.3 & 0.0220 & 24.095 & 1034.4 & 0.0221 & 26.181 & 1008.5 & 0.0221 \\
\hline 13 & 20.405 & 1027.8 & 0.0220 & 24.081 & 1025.3 & 0.0220 & 26.162 & 1005.3 & 0.0220 \\
\hline 14 & 20.391 & 1010.1 & 0.0219 & 23.974 & 1011.7 & 0.0219 & 26.037 & 1000.0 & 0.0219 \\
\hline 15 & 20.189 & 986.0 & 0.0218 & 23.618 & 992.1 & 0.0218 & 25.634 & 990.1 & 0.0218 \\
\hline 16 & 19.414 & 953.6 & 0.0217 & 22.588 & 963.6 & 0.0217 & 24.497 & 972.3 & 0.0218 \\
\hline 17 & 17.011 & 906.6 & 0.0217 & 19.728 & 918.9 & 0.0217 & 21.397 & 936.6 & 0.0217 \\
\hline 18 & 10.912 & 810.2 & 0.0216 & 12.692 & 822.3 & 0.0216 & 13.805 & 843.2 & 0.0216 \\
\hline
\end{tabular}


Table 4-89. Burnup and TH Feedback Parameters by Axial Node for Assembly J25a

\begin{tabular}{|c|c|c|c|c|c|c|c|c|c|}
\hline \multirow[b]{2}{*}{$\begin{array}{l}\text { Axial } \\
\text { Node }\end{array}$} & \multicolumn{3}{|c|}{ Datapoint (214.0 Cy9) } & \multicolumn{3}{|c|}{ Datapoint (425.0 Cy9) } & \multicolumn{3}{|c|}{ Statepoint 69 (BOC Cy10) } \\
\hline & $\begin{array}{c}\text { Burnup } \\
\text { (GWd/MTU) }\end{array}$ & $\begin{array}{l}\text { T-Fuel } \\
{ }^{\circ} \mathrm{F}\end{array}$ & $\begin{array}{c}\text { Spec. Vol. } \\
\mathrm{ft}^{3} / \mathrm{lbm}\end{array}$ & $\begin{array}{c}\text { Burnup } \\
\text { (GWd/MTU) }\end{array}$ & $\begin{array}{l}\text { T-Fuel } \\
{ }^{\circ} \mathrm{F}\end{array}$ & $\begin{array}{l}\text { Spec. Vol. } \\
\mathrm{ft}^{3} / \mathrm{lbm}\end{array}$ & $\begin{array}{c}\text { Burnup } \\
\text { (GWd/MTU) }\end{array}$ & $\begin{array}{l}\text { T-Fuel } \\
{ }^{\circ} \mathrm{F}\end{array}$ & $\begin{array}{c}\text { Spec. Vol. } \\
\mathrm{ft}^{3} / \mathrm{lbm}\end{array}$ \\
\hline & 214.0 Cyg & 109.3 Cy9 & 109.3 Cy9 & 425.0 Cy9 & 328.7 Cy 9 & 328.7 Cy 9 & BOC Cy 10 & 549.2 Cy9 & 549.2 Cy 9 \\
\hline 1 & 2.676 & 874.3 & 0.0230 & 5.904 & 912.2 & 0.0230 & 9.645 & 951.8 & 0.0231 \\
\hline 2 & 4.549 & 1051.9 & 0.0229 & 9.611 & 1047.3 & 0.0229 & 15.042 & 1054.2 & 0.0230 \\
\hline 3 & 5.632 & 1140.2 & 0.0229 & 11.487 & 1092.3 & 0.0228 & 17.851 & 1074.9 & 0.0229 \\
\hline 4 & 6.220 & 1181.6 & 0.0228 & 12.344 & 1102.0 & 0.0228 & 18.899 & 1069.5 & 0.0228 \\
\hline 5 & 6.532 & 1199.4 & 0.0227 & 12.713 & 1099.2 & 0.0227 & 19.252 & 1060.3 & 0.0227 \\
\hline 6 & 6.698 & 1205.7 & 0.0226 & 12.867 & 1093.4 & 0.0226 & 19.351 & 1052.8 & 0.0226 \\
\hline 7 & 6.786 & 1207.0 & 0.0225 & 12.933 & 1088.2 & 0.0225 & 19.376 & 1047.8 & 0.0225 \\
\hline 8 & 6.832 & 1206.0 & 0.0224 & 12.967 & 1084.4 & 0.0224 & 19.388 & 1044.9 & 0.0225 \\
\hline 9 & 6.854 & 1204.2 & 0.0223 & 12.991 & 1082.2 & 0.0223 & 19.404 & 1043.2 & 0.0224 \\
\hline 10 & 6.859 & 1202.1 & 0.0222 & 13.016 & 1081.6 & 0.0222 & 19.429 & 1042.3 & 0.0223 \\
\hline 11 & 6.853 & 1200.0 & 0.0221 & 13.047 & 1082.5 & 0.0222 & 19.468 & 1042.1 & 0.0222 \\
\hline 12 & 6.832 & 1197.5 & 0.0221 & 13.082 & 1085.1 & 0.0221 & 19.522 & 1042.9 & 0.0221 \\
\hline 13 & 6.777 & 1193.3 & 0.0220 & 13.096 & 1089.3 & 0.0220 & 19.580 & 1045.7 & 0.0220 \\
\hline 14 & 6.651 & 1184.3 & 0.0219 & 13.035 & 1094.3 & 0.0219 & 19.596 & 1051.3 & 0.0219 \\
\hline 15 & 6.392 & 1165.1 & 0.0218 & 12.783 & 1097.3 & 0.0218 & 19.439 & 1059.5 & 0.0219 \\
\hline 16 & 5.873 & 1124.2 & 0.0217 & 12.081 & 1089.2 & 0.0218 & 18.729 & 1065.3 & 0.0218 \\
\hline 17 & 4.830 & 1036.6 & 0.0217 & 10.306 & 1045.0 & 0.0217 & 16.432 & 1048.2 & 0.0217 \\
\hline 18 & 2.834 & 849.3 & 0.0216 & 6.329 & 900.7 & 0.0216 & 10.536 & 938.2 & 0.0216 \\
\hline
\end{tabular}

\begin{tabular}{|c|c|c|c|c|c|c|c|c|c|}
\hline \multirow[b]{2}{*}{$\begin{array}{l}\text { Axial } \\
\text { Node }\end{array}$} & \multicolumn{3}{|c|}{ Statepoint 70 (27.0 Cy10) } & \multicolumn{3}{|c|}{ Statepoint 71 ( 143.0 Cy10) } & \multicolumn{3}{|c|}{ Statepoint 72 (209.0 Cy10) } \\
\hline & $\begin{array}{c}\text { Burnup } \\
\text { (GWd/MTU) }\end{array}$ & $\begin{array}{l}\text { T-Fuel } \\
{ }^{\circ} \mathrm{F}\end{array}$ & $\begin{array}{c}\text { Spec. Vol. } \\
\mathrm{tt}^{3} / \mathrm{bm}\end{array}$ & $\begin{array}{c}\text { Burnup } \\
\text { (GWd/MTU) }\end{array}$ & $\begin{array}{l}\text { T-Fuel } \\
{ }^{\circ} \mathrm{F}\end{array}$ & $\begin{array}{l}\text { Spec. Vol. } \\
\mathrm{ft}^{3} / \mathrm{lbm}\end{array}$ & $\begin{array}{c}\text { Burnup } \\
\text { (GWd/MTU) }\end{array}$ & $\begin{array}{c}\text { T-Fuel } \\
{ }^{\circ} \mathrm{F}\end{array}$ & $\begin{array}{l}\text { Spec. Vol. } \\
\mathrm{ft}^{3} / \mathrm{lbm}\end{array}$ \\
\hline & 27.0 Cy 10 & 18.0 Cy 10 & $18.0 \mathrm{Cy} 10$ & 143.0 Cy 10 & 82.9 Cy10 & 82.9 Cy 10 & 209.0 Cy 10 & 176.7 Cy 10 & 176.7 Cy 10 \\
\hline 1 & 10.093 & 917.8 & 0.0234 & 12.064 & 909.0 & 0.0234 & 13.286 & 918.9 & 0.0234 \\
\hline 2 & 15.753 & 1045.8 & 0.0233 & 18.843 & 1030.7 & 0.0233 & 20.718 & 1028.4 & 0.0233 \\
\hline 3 & 18.713 & 1102.5 & 0.0232 & 22.444 & 1085.2 & 0.0232 & 24.651 & 1072.6 & 0.0232 \\
\hline 4 & 19.842 & 1130.6 & 0.0231 & 23.900 & 1110.9 & 0.0231 & 26.246 & 1089.2 & 0.0231 \\
\hline 5 & 20.243 & 1147.6 & 0.0230 & 24.481 & 1124.9 & 0.0230 & 26.887 & 1095.0 & 0.0229 \\
\hline 6 & 20.373 & 1158.2 & 0.0228 & 24.719 & 1132.4 & 0.0228 & 27.151 & 1096.3 & 0.0228 \\
\hline 7 & 20.419 & 1164.0 & 0.0227 & 24.829 & 1135.6 & 0.0227 & 27.272 & 1095.2 & 0.0227 \\
\hline 8 & 20.442 & 1165.7 & 0.0226 & 24.887 & 1135.8 & 0.0226 & 27.333 & 1092.8 & 0.0226 \\
\hline 9 & 20.462 & 1163.9 & 0.0225 & 24.919 & 1133.6 & 0.0225 & 27.366 & 1089.9 & 0.0225 \\
\hline 10 & 20.484 & 1158.9 & 0.0224 & 24.936 & 1129.4 & 0.0224 & 27.381 & 1086.8 & 0.0224 \\
\hline 11 & 20.514 & 1150.8 & 0.0223 & 24.943 & 1123.2 & 0.02 .23 & 27.386 & 1083.6 & 0.0223 \\
\hline 12 & 20.552 & 1139.2 & 0.0221 & 24.938 & 1114.8 & 0.0222 & 27.376 & 1079.9 & 0.0222 \\
\hline 13 & 20.586 & 1123.4 & 0.0220 & 24.903 & 1103.3 & 0.0221 & 27.329 & 1075.1 & 0.0221 \\
\hline 14 & 20.566 & 1102.7 & 0.0219 & 24.777 & 1087.9 & 0.0220 & 27.178 & 1068.1 & 0.0220 \\
\hline 15 & 20.360 & 1076.4 & 0.0219 & 24.410 & 1067.3 & 0.0219 & 26.761 & 1057.1 & 0.0219 \\
\hline 16 & 19.583 & 1043.4 & 0.0218 & 23.380 & 1039.8 & 0.0218 & 25.628 & 1039.6 & 0.0218 \\
\hline 17 & 17.169 & 994.0 & 0.0217 & 20.488 & 995.7 & 0.0217 & 22.488 & 1004.8 & 0.0217 \\
\hline 18 & 11.019 & 877.7 & 0.0216 & 13.226 & 884.8 & 0.0216 & 14.585 & 903.9 & 0.0216 \\
\hline
\end{tabular}


Table 4-90. Burnup and TH Feedback Parameters by Axial Node for Assembly J27

\begin{tabular}{|c|c|c|c|c|c|c|c|c|c|}
\hline \multirow[b]{2}{*}{$\begin{array}{l}\text { Axial } \\
\text { Node }\end{array}$} & \multicolumn{3}{|c|}{ Datapoint (214.0 Cy9) } & \multicolumn{3}{|c|}{ Datapoint (425.0 Cy9) } & \multicolumn{3}{|c|}{ Statepoint 69 (BOC Cy10) } \\
\hline & $\begin{array}{c}\text { Burnup } \\
\text { (GWd/MTU) }\end{array}$ & $\begin{array}{c}\text { T-Fuel } \\
{ }^{\circ} \mathrm{F}\end{array}$ & $\begin{array}{c}\text { Spec. Vol. } \\
\mathrm{ft}^{3} / \mathrm{lbm}\end{array}$ & $\begin{array}{c}\text { Burnup } \\
\text { (GWd/MTU) }\end{array}$ & $\begin{array}{c}\text { T-Fuel } \\
{ }^{\circ} \mathrm{F}\end{array}$ & $\begin{array}{c}\text { Spec. Vol. } \\
\mathrm{ft}^{3} / / \mathrm{bm}\end{array}$ & $\begin{array}{c}\text { Burnup } \\
\text { (GWd/MTU) }\end{array}$ & $\begin{array}{c}\text { T-Fuel } \\
{ }^{\circ} \mathrm{F} \\
\end{array}$ & $\begin{array}{c}\text { Spec. Vol. } \\
\mathrm{ft}^{3} / / \mathrm{bm}\end{array}$ \\
\hline & 214.0 Cy9 & 109.3 Су9 & 109.3 Су9 & 425.0 Cyg & 328.7 Cy9 & 328.7 Cy 9 & BOC Cy 10 & 549.2 Cy9 & 549.2 Cy 9 \\
\hline 1 & 2.895 & 905.3 & 0.0232 & 6.354 & 937.7 & 0.0232 & 10.314 & 969.2 & 0.0232 \\
\hline 2 & 5.044 & 1108.5 & 0.0232 & 10.580 & 1084.8 & 0.0231 & 16.369 & 1079.7 & 0.0232 \\
\hline 3 & 6.449 & 1215.6 & 0.0231 & 13.003 & 1139.4 & 0.0230 & 19.942 & 1106.1 & 0.0231 \\
\hline 4 & 7.214 & 1264.8 & 0.0230 & 14.126 & 1153.1 & 0.0229 & 21.341 & 1101.6 & 0.0230 \\
\hline 5 & 7.598 & 1284.7 & 0.0229 & 14.584 & 1150.3 & 0.0228 & 21.791 & 1091.4 & 0.0229 \\
\hline 6 & 7.796 & 1291.6 & 0.0228 & 14.770 & 1143.9 & 0.0227 & 21.918 & 1083.1 & 0.0227 \\
\hline 7 & 7.903 & 1292.9 & 0.0227 & 14.853 & 1138.1 & 0.0226 & 21.955 & 1077.7 & 0.0226 \\
\hline 8 & 7.961 & 1291.8 & 0.0226 & 14.897 & 1133.9 & 0.0225 & 21.975 & 1074.4 & 0.0226 \\
\hline 9 & 7.990 & 1289.8 & 0.0224 & 14.930 & 1131.4 & 0.0224 & 21.998 & 1072.5 & 0.0225 \\
\hline 10 & 8.001 & 1287.6 & 0.0223 & 14.963 & 1130.7 & 0.0223 & 22.031 & 1071.4 & 0.0224 \\
\hline 11 & 7.999 & 1285.5 & 0.0222 & 15.004 & 1131.6 & 0.0222 & 22.078 & 1070.9 & 0.0223 \\
\hline 12 & 7.979 & 1283.1 & 0.0221 & 15.048 & 1134.4 & 0.0221 & 22.142 & 1071.6 & 0.0222 \\
\hline 13 & 7.921 & 1279.0 & 0.0220 & 15.070 & 1139.0 & 0.0221 & 22.211 & 1074.4 & 0.0221 \\
\hline 14 & 7.785 & 1269.9 & 0.0219 & 15.009 & 1144.7 & 0.0220 & 22.236 & 1080.2 & 0.0220 \\
\hline 15 & 7.500 & 1250.2 & 0.0218 & 14.744 & 1148.5 & 0.0219 & 22.080 & 1089.3 & 0.0219 \\
\hline 16 & 6.927 & 1208.0 & 0.0218 & 13.989 & 1141.3 & 0.0218 & 21.338 & 1097.4 & 0.0218 \\
\hline 17 & 5.757 & 1116.1 & 0.0217 & 12.053 & 1097.6 & 0.0217 & 18.893 & 1084.7 & 0.0217 \\
\hline 18 & 3.547 & 919.0 & 0.0216 & 7.783 & 961.2 & 0.0216 & 12.752 & 987.1 & 0.0216 \\
\hline
\end{tabular}

\begin{tabular}{|c|c|c|c|c|c|c|c|c|c|}
\hline \multirow[b]{2}{*}{$\begin{array}{l}\text { Axial } \\
\text { Node }\end{array}$} & \multicolumn{3}{|c|}{ Statepoint $70(27.0$ Cy10) } & \multicolumn{3}{|c|}{ Statepoint $71(143.0$ Cy10) } & \multicolumn{3}{|c|}{ Statepoint 72 (209.0 Cy10) } \\
\hline & $\begin{array}{c}\text { Burnup } \\
\text { (GWd/MTU) }\end{array}$ & $\begin{array}{c}\text { T-Fuel } \\
{ }^{\circ} \mathbf{F}\end{array}$ & $\begin{array}{c}\text { Spec. Vol. } \\
\mathrm{ft}^{3} / \mathrm{lbm}\end{array}$ & $\begin{array}{c}\text { Burnup } \\
\text { (GWd/MTU) }\end{array}$ & $\begin{array}{c}\text { T-Fuel } \\
{ }^{\circ} \mathrm{F} \\
\end{array}$ & $\begin{array}{c}\text { Spec. Vol. } \\
\mathrm{ft}^{3} / \mathrm{lbm}\end{array}$ & $\begin{array}{c}\text { Burnup } \\
\text { (GWd/MTU) }\end{array}$ & $\begin{array}{c}\text { T-Fuel } \\
{ }^{\circ} \mathrm{F} \\
\end{array}$ & $\begin{array}{l}\text { Spec. Vol. } \\
\mathrm{ft}^{3} / \mathrm{lbm}\end{array}$ \\
\hline & 27.0 Cy 10 & 18.0 Cy 10 & 18.0 Cy 10 & $143.0 \mathrm{Cy} 10$ & $82.9 \mathrm{Cy} 10$ & 82.9 Cy 10 & 209.0 Cy 10 & 176.7 Cy 10 & 176.7 Cy10 \\
\hline 1 & 10.667 & 865.8 & 0.0234 & 12.173 & 837.5 & 0.0235 & 13.119 & 850.5 & 0.0235 \\
\hline 2 & 17.032 & 1038.1 & 0.0234 & 19.826 & 1009.3 & 0.0234 & 21.627 & 1007.4 & 0.0234 \\
\hline 3 & 20.814 & 1096.0 & 0.0233 & 24.626 & 1084.6 & 0.0233 & 26.925 & 1080.5 & 0.0233 \\
\hline 4 & 22.307 & 1124.4 & 0.0231 & 26.531 & 1115.4 & 0.0232 & 29.007 & 1102.3 & 0.0232 \\
\hline 5 & 22.811 & 1142.5 & 0.0230 & 27.244 & 1131.6 & 0.0230 & 29.793 & 1110.4 & 0.0231 \\
\hline 6 & 22.973 & 1154.2 & 0.0229 & 27.534 & 1141.0 & 0.0229 & 30.115 & 1112.7 & 0.0229 \\
\hline 7 & 23.033 & 1161.1 & 0.0228 & 27.673 & 1145.6 & 0.0228 & 30.270 & 1112.2 & 0.0228 \\
\hline 8 & 23.067 & 1163.8 & 0.0226 & 27.754 & 1147.0 & 0.0227 & 30.359 & 1110.3 & 0.0227 \\
\hline 9 & 23.097 & 1163.0 & 0.0225 & 27.808 & 1145.8 & 0.0225 & 30.417 & 1107.9 & 0.0226 \\
\hline 10 & 23.129 & 1159.1 & 0.0224 & 27.845 & 1142.5 & 0.0224 & 30.458 & 1105.4 & 0.0224 \\
\hline 11 & 23.170 & 1152.3 & 0.0223 & 27.873 & 1137.4 & 0.0223 & 30.490 & 1103.0 & 0.0223 \\
\hline 12 & 23.221 & 1142.3 & 0.0222 & 27.894 & 1130.2 & 0.0222 & 30.514 & 1100.6 & 0.0222 \\
\hline 13 & 23.268 & 1128.1 & 0.0221 & 27.883 & 1119.9 & 0.0221 & 30.498 & 1097.2 & 0.0221 \\
\hline 14 & 23.258 & 1107.8 & 0.0220 & 27.766 & 1104.4 & 0.0220 & 30.361 & 1090.8 & 0.0220 \\
\hline 15 & 23.051 & 1080.3 & 0.0219 & 27.381 & 1081.8 & 0.0219 & 29.920 & 1079.0 & 0.0219 \\
\hline 16 & 22.233 & 1044.5 & 0.0218 & 26.272 & 1049.8 & 0.0218 & 28.690 & 1057.4 & 0.0218 \\
\hline 17 & 19.665 & 994.2 & 0.0217 & 23.181 & 1001.8 & 0.0217 & 25.321 & 1016.6 & 0.0217 \\
\hline 18 & 13.266 & 882.4 & 0.0216 & 15.620 & 892.9 & 0.0216 & 17.074 & 912.8 & 0.0216 \\
\hline
\end{tabular}


Table 4-91. Burnup and TH Feedback Parameters by Axial Node for Assembly J27a

\begin{tabular}{|c|c|c|c|c|c|c|c|c|c|}
\hline \multirow[b]{2}{*}{$\begin{array}{l}\text { Axial } \\
\text { Node } \\
\end{array}$} & \multicolumn{3}{|c|}{ Datapoint (214.0 Cy9) } & \multicolumn{3}{|c|}{ Datapoint (425.0 Cy9) } & \multicolumn{3}{|c|}{ Statepoint 69 (BOC Cy10) } \\
\hline & $\begin{array}{c}\text { Burnup } \\
\text { (GWd/MTU) }\end{array}$ & $\begin{array}{c}\text { T-Fuel } \\
{ }^{\circ} \mathrm{F} \\
\end{array}$ & $\begin{array}{c}\text { Spec. Vol. } \\
\mathrm{ft}^{3} / \mathrm{lbm}\end{array}$ & $\begin{array}{c}\text { Burnup } \\
\text { (GWd/MTU) }\end{array}$ & $\begin{array}{l}\text { T-Fuel } \\
{ }^{\circ} \mathrm{F} \\
\end{array}$ & $\begin{array}{c}\text { Spec. Vol. } \\
\mathrm{ft}^{3} / \mathrm{lbm}\end{array}$ & $\begin{array}{c}\text { Burnup } \\
\text { (GWd/MTU) }\end{array}$ & $\begin{array}{l}\text { T-Fuel } \\
{ }^{\circ} \mathrm{F} \\
\end{array}$ & $\begin{array}{c}\text { Spec. Vol. } \\
\mathrm{ft}^{3} / \mathrm{lbm}\end{array}$ \\
\hline & 214.0 Cy9 & 109.3 Сy9 & 109.3 Cy9 & 425.0 Cy 9 & 328.7 Cyg & 328.7 Cy 9 & BOC Cy 10 & 549.2 Cy9 & 549.2 Cy 9 \\
\hline 1 & 2.895 & 905.3 & 0.0232 & 6.354 & 937.7 & 0.0232 & 10.316 & 969.2 & 0.0232 \\
\hline 2 & 5.044 & 1108.5 & 0.0232 & 10.580 & 1084.8 & 0.0231 & 16.375 & 1079.7 & 0.0232 \\
\hline 3 & 6.449 & 1215.6 & 0.0231 & 13.003 & 1139.4 & 0.0230 & 19.956 & 1106.1 & 0.0231 \\
\hline 4 & 7.214 & 1264.8 & 0.0230 & 14.126 & 1153.1 & 0.0229 & 21.360 & 1101.6 & 0.0230 \\
\hline 5 & 7.598 & 1284.7 & 0.0229 & 14.584 & 1150.3 & 0.0228 & 21.812 & 1091.4 & 0.0229 \\
\hline 6 & 7.796 & 1291.6 & 0.0228 & 14.770 & 1143.9 & 0.0227 & 21.940 & 1083.1 & 0.0227 \\
\hline 7 & 7.903 & 1292.9 & 0.0227 & 14.853 & 1138.1 & 0.0226 & 21.978 & 1077.7 & 0.0226 \\
\hline 8 & 7.961 & 1291.8 & 0.0226 & 14.897 & 1133.9 & 0.0225 & 21.999 & 1074.4 & 0.0226 \\
\hline 9 & 7.990 & 1289.8 & 0.0224 & 14.930 & 1131.4 & 0.0224 & 22.023 & 1072.5 & 0.0225 \\
\hline 10 & 8.001 & 1287.6 & 0.0223 & 14.963 & 1130.7 & 0.0223 & 22.057 & 1071.4 & 0.0224 \\
\hline 11 & 7.999 & 1285.5 & 0.0222 & 15.004 & 1131.6 & 0.0222 & 22.105 & 1070.9 & 0.0223 \\
\hline 12 & 7.979 & 1283.1 & 0.0221 & 15.048 & 1134.4 & 0.0221 & 22.170 & 1071.6 & 0.0222 \\
\hline 13 & 7.921 & 1279.0 & 0.0220 & 15.070 & 1139.0 & 0.0221 & 22.239 & 1074.4 & 0.0221 \\
\hline 14 & 7.785 & 1269.9 & 0.0219 & 15.009 & 1144.7 & 0.0220 & 22.264 & 1080.2 & 0.0220 \\
\hline 15 & 7.500 & 1250.2 & 0.0218 & 14.744 & 1148.5 & 0.0219 & 22.109 & 1089.3 & 0.0219 \\
\hline 16 & 6.927 & 1208.0 & 0.0218 & 13.989 & 1141.3 & 0.0218 & 21.366 & 1097.4 & 0.0218 \\
\hline 17 & 5.757 & 1116.1 & 0.0217 & 12.053 & 1097.6 & 0.0217 & 18.917 & 1084.7 & 0.0217 \\
\hline 18 & 3.547 & 919.0 & 0.0216 & 7.783 & 961.2 & 0.0216 & 12.768 & 987.1 & 0.0216 \\
\hline
\end{tabular}

\begin{tabular}{|c|c|c|c|c|c|c|c|c|c|}
\hline \multirow[b]{2}{*}{$\begin{array}{l}\text { Axial } \\
\text { Node }\end{array}$} & \multicolumn{3}{|c|}{ Statepoint $70(27.0$ Cy10) } & \multicolumn{3}{|c|}{ Statepoint 71 ( 143.0 Cy10) } & \multicolumn{3}{|c|}{ Statepoint $72(209.0$ Cy 10$)$} \\
\hline & $\begin{array}{c}\text { Burnup } \\
\text { (GWd/MTU) }\end{array}$ & $\begin{array}{c}\text { T-Fuel } \\
{ }^{\circ} \mathrm{F}\end{array}$ & $\begin{array}{c}\text { Spec. Vol. } \\
\mathrm{ft}^{3} / / \mathrm{bm}\end{array}$ & $\begin{array}{c}\text { Burnup } \\
\text { (GWd/MTU) }\end{array}$ & $\begin{array}{c}\text { T-Fuel } \\
{ }^{\circ} \mathrm{F} \\
\end{array}$ & $\begin{array}{c}\text { Spec. Vol. } \\
\mathrm{ft}^{3} / \mathrm{lbm}\end{array}$ & \begin{tabular}{|c|} 
Burnup \\
(GWd/MTU) \\
\end{tabular} & $\begin{array}{l}\text { T-Fuel } \\
{ }^{\circ} \mathrm{F}\end{array}$ & \begin{tabular}{|c} 
Spec. Vol. \\
$\mathrm{ft}^{3} / \mathrm{lbm}$
\end{tabular} \\
\hline & 27.0 Cy 10 & 18.0 Cy 10 & 18.0 Cy 10 & 143.0 Cy 10 & 82.9 Cy 10 & 82.9 Cy 10 & 209.0 Cy 10 & 176.7 Cy 10 & 176.7 Cy 10 \\
\hline 1 & 10.642 & 847.5 & 0.0235 & 11.992 & 812.5 & 0.0234 & 12.823 & 816.9 & 0.0233 \\
\hline 2 & 17.020 & 1025.0 & 0.0234 & 19.629 & 982.7 & 0.0233 & 21.258 & 966.2 & 0.0232 \\
\hline 3 & 20.826 & 1093.1 & 0.0233 & 24.451 & 1059.9 & 0.0232 & 26.547 & 1034.1 & 0.0231 \\
\hline 4 & 22.339 & 1128.0 & 0.0232 & 26.385 & 1091.4 & 0.0231 & 28.643 & 1053.2 & 0.0230 \\
\hline 5 & 22.854 & 1150.2 & 0.0231 & 27.114 & 1107.6 & 0.0230 & 29.435 & 1059.6 & 0.0229 \\
\hline 6 & 23.025 & 1164.7 & 0.0229 & 27.410 & 1116.5 & 0.0229 & 29.758 & 1060.7 & 0.0228 \\
\hline 7 & 23.091 & 1173.6 & 0.0228 & 27.555 & 1120.8 & 0.0227 & 29.915 & 1059.5 & 0.0227 \\
\hline 8 & 23.130 & 1178.1 & 0.0227 & 27.643 & 1122.2 & 0.0226 & 30.008 & 1057.3 & 0.0226 \\
\hline 9 & 23.164 & 1179.1 & 0.0226 & 27.705 & 1121.3 & 0.0225 & 30.073 & 1054.9 & 0.0225 \\
\hline 10 & 23.202 & 1176.8 & 0.0224 & 27.753 & 1118.8 & 0.0224 & 30.124 & 1052.6 & 0.0224 \\
\hline 11 & 23.247 & 1171.6 & 0.0223 & 27.795 & 1114.8 & 0.0223 & 30.171 & 1050.6 & 0.0223 \\
\hline 12 & 23.301 & 1163.1 & 0.0222 & 27.831 & 1109.1 & 0.0222 & 30.212 & 1048.9 & 0.0222 \\
\hline 13 & 23.351 & 1149.6 & 0.0221 & 27.835 & 1100.5 & 0.0221 & 30.216 & 1046.4 & 0.0221 \\
\hline 14 & 23.340 & 1129.0 & 0.0220 & 27.729 & 1086.8 & 0.0220 & 30.096 & 1041.3 & 0.0220 \\
\hline 15 & 23.126 & 1098.8 & 0.0219 & 27.343 & 1065.3 & 0.0219 & 29.661 & 1031.2 & 0.0219 \\
\hline 16 & 22.293 & 1056.1 & 0.0218 & 26.206 & 1032.4 & 0.0218 & 28.410 & 1011.4 & 0.0218 \\
\hline 17 & 19.694 & 992.4 & 0.0217 & 23.039 & 979.5 & 0.0217 & 24.974 & 972.4 & 0.0217 \\
\hline 18 & 13.258 & 865.6 & 0.0216 & 15.417 & 864.6 & 0.0216 & 16.702 & 872.8 & 0.0216 \\
\hline
\end{tabular}


Table 4-92. Burnup and TH Feedback Parameters by Axial Node for Assembly K2

\begin{tabular}{|c|c|c|c|c|c|c|c|c|c|}
\hline \multirow[b]{2}{*}{$\begin{array}{l}\text { Axial } \\
\text { Node }\end{array}$} & \multicolumn{3}{|c|}{ Statepoint $70(27.0$ Cy10) } & \multicolumn{3}{|c|}{ Statepoint $71(143.0$ Cy10) } & \multicolumn{3}{|c|}{ Statepoint 72 (209.0 Cy10) } \\
\hline & $\begin{array}{c}\text { Burnup } \\
\text { (GWd/MTU) }\end{array}$ & $\begin{array}{l}\text { T-Fuel } \\
{ }^{\circ} \mathrm{F} \\
\end{array}$ & $\begin{array}{c}\text { Spec. Vol. } \\
\mathrm{tt}^{3} / \mathrm{bm}\end{array}$ & $\begin{array}{c}\text { Burnup } \\
\text { (GWd/MTU) }\end{array}$ & $\begin{array}{c}\text { T-Fuel } \\
{ }^{\circ} \mathrm{F} \\
\end{array}$ & $\begin{array}{c}\text { Spec. Vol. } \\
\mathrm{ft}^{3} / \mathrm{lbm}\end{array}$ & $\begin{array}{c}\text { Burnup } \\
\text { (GWd/MTU) }\end{array}$ & $\begin{array}{l}\text { T-Fuel } \\
{ }^{\circ} \mathrm{F}\end{array}$ & $\begin{array}{c}\text { Spec. Vol. } \\
\mathrm{ft}^{3} / \mathrm{lbm}\end{array}$ \\
\hline & 27.0 Cy 10 & 18.0 Cy 10 & 18.0 Cy 10 & 143.0 Cy 10 & 82.9 Cy 10 & $82.9 C_{y} 10$ & 209.0 Cy 10 & 176.7 Cy 10 & 176.7 Cy 10 \\
\hline 1 & 0.506 & 1043.7 & 0.0236 & 2.724 & 1035.8 & 0.0237 & 4.096 & 1049.0 & 0.0237 \\
\hline 2 & 0.764 & 1238.0 & 0.0235 & 4.116 & 1224.1 & 0.0236 & 6.174 & 1220.1 & 0.0236 \\
\hline 3 & 0.950 & 1364.4 & 0.0234 & 5.112 & 1344.1 & 0.0235 & 7.613 & 1321.4 & 0.0235 \\
\hline 4 & 1.057 & 1432.4 & 0.0233 & 5.669 & 1405.3 & 0.0233 & 8.384 & 1365.9 & 0.0234 \\
\hline 5 & 1.121 & 1469.8 & 0.0232 & 5.979 & 1436.1 & 0.0232 & 8.793 & 1383.6 & 0.0232 \\
\hline 6 & 1.160 & 1490.7 & 0.0230 & 6.162 & 1451.1 & 0.0230 & 9.024 & 1389.1 & 0.0231 \\
\hline 7 & 1.185 & 1500.7 & 0.0229 & 6.272 & 9457.0 & 0.0229 & 9.157 & 1389.4 & 0.0229 \\
\hline 8 & 1.199 & 1504.0 & 0.0227 & 6.333 & 1458.1 & 0.0228 & 9.230 & 1387.7 & 0.0228 \\
\hline 9 & 1.204 & 1502.6 & 0.0226 & 6.358 & 1456.3 & 0.0226 & 9.261 & 1385.4 & 0.0227 \\
\hline 10 & 1.201 & 1497.2 & 0.0225 & 6.353 & 1452.2 & 0.0225 & 9.259 & 1383.0 & 0.0225 \\
\hline 11 & 1.191 & 1487.7 & 0.0223 & 6.318 & 1445.6 & 0.0224 & 9.224 & 1380.4 & 0.0224 \\
\hline 12 & 1.172 & 1472.8 & 0.0222 & 6.248 & 1436.1 & 0.0222 & 9.150 & 1377.2 & 0.0223 \\
\hline 13 & 1.143 & 1450.7 & 0.0221 & 6.137 & 1421.7 & 0.0221 & 9.023 & 1372.7 & 0.0222 \\
\hline 14 & 1.103 & 1419.9 & 0.0220 & 5.968 & 1399.8 & 0.0220 & 8.821 & 1364.8 & 0.0220 \\
\hline 15 & 1.045 & 1377.2 & 0.0219 & 5.710 & 1366.8 & 0.0219 & 8.491 & 1347.8 & 0.0219 \\
\hline 16 & 0.957 & 1313.2 & 0.0218 & 5.282 & 1312.7 & 0.0218 & 7.906 & 1310.5 & 0.0218 \\
\hline 17 & 0.813 & 1207.5 & 0.0217 & 4.517 & 1215.3 & 0.0217 & 6.800 & 1228.8 & 0.0217 \\
\hline 18 & 0.574 & 1032.4 & 0.0216 & 3.191 & 1043.4 & 0.0216 & 4.812 & 1065.0 & 0.0216 \\
\hline
\end{tabular}

\begin{tabular}{|c|c|c|c|c|c|c|c|c|c|}
\hline \multirow[b]{2}{*}{$\begin{array}{l}\text { Axial } \\
\text { Node }\end{array}$} & \multicolumn{3}{|c|}{ Datapoint (430.4 Cy10) } & \multicolumn{3}{|c|}{ Statepoint 73 (BOC Cy11) } & \multicolumn{3}{|c|}{ Datapoint (208.7 Cy11) } \\
\hline & $\begin{array}{c}\text { Burnup } \\
\text { (GWd/MTU) }\end{array}$ & $\begin{array}{c}\text { T-Fuel } \\
{ }^{\circ} \mathrm{F}\end{array}$ & $\begin{array}{c}\text { Spec. Vol. } \\
\mathrm{ft}^{3} / \mathrm{lbm}\end{array}$ & $\begin{array}{c}\text { Burnup } \\
\text { (GWd/MTU) }\end{array}$ & $\begin{array}{c}\text { T-Fuel } \\
{ }^{\circ} \mathrm{F}\end{array}$ & $\begin{array}{c}\text { Spec. Vol. } \\
\mathrm{ft}^{3} / \mathrm{lbm}\end{array}$ & $\begin{array}{c}\text { Burnup } \\
\text { (GWd/MTU) }\end{array}$ & $\begin{array}{c}\text { T-Fuel } \\
{ }^{\circ} \mathrm{F}\end{array}$ & $\begin{array}{l}\text { Spec. Vol. } \\
\mathrm{ft}^{3} / \mathrm{lbm}\end{array}$ \\
\hline & 430.4 Cy 10 & 319.5 Cy 10 & 319.5 Cy10 & BOC Cy 11 & 549.3 Cy 10 & 549.3 Cy 10 & 208.7 Cy11 & 103.8 Cy 11 & $103.8 \mathrm{Cy} 11$ \\
\hline 1 & 9.329 & 1073.0 & 0.0239 & 15.551 & 1074.4 & 0.0239 & 18.682 & 834.3 & 0.0230 \\
\hline 2 & 13.896 & 1227.6 & 0.0238 & 22.720 & 1197.4 & 0.0238 & 27.476 & 910.3 & 0.0230 \\
\hline 3 & 16.721 & 1307.0 & 0.0236 & 26.646 & 1234.1 & 0.0236 & 32.243 & 940.0 & 0.0229 \\
\hline 4 & 18.007 & 1328.0 & 0.0235 & 28.126 & 1232.6 & 0.0235 & 34.147 & 960.6 & 0.0228 \\
\hline 5 & 18.574 & 1328.2 & 0.0233 & 28.637 & 1221.7 & 0.0233 & 34.893 & 972.9 & 0.0227 \\
\hline 6 & 18.839 & 1323.8 & 0.0232 & 28.822 & 1211.6 & 0.0232 & 35.208 & 979.0 & 0.0226 \\
\hline 7 & 18.974 & 1318.9 & 0.0230 & 28.905 & 1204.2 & 0.0230 & 35.364 & 981.2 & 0.0225 \\
\hline 8 & 19.049 & 1315.0 & 0.0229 & 28.965 & 1199.3 & 0.0229 & 35.458 & 981.1 & 0.0224 \\
\hline 9 & 19.097 & 1312.4 & 0.0227 & 29.026 & 1196.4 & 0.0228 & 35.528 & 979.2 & 0.0223 \\
\hline 10 & 19.128 & 1311.4 & 0.0226 & 29.095 & 1194.8 & 0.0226 & 35.585 & 976.1 & 0.0222 \\
\hline 11 & 19.143 & 1311.7 & 0.0225 & 29.169 & 1194.7 & 0.0225 & 35.631 & 971.8 & 0.0221 \\
\hline 12 & 19.132 & 1313.4 & 0.0223 & 29.244 & 1196.3 & 0.0224 & 35.656 & 966.1 & 0.0221 \\
\hline 13 & 19.072 & 1315.9 & 0.0222 & 29.305 & 1200.4 & 0.0222 & 35.638 & 958.3 & 0.0220 \\
\hline 14 & 18.917 & 1318.1 & 0.0221 & 29.311 & 1207.7 & 0.0221 & 35.516 & 947.4 & 0.0219 \\
\hline 15 & 18.545 & 1316.7 & 0.0220 & 29.111 & 1217.3 & 0.0220 & 35.105 & 931.4 & 0.0218 \\
\hline 16 & 17.643 & 1302.5 & 0.0218 & 28.227 & 1222.1 & 0.0219 & 33.854 & 906.2 & 0.0217 \\
\hline 17 & 15.492 & 1241.7 & 0.0217 & 25.383 & 1195.5 & 0.0217 & 30.277 & 870.1 & 0.0217 \\
\hline 18 & 11.062 & 1086.4 & 0.0216 & 18.453 & 1084.6 & 0.0216 & 21.671 & 791.2 & 0.0216 \\
\hline
\end{tabular}


Table 4-92. Burnup and TH Feedback Parameters by Axial Node for Assembly K2 (Cont.)

\begin{tabular}{|c|c|c|c|c|c|c|}
\hline \multirow{2}{*}{$\begin{array}{c}\text { Axial } \\
\text { Node }\end{array}$} & $\begin{array}{c}\text { Burnup } \\
\text { (GWd/MTU) }\end{array}$ & $\begin{array}{c}\text { T-Fuel } \\
{ }^{\circ} \mathrm{F}\end{array}$ & $\begin{array}{c}\text { Spec. Vol. } \\
\mathrm{ft}^{3} / \mathrm{lbm}\end{array}$ & $\begin{array}{c}\text { Burnup } \\
(\mathrm{GWd} / \mathrm{MTU})\end{array}$ & $\begin{array}{c}\text { T-Fuel } \\
{ }^{\circ} \mathrm{F}\end{array}$ & $\begin{array}{c}\text { Spec. Vol. } \\
\mathrm{ft}^{3} / \mathrm{lbm}\end{array}$ \\
\hline & $417.8 \mathrm{Cy} 11$ & $312.8 \mathrm{Cy} 11$ & $312.8 \mathrm{Cy} 11$ & $615.0 \mathrm{Cy} 11$ & 515.7 Cy11 & $515.7 \mathrm{Cy} 11$ \\
\hline 2 & 22.405 & 861.0 & 0.0230 & 26.337 & 872.1 & 0.0230 \\
\hline 3 & 32.854 & 920.7 & 0.0230 & 38.216 & 929.2 & 0.0229 \\
\hline 4 & 38.289 & 951.9 & 0.0229 & 44.081 & 945.8 & 0.0228 \\
\hline 5 & 40.393 & 958.7 & 0.0228 & 46.214 & 941.7 & 0.0228 \\
\hline 6 & 41.182 & 957.9 & 0.0227 & 46.944 & 934.4 & 0.0227 \\
\hline 7 & 41.492 & 954.8 & 0.0226 & 47.193 & 927.9 & 0.0226 \\
\hline 8 & 41.631 & 951.4 & 0.0225 & 47.289 & 922.9 & 0.0225 \\
\hline 9 & 41.712 & 948.2 & 0.0224 & 47.345 & 919.1 & 0.0224 \\
\hline 10 & 41.775 & 945.6 & 0.0223 & 47.398 & 916.3 & 0.0223 \\
\hline 11 & 41.893 & 943.5 & 0.0223 & 47.459 & 914.2 & 0.0222 \\
\hline 12 & 41.937 & 942.0 & 0.0222 & 47.528 & 912.7 & 0.0222 \\
\hline 13 & 41.941 & 941.0 & 0.0221 & 47.596 & 912.1 & 0.0221 \\
\hline 14 & 41.837 & 940.3 & 0.0220 & 47.641 & 912.7 & 0.0220 \\
\hline 15 & 41.408 & 939.5 & 0.0219 & 47.597 & 914.7 & 0.0219 \\
\hline 16 & 40.014 & 937.2 & 0.0218 & 47.238 & 917.8 & 0.0218 \\
\hline 17 & 35.878 & 928.8 & 0.0218 & 45.855 & 919.0 & 0.0218 \\
\hline 18 & 25.537 & 898.1 & 0.0217 & 41.395 & 903.3 & 0.0217 \\
\hline
\end{tabular}

Table 4-93. Burnup and TH Feedback Parameters by Axial Node for Assembly K6

\begin{tabular}{|c|c|c|c|c|c|c|c|c|c|}
\hline \multirow[b]{2}{*}{$\begin{array}{l}\text { Axial } \\
\text { Node }\end{array}$} & \multicolumn{3}{|c|}{ Statepoint 70 (27.0 Cy10) } & \multicolumn{3}{|c|}{ Statepoint 71 (143.0 Cy10) } & \multicolumn{3}{|c|}{ Statepoint 72 (209.0 Cy10) } \\
\hline & $\begin{array}{c}\text { Burnup } \\
\text { (GWd/MTU) }\end{array}$ & $\begin{array}{c}\text { T-Fuel } \\
{ }^{\circ} \mathrm{F} \\
\end{array}$ & $\begin{array}{c}\text { Spec. Vol. } \\
\mathrm{ft}^{3} / / \mathrm{bm}\end{array}$ & $\begin{array}{c}\text { Burnup } \\
\text { (GWd/MTU) }\end{array}$ & $\begin{array}{c}\text { T-Fuel } \\
{ }^{\circ} \mathbf{F} \\
\end{array}$ & $\begin{array}{c}\text { Spec. Vol. } \\
\mathrm{ft}^{3} / \mathrm{lbm}\end{array}$ & $\begin{array}{c}\text { Burnup } \\
\text { (GWd/MTU) } \\
\end{array}$ & $\begin{array}{l}\text { T-Fuel } \\
{ }^{\circ} \mathrm{F}\end{array}$ & $\begin{array}{c}\text { Spec. Vol. } \\
\mathrm{ft}^{3} / \mathrm{bm}\end{array}$ \\
\hline & $27.0 \mathrm{Cy} 10$ & 18.0 Cy 10 & $18.0 \mathrm{Cy} 10$ & 143.0 Cy 10 & 82.9 Cy 10 & 82.9 Cy 10 & 209.0 Cy 10 & 176.7 Cy 10 & 176.7 Cy 10 \\
\hline 1 & 0.497 & 1039.0 & 0.0237 & 2.703 & 1037.7 & 0.0238 & 4.089 & 1057.7 & 0.0239 \\
\hline 2 & 0.751 & 1231.5 & 0.0236 & 4.120 & 1231.4 & 0.0237 & 6.243 & 1241.7 & 0.0238 \\
\hline 3 & 0.946 & 1364.2 & 0.0235 & 5.206 & 1364.2 & 0.0236 & 7.831 & 1356.9 & 0.0237 \\
\hline 4 & 1.064 & 1439.3 & 0.0234 & 5.845 & 1436.1 & 0.0235 & 8.721 & 1407.0 & 0.0235 \\
\hline 5 & 1.136 & 1482.6 & 0.0232 & 6.213 & 1471.4 & 0.0233 & 9.206 & 1422.8 & 0.0234 \\
\hline 6 & 1.183 & 1506.7 & 0.0231 & 6.441 & 1487.9 & 0.0232 & 9.491 & 1427.6 & 0.0232 \\
\hline 7 & 1.214 & 1519.7 & 0.0230 & 6.589 & 1496.9 & 0.0230 & 9.670 & 1427.9 & 0.0231 \\
\hline 8 & 1.235 & 1526.7 & 0.0228 & 6.686 & 1501.3 & 0.0229 & 9.786 & 1426.7 & 0.0229 \\
\hline 9 & 1.246 & 1529.0 & 0.0227 & 6.745 & 1502.5 & 0.0227 & 9.860 & 1425.2 & 0.0228 \\
\hline 10 & 1.250 & 1527.3 & 0.0225 & 6.773 & 1501.2 & 0.0226 & 9.902 & 1424.0 & 0.0226 \\
\hline 11 & 1.247 & 1521.8 & 0.0224 & 6.774 & 1497.8 & 0.0224 & 9.917 & 1423.5 & 0.0225 \\
\hline 12 & 1.235 & 1512.0 & 0.0223 & 6.743 & 1491.8 & 0.0223 & 9.899 & 1423.2 & 0.0223 \\
\hline 13 & 1.213 & 1496.1 & 0.0221 & 6.664 & 1481.5 & 0.0222 & 9.823 & 1422.2 & 0.0222 \\
\hline 14 & 1.175 & 1470.0 & 0.0220 & 6.503 & 1463.5 & 0.0220 & 9.642 & 1417.6 & 0.0221 \\
\hline 15 & 1.112 & 1425.1 & 0.0219 & 6.214 & 1432.7 & 0.0219 & 9.281 & 1404.3 & 0.0219 \\
\hline 16 & 1.014 & 1352.7 & 0.0218 & 5.708 & 1370.8 & 0.0218 & 8.593 & 1371.0 & 0.0218 \\
\hline 17 & 0.854 & 1236.3 & 0.0217 & 4.828 & 1258.2 & 0.0217 & 7.310 & 1278.4 & 0.0217 \\
\hline 18 & 0.607 & 1056.1 & 0.0216 & 3.399 & 1071.7 & 0.0216 & 5.129 & 1092.4 & 0.0216 \\
\hline
\end{tabular}


Table 4-93. Burnup and TH Feedback Parameters by Axial Node for Assembly K6 (Cont.)

\begin{tabular}{|c|c|c|c|c|c|c|c|c|c|}
\hline \multirow{2}{*}{$\begin{array}{c}\text { Axial } \\
\text { Node }\end{array}$} & \multicolumn{2}{|c|}{ Datapoint (430.4 Cy10) } & \multicolumn{2}{c|}{ Statepoint 73 (BOC Cy11) } & \multicolumn{3}{c|}{ Datapoint (208.7 Cy11) } \\
\hline & $\begin{array}{c}\text { Burnup } \\
\text { (GW/MTU) }\end{array}$ & $\begin{array}{c}\text { T-Fuel } \\
{ }^{\circ} \mathrm{F}\end{array}$ & $\begin{array}{c}\text { Spec. Vol. } \\
\mathrm{ft}^{3} / \mathrm{lbm}\end{array}$ & $\begin{array}{c}\text { Burnup } \\
\text { (GWd/MTU) }\end{array}$ & $\begin{array}{c}\text { T-Fuel } \\
{ }^{\circ} \mathrm{F}\end{array}$ & $\begin{array}{c}\text { Spec. Vol. } \\
\mathrm{ft}^{3} / \mathrm{lbm}\end{array}$ & $\begin{array}{c}\text { Burnup } \\
\text { (GWd/MTU) }\end{array}$ & $\begin{array}{c}\text { T-Fuel } \\
{ }^{\circ} \mathrm{F}\end{array}$ & $\begin{array}{c}\text { Spec. Vol. } \\
\mathrm{ft}^{3} / \mathrm{lbm}\end{array}$ \\
\hline 1 & 430.4 Cy10 & 319.5 Cy10 & 319.5 Cy10 & BOC Cy11 & 549.3 Cy10 & 549.3 Cy10 & 208.7 Cy11 & 103.8 Cy11 & $103.8 \mathrm{Cy} 11$ \\
\hline 2 & 9.325 & 1073.6 & 0.0239 & 15.460 & 1066.7 & 0.0238 & 18.502 & 837.5 & 0.0235 \\
\hline 3 & 14.119 & 1237.5 & 0.0238 & 22.919 & 1191.1 & 0.0237 & 28.567 & 977.4 & 0.0234 \\
\hline 4 & 18.543 & 1333.0 & 0.0235 & 28.534 & 1219.0 & 0.0234 & 36.305 & 1078.5 & 0.0232 \\
\hline 5 & 19.148 & 1329.3 & 0.0233 & 29.044 & 1205.7 & 0.0233 & 37.084 & 1091.4 & 0.0230 \\
\hline 6 & 19.435 & 1321.9 & 0.0232 & 29.227 & 1194.3 & 0.0231 & 37.409 & 1096.8 & 0.0229 \\
\hline 7 & 19.594 & 1314.9 & 0.0230 & 29.323 & 1186.1 & 0.0230 & 37.578 & 1098.2 & 0.0228 \\
\hline 8 & 19.701 & 1309.5 & 0.0229 & 29.404 & 1180.6 & 0.0229 & 37.689 & 1096.8 & 0.0227 \\
\hline 9 & 19.786 & 1305.9 & 0.0228 & 29.492 & 1176.9 & 0.0227 & 37.776 & 1093.5 & 0.0225 \\
\hline 10 & 19.863 & 1304.2 & 0.0226 & 29.595 & 1174.4 & 0.0226 & 37.854 & 1088.8 & 0.0224 \\
\hline 11 & 19.938 & 1304.4 & 0.0225 & 29.717 & 1173.1 & 0.0225 & 37.934 & 1083.0 & 0.0223 \\
\hline 12 & 20.006 & 1306.5 & 0.0224 & 29.861 & 1173.5 & 0.0223 & 38.019 & 1076.1 & 0.0222 \\
\hline 13 & 20.034 & 1310.3 & 0.0222 & 30.003 & 1176.5 & 0.0222 & 38.073 & 1067.4 & 0.0221 \\
\hline 14 & 19.945 & 1314.8 & 0.0221 & 30.075 & 1183.3 & 0.0221 & 38.003 & 1055.3 & 0.0220 \\
\hline 15 & 19.590 & 1317.0 & 0.0220 & 29.909 & 1193.8 & 0.0220 & 37.605 & 1038.1 & 0.0219 \\
\hline 16 & 18.618 & 1306.9 & 0.0218 & 29.003 & 1201.8 & 0.0219 & 36.312 & 1012.5 & 0.0218 \\
\hline 17 & 16.260 & 1251.3 & 0.0217 & 26.026 & 1180.6 & 0.0217 & 32.557 & 969.0 & 0.0217 \\
\hline 18 & 11.463 & 1086.5 & 0.0216 & 18.716 & 1071.1 & 0.0216 & 23.189 & 876.5 & 0.0216 \\
\hline
\end{tabular}

\begin{tabular}{|c|c|c|c|c|c|c|}
\hline \multirow[b]{2}{*}{$\begin{array}{l}\text { Axial } \\
\text { Node }\end{array}$} & \multicolumn{3}{|c|}{ Datapoint (417.8 Cy11) } & \multicolumn{3}{|c|}{ Statepoint 74 (615.0 Cy11) } \\
\hline & $\begin{array}{c}\text { Burnup } \\
\text { (GWd/MTU) }\end{array}$ & $\begin{array}{c}\text { T-Fuel } \\
{ }^{\circ} \mathrm{F}\end{array}$ & $\begin{array}{c}\text { Spec. Vol. } \\
\mathrm{ft}^{3} / \mathrm{lbm}\end{array}$ & $\begin{array}{c}\text { Burnup } \\
\text { (GWd/MTU) }\end{array}$ & $\begin{array}{c}\text { T-Fuel } \\
{ }^{\circ} \mathrm{F}\end{array}$ & $\begin{array}{c}\text { Spec. Vol. } \\
\mathrm{ft}^{3} / \mathrm{bm}\end{array}$ \\
\hline & $417.8 \mathrm{Cy} 11$ & 312.8 Cy 11 & 312.8 Cy11 & 615.0 Cy 11 & 515.7 Cy 11 & 515.7 Cy11 \\
\hline 1 & 21.940 & 848.5 & 0.0234 & 25.517 & 857.4 & 0.0234 \\
\hline 2 & 34.666 & 965.3 & 0.0233 & 40.716 & 976.9 & 0.0233 \\
\hline 3 & 41.605 & 1038.1 & 0.0232 & 48.623 & 1024.6 & 0.0232 \\
\hline 4 & 44.027 & 1052.2 & 0.0231 & 51.161 & 1024.9 & 0.0231 \\
\hline 5 & 44.874 & 1052.3 & 0.0230 & 51.955 & 1017.3 & 0.0229 \\
\hline 6 & 45.198 & 1049.1 & 0.0229 & 52.209 & 1009.9 & 0.0228 \\
\hline 7 & 45.348 & 1045.2 & 0.0228 & 52.309 & 1004.0 & 0.0227 \\
\hline 8 & 45.442 & 1041.4 & 0.0226 & 52.373 & 999.4 & 0.0226 \\
\hline 9 & 45.519 & 1038.0 & 0.0225 & 52.436 & 995.9 & 0.0225 \\
\hline 10 & 45.596 & 1035.3 & 0.0224 & 52.514 & 993.3 & 0.0224 \\
\hline 11 & 45.687 & 1033.2 & 0.0223 & 52.620 & 991.6 & 0.0223 \\
\hline 12 & 45.795 & 1031.7 & 0.0222 & 52.760 & 990.9 & 0.0222 \\
\hline 13 & 45.878 & 1030.6 & 0.0221 & 52.896 & 991.5 & 0.0221 \\
\hline 14 & 45.823 & 1029.1 & 0.0220 & 52.914 & 993.6 & 0.0220 \\
\hline 15 & 45.398 & 1025.7 & 0.0219 & 52.563 & 996.8 & 0.0219 \\
\hline 16 & 43.945 & 1016.3 & 0.0218 & 51.116 & 997.7 & 0.0218 \\
\hline 17 & 39.601 & 985.6 & 0.0217 & 46.403 & 981.2 & 0.0217 \\
\hline 18 & 28.208 & 888.2 & 0.0216 & 33.278 & 885.4 & 0.0216 \\
\hline
\end{tabular}


Table 4-94. Burnup and TH Feedback Parameters by Axial Node for Assembly K10

\begin{tabular}{|c|c|c|c|c|c|c|c|c|c|}
\hline \multirow[b]{2}{*}{$\begin{array}{l}\text { Axial } \\
\text { Node }\end{array}$} & \multicolumn{3}{|c|}{ Statepoint 70 (27.0 Cy10) } & \multicolumn{3}{|c|}{ Statepoint $71(143.0$ Cy10) } & \multicolumn{3}{|c|}{ Statepoint 72 (209.0 Cy10) } \\
\hline & \begin{tabular}{c|} 
Burnup \\
(GWd/MTU)
\end{tabular} & $\begin{array}{l}\text { T-Fuel } \\
{ }^{\circ} \mathrm{F}\end{array}$ & $\begin{array}{c}\text { Spec. Vol. } \\
\mathrm{ft}^{3} / \mathrm{lbm}\end{array}$ & \begin{tabular}{|c|} 
Burnup \\
(GWd/MTU)
\end{tabular} & $\begin{array}{l}\text { T-Fuel } \\
{ }^{\circ} \mathrm{F}\end{array}$ & $\begin{array}{c}\text { Spec. Vol. } \\
\mathrm{ft}^{3} / \mathrm{lbm}\end{array}$ & $\begin{array}{c}\text { Burnup } \\
\text { (GWd/MTU) }\end{array}$ & $\begin{array}{l}\text { T-Fuel } \\
{ }^{\circ} \mathrm{F}\end{array}$ & $\begin{array}{c}\text { Spec. Vol. } \\
\mathrm{ft}^{3} / \mathrm{lbm}\end{array}$ \\
\hline & 27.0 Cy 10 & $18.0 \mathrm{Cy} 10$ & 18.0 Cy 10 & 143.0 Cy 10 & 82.9 Cy 10 & 82.9 Cy10 & 209.0 Cy 10 & 176.7 Cy 10 & 176.7 Cy 10 \\
\hline 1 & 0.547 & 1074.0 & 0.0237 & 2.955 & 1070.6 & 0.0238 & 4.429 & 1078.5 & 0.0238 \\
\hline 2 & 0.812 & 1269.3 & 0.0236 & 4.391 & 1256.9 & 0.0237 & 6.561 & 1247.7 & 0.0237 \\
\hline 3 & 0.995 & 1396.7 & 0.0235 & 5.352 & 1373.3 & 0.0235 & 7.946 & 1343.3 & 0.0236 \\
\hline 4 & 1.101 & 1465.4 & 0.0234 & 5.888 & 1432.7 & 0.0234 & 8.684 & 1384.6 & 0.0234 \\
\hline 5 & 1.165 & 1502.2 & 0.0232 & 6.196 & 1461.5 & 0.0233 & 9.087 & 1399.0 & 0.0233 \\
\hline 6 & 1.206 & 1520.5 & 0.0231 & 6.382 & 1473.8 & 0.0231 & 9.318 & 1402.3 & 0.0231 \\
\hline 7 & 1.232 & 1530.1 & 0.0229 & 6.496 & 1479.3 & 0.0230 & 9.454 & 1401.8 & 0.0230 \\
\hline 8 & 1.248 & 1533.9 & 0.0228 & 6.561 & 1480.6 & 0.0228 & 9.532 & 1399.8 & 0.0228 \\
\hline 9 & 1.254 & 1533.1 & 0.0227 & 6.591 & 1479.1 & 0.0227 & 9.569 & 1397.4 & 0.0227 \\
\hline 10 & 1.253 & 1528.3 & 0.0225 & 6.591 & 1475.2 & 0.0225 & 9.573 & 1395.0 & 0.0226 \\
\hline 11 & 1.243 & 1519.4 & 0.0224 & 6.562 & 1469.1 & 0.0224 & 9.546 & 1392.7 & 0.0224 \\
\hline 12 & 1.226 & 1505.9 & 0.0223 & 6.500 & 1460.2 & 0.0223 & 9.482 & 1390.0 & 0.0223 \\
\hline 13 & 1.198 & 1486.7 & 0.0221 & 6.393 & 1447.4 & 0.0221 & 9.363 & 1386.1 & 0.0222 \\
\hline 14 & 1.157 & 1458.2 & 0.0220 & 6.226 & 1428.6 & 0.0220 & 9.165 & 1379.3 & 0.0220 \\
\hline 15 & 1.097 & 1414.6 & 0.0219 & 5.963 & 1396.8 & 0.0219 & 8.832 & 1365.2 & 0.0219 \\
\hline 16 & 1.006 & 1347.7 & 0.0218 & 5.520 & 1341.3 & 0.0218 & 8.231 & 1329.6 & 0.0218 \\
\hline 17 & 0.853 & 1236.2 & 0.0217 & 4.718 & 1239.9 & 0.0217 & 7.077 & 1246.2 & 0.0217 \\
\hline 18 & 0.600 & 1051.6 & 0.0216 & 3.322 & 1059.9 & 0.0216 & 4.993 & 1077.0 & 0.0216 \\
\hline
\end{tabular}

\begin{tabular}{|c|c|c|c|c|c|c|c|c|c|}
\hline \multirow[b]{2}{*}{$\begin{array}{l}\text { Axial } \\
\text { Node }\end{array}$} & \multicolumn{3}{|c|}{ Datapoint (430.4 Cy10) } & \multicolumn{3}{|c|}{ Statepoint 73 (BOC Cy11) } & \multicolumn{3}{|c|}{ Datapoint (208.7 Cy11) } \\
\hline & $\begin{array}{c}\text { Burnup } \\
\text { (GWd/MTU) }\end{array}$ & $\begin{array}{c}\text { T-Fuel } \\
{ }^{\circ} \mathrm{F}\end{array}$ & $\begin{array}{c}\text { Spec. Vol. } \\
\mathrm{ft}^{3} / \mathrm{lbm}\end{array}$ & $\begin{array}{c}\text { Burnup } \\
\text { (GWd/MTU) }\end{array}$ & $\begin{array}{c}\begin{array}{c}\text { T-Fuel } \\
{ }^{\circ} \mathrm{F}\end{array} \\
\end{array}$ & $\begin{array}{c}\text { Spec. Vol. } \\
\mathrm{ft}^{3} / \mathrm{lbm}\end{array}$ & $\begin{array}{c}\text { Burnup } \\
\text { (GWd/MTU) }\end{array}$ & $\begin{array}{c}\text { T-Fuel } \\
{ }^{\circ} \mathrm{F}\end{array}$ & $\begin{array}{c}\text { Spec. Vol. } \\
\mathrm{ft}^{3} / \mathrm{lbm}\end{array}$ \\
\hline & $430.4 \mathrm{Cy} 10$ & 319.5 Cy10 & 319.5 Cy 10 & BOC Cy 11 & 549.3 Cy 10 & 549.3 Cy 10 & 208.7 Cy 11 & 103.8 Cy 11 & 103.8 Cy 11 \\
\hline 1 & 9.955 & 1090.4 & 0.0239 & 16.430 & 1086.9 & 0.0239 & 20.703 & 922.6 & 0.0235 \\
\hline 2 & 14.550 & 1241.1 & 0.0238 & 23.525 & 1199.6 & 0.0238 & 29.806 & 1011.9 & 0.0234 \\
\hline 3 & 17.229 & 1314.2 & 0.0236 & 27.176 & 1231.3 & 0.0236 & 34.489 & 1054.5 & 0.0233 \\
\hline 4 & 18.430 & 1330.7 & 0.0235 & 28.519 & 1227.1 & 0.0235 & 36.348 & 1081.3 & 0.0232 \\
\hline 5 & 18.963 & 1329.4 & 0.0233 & 28.975 & 1215.2 & 0.0233 & 37.062 & 1093.1 & 0.0231 \\
\hline 6 & 19.212 & 1323.9 & 0.0232 & 29.132 & 1204.5 & 0.0232 & 37.359 & 1098.1 & 0.0229 \\
\hline 7 & 19.339 & 1318.2 & 0.0230 & 29.201 & 1196.9 & 0.0230 & 37.509 & 1099.5 & 0.0228 \\
\hline 8 & 19.412 & 1313.8 & 0.0229 & 29.252 & 1191.7 & 0.0229 & 37.600 & 1098.6 & 0.0227 \\
\hline 9 & 19.459 & 1310.8 & 0.0228 & 29.308 & 1188.4 & 0.0227 & 37.667 & 1096.0 & 0.0226 \\
\hline 10 & 19.493 & 1309.5 & 0.0226 & 29.373 & 1186.5 & 0.0226 & 37.724 & 1092.3 & 0.0224 \\
\hline 11 & 19.516 & 1309.7 & 0.0225 & 29.447 & 1185.9 & 0.0225 & 37.783 & 1088.3 & 0.0223 \\
\hline 12 & 19.515 & 1311.3 & 0.0223 & 29.525 & 1187.0 & 0.0224 & 37.849 & 1084.5 & 0.0222 \\
\hline 13 & 19.468 & 1313.9 & 0.0222 & 29.592 & 1190.6 & 0.0222 & 37.889 & 1079.9 & 0.0221 \\
\hline 14 & 19.325 & 1316.5 & 0.0221 & 29.605 & 1197.6 & 0.0221 & 37.796 & 1070.5 & 0.0220 \\
\hline 15 & 18.963 & 1316.0 & 0.0220 & 29.417 & 1207.3 & 0.0220 & 37.383 & 1054.2 & 0.0219 \\
\hline 16 & 18.063 & 1303.2 & 0.0218 & 28.553 & 1213.1 & 0.0219 & 36.115 & 1028.1 & 0.0218 \\
\hline 17 & 15.872 & 1245.2 & 0.0217 & 25.705 & 1188.7 & 0.0217 & 32.475 & 984.7 & 0.0217 \\
\hline 18 & 11.314 & 1088.8 & 0.0216 & 18.680 & 1080.3 & 0.0216 & 23.374 & 890.7 & 0.0216 \\
\hline
\end{tabular}


Table 4-94. Burnup and TH Feedback Parameters by Axial Node for Assembly K10 (Cont.)

\begin{tabular}{|c|c|c|c|c|c|c|}
\hline \multirow{2}{*}{$\begin{array}{c}\text { Axial } \\
\text { Node }\end{array}$} & $\begin{array}{c}\text { Burnup } \\
\text { (GWd/MTU) }\end{array}$ & $\begin{array}{c}\text { T-Fuel } \\
{ }^{\circ} \mathrm{F}\end{array}$ & $\begin{array}{c}\text { Spec. Vol. } \\
\mathrm{ft}^{3} / \mathrm{lbm}\end{array}$ & $\begin{array}{c}\text { Burnup } \\
(\mathrm{GWd} / \mathrm{MTU})\end{array}$ & $\begin{array}{c}\text { S-Fuel } \\
{ }^{\circ} \mathrm{F}\end{array}$ & $\begin{array}{c}\text { Spec. Vol. } \\
\mathrm{ft}^{3} / \mathrm{lbm}\end{array}$ \\
\hline & $417.8 \mathrm{Cy} 11$ & $312.8 \mathrm{Cy} 11$ & $312.8 \mathrm{Cy} 11$ & $615.0 \mathrm{Cy} 11$ & 515.7 Cy11 & $515.7 \mathrm{Cy} 11$ \\
\hline 1 & 25.404 & 924.0 & 0.0235 & 30.277 & 929.0 & 0.0235 \\
\hline 2 & 36.424 & 1002.2 & 0.0234 & 43.020 & 1013.6 & 0.0234 \\
\hline 3 & 41.939 & 1042.3 & 0.0233 & 49.167 & 1040.3 & 0.0232 \\
\hline 4 & 44.088 & 1053.9 & 0.0231 & 51.433 & 1040.4 & 0.0231 \\
\hline 5 & 44.875 & 1054.5 & 0.0230 & 52.175 & 1033.5 & 0.0230 \\
\hline 6 & 45.176 & 1051.8 & 0.0229 & 52.414 & 1026.4 & 0.0229 \\
\hline 7 & 45.316 & 1048.4 & 0.0228 & 52.510 & 1020.9 & 0.0228 \\
\hline 8 & 45.398 & 1045.2 & 0.0227 & 52.570 & 1016.8 & 0.0227 \\
\hline 9 & 45.463 & 1042.4 & 0.0225 & 52.629 & 1013.7 & 0.0225 \\
\hline 10 & 45.529 & 1040.2 & 0.0224 & 52.706 & 1011.7 & 0.0224 \\
\hline 11 & 45.616 & 1039.2 & 0.0223 & 52.821 & 1010.6 & 0.0223 \\
\hline 12 & 45.735 & 1039.6 & 0.0222 & 52.997 & 1011.3 & 0.0222 \\
\hline 13 & 45.842 & 1040.8 & 0.0221 & 53.187 & 1013.7 & 0.0221 \\
\hline 14 & 45.785 & 1040.4 & 0.0220 & 53.221 & 1016.9 & 0.0220 \\
\hline 15 & 45.339 & 1036.5 & 0.0219 & 52.850 & 1020.0 & 0.0219 \\
\hline 16 & 43.886 & 1025.4 & 0.0218 & 51.375 & 1019.2 & 0.0218 \\
\hline 17 & 39.633 & 992.9 & 0.0217 & 46.697 & 998.9 & 0.0217 \\
\hline 18 & 28.536 & 896.1 & 0.0216 & 33.815 & 899.5 & 0.0216 \\
\hline
\end{tabular}

Table 4-95. Burnup and TH Feedback Parameters by Axial Node for Assembly K10a

\begin{tabular}{|c|c|c|c|c|c|c|c|c|c|}
\hline \multirow[b]{2}{*}{$\begin{array}{l}\text { Axlal } \\
\text { Node }\end{array}$} & \multicolumn{3}{|c|}{ Statepolnt 70 (27.0 Cy10) } & \multicolumn{3}{|c|}{ Statepoint 71 (143.0 Cy10) } & \multicolumn{3}{|c|}{ Statepoint 72 (209.0 Cy10) } \\
\hline & $\begin{array}{c}\text { Burnup } \\
\text { (GWd/MTU) } \\
\end{array}$ & $\begin{array}{c}\text { T-Fuel } \\
{ }^{\circ} \mathrm{F} \\
\end{array}$ & $\begin{array}{c}\text { Spec. Vol. } \\
\mathrm{ft}^{3} / \mathrm{lbm}\end{array}$ & $\begin{array}{c}\text { Burnup } \\
\text { (GWd/MTU) }\end{array}$ & $\begin{array}{l}\text { T-Fuel } \\
{ }^{\circ} \mathrm{F}\end{array}$ & $\begin{array}{c}\text { Spec. Vol. } \\
\mathrm{ft}^{3} / \mathrm{lbm}\end{array}$ & \begin{tabular}{|c|} 
Burnup \\
(GWd/MTU)
\end{tabular} & $\begin{array}{l}\text { T-Fuel } \\
{ }^{\circ} \mathrm{F}\end{array}$ & $\begin{array}{l}\text { Spec. Vol. } \\
\mathrm{ft}^{3} / \mathrm{lbm}\end{array}$ \\
\hline & 27.0 Cy 10 & $18.0 \mathrm{Cy} 10$ & 18.0 Cy10 & 143.0 Cy 10 & 82.9 Cy 10 & 82.9 Cy 10 & 209.0 Cy 10 & 176.7 Cy 10 & 176.7 Cy 10 \\
\hline 1 & 0.547 & 1074.0 & 0.0237 & 2.955 & 1070.6 & 0.0238 & 4.429 & 1078.5 & 0.0238 \\
\hline 2 & 0.812 & 1269.3 & 0.0236 & 4.391 & 1256.9 & 0.0237 & 6.561 & 1247.7 & 0.0237 \\
\hline 3 & 0.995 & 1396.7 & 0.0235 & 5.352 & 1373.3 & 0.0235 & 7.946 & 1343.3 & 0.0236 \\
\hline 4 & 1.101 & 1465.4 & 0.0234 & 5.888 & 1432.7 & 0.0234 & 8.684 & 1384.6 & 0.0234 \\
\hline 5 & 1.165 & 1502.2 & 0.0232 & 6.196 & 1461.5 & 0.0233 & 9.087 & 1399.0 & 0.0233 \\
\hline 6 & 1.206 & 1520.5 & 0.0231 & 6.382 & 1473.8 & 0.0231 & 9.318 & 1402.3 & 0.0231 \\
\hline 7 & 1.232 & 1530.1 & 0.0229 & 6.496 & 1479.3 & 0.0230 & 9.454 & 1401.8 & 0.0230 \\
\hline 8 & 1.248 & 1533.9 & 0.0228 & 6.561 & 1480.6 & 0.0228 & 9.532 & 1399.8 & 0.0228 \\
\hline 9 & 1.254 & 1533.1 & 0.0227 & 6.591 & 1479.1 & 0.0227 & 9.569 & 1397.4 & 0.0227 \\
\hline 10 & 1.253 & 1528.3 & 0.0225 & 6.591 & 1475.2 & 0.0225 & 9.573 & 1395.0 & 0.0226 \\
\hline 11 & 1.243 & 1519.4 & 0.0224 & 6.562 & 1469.1 & 0.0224 & 9.546 & 1392.7 & 0.0224 \\
\hline 12 & 1.226 & 1505.9 & 0.0223 & 6.500 & 1460.2 & 0.0223 & 9.482 & 1390.0 & 0.0223 \\
\hline 13 & 1.198 & 1486.7 & 0.0221 & 6.393 & 1447.4 & 0.0221 & 9.363 & 1386.1 & 0.0222 \\
\hline 14 & 1.157 & 1458.2 & 0.0220 & 6.226 & 1428.6 & 0.0220 & 9.165 & 1379.3 & 0.0220 \\
\hline 15 & 1.097 & 1414.6 & 0.0219 & 5.963 & 1396.8 & 0.0219 & 8.832 & 1365.2 & 0.0219 \\
\hline 16 & 1.006 & 1347.7 & 0.0218 & 5.520 & 1341.3 & 0.0218 & 8.231 & 1329.6 & 0.0218 \\
\hline 17 & 0.853 & 1236.2 & 0.0217 & 4.718 & 1239.9 & 0.0217 & 7.077 & 1246.2 & 0.0217 \\
\hline 18 & 0.600 & 1051.6 & 0.0216 & 3.322 & 1059.9 & 0.0216 & 4.993 & 1077.0 & 0.0216 \\
\hline
\end{tabular}


Table 4-95. Burnup and TH Feedback Parameters by Axial Node for Assembly K10a (Cont.)

\begin{tabular}{|c|c|c|c|c|c|c|c|c|c|}
\hline \multirow[b]{2}{*}{$\begin{array}{l}\text { Axial } \\
\text { Node }\end{array}$} & \multicolumn{3}{|c|}{ Datapoint (430.4 Cy10) } & \multicolumn{3}{|c|}{ Statepoint 73 (BOC Cy11) } & \multicolumn{3}{|c|}{ Datapoint (208.7 Cy11) } \\
\hline & $\begin{array}{c}\text { Burnup } \\
\text { (GWd/MTU) }\end{array}$ & $\begin{array}{l}\text { T-Fuel } \\
{ }^{\circ} \mathrm{F}\end{array}$ & $\begin{array}{c}\text { Spec. Vol. } \\
\mathrm{ft}^{3} / \mathrm{lbm}\end{array}$ & \begin{tabular}{|c|} 
Burnup \\
(GWd/MTU)
\end{tabular} & $\begin{array}{l}\text { T-Fuel } \\
{ }^{\circ} \mathrm{F}\end{array}$ & $\begin{array}{c}\text { Spec. Vol. } \\
\mathrm{ft}^{3} / \mathrm{lbm}\end{array}$ & $\begin{array}{c}\text { Burnup } \\
\text { (GWd/MTU) }\end{array}$ & $\begin{array}{l}\text { T-Fuel } \\
{ }^{\circ} \mathrm{F}\end{array}$ & $\begin{array}{c}\text { Spec. Vol. } \\
\mathrm{ft}^{3} / \mathrm{lbm}\end{array}$ \\
\hline & 430.4 Cy 10 & 319.5 Cy 10 & 319.5 Cy 10 & BOC Cy 11 & 549.3 Cy 10 & 549.3 Cy 10 & 208.7 Cy 11 & 103.8 Cy 11 & 103.8 Cy 11 \\
\hline 1 & 9.955 & 1090.4 & 0.0239 & 16.469 & 1086.9 & 0.0239 & 21.007 & 942.1 & 0.0236 \\
\hline 2 & 14.550 & 1241.1 & 0.0238 & 23.582 & 1199.6 & 0.0238 & 30.253 & 1036.8 & 0.0235 \\
\hline 3 & 17.229 & 1314.2 & 0.0236 & 27.238 & 1231.3 & 0.0236 & 34.952 & 1080.3 & 0.0234 \\
\hline 4 & 18.430 & 1330.7 & 0.0235 & 28.579 & 1227.1 & 0.0235 & 36.797 & 1105.7 & 0.0232 \\
\hline 5 & 18.963 & 1329.4 & 0.0233 & 29.033 & 1215.2 & 0.0233 & 37.516 & 1117.5 & 0.0231 \\
\hline 6 & 19.212 & 1323.9 & 0.0232 & 29.189 & 1204.5 & 0.0232 & 37.814 & 1122.3 & 0.0230 \\
\hline 7 & 19.339 & 1318.2 & 0.0230 & 29.259 & 1196.9 & 0.0230 & 37.954 & 1122.9 & 0.0228 \\
\hline 8 & 19.412 & 1313.8 & 0.0229 & 29.312 & 1191.7 & 0.0229 & 38.031 & 1120.9 & 0.0227 \\
\hline 9 & 19.459 & 1310.8 & 0.0228 & 29.368 & 1188.4 & 0.0227 & 38.080 & 1117.0 & 0.0226 \\
\hline 10 & 19.493 & 1309.5 & 0.0226 & 29.435 & 1186.5 & 0.0226 & 38.113 & 1111.6 & 0.0225 \\
\hline 11 & 19.516 & 1309.7 & 0.0225 & 29.512 & 1185.9 & 0.0225 & 38.134 & 1105.0 & 0.0223 \\
\hline 12 & 19.515 & 1311.3 & 0.0223 & 29.596 & 1187.0 & 0.0224 & 38.138 & 1097.0 & 0.0222 \\
\hline 13 & 19.468 & 1313.9 & 0.0222 & 29.671 & 1190.6 & 0.0222 & 38.102 & 1087.2 & 0.0221 \\
\hline 14 & 19.325 & 1316.5 & 0.0221 & 29.689 & 1197.6 & 0.0221 & 37.966 & 1074.9 & 0.0220 \\
\hline 15 & 18.963 & 1316.0 & 0.0220 & 29.502 & 1207.3 & 0.0220 & 37.549 & 1058.5 & 0.0219 \\
\hline 16 & 18.063 & 1303.2 & 0.0218 & 28.635 & 1213.1 & 0.0219 & 36.300 & 1033.9 & 0.0218 \\
\hline 17 & 15.872 & 1245.2 & 0.0217 & 25.777 & 1188.7 & 0.0217 & 32.639 & 989.4 & 0.0217 \\
\hline 18 & 11.314 & 1088.8 & 0.0216 & 18.728 & 1080.3 & 0.0216 & 23.436 & 891.0 & 0.0216 \\
\hline
\end{tabular}

\begin{tabular}{|c|c|c|c|c|c|c|}
\hline \multirow[b]{2}{*}{$\begin{array}{l}\text { Axial } \\
\text { Node }\end{array}$} & \multicolumn{3}{|c|}{ Datapoint (417.8 Cy11) } & \multicolumn{3}{|c|}{ Statepoint $74(615.0$ Cy11) } \\
\hline & $\begin{array}{c}\text { Burnup } \\
\text { (GWd/MTU) }\end{array}$ & $\begin{array}{c}\text { T-Fuel } \\
{ }^{\circ} \mathbf{F}\end{array}$ & $\begin{array}{c}\text { Spec. Vol. } \\
\mathrm{tt}^{3} / \mathrm{lbm}\end{array}$ & $\begin{array}{c}\text { Burnup } \\
\text { (GWd/MTU) }\end{array}$ & $\begin{array}{c}\text { T-Fuel } \\
{ }^{\circ} \mathrm{F}\end{array}$ & $\begin{array}{c}\text { Spec. Vol. } \\
\mathrm{ft}^{3} / \mathrm{lbm}\end{array}$ \\
\hline & $417.8 \mathrm{Cy} 11$ & 312.8 Cy 11 & 312.8 Cy 11 & $615.0 \mathrm{Cy} 11$ & 515.7 Cy 11 & 515.7 Cy 11 \\
\hline 1 & 25.852 & 932.5 & 0.0235 & 30.756 & 927.4 & 0.0234 \\
\hline 2 & 37.054 & 1013.8 & 0.0234 & 43.628 & 1009.5 & 0.0233 \\
\hline 3 & 42.555 & 1051.7 & 0.0233 & 49.677 & 1030.5 & 0.0232 \\
\hline 4 & 44.668 & 1061.7 & 0.0231 & 51.860 & 1027.8 & 0.0231 \\
\hline 5 & 45.462 & 1062.4 & 0.0230 & 52.600 & 1020.2 & 0.0230 \\
\hline 6 & 45.766 & 1059.8 & 0.0229 & 52.838 & 1012.9 & 0.0228 \\
\hline 7 & 45.894 & 1056.3 & 0.0228 & 52.915 & 1007.0 & 0.0227 \\
\hline 8 & 45.957 & 1052.8 & 0.0227 & 52.949 & 1002.5 & 0.0226 \\
\hline 9 & 45.997 & 1049.6 & 0.0226 & 52.977 & 999.2 & 0.0225 \\
\hline 10 & 46.029 & 1046.9 & 0.0224 & 53.012 & 996.8 & 0.0224 \\
\hline 11 & 46.057 & 1044.6 & 0.0223 & 53.057 & 995.3 & 0.0223 \\
\hline 12 & 46.074 & 1042.8 & 0.0222 & 53.106 & 994.8 & 0.0222 \\
\hline 13 & 46.053 & 1041.0 & 0.0221 & 53.136 & 995.5 & 0.0221 \\
\hline 14 & 45.922 & 1038.9 & 0.0220 & 53.076 & 997.7 & 0.0220 \\
\hline 15 & 45.471 & 1034.9 & 0.0219 & 52.698 & 1000.8 & 0.0219 \\
\hline 16 & 44.054 & 1024.8 & 0.0218 & 51.283 & 1001.5 & 0.0218 \\
\hline 17 & 39.789 & 992.7 & 0.0217 & 46.642 & 984.4 & 0.0217 \\
\hline 18 & 28.562 & 893.9 & 0.0216 & 33.698 & 889.4 & 0.0216 \\
\hline
\end{tabular}


Table 4-96. Burnup and TH Feedback Parameters by Axial Node for Assembly K12

\begin{tabular}{|c|c|c|c|c|c|c|c|c|c|}
\hline \multirow[b]{2}{*}{$\begin{array}{l}\text { Axial } \\
\text { Node } \\
\end{array}$} & \multicolumn{3}{|c|}{ Statepoint 70 (27.0 Cy10) } & \multicolumn{3}{|c|}{ Statepoint 71 (143.0 Cy10) } & \multicolumn{3}{|c|}{ Statepoint 72 (209.0 Cy10) } \\
\hline & $\begin{array}{c}\text { Burnup } \\
\text { (GWd/MTU) }\end{array}$ & $\begin{array}{c}\text { T-Fuel } \\
{ }^{\circ} \mathrm{F} \\
\end{array}$ & $\begin{array}{c}\text { Spec. Vol. } \\
\mathrm{ft}^{3} / \mathrm{lbm}\end{array}$ & $\begin{array}{c}\text { Burnup } \\
\text { (GWd/MTU) }\end{array}$ & $\begin{array}{c}\text { T-Fuel } \\
{ }^{\circ} \mathrm{F} \\
\end{array}$ & $\begin{array}{c}\text { Spec. Vol. } \\
\mathrm{tt}^{3} / \mathrm{lbm}\end{array}$ & $\begin{array}{c}\text { Burnup } \\
\text { (GWd/MTU) }\end{array}$ & $\begin{array}{c}\text { T-Fuel } \\
{ }^{\circ} \mathrm{F} \\
\end{array}$ & $\begin{array}{c}\text { Spec. Vol. } \\
\mathrm{ft}^{3} / \mathrm{lbm}\end{array}$ \\
\hline & 27.0 Cy 10 & 18.0 Cy 10 & 18.0 Cy 10 & 143.0 Cy 10 & 82.9 Cy 10 & 82.9 Cy 10 & $209.0 \mathrm{Cy} 10$ & 176.7 Cy10 & 176.7 Cy 10 \\
\hline 1 & 0.520 & 1059.1 & 0.0237 & 2.813 & 1053.9 & 0.0238 & 4.242 & 1069.0 & 0.0239 \\
\hline 2 & 0.776 & 1250.4 & 0.0236 & 4.234 & 1245.2 & 0.0237 & 6.395 & 1250.1 & 0.0238 \\
\hline 3 & 0.968 & 1380.7 & 0.0235 & 5.296 & 1374.3 & 0.0236 & 7.945 & 1361.5 & 0.0237 \\
\hline 4 & 1.079 & 1450.4 & 0.0234 & 5.894 & 1440.8 & 0.0235 & 8.776 & 1407.8 & 0.0235 \\
\hline 5 & 1.142 & 1487.1 & 0.0232 & 6.213 & 1470.0 & 0.0233 & 9.193 & 1420.0 & 0.0234 \\
\hline 6 & 1.183 & 1507.0 & 0.0231 & 6.410 & 1483.4 & 0.0232 & 9.437 & 1423.3 & 0.0232 \\
\hline 7 & 1.211 & 1517.8 & 0.0230 & 6.540 & 1490.6 & 0.0230 & 9.593 & 1423.0 & 0.0231 \\
\hline 8 & 1.228 & 1523.3 & 0.0228 & 6.622 & 1493.6 & 0.0229 & 9.691 & 1421.3 & 0.0229 \\
\hline 9 & 1.238 & 1524.2 & 0.0227 & 6.668 & 1493.6 & 0.0227 & 9.749 & 1419.3 & 0.0228 \\
\hline 10 & 1.240 & 1521.4 & 0.0225 & 6.686 & 1491.3 & 0.0226 & 9.777 & 1417.7 & 0.0226 \\
\hline 11 & 1.235 & 1515.4 & 0.0224 & 6.681 & 1487.3 & 0.0224 & 9.784 & 1416.9 & 0.0225 \\
\hline 12 & 1.227 & 1507.4 & 0.0223 & 6.663 & 1482.4 & 0.0223 & 9.784 & 1417.4 & 0.0223 \\
\hline 13 & 1.212 & 1495.7 & 0.0221 & 6.620 & 1475.7 & 0.0222 & 9.757 & 1418.4 & 0.0222 \\
\hline 14 & 1.177 & 1472.0 & 0.0220 & 6.478 & 1459.6 & 0.0220 & 9.600 & 1414.4 & 0.0221 \\
\hline 15 & 1.117 & 1428.7 & 0.0219 & 6.202 & 1429.9 & 0.0219 & 9.253 & 1401.2 & 0.0219 \\
\hline 16 & 1.022 & 1359.0 & 0.0218 & 5.721 & 1370.9 & 0.0218 & 8.597 & 1368.9 & 0.0218 \\
\hline 17 & 0.868 & 1246.5 & 0.0217 & 4.877 & 1263.1 & 0.0217 & 7.367 & 1279.8 & 0.0217 \\
\hline 18 & 0.624 & 1068.6 & 0.0216 & 3.474 & 1080.7 & 0.0216 & 5.230 & 1098.4 & 0.0216 \\
\hline
\end{tabular}

\begin{tabular}{|c|c|c|c|c|c|c|c|c|c|}
\hline \multirow[b]{2}{*}{$\begin{array}{l}\text { Axial } \\
\text { Node }\end{array}$} & \multicolumn{3}{|c|}{ Datapoint (430.4 Cy10) } & \multicolumn{3}{|c|}{ Statepoint 73 (BOC Cy11) } & \multicolumn{3}{|c|}{ Datapoint (208.7 Cy11) } \\
\hline & $\begin{array}{c}\text { Burnup } \\
\text { (GWd/MTU) }\end{array}$ & $\begin{array}{c}\text { T-Fuel } \\
{ }^{\circ} \mathrm{F}\end{array}$ & $\begin{array}{c}\text { Spec. Vol. } \\
\mathrm{ft}^{3} \mathrm{mbm}\end{array}$ & $\begin{array}{c}\text { Burnup } \\
\text { (GWd/MTU) }\end{array}$ & $\begin{array}{c}\text { T-Fuel } \\
{ }^{\circ} \mathrm{F}\end{array}$ & $\begin{array}{c}\text { Spec. Vol. } \\
\mathrm{ft}^{3} / \mathrm{lbm}\end{array}$ & $\begin{array}{c}\text { Burnup } \\
\text { (GWd/MTU) }\end{array}$ & $\begin{array}{c}\text { T-Fuel } \\
{ }^{\circ} \mathrm{F}\end{array}$ & $\begin{array}{c}\text { Spec. Vol. } \\
\mathrm{ft}^{3} / \mathrm{lbm}\end{array}$ \\
\hline & 430.4 Cy 10 & 319.5 Cy 10 & 319.5 Cy10 & BOC Cy11 & 549.3 Cy 10 & 549.3 Cy 10 & 208.7 Cy 11 & $103.8 \mathrm{Cy} 11$ & 103.8 Cy 11 \\
\hline 1 & 9.623 & 1083.3 & 0.0239 & 15.903 & 1075.2 & 0.0239 & 20.051 & 914.2 & 0.0234 \\
\hline 2 & 14.415 & 1247.1 & 0.0238 & 23.374 & 1198.4 & 0.0238 & 29.443 & 997.1 & 0.0233 \\
\hline 3 & 17.398 & 1326.4 & 0.0237 & 27.417 & 1233.1 & 0.0236 & 34.428 & 1032.8 & 0.0232 \\
\hline 4 & 18.704 & 1339.2 & 0.0235 & 28.843 & 1227.4 & 0.0235 & 36.250 & 1052.4 & 0.0231 \\
\hline 5 & 19.210 & 1334.9 & 0.0234 & 29.226 & 1213.2 & 0.0233 & 36.628 & 1048.3 & 0.0230 \\
\hline 6 & 19.443 & 1327.4 & 0.0232 & 29.345 & 1201.4 & 0.0232 & 36.851 & 1051.8 & 0.0228 \\
\hline 7 & 19.576 & 1320.7 & 0.0231 & 29.412 & 1193.2 & 0.0230 & 36.989 & 1053.3 & 0.0227 \\
\hline 8 & 19.663 & 1315.4 & 0.0229 & 29.472 & 1187.7 & 0.0229 & 37.083 & 1052.4 & 0.0226 \\
\hline 9 & 19.729 & 1312.0 & 0.0228 & 29.541 & 1184.1 & 0.0227 & 37.159 & 1049.8 & 0.0225 \\
\hline 10 & 19.790 & 1310.4 & 0.0226 & 29.628 & 1181.7 & 0.0226 & 37.234 & 1046.1 & 0.0224 \\
\hline 11 & 19.860 & 1311.0 & 0.0225 & 29.751 & 1180.7 & 0.0225 & 37.341 & 1042.2 & 0.0223 \\
\hline 12 & 19.966 & 1314.3 & 0.0224 & 29.955 & 1182.0 & 0.0224 & 37.610 & 1043.1 & 0.0222 \\
\hline 13 & 20.084 & 1319.6 & 0.0222 & 30.229 & 1186.9 & 0.0222 & 38.273 & 1064.3 & 0.0221 \\
\hline 14 & 20.029 & 1324.2 & 0.0221 & 30.351 & 1194.4 & 0.0221 & 38.326 & 1056.8 & 0.0220 \\
\hline 15 & 19.683 & 1325.6 & 0.0220 & 30.194 & 1204.9 & 0.0220 & 37.939 & 1039.8 & 0.0219 \\
\hline 16 & 18.739 & 1314.6 & 0.0218 & 29.311 & 1212.4 & 0.0219 & 36.651 & 1013.2 & 0.0218 \\
\hline 17 & 16.444 & 1260.3 & 0.0217 & 26.386 & 1190.0 & 0.0217 & 32.948 & 968.2 & 0.0217 \\
\hline 18 & 11.702 & 1095.7 & 0.0216 & 19.108 & 1079.0 & 0.0216 & 23.642 & 878.2 & 0.0216 \\
\hline
\end{tabular}


Table 4-96. Burnup and TH Feedback Parameters by Axial Node for Assembly K12 (Cont.)

\begin{tabular}{|c|c|c|c|c|c|c|}
\hline \multirow[b]{2}{*}{$\begin{array}{l}\text { Axial } \\
\text { Node }\end{array}$} & \multicolumn{3}{|c|}{ Datapoint (417.8 Cy11) } & \multicolumn{3}{|c|}{ Statepoint 74 (615.0 Cy11) } \\
\hline & $\begin{array}{c}\text { Burnup } \\
\text { (GWd/MTU) }\end{array}$ & $\begin{array}{l}\text { T-Fuel } \\
{ }^{\circ} \mathrm{F}\end{array}$ & $\begin{array}{l}\text { Spec. Vol. } \\
\mathrm{ft}^{3} / \mathrm{lbm}\end{array}$ & $\begin{array}{c}\text { Burnup } \\
\text { (GWd/MTU) }\end{array}$ & $\begin{array}{l}\text { T-Fuel } \\
{ }^{\circ} \mathrm{F}\end{array}$ & $\begin{array}{c}\text { Spec. Vol. } \\
\mathrm{ft}^{3} / \mathrm{lbm}\end{array}$ \\
\hline & 417.8 Cy 11 & 312.8 Cy 11 & 312.8 Cy 11 & $615.0 \mathrm{Cy} 11$ & 515.7 Cy 11 & 515.7 Cy11 \\
\hline 1 & 24.671 & 920.9 & 0.0234 & 29.451 & 924.9 & 0.0234 \\
\hline 2 & 35.949 & 994.2 & 0.0233 & 42.411 & 1003.7 & 0.0233 \\
\hline 3 & 41.697 & 1029.5 & 0.0232 & 48.726 & 1025.9 & 0.0232 \\
\hline 4 & 43.690 & 1033.3 & 0.0231 & 50.734 & 1020.5 & 0.0230 \\
\hline 5 & 43.919 & 1021.8 & 0.0229 & 50.725 & 1002.5 & 0.0229 \\
\hline 6 & 44.122 & 1017.9 & 0.0228 & 50.847 & 994.6 & 0.0228 \\
\hline 7 & 44.248 & 1014.6 & 0.0227 & 50.930 & 989.3 & 0.0227 \\
\hline 8 & 44.331 & 1011.4 & 0.0226 & 50.990 & 985.4 & 0.0226 \\
\hline 9 & 44.402 & 1008.5 & 0.0225 & 51.053 & 982.4 & 0.0225 \\
\hline 10 & 44.483 & 1006.3 & 0.0224 & 51.141 & 980.3 & 0.0224 \\
\hline 11 & 44.617 & 1005.5 & 0.0223 & 51.303 & 979.3 & 0.0223 \\
\hline 12 & 45.022 & 1011.4 & 0.0222 & 51.827 & 983.2 & 0.0222 \\
\hline 13 & 46.106 & 1032.1 & 0.0221 & 53.312 & 1003.7 & 0.0221 \\
\hline 14 & 46.228 & 1033.3 & 0.0220 & 53.554 & 1008.6 & 0.0220 \\
\hline 15 & 45.809 & 1029.5 & 0.0219 & 53.210 & 1011.3 & 0.0219 \\
\hline 16 & 44.339 & 1018.7 & 0.0218 & 51.721 & 1010.8 & 0.0218 \\
\hline 17 & 40.029 & 986.9 & 0.0217 & 46.994 & 991.2 & 0.0217 \\
\hline 18 & 28.709 & 888.6 & 0.0216 & 33.885 & 892.0 & 0.0216 \\
\hline
\end{tabular}

Table 4-97. Burnup and TH Feedback Parameters by Axial Node for Assembly K14

\begin{tabular}{|c|c|c|c|c|c|c|c|c|c|}
\hline \multirow[b]{2}{*}{$\begin{array}{l}\text { Axial } \\
\text { Node }\end{array}$} & \multicolumn{3}{|c|}{ Statepoint 70 (27.0 Cy10) } & \multicolumn{3}{|c|}{ Statepoint 71 (143.0 Cy10) } & \multicolumn{3}{|c|}{ Statepoint 72 (209.0 Cy10) } \\
\hline & $\begin{array}{c}\text { Burnup } \\
\text { (GWd/MTU) }\end{array}$ & $\begin{array}{l}\text { T-Fuel } \\
{ }^{\circ} \mathrm{F}\end{array}$ & $\begin{array}{c}\text { Spec. Vol. } \\
\mathrm{ft}^{3} / \mathrm{lbm}\end{array}$ & $\begin{array}{c}\text { Burnup } \\
\text { (GWd/MTU) }\end{array}$ & $\begin{array}{l}\text { T-Fuel } \\
{ }^{\circ} \mathrm{F}\end{array}$ & $\begin{array}{l}\text { Spec. Vol. } \\
\mathrm{ft}^{3} / \mathrm{lbm}\end{array}$ & $\begin{array}{c}\text { Burnup } \\
\text { (GWd/MTU) }\end{array}$ & $\begin{array}{l}\text { T-Fuel } \\
{ }^{\circ} \mathrm{F}\end{array}$ & $\begin{array}{c}\text { Spec. Vol. } \\
\mathrm{ft}^{3} / \mathrm{lbm}\end{array}$ \\
\hline & 27.0 Cy 10 & 18.0 Cy 10 & 18.0 Cy 10 & 143.0 Cy 10 & 82.9 Cy 10 & 82.9 Cy 10 & 209.0 Cy 10 & 176.7 Cy 10 & 176.7 Cy 10 \\
\hline 1 & 0.419 & 960.9 & 0.0235 & 2.300 & 968.3 & 0.0236 & 3.477 & 987.6 & 0.0236 \\
\hline 2 & 0.691 & 1172.8 & 0.0235 & 3.776 & 1174.5 & 0.0235 & 5.674 & 1178.2 & 0.0235 \\
\hline 3 & 0.874 & 1301.5 & 0.0233 & 4.747 & 1294.9 & 0.0234 & 7.068 & 1275.4 & 0.0234 \\
\hline 4 & 0.986 & 1375.0 & 0.0232 & 5.309 & 1359.5 & 0.0233 & 7.834 & 1317.3 & 0.0232 \\
\hline 5 & 1.056 & 1417.2 & 0.0231 & 5.648 & 1392.8 & 0.0231 & 8.269 & 1332.7 & 0.0231 \\
\hline 6 & 1.103 & 1441.8 & 0.0230 & 5.861 & 1410.1 & 0.0230 & 8.528 & 1336.9 & 0.0230 \\
\hline 7 & 1.135 & 1456.5 & 0.0228 & 6.000 & 1419.6 & 0.0229 & 8.692 & 1337.0 & 0.0229 \\
\hline 8 & 1.156 & 1464.9 & 0.0227 & 6.092 & 1424.4 & 0.0227 & 8.798 & 1335.8 & 0.0227 \\
\hline 9 & 1.168 & 1468.4 & 0.0226 & 6.149 & 1426.2 & 0.0226 & 8.868 & 1334.4 & 0.0226 \\
\hline 10 & 1.173 & 1467.7 & 0.0224 & 6.180 & 1425.6 & 0.0225 & 8.909 & 1333.4 & 0.0225 \\
\hline 11 & 1.171 & 1462.8 & 0.0223 & 6.183 & 1422.7 & 0.0223 & 8.925 & 1332.9 & 0.0224 \\
\hline 12 & 1.160 & 1452.9 & 0.0222 & 6.154 & 1416.8 & 0.0222 & 8.905 & 1332.6 & 0.0222 \\
\hline 13 & 1.138 & 1436.2 & 0.0221 & 6.077 & 1406.3 & 0.0221 & 8.831 & 1331.4 & 0.0221 \\
\hline 14 & 1.100 & 1409.8 & 0.0220 & 5.928 & 1388.5 & 0.0220 & 8.666 & 1327.3 & 0.0220 \\
\hline 15 & 1.039 & 1367.5 & 0.0219 & 5.659 & 1357.7 & 0.0219 & 8.336 & 1315.0 & 0.0219 \\
\hline 16 & 0.940 & 1296.2 & 0.0218 & 5.176 & 1299.5 & 0.0218 & 7.695 & 1282.0 & 0.0218 \\
\hline 17 & 0.771 & 1173.9 & 0.0217 & 4.288 & 1186.6 & 0.0217 & 6.432 & 1193.7 & 0.0217 \\
\hline 18 & 0.488 & 959.7 & 0.0216 & 2.722 & 976.5 & 0.0216 & 4.105 & 999.4 & 0.0216 \\
\hline
\end{tabular}


Table 4-97. Burnup and TH Feedback Parameters by Axial Node for Assembly K14 (Cont.)

\begin{tabular}{|c|c|c|c|c|c|c|c|c|c|}
\hline \multirow[b]{2}{*}{$\begin{array}{l}\text { Axial } \\
\text { Node }\end{array}$} & \multicolumn{3}{|c|}{ Datapoint (430.4 Cy10) } & \multicolumn{3}{|c|}{ Statepoint 73 (BOC Cy11) } & \multicolumn{3}{|c|}{ Datapoint (208.7 Cy 11) } \\
\hline & $\begin{array}{c}\text { Burnup } \\
\text { (GWd/MTU) }\end{array}$ & $\begin{array}{c}\text { T-Fuel } \\
{ }^{\circ} \mathrm{F}\end{array}$ & $\begin{array}{c}\text { Spec. Vol. } \\
\mathrm{ft}^{3} / \mathrm{lbm}\end{array}$ & $\begin{array}{c}\text { Burnup } \\
\text { (GWd/MTU) }\end{array}$ & $\begin{array}{c}\text { T-Fuel } \\
{ }^{\circ} \mathrm{F} \\
\end{array}$ & \begin{tabular}{|c|} 
Spec. Vol. \\
$\mathrm{ft}^{3} / \mathrm{lbm}$ \\
\end{tabular} & $\begin{array}{c}\text { Burnup } \\
\text { (GWd/MTU) }\end{array}$ & $\begin{array}{l}\text { T-Fuel } \\
{ }^{\circ} \mathrm{F}\end{array}$ & $\begin{array}{l}\text { Spec. Vol. } \\
\mathrm{ft}^{3} / \mathrm{lbm}\end{array}$ \\
\hline & 430.4 Cy 10 & 319.5 Cy 10 & 319.5 Cy 10 & BOC Cy 11 & 549.3 Cy 10 & 549.3 Cy 10 & 208.7 Cy 11 & 103.8 Cy 11 & 103.8 Cy 11 \\
\hline 1 & 7.926 & 1007.6 & 0.0235 & 13.257 & 1014.1 & 0.0234 & 17.812 & 963.2 & 0.0236 \\
\hline 2 & 12.537 & 1161.5 & 0.0234 & 20.154 & 1126.4 & 0.0233 & 26.857 & 1061.5 & 0.0235 \\
\hline 3 & 15.072 & 1222.5 & 0.0233 & 23.486 & 1149.6 & 0.0232 & 31.263 & 1105.8 & 0.0234 \\
\hline 4 & 16.205 & 1235.6 & 0.0232 & 24.706 & 1142.6 & 0.0231 & 33.003 & 1129.6 & 0.0233 \\
\hline 5 & 16.719 & 1232.2 & 0.0230 & 25.134 & 1130.5 & 0.0230 & 33.694 & 1140.7 & 0.0231 \\
\hline 6 & 16.966 & 1224.8 & 0.0229 & 25.291 & 1120.4 & 0.0229 & 33.992 & 1145.3 & 0.0230 \\
\hline 7 & 17.101 & 1217.8 & 0.0228 & 25.369 & 1113.2 & 0.0228 & 34.146 & 1146.2 & 0.0229 \\
\hline 8 & 17.190 & 1212.4 & 0.0227 & 25.434 & 1108.2 & 0.0226 & 34.243 & 1144.5 & 0.0227 \\
\hline 9 & 17.261 & 1208.9 & 0.0226 & 25.505 & 1104.8 & 0.0225 & 34.314 & 1141.0 & 0.0226 \\
\hline 10 & 17.325 & 1207.2 & 0.0224 & 25.587 & 1102.5 & 0.0224 & 34.373 & 1136.1 & 0.0225 \\
\hline 11 & 17.384 & 1207.4 & 0.0223 & 25.680 & 1101.1 & 0.0223 & 34.427 & 1130.3 & 0.0224 \\
\hline 12 & 17.428 & 1209.3 & 0.0222 & 25.781 & 1101.1 & 0.0222 & 34.477 & 1123.9 & 0.0222 \\
\hline 13 & 17.432 & 1212.9 & 0.0221 & 25.874 & 1103.3 & 0.0221 & 34.497 & 1116.2 & 0.0221 \\
\hline 14 & 17.343 & 1217.3 & 0.0220 & 25.915 & 1108.7 & 0.0220 & 34.403 & 1104.9 & 0.0220 \\
\hline 15 & 17.027 & 1219.8 & 0.0219 & 25.753 & 1117.6 & 0.0219 & 34.013 & 1088.5 & 0.0219 \\
\hline 16 & 16.163 & 1210.1 & 0.0218 & 24.935 & 1125.1 & 0.0218 & 32.803 & 1064.5 & 0.0218 \\
\hline 17 & 13.977 & 1158.7 & 0.0217 & 22.216 & 1108.7 & 0.0217 & 29.274 & 1025.0 & 0.0217 \\
\hline 18 & 9.205 & 1005.2 & 0.0216 & 15.237 & 1006.6 & 0.0216 & 20.110 & 923.5 & 0.0216 \\
\hline
\end{tabular}

\begin{tabular}{|c|c|c|c|c|c|c|}
\hline \multirow[b]{2}{*}{$\begin{array}{l}\text { Axial } \\
\text { Node }\end{array}$} & \multicolumn{3}{|c|}{ Datapoint (417.8 Cy11) } & \multicolumn{3}{|c|}{ Statepoint 74 (615.0 Cy11) } \\
\hline & $\begin{array}{c}\text { Burnup } \\
\text { (GWd/MTU) }\end{array}$ & $\begin{array}{c}\text { T-Fuel } \\
{ }^{\circ} \mathrm{F}\end{array}$ & $\begin{array}{c}\text { Spec. Vol. } \\
\mathrm{ft}^{3} / 1 \mathrm{bm}\end{array}$ & $\begin{array}{c}\text { Burnup } \\
\text { (GWd/MTU) }\end{array}$ & $\begin{array}{c}\text { T-Fuel } \\
{ }^{\circ} \mathbf{F}\end{array}$ & $\begin{array}{c}\text { Spec. Vol. } \\
\mathrm{ft}^{3} / \mathrm{lbm}\end{array}$ \\
\hline & 417.8 Cy 11 & 312.8 Cy 11 & 312.8 Cy 11 & $615.0 \mathrm{Cy} 11$ & 515.7 Cy 11 & 515.7 Cy 11 \\
\hline 1 & 22.744 & 957.3 & 0.0235 & 27.772 & 955.1 & 0.0235 \\
\hline 2 & 33.800 & 1033.7 & 0.0234 & 40.570 & 1030.5 & 0.0234 \\
\hline 3 & 39.049 & 1071.8 & 0.0233 & 46.416 & 1055.6 & 0.0233 \\
\hline 4 & 41.068 & 1083.0 & 0.0232 & 48.526 & 1054.3 & 0.0231 \\
\hline 5 & 41.828 & 1083.4 & 0.0231 & 49.232 & 1046.7 & 0.0230 \\
\hline 6 & 42.129 & 1080.5 & 0.0229 & 49.466 & 1039.3 & 0.0229 \\
\hline 7 & 42.270 & 1076.8 & 0.0228 & 49.560 & 1033.4 & 0.0228 \\
\hline 8 & 42.355 & 1073.3 & 0.0227 & 49.618 & 1028.9 & 0.0227 \\
\hline 9 & 42.420 & 1070.1 & 0.0226 & 49.674 & 1025.7 & 0.0226 \\
\hline 10 & 42.482 & 1067.6 & 0.0225 & 49.743 & 1023.4 & 0.0224 \\
\hline 11 & 42.553 & 1065.8 & 0.0224 & 49.837 & 1022.0 & 0.0223 \\
\hline 12 & 42.637 & 1065.0 & 0.0222 & 49.965 & 1022.1 & 0.0222 \\
\hline 13 & 42.698 & 1064.7 & 0.0221 & 50.095 & 1023.7 & 0.0221 \\
\hline 14 & 42.627 & 1063.4 & 0.0220 & 50.106 & 1026.4 & 0.0220 \\
\hline 15 & 42.207 & 1059.7 & 0.0219 & 49.765 & 1029.8 & 0.0219 \\
\hline 16 & 40.828 & 1049.3 & 0.0218 & 48.383 & 1030.1 & 0.0218 \\
\hline 17 & 36.691 & 1016.5 & 0.0217 & 43.853 & 1011.5 & 0.0217 \\
\hline 18 & 25.448 & 926.5 & 0.0216 & 30.825 & 919.4 & 0.0216 \\
\hline
\end{tabular}


Table 4-98. Burnup and TH Feedback Parameters by Axial Node for Assembly K17

\begin{tabular}{|c|c|c|c|c|c|c|c|c|c|}
\hline \multirow[b]{2}{*}{$\begin{array}{l}\text { Axial } \\
\text { Node }\end{array}$} & \multicolumn{3}{|c|}{ Statepoint 70 (27.0 Cy10) } & \multicolumn{3}{|c|}{ Statepoint 71 (143.0 Cy10) } & \multicolumn{3}{|c|}{ Statepoint 72 (209.0 Cy10) } \\
\hline & $\begin{array}{c}\text { Burnup } \\
\text { (GWd/MTU) }\end{array}$ & $\begin{array}{c}\text { T-Fuel } \\
{ }^{\circ} \mathrm{F} \\
\end{array}$ & $\begin{array}{c}\text { Spec. Vol. } \\
\mathrm{ft}^{3} / \mathrm{lbm}\end{array}$ & \begin{tabular}{|c|} 
Burnup \\
(GWd/MTU)
\end{tabular} & $\begin{array}{c}\text { T-Fuel } \\
{ }^{\circ} \mathrm{F} \\
\end{array}$ & $\begin{array}{c}\text { Spec. Vol. } \\
\mathrm{ft}^{3} / \mathrm{lbm}\end{array}$ & $\begin{array}{c}\text { Burnup } \\
\text { (GWd/MTU) }\end{array}$ & $\begin{array}{l}\text { T-Fuel } \\
{ }^{\circ} \mathrm{F} \\
\end{array}$ & $\begin{array}{c}\text { Spec. Vol. } \\
\mathrm{ft}^{3} / \mathrm{lbm}\end{array}$ \\
\hline & 27.0 Cy 10 & 18.0 Cy 10 & 18.0 Cy 10 & 143.0 Cy 10 & 82.9 Cy 10 & 82.9 Cy 10 & $209.0 \mathrm{Cy} 10$ & 176.7 Cy 10 & 176.7 Cy 10 \\
\hline 1 & 0.565 & 1091.5 & 0.0238 & 3.051 & 1086.9 & 0.0239 & 4.572 & 1092.3 & 0.0239 \\
\hline 2 & 0.839 & 1291.0 & 0.0238 & 4.534 & 1277.8 & 0.0238 & 6.776 & 1266.6 & 0.0238 \\
\hline 3 & 1.029 & 1422.8 & 0.0236 & 5.535 & 1398.8 & 0.0236 & 8.218 & 1365.6 & 0.0237 \\
\hline 4 & 1.138 & 1493.0 & 0.0235 & 6.088 & 1459.7 & 0.0235 & 8.977 & 1405.4 & 0.0235 \\
\hline 5 & 1.201 & 1525.1 & 0.0233 & 6.390 & 1483.2 & 0.0233 & 9.366 & 1415.5 & 0.0234 \\
\hline 6 & 1.243 & 1542.9 & 0.0232 & 6.576 & 1495.0 & 0.0232 & 9.595 & 1417.7 & 0.0232 \\
\hline 7 & 1.271 & 1553.6 & 0.0230 & 6.699 & 1501.0 & 0.0230 & 9.741 & 1416.9 & 0.0231 \\
\hline 8 & 1.289 & 1558.8 & 0.0229 & 6.776 & 1503.2 & 0.0229 & 9.831 & 1414.9 & 0.0229 \\
\hline 9 & 1.298 & 1559.4 & 0.0227 & 6.818 & 1502.5 & 0.0227 & 9.882 & 1412.7 & 0.0228 \\
\hline 10 & 1.300 & 1556.3 & 0.0226 & 6.832 & 1499.7 & 0.0226 & 9.905 & 1410.8 & 0.0226 \\
\hline 11 & 1.296 & 1550.0 & 0.0224 & 6.825 & 1495.4 & 0.0225 & 9.908 & 1409.6 & 0.0225 \\
\hline 12 & 1.287 & 1541.7 & 0.0223 & 6.805 & 1490.4 & 0.0223 & 9.904 & 1409.8 & 0.0223 \\
\hline 13 & 1.271 & 1529.6 & 0.0222 & 6.762 & 1483.7 & 0.0222 & 9.876 & 1410.4 & 0.0222 \\
\hline 14 & 1.235 & 1505.8 & 0.0220 & 6.623 & 1468.1 & 0.0220 & 9.720 & 1406.1 & 0.0221 \\
\hline 15 & 1.173 & 1466.5 & 0.0219 & 6.350 & 1439.7 & 0.0219 & 9.379 & 1393.2 & 0.0219 \\
\hline 16 & 1.074 & 1395.1 & 0.0218 & 5.872 & 1384.2 & 0.0218 & 8.732 & 1362.7 & 0.0218 \\
\hline 17 & 0.910 & 1276.3 & 0.0217 & 5.015 & 1276.8 & 0.0217 & 7.502 & 1276.0 & 0.0217 \\
\hline 18 & 0.642 & 1081.0 & 0.0216 & 3.535 & 1087.0 & 0.0216 & 5.296 & 1098.7 & 0.0216 \\
\hline
\end{tabular}

\begin{tabular}{|c|c|c|c|c|c|c|c|c|c|}
\hline \multirow[b]{2}{*}{$\begin{array}{l}\text { Axial } \\
\text { Node }\end{array}$} & \multicolumn{3}{|c|}{ Datapoint (430.4 Cy10) } & \multicolumn{3}{|c|}{ Statepoint 73 (BOC Cy11) } & \multicolumn{3}{|c|}{ Datapoint (208.7 Cy11) } \\
\hline & $\begin{array}{c}\text { Burnup } \\
\text { (GWd/MTU) }\end{array}$ & $\begin{array}{l}\text { T-Fuel } \\
{ }^{\circ} \mathrm{F}\end{array}$ & $\begin{array}{c}\text { Spec. Vol. } \\
\mathrm{ft}^{3} / \mathrm{lbm}\end{array}$ & \begin{tabular}{|c|} 
Burnup \\
(GWd/MTU)
\end{tabular} & $\begin{array}{l}\text { T-Fuel } \\
{ }^{\circ} \mathrm{F}\end{array}$ & $\begin{array}{c}\text { Spec. Vol. } \\
\mathrm{ft}^{3} / \mathrm{lbm}\end{array}$ & $\begin{array}{c}\text { Burnup } \\
\text { (GWd/MTU) }\end{array}$ & $\begin{array}{l}\text { T-Fuel } \\
{ }^{\circ} \mathrm{F}\end{array}$ & $\begin{array}{l}\text { Spec. Vol. } \\
\mathrm{ft}^{3} / \mathrm{lbm}\end{array}$ \\
\hline & 430.4 Cy 10 & 319.5 Cy 10 & 319.5 Cy 10 & BOC Cy11 & 549.3 Cy 10 & 549.3 Cy 10 & 208.7 Cy 11 & 103.8 Cy 11 & 103.8 Cy 11 \\
\hline 1 & 10.232 & 1099.5 & 0.0240 & 16.813 & 1092.2 & 0.0239 & 19.042 & 753.4 & 0.0226 \\
\hline 2 & 14.969 & 1254.4 & 0.0238 & 24.095 & 1204.2 & 0.0238 & 27.560 & 810.9 & 0.0226 \\
\hline 3 & 17.734 & 1327.3 & 0.0237 & 27.833 & 1236.5 & 0.0236 & 31.966 & 834.4 & 0.0225 \\
\hline 4 & 18.939 & 1339.5 & 0.0235 & 29.146 & 1230.6 & 0.0235 & 33.625 & 851.7 & 0.0225 \\
\hline 5 & 19.416 & 1335.8 & 0.0234 & 29.505 & 1216.7 & 0.0233 & 34.174 & 861.1 & 0.0224 \\
\hline 6 & 19.638 & 1328.9 & 0.0232 & 29.617 & 1205.1 & 0.0232 & 34.387 & 865.4 & 0.0223 \\
\hline 7 & 19.765 & 1322.5 & 0.0231 & 29.679 & 1197.1 & 0.0230 & 34.503 & 866.7 & 0.0223 \\
\hline 8 & 19.847 & 1317.6 & 0.0229 & 29.737 & 1191.7 & 0.0229 & 34.585 & 866.2 & 0.0222 \\
\hline 9 & 19.908 & 1314.4 & 0.0228 & 29.804 & 1188.1 & 0.0228 & 34.658 & 864.6 & 0.0221 \\
\hline 10 & 19.964 & 1313.0 & 0.0226 & 29.889 & 1185.9 & 0.0226 & 34.735 & 862.2 & 0.0221 \\
\hline 11 & 20.031 & 1313.8 & 0.0225 & 30.012 & 1185.1 & 0.0225 & 34.838 & 859.0 & 0.0220 \\
\hline 12 & 20.134 & 1317.1 & 0.0224 & 30.217 & 1186.7 & 0.0224 & 35.006 & 854.8 & 0.0219 \\
\hline 13 & 20.248 & 1322.3 & 0.0222 & 30.489 & 1191.8 & 0.0222 & 35.217 & 848.9 & 0.0219 \\
\hline 14 & 20.189 & 1326.5 & 0.0221 & 30.607 & 1199.4 & 0.0221 & 35.240 & 841.2 & 0.0218 \\
\hline 15 & 19.837 & 1327.1 & 0.0220 & 30.441 & 1209.8 & 0.0220 & 34.914 & 829.8 & 0.0218 \\
\hline 16 & 18.893 & 1315.1 & 0.0218 & 29.548 & 1216.6 & 0.0219 & 33.737 & 811.7 & 0.0217 \\
\hline 17 & 16.596 & 1260.7 & 0.0217 & 26.605 & 1193.2 & 0.0217 & 30.223 & 783.4 & 0.0217 \\
\hline 18 & 11.826 & 1099.4 & 0.0216 & 19.331 & 1084.3 & 0.0216 & 21.673 & 726.0 & 0.0216 \\
\hline
\end{tabular}


Table 4-98. Burnup and TH Feedback Parameters by Axial Node for Assembly K17 (Cont.)

\begin{tabular}{|c|c|c|c|c|c|c|}
\hline \multirow[b]{2}{*}{$\begin{array}{l}\text { Axial } \\
\text { Node }\end{array}$} & \multicolumn{3}{|c|}{ Datapoint (417.8 Cy11) } & \multicolumn{3}{|c|}{ Statepoint 74 (615.0 Cy11) } \\
\hline & $\begin{array}{c}\text { Burnup } \\
\text { (GWd/MTU) }\end{array}$ & $\begin{array}{c}\text { T-Fuel } \\
{ }^{\circ} \mathrm{F}\end{array}$ & $\begin{array}{c}\text { Spec. Vol. } \\
\text { ft }^{3} / / \mathrm{bm}\end{array}$ & $\begin{array}{c}\text { Burnup } \\
\text { (GWd/MTU) }\end{array}$ & $\begin{array}{c}\text { T-Fuel } \\
{ }^{\circ} \mathrm{F}\end{array}$ & $\begin{array}{c}\text { Spec. Vol. } \\
\mathrm{ft}^{3} / / \mathrm{bm}\end{array}$ \\
\hline & 417.8 Cy 11 & 312.8 Cy 11 & 312.8 Cy 11 & 615.0 Cy11 & 515.7 Cy 11 & 515.7 Cy 11 \\
\hline 1 & 21.696 & 775.3 & 0.0226 & 24.608 & 796.0 & 0.0227 \\
\hline 2 & 31.464 & 821.1 & 0.0226 & 35.522 & 838.6 & 0.0226 \\
\hline 3 & 36.406 & 844.6 & 0.0225 & 40.848 & 854.8 & 0.0225 \\
\hline 4 & 38.244 & 851.5 & 0.0225 & 42.742 & 854.1 & 0.0225 \\
\hline 5 & 38.841 & 852.1 & 0.0224 & 43.311 & 849.9 & 0.0224 \\
\hline 6 & 39.055 & 850.3 & 0.0223 & 43.484 & 845.5 & 0.0223 \\
\hline 7 & 39.158 & 847.7 & 0.0223 & 43.555 & 841.8 & 0.0223 \\
\hline 8 & 39.228 & 845.3 & 0.0222 & 43.605 & 838.8 & 0.0222 \\
\hline 9 & 39.292 & 843.1 & 0.0221 & 43.660 & 836.5 & 0.0222 \\
\hline 10 & 39.368 & 841.3 & 0.0221 & 43.733 & 834.7 & 0.0221 \\
\hline 11 & 39.475 & 839.8 & 0.0220 & 43.846 & 833.3 & 0.0220 \\
\hline 12 & 39.650 & 838.4 & 0.0220 & 44.034 & 832.2 & 0.0220 \\
\hline 13 & 39.867 & 836.9 & 0.0219 & 44.272 & 831.7 & 0.0219 \\
\hline 14 & 39.893 & 835.5 & 0.0218 & 44.337 & 832.4 & 0.0218 \\
\hline 15 & 39.543 & 832.9 & 0.0218 & 44.028 & 833.8 & 0.0218 \\
\hline 16 & 38.242 & 825.7 & 0.0217 & 42.715 & 833.3 & 0.0217 \\
\hline 17 & 34.296 & 802.5 & 0.0217 & 38.485 & 819.3 & 0.0217 \\
\hline 18 & 24.453 & 748.8 & 0.0216 & 27.468 & 765.2 & 0.0216 \\
\hline
\end{tabular}

Table 4-99. Burnup and TH Feedback Parameters by Axial Node for Assembly K19

\begin{tabular}{|c|c|c|c|c|c|c|c|c|c|}
\hline \multirow[b]{2}{*}{$\begin{array}{l}\text { Axial } \\
\text { Node }\end{array}$} & \multicolumn{3}{|c|}{ StatepoInt 70 (27.0 Cy10) } & \multicolumn{3}{|c|}{ Statepoint 71 (143.0 Cy10) } & \multicolumn{3}{|c|}{ Statepoint 72 (209.0 Cy10) } \\
\hline & $\begin{array}{c}\text { Burnup } \\
\text { (GWd/MTU) }\end{array}$ & $\begin{array}{c}\text { T-Fuel } \\
\quad{ }^{\circ} \mathrm{F} \\
\end{array}$ & $\begin{array}{c}\text { Spec. Vol. } \\
\mathrm{ft}^{3} / \mathrm{bm}\end{array}$ & $\begin{array}{c}\text { Burnup } \\
\text { (GWd/MTU) }\end{array}$ & $\begin{array}{c}\text { T-Fuel } \\
{ }^{\circ} \mathrm{F}\end{array}$ & $\begin{array}{c}\text { Spec. Vol. } \\
\mathrm{ft}^{3} / \mathrm{lbm}\end{array}$ & $\begin{array}{c}\text { Burnup } \\
\text { (GWd/MTU) }\end{array}$ & $\begin{array}{c}\text { T-Fuel } \\
{ }^{\circ} \mathrm{F}\end{array}$ & $\begin{array}{c}\text { Spec. Vol. } \\
\mathrm{ft}^{3} / \mathrm{lbm}\end{array}$ \\
\hline & 27.0 Cy 10 & 18.0 Cy 10 & $18.0 \mathrm{Cy} 10$ & 143.0 Cy 10 & 82.9 Cy 10 & 82.9 Cy 10 & 209.0 Cy 10 & 176.7 Cy 10 & 176.7 Cy 10 \\
\hline 1 & 0.519 & 1051.0 & 0.0238 & 2.813 & 1051.8 & 0.0239 & 4.231 & 1065.5 & 0.0239 \\
\hline 2 & 0.779 & 1244.0 & 0.0237 & 4.244 & 1241.6 & 0.0238 & 6.381 & 1242.8 & 0.0238 \\
\hline 3 & 0.977 & 1382.2 & 0.0236 & 5.305 & 1372.0 & 0.0236 & 7.917 & 1350.5 & 0.0237 \\
\hline 4 & 1.098 & 1461.9 & 0.0235 & 5.930 & 1442.8 & 0.0235 & 8.776 & 1397.4 & 0.0235 \\
\hline 5 & 1.171 & 1504.9 & 0.0233 & 6.284 & 1474.8 & 0.0233 & 9.232 & 1411.3 & 0.0234 \\
\hline 6 & 1.220 & 1527.4 & 0.0232 & 6.508 & 1490.3 & 0.0232 & 9.506 & 1414.6 & 0.0232 \\
\hline 7 & 1.254 & 1541.5 & 0.0230 & 6.658 & 1499.0 & 0.0230 & 9.685 & 1414.4 & 0.0231 \\
\hline 8 & 1.277 & 1549.5 & 0.0229 & 6.759 & 1503.4 & 0.0229 & 9.804 & 1412.9 & 0.0229 \\
\hline 9 & 1.290 & 1552.8 & 0.0227 & 6.822 & 1504.7 & 0.0227 & 9.882 & 1411.3 & 0.0228 \\
\hline 10 & 1.296 & 1552.1 & 0.0226 & 6.857 & 1503.9 & 0.0226 & 9.932 & 1410.2 & 0.0226 \\
\hline 11 & 1.296 & 1548.0 & 0.0224 & 6.869 & 1501.4 & 0.0225 & 9.961 & 1410.1 & 0.0225 \\
\hline 12 & 1.290 & 1541.3 & 0.0223 & 6.865 & 1497.9 & 0.0223 & 9.980 & 1411.5 & 0.0223 \\
\hline 13 & 1.276 & 1530.2 & 0.0222 & 6.832 & 1492.3 & 0.0222 & 9.970 & 1413.5 & 0.0222 \\
\hline 14 & 1.239 & 1506.1 & 0.0220 & 6.692 & 1476.6 & 0.0220 & 9.819 & 1410.7 & 0.0221 \\
\hline 15 & 1.172 & 1463.6 & 0.0219 & 6.395 & 1445.9 & 0.0219 & 9.452 & 1398.3 & 0.0219 \\
\hline 16 & 1.061 & 1383.9 & 0.0218 & 5.851 & 1383.9 & 0.0218 & 8.722 & 1364.5 & 0.0218 \\
\hline 17 & 0.883 & 1254.5 & 0.0217 & 4.900 & 1264.1 & 0.0217 & 7.357 & 1270.1 & 0.0217 \\
\hline 18 & 0.618 & 1062.2 & 0.0216 & 3.406 & 1070.9 & 0.0216 & 5.108 & 1084.2 & 0.0216 \\
\hline
\end{tabular}


Table 4-99. Burnup and TH Feedback Parameters by Axial Node for Assembly K19 (Cont.)

\begin{tabular}{|c|c|c|c|c|c|c|c|c|c|}
\hline \multirow[b]{2}{*}{$\begin{array}{l}\text { Axial } \\
\text { Node }\end{array}$} & \multicolumn{3}{|c|}{ Datapoint (430.4 Cy10) } & \multicolumn{3}{|c|}{ Statepoint 73 (BOC Cy11) } & \multicolumn{3}{|c|}{ Datapoint (208.7 Cy11) } \\
\hline & $\begin{array}{c}\text { Burnup } \\
\text { (GWd/MTU) }\end{array}$ & $\begin{array}{c}\text { T-Fuel } \\
{ }^{\circ} \mathbf{F}\end{array}$ & $\begin{array}{c}\text { Spec. Vol. } \\
\mathrm{ft}^{3} / \mathrm{lbm}\end{array}$ & $\begin{array}{c}\text { Burnup } \\
\text { (GWd/MTU) }\end{array}$ & $\begin{array}{c}\text { T-Fuel } \\
{ }^{\circ} \mathrm{F}\end{array}$ & $\begin{array}{c}\text { Spec. Vol. } \\
\mathrm{ft}^{3} / \mathrm{lbm}\end{array}$ & $\begin{array}{c}\text { Burnup } \\
\text { (GWd/MTU) }\end{array}$ & $\begin{array}{l}\text { T-Fuel } \\
{ }^{\circ} \mathrm{F}\end{array}$ & $\begin{array}{l}\text { Spec. Vol. } \\
\mathrm{ft}^{3} / \mathrm{lbm}\end{array}$ \\
\hline & 430.4 Cy 10 & 319.5 Cy 10 & 319.5 Cy10 & BOC Cy 11 & 549.3 Cy 10 & 549.3 Cy 10 & 208.7 Cy 11 & 103.8 Cy 11 & 103.8 Cy 11 \\
\hline 1 & 9.529 & 1074.6 & 0.0239 & 15.755 & 1071.9 & 0.0238 & 20.228 & 941.6 & 0.0236 \\
\hline 2 & 14.210 & 1229.7 & 0.0238 & 22.995 & 1190.4 & 0.0237 & 29.629 & 1038.1 & 0.0235 \\
\hline 3 & 17.108 & 1305.6 & 0.0236 & 26.888 & 1220.9 & 0.0236 & 34.587 & 1080.1 & 0.0234 \\
\hline 4 & 18.438 & 1321.3 & 0.0235 & 28.343 & 1215.1 & 0.0234 & 36.566 & 1106.6 & 0.0232 \\
\hline 5 & 18.989 & 1317.2 & 0.0233 & 28.784 & 1201.3 & 0.0233 & 37.287 & 1119.4 & 0.0231 \\
\hline 6 & 19.255 & 1309.6 & 0.0232 & 28.946 & 1189.9 & 0.0231 & 37.596 & 1124.5 & 0.0230 \\
\hline 7 & 19.414 & 1302.6 & 0.0230 & 29.046 & 1182.1 & 0.0230 & 37.766 & 1125.0 & 0.0228 \\
\hline 8 & 19.524 & 1297.3 & 0.0229 & 29.135 & 1176.9 & 0.0228 & 37.877 & 1122.6 & 0.0227 \\
\hline 9 & 19.615 & 1293.9 & 0.0227 & 29.234 & 1173.4 & 0.0227 & 37.961 & 1118.2 & 0.0226 \\
\hline 10 & 19.701 & 1292.4 & 0.0226 & 29.349 & 1171.1 & 0.0226 & 38.037 & 1112.3 & 0.0225 \\
\hline 11 & 19.798 & 1293.2 & 0.0225 & 29.502 & 1170.1 & 0.0225 & 38.121 & 1104.7 & 0.0223 \\
\hline 12 & 19.931 & 1296.6 & 0.0223 & 29.736 & 1171.4 & 0.0223 & 38.253 & 1095.0 & 0.0222 \\
\hline 13 & 20.075 & 1302.2 & 0.0222 & 30.038 & 1176.2 & 0.0222 & 38.413 & 1082.8 & 0.0221 \\
\hline 14 & 20.036 & 1307.4 & 0.0221 & 30.176 & 1183.6 & 0.0221 & 38.378 & 1069.0 & 0.0220 \\
\hline 15 & 19.674 & 1309.5 & 0.0220 & 30.003 & 1194.2 & 0.0220 & 37.966 & 1052.0 & 0.0219 \\
\hline 16 & 18.646 & 1299.0 & 0.0218 & 29.029 & 1201.9 & 0.0219 & 36.607 & 1027.5 & 0.0218 \\
\hline 17 & 16.181 & 1241.2 & 0.0217 & 25.913 & 1179.6 & 0.0217 & 32.708 & 984.5 & 0.0217 \\
\hline 18 & 11.326 & 1078.0 & 0.0216 & 18.522 & 1068.8 & 0.0216 & 23.182 & 889.1 & 0.0216 \\
\hline
\end{tabular}

\begin{tabular}{|c|c|c|c|c|c|c|}
\hline \multirow[b]{2}{*}{$\begin{array}{l}\text { Axlal } \\
\text { Node }\end{array}$} & \multicolumn{3}{|c|}{ Datapoint (417.8 Cyl1) } & \multicolumn{3}{|c|}{ Statepoint 74 (615.0 Cy11) } \\
\hline & $\begin{array}{c}\text { Burnup } \\
\text { (GWd/MTU) }\end{array}$ & $\begin{array}{c}\text { T-Fuel } \\
{ }^{\circ} \mathrm{F} \\
\end{array}$ & $\begin{array}{c}\text { Spec. Vol. } \\
\mathrm{ft}^{3} / \mathrm{lbm}\end{array}$ & $\begin{array}{c}\text { Burnup } \\
\text { (GWd/MTU) }\end{array}$ & $\begin{array}{c}\text { T-Fuel } \\
{ }^{\circ} \mathbf{F} \\
\end{array}$ & $\begin{array}{c}\text { Spec. Vol. } \\
\mathrm{ft}^{3} / \mathrm{bm}\end{array}$ \\
\hline & 417.8 Cy 11 & 312.8 Cy11 & $312.8 \mathrm{Cy} 11$ & 615.0 Cy 11 & 515.7 Cy 11 & 515.7 Cy11 \\
\hline 1 & 25.025 & 934.2 & 0.0235 & 29.894 & 930.1 & 0.0234 \\
\hline 2 & 36.448 & 1016.5 & 0.0234 & 43.062 & 1013.5 & 0.0233 \\
\hline 3 & 42.249 & 1056.6 & 0.0233 & 49.445 & 1036.1 & 0.0232 \\
\hline 4 & 44.521 & 1067.9 & 0.0232 & 51.802 & 1033.8 & 0.0231 \\
\hline 5 & 45.333 & 1069.6 & 0.0230 & 52.567 & 1026.6 & 0.0230 \\
\hline 6 & 45.656 & 1067.4 & 0.0229 & 52.825 & 1019.3 & 0.0229 \\
\hline 7 & 45.815 & 1063.9 & 0.0228 & 52.934 & 1013.3 & 0.0227 \\
\hline 8 & 45.912 & 1060.3 & 0.0227 & 53.001 & 1008.7 & 0.0226 \\
\hline 9 & 45.987 & 1057.0 & 0.0226 & 53.064 & 1005.2 & 0.0225 \\
\hline 10 & 46.059 & 1054.0 & 0.0224 & 53.138 & 1002.7 & 0.0224 \\
\hline 11 & 46.145 & 1051.3 & 0.0223 & 53.238 & 1001.0 & 0.0223 \\
\hline 12 & 46.277 & 1048.3 & 0.0222 & 53.395 & 1000.0 & 0.0222 \\
\hline 13 & 46.431 & 1044.8 & 0.0221 & 53.586 & 999.7 & 0.0221 \\
\hline 14 & 46.387 & 1041.5 & 0.0220 & 53.606 & 1001.3 & 0.0220 \\
\hline 15 & 45.931 & 1036.9 & 0.0219 & 53.222 & 1003.7 & 0.0219 \\
\hline 16 & 44.395 & 1026.5 & 0.0218 & 51.684 & 1004.4 & 0.0218 \\
\hline 17 & 39.886 & 994.5 & 0.0217 & 46.788 & 987.3 & 0.0217 \\
\hline 18 & 28.299 & 895.0 & 0.0216 & 33.434 & 889.8 & 0.0216 \\
\hline
\end{tabular}


Table 4-100. Burnup and TH Feedback Parameters by Axial Node for Assembly K19a

\begin{tabular}{|c|c|c|c|c|c|c|c|c|c|}
\hline \multirow[b]{2}{*}{$\begin{array}{l}\text { Axial } \\
\text { Node }\end{array}$} & \multicolumn{3}{|c|}{ Statepoint $70(27.0$ Cy10) } & \multicolumn{3}{|c|}{ Statepoint 71 (143.0 Cy10) } & \multicolumn{3}{|c|}{ Statepoint $72(209.0$ Cy10) } \\
\hline & $\begin{array}{c}\text { Burnup } \\
\text { (GWd/MTU) }\end{array}$ & $\begin{array}{c}\text { T-Fuel } \\
{ }^{\circ} \mathrm{F} \\
\end{array}$ & \begin{tabular}{|c} 
Spec. Vol. \\
$\mathrm{ft}^{3} / \mathrm{lbm}$
\end{tabular} & $\begin{array}{c}\text { Burnup } \\
\text { (GWd/MTU) }\end{array}$ & $\begin{array}{c}\text { T-Fuel } \\
{ }^{\circ} \mathrm{F}\end{array}$ & $\begin{array}{c}\text { Spec. Vol. } \\
\mathrm{ft}^{3} / \mathrm{lbm}\end{array}$ & $\begin{array}{c}\text { Burnup } \\
\text { (GWd/MTU) }\end{array}$ & $\begin{array}{c}\text { T-Fuel } \\
{ }^{\circ} \mathrm{F}\end{array}$ & \begin{tabular}{|c|} 
Spec. Vol. \\
$\mathrm{tt}^{3} / \mathrm{lbm}$ \\
\end{tabular} \\
\hline & 27.0 Cy 10 & 18.0 Cy 10 & 18.0 Cy 10 & 143.0 Cy 10 & 82.9 Cy 10 & 82.9 Cy 10 & 209.0 Cy 10 & 176.7 Cy 10 & 176.7 Cy 10 \\
\hline 1 & 0.519 & 1051.0 & 0.0238 & 2.813 & 1051.8 & 0.0239 & 4.231 & 1065.5 & 0.0239 \\
\hline 2 & 0.779 & 1244.0 & 0.0237 & 4.244 & 1241.6 & 0.0238 & 6.381 & 1242.8 & 0.0238 \\
\hline 3 & 0.977 & 1382.2 & 0.0236 & 5.305 & 1372.0 & 0.0236 & 7.917 & 1350.5 & 0.0237 \\
\hline 4 & 1.098 & 1461.9 & 0.0235 & 5.930 & 1442.8 & 0.0235 & 8.776 & 1397.4 & 0.0235 \\
\hline 5 & 1.171 & 1504.9 & 0.0233 & 6.284 & 1474.8 & 0.0233 & 9.232 & 1411.3 & 0.0234 \\
\hline 6 & 1.220 & 1527.4 & 0.0232 & 6.508 & 1490.3 & 0.0232 & 9.506 & 1414.6 & 0.0232 \\
\hline 7 & 1.254 & 1541.5 & 0.0230 & 6.658 & 1499.0 & 0.0230 & 9.685 & 1414.4 & 0.0231 \\
\hline 8 & 1.277 & 1549.5 & 0.0229 & 6.759 & 1503.4 & 0.0229 & 9.804 & 1412.9 & 0.0229 \\
\hline 9 & 1.290 & 1552.8 & 0.0227 & 6.822 & 1504.7 & 0.0227 & 9.882 & 1411.3 & 0.0228 \\
\hline 10 & 1.296 & 1552.1 & 0.0226 & 6.857 & 1503.9 & 0.0226 & 9.932 & 1410.2 & 0.0226 \\
\hline 11 & 1.296 & 1548.0 & 0.0224 & 6.869 & 1501.4 & 0.0225 & 9.961 & 1410.1 & 0.0225 \\
\hline 12 & 1.290 & 1541.3 & 0.0223 & 6.865 & 1497.9 & 0.0223 & 9.980 & 1411.5 & 0.0223 \\
\hline 13 & 1.276 & 1530.2 & 0.0222 & 6.832 & 1492.3 & 0.0222 & 9.970 & 1413.5 & 0.0222 \\
\hline 14 & 1.239 & 1506.1 & 0.0220 & 6.692 & 1476.6 & 0.0220 & 9.819 & 1410.7 & 0.0221 \\
\hline 15 & 1.172 & 1463.6 & 0.0219 & 6.395 & 1445.9 & 0.0219 & 9.452 & 1398.3 & 0.0219 \\
\hline 16 & 1.061 & 1383.9 & 0.0218 & 5.851 & 1383.9 & 0.0218 & 8.722 & 1364.5 & 0.0218 \\
\hline 17 & 0.883 & 1254.5 & 0.0217 & 4.900 & 1264.1 & 0.0217 & 7.357 & 1270.1 & 0.0217 \\
\hline 18 & 0.618 & 1062.2 & 0.0216 & 3.406 & 1070.9 & 0.0216 & 5.108 & 1084.2 & 0.0216 \\
\hline
\end{tabular}

\begin{tabular}{|c|c|c|c|c|c|c|c|c|c|}
\hline \multirow[b]{2}{*}{$\begin{array}{l}\text { Axlal } \\
\text { Node }\end{array}$} & \multicolumn{3}{|c|}{ Datapoint (430.4 Cy10) } & \multicolumn{3}{|c|}{ Statepoint 73 (BOC Cy 11$)$} & \multicolumn{3}{|c|}{ Datapoint (208.7 Cy11) } \\
\hline & \begin{tabular}{|c|} 
Burnup \\
(GWd/MTU)
\end{tabular} & $\begin{array}{c}\text { T-Fuel } \\
{ }^{\circ} \mathrm{F} \\
\end{array}$ & \begin{tabular}{|c|}
$\begin{array}{c}\text { Spec. Vol. } \\
\mathrm{ft}^{3} / \mathrm{lbm}\end{array}$ \\
\end{tabular} & $\begin{array}{c}\text { Burnup } \\
\text { (GWd/MTU) }\end{array}$ & $\begin{array}{c}\text { T-Fuel } \\
{ }^{\circ} \mathrm{F}\end{array}$ & $\begin{array}{c}\text { Spec. Vol. } \\
\mathrm{ft}^{3} / 1 \mathrm{bm}\end{array}$ & $\begin{array}{c}\text { Burnup } \\
\text { (GWd/MTU) }\end{array}$ & $\begin{array}{c}\text { T-Fuel } \\
{ }^{\circ} \mathrm{F} \\
\end{array}$ & $\begin{array}{c}\text { Spec. Vol. } \\
\mathrm{ft}^{3} / \mathrm{lbm}\end{array}$ \\
\hline & 430.4 Cy 10 & 319.5 Cy 10 & 319.5 Cy 10 & BOC Cy11 & 549.3 Cy 10 & 549.3 Cy 10 & 208.7 Cy 11 & 103.8 Cy 11 & 103.8 Cy 11 \\
\hline 1 & 9.529 & 1074.6 & 0.0239 & 15.760 & 1071.9 & 0.0238 & 20.328 & 948.0 & 0.0236 \\
\hline 2 & 14.210 & 1229.7 & 0.0238 & 23.004 & 1190.4 & 0.0237 & 29.669 & 1039.7 & 0.0235 \\
\hline 3 & 17.108 & 1305.6 & 0.0236 & 26.902 & 1220.9 & 0.0236 & 34.550 & 1076.6 & 0.0233 \\
\hline 4 & 18.438 & 1321.3 & 0.0235 & 28.360 & 1215.1 & 0.0234 & 36.493 & 1100.7 & 0.0232 \\
\hline 5 & 18.989 & 1317.2 & 0.0233 & 28.802 & 1201.3 & 0.0233 & 37.199 & 1112.7 & 0.0231 \\
\hline 6 & 19.255 & 1309.6 & 0.0232 & 28.964 & 1189.9 & 0.0231 & 37.502 & 1117.4 & 0.0230 \\
\hline 7 & 19.414 & 1302.6 & 0.0230 & 29.065 & 1182.1 & 0.0230 & 37.672 & 1118.0 & 0.0228 \\
\hline 8 & 19.524 & 1297.3 & 0.0229 & 29.154 & 1176.9 & 0.0228 & 37.787 & 1116.0 & 0.0227 \\
\hline 9 & 19.615 & 1293.9 & 0.0227 & 29.252 & 1173.4 & 0.0227 & 37.879 & 1112.1 & 0.0226 \\
\hline 10 & 19.701 & 1292.4 & 0.0226 & 29.368 & 1171.1 & 0.0226 & 37.966 & 1106.9 & 0.0225 \\
\hline 11 & 19.798 & 1293.2 & 0.0225 & 29.521 & 1170.1 & 0.0225 & 38.068 & 1100.5 & 0.0223 \\
\hline 12 & 19.931 & 1296.6 & 0.0223 & 29.754 & 1171.4 & 0.0223 & 38.228 & 1092.5 & 0.0222 \\
\hline 13 & 20.075 & 1302.2 & 0.0222 & 30.056 & 1176.2 & 0.0222 & 38.420 & 1082.2 & 0.0221 \\
\hline 14 & 20.036 & 1307.4 & 0.0221 & 30.194 & 1183.6 & 0.0221 & 38.407 & 1069.8 & 0.0220 \\
\hline 15 & 19.674 & 1309.5 & 0.0220 & 30.020 & 1194.2 & 0.0220 & 38.005 & 1053.4 & 0.0219 \\
\hline 16 & 18.646 & 1299.0 & 0.0218 & 29.046 & 1201.9 & 0.0219 & 36.654 & 1029.4 & 0.0218 \\
\hline 17 & 16.181 & 1241.2 & 0.0217 & 25.929 & 1179.6 & 0.0217 & 32.784 & 988.2 & 0.0217 \\
\hline 18 & 11.326 & 1078.0 & 0.0216 & 18.534 & 1068.8 & 0.0216 & 23.281 & 894.9 & 0.0216 \\
\hline
\end{tabular}


Table 4-100. Burnup and TH Feedback Parameters by Axial Node for Assembly K19a (Cont.)

\begin{tabular}{|c|c|c|c|c|c|c|}
\hline \multirow{2}{*}{$\begin{array}{c}\text { Axial } \\
\text { Node }\end{array}$} & \multicolumn{3}{|c|}{ Datapoint (417.8 Cy11) } & \multicolumn{2}{c|}{ Statepoint 74 (615.0 Cy11) } \\
\cline { 2 - 7 } & $\begin{array}{c}\text { Burnup } \\
\text { (GWd/MTU) }\end{array}$ & $\begin{array}{c}\text { T-Fuel } \\
{ }^{\circ} \mathrm{F}\end{array}$ & $\begin{array}{c}\text { Spec. Vol. } \\
\mathrm{ft}^{3} / \mathrm{lbm}\end{array}$ & $\begin{array}{c}\text { Burnup } \\
(\mathrm{GWd} / \mathrm{MTU})\end{array}$ & $\begin{array}{c}\text { T-Fuel } \\
{ }^{\circ} \mathrm{F}\end{array}$ & $\begin{array}{c}\text { Spec. Vol. } \\
\mathrm{ft}^{3} / \mathrm{lbm}\end{array}$ \\
\hline 1 & $417.8 \mathrm{Cy} 11$ & $312.8 \mathrm{Cy} 11$ & $312.8 \mathrm{Cy} 11$ & $615.0 \mathrm{Cy} 11$ & 515.7 Cy11 & $515.7 \mathrm{Cy} 11$ \\
\hline 2 & 25.240 & 941.1 & 0.0235 & 30.250 & 939.6 & 0.0235 \\
\hline 3 & 36.548 & 1020.1 & 0.0234 & 43.293 & 1023.2 & 0.0234 \\
\hline 4 & 42.209 & 1056.1 & 0.0233 & 49.530 & 1045.5 & 0.0233 \\
\hline 5 & 44.423 & 1065.8 & 0.0232 & 51.837 & 1043.9 & 0.0231 \\
\hline 6 & 45.211 & 1067.0 & 0.0230 & 52.586 & 1037.1 & 0.0230 \\
\hline 7 & 45.524 & 1064.6 & 0.0229 & 52.838 & 1030.1 & 0.0229 \\
\hline 8 & 45.682 & 1061.0 & 0.0228 & 52.950 & 1024.2 & 0.0228 \\
\hline 9 & 45.784 & 1057.4 & 0.0227 & 53.024 & 1019.8 & 0.0227 \\
\hline 10 & 45.867 & 1054.1 & 0.0226 & 53.098 & 1016.4 & 0.0225 \\
\hline 11 & 45.953 & 1051.3 & 0.0224 & 53.188 & 1013.9 & 0.0224 \\
\hline 12 & 46.064 & 1048.9 & 0.0223 & 53.318 & 1012.3 & 0.0223 \\
\hline 13 & 46.237 & 1046.6 & 0.0222 & 53.522 & 1011.5 & 0.0222 \\
\hline 14 & 46.438 & 1043.9 & 0.0221 & 53.769 & 1011.8 & 0.0221 \\
\hline 15 & 46.427 & 1041.2 & 0.0220 & 53.826 & 1013.6 & 0.0220 \\
\hline 16 & 44.982 & 1036.6 & 0.0219 & 53.451 & 1015.6 & 0.0219 \\
\hline 17 & 39.999 & 1026.3 & 0.0218 & 51.910 & 1015.4 & 0.0218 \\
\hline 18 & 28.471 & 996.0 & 0.0217 & 47.048 & 996.9 & 0.0217 \\
\hline
\end{tabular}

Table 4-101. Burnup and TH Feedback Parameters by Axial Node for Assembly K20

\begin{tabular}{|c|c|c|c|c|c|c|c|c|c|}
\hline \multirow[b]{2}{*}{$\begin{array}{l}\text { Axial } \\
\text { Node } \\
\end{array}$} & \multicolumn{3}{|c|}{ Statepoint 70 (27.0 Cy10) } & \multicolumn{3}{|c|}{ Statepoint 71 (143.0 Cy10) } & \multicolumn{3}{|c|}{ Statepoint 72 (209.0 Cy10) } \\
\hline & $\begin{array}{c}\text { Burnup } \\
\text { (GWd/MTU) }\end{array}$ & $\begin{array}{l}\text { T-Fuel } \\
{ }^{\circ} \mathrm{F}\end{array}$ & $\begin{array}{l}\text { Spec. Vol. } \\
\mathrm{ft}^{3} / \mathrm{lbm}\end{array}$ & \begin{tabular}{|c|} 
Burnup \\
(GWd/MTU)
\end{tabular} & $\begin{array}{l}\text { T-Fuel } \\
{ }^{\circ} \mathrm{F}\end{array}$ & $\begin{array}{c}\text { Spec. Vol. } \\
\mathrm{tt}^{3} / \mathrm{lbm}\end{array}$ & $\begin{array}{c}\text { Burnup } \\
\text { (GWd/MTU) }\end{array}$ & $\begin{array}{l}\text { T-Fuel } \\
{ }^{\circ} \mathrm{F}\end{array}$ & $\begin{array}{c}\text { Spec. Vol. } \\
\mathrm{ft}^{3} / \mathrm{lbm}\end{array}$ \\
\hline & $27.0 \mathrm{Cy} 10$ & $18.0 \mathrm{Cy} 10$ & $18.0 \mathrm{Cy} 10$ & 143.0 Cy 10 & 82.9 Cy 10 & 82.9 Cy 10 & 209.0 Cy 10 & 176.7 Cy 10 & 176.7 Cy 10 \\
\hline 1 & 0.397 & 941.9 & 0.0235 & 2.167 & 946.4 & 0.0235 & 3.267 & 961.6 & 0.0235 \\
\hline 2 & 0.682 & 1165.1 & 0.0235 & 3.676 & 1158.9 & 0.0235 & 5.483 & 1152.3 & 0.0234 \\
\hline 3 & 0.877 & 1301.9 & 0.0234 & 4.673 & 1281.6 & 0.0233 & 6.892 & 1246.6 & 0.0233 \\
\hline 4 & 0.997 & 1380.9 & 0.0233 & 5.250 & 1347.1 & 0.0232 & 7.666 & 1286.9 & 0.0232 \\
\hline 5 & 1.072 & 1425.1 & 0.0231 & 5.594 & 1380.7 & 0.0231 & 8.101 & 1302.0 & 0.0230 \\
\hline 6 & 1.122 & 1450.6 & 0.0230 & 5.807 & 1397.4 & 0.0230 & 8.356 & 1306.0 & 0.0229 \\
\hline 7 & 1.155 & 1466.1 & 0.0229 & 5.944 & 1406.2 & 0.0228 & 8.514 & 1305.9 & 0.0228 \\
\hline 8 & 1.177 & 1475.0 & 0.0227 & 6.035 & 1410.6 & 0.0227 & 8.617 & 1304.4 & 0.0227 \\
\hline 9 & 1.191 & 1479.1 & 0.0226 & 6.093 & 1412.2 & 0.0226 & 8.685 & 1302.9 & 0.0225 \\
\hline 10 & 1.197 & 1479.0 & 0.0225 & 6.125 & 1411.7 & 0.0225 & 8.728 & 1302.0 & 0.0224 \\
\hline 11 & 1.196 & 1474.7 & 0.0223 & 6.132 & 1409.1 & 0.0223 & 8.746 & 1301.6 & 0.0223 \\
\hline 12 & 1.186 & 1465.3 & 0.0222 & 6.107 & 1403.7 & 0.0222 & 8.731 & 1301.3 & 0.0222 \\
\hline 13 & 1.164 & 1448.7 & 0.0221 & 6.036 & 1394.1 & 0.0221 & 8.664 & 1300.4 & 0.0221 \\
\hline 14 & 1.125 & 1422.1 & 0.0220 & 5.892 & 1377.2 & 0.0220 & 8.506 & 1296.7 & 0.0220 \\
\hline 15 & 1.061 & 1379.3 & 0.0219 & 5.623 & 1347.2 & 0.0219 & 8.181 & 1285.0 & 0.0219 \\
\hline 16 & 0.954 & 1303.7 & 0.0218 & 5.126 & 1288.3 & 0.0218 & 7.531 & 1251.7 & 0.0218 \\
\hline 17 & 0.770 & 1171.2 & 0.0217 & 4.195 & 1171.8 & 0.0217 & 6.232 & 1165.1 & 0.0217 \\
\hline 18 & 0.451 & 928.7 & 0.0216 & 2.491 & 941.2 & 0.0216 & 3.740 & 960.6 & 0.0216 \\
\hline
\end{tabular}


Table 4-102. Burnup and TH Feedback Parameters by Axial Node for Assembly K23

\begin{tabular}{|c|c|c|c|c|c|c|c|c|c|}
\hline \multirow[b]{2}{*}{$\begin{array}{l}\text { Axial } \\
\text { Node }\end{array}$} & \multicolumn{3}{|c|}{ Statepoint $70(27.0$ Cy10) } & \multicolumn{3}{|c|}{ Statepoint 71 (143.0 Cy10) } & \multicolumn{3}{|c|}{ Statepoint $72(209.0$ Cy10) } \\
\hline & $\begin{array}{c}\text { Burnup } \\
\text { (GWd/MTU) }\end{array}$ & $\begin{array}{c}\text { T-Fuel } \\
{ }^{\circ} \mathrm{F} \\
\end{array}$ & $\begin{array}{c}\text { Spec. Vol. } \\
\mathrm{ft}^{3} / \mathrm{lbm}\end{array}$ & $\begin{array}{c}\text { Burnup } \\
\text { (GWd/MTU) }\end{array}$ & $\begin{array}{l}\text { T-Fuel } \\
{ }^{\circ} \mathrm{F}\end{array}$ & $\begin{array}{l}\text { Spec. Vol. } \\
\mathrm{ft}^{3} / \mathrm{lbm}\end{array}$ & $\begin{array}{c}\text { Burnup } \\
\text { (GWd/MTU) }\end{array}$ & $\begin{array}{l}\text { T-Fuel } \\
{ }^{\circ} \mathrm{F}\end{array}$ & $\begin{array}{c}\text { Spec. Vol. } \\
\mathrm{t}^{3} / \mathrm{bm}\end{array}$ \\
\hline & 27.0 Cy 10 & $18.0 \mathrm{Cy} 10$ & 18.0 Cy 10 & 143.0 Cy 10 & 82.9 Cy 10 & $82.9 \mathrm{Cy} 10$ & 209.0 Cy 10 & 176.7 Cy 10 & 176.7 Cy 10 \\
\hline 1 & 0.523 & 1063.8 & 0.0239 & 2.790 & 1049.9 & 0.0238 & 4.184 & 1056.6 & 0.0238 \\
\hline 2 & 0.803 & 1272.2 & 0.0238 & 4.282 & 1249.3 & 0.0238 & 6.397 & 1234.7 & 0.0237 \\
\hline 3 & 1.013 & 1414.3 & 0.0236 & 5.384 & 1380.8 & 0.0236 & 7.970 & 1341.0 & 0.0236 \\
\hline 4 & 1.136 & 1491.3 & 0.0235 & 6.002 & 1447.2 & 0.0235 & 8.807 & 1385.2 & 0.0235 \\
\hline 5 & 1.208 & 1527.4 & 0.0234 & 6.333 & 1474.6 & 0.0233 & 9.227 & 1397.5 & 0.0233 \\
\hline 6 & 1.255 & 1548.0 & 0.0232 & 6.536 & 1487.4 & 0.0232 & 9.472 & 1399.7 & 0.0232 \\
\hline 7 & 1.288 & 1561.0 & 0.0230 & 6.672 & 1494.2 & 0.0230 & 9.630 & 1398.7 & 0.0230 \\
\hline 8 & 1.309 & 1568.1 & 0.0229 & 6.760 & 1497.1 & 0.0229 & 9.732 & 1396.6 & 0.0229 \\
\hline 8 & 1.322 & 1570.6 & 0.0227 & 6.814 & 1497.3 & 0.0227 & 9.796 & 1394.5 & 0.0227 \\
\hline 10 & 1.327 & 1569.2 & 0.0226 & 6.840 & 1495.5 & 0.0226 & 9.833 & 1392.9 & 0.0226 \\
\hline 11 & 1.326 & 1564.7 & 0.0224 & 6.846 & 1492.4 & 0.0224 & 9.853 & 1392.2 & 0.0225 \\
\hline 12 & 1.320 & 1558.0 & 0.0223 & 6.841 & 1488.7 & 0.0223 & 9.868 & 1393.1 & 0.0223 \\
\hline 13 & 1.306 & 1547.1 & 0.0222 & 6.811 & 1483.4 & 0.0222 & 9.859 & 1394.7 & 0.0222 \\
\hline 14 & 1.270 & 1523.2 & 0.0220 & 6.677 & 1468.7 & 0.0220 & 9.713 & 1391.5 & 0.0221 \\
\hline 15 & 1.203 & 1482.0 & 0.0219 & 6.395 & 1439.9 & 0.0219 & 9.365 & 1379.4 & 0.0219 \\
\hline 16 & 1.094 & 1406.6 & 0.0218 & 5.885 & 1382.0 & 0.0218 & 8.686 & 1346.7 & 0.0218 \\
\hline 17 & 0.916 & 1278.0 & 0.0217 & 4.976 & 1269.4 & 0.0217 & 7.398 & 1258.6 & 0.0217 \\
\hline 18 & 0.637 & 1075.6 & 0.0216 & 3.466 & 1076.6 & 0.0216 & 5.167 & 1083.1 & 0.0216 \\
\hline
\end{tabular}

\begin{tabular}{|c|c|c|c|c|c|c|c|c|c|}
\hline \multirow[b]{2}{*}{$\begin{array}{l}\text { Axial } \\
\text { Node }\end{array}$} & \multicolumn{3}{|c|}{ Datapoint (430.4 Cy10) } & \multicolumn{3}{|c|}{ Statepoint 73 (BOC Cy11) } & \multicolumn{3}{|c|}{ Datapoint (208.7 Cy11) } \\
\hline & $\begin{array}{c}\text { Burnup } \\
\text { (GWd/MTU) }\end{array}$ & $\begin{array}{c}\text { T-Fuel } \\
{ }^{\circ} \mathrm{F}\end{array}$ & $\begin{array}{c}\text { Spec. Vol. } \\
\mathrm{ft}^{3} / \mathrm{lbm}\end{array}$ & $\begin{array}{c}\text { Burnup } \\
\text { (GWd/MTU) }\end{array}$ & $\begin{array}{c}\text { T-Fuel } \\
{ }^{\circ} \mathrm{F}\end{array}$ & $\begin{array}{c}\text { Spec. Vol. } \\
\mathrm{ft}^{3} / \mathrm{lbm}\end{array}$ & $\begin{array}{c}\text { Burnup } \\
\text { (GWd/MTU) }\end{array}$ & $\begin{array}{l}\text { T-Fuel } \\
{ }^{\circ} \mathrm{F}\end{array}$ & $\begin{array}{c}\text { Spec. Vol. } \\
\mathrm{ft}^{3} / \mathrm{bm}\end{array}$ \\
\hline & 430.4 Cy 10 & 319.5 Cy 10 & 319.5 Cy 10 & BOC Cy11 & $549.3 \mathrm{Cy} 10$ & 549.3 Cy 10 & 208.7 Cy 11 & $103.8 \mathrm{Cy} 11$ & $103.8 \mathrm{Cy} 11$ \\
\hline 1 & 9.404 & 1069.8 & 0.0238 & 15.556 & 1067.1 & 0.0238 & 19.544 & 906.2 & 0.0234 \\
\hline 2 & 14.149 & 1225.5 & 0.0237 & 22.886 & 1187.5 & 0.0237 & 28.833 & 994.7 & 0.0234 \\
\hline 3 & 17.102 & 1301.8 & 0.0236 & 26.887 & 1221.2 & 0.0236 & 33.877 & 1035.8 & 0.0232 \\
\hline 4 & 18.393 & 1317.0 & 0.0234 & 28.304 & 1216.1 & 0.0234 & 35.840 & 1065.3 & 0.0231 \\
\hline 5 & 18.892 & 1312.6 & 0.0233 & 28.689 & 1202.5 & 0.0233 & 36.521 & 1080.8 & 0.0230 \\
\hline 6 & 19.123 & 1305.1 & 0.0231 & 28.813 & 1191.4 & 0.0231 & 36.809 & 1088.3 & 0.0229 \\
\hline 7 & 19.257 & 1298.3 & 0.0230 & 28.887 & 1183.6 & 0.0230 & 36.975 & 1091.1 & 0.0228 \\
\hline 8 & 19.347 & 1293.1 & 0.0229 & 28.955 & 1178.5 & 0.0229 & 37.090 & 1091.0 & 0.0226 \\
\hline 9 & 19.418 & 1289.7 & 0.0227 & 29.034 & 1175.0 & 0.0227 & 37.183 & 1088.9 & 0.0225 \\
\hline 10 & 19.487 & 1288.2 & 0.0226 & 29.132 & 1172.8 & 0.0226 & 37.272 & 1085.3 & 0.0224 \\
\hline 11 & 19.570 & 1289.0 & 0.0225 & 29.270 & 1172.0 & 0.0225 & 37.383 & 1080.3 & 0.0223 \\
\hline 12 & 19.695 & 1292.5 & 0.0223 & 29.497 & 1173.3 & 0.0223 & 37.562 & 1073.8 & 0.0222 \\
\hline 13 & 19.834 & 1297.8 & 0.0222 & 29.794 & 1178.2 & 0.0222 & 37.776 & 1064.9 & 0.0221 \\
\hline 14 & 19.792 & 1302.4 & 0.0221 & 29.927 & 1185.5 & 0.0221 & 37.767 & 1052.4 & 0.0220 \\
\hline 15 & 19.442 & 1303.7 & 0.0220 & 29.761 & 1195.8 & 0.0220 & 37.353 & 1033.7 & 0.0219 \\
\hline 16 & 18.474 & 1292.8 & 0.0218 & 28.844 & 1203.0 & 0.0219 & 36.002 & 1004.6 & 0.0218 \\
\hline 17 & 16.141 & 1235.7 & 0.0217 & 25.877 & 1180.7 & 0.0217 & 32.192 & 957.5 & 0.0217 \\
\hline 18 & 11.422 & 1080.1 & 0.0216 & 18.710 & 1074.2 & 0.0216 & 23.003 & 864.7 & 0.0216 \\
\hline
\end{tabular}


Table 4-102. Burnup and TH Feedback Parameters by Axial Node for Assembly K23 (Cont.)

\begin{tabular}{|c|c|c|c|c|c|c|}
\hline \multirow[b]{2}{*}{$\begin{array}{l}\text { Axial } \\
\text { Node }\end{array}$} & \multicolumn{3}{|c|}{ Datapoint (417.8 Cy11) } & \multicolumn{3}{|c|}{ Statepoint 74 (615.0 Cyt1) } \\
\hline & $\begin{array}{c}\text { Burnup } \\
\text { (GWd/MTU) }\end{array}$ & $\begin{array}{c}\text { T-Fuel } \\
{ }^{\circ} \mathrm{F} \\
\end{array}$ & $\begin{array}{c}\text { Spec. Vol. } \\
\mathrm{ft}^{3} / \mathrm{lbm}\end{array}$ & $\begin{array}{c}\text { Burnup } \\
\text { (GWd/MTU) }\end{array}$ & $\begin{array}{c}\text { T-Fuel } \\
{ }^{\circ} \mathrm{F} \\
\end{array}$ & $\begin{array}{c}\text { Spec. Vol. } \\
\mathrm{ft}^{3} / \mathrm{lbm}\end{array}$ \\
\hline & 417.8 Cy 11 & 312.8 Cy 11 & 312.8 Cy 11 & 615.0 Cy11 & 515.7 Cy 11 & 515.7 Cy 11 \\
\hline 1 & 24.109 & 921.2 & 0.0234 & 28.829 & 924.0 & 0.0234 \\
\hline 2 & 35.348 & 997.5 & 0.0233 & 41.758 & 1001.4 & 0.0233 \\
\hline 3 & 41.229 & 1037.3 & 0.0232 & 48.213 & 1023.9 & 0.0232 \\
\hline 4 & 43.475 & 1048.0 & 0.0231 & 50.541 & 1021.8 & 0.0230 \\
\hline 5 & 44.233 & 1048.8 & 0.0230 & 51.250 & 1014.7 & 0.0229 \\
\hline 6 & 44.527 & 1046.0 & 0.0229 & 51.481 & 1007.7 & 0.0228 \\
\hline 7 & 44.681 & 1042.4 & 0.0227 & 51.590 & 1002.0 & 0.0227 \\
\hline 8 & 44.786 & 1039.0 & 0.0226 & 51.670 & 997.8 & 0.0226 \\
\hline 9 & 44.877 & 1036.1 & 0.0225 & 51.752 & 994.6 & 0.0225 \\
\hline 10 & 44.974 & 1033.8 & 0.0224 & 51.855 & 992.3 & 0.0224 \\
\hline 11 & 45.105 & 1032.2 & 0.0223 & 52.004 & 990.7 & 0.0223 \\
\hline 12 & 45.314 & 1031.0 & 0.0222 & 52.249 & 989.9 & 0.0222 \\
\hline 13 & 45.562 & 1029.9 & 0.0221 & 52.551 & 990.3 & 0.0221 \\
\hline 14 & 45.577 & 1028.8 & 0.0220 & 52.642 & 992.5 & 0.0220 \\
\hline 15 & 45.138 & 1025.7 & 0.0219 & 52.283 & 995.9 & 0.0219 \\
\hline 16 & 43.611 & 1015.6 & 0.0218 & 50.758 & 996.9 & 0.0218 \\
\hline 17 & 39.161 & 981.8 & 0.0217 & 45.916 & 979.1 & 0.0217 \\
\hline 18 & 27.936 & 883.7 & 0.0216 & 32.954 & 882.0 & 0.0216 \\
\hline
\end{tabular}

Table 4-103. Burnup and TH Feedback Parameters by Axial Node for Assembly K25

\begin{tabular}{|c|c|c|c|c|c|c|c|c|c|}
\hline \multirow[b]{2}{*}{$\begin{array}{l}\text { Axial } \\
\text { Node }\end{array}$} & \multicolumn{3}{|c|}{ Statepoint $70(27.0$ Cy10) } & \multicolumn{3}{|c|}{ StatepoInt 71 (143.0 Cy10) } & \multicolumn{3}{|c|}{ Statepoint 72 (209.0 Cy 10$)$} \\
\hline & $\begin{array}{c}\text { Burnup } \\
\text { (GWd/MTU) }\end{array}$ & $\begin{array}{l}\text { T-Fuel } \\
{ }^{\circ} \mathrm{F}\end{array}$ & $\begin{array}{c}\text { Spec. Vol. } \\
\mathrm{ft}^{3} / / \mathrm{bm}\end{array}$ & $\begin{array}{c}\text { Burnup } \\
\text { (GWd/MTU) }\end{array}$ & $\begin{array}{l}\text { T-Fuel } \\
{ }^{\circ} \mathrm{F}\end{array}$ & $\begin{array}{l}\text { Spec. Vol. } \\
\mathrm{ft}^{3} / \mathrm{lbm}\end{array}$ & $\begin{array}{c}\text { Burnup } \\
\text { (GWd/MTU) }\end{array}$ & $\begin{array}{l}\text { T-Fuel } \\
{ }^{\circ} \mathrm{F}\end{array}$ & $\begin{array}{c}\text { Spec. Vol. } \\
\mathrm{ft}^{3} / \mathrm{lbm}\end{array}$ \\
\hline & 27.0 Cy 10 & 18.0 Cy 10 & $18.0 \mathrm{Cy} 10$ & 143.0 Cy 10 & 82.9 Cy 10 & 82.9 Cy 10 & 209.0 Cy 10 & 176.7 Cy 10 & 176.7 Cy 10 \\
\hline 1 & 0.335 & 884.3 & 0.0232 & 1.810 & 884.0 & 0.0232 & 2.722 & 895.7 & 0.0231 \\
\hline 2 & 0.587 & 1086.0 & 0.0232 & 3.126 & 1075.0 & 0.0231 & 4.647 & 1069.4 & 0.0231 \\
\hline 3 & 0.761 & 1210.9 & 0.0231 & 3.996 & 1186.1 & 0.0230 & 5.870 & 1155.3 & 0.0230 \\
\hline 4 & 0.865 & 1279.8 & 0.0230 & 4.492 & 1242.7 & 0.0229 & 6.530 & 1190.3 & 0.0229 \\
\hline 5 & 0.931 & 1319.7 & 0.0229 & 4.782 & 1272.1 & 0.0228 & 6.894 & 1203.1 & 0.0228 \\
\hline 6 & 0.973 & 1342.2 & 0.0228 & 4.957 & 1287.4 & 0.0227 & 7.101 & 1206.5 & 0.0227 \\
\hline 7 & 1.002 & 1355.7 & 0.0227 & 5.068 & 1294.6 & 0.0226 & 7.226 & 1206.1 & 0.0226 \\
\hline 8 & 1.020 & 1363.2 & 0.0226 & 5.138 & 1297.9 & 0.0225 & 7.304 & 1204.5 & 0.0225 \\
\hline 9 & 1.031 & 1366.4 & 0.0224 & 5.181 & 1298.6 & 0.0224 & 7.353 & 1202.8 & 0.0224 \\
\hline 10 & 1.035 & 1365.8 & 0.0223 & 5.202 & 1297.5 & 0.0223 & 7.380 & 1201.6 & 0.0223 \\
\hline 11 & 1.033 & 1361.4 & 0.0222 & 5.202 & 1294.6 & 0.0222 & 7.387 & 1200.9 & 0.0222 \\
\hline 12 & 1.024 & 1352.3 & 0.0221 & 5.175 & 1289.3 & 0.0221 & 7.366 & 1200.3 & 0.0221 \\
\hline 13 & 1.004 & 1336.9 & 0.0220 & 5.109 & 1280.2 & 0.0220 & 7.300 & 1199.0 & 0.0220 \\
\hline 14 & 0.969 & 1312.3 & 0.0219 & 4.982 & 1264.8 & 0.0219 & 7.159 & 1194.9 & 0.0219 \\
\hline 15 & 0.913 & 1272.5 & 0.0218 & 4.750 & 1236.5 & 0.0218 & 6.877 & 1183.1 & 0.0218 \\
\hline 16 & 0.818 & 1204.9 & 0.0217 & 4.324 & 1183.9 & 0.0217 & 6.321 & 1152.5 & 0.0218 \\
\hline 17 & 0.655 & 1084.6 & 0.0217 & 3.516 & 1079.8 & 0.0217 & 5.199 & 1074.5 & 0.0217 \\
\hline 18 & 0.375 & 866.0 & 0.0216 & 2.048 & 873.2 & 0.0216 & 3.064 & 889.1 & 0.0216 \\
\hline
\end{tabular}


Table 4-103. Burnup and TH Feedback Parameters by Axial Node for Assembly K25 (Cont.)

\begin{tabular}{|c|c|c|c|c|c|c|c|c|c|}
\hline \multirow[b]{2}{*}{$\begin{array}{l}\text { Axial } \\
\text { Node }\end{array}$} & \multicolumn{3}{|c|}{ Datapoint (430.4 Cy10) } & \multicolumn{3}{|c|}{ Statepoint 73 (BOC Cy11) } & \multicolumn{3}{|c|}{ Datapoint (208.7 Cy11) } \\
\hline & $\begin{array}{c}\text { Burnup } \\
\text { (GWd/MTU) }\end{array}$ & $\begin{array}{c}\text { T-Fuel } \\
{ }^{\circ} \mathrm{F} \\
\end{array}$ & $\begin{array}{c}\text { Spec. Vol. } \\
\mathrm{ft}^{3} / \mathrm{bm}\end{array}$ & $\begin{array}{c}\text { Burnup } \\
\text { (GWd/MTU) }\end{array}$ & $\begin{array}{l}\text { T-Fuel } \\
{ }^{\circ} \mathrm{F}\end{array}$ & $\begin{array}{c}\text { Spec. Vol. } \\
\mathrm{ft}^{3} / \mathrm{lbm}\end{array}$ & $\begin{array}{c}\text { Burnup } \\
\text { (GWd/MTU) }\end{array}$ & $\begin{array}{l}\text { T-Fuel } \\
{ }^{\circ} \mathrm{F}\end{array}$ & $\begin{array}{c}\text { Spec. Vol. } \\
\mathrm{ft}^{3} / \mathrm{bm}\end{array}$ \\
\hline & 430.4 Cy 10 & 319.5 Cy 10 & 319.5 Cy 10 & BOC Cy 11 & 549.3 Cy 10 & 549.3 Cy 10 & 208.7 Cy 11 & 103.8 Cy 11 & 103.8 Cy 11 \\
\hline 1 & 6.196 & 920.0 & 0.0231 & 10.510 & 946.3 & 0.0231 & 14.287 & 915.3 & 0.0234 \\
\hline 2 & 10.129 & 1062.0 & 0.0230 & 16.423 & 1052.6 & 0.0230 & 22.195 & 1023.7 & 0.0234 \\
\hline 3 & 12.321 & 1116.6 & 0.0229 & 19.354 & 1080.1 & 0.0229 & 26.265 & 1076.5 & 0.0233 \\
\hline 4 & 13.300 & 1128.6 & 0.0228 & 20.445 & 1076.9 & 0.0228 & 27.949 & 1103.7 & 0.0231 \\
\hline 5 & 13.731 & 1126.1 & 0.0227 & 20.820 & 1067.6 & 0.0227 & 28.637 & 1117.6 & 0.0230 \\
\hline 6 & 13.926 & 1120.0 & 0.0226 & 20.946 & 1059.4 & 0.0226 & 28.940 & 1124.6 & 0.0229 \\
\hline 7 & 14.023 & 1114.0 & 0.0225 & 20.998 & 1053.5 & 0.0226 & 29.098 & 1127.6 & 0.0228 \\
\hline 8 & 14.082 & 1109.3 & 0.0224 & 21.037 & 1049.4 & 0.0225 & 29.199 & 1128.0 & 0.0227 \\
\hline 9 & 14.126 & 1106.1 & 0.0224 & 21.081 & 1046.6 & 0.0224 & 29.277 & 1126.6 & 0.0225 \\
\hline 10 & 14.166 & 1104.6 & 0.0223 & 21.136 & 1044.8 & 0.0223 & 29.344 & 1124.1 & 0.0224 \\
\hline 11 & 14.202 & 1104.6 & 0.0222 & 21.203 & 1043.8 & 0.0222 & 29.411 & 1120.8 & 0.0223 \\
\hline 12 & 14.227 & 1106.0 & 0.0221 & 21.275 & 1044.0 & 0.0221 & 29.473 & 1117.1 & 0.0222 \\
\hline 13 & 14.218 & 1108.6 & 0.0220 & 21.338 & 1046.0 & 0.0220 & 29.501 & 1111.9 & 0.0221 \\
\hline 14 & 14.129 & 1111.9 & 0.0219 & 21.354 & 1050.7 & 0.0219 & 29.405 & 1102.1 & 0.0220 \\
\hline 15 & 13.843 & 1113.0 & 0.0218 & 21.183 & 1057.8 & 0.0219 & 28.999 & 1085.5 & 0.0219 \\
\hline 16 & 13.077 & 1102.7 & 0.0218 & 20.422 & 1062.0 & 0.0218 & 27.787 & 1059.7 & 0.0218 \\
\hline 17 & 11.146 & 1054.7 & 0.0217 & 17.954 & 1042.1 & 0.0217 & 24.408 & 1015.4 & 0.0217 \\
\hline 18 & 6.864 & 906.9 & 0.0216 & 11.574 & 930.9 & 0.0216 & 15.916 & 906.8 & 0.0216 \\
\hline
\end{tabular}

\begin{tabular}{|c|c|c|c|c|c|c|}
\hline \multirow[b]{2}{*}{$\begin{array}{l}\text { Axial } \\
\text { Node }\end{array}$} & \multicolumn{3}{|c|}{ Datapoint (417.8 Cy11) } & \multicolumn{3}{|c|}{ Statepoint $74(615.0$ Cy11) } \\
\hline & $\begin{array}{c}\text { Burnup } \\
\text { (GWd/MTU) }\end{array}$ & $\begin{array}{c}\text { T-Fuel } \\
{ }^{\circ} \mathrm{F}\end{array}$ & $\begin{array}{c}\text { Spec. Vol. } \\
\mathrm{ft}^{3} / \mathrm{lbm}\end{array}$ & $\begin{array}{c}\text { Burnup } \\
\text { (GWd/MTU) }\end{array}$ & $\begin{array}{c}\text { T-Fuel } \\
{ }^{\circ} \mathrm{F}\end{array}$ & $\begin{array}{c}\text { Spec. Vol. } \\
\mathrm{ft}^{3} / \mathrm{lbm}\end{array}$ \\
\hline & $417.8 \mathrm{Cy} 11$ & 312.8 Cy 11 & 312.8 Cy 11 & $615.0 \mathrm{Cy} 11$ & 515.7 Cy 11 & 515.7 Cy 11 \\
\hline 1 & 18.581 & 930.0 & 0.0234 & 23.111 & 941.6 & 0.0233 \\
\hline 2 & 28.404 & 1008.6 & 0.0233 & 34.616 & 1003.9 & 0.0233 \\
\hline 3 & 33.349 & 1042.0 & 0.0232 & 40.182 & 1030.9 & 0.0231 \\
\hline 4 & 35.338 & 1051.9 & 0.0231 & 42.280 & 1031.6 & 0.0230 \\
\hline 5 & 36.106 & 1052.1 & 0.0229 & 43.009 & 1025.1 & 0.0229 \\
\hline 6 & 36.419 & 1049.4 & 0.0228 & 43.267 & 1018.5 & 0.0228 \\
\hline 7 & 36.573 & 1046.1 & 0.0227 & 43.384 & 1013.5 & 0.0227 \\
\hline 8 & 36.672 & 1043.2 & 0.0226 & 43.466 & 1009.8 & 0.0226 \\
\hline 9 & 36.754 & 1040.8 & 0.0225 & 43.548 & 1007.1 & 0.0225 \\
\hline 10 & 36.839 & 1039.2 & 0.0224 & 43.645 & 1005.3 & 0.0224 \\
\hline 11 & 36.937 & 1038.5 & 0.0223 & 43.771 & 1004.5 & 0.0223 \\
\hline 12 & 37.051 & 1039.0 & 0.0222 & 43.935 & 1005.0 & 0.0222 \\
\hline 13 & 37.140 & 1040.2 & 0.0221 & 44.099 & 1007.1 & 0.0221 \\
\hline 14 & 37.087 & 1040.6 & 0.0220 & 44.134 & 1010.5 & 0.0220 \\
\hline 15 & 36.667 & 1038.6 & 0.0219 & 43.798 & 1014.4 & 0.0219 \\
\hline 16 & 35.282 & 1030.0 & 0.0218 & 42.414 & 1014.9 & 0.0218 \\
\hline 17 & 31.262 & 1001.3 & 0.0217 & 37.998 & 994.9 & 0.0217 \\
\hline 18 & 20.780 & 920.1 & 0.0216 & 25.807 & 922.4 & 0.0216 \\
\hline
\end{tabular}


Table 4-104. Burnup and TH Feedback Parameters by Axial Node for Assembly K27

\begin{tabular}{|c|c|c|c|c|c|c|c|c|c|}
\hline \multirow{2}{*}{$\begin{array}{c}\text { Axial } \\
\text { Node }\end{array}$} & \multicolumn{3}{|c|}{ Statepoint 70 (27.0 Cy10) } & \multicolumn{2}{c|}{ Statepoint 71 (143.0 Cy10) } & \multicolumn{2}{c|}{ Statepoint 72 (209.0 Cy10) } \\
\cline { 2 - 10 } \\
\hline
\end{tabular}

\begin{tabular}{|c|c|c|c|c|c|c|c|c|c|}
\hline \multirow[b]{2}{*}{$\begin{array}{l}\text { Axial } \\
\text { Node }\end{array}$} & \multicolumn{3}{|c|}{ Datapoint (430.4 Cy10) } & \multicolumn{3}{|c|}{ Statepoint 73 (BOC Cy11) } & \multicolumn{3}{|c|}{ Datapoint (208.7 Cy11) } \\
\hline & $\begin{array}{c}\text { Burnup } \\
\text { (GWd/MTU) }\end{array}$ & $\begin{array}{l}\text { T-Fuel } \\
{ }^{\circ} \mathrm{F}\end{array}$ & \begin{tabular}{|c|} 
Spec. Vol. \\
$\mathrm{ft}^{3} / \mathrm{lbm}$ \\
\end{tabular} & $\begin{array}{c}\text { Burnup } \\
\text { (GWd/MTU) }\end{array}$ & $\begin{array}{c}\text { T-Fuel } \\
{ }^{\circ} \mathrm{F} \\
\end{array}$ & $\begin{array}{c}\text { Spec. Vol. } \\
\mathrm{ft}^{3} / \mathrm{lbm}\end{array}$ & $\begin{array}{c}\text { Burnup } \\
\text { (GWd/MTU) }\end{array}$ & $\begin{array}{c}\text { T-Fuel } \\
{ }^{\circ} \mathrm{F}\end{array}$ & $\begin{array}{c}\text { Spec. Vol. } \\
\mathrm{tt}^{3} / \mathrm{lbm}\end{array}$ \\
\hline & 430.4 Cy 10 & 319.5 Cy 10 & 319.5 Cy10 & BOC Cy11 & 549.3 Cy 10 & 549.3 Cy 10 & 208.7 Cy11 & 103.8 Cy 11 & $103.8 \mathrm{Cy} 11$ \\
\hline 1 & 6.714 & 949.0 & 0.0232 & 11.325 & 967.6 & 0.0233 & 14.178 & 839.9 & 0.0235 \\
\hline 2 & 11.150 & 1103.1 & 0.0232 & 17.967 & 1082.8 & 0.0232 & 23.478 & 999.0 & 0.0235 \\
\hline 3 & 13.729 & 1162.2 & 0.0231 & 21.412 & 1112.9 & 0.0231 & 28.635 & 1083.6 & 0.0234 \\
\hline 4 & 14.842 & 1174.4 & 0.0230 & 22.650 & 1109.0 & 0.0230 & 30.582 & 1116.3 & 0.0232 \\
\hline 5 & 15.306 & 1171.0 & 0.0229 & 23.050 & 1098.8 & 0.0229 & 31.331 & 1132.0 & 0.0231 \\
\hline 6 & 15.512 & 1164.1 & 0.0227 & 23.180 & 1090.0 & 0.0228 & 31.654 & 1139.5 & 0.0230 \\
\hline 7 & 15.617 & 1157.6 & 0.0226 & 23.238 & 1083.8 & 0.0227 & 31.825 & 1142.5 & 0.0228 \\
\hline 8 & 15.682 & 1152.7 & 0.0225 & 23.283 & 1079.5 & 0.0226 & 31.938 & 1142.8 & 0.0227 \\
\hline 9 & 15.731 & 1149.4 & 0.0224 & 23.335 & 1076.7 & 0.0225 & 32.026 & 1141.4 & 0.0226 \\
\hline 10 & 15.776 & 1147.7 & 0.0223 & 23.399 & 1074.8 & 0.0224 & 32.106 & 1138.8 & 0.0225 \\
\hline 11 & 15.819 & 1147.7 & 0.0222 & 23.477 & 1073.8 & 0.0223 & 32.186 & 1135.5 & 0.0224 \\
\hline 12 & 15.853 & 1149.4 & 0.0222 & 23.566 & 1074.1 & 0.0222 & 32.264 & 1131.4 & 0.0222 \\
\hline 13 & 15.853 & 1152.4 & 0.0221 & 23.651 & 1076.4 & 0.0221 & 32.304 & 1125.5 & 0.0221 \\
\hline 14 & 15.765 & 1156.0 & 0.0220 & 23.680 & 1081.5 & 0.0220 & 32.217 & 1115.6 & 0.0220 \\
\hline 15 & 15.467 & 1157.3 & 0.0219 & 23.514 & 1089.2 & 0.0219 & 31.810 & 1099.0 & 0.0219 \\
\hline 16 & 14.670 & 1147.2 & 0.0218 & 22.740 & 1095.1 & 0.0218 & 30.567 & 1071.3 & 0.0218 \\
\hline 17 & 12.640 & 1099.5 & 0.0217 & 20.188 & 1079.1 & 0.0217 & 27.039 & 1023.5 & 0.0217 \\
\hline 18 & 8.205 & 961.3 & 0.0216 & 13.730 & 979.8 & 0.0216 & 18.296 & 910.1 & 0.0216 \\
\hline
\end{tabular}


Table 4-104. Burnup and TH Feedback Parameters by Axial Node for Assembly K27 (Cont.)

\begin{tabular}{|c|c|c|c|c|c|c|}
\hline \multirow{2}{*}{$\begin{array}{c}\text { Axial } \\
\text { Node }\end{array}$} & $\begin{array}{c}\text { Burnup } \\
\text { (GWd/MTU) }\end{array}$ & $\begin{array}{c}\text { T-Fuel } \\
{ }^{\circ} \mathrm{F}\end{array}$ & $\begin{array}{c}\text { Spec. Vol. } \\
\mathrm{ft}^{3} / \mathrm{lbm}\end{array}$ & $\begin{array}{c}\text { Burnup } \\
\text { (GWd/MTU) }\end{array}$ & $\begin{array}{c}\text { T-Fuel } \\
{ }^{\circ} \mathrm{F}\end{array}$ & $\begin{array}{c}\text { Spec. Vol. } \\
\mathrm{ft}^{3} / \mathrm{lbm}\end{array}$ \\
\hline & $417.8 \mathrm{Cy} 11$ & $312.8 \mathrm{Cy} 11$ & $312.8 \mathrm{Cy} 11$ & $615.0 \mathrm{Cy} 11$ & $515.7 \mathrm{Cy} 11$ & $515.7 \mathrm{Cy} 11$ \\
\hline 1 & 17.408 & 851.0 & 0.0234 & 20.846 & 867.8 & 0.0234 \\
\hline 3 & 29.333 & 973.4 & 0.0233 & 35.238 & 978.9 & 0.0233 \\
\hline 3 & 35.855 & 1039.9 & 0.0232 & 42.794 & 1032.2 & 0.0232 \\
\hline 4 & 38.181 & 1056.8 & 0.0231 & 45.280 & 1036.1 & 0.0231 \\
\hline 5 & 39.025 & 1058.9 & 0.0230 & 46.093 & 1029.9 & 0.0230 \\
\hline 6 & 39.362 & 1056.7 & 0.0229 & 46.376 & 1023.3 & 0.0228 \\
\hline 7 & 39.527 & 1053.5 & 0.0228 & 46.502 & 1018.1 & 0.0227 \\
\hline 8 & 39.636 & 1050.5 & 0.0226 & 46.592 & 1014.2 & 0.0226 \\
\hline 9 & 39.729 & 1048.0 & 0.0225 & 46.682 & 1011.3 & 0.0225 \\
\hline 10 & 39.824 & 1046.3 & 0.0224 & 46.789 & 1009.3 & 0.0224 \\
\hline 11 & 39.935 & 1045.4 & 0.0223 & 46.926 & 1008.2 & 0.0223 \\
\hline 12 & 40.059 & 1045.5 & 0.0222 & 47.095 & 1008.3 & 0.0222 \\
\hline 13 & 40.154 & 1046.1 & 0.0221 & 47.256 & 1009.8 & 0.0221 \\
\hline 14 & 40.106 & 1046.1 & 0.0220 & 47.293 & 1012.8 & 0.0220 \\
\hline 15 & 39.684 & 1043.5 & 0.0219 & 46.956 & 1016.6 & 0.0219 \\
\hline 16 & 38.268 & 1033.1 & 0.0218 & 45.541 & 1017.3 & 0.0218 \\
\hline 17 & 34.090 & 1000.8 & 0.0217 & 40.965 & 997.9 & 0.0217 \\
\hline 18 & 23.287 & 913.8 & 0.0216 & 28.411 & 914.5 & 0.0216 \\
\hline
\end{tabular}

Table 4-105. Burnup and TH Feedback Parameters by Axial Node for Assembly K27a

\begin{tabular}{|c|c|c|c|c|c|c|c|c|c|}
\hline \multirow[b]{2}{*}{$\begin{array}{l}\text { Axial } \\
\text { Node }\end{array}$} & \multicolumn{3}{|c|}{ Statepoint 70 (27.0 Cy10) } & \multicolumn{3}{|c|}{ Statepoint 71 ( 143.0 Cy10) } & \multicolumn{3}{|c|}{ Statepoint $72(209.0$ Cy10) } \\
\hline & $\begin{array}{c}\text { Burnup } \\
\text { (GWd/MTU) }\end{array}$ & $\begin{array}{c}\text { T-Fuel } \\
{ }^{\circ} \mathrm{F}\end{array}$ & $\begin{array}{c}\text { Spec. Vol. } \\
\mathrm{ft}^{3} / \mathrm{lbm}\end{array}$ & $\begin{array}{c}\text { Burnup } \\
\text { (GWd/MTU) }\end{array}$ & $\begin{array}{c}\text { T-Fuel } \\
{ }^{\circ} \mathrm{F}\end{array}$ & $\begin{array}{c}\text { Spec. Vol. } \\
\mathrm{ft}^{3} / \mathrm{lbm}\end{array}$ & $\begin{array}{c}\text { Burnup } \\
\text { (GWd/MTU) }\end{array}$ & $\begin{array}{l}\text { T-Fuel } \\
{ }^{\circ} \mathrm{F} \\
\end{array}$ & $\begin{array}{c}\text { Spec. Vol. } \\
\mathrm{ft}^{3} / \mathrm{lbm}\end{array}$ \\
\hline & 27.0 Cy 10 & $18.0 \mathrm{Cy} 10$ & 18.0 Cy 10 & 143.0 Cy 10 & 82.9 Cy 10 & 82.9 Cy 10 & 209.0 Cy 10 & 176.7 Cy 10 & 176.7 Cy 10 \\
\hline 1 & 0.374 & 932.6 & 0.0235 & 1.974 & 917.1 & 0.0234 & 2.960 & 922.7 & 0.0233 \\
\hline 2 & 0.666 & 1165.7 & 0.0235 & 3.468 & 1133.4 & 0.0233 & 5.138 & 1113.2 & 0.0232 \\
\hline 3 & 0.876 & 1307.2 & 0.0234 & 4.515 & 1259.1 & 0.0233 & 6.596 & 1208.4 & 0.0231 \\
\hline 4 & 0.999 & 1383.6 & 0.0232 & 5.096 & 1320.8 & 0.0231 & 7.361 & 1245.8 & 0.0230 \\
\hline 5 & 1.074 & 1425.2 & 0.0231 & 5.418 & 1351.1 & 0.0230 & 7.760 & 1258.3 & 0.0229 \\
\hline 6 & 1.122 & 1450.1 & 0.0230 & 5.610 & 1365.6 & 0.0229 & 7.984 & 1261.0 & 0.0228 \\
\hline 7 & 1.154 & 1464.7 & 0.0228 & 5.731 & 1372.8 & 0.0228 & 8.119 & 1260.1 & 0.0227 \\
\hline 8 & 1.176 & 1472.9 & 0.0227 & 5.809 & 1376.0 & 0.0226 & 8.204 & 1258.0 & 0.0226 \\
\hline 9 & 1.189 & 1476.4 & 0.0226 & 5.856 & 1376.5 & 0.0225 & 8.258 & 1256.0 & 0.0225 \\
\hline 10 & 1.194 & 1475.9 & 0.0225 & 5.880 & 1375.1 & 0.0224 & 8.288 & 1254.5 & 0.0224 \\
\hline 11 & 1.193 & 1471.4 & 0.0223 & 5.881 & 1372.0 & 0.0223 & 8.296 & 1253.6 & 0.0223 \\
\hline 12 & 1.183 & 1462.1 & 0.0222 & 5.856 & 1366.6 & 0.0222 & 8.277 & 1253.1 & 0.0222 \\
\hline 13 & 1.162 & 1446.1 & 0.0221 & 5.789 & 1357.4 & 0.0221 & 8.212 & 1252.0 & 0.0221 \\
\hline 14 & 1.124 & 1419.6 & 0.0220 & 5.654 & 1341.3 & 0.0220 & 8.063 & 1247.9 & 0.0220 \\
\hline 15 & 1.060 & 1376.0 & 0.0219 & 5.405 & 1312.8 & 0.0219 & 7.765 & 1236.3 & 0.0219 \\
\hline 16 & 0.956 & 1302.6 & 0.0218 & 4.949 & 1258.1 & 0.0218 & 7.176 & 1205.8 & 0.0218 \\
\hline 17 & 0.772 & 1171.4 & 0.0217 & 4.073 & 1150.5 & 0.0217 & 5.973 & 1129.0 & 0.0217 \\
\hline 18 & 0.463 & 938.1 & 0.0216 & 2.493 & 938.5 & 0.0216 & 3.705 & 948.4 & 0.0216 \\
\hline
\end{tabular}


Table 4-105. Burnup and TH Feedback Parameters by Axial Node for Assembly K27a (Cont.)

\begin{tabular}{|c|c|c|c|c|c|c|c|c|c|}
\hline \multirow[b]{2}{*}{$\begin{array}{l}\text { Axial } \\
\text { Node }\end{array}$} & \multicolumn{3}{|c|}{ Datapoint (430.4 Cy10) } & \multicolumn{3}{|c|}{ Statepoint 73 (BOC Cy11) } & \multicolumn{3}{|c|}{ Datapoint (208.7 Cy11) } \\
\hline & $\begin{array}{c}\text { Burnup } \\
\text { (GWd/MTU) }\end{array}$ & $\begin{array}{l}\text { T-Fuel } \\
{ }^{\circ} \mathrm{F}\end{array}$ & $\begin{array}{c}\text { Spec. Vol. } \\
\mathrm{ft}^{3} / \mathrm{lbm}\end{array}$ & $\begin{array}{c}\text { Burnup } \\
\text { (GWd/MTU) }\end{array}$ & $\begin{array}{l}\text { T-Fuel } \\
{ }^{\circ} \mathrm{F}\end{array}$ & $\begin{array}{c}\text { Spec. Vol. } \\
\mathrm{ft}^{3} / \mathrm{lbm}\end{array}$ & $\begin{array}{c}\text { Burnup } \\
\text { (GWd/MTU) }\end{array}$ & $\begin{array}{l}\text { T-Fuel } \\
{ }^{\circ} \mathrm{F}\end{array}$ & $\begin{array}{l}\text { Spec. Vol. } \\
\mathrm{ft}^{3} / \mathrm{lbm}\end{array}$ \\
\hline & 430.4 Cy 10 & 319.5 Cy 10 & 319.5 Cy 10 & BOC Cy11 & 549.3 Cy 10 & 549.3 Cy 10 & 208.7 Cy 11 & 103.8 Cy 11 & $103.8 \mathrm{Cy} 11$ \\
\hline 1 & 6.714 & 949.0 & 0.0232 & 11.322 & 967.6 & 0.0233 & 15.829 & 971.7 & 0.0236 \\
\hline 2 & 11.150 & 1103.1 & 0.0232 & 17.967 & 1082.8 & 0.0232 & 24.716 & 1080.1 & 0.0236 \\
\hline 3 & 13.729 & 1162.2 & 0.0231 & 21.419 & 1112.9 & 0.0231 & 29.313 & 1127.5 & 0.0234 \\
\hline 4 & 14.842 & 1174.4 & 0.0230 & 22.663 & 1109.0 & 0.0230 & 31.087 & 1148.9 & 0.0233 \\
\hline 5 & 15.306 & 1171.0 & 0.0229 & 23.065 & 1098.8 & 0.0229 & 31.759 & 1159.9 & 0.0232 \\
\hline 6 & 15.512 & 1164.1 & 0.0227 & 23.197 & 1090.0 & 0.0228 & 32.038 & 1164.7 & 0.0230 \\
\hline 7 & 15.617 & 1157.6 & 0.0226 & 23.255 & 1083.8 & 0.0227 & 32.174 & 1165.6 & 0.0229 \\
\hline 8 & 15.682 & 1152.7 & 0.0225 & 23.301 & 1079.5 & 0.0226 & 32.253 & 1163.9 & 0.0228 \\
\hline 9 & 15.731 & 1149.4 & 0.0224 & 23.353 & 1076.7 & 0.0225 & 32.306 & 1160.3 & 0.0226 \\
\hline 10 & 15.776 & 1147.7 & 0.0223 & 23.417 & 1074.8 & 0.0224 & 32.346 & 1155.3 & 0.0225 \\
\hline 11 & 15.819 & 1147.7 & 0.0222 & 23.495 & 1073.8 & 0.0223 & 32.380 & 1149.2 & 0.0224 \\
\hline 12 & 15.853 & 1149.4 & 0.0222 & 23.585 & 1074.1 & 0.0222 & 32.407 & 1141.8 & 0.0222 \\
\hline 13 & 15.853 & 1152.4 & 0.0221 & 23.671 & 1076.4 & 0.0221 & 32.399 & 1132.8 & 0.0221 \\
\hline 14 & 15.765 & 1156.0 & 0.0220 & 23.700 & 1081.5 & 0.0220 & 32.286 & 1121.0 & 0.0220 \\
\hline 15 & 15.467 & 1157.3 & 0.0219 & 23.535 & 1089.2 & 0.0219 & 31.896 & 1105.1 & 0.0219 \\
\hline 16 & 14.670 & 1147.2 & 0.0218 & 22.761 & 1095.1 & 0.0218 & 30.741 & 1082.6 & 0.0218 \\
\hline 17 & 12.640 & 1099.5 & 0.0217 & 20.206 & 1079.1 & 0.0217 & 27.344 & 1042.9 & 0.0217 \\
\hline 18 & 8.205 & 961.3 & 0.0216 & 13.742 & 979.8 & 0.0216 & 18.611 & 932.7 & 0.0216 \\
\hline
\end{tabular}

\begin{tabular}{|c|c|c|c|c|c|c|}
\hline \multirow{2}{*}{$\begin{array}{c}\text { Axial } \\
\text { Node }\end{array}$} & $\begin{array}{c}\text { Burnup } \\
\text { (GWd/MTU) }\end{array}$ & $\begin{array}{c}\text { T-Fuel } \\
{ }^{\circ} \mathbf{F}\end{array}$ & $\begin{array}{c}\text { Spec. Vol. } \\
\mathrm{ft}^{3} / \mathrm{lbm}\end{array}$ & $\begin{array}{c}\text { Burnup } \\
(\text { GW d/MTU) }\end{array}$ & $\begin{array}{c}\text { T-Fuel } \\
{ }^{\circ} \mathrm{F}\end{array}$ & $\begin{array}{c}\text { Spec. Vol. } \\
\mathrm{ft}^{3} / \mathrm{lbm}\end{array}$ \\
\hline & 417.8 Cy11 & 312.8 Cy11 & $312.8 \mathrm{Cy} 11$ & 615.0 Cy11 & 515.7 Cy11 & 515.7 Cy11 \\
\hline 1 & 20.698 & 964.8 & 0.0235 & 25.652 & 960.9 & 0.0235 \\
\hline 2 & 31.638 & 1041.1 & 0.0234 & 38.324 & 1028.8 & 0.0234 \\
\hline 3 & 37.103 & 1076.4 & 0.0233 & 44.380 & 1053.5 & 0.0232 \\
\hline 4 & 39.146 & 1087.3 & 0.0232 & 46.495 & 1051.2 & 0.0231 \\
\hline 5 & 39.883 & 1087.5 & 0.0231 & 47.174 & 1043.3 & 0.0230 \\
\hline 6 & 40.163 & 1084.5 & 0.0229 & 47.384 & 1035.7 & 0.0229 \\
\hline 7 & 40.284 & 1080.7 & 0.0228 & 47.455 & 1029.7 & 0.0228 \\
\hline 8 & 40.349 & 1077.1 & 0.0227 & 47.491 & 1025.2 & 0.0226 \\
\hline 9 & 40.394 & 1073.9 & 0.0226 & 47.525 & 1021.9 & 0.0225 \\
\hline 10 & 40.436 & 1071.3 & 0.0225 & 47.571 & 1019.4 & 0.0224 \\
\hline 11 & 40.483 & 1069.3 & 0.0224 & 47.635 & 1017.9 & 0.0223 \\
\hline 12 & 40.533 & 1068.0 & 0.0222 & 47.721 & 1017.5 & 0.0222 \\
\hline 13 & 40.554 & 1067.0 & 0.0221 & 47.798 & 1018.5 & 0.0221 \\
\hline 14 & 40.459 & 1065.6 & 0.0220 & 47.778 & 1021.0 & 0.0220 \\
\hline 15 & 40.048 & 1062.4 & 0.0219 & 47.449 & 1024.6 & 0.0219 \\
\hline 16 & 38.745 & 1053.2 & 0.0218 & 46.162 & 1026.0 & 0.0218 \\
\hline 17 & 34.747 & 1022.5 & 0.0217 & 41.805 & 1009.0 & 0.0217 \\
\hline 18 & 23.926 & 934.0 & 0.0216 & 29.255 & 925.1 & 0.0216 \\
\hline
\end{tabular}


Table 4-106. Burnup and TH Feedback Parameters by Axial Node for Assembly $L 2$

\begin{tabular}{|c|c|c|c|c|c|c|c|c|c|}
\hline \multirow[b]{2}{*}{$\begin{array}{l}\text { Axial } \\
\text { Node }\end{array}$} & \multicolumn{3}{|c|}{ Datapoint (208.7 Cy11) } & \multicolumn{3}{|c|}{ Datapoint (417.8 Cy11) } & \multicolumn{3}{|c|}{ Statepoint 74 (615.0 Cyl1) } \\
\hline & $\begin{array}{c}\text { Burnup } \\
\text { (GWd/MTU) }\end{array}$ & $\begin{array}{l}\text { T-Fuel } \\
{ }^{\circ} \mathrm{F}\end{array}$ & $\begin{array}{c}\text { Spec. Vol. } \\
\mathrm{ft}^{3} / \mathrm{lbm}\end{array}$ & $\begin{array}{c}\text { Burnup } \\
\text { (GWd/MTU) }\end{array}$ & $\begin{array}{l}\text { T-Fuel } \\
{ }^{\circ} \mathrm{F}\end{array}$ & $\begin{array}{c}\text { Spec. Vol. } \\
\mathrm{ft}^{3} / \mathrm{bm}\end{array}$ & $\begin{array}{c}\text { Burnup } \\
\text { (GWd/MTU) }\end{array}$ & $\begin{array}{c}\text { T-Fuel } \\
{ }^{\circ} \mathrm{F}\end{array}$ & $\begin{array}{c}\text { Spec. Vol. } \\
\mathrm{ft}^{3} / \mathrm{lbm}\end{array}$ \\
\hline & 208.7 Cy 11 & $103.8 \mathrm{Cy} 11$ & $103.8 \mathrm{Cy} 11$ & 417.8 Cy 11 & 312.8 Cy 11 & 312.8 Cy 11 & $615.0 \mathrm{Cy} 11$ & 515.7 Cy 11 & 515.7 Cy 11 \\
\hline 1 & 4.483 & 1074.0 & 0.0238 & 9.323 & 1054.8 & 0.0238 & 14.242 & 1036.4 & 0.0237 \\
\hline 2 & 6.636 & 1257.6 & 0.0237 & 13.734 & 1200.7 & 0.0237 & 20.753 & 1159.4 & 0.0236 \\
\hline 3 & 8.045 & 1370.2 & 0.0235 & 16.396 & 1279.4 & 0.0236 & 24.332 & 1194.6 & 0.0235 \\
\hline 4 & 8.750 & 1422.7 & 0.0234 & 17.575 & 1304.4 & 0.0234 & 25.704 & 1197.0 & 0.0233 \\
\hline 5 & 9.104 & 1445.0 & 0.0232 & 18.084 & 1309.4 & 0.0233 & 26.194 & 1189.2 & 0.0232 \\
\hline 6 & 9.290 & 1452.7 & 0.0231 & 18.316 & 1307.7 & 0.0231 & 26.370 & 1181.0 & 0.0230 \\
\hline 7 & 9.387 & 1454.7 & 0.0230 & 18.423 & 1304.6 & 0.0230 & 26.437 & 1174.6 & 0.0229 \\
\hline 8 & 9.429 & 1453.5 & 0.0228 & 18.470 & 1301.8 & 0.0228 & 26.466 & 1170.2 & 0.0228 \\
\hline 9 & 9.433 & 1450.3 & 0.0227 & 18.485 & 1299.6 & 0.0227 & 26.484 & 1167.5 & 0.0227 \\
\hline 10 & 9.408 & 1445.4 & 0.0225 & 18.479 & 1298.1 & 0.0226 & 26.499 & 1166.2 & 0.0225 \\
\hline 11 & 9.355 & 1439.0 & 0.0224 & 18.452 & 1297.5 & 0.0224 & 26.509 & 1166.1 & 0.0224 \\
\hline 12 & 9.271 & 1430.9 & 0.0223 & 18.399 & 1297.3 & 0.0223 & 26.511 & 1167.5 & 0.0223 \\
\hline 13 & 9.150 & 1420.6 & 0.0222 & 18.307 & 1297.6 & 0.0222 & 26.496 & 1170.6 & 0.0222 \\
\hline 14 & 8.977 & 1405.9 & 0.0220 & 18.147 & 1297.3 & 0.0221 & 26.433 & 1175.6 & 0.0221 \\
\hline 15 & 8.706 & 1382.4 & 0.0219 & 17.819 & 1294.0 & 0.0219 & 26.194 & 1181.4 & 0.0220 \\
\hline 16 & 8.206 & 1339.7 & 0.0218 & 17.037 & 1278.1 & 0.0218 & 25.367 & 1181.2 & 0.0218 \\
\hline 17 & 7.171 & 1252.6 & 0.0217 & 15.085 & 1217.6 & 0.0217 & 22.810 & 1153.9 & 0.0217 \\
\hline 18 & 5.162 & 1080.6 & 0.0216 & 10.865 & 1068.0 & 0.0216 & 16.565 & 1040.5 & 0.0216 \\
\hline
\end{tabular}

Table 4-107. Burnup and TH Feedback Parameters by Axial Node for Assembly L6

\begin{tabular}{|c|c|c|c|c|c|c|c|c|c|}
\hline \multirow[b]{2}{*}{$\begin{array}{l}\text { Axlal } \\
\text { Node }\end{array}$} & \multicolumn{3}{|c|}{ Datapoint (208.7 Cy11) } & \multicolumn{3}{|c|}{ Datapoint (417.8 Cy11) } & \multicolumn{3}{|c|}{ Statepoint 74 (615.0 Cy11) } \\
\hline & $\begin{array}{c}\text { Burnup } \\
\text { (GWd/MTU) }\end{array}$ & $\begin{array}{c}\text { T-Fuel } \\
{ }^{\circ} \mathrm{F}\end{array}$ & $\begin{array}{c}\text { Spec. Vol. } \\
\mathrm{ft}^{3} / \mathrm{lbm}\end{array}$ & $\begin{array}{c}\text { Burnup } \\
\text { (GWd/MTU) }\end{array}$ & $\begin{array}{c}\text { T-Fuel } \\
{ }^{\circ} \mathrm{F}\end{array}$ & $\begin{array}{c}\text { Spec. Vol. } \\
\mathrm{ft}^{3} / \mathrm{lbm}\end{array}$ & $\begin{array}{c}\text { Burnup } \\
\text { (GWd/MTU) }\end{array}$ & $\begin{array}{c}\text { T-Fuel } \\
{ }^{\circ} \mathrm{F}\end{array}$ & $\begin{array}{c}\text { Spec. Vol. } \\
\mathrm{ft}^{3} / \mathrm{lbm}\end{array}$ \\
\hline & 208.7 Cy 11 & 103.8 Cy 11 & 103.8 Cy 11 & 417.8 Cy 11 & 312.8 Cy 11 & 312.8 Cy 11 & 615.0 Cy 11 & 515.7 Cy11 & 515.7 Cyt1 \\
\hline 1 & 4.195 & 1039.8 & 0.0237 & 9.010 & 1049.5 & 0.0237 & 13.984 & 1037.0 & 0.0237 \\
\hline 2 & 6.244 & 1217.8 & 0.0236 & 13.300 & 1192.8 & 0.0236 & 20.396 & 1161.4 & 0.0236 \\
\hline 3 & 7.655 & 1331.7 & 0.0235 & 15.984 & 1271.0 & 0.0235 & 24.030 & 1197.4 & 0.0235 \\
\hline 4 & 8.393 & 1388.4 & 0.0233 & 17.200 & 1295.3 & 0.0234 & 25.447 & 1199.5 & 0.0233 \\
\hline 5 & 8.776 & 1415.8 & 0.0232 & 17.729 & 1298.8 & 0.0232 & 25.956 & 1191.7 & 0.0232 \\
\hline 6 & 8.987 & 1428.9 & 0.0231 & 17.977 & 1296.3 & 0.0231 & 26.151 & 1183.6 & 0.0231 \\
\hline 7 & 9.108 & 1434.3 & 0.0229 & 18.106 & 1292.7 & 0.0230 & 26.241 & 1177.3 & 0.0229 \\
\hline 8 & 9.175 & 1435.9 & 0.0228 & 18.180 & 1289.6 & 0.0228 & 26.301 & 1173.0 & 0.0228 \\
\hline 9 & 9.206 & 1435.0 & 0.0226 & 18.228 & 1287.4 & 0.0227 & 26.354 & 1170.3 & 0.0227 \\
\hline 10 & 9.212 & 1432.3 & 0.0225 & 18.261 & 1286.2 & 0.0226 & 26.410 & 1168.7 & 0.0225 \\
\hline 11 & 9.195 & 1428.2 & 0.0224 & 18.286 & 1286.3 & 0.0224 & 26.475 & 1168.4 & 0.0224 \\
\hline 12 & 9.153 & 1422.2 & 0.0223 & 18.299 & 1287.3 & 0.0223 & 26.548 & 1169.5 & 0.0223 \\
\hline 13 & 9.070 & 1413.2 & 0.0221 & 18.275 & 1289.2 & 0.0222 & 26.609 & 1172.5 & 0.0222 \\
\hline 14 & 8.914 & 1398.6 & 0.0220 & 18.159 & 1290.7 & 0.0221 & 26.600 & 1177.7 & 0.0221 \\
\hline 15 & 8.624 & 1372.1 & 0.0219 & 17.833 & 1289.0 & 0.0219 & 26.374 & 1184.0 & 0.0220 \\
\hline 16 & 8.069 & 1323.8 & 0.0218 & 16.994 & 1274.0 & 0.0218 & 25.499 & 1184.7 & 0.0218 \\
\hline 17 & 6.976 & 1231.1 & 0.0217 & 14.953 & 1213.6 & 0.0217 & 22.836 & 1158.0 & 0.0217 \\
\hline 18 & 4.999 & 1062.3 & 0.0216 & 10.755 & 1066.5 & 0.0216 & 16.576 & 1043.8 & 0.0216 \\
\hline
\end{tabular}


Table 4-108. Burnup and TH Feedback Parameters by Axial Node for Assembly L10

\begin{tabular}{|c|c|c|c|c|c|c|c|c|c|}
\hline \multirow[b]{2}{*}{$\begin{array}{l}\text { Axial } \\
\text { Node }\end{array}$} & \multicolumn{3}{|c|}{ Datapoint (208.7 Cy11) } & \multicolumn{3}{|c|}{ Datapoint (417.8 Cy11) } & \multicolumn{3}{|c|}{ Statepoint 74 (615.0 Cy11) } \\
\hline & $\begin{array}{c}\text { Burnup } \\
\text { (GWd/MTU) }\end{array}$ & $\begin{array}{l}\text { T-Fuel } \\
{ }^{\circ} \mathrm{F}\end{array}$ & $\begin{array}{c}\text { Spec. Vol. } \\
\mathrm{ft}^{3} / \mathrm{lbm}\end{array}$ & $\begin{array}{c}\text { Burnup } \\
\text { (GWd/MTU) }\end{array}$ & $\begin{array}{l}\text { T-Fuel } \\
{ }^{\circ} \mathrm{F}\end{array}$ & $\begin{array}{c}\text { Spec. Vol. } \\
\mathrm{ft}^{3} / \mathrm{lbm}\end{array}$ & $\begin{array}{c}\text { Burnup } \\
\text { (GWd/MTU) }\end{array}$ & $\begin{array}{l}\text { T-Fuel } \\
{ }^{\circ} \mathrm{F}\end{array}$ & $\begin{array}{l}\text { Spec. Vol. } \\
\mathrm{ft}^{3} / / \mathrm{bm}\end{array}$ \\
\hline & 208.7 Cy 11 & 103.8 Cy 11 & 103.8 Cy 11 & 417.8 Cy 11 & 312.8 Cy11 & $312.8 \mathrm{Cy} 11$ & $615.0 \mathrm{Cy} 11$ & 515.7 Cy11 & 515.7 Cy 11 \\
\hline 1 & 4.868 & 1108.0 & 0.0238 & 10.067 & 1078.7 & 0.0238 & 15.331 & 1060.7 & 0.0238 \\
\hline 2 & 6.799 & 1269.9 & 0.0237 & 14.000 & 1206.9 & 0.0237 & 21.222 & 1173.5 & 0.0237 \\
\hline 3 & 8.059 & 1369.7 & 0.0235 & 16.379 & 1276.1 & 0.0235 & 24.495 & 1209.7 & 0.0235 \\
\hline 4 & 8.706 & 1417.5 & 0.0234 & 17.465 & 1298.8 & 0.0234 & 25.794 & 1214.0 & 0.0234 \\
\hline 5 & 41 & 1439.4 & 0.0232 & 17.950 & 1303.5 & 0.0232 & 26.275 & 1207.6 & 0.0232 \\
\hline 6 & 9.221 & 1446.8 & 0.0231 & 18.174 & 1302.2 & 0.0231 & 26.453 & 1200.0 & 0.0231 \\
\hline 7 & 9.317 & 1448.8 & 0.0230 & 18.280 & 1299.2 & 0.0230 & 26.524 & 1194.0 & 0.0230 \\
\hline 8 & 9.362 & 1447.8 & 0.0228 & 18.329 & 1296.4 & 0.0228 & 26.559 & 1189.8 & 0.0228 \\
\hline 9 & 9.371 & 1444.8 & 0.0227 & 18.348 & 1294.2 & 0.0227 & 26.585 & 1187.3 & 0.0227 \\
\hline 10 & 9.354 & 1440.4 & 0.0225 & 18.349 & 1292.7 & 0.0226 & 26.609 & 1186.0 & 0.0226 \\
\hline 11 & 9.313 & 1434.8 & 0.0224 & 18.335 & 1292.0 & 0.0224 & 26.637 & 1186.1 & 0.0224 \\
\hline 12 & 9.247 & 1427.9 & 0.0223 & 18.303 & 1292.0 & 0.0223 & 26.665 & 1187.5 & 0.0223 \\
\hline 13 & 9.146 & 1418.8 & 0.0222 & 18.236 & 1292.1 & 0.0222 & 26.678 & 1190.7 & 0.0222 \\
\hline 14 & 8.989 & 1405.6 & 0.0220 & 18.092 & 1291.7 & 0.0221 & 26.632 & 1195.5 & 0.0221 \\
\hline 15 & 8.730 & 1382.7 & 0.0219 & 17.775 & 1288.0 & 0.0219 & 26.397 & 1200.4 & 0.0220 \\
\hline 16 & 8.244 & 1341.5 & 0.0218 & 17.007 & 1270.7 & 0.0218 & 25.557 & 1198.1 & 0.0218 \\
\hline 17 & 7.260 & 1259.2 & 0.0217 & 15.154 & 1213.6 & 0.0217 & 23.051 & 1166.3 & 0.0217 \\
\hline 18 & 5.441 & 1104.3 & 0.0216 & 11.364 & 1081.0 & 0.0216 & 17.304 & 1055.5 & 0.0216 \\
\hline
\end{tabular}

Table 4-109. Burnup and TH Feedback Parameters by Axial Node for Assembly L12

\begin{tabular}{|c|c|c|c|c|c|c|c|c|c|}
\hline \multirow[b]{2}{*}{$\begin{array}{l}\text { Axial } \\
\text { Node }\end{array}$} & \multicolumn{3}{|c|}{ Datapoint (208.7 Cyl1) } & \multicolumn{3}{|c|}{ Datapoint (417.8 Cy 11) } & \multicolumn{3}{|c|}{ Statepoint 74 (615.0 Cy 11$)$} \\
\hline & \begin{tabular}{|c|} 
Burnup \\
(GWd/MTU)
\end{tabular} & $\begin{array}{c}\text { T-Fuel } \\
{ }^{\circ} \mathrm{F} \\
\end{array}$ & $\begin{array}{c}\text { Spec. Vol. } \\
\mathrm{ft}^{3} / \mathrm{lbm}\end{array}$ & \begin{tabular}{|c|} 
Burnup \\
(GWd/MTU)
\end{tabular} & $\begin{array}{c}\text { T-Fuel } \\
{ }^{\circ} \mathrm{F} \\
\end{array}$ & $\begin{array}{c}\text { Spec. Vol. } \\
\mathrm{tt}^{3} / \mathrm{lbm}\end{array}$ & $\begin{array}{c}\text { Burnup } \\
\text { (GWd/MTU) }\end{array}$ & $\begin{array}{l}\text { T-Fuel } \\
{ }^{\circ} \mathrm{F}\end{array}$ & $\begin{array}{c}\text { Spec. Vol. } \\
\mathrm{ft}^{3} / \mathrm{lbm}\end{array}$ \\
\hline & 208.7 Cy 11 & 103.8 Cy 11 & 103.8 Cy 11 & 417.8 Cy 11 & 312.8 Cy 11 & 312.8 Cy11 & 615.0 Cy11 & 515.7 Cy11 & 515.7 Cy 11 \\
\hline 1 & 4.345 & 1059.3 & 0.0236 & 9.194 & 1055.3 & 0.0237 & 14.180 & 1043.3 & 0.0237 \\
\hline 2 & 6.168 & 1217.3 & 0.0235 & 13.062 & 1188.4 & 0.0236 & 20.069 & 1165.5 & 0.0236 \\
\hline 3 & 7.462 & 1323.4 & 0.0234 & 15.551 & 1263.2 & 0.0235 & 23.534 & 1206.9 & 0.0235 \\
\hline 4 & 8.118 & 1374.2 & 0.0233 & 16.655 & 1287.3 & 0.0233 & 24.866 & 1210.7 & 0.0234 \\
\hline 5 & 8.428 & 1396.1 & 0.0231 & 17.085 & 1290.4 & 0.0232 & 25.273 & 1203.5 & 0.0232 \\
\hline 6 & 8.597 & 1406.4 & 0.0230 & 17.281 & 1287.9 & 0.0231 & 25.415 & 1195.6 & 0.0231 \\
\hline 7 & 8.696 & 1411.0 & 0.0229 & 17.386 & 1284.6 & 0.0229 & 25.485 & 1189.7 & 0.0229 \\
\hline 8 & 8.748 & 1411.9 & 0.0227 & 17.442 & 1281.6 & 0.0228 & 25.528 & 1185.7 & 0.0228 \\
\hline 9 & 8.767 & 1410.3 & 0.0226 & 17.472 & 1279.5 & 0.0227 & 25.567 & 1183.2 & 0.0227 \\
\hline 10 & 8.764 & 1407.1 & 0.0225 & 17.493 & 1278.4 & 0.0225 & 25.612 & 1181.9 & 0.0226 \\
\hline 11 & 8.749 & 1402.9 & 0.0224 & 17.519 & 1278.9 & 0.0224 & 25.685 & 1182.0 & 0.0224 \\
\hline 12 & 8.736 & 1399.1 & 0.0222 & 17.581 & 1281.6 & 0.0223 & 25.829 & 1184.4 & 0.0223 \\
\hline 13 & 8.714 & 1394.4 & 0.0221 & 17.656 & 1285.9 & 0.0222 & 26.030 & 1189.7 & 0.0222 \\
\hline 14 & 8.595 & 1381.8 & 0.0220 & 17.587 & 1287.7 & 0.0221 & 26.079 & 1195.5 & 0.0221 \\
\hline 15 & 8.336 & 1357.7 & 0.0219 & 17.282 & 1284.6 & 0.0219 & 25.862 & 1200.7 & 0.0220 \\
\hline 16 & 7.837 & 1313.7 & 0.0218 & 16.503 & 1267.0 & 0.0218 & 25.011 & 1198.4 & 0.0218 \\
\hline 17 & 6.870 & 1230.2 & 0.0217 & 14.663 & 1209.1 & 0.0217 & 22.516 & 1168.1 & 0.0217 \\
\hline 18 & 5.172 & 1082.8 & 0.0216 & 10.975 & 1074.7 & 0.0216 & 16.841 & 1052.8 & 0.0216 \\
\hline
\end{tabular}


Table 4-110. Burnup and TH Feedback Parameters by Axial Node for Assembly L14

\begin{tabular}{|c|c|c|c|c|c|c|c|c|c|}
\hline \multirow[b]{2}{*}{$\begin{array}{l}\text { Axial } \\
\text { Node }\end{array}$} & \multicolumn{3}{|c|}{ Datapoint (208.7 Cy11) } & \multicolumn{3}{|c|}{ Datapoint (417.8 Cy11) } & \multicolumn{3}{|c|}{ Statepoint 74 (615.0 Cy 11$)$} \\
\hline & $\begin{array}{c}\text { Burnup } \\
\text { (GWd/MTU) } \\
\end{array}$ & $\begin{array}{c}\text { T-Fuel } \\
{ }^{\circ} \mathrm{F} \\
\end{array}$ & $\begin{array}{c}\text { Spec. Vol. } \\
\mathrm{ft}^{3} / \mathrm{lbm}\end{array}$ & $\begin{array}{c}\text { Burnup } \\
\text { (GWd/MTU) }\end{array}$ & $\begin{array}{c}\text { T-Fuel } \\
{ }^{\circ} \mathrm{F} \\
\end{array}$ & \begin{tabular}{|c} 
Spec. Vol. \\
$\mathrm{ft}^{3} / \mathrm{lbm}$
\end{tabular} & $\begin{array}{c}\text { Burnup } \\
\text { (GWd/MTU) }\end{array}$ & $\begin{array}{c}\text { T-Fuel } \\
{ }^{\circ} \mathrm{F} \\
\end{array}$ & $\begin{array}{c}\text { Spec. Vol. } \\
\mathrm{ft}^{3} / \mathrm{bm}\end{array}$ \\
\hline & 208.7 Cy 11 & 103.8 Cy 11 & 103.8 Cy 11 & 417.8 Cy 11 & 312.8 Cy 11 & 312.8 Cy 11 & $615.0 \mathrm{Cy} 11$ & 515.7 Cy 11 & 515.7 Cy 11 \\
\hline 1 & 3.560 & 978.2 & 0.0235 & 7.776 & 1007.9 & 0.0235 & 12.205 & 1002.9 & 0.0234 \\
\hline 2 & 5.690 & 1174.2 & 0.0234 & 12.214 & 1164.9 & 0.0234 & 18.642 & 1122.9 & 0.0233 \\
\hline 3 & 6.991 & 1283.3 & 0.0233 & 14.584 & 1227.1 & 0.0233 & 21.734 & 1150.5 & 0.0232 \\
\hline 4 & 7.670 & 1338.0 & 0.0232 & 15.624 & 1243.3 & 0.0232 & 22.902 & 1148.4 & 0.0231 \\
\hline 5 & 8.037 & 1365.7 & 0.0231 & 16.095 & 1244.2 & 0.0231 & 23.341 & 1140.0 & 0.0230 \\
\hline 6 & 8.245 & 1379.2 & 0.0229 & 16.324 & 1240.7 & 0.0229 & 23.517 & 1132.0 & 0.0229 \\
\hline 7 & 8.368 & 1385.4 & 0.0228 & 16.447 & 1236.7 & 0.0228 & 23.605 & 1126.1 & 0.0228 \\
\hline 8 & 8.441 & 1387.8 & 0.0227 & 16.524 & 1233.5 & 0.0227 & 23.668 & 1122.0 & 0.0226 \\
\hline 9 & 8.481 & 1387.9 & 0.0226 & 16.578 & 1231.4 & 0.0226 & 23.726 & 1119.4 & 0.0225 \\
\hline 10 & 8.496 & 1386.1 & 0.0224 & 16.621 & 1230.6 & 0.0225 & 23.788 & 1117.8 & 0.0224 \\
\hline 11 & 8.491 & 1382.8 & 0.0223 & 16.657 & 1230.9 & 0.0223 & 23.858 & 1117.3 & 0.0223 \\
\hline 12 & 8.459 & 1377.5 & 0.0222 & 16.678 & 1232.2 & 0.0222 & 23.930 & 1118.1 & 0.0222 \\
\hline 13 & 8.386 & 1369.0 & 0.0221 & 16.664 & 1234.5 & 0.0221 & 23.989 & 1120.7 & 0.0221 \\
\hline 14 & 8.240 & 1354.8 & 0.0220 & 16.567 & 1236.9 & 0.0220 & 23.986 & 1125.5 & 0.0220 \\
\hline 15 & 7.958 & 1329.1 & 0.0219 & 16.271 & 1236.7 & 0.0219 & 23.784 & 1131.8 & 0.0219 \\
\hline 16 & 7.399 & 1278.9 & 0.0218 & 15.482 & 1224.4 & 0.0218 & 22.974 & 1134.1 & 0.0218 \\
\hline 17 & 6.240 & 1177.4 & 0.0217 & 13.429 & 1170.0 & 0.0217 & 20.376 & 1111.4 & 0.0217 \\
\hline 18 & 3.913 & 966.3 & 0.0216 & 8.566 & 996.9 & 0.0216 & 13.368 & 984.3 & 0.0216 \\
\hline
\end{tabular}

Table 4-111. Burnup and TH Feedback Parameters by Axial Node for Assembly L17

\begin{tabular}{|c|c|c|c|c|c|c|c|c|c|}
\hline \multirow[b]{2}{*}{$\begin{array}{l}\text { Axial } \\
\text { Node }\end{array}$} & \multicolumn{3}{|c|}{ Datapoint (208.7 Cy11) } & \multicolumn{3}{|c|}{ Datapoint (417.8 Cy11) } & \multicolumn{3}{|c|}{ Statepoint 74 (615.0 Cy11) } \\
\hline & $\begin{array}{c}\text { Burnup } \\
\text { (GWd/MTU) }\end{array}$ & $\begin{array}{c}\text { T-Fuel } \\
{ }^{\circ} \mathrm{F}\end{array}$ & $\begin{array}{c}\text { Spec. Vol. } \\
\mathrm{ft}^{3} / \mathrm{lbm}\end{array}$ & $\begin{array}{c}\text { Burnup } \\
\text { (GWd/MTU) }\end{array}$ & $\begin{array}{c}\text { T-Fuel } \\
{ }^{\circ} \mathrm{F}\end{array}$ & $\begin{array}{c}\text { Spec. Vol. } \\
\mathrm{ft}^{3} / \mathrm{lbm}\end{array}$ & $\begin{array}{c}\text { Burnup } \\
\text { (GWd/MTU) }\end{array}$ & $\begin{array}{c}\text { T-Fuel } \\
{ }^{\circ} \mathrm{F}\end{array}$ & $\begin{array}{c}\text { Spec. Vol. } \\
\mathrm{ft}^{3} / \mathrm{lbm}\end{array}$ \\
\hline & 208.7 Cy 11 & 103.8 Cy 11 & $103.8 \mathrm{Cy} 11$ & 417.8 Cy 11 & 312.8 Cy 11 & 312.8 Cy11 & 615.0 Cy11 & 515.7 Cy11 & 515.7 Cy 11 \\
\hline 1 & 4.715 & 1093.9 & 0.0237 & 9.850 & 1074.8 & 0.0237 & 15.114 & 1062.9 & 0.0238 \\
\hline 2 & 6.564 & 1249.9 & 0.0236 & 13.663 & 1200.6 & 0.0236 & 20.895 & 1178.3 & 0.0237 \\
\hline 3 & 7.777 & 1347.1 & 0.0235 & 15.983 & 1269.8 & 0.0235 & 24.140 & 1216.5 & 0.0236 \\
\hline 4 & 8.396 & 1393.4 & 0.0233 & 17.027 & 1292.0 & 0.0234 & 25.408 & 1221.8 & 0.0234 \\
\hline 5 & 8.696 & 1413.3 & 0.0232 & 17.450 & 1295.4 & 0.0232 & 25.816 & 1215.0 & 0.0233 \\
\hline 6 & 8.860 & 1422.4 & 0.0231 & 17.646 & 1293.4 & 0.0231 & 25.963 & 1207.5 & 0.0231 \\
\hline 7 & 8.956 & 1425.8 & 0.0229 & 17.750 & 1290.4 & 0.0230 & 26.036 & 1201.7 & 0.0230 \\
\hline 8 & 9.007 & 1425.8 & 0.0228 & 17.807 & 1287.6 & 0.0228 & 26.083 & 1197.8 & 0.0228 \\
\hline 9 & 9.026 & 1423.8 & 0.0227 & 17.839 & 1285.6 & 0.0227 & 26.125 & 1195.4 & 0.0227 \\
\hline 10 & 9.023 & 1420.4 & 0.0225 & 17.861 & 1284.6 & 0.0226 & 26.175 & 1194.4 & 0.0226 \\
\hline 11 & 9.011 & 1416.6 & 0.0224 & 17.892 & 1285.2 & 0.0224 & 26.257 & 1194.7 & 0.0225 \\
\hline 12 & 9.003 & 1413.4 & 0.0223 & 17.961 & 1287.8 & 0.0223 & 26.414 & 1197.3 & 0.0223 \\
\hline 13 & 8.987 & 1409.7 & 0.0221 & 18.044 & 1291.4 & 0.0222 & 26.625 & 1202.5 & 0.0222 \\
\hline 14 & 8.875 & 1398.4 & 0.0220 & 17.979 & 1292.3 & 0.0221 & 26.681 & 1208.2 & 0.0221 \\
\hline 15 & 8.624 & 1375.8 & 0.0219 & 17.675 & 1288.9 & 0.0219 & 26.460 & 1213.0 & 0.0220 \\
\hline 16 & 8.134 & 1333.9 & 0.0218 & 16.895 & 1271.1 & 0.0218 & 25.594 & 1209.6 & 0.0218 \\
\hline 17 & 7.169 & 1252.5 & 0.0217 & 15.058 & 1213.7 & 0.0217 & 23.075 & 1176.2 & 0.0217 \\
\hline 18 & 5.398 & 1101.1 & 0.0216 & 11.331 & 1082.0 & 0.0216 & 17.351 & 1062.2 & 0.0216 \\
\hline
\end{tabular}


Table 4-112. Burnup and TH Feedback Parameters by Axial Node for Assembly L19

\begin{tabular}{|c|c|c|c|c|c|c|c|c|c|}
\hline \multirow[b]{2}{*}{$\begin{array}{l}\text { Axial } \\
\text { Node }\end{array}$} & \multicolumn{3}{|c|}{ Datapoint (208.7 Cy11) } & \multicolumn{3}{|c|}{ Datapoint (417.8 Cy11) } & \multicolumn{3}{|c|}{ Statepoint 74 (615.0 Cy11) } \\
\hline & $\begin{array}{c}\text { Burnup } \\
\text { (GWd/MTU) }\end{array}$ & $\begin{array}{c}\text { T-Fuel } \\
{ }^{\circ} \mathrm{F}\end{array}$ & $\begin{array}{c}\text { Spec. Vol. } \\
\mathrm{ft}^{3} / \mathrm{lbm}\end{array}$ & $\begin{array}{c}\text { Burnup } \\
\text { (GWd/MTU) }\end{array}$ & $\begin{array}{c}\text { T-Fuel } \\
{ }^{\circ} \mathrm{F} \\
\end{array}$ & $\begin{array}{c}\text { Spec. Vol. } \\
\mathrm{ft}^{3} / / \mathrm{bm}\end{array}$ & $\begin{array}{c}\text { Burnup } \\
\text { (GWd/MTU) }\end{array}$ & $\begin{array}{l}\text { T-Fuel } \\
{ }^{\circ} \mathrm{F}\end{array}$ & $\begin{array}{c}\text { Spec. Vol. } \\
\mathrm{ft}^{3} / \mathrm{lbm}\end{array}$ \\
\hline & 208.7 Cy 11 & 103.8 Cy 11 & 103.8 Cy 11 & $417.8 \mathrm{Cy} 11$ & $312.8 \mathrm{Cy} 11$ & 312.8 Cy 11 & 615.0 Cy 11 & 515.7 Cy11 & $515.7 \mathrm{Cy} 11$ \\
\hline 1 & 4.264 & 1050.8 & 0.0236 & 9.110 & 1056.5 & 0.0237 & 14.135 & 1046.9 & 0.0237 \\
\hline 2 & 6.047 & 1206.5 & 0.0235 & 12.876 & 1185.9 & 0.0236 & 19.838 & 1163.8 & 0.0236 \\
\hline 3 & 7.308 & 1310.6 & 0.0234 & 15.268 & 1255.1 & 0.0235 & 23.142 & 1201.9 & 0.0235 \\
\hline 4 & 7.988 & 1363.9 & 0.0233 & 16.384 & 1277.4 & 0.0233 & 24.478 & 1204.1 & 0.0233 \\
\hline 5 & 8.331 & 1388.8 & 0.0231 & 16.851 & 1280.1 & 0.0232 & 24.930 & 1196.8 & 0.0232 \\
\hline 6 & 8.528 & 1401.4 & 0.0230 & 17.080 & 1277.7 & 0.0230 & 25.113 & 1189.3 & 0.0231 \\
\hline 7 & 8.649 & 1407.9 & 0.0229 & 17.213 & 1274.5 & 0.0229 & 25.218 & 1183.8 & 0.0229 \\
\hline 8 & 8.722 & 1410.6 & 0.0228 & 17.298 & 1271.8 & 0.0228 & 25.297 & 1180.0 & 0.0228 \\
\hline 9 & 8.762 & 1410.7 & 0.0226 & 17.359 & 1270.1 & 0.0227 & 25.372 & 1177.8 & 0.0227 \\
\hline 10 & 8.781 & 1409.1 & 0.0225 & 17.411 & 1269.5 & 0.0225 & 25.455 & 1176.7 & 0.0226 \\
\hline 11 & 8.788 & 1406.7 & 0.0224 & 17.472 & 1270.6 & 0.0224 & 25.569 & 1177.1 & 0.0224 \\
\hline 12 & 8.797 & 1404.5 & 0.0223 & 17.570 & 1274.1 & 0.0223 & 25.756 & 1179.7 & 0.0223 \\
\hline 13 & 8.794 & 1401.3 & 0.0221 & 17.680 & 1279.3 & 0.0222 & 26.000 & 1185.2 & 0.0222 \\
\hline 14 & 8.682 & 1389.1 & 0.0220 & 17.632 & 1282.0 & 0.0221 & 26.078 & 1191.3 & 0.0221 \\
\hline 15 & 8.406 & 1363.5 & 0.0219 & 17.319 & 1279.8 & 0.0219 & 25.856 & 1196.8 & 0.0220 \\
\hline 16 & 7.850 & 1314.7 & 0.0218 & 16.470 & 1262.5 & 0.0218 & 24.930 & 1194.6 & 0.0218 \\
\hline 17 & 6.789 & 1223.3 & 0.0217 & 14.498 & 1203.2 & 0.0217 & 22.286 & 1164.7 & 0.0217 \\
\hline 18 & 5.021 & 1069.8 & 0.0216 & 10.719 & 1068.4 & 0.0216 & 16.535 & 1050.6 & 0.0216 \\
\hline
\end{tabular}

Table 4-113. Burnup and TH Feedback Parameters by Axial Node for Assembly L20

\begin{tabular}{|c|c|c|c|c|c|c|c|c|c|}
\hline \multirow[b]{2}{*}{$\begin{array}{l}\text { Axlal } \\
\text { Node } \\
\end{array}$} & \multicolumn{3}{|c|}{ Datapoint (208.7 Cy/1) } & \multicolumn{3}{|c|}{ Datapoint (417.8 Cy11) } & \multicolumn{3}{|c|}{ Statepoint 74 (615.0 Cy11) } \\
\hline & $\begin{array}{c}\text { Burnup } \\
\text { (GWd/MTU) }\end{array}$ & $\begin{array}{c}\text { T-Fuel } \\
{ }^{\circ} \mathrm{F}\end{array}$ & $\begin{array}{c}\text { Spec. Vol. } \\
\mathrm{ft}^{3} / \mathrm{lbm}\end{array}$ & $\begin{array}{c}\text { Burnup } \\
\text { (GWd/MTU) }\end{array}$ & $\begin{array}{c}\text { T-Fuel } \\
{ }^{\circ} \mathrm{F} \\
\end{array}$ & \begin{tabular}{|c} 
Spec. Vol. \\
$\mathrm{ft}^{3} / / \mathrm{bm}$
\end{tabular} & $\begin{array}{c}\text { Burnup } \\
\text { (GWd/MTU) } \\
\end{array}$ & $\begin{array}{c}\text { T-Fuel } \\
{ }^{\circ} \mathrm{F}\end{array}$ & $\begin{array}{c}\text { Spec. Vol. } \\
\mathrm{tt}^{3} / \mathrm{lbm}\end{array}$ \\
\hline & 208.7 Cy 11 & 103.8 Cy 11 & 103.8 Cy 11 & 417.8 Cy 11 & $312.8 \mathrm{Cy} 11$ & 312.8 Cy 11 & 615.0 Cy 11 & 515.7 Cy 11 & 515.7 Cy 11 \\
\hline 1 & 3.200 & 937.6 & 0.0233 & 6.969 & 965.4 & 0.0233 & 10.965 & 968.6 & 0.0232 \\
\hline 2 & 5.226 & 1129.7 & 0.0232 & 11.017 & 1108.7 & 0.0232 & 16.786 & 1078.9 & 0.0232 \\
\hline 3 & 6.426 & 1230.9 & 0.0232 & 13.174 & 1165.3 & 0.0231 & 19.625 & 1109.5 & 0.0231 \\
\hline 4 & 7.048 & 1280.5 & 0.0230 & 14.129 & 1180.6 & 0.0230 & 20.717 & 1108.6 & 0.0230 \\
\hline 5 & 7.380 & 1305.1 & 0.0229 & 14.557 & 1181.7 & 0.0229 & 21.124 & 1101.3 & 0.0228 \\
\hline 6 & 7.566 & 1317.5 & 0.0228 & 14.761 & 1178.6 & 0.0228 & 21.282 & 1094.2 & 0.0227 \\
\hline 7 & 7.676 & 1323.1 & 0.0227 & 14.870 & 1174.9 & 0.0227 & 21.360 & 1088.8 & 0.0226 \\
\hline 8 & 7.741 & 1325.1 & 0.0226 & 14.936 & 1172.0 & 0.0226 & 21.414 & 1085.1 & 0.0225 \\
\hline 9 & 7.777 & 1325.1 & 0.0225 & 14.984 & 1170.0 & 0.0225 & 21.465 & 1082.7 & 0.0224 \\
\hline 10 & 7.793 & 1323.6 & 0.0224 & 15.022 & 1169.2 & 0.0224 & 21.521 & 1081.3 & 0.0223 \\
\hline 11 & 7.793 & 1320.9 & 0.0223 & 15.058 & 1169.4 & 0.0223 & 21.588 & 1080.8 & 0.0223 \\
\hline 12 & 7.774 & 1316.8 & 0.0222 & 15.088 & 1170.8 & 0.0222 & 21.665 & 1081.6 & 0.0222 \\
\hline 13 & 7.720 & 1310.1 & 0.0220 & 15.089 & 1173.1 & 0.0221 & 21.735 & 1084.1 & 0.0221 \\
\hline 14 & 7.596 & 1297.8 & 0.0219 & 15.008 & 1175.3 & 0.0220 & 21.739 & 1088.4 & 0.0220 \\
\hline 15 & 7.342 & 1274.4 & 0.0219 & 14.735 & 1174.5 & 0.0219 & 21.543 & 1093.9 & 0.0219 \\
\hline 16 & 6.831 & 1228.9 & 0.0218 & 14.005 & 1162.2 & 0.0218 & 20.775 & 1094.9 & 0.0218 \\
\hline 17 & 5.764 & 1135.9 & 0.0217 & 12.138 & 1111.8 & 0.0217 & 18.389 & 1071.1 & 0.0217 \\
\hline 18 & 3.552 & 929.4 & 0.0216 & 7.726 & 958.2 & 0.0216 & 12.081 & 953.9 & 0.0216 \\
\hline
\end{tabular}


Table 4-114. Burnup and TH Feedback Parameters by Axial Node for Assembly L23

\begin{tabular}{|c|c|c|c|c|c|c|c|c|c|}
\hline \multirow[b]{2}{*}{$\begin{array}{l}\text { Axial } \\
\text { Node } \\
\end{array}$} & \multicolumn{3}{|c|}{ Datapoint (208.7 Cy11) } & \multicolumn{3}{|c|}{ Datapoint (417.8 Cy11) } & \multicolumn{3}{|c|}{ Statepoint 74 (615.0 Cy11) } \\
\hline & $\begin{array}{c}\text { Burnup } \\
\text { (GWd/MTU) }\end{array}$ & \begin{tabular}{|c|}
$\begin{array}{c}\text { T-Fuel } \\
{ }^{\circ} \mathrm{F}\end{array}$ \\
\end{tabular} & $\begin{array}{c}\text { Spec. Vol. } \\
\mathrm{ft}^{3} / \mathrm{lbm}\end{array}$ & $\begin{array}{c}\text { Burnup } \\
\text { (GWd/MTU) }\end{array}$ & $\begin{array}{c}\text { T-Fuel } \\
{ }^{\circ} \mathrm{F} \\
\end{array}$ & $\begin{array}{c}\text { Spec. Vol. } \\
\mathrm{ft}^{3} / \mathrm{lbm}\end{array}$ & $\begin{array}{c}\text { Burnup } \\
\text { (GWd/MTU) }\end{array}$ & $\begin{array}{c}\text { T-Fuel } \\
{ }^{\circ} \mathrm{F} \\
\end{array}$ & $\begin{array}{c}\text { Spec. Vol. } \\
\mathrm{ft}^{3} / \mathrm{lbm}\end{array}$ \\
\hline & 208.7 Cy 11 & 103.8 Cy11 & 103.8 Cy 11 & 417.8 Cy 11 & 312.8 Cy 11 & 312.8 Cy 11 & 615.0 Cy 11 & 515.7 Cy 11 & 515.7 Cy 11 \\
\hline 1 & 4.236 & 1048.7 & 0.0236 & 8.954 & 1045.3 & 0.0237 & 13.871 & 1039.3 & 0.0237 \\
\hline 2 & 6.115 & 1212.5 & 0.0236 & 12.815 & 1173.7 & 0.0236 & 19.712 & 1160.1 & 0.0236 \\
\hline 3 & 7.467 & 1321.9 & 0.0234 & 15.371 & 1248.4 & 0.0234 & 23.273 & 1203.8 & 0.0235 \\
\hline 4 & 8.175 & 1375.4 & 0.0233 & 16.545 & 1273.6 & 0.0233 & 24.703 & 1208.3 & 0.0234 \\
\hline 5 & 8.517 & 1398.7 & 0.0232 & 17.019 & 1277.3 & 0.0232 & 25.169 & 1201.9 & 0.0232 \\
\hline 6 & 8.707 & 1409.7 & 0.0230 & 17.243 & 1275.2 & 0.0230 & 25.347 & 1194.4 & 0.0231 \\
\hline 7 & 8.824 & 1415.0 & 0.0229 & 17.371 & 1272.2 & 0.0229 & 25.447 & 1188.8 & 0.0229 \\
\hline 8 & 8.894 & 1416.8 & 0.0228 & 17.452 & 1269.5 & 0.0228 & 25.521 & 1185.1 & 0.0228 \\
\hline 9 & 8.933 & 1416.4 & 0.0226 & 17.509 & 1267.7 & 0.0227 & 25.591 & 1182.7 & 0.0227 \\
\hline 10 & 8.953 & 1414.7 & 0.0225 & 17.560 & 1267.0 & 0.0225 & 25.672 & 1181.6 & 0.0226 \\
\hline 11 & 8.965 & 1412.5 & 0.0224 & 17.625 & 1268.0 & 0.0224 & 25.789 & 1181.9 & 0.0224 \\
\hline 12 & 8.984 & 1411.1 & 0.0223 & 17.734 & 1271.4 & 0.0223 & 25.989 & 1184.4 & 0.0223 \\
\hline 13 & 8.993 & 1409.2 & 0.0221 & 17.858 & 1276.6 & 0.0222 & 26.246 & 1189.9 & 0.0222 \\
\hline 14 & 8.895 & 1399.0 & 0.0220 & 17.821 & 1279.2 & 0.0221 & 26.334 & 1195.8 & 0.0221 \\
\hline 15 & 8.636 & 1375.9 & 0.0219 & 17.521 & 1276.2 & 0.0219 & 26.122 & 1201.0 & 0.0220 \\
\hline 16 & 8.105 & 1330.9 & 0.0218 & 16.704 & 1258.6 & 0.0218 & 25.228 & 1198.5 & 0.0218 \\
\hline 17 & 7.069 & 1243.9 & 0.0217 & 14.791 & 1201.5 & 0.0217 & 22.649 & 1167.5 & 0.0217 \\
\hline 18 & 5.251 & 1088.4 & 0.0216 & 11.028 & 1071.4 & 0.0216 & 16.927 & 1055.0 & 0.0216 \\
\hline
\end{tabular}

Table 4-115. Burnup and TH Feedback Parameters by Axial Node for Assembly L27

\begin{tabular}{|c|c|c|c|c|c|c|c|c|c|}
\hline \multirow[b]{2}{*}{$\begin{array}{l}\text { Axial } \\
\text { Node }\end{array}$} & \multicolumn{3}{|c|}{ Datapoint (208.7 Cy11) } & \multicolumn{3}{|c|}{ Datapoint (417.8 Cy11) } & \multicolumn{3}{|c|}{ Statepoint 74 (615.0 Cy11) } \\
\hline & $\begin{array}{c}\text { Burnup } \\
\text { (GWd/MTU) }\end{array}$ & $\begin{array}{c}\text { T-Fuel } \\
{ }^{\circ} \mathrm{F} \\
\end{array}$ & $\begin{array}{c}\text { Spec. Vol. } \\
\mathrm{ft}^{3} / \mathrm{lbm}\end{array}$ & $\begin{array}{c}\text { Burnup } \\
\text { (GWd/MTU) }\end{array}$ & $\begin{array}{c}\text { T-Fuel } \\
{ }^{\circ} \mathrm{F} \\
\end{array}$ & $\begin{array}{c}\text { Spec. Vol. } \\
\mathrm{ft}^{3} / \mathrm{lbm}\end{array}$ & $\begin{array}{c}\text { Burnup } \\
\text { (GWd/MTU) }\end{array}$ & $\begin{array}{c}\text { T-Fuel } \\
{ }^{\circ} \mathrm{F} \\
\end{array}$ & $\begin{array}{c}\text { Spec. Vol. } \\
\mathrm{ft}^{3} / \mathrm{lbm} \\
\end{array}$ \\
\hline & 208.7 Cy 11 & $103.8 \mathrm{Cy} 11$ & 103.8 Cy 11 & 417.8 Cy 11 & 312.8 Cy 11 & 312.8 Cy11 & 615.0 Cy11 & 515.7 Cy 11 & 515.7 Cy 11 \\
\hline 1 & 3.073 & 926.4 & 0.0234 & 6.615 & 944.5 & 0.0233 & 10.433 & 956.6 & 0.0233 \\
\hline 2 & 5.277 & 1135.2 & 0.0233 & 10.931 & 1095.9 & 0.0232 & 16.629 & 1074.4 & 0.0232 \\
\hline 3 & 6.699 & 1252.1 & 0.0232 & 13.447 & 1160.7 & 0.0231 & 19.941 & 1110.9 & 0.0231 \\
\hline 4 & 7.428 & 1307.6 & 0.0231 & 14.567 & 1181.1 & 0.0230 & 21.237 & 1111.6 & 0.0230 \\
\hline 5 & 7.801 & 1333.3 & 0.0230 & 15.053 & 1183.3 & 0.0229 & 21.710 & 1104.7 & 0.0229 \\
\hline 6 & 8.005 & 1344.9 & 0.0229 & 15.278 & 1180.4 & 0.0228 & 21.893 & 1097.5 & 0.0228 \\
\hline 7 & 8.125 & 1349.9 & 0.0228 & 15.397 & 1176.8 & 0.0227 & 21.981 & 1092.0 & 0.0227 \\
\hline 8 & 8.198 & 1351.7 & 0.0226 & 15.472 & 1173.7 & 0.0226 & 22.042 & 1088.2 & 0.0226 \\
\hline 9 & 8.243 & 1351.6 & 0.0225 & 15.526 & 1171.6 & 0.0225 & 22.100 & 1085.7 & 0.0225 \\
\hline 10 & 8.268 & 1350.3 & 0.0224 & 15.574 & 1170.6 & 0.0224 & 22.164 & 1084.1 & 0.0224 \\
\hline 11 & 8.279 & 1348.2 & 0.0223 & 15.619 & 1170.6 & 0.0223 & 22.239 & 1083.5 & 0.0223 \\
\hline 12 & 8.271 & 1345.0 & 0.0222 & 15.660 & 1171.8 & 0.0222 & 22.327 & 1084.0 & 0.0222 \\
\hline 13 & 8.228 & 1339.5 & 0.0221 & 15.672 & 1173.9 & 0.0221 & 22.405 & 1086.2 & 0.0221 \\
\hline 14 & 8.114 & 1328.8 & 0.0220 & 15.600 & 1175.9 & 0.0220 & 22.417 & 1090.3 & 0.0220 \\
\hline 15 & 7.864 & 1307.9 & 0.0219 & 15.332 & 1175.2 & 0.0219 & 22.227 & 1095.7 & 0.0219 \\
\hline 16 & 7.337 & 1263.9 & 0.0218 & 14.594 & 1163.1 & 0.0218 & 21.455 & 1097.2 & 0.0218 \\
\hline 17 & 6.186 & 1167.3 & 0.0217 & 12.643 & 1113.1 & 0.0217 & 18.990 & 1074.8 & 0.0217 \\
\hline 18 & 3.764 & 949.0 & 0.0216 & 7.986 & 960.7 & 0.0216 & 12.412 & 957.4 & 0.0216 \\
\hline
\end{tabular}


Table 4-116. Burnup and TH Feedback Parameters by Axial Node for Assembly L28

\begin{tabular}{|c|c|c|c|c|c|c|c|c|c|}
\hline \multirow[b]{2}{*}{$\begin{array}{l}\text { Axial } \\
\text { Node } \\
\end{array}$} & \multicolumn{3}{|c|}{ Datapoint (208.7 Cy11) } & \multicolumn{3}{|c|}{ Datapoint (417.8 Cy11) } & \multicolumn{3}{|c|}{ Statepoint 74 (615.0 Cy11) } \\
\hline & $\begin{array}{c}\text { Burnup } \\
\text { (GWd/MTU) }\end{array}$ & $\begin{array}{c}\text { T-Fuel } \\
{ }^{\circ} \mathrm{F} \\
\end{array}$ & $\begin{array}{c}\text { Spec. Vol. } \\
\mathrm{ft}^{3} / \mathrm{lbm}\end{array}$ & $\begin{array}{c}\text { Burnup } \\
\text { (GWd/MTU) }\end{array}$ & $\begin{array}{c}\text { T-Fuel } \\
{ }^{\circ} \mathrm{F} \\
\end{array}$ & $\begin{array}{c}\text { Spec. Vol. } \\
\mathrm{ft}^{3} / \mathrm{lbm}\end{array}$ & $\begin{array}{c}\text { Burnup } \\
\text { (GWd/MTU) }\end{array}$ & $\begin{array}{c}\text { T-Fuel } \\
{ }^{\circ} \mathrm{F}\end{array}$ & $\begin{array}{c}\text { Spec. Vol. } \\
\mathrm{ft}^{3} / \mathrm{lbm}\end{array}$ \\
\hline & 208.7 Cy 11 & 103.8 Cy 11 & 103.8 Cy 11 & 417.8 Cy 11 & $312.8 \mathrm{Cy} 11$ & 312.8 Cy 11 & 615.0 Cy 11 & 515.7 Cy 11 & $515.7 \mathrm{Cy} 11$ \\
\hline 1 & 1.734 & 766.9 & 0.0225 & 3.839 & 797.9 & 0.0226 & 6.223 & 828.4 & 0.0226 \\
\hline 2 & 2.866 & 882.2 & 0.0225 & 6.242 & 910.9 & 0.0225 & 9.902 & 927.5 & 0.0226 \\
\hline 3 & 3.614 & 954.5 & 0.0224 & 7.675 & 964.0 & 0.0225 & 11.872 & 960.9 & 0.0225 \\
\hline 4 & 4.015 & 991.1 & 0.0224 & 8.337 & 980.8 & 0.0224 & 12.662 & 964.9 & 0.0224 \\
\hline 5 & 4.224 & 1008.6 & 0.0223 & 8.628 & 983.9 & 0.0223 & 12.952 & 961.0 & 0.0224 \\
\hline 6 & 4.335 & 1016.7 & 0.0223 & 8.755 & 982.6 & 0.0223 & 13.051 & 956.3 & 0.0223 \\
\hline 7 & 4.395 & 1020.1 & 0.0222 & 8.813 & 980.2 & 0.0222 & 13.086 & 952.4 & 0.0223 \\
\hline 8 & 4.426 & 1021.0 & 0.0221 & 8.841 & 978.1 & 0.0222 & 13.102 & 949.7 & 0.0222 \\
\hline 9 & 4.441 & 1020.5 & 0.0221 & 8.856 & 976.5 & 0.0221 & 13.116 & 947.9 & 0.0221 \\
\hline 10 & 4.443 & 1019.0 & 0.0220 & 8.866 & 975.6 & 0.0220 & 13.133 & 946.8 & 0.0221 \\
\hline 11 & 4.436 & 1016.7 & 0.0220 & 8.872 & 975.3 & 0.0220 & 13.154 & 946.5 & 0.0220 \\
\hline 12 & 4.416 & 1013.3 & 0.0219 & 8.871 & 975.6 & 0.0219 & 13.178 & 946.9 & 0.0220 \\
\hline 13 & 4.373 & 1008.0 & 0.0218 & 8.849 & 976.2 & 0.0219 & 13.191 & 948.5 & 0.0219 \\
\hline 14 & 4.291 & 999.1 & 0.0218 & 8.777 & 976.5 & 0.0218 & 13.165 & 951.3 & 0.0218 \\
\hline 15 & 4.133 & 983.1 & 0.0217 & 8.583 & 974.1 & 0.0218 & 13.007 & 954.3 & 0.0218 \\
\hline 16 & 3.813 & 951.4 & 0.0217 & 8.088 & 961.3 & 0.0217 & 12.455 & 952.1 & 0.0217 \\
\hline 17 & 3.161 & 887.4 & 0.0216 & 6.866 & 916.4 & 0.0217 & 10.818 & 925.0 & 0.0217 \\
\hline 18 & 1.940 & 762.5 & 0.0216 & 4.272 & 795.3 & 0.0216 & 6.870 & 820.4 & 0.0216 \\
\hline
\end{tabular}




\subsection{CORE FOLLOW-CONTROL ROD HISTORY AND BORON DATA}

Control rod insertion time (by axial node) for each assembly with a control rod inserted during core operation is provided in Tables 4-117 through 4-146. Each SP is defined by EFPD and cycle. The boron data is provided as all-rods-out-critical-boron (AROCB) concentrations at hot full power conditions (Table 4-147). The power level is $100 \%$ of full power (\%FP) and the control rod insertion data (for rod banks 6,7, and 8) are expressed as \% withdrawn (\%WD). For banks 6 and 7, the \%WD can be related to position relative to active fuel length by referring to Figure 2-22 (for 0\%WD) and Figure 2-23 (for 100\%WD). Figures 2-24 and 2-25 provide similar information for bank 8 (APSRs).

Table 4-117. Rod Insertion Time by Axial Node for Assembly B15

\begin{tabular}{|c|c|c|}
\hline \multirow{2}{*}{$\begin{array}{c}\text { Axial } \\
\text { Node }\end{array}$} & \multicolumn{2}{|c|}{ Time Rod Inserted (EFPD) } \\
\cline { 2 - 3 } & BOC3 to 142.8 EFPD & $\mathbf{1 4 2 . 8}$ to 287.47 EFPD(EOC3) \\
\hline 1 & 0.0 & 0.0 \\
\hline 2 & 0.0 & 0.0 \\
\hline 3 & 0.0 & 0.0 \\
\hline 4 & 0.0 & 0.0 \\
\hline 5 & 0.0 & 0.0 \\
\hline 6 & 0.0 & 0.0 \\
\hline 7 & 0.9 & 0.0 \\
\hline 8 & 1.0 & 0.0 \\
\hline 9 & 15.6 & 26.7 \\
\hline 10 & 141.4 & 131.0 \\
\hline 11 & 142.3 & 144.7 \\
\hline 12 & 139.3 & 144.7 \\
\hline 13 & 137.2 & 141.9 \\
\hline 14 & 64.7 & 60.2 \\
\hline 15 & 0.0 & 0.3 \\
\hline 16 & 0.0 & 0.0 \\
\hline 17 & 0.0 & 0.0 \\
\hline 18 & 0.0 & 0.0 \\
\hline
\end{tabular}


Table 4-118. Rod Insertion Time by Axial Node for Assembly B21

\begin{tabular}{|c|c|c|}
\hline \multirow{2}{*}{$\begin{array}{c}\text { Axial } \\
\text { Node }\end{array}$} & \multicolumn{2}{|c|}{ Time Rod Inserted (EFPD) } \\
\hline 1 & BOC4 to 126.6 EFPD & 126.6 to 273.96 EFPD(EOC4) \\
\hline 2 & 124.9 & 124.3 \\
\hline 3 & 23.1 & 0.7 \\
\hline 4 & 3.5 & 0.0 \\
\hline 5 & 0.0 & 0.0 \\
\hline 6 & 0.0 & 0.0 \\
\hline 7 & 0.0 & 0.0 \\
\hline 8 & 0.0 & 0.0 \\
\hline 9 & 0.0 & 0.0 \\
\hline 10 & 0.0 & 0.0 \\
\hline 11 & 0.0 & 0.0 \\
\hline 12 & 0.0 & 0.0 \\
\hline 13 & 0.0 & 0.0 \\
\hline 14 & 0.0 & 0.0 \\
\hline 15 & 0.0 & 0.0 \\
\hline 16 & 0.0 & 0.0 \\
\hline 17 & 0.0 & 0.0 \\
\hline 18 & 0.0 & 0.0 \\
\hline & 0.0 & 0.0 \\
\hline
\end{tabular}

Table 4-119. Rod Insertion Time by Axial Node for Assembly B21a

\begin{tabular}{|c|c|}
\hline \multirow{2}{*}{$\begin{array}{c}\text { Axial } \\
\text { Node }\end{array}$} & Time Rod Inserted (EFPD) \\
\cline { 2 - 2 } & BOC5 to 114.4 EFPD(SP61) \\
\hline 1 & 103.3 \\
\hline 2 & 46.6 \\
\hline 3 & 3.0 \\
\hline 4 & 0.0 \\
\hline 5 & 0.0 \\
\hline 6 & 0.0 \\
\hline 7 & 0.0 \\
\hline 8 & 0.0 \\
\hline 9 & 0.0 \\
\hline 10 & 0.0 \\
\hline 11 & 0.0 \\
\hline 13 & 0.0 \\
\hline 14 & 0.0 \\
\hline 15 & 0.0 \\
\hline 16 & 0.0 \\
\hline 17 & 0.0 \\
\hline 18 & 0.0 \\
\hline & 0.0 \\
\hline
\end{tabular}


Table 4-120. Rod Insertion Time by Axial Node for Assembly B29

\begin{tabular}{|c|c|c|}
\hline \multirow{2}{*}{$\begin{array}{c}\text { Axial } \\
\text { Node }\end{array}$} & BOC4 to 126.6 EFPD & $\mathbf{1 2 6 . 6}$ to 274.0 EFPD \\
\cline { 2 - 3 } & 124.9 & 124.3 \\
\hline 1 & 23.0 & 0.7 \\
\hline 2 & 3.4 & 0.0 \\
\hline 3 & 0.0 & 0.0 \\
\hline 4 & 0.0 & 0.0 \\
\hline 5 & 0.0 & 0.0 \\
\hline 6 & 0.0 & 0.0 \\
\hline 7 & 0.0 & 0.0 \\
\hline 8 & 0.0 & 0.0 \\
\hline 9 & 0.0 & 0.0 \\
\hline 10 & 0.0 & 0.0 \\
\hline 11 & 0.0 & 0.0 \\
\hline 12 & 0.0 & 0.0 \\
\hline 13 & 0.0 & 0.0 \\
\hline 14 & 0.0 & 0.0 \\
\hline 15 & 0.0 & 0.0 \\
\hline 16 & 0.0 & 0.0 \\
\hline 17 & 0.0 & 0.0 \\
\hline 18 & & \\
\hline
\end{tabular}

Table 4-121. Rod Insertion Time by Axial Node for Assembly C21

\begin{tabular}{|c|c|c|}
\hline \multirow{2}{*}{$\begin{array}{c}\text { Axial } \\
\text { Node }\end{array}$} & BOC4 to 126.6 EFPD & Time Rod Inserted (EFPD) \\
\cline { 2 - 3 } & 0.0 & 0.0 \\
\hline 1 & 0.0 & 0.0 \\
\hline 3 & 0.0 & 0.0 \\
\hline 4 & 0.0 & 0.0 \\
\hline 5 & 0.0 & 0.0 \\
\hline 6 & 0.0 & 0.0 \\
\hline 7 & 0.4 & 0.0 \\
\hline 8 & 16.3 & 0.0 \\
\hline 9 & 92.2 & 28.7 \\
\hline 10 & 126.6 & 146.5 \\
\hline 11 & 126.6 & 147.4 \\
\hline 12 & 118.3 & 147.4 \\
\hline 13 & 72.3 & 147.4 \\
\hline 14 & 5.1 & 52.6 \\
\hline 15 & 0.0 & 0.0 \\
\hline 16 & 0.0 & 0.0 \\
\hline 17 & 0.0 & 0.0 \\
\hline 18 & 0.0 & 0.0 \\
\hline & & \\
\hline
\end{tabular}


Table 4-122. Rod Insertion Time by Axial Node for Assembly C28

\begin{tabular}{|c|c|c|}
\hline \multirow{2}{*}{$\begin{array}{c}\text { Axial } \\
\text { Node }\end{array}$} & BOC4 to 126.6 EFPD & Time Rod Inserted (EFPD) \\
\cline { 2 - 3 } & 10.2 & 3.1 \\
\hline 1 & 0.0 & 0.0 \\
\hline 2 & 0.0 & 0.0 \\
\hline 3 & 0.0 & 0.0 \\
\hline 4 & 0.0 & 0.0 \\
\hline 5 & 0.0 & 0.0 \\
\hline 6 & 0.0 & 0.0 \\
\hline 7 & 0.0 & 0.0 \\
\hline 8 & 0.0 & 0.0 \\
\hline 9 & 0.0 & 0.0 \\
\hline 10 & 0.0 & 0.0 \\
\hline 11 & 0.0 & 0.0 \\
\hline 12 & 0.0 & 0.0 \\
\hline 13 & 0.0 & 0.0 \\
\hline 14 & 0.0 & 0.0 \\
\hline 15 & 0.0 & 0.0 \\
\hline 16 & 0.0 & 0.0 \\
\hline 17 & 0.0 & 0.0 \\
\hline 18 & & \\
\hline & & \\
\hline
\end{tabular}

Table 4-123. Rod Insertion Time by Axial Node for Assembly C29

\begin{tabular}{|c|c|c|}
\hline \multirow{2}{*}{$\begin{array}{c}\text { Axial } \\
\text { Node }\end{array}$} & BOC4 to 126.6 EFPD & $\mathbf{1 2 6 . 6}$ to 274.0 EFPD \\
\cline { 2 - 3 } & 124.9 & 124.3 \\
\hline 1 & 23.3 & 0.7 \\
\hline 3 & 3.6 & 0.0 \\
\hline 4 & 0.0 & 0.0 \\
\hline 5 & 0.0 & 0.0 \\
\hline 6 & 0.0 & 0.0 \\
\hline 7 & 0.0 & 0.0 \\
\hline 8 & 0.0 & 0.0 \\
\hline 9 & 0.0 & 0.0 \\
\hline 10 & 0.0 & 0.0 \\
\hline 11 & 0.0 & 0.0 \\
\hline 12 & 0.0 & 0.0 \\
\hline 13 & 0.0 & 0.0 \\
\hline 14 & 0.0 & 0.0 \\
\hline 15 & 0.0 & 0.0 \\
\hline 16 & 0.0 & 0.0 \\
\hline 17 & 0.0 & 0.0 \\
\hline 18 & 0.0 & 0.0 \\
\hline
\end{tabular}


Table 4-124. Rod Insertion Time by Axial Node for Assembly D15

\begin{tabular}{|c|c|c|c|c|}
\hline \multirow{2}{*}{$\begin{array}{c}\text { Axial } \\
\text { Node }\end{array}$} & \multicolumn{4}{|c|}{ Time Rod inserted (EFPD) } \\
\cline { 2 - 5 } & $\begin{array}{c}\text { BOC6 (SP62) to } \\
\text { 69.1 EFPD (SP63) }\end{array}$ & $\begin{array}{c}69.1 \text { (SP63) to } \\
176.7 \text { EFPD }\end{array}$ & $\begin{array}{c}176.7 \text { to 320.3 } \\
\text { EFPD }\end{array}$ & $\begin{array}{c}\text { 320.3 to 421.2 } \\
\text { EFPD (EOC6) }\end{array}$ \\
\hline 1 & 68.21 & 107.52 & 143.67 & 91.03 \\
\hline 2 & 26.43 & 31.34 & 20.25 & 2.33 \\
\hline 3 & 0.00 & 0.00 & 0.00 & 0.00 \\
\hline 4 & 0.00 & 0.00 & 0.00 & 0.00 \\
\hline 5 & 0.00 & 0.00 & 0.00 & 0.00 \\
\hline 6 & 0.00 & 0.00 & 0.00 & 0.00 \\
\hline 7 & 0.00 & 0.00 & 0.00 & 0.00 \\
\hline 8 & 0.00 & 0.00 & 0.00 & 0.00 \\
\hline 9 & 0.00 & 0.00 & 0.00 & 0.00 \\
\hline 10 & 0.00 & 0.00 & 0.00 & 0.00 \\
\hline 11 & 0.00 & 0.00 & 0.00 & 0.00 \\
\hline 12 & 0.00 & 0.00 & 0.00 & 0.00 \\
\hline 13 & 0.00 & 0.00 & 0.00 & 0.00 \\
\hline 14 & 0.00 & 0.00 & 0.00 & 0.00 \\
\hline 15 & 0.00 & 0.00 & 0.00 & 0.00 \\
\hline 16 & 0.00 & 0.00 & 0.00 & 0.00 \\
\hline 17 & 0.00 & 0.00 & 0.00 & 0.00 \\
\hline 18 & 0.00 & 0.00 & 0.00 & 0.00 \\
\hline
\end{tabular}

Table 4-125. Rod Insertion Time by Axial Node for Assembly D15a

\begin{tabular}{|c|c|c|c|c|}
\hline \multirow{2}{*}{$\begin{array}{c}\text { Axial } \\
\text { Node }\end{array}$} & \multicolumn{4}{|c|}{ Time Rod Inserted (EFPD) } \\
\cline { 2 - 5 } & $\begin{array}{c}\text { BOC6 (SP62) to } \\
69.1 \text { EFPD (SP63) }\end{array}$ & $\begin{array}{c}\text { 69.1 (SP63) to } \\
176.7 \text { EFPD }\end{array}$ & $\begin{array}{c}176.7 \text { to 320.3 } \\
\text { EFPD }\end{array}$ & $\begin{array}{c}\text { 320.3 to 421.2 } \\
\text { EFPD (EOC6) }\end{array}$ \\
\hline 1 & 68.09 & 107.60 & 143.68 & 90.98 \\
\hline 2 & 26.29 & 31.29 & 20.23 & 2.32 \\
\hline 3 & 0.00 & 0.00 & 0.00 & 0.00 \\
\hline 4 & 0.00 & 0.00 & 0.00 & 0.00 \\
\hline 5 & 0.00 & 0.00 & 0.00 & 0.00 \\
\hline 6 & 0.00 & 0.00 & 0.00 & 0.00 \\
\hline 7 & 0.00 & 0.00 & 0.00 & 0.00 \\
\hline 8 & 0.00 & 0.00 & 0.00 & 0.00 \\
\hline 9 & 0.00 & 0.00 & 0.00 & 0.00 \\
\hline 10 & 0.00 & 0.00 & 0.00 & 0.00 \\
\hline 11 & 0.00 & 0.00 & 0.00 & 0.00 \\
\hline 12 & 0.00 & 0.00 & 0.00 & 0.00 \\
\hline 13 & 0.00 & 0.00 & 0.00 & 0.00 \\
\hline 14 & 0.00 & 0.00 & 0.00 & 0.00 \\
\hline 15 & 0.00 & 0.00 & 0.00 & 0.00 \\
\hline 16 & 0.00 & 0.00 & 0.00 & 0.00 \\
\hline 17 & 0.00 & 0.00 & 0.00 & 0.00 \\
\hline 18 & 0.00 & 0.00 & 0.00 & 0.00 \\
\hline
\end{tabular}


Table 4-126. Rod Insertion Time by Axial Node for Assembly D20a

\begin{tabular}{|c|c|c|c|}
\hline \multirow{2}{*}{$\begin{array}{c}\text { Axial } \\
\text { Node }\end{array}$} & \multicolumn{3}{|c|}{ Time Rod Inserted (EFPD) } \\
\cline { 2 - 4 } & $\begin{array}{c}\text { BOC5 (SP60) to } \\
114.4 \text { EFPD (SP61) }\end{array}$ & $\begin{array}{c}114.4 \text { (SP61) to } \\
\text { 206.7 EFPD }\end{array}$ & $\begin{array}{c}\text { 206.7 to 302.4 } \\
\text { EFPD (EOC5) }\end{array}$ \\
\hline 1 & 102.26 & 91.97 & 82.37 \\
\hline 2 & 46.52 & 5.98 & 1.43 \\
\hline 3 & 2.96 & 0.00 & 0.00 \\
\hline 4 & 0.00 & 0.00 & 0.00 \\
\hline 5 & 0.00 & 0.00 & 0.00 \\
\hline 6 & 0.00 & 0.00 & 0.00 \\
\hline 7 & 0.00 & 0.00 & 0.00 \\
\hline 8 & 0.00 & 0.00 & 0.00 \\
\hline 9 & 0.00 & 0.00 & 0.00 \\
\hline 10 & 0.00 & 0.00 & 0.00 \\
\hline 11 & 0.00 & 0.00 & 0.00 \\
\hline 12 & 0.00 & 0.00 & 0.00 \\
\hline 13 & 0.00 & 0.00 & 0.00 \\
\hline 14 & 0.00 & 0.00 & 0.00 \\
\hline 15 & 0.00 & 0.00 & 0.00 \\
\hline 16 & 0.00 & 0.00 & 0.00 \\
\hline 17 & 0.00 & 0.00 & 0.00 \\
\hline 18 & 0.00 & 0.00 & 0.00 \\
\hline
\end{tabular}

Table 4-127. Rod Insertion Time by Axial Node for Assembly D21

\begin{tabular}{|c|c|c|c|}
\hline \multirow{2}{*}{$\begin{array}{c}\text { Axial } \\
\text { Node }\end{array}$} & \multicolumn{3}{|c|}{ Time Rod Inserted (EFPD) } \\
\cline { 2 - 4 } & $\begin{array}{c}\text { BOC5 (SP60) to } \\
114.4 \text { EFPD (SP61) }\end{array}$ & $\begin{array}{c}114.4 \text { (SP61) to } \\
\text { 206.7 EFPD }\end{array}$ & $\begin{array}{c}\text { 206.7 to 302.4 } \\
\text { EFPD (EOC5) }\end{array}$ \\
\hline 1 & 0.00 & 0.00 & 0.00 \\
\hline 2 & 0.00 & 0.00 & 0.00 \\
\hline 3 & 0.00 & 0.00 & 0.00 \\
\hline 4 & 0.00 & 0.00 & 0.00 \\
\hline 5 & 0.00 & 0.00 & 0.00 \\
\hline 6 & 0.00 & 0.00 & 0.00 \\
\hline 7 & 4.96 & 0.00 & 0.00 \\
\hline 8 & 36.68 & 11.45 & 4.56 \\
\hline 9 & 105.43 & 92.26 & 30.16 \\
\hline 10 & 114.40 & 92.26 & 27.59 \\
\hline 11 & 114.40 & 92.30 & 27.30 \\
\hline 12 & 81.74 & 92.30 & 28.86 \\
\hline 13 & 32.77 & 40.67 & 16.44 \\
\hline 14 & 1.69 & 0.00 & 0.00 \\
\hline 15 & 0.00 & 0.00 & 0.00 \\
\hline 16 & 0.00 & 0.00 & 0.00 \\
\hline 17 & 0.00 & 0.00 & 0.00 \\
\hline 18 & 0.00 & 0.00 & 0.00 \\
\hline
\end{tabular}


Table 4-128. Rod Insertion Time by Axial Node for Assembly D25a

\begin{tabular}{|c|c|c|c|}
\hline \multirow{2}{*}{$\begin{array}{c}\text { Axial } \\
\text { Node }\end{array}$} & \multicolumn{3}{|c|}{ Time Rod Inserted (EFPD) } \\
\cline { 2 - 4 } & $\begin{array}{c}\text { BOC5 (SP60) to } \\
114.4 \text { EFPD (SP61) }\end{array}$ & $\begin{array}{c}114.4 \text { (SP61) to } \\
\text { 206.7 EFPD }\end{array}$ & $\begin{array}{c}\text { 206.7 to 302.4 } \\
\text { EFPD (EOC5) }\end{array}$ \\
\hline 1 & 102.55 & 91.98 & 82.57 \\
\hline 2 & 47.02 & 5.99 & 1.43 \\
\hline 3 & 3.14 & 0.00 & 0.00 \\
\hline 4 & 0.00 & 0.00 & 0.00 \\
\hline 5 & 0.00 & 0.00 & 0.00 \\
\hline 6 & 0.00 & 0.00 & 0.00 \\
\hline 7 & 0.00 & 0.00 & 0.00 \\
\hline 8 & 0.00 & 0.00 & 0.00 \\
\hline 9 & 0.00 & 0.00 & 0.00 \\
\hline 10 & 0.00 & 0.00 & 0.00 \\
\hline 11 & 0.00 & 0.00 & 0.00 \\
\hline 12 & 0.00 & 0.00 & 0.00 \\
\hline 13 & 0.00 & 0.00 & 0.00 \\
\hline 14 & 0.00 & 0.00 & 0.00 \\
\hline 15 & 0.00 & 0.00 & 0.00 \\
\hline 16 & 0.00 & 0.00 & 0.00 \\
\hline 17 & 0.00 & 0.00 & 0.00 \\
\hline 18 & 0.00 & 0.00 & 0.00 \\
\hline
\end{tabular}

Table 4-129. Rod Insertion Time by Axial Node for Assembly D28

\begin{tabular}{|c|c|c|c|c|}
\hline \multirow{2}{*}{$\begin{array}{c}\text { Axial } \\
\text { Node }\end{array}$} & \multicolumn{4}{|c|}{ Time Rod Inserted (EFPD) } \\
\cline { 2 - 5 } & $\begin{array}{c}\text { BOC6 (SP62) to } \\
69.1 \text { EFPD (SP63) }\end{array}$ & $\begin{array}{c}\mathbf{6 9 . 1} \text { (SP63) to } \\
\mathbf{1 7 6 . 7} \text { EFPD }\end{array}$ & $\begin{array}{c}\mathbf{1 7 6 . 7} \text { to 320.3 } \\
\text { EFPD }\end{array}$ & $\begin{array}{c}\text { 320.3 to 421.2 } \\
\text { EFPD EOC6 }\end{array}$ \\
\hline 1 & 0.00 & 0.00 & 0.00 & 0.00 \\
\hline 2 & 0.00 & 0.00 & 0.00 & 0.00 \\
\hline 3 & 0.00 & 0.00 & 0.00 & 0.00 \\
\hline 4 & 13.24 & 2.93 & 19.19 & 6.55 \\
\hline 5 & 62.32 & 91.08 & 143.57 & 100.88 \\
\hline 6 & 69.10 & 107.60 & 143.60 & 100.85 \\
\hline 7 & 69.07 & 107.63 & 143.60 & 100.88 \\
\hline 8 & 69.10 & 107.60 & 143.60 & 100.88 \\
\hline 9 & 69.10 & 107.60 & 143.60 & 100.88 \\
\hline 10 & 69.13 & 107.57 & 143.60 & 100.88 \\
\hline 11 & 69.10 & 107.63 & 143.57 & 100.88 \\
\hline 12 & 60.10 & 107.35 & 139.17 & 100.85 \\
\hline 13 & 10.82 & 27.41 & 3.35 & 6.27 \\
\hline 14 & 0.00 & 0.00 & 0.00 & 0.00 \\
\hline 15 & 0.00 & 0.00 & 0.00 & 0.00 \\
\hline 16 & 0.00 & 0.00 & 0.00 & 0.00 \\
\hline 17 & 0.00 & 0.00 & 0.00 & 0.00 \\
\hline 18 & 0.00 & 0.00 & 0.00 & 0.00 \\
\hline
\end{tabular}


Table 4-130. Rod Insertion Time by Axial Node for Assembly F2

\begin{tabular}{|c|c|c|c|c|c|}
\hline & \multicolumn{5}{|c|}{ Time Rod Inserted (EFPD) } \\
\cline { 2 - 6 } $\begin{array}{c}\text { Axial } \\
\text { Node }\end{array}$ & $\begin{array}{c}\text { BOC7 (SP64) to } \\
\text { 30.0 EFPD } \\
\text { (SP65) }\end{array}$ & $\begin{array}{c}\text { 30.0 (SP65) to } \\
\text { 107.8 EFPD } \\
\text { (SP66) }\end{array}$ & $\begin{array}{c}1 \text { 107.8(SP66) } \\
\text { to 227.4 } \\
\text { EFPD }\end{array}$ & $\begin{array}{c}\text { 227.4 to } \\
367.3 \text { EFPD }\end{array}$ & $\begin{array}{c}\text { 474.7 EFPD } \\
\text { (EOC7) }\end{array}$ \\
\hline 1 & 30.00 & 78.00 & 119.31 & 139.90 & 93.28 \\
\hline 2 & 13.12 & 25.32 & 31.76 & 47.51 & 20.07 \\
\hline 3 & 0.00 & 0.00 & 0.00 & 0.00 & 0.00 \\
\hline 4 & 0.00 & 0.00 & 0.00 & 0.00 & 0.00 \\
\hline 5 & 0.00 & 0.00 & 0.00 & 0.00 & 0.00 \\
\hline 6 & 0.00 & 0.00 & 0.00 & 0.00 & 0.00 \\
\hline 7 & 0.00 & 0.00 & 0.00 & 0.00 & 0.00 \\
\hline 8 & 0.00 & 0.00 & 0.00 & 0.00 & 0.00 \\
\hline 9 & 0.00 & 0.00 & 0.00 & 0.00 & 0.00 \\
\hline 10 & 0.00 & 0.00 & 0.00 & 0.00 & 0.00 \\
\hline 11 & 0.00 & 0.00 & 0.00 & 0.00 & 0.00 \\
\hline 12 & 0.00 & 0.00 & 0.00 & 0.00 & 0.00 \\
\hline 13 & 0.00 & 0.00 & 0.00 & 0.00 & 0.00 \\
\hline 14 & 0.00 & 0.00 & 0.00 & 0.00 & 0.00 \\
\hline 15 & 0.00 & 0.00 & 0.00 & 0.00 & 0.00 \\
\hline 16 & 0.00 & 0.00 & 0.00 & 0.00 & 0.00 \\
\hline 17 & 0.00 & 0.00 & 0.00 & 0.00 & 0.00 \\
\hline 18 & 0.00 & 0.00 & 0.00 & 0.00 & 0.00 \\
\hline
\end{tabular}

Table 4-131. Rod Insertion Time by Axial Node for Assembly F4

\begin{tabular}{|c|c|c|c|c|c|}
\hline & \multicolumn{5}{|c|}{ Time Rod Inserted (EFP) } \\
\cline { 2 - 6 } $\begin{array}{c}\text { Axial } \\
\text { Node }\end{array}$ & $\begin{array}{c}\text { BOC7 (SP64) } \\
\text { to 30.0 EFPD } \\
\text { (SP65) }\end{array}$ & $\begin{array}{c}\text { 30.0 (SP65) to } \\
107.8 \text { EFPD } \\
\text { (SP66) }\end{array}$ & $\begin{array}{c}\text { 107.8(SP66) } \\
\text { to 227.4 } \\
\text { EFPD }\end{array}$ & $\begin{array}{c}\text { 227.4 to } \\
\mathbf{3 6 7 . 3} \text { EFPD }\end{array}$ & $\begin{array}{c}\text { 367.3 } \\
\text { (EOC7) EFP }\end{array}$ \\
\hline 1 & 30.00 & 78.00 & 119.40 & 139.83 & 93.60 \\
\hline 2 & 13.09 & 25.29 & 31.78 & 47.48 & 20.24 \\
\hline 3 & 0.00 & 0.00 & 0.00 & 0.00 & 0.00 \\
\hline 4 & 0.00 & 0.00 & 0.00 & 0.00 & 0.00 \\
\hline 5 & 0.00 & 0.00 & 0.00 & 0.00 & 0.00 \\
\hline 6 & 0.00 & 0.00 & 0.00 & 0.00 & 0.00 \\
\hline 7 & 0.00 & 0.00 & 0.00 & 0.00 & 0.00 \\
\hline 8 & 0.00 & 0.00 & 0.00 & 0.00 & 0.00 \\
\hline 9 & 0.00 & 0.00 & 0.00 & 0.00 & 0.00 \\
\hline 10 & 0.00 & 0.00 & 0.00 & 0.00 & 0.00 \\
\hline 11 & 0.00 & 0.00 & 0.00 & 0.00 & 0.00 \\
\hline 12 & 0.00 & 0.00 & 0.00 & 0.00 & 0.00 \\
\hline 13 & 0.00 & 0.00 & 0.00 & 0.00 & 0.00 \\
\hline 14 & 0.00 & 0.00 & 0.00 & 0.00 & 0.00 \\
\hline 15 & 0.00 & 0.00 & 0.00 & 0.00 & 0.00 \\
\hline 16 & 0.00 & 0.00 & 0.00 & 0.00 & 0.00 \\
\hline 17 & 0.00 & 0.00 & 0.00 & 0.00 & 0.00 \\
\hline 18 & 0.00 & 0.00 & 0.00 & 0.00 & 0.00 \\
\hline
\end{tabular}


Table 4-132. Rod Insertion Time by Axial Node for Assembly F23

\begin{tabular}{|c|c|c|c|c|c|}
\hline & \multicolumn{5}{|c|}{ Time Rod Inserted (EFPD) } \\
\cline { 2 - 6 } $\begin{array}{c}\text { Axial } \\
\text { Node }\end{array}$ & $\begin{array}{c}\text { BOC7 (SP64) } \\
\text { to 30.0 EFPD } \\
\text { (SP65) }\end{array}$ & $\begin{array}{c}\mathbf{3 0 . 0} \text { (SP65) to } \\
107.8 \text { EFPD } \\
\text { (SP66) }\end{array}$ & $\begin{array}{c}107.8(\text { SP66) } \\
\text { to 227.4 } \\
\text { EFPD }\end{array}$ & $\begin{array}{c}\text { 227.4 to } \\
\mathbf{3 6 7 . 3} \text { EFPD }\end{array}$ & $\begin{array}{c}\text { 474.7 EFPD } \\
\text { (EOC7) }\end{array}$ \\
\hline 1 & 0.00 & 0.00 & 0.00 & 0.00 & 0.00 \\
\hline 2 & 0.00 & 0.00 & 0.00 & 0.00 & 0.00 \\
\hline 3 & 0.00 & 0.00 & 0.00 & 0.00 & 0.00 \\
\hline 4 & 19.59 & 69.57 & 45.14 & 3.65 & 18.94 \\
\hline 5 & 30.00 & 77.97 & 119.43 & 16.64 & 78.32 \\
\hline 6 & 30.00 & 78.00 & 119.40 & 16.64 & 78.11 \\
\hline 7 & 30.00 & 78.00 & 119.40 & 16.64 & 78.11 \\
\hline 8 & 30.00 & 78.00 & 119.40 & 16.64 & 78.26 \\
\hline 9 & 30.00 & 78.03 & 119.37 & 16.64 & 78.45 \\
\hline 10 & 30.00 & 78.00 & 119.40 & 16.64 & 78.81 \\
\hline 11 & 30.00 & 78.00 & 119.40 & 16.64 & 79.37 \\
\hline 12 & 14.11 & 18.28 & 88.94 & 15.08 & 71.53 \\
\hline 13 & 0.00 & 0.00 & 0.00 & 0.01 & 0.00 \\
\hline 14 & 0.00 & 0.00 & 0.00 & 0.00 & 0.00 \\
\hline 15 & 0.00 & 0.00 & 0.00 & 0.00 & 0.00 \\
\hline 16 & 0.00 & 0.00 & 0.00 & 0.00 & 0.00 \\
\hline 17 & 0.00 & 0.00 & 0.00 & 0.00 & 0.00 \\
\hline 18 & 0.00 & 0.00 & 0.00 & 0.00 & 0.00 \\
\hline
\end{tabular}

Table 4-133. Rod Insertion Time by Axial Node for Assembly G2

\begin{tabular}{|c|c|c|c|}
\hline \multirow{3}{*}{$\begin{array}{c}\text { Axial } \\
\text { Node }\end{array}$} & \multicolumn{3}{|c|}{ Time Rod Inserted (EFPD) } \\
\cline { 2 - 4 } & $\begin{array}{c}\text { BOC8 (SP67) to 171.1 } \\
\text { EFPD }\end{array}$ & $\begin{array}{c}\text { 171.1 to 338.1 } \\
\text { EFPD }\end{array}$ & $\begin{array}{c}\text { 338.1 to 508.8 } \\
\text { EFPD (EOC8) }\end{array}$ \\
\hline 1 & 171.10 & 167.00 & 170.76 \\
\hline 2 & 84.97 & 54.20 & 49.13 \\
\hline 3 & 0.00 & 0.00 & 0.00 \\
\hline 4 & 0.00 & 0.00 & 0.00 \\
\hline 5 & 0.00 & 0.00 & 0.00 \\
\hline 6 & 0.00 & 0.00 & 0.00 \\
\hline 7 & 0.00 & 0.00 & 0.00 \\
\hline 8 & 0.00 & 0.00 & 0.00 \\
\hline 9 & 0.00 & 0.00 & 0.00 \\
\hline 10 & 0.00 & 0.00 & 0.00 \\
\hline 11 & 0.00 & 0.00 & 0.00 \\
\hline 12 & 0.00 & 0.00 & 0.00 \\
\hline 13 & 0.00 & 0.00 & 0.00 \\
\hline 14 & 0.00 & 0.00 & 0.00 \\
\hline 15 & 0.00 & 0.00 & 0.00 \\
\hline 16 & 0.00 & 0.00 & 0.00 \\
\hline 17 & 0.00 & 0.00 & 0.00 \\
\hline 18 & 0.00 & 0.00 & 0.00 \\
\hline
\end{tabular}


Table 4-134. Rod Insertion Time by Axial Node for Assembly G10

\begin{tabular}{|c|c|c|c|}
\hline \multirow{2}{*}{$\begin{array}{c}\text { Axial } \\
\text { Node }\end{array}$} & \multicolumn{3}{|c|}{ Time Rod Inserted (EFPD) } \\
\cline { 2 - 4 } & $\begin{array}{c}\text { BOC8 (SP67) to 171.1 } \\
\text { EFPD }\end{array}$ & $\begin{array}{c}171.1 \text { to 338.1 } \\
\text { EFPD }\end{array}$ & $\begin{array}{c}\text { 338.1 to 508.8 } \\
\text { EFPD (EOC8) }\end{array}$ \\
\hline 1 & 0.00 & 0.00 & 0.00 \\
\hline 2 & 0.00 & 0.00 & 0.00 \\
\hline 3 & 0.00 & 0.00 & 0.00 \\
\hline 4 & 17.72 & 31.58 & 39.90 \\
\hline 5 & 171.07 & 167.03 & 170.67 \\
\hline 6 & 171.10 & 167.00 & 170.70 \\
\hline 7 & 171.10 & 167.00 & 170.70 \\
\hline 8 & 171.10 & 167.00 & 170.73 \\
\hline 9 & 171.10 & 166.97 & 170.73 \\
\hline 10 & 171.10 & 167.00 & 170.70 \\
\hline 11 & 171.10 & 167.00 & 170.70 \\
\hline 12 & 166.35 & 155.00 & 150.51 \\
\hline 13 & 8.93 & 1.39 & 1.75 \\
\hline 14 & 0.00 & 0.00 & 0.00 \\
\hline 15 & 0.00 & 0.00 & 0.00 \\
\hline 16 & 0.00 & 0.00 & 0.00 \\
\hline 17 & 0.00 & 0.00 & 0.00 \\
\hline 18 & 0.00 & 0.00 & 0.00 \\
\hline
\end{tabular}

Table 4-135. Rod Insertion Time by Axial Node for Assembly G27a

\begin{tabular}{|c|c|c|c|}
\hline \multirow{2}{*}{$\begin{array}{c}\text { Axial } \\
\text { Node }\end{array}$} & \multicolumn{3}{|c|}{ Time Rod Inserted (EFPD) } \\
\cline { 2 - 4 } & $\begin{array}{c}\text { BOC8 (SP67) to 171.1 } \\
\text { EFPD }\end{array}$ & $\begin{array}{c}171.1 \text { to 338.1 } \\
\text { EFPD }\end{array}$ & $\begin{array}{c}\text { 338.1 to 508.8 } \\
\text { EFPD (EOC8) }\end{array}$ \\
\hline 1 & 171.10 & 167.00 & 13.64 \\
\hline 2 & 84.99 & 54.14 & 3.92 \\
\hline 3 & 0.00 & 0.00 & 0.00 \\
\hline 4 & 0.00 & 0.00 & 0.00 \\
\hline 5 & 0.00 & 0.00 & 0.00 \\
\hline 6 & 0.00 & 0.00 & 0.00 \\
\hline 7 & 0.00 & 0.00 & 0.00 \\
\hline 8 & 0.00 & 0.00 & 0.00 \\
\hline 9 & 0.00 & 0.00 & 0.00 \\
\hline 10 & 0.00 & 0.00 & 0.00 \\
\hline 11 & 0.00 & 0.00 & 0.00 \\
\hline 12 & 0.00 & 0.00 & 0.00 \\
\hline 13 & 0.00 & 0.00 & 0.00 \\
\hline 14 & 0.00 & 0.00 & 0.00 \\
\hline 15 & 0.00 & 0.00 & 0.00 \\
\hline 16 & 0.00 & 0.00 & 0.00 \\
\hline 17 & 0.00 & 0.00 & 0.00 \\
\hline 18 & 0.00 & 0.00 & 0.00 \\
\hline
\end{tabular}


Table 4-136. Rod Insertion Time by Axial Node for Assembly $\mathrm{H} 10 \mathrm{a}$

\begin{tabular}{|c|c|c|c|c|c|}
\hline & \multicolumn{5}{|c|}{ Time Rod inserted (EFP) } \\
\cline { 2 - 6 } $\begin{array}{c}\text { Axial } \\
\text { Node }\end{array}$ & $\begin{array}{c}\text { BOC10 (SP69) } \\
\text { to 27.0 EFPD } \\
(\text { SP70) }\end{array}$ & $\begin{array}{c}27.0 \text { (SP70) to } \\
143.0 \text { EFPD } \\
\text { (SP71) }\end{array}$ & $\begin{array}{c}143.0 \text { (SP71) to } \\
\text { 209.0 EFPD } \\
\text { (SP72) }\end{array}$ & $\begin{array}{c}\text { 209.0 (SP72) } \\
\text { to 430.4 } \\
\text { EFPD }\end{array}$ & $\begin{array}{c}\text { 430.4 to } \\
660.3 \text { EFPD } \\
\text { EOC10 }\end{array}$ \\
\hline 1 & 25.07 & 113.50 & 66.08 & 220.68 & 229.66 \\
\hline 2 & 7.37 & 50.04 & 19.63 & 32.65 & 26.51 \\
\hline 3 & 0.00 & 0.00 & 0.00 & 0.00 & 0.00 \\
\hline 4 & 0.00 & 0.00 & 0.00 & 0.00 & 0.00 \\
\hline 5 & 0.00 & 0.00 & 0.00 & 0.00 & 0.00 \\
\hline 6 & 0.00 & 0.00 & 0.00 & 0.00 & 0.00 \\
\hline 7 & 0.00 & 0.00 & 0.00 & 0.00 & 0.00 \\
\hline 8 & 0.00 & 0.00 & 0.00 & 0.00 & 0.00 \\
\hline 9 & 0.00 & 0.00 & 0.00 & 0.00 & 0.00 \\
\hline 10 & 0.00 & 0.00 & 0.00 & 0.00 & 0.00 \\
\hline 11 & 0.00 & 0.00 & 0.00 & 0.00 & 0.00 \\
\hline 12 & 0.00 & 0.00 & 0.00 & 0.00 & 0.00 \\
\hline 13 & 0.00 & 0.00 & 0.00 & 0.00 & 0.00 \\
\hline 14 & 0.00 & 0.00 & 0.00 & 0.00 & 0.00 \\
\hline 15 & 0.00 & 0.00 & 0.00 & 0.00 & 0.00 \\
\hline 16 & 0.00 & 0.00 & 0.00 & 0.00 & 0.00 \\
\hline 17 & 0.00 & 0.00 & 0.00 & 0.00 & 0.00 \\
\hline 18 & 0.00 & 0.00 & 0.00 & 0.00 & 0.00 \\
\hline
\end{tabular}

Table 4-137. Rod Insertion Time by Axial Node for Assembly H1Ob

\begin{tabular}{|c|c|c|c|c|}
\hline \multirow{2}{*}{$\begin{array}{c}\text { Axial } \\
\text { Node }\end{array}$} & \multicolumn{5}{|c|}{ Time Rod Inserted (EFPD) } \\
\cline { 2 - 5 } & $\begin{array}{c}\text { BOC 11 (SP73) } \\
\text { to 208.7 EFPD }\end{array}$ & $\begin{array}{c}\text { 208.7 to 417.8 } \\
\text { EFPD }\end{array}$ & $\begin{array}{c}\text { 417.8 to 615.0 } \\
\text { EFPD (SP74) }\end{array}$ & $\begin{array}{c}\mathbf{6 1 5 . 0} \text { (SP74) to } \\
\text { 683.0 EFPD EOC11 }\end{array}$ \\
\hline 1 & 208.70 & 209.10 & 197.20 & 60.67 \\
\hline 2 & 62.43 & 55.84 & 45.43 & 13.83 \\
\hline 3 & 0.00 & 0.00 & 0.00 & 0.00 \\
\hline 4 & 0.00 & 0.00 & 0.00 & 0.00 \\
\hline 5 & 0.00 & 0.00 & 0.00 & 0.00 \\
\hline 6 & 0.00 & 0.00 & 0.00 & 0.00 \\
\hline 7 & 0.00 & 0.00 & 0.00 & 0.00 \\
\hline 8 & 0.00 & 0.00 & 0.00 & 0.00 \\
\hline 9 & 0.00 & 0.00 & 0.00 & 0.00 \\
\hline 10 & 0.00 & 0.00 & 0.00 & 0.00 \\
\hline 11 & 0.00 & 0.00 & 0.00 & 0.00 \\
\hline 12 & 0.00 & 0.00 & 0.00 & 0.00 \\
\hline 13 & 0.00 & 0.00 & 0.00 & 0.00 \\
\hline 14 & 0.00 & 0.00 & 0.00 & 0.00 \\
\hline 15 & 0.00 & 0.00 & 0.00 & 0.00 \\
\hline 16 & 0.00 & 0.00 & 0.00 & 0.00 \\
\hline 17 & 0.00 & 0.00 & 0.00 & 0.00 \\
\hline 18 & 0.00 & 0.00 & 0.00 & 0.00 \\
\hline
\end{tabular}


Table 4-138. Rod Insertion Time by Axial Node for Assembly H12

\begin{tabular}{|c|c|c|c|}
\hline \multirow{2}{*}{$\begin{array}{c}\text { Axial } \\
\text { Node }\end{array}$} & \multicolumn{3}{|c|}{ Time Rod Inserted (EFPD) } \\
\cline { 2 - 4 } & $\begin{array}{c}\text { BOC9 (SP68) to } \\
214.0 \text { EFPD }\end{array}$ & $\begin{array}{c}214.0 \text { to 425.0 } \\
\text { EFPD }\end{array}$ & $\begin{array}{c}\text { 425.0 to 639.4 } \\
\text { EFPD (EOC9) }\end{array}$ \\
\hline 1 & 214.00 & 211.00 & 213.69 \\
\hline 2 & 127.88 & 103.85 & 86.25 \\
\hline 3 & 0.00 & 0.00 & 0.00 \\
\hline 4 & 0.00 & 0.00 & 0.00 \\
\hline 5 & 0.00 & 0.00 & 0.00 \\
\hline 6 & 0.00 & 0.00 & 0.00 \\
\hline 7 & 0.00 & 0.00 & 0.00 \\
\hline 8 & 0.00 & 0.00 & 0.00 \\
\hline 9 & 0.00 & 0.00 & 0.00 \\
\hline 10 & 0.00 & 0.00 & 0.00 \\
\hline 11 & 0.00 & 0.00 & 0.00 \\
\hline 12 & 0.00 & 0.00 & 0.00 \\
\hline 13 & 0.00 & 0.00 & 0.00 \\
\hline 14 & 0.00 & 0.00 & 0.00 \\
\hline 15 & 0.00 & 0.00 & 0.00 \\
\hline 16 & 0.00 & 0.00 & 0.00 \\
\hline 17 & 0.00 & 0.00 & 0.00 \\
\hline 18 & 0.00 & 0.00 & 0.00 \\
\hline
\end{tabular}

Table 4-139. Rod Insertion Time by Axial Node for Assembly H23

\begin{tabular}{|c|c|c|c|}
\hline \multirow{2}{*}{$\begin{array}{c}\text { Axial } \\
\text { Node }\end{array}$} & \multicolumn{3}{|c|}{ Time Rod Inserted (EFPD) } \\
\cline { 2 - 4 } & $\begin{array}{c}\text { BOC9 (SP68) to } \\
\text { 214.0 EFPD }\end{array}$ & $\begin{array}{c}\text { 214.0 to 425.0 } \\
\text { EFPD }\end{array}$ & $\begin{array}{c}\text { 425.0 to 639.4 } \\
\text { EFPD (EOC9) }\end{array}$ \\
\hline 1 & 0.00 & 0.00 & 1.67 \\
\hline 2 & 0.00 & 0.00 & 1.67 \\
\hline 3 & 0.00 & 0.00 & 3.31 \\
\hline 4 & 51.93 & 56.47 & 46.53 \\
\hline 5 & 214.00 & 211.00 & 158.37 \\
\hline 6 & 214.00 & 211.00 & 157.86 \\
\hline 7 & 214.00 & 211.00 & 157.86 \\
\hline 8 & 214.00 & 211.03 & 158.01 \\
\hline 9 & 214.00 & 211.00 & 158.39 \\
\hline 10 & 214.03 & 210.97 & 159.03 \\
\hline 11 & 214.03 & 211.00 & 158.47 \\
\hline 12 & 189.27 & 181.27 & 136.97 \\
\hline 13 & 0.00 & 0.00 & 0.00 \\
\hline 14 & 0.00 & 0.00 & 0.00 \\
\hline 15 & 0.00 & 0.00 & 0.00 \\
\hline 16 & 0.00 & 0.00 & 0.00 \\
\hline 17 & 0.00 & 0.00 & 0.00 \\
\hline 18 & 0.00 & 0.00 & 0.00 \\
\hline
\end{tabular}


Table 4-140. Rod Insertion Time by Axial Node for Assembly H27a

\begin{tabular}{|c|c|c|c|}
\hline \multirow[b]{2}{*}{$\begin{array}{l}\text { Axial } \\
\text { Node }\end{array}$} & \multicolumn{3}{|c|}{ Time Rod Inserted (EFPD) } \\
\hline & $\begin{array}{c}\text { BOC9 (SP68) to } \\
214.0 \text { EFPD }\end{array}$ & $\begin{array}{c}214.0 \text { to } 425.0 \\
\text { EFPD }\end{array}$ & $\begin{array}{l}425.0 \text { to } 639.4 \\
\text { EFPD (EOC9) }\end{array}$ \\
\hline 1 & 214.00 & 211.00 & 213.70 \\
\hline 2 & 127.88 & 103.77 & 85.85 \\
\hline 3 & 0.00 & 0.00 & 0.00 \\
\hline 4 & 0.00 & 0.00 & 0.00 \\
\hline 5 & 0.00 & 0.00 & 0.00 \\
\hline 6 & 0.00 & 0.00 & 0.00 \\
\hline 7 & 0.00 & 0.00 & 0.00 \\
\hline 8 & 0.00 & 0.00 & 0.00 \\
\hline 9 & 0.00 & 0.00 & 0.00 \\
\hline 10 & 0.00 & 0.00 & 0.00 \\
\hline 11 & 0.00 & 0.00 & 0.00 \\
\hline 12 & 0.00 & 0.00 & 0.00 \\
\hline 13 & 0.00 & 0.00 & 0.00 \\
\hline 14 & 0.00 & 0.00 & 0.00 \\
\hline 15 & 0.00 & 0.00 & 0.00 \\
\hline 16 & 0.00 & 0.00 & 0.00 \\
\hline 17 & 0.00 & 0.00 & 0.00 \\
\hline 18 & 0.00 & 0.00 & 0.00 \\
\hline
\end{tabular}

Table 4-141. Rod Insertion Time by Axial Node for Assembly J20

\begin{tabular}{|c|c|c|c|c|c|}
\hline \multirow[b]{2}{*}{$\begin{array}{l}\text { Axial } \\
\text { Node }\end{array}$} & \multicolumn{5}{|c|}{ Time Rod Inserted (EFPD) } \\
\hline & $\begin{array}{c}\text { BOC10 (SP69) } \\
\text { to } 27.0 \text { EFPD } \\
\text { (SP70) }\end{array}$ & $\begin{array}{c}27.0 \text { (SP70) to } \\
143.0 \text { EFPD } \\
\text { (SP71) }\end{array}$ & $\begin{array}{c}143.0 \text { (SP71) to } \\
209.0 \text { EFPD } \\
\text { (SP72) }\end{array}$ & $\begin{array}{c}209.0 \text { (SP72) to } \\
430.4 \text { EFPD }\end{array}$ & $\begin{array}{c}430.4 \text { to } \\
660.3 \text { EFPD } \\
\text { EOC10 }\end{array}$ \\
\hline 1 & 0.00 & 0.00 & 0.00 & 0.00 & 0.00 \\
\hline 2 & 0.00 & 0.00 & 0.00 & 0.00 & 0.00 \\
\hline 3 & 0.00 & 0.00 & 0.00 & 0.00 & 0.00 \\
\hline 4 & 5.77 & 17.87 & 14.86 & 51.02 & 59.87 \\
\hline 5 & 27.00 & 115.59 & 66.00 & 221.40 & 229.90 \\
\hline 6 & 27.03 & 115.97 & 66.00 & 221.40 & 229.90 \\
\hline 7 & 27.00 & 116.00 & 66.00 & 221.40 & 229.87 \\
\hline 8 & 27.03 & 115.97 & 66.00 & 221.40 & 229.93 \\
\hline 9 & 26.97 & 116.00 & 66.03 & 221.40 & 229.90 \\
\hline 10 & 27.00 & 116.00 & 66.00 & 221.40 & 229.90 \\
\hline 11 & 27.00 & 116.00 & 66.00 & 221.40 & 229.90 \\
\hline 12 & 24.68 & 111.35 & 59.46 & 198.33 & 199.22 \\
\hline 13 & 0.00 & 1.83 & 0.00 & 0.19 & 0.00 \\
\hline 14 & 0.00 & 0.00 & 0.00 & 0.00 & 0.00 \\
\hline 15 & 0.00 & 0.00 & 0.00 & 0.00 & 0.00 \\
\hline$\overline{16}$ & 0.00 & 0.00 & 0.00 & 0.00 & 0.00 \\
\hline 17 & 0.00 & 0.00 & 0.00 & 0.00 & 0.00 \\
\hline 18 & 0.00 & 0.00 & 0.00 & 0.00 & 0.00 \\
\hline
\end{tabular}


Table 4-142. Rod Insertion Time by Axial Node for Assembly J27

\begin{tabular}{|c|c|c|c|c|c|}
\hline & \multicolumn{5}{|c|}{ Time Rod Inserted (EFPD) } \\
\cline { 2 - 6 } Axial \\
Node & $\begin{array}{c}\text { BOC10 (SP69) } \\
\text { to 27.0 EFPD } \\
(\text { SP70) }\end{array}$ & $\begin{array}{c}\text { 27.0 (SP70) to } \\
143.0 \text { EFPD } \\
\text { (SP71) }\end{array}$ & $\begin{array}{c}143.0 \text { (SP71) to } \\
\text { 209.0 EFPD } \\
\text { (SP72) }\end{array}$ & $\begin{array}{c}\text { 430.4 to } \\
\text { 209.0 (SP72) to } \\
430.4 \text { EFPD }\end{array}$ & $\begin{array}{c}660.3 \text { EFPD } \\
\text { EOC10 }\end{array}$ \\
\hline 1 & 25.09 & 113.54 & 66.00 & 220.79 & 229.58 \\
\hline 2 & 7.33 & 49.95 & 19.61 & 32.85 & 26.50 \\
\hline 3 & 0.00 & 0.00 & 0.00 & 0.00 & 0.00 \\
\hline 4 & 0.00 & 0.00 & 0.00 & 0.00 & 0.00 \\
\hline 5 & 0.00 & 0.00 & 0.00 & 0.00 & 0.00 \\
\hline 6 & 0.00 & 0.00 & 0.00 & 0.00 & 0.00 \\
\hline 7 & 0.00 & 0.00 & 0.00 & 0.00 & 0.00 \\
\hline 8 & 0.00 & 0.00 & 0.00 & 0.00 & 0.00 \\
\hline 9 & 0.00 & 0.00 & 0.00 & 0.00 & 0.00 \\
\hline 10 & 0.00 & 0.00 & 0.00 & 0.00 & 0.00 \\
\hline 11 & 0.00 & 0.00 & 0.00 & 0.00 & 0.00 \\
\hline 12 & 0.00 & 0.00 & 0.00 & 0.00 & 0.00 \\
\hline 13 & 0.00 & 0.00 & 0.00 & 0.00 & 0.00 \\
\hline 14 & 0.00 & 0.00 & 0.00 & 0.00 & 0.00 \\
\hline 15 & 0.00 & 0.00 & 0.00 & 0.00 & 0.00 \\
\hline 16 & 0.00 & 0.00 & 0.00 & 0.00 & 0.00 \\
\hline 17 & 0.00 & 0.00 & 0.00 & 0.00 & 0.00 \\
\hline 18 & 0.00 & 0.00 & 0.00 & 0.00 & 0.00 \\
\hline
\end{tabular}

Table 4-143. Rod Insertion Time by Axial Node for Assembly J27a

\begin{tabular}{|c|c|c|c|c|c|}
\hline \multirow[b]{2}{*}{$\begin{array}{l}\text { Axial } \\
\text { Node }\end{array}$} & \multicolumn{5}{|c|}{ Time Rod Inserted (EFPD) } \\
\hline & $\begin{array}{c}\text { BOC10 (SP69) } \\
\text { to } 27.0 \text { EFPD } \\
\text { (SP70) }\end{array}$ & $\begin{array}{c}27.0 \text { (SP70) to } \\
143.0 \text { EFPD } \\
\text { (SP71) }\end{array}$ & $\begin{array}{c}143.0 \text { (SP71) to } \\
209.0 \text { EFPD } \\
\text { (SP72) }\end{array}$ & $\begin{array}{c}209.0 \text { (SP72) to } \\
430.4 \text { EFPD }\end{array}$ & $\begin{array}{c}430.4 \text { to } \\
660.3 \text { EFPD } \\
\text { EOC10 }\end{array}$ \\
\hline 1 & 25.10 & 113.42 & 66.00 & 220.77 & 20.97 \\
\hline 2 & 7.33 & 50.06 & 19.61 & 32.78 & 2.42 \\
\hline 3 & 0.00 & 0.00 & 0.00 & 0.00 & 0.00 \\
\hline 4 & 0.00 & 0.00 & 0.00 & 0.00 & 0.00 \\
\hline 5 & 0.00 & 0.00 & 0.00 & 0.00 & 0.00 \\
\hline 6 & 0.00 & 0.00 & 0.00 & 0.00 & 0.00 \\
\hline 7 & 0.00 & 0.00 & 0.00 & 0.00 & 0.00 \\
\hline 8 & 0.00 & 0.00 & 0.00 & 0.00 & 0.00 \\
\hline 9 & 0.00 & 0.00 & 0.00 & 0.00 & 0.00 \\
\hline 10 & 0.00 & 0.00 & 0.00 & 0.00 & 0.00 \\
\hline 11 & 0.00 & 0.00 & 0.00 & 0.00 & 0.00 \\
\hline 12 & 0.00 & 0.00 & 0.00 & 0.00 & 0.00 \\
\hline 13 & 0.00 & 0.00 & 0.00 & 0.00 & 0.00 \\
\hline 14 & 0.00 & 0.00 & 0.00 & 0.00 & 0.00 \\
\hline 15 & 0.00 & 0.00 & 0.00 & 0.00 & 0.00 \\
\hline 16 & 0.00 & 0.00 & 0.00 & 0.00 & 0.00 \\
\hline 17 & 0.00 & 0.00 & 0.00 & 0.00 & 0.00 \\
\hline 18 & 0.00 & 0.00 & 0.00 & 0.00 & 0.00 \\
\hline
\end{tabular}


Table 4-144. Rod Insertion Time by Axial Node for Assembly K6

\begin{tabular}{|c|c|c|c|c|}
\hline \multirow{2}{*}{$\begin{array}{c}\text { Axial } \\
\text { Node }\end{array}$} & $\begin{array}{c}\text { BOC11 (SP73) to } \\
\text { 208.7 EFPD }\end{array}$ & $\begin{array}{c}\text { 208.7 to 417.8 } \\
\text { EFPD }\end{array}$ & $\begin{array}{c}\text { 417.8 to 615.0 } \\
\text { EFPD (SP74) }\end{array}$ & $\begin{array}{c}\text { 615.0 (SP74) to } \\
\text { 683.0 EFPD EOC11 }\end{array}$ \\
\hline 1 & 208.70 & 5.87 & 197.20 & 60.68 \\
\hline 2 & 62.26 & 1.57 & 45.44 & 13.77 \\
\hline 3 & 0.00 & 0.00 & 0.00 & 0.00 \\
\hline 4 & 0.00 & 0.00 & 0.00 & 0.00 \\
\hline 5 & 0.00 & 0.00 & 0.00 & 0.00 \\
\hline 6 & 0.00 & 0.00 & 0.00 & 0.00 \\
\hline 7 & 0.00 & 0.00 & 0.00 & 0.00 \\
\hline 8 & 0.00 & 0.00 & 0.00 & 0.00 \\
\hline 9 & 0.00 & 0.00 & 0.00 & 0.00 \\
\hline 10 & 0.00 & 0.00 & 0.00 & 0.00 \\
\hline 11 & 0.00 & 0.00 & 0.00 & 0.00 \\
\hline 12 & 0.00 & 0.00 & 0.00 & 0.00 \\
\hline 13 & 0.00 & 0.00 & 0.00 & 0.00 \\
\hline 14 & 0.00 & 0.00 & 0.00 & 0.00 \\
\hline 15 & 0.00 & 0.00 & 0.00 & 0.00 \\
\hline 16 & 0.00 & 0.00 & 0.00 & 0.00 \\
\hline 17 & 0.00 & 0.00 & 0.00 & 0.00 \\
\hline 18 & 0.00 & 0.00 & 0.00 & 0.00 \\
\hline
\end{tabular}

Table 4-145. Rod insertion Time by Axial Node for Assembly K12

\begin{tabular}{|c|c|c|c|c|}
\hline \multirow{2}{*}{$\begin{array}{c}\text { Axial } \\
\text { Node }\end{array}$} & $\begin{array}{c}\text { Time Rod Inserted (EFPD) } \\
\text { 208.7 EFPD }\end{array}$ & $\begin{array}{c}\text { 208.7 to 417.8 } \\
\text { EFPD }\end{array}$ & $\begin{array}{c}\text { 417.8 to 615.0 } \\
\text { EFPD (SP74) }\end{array}$ & $\begin{array}{c}\text { 615.0 (SP74) to } \\
\mathbf{6 8 3 . 0} \text { EFPD EOC11 }\end{array}$ \\
\hline 1 & 0.00 & 0.00 & 0.00 & 6.44 \\
\hline 2 & 0.00 & 0.00 & 0.00 & 6.42 \\
\hline 3 & 0.00 & 0.00 & 0.00 & 6.45 \\
\hline 4 & 45.70 & 52.61 & 45.58 & 15.95 \\
\hline 5 & 208.67 & 209.13 & 197.20 & 50.88 \\
\hline 6 & 208.70 & 209.10 & 197.20 & 44.29 \\
\hline 7 & 208.73 & 209.10 & 197.20 & 44.10 \\
\hline 8 & 208.70 & 209.10 & 197.20 & 44.05 \\
\hline 9 & 208.70 & 209.10 & 197.20 & 44.10 \\
\hline 10 & 208.70 & 209.10 & 197.23 & 44.21 \\
\hline 11 & 208.70 & 209.13 & 197.17 & 44.49 \\
\hline 12 & 188.58 & 183.06 & 176.63 & 41.71 \\
\hline 13 & 0.78 & 0.00 & 0.00 & 0.00 \\
\hline 14 & 0.00 & 0.00 & 0.00 & 0.00 \\
\hline 15 & 0.00 & 0.00 & 0.00 & 0.00 \\
\hline 16 & 0.00 & 0.00 & 0.00 & 0.00 \\
\hline 17 & 0.00 & 0.00 & 0.00 & 0.00 \\
\hline 18 & 0.00 & 0.00 & 0.00 & 0.00 \\
\hline
\end{tabular}


Table 4-146. Rod Insertion Time by Axial Node for Assembly K27

\begin{tabular}{|c|c|c|c|c|}
\hline \multirow[b]{2}{*}{$\begin{array}{l}\text { Axial } \\
\text { Node }\end{array}$} & \multicolumn{4}{|c|}{ Time Rod Inserted (EFPD) } \\
\hline & $\begin{array}{c}\text { BOC11 (SP73) to } \\
208.7 \text { EFPD }\end{array}$ & $\begin{array}{c}208.7 \text { to } 417.8 \\
\text { EFPD }\end{array}$ & $\begin{array}{l}417.8 \text { to } 615.0 \\
\text { EFPD (SP74) }\end{array}$ & $\begin{array}{c}615.0 \text { (SP74) to } \\
683.0 \text { EFPD EOC11 }\end{array}$ \\
\hline 1 & 208.70 & 209.04 & 197.26 & 60.52 \\
\hline 2 & $62 . \overline{33}$ & 55.78 & 45.45 & 13.74 \\
\hline 3 & 0.00 & 0.00 & 0.00 & 0.00 \\
\hline 4 & 0.00 & 0.00 & 0.00 & 0.00 \\
\hline 5 & 0.00 & 0.00 & 0.00 & 0.00 \\
\hline 6 & 0.00 & 0.00 & 0.00 & 0.00 \\
\hline 7 & 0.00 & 0.00 & 0.00 & 0.00 \\
\hline 8 & 0.00 & 0.00 & 0.00 & 0.00 \\
\hline 9 & 0.00 & 0.00 & 0.00 & 0.00 \\
\hline 10 & 0.00 & 0.00 & 0.00 & 0.00 \\
\hline 11 & 0.00 & 0.00 & 0.00 & 0.00 \\
\hline 12 & 0.00 & 0.00 & 0.00 & 0.00 \\
\hline 13 & 0.00 & 0.00 & 0.00 & 0.00 \\
\hline 14 & 0.00 & 0.00 & 0.00 & 0.00 \\
\hline 15 & 0.00 & 0.00 & 0.00 & 0.00 \\
\hline 16 & 0.00 & 0.00 & 0.00 & 0.00 \\
\hline 17 & 0.00 & 0.00 & 0.00 & 0.00 \\
\hline 18 & 0.00 & 0.00 & 0.00 & 0.00 \\
\hline
\end{tabular}


Table 4-147. All-Rods-Out-Critical-Boron Data for TMl-1

\begin{tabular}{|c|c|}
\hline \multicolumn{2}{|c|}{ Cycle 2 } \\
\hline EFPD & ppmB $^{\mathbf{a}}$ \\
\hline 25 & 912 \\
\hline 50 & 840 \\
\hline 75 & 767 \\
\hline 100 & 695 \\
\hline 125 & 622 \\
\hline 150 & 550 \\
\hline 175 & 477 \\
\hline 200 & 405 \\
\hline 225 & 332 \\
\hline 250 & 260 \\
\hline 256.2 & 242 \\
\hline
\end{tabular}

\begin{tabular}{|c|c|}
\hline \multicolumn{2}{|c|}{ Cycle 3 } \\
\hline EFPD & ppmB \\
\hline 25 & 788 \\
\hline 50 & 717 \\
\hline 75 & 645 \\
\hline 100 & 573 \\
\hline 125 & 502 \\
\hline 150 & 430 \\
\hline 175 & 358 \\
\hline 200 & 287 \\
\hline 225 & 215 \\
\hline 250 & 143 \\
\hline 275 & 72 \\
\hline 287.1 & 37 \\
\hline
\end{tabular}

\begin{tabular}{|c|c|}
\hline \multicolumn{2}{|c|}{ Cycle 4 } \\
\hline EFPD & ppmB \\
\hline 25 & 765 \\
\hline 50 & 694 \\
\hline 75 & 624 \\
\hline 100 & 554 \\
\hline 125 & 483 \\
\hline 150 & 413 \\
\hline 175 & 343 \\
\hline 200 & 273 \\
\hline 225 & 202 \\
\hline 250 & 132 \\
\hline 274 & 64 \\
\hline
\end{tabular}

\begin{tabular}{|c|c|}
\hline \multicolumn{2}{|c|}{ Cycle 5 } \\
\hline EFPD & ppmB \\
\hline 25 & 749 \\
\hline 50 & 677 \\
\hline 75 & 604 \\
\hline 100 & 532 \\
\hline 125 & 459 \\
\hline 150 & 386 \\
\hline 175 & 314 \\
\hline 200 & 241 \\
\hline 225 & 168 \\
\hline 250 & 96 \\
\hline 275 & 23 \\
\hline
\end{tabular}

\begin{tabular}{|c|c|}
\hline \multicolumn{2}{|c|}{ Cycle 6 } \\
\hline EFPD & ppmB \\
\hline 25 & 960 \\
\hline 50 & 935 \\
\hline 75 & 900 \\
\hline 100 & 855 \\
\hline 125 & 805 \\
\hline 150 & 750 \\
\hline 175 & 690 \\
\hline 200 & 625 \\
\hline 225 & 560 \\
\hline 250 & 495 \\
\hline 275 & 425 \\
\hline 300 & 350 \\
\hline 325 & 270 \\
\hline 350 & 180 \\
\hline 375 & 90 \\
\hline 400 & 40 \\
\hline 420 & 10 \\
\hline
\end{tabular}

\begin{tabular}{|c|c|}
\hline \multicolumn{2}{|c|}{ Cycle 7 } \\
\hline EFPD & ppmB \\
\hline 25 & 1150 \\
\hline 50 & 1125 \\
\hline 75 & 1080 \\
\hline 100 & 1020 \\
\hline 125 & 955 \\
\hline 150 & 890 \\
\hline 175 & 820 \\
\hline 200 & 755 \\
\hline 225 & 680 \\
\hline 250 & 600 \\
\hline 275 & 515 \\
\hline 300 & 430 \\
\hline 325 & 350 \\
\hline 350 & 265 \\
\hline 375 & 185 \\
\hline 400 & 105 \\
\hline 425 & 30 \\
\hline 450 & 5 \\
\hline
\end{tabular}

\begin{tabular}{|c|c|}
\hline \multicolumn{2}{|c|}{ Cycle 8 } \\
\hline EFPD & ppmB \\
\hline 25 & 1290 \\
\hline 50 & 1250 \\
\hline 75 & 1200 \\
\hline 100 & 1155 \\
\hline 125 & 1105 \\
\hline 150 & 1055 \\
\hline 175 & 1000 \\
\hline 200 & 940 \\
\hline 225 & 880 \\
\hline 250 & 815 \\
\hline 275 & 745 \\
\hline 300 & 675 \\
\hline 325 & 605 \\
\hline 350 & 535 \\
\hline 375 & 460 \\
\hline 400 & 385 \\
\hline 425 & 305 \\
\hline 450 & 220 \\
\hline 475 & 130 \\
\hline 500 & 40 \\
\hline
\end{tabular}

\begin{tabular}{|c|c|}
\hline \multicolumn{2}{|c|}{ Cycle 9 } \\
\hline EFPD & ppmB \\
\hline 25 & 1600 \\
\hline 50 & 1525 \\
\hline 75 & 1475 \\
\hline 100 & 1420 \\
\hline 125 & 1355 \\
\hline 150 & 1290 \\
\hline 175 & 1230 \\
\hline 200 & 1175 \\
\hline 225 & 1120 \\
\hline 250 & 1065 \\
\hline 275 & 980 \\
\hline 300 & 900 \\
\hline 325 & 825 \\
\hline 350 & 740 \\
\hline 375 & 670 \\
\hline 400 & 600 \\
\hline 425 & 530 \\
\hline 450 & 470 \\
\hline 475 & 410 \\
\hline 500 & 345 \\
\hline 550 & 210 \\
\hline 600 & 20 \\
\hline
\end{tabular}


Table 4-147. All-Rods-Out-Critical-Boron Data for TMl-1 (Cont.)

\begin{tabular}{|c|c|}
\hline \multicolumn{2}{|c|}{ Cycle 10} \\
\hline EFPD & ppmB \\
\hline 25.0 & 1737 \\
\hline 48.0 & 1696 \\
\hline 68.0 & 1656 \\
\hline 103.9 & 1580 \\
\hline 124.8 & 1535 \\
\hline 147.7 & 1486 \\
\hline 169.7 & 1419 \\
\hline 204.6 & 1334 \\
\hline 223.2 & 1296 \\
\hline 244.1 & 1226 \\
\hline 265.1 & 1159 \\
\hline 299.8 & 1068 \\
\hline 333.4 & 964 \\
\hline 354.5 & 906 \\
\hline 382.5 & 826 \\
\hline 402.5 & 770 \\
\hline 430.4 & 688 \\
\hline 465.4 & 587 \\
\hline 479.4 & 539 \\
\hline 500.4 & 480 \\
\hline 556.3 & 309 \\
\hline 605.2 & 167 \\
\hline 652.8 & 35 \\
\hline
\end{tabular}

\begin{tabular}{|c|c|}
\hline \multicolumn{2}{|c|}{ Cycle 11} \\
\hline EFPD & ppmB \\
\hline 26.9 & 1670 \\
\hline 48.0 & 1575 \\
\hline 82.9 & 1508 \\
\hline 103.8 & 1453 \\
\hline 124.8 & 1401 \\
\hline 145.8 & 1358 \\
\hline 180.7 & 1265 \\
\hline 201.7 & 1211 \\
\hline 222.8 & 1154 \\
\hline 243.7 & 1103 \\
\hline 277.9 & 1016 \\
\hline 298.9 & 966 \\
\hline 319.9 & 906 \\
\hline 354.8 & 823 \\
\hline 375.9 & 764 \\
\hline 396.9 & 709 \\
\hline 417.8 & 660 \\
\hline 452.8 & 565 \\
\hline 473.8 & 510 \\
\hline 494.7 & 445 \\
\hline 550.4 & 308 \\
\hline 592.4 & 195 \\
\hline 647.6 & 48 \\
\hline 682.5 & 2 \\
\hline
\end{tabular}

NOTE: ${ }^{a}$ ppmB $=$ Parts per million Boron by mass

\subsection{STATEPOINT CRITICAL CONDITIONS AND MEASUREMENTS}

Measured critical conditions for 16 reactor startups (or SPs) are provided in Table 4-148. The data includes the initial startup of the reactor or beginning-of-life, the beginning-of-cycle (BOC) of each reload core for Cycles 2 through 11 , and 8 reactor restarts during the first 11 cycles of TMI-1. The cycle and SP number, along with the effective-full-power-days (EFPD) during the cycle for which the startup occurred, is provided. The elapsed time (in hours) since the reactor was shutdown (downtime) prior to the startup is also given for each SP. In addition, Table 4-148 provides the measured soluble boron concentration (ppmB), rod bank positions, and temperature of the moderator or coolant in the reactor (for each SP) when criticality was achieved.

Table 4-149 provides shutdown and startup dates for each cycle and SP. The cycle shutdown and startup dates can be used in determining the downtime for fuel assemblies that are out of the reactor for one or more cycles and are then reinserted in a later cycle. 
Table 4-148. Statepoint Data for TMI-1 - Measured Critical Conditions

\begin{tabular}{|c|c|c|c|c|c|c|c|c|}
\hline \multirow[b]{2}{*}{ Cycle(SP } & \multirow[b]{2}{*}{$\begin{array}{c}\text { Burnup } \\
\text { EFPD }\end{array}$} & \multirow[b]{2}{*}{$\begin{array}{c}\text { Downtime } \\
\text { (Hours) }\end{array}$} & \multirow{2}{*}{$\begin{array}{l}\text { Boron } \\
\text { Concent- } \\
\text { ration } \\
\text { ppmB }\end{array}$} & \multicolumn{4}{|c|}{$\begin{array}{l}\text { Rod Bank Position } \\
\text { 100\% Rod Withdrawn (WD) Or The Distance } \\
\text { In cm Above The Bottom Of Active Fuel }\end{array}$} & \multirow{2}{*}{$\begin{array}{c}\text { RCS } \\
\mathrm{T}(\text { Coolant }) \\
\left({ }^{\circ} \mathrm{F}\right) \\
\end{array}$} \\
\hline & & & & Bank 5 & Bank 6 & Bank 7 & $\begin{array}{c}\text { Bank } 8 \\
\text { APSR }\end{array}$ & \\
\hline 1 (SP59) & 0.0 & 0.0 & 1609 & $W D^{a}$ & WD & 274.9 & WD & 532 \\
\hline 5 (SP60) & 0.0 & 58080 & 1178 & WD & WD & 338.3 & 140.0 & 532 \\
\hline 5 (SP61) & 114.4 & 792 & 777 & WD & 324.3 & 62.4 & 103.3 & 532 \\
\hline 6 (SP62) & 0.0 & 3432 & 1453 & WD & $W D$ & 313.8 & 104.3 & 532 \\
\hline 6 (SP63) & 69.1 & 360 & 1194 & WD & 146.2 & 17.0 & 118.2 & 531 \\
\hline 7 (SP64) & 0.0 & 1392 & 1636 & WD & WD & 156.7 & 104.3 & 532 \\
\hline 7 (SP65) & 30.0 & 216 & 1536 & WD & 357.5 & 95.6 & 135.7 & 532.2 \\
\hline 7 (SP66) & 107.8 & 312 & 1380 & WD & 285.9 & 23.9 & 121.7 & 534.5 \\
\hline 8 (SP67) & 0.0 & 1368 & 1813 & WD & WD & 272.3 & 104.3 & 532 \\
\hline 9 (SP68) & 0.0 & 1152 & 2162 & WD & WD & 334.8 & 104.3 & 532 \\
\hline 10 (SP69) & 0.0 & 840 & 2407 & WD & WD & 311.1 & 116.3 & 532 \\
\hline 10 (SP70) & 27.0 & 120 & 2187 & WD & WD & 99.9 & 122.6 & 528.8 \\
\hline 10 (SP71) & 143.0 & 216 & 1983 & WD & 346.1 & 84.1 & 120.8 & 537.4 \\
\hline 10 (SP72) & 209.0 & 192 & 1719 & WD & 145.3 & 16.0 & 120.8 & 534 \\
\hline 11 (SP73) & 0.0 & 816 & 2249 & WD & WD & 229.1 & 120.8 & 532 \\
\hline 11 (SP74) & 615.0 & 168 & 555 & 355.2 & 93.2 & 16.0 & 121.5 & 535.9 \\
\hline
\end{tabular}

NOTE: ${ }^{a}$ WD $=\operatorname{Rod} 100 \%$ Withdrawn

Table 4-149. Statepoint Data for TMI 1 - Shutdown and Startup Dates

\begin{tabular}{|c|c|c|c|}
\hline Cycle(SP) & EFPD & Shutdown Date & Startup Time \\
\hline 1 (SP59) & 0.0 & - & 05-Jun-74 \\
\hline 5 (SP60) & 0.0 & 17-Feb-79 & $03-0 c t-85 @ 1330$ \\
\hline 5 (SP61) & 114.4 & 21-Mar-86 & 23-Apr-86@0410 \\
\hline 6 (SP62) & 0.0 & 31-Oct-86 & 23-Mar-87 @ 1731 \\
\hline 6 (SP63) & 69.1 & 12-Jun-87 & 27-Jun-87 @ 0532 \\
\hline 7 (SP64) & 0.0 & 17-Jun-88 & 14-Aug-88@ 1500 \\
\hline 7 (SP65) & 30.0 & 17-Sep-88 & 26-Sep-88@ 0630 \\
\hline 7 (SP66) & 107.8 & 16-Dec-88 & 29-Dec-88 @ 0155 \\
\hline 8 (SP67) & 0.0 & 05-Jan-90 & 03-Mar-90 @ 0600 \\
\hline 9 (SP68) & 0.0 & 27-Sep-91 & 14-Nov-91@1230 \\
\hline 10 (SP69) & 0.0 & 10-Sep-93 & 15-Oct-93@0852 \\
\hline 10 (SP70) & 27.0 & 14-Nov-93 & 19-Nov-93 @ 0339 \\
\hline 10 (SP71) & 143.0 & 17-Mar-94 & 26-Mar-94 @ 1404 \\
\hline 10 (SP72) & 209.0 & 01-Jun-94 & 09-Jun-94 @ 1115 \\
\hline 11 (SP73) & 0.0 & 08-Sep-95 & 12-Oct-95 @ 1250 \\
\hline 11 (SP74) & 615.0 & 21-Jun-97 & 28-Jun-97@ 1435 \\
\hline
\end{tabular}


INTENTIONALLY LEFT BLANK 


\section{CONCLUSIONS}

The information reported herein is acceptable for quality affecting activities and for use in evaluations concerning procurement, construction, or fabrication. The components of the engineered barrier system have been identified as items important to radiological safety and waste isolation in a number of classification analyses (e.g., CRWMS M\&O 1999). 
INTENTIONALLY LEFT BLANK 


\section{REFERENCES}

\subsection{DOCUMENTS CITED}

BSC (Bechtel SAIC Company) 2001. Technical Work Plan for: Waste Package Design Description for LA. TWP-EBS-MD-000004 REV 01. Las Vegas, Nevada: Bechtel SAIC Company. ACC: MOL.20010702.0152.

CRWMS (Civilian Radioactive Waste Management System) M\&O (Management and Operating Contractor) 1999. Classification of the MGR Uncanistered Spent Nuclear Fuel Disposal Container System. ANL-UDC-SE-000001 REV 00. Las Vegas, Nevada: CRWMS M\&O. ACC: MOL.19990928.0216.

DOE (U.S. Department of Energy) 2000. Quality Assurance Requirements and Description. DOE/RW-0333P, Rev. 10. Washington, D.C.: U.S. Department of Energy, Office of Civilian Radioactive Waste Management. ACC: MOL.20000427.0422.

Framatome Cogema Fuels 2000. Quality Assurance Program for Framatome Cogema Fuels. 561177617-06. Lynchburg, Virginia: Framatome Cogema Fuels. TIC: 249160.

Mellington, S.P. 1999. "Office of Project Execution (OPE) Approval of LV.WP.TWD.08/99-131 as Accepted Data." Letter from S.P. Mellington (DOE/YMSCO) to T.W. Doering (CRWMS M\&O), September 29, 1999 ACC: MOL.19990927.0472.

YMP (Yucca Mountain Site Characterization Project) 2000. Disposal Criticality Analysis Methodology Topical Report. YMP/TR-004Q, Rev. 01. Las Vegas, Nevada: Yucca Mountain Site Characterization Office. ACC: MOL.20001214.0001.

\subsection{CODES, STANDARDS, REGULATIONS, AND PROCEDURES}

ASTM A533/A 533M-93 (Reapproved 1999). 1993. Standard Specification for Pressure Vessel Plates, Alloy Steel, Quenched and Tempered, Manganese-Molybdenum and ManganeseMolybdenum-Nickel. West Conshohocken, Pennsylvania: American Society for Testing and Materials. TIC: 249680.

\subsection{SOURCE DATA, LISTED BY DATA TRACKING NUMBER}

MO0106SPADEP01.007. TMI-1 Nemo Depletion and State-Points. Submittal date: 06/21/01. 
INTENTIONALLY LEFT BLANK 


\section{APPENDIX A}

FUEL ROD DATA FOR ASSEMBLIES NJ05YU \& NJ070G 
INTENTIONALLY LEFT BLANK 


\section{APPENDIX A - FUEL ROD DATA FOR ASSEMBLIES NJ05YU \& NJ070G}

At the end of cycle 10, several fuel rods were removed from once burned and twice burned fuel assemblies. A chemical analysis was performed on a few of the rods that were removed. This section provides information on six of these rods, two from assembly NJ05YU, and four from assembly NJ070G.

Assembly NJ05YU was a fresh assembly at the beginning of cycle 9 and was inserted in the core during both cycles 9 and 10 . The assembly was removed at the end of cycle 10 . The data for two of the fuel rods that were removed are presented in this section.

Assembly NJ070G was a fresh assembly at the beginning of cycle 10 and was inserted in the core during both cycles 10 and 11 . At the end of cycle 10, some of the fuel rods were removed and replaced with stainless steel rods. The assembly was re-inserted into the core for cycle 11 . The data for four of the fuel rods that were removed at the end of cycle 10 are presented in this section.

The locations of the assemblies in the core as a function of cycle are provided in Figures A-1 and A-2.

The rods that were removed from assembly NJ05YU were located in position D5 and H6 (Figure A-3). The rods that were removed from assembly NJ070G were located in position O1, O11, O12, and 013 (Figure A-4).

The fuel assembly and selected rod Uranium loading are provided in Table A-1. Table A-2 provides the geometric, material, and identification information for the fuel rods. Table A-3 provides the fuel pellet Uranium isotopic weight percent. The rod burnup history (GWd/MTU) is provided in Tables A-4 and A-5.

Although assembly NJ070G contained Gadolinia fuel rods, none of the rods presented in this section contained Gadolinia. 
$\longleftarrow$ NORTH

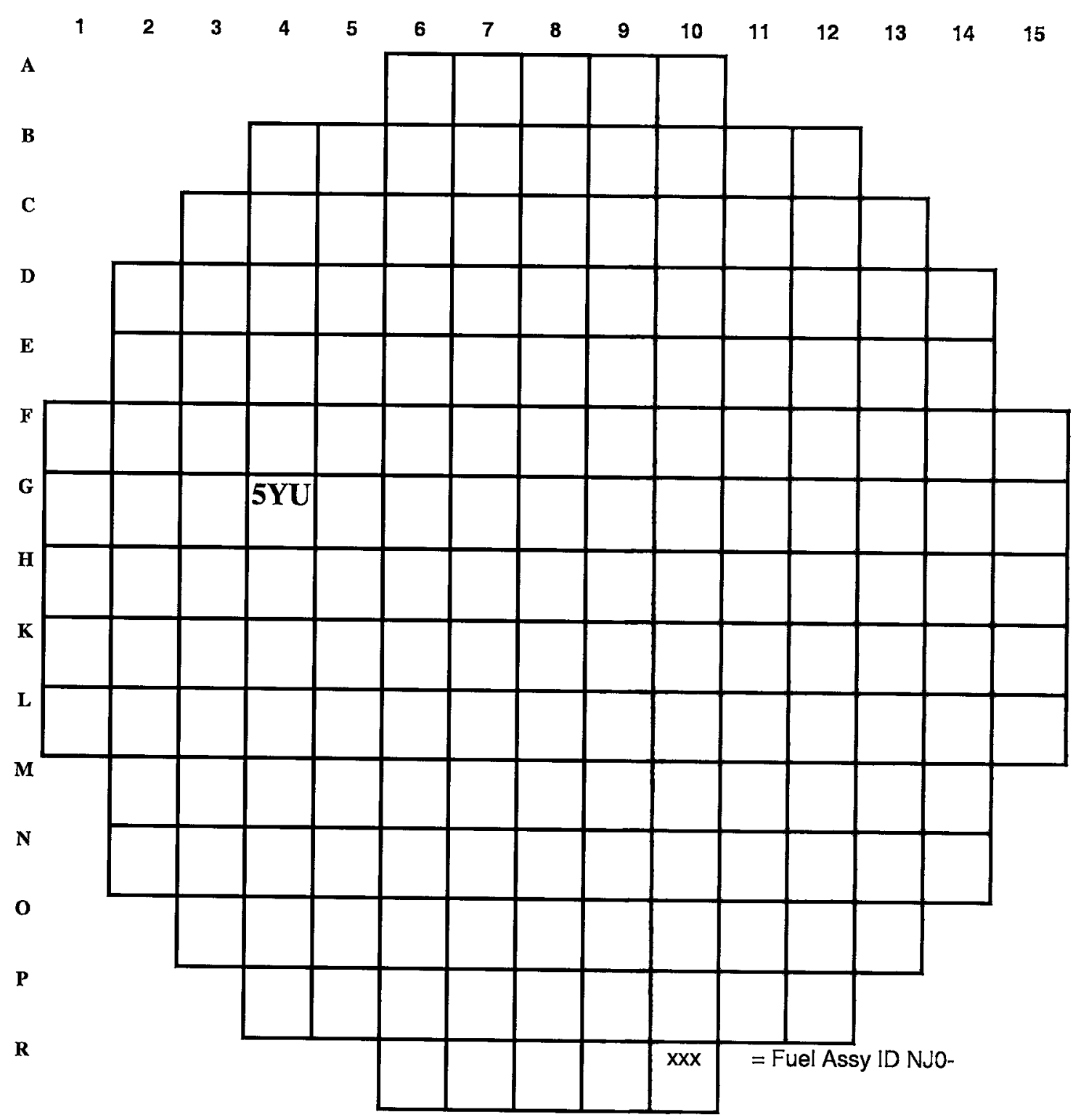

Figure A-1. TMI-1 Cycle 9 Assembly NJ05YU Location 


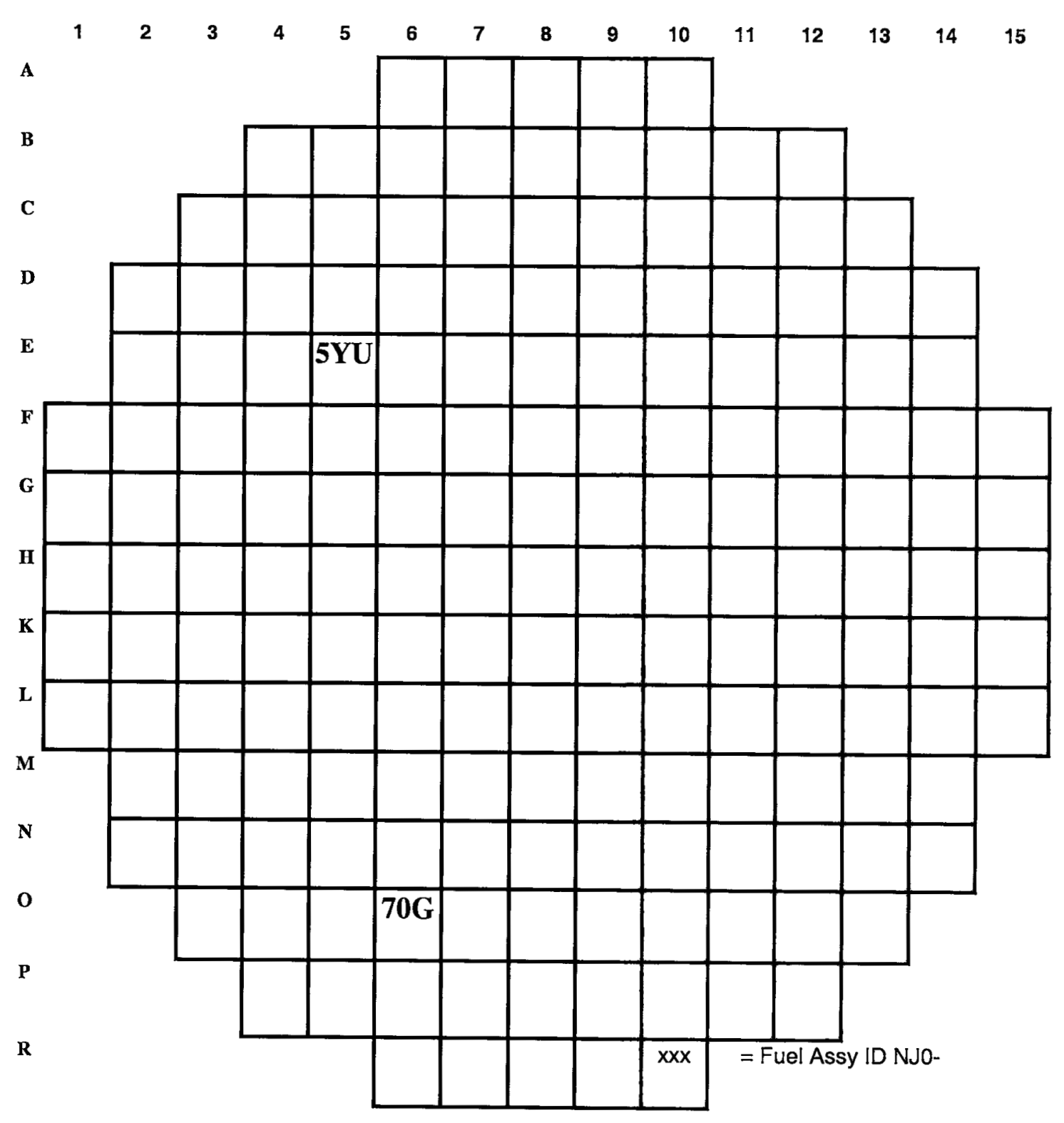

Figure A-2. TMI-1 Cycle 10 Assembly NJ05YU and NJ070G Location 


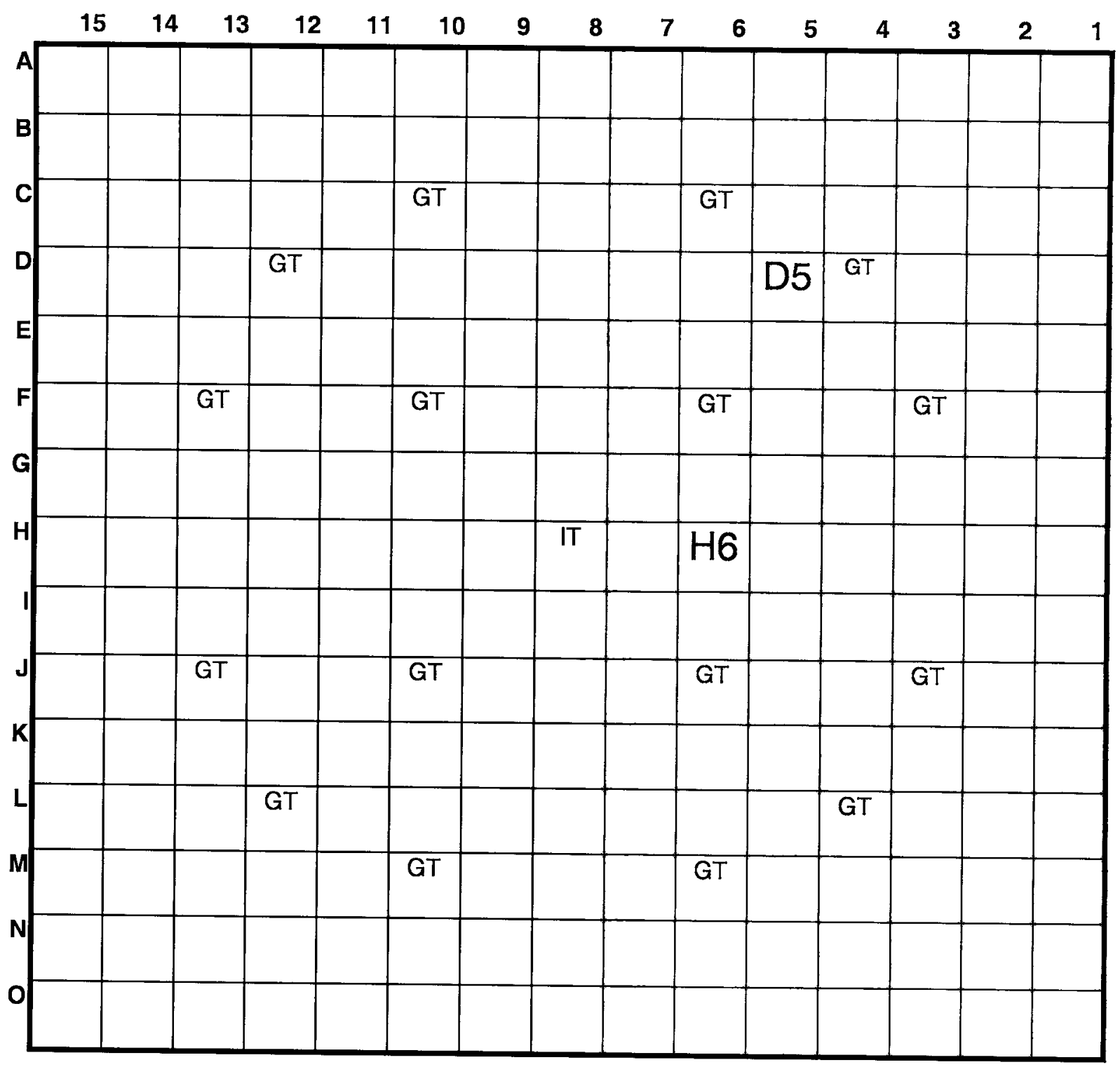

Figure A-3. TMI-1 Fuel Rod Locations in Assembly NJ05YU 


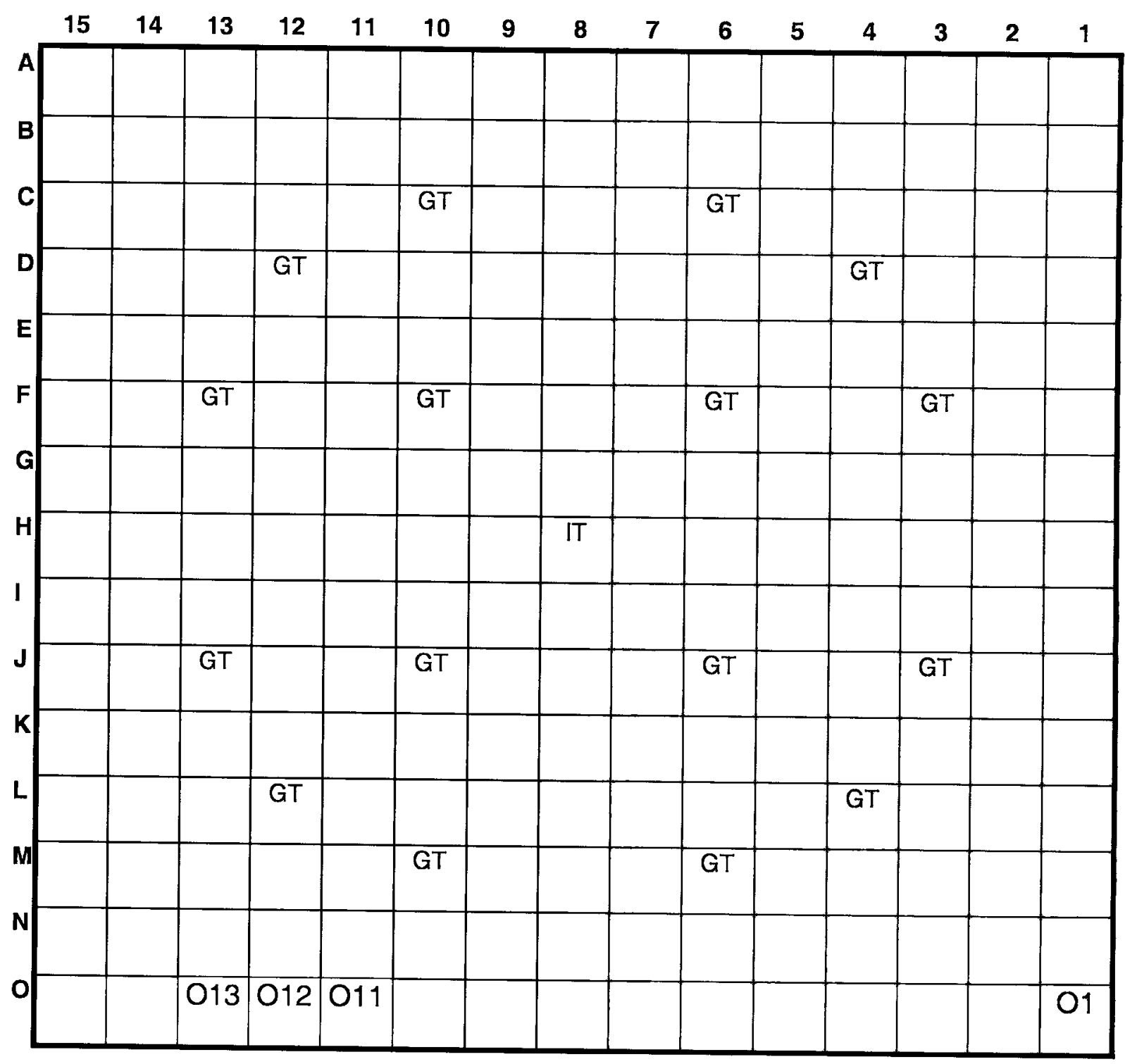

Figure A-4. TMI-1 Fuel Rod Locations in Assembly NJ070G 
Table A-1. Assembly \& Fuel Rod Uranium Loading (Assemblies NJ05YU \& NJ070G)

\begin{tabular}{|r|c|c|c|c|c|}
\hline Fuel Assembly/Rod & $\begin{array}{c}\text { Position } \\
\text { In FA }\end{array}$ & $\begin{array}{c}\text { Initial UO } \\
\text { (gm) }\end{array}$ & $\begin{array}{c}\text { Uranium } \\
(\mathbf{g m})\end{array}$ & $\begin{array}{c}{ }^{235} \mathrm{U} \\
(\mathbf{g m})\end{array}$ & $\begin{array}{c}\text { Enrichment } \\
\text { wt \% }{ }^{235} \mathbf{U}\end{array}$ \\
\hline FA NJ05YU & & & $463,167.8$ & $18,587.09$ & 4.00 \\
\hline Fuel Rod I05381 & $\mathrm{D} 5$ & 2,531 & $2,229.8$ & 89.48 & 4.00 \\
\hline Fuel Rod I05243 & $\mathrm{H} 6$ & 2,528 & $2,227.2$ & 89.38 & 4.00 \\
\hline & & & & & \\
\hline FA NJ070G & & & $462,975.3$ & $21,487.29$ & $4.65 / 4.19 \mathrm{Gd}^{\mathrm{a}}$ \\
\hline Fuel Rod C53923 & $\mathrm{O}$ & 2,531 & $2,230.8$ & 103.89 & 4.65 \\
\hline Fuel Rod C53927 & $\mathrm{O} 11$ & 2,522 & $2,222.9$ & 103.52 & 4.65 \\
\hline Fuel Rod C53928 & $\mathrm{O} 13$ & 2,528 & $2,228.2$ & 103.77 & 4.65 \\
\hline
\end{tabular}

NOTE: ${ }^{a}$ Four of the fuel rods contain $2.0 \mathrm{wt} \% \mathrm{Gd}$ and $4.19 \mathrm{wt} \%$ U235

Table A-2. Fuel Rod Geometric \& Material Data (Assemblies NJ05YU \& NJ070G)

\begin{tabular}{|c|c|c|}
\hline \multicolumn{3}{|l|}{ Fuel Assembly } \\
\hline Fuel Assembly Identification & NJ05YU & NJO70G \\
\hline Fuel Assembly Type & MK-B8 & MK-B9Gd \\
\hline Fuel Batch & 11 & 12 \\
\hline \multicolumn{3}{|l|}{ Fuel Rod } \\
\hline Clad OD (cm) & 1.0922 & 1.0922 \\
\hline Clad ID $(\mathrm{cm})$ & 0.9576 & 0.9576 \\
\hline Fuel Rod Length $(\mathrm{cm})$ & 391.5664 & 391.5664 \\
\hline Fill Gas & Helium & Helium \\
\hline \multicolumn{3}{|l|}{ Fuel Pellet } \\
\hline Diameter $(\mathrm{cm})$ & 0.9362 & 0.9398 \\
\hline Active Fuel Length (cm) & 360.172 & 357.124 \\
\hline $\mathrm{LAF}^{\mathrm{b}}$ to Fuel Rod Bottom (cm) & 13.0810 & 13.0302 \\
\hline${ }^{235} \mathrm{U}$ wt $\%$ & 4.00 & $4.65 / 4.19(\mathrm{Gd})^{\mathrm{a}}$ \\
\hline \% Theoretical Density & 95 & 95 \\
\hline \multicolumn{3}{|l|}{ Volumes } \\
\hline Upper Cap $\left(\mathrm{cm}^{3}\right)$ & 0.7194 & 0.7194 \\
\hline Upper Spring $\left(\mathrm{cm}^{3}\right)$ & 2.1631 & 2.5236 \\
\hline Upper Plenum Gas $\left(\mathrm{cm}^{3}\right)$ & 10.3320 & 12.1756 \\
\hline Lower Plenum Gas $\left(\mathrm{cm}^{3}\right)$ & 3.1955 & 3.1955 \\
\hline Lower Spring $\left(\mathrm{cm}^{3}\right)$ & 0.7915 & 0.7915 \\
\hline Lower Cap $\left(\mathrm{cm}^{3}\right)$ & 6.4778 & 6.4778 \\
\hline \multicolumn{3}{|l|}{ Materials } \\
\hline Fuel Rod Clad & Zircaloy & Zircaloy \\
\hline Fuel Rod Upper Cap & Zircaloy-4 & Zircaloy-4 \\
\hline Fuel Rod Upper Spring & 302SS & $3025 S$ \\
\hline Fuel Rod Lower Spring & A286 Alloy & A286 Alloy \\
\hline Fuel Rod Lower Cap & Zircaloy-4 & Zircaloy -4 \\
\hline
\end{tabular}

NOTES: ${ }^{a}$ Four of the fuel rods contain $2.0 \mathrm{wt} \%$ Gd and $4.19 \mathrm{wt} \%$ U235

${ }^{b}$ Lower Active Fuel 
Table A-3. Fuel Pellet Uranium Isotopic (wt \%)

\begin{tabular}{|c|c|c|c|c|}
\hline Identification No. & ${ }^{234} U$ & ${ }^{235} \mathbf{U}$ & ${ }^{236} \mathrm{U}$ & ${ }^{237} \mathrm{U}$ \\
\hline FA NJ05YU & 0.040 & 4.013 & $<0.001$ & 95.947 \\
\hline FA NJ070G & 0.045 & 4.657 & $<0.001$ & 95.298 \\
\hline
\end{tabular}

Table A-4. Fuel Rod Data for Assembly NJ05YU

Cycle 9, EFPD: 0.0, Boron (ppmB): 1670

\begin{tabular}{|c|c|c|c|c|c|c|c|}
\hline & \multicolumn{2}{|c|}{ Elevation } & \multicolumn{3}{|c|}{} & Fuel Rod D5 & Fuel Rod H6 \\
\hline Axial & Node Height & Bottom & Top & Spec. Vol. & T-Fuel & Burnup & Burnup \\
\hline Node & $(\mathbf{c m})$ & $(\mathbf{c m})$ & $(\mathbf{c m})$ & $\left(\mathrm{ft}^{3} / \mathrm{lbm}\right)$ & $\left({ }^{\mathrm{F}}\right)$ & $(\mathrm{GWd} / \mathrm{MTU})$ & $(\mathrm{GWd} / \mathrm{MTU})$ \\
\hline 1 (Top) & 20.066 & 340.1060 & 360.1720 & 0.0238 & 1041.0 & 0.000 & 0.000 \\
\hline 2 & 20.0025 & 320.1035 & 340.1060 & 0.0237 & 1243.4 & 0.000 & 0.000 \\
\hline 3 & 20.0025 & 300.1010 & 320.1035 & 0.0236 & 1390.6 & 0.000 & 0.000 \\
\hline 4 & 20.0025 & 280.0985 & 300.1010 & 0.0234 & 1481.0 & 0.000 & 0.000 \\
\hline 5 & 20.0025 & 260.0960 & 280.0985 & 0.0233 & 1526.5 & 0.000 & 0.000 \\
\hline 6 & 20.0025 & 240.0935 & 260.0960 & 0.0231 & 1550.6 & 0.000 & 0.000 \\
\hline 7 & 20.0025 & 220.0910 & 240.0935 & 0.0230 & 1564.2 & 0.000 & 0.000 \\
\hline 8 & 20.0025 & 200.0885 & 220.0910 & 0.0228 & 1569.6 & 0.000 & 0.000 \\
\hline 9 & 20.0025 & 180.0860 & 200.0885 & 0.0227 & 1568.3 & 0.000 & 0.000 \\
\hline 10 & 20.0025 & 160.0835 & 180.0860 & 0.0225 & 1561.3 & 0.000 & 0.000 \\
\hline 11 & 20.0025 & 140.0810 & 160.0835 & 0.0224 & 1549.6 & 0.000 & 0.000 \\
\hline 12 & 20.0025 & 120.0785 & 140.0810 & 0.0223 & 1534.7 & 0.000 & 0.000 \\
\hline 13 & 20.0025 & 100.0760 & 120.0785 & 0.0221 & 1516.2 & 0.000 & 0.000 \\
\hline 14 & 20.0025 & 80.0735 & 100.0760 & 0.0220 & 1486.5 & 0.000 & 0.000 \\
\hline 15 & 20.0025 & 60.0710 & 80.0735 & 0.0219 & 1436.8 & 0.000 & 0.000 \\
\hline 16 & 20.0025 & 40.0685 & 60.0710 & 0.0218 & 1359.3 & 0.000 & 0.000 \\
\hline 17 & 20.0025 & 20.0660 & 40.0685 & 0.0217 & 1236.4 & 0.000 & 0.000 \\
\hline 18 (Bottom) & 20.066 & 0.0000 & 20.0660 & 0.0216 & 1042.8 & 0.000 & 0.000 \\
\hline
\end{tabular}


TABLE A-4. Fuel Rod Data for Assembly NJ05YU (Cont.)

Cycle 9, EFPD: 74.2, Boron (ppmB): 1481

\begin{tabular}{|c|c|c|c|c|c|c|c|}
\hline & & Ele & ion & & & Fuel Rod D5 & Fuel Rod H6 \\
\hline Axial & Node Height & Bottom & Top & Spec. Vol. & T-Fuel & Burnup & Burnup \\
\hline Node & (cm) & $(\mathrm{cm})$ & (cm) & $\left(\mathrm{ft}^{3} / \mathrm{lbm}\right)$ & $\left({ }^{\circ} \mathrm{F}\right)$ & (GWd/MTU) & (GWd/MTU) \\
\hline 1 (Top) & 20.066 & 340.1060 & 360.1720 & 0.0238 & 1068.7 & 1.600 & 1.504 \\
\hline 2 & 20.0025 & 320.1035 & 340.1060 & 0.0237 & 1269.3 & 2.183 & 2.283 \\
\hline 3 & 20.0025 & 300.1010 & 320.1035 & 0.0236 & 1394.5 & 2.679 & 2.839 \\
\hline 4 & 20.0025 & 280.0985 & 300.1010 & 0.0235 & 1458.4 & 2.980 & 3.179 \\
\hline 5 & 20.0025 & 260.0960 & 280.0985 & 0.0233 & 1482.4 & 3.145 & 3.365 \\
\hline 6 & 20.0025 & 240.0935 & 260.0960 & 0.0231 & 1492.7 & 3.246 & 3.476 \\
\hline 7 & 20.0025 & 220.0910 & 240.0935 & 0.0230 & 1496.8 & 3.309 & 3.543 \\
\hline 8 & 20.0025 & 200.0885 & 220.0910 & 0.0229 & 1496.9 & 3.342 & 3.578 \\
\hline 9 & 20.0025 & 180.0860 & 200.0885 & 0.0227 & 1494.4 & 3.352 & 3.588 \\
\hline 10 & 20.0025 & 160.0835 & 180.0860 & 0.0226 & 1490.3 & 3.343 & 3.577 \\
\hline 11 & 20.0025 & 140.0810 & 160.0835 & 0.0224 & 1485.1 & 3.319 & 3.551 \\
\hline 12 & 20.0025 & 120.0785 & 140.0810 & 0.0223 & 1479.5 & 3.292 & 3.513 \\
\hline 13 & 20.0025 & 100.0760 & 120.0785 & 0.0222 & 1471.8 & 3.257 & 3.456 \\
\hline 14 & 20.0025 & 80.0735 & 100.0760 & 0.0220 & 1456.1 & 3.165 & 3.353 \\
\hline 15 & 20.0025 & 60.0710 & 80.0735 & 0.0219 & 1428.0 & 3.004 & 3.184 \\
\hline 16 & 20.0025 & 40.0685 & 60.0710 & 0.0218 & 1369.2 & 2.742 & 2.909 \\
\hline 17 & 20.0025 & 20.0660 & 40.0685 & 0.0217 & 1259.4 & 2.306 & 2.450 \\
\hline 18 (Bottom) & 20.066 & 0.0000 & 20.0660 & 0.0216 & 1066.1 & 1.826 & 1.693 \\
\hline
\end{tabular}

Cycle 9, EFPD: 144.1, Boron (ppmB): 1342

\begin{tabular}{|c|c|c|c|c|c|c|c|}
\hline & \multicolumn{2}{|c|}{ Elevation } & \multicolumn{2}{|c|}{} & Fuel Rod D5 & Fuel Rod H6 \\
\hline Axial & Node Height & Bottom & Top & Spec. Vol. & T-Fuel & Burnup & Burnup \\
\hline Node & $\mathbf{( c m )}$ & $\mathbf{( c m )}$ & $\mathbf{( c m )}$ & $\mathbf{f t}^{3}$ / $\left./ \mathbf{b m}\right)$ & $(\mathbf{F})$ & $(\mathbf{G W d / M T U )}$ & (GWd/MTU) \\
\hline 1 (Top) & 20.066 & $\mathbf{3 4 0 . 1 0 6 0}$ & 360.1720 & 0.0238 & 1080.7 & 3.216 & 3.035 \\
\hline 2 & 20.0025 & 320.1035 & 340.1060 & 0.0237 & 1271.0 & 4.423 & 4.594 \\
\hline 3 & 20.0025 & 300.1010 & 320.1035 & 0.0236 & 1376.9 & 5.379 & 5.639 \\
\hline 4 & 20.0025 & 280.0985 & 300.1010 & 0.0235 & 1421.6 & 5.922 & 6.235 \\
\hline 5 & 20.0025 & 260.0960 & 280.0985 & 0.0233 & 1432.4 & 6.195 & 6.540 \\
\hline 6 & 20.0025 & 240.0935 & 260.0960 & 0.0232 & 1434.2 & 6.352 & 6.709 \\
\hline 7 & 20.0025 & 220.0910 & 240.0935 & 0.0230 & 1432.7 & 6.445 & 6.805 \\
\hline 8 & 20.0025 & 200.0885 & 220.0910 & 0.0229 & 1429.9 & 6.492 & 6.853 \\
\hline 9 & 20.0025 & 180.0860 & 200.0885 & 0.0227 & 1426.9 & 6.505 & 6.866 \\
\hline 10 & 20.0025 & 160.0835 & 180.0860 & 0.0226 & 1424.1 & 6.492 & 6.852 \\
\hline 11 & 20.0025 & 140.0810 & 160.0835 & 0.0224 & 1422.0 & 6.464 & 6.819 \\
\hline 12 & 20.0025 & 120.0785 & 140.0810 & 0.0223 & 1421.1 & 6.437 & 6.774 \\
\hline 13 & 20.0025 & 100.0760 & 120.0785 & 0.0222 & 1420.5 & 6.402 & 6.701 \\
\hline 14 & 20.0025 & 80.0735 & 100.0760 & 0.0221 & 1414.8 & 6.264 & 6.546 \\
\hline 15 & 20.0025 & 60.0710 & 80.0735 & 0.0219 & 1400.0 & 5.993 & 6.269 \\
\hline 16 & 20.0025 & 40.0685 & 60.0710 & 0.0218 & 1362.0 & 5.515 & 5.780 \\
\hline 17 & 20.0025 & 20.0660 & 40.0685 & 0.0217 & 1268.2 & 4.664 & 4.907 \\
\hline 18 (Bottom) & 20.066 & 0.0000 & 20.0660 & 0.0216 & 1081.8 & 3.662 & 3.399 \\
\hline
\end{tabular}


TABLE A-4. Fuel Rod Data for Assembly NJ05YU (Cont.)

Cycle 9, EFPD: 214, Boron (ppmB): 1175

\begin{tabular}{|c|c|c|c|c|c|c|c|}
\hline & & \multicolumn{2}{|c|}{ Elevation } & & & Fuel Rod D5 & Fuel Rod $\mathrm{H} 6$ \\
\hline Axial & Node Height & Bottom & Top & Spec. Vol. & T-Fuel & Burnup & Burnup \\
\hline Node & (cm) & $(\mathrm{cm})$ & (cm) & $\left(\mathrm{ft}^{3} / \mathrm{lbm}\right)$ & ( $\mathrm{F})$ & (GWd/MTU) & (GWd/MTU) \\
\hline i (Top) & 20.066 & 340.1060 & 360.1720 & 0.0239 & 1072.1 & 4.880 & 4.614 \\
\hline 2 & 20.0025 & 320.1035 & 340.1060 & 0.0238 & 1244.7 & 6.764 & 6.963 \\
\hline 3 & 20.0025 & 300.1010 & 320.1035 & 0.0236 & 1337.4 & 8.173 & 8.474 \\
\hline 4 & 20.0025 & 280.0985 & 300.1010 & 0.0235 & 1372.5 & 8.934 & 9.293 \\
\hline 5 & 20.0025 & 260.0960 & 280.0985 & 0.0233 & 1377.2 & 9.290 & 9.687 \\
\hline 6 & 20.0025 & 240.0935 & 260.0960 & 0.0232 & 1375.3 & 9.483 & 9.892 \\
\hline 7 & 20.0025 & 220.0910 & 240.0935 & 0.0230 & 1371.9 & 9.593 & 10.004 \\
\hline 8 & 20.0025 & 200.0885 & 220.0910 & 0.0229 & 1368.6 & 9.647 & 10.059 \\
\hline 9 & 20.0025 & 180.0860 & 200.0885 & 0.0227 & 1366.0 & 9.665 & 10.077 \\
\hline 10 & 20.0025 & 160.0835 & 180.0860 & 0.0226 & 1364.5 & 9.659 & 10.069 \\
\hline 11 & 20.0025 & 140.0810 & 160.0835 & 0.0225 & 1364.4 & 9.640 & 10.045 \\
\hline$\overline{12}$ & 20.0025 & 120.0785 & 140.0810 & 0.0223 & 1366.2 & 9.635 & 10.014 \\
\hline 13 & 20.0025 & 100.0760 & 120.0785 & 0.0222 & 1369.4 & 9.630 & 9.953 \\
\hline 14 & 20.0025 & 80.0735 & 100.0760 & 0.0221 & 1370.0 & 9.481 & 9.786 \\
\hline 15 & 20.0025 & 60.0710 & 80.0735 & 0.0220 & 1364.6 & 9.142 & 9.446 \\
\hline 16 & 20.0025 & 40.0685 & 60.0710 & 0.0218 & 1343.5 & 8.483 & 8.786 \\
\hline 17 & 20.0025 & 20.0660 & 40.0685 & 0.0217 & 1266.1 & 7.223 & 7.521 \\
\hline 18 (Bottom) & 20.066 & 0.0000 & 20.0660 & 0.0216 & 1088.3 & 5.616 & 5.225 \\
\hline
\end{tabular}

Cycle 9, EFPD: 283.9, Boron (ppmB): 990

\begin{tabular}{|c|c|c|c|c|c|c|c|}
\hline & & \multicolumn{2}{|c|}{ Elevation } & \multicolumn{2}{|c|}{} & Fuel Rod D5 & Fuel Rod H6 \\
\hline Axial & Node Height & Bottom & Top & Spec. Vol. & T-Fuel & Burnup & Burnup \\
\hline Node & $\mathbf{( c m )}$ & $\mathbf{( c m )}$ & $\mathbf{( c m )}$ & $\left(\mathbf{f t}^{3} / / \mathbf{b m}\right)$ & $(\mathrm{F})$ & $($ GWd/MTU) & (GWd/MTU) \\
\hline 1 (Top) & 20.066 & 340.1060 & 360.1720 & 0.0239 & 1087.3 & 6.610 & 6.259 \\
\hline 2 & 20.0025 & 320.1035 & 340.1060 & 0.0237 & 1255.2 & 9.225 & 9.411 \\
\hline 3 & 20.0025 & 300.1010 & 320.1035 & 0.0236 & 1327.6 & 11.073 & 11.359 \\
\hline 4 & 20.0025 & 280.0985 & 300.1010 & 0.0234 & 1341.2 & 12.016 & 12.363 \\
\hline 5 & 20.0025 & 260.0960 & 280.0985 & 0.0233 & 1335.8 & 12.424 & 12.813 \\
\hline 6 & 20.0025 & 240.0935 & 260.0960 & 0.0232 & 1327.5 & 12.628 & 13.030 \\
\hline 7 & 20.0025 & 220.0910 & 240.0935 & 0.0230 & 1320.1 & 12.740 & 13.142 \\
\hline 8 & 20.0025 & 200.0885 & 220.0910 & 0.0229 & 1314.4 & 12.794 & 13.197 \\
\hline 9 & 20.0025 & 180.0860 & 200.0885 & 0.0227 & 1310.8 & 12.816 & 13.218 \\
\hline 10 & 20.0025 & 160.0835 & 180.0860 & 0.0226 & 1309.1 & 12.818 & 13.219 \\
\hline 11 & 20.0025 & 140.0810 & 160.0835 & 0.0225 & 1309.7 & 12.818 & 13.213 \\
\hline 12 & 20.0025 & 120.0785 & 140.0810 & 0.0223 & 1312.9 & 12.849 & 13.209 \\
\hline 13 & 20.0025 & 100.0760 & 120.0785 & 0.0222 & 1318.3 & 12.893 & 13.178 \\
\hline 14 & 20.0025 & 80.0735 & 100.0760 & 0.0221 & 1322.8 & 12.762 & 13.025 \\
\hline 15 & 20.0025 & 60.0710 & 80.0735 & 0.0220 & 1323.4 & 12.391 & 12.660 \\
\hline 16 & 20.0025 & 40.0685 & 60.0710 & 0.0218 & 1310.8 & 11.592 & 11.873 \\
\hline 17 & 20.0025 & 20.0660 & 40.0685 & 0.0217 & 1247.1 & 9.938 & 10.245 \\
\hline 18 (Bottom) & 20.066 & 0.0000 & 20.0660 & 0.0216 & 1083.1 & 7.662 & 7.141 \\
\hline
\end{tabular}


TABLE A-4. Fuel Rod Data for Assembly NJ05YU (Cont.)

Cycle 9, EFPD: 349.7, Boron (ppmB): 772

\begin{tabular}{|c|c|c|c|c|c|c|c|}
\hline & & \multicolumn{2}{|c|}{ Elevation } & & & Fuel Rod D5 & Fuel Rod H6 \\
\hline Axial & Node Height & Bottom & Top & Spec. Vol. & T-Fuel & Burnup & Burnup \\
\hline Node & $(\mathrm{cm})$ & (cm) & $(\mathrm{cm})$ & $\left(\mathrm{ft}^{3} / \mathrm{lbm}\right)$ & (F) & (GWd/MTU) & (GWd/MTU) \\
\hline 1 (Top) & 20.066 & 340.1060 & 360.1720 & 0.0238 & 1073.0 & 8.320 & 7.884 \\
\hline 2 & 20.0025 & 320.1035 & 340.1060 & 0.0237 & 1228.0 & 11.666 & 11.805 \\
\hline 3 & 20.0025 & 300.1010 & 320.1035 & 0.0236 & 1290.3 & 13.892 & 14.123 \\
\hline 4 & 20.0025 & 280.0985 & 300.1010 & 0.0234 & 1299.0 & 14.959 & 15.249 \\
\hline 5 & 20.0025 & 260.0960 & 280.0985 & 0.0233 & 1291.1 & 15.376 & 15.715 \\
\hline 6 & 20.0025 & 240.0935 & 260.0960 & 0.0231 & 1281.8 & 15.568 & 15.922 \\
\hline 7 & 20.0025 & 220.0910 & 240.0935 & 0.0230 & 1274.2 & 15.668 & 16.021 \\
\hline 8 & 20.0025 & 200.0885 & 220.0910 & 0.0229 & 1269.1 & 15.716 & 16.070 \\
\hline 9 & 20.0025 & 180.0860 & 200.0885 & 0.0227 & 1266.3 & 15.739 & 16.092 \\
\hline 10 & 20.0025 & 160.0835 & 180.0860 & 0.0226 & 1265.8 & 15.753 & 16.104 \\
\hline 11 & 20.0025 & 140.0810 & 160.0835 & 0.0225 & 1267.2 & 15.775 & 16.119 \\
\hline 12 & 20.0025 & 120.0785 & 140.0810 & 0.0223 & 1271.2 & 15.847 & 16.147 \\
\hline 13 & 20.0025 & 100.0760 & 120.0785 & 0.0222 & 1278.2 & 15.953 & 16.158 \\
\hline 14 & 20.0025 & 80.0735 & 100.0760 & 0.0221 & 1286.1 & 15.865 & 16.043 \\
\hline 15 & 20.0025 & 60.0710 & 80.0735 & 0.0220 & 1292.8 & 15.502 & 15.690 \\
\hline 16 & 20.0025 & 40.0685 & 60.0710 & 0.0218 & 1289.8 & 14.619 & 14.831 \\
\hline 17 & 20.0025 & 20.0660 & 40.0685 & 0.0217 & 1245.1 & 12.632 & 12.900 \\
\hline 18 (Bottom) & 20.066 & 0.0000 & 20.0660 & 0.0216 & 1085.4 & 9.678 & 9.027 \\
\hline
\end{tabular}

Cycle 9, EFPD: 425, Boron (ppmB): 545

\begin{tabular}{|c|c|c|c|c|c|c|c|}
\hline & & \multicolumn{2}{|c|}{ Elevation } & & & Fuel Rod D5 & Fuel Rod H6 \\
\hline Axial & Node Height & Bottom & Top & Spec. Vol. & T-Fuel & Burnup & Burnup \\
\hline Node & $(\mathbf{c m})$ & $\mathbf{( c m )}$ & $\mathbf{( c m )}$ & $\left(\mathbf{f t}^{3} / \mathbf{h b m}\right)$ & $(\mathbf{F})$ & $($ GWd/MTU) & (GWd/MTU) \\
\hline 1 (Top) & 20.066 & 340.1060 & 360.1720 & 0.0238 & 1069.8 & 10.325 & 9.788 \\
\hline 2 & 20.0025 & 320.1035 & 340.1060 & 0.0237 & 1217.3 & 14.528 & 14.573 \\
\hline 3 & 20.0025 & 300.1010 & 320.1035 & 0.0236 & 1259.7 & 17.142 & 17.275 \\
\hline 4 & 20.0025 & 280.0985 & 300.1010 & 0.0234 & 1256.6 & 18.311 & 18.506 \\
\hline 5 & 20.0025 & 260.0960 & 280.0985 & 0.0233 & 1243.2 & 18.715 & 18.971 \\
\hline 6 & 20.0025 & 240.0935 & 260.0960 & 0.0231 & 1231.2 & 18.881 & 19.155 \\
\hline 7 & 20.0025 & 220.0910 & 240.0935 & 0.0230 & 1222.6 & 18.963 & 19.237 \\
\hline 8 & 20.0025 & 200.0885 & 220.0910 & 0.0228 & 1216.9 & 19.004 & 19.278 \\
\hline 9 & 20.0025 & 180.0860 & 200.0885 & 0.0227 & 1213.6 & 19.031 & 19.305 \\
\hline 10 & 20.0025 & 160.0835 & 180.0860 & 0.0226 & 1212.2 & 19.059 & 19.331 \\
\hline 11 & 20.0025 & 140.0810 & 160.0835 & 0.0225 & 1212.7 & 19.109 & 19.370 \\
\hline 12 & 20.0025 & 120.0785 & 140.0810 & 0.0223 & 1216.1 & 19.232 & 19.439 \\
\hline 13 & 20.0025 & 100.0760 & 120.0785 & 0.0222 & 1222.6 & 19.417 & 19.505 \\
\hline 14 & 20.0025 & 80.0735 & 100.0760 & 0.0221 & 1231.2 & 19.396 & 19.448 \\
\hline 15 & 20.0025 & 60.0710 & 80.0735 & 0.0220 & 1241.7 & 19.077 & 19.139 \\
\hline 16 & 20.0025 & 40.0685 & 60.0710 & 0.0219 & 1247.7 & 18.151 & 18.246 \\
\hline 17 & 20.0025 & 20.0660 & 40.0685 & 0.0217 & 1221.3 & 15.840 & 16.023 \\
\hline 18 (Bottom) & 20.066 & 0.0000 & 20.0660 & 0.0216 & 1080.0 & 12.080 & 11.278 \\
\hline
\end{tabular}


TABLE A-4. Fuel Rod Data for Assembly NJ05YU (Cont.)

Cycle 9, EFPD: 483.9, Boron (ppmB): 352

\begin{tabular}{|c|c|c|c|c|c|c|c|}
\hline & & Ele & tion & & & Fuel Rod D5 & Fuel Rod H6 \\
\hline Axial & Node Height & Bottom & Top & Spec. Vol. & T-Fuel & Burnup & Burnup \\
\hline Node & $(\mathrm{cm})$ & $(\mathrm{cm})$ & $(\mathrm{cm})$ & $\left(\mathrm{ft}^{3} / \mathrm{lbm}\right)$ & (F) & (GWd/MTU) & (GWd/MTU) \\
\hline 1 (Top) & 20.066 & 340.1060 & 360.1720 & 0.0238 & 1066.6 & 11.953 & 11.338 \\
\hline 2 & 20.0025 & 320.1035 & 340.1060 & 0.0237 & 1197.7 & 16.837 & 16.792 \\
\hline 3 & 20.0025 & 300.1010 & 320.1035 & 0.0235 & 1225.6 & 19.715 & 19.756 \\
\hline 4 & 20.0025 & 280.0985 & 300.1010 & 0.0234 & 1220.8 & 20.926 & 21.038 \\
\hline 5 & 20.0025 & 260.0960 & 280.0985 & 0.0232 & 1207.9 & 21.300 & 21.483 \\
\hline 6 & 20.0025 & 240.0935 & 260.0960 & 0.0231 & 1197.0 & 21.435 & 21.639 \\
\hline 7 & 20.0025 & 220.0910 & 240.0935 & 0.0230 & 1189.7 & 21.498 & 21.703 \\
\hline 8 & 20.0025 & 200.0885 & 220.0910 & 0.0228 & 1185.1 & 21.531 & 21.736 \\
\hline 9 & 20.0025 & 180.0860 & 200.0885 & 0.0227 & 1182.6 & 21.558 & 21.764 \\
\hline 10 & 20.0025 & 160.0835 & 180.0860 & 0.0226 & 1181.6 & 21.594 & 21.798 \\
\hline 11 & 20.0025 & 140.0810 & 160.0835 & 0.0225 & 1182.1 & 21.662 & 21.854 \\
\hline 12 & 20.0025 & 120.0785 & 140.0810 & 0.0223 & 1185.2 & 21.821 & 21.951 \\
\hline 13 & 20.0025 & 100.0760 & 120.0785 & 0.0222 & 1191.3 & 22.065 & 22.055 \\
\hline 14 & 20.0025 & 80.0735 & 100.0760 & 0.0221 & 1199.6 & 22.098 & 22.047 \\
\hline 15 & 20.0025 & 60.0710 & 80.0735 & 0.0220 & 1210.4 & 21.828 & 21.785 \\
\hline 16 & 20.0025 & 40.0685 & 60.0710 & 0.0219 & 1217.0 & 20.902 & 20.894 \\
\hline 17 & 20.0025 & 20.0660 & 40.0685 & 0.0217 & 1198.6 & 18.385 & 18.484 \\
\hline 18 (Bottom) & 20.066 & 0.0000 & 20.0660 & 0.0216 & 1077.9 & 13.999 & 13.081 \\
\hline
\end{tabular}

Cycle 9, EFPD: 549.2, Boron (ppmB): 134

\begin{tabular}{|c|c|c|c|c|c|c|c|}
\hline & \multicolumn{2}{|c|}{ Elevation } & \multicolumn{3}{|c|}{} & Fuel Rod D5 & Fuel Rod H6 \\
\hline Axial & Node Height & Bottom & Top & Spec. Vol. & T-Fuel & Burnup & Burnup \\
\hline Node & $(\mathbf{c m})$ & $(\mathbf{c m})$ & $(\mathbf{c m})$ & $\left(\mathrm{ft}^{3} / \mathbf{l b m}\right)$ & (F) & (GWd/MTU) & (GWd/MTU) \\
\hline 1 (Top) & 20.066 & 340.1060 & 360.1720 & 0.0238 & 1066.1 & 13.811 & 13.106 \\
\hline 2 & 20.0025 & 320.1035 & $\mathbf{3 4 0 . 1 0 6 0}$ & 0.0236 & 1176.3 & 19.434 & 19.277 \\
\hline 3 & 20.0025 & 300.1010 & 320.1035 & 0.0235 & 1200.4 & 22.554 & 22.490 \\
\hline 4 & 20.0025 & 280.0985 & 300.1010 & 0.0233 & 1191.4 & 23.779 & 23.797 \\
\hline 5 & 20.0025 & 260.0960 & 280.0985 & 0.0232 & 1176.8 & 24.104 & 24.208 \\
\hline 6 & 20.0025 & 240.0935 & 260.0960 & 0.0231 & 1165.9 & 24.201 & 24.331 \\
\hline 7 & 20.0025 & 220.0910 & 240.0935 & 0.0229 & 1159.0 & 24.244 & 24.376 \\
\hline 8 & 20.0025 & 200.0885 & 220.0910 & 0.0228 & 1155.0 & 24.270 & 24.403 \\
\hline 9 & 20.0025 & 180.0860 & 200.0885 & 0.0227 & 1152.6 & 24.300 & 24.433 \\
\hline 10 & 20.0025 & 160.0835 & 180.0860 & 0.0226 & 1151.2 & 24.346 & 24.477 \\
\hline 11 & 20.0025 & 140.0810 & 160.0835 & 0.0224 & 1150.8 & 24.432 & 24.550 \\
\hline 12 & 20.0025 & 120.0785 & 140.0810 & 0.0223 & 1152.4 & 24.628 & 24.675 \\
\hline 13 & 20.0025 & 100.0760 & 120.0785 & 0.0222 & 1157.4 & 24.934 & 24.819 \\
\hline 14 & 20.0025 & 80.0735 & 100.0760 & 0.0221 & 1165.2 & 25.027 & 24.863 \\
\hline 15 & 20.0025 & 60.0710 & 80.0735 & 0.0220 & 1176.7 & 24.820 & 24.662 \\
\hline 16 & 20.0025 & 40.0685 & 60.0710 & 0.0219 & 1187.5 & 23.922 & 23.799 \\
\hline 17 & 20.0025 & 20.0660 & 40.0685 & 0.0217 & 1172.2 & 21.232 & 21.227 \\
\hline 18 (Bottom) & 20.066 & 0.0000 & 20.0660 & 0.0216 & 1071.2 & 16.177 & 15.130 \\
\hline
\end{tabular}


TABLE A-4. Fuel Rod Data for Assembly NJ05YU (Cont.)

Cycle 9, EFPD: 608, Boron (ppmB): 13

\begin{tabular}{|c|c|c|c|c|c|c|c|}
\hline & & \multicolumn{2}{|c|}{ Elevation } & & & Fuel Rod D5 & Fuel Rod H6 \\
\hline Axial & Node Height & Bottom & Top & Spec. Vol. & T-Fuel & Burnup & Burnup \\
\hline Node & $(\mathrm{cm})$ & $(\mathrm{cm})$ & $(\mathrm{cm})$ & $\left(\mathrm{ft}^{3} / \mathrm{lbm}\right)$ & (F) & (GWd/MTU) & (GWd/MTU) \\
\hline 1 (Top) & 20.066 & 340.1060 & 360.1720 & 0.0235 & 1087.3 & 15.525 & 14.738 \\
\hline 2 & 20.0025 & 320.1035 & 340.1060 & 0.0234 & 1194.3 & 21.793 & 21.528 \\
\hline 3 & 20.0025 & 300.1010 & 320.1035 & 0.0232 & 1207.9 & 25.092 & 24.933 \\
\hline 4 & 20.0025 & 280.0985 & 300.1010 & 0.0231 & 1192.6 & 26.325 & 26.256 \\
\hline 5 & 20.0025 & 260.0960 & 280.0985 & 0.0230 & 1179.2 & 26.621 & 26.645 \\
\hline 6 & 20.0025 & 240.0935 & 260.0960 & 0.0228 & 1169.7 & 26.699 & 26.750 \\
\hline 7 & 20.0025 & 220.0910 & 240.0935 & 0.0227 & 1162.5 & 26.734 & 26.788 \\
\hline 8 & 20.0025 & 200.0885 & 220.0910 & 0.0226 & 1156.5 & 26.759 & 26.814 \\
\hline 9 & 20.0025 & 180.0860 & 200.0885 & 0.0224 & 1150.3 & 26.791 & 26.846 \\
\hline 10 & 20.0025 & 160.0835 & 180.0860 & 0.0223 & 1142.6 & 26.839 & 26.891 \\
\hline 11 & 20.0025 & 140.0810 & 160.0835 & 0.0222 & 1132.5 & 26.927 & 26.966 \\
\hline 12 & 20.0025 & 120.0785 & 140.0810 & 0.0221 & 1119.2 & 27.126 & 27.091 \\
\hline 13 & 20.0025 & 100.0760 & 120.0785 & 0.0220 & 1105.7 & 27.449 & 27.240 \\
\hline 14 & 20.0025 & 80.0735 & 100.0760 & 0.0219 & 1099.1 & 27.568 & 27.308 \\
\hline 15 & 20.0025 & 60.0710 & 80.0735 & 0.0218 & 1101.5 & 27.404 & 27.148 \\
\hline 16 & 20.0025 & 40.0685 & 60.0710 & 0.0217 & 1108.8 & 26.536 & 26.313 \\
\hline 17 & 20.0025 & 20.0660 & 40.0685 & 0.0216 & 1099.8 & 23.726 & 23.628 \\
\hline 18 (Bottom) & 20.066 & 0.0000 & 20.0660 & 0.0215 & 1015.6 & 18.116 & 16.960 \\
\hline
\end{tabular}

Cycle 9, EFPD: 639.4, Boron (ppmB): 2 (EOC9)

\begin{tabular}{|c|c|c|c|c|c|c|c|}
\hline & & & tion & & & Fuel Rod D5 & Fuel Rod H6 \\
\hline Axial & Node Height & Bottom & Top & Spec. Vol. & T-Fuel & Burnup & Burnup \\
\hline Node & (cm) & (cm) & (cm) & $\left(\mathrm{ft}^{3} / \mathrm{bm}\right)$ & ( $\mathrm{F})$ & (GWd/MTU) & (GWd/MTU) \\
\hline 1 (Top) & 20.0660 & 340.1060 & 360.1720 & 0.0229 & 842.5 & 16.567 & 15.732 \\
\hline 2 & 20.0025 & 320.1035 & 340.1060 & 0.0228 & 891.3 & 23.205 & 22.880 \\
\hline 3 & 20.0025 & 300.1010 & 320.1035 & 0.0228 & 886.0 & 26.583 & 26.366 \\
\hline 4 & 20.0025 & 280.0985 & 300.1010 & 0.0227 & 870.2 & 27.799 & 27.672 \\
\hline 5 & 20.0025 & 260.0960 & 280.0985 & 0.0226 & 857.1 & 28.068 & 28.034 \\
\hline 6 & 20.0025 & 240.0935 & 260.0960 & 0.0226 & 845.9 & 28.121 & 28.115 \\
\hline 7 & 20.0025 & 220.0910 & 240.0935 & 0.0225 & 835.7 & 28.136 & 28.132 \\
\hline 8 & 20.0025 & 200.0885 & 220.0910 & 0.0225 & 826.0 & 28.143 & 28.141 \\
\hline 9 & 20.0025 & 180.0860 & 200.0885 & 0.0224 & 816.3 & 28.157 & 28.155 \\
\hline 10 & 20.0025 & 160.0835 & 180.0860 & 0.0223 & 806.3 & 28.183 & 28.181 \\
\hline 11 & 20.0025 & 140.0810 & 160.0835 & 0.0223 & 795.7 & 28.244 & 28.230 \\
\hline 12 & 20.0025 & 120.0785 & 140.0810 & 0.0223 & 784.4 & 28.410 & 28.325 \\
\hline 13 & 20.0025 & 100.0760 & 120.0785 & 0.0222 & 773.5 & 28.701 & 28.444 \\
\hline 14 & 20.0025 & 80.0735 & 100.0760 & 0.0222 & 765.6 & 28.802 & 28.497 \\
\hline 15 & 20.0025 & 60.0710 & 80.0735 & 0.0221 & 761.6 & 28.639 & 28.338 \\
\hline 16 & 20.0025 & 40.0685 & 60.0710 & 0.0221 & 761.0 & 27.774 & 27.507 \\
\hline 17 & 20.0025 & 20.0660 & 40.0685 & 0.0220 & 758.8 & 24.907 & 24.767 \\
\hline 18 (Bottom) & 20.0660 & 0.0000 & 20.0660 & 0.0220 & 732.0 & 19.041 & 17.836 \\
\hline
\end{tabular}


TABLE A-4. Fuel Rod Data for Assembly NJ05YU (Cont.)

Cycle 10, EFPD: 0.0 , Boron (ppmB): 1800

\begin{tabular}{|c|c|c|c|c|c|c|c|}
\hline & & & tion & & & Fuel Rod D5 & Fuel Rod H6 \\
\hline Axial & Node Height & Bottom & Top & Spec. Vol. & T-Fuel & Burnup & Burnup \\
\hline Node & (cm) & $(\mathrm{cm})$ & $(\mathrm{cm})$ & $\left(\mathrm{ft}^{3} / \mathrm{lbm}\right)$ & ( $\mathrm{F})$ & (GWd/MTU) & (GWd/MTU) \\
\hline 1 (Top) & 17.4777 & 339.6336 & 357.1113 & 0.0234 & 877.4 & 15.868 & 15.238 \\
\hline 2 & 20.0025 & 319.6311 & 339.6336 & 0.0234 & 978.8 & 22.757 & 22.164 \\
\hline 3 & 20.0025 & 299.6286 & 319.6311 & 0.0233 & 1030.6 & 26.477 & 26.122 \\
\hline 4 & 20.0025 & 279.6261 & 299.6286 & 0.0231 & 1065.9 & 27.808 & 27.605 \\
\hline 5 & 20.0025 & 259.6236 & 279.6261 & 0.0230 & 1091.6 & 28.115 & 28.021 \\
\hline 6 & 20.0025 & 239.6211 & 259.6236 & 0.0229 & 1109.8 & 28.172 & 28.115 \\
\hline 7 & 20.0025 & 219.6186 & 239.6211 & 0.0228 & 1122.1 & 28.188 & 28.136 \\
\hline 8 & 20.0025 & 199.6161 & 219.6186 & 0.0227 & 1129.2 & 28.194 & 28.144 \\
\hline 9 & 20.0025 & 179.6136 & 199.6161 & 0.0225 & 1131.7 & 28.207 & 28.158 \\
\hline 10 & 20.0025 & 159.6111 & 179.6136 & 0.0224 & 1130.1 & 28.232 & 28.183 \\
\hline 11 & 20.0025 & 139.6086 & 159.6111 & 0.0223 & 1125.0 & 28.289 & 28.231 \\
\hline 12 & 20.0025 & 119.6061 & 139.6086 & 0.0222 & 1116.3 & 28.445 & 28.325 \\
\hline 13 & 20.0025 & 99.6036 & 119.6061 & 0.0221 & 1102.5 & 28.727 & 28.448 \\
\hline 14 & 20.0025 & 79.6011 & 99.6036 & 0.0220 & 1081.0 & 28.830 & 28.503 \\
\hline 15 & 20.0025 & 59.5986 & 79.6011 & 0.0219 & 1050.0 & 28.676 & 28.345 \\
\hline 16 & 20.0025 & 39.5961 & 59.5986 & 0.0218 & 1010.3 & 27.840 & 27.506 \\
\hline 17 & 20.0025 & 19.5936 & 39.5961 & 0.0217 & 958.6 & 25.036 & 24.716 \\
\hline 18 (Bottom) & 19.5936 & 0.0000 & 19.5936 & 0.0216 & 853.5 & 18.045 & 17.761 \\
\hline
\end{tabular}

NOTE: The EOC9 burnup is slightly different from BOC10 due to the non-symmetric one-quarter core model relative to the full core and the difference in axial nodal height between the Cycle 9 and Cycle 10.

Cycle 10, EFPD: 68, Boron (ppmB): 1649

\begin{tabular}{|c|c|c|c|c|c|c|c|}
\hline & & & tion & & & Fuel Rod D5 & Fuel Rod H6 \\
\hline Axial & Node Height & Bottom & Top & Spec. Vol. & T-Fuel & Burnup & Burnup \\
\hline Node & $(\mathrm{cm})$ & $(\mathrm{cm})$ & $(\mathrm{cm})$ & $\left(\mathrm{ft}^{3} / \mathrm{bm}\right)$ & (F) & (GWd/MTU) & (GWd/MTU) \\
\hline 1 (Top) & 17.4777 & 339.6336 & 357.1113 & 0.0234 & 877.9 & 17.136 & 16.393 \\
\hline 2 & 20.0025 & 319.6311 & 339.6336 & 0.0233 & 973.3 & 24.694 & 23.948 \\
\hline 3 & 20.0025 & 299.6286 & 319.6311 & 0.0232 & 1020.3 & 28.799 & 28.260 \\
\hline 4 & 20.0025 & 279.6261 & 299.6286 & 0.0231 & 1050.8 & 30.346 & 29.947 \\
\hline 5 & 20.0025 & 259.6236 & 279.6261 & 0.0230 & 1073.0 & 30.785 & 30.489 \\
\hline 6 & 20.0025 & 239.6211 & 259.6236 & 0.0229 & 1086.4 & 30.927 & 30.667 \\
\hline 7 & 20.0025 & 219.6186 & 239.6211 & 0.0228 & 1094.2 & 30.999 & 30.743 \\
\hline 8 & 20.0025 & 199.6161 & 219.6186 & 0.0226 & 1097.9 & 31.041 & 30.786 \\
\hline 9 & $20 . \overline{0025}$ & 179.6136 & 199.6161 & 0.0225 & 1098.4 & 31.071 & 30.817 \\
\hline 10 & $20 . \overline{0025}$ & 159.6111 & 179.6136 & 0.0224 & 1096.4 & 31.099 & 30.844 \\
\hline 11 & 20.0025 & 139.6086 & 159.6111 & 0.0223 & 1092.4 & 31.146 & 30.883 \\
\hline 12 & 20.0025 & 119.6061 & 139.6086 & 0.0222 & 1086.6 & 31.282 & 30.956 \\
\hline 13 & 20.0025 & 99.6036 & 119.6061 & 0.0221 & 1077.8 & 31.523 & 31.040 \\
\hline 14 & 20.0025 & 79.6011 & 99.6036 & 0.0220 & 1062.8 & 31.552 & 31.021 \\
\hline 15 & 20.0025 & 59.5986 & 79.6011 & 0.0219 & 1038.6 & 31.274 & 30.741 \\
\hline 16 & 20.0025 & 39.5961 & 59.5986 & 0.0218 & 1003.4 & 30.248 & 29.716 \\
\hline 17 & 20.0025 & $19.5 \overline{936}$ & 39.5961 & 0.0217 & 958.4 & 27.125 & 26.617 \\
\hline 18 (Bottom) & 19.5936 & 0.0000 & 19.5936 & 0.0216 & 858.3 & 19.441 & 19.008 \\
\hline
\end{tabular}


TABLE A-4. Fuel Rod Data for Assembly NJ05YU (Cont.)

Cycle 10, EFPD: 131.8, Boron (ppmB): 1521

\begin{tabular}{|c|c|c|c|c|c|c|c|}
\hline & & \multicolumn{2}{|c|}{ Elevation } & & & Fuel Rod D5 & Fuel Rod $\mathrm{H} 6$ \\
\hline Axial & Node Height & Bottom & Top & Spec. Vol. & T-Fuel & Burnup & Burnup \\
\hline Node & $(\mathrm{cm})$ & (cm) & $(\mathrm{cm})$ & $\left(\mathrm{ft}^{3} / \mathrm{lbm}\right)$ & ( $\mathrm{F})$ & (GWd/MTU) & (GWd/MTU) \\
\hline 1 (Top) & 17.4777 & 339.6336 & 357.1113 & 0.0234 & 902.1 & 18.364 & 17.518 \\
\hline 2 & 20.0025 & 319.6311 & 339.6336 & 0.0233 & 995.4 & 26.552 & 25.669 \\
\hline 3 & 20.0025 & 299.6286 & 319.6311 & 0.0232 & 1032.3 & 31.001 & 30.302 \\
\hline 4 & 20.0025 & 279.6261 & 299.6286 & 0.0231 & 1055.6 & 32.725 & 32.156 \\
\hline 5 & 20.0025 & 259.6236 & 279.6261 & 0.0230 & 1065.6 & 33.260 & 32.790 \\
\hline 6 & 20.0025 & 239.6211 & 259.6236 & 0.0228 & 1069.5 & 33.457 & 33.022 \\
\hline 7 & 20.0025 & 219.6186 & 239.6211 & 0.0227 & 1070.3 & 33.561 & 33.131 \\
\hline 8 & 20.0025 & 199.6161 & 219.6186 & 0.0226 & 1069.1 & 33.622 & 33.192 \\
\hline 9 & 20.0025 & 179.6136 & 199.6161 & 0.0225 & 1066.9 & 33.661 & 33.232 \\
\hline 10 & 20.0025 & 159.6111 & 179.6136 & 0.0224 & 1063.9 & 33.690 & 33.260 \\
\hline 11 & 20.0025 & 139.6086 & 159.6111 & 0.0223 & 1060.7 & 33.733 & 33.294 \\
\hline 12 & 20.0025 & 119.6061 & 139.6086 & 0.0222 & 1057.2 & 33.860 & 33.358 \\
\hline 13 & 20.0025 & 99.6036 & 119.6061 & 0.0221 & 1051.9 & 34.080 & 33.423 \\
\hline 14 & 20.0025 & 79.6011 & 99.6036 & 0.0220 & 1042.1 & 34.063 & 33.358 \\
\hline 15 & 20.0025 & 59.5986 & 79.6011 & 0.0219 & 1025.0 & 33.699 & 32.991 \\
\hline 16 & 20.0025 & 39.5961 & 59.5986 & 0.0218 & 996.7 & 32.523 & 31.818 \\
\hline 17 & 20.0025 & 19.5936 & 39.5961 & 0.0217 & 951.1 & 29.119 & 28.445 \\
\hline 18 (Bottom) & 19.5936 & 0.0000 & 19.5936 & 0.0216 & 858.7 & 20.788 & 20.221 \\
\hline
\end{tabular}

Cycle 10, EFPD: 209, Boron (ppmB): 1322

\begin{tabular}{|c|c|c|c|c|c|c|c|}
\hline & & \multicolumn{2}{|c|}{ Elevation } & & & Fuel Rod D5 & Fuel Rod $\mathrm{H} 6$ \\
\hline Axial & Node Height & Bottom & Top & Spec. Vol. & T-Fuel & Burnup & Burnup \\
\hline Node & (cm) & (cm) & $(\mathrm{cm})$ & $\left(\mathrm{ft}^{3} / \mathrm{bbm}\right)$ & $\left({ }^{\circ} \mathrm{F}\right)$ & (GWd/MTU) & (GWd/MTU) \\
\hline 1 (Top) & 17.4777 & 339.6336 & 357.1113 & 0.0234 & 910.9 & 19.935 & 18.970 \\
\hline 2 & 20.0025 & 319.6311 & 339.6336 & 0.0233 & 996.7 & 28.892 & 27.851 \\
\hline 3 & 20.0025 & 299.6286 & 319.6311 & 0.0232 & 1036.3 & 33.719 & 32.841 \\
\hline 4 & 20.0025 & 279.6261 & 299.6286 & 0.0231 & 1052.2 & 35.610 & 34.852 \\
\hline 5 & 20.0025 & 259.6236 & 279.6261 & 0.0230 & 1055.7 & 36.218 & 35.557 \\
\hline 6 & 20.0025 & 239.6211 & 259.6236 & 0.0228 & 1054.3 & 36.448 & 35.820 \\
\hline 7 & 20.0025 & 219.6186 & 239.6211 & 0.0227 & 1051.2 & 36.567 & 35.944 \\
\hline 8 & 20.0025 & 199.6161 & 219.6186 & 0.0226 & 1047.7 & 36.634 & 36.012 \\
\hline 9 & 20.0025 & 179.6136 & 199.6161 & 0.0225 & 1044.4 & 36.676 & 36.053 \\
\hline 10 & 20.0025 & 159.6111 & 179.6136 & 0.0224 & 1041.5 & 36.707 & 36.083 \\
\hline 11 & 20.0025 & 139.6086 & 159.6111 & 0.0223 & 1039.5 & 36.754 & 36.121 \\
\hline 12 & 20.0025 & 119.6061 & 139.6086 & 0.0222 & 1038.3 & 36.885 & 36.190 \\
\hline $1 \overline{3}$ & 20.0025 & 99.6036 & 119.6061 & 0.0221 & 1037.1 & 37.101 & 36.253 \\
\hline 14 & 20.0025 & 79.6011 & 99.6036 & 0.0220 & 1033.2 & 37.061 & 36.165 \\
\hline 15 & 20.0025 & 59.5986 & 79.6011 & 0.0219 & 1023.9 & 36.630 & 35.730 \\
\hline 16 & 20.0025 & 39.5961 & 59.5986 & 0.0218 & 1004.2 & 35.313 & 34.419 \\
\hline 17 & 20.0025 & 19.5936 & 39.5961 & 0.0217 & 960.0 & 31.601 & 30.742 \\
\hline 18 (Bottom) & 19.5936 & 0.0000 & 19.5936 & 0.0216 & 870.0 & 22.490 & 21.768 \\
\hline
\end{tabular}


TABLE A-4. Fuel Rod Data for Assembly NJ05YU (Cont.)

Cycle 10, EFPD: 272.1, Boron (ppmB): 1140

\begin{tabular}{|c|c|c|c|c|c|c|c|}
\hline \multirow[b]{2}{*}{ Axial } & \multirow[b]{2}{*}{ Node Height } & \multicolumn{2}{|c|}{ Elevation } & \multirow[b]{2}{*}{ Spec. Vol. } & \multirow[b]{2}{*}{ T-Fuel } & \multirow{2}{*}{\begin{tabular}{|c|} 
Fuel Rod D5 \\
Burnup \\
\end{tabular}} & \multirow{2}{*}{\begin{tabular}{|c|} 
Fuel Rod HE \\
Burnup
\end{tabular}} \\
\hline & & Bottom & Top & & & & \\
\hline Node & (cm) & (cm) & (cm) & $\left(\mathrm{ft}^{3} / \mathrm{lbm}\right)$ & ( $\mathrm{F})$ & (GWd/MTU) & (GWd/MTU) \\
\hline 1 (Top) & 17.4777 & 339.6336 & 357.1113 & 0.0234 & 918.2 & 21.287 & 20.227 \\
\hline 2 & 20.0025 & 319.6311 & 339.6336 & 0.0233 & 997.7 & 30.869 & 29.709 \\
\hline 3 & 20.0025 & 299.6286 & 319.6311 & 0.0232 & 1038.6 & 35.982 & 34.970 \\
\hline 4 & 20.0025 & 279.6261 & 299.6286 & 0.0231 & 1048.6 & 37.979 & 37.080 \\
\hline 5 & 20.0025 & 259.6236 & 279.6261 & 0.0229 & 1047.6 & 38.620 & 37.816 \\
\hline 6 & 20.0025 & 239.6211 & 259.6236 & 0.0228 & 1043.2 & 38.856 & 38.084 \\
\hline 7 & 20.0025 & 219.6186 & 239.6211 & 0.0227 & 1038.3 & 38.973 & 38.205 \\
\hline 8 & 20.0025 & 199.6161 & 219.6186 & 0.0226 & 1033.9 & 39.037 & 38.270 \\
\hline 9 & 20.0025 & 179.6136 & 199.6161 & 0.0225 & 1030.4 & 39.076 & 38.309 \\
\hline 10 & 20.0025 & 159.6111 & 179.6136 & 0.0224 & 1027.9 & 39.109 & 38.341 \\
\hline 11 & 20.0025 & 139.6086 & 159.6111 & 0.0223 & 1026.6 & 39.162 & 38.384 \\
\hline 12 & 20.0025 & 119.6061 & 139.6086 & 0.0222 & 1026.8 & 39.305 & 38.466 \\
\hline 13 & 20.0025 & 99.6036 & 119.6061 & 0.0221 & 1027.5 & 39.532 & 38.541 \\
\hline 14 & 20.0025 & 79.6011 & 99.6036 & 0.0220 & 1026.9 & 39.492 & 38.454 \\
\hline 15 & 20.0025 & 59.5986 & 79.6011 & 0.0219 & 1022.3 & 39.035 & 37.994 \\
\hline 16 & 20.0025 & 39.5961 & 59.5986 & 0.0218 & 1008.5 & 37.635 & 36.600 \\
\hline 17 & 20.0025 & 19.5936 & 39.5961 & 0.0217 & 969.8 & 33.696 & 32.698 \\
\hline 18 (Bottom) & 19.5936 & 0.0000 & 19.5936 & 0.0216 & 877.2 & 23.951 & 23.107 \\
\hline
\end{tabular}

Cycle 10, EFPD: 347.4 , Boron (ppmB): 918

\begin{tabular}{|c|c|c|c|c|c|c|c|}
\hline & & \multicolumn{2}{|c|}{ Elevation } & & & Fuel Rod D5 & Fuel Rod H6 \\
\hline Axial & Node Height & Bottom & Top & Spec. Vol. & T-Fuel & Burnup & Burnup \\
\hline Node & (cm) & (cm) & (cm) & $\left(\mathrm{ft}^{3} / \mathrm{bm}\right)$ & ( $\mathrm{F})$ & (GWd/MTU) & (GWd/MTU) \\
\hline 1 (Top) & 17.4777 & 339.6336 & 357.1113 & 0.0234 & 923.0 & 22.978 & 21.810 \\
\hline 2 & 20.0025 & 319.6311 & 339.6336 & 0.0233 & 1002.4 & 33.300 & 32.005 \\
\hline 3 & 20.0025 & 299.6286 & 319.6311 & 0.0232 & 1037.7 & 38.722 & 37.561 \\
\hline 4 & 20.0025 & 279.6261 & 299.6286 & 0.0231 & 1041.9 & 40.810 & 39.756 \\
\hline 5 & 20.0025 & 259.6236 & 279.6261 & 0.0229 & 1037.1 & 41.465 & 40.502 \\
\hline 6 & 20.0025 & 239.6211 & 259.6236 & 0.0228 & 1030.7 & 41.690 & 40.759 \\
\hline 7 & 20.0025 & 219.6186 & 239.6211 & 0.0227 & 1024.8 & 41.795 & 40.867 \\
\hline 8 & 20.0025 & 199.6161 & 219.6186 & 0.0226 & 1020.1 & 41.849 & 40.922 \\
\hline 9 & 20.0025 & 179.6136 & 199.6161 & 0.0225 & 1016.6 & 41.885 & 40.958 \\
\hline 10 & 20.0025 & 159.6111 & 179.6136 & 0.0224 & 1014.4 & 41.921 & 40.992 \\
\hline 11 & 20.0025 & 139.6086 & 159.6111 & 0.0223 & 1013.6 & 41.985 & 41.047 \\
\hline 12 & 20.0025 & 119.6061 & 139.6086 & 0.0222 & 1014.5 & 42.147 & 41.149 \\
\hline 13 & 20.0025 & 99.6036 & 119.6061 & 0.0221 & 1016.4 & 42.396 & 41.249 \\
\hline 14 & 20.0025 & 79.6011 & $99 . \overline{6036}$ & 0.0220 & 1018.3 & 42.374 & 41.180 \\
\hline 15 & 20.0025 & 59.5986 & 79.6011 & 0.0219 & 1018.0 & 41.912 & 40.714 \\
\hline 16 & 20.0025 & 39.5961 & 59.5986 & 0.0218 & 1010.4 & 40.445 & 39.255 \\
\hline 17 & 20.0025 & 19.5936 & 39.5961 & 0.0217 & 978.4 & 36.264 & 35.112 \\
\hline 18 (Bottom) & 19.5936 & 0.0000 & 19.5936 & 0.0216 & 883.7 & 25.771 & 24.789 \\
\hline
\end{tabular}


TABLE A-4. Fuel Rod Data for Assembly NJ05YU (Cont.)

Cycle 10, EFPD: 416.4, Boron (ppmB): 718

\begin{tabular}{|c|c|c|c|c|c|c|c|}
\hline & \multicolumn{2}{|c|}{ Elevation } & \multicolumn{3}{c|}{} & Fuel Rod D5 & Fuel Rod H6 \\
\hline Axial & Node Height & Bottom & Top & Spec. Vol. & T-Fuel & Burnup & Burnup \\
\hline Node & (cm) & $(\mathbf{c m})$ & $(\mathbf{c m})$ & $\left(\mathrm{ft}^{3} / \mathrm{lbm}\right)$ & $($ F) & $($ GWd/MTU) & $($ GWd/MTU) \\
\hline 1 (Top) & 17.4777 & 339.6336 & 357.1113 & 0.0234 & 926.9 & 24.596 & 23.331 \\
\hline 2 & 20.0025 & 319.6311 & 339.6336 & 0.0233 & 1007.0 & 35.582 & 34.172 \\
\hline 3 & 20.0025 & 299.6286 & 319.6311 & 0.0232 & 1036.2 & 41.259 & 39.971 \\
\hline 4 & 20.0025 & 279.6261 & 299.6286 & 0.0230 & 1035.7 & 43.402 & 42.215 \\
\hline 5 & 20.0025 & 259.6236 & 279.6261 & 0.0229 & 1028.2 & 44.049 & 42.951 \\
\hline 6 & 20.0025 & 239.6211 & 259.6236 & 0.0228 & 1020.3 & 44.253 & 43.186 \\
\hline 7 & 20.0025 & 219.6186 & 239.6211 & 0.0227 & 1013.8 & 44.340 & 43.276 \\
\hline 8 & 20.0025 & 199.6161 & 219.6186 & 0.0226 & 1008.8 & 44.382 & 43.319 \\
\hline 9 & 20.0025 & 179.6136 & 199.6161 & 0.0225 & 1005.1 & 44.414 & 43.350 \\
\hline 10 & 20.0025 & 159.6111 & 179.6136 & 0.0224 & 1002.7 & 44.452 & 43.386 \\
\hline 11 & 20.0025 & 139.6086 & 159.6111 & 0.0223 & 1001.7 & 44.527 & 43.451 \\
\hline 12 & 20.0025 & 119.6061 & 139.6086 & 0.0222 & 1002.3 & 44.708 & 43.574 \\
\hline 13 & 20.0025 & 99.6036 & 119.6061 & 0.0221 & 1004.4 & 44.982 & 43.701 \\
\hline 14 & 20.0025 & 79.6011 & 99.6036 & 0.0220 & 1007.5 & 44.986 & 43.660 \\
\hline 15 & 20.0025 & 59.5986 & 79.6011 & 0.0219 & 1010.0 & 44.539 & 43.209 \\
\hline 16 & 20.0025 & 39.5961 & 59.5986 & 0.0218 & 1007.2 & 43.041 & 41.720 \\
\hline 17 & 20.0025 & 19.5936 & 39.5961 & 0.0217 & 981.7 & 38.670 & 37.386 \\
\hline 18 (Bottom) & 19.5936 & 0.0000 & 19.5936 & 0.0216 & 886.5 & 27.508 & 26.403 \\
\hline
\end{tabular}

Cycle 10, EFPD: 486.4, Boron (ppmB): 506

\begin{tabular}{|c|c|c|c|c|c|c|c|}
\hline & & \multicolumn{2}{|c|}{ Elevation } & & & Fuel Rod D5 & Fuel Rod H6 \\
\hline Axial & Node Height & Bottom & Top & Spec. Vol. & T-Fuel & Burnup & Burnup \\
\hline Node & (cm) & (cm) & (cm) & $\left(\mathrm{ft}^{3} / \mathrm{lbm}\right)$ & (F) & (GWd/MTU) & (GWd/MTU) \\
\hline 1 (Top) & 17.4777 & 339.6336 & 357.1113 & 0.0234 & 929.8 & 26.297 & 24.938 \\
\hline 2 & 20.0025 & 319.6311 & 339.6336 & 0.0233 & 1009.6 & 37.937 & 36.419 \\
\hline 3 & 20.0025 & 299.6286 & 319.6311 & 0.0232 & 1032.2 & 43.843 & 42.435 \\
\hline 4 & 20.0025 & 279.6261 & 299.6286 & 0.0230 & 1027.3 & 46.018 & 44.706 \\
\hline 5 & 20.0025 & 259.6236 & 279.6261 & 0.0229 & 1017.4 & 46.642 & 45.416 \\
\hline 6 & 20.0025 & 239.6211 & 259.6236 & 0.0228 & 1008.6 & 46.818 & 45.621 \\
\hline 7 & 20.0025 & 219.6186 & 239.6211 & 0.0227 & 1001.9 & 46.883 & 45.689 \\
\hline 8 & 20.0025 & 199.6161 & 219.6186 & 0.0226 & 997.0 & 46.913 & 45.718 \\
\hline 9 & 20.0025 & 179.6136 & 199.6161 & 0.0225 & 993.4 & 46.939 & 45.744 \\
\hline 10 & 20.0025 & 159.6111 & 179.6136 & 0.0224 & 991.0 & 46.979 & 45.782 \\
\hline 11 & 20.0025 & 139.6086 & 159.6111 & 0.0223 & 989.7 & 47.063 & 45.856 \\
\hline 12 & 20.0025 & 119.6061 & 139.6086 & 0.0222 & 990.0 & 47.263 & 45.998 \\
\hline 13 & 20.0025 & 99.6036 & 119.6061 & 0.0221 & 992.0 & 47.563 & 46.153 \\
\hline 14 & 20.0025 & 79.6011 & 99.6036 & 0.0220 & 995.7 & 47.601 & 46.147 \\
\hline 15 & 20.0025 & $59 . \overline{5986}$ & 79.6011 & 0.0219 & 1000.4 & 47.184 & 45.728 \\
\hline 16 & 20.0025 & 39.5961 & 59.5986 & 0.0218 & 1002.1 & 45.680 & 44.234 \\
\hline 17 & 20.0025 & 19.5936 & 39.5961 & 0.0217 & 983.4 & 41.149 & 39.740 \\
\hline 18 (Bottom) & 19.5936 & 0.0000 & 19.5936 & 0.0216 & 888.7 & 29.331 & 28.107 \\
\hline
\end{tabular}


TABLE A-4. Fuel Rod Data for Assembly NJ05YU (Cont.)

Cycle 10, EFPD: 556.3, Boron (ppmB): 298

\begin{tabular}{|c|c|c|c|c|c|c|c|}
\hline & & \multicolumn{2}{|c|}{ Elevation } & \multicolumn{2}{c|}{} & Fuel Rod D5 & Fuel Rod H6 \\
\hline Axial & Node Height & Bottom & Top & Spec. Vol. & T-Fuel & Burnup & Burnup \\
\hline Node & $(\mathbf{c m})$ & $(\mathrm{cm})$ & $(\mathbf{c m})$ & $\left(\mathrm{ft}^{3} / \mathrm{lbm}\right)$ & $(\mathrm{F})$ & $(\mathrm{GWd} / \mathrm{MTU})$ & (GWd/MTU) \\
\hline 1 (Top) & 17.4777 & 339.6336 & 357.1113 & 0.0233 & 932.5 & 28.049 & 26.602 \\
\hline 2 & 20.0025 & 319.6311 & 339.6336 & 0.0232 & 1011.5 & 40.314 & 38.696 \\
\hline 3 & 20.0025 & 299.6286 & 319.6311 & 0.0231 & 1027.0 & 46.420 & 44.900 \\
\hline 4 & 20.0025 & 279.6261 & 299.6286 & 0.0230 & 1017.4 & 48.605 & 47.174 \\
\hline 5 & 20.0025 & 259.6236 & 279.6261 & 0.0229 & 1005.4 & 49.196 & 47.848 \\
\hline 6 & 20.0025 & 239.6211 & 259.6236 & 0.0228 & 995.8 & 49.340 & 48.020 \\
\hline 7 & 20.0025 & 219.6186 & 239.6211 & 0.0227 & 989.1 & 49.384 & 48.065 \\
\hline 8 & 20.0025 & 199.6161 & 219.6186 & 0.0226 & 984.3 & 49.402 & 48.083 \\
\hline 9 & 20.0025 & 179.6136 & 199.6161 & 0.0225 & 980.8 & 49.425 & 48.104 \\
\hline 10 & 20.0025 & 159.6111 & 179.6136 & 0.0224 & 978.3 & 49.466 & 48.144 \\
\hline 11 & 20.0025 & 139.6086 & 159.6111 & 0.0223 & 976.7 & 49.559 & 48.227 \\
\hline 12 & 20.0025 & 119.6061 & 139.6086 & 0.0222 & 976.4 & 49.775 & 48.386 \\
\hline 13 & 20.0025 & 99.6036 & 119.6061 & 0.0221 & 978.0 & 50.100 & 48.568 \\
\hline 14 & 20.0025 & 79.6011 & 99.6036 & 0.0220 & 981.7 & 50.173 & 48.597 \\
\hline 15 & 20.0025 & 59.5986 & 79.6011 & 0.0219 & 987.7 & 49.797 & 48.220 \\
\hline 16 & 20.0025 & 39.5961 & 59.5986 & 0.0218 & 993.0 & 48.309 & 46.744 \\
\hline 17 & 20.0025 & 19.5936 & 39.5961 & 0.0217 & 980.8 & 43.649 & 42.123 \\
\hline 18 (Bottom) & 19.5936 & 0.0000 & 19.5936 & 0.0216 & 888.5 & 31.208 & 29.870 \\
\hline
\end{tabular}

Cycle 10, EFPD: 626.1, Boron (ppmB): 103

\begin{tabular}{|c|c|c|c|c|c|c|c|}
\hline & & \multicolumn{2}{|c|}{ Elevation } & & & Fuel Rod D5 & Fuel Rod H6 \\
\hline Axial & Node Height & Bottom & Top & Spec. Vol. & T-Fuel & Burnup & Burnup \\
\hline Node & (cm) & (cm) & $(\mathrm{cm})$ & $\left(\mathrm{ft}^{3} / \mathrm{bm}\right)$ & (F) & (GWd/MTU) & (GWd/MTU) \\
\hline 1 (Top) & 17.4777 & 339.6336 & 357.1113 & 0.0233 & 932.5 & 29.858 & 28.326 \\
\hline 2 & 20.0025 & 319.6311 & 339.6336 & 0.0232 & 1010.0 & 42.718 & 41.005 \\
\hline 3 & 20.0025 & 299.6286 & 319.6311 & 0.0231 & 1019.2 & 48.990 & 47.363 \\
\hline 4 & 20.0025 & 279.6261 & 299.6286 & 0.0230 & 1007.1 & 51.164 & 49.620 \\
\hline 5 & 20.0025 & 259.6236 & 279.6261 & 0.0229 & 996.0 & 51.712 & 50.248 \\
\hline 6 & 20.0025 & 239.6211 & 259.6236 & 0.0228 & 987.2 & 51.822 & 50.383 \\
\hline 7 & 20.0025 & 219.6186 & 239.6211 & 0.0227 & 981.1 & 51.845 & 50.407 \\
\hline 8 & 20.0025 & 199.6161 & 219.6186 & 0.0226 & 976.9 & 51.853 & 50.415 \\
\hline 9 & 20.0025 & 179.6136 & 199.6161 & 0.0225 & 973.9 & 51.872 & 50.432 \\
\hline 10 & 20.0025 & 159.6111 & 179.6136 & 0.0224 & 971.6 & 51.915 & 50.473 \\
\hline 11 & 20.0025 & 139.6086 & 159.6111 & 0.0223 & 970.1 & 52.015 & 50.563 \\
\hline 12 & 20.0025 & 119.6061 & 139.6086 & 0.0222 & 970.0 & 52.245 & 50.735 \\
\hline 13 & 20.0025 & 99.6036 & 119.6061 & 0.0221 & 971.6 & 52.591 & 50.941 \\
\hline 14 & 20.0025 & 79.6011 & 99.6036 & 0.0220 & 974.9 & 52.699 & 51.005 \\
\hline 15 & 20.0025 & 59.5986 & 79.6011 & 0.0219 & 980.0 & 52.367 & 50.674 \\
\hline 16 & 20.0025 & 39.5961 & 59.5986 & 0.0218 & 983.8 & 50.911 & 49.232 \\
\hline 17 & 20.0025 & 19.5936 & 39.5961 & 0.0217 & 976.9 & 46.153 & 44.515 \\
\hline 18 (Bottom) & 19.5936 & 0.0000 & 19.5936 & 0.0216 & 894.0 & 33.126 & 31.679 \\
\hline
\end{tabular}


TABLE A-4. Fuel Rod Data for Assembly NJ05YU (Cont.)

Cycle 10, EFPD: 660.3 , Boron (ppmB): 1.8 (EOC10)

\begin{tabular}{|c|c|c|c|c|c|c|c|}
\hline & \multicolumn{2}{|c|}{ Elevation } & \multicolumn{3}{|c|}{} & Fuel Rod D5 & Fuel Rod H6 \\
\hline Axial & Node Height & Bottom & Top & Spec. Vol. & T-Fuel & Burnup & Burnup \\
\hline Node & $(\mathbf{c m})$ & $(\mathbf{c m})$ & $(\mathrm{cm})$ & $\left(\mathrm{ft}^{3} / \mathbf{l b m}\right)$ & $(\mathbf{F})$ & $(\mathrm{GWd} / \mathrm{MTU})$ & $(\mathrm{GWd} / \mathrm{MTU})$ \\
\hline 1 (Top) & 17.4777 & 339.6336 & 357.1113 & 0.0233 & 927.7 & 30.765 & 29.193 \\
\hline 2 & 20.0025 & 319.6311 & 339.6336 & 0.0232 & 1003.4 & 43.904 & 42.146 \\
\hline 3 & 20.0025 & 299.6286 & 319.6311 & 0.0231 & 1010.8 & 50.247 & 48.569 \\
\hline 4 & 20.0025 & 279.6261 & 299.6286 & 0.0230 & 1002.2 & 52.409 & 50.810 \\
\hline 5 & 20.0025 & 259.6236 & 279.6261 & 0.0229 & 992.1 & 52.933 & 51.413 \\
\hline 6 & 20.0025 & 239.6211 & 259.6236 & 0.0228 & 984.3 & 53.025 & 51.530 \\
\hline 7 & 20.0025 & 219.6186 & 239.6211 & 0.0227 & 979.1 & 53.038 & 51.545 \\
\hline 8 & 20.0025 & 199.6161 & 219.6186 & 0.0226 & 975.7 & 53.042 & 51.547 \\
\hline 9 & 20.0025 & 179.6136 & 199.6161 & 0.0225 & 973.3 & 53.060 & 51.563 \\
\hline 10 & 20.0025 & 159.6111 & 179.6136 & 0.0224 & 971.4 & 53.103 & 51.604 \\
\hline 11 & 20.0025 & 139.6086 & 159.6111 & 0.0223 & 970.3 & 53.205 & 51.696 \\
\hline 12 & 20.0025 & 119.6061 & 139.6086 & 0.0222 & 970.2 & 53.440 & 51.874 \\
\hline 13 & 20.0025 & 99.6036 & 119.6061 & 0.0221 & 971.7 & 53.796 & 52.089 \\
\hline 14 & 20.0025 & 79.6011 & 99.6036 & 0.0220 & 974.9 & 53.920 & 52.170 \\
\hline 15 & 20.0025 & 59.5986 & 79.6011 & 0.0219 & 980.3 & 53.611 & 51.861 \\
\hline 16 & 20.0025 & 39.5961 & 59.5986 & 0.0218 & 983.9 & 52.174 & 50.440 \\
\hline 17 & 20.0025 & 19.5936 & 39.5961 & 0.0217 & 976.4 & 47.377 & 45.687 \\
\hline 18 (Bottom) & 19.5936 & 0.0000 & 19.5936 & 0.0216 & 897.8 & 34.078 & 32.579 \\
\hline
\end{tabular}

Table A-5. Fuel Rod Data for Assembly NJ070G

Cycle 10, EFPD: 0.0, Boron (ppmB): 1800

\begin{tabular}{|c|c|c|c|c|c|c|c|c|c|}
\hline & & \multicolumn{2}{|c|}{ Elevation } & Spec. & Fuel & Rod O1 & Rod O11 & Rod O12 & Rod O13 \\
\hline Axial & Node Height & Bottom & Top & Vol. & T-Fuel & Burnup & Burnup & Burnup & Burnup \\
\hline Node & $(\mathbf{c m})$ & $\mathbf{( c m )}$ & $(\mathbf{c m})$ & $\left(\mathbf{f t}^{3} / \mathbf{l b m}\right)$ & $(\mathbf{F})$ & $($ GWd/MTU) & $($ GWd/MTU) & (GWd/MTU) & (GWd/MTU) \\
\hline 1 (Top) & 17.4777 & 339.6336 & 357.1113 & 0.0238 & 1022.7 & 0 & 0 & 0 & 0 \\
\hline 2 & 20.0025 & 319.6311 & 339.6336 & 0.0237 & 1212.7 & 0 & 0 & 0 & 0 \\
\hline 3 & 20.0025 & 299.6286 & 319.6311 & 0.0236 & 1356.3 & 0 & 0 & 0 & 0 \\
\hline 4 & 20.0025 & 279.6261 & 299.6286 & 0.0235 & 1448.2 & 0 & 0 & 0 & 0 \\
\hline 5 & 20.0025 & 259.6236 & 279.6261 & 0.0233 & 1505.0 & 0 & 0 & 0 & 0 \\
\hline 6 & 20.0025 & 239.6211 & 259.6236 & 0.0232 & 1538.4 & 0 & 0 & 0 & 0 \\
\hline 7 & 20.0025 & 219.6186 & 239.6211 & 0.0230 & 1560.8 & 0 & 0 & 0 & 0 \\
\hline 8 & 20.0025 & 199.6161 & 219.6186 & 0.0229 & 1575.3 & 0 & 0 & 0 & 0 \\
\hline 9 & 20.0025 & 179.6136 & 199.6161 & 0.0227 & 1583.3 & 0 & 0 & 0 & 0 \\
\hline 10 & 20.0025 & 159.6111 & 179.6136 & 0.0226 & 1585.5 & 0 & 0 & 0 & 0 \\
\hline 11 & 20.0025 & 139.6086 & 159.6111 & 0.0224 & 1582.6 & 0 & 0 & 0 & 0 \\
\hline 12 & 20.0025 & 119.6061 & 139.6086 & 0.0223 & 1575.5 & 0 & 0 & 0 & 0 \\
\hline 13 & 20.0025 & 99.6036 & 119.6061 & 0.0222 & 1562.3 & 0 & 0 & 0 & 0 \\
\hline 14 & 20.0025 & 79.6011 & 99.6036 & 0.0220 & 1534.2 & 0 & 0 & 0 & 0 \\
\hline 15 & 20.0025 & 59.5986 & 79.6011 & 0.0219 & 1486.7 & 0 & 0 & 0 & 0 \\
\hline 16 & 20.0025 & 39.5961 & 59.5986 & 0.0218 & 1401.5 & 0 & 0 & 0 & 0 \\
\hline 17 & 20.0025 & 19.5936 & 39.5961 & 0.0217 & 1264.9 & 0 & 0 & 0 & 0 \\
\hline 18 (Bottom) & 19.5936 & 0.0000 & 19.5936 & 0.0216 & 1067.8 & 0 & 0 & 0 & 0 \\
\hline
\end{tabular}


TABLE A-5. Fuel Rod Data for Assembly NJ070G (Cont.)

Cycle 10, EFPD: 68, Boron (ppmB): 1649

\begin{tabular}{|c|c|c|c|c|c|c|c|c|c|}
\hline & & \multicolumn{2}{|c|}{ Elevation } & Spec. & Fuel & Rod 01 & Rod 011 & Rod O12 & Rod O13 \\
\hline Axial & Node Height & Bottom & Top & Vol. & T-Fuel & Burnup & Burnup & Burnup & Burnup \\
\hline Node & $\mathbf{( c m )}$ & $(\mathbf{c m})$ & $\mathbf{( c m )}$ & $\left(\mathbf{f t}^{3} /\right.$ /bm) & $\left({ }^{\circ}\right.$ F) & $($ GWd/MTU) & (GWd/MTU) & (GWd/MTU) & (GWd/MTU) \\
\hline 1 (Top) & 17.4777 & 339.6336 & 357.1113 & 0.0239 & 1028.5 & 1.363 & 1.261 & 1.255 & 1.262 \\
\hline 2 & 20.0025 & 319.6311 & 339.6336 & 0.0238 & 1215.9 & 2.138 & 2.079 & 2.051 & 2.000 \\
\hline 3 & 20.0025 & 299.6286 & 319.6311 & 0.0237 & 1351.1 & 2.722 & 2.648 & 2.616 & 2.553 \\
\hline 4 & 20.0025 & 279.6261 & 299.6286 & 0.0235 & 1432.3 & 3.084 & 3.001 & 2.966 & 2.897 \\
\hline 5 & 20.0025 & 259.6236 & 279.6261 & 0.0234 & 1476.8 & 3.312 & 3.221 & 3.184 & 3.112 \\
\hline 6 & 20.0025 & 239.6211 & 259.6236 & 0.0232 & 1499.9 & 3.462 & 3.365 & 3.327 & 3.253 \\
\hline 7 & 20.0025 & 219.6186 & 239.6211 & 0.0231 & 1514.5 & 3.563 & 3.462 & 3.424 & 3.349 \\
\hline 8 & 20.0025 & 199.6161 & 219.6186 & 0.0229 & 1523.2 & 3.632 & 3.529 & 3.490 & 3.414 \\
\hline 9 & 20.0025 & 179.6136 & 199.6161 & 0.0228 & 1527.6 & 3.676 & 3.571 & 3.532 & 3.455 \\
\hline 10 & 20.0025 & 159.6111 & 179.6136 & 0.0226 & 1528.5 & 3.699 & 3.594 & 3.554 & 3.477 \\
\hline 11 & 20.0025 & 139.6086 & 159.6111 & 0.0225 & 1526.7 & 3.702 & 3.597 & 3.558 & 3.480 \\
\hline 12 & 20.0025 & 119.6061 & 139.6086 & 0.0223 & 1523.0 & 3.682 & 3.581 & 3.541 & 3.463 \\
\hline 13 & 20.0025 & 99.6036 & 119.6061 & 0.0222 & 1516.3 & 3.628 & 3.534 & 3.493 & 3.415 \\
\hline 14 & 20.0025 & 79.6011 & 99.6036 & 0.0221 & 1498.2 & 3.525 & 3.435 & 3.395 & 3.318 \\
\hline 15 & 20.0025 & 59.5986 & 79.6011 & 0.0219 & 1463.8 & 3.341 & 3.256 & 3.217 & 3.143 \\
\hline 16 & 20.0025 & 39.5961 & 59.5986 & 0.0218 & 1397.8 & 3.028 & 2.946 & 2.910 & 2.841 \\
\hline 17 & 20.0025 & 19.5936 & 39.5961 & 0.0217 & 1272.8 & 2.488 & 2.418 & 2.384 & 2.323 \\
\hline 18 (Bottom) & 19.5936 & 0.0000 & 19.5936 & 0.0216 & 1076.4 & 1.599 & 1.503 & 1.486 & 1.485 \\
\hline
\end{tabular}

Cycle 10, EFPD: 131.8, Boron (ppmB): 1521

\begin{tabular}{|c|c|c|c|c|c|c|c|c|c|}
\hline & & \multicolumn{2}{|c|}{ Elevation } & Spec. & Fuel & Rod O1 & Rod O11 & Rod O12 & Rod O13 \\
\hline Axial & Node Height & Bottom & Top & Vol. & T-Fuel & Burnup & Burnup & Burnup & Burnup \\
\hline Node & $\mathbf{( c m )}$ & $(\mathbf{c m})$ & $(\mathbf{c m})$ & $\left(\mathbf{f t}^{3} / / \mathbf{l b m}\right)$ & $(\mathbf{F})$ & $($ GWd/MTU) & $($ GWd/MTU) & $($ GWd/MTU) & (GWd/MTU) \\
\hline 1 (Top) & 17.4777 & 339.6336 & 357.1113 & 0.0239 & 1064.2 & 2.704 & 2.502 & 2.486 & 2.495 \\
\hline 2 & 20.0025 & 319.6311 & 339.6336 & 0.0238 & 1249.6 & 4.259 & 4.100 & 4.049 & 3.956 \\
\hline 3 & 20.0025 & 299.6286 & 319.6311 & 0.0237 & 1368.0 & 5.393 & 5.179 & 5.122 & 5.018 \\
\hline 4 & 20.0025 & 279.6261 & 299.6286 & 0.0235 & 1425.5 & 6.074 & 5.823 & 5.762 & 5.653 \\
\hline 5 & 20.0025 & 259.6236 & 279.6261 & 0.0234 & 1446.2 & 6.485 & 6.206 & 6.143 & 6.033 \\
\hline 6 & 20.0025 & 239.6211 & 259.6236 & 0.0232 & 1454.6 & 6.744 & 6.447 & 6.383 & 6.271 \\
\hline 7 & 20.0025 & 219.6186 & 239.6211 & 0.0231 & 1458.0 & 6.916 & 6.606 & 6.542 & 6.429 \\
\hline 8 & 20.0025 & 199.6161 & 219.6186 & 0.0229 & 1458.8 & 7.032 & 6.714 & 6.648 & 6.535 \\
\hline 9 & 20.0025 & 179.6136 & 199.6161 & 0.0228 & 1458.3 & 7.107 & 6.783 & 6.718 & 6.604 \\
\hline 10 & 20.0025 & 159.6111 & 179.6136 & 0.0226 & 1457.3 & 7.149 & 6.824 & 6.757 & 6.643 \\
\hline 11 & 20.0025 & 139.6086 & 159.6111 & 0.0225 & 1456.1 & 7.162 & 6.837 & 6.770 & 6.655 \\
\hline 12 & 20.0025 & 119.6061 & 139.6086 & 0.0223 & 1455.4 & 7.140 & 6.820 & 6.752 & 6.637 \\
\hline 13 & 20.0025 & 99.6036 & 119.6061 & 0.0222 & 1453.8 & 7.061 & 6.755 & 6.686 & 6.568 \\
\hline 14 & 20.0025 & 79.6011 & 99.6036 & 0.0221 & 1444.6 & 6.894 & 6.601 & 6.532 & 6.414 \\
\hline 15 & 20.0025 & 59.5986 & 79.6011 & 0.0219 & 1422.9 & 6.575 & 6.301 & 6.233 & 6.116 \\
\hline 16 & 20.0025 & 39.5961 & 59.5986 & 0.0218 & 1375.4 & 5.998 & 5.747 & 5.682 & 5.570 \\
\hline 17 & 20.0025 & 19.5936 & 39.5961 & 0.0217 & 1266.4 & 4.955 & 4.754 & 4.693 & 4.586 \\
\hline $18($ Bottom) & 19.5936 & 0.0000 & 19.5936 & 0.0216 & 1076.4 & 3.176 & 2.975 & 2.938 & 2.930 \\
\hline
\end{tabular}


TABLE A-5. Fuel Rod Data for Assembly NJ070G (Cont.)

Cycle 10, EFPD: 209, Boron (ppmB): 1322

\begin{tabular}{|c|c|c|c|c|c|c|c|c|c|}
\hline & & \multicolumn{2}{|c|}{ Elevation } & Spec. & Fuel & Rod O1 & Rod O11 & Rod O12 & Rod 013 \\
\hline Axial & Node Height & Bottom & Top & Vol. & T-Fuel & Burnup & Burnup & Burnup & Burnup \\
\hline Node & $(\mathbf{c m})$ & $(\mathbf{c m})$ & $(\mathbf{c m})$ & $(\mathbf{f t}$ ///bm) & $($ F) & $($ GWd/MTU) & (GWd/MTU) & (GWd/MTU) & (GWd/MTU) \\
\hline 1 (Top) & 17.4777 & 339.6336 & 357.1113 & 0.0239 & 1076.1 & 4.443 & 4.107 & 4.079 & 4.087 \\
\hline 2 & 20.0025 & 319.6311 & 339.6336 & 0.0238 & 1251.2 & 7.001 & 6.654 & 6.582 & 6.459 \\
\hline 3 & 20.0025 & 299.6286 & 319.6311 & 0.0236 & 1350.7 & 8.772 & 8.306 & 8.227 & 8.099 \\
\hline 4 & 20.0025 & 279.6261 & 299.6286 & 0.0235 & 1385.5 & 9.767 & 9.231 & 9.147 & 9.016 \\
\hline 5 & 20.0025 & 259.6236 & 279.6261 & 0.0233 & 1390.9 & 10.328 & 9.745 & 9.659 & 9.528 \\
\hline 6 & 20.0025 & 239.6211 & 259.6236 & 0.0232 & 1388.8 & 10.659 & 10.049 & 9.961 & 9.829 \\
\hline 7 & 20.0025 & 219.6186 & 239.6211 & 0.0230 & 1384.9 & 10.869 & 10.242 & 10.153 & 10.020 \\
\hline 8 & 20.0025 & 199.6161 & 219.6186 & 0.0229 & 1381.1 & 11.010 & 10.371 & 10.281 & 10.148 \\
\hline 9 & 20.0025 & 179.6136 & 199.6161 & 0.0228 & 1378.0 & 11.106 & 10.459 & 10.368 & 10.234 \\
\hline 10 & 20.0025 & 159.6111 & 179.6136 & 0.0226 & 1376.2 & 11.168 & 10.517 & 10.425 & 10.290 \\
\hline 11 & 20.0025 & 139.6086 & 159.6111 & 0.0225 & 1375.7 & 11.201 & 10.550 & 10.457 & 10.321 \\
\hline 12 & 20.0025 & 119.6061 & 139.6086 & 0.0223 & 1377.4 & 11.199 & 10.553 & 10.458 & 10.320 \\
\hline 13 & 20.0025 & 99.6036 & 119.6061 & 0.0222 & 1380.7 & 11.127 & 10.499 & 10.401 & 10.260 \\
\hline 14 & 20.0025 & 79.6011 & 99.6036 & 0.0221 & 1380.6 & 10.938 & 10.327 & 10.229 & 10.087 \\
\hline 15 & 20.0025 & 59.5986 & 79.6011 & 0.0219 & 1373.5 & 10.529 & 9.944 & 9.848 & 9.706 \\
\hline 16 & 20.0025 & 39.5961 & 59.5986 & 0.0218 & 1348.6 & 9.711 & 9.169 & 9.078 & 8.938 \\
\hline 17 & 20.0025 & 19.5936 & 39.5961 & 0.0217 & 1265.5 & 8.108 & 7.672 & 7.583 & 7.443 \\
\hline 18 (Bottom) & 19.5936 & 0.0000 & 19.5936 & 0.0216 & 1084.3 & 5.183 & 4.854 & 4.788 & 4.763 \\
\hline
\end{tabular}

Cycle 10, EFPD: 272.1, Boron (ppmB): 1140

\begin{tabular}{|c|c|c|c|c|c|c|c|c|c|}
\hline & & \multicolumn{2}{|c|}{ Elevation } & Spec. & Fuel & Rod O1 & Rod O11 & Rod O12 & Rod O13 \\
\hline Axial & Node Height & Bottom & Top & Vol. & T-Fuel & Burnup & Burnup & Burnup & Burnup \\
\hline Node & $(\mathbf{c m})$ & $(\mathbf{c m})$ & $\mathbf{( c m )}$ & $(\mathbf{f t}$ //lbm) & (F) & (GWd/MTU) & (GWd/MTU) & (GWd/MTU) & (GWd/MTU) \\
\hline 1 (Top) & 17.4777 & 339.6336 & 357.1113 & 0.0239 & 1078.0 & 5.952 & 5.498 & 5.457 & 5.463 \\
\hline 2 & 20.0025 & 319.6311 & 339.6336 & 0.0238 & 1242.9 & 9.353 & 8.817 & 8.729 & 8.595 \\
\hline 3 & 20.0025 & 299.6286 & 319.6311 & 0.0236 & 1325.4 & 11.587 & 10.893 & 10.795 & 10.657 \\
\hline 4 & 20.0025 & 279.6261 & 299.6286 & 0.0235 & 1349.4 & 12.770 & 11.993 & 11.889 & 11.747 \\
\hline 5 & 20.0025 & 259.6236 & 279.6261 & 0.0233 & 1348.9 & 13.397 & 12.568 & 12.462 & 12.320 \\
\hline 6 & 20.0025 & 239.6211 & 259.6236 & 0.0232 & 1343.0 & 13.747 & 12.890 & 12.782 & 12.638 \\
\hline 7 & 20.0025 & 219.6186 & 239.6211 & 0.0230 & 1336.9 & 13.961 & 13.088 & 12.978 & 12.832 \\
\hline 8 & 20.0025 & 199.6161 & 219.6186 & 0.0229 & 1331.8 & 14.104 & 13.220 & 13.108 & 12.962 \\
\hline 9 & 20.0025 & 179.6136 & 199.6161 & 0.0227 & 1328.2 & 14.205 & 13.314 & 13.201 & 13.053 \\
\hline 10 & 20.0025 & 159.6111 & 179.6136 & 0.0226 & 1326.4 & 14.279 & 13.383 & 13.268 & 13.119 \\
\hline 11 & 20.0025 & 139.6086 & 159.6111 & 0.0225 & 1326.6 & 14.331 & 13.433 & 13.317 & 13.167 \\
\hline 12 & 20.0025 & 119.6061 & 139.6086 & 0.0223 & 1329.4 & 14.353 & 13.460 & 13.342 & 13.189 \\
\hline 13 & 20.0025 & 99.6036 & 119.6061 & 0.0222 & 1334.7 & 14.306 & 13.431 & 13.310 & 13.152 \\
\hline 14 & 20.0025 & 79.6011 & 99.6036 & 0.0221 & 1338.3 & 14.132 & 13.273 & 13.151 & 12.992 \\
\hline 15 & 20.0025 & 59.5986 & 79.6011 & 0.0220 & 1336.7 & 13.701 & 12.867 & 12.747 & 12.589 \\
\hline 16 & 20.0025 & 39.5961 & 59.5986 & 0.0218 & 1319.9 & 12.758 & 11.969 & 11.854 & 11.700 \\
\hline 17 & 20.0025 & 19.5936 & 39.5961 & 0.0217 & 1250.2 & 10.769 & 10.114 & 10.004 & 9.849 \\
\hline $18($ (Bottom) & 19.5936 & 0.0000 & 19.5936 & 0.0216 & 1082.3 & 6.906 & 6.464 & 6.372 & 6.334 \\
\hline
\end{tabular}


TABLE A-5. Fuel Rod Data for Assembly NJ070G (Cont.)

Cycle 10, EFPD: 347.4, Boron (ppmB): 918

\begin{tabular}{|c|c|c|c|c|c|c|c|c|c|}
\hline & & \multicolumn{2}{|c|}{ Elevation } & Spec. & Fuel & Rod O1 & Rod O11 & Rod O12 & Rod O13 \\
\hline Axial & Node Height & Bottom & Top & Vol. & T-Fuel & Burnup & Burnup & Burnup & Burnup \\
\hline Node & $\mathbf{( c m )}$ & $(\mathbf{c m})$ & $(\mathbf{c m})$ & $\left(\mathrm{ft}^{3} / \mathrm{lbm}\right)$ & $(\mathrm{F})$ & $(\mathrm{GWd} / \mathrm{MTU})$ & (GWd/MTU) & (GWd/MTU) & (GWd/MTU) \\
\hline 1 (Top) & 17.4777 & 339.6336 & 357.1113 & 0.0239 & 1074.3 & 7.837 & 7.238 & 7.181 & 7.185 \\
\hline 2 & 20.0025 & 319.6311 & 339.6336 & 0.0237 & 1228.2 & 12.230 & 11.462 & 11.357 & 11.213 \\
\hline 3 & 20.0025 & 299.6286 & 319.6311 & 0.0236 & 1297.4 & 14.945 & 13.992 & 13.873 & 13.722 \\
\hline 4 & 20.0025 & 279.6261 & 299.6286 & 0.0235 & 1308.3 & 16.290 & 15.250 & 15.121 & 14.964 \\
\hline 5 & 20.0025 & 259.6236 & 279.6261 & 0.0233 & 1301.7 & 16.954 & 15.862 & 15.729 & 15.570 \\
\hline 6 & 20.0025 & 239.6211 & 259.6236 & 0.0232 & 1292.6 & 17.299 & 16.181 & 16.046 & 15.885 \\
\hline 7 & 20.0025 & 219.6186 & 239.6211 & 0.0230 & 1284.9 & 17.502 & 16.370 & 16.232 & 16.070 \\
\hline 8 & 20.0025 & 199.6161 & 219.6186 & 0.0229 & 1279.1 & 17.639 & 16.497 & 16.358 & 16.194 \\
\hline 9 & 20.0025 & 179.6136 & 199.6161 & 0.0227 & 1275.3 & 17.742 & 16.594 & 16.453 & 16.288 \\
\hline 10 & 20.0025 & 159.6111 & 179.6136 & 0.0226 & 1273.5 & 17.828 & 16.675 & 16.533 & 16.366 \\
\hline 11 & 20.0025 & 139.6086 & 159.6111 & 0.0225 & 1273.9 & 17.904 & 16.748 & 16.603 & 16.434 \\
\hline 12 & 20.0025 & 119.6061 & 139.6086 & 0.0223 & 1277.1 & 17.959 & 16.807 & 16.660 & 16.487 \\
\hline 13 & 20.0025 & 99.6036 & 119.6061 & 0.0222 & 1283.1 & 17.950 & 16.817 & 16.665 & 16.487 \\
\hline 14 & 20.0025 & 79.6011 & 99.6036 & 0.0221 & 1289.4 & 17.814 & 16.695 & 16.542 & 16.361 \\
\hline 15 & 20.0025 & 59.5986 & 79.6011 & 0.0220 & 1293.7 & 17.393 & 16.294 & 16.143 & 15.963 \\
\hline 16 & 20.0025 & 39.5961 & 59.5986 & 0.0218 & 1287.0 & 16.355 & 15.299 & 15.155 & 14.981 \\
\hline 17 & 20.0025 & 19.5936 & 39.5961 & 0.0217 & 1236.5 & 13.976 & 13.072 & 12.937 & 12.764 \\
\hline 18 (Bottom) & 19.5936 & 0.0000 & 19.5936 & 0.0216 & 1075.8 & 9.034 & 8.458 & 8.336 & 8.282 \\
\hline
\end{tabular}

Cycle 10, EFPD: 416.4, Boron (ppmB): 718

\begin{tabular}{|c|c|c|c|c|c|c|c|c|c|}
\hline & & \multicolumn{2}{|c|}{ Elevation } & Spec. & Fuel & Rod O1 & Rod O11 & Rod O12 & Rod O13 \\
\hline Axial & Node Height & Bottom & Top & Vol. & T-Fuel & Burnup & Burnup & Burnup & Burnup \\
\hline Node & $(\mathbf{c m})$ & $(\mathbf{c m})$ & $\mathbf{( c m )}$ & $\left(\mathbf{f t}^{3} / \mathbf{l b m}\right)$ & (F) & (GWd/MTU) & (GWd/MTU) & (GWd/MTU) & (GWd/MTU) \\
\hline 1 (Top) & 17.4777 & 339.6336 & 357.1113 & 0.0238 & 1070.1 & 9.637 & 8.903 & 8.833 & 8.833 \\
\hline 2 & 20.0025 & 319.6311 & 339.6336 & 0.0237 & 1218.5 & 14.906 & 13.937 & 13.816 & 13.661 \\
\hline 3 & 20.0025 & 299.6286 & 319.6311 & 0.0236 & 1270.6 & 18.012 & 16.841 & 16.701 & 16.536 \\
\hline 4 & 20.0025 & 279.6261 & 299.6286 & 0.0234 & 1271.9 & 19.459 & 18.204 & 18.052 & 17.878 \\
\hline 5 & 20.0025 & 259.6236 & 279.6261 & 0.0233 & 1260.9 & 20.127 & 18.823 & 18.666 & 18.490 \\
\hline 6 & 20.0025 & 239.6211 & 259.6236 & 0.0231 & 1249.9 & 20.450 & 19.126 & 18.966 & 18.787 \\
\hline 7 & 20.0025 & 219.6186 & 239.6211 & 0.0230 & 1241.3 & 20.634 & 19.299 & 19.136 & 18.955 \\
\hline 8 & 20.0025 & 199.6161 & 219.6186 & 0.0229 & 1235.1 & 20.761 & 19.419 & 19.254 & 19.071 \\
\hline 9 & 20.0025 & 179.6136 & 199.6161 & 0.0227 & 1231.0 & 20.865 & 19.516 & 19.350 & 19.164 \\
\hline 10 & 20.0025 & 159.6111 & 179.6136 & 0.0226 & 1228.8 & 20.960 & 19.607 & 19.438 & 19.251 \\
\hline 11 & 20.0025 & 139.6086 & 159.6111 & 0.0225 & 1228.4 & 21.054 & 19.698 & 19.527 & 19.338 \\
\hline 12 & 20.0025 & 119.6061 & 139.6086 & 0.0223 & 1230.6 & 21.139 & 19.788 & 19.613 & 19.419 \\
\hline 13 & 20.0025 & 99.6036 & 119.6061 & 0.0222 & 1236.2 & 21.168 & 19.837 & 19.656 & 19.456 \\
\hline 14 & 20.0025 & 79.6011 & 99.6036 & 0.0221 & 1243.3 & 21.079 & 19.757 & 19.575 & 19.372 \\
\hline 15 & 20.0025 & 59.5986 & 79.6011 & 0.0220 & 1251.2 & 20.690 & 19.384 & 19.203 & 19.001 \\
\hline 16 & 20.0025 & 39.5961 & 59.5986 & 0.0218 & 1252.4 & 19.611 & 18.340 & 18.169 & 17.973 \\
\hline 17 & 20.0025 & 19.5936 & 39.5961 & 0.0217 & 1217.9 & 16.936 & 15.821 & 15.663 & 15.472 \\
\hline 18 (Bottom) & 19.5936 & 0.0000 & 19.5936 & 0.0216 & 1070.4 & 11.050 & 10.353 & 10.205 & 10.134 \\
\hline
\end{tabular}


TABLE A-5. Fuel Rod Data for Assembly NJ070G (Cont.)

Cycle 10, EFPD: 486.4, Boron (ppmB): 506

\begin{tabular}{|c|c|c|c|c|c|c|c|c|c|}
\hline & & \multicolumn{2}{|c|}{ Elevation } & Spec. & Fuel & Rod O1 & Rod O11 & Rod O12 & \multicolumn{2}{|c|}{ Rod O13 } \\
\hline Axial & Node Height & Bottom & Top & Vol. & T-Fuel & Burnup & Burnup & Burnup & Burnup \\
\hline Node & $(\mathbf{c m})$ & $(\mathbf{c m})$ & $(\mathbf{c m})$ & $\left(\mathrm{ft}^{3}\right.$ /bm) & $(\mathrm{F})$ & $($ GWd/MTU) & $($ GWd/MTU) & (GWd/MTU) & $($ GWd/MTU) \\
\hline 1 (Top) & 17.4777 & 339.6336 & 357.1113 & 0.0238 & 1069.6 & 11.528 & 10.658 & 10.575 & 10.571 \\
\hline 2 & 20.0025 & 319.6311 & 339.6336 & 0.0237 & 1205.0 & 17.652 & 16.493 & 16.355 & 16.189 \\
\hline 3 & 20.0025 & 299.6286 & 319.6311 & 0.0236 & 1239.8 & 21.100 & 19.738 & 19.575 & 19.392 \\
\hline 4 & 20.0025 & 279.6261 & 299.6286 & 0.0234 & 1237.0 & 22.619 & 21.175 & 20.998 & 20.805 \\
\hline 5 & 20.0025 & 259.6236 & 279.6261 & 0.0233 & 1225.3 & 23.274 & 21.784 & 21.603 & 21.407 \\
\hline 6 & 20.0025 & 239.6211 & 259.6236 & 0.0231 & 1214.3 & 23.568 & 22.061 & 21.877 & 21.678 \\
\hline 7 & 20.0025 & 219.6186 & 239.6211 & 0.0230 & 1206.2 & 23.731 & 22.216 & 22.029 & 21.828 \\
\hline 8 & 20.0025 & 199.6161 & 219.6186 & 0.0229 & 1200.6 & 23.847 & 22.327 & 22.138 & 21.935 \\
\hline 9 & 20.0025 & 179.6136 & 199.6161 & 0.0227 & 1197.0 & 23.950 & 22.425 & 22.234 & 22.028 \\
\hline 10 & 20.0025 & 159.6111 & 179.6136 & 0.0226 & 1194.9 & 24.053 & 22.525 & 22.331 & 22.123 \\
\hline 11 & 20.0025 & 139.6086 & 159.6111 & 0.0225 & 1194.6 & 24.164 & 22.633 & 22.437 & 22.227 \\
\hline 12 & 20.0025 & 119.6061 & 139.6086 & 0.0223 & 1196.9 & 24.276 & 22.751 & 22.550 & 22.335 \\
\hline 13 & 20.0025 & 99.6036 & 119.6061 & 0.0222 & 1202.5 & 24.343 & 22.839 & 22.632 & 22.408 \\
\hline 14 & 20.0025 & 79.6011 & 99.6036 & 0.0221 & 1210.1 & 24.305 & 22.809 & 22.599 & 22.372 \\
\hline 15 & 20.0025 & 59.5986 & 79.6011 & 0.0220 & 1219.0 & 23.966 & 22.479 & 22.271 & 22.044 \\
\hline 16 & 20.0025 & 39.5961 & 59.5986 & 0.0219 & 1221.9 & 22.878 & 21.419 & 21.220 & 21.001 \\
\hline 17 & 20.0025 & 19.5936 & 39.5961 & 0.0217 & 1196.5 & 19.951 & 18.653 & 18.468 & 18.257 \\
\hline $18($ Bottom) & 19.5936 & 0.0000 & 19.5936 & 0.0216 & 1070.4 & 13.159 & 12.348 & 12.173 & 12.084 \\
\hline
\end{tabular}

Cycle 10, EFPD: 556.3, Boron (ppmB): 298

\begin{tabular}{|c|c|c|c|c|c|c|c|c|c|}
\hline & & \multicolumn{2}{|c|}{ Elevation } & Spec. & Fuel & Rod 01 & Rod 011 & Rod 012 & Rod 013 \\
\hline Axial & Node Height & Bottom & Top & Vol. & T-Fuel & Burnup & Burnup & Burnup & Burnup \\
\hline Node & $(\mathrm{cm})$ & $(\mathrm{cm})$ & (cm) & $\left(\mathrm{ft}^{3} / \mathrm{lbm}\right)$ & ( $\mathrm{F})$ & (GWd/MTU) & (GWd/MTU) & (GWd/MTU) & (GWd/MTU) \\
\hline 1 (Top) & 17.4777 & 339.6336 & 357.1113 & 0.0238 & 1073.1 & 13.480 & 12.477 & 12.381 & 12.374 \\
\hline 2 & 20.0025 & 319.6311 & 339.6336 & $0 . \overline{0237}$ & 1189.3 & 20.413 & 19.087 & 18.930 & 18.750 \\
\hline 3 & 20.0025 & 299.6286 & 319.6311 & 0.0236 & 1218.7 & 24.166 & 22.631 & 22.446 & 22.246 \\
\hline 4 & 20.0025 & 279.6261 & 299.6286 & 0.0234 & 1211.1 & 25.728 & 24.116 & 23.917 & 23.705 \\
\hline 5 & 20.0025 & 259.6236 & 279.6261 & 0.0233 & 1196.3 & 26.355 & 24.703 & 24.499 & 24.285 \\
\hline 6 & 20.0025 & 239.6211 & 259.6236 & 0.0231 & 1184.4 & 26.618 & 24.953 & 24.746 & 24.528 \\
\hline 7 & 20.0025 & 219.6186 & 239.6211 & 0.0230 & 1176.3 & 26.759 & 25.090 & 24.881 & 24.660 \\
\hline 8 & 20.0025 & 199.6161 & 219.6186 & 0.0228 & 1170.9 & 26.867 & 25.195 & 24.983 & 24.760 \\
\hline 9 & 20.0025 & 179.6136 & 199.6161 & 0.0227 & 1167.4 & 26.970 & 25.295 & 25.081 & 24.856 \\
\hline 10 & 20.0025 & 159.6111 & 179.6136 & 0.0226 & 1165.1 & 27.082 & 25.403 & 25.187 & 24.959 \\
\hline 11 & 20.0025 & 139.6086 & 159.6111 & 0.0225 & 1164.0 & 27.209 & 25.528 & 25.309 & 25.078 \\
\hline 12 & 20.0025 & 119.6061 & 139.6086 & 0.0223 & 1165.2 & 27.345 & 25.671 & 25.447 & 25.210 \\
\hline 13 & 20.0025 & 99.6036 & 119.6061 & 0.0222 & 1169.9 & 27.446 & 25.796 & 25.564 & 25.318 \\
\hline 14 & 20.0025 & 79.6011 & 99.6036 & 0.0221 & 1177.4 & 27.460 & 25.815 & 25.580 & 25.331 \\
\hline 15 & 20.0025 & 59.5986 & 79.6011 & 0.0220 & 1188.3 & 27.181 & 25.541 & 25.306 & 25.057 \\
\hline 16 & 20.0025 & 39.5961 & 59.5986 & 0.0219 & 1196.8 & 26.111 & 24.491 & 24.266 & 24.025 \\
\hline 17 & 20.0025 & $19 . \overline{5936}$ & 39.5961 & 0.0217 & 1175.9 & 22.982 & 21.520 & 21.311 & 21.079 \\
\hline 18 (Bottom) & 19.5936 & 0.0000 & 19.5936 & 0.0216 & 1066.8 & 15.335 & 14.416 & 14.215 & 14.108 \\
\hline
\end{tabular}


TABLE A-5. Fuel Rod Data for Assembly NJ070G (Cont.)

Cycle 10, EFPD: 626.1, Boron (ppmB): 103

\begin{tabular}{|c|c|c|c|c|c|c|c|c|c|}
\hline & & \multicolumn{2}{|c|}{ Elevation } & Spec. & Fuel & Rod O1 & Rod O11 & Rod O12 & Rod O13 \\
\hline Axial & Node Height & Bottom & Top & Vol. & T-Fuel & Burnup & Burnup & Burnup & Burnup \\
\hline Node & $(\mathbf{c m})$ & $(\mathbf{c m})$ & $(\mathbf{c m})$ & $\left(\mathbf{f t}^{3} / / \mathbf{b m}\right)$ & $($ F) & $($ GWd/MTU) & $($ GWd/MTU) & (GWd/MTU) & (GWd/MTU) \\
\hline 1 (Top) & 17.4777 & 339.6336 & 357.1113 & 0.0238 & 1073.2 & 15.502 & 14.369 & 14.261 & 14.250 \\
\hline 2 & 20.0025 & 319.6311 & 339.6336 & 0.0237 & 1174.8 & 23.204 & 21.724 & 21.547 & 21.354 \\
\hline 3 & 20.0025 & 299.6286 & 319.6311 & 0.0235 & 1196.6 & 27.215 & 25.531 & 25.323 & 25.106 \\
\hline 4 & 20.0025 & 279.6261 & 299.6286 & 0.0234 & 1183.9 & 28.792 & 27.037 & 26.815 & 26.586 \\
\hline 5 & 20.0025 & 259.6236 & 279.6261 & 0.0232 & 1168.0 & 29.382 & 27.591 & 27.365 & 27.133 \\
\hline 6 & 20.0025 & 239.6211 & 259.6236 & 0.0231 & 1156.7 & 29.610 & 27.810 & 27.581 & 27.347 \\
\hline 7 & 20.0025 & 219.6186 & 239.6211 & 0.0230 & 1149.6 & 29.732 & 27.931 & 27.699 & 27.462 \\
\hline 8 & 20.0025 & 199.6161 & 219.6186 & 0.0228 & 1145.0 & 29.833 & 28.031 & 27.797 & 27.557 \\
\hline 9 & 20.0025 & 179.6136 & 199.6161 & 0.0227 & 1141.9 & 29.938 & 28.134 & 27.898 & 27.655 \\
\hline 10 & 20.0025 & 159.6111 & 179.6136 & 0.0226 & 1139.6 & 30.057 & 28.251 & 28.012 & 27.767 \\
\hline 11 & 20.0025 & 139.6086 & 159.6111 & 0.0225 & 1138.3 & 30.197 & 28.390 & 28.148 & 27.899 \\
\hline 12 & 20.0025 & 119.6061 & 139.6086 & 0.0223 & 1138.6 & 30.354 & 28.555 & 28.307 & 28.053 \\
\hline 13 & 20.0025 & 99.6036 & 119.6061 & 0.0222 & 1141.6 & 30.484 & 28.712 & 28.456 & 28.191 \\
\hline 14 & 20.0025 & 79.6011 & 99.6036 & 0.0221 & 1147.7 & 30.548 & 28.780 & 28.520 & 28.251 \\
\hline 15 & 20.0025 & 59.5986 & 79.6011 & 0.0220 & 1158.9 & 30.333 & 28.565 & 28.306 & 28.036 \\
\hline 16 & 20.0025 & 39.5961 & 59.5986 & 0.0219 & 1171.1 & 29.303 & 27.548 & 27.297 & 27.035 \\
\hline 17 & 20.0025 & 19.5936 & 39.5961 & 0.0217 & 1159.5 & 26.015 & 24.413 & 24.179 & 23.927 \\
\hline 18 (Bottom) & 19.5936 & 0.0000 & 19.5936 & 0.0216 & 1061.0 & 17.566 & 16.550 & 16.324 & 16.199 \\
\hline
\end{tabular}

Cycle 10, EFPD: 660.3 , Boron (ppmB): 1.8 (EOC10)

\begin{tabular}{|c|c|c|c|c|c|c|c|c|c|}
\hline & & \multicolumn{2}{|c|}{ Elevation } & Spec. & Fuel & Rod O1 & Rod O11 & Rod O12 & Rod O13 \\
\hline Axial & Node Height & Bottom & Top & Vol. & TrFuel & Burnup & Burnup & Burnup & Burnup \\
\hline Node & $(\mathbf{c m})$ & $\mathbf{( c m )}$ & $\mathbf{( c m )}$ & $\left(\mathbf{f t t}^{3} /\right.$ /bm) & (F) & (GWd/MTU) & (GWd/MTU) & (GWd/MTU) & (GWd/MTU) \\
\hline 1 (Top) & 17.4777 & 339.6336 & 357.1113 & 0.0238 & 1067.7 & 16.518 & 15.324 & 15.209 & 15.197 \\
\hline 2 & 20.0025 & 319.6311 & 339.6336 & 0.0237 & 1162.7 & 24.580 & 23.031 & 22.845 & 22.645 \\
\hline 3 & 20.0025 & 299.6286 & 319.6311 & 0.0235 & 1179.8 & 28.703 & 26.953 & 26.735 & 26.510 \\
\hline 4 & 20.0025 & 279.6261 & 299.6286 & 0.0234 & 1166.3 & 30.279 & 28.462 & 28.229 & 27.992 \\
\hline 5 & 20.0025 & 259.6236 & 279.6261 & 0.0232 & 1151.7 & 30.848 & 28.997 & 28.760 & 28.521 \\
\hline 6 & 20.0025 & 239.6211 & 259.6236 & 0.0231 & 1141.9 & 31.059 & 29.201 & 28.961 & 28.719 \\
\hline 7 & 20.0025 & 219.6186 & 239.6211 & 0.0230 & 1136.0 & 31.172 & 29.314 & 29.072 & 28.827 \\
\hline 8 & 20.0025 & 199.6161 & 219.6186 & 0.0228 & 1132.6 & 31.271 & 29.412 & 29.168 & 28.920 \\
\hline 9 & 20.0025 & 179.6136 & 199.6161 & 0.0227 & 1130.3 & 31.377 & 29.518 & 29.271 & 29.020 \\
\hline 10 & 20.0025 & 159.6111 & 179.6136 & 0.0226 & 1128.6 & 31.500 & 29.639 & 29.389 & 29.136 \\
\hline 11 & 20.0025 & 139.6086 & 159.6111 & 0.0225 & 1127.5 & 31.645 & 29.784 & 29.531 & 29.274 \\
\hline 12 & 20.0025 & 119.6061 & 139.6086 & 0.0223 & 1128.1 & 31.810 & 29.958 & 29.699 & 29.436 \\
\hline 13 & 20.0025 & 99.6036 & 119.6061 & 0.0222 & 1131.4 & 31.953 & 30.130 & 29.862 & 29.588 \\
\hline 14 & 20.0025 & 79.6011 & 99.6036 & 0.0221 & 1137.4 & 32.039 & 30.220 & 29.948 & 29.669 \\
\hline 15 & 20.0025 & 59.5986 & 79.6011 & 0.0220 & 1148.1 & 31.855 & 30.035 & 29.763 & 29.484 \\
\hline 16 & 20.0025 & 39.5961 & 59.5986 & 0.0219 & 1160.9 & 30.849 & 29.038 & 28.775 & 28.504 \\
\hline 17 & 20.0025 & 19.5936 & 39.5961 & 0.0217 & 1153.3 & 27.498 & 25.838 & 25.592 & 25.331 \\
\hline 18 (Bottom) & 19.5936 & 0.0000 & 19.5936 & 0.0216 & 1059.6 & 18.678 & 17.620 & 17.380 & 17.247 \\
\hline
\end{tabular}


INTENTIONALLY LEFT BLANK 